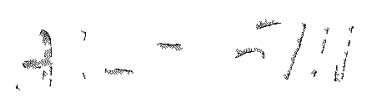

\title{
ANNOTED BIBLIOGRAPHY
}

\section{IN RADIOBIOLOGY}

\author{
Compiled by \\ Sophie V. Stephens \\ Argonne National Laboratory \\ and
}

Robert D. Boche

U. S. Air Force Radiation Laboratory, University of Chicago

and

Department of Physiology, University of Chicago

I 


\section{DISCLAIMER}

This report was prepared as an account of work sponsored by an agency of the United States Government. Neither the United States Government nor any agency Thereof, nor any of their employees, makes any warranty, express or implied, or assumes any legal liability or responsibility for the accuracy, completeness, or usefulness of any information, apparatus, product, or process disclosed, or represents that its use would not infringe privately owned rights. Reference herein to any specific commercial product, process, or service by trade name, trademark, manufacturer, or otherwise does not necessarily constitute or imply its endorsement, recommendation, or favoring by the United States Government or any agency thereof. The views and opinions of authors expressed herein do not necessarily state or reflect those of the United States Government or any agency thereof. 


\section{DISCLAIMER}

Portions of this document may be illegible in electronic image products. Images are produced from the best available original document. 


\section{TABLE OF CONTENTS}

Foreword

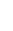

\section{III}

\section{GENERAL INFORMATION}

\section{EFTLCTS OF EXTERNAL RADIATION}

Time-Intensity studies

Lethal Dose Studies

Wavelength Studies

Deptli Dose Studies

Efferts of Beta Rays

Effects of Neutrons

Eifects of Pile and Isssion Radiations

Chronic Whole-Body Irradiation

X- and Gamma Regional Irradiation

Radrogensitivity

Comparative $\mathbb{E}$ fects

Pathology

Carcinogenesis

Effects on Immunity of Organisms

Injurious Effects of Radiation Therapy

Difiects on Blood

Hemopolesis - Humans

Hemoponesis - Animals

Clottng Mechansms - Humans

Clotting Mechanisms - Animals

Miscellaneous

Page

vii

1

10

10

14

21

24

25

28

34

34

37

45

55

83

73

81

87

92

95

100

111

112

116

III E'FECTS OF INTERNAL RADIATION AND METABLOISM AND TOXICOLOGY OF INTERNALLY DEPOSITED RADIO-ELEMENTS

General Dosage Studies

Arsenic

Barium-Ianthanum

118

123

124

Beryllium 124

Calchm 125

Carbon $\quad 126$

Cerium 128

Cobalt 128

Europium 129

Fission Products and Heavy Metals 129

Gallium 133

Gold 134

Iodine 135

Iron $\quad 138$

Lanthanum 138

Phosphorus 138

Plutonium
Polonium 140

Radium

Radon 155

Sodium 156

Stronthuma $\quad 156$

Tellurium 160

Thallium 161

Thorium 161

Tritium 162

Uranium 163

Tttrium 165

$\begin{array}{ll}\text { Zirconiumas } & 167\end{array}$

IV RADIATION SICKNESS 167

Syndrome in Humans - General $10 \%$

Syndrome in Humans - Miroshima and Nagasaki 170

Syndrome in Animals 175

Prophylasis and Therapy - General Reviews

Anoxia 185

Antiobiotics 186

Antihistamines 188

Cyanide Studies 190

Flavonoids 192

Hormones 194

Sulthydryl Compounds 200

Surgical Treatment 204

Vitamin studies $20 \%$

Mascelianeous Treatment 210

V MECHMNISM OF RADATION EFFECTS

General Theoretical Considerations

General Blochemical Studies

Studies on Nucleoprotelns and Nuclele Acids 237

Enzyme Studies

$\mathrm{H}_{2} \mathrm{O}_{2}$ Problem

Low Temperature Studies 251

Radiation Chemistry of Aqueous Solutions 25s

VI GROWTI AND DEVELOPMENT

VII GENTIICS 276

VII CYTOLOGY

Author Index 344

Numerical Index

Source of Abstracts Index 360 


\section{FOREWORD}

Biological scientists, in considerable numbers, continue to be drawn into the field of radiobiology, either as a full-time vocation or as an adjunct to other brological activities. And more and more graduate students are preparing for careers in which knowledge of the biological effects of radiation is of the utmost importance.

This annotated bibliography was initially conceived as a reading guide for those seeking orientation in a complex and rapidly growing field of scientific interest. It still will serve this original purpose, but its scope has been broadened to increase its usefulness as a work of reference, so that it may find a place on the shelves of those who are already established in the field which it repregents.

It will be noted that the volume omits consideration of tracer studies on topics not directly concerned with the biological effects of radiation. In the opinion of the editors, this is a desirable limitation, but opinion to the contrary will be given due consideration.

The preparation of this material was sponsored jointly by the Argonne National Laboratory of the Atomic Energy Commission and the U. S. Air Force Radiation Laboratory, University of Chicago. The compilation was made by Sophie V. Stephens, Argonne National Laboratory, and Robert D. Boche, U. S. Air Force Radiation Laboratory, University of Chicago. Edith Goldfarb and Valegka Evertsbusch assisted materially in the collection of abstracts. Margaret Fieldhouse helped to put the compilation in final form. Grateful acknowledgment is made to the sources of the abstracts, as noted in each instance.

\section{Qustan the Brwen \\ Austin M. Brues, M. D., Director}

Division of Biological and Medical Research

Argonne National Laboratory

Lemont, Illinois

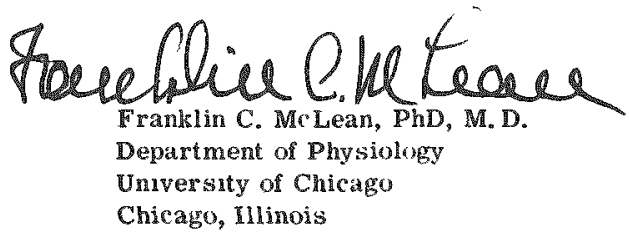




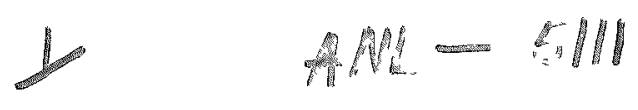

\section{REFERENCES}

Behrens, C. E. ed. ATOMIC MEDICINE. Thomas Nelson and Sons, New York. 1949.

This collection of 21 artictes ancludes a few general discussions of nuclear theory, but most of the material relates to brological and medical application of nuclear research. Thus, among the lopies treated are radiobiolcgy and patholugy, the hematology of irradiation, raduation illness, the defection and measurement of radiations, atomic disaster planning, tracer methods in biology, radiophosphorus and radionodine, radioistopes of medical interest, radiation effects in dentistry, and research in atomic medicne. Symbols, units, and defintions pertainmg to the subject are given in a three-page appendix. A 21 patge table of 1sotopes and their hall-lives and emissions is also gaven. (NSA)

Blist, R. W. WHAT YOU SHOULD KNOW ABOUT THE ATOMIC BOMB. A MESSAGE FROM THE SURGEON GENERAL. Army Medical Department, Washington, D.C. Revised 1950.

This pamphlet contans the follown serass of artucles based on lectures delwered durng a course in atomic medicune sponsored by the Armed Forces Special Weapons Project: I. Introduction to Nuclear Physics: 11. Biologic Efferts of Nuclear Radiation from an Atomic Explosion: 111. Medical Lffects of Atomic Explosion; IV. The Doctor's Problem in Atomic Warfare; V. The Atomic Explosion; VI. Fundamentals of Radation Pathology: VII. Pathologic Anat omy of Radiation Lffects of Alome Esplosion; VIII. Detection of Overexposure to Ionizing Radiation; IX. Public Health Aspects of Atome Explosion; X. Psychological Factors in Atume Warfare; XI. Hissentials of Instrumentation; XII. Protection Against Atomic I3ombs. (NSA) 3

Bloom, William, «d. HISTOPATHOLOGY OF IRRADITION FROM EXTERNAL AND INTERNAL SOURCES. National Nuclear Energy Series, Division IV, Volume 22H, MeGraw-Hill Book Company, Ine., New York, 1948.

This volume was prepared by the Histological Group of the Mealth Division at the Unversity of Chrage. It covers a wide variety of experiments and gives detalled informathon on techniques and matteruals. Among the radioactive agents used in these experuments were, and, rays, fast and slow neutrons, and 8 -rays to establish a base line for comparison. The effects of sudum 24, carbon 14, yttrum 91, radium and many others were studied. The isotopes vere adminstered intramuscularly, intravenously, intracardlally, or intraperitoneally. The volume describes the histopathological and cytologral effect of radioative agents in the cells, skin. bone, bone marrow, spleen, lymph node and intestinal lymphatic tissue, the thymus, gastrointestinal trael, testis, ovary, kudney, lung, vascular system, adrenal, nervous system, etc. (NSA)

4

British Mission to Japan. THE $\mathbb{E}$ FFECTS OF THE ATOMIC BOMBS AT HIROSHMA AND NAGASAKI. [nd] $34 \mathrm{p} . \quad(\mathrm{NP}-1156)$
Report by the British Mission to Japan on Hiroshima and Nagasaki three months after the bombing of these two cities. Includes sections on the two cities before the attack; the lwo cilies after the attack; the action of the atomie bomb; blast effects; heat effets; radio-active effects; casualties; conclusions. (S.V.S.)

5 Brues, Austin M. ISOTOPES: RADIOACTIVE; TOXICITY, Volume 2, p.465-71 of MEDICAL PHYSICS, 0 . classer, ed. The Year Buok Publishers, Inc., Chicago. 1950.

Discussion nature of the hazards, chronic radiation toxicity, changes induced by isotopes, dosimetry and clinical use of isotopes. (H.D.B.)

6 Clark, George L. A SURVEY OF THE BIOLOGICAL EFTECTS OF X-RADIATION. Radiology 26, 295-312(1936).

A summary of the brological effects of radiation, to date, under the following headings: Bactericidal and general lethal effects: Biologic dosimeters; Bffects on the hereditary mater1al; Fifeets on normal cells; Hadiosensitiveness of cells; Recovery and the time factor; $X$-ray effects on human tissues; Stimulating effect of X-rays; Photochemical experiments with a possible bearing on biologic effects; Medical implications of cell radiosensitivity and reactions to radiation with special reference to cancer; and a new X-ray method of pathologic diagnosis. (S.V.S.) 7

Clark, Janet Howell. THE BOLOGCAL EFECTS OF FADIATON. Ann. Rev. Physiol. 1, 21-40(1939).

A review, chielly of work published between January 1936 and July 1938.94 references. (V.r.)

8

COpp, D. Harold. RESEARCR AND TRAINING FACRLITIES FOR RADIOBIOLOGT AND FOR USE OF ISOTOPES IN BIOLOGY AND MEDICINE IN THE UNITED STATES AND CANADA. National Research Council, Washington, D. C. 1950.

The book lists personnel and facilities for research, clinical use, training and health protection and lists isotopes employed in various projects. 151 pages. (R.D.B.) 9

Cronkite, Eugene P. THE DIAGNOSIS OIP IONIZING RADIATION INJURY BY PHYSICAL EXAMINATION AND CLINICAL IABORATORY PROCEDURES. NaVal Medical Research Institute. Apr.8, 1948. 15p. (NP-573; Report No. 8 ; NM-007-039)

The symptomatology and diagnosis of acute and chronic radiation illness is discussed. Characterishe symptoms of the acute illness are nausea, vomiting, diarrhea, hemorrhagic manifestations, agranulocytosis followed by nonpurulent necrotizing infections, and epilation. The blood picture is the most sensitive biologic index of exposure to ionizing radiation. Lymphocylopenia appears promptly after the single intense exposure. The symptomatology and blood picture of chronic exposure is protean. In order to evaluate the blood changes of chronic exposure, rigid con trol data must be establiahed. The most significant blood change following chronic exposure is a depression of the 
mean leukocyte count of the exposed groups below the conlrol groups. Dosimetry, the measurement of radiation intensities, must pemain the basis of all protective meas10 ures. (Author Summary)

Crontite, Eugene 1 and Ceorge Brecher. RADIOLOGY ANO RADIOACTIVTY IRRADIATION. NOV. 15, 1951. 25p. (Lecture and Review Series 51-11; NP-3796; : 1 -6317)

Biological effects of whole-body irsadiation are revieved. Topics covered include dose measurements and lethal dose, survival time and chinical sympomes, intestimal injury, bone marrow injuxy, anemia, hemorxhage, in fection, late effects, modification of response, and mechanics of protective action. A brief discussion of the medical aspects of atomic warfare is included. $165 \mathrm{ref}-$ erences. (NSA)

11.

Cronkite, Ẻugene P. and Williar H. Chapman. A CRITICAL ANALYSIS OF THE SYNDROME OY ACUTF TOTAL DODE RADLATON ILLNESS, ITS ROLE IN ATOMUC WARE ARE AND ITS INILUENCE ON THE FUTURE PRACTICE OF MILITARY MEDICINE. Mintary Surgeon 104, 7-21(1949).

This analysis includes the following material: There is a clinical description of acute lotal body yadiation illness based on bombing experiences at Ilitoshima and Nagasaki and on accumulated experimental laboratory data. The pathogenesis of radiation illness is discussed primarily from the standpoint of the initial injury of sensitive callular systems. The possible presence and influence of circulating loxins from injured lissues and the bowel is discussed. A program for the study and care of human radiation casualties under ideal conditions is outined, and a generd analysis of the therapeutic challenge that atomic warare presents $t 0$ military medicine is presented. It is concluded that investigation is urgently needed along many avenues lo improve our understanding of this illness: such as (a) Information on the infuence of diet and envirommen1al factors on survival after exposure to doses in the letha. range is needed; (b) Information concerning the relation5hip of the adaptation syndrome and the pituitary-adrenocortical mechaniam to radiation Hlness is desirable: $(\mathrm{c})$ The role of autosensitization and anaphylactoid phenomena in radiation illness mat be clarified. And in regard to defensive measures in the event of an atomic war, the most critical and practical needs today are these: (a) The development of an easily read dosimeter; (b) The continuation of basic and applied research along the lines discussed in this paper. (INSA)

12

CrOwther, J.A. THE BHOLOGICAL ACTION OF K-RATS. Brit. T. Radiol. 11, 132-45(1030).

The author deals with the possible modes of action of X- T2ys on single cella and unicellula" organisms, asmuming that the effect is due to some specific reactiun in the nucleus. Explanations fall into two classes, which he calls the "poison" hypothesis and the "larget" hypothesis, the firgt assuming the formation by photochemical action of some toxic substane within the cell; the second postulating that something happang to the coll whenever it is "hit" by a photon. Application of the wo theories to known biological reactors are diseussed. (BA)

13

Curlis, Howard J. THE BIOLOCICAL IDETCTS OF RADLITON. AnM. Rev. Physiol. 13, 41-56(1051).

Review with 142 references. (V.R.)
14

Curtis, Howard J. THE POLOCICAL EFECTS OR RADIATONS, Vulune 2, 1. 1-51 of ADVANCES IN BIOLOGICAL AND MEDCAL PHYSICS, John H. Lat Pence and Joseph G. Mamilton, eds. Academic Press, Ine., New York. 1951.

This review is a survey of the problems encountered on the wartume Metallurgical Project, the experimental methods developed, and the chief results attainad. It ancludes sections on (1) the physical properties of radiations: (2) methods of measurement; (3) comparative effectiveness of radiations: (4) and physiological effects. No attempt has been made to review the vast literature of the biological effects of radiations and only such references are made as seems necessary for a cohrent account of the general 15 nuolem. 38 geterences. (S.V.S.)

Dobson, R. Lowry and John H. Lawrence. PHYSIOLOGEAL IFIECTS OF RADTANT ENERGY. Ann. ROV. Physiol. 10, $479-500(1948)$.

This zeview covers the period from August 1946 to August 1947. An attempt has been made to bing out the highlights of recent developments in the field of radiation and to mention pertinent work which has been brought out in this period. Included are sections on the mechanism of action of radiations, neutrons, slow neutrons and fission fragments, protons, $\beta$ rays, $\gamma$ rays and $\mathrm{X}-\mathrm{rays}$; fifects on blood and hematopoietic tissue; immunity; carcinogenic effects; and effects of the atomic bomb. (S.V.S.)

16

Dowdy, Andrew M. MEDICAL AND BIOLOGICAL ASPECTS OF NUCLEAR ENERGY. Univ. of Rochenter. Aug. 13, 1947. (RAD-202)

In this review paper University of Rochester oxperments and results are described for the effects of chrons: and acute s-radiation and chronic neut in in Iadiation upor peripheral blood changes (red blood cell count, lymphocyte count, polymorphonuclear count and platelet count). mc1dence of leukemia, mortality, body werght changes, sterility in the male (aspermia, sperm reduction, sperm motility, and abnormal sperm form), genetic changes (mutations) and histognetic changes (chromosome abnormali ties). Dogs, rabbits, monkeys, rats, and frut fljes were the subject of experimentation. It was coneluded that genetic changes are the most sensitive indicators of $\mathrm{x}-\mathrm{s}$ adi ation, that chromosome damage is directy proportional to cotal number of roentgens received, and that alteration in æperm could be detected in cases of chronic radiation down lo dosage levels as low as $0.1 \mathrm{r} / \mathrm{day}$. Lymohocytes are the most sensitive to radiation, followed closely by polymor phonuclean and platelat; red blood cells a me more re git stant. Chronic $x$ - and neutron irradiation increases the incidence of leukemia; the life span of mmall animals is reduced in proportion to the number of roentgens of $x$ rediation gecerved. It appears that any amount of penetrating radiation is deterimental and that many of the reæwonses studied fall into the non-threshold group. (NSA) 17

DOWdy, Andrew H. TABULATION OF AVALABLED DATA RELATIVE TO RADITION BIOLOGY. Feb. 1, 1949. $48 \mathrm{p}$ (AECU-353: UCLA-22)

The greport tabulates data on the following subjects for several speciss including human, rats, micc, gunea pigs, dogs, fuit flics, and grasshoppers: histogenetic and genetic offect of radiations; ffect of igadiation upon gonads: meidenee of leukem: hematolomisal effects othe than leukemia; acute total body iradiotion of rats with $250 \mathrm{kVp}$ J-1ays; embryolugical effects of urradiation; neutron- 
roentgen ratios; mortality table for $x$-radiatuon; mortalitychronic $x$-radiation from $3 \mathrm{x}$ to $10 \mathrm{x} / \mathrm{day}$; longevity table for $\mathrm{x}$ - and $\mathrm{z}$-radiation; effect of radiation--human cases; effect of total body irraduation - human cases; comparison of radiation effect by total dose, 26 cases; technques employed for total irradiation; summary - polycythemic and leukemic patients living one year or more; chemical aspects of $\mathrm{x}$-radiation. The data tabulated in the several tables listed above is summarized. The estimated results to humans exposed to filtered 200-1,000 kvp s-rays are listed. The appendix includes discussions of the general background material concerning the effects of radiation on chromosomes, genetic changes in human sperm and eggs caused by single exposures of 300 r or less, and induced 18 mutation rates. (NVSA)

Draeger, R. H. and S. Warren. MEDICINE AT THE CROSSROADS. A REPORT BY THE MEDICAL CORPS OT THE NAVY ON THE STUDY OF ANMMAS USED IN THE BISINI TESTS. U. S. Naval Med. Bull. 47, 219-25(1247).

The biological experiments conducted durmg Operations Crossroads are outlined and then results summarized. Twenty-two scientists, 200 goats, 200 pugs, 5,000 rats, 120 mice, and 60 guinea pigs were transported to Bikini on U.S.S. Burleson, which served as the laboratory. In addition to the manmals, other biological material - insects, bacteria, seeds, sera, hormones, ete.-and inorganic substances guch as soil and drugs were included. Most of the animals were used in Test Able, where the atomic bomb was exploded above the ground. All were retrieved within two days. It was found that the majority of early deahts were due to blast injury, radiation effects being a lesser casue of death at this time and flash burns being minimal because of the furry conts of the anmals. The animals which were exposed to appreciable amounts of radiation weveloped the same signs and symptoms as were noted in the human survivors of Hiroshima and Nagasaki. The first sign was atrophy of lymphoid tissue; then appeared a hemorrhagic diathesis and severe enteritis, often with necrosis of mucosa and submucosa. Marked changes in the blood picture occurred and, some veeks later, partial depilation. The sequence of the pathologic changes is being studied by means of sections from the tissues of animals sacrificed at intervals after the exposure. Information obtained by placing swatches of different clotha on fir plywood confirmed the impression gained in Japan that white material protects against flash burns better than dark cloth. After Test Baker, in which the bomb was exploded under water, the test subjects could not be recovered for several days because of the intense residual radioactivity resulting from the flooding of the target ships with radioactive "rain". The animals thus received not only a more intense radiation but also a much longer exposure than those al Test Able. The surviving animals and the inert materials are now being studied at the Naval Medical Research Institute. (BA)

19

Duggar, Benjamin M., ed. BIOLOGICAL EFFECTS OF RADIATION. McGraw-Hill Book Company, Inc., New Yorka 1936.

This book constitutes a collective contribution from represenatives of the fields of physies, zoology, botany, etc. The material is carefully selected so that an adequate comprehensive view of the whole field may be obtained. Chapters included are: Measurement of $X$-Rays and Radium, by Lauriston S. Taylor; Ionization and its Bearing on the Biological Effects of Radiation, by G. Failla; Statistical Treatment of Biological Problems in Irradiation, by Lowell J. Reed; The Effect of Radiation on Proteins, by
Janet Howell Clark; Radiation and the Vitamins, by Charles E. Bills; The Effects of Ir radiation on Venoms, Toxins, Antibodies, and Related Substances, by S. C. Brooks; The Rffects of Radium and X-Rays on Embryonic Development, by Elmer G. Butler; Effects of X-Rays and Radium Upon Regeneration, by W. C. Curtig; The Biological ERfectiveness of $\mathrm{X}$-Ray Wave-Lengths, by Charles Packard; The Physiological Effects of Radiation upon Organ and Body Systems, by Stafford L. Warren; Biological rffects of Alpha Particles, by R. E. Zirkle; The Action of Radiations on Living Protoplasm, by L. V. Heilbrunn and Daniel Mazia; The effects of Radiation on Fungi, by Elizabeth $C$. Smith; fifects of X-Rays Upon Green Rlants, by Edna L. Johnson; The Effects of Radium Rays on Plants, by C. Stuart Gager; The Influence of Radiation on Plant Respiration and Fermentation, by Charles J. Lyon; Growth Movements in Relation to Radiation, by Earl S. Johnston; Chlorophyll and Chlorophyll Development in Relation to Radiation, by 0 . L. Inman, Paul Rothemund and C. I. Kettering; Radiation and Anthocyanin Rigments, by John M. Arthur; Effects of Radiation on Bacteria, by B. M. Duggar; The Effects of Radiation on Enzymes, by Harold A. Schomer; Radiation and the Study of Mutation in Animas, by Jack Schultz; Induced Chromosomal Alterations in Maize, by E. G. Anderson; and Biological Aspects of the Quantum Theory of Radiation Absorption in Tissues, by John W. Gower. (S.V.S.)

20

Edelmann, Abraham. RADIANT ENERGY. Ann. Rev. Physiol. 12. 27-46(1950).

The review deals chiefly with physiological effects of ionizing radiations. 172 references. (CA)

21

Edelmann, Abraham. AAAS SYMPOSIUM ON RADIOBIOLOGY. Nucleonics 3, 28-36(1951). Apr.

A summary is presented of 22 papers offered at the 117 th meeting of the American Association for the Advancement of Science. The subject matter covered in these papers includes the effects of ionizing radiation on plant growth and development, radiation-induced mutations, neutron irradiation of compounds, action of 8 rays on cellg and rodent testicles, effect of $\mathbb{1}^{131}$ on thyroid tissue spleen shielding, $\mathrm{C}^{14}$ metabolism, and radiation-sickness therapy. (NSA)

22

ELInger, Fedrick. THE BIOLOGIC FUNDAMENTALS OF RADIATION TWERAPY. Elsevier Pub. Co., New York. 1911.

Included are effects of roentgen rays and the gamma radiation of radium on vaxious parts of the body, i.e.s skin, central nervous system, eye, endocrine glands; direct and indirect effects of uitraviolet light; effects of corpuseular rays; action of visible light and infra-red says. (M.F.)

23

Evans, Robley D. REALTH PKXSICS: INSTRUMENTANON AND HAZARD EVALUATION, Volume If, Chapter 16, p.249m 74 of THE SCLENCE AND ENGINEERING OF NUCLEAR POWER, Clark Goodman, ed. Addison-Wesley Press, Inc., Cambridge, Mass. 1849.

The author presents a brief review of the function of the health physicist. A brief discusgion of the biological sfects of radiation follows. Ineluded in the discussion are sections on blood changes duse to irradiation, leukemia in radiologista, chronic radium poironing, lung cancer and radon inhalation, genetic effects, occupational exposure to radiation, and masimum permissible dosage-rate. A brief description is given of instruments for the measurement of gamma-ray ionization intensilies in mr/hr, for very small gamma-ray ionization intensities, and for personnel moni- 
toring (film-badge and pocket, condenser-type ionization chamber). Included is an evaluation of some of the commercially available instruments. (S.V.S.)

24

FaIla, G. IONIZATION AND ITS BEARING ON THE BIOLOGICAL EFFECTS OF RADIATION, Volume 1, p.87122 Of BIOLOGICAL EFISCTS OF RADIATION, B. M. Duggar, ed. McGraw-Mill Book Company, Inc., New York. 1936.

A discussion of the fundamental character of ionizat ton and its bearing on the biological effecta of radiation. Includes sections on (1) the distribution of ions in an ionized medium, (2) interaction of radiation and matter, (3) influence of the quality of radiation on the distribution of ions in living matter; (4) intensity of radiation and inverse square law; (5) influence of matter on the intensity of radiation; (6) secondary phenomena; (7) fillration; (8) essential factors for the correlation of ionization and biological effects; (9) effective intensity of radiation: (10) significance of Lonization measurements; (11) knowledge of ionization produced in irradiated organism all important; and (12) effective quantity of radiation. (S.V.S.)

25

Fint, Robert M., ed. BIOLOGICAL STUDIES WITH POLONUM, RADUM, AND PLUTONIUM. MCGraw-Hill Book Company, Inc., New York. 1950. (National Nuclear Energy Series, Division VI, Volume 3)

The general problem undertaken is a comparison of the biological effects of the three a particle emitters, polonium, plutonium, and radiun. Part I deals with the distribution and excretion of polonium, Part II discusses the distribution and exeretion of radium, and Part III deals with the comparative loxicities of polonium, plutonium, and radium. The book includes a critical survey of available literature concerning these elements. It also describes carefully controlled rat experiments designed to determine accurately the relative toxicity of single injections of radium, polonium, and plutonium over a very wide range of doses, and discusses further the probable correlations between the toxicity results and retention and distribution data. (NSA)

26

Forssberg Arne. SOME RECENT PINDINGS IN RADIOBIOLOGY. Strahlentherapie 81, 161-72(1950).

This is a review of some of the experimental work done recently on the fundamental phenomena of radiobiology. The dependence of mutation frequencies on the dose in the small-dose region, the chemical changes produced by radiations in the chromosomes, the part played by the solvent in a radio-reaction, the effects of very small doses, are gome of the subjects discusged. 18 references. (NSA)

27

Classer, O., I. I. Quimby, L. S. Taylor, and J. L. Weatherwax, eds. PHYSICAL FOUNDATIONS OF RADIOLOGY. Paul B. Hoeber, Inc., New York and London. 1250.

The Iirst chapters cover the basic concepts of radiation (fundamental concepts of matter, fundamentals of electricity and magnetism etc.), while the later chapters deal with principles of $x$-ray diagnostic procedure, measurement of $x-r a y$ and $\gamma$ doses, measurement of $x-$ ray and $\gamma$ 8 ray quality, and dosage in $x-r a y$ and $\gamma$ ray therapy. (M.F.)

Glasstone, Samuel, ed. THE WEAPONS. D. S. Government Printing Office, Whington, D. C. June 1950.

This books was prepared for the Department of Defense and the $\mathrm{ArC}$ by a board of editors under the direction of the Los Alamos Scientific Laboratory. It presents a technical summary of the regulls to be expected from the deto- nation of atomic weapons, with chapter descrabing an atomic explosion, the shock from air, underwater, and underground bursts; blast, raduation, and fure efferts; methods of protecting personnel and decontamination methods. One chapter is devoted to the effects on personnel and discusses the types of injuries, nuclear radiation effects, clinical syndrome of radiation sickness (acute radiation syndrome), the pathology of radiation sickness, residual radiation hazards, and genetir effects of raduation. (S.V.S.)

29

Goldsmith, H. H. BIBLIOGRAPHY ON RADIATION

PROTECTION. Nucleonies 4, 62-9(1949)

This biblography includes several hundred references under the following general headings: (1) revews, (2) general biological effects of radiations, (3) blood changes due to radiation, (4) radiation sickness, (5) atomic bomb effects, (6) tolerances doses and dusage measurements, (7) radiation exposure monitoring, (8) health protection measures, (9) dust monitoring and hazards, and (10) laboratory designs. (To appear as part of a report from the Committee on Nuclear Science of the National Research Council.) A more comprehensive bibliography on this subject is being compiled by the author for the United Nations as "An International Biblography on Atomic Energy. Vol. IIScientific Aspects". (NSA)

30

Gray, L. H. ELECTRONS, NEUTRONS, AND ALPHA PARTICLES, Chapter XV, p.491-560 of BIOPHYSICAL RPSEARCH ME'TODS, F.M. Uber, ed. Interscience Publishers, New York. 1950.

Ionizing radiations are diseussed with regard to: effects on living cells; health hazards; effects as mutagente and carcinogenic agents. Other subjects are physical characteristics of different ionizing radiations, sources, and measurement. (M.F.)

31

Gray, L. H. BIOLOGICAL ACTIONS OF IONIZING

RADIATONS, Volume 2, Chapter 8, p.240-305 of PROGRESS IN BIOPHYSICS AND BIOPHYSICAL CHEMISTRY, J. A. V. Butler and J. T. Randall, eds. Academic Press, New York. 1951.

Whole body exposure to radiation damages the haemopoietie and lymphoid tissues. Anoxia reduces the sensitivity of the shin whereas increased osygen durung irradiation increases damage. Protection is also afforded by cysteine and cyanide. Tissues most easily damaged by radiation have in common constant proliferation of germnative daughter cells. Probably metabolism plays an essential role in injury to structural components of the mucleus; damage may result from diffusion of toxic products of an irsadiated cytoplasm into the nuclens. InhIbition of mitosis is an immediate effect of even low levels of radiation. The visible physical changes nay be related to changes in the DNA molecule. Reduction is the rate of synthesis of the purines of DNA has been established. Radiobiology has been greatly influenced by the progress made in the study of reactions induced in aqueous solutions by ionizing radiations, which the author has summarized. (M.F.)

32

Menshaw, Paul S. RADIATION AND THE CELL. I. Nat. Cancer Inst. 1, 277-90(1940).

This paper deals briefly with the nature of matter in general and how radiation acts to modify 11 . The author discusses the action of high energy radiation on living matter and the kinds of radiobiologic responses. The following types of responses are postulated: Type I. SingleEvent Action; Type 11. Multiple-Event Action; Effect Mani- 
fest in Holistic lashion; Type III. Multiple-Fent Action; 33 Effect Manifest by Degree (S.V.S.)

Hueper, W. C. OCCUPATIONAL TUMORS AND ALLIED DISEASES. Charles C. Thomas, Springrield, Hlinois, 1342 .

Ths section is devoted to a general review of occupational roentgen and radum-cancers of the skin. Included are discussions on the geographical distrubution and incidence, age distribution, multiplicity, occupational duslribution, sex distribution, exposure time, symptomatology, histology, susceptiblity, prognosis, experimental roentgen and radiun cancer, causatuve mechanism, therapy, preventive, protective, and sanitary aspects, and medico-legal aspects. A representative bibliography is appended. (S.V.S.)

34

Jacobson, Leon O., R. S. Stone, and J. Garrott Allen. PHYSICIANS IN AN ATOMIC WAR. J. Am. Med. Astive. 139, $138-40(1949)$.

Authors discuss the role of the physicians in the event of an atomic war and cite facts which permit formulation of certain broad prineiples to be followed if large cities are bombed; also discuss research work in progress on biologic effects of radiation. (S.V.S.)

35

Tamen, M. D. HADIOACTIVE TRACERS IN BIOLOGY. 2nd Ed. 429p. Academîc Press, Inc., New York, 1951.

The second edition as the first (1948) was wrilten to serve as an introduction to tracer methodology in general and as a gunde book for those, particularly in biological fields, employing such technics. The first chapters provide an introduction to the properties of atomic nuclei and nuclear reartions, the nature of tracer radiation, methods of measurement of radioactivity, and tracer methodology in biochemstry, physiology, and medicine. Subsequent chapters deal with particular radioactive isotopes such as $\mathrm{H}^{3}, \mathrm{C}^{11}, \mathrm{C}^{14}, \mathrm{P}^{32}, \mathrm{~S}^{35}$, etc. The general plan of the first edition has been retained, although important revisions and enlargements have been made, chief among which is the addition of a chapter on radiation hazards. New appendices have been included, these being, a descruption of radioactivity units, a listing of worlsing laboratory rules when using tracers, a listing of useful radioactive nuclides, and a pertinent bibliography. (BA)

36

Lavedan, Jacques. THE EFPECT OF RADIATIONS ON THE NORMAL CELL. Gauthier-Villars, Paris, 1945.

The book gives a quick survey of the influence of radiation on the normal cell. The main emphasis 18 on the ef fect of ionizing radiation ( $\mathrm{x}$-rays, $\alpha, \beta$ and $\gamma$ rays) rather than on the effect of ultraviolet radiation. A short introduction gives the physical aspects and the following chapters discuss the biological effects under the litles: Action of radiation on the cytological structure of the cell; the radiosensilivity of the cell and the factors which modify it: effecta of radiation on the biological function of the cell. The physical inferpretation of the radiation sensitivity is given under the chapter headings: The inactivation curve and its interpretation; the influence of different factors like wave-length, lime, latent periods, etc.; radiomutalions, the primary physical effect, etc. An addendum discusses recent concept of the mechanism of radiation action. Very little of the literature which appeared during the last 10 yeara is diseussed. (BA)

37

Lawrence, John H. THE NEW NUCLEAR PHYSICS AND MEDICIN (THE CALDWELL LECTURE OF THE AMER-
ICAN ROLNTGEN RAY SOCIETY). Am. J. Roentgenol. Radium Therapy 48, 283-301(1942).

This paper presents no new material but is an excellent summary of the subject to date. After a historical introduction lsawrence esplains in detail the use of radiosctive tracers and radioassay of biological material, amply illustrated by accounts of various experiments. Possibilities of clinical usage of the radioisotopes and of neutrons are emphasized by statements of results already obtained in certain types of disease. A well-selected bibliography is appended. (BA)

38

Lawrence, John h. and Joseph G. Hamilton. ADVANCES IN BIOLOGICAL AND MEDICAL PHWSICS. Academic Press, Inc., New York. 1048.

The topics discussed are as follows: heavy and radioactive isolopes in clinical and experimental medicine; nim trogen and carbon isotopes, their application in viwo to the study of the animal organism; the nature and production of artificial radioactivity; fundamentals of radioactivity and its instrumentation; health physics instrumentation and radiation proteclion; the use of radioactive isotopes in the study of iron and hemoglobin metabolism and the physiology of the eryhrocyte; radioactive phosphorus, its application to the study of phospholipid metabolism; iodine metabolism; the effects of the atomir bomb ingradiation on the Japanese; and nucleic acid metabolism. (NSA)

39

LEA, D. $1:$ ACTHONS OF RADIATIONS ON LIVHNG CELIS. The Macmillan Company, New Tork. 104\%.

Data on biologic effects which have been produced by $\mathrm{X}$-rays, $\gamma$-rays, electrons, neutrons and, to a much lesser extent, by u. $-\mathrm{v}$. rays, and which have been investigated quantitatively, are assembled and correlated in an important effort to achieve an acceptable meehanism for their production. About $1 / 3$ of the volume is devoted to a presentation of physical and chemical factors, ineluding extensive tabular and graphical data concerning the dissipation of energy by high speed parlicles and X-rays. Much of this information was previously lacking or inaceessiole. Organisms which have been studied quantitatively are limited so that the discussion centers primarily around effects on Drosophila, maize, Tradescanta, viruses, bacteria, and Arbacia. Genetic and lethal झtudies on Drosophila, and chromosomal alterations in D rosophida and Tradescantia are exhaustively treated, and occupy nearly half of the book. There is also a chapter each on viruses, delayed cell division (mostly in Arbacia eggs), lethal effects (in bacteria, Drosophila, Tradescantia), and on the "farget" hypothesis or "hil" theory of radiation effects. The "target" hypothesis is considered valid for ezplaining various genic and chromosomal effects of high energy particles and quanta. (BA)

40

Martin, J. R. RADHTION-ITS BIOLOGICAL EFFECWS AND PROBLIRS. Mech. Eng. 71, 823-98(1840).

The biological effects of radiation are reviewed. Tolerance concentrations of radioactive isotopes in air to produce an average tolerance dose to the affected ongan at the end of 1 year are in the order of a millionth of a $y$ per cC of inspired air for centain emitters. For $\beta$-emiliers of long half-life, the amounts which must be detected are minute, ranging from a thousandth to a hundred thousandth of a $\gamma$. Ionization chambers and Geiger-Muller Lubes are capable of measuring these very minute quantities. The protective measures for afe handling are gimilar to those used in industries handingg toxic chemicals. (CA) 
4

McDonnel, G. M. MEDICAL ASPECTS OF THE ATOMIC BOMB. Ordnance 34, 300-10(1950).

Alomic casualties, which are produced as a result of blast, heat, and ionizing radiation, are discussed. The relative value of these agents determined by a scale of biolofical significance will vary primarily with the type of detonation, namely, in the air, on or near the ground, and under water. With an air detonation, the ranges of the blast and thermal effects are distributed over the greatest possible area. In the ground detonation the ranges of blast, heat, and ionizing radiation are less than the ail - detonation values. The heat and initial ionizing radiation produced as a result of an underwater detonation are largely contained within the surrounding water. It is stated that the danger 42. from radiation has been highly overrated. (NSA)

Mitchell, J. \$. APPLICATIONS OF RECENT ADVANCES IN NUCLEAR PHYSICS TO CANCER RESEARCH. Brit. J. Cancer 1, 1-14(1947).

This review surnmarizes information on the following subjects: (1) Properties and production of isotopes of interest, mainly radioactive, with Tables. (2) Isotopic tracer research, with precautions necessary, including avoidance of risk of carcinogenesis in possible clinical applications. (3) Artificial radioactive sources for use in radiotherapy made by means of the pile. The most promising substitute for radium appears to be radio-cobalt, $\mathrm{Co}^{60}$, of half-life 5.3 years. Unseparated fission products should be investigated as an alternative. (4) Therapeutic possibilities of selectively concentrated radioactive agents, including the present position with regard to therapeutic applications of radio-phosphorus and the radio-iodines. (5) Therapeutic application of high energy $(30 \mathrm{Mev}) \gamma$ radiation and the development of the synchrotron. (Authors' Summary)

43

Morgan, K. Z. MAXIMUM PERMSSIBLE LEVELS OF EXPOSURE AGREED ON AT CHALK IIVER CONS ERRNCE. Am. Ind. Hyg. Assoc. Quart. 11, 116(1950).

In September, 1949, representatives of the U. S., Great Britain, and Canada proposed maximum permissible tissue dose limits for ionizing radiation at any point within the body of 0.3 roentgen equivalents physical/week for $\mathrm{x}$-and $\gamma$-rays, 0.3 for $\beta-, 0.03$ for protons, 0.015 for $\alpha$-particles, 0.03 for fast neutrons, and 0.06 for thermal neutrons. Data for exposure of the entire body and of hand only are also cited. (CA)

National Security Resources Board. MEDICAL ASPECTS OF ATOMIC WEAPONS. U.S. Government printing Office, Washington, D. C. 1950.

This is one of a series of reports which assess, in general terms, what is known about the results of an atomic explosion, what damage and injuries a bomb can cause, and what can feasibly be done to protect people and structures. This first report deals particularly with the effects of an atomic bomb explosion upon people within its range, the medical and biological aspects of injuries and their treatment. A general discussion is given of (a) the type of injuries caused by an atomic explosion, and the extent to which they occurred in Japan at various distances from ground zero, $(b)$ the nature of the radiation hazard, the ef fects of exposure, and some of the treatments for acute radiation sickness, and (c) the nature of the hazard from radiological contamination - not encountered in Japan - and some of the precautions that can be taken against it. (S.V.S.)
45

Nims, Leslie f. RADIATION. Ann. Rev. Physiol. 11, $527-44(1949)$.

This review covers the period from duly 1947 to July 1948. The author covers the fields of (1) the mechanism of action of ionizing radiation on living cells and materials; (2) whole-body radiation; (3) blood and hemopoiesis; (4) skin; (5) other tissues; (6) internal radiations. No altempt has been made to cover the whole lield of radiobiology in this review - genetic effects are not included. Bibliography of 156 references appended. (S.V.S.) 46

Rajewsky, B., A. Schxaub, G. Kahlau, W. Dreblow, A. Krebs, and H. Schaefer. DAMAGES BY RADLTION, in IIAT Review of German Science, 1939-1946: Biophysîcs, Part I, p. 123-75. Office of Military Government for Germany, Field Information Agrencies Technical, 1948.

Radiation injuries are discussed under the following headings: (1) Lethal Dosage Curves; (2) Production of Cancer by Radiation, Especially as Observed in the Radium Mines at Schneeberg; (3) Changes in Blood Produced by Radiation; (4) Radium Poisoning and Its Physical Diagnosis; (5) Measurement of the Content and of the Distribution of Radioactive Substances in the Body; (6) Radiography and the Counting of Single Rarticle Tracks as Used in RadioT biology. 90 references. (NSA)

Robinson, Radeliffe F., Michael D. Phillips, and Margaret G. Nagelsen. A BIBLIOCRAPHY ON "THE EFTECTS OR X-RAY ON BACTERLA" (I896 - August 1951). Battelle Memorial Inst. Aug. 20, 1951. 18p. (NP-3610)

A list of 178 tilles on the effects of $x$-radiation on bacteria was compiled from abstract journals and indexes. published articles from 1896 through August 1951 are included. (NSA)

48

Rossi, Harald H. THF BLOLOGICAL SIGNIHICANCE OF THE TISSUE DOSE OF IONIZING RADIATIONS. Trans. New York Acad. Sci. 2, 270-5(1949).

A general discussion of ionizing radiations is given, with special reference to the problem of a quantitative measurement of radiation and the question as to how far the result of such measurements serves to define the conditions under which a radiobiological experiment is performed. Spatial and temporal considerations of dosimetry in connection with ionization and induced electromagnetic fields are qualitatively discussed; the author suggests the further collaboration of physicists with biologists for more exact dosimetry measurementz and for discussion of the comples theoretical problems which arise in treatment of biological material. (NSA)

49

Smyth, PURPOSES. U. S. Government Printing Office, Washington, D. C. 1945.

The purpose of this report is to describe the scientific and technical development in this country since 1940 directed toward the military usses of energy from atomic nuclei. It includes a statement of the chain-reaction problem; administrative history of the uranium project beginning with the inatial approaches of a group of scientists to the government and ineluding the administrative history to 1945; the Metallurgical Project at Chicago in 1942; the plutonium production problem; a general discussion of the separation of isotopes; gaseous diffusion and electromagnetic separation of uranium isotopes. The author states in his preface that this book is neither a documental official 
history nor a technical treatise for experts but rather is a matter-of-fact, general account of work in the United States since 1939 aimed at the production of atomic bornbs. As such at represents the official report on the development of the atomic bomb under the auspices of the United 50 States Government, 1940-1945. (S.V.S.)

Sparrow, A. H. and B. A. Rubin. EFFECTS OF RADIATIONS ON BIOLOGICAL SYSTEMS. Feb. 15, 1951. $94 \mathrm{p}$. (ABCU-1207)

An extensive review covering types of radiation, radiation dosage units, radiation energy transfer mechanisms, the target theory, relation between chemical and biological effects of radiations, effects of radiation on microorganisms, cytological and genetic effects, carcinogenesis, physiological and embryological effects, and factors determuning radiation sensitivity is presented. The selected bibliography favors recent publications, especially reviews. (NSA)

51

Spear, F. G., ed. RADIOBIOLOGY: EXPERIMENTAL AND APPLIED. Brit. Med. Bull. 4, No. 1 (1946).

A comprehensive survey of the field of radiology by $F$. G. Spear, covers the following subjects: (1) Background theory; (2) Physical dose and biological response; (3) Hadio-chemistry; (4) Biological indrators; (5) Genetic effects of radiation; (6) Lethal and injurious actions of radiations; (7) Summary of effects on normal tissues; (8) Radiations and malignancy. In a comparative study of biological effects of ionizing radiations $\mathbb{L}_{\text {. }} \mathrm{H}$. Gray discusses: (1) Linear ion density, the distinguishing feature of an ionizing radiation, from the biological standpoint. (2) Ion densily produced by different ionizing particles. (3) The influence of ion density on radiochemical yield. (4) Ion density in relation to the inactivations of elementary biologic units. (5) The structural changes induced in chromosomes by different types of ionizing radiation. (6) Comparative studies with other biologic material (Drosophila eggs, root-tips, of Vicia faba, animal embryonic tissue, tumor tissue. (7) Neulron therapy. The topics of genetic effects of radiation discussed by D.G. Catcheside are: (1) Stability of chromosomes and genes. (2) Radiation induced mutation in Drosophila melanogaster. (3) Induced chromosome aberrations. Inactivation and other efferts of radiation on viruses and bacteria are discussed by D. . Lea. G. J. Mary discussed the "ionization method" of radiation measurement and other forms of measurement. (TIS)

52

Spear, . G., ed. CERTAIN ASPECTS OF THE ACTION OF RAOIATION ON LIVING CELLS. Brit. J. Radiol.g Suppl. No. 1, 1947.

This is a report of the London Conference held May 13-14, 1946 on certain aspects of the action of radiation on living cells. The following papers are included: (1) The Distribution of the Ions Resulting from the Irradiation of Living Cells, by L. H. Gray; (2) The Nature of Viruses and the Irradiation of Plant Viruses, by Roy Markham; (3) The Irradiation of Viruses, by P. Bonet-Maury; (4) The Effect of Radiation on Vaccinia Virus, and on Bacteriophages, by M. I. Salaman; (5) The Inactivation of Viruses, in Relation to the Mechanism of Biological Actions of Radiation, by D. E. Lea; (6) Introductory Survey of Isperimental Data, by C. B. Allsopp; (7) Action of Radiation on Aqueous Solutions: Experimental Work with Enzymes in Solution, by Walter M. Dale; (8) The Radiochemistry of Aqueous Solutions: A Survey of Recent French Research, by M. Frilley; (9) Some Aspects of the Action of Radiations on Aqueous Solutions, by Joseph Weiss; (10) The Action of
Radiations on Dilute Aqueous Solutions: The Spatial Distribution of H and OH, by D. L. Lea; (11) Survey of Effects of Radiation on Chromosomes, by D. G. Catcheside; (12) The Induction of Chromosome Structural Changes by Radiation: Detailed Quantitative Interpretation, by D. L. Lea; (13) The Effect of Radiation on the Normal and Malignant Cell in Man, by P. C. Koller; (14) The Effects of Radiation on Reproductive Organs, by A. Glucksmann; (15) Genetic Effects of Radiations, by D. G. Catcheside; (18) Iffects on Early Embryonic Development of Ir radiation of Spermatozoa; (17) Effects of Radiation on Germ Cells: Dominant Lethals and Hereditary Partial Sterility, by D. E. Lea. 3 (S.V.S.)

Stone, Robert S. RADIATION INJURY. Radiology 46: 59-62(1946).

This paper is one of a combined report of the Standardization Commitfees of the American Roentgen Ray Society and the Radiological society of North America. The purpose of these reports is to acquaint recent users of $x$-rays and radium with the actual hazards involved. This report is necessarily a very generalized one. It describes the types of injuries which may result from overexposure to "radiation": injuries from acute local overexposure; injuries caused by repeated or chronic local overexposure; injuries from local irradiation of hair-bearing areas; injurious effects on the testicles and ovaries; radiation effects from radiation to the entipe body or large portions of the body om effects on the blood, lymphatic tissues, bone, general symptoms such as nausea, vomiting, etc. In conclusion the author points out that all of the above conditions are preventable. (S.V.S.)

\section{4}

Stone, Robert S., ed. INDUSTRIAL MEDICINE ON THE PLUTONIUM PROJECT. MeGraw-Hill Book Company, Inc., New York. 1951. (National Nuclear Energy Series, Division IV, Volume 20)

This book is a comprehensive survey of the medical activities of the Manhatian Project. Ineluded are chapters on the medical services of the Plutonium Project, biological bases for permissible exposures, laboratory examanation of project personnel, hematological effect of ionizing radiations, biochemical studies, uranium excretion studies, distribution and excretion of plutonium, management and treatment of exposed personnel, industrial medical program at the Hanford Engineer Works, blood changes in human beings following total-body ir radiation, hematological studies on patients treated by total-body exposure to $\mathrm{X}$-rays, biometric analysis of blood variability, determination of radium in excreta, health protection at Ianford, tolerance to whole-body irradiation of patients with advanced cancer. (S.V.S.) 55

Uber, F. M., ed. BIOPHYSICAL RESEARCH METHODS. Interscience Publications, Inc. N New York. 1950.

This book contains chapters on various topics in biophysics by experts in the field, including the following: Xrays and $\mathrm{X}$-irradiation, J.W. Gowen; Electrons, Neutrons, and $\alpha$ Particles, L. H. Gray; Stable Isotopes as Tracers, 50 F. M. Uber; and Radioactive rracers, A. F. Voigt. (NSA)

United Nations Atomic Energy Commission Group. ISOTOPES IN BIOLOGY AND MEDICINE, PART IV, of the BIOLOGICAL AND MEDICAL EFECTS OF HIGI IENERCY RADIATION in AN INTERNA TIONAL BIBLIOGRAPEY ON ATOMIC ENERGY, VOLUME 2, SCIENTIFIC ASPECTS. New York. 1951.

This document comprises Parts III and IV of "An lnternational Bibliography on Atomic Energy." References are 
listed under the followng headung in part III: Eflects of high energy radiations on chomelal commonds, on nacreorgannms, or cells, on genetres and mutations, on the growth and development of organisms, an organ systems, on physiology, and on botany and as $\mathrm{r}^{\circ}$ allure; nudreal

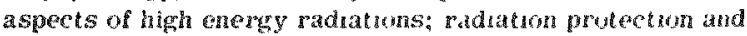
dosage measurement; and tedincal aspects. Part IV uncludes antroductory ssays on stable lsotopte traceses and

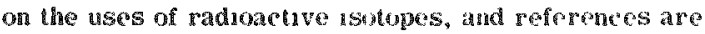
listed under the follownry headngs: Goneral articles on tracers; agroculture and ecology; physuloglcal chenstry; 57 physiology; medicine and toxroology; tochlonue. (NSA)

United States Atomic Hnergy Commisiun. ATOMIC FNERGI AND THE LIFE SCIFNCES. U. S. GOPErnment Printing Office, Washington, D. C. July 1949.

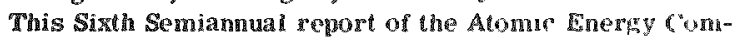
mission sums up briefly the majur developments in the

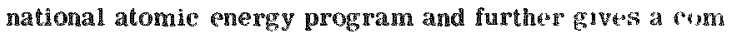
prehensive review of one of its major phases - the biological and medical activitues. This report outlunes the ACC program in all phases of medicing and biology. There is a section deseribing research on the chemistry of living things, the effects of radiation on living cells and tissue, organs of the body, and the body as whole. Another section describes research into the effects of radiation upon the mechanism of heredity in living things and upon the precesses involved in heredity. The results of analysis of bomb effects at Horoshima and Nagasaki and biological surveys at Bikini and Alamogordo two and throe years after atomic bomb tests there are discussed in this report. One section of the report is devoted to protective rneasures for personnel and includes a discussion of the handling of zadioactive wastes from atomic cnergy plants and other sources. Included lso are sections on the use of radioactive lracers for chemical studies; the use of radioisotope to diagnose and treat disease, outline of the AEC cancer program, and padiation and agriculture. (S.V.S.)

\section{8}

United States Atomic Energy Commission. CONTROL OR' RADUATON HAZARDS IN THE ATOML INERGI PROGRAM. U. S. Government Pxinting Office, Washington, D. C. July 1950.

This Eighth Semianumal Report of the Alomic Enorgy Commission revievs in some detail the control of radiation hazards in the atomic energy program. The report includes sections on radiation safety, the problems, the progsess, and the record (hazards of radiation, methods of radiation control, permigsible limits on exposure, the recond of radiological safety); radiation safety in production operations (processing saw and feed materials; protection in pile buildings, protection in chemical separetion plants, protection in platonium netal work, radiation control at Los Alamos, etc.); safe handling of tadioisotopes: control of radiation in sesearch and development (protection in laboratories, choice of building materials, protection against gamma rays, remote control enginer ing etc.); environmental safeguards (handling liquid wates, controlling gaseous wastes, etc.); control of hazards at weapons tegt; the ACC program for radialion control; and defection instruments for gadiation protection.$$
\text { (S.V.S.) }
$$

59

United States Atomic Energy Commission. TSOTOLPS--

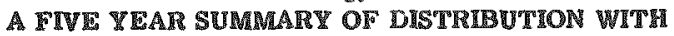
BIBLIOGRAPHY, U. S. Government Printing Ofice, Washington, D.C. 1951 .
An acecun of the sotope distribution propram. 'lables shuw what isutopes have been distribut fo whe th mvest 1 . getors, where, for what purpose, and where the results aro 60 published. 451 pp. 1402 referenges. (1R.D.H.)

U. S. Army Medic Doparinant. WHAT EVERY MEDTCAL OFTICER SHOULD KNOW ABOUT THE ATOMIC BOMB.

Bull. U. S. An my Med. Devt. 8, 350-6; 357-62; 422-8; 428-31(1948)

Lethal efferts will be produced by alr-burst atomit

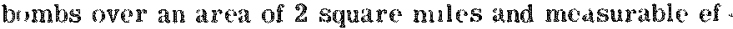
fect nver an aren of 7 square miles as a result of the

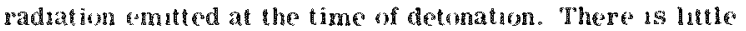
mpotane in the residual radiogetavity escept in the area

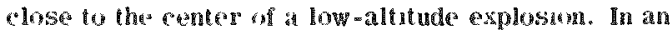
und - wato detmatton. padoact ive fission products and

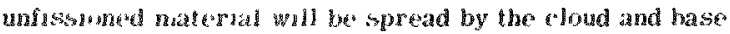

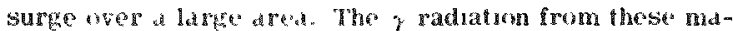
tewals whll be lethal to chosed personnel more than 2

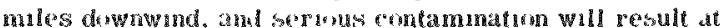
much grealer distande. A sasuos hazard will be provided

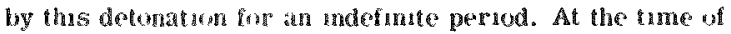
the detunation promut posive artion will permut the res duction of asuallues. The pathologrce effects of radation

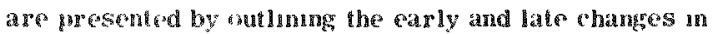
tissue cells, mrgan systems, total body ir radiation, and intarnal radation by radoactive materials introduced into the body either aceidentally or the rapeutically. The sensttivity of various body lissues has been cstablished and has been expressed largely as the relation of one thsur to another. A table is given which shows the relative radiom sensitivitues of various body llssues listed in decreasung order. The affects of overesposure may be acute or chronic. The exposurss may result from any type of gadi ation applied extermily or internally. The clinical preture will depend on the amount, rate of delivery and depth of the dose. The signs and symptoms surh as themal burns, diarshea, nausea, vomitig, headache, anur 1a, purpura, blood changes, fingernail brittleness, prgmentat an, etc., are discussed. A procedure to be follow.d in studymg a person suspected of being overexposed is given. (NSA) 61

University of Rochester. VALIDITY AND USERULNESS OF" CURRENTL ACCEPTED "TOLERANCE DOSE" FOR $X$ - and $\gamma$-Radiation. Indust rial Tolerance Conerence. ARCD-2832, 98p. 1945 .

The main purpose of this conferance was to consuder the validity and usc fulness of the currently accepted human lolerance dusages for $x$ - and $\gamma$-Fadialion, namely, 0.1 $r /$ day for an unlimited length of exposure. Papers were presented on the following subjects: chronic sperments correlating near-loletance dosages with survival, blond changer, aging, genetic mutatuons, and skin changes; the mechanism of radiation effects; and the human tolerance dose. (NSA)

62

Warren, Shiclds. EF ECTS OF RADIATON ON NORMAL HCSUL:S. Arch. Pahol. 34, 443,562,749, 917, 1070 , and $35,121,305,323,340(1942)$.

This review is limited to the ffects on normal anmal and human lissues of the apcatic wadion fom the more important sources - roentgen rays (produced at low as Well as high voltages), radium, yadon, neut lons, and cettain of the lemporatily rad lodet ve isolopes. Biblogra$63^{2}$ phuss an Gen at the end of each part. (S.V.S.)

Warren, Shields, "HHE' PISTOPATMOLOGX OF RADATION LESIONS. physiol. Revs. 24, 225-38(1944). 
Thus sinule of unformative revew, cheng only 35 refercuces, deals first with the the rapeutic types of radatuon, electro-maknetic, corpuseular (aneluduge the recently used radroactive 1sostopes), and neutrons. by way of ntroduction, it tells brefly how these raduation methods can be coututed

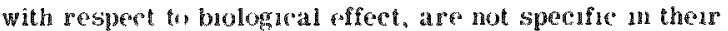
results, are never directly stimulatung but only destructive, fail to reach all of the rells of a tussue, atways reach some cells of adjacent tussues; how ceells duffer from one another in susteptibnlity to damage by irraduation, and differ withn themselves from one stage of a life history or a mitosis to ant her. Then it deserubes for normal tissues the apparent and real latent periods of injury, the types of visible change (mutotic, cytoplasmic, functuonal, vascular, untracellular): the order of sensitivity of different cells, with special ment ion of the germ plasm; the role of infection and trauma in radratsun damage. It diseusses padration mujury a a cause of neoplasms. Finally if gaves separate descruptans uf the radratun changes in examples of the

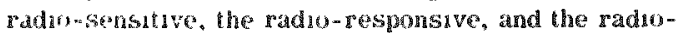
resistant tumor: and comments on curability an relation to 64 radere-sentolduty. (BA

Warren, Shields. PHYSTOLOGIC EFFLCTS OR NUCLEAR ENERGY. Occupational Med. $2,447-51$ 1946).

Ths is a general revew of the physuglogical effect of nuelear energy. The auther states that the whe blood cell count, and partucularly lymphocyte count is the nust sensitive andex for possible nnjury. He distusses briefly other thssues which are affected and trouches on the use of radioactuve 1 sutopes as therapeutur arents; brief discussion on use of radioactive phosphorus as a radrotherapeutic agent. (S.V.S.)

Warten, Shelds. THE PATHOGENESIS OF IRRADIATION SICKNESS. Chicago Med. Suc. Bull. 51: 957-9(1949).

The syndrome of radiafun suchness is discussed as it applies to mass radiatuon by atomac weapuns. The cells of the body which have a short life-span are more affected by the radiation that those with long life-spans, and rapudly divading cells, or those in mutusis, are more affected than cells in the restung phase. A doste of $400 \mathrm{r}$ is usually fatal 10 about $1 / 2$ of the human population present. There is great variabulty in the sqnsutuvity of different cells and organisms in general. It is beleved that the heaviest types of doses cause dasruption in the conzyme systems and vital functums of most of the body cells and dicath recurs prompty without much evidence of morphological change. Slides are presented which indrate specific and individual reartions to the radiation doses. These melude multuple leukopenc abscesses and cellulıt of the neck. The different stages of development of the symptoms are pounted out. If the radiation dose is more than $500-600 \mathrm{p}$, the chances of survival are small. Future problems connected with mass effective medical care of radiation victims are discussed. prevention rather than cure is suggested as the most promising method of handing the padation sickness problem. Leen w w wh slides. (NSA)

66

Warren, Shields and Austin M. Bruss. PROTECTION AGAINST RADIATION HAZARTS. Nucleonics 7: 70-6 (1950). Oct.

A general review of the principles on whoh are based present 1deas of radiation safety whth special reference to the pecularities arising from radioactive 1 sotopes an the body. (BA)
67

Warren, Staffurd L., Fred A. Bryan, and Robert J. Buettner. THE ATOM BOMB: HAZARDS INCDENT TO ITS USE. Ind. Med. 16: 276-8(1947).

This paper was pre'sented at a joint meeting of the American Association of Industral Physicians and Sur geons and the Amertcin Industral llygiene Association, Buffalo. New York, Anril 30, 1947. The author's discuss the physical aspects of the atomic lomb and projected control measures. A general discussion of the physiological aspects is presented. The four major causative factors under which all the pathological results may be classified - blast effects, thermal effects, effects of radiation, and psychological effects - are reviewed. The authors conclude that the physucian, and especially the ndustrial physician, had had a whole new set of problems placed before him with the use of atomic energy and its by-products of radioisotopes. (S.V.S.)

68

Van der Werf J.T. BIOLOGICAL REACTIONS CAUSTD BY ELECTRIC CURRENTS AND BY X-RAYS. EISEVIE Publishing Company, Amsterdam and London.

The main thesis of this book, which concerns itself with the experimental data accumulated by both radiologists and physiologists, is that in all living organisms parts are continuously being destroyed and then regenerated. A beam of radiation is thought to change the dissimilation coefficient and thereby the assimilation process. This premise is the basis of mathematical derivations which correlate the data from the two sources. The derived curves often fit the actual observations. (NSA) 69

Zirkle, Raymond E., et al. PLUTONIUM PROJECT SYMPOSIUM. Radiology $49,269-365$ (1947).

A study of the acute lethal action of slow neutrons established the $\mathrm{LD}_{50}$ as $4.2 \times 10^{12}$ slow neutrons per $\mathrm{cm}^{2}$ of mouse. Results of chronic radiation on mice, guinea pigs, and rabbits are reported. Carcinogenic action and aterility fefect dominate the picture of radiation injury. As far as genetic changes are concerned, the data indicate that the present permissible dose $(0.1 \mathrm{r} /$ day $)$ gives a sufficiently wide range of safety that a detectable increase in rate of gene mutation and translocations will not occur. The data also suggest that an intensity factor exists. No hematological change is detected in mice, rabbits, or guinea pigs with a daily whole-body exposure to $0.1 \mathrm{r}$ of $\gamma$ radiation extending over 3 years. Changes may be observed for doses above $1.1 \mathrm{x}$ per day with guinea pigs most sensitive, fol lowed by mice and rabbits. Muman beings subjected to whole-body $x-r a y$ exposure totaling $300 \mathrm{r}$ given in divided daily doses of 5 to $20 \mathrm{r}$ showed a degree of sensitivity of the hemopoietic system roughly compatible to that of the guinea pig and the dog. The clinical sequence of physiological effects of lonizing radiations is briefly reviewed. The effects of total surface $\beta$-irradiation in doses below the acute lethal range are shown to result in greater grosg and supericicial damage than that following ir radiation with the more penetrating $x$ and $\gamma-$ rays. The metabolism of fission products and the heaviest elements are reviewed. Histological changes following a great variety of radiation exposure are discussed. Experiments are reported that have been carried out with mice to determine the late ef fects of periodic and single exposures of fast neutrons, slow neutrons, $\gamma-$ rays, and $\beta$-rays (limited to the skin, from pile radiation. A general report is given on "Carcinogenic Properties of Radioactive rission Products and of Rlutonium". (TIS) 
70

Abele, Adolph. EXPERIMENTS ON FHYTHMIC IRRADIATION OF THE ECGS OF DROSOPHILA MELANOGASTER MEIG. Strahlentherapie 67: 293-306(1040).

A study was made of the rate of injury to eggs of Drosophila produced by ir radiation with the same lotal dose of x-rays when administered at different intensities $(95,47.6$, and $23.7 \mathrm{r}$ per min) and with both continuous and rhythmically inter rupted irradiation (intervals of $1 / 13^{1} 0^{1} / 2 \mathrm{me}$ sec). (CA) 70a

BIESS, A. A. THE BIOLOGICAL EFTECTS OF W-RAYS AS A FUNCTION OF INTENSITT. Proc. Nat. Acad. Sci. Wash. 30, 118-21(1944).

The seeds (wheat) were soaked in water for three hours, and then spread in trays over moist filter paper. After 12 hours the seeds were placed on plates and exposed to srays. After 48 hours of sprouting, the length of the stems was measured; this was used as the biological indicator. Graphs of results show that different intensities do not produce any marled differences. (PA) $70 \mathrm{~b}$

Brasch, Arno and Wolfgang Huber. ULTRASHORT APPLICATION TIME OF PENETRATING ELECTRONS. Science 105: 112-17(1947).

Many drugs, foodstufis, and cultures of microorganisms were exposed to penetrating electrons which were released during a time period of about $10^{-6}$ sec. The electronic intensity was about $30,000-50,000$ amps. To determine the over-all extent of side reactions, distilled and tap water were irradiated, and it was found that with dosages comparable to those causing complete gterilization, the $\mathrm{H}_{2} \mathrm{O}_{2}$ formation was less than $0.005 \%$.

The experiments were done at room temperature. At $-100^{\circ} \mathrm{C}$, the final $\mathrm{H}_{2} \mathrm{O}_{2} \%$ was only about $\%$ that formed at room temperature. This method in combination with ultrachort exposure times provided a mean to preserve many raw foodstufis, without changes in appearance, taste, or odor. Not only oxidation but also all other processes, except for very rapid reactions were suppressed. An apparatus called the "Capacitron," based on these principles, which has a high sterilizing capacity is discussed. This device carries sufficient intensities into the targets in such short times that most chemical reactions can be eliminated while the effective biologic impact is maintained. (BA) 7oc

Brunst, V. V. and E. A. Sheremetieva-Brunst. THF THME FACTOR IN LETHAL EFECTS OF TOTAL ROENTGEN IRRADIATION IN TRTON. Am. J. Roentgenol. Fadium Therapy 62, 550-4(1949).

The results of an investigation to determine variations in the effect on adult tritons (Triton cristatus, Amphibia, Urodels) with variations in the length of time used to distribute a definite total dosage of radiations are presented. The radiations were delivered by a 120-isv source with a filtration of $3 \mathrm{~mm} \mathrm{Al}_{\text {, }}$ and at variable target distance; in the irst series of experiments, all groups were given $3,000 \mathrm{r}$ total body irradiation, fractionated in daily doses of 150$3,000 \mathrm{r}$; in the second series all animals received a tolal of $2,000 \mathrm{r}$, delivered in daily doges of 100-2,000 $\mathrm{r}$. The results indicate that the lethal effect of total body ir radiation on the adult animals is not dependent on the distribution of the dosage in time; the triton apparently accumulates even small doses during a long period of protracted or fractionated treatments. 21 references. (NSA)
Casarett, George W. PLLOT EXPERIMENTS ON INDICATIONS OF RADIATION SICINESS AND COMPARISON OF LETHAC DOSES OF X-RAYS FOR RATS AND DOGS AT INTENSITIES OF 160r PER MOUR AND WHEN DELIVERED IN 24 HOURS. University of Rochester. Apr. 17, 1950. $88 \mathrm{p} . \quad(\mathrm{UR}-113)$

Pilot experiments were carried out to investigate correlations among factors including radiation sickness and mortality, and dose and intensity of $x$-radiation (total-bodyexposure). The source of radiation was a $1000-\mathrm{kv}$ peak generator, equipped with a lead parabolic filter, and having a. half value layer equivalent to $5.12 \mathrm{~mm}$ of lead. In a study of radiation sickness, single doses of $75,150,300$, and 550 r were administered to groups of 12 rats each; and single doses of $40,80,120$, and $200 \mathrm{r}$ were administered to grouns of 4 dogs each, at an approwimate rate of $160 \mathrm{r} / \mathrm{hr}$. Body weight determinatrons, food consumption measurement, and superficial physical paminations were made daily. There were no deaths among these animals. In rats, a dose of $500 \mathrm{r}$ produced a transient radiation sickness of moderate degree, and a dose of $300 \mathrm{r}$ produced a mild, transient radiation sickness. The effects of doses of $150 \mathrm{r}$ and $75 \mathrm{r}$ were sight and transient. In dogs, a dose of $200 \mathrm{r}$ produced only a mild, very transient reduction in food consumption, and lower doses resulted in no significant change with refa rd to measurements made. In a comparison of lethal effects of $x$-rays for rats and dogs, with intensities of 160 $\mathrm{r} / \mathrm{hr}$. and when the dose was delivered in 24 hours, single doses of $600,750,900$, and $1050 \mathrm{r}$ were administered to groups of 20 rats each al a rate of about $160 \mathrm{r} / \mathrm{hr}$., and single doses of $600,750,900,1050,1300$, and $1500 \mathrm{r}$ were administered to groups of 20 rats each at rates such that each dose was delivered in 24 hours. Single doses of 250 , 310,370 , and $430 \mathrm{~g}$ were administered to groups of 6 dogs each at a rate of about $160 \mathrm{r} / \mathrm{hr}$. Single doses of 300,400 , $500,600,750$, and $1000 \mathrm{r}$ were administered to groups of 6 dog each at rates such that each does was delivered in 24 hours. The data obtained, together with data from other sources, aggest that up to a certain point, decrease in intensity of a single dose of $\mathrm{x}-$ radiation may result in decreased mortallity to aome extent. The data suggest, also, that a dose high enough to produce severe signs of radiation sickness will also have associated with it considerable mortaility. Survival times, weights, clinical observations, and gross pathology observed at autopsy are described for these animals, as well as mortality. (NSA)

72

Forssberg, A. TRE TIME ICTOR IN BIOLOGICAL EFITCTS OF W-RAYS. II. INVESTIGATIONS OF ALGAE AND DROSOPUILA-PUPAE. Acta Radiol. 14, 399-407(1933).

In experiments on unicellular algae, 5,000 and $10,000 \mathrm{r}$ $x$ rays at a rate of 22 to $3,235 \mathrm{r} / \mathrm{min}$ were used, on Drosophila chrysalides $2,000 \mathrm{r}$ at a rate of 5 to $3,235 \mathrm{r} / \mathrm{min}$. The radiation effects on mortality and cell division retardation of the algae increased at first rapidly with increasing intensity until a maximum was reached at $1,620 \mathrm{r} / \mathrm{min}$. In Drosophila chrysalides, the limit of effect was reached with about $20 \mathrm{r} / \mathrm{min}$. The amount of effect produced by irradiations of from 22 to $3,235 \mathrm{r} / \mathrm{min}$ was more or less the same. (TIS)

73

Furth, F. W., M. P. Coulter, and J. W. Howland. EFFESS OF A SECOND LARGE DOSE OF X-RADIATION ON DOGS SURVIING ONE DOSE OF X-RADIATION, D.47-53 of QUARTERLY TECHNICAL REPORT; OCTOBER 1, 1950 THPOUCH DECTMBER 31, 1950. University of Rochester. Feb. 1, 1951. 7p. (UR-152 (p.47-53)) 
Nineteen dogs were given two dosages of $450 \mathrm{r}$ of $\mathrm{x}$ radiation with intervals of 5 to 12 months between them. Following the firgt dose of radiation, only $30 \%$ of the dogs showed any signs of radiation toxicity, and all dogg appeared normal at the time of the second radiation dose. The erythrocyte and platelet counts after the first dose of radiation returned to normal within 60 to 90 days, but the leukocyte counts returned to only $70 \%$ of normal preradiation value. After the second dose of radiation, all the dogs showed signs of severe radiation illnegs, and only one animal survived. The weight loss was greater after the second dosage of radiation. The rate of fall of erythrocyte, leukocyte, and platelet counts after the second irradiation was essentially the same as after the first. (NSA)

74

Gershon-Cohen, J., Harry Shay, and S. S. Fels. TXPERIMENTAL STUDIES WITH "CONTACT" ROENTGEN RAYS: THE TIME-INTENSITY FACTOR OF THE

"TUMOR DOSE" FOR RAT' SARCOMA 39 IN SITU. Am.

J. Reent gegenul. Radium The rapy 45, 600-4(1941).

Using a Chaoul contact $x$-ray apparatus operating at 60 kv with $0.12 \mathrm{~mm}$ Cu filter, $3 \mathrm{~cm}$ focal-skin distance, $450 \mathrm{r}$ per min, the lethal dose for rat sarcoma 39 in situ was $10,000 \mathrm{r}$ administered in a single dose, if the tumor was about 10-15 $\mathrm{mm}$ in diameter. This condition usually existed about 15-19 days after transplantation. Under these cir cumstances the fumor disappeared with survival of the animal. With divided doses the effect was less, and the decrease in effect was more marked as the lotal irradiation period became greater. (BA)

75

Glucksmann, A., K. Tansley, and C.W. Wilson. THE EFFECT OF VARIATIONS IN THE DOSE-RATE OF GAMMA RADIATION ON CELL DEGENERATION IN THE FROG TADPOLE. Brit. J. Radiol. 18, 158-64(1945).

Tadpoles were exposed to al dose of $336 \mathrm{r}$ of gamma radiation, given at 5 different dose-rates varying from 5 to 20 $\mathrm{r} / \mathrm{min}$. The effects, mreasured by the number of mitotic and degenerate cells present at different intervals after exposure in the brain and the eye, varied with the doserate as follows: (a) The maximal degeneration count increased with the dose rate. (b) The interval between irradiation and the time at which degeneration reached its maximum increased with the dose rate. (c) The total amount of degeneration was highest for a dose-rate of $15 \mathrm{r} / \mathrm{min}$. The results show that under the conditions of these experiments there is an optimal dose-rate. The experimental results are discussed in terms of the interac76 tion of the various biological processes involved. (auth)

Goldfeder, A. FURTHER STUDIES ON THE FFFETS OF IRRADIATION ON PROLIFERATION AND METABOL IC PROCESSES OF NORMAL AND MALIGNANT TISSUES. IV. EFIECTS PRODUCED BY DUF FERENT DOSAGE RATES OF X-RAY PADIATION ON THE PROLIFERATION OF VARIOUS TISSUES GROWN IN VITRO. Radiology 35 , 210-28(1940).

The tissues studied were kidneys and spleens of $1 / 2$ day old rats, mouse sarcoma 180 of $8-10-$ day growng tumours in the animal body, and 8-day-old chick embryo hearts. Freshly excised tissue fragments of $2.5 \mathrm{ng}$ were placed in a thin layer of nutritive medium, irradiated, washed in Tyrode solution, placed in a freshly prepared medium, and uncubated. The dosage rates employed were 234 and $936 \mathrm{r}$ per min. produced at $200 \mathrm{kv}, 20 \mathrm{ma}, 0.5 \mathrm{~mm}$ Cu plus $1 \mathrm{~mm}$ Al filter with a half-value layer of $0.85 \mathrm{~mm}$ of $\mathrm{Cu}$, the tissure target distance being 25 and $12.5 \mathrm{~cm}$, respectively, and $10,000 \mathrm{r}$ per min. produred by at contact therapy tube (cl. Braestrup, A., 1941, III, 46). The critical doses preventing proliferation rose from 80,000 to $140,000 \mathrm{a}$ for rat kidneys and the three dosage rates, from 130,000 to 260,000 $r$ for chick embryo heart using the two higher dosage rates, from 50,000 to 90,000 for sarcoma 180 at the same rates, and lay at $60,000 \mathrm{r}$ for rat spleen at $936 \mathrm{r}$ per min. whil st the first growth-inhibiting effectr were seen at $50,000-90,000,90,000-100,000,30,000-50,000$, and $40,000 \mathrm{r}$, respectively, representing a drop of $15-25 \%$ of proliferating explants against the controls. In addition to the decrease in per cent of growing explants within the range of critical dosege, a decrease in size of the new growth, a latent period of $48-72 \mathrm{hr}$, and liquefaction of 77 the nutrifive medium were also observed. (BCPA)

Heeren, I. G. THE EFFECT OF DISCONTWUOUS XIREADIATION ON RETICULOCYTE COUNTS IN TTE MOUSE. Strahlentherapie 77, 383-6(1048).

The course of the retieulocyte count in male white mice was used to compare the effects of continuous and discontinuous irradiation. In both cases the dose was 100 in nine minutes. The lowering of the reticulocyte value was less after discontinuous irradiation. The reticulocyte count fell from an average of 5.5 to $2.5 \%$ two to three days after continuous ir radiation. It had risen to 10.6 to $18.5 \%$ six days after and subsequently moved rhythmically between these values. However, after discontinuous irradiation, produced by a rotating lead shutter with an irradiation-interval relation of 1 to 9 , the fall was less than three days after, namely 3.6 to $4 \%$, and subsequent variations lay between 12.3 and $15.4 \%$. With 16 intervals per second the figures were 3.6 and 16.5 to $31.2 \%$. The author concludes that it is possible through rhythmical interruption of irradiation to regulate the irradiation reactions of tissue. (NSA)

78

Lanarque, Paul. STUDY OF RECOVRRY AITER IRRADIATION WTTH X RAYS. J. chim. phys. $48,252-5$ (1951).

Hatching-rate curves of repeatedly $\mathrm{x}$-irradiated eggs of the common silkworm (Bombyx mori) point to the existence of a recovery effects; the total dose of two irradiations, separated by a time interval, is larger than the dose of a single irradiation producing the same damaging effect. For a 7-hr interval the optimal recovery is observed when the ratio between the first partial dose and the total dose is equal to $\%$. Recovery processes are ascribed to metabolic activities of the cell. (NSA)

79

Lorenz, Egon, Allen B. Eschenbrenner, M. Derringer, and W. E. Meston. BIOLOGICAL ACTION OF GAMMA AND IX-RAYS. I. EXXPOSURE OF MCE TO DAILY DOSES OP GAMMA RADIATION AT TWO RATES: $5.5 \mathrm{r} / \mathrm{hR}$. AND 0.11 r/8 MR. J. Nat1. Cancer Inst. 6, 349-53(1946). (MODC-907; CH-2576)

Haematology: Female LAF-1 mice were exposed to dally doses of $5.5 \mathrm{r}$ in one hour $\left(\mathrm{I}_{1}\right)$ and $5.5 \mathrm{r}$ in one hour, plus $0.11 \mathrm{r}$ in eight hours. Biweekly blood counts were taken on these animals. They came to autopsy after having received total doses ranging from 600 to $1,600 \mathrm{r}$. The blood picture of these animals is conparable to that of controls of the same age with the exception that the neutrophil count was higher than normal for most of the experimental animals. This increase in neutrophils was probably due to a low grade pneumonia and not due to the irradiation. Radiation effects were observed only in lymph nodes, apleen and ovuries while all other organs were within normal limits histologically. An exposure of mice to $5.5 \mathrm{r} / \mathrm{hr} /$ day up to total doses of approximately $1,600 \mathrm{~g}$ produces radiation 
effects on the ovaries similar to and not more pronounced than those observed when similar doses are given at the rates of $4.4 \mathrm{x} / \mathrm{day}, 8.8 \mathrm{r} / \mathrm{dzy}$ and $8.8 \mathrm{~s} / 8 \mathrm{hr} / \mathrm{day}$. The discrepancy between a single sterilization dose of $300 \mathrm{r}$ and a dose of approximately $900 \mathrm{r}$ given daily at the above rate seems to indicate that a certain amount of recovery of the ovary is possible when doses are subdivided. Whether the subdivision of the dose or the rate of administration of the dose is more important in producing 80 sterility has to be decided by further experiments. (ADD)

MacKee, C., A. Mutschellex, and A. C. Cipollaro. TME FACTOR IN MREADIATION. Arch. Dermatol. and Syphilol, 47, 490-7(1943).

Two series of experiments were performed on at total of 29 persons: in the first series two adjacent skin areas of the inner surfaces of the forearm or the thigh were exposed to non-filtered $\mathrm{X}$-ray radiation of $52 \mathrm{kV}$ and $10 \mathrm{~mA}$, in the second series $100 \mathrm{kV}$ and $5 \mathrm{~mA}$ were used. The hali-value layer of the first experimental series amounted to $0.5 \mathrm{~mm}$ of aluminum, the second series $100.8 \mathrm{~mm}$ of aluminum. The variable factors were represented by distance and time of exposure. The experiments confirmed the general validity of Holthusen' 3 radiation intensity curve and suggested the logarithmic formula for the definition of radiation quantity of $\log \mathrm{x} / \mathrm{m}=\mathrm{IS}(\mathrm{log}$ $\left.\left[r_{t}-r_{1}\right]\right)$, in which formula $r / m$ represents roentgens per minute, $x_{t}$ the total exposure time, and $r_{1}$, the value of latent radiation. The generally observed decreases in the effectiveness of radiation with lower intensities is explained as being due in reality to the healing or recovery of the tissues during the course of irradiation. 81 (EM)

Noonan, T., F. Van Slyke, and J. Hursh. WFFCT OF SINGLE DOSES OF X-RAY ON THE SURVIVAL OF RATS. University of Rochester. Apr. 20, 1951. 12p. (UR-161)

Parallel groups of male and female 5 -month-old Wistar albino rats were used. Three groups, each of 24 rats, received respectively 150,300 , and $600 \times$ in single doses of 250-kvp $x$ irradiation delivered at $18.5 \mathrm{r} / \mathrm{min}$. Twentyfour male and 24 female rats served as controls. The mean survival times appear to be reduced by an amount proportional to the dose in both the male and female series. The spread of the data does not permit the exclusion of a possible threshold effect on survival as tested for the 150 $\mathrm{r}$ groups $\mathrm{Vs}$. the controls. Tumors which were grossly visible occurred in a larger number of 150 - and 300-r female rats than in the control group. (NSA) 82

Paterson, Edith. THE TIME-INTENSTYY FACTOR IN X-RAY IRPADLATION. Bril. J. Radiol. 17, 26-30(1944).

Studies of experiments are recorded on the effect of varying the over-all time, dose and dosage-rate per session being lrept constant. Culture of chick fibroblasts, maintained by the hanging drop method as described previously by Paterson (1942) were divided into two or more groups and were $x$ irradiated. The dosage levels chosen were those found by experience to give, on a short term basis, a moderate proportion of surviving cultures. The dosage rate per session of irradiation was the same for the short-term and long-term parts of any one experiment. Three variations of short-term irradiation were employed: (a) short continuous ir radiation which was completed in a matter of hours; (b) short-term intermittent irradiation in which the total dose was given in two sessions over a period of three days; and (e) short-term ipradiation in which the total dose was given in three sessions covering a period of 4 days. In all cases the long-term irradiation covered a period of 21 days. The criteria of effect employed was the survival rate and survival tume of the cultures. An increase of the over-all time of radiation to 21 days increased the lethal effect when the dose was split into 11 sessions. This increased effect was seen as a decreased proportion of survivors and a decreased survival time if the latter was calculated for long-term experiments from any point subsequent to the beginning of radiation. A qualitative difference was also shown by a lessened variation from the median point of death and a failure of cultures to show the recovery phenomena after long-term radiation. The degree of splitting of long-term radiation was of importance in influencing the effects. Some of the implications of these findings are discussed. (S.V.S)

\section{3}

Paterson, Edith and M. V. Thompson. TME-INTENSITY FACTORS IN X-IRPADIATION. PART II. THE INFLUENCE OF TRE DOSAGE RATE. Brit. J. Radiology 21, $414-19(1948)$.

Four groups of experiments were made on chick fibroblasts grown in vitro with the purpose of determining the lethal influence of dosage-rate uncomplicated by other factors. The over-all time was kept constant for all the sections of any one experiment. Dose levels of $10,000 \mathrm{r}$ to $12,000 \mathrm{r}$ were given by $250 \mathrm{kv}$ continuously evacuated X-ray tubes. Dose-rates of $4 \mathrm{r} / \mathrm{min}$. to $5 \mathrm{r}, \mathrm{min}$. were contrasted with rates of over $100 \mathrm{r} /$ min. The lethal effect of irradiation by a high dosage-rate, highly fractionated, was found to be the same as that of low dosage contnuous ir radiation. The effect of highly fractionated high dosagerate irradiation, however, was less than that of irradiation given at the same dosage-rate in fewer fractions but with an interval of over 40 hours. Therefore, dosage-rate as such is of less importance than the dose per fraction or the intervals between fractions, and it may be concluded that, in comparisons between high dosage-rate ir radiation given over a short time and that at low dosage-rate continued over a longer period, the operative factor ts the over-all time. (NSA)

84

paterson, Edith. TME-INTENSITE FACTORS IN TOTAL BODY IRRADIATION, p.161 Of INTERNATIONAL CONGRESS OF RADIOLOGY, 6th Congress, London. 1950.

The effect of prolonging the over-all time of irradiation has been studied in two pure-bred strains of mice, usmg $250 \mathrm{kv}$. X-radiation. Various biological criteria have been adopted, including the L.D.50/30 days, the Mean Survival time and the Variance. Over-all times of about 10 minutes are compared with thos of 24 and 48 hours, and also with periods of 5, 10 and 21 days. For any given time factor the probits of mortality percentages plotted against dose give a straight line. As the over-all time of irradiation is extended by fractionation of the dose into daily sessions, the L.D.50 rises; with an over-all time of 21 days the L.D.50 dose is nearly double that for single session radiation given in both cases at the same dosage rate. Another approach has been made by finding the L.D.50 when irradiation is given in two sessions at varying intervals.

85

Poulsen, B. R. INVESTIGATION INTO TKE TMME FACTOR IN THE ROENTGEN IPRADIATON ON CAMCER CELIS. PROTRACTION EXPERIMENTS WTTP A TRANSPLANTABLE MOUSE CARCINOMA. Acta radiol. 26, 463-93(1945).

Protraction experiments have been made with two mousetransplantation tumour: a rapidly growing: gensitive 
round-cell sarcoma, and a slowly-growing, resistance carcinoma. The criterion of the effect was the percentage of takes and the latent period for the development of the tumours. The investigations were carried out with a new experimental method which gives great statistic reliability. The objects were ir radiated with the middle dose at the intensities of $58-13.3-3.3-$ and $1 \mathrm{r} / \mathrm{min}$. In the case of carcinoma there was found (expressed both by the percentage of takes and by the latent period) a steadily decreasing effect with increasing protraction. The percentage of takes falls about 20\% when the intensity is varied from $58 \mathrm{r} / \mathrm{min}$ to $1 \mathrm{r} / \mathrm{min}$. The latent period for the development of the tumours was decreased at $1 \mathrm{r} / \mathrm{min}$. to aboul half of what it was at $58 \mathrm{r} / \mathrm{min}$. The round-cell sarcoma shows unchanged effect when the intensity is varied from $58 \mathrm{r} / \mathrm{min}$. $103.3 \mathrm{r} / \mathrm{min}$. but at $1 \mathrm{r} / \mathrm{min}$. the re is a distunctly increased effect, the percentage of takes in the two experiments being lowered by at least $25 \%$. This increased effect is also observable in the latent period for the development of the tumours. The decreasing effect on the carcinoma with increasing protraction is easily explained as an incomplete cumulation as a result of regeneration during irradiation. The increased effect of a low intensity on the round-cell sarcoma must be assumed to express that at this intensity (round about $1 \mathrm{r} / \mathrm{min}$ ) a crilical intensity has been demonstrated, as with steadily increasing protraction the effect nust again decrease at some low intensity or other. Two hypothesis are advanced in explanation of the critical intensity. (auth) 86

Russ, S. and G. M. Scutt. BROLOGICAL EFFECTS OF CAMMA IRRADIATION (SERIES II). Brit. J. Radiol. 12, 440-1(1939).

In a previous paper (Brit. J. Radiology 10, 1937) the authors reported biological effects of continuous exposure of rats to $\gamma$ rays. In the present experiments the same daily dose and the same total dose were administered, but on two days no irradiation was given, the lost dayg being made up by extending the time over which the treatment series lasted. The biological effects were just as marked as when daily irradiation was performed. There was a considerably greater death rate among the irradiated rats during the period of ir radiation (45.8\% to $14.8 \%)$; more rapid aging with persistence of the higher death rate; and great damage to breeding powers. (BA)

87

Sacher, George A. A NEW PROCEDURE FOR THE INVESTIGATION OF X-RAY INJURY AND RECOVERY, AND SOME PRELIMINARY RESULTS, p.83-5 of BIOLOGICAL AND MEDICAL DIVISIONS; QUARTERLY REPORT, FRBRUARY, MARCH, ARRIL, 1948. Argonne National Lab. May 1, 1948. 3p. (ANL-4163(p.83-5))

Author describes preliminary experiments carried out on mice and rabbits in which the animals were given single "conditioning" X-ray doses in the fractionally lethal range. After a period of time sufficient for the initial phase response to subside, the surviving animals were subjected to daily $\mathrm{X}$-ray exposures at a relatively high dose rate for the duration of life. The results to date are summarized in a table. (S.V.S)

88

Sacher, George A. THE SURVIVAL OF MIC UNDER DURATION-OF-LIFE EXPOSURE TO X RAYS AT VARIOUS DOSE RATES. Univ. of Chicago Metallurgical Lab. Apr. 1950. 43p. (CH-3900)

The survival of two strains of mice is reported for dose rates extending from 20 to $1000 \mathrm{r} /$ day. The relations between mean survival time, mean accumulated dose, and dose rate are exhibited. The relation of repeated-dose lethality to : ingle-dose lethality is investigated and the quantitative consistency is discussed. The hypothesis of a lethality process is introduced. On this hypothesis a lethality function is dedured, and its characteristics discussed. The deduction of the lethality function required certain mediating assumptions about linearity and about the properties of a defined lethal bound of injury. These assumptions are discussed. Applications of the conceptions developed are 89 considered briefly. 18 references. (NSA)

Schottelndreyer, H. STUDIES OF THE EFECT OF THE PROTRACTION OF X-RADIATION BY 12 FRACTIONATIONS ON THE ERYTHLMA REACTIONS. Brit. J.

Radiol. 22, 386-92(1949).

Three experiments were carried out on women patients in order to study the effects of the protraction of $\mathrm{x}$-radiathons on the erythema reactions. Two of the patients were under Ireatment for gynecological carcinomas and the third was being treated for an inflammation of the adnesa; all had normal skin conditions at the time of treatment. Each experiment is described in detail. The results show that the biological effect of a single irradiation with $631 \mathrm{x}$ on the skin is equivalent to that of $2064 \mathrm{r}$ when given in 12 fractions irrespective of whether an effective dose rate of $5.25 \mathrm{r} / \mathrm{min}$. or ate of $112 \mathrm{r} / \mathrm{min}$. is used, confirming the observation of Kepp (Strahlentherapie 72, 195 (1942)) that, when using 12 fractionating doses, protraction of the delivery time has practically no effect on the biological erythema dose. Similarly, it was found that a similar protraction does not have any detectable influence on the erythema effect using 12 fractions when the above doses are increased or decreased by $20 \%$. The sensitivity of the react 0

Sievert, R. M. and A. Forssberg. TRE TIME FACTOR IN THE BIOLOGICAL ACTION OF ROENTGEN RAYS. III. INVESTIGATIONS AT VERY SHORT IRRADIATION TMES. Acta Radiol. 17, 290-8(1936).

The authors describe investigations of the death rate of Drosophila eggs after irradiation with about $165 \mathrm{r}$ in very short periods, varying from 0.39 to 1.18 seconds. This corresponds to radiation intensities of $24,000 \mathrm{~m}, 800 \mathrm{r} / \mathrm{min}$. They compare the reaults with those previously obtained with the same dose given in from 2.1 seconds-30 minutes. A critical study of the possible sources of error indicates the probability that the irradiation effect diminishes at very short radiation times. The experiments show that the death rate is reduced at 0.39 seconds by more than $20 \%$. The authors are of the opinion, however, that experiments with still greater intensilies are advisable in order to confirm the results. (AR)

21

Stearnex, S. Phyllis. THE FFECT OF VARIATION IN DOSAGE RATE OF ROENTGEN RAYS ON SURVIVAL, IN YOUNG BRDS. Am. J. Roentgenol. Radium Theray $65,265-71(1951)$.

Survival following roentgen irradiation of young birds depends on total dose, dose rate at which the exposure is made, and age at time of exposure. Exposure of $2-10$ 3-day chichs to $1000 \mathrm{r}$ at a rate of $15 \mathrm{r} / \mathrm{min}$ or more resulted in a $75 \%$ mortality within $24 \mathrm{hr}$. Exposure to the same dose at $6 \mathrm{r} / \mathrm{min}$ resulted in only $1 \%$ mortality within $24 \mathrm{hr}$, and $46 \%$ between 5 and 10 days after ir radiation. The mechanismas leading to the early deaths are not clearly understood. It is suggested that the biological effects of variation in dose rate indicates that recovery in taking place during an exposure at the low rate so that the total 
effect of the dose is less than if it were given at a high 92 rate. (NSA)

Stearner, S. Phyllis and Emily J. B. Christian. PROGRESS REPORT; INFLUENCE OF DOSAGE RATE ON X-RAY EFPECTS IN YOUNG CHICKS AND RATS, p.125-9 Of BIOLOGICAL AND MEDICAL DIVISIONS; QUARTERLY REPORT, NOVEMBER, DECEMBER, JANUARY, 1949-50. Argonne National Lab. [nd] 5p. (ANL-4401(p.125-9))

Mortality of 2- to 3-day chicks within 24 hours after $x-$ irradiation $(1,000 \mathrm{x}$ at $43 \mathrm{r}$ per minute) has been described earlier. Administration of vitamin $\mathbb{P}$ substances had no effect on survival or hemorrhagic tendencies. Histamine is probably not an important factor in the reactions leading to the early deaths, for the chick shows a relatively low sensitivity to histamine shock. Although the speed with which death follows irradiation suggests that some part of the effect might be mediated through the autonomic nervous system, prophylactic and therapeutic treatment with atropine and physostigmine was without effect. Adrenaline was effective in increasing survival and in decreasing the severity and number of hemor rhages when its time of administration was close to that of 8 -irradiation. Further experiments are under way to determine more prectsely its time of action. Hematocrit determinations indicated that a hemoconcentration was present at 3 and 5 hours after exposure at the high dosage rate. Exposure of 1 - to 5 -hour rats to $600 \mathrm{r}$ at $80 \mathrm{r}$ per minute resulted in $48 \%$ mortality within four days, but no deaths occurred during this period if the exposure rate was $6 r$ per minute. In all animals, cerebral hemorrhages developed within a few hours after irradiation, but the hemorrhages were greater (more diffuse) and more numerous after high rate exposure. Histopathological studies are planned following exposure of newborn rats. Experiments are in progress to determine effects of other dosages and intermediate 93 dosage rates. (auth)

Stearner, S. Phyllis and Emily J. B. Christian. THE EFFECT OF OVER-ALL TIME OF EXPOSURE UPON SURVIVAL OF YOUNG CHICKS FOLLOWING ROENTGEN IRRADIATION. Am. J. Roentgenol. Radium Therapy 65, 272-6(1951).

Survival following exposure to fractionated roentgen-ray dosages indicates that over-all time is of greater importance than dosage rate in determining the lethality of a given dosage for newly hatched chicks. Survival is increased as the over-all time of exposure is increased, but 94 not at a proportional rate. (NSA)

Van, Roojen, J. INTENSITY OF RADIATION AND SELECTIVE ACTION. Brit. I. Radiol. 12, 547-53(1939).

It is now generally agreed that the action of radiation is independent of the wave length. If there is a diference between the action of the gamma and $X-r a y s$, it must be due to some other factor. The only other possible variation is intensity. Experiments have indicated that variation in effect might be due to variation in the periodicity of sensitivity of cells or to a process of recovery taking place while the destructive process is going on. The shape of dose-intensity curves indicates that the latter takes place. With high intensities the dose is independent of the intensily. With low intensity the dose must be increased. These facts are confirmed by clinical experience. Equations are derived showing these effects mathematically, disregarding recovery effects, making use of several reasonable assumptions. The concept of a specific zensitivity of cells so that they can be arranged in an order of susceptibility does not hold for low intensities. Selectivity of action appears when the intensity is so low that the factor of recovery comes in. It is possible that we may be able to improve results by further decreasing the intensity but we do not yet know if this is so, nor how much recluction is best. Lowering of the intensity cannot be pushed beyond a certain point if the desired result is to be obtained. The destruction must be greater than the normal rate of growth. Reducing the intensity may allow us to continue the radiation until the tumor has vanished, thus relieving us of the uncertainty about the total dose required. Geometrical homogeneity of distribution is important. Successive application of beams from various ports is not equivalent to continuous action in a bomogeneous field. (R) 95

Warren, S. L. and G. H. Whipple. THE CUMULATIVE EFTECT OR SUMMATION OF X-RAY EXPOSURES GIVEN AT VARXING INTERVALS. J. ExpLL. Med. 38, $725(1923)$.

It is generally accepted that $\mathbb{X}$-ray exposures repeated at short intervals give a summation effect, but the observations are largely concerned with slin injury. Accurate measurements have been made on the dose necessary for destruction of the mucosa of the small intestines in dogs. From experiments performed by dividing the maximum sublethal dose in dogs, it was found that the ordinary cumulative effect noted in skin reactions does not apply to depth reactions. A single large dose of $\mathrm{X}$-rays over the abdomen will cause a definite injury of the mucosa of the small intestine and the severity of the clinical intoxication seems to parallel this recognizable epithelial injury. This clinical intoxication lasts 4 to 6 days, if the $\mathrm{X}$-ray dose is sublethal. Subsequent doses of radiation given with this period of clinical intoxication give recognizable evidence of summation or a cumulative effect. Small but repeated doses of radiation given within a 5 or 6 -day period will cause practically the same cell injury and clinical intoxication as will a single dose representing the sum of small doses expressed in milliampere minutes. Doses of radiation given at 6-day or longer intervals show no evidence of summation. The reaction of this relatively sensitive in testinal epithelium to radiation may be similar to the reaction of certain deey-lying tumor tissues to X-ray therapy, and our experments may give information of value to physicians concerned with $X-$ ray or $R$ a therapy. (R)

\section{Lethal Dose Studies}

96

Alland, Paul D., Benjamin Highman, and Betty Wood. SOME EFFECTS OF X-IRRADIATION ON TURTLES. J. Exptl. Z001. 118, 4-14(1951).

Box turtles were given whole-body $x$ irradiation with doses ranging from 500 to $10,000 \mathrm{r}$. In general the effects of $x$ irradiation on turtles appeared to be similar to those on mammals, but they occurred at a much slower rate. Turtles survived for 270 days (alive at writing) with doses of $500 \mathrm{r} ; 4$ of 6 survived longer than 4 months after $1000 \mathrm{r}$; 2 of 6 turtles receiving $1500 \mathrm{r}$ survived 4 months; higher doses up to $10,000 \times$ caused death in from 18 to 48 days. The carapace and plastron reduced the tissue dose by approximately $10 \%$. Effects on circulatory and general systems are noted. (NSA)

97

Betz, H. THE IMPORTANCE OF TRE RESISTANCE PHASE OF THE ORGANISM AT THE TME OF APPLICATION OF A LETHAL DOSE OF X RADIATION. Compt. rend. soc. biol. 144, 1439-42(1950). 
In order to determine whether a radiation-resistance phase could be produced, three lots of $C 57$ mice were irradiated with a nonlethal dose of $500 \mathrm{r}$ of $x$ rays followed at 7,10 , and 15 days, respectively, with a $700-\mathrm{g}^{\circ}$ dose. In contrast to a $700-r$-irradiated control lot, all of which succumbed by 10 days, the lots receiving the $7-, 10-$, and 15 day fractionated exposures showed decreased rates of mortality and $20,26.5$, and $36 \%$ survival, respectively. The protective action of the nonlethal exposure is discussed inconclusively. (NSA)

98

Boche, R. D. and F. W. Bishop. STUDIES ON THE EFFECTS OF MASSIVE DOSES OF ROENTGEN RADIATION ON MORTALITY IN LABORA TORY ANMMLS. Univ. of Chicago Metallurgical Lab. [nd] 13p. (MDDC-250)

The object of these experiments was to supplement the exnsting literature on the effects of single acute exposures of animals with a view of forming an estimate of what variations exist among different mammalian sperieg. The applicability to man is uncertain but one may assume as a first approximation that his sensitivity lies within the range of other species. Systematic studies have been made on the behavior of peripheral blood elements in the rat, dog, and monkey following single exposures. Tissue studies on animals sacrificed serially have been carried out for the rat and farly complete histology has been done on animals at death. It is now well established that curves relating mortalkty to dose are of the sigmoid type for most organsms studied and the mammals are no exception in this respect. Such sigmoid curves may be conveniently iransformed to stranght lines by the method of Bliss which is based on the assumption (a) of normally distributed susceptibility of the individuals radiated and usually (b) geometric increase in effect with increasing dose. Such straight lines proved the simplest method of estimating the median lethal dose for a given set of conditions and variam tions in $L D \cdot 50^{\circ} \mathrm{s}$ constitute a convenient basis for comparison of species or other factors. Mortalities and average survival tumes are tabulated and plotted for various 99 animals given varying doses of roentgen radiation. (ADD)

Bonet-Maury, P. and F. Patti. LETHAL IRRADLATION OF MICE BY Y-RAYS AND GAMMA-RAYS. THERA-

PEUTIC TRIALS. J. radiol et electrol. 31, 286-9(1950).

A study on the ffects of $\mathrm{X}$-rays administered in doses vary ing between 400 and $100,000 r$, on the survival of mice (heterogencous groups). For $100 \%$ of the mice the lethal dose is attained at $600 \mathrm{r}$. At 800 to $13,000 \mathrm{r}$ the mean time of survival remains constant $(80 \mathrm{hr})$. From $13,000 \mathrm{r}$ onwards a new rapid fall in the survival time develops according to the dose. The furst deaths during irradiation appear at doses of $60,000 \mathrm{r}$ (output: $470 \mathrm{r} / \mathrm{min}$ ). At $100,000 \mathrm{r}$ the mean survival time is 2 hours and 30 mir. The variations due to age, weight, and sex proved negligible. Attempts to protect mice exposed to 1,000 $\mathrm{r}$ by using various substances (DOCA, horse serum) and certain methods of physical therapy (short waves, UV rays, grenz rays, darkness, illumination) did not meet with success. The effects of gamma radiation were submitted to similar investigations. The gamma $X$-ray ratio of effectiveness varied between 2.1 and 3.6 . (EM)

\section{0}

Bonham, Kelshaw, Allyn H. Seymour, Lauren R. Donaldson, and Arthur D. Welander. LETHAL EFFET OF X-RAYS ON MARINE MICROPLANRTON ORGANESMS. Science 106, 245-6(1947). (MDDC-1158)

Literature on the effect of $x$-rays on bacteria, yeasts, and Protista is summarized in a table showing that the average immediately lethal dose is 595,000 roentgen
$(20,800-1,250,000)$ and that the average dose capable of stopping growth or cell division of these micro-organisms is $93,000 \mathrm{r}(12,000-400,000)$. In this experiment vialed samples of Chiorella sp., Nitzschia clostarium, a carterild, and another mastigophoran (unidentified, brown) were each subjected to five exposures from 1,000 r to $100,000 \mathrm{r}$. Numbers of organisms per unit volume of culture were counted using ruled blood counting chambers. Immediate immobilization of the brown mastigophoran was $50 \%$ effective at $50,000 \mathrm{r}$, and $100 \%$ at $100,000 \mathrm{r}$. Counts for an organism were comparable only with other counts for different irradiation samples of the same organism on the stume date. Counts over a period of four months were most nearly as might be expected in the case of Chlorella where the concentration of individuals remained inversely proportional to the amount of irradiation for practically the entire time, except in the case of the $1,000 \mathrm{r}$ sample which exceeded one of the two controls. Nitzschia counts in the higher irradiations decreased earlier and more drastically than did those of Chlorella, but later these more heavily irradiated samples gave higher counts than the lower dosages. Counts of the two Mastigophora were arranged erratically with only a general tendency for count to be inversely related to exposure. In no case did a sample count drop to and remain at zero. (auth) 101

Clark, W. G. and R. P. Uncapher. DOSAGE-MORTALITY IN RATS GIVEN TOTAL BODY ROENTGEN IRRADIATION. Proc. Soc. Expt1. Biol. Med. 71, 214-8(1949).

Over 500 young male rats were used in studies to determine the dosage-mortality ratio for total body roentgen irradiation. The doses were delivered at diferent lengths of time by a $200 \mathrm{kv}$ machine, at $52.5 \mathrm{~cm}$ target distance, and through $1 / 2 \mathrm{~mm}$ Cu and $1 \mathrm{~mm}$ Al fllters; the dose-rate was $30.4 \mathrm{r} / \mathrm{min}$. in air. The mortality data represent indefinite survival of the rats (past $30-60$ days) and are included in three graphs. The results ghow that the maximum dosage for rats in this geries is $640 \pm 5 \mathrm{r}$ with a 30-day end-point. Becauge of the steep mortality curvea in the rat groups, and the consequent errors in calculation, dosages in the lethal range should not be used. (NSA) 102

Cronkite, Eugene P., Clyde R. Sipe, Dean Eltzholtz, Wulliam H. Chapman, and F. W. Chambers. THE INCREASED TOLERANCE OF MICE TO A LETHAL DOSE OF X-RAY RADIATION AS A RESULT OF PREVIOUS SUBLETHAL DOSES. Naval Medical Research Inst.

Report Number 15. Aug. 17, 1948. 6p. (NP-589)

It has been demongtrated with a fair degree of certatnty (probability that results are due to chance, calculated by a pooled Chi aquare found to be between 0.02 and 0.05 ) that three weekly exposures of $144 \mathrm{r}$ followed by a $30-d a y$ rest period increases the resistence of mice to a subsequent lethal dose of $x$-radiation. It is concluded that no adeguate explanation for this ghenomenon of increased tolerance to X-ray radiation is available at the present time. (NSA) 103

Ellinger, Friedrich. LETHAL DOSE STUDIES WITH XRATS. Radiology 44, 125-41(1945).

The applicability of a method for the determination of lethal doses of $X$-rays derived from the study of mice has been demonstrated for a larger animal, the guinea pig. With increasing doses of $\mathrm{X}$-rays, an increase in the percentage mortality was found up to $500 \mathrm{x}$ in alr. This dose represents the absolute lethal dose (ALD) for guinea pigs under the conditions of the exposure used. $A$ in in mice and goldish, the relationship between dose of $\mathrm{X}$-rays and percentage mortality is expressed by an S-ghaped curve. Comparison of the mortality curves of mice and guinea 
pigs shows a steeper slope of the curve for guinea pigs. In connection with some observations concerning the latent period, this is considered to be an expression of species specifzeity. (R)

104

Ellinger, Fitedrich. INFLUENCE OF DOSE FRACTIONATION ON THE LETHAL X-RAY EFFECT PHODUCED BY TOTAL BODY IRRADIATION IN MICE; A PRELIMINARY NOTE. Radiology $49,238-41$ (1947).

Data are presented on the lethal effect upon mice of total body irradiation by a single dose, and a comparison is made with the effect of the same dose fractionated, 252 white male mice having been irradiated wh $1,000,500$, and $400 \mathrm{r}$ in air. Fractionation consisted in giving a dose of 100 r on successive days. The results are given in graphs, and show that there was in every case a reduced mortality rate after the fractionated dose, as compared with the mortality rate after the same dose given as a single exposure. The effect was found to be most pronounced for that dose which, when given unfractionated, resulted in the lowest mortality rate - in this case the $400 \mathrm{r}$ dose. The author consideres the result significant from the point of view of the physician, since there is here some suggestion that the living organism may tolerate as general radiation, multiple applications of a dose which is high enough to have 105 antiple applications of a dose which is
n effect upon neoplastic tissue. (NSA)

Ellinger, Friedrich and James C. Barnett. FURTHER STUDIES ON THE INFLUENCE OF DOSE FRACTIONATION ON THE LETHAL X-RAY EFTECT PRODUCED BI TOTAL BODY IRRADIATION IN MCE. Radiology 54, 90-2(1950).

The influence of the size of the single exposure and overall exposure time on the lethal effect of total body irradiation of mice with $x$-rays was sutided. A total of 220 white male Swiss mice were used in this esperiment, 20 of which served as unirradiated controls. The irradiation was delivered by a $200 \mathrm{kv}$ source thorugh $0.25 \mathrm{~mm}$ Cu and $1.0 \mathrm{~mm}$ Al total filtration at a target distance of $50 \mathrm{~cm}$ and a dosage rate of $23.4 \mathrm{~g} / \mathrm{min}$. The effect of $1,000 \mathrm{In}$ in in one exposure (LD-100) was compared with that of the administration of the same dose when given in single fxactions of 100-50 in air within 10-20 days. It was found that the effect of dose fractionation in total body irradiation depends on the size of the single fraction as well as on the over-all exposure time; of these two factors, the size of the fraction appears as the more important. It also appears that, as with observations on tumor destruction, there exists a certain optimum for dose fractionation in total body irradiation. (NSA)

106

Illinger, Friedrich, Jasper E. Morgan, Erancis W. Chambers, Jr., C. R. Biles, and J. T. Istock. THE USE OF SMALL LABORATORY ANMALS IN MEDICAL RADLATION BIOLOGY. I. APPLICATION OF A 200 KVP X-RAY THERAPY UNIT TO LTTHAL DOSE STUDIES. Naval Medical Research Inst. Bethesda. Apr. 30, 1952. 35p. (NP-4120; U23980; NM-006-012.04.43)

A critical study of irradiation techniques and apparatus has been undertaken, with particular reference to the effective application of conventional X-ray therapy equipment. Major emphasis has been assigned to details of accurate physical doismetry, with the goal of consistently reproducible interlaboratory comparison of experimental data. By automatic regulation of input line voltage fluctuations to less than $1.0 \%$, plus maximum care in manual control and timing, individual calibration readings are reproduced by the same Victoreen chamber to the order of
$10.5 \%$. Approximately uniform simultaneous rexposures are obtained in animal containers designed for twolve mee, and for two guinea pigs. The radiation quality is varable from H.V.L. of $2.3 \mathrm{~mm}$ Al to $1.8 \mathrm{~mm}$ Cu. The ine tdent backscatter becomes constant in each case after approxnmately a $10 \mathrm{~cm}$ thickness of Masonite, while the harde's radiations approach an H.V.L. near $0.25 \mathrm{~mm}$ Cu after traversing large thicknesses of Masonite. (auth) $10 \%$

Engelhard, Hermann and Thea Houtermans. ACTION OF ALPHA RAYS AND OF ULTRAVIOLET LIGHT ON BACTERIUM COLI. Z. Naturforsch.5b, 264-9(1950).

By combining phase-contrast microscopic observatuons with a counter technique, the knlling effect of uitraviolet light and $\alpha$ radiations on Bacterum cols was determined as a function of the absolute dose. It was found that, at equal doses, the effect is independent of the radration intensity. The survival versus dose curve falls off exponenvially at small and medum doses and becomes steeper at higher doses. In the case of ultravolet light the curve terminates 1 a borizontal tal possible reflectung the presence of bacteria resistant to ultraviolel radiations. Morphologral pictures pont to a many-hit radiobiological process, while the shape of the curves indicates the presence of two separate mechansims, one of the one-hit and another of the many-hit nature. (NSA) 108

Fram, Harvey, Bernard E. Proctor, and Cecul G. Dunn. IFECTS OF X-RAXS RRODUCED AT 50 IILOVOLTS ON DIFIERENT SPECIES OF BACTERIA. J. Bacteriol. 60, $263-7(1950)$.

The effects of irradiatuon with $\mathrm{x}-\mathrm{rays}$ produced at $50 \mathrm{kV}$ were studied with six bacteria, Escherichia coli. Aerobacter aerogenes, Staphylococcus aureus, Serratia marcescens, Pseudomonas aeruginosa, and Pseudomonas fluorescens. The gate of destruction followed a first-order reaction. The "ar? dose" for each sepcies of bacteria was caleulated. S. aureus was found to be the most radioresistant of those studied. The percentage of bacteria of a given specass killed by a specific total dosage of $x$-rays was the same regardless of the initial concentration of bacteria in the suspension irradiated. These results indicate that the bacteria were destroyed by soft $\mathrm{x}$-rays 109 according to the "direct hit" theory of radation. (NSA)

Gray, L. H. and John Read. THE LFECT OF IONIZING RADIATONS ON THE BROAD BEAN ROOT. I. GENERAL CONSIOERATIONS. II. THE LETHAL ACTION OF GAMMA RADIATION III. THE LETHAL ACTION OF NEUTRON RADIATION. Bxit. J. Radiol. 15, 11-17, 39-43, $72-7(1842)$.

The technique of growing and measuring the roots, and the statistical method of handling the mortality data are given in delall. For gamma radiation, data on 50 beans give the mean lethal dose; for neutron radiation data on 146 beans give a lethal dose about $\%$ as great as for gamma rays. Statistical considerations of these data and of the mortality curves drawn from them indicate that the difference in shape of mortalify curves for gamma and neutron rays, previously published is not real. (BA) 110

Gray, L H., John Read, and M. Paynter. THE LFECT OF IONIZING RADIATIONS ON THE BROAD BEAN ROOT, $\checkmark$ THE LETHAL ACTION OF RADIATIONS. Brit. J. Radiol. 16, 125-8(1943).

Roots of Vicia faba were exposed to $X$ rays having a mean wavelength of about $0.16 \mathrm{~A}^{\circ}$. The duration of exposure ranged from 52 to 230 minutes for $X-r a y$, with a 
mean tume of 145 minutes for doses in the ne aghborhoot of the mean lethal dose. Corresponding averaye durations of exposure for gamma rays, neutrons, and alpha partacle experiments were 131 munutes, 133 minutes, and 81 minutes, resyectively. Relative efficiencses of the different radiations (reciprocal of mean lethal dose relative to that for gamma rays) are (gamma rays 1.0$)$, $\mathrm{X}$ rays $1.5 \div 0.1$, neutrons $8.7 \div 1.6$, alpha particles $8.9 \div 0.8$. Relationshp of the findings to radiology and radiation mjury is discussed. (V.E.)

111

Gray, L. H. and John Rrad. THE EFFHCT OF IONIZING RADIATION ON THE BROAD BEAN ROOT. IV. SUMMATION OF THE EFECTS OF RADIATION OF DIFELRENT ION DENSITY. A. NEUTRON PLUS GAMMA RADIATION. B. ALPHA PLUS X-RADIATION. Brit. J. Radiol. 17, 265-71(1944).

Bean roots were exposed in succession to two donizing radiations of dufferent types, either gamma is neutron, or $x$-rays + alpha. Data on the furst pair of radiations are anconelusive. For the combination $x$-rays + inlpha, to kill $1 / 2$ the roots it was necessary to administer slightly more than half a mean lethal dose of each. The primary cell injury responsible for the death of the root is apparently the same, whichever radiation is used, so that large differences among mean lethal doses for various types of radiation must be asc ribed to a difference in the efficiency of the radiations in bringing about the primary injury. (BA)

112

Gray, L H. and J. Read. IONIZING RADIATON ON THE BROAD BEAN ROOT. VI. SUMMATION OF THE ETIECTS OF RADIATION OF DHFTERENT ION INTENSITY. A. NEUTRON AND GAMMA RADIATION. B. ALPHA AND X-RADIATION. Brit. J.Radiol. 17, 271-3(1944).

The authors ${ }^{8}$ earlier work showed that in killing the bean root densely lonizng radiations (neutrons, $x$-rays) were more effective, per lonization, than less densely ionizing radiation $(z-r a y s, x-r a y s)$. It is now shown that the effects of successive exposures of bean roots to partial doses of $x-r a y s$ and $\alpha$ rays, or to partial doses of neutrons and $\gamma$-rays, are additive. It is considered that the lethal effect of all four radration is due to the same type of primary injury, and that differences in the efficiency of different radiations arise from differences in the amount of ionization needed to produce this particular injury, due to the spatial distribution of the ions. (EM)

113

Magen, C. W. Jro L. O. Jacobson, R. Murray, and P. Lear. EFPECTS OF SINGLE DOSES OF X-RAYS ON RABBITS. Univ. of Chicago Metallurgical Lab. Sept. 9, 1944. Decl. June 4, 1947. 47p. (MDDC-999)

Rabbits were exposed to $200 \mathrm{kvp} x$ rays. The median lethal dose (based on survival for 30 days) was $790 \mathrm{r}+20 \mathrm{r}$. Heavy (older?) rabbits were more resistant to the lethal effects of $x$ rays than were hght rabbits. Deaths occurred most frequently at 1 day, at 5 to 15 days, and at 35 to 60 days after exposure. Chncial effects were also most severe on the first day, at 5 to 15 days and between 35 and 60 days after irradiation. The most conspicuous changes observed were listlessness, duarrhea, excessive salivation and lachrymation during the first day, epilation, paleness, edema, diarrhea, occasional paralysis of the hind legs, and bloody discharge from the mouth at 5 to 15 days; and emaciation and diarrhea at 35 to 60 days. After $800 \mathrm{I}$ and sometimes after $400 \mathrm{x}$, rabbits lost weight for the fir st 2 days. This loss of weight was associated with a decrease in food mikle. The werght changes after the first 2 days varied with the ndividual rabbit but in general consisted of one or more consecutive periods of gain and loss. The period of weught minima correpsonded roughly with the periods at which deaths were most fruquent. Following single doses of $400 x$ and $800 \mathrm{x}$ on rabbits the blood forming tissues showed the most severe damage. Histological changes of various tissues and of the blood picture are described to some detail. (TIS)

114

Hagen, C. W., Jr. and George A. Sacher, EHFETS OF X-RAYS ON RABBITS. PART I. MORTALITY AFTER SINGLE AND PAIRED DOSES. Argonne National Lalo. Dec. 9, 1946. Decl. Aug. 29, 1947. 38p. (MDDC-1252; C. $1-3754$ )

Mortality statistics were collected on 227 young adulf rabbits treated with single doses of $x$ rays. The distributions of times of death for males and females show that there are 3 distinct modal tumes of death in the first days after exposure. The first peak of deaths occurred in the 1 st day after ireatment, and males were more sensitive than females at this time. The second peak appeared between the 4th and 8th days, and was shown only in the females. The main peak of mortalnty occurred at 10 days and showed little difference between the sexes. The $L D_{60}$ for the entire population, based upon all deaths in the furst 20 days, was $800 \mathrm{r}$. When animals which died during the first 3 days were excluded from the population, the $L D_{5 s}$ for the remaining group was $810 \mathrm{r}$, and the standard deviation of the survival curve was $145 \mathrm{x}$. The effects of dose and of weight and sex of the rabbits on the $\mathrm{LD}_{\mathrm{g}}$ at varlous intervals is discussed to some detail. (TIS) 115

Hagen, C. W. Jr., and George A. Sacher. THE ACUTE LETHAL ACTION OF SMGLE DOSES OF W-RAYS ON GUINEA PIGS. Univ, of Chicago Metallurgical Lab. Aug. 1951. 12p. (CH-3907)

Groups of guinea pigs were exposed to $x$-ray doses ranging from 0 to $800 \mathrm{x}$ and their survival followed for 90 days thereaiter. The guinea pig dosage survtval curve 1 s very broad, almost exponential in form. The LD-50 is about $200 \mathrm{r}$. The relation of mean gurvival the to dose was determined and it was found that the mean survival time of guinea pigs exposed to lethal doses are quantitatively similar to those of the other experimental animals. The relation of mean survival time of 30-day decedents to proportion killed in cage groups was also exhibited. An unusual phenomenon was noted in that the survival time of decedents is on the average longer in cages which sustained about 50\% 30-day killing than in cages in which the 16 30-day mortality was either higher or lower. (auth)

Hagen, C. W. J $r$ and E. L. Simmons. EFTECTS OF TOYAL BODY X-IRRADIATION ON RATS. PART 1. LTTHAL ACTION OF SINGLE, PAIRED, AND PERIODIC Doses. Univ. of Chicago Metallurgical Lab. May $184 \%$. $47 \mathrm{p}$. (MDDC-1210; C

Exposures of Sprague-Dawley white rats to single doses of $200 \mathrm{kvp}$ x-radiation demonstrated a median lethal dose of $600 \mathrm{r}$ which was not markedly dependent upon sex, weight, or, in the case of females, stage of the estrous cycle at the time of Irradiation. Deaths within 30 days were most frequent between the fourth and eighth, and between the tenth and fifteenth post-irradiation days, indicating the existence of at least two processes leading to death in the "acute" period. Male rats exposed to $300 \mathrm{r}$ were less resistant to $\mathrm{X}$-rays than unexposed animals for at least six weeks. During the first three weeks after the initial exposure, recovery was exponential at a rate of $8.2 \%$ per day. Beyond three weeks, the rate of recovery apparently decreased. Deaths that occurged after 
daily exposure to doses greater than $50 \mathrm{r}$ per day occurred in less than 30 days; whereas with lower daily doses, the period of survival was greatly extended. These results are compatible with the recovery rate of $8.2 \%$ per day determined by paired-dose studies and indicate that after high daily doses, the lethal mechanisms are the same as those after single doses. With low daily doses, other mechanisms may operate. A bibliography of 19 references is appended. (ADD)

117

Halberstaedter, L. and A. Back. THE EFECT OF XRAYS ON SINGLE COLONIES OI PANDORINA. Brit.

J. Radiol. $15,124-8(1942)$.

In the green alga Pandorina, death is produced within 45 minutes after irradiation with $300,000-600,000$ roent gens of $35 \mathrm{kv} x$-rays. All daughter cells of the same clan are killed by approximately the same dose, while in wild cultures the lethal dose varies over the above range, indicating that different colonies have varying degrees of radio-sensitivity. With doses of $3,000-300,000 \mathrm{r}$, the cells behave normally until the time for division, then become immobile, and undergo cytolysis. Doses $<3,000-4,000 \mathrm{r}$ do not produce demonstrable effects. No recovery was observed. (BA)

\section{8}

Jacquez, John A. and David A. Karnofsky. THP TOCICITY AND PATHOLOGICAL EFFECTS OF ROENTGEN RAYS IN THE CHICKEN. Am. J. Roentgenol. Radium Therapy 64, 289-97(1950).

Acute deaths are produced by roentgen rays in young chicks at dose rates above $10 \mathrm{r} / \mathrm{min}$. and tolal doses of 1,000 $\mathrm{r}$ or higher. The delayed LD-50 dose, in those chicks surviving the acute effects of high dose rates, is about $800 \mathrm{r}$, and a low dose rates it is about $1,100101,200$ r. Adult chickens of both sexes may die acutely at total doses of 1,250 to $3,000 \mathrm{r}$, but they have often survived a dose of $5,000 \times$ for several days. Chickens surviving the acute effects succumb within 3 to 10 days, and the LD-50 is in the range 600 to $800 \mathrm{r}$. The chickens dying acutely following irradiation show, within two hours after irradiation, necrotic foci in their livers, and pyknosis and fragmentation of their lymphatic cells. The delayed deaths are not associated with liver injury, but the lymphatic tissues, including the thymus and spleen, involute, the bone marrow becomes aplastic, erosions and degenerative changes occur in the intestinal tract, and there is frequent evidence of bacterial infections. These observations on the delayed effects of roentgen rays parallel those reported 120 for manmals. (NSA)

Lambert, G., J. Maisin, M. Mandart, Meersseman, G. Podio, and E. Pluygers. INILUENCE OF CERTAIN METALLIC IONS ON THE SURVIVAL OF RATS RECEIVING A LETHAL DOSE OF I-RAYS. Compt. rend. soc. biol. 144, 444-8(1950).

$B i$ and Li ions injected in non-lethal doses into rats receiving a lethal dose of $\mathrm{X}$-rays hasten their death. The mercuric lons tend to delay death and decrease the per120 contage of deaths. (BA)

Lorenz, Egon, Delta Uphoff, Marriet Sutton, and R. Schweisthal. PROGRESS REPORT: TIIID 30-DAY LD FOR HYBRID GUINEA PIGS, p. 0 0-2 Of BIOLOGICAL AND MEDICAL DIVISIONS: QUARTERLY REPORT, PEBRUARY, MARCH, APRIL, 1950. Argonne National Lab. [nd] $3 \mathrm{p}, \quad(A N L=4451(\mathrm{p}, 50-2)$ )

In previous experiments designed to determine the 30-day LD-50 for hybrid guinea pigs groups of animals were given single total-body exposures to $220 \mathrm{r}, 260 \mathrm{r}$, and $310 \mathrm{~g} x$-radiation. This study has bean concluded with two additional cxposure groups of $360 \mathrm{r}$ and $420 \mathrm{r}$. In the first three groups the was no sex difference in survival but in the last two groups, the female secmed more radiosensitive than the males. However, this finding is probably duc to chance since the experiments were spread over a year and hybrid stock was used. The 30-day LD-50 is ralculated to be $409 x$ with 20 limits of $307 x$ and 543 . This value is at variance with previously published data of other investigators and it is sugrested that the low figures rported by these invest 1 gators may have been caused by irradiating duseased gunea pigs. (auth) 121

Lorenz, Egon, Delta Uphoff, and llarriet Sutton. PRELIMINARY REPORT: THE 30-DAV LD 50 AND ACCOMPANEING BLOOD PICTURE OF TYBRID GUINEA PIGS, p.57-65 of BIOLOGICAL AND MEDICAL DIVISIONS; QUARTERLY REPORT, MAY, JUNE, JULY 1950. Argonne National Lab. July 1950. 9p. (ANL-4333(p.$57-65)$ )

Tor the determination of the 30 -day ${ }^{-D_{50}}$ dose, two groups of animals, each consisting of 30 males and 30 females, approximately 3 months of age, was given $220 \mathrm{r}$ and $260 \mathrm{r}$, respectively, of $186 \mathrm{kv}, \mathrm{x}-\mathrm{irradiat}$ ton. To obtain as uniform distribution of energy within the animal as possible, a double tube sctup, irradiath the animal from above and below simultaneously, was used. Ir radiation was delivered at the rate of $63 \mathrm{~F}$ per minute. The exposure to $220 \mathrm{r}$ killed $27 \%$ of the animals within 30 days, and the $260 \mathrm{r}$ dose killed $38 \%$. It can be stated fom these two experiments that the 30 -day $\mathrm{LO}_{\mathrm{g} 0}$ will lie between 250 and $300 \mathrm{r}$ for the hybrid guinea pigs used. Mortality in the two groups reached a peak between the $10 \mathrm{th}$ and 12 th days, corresponding with the lowest values in blood counts, except for a second peak in the 260 r group on the 20 th day, after blood values had almost returned to normal. Clotting time was not materially affected. The decrease in blood cells started shortly after exposure and reached a maximum on the 12 th day, the abrupt fall resembling that seen in chronic exposures when the dose approaches lethal levels. However, recoveries here were much more rapid than those seen in chronic exposures. The acute 30 -day $\mathrm{LD}_{50}$ is comparable with the mean survival time and mean accumulated dose causing death fom pancytopenia in chronic exposures (at $8.8 \times$ dose level, 187 days, and $1700 \mathrm{r}$, at $4.4 \mathrm{r}$ dose level, 653 days and $2900 \mathrm{r}$, and at. 2.2 dose level, 987 days and $2200 \mathrm{r}$, respectively). (V.E.)

\section{2}

Lorenz, K. P. and Paul Menshaw. RADIODIOLOGC ACTION AND THE KILLING EFECTS OF X-IRAYS ON ACHROMOBACTER FISCMER1. Tadiology 36, 471-81 (1941).

A short historical review has been presented of the prevalling ideas and experimental studies on the biological action of radiation. Experiments are recorded in which the Gurvival curve for Achromobacter fischeri after treat ment with $200 \mathrm{kv} x-\mathrm{rays}$ was found to be of the exponential type. (auth)

123

Pack RADIATIONS. Am. J. Rocntgenol. Radium Therapy 41 , 술셩 $1-3(1939)$.

Arguments are presented in favor of the use of the biologic method of measurement of radiations. Survival data obtained from experiments with the fruit fly, Drosophila, are cited as proof of the constancy of response of biological material. The biological method of measurement can be used to determine surface and depth doses and is 
the only method that can be employed effectively to measure the effectiveness of different wave lengths. It is concluded that this method of measurement is logical, it 124 is accurate, and it ean be put to practical use. (S.V.S)

Powers, E. L., Carlene M. Allen, Charles F. Ehret, Sylvia D. Mayer, Richard Sato, and D. Sheiner. LETHAL CHANCES INDUCED BY X-RAYS IN PARAMECIUM AURELIA, p.159-64 of BIOLOGICAL AND MEDICAL DIVISIONS; QUARTERLY REPORT, FEBRUARY, MARCH APIIL, 1949. Argonne National Lab. [nd] $6 \mathrm{p}$. ANL-4291(p.159-64))

Paramecuum aurelia, variety 4 , was exposed to increasing amounts of $250 \mathrm{kv} x$-rays, ranging from $500 \mathrm{r}$ to 25,000 r. No untoward physiological effects such ag depression of fission rate or delay of entrance into autogamy were observed in the anmals isolated after ir radiation. When the clones arising from these isolations were allowed to go into autogamy the proportion surviving autogany decreased regularly with increasing dosage of $\mathrm{x}-\mathrm{ray}$. Comparison of this dose response with that determined by $\mathrm{SI}^{88}{ }^{80} \mathrm{Y}^{30}$ in solution reveals no significant dufference botween the types of radiation even though the dosage rates differed greatly. (authis

125

Powers, R. L. and Deborah Shefner. BFFCTS OF HIGH DOSAGES OF X-RAYS IN PARAMECIUM AURELIA. Genetics 35, 131(1950).

Stock 51 Paramecium aurelia was given in culture solution dosages of unfiltered $50 \mathrm{kv} X$-rays up to $1,000,000 \mathrm{r}$ at $62,200 \mathrm{r}$ per minute. Fifty per cent of the cells died after $650,000 \mathrm{r}$, and fission rate of survivors was $50 \%$ of control rate at the same level. Death after autogamy increased to $98 \%$ at $50,000 \mathrm{I}$, followed by a decrease to about $80 \%$ at $250,000 \mathrm{r}$ and a subsequent slow rise at higher doses. These regnonses are like those observed lollowing mustard treatment. However, in contrast, the peak in the autogamy curve induced by $\mathrm{X}$-rays is highes $\mathrm{s}$, and it occurs at doses below those causing physiological effects of any magnitude.

126

Quastler, Henry. STUDIES ON ROENTGEN DEATH IN

MCE. I. SURVIVAL TIME AND DOSAGE. AM. J.

Roentgenol. Radium Therapy 54, 449-56(1945).

Mice of $20 \mathrm{gm}$. weight were irgadiated with roentges rays. The doses ranged from 500 to $25,000 \mathrm{r}$. The tissue doses were about homogeneous throughout the whole body. The reactions produced are classified as subacute, acute, and hyperacute reactions. The survival time (between ixradiation and death) is used as a quantitative measure of the irradiation effect. To characterize the reaction of a whole class of mice, we use the mean logarithmic aurvival time, or the almost identical median survival time, i.e., the time after whech one-half of all fatalitios have occurred. The relation between survival time and dose administered shows four zones with distinctive dose-response characteristic3. There are two zones where the survival time decreases with increasing dose, and two plateaus where the survival time aremains constant over considerable dosage ranges. Simple mathematical formulae for the survival tim 127 ime-dosage relations are given. (auth)

Ralston, H. J. THE IMMEOIATE AND DRLAYED ACTION OF X-RAYS UPON THE PROTOZOAN DUNALIELIA SALINA. Am. J. Cancer 37, 288-97(1939).

The survival curves of Dunaluella populations were determined after $\mathrm{x}=1 \mathrm{rradiat}$ ron. The experimental curve changes in form with tume after irradiation. The sugges- tion is advanced, and quantitatively supported, that two separate processes are involved, one causing immediate death and affecting the cytoplasm of the cell, and the other acting indirectly by way of the nueleus, inhibiting reproduction and thus representing death after some time has elapsed. The analysis of survival curves as exemplified by the work of previous investigators is discussed and criticized. (BA)

128

Rekers, Paul E. THE EFIECT OF X-IRRADIATION ON RATS WITH AND WITHOUT BARTONELLA MURIS.

J. Infectious Diseases 88, 224-9(1951).

Rats free of a conmon laboratory infection such as Bartonella muris, showed a mortality rate of $00 \%$ when exposed to single dose, total-body X-irradiation. When these rats had become infected with $B$. muris for 10 days or longer and then irradiated, there was not only prolongation of the survival period but a reduction in the mortality rate to $50 \%$. It seems that the infection not only conferred a resistance to the infection but an increased tolerance to the physical agent $\mathrm{A}$ irradiation. (BA) 129

Sacher, George A. THE ACTUARIAL CALCULUS OF CHRONIC RADIATION LETHALITY, D.82-3 of BIOLOGICAL AND MEDICAL OIVISIONS; QUARTRRLY REPORT, FEBRUARY, MARCH, APRI, 1950. Argonne National Lab. [nd] 2p. (ANL-A151(p.82-3))

The logarithm of the rate of mortality (compertz function) is (except for a short period after birth) approximately a linearly fncreasing function of age for all apecies of mammals investigated and for many other metazoan forms. If the Gomperte function is a linear function of age, all the commonly used actuarial functions can be evaluated exw plicitly. A simplified picture of the effect of exposure to radiations on the Gomgertz function is presented. The ef fect of single exposure may be described as consisting primarily of a displacement upward of the Compertz funetion without change in alope, while the effect of duration of -life exposure is to increase the slope of the Compertz function subsequent to exposure. These effects have been confirmed in investigations on populations of mice and drosophila images. Various consequences of this achema with respect to effects of a single dose at a given age, continuous exposure, ano specie difference are presented. (NSA)

130

Sacher, Grorge A., John Sackis, and Austin M. Brues. ANALYTIC \$TUDY ON ERTECTS OF VAROUS PATTERNS OF X-IRRADIATION ON SURVIVAL. Federation Proc. 8, 136(1949). (ADCU 468)

This document is in abstract form; it is reproduced here in it entirety. Sixteen-hundred female $\mathrm{CF}-1$ mice were treated with $250 \mathrm{lg}$ total-body $\mathrm{z}-$ radiation according to the following dosage patterns: $400 \mathrm{~g}$ in a single dosage, or in 10 or 40 daily treatments; $800 \mathrm{x}$ in 10 or 40 ireatment 1200 r in 40 treatments, $5 \mathrm{~g}$ daily throughout life; $800 \mathrm{r}$ in 40 treatments superimposed upon $5 \mathrm{r}$ daily throughout life. There were 1400 control mice. Gross mortalily rates have been computed as a function of thme, in addition to rates associated with certain prominent types of pathologic change. As a general rule, all patterns of treatment increase the incidence of all types of pathology at all ages. The effect of radiation on rates of mortality is best expressed by means of the Compertzian diagram (logarithm of rate of mortality against time). In this form, all categories of pathology in control animals show a linear in creass with age, as is well known in the case of many human diseases. A dosage administered to youmg adult 
mice within a short interval (weeks) displaces these lines upward by a constant amount. The incidence of lymphoid tumors is phasic and constitutes an exception to this rule. The effect of daily small exposures throughout life combined with a heavy exposure early un life is discussed in terms of gross mortality excluding Iymphoid tumors. To a first approximation, the resulfs are additive on the Compertzian diagram. The results indicate lines along which a calculus of radiation mortality may be developed 131 for arbitrary time patierns of axposure. (auth)

Sacher, George and Sylvamus A. Tyler. SOME BIOLOGICAL PIEIOMENA UNDERL ITG RADITION MORTALITY. Argonme National Lab. Aug. 1, 1947. 25p. (AECD$2024-\mathrm{T})$

In recent years much progress has been made in the applicalion of modern statistical methods to the evaluation and comparison of dosage-morlality curves based on small samples. In this paper an extensive body of dosage-mortality data is analyzed. Records on some thousands of mice which received single doses of $\mathrm{x}$-rays were available. These mice were ir radiated in groups which were kept togethes throughout the period of observation. On each group of mice, information was obtained on (1) the X-ray dose given, (2) age, (3) the propostion of the group which died within 20 days after treatment, and (4) the mear survival time of the nonsurvivors. The analysis was directed toward exhibiting the relations between these variables as clealy and completely as possible. The aim was to determine whether mortality could be investigated as a physiological variable, i.e., whether degree of killing and mean time to death could reveal anything about the functional nature of the mechanisms of radiation injury and mortality. There are several observations which can be made. First, the existence of successive orders of injury can be postulated. A given order of injury can appear only when injury of the previous order has reached a certain degree of severity. Second, if successive orders of injury axist, and if the appearance or non-appearance of a certain order of injury depends on external factors other than the administered dose, then multiple-valued relations betweers dose and mortality can exist. Present evidence indicates that the

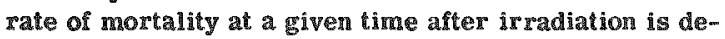
pendent on the instantansous intensity of injury exigting at that time, and further that rate of mortality is a rapidiy increasing function of intensity of injury. The results so far Ireported are sufficient lo justiry the further investigation of the process of adiation injury and mortality, by method which could be developed for use with data from 13 properly designed experiments. (NSA)

stearner, 5. Phyllis. THE EIF FETS OF X IRRADIATON ON RANA PIPIENS (LEOPARD FROG), WTY SPECLA REREREMCE TO SUEVIVAL AND TO THI RESPONSE OT TIE PERPHERAL BCOOD. J. EXPLI. ZOOL. 115, 251-82(1950).

Survival of the Irog, Rana piniens, was observed following x-xay dosages ranging from 000 to $2000 \mathrm{~g}$. The greatest number of deaths occured between 2 and 5 weeks after cxposure to dosages helow $1200 \mathrm{~F}$, and deaths reached a minimum after 6 weeks. Exposure 10700 r results in $50 \%$ moxtality at 6 weeks. Comparison with survival gtudies on is there is a wider range of dosages producing less than $100 \%$ mortality and that the slope of the survival curve is not as great. The cells of the circulating blood of the frog include the types characteristic of higher vertebrates. Peripheral blood counts following exposure to 600 and 900 indicate that the relative sensativitites of the leukocytes to radiation injury were smilar to those of most mammals; however, the response followng irradiation oceured more slowly than an most mammals and indicates that the lower metatbolic rate of the frog may nerease the duration of the socalled latent period. No significant effect wiss seen on the number of thrombocytes or erythrocytes, on on the hemoglobin roncentration. It is suggested that the selatuvely long life span of the erythrocyles would mask any but a prolonged decrease in exythropoiesis. (NSA) 133

Tobias, Cormeliuss A. Hal Anger, P. P. Weymouth, and R. Lowry Dolbson. THE RADIOLOGICAL USE OF HIGH ENERGY DEUTERON BEAMS. Badiation Lab. Univ. of Calif. May 28, 1948. Decl. July 9, 1948. 19p. (AECD-2093-A)

The suitability of the 190 Mev deuteron beam as a source of radiation for producing biological effects was unvestigated. There is a good indication that fast particle beams wll become precision tools un the study of the biological effects of radiation and in experimental tumor therapy. The lethal ef fect of the high energy deuteron beam on Bagg Albino muce was measured. The LD 50 was 150 rep when the length of exposure was 15 seconds. The LD 50 appeared to change approximately proportionately to the fourth root of the length of exposure time. (NSA) 134

Tullis, John L., Carl F. Tessmex, Eugene P. Cronkî́, and 5 . Chambers, JP. THE LETMAL DOSE OF TOTAL-BODY X-RAY IRRADIATION IN SWINE. Radiolory $52,396-400(1949)$.

Adult, hethlty, hybrid swine were exposed in groups of eight (four males and four females) 10 predetermined amounts of total-body radiation delivered in succession to each lateral aspect of the body one meter from a $1,000 \mathrm{kv}$, 3-Ima, X-ray source. Within 30-day period following exposure, animals died from the effects of irtadiation in the proportions of 1 of (12.5\%) that received $200 \mathrm{r}$, 5 of 8 $(62.5 \%)$ that received $300 \mathrm{x}, 7$ of $8(87.5 \%)$ that received $400 \mathrm{r}$, and 8 of $8(100 \%)$ that received $600 \mathrm{r}$. The 30 -day per cent mortality plotted as a function of the bilaterally administered dose gave an " ${ }^{3 "}$-shaped curve, the LD $50 / 30$ of which was taken by interpolation to be $275 x$. (NSA)

\section{5}

Tyle:, Sylvanus A. PROGRESS REPORT (BIOMETRICS) p.61- Of BIOLOCICAL AND MEDICAL DTVISONS; QUARTERLY REPORT, NOVEMBER, DECEMBER, JANUARY, 1948-1949. Argonne National Lab. Feb. 1, 1949. 9p. (ANL-4253(p.61-9))

Statistical investigation of survival al varying single dosages of $\mathrm{x}$-rays, observed for 9.5 young mice by shipmert groups, disclosed a non-significan difference between group survival patterns. By the methods of probit analysis, the 20-day LD-50 and associated standard error for the total group were computed. The problem of determining the mean length of the totality of rays emanating from a point to the bounding sureces of degula configurations is underway. The required mathematical expressions are set down and the mean radial lengths for a circle with point of emanation (1) at the center of the circle and (2) on the boundary (circumference) of the circle are computed. The excretion time relationship, $\mathrm{b}^{-}-\mathbb{E}_{\mathrm{a}} t^{\mathrm{b}}(\mathrm{b} O)$, for animals injected with a selected class of radioisotopes is considered. A modification and extension of this excretion function that ensures, over the lime continum, (1) a finite mean retention fime and (2) a total excretion equal to the injected dose is proposed. (ACD) 


\section{Wavelength Studiss}

136

Chate, H. B., Honry Quastler, and L. S. Skagg. BIOLOGICAL VALUATON OF 20 MLLION VOLT ROENTGEN RAXS. II. DECOLORATION OF MAIR IN MICE.

Am. J. Roentfenol. Radium Therapy 57, 359-61(1947).

Grayung of the coat in mice is produced by appropriate dosas of roentgen rays. It occurs with high and low energy rays (20 million volt and 200 kilovolt, respectively). Quantutat 1 ely, the two radiations are not cqually affective. If the effectiveness of high and low energy pays with regard to the virtoreen $\mathrm{r}$-meter is considered identical, then the effectiveness of high energy rays with regard to graying of the coat $150 \%$ of the effectiveness of low energy rays. (auth)

137

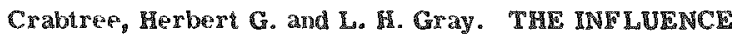
OF WAVE LENGTH ON THE BIOLOGLAL HECTIVENESS OF RADIATION. Brit. J. Radiol. 12,39-53(1939).

The rate of an aerobic glycolysis in nomal and ix rat retinas was studied at trmperatures of $3-8^{\circ} \mathrm{C}$, for bota, famman, and $\mathrm{X}$-1 Iradiation. Ths material is rodiosensitive, a dose of 1000 producing easily measured effects in a few mir. Consuderations due to mitosis do not enter, since the tissue consist of non dividing cells. Detantu are given of the physical measurements made to ensure the equality of dose for the different qualities of radiation. The results of the expts. show no significant variation between the effects of the various qualities of radiation on the rate of glycolysis. (BA)

\section{8}

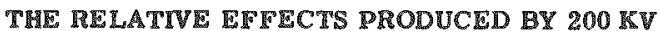
ROENTGEN TAYS, 700 IVV ROENTGEN RAYS, AND GAMMA RAYS, SYMPOSIUM HELD ON THE $33 \mathrm{Yd}$ ANNUA MEETING OI TIIE ANNUAL ROENTGEN RAY SOCIETY, OETROHT, MCH. SEPT. $2 \%$ - 304h 1932.

Am. J. Roentgenol. Radium Thesipy 29, (3), 293-367(1933).

The work reported in this symposium was undertaken to determine whether he effect of these types of radiation were were due to quantity of energy measured only, or whether there was a differential effect due to wave length, especial attent ion being paid to the possible bearing of the results on the radiation therapy of cancer. I. The materal of the ionzation chamber influences greatly the measurements of depth doses in a water phanion. The variation from one type of radiation to another is different for differant chambers. I. The chemical effects studied show some diferental action among themselves and lack of agreement with the small celluloid $\left({ }^{66}\right.$ ain - wall $\left.{ }^{p p}\right)$ Ionization chamber which has besn taken as standard. II and IV. The various biological effects agree fairly well among themselves and with the small celluloid chamber. V. The production of erythema on the human skin does not at all agree with the other biological effects or with the standard ionization chamber. 1t indicates that the akin is more resigtant 10 shoter wave longth (higher voltage) radiation. V. The clinical impression is that, whereas the shin is more resistant to shorter wave lengh radiation, malignant tissue is not. Therefore a selective action of radiation between the wo types of lissue is more marked for the higher voltage. VI. The fact that two or thre biological effects taken by themselves, or even with ionization messurement show no differential action of radiation with respect to wave length, is rot sufficient to war rant the generalizat ion that such action does not exist. Experiments with shin erythoma show that there is a very marlsed differential action in some cases. Explanations are offered for this phenomenon. It is concluded that beter clinical results may be expected from the use of high voltage $\mathrm{X}-\mathrm{day}$ and $;-$ tays. (BA)
139

Gall, E. A., J. R. Lingley, and J. A. Hillden. COMPARATIVE EXPERIMENTAL STUDIES OF $200 \mathrm{KV}$ AND 1000 KV ROENTGEN RAYS. I. THE BIOLOGIC RFIPCTS ON THE EPIPHYSIS OF THE ALBINO RAT. Am. J. Pathol. 16, $805-18(1940)$.

Ninety-five albino rats, 50 to 54 days old, were treated with single dosages of roentgen rays ranging from $600 \mathrm{r}$ to $3000 \mathrm{r}$. The rays were directed at the thighs and knees of one hind extremily. The animals were divided into two groups, one of which received i reatment from a $200 \mathrm{kv}$ machine, and the other from an apparatus operated at $1,000 \mathrm{kv}$. Growth was impeded by all doses utilized and completely inhibited with the higher dosages. Major damage was inflicted upon the epiphyseal cartilage in the zone of chonodroblastic proliferation. Despite the extent of injury to the epiphyseal cartilage neither the articular cartilage nor osteoblastic function was significantly affected. There were no detectable differences in the degree of histological modification produced by equal doses from either the $200 \mathrm{kv}$ or $1,000 \mathrm{kv}$ generators. The ultimate ef fects and the entire sequence of events were exactly similar 140 regardless of the type of rays employed. (BA)

Gall, E. A., J. R. Lingley, and J. A. Hileken. COMPARATIV FXPERMMENTAL STUDIES OF' 200 RV AND 1000 KV ROENTGEN RAYS. III. TIE BIOLOGICAL FIFECT ON THE SKIN OF THE ALBINO RAT. Am. J. Pathol. 17. 319-40(1943).

Equal amounts of roentgen rays, as measured with the Virtoreen dosimeter were obtained from a $200 \mathrm{kv}$ and a $1000 \mathrm{kv}$ generator. Two large groups of albino rats were given graded amounts of irradiation to the glkin of the thigh fron either of these sources. The character of the histologic changeg produced at periods of 1 to 19 weels after irradiation are described. It was noted that high dosages of $200 \mathrm{kv}$ rays $(1800$ to $3000 \mathrm{r})$ caused severe and permanent injury to the oksin and its appendages. Equal doses of aupervoltage rays, however, caused only mild transient morphologic changes. The differences apparent in the lesions produced by the two types of rays are far greater than the amount of error attributable to the Victoreen dosimeter. The hypothesis is therefore advanced that the discrepancy must than result from either a specific cutaneous tolerance for aupervoltage rays (wave length ef fect) or as enhanced slipin penetrability, inherent upon the physical qualities of the rays themselves, due fundamentally to the difference in the degrees of ioxization produced by the two types of rays at the level of the gkin. (auth) 141

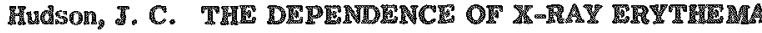
ON WAVI LENGTH. Radiology 29, $95-8(1937)$.

Experimental data are presented showing the variation of erythema dose in roentgens with effective wave lengh over the region from $200 \mathrm{kv}, 0.5 \mathrm{~mm}$ copper filter, to $100 \mathrm{kv}$ unfiltered radiation. Calculations indicate that the difference in doge necessary for an erythema at the various wave lengths is due chiefly to the difference in abeorpton by the tissue. (auh) 142

Lacassagne, A. THE INELUENCE OF WFVIE-IENCTRS ON CERTAIN LESIONS PRODUCED BI TYE IRRADATION OF MCE. Proc. ROy. Soc. Med. 32, 605-12(1946).

Irradiation of a limited area of the dormal lumbar reglon in mice wag carried out with 3 types of radiation of different wave-lenghth: $\mathbb{K}$ radiation of molybdenum, L radiation of ailver and ultraviolet rays. Naturally deep-seated histological lesions, apart from those of the glin, were observed only in the first case. (1) With a dose of $\mathrm{Ir}$ radiation of molybdenum, $3,000 \mathrm{r}$ on the surface of the skin (correspond- 
ing to an incident energy of $760 \mathrm{ergs}$ per square $\mathrm{mm}$ ) the only effect produced on the skin of the adult mouse is a clearly defined loss of hair, effected by selection, without ulceration of any kind. On the other hand, the ovary and parts of the kidney, situated about $5 \mathrm{~mm}$ below the surface, are clearly and seriously affected. There is complete sterilization of the ovary and destruction of the uriniferous tubules, the glomeruli being unaffected. The fact that the intensity of the rays at a depth of $4 \mathrm{~mm}$ is only about $25 \%$ of that on the surface malses it very clear that the epithelial tissues of the ovary and kidney are far more sensilive to irradiation than those of the epidermis. (2) With $\mathrm{L}$ radiation of silver, a dose producing an incident energy of 500 ergs per sq mm applied to the skin of a newborn mouse causes destruction of the epidermis by a histological process of selectrve radioepidermilis. The effect on the skin is thus much nore severe than that caused by a stronger dose of If radiation of molybdenum. This difference is easily explained by the fact that the absorption of the rays by the gkin is proportionately much higher in the case of the longer wave-length. (3) Two radiations of different wave-lengths 1 radiation of silver and ultraviolet rays - absorbed in depth in considerable proportions-- both cause destruction of the skin of the newborn mouse, but the histological process 143 in the two cases is totally different. (auth)

Lasnitzki, Ise and D. E. Lea. VARIATION WIH WAVELENGTHS OF THF BIOLOGICAL EFFET OF RADIATION. Brit. J. Radiol. 13, 149-62(1940).

The effects were compared of radium gamma-rays, and of X-rays of effective wavelengths $0.170,0.150,0.363 \mathrm{~A}$. Dose-rates ranged from 8 to $29 \mathrm{r} / \mathrm{min}$., doses from 20 to $200 \mathrm{r}$. Chick fibroblasts were grown in tissue culture, ixradiated and fixed 80 minutes later. Cells in mitotic prophase, metaphase and telophase were counted in the stained cultures and the mitotic count of an irradiated culture expressed as a percentage of the mitotic count in an unirradiated matched control culture. Usually six pairs of cultures were used for each exposure time. The mitotic percentage diminished with increasing exposure time, the shapes of the curves being similar with the different radiations. The doses in roentgens required for reduction of mitotic percentages to 50 per cent were determined by a least squares method, and were: Gammarays (0.014 A, 26.9 g/min.) 85 +7; X-rays (0.107 A, 8.25 $\mathrm{x} / \mathrm{mins}$ ) $46 \pm 6 ; \mathrm{X}$-rays $\left(0.150 \mathrm{~A}, 29.0 \mathrm{r} / \mathrm{min}_{\mathrm{s}}\right) 42 \pm 3 ; \mathrm{x}-\mathrm{rays}$ $(0.363 \mathrm{~A}, 9.50 \mathrm{r} / \mathrm{min}) 43 \pm 5$. It was concluded that the three wavelengths of $\mathrm{X}$-rays do not difer significantly in efficiency, and that gamma-rays are, per roentgen, less effective than X-rays. Data by Spear and other author are quoted for geutrons on lissue cultures, suggesting that for equal ionization in the lissue cultures, neutron are about as efiective as $\mathrm{X}$-rays. The relation between dosage in roentgens and energy dissipation in the cultures is discussed, with consideration of secondary electrons from the glass coverslips on which the cultures are grown. The precision obtainable in tissue culture experiments is discussed. (GPR)

144

LE, D. E. THE DEPENDENCE OF THE BIOLOGICAL EFFECT OF RADLATION ON WNTENSITYY AND WAVE LENGTH, AS MEASURED BY THE DELAYED LETIEAL ACTION ON CHICI IMBRYOS. Am. J. Roentgenol. Radium Therapy 45, 605-13(1941).

Chick embryos were irradiated after 6 days' incubation, with $\gamma$ rays, $160 \mathrm{kv} x$-rays, or $60 \mathrm{kv}$ s-rays; the criterion of effect was death $2-3$ days afterward. The doses of these 3 qualities of radiation necessary to produce the \$ame ef - fect were in the ratio $1: 0: 0.6: 0.55$. When the given dose was delivered in a single exposure at ntensity greater than $10 x$ per min, the effect was practically independent of intensity. For lower mtensities, or for divided doses, the effect of a given dose was less. A theory of recovery 145 under such conditions is elaborated. (BA)

Lingley, J. R., E. A. Gall, and J. A. Hilcken. COMPARATVE EXPERIMENTAL STUDIES OF 200 KV AND 1000 KV ROENTGEN RAYS. H. THE BIOLOGICAL EFTECTS ON THE BONE MARROW OF THE ALBINO RAT. Am.J. Pathol. 16, 845-54(1940).

Graded doses $(600 r-300 r)$ of irradiation derived in one group from a $200 \mathrm{kv}$ machine and in another from a 1,000 $\mathrm{kv}$ apparatus were directed at the femoral bone marrow of albino rats. A prompt diminution in cellular content proportional to the dose was noted. This was initially contributed to almost wholly by normoblasts and was invar. ably followed by a brief period of apparent regeneration which subsequently gave place to a progressive decline in cellularity. The degree of hypocellularity ulimately attained was again proportional to the dose administered. Qualitatively there was a depression of normoblasts, primitive cells, and myelocytes, with a persistent predominance of mature granulocytes. Comparative analyses after irradiation with $200 \mathrm{lkv}$ and $1,000 \mathrm{kv}$ roentgen rays demonstrated no essential difference in the effects on the marrow of equal amounts of both types of rays. (BA) 146

Luce, W. M., H. Quastler, and E. F. Lanzl. BIOLOGICAL EVALUATION OF 20 MILLION VOLT ROENTGEN RAYS.

V. BAR EFFECT IN DROSOPHLA. Am. J. Roentgenol.

Radium The rapy 64, 963-7(1950).

The reduction of eye facet number in Drosophila melanogaster after irradiation during the sensitive period was used to compare effects of high-and low-energy roentgen rays. It was found that the eficiency $\eta$ (20 million volt at $200 \mathrm{kv}$ ) $\approx 0.85$, the amounts of both radiations being measured by a certain thimble chamber. The result falls in line with values previously obtained with responses to single doses: 0.79 for acute roentgen death in mice, 0.72 for graying of hair in mice, 0.75 for production of sex-linged lethals in Drosophila. (NSA)

147

Mackee, G. M., A. Mutscheller, and A. C. Cipollaro. THE AREA FACTOR IN ROENTGEN IRRADIATION.

Arch Dermatol. and Syphilol. 47, 657-64(1943).

Continuing their previous work on the effects of variations in radiation intensity, the authors studied the radiotherapeutic importance of the area factor in a tolal of 35 persons. Two areas were exposed on the inner surfaces of the forearm or the thigh of both males and females with various complexions and different ages. An area 2.5 cm. squared was used as standard; the size of additional areas was increased or decreased accotdingly. Three series of observations were made: one with 52 kilovolts unfiltered; one with 100 kilovolts unfiltered, and one with 120 kilovolts filtered through $3 \mathrm{~mm}$ aluminium. The results of the $52 \mathrm{kv}$ and $100 \mathrm{kv}$ experiments coincided so closely that the drawing of a third curve could be dispensed with. For nonfiltered or only slightly filtered radiation the area factor above $2 \mathrm{~cm}$. squared showed no appreciable change; in other words, in order to obtain equal biological reactions in areas larger than $2 \mathrm{~cm}$. in diameter considerably increased quantities of radiation are needed to obtain the same result. For highly filtered radiation of hgih voltage, the effect of the area variation is slight in areas above 10 $\mathrm{cm}$. squared; in order to achieve comparable results in 
areas below $16 \mathrm{~cm}$. squared, increased radiation quantites must be applied. Graphs demonstrate the respective factors undirating, e.f., that identical skin reaction will ensue in areas of 2.5 cm. squared, regardless whether $330 \mathrm{r}$ non-filtered or $540 \mathrm{r}$ filtered through $3 \mathrm{~mm}$ of aluminum are administered. Special attention is called to the fact that the tunted area of shin reaction does not exactly correspond to the opening of the lead mask or diaphragm employed. When unfultered radiation of 52 or $100 \mathrm{kv}$ was used, the area of reaction was $1.5 \mathrm{~mm}$ smaller than the mask opening; with $120 \mathrm{kv}$ and $3 \mathrm{~km}$ aluminum filtration of the area of reaction was 3 min smaller than the mask. With further increase of voltage and filtration the area of skun reaction decreased to a greater degree. In practice it wil therefore be necassary to inclease the diameters of the esposed fields at least by the same measurements (approxmately $1.5 \mathrm{~mm}$ for unfilted radiation and $3 \mathrm{~mm}$ for aluminium fltered rays). (GPL)

148

Mîtchell, J. S. WAVE-LENGTH E E IOECW IN THE REACTION OF HUMAN SRIN TO X-AND GAMMA RADLATION. Nature $145,105-6(1950)$.

In the production of most desquamation of normal human gkin, 0.7-0.8 wnit of $\mathrm{k}$ - tadiation of ef fective wave length $145 \mathrm{x} . \mathrm{u}$. is equivalent to $1 \mathrm{r}$ unt of gamma-radiation of $\mathrm{f}$ fective wave lergih $15 \mathrm{xu}$. Attermpted physical and chemical 149 explanations of this fact are not antirely adequate. (CA)

Mottram, T. C. and L. A. Gray, THE RELATIVE RESPONSE ON THE SILIN OT MCE TO X-RADIATION AND GAMMA RADHTION. Brît. J. Radiol. 13, 31-4(1940).

Equal-\$ized sections of the tails of mice were ir radiated with equal quantities of $\mathrm{x}$-radiation and gamma radiation, measured in roentgens. The time factor was the same for both fypes. In each instance the effect from $x$-radiation was the greater. Alhough the dose applied was the same, it does not follow that the energy affecting the tissues was the same. The calcium in the bones and the $S$ in the ghin would give rise to different amounts of photo-electrons with the two applied radiations. As under $x-r a d i a t i o n$ the photo-electron emission is higher, which may account for the difference in observed effect. (R)

150

Packard, C. TIE BIOLOGLAL MEASUREMENT OT MGE VOLTAGE RADIATIONS. Radiology 34, 17-23(1240).

Studies about the biologic effect of roentgen rays on the fruit fly, Orosophilla, which previousiy were made in the range 12 to 220 kilovolts were extended to the range 220 to 900 kilovolt. A Victoreen Dosimeter was calbrated in terms of the biologic dose in such a manne? that the $\%$ of survival of eggs exposed to the radiations between 220 to 900 lillovolts was compared with the survival rate, which follows the same doses of low vollage nadiation. The results were tabulated. With voltages from 400 to $900 \mathrm{kilo}$ volts the biologic dose is about $80 \%$ of the physical dose. No explanation for this descrepancy 15 advanced. Depth dosage measurements indicated that there is but little increase in the biologically measured depth dose when the voltage is increased from 500-900 kilovolts. Depth dosage measurements have been made in $10 \mathrm{~cm}$ depth of a water phantom. The eggrs were covered by a celluloid sheat, for the measurements on he surface of the phantom, and were used in a bakelite contaner sealed with cellophane and beeswax, in the depths. The submersion of the eggs for. periods up to 15 min. did not produre ill elfects, provided the chambers contained not less than 1 ce of air. (BA)

151

Potter, J. Craig. THE BIOLOGICAL FFECT OF ROENGEN RAYS OT LONG AND SHORT WAVELENGTH
ON THE TOTALLY IRRADIATED RAT. Radiology 37, $724-5(1941)$

Experiments were performed in order to determine the comparative lethal effects of total irradiation of the rat by 3 different wavelengths. At the same time the effects on the fertility of rats by irradiation of the ovaries were also investigated. The study was carried out over a period of 8 152 years and 500 rats were used. (BA)

Quastler, Henry. THE BIOLOGC EFFETS OF HIG ENERGY ROENTGEN RAYS. Acta Unio Intern. contra Cancrum 6, 825-30(1949).

The results of an investigation to determine the specific effects of high energy roentgen rays, the qualitative efficiency of such rays and whether the efficiency of the rays varies with regard to different reactions are reported. $A$ clinical and histological study of mice irradiated with 20 Mev roentgen rays, and other investigations on many plant seedings irradiated with high energy indicated that no specific effect of high energy rays could be found. The survival time of irradiated mice, the greying of hair in mice, the stunting of growth in beans and the ses-linked mutations in prosophila were some biologic tests used in reaching the conclusions that the biologic eficiency of high energy photons is roughly three-fourths that of conventional roentgen rays, if both doses are measured and referred to the $25 \mathrm{r}$ thimble chamber of an ionmeter. Bioligic investigations undertalsen to determine whether the differences found in the factor of equivalence are due to random sampling or to actual differences indicated that the qualitative effectiveness of the hish energy rays essms to vary slightly with regard to various biologic reactions. If is concluded that the main advantage of this type of ray will reside in the possibility of administration of optimum tumor doses anywhere within the human body without skin burns and without very severe systemic reactions. (NSA)

153

Quastler, Henry and Robert $\mathrm{K}$. Clark. BOLOGICAL EVALUATION OF 20 MILLION VOLT ROENTGEN RAYS. I. ACUTE ROENTGEN DEATH IN MICE. Am. J.

Roentgenol. Radium Therapy 54, 723-7(1245).

It can be predicted, on the basis of established depth dose curves, that the high energy radiation of the betatron will be useful in the treatment of deep-seated cancer. It camnot be predicted how the betatron radiation will act on any element of living tissue. The elementary actiong of high energy roentgen rays must be established by biological evaluation, conducted in guch a manner that the influence of the denth dose distribution is eliminated. Mice were subject to irradiation of the whole body with a homogeneous dose of 20 million volt roentgen rays. Qualitatively, the elinical and pathological effects observed were the rame as those attending the use of conventional roentgen rays. A quantitative evaluation of effectiveness was obtained, using the Victoreen $r-m e t e r$ as a physical measure and the survival time of mice (between irradiation and death) as a biological measure. It was found that, under the conditions stated, 1 I of the 20 million volt rays of the betatron, is equivalent to about $0.65 \mathrm{r}$ of the conventional $200 \mathrm{kw}$ rays. The significance of this conversion factor will be established only by further experimental investigation. (auth)

154

Quastler, Henry and Elisabeth F. Lanzl. BIOLOGICAL EVALUATION OF 20 MILLION VOLT ROENTGEN RAYS. IV. EFIICIENCY AND DOSAGR RANGE. AM. J.

Roentgenol. Radium Therapy 63, 566-7\$(1950).

The efficiency of 20 million volt roentgen rays produced by the betatron was compared with that of conventional roentgen rays at different dose levels. The lethal effect of 
divided doses on mice was used as test reaction. At a dogage level which causes death after about one week of daily treatments, high-onergy rays are about as efficient as in the nassive dose lest; al a dosage level which causes death after about three weeks of daily treatments, their efficiency significanty lowered. The lower relative efficiency of high-energy roentgen rays at small doses is compared to the higher relative efficiency of neutrons under similas conditions. (auth)

155

Splers, $5 . W$. EFECTIVE ATOMC NUMBER AND ENERGY ABSORPTION IN TISSUES. Brit. J. Tadiol. 19, $52-63(1946)$.

Measurement of the effective atomic number of excised tigsues were made by an absorption method. The results of these studies were used to calculate the linear absorpthon coeficients and the true energy absorption of the tissues for a wide range of wave lengths. From the resulis thus obtained, approximations were made for conditions of actual treatment, indicating that there is a significant dif ference in energy absorption batween fat, muscle, and bone. (2)

156

Stone, Robert 5. and T. M. Robinson. A COMPARISON ON SRIN REACTIONS PRODUCED DI $200 \mathrm{KV}$ AND $1000 \mathrm{KV}$ RAOTATONS. Am. T. Roentgenol. Radum Therapy 44 $601-9(1940)$.

Similar skin reactions can be produced by $200 \mathrm{kv}$ and $1,000 \mathrm{kv}$ radiations on opposite sides of the abdomen of the ame patient. The similarity includes: (1) time of appear ance; (2) degxee of erythema; (3) extent of blistering and peeling; (4) degree of pigmentation: (5) subjective symptom. The physical doses as measured by a thimble chamber are not the same. It required approximately $25 \%$ more roentgens in "total skin dose" of the $1,000 \mathrm{kV}$ radiations. To produce these similar reactions the fractonal doses as well as the total doses must be "biologicaly equivalent". "The aistribution of the doge in days, and the lotal clapsed time mugt also be the same. The difference in the number of roentgens, as measured by a thimble chamber, required to produce "biologically equivalent" sum reactions with the two qualities of radiation is approwmately the same as that sound by Packard using Drosophila eggs. "Biologically equivalent" doses, merely because they mequire greater number of roentgens of higher voliage sadiations, should 15 not be described as "larger doses". (auth)

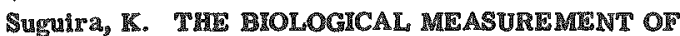
GAMMA RAYS IN "EQUTALENT ROLNTGENS" WITI MOUSE SARCOM 180 AS THE TEST OBJECT. AR.J. Cancer 37, 셩년 $-52(1939)$.

A quantitative comparison of effectiveness of $200 \mathrm{kv}$ $x$-rays and $\gamma$-rays wa made on mouse sarcoma 180. The aurvival curves obtained for 200 lav $\mathrm{x}$-rays and $y$ rays showed an almozt identical course, indicating that the nature of the effect produced by hard $z$-rays and $\gamma$ rays on mouse garcoma 180 irradiated in vitro is saentially the same. An "equivalent poentgen" value for $y$ rays of 96 lo 114 $\mathrm{r} / \mathrm{min} / \mathrm{s}$ was obtained for the standard conditions of $1 \mathrm{~cm}$ distance from an effective point source of $\gamma$ radiation, with a filtation of $0.5 \mathrm{~mm}$ platinum. The corresponding value obtained by Falla and Marinelli with physical measurements was $11 \mathrm{~s} / \mathrm{min} / \mathrm{g}$. The aquivalent rosntgen value of $93.2 \mathrm{r} / \mathrm{min} \mathrm{g}$ was found by Henshaw and Frances for Drosophila Cggs, and $103 \mathrm{r} / \mathrm{min} / \mathrm{g}$ by Muller $10 \mathrm{x}$ Drom sophila pupae. (DA)
Depth Dose Studies

\section{8}

Braestrup, Carl B. Gordon H. Cameron, and Patricia McClement. OFPTP DOSF MEASUREMENTS FOR 250 KV ROENTCEN RAYS. Am. J. Roentgenol. Radium Therapy $61,397-401$ (1949).

Farlier depth dose measurements made by Braest mup have been exicnded to include $250 \mathrm{kv}$ roentgen radiation. The measurements were made in a "presdwood" phantom with a densily of $0.985 \mathrm{gm} / \mathrm{cc}$. as previeously described (Radiology, 42: 258 (1944)). A thimble-type ionization chamber, in conjunction with a projection-type leadshelded elfectrometer, was used to measure the radiations. The results obtained ase shown graphically in the form of curves of "roengers per $100 \mathrm{r}$ in air" as a function of the uepth in cur for a range of $01020 \mathrm{~cm}$. Tabulated dosage calculation for target sin astances of 50,70, and $80 \mathrm{~cm}$ are given in terres of skin dose to permin direct calculathon of the lumor dose tri pereantage of the radation received by the skin. Another table is also given in terms of 100 In ain for the conventence of adiologists who calcu late in terms of the air dose, The results obtained are in general agreement with those obtained by rmen and Packard (Radiology, 44: 367,1945 ), although the depth dowe values obtained by the author (using consterably diffegent equipment) are sowewhat higher. This discrepancy is consigtent with a similar discrepancy observed at $200 \mathrm{kv}$. The results obtained indicate a definite improvement in the depth dose as the quality in increased from a hall-value layer of 2 to $3.2 \mathrm{~mm}$ of $\mathrm{Cu}_{3}$.e., it is possible to thus increase the tumor dose at a given depth for the same exposure of the kin. (N⿴囗十)

\section{9}

Ellinger, Friedrich, and R. Gross. DRPTH DOSAGE MHASURE MENTS BY MEANS OF GOLDF'IS. Radiology 37. $17-21(1941)$.

Conparing the mortality curves obtained by lethal doses administered at the surface and those obtained on the basis of the calculated depth dose it was apparent that the anticlpated depth effect did not occur. There was a greater lethal sfect rom the depth dose than was predicted on the basis of the calculated data. Feneared that the depth dose howed a biologic activily which was $20 \%$ greater than hat 160 calculated for the given surface messurement. (BA)

Jokns, T. W. W. Daxby, T. A. Watsong and C.C. Burkell. COMPARISON OF DOSAGE DISTRIBUTIONS ORTAINADLE WITH 400 RVP W RAYS AND 22 MEV MAYS. Brit. 3. Radiol. 23, 290-9(1050).

laodose curves made in a water phantom with $400 \mathrm{kv}$. rays and 22 Mev rays (from a betat ron) were compared for single and multiple fields. The depth dose was from 2 to 4 times greater with the higher voltage. The ratio of integral dose to tumor dose is about 4 timer as high for 22 mev as for $400 \mathrm{kv}$. The isodose curves with the higher voltage are llat, making the distribution more homogeneous. The depth dose with $\mathrm{H}$-xay rrom the betatron are independent of the gize of the field, which simplifies clinical applications. (R) 161

KIUmpar, J. RELATION BETWE EN THE RADLATON DOSE TOLEHATED DY THE SEIN AND THE DISTANCE

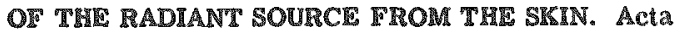
Madiol. 31, 223-32(1949).

The surace cosage of $\gamma$ grays recuired to produce a certain degree of skin reaction by radium therapy depends on the 
distance between the radiophore and the surface of the slin; at small distances a five times greater surface dose may be applied than at yreater distances. On the basis of empirically determined application times and of graphs showing the dependence of the untensity of radiation on distance, it could be demonstrated that the depth dose $4 \mathrm{~mm}$ below the surface was responsible for the reaction of the skin. In all cases, with distances of 0.25 to $0.28 \mathrm{~cm}$, the same degree of reaction was obtained if the dose $4 \mathrm{~mm}$ below the suriace was $3,500: 600 \mathrm{x}$. It is concluded that dosage figures obtained with ionization chambers of an external diameter of 7 to $8 \mathrm{~mm}$ 162 are convenient for all types of surface applicators. (NSA)

IKoch, E. W., D. W. Kerst, and P. Morrîson. RXPERIMENTAL DEPTH DOSE FOR $5,000,000,10,000,000$, $15,000,000$, and $20,000,000$ VOLT X-RAYS. Fadiology 40, 120-7(1243).

X-ray specimens from the betatron are used for depthdose measurements of ionization in a Pressdwood phantom. Data are plotted as percentage of peak ionization with respect to distance of the surface from the phantom. The point of maximum ionization moves into the phantom with increasing energy and the maximum becomes broader. $\mathbb{A}$ dose as high as $75 \%$ is found at $20,000,000 \mathrm{v}$ with the targetto-surface distance only $45 \mathrm{~cm}$. (CA) 163

Laughlin, J. S. and W. D. Davies. PROCFDURE IN DOSE DISTRIBUTION MEASUREMENT OF 25-MEV X-RAYS. Science 111, 514-16(1950).

In preparation of a $25-\mathrm{Mev}$ betatron for human therapy and animal experimentation, extensive measurements of depth-dose and isodose distributions were made under a variety of conditions. Two general techniques were cmployed, one based on measurements with small ionization chambers and the other on $\mathrm{x}-\mathrm{ray}$ film density response. The lonization measurements were carried out in a remotecontrolled water phanton whose construction and mode of operation are described in detail. A series of measurements of the dose-density relation of industrial x-ray films establighed a linear relation $r$. const $\times D$ for type $A$ and type films, where $D$ refers to the net density measured with an electronc densitometer employing a photocell and amplifier, and 1 refers to the dose as recorded by a Victoreen thimble chamber. (NSA)

184

Mayneotd, W. V. and L. F. Lamerton. A SURVET OF DEPTH DOSE DATA. Brit. J. Radiol. 14, 255-04(1041).

A survey has been made of published depth dose and backgcatter data. The influence of (1) focal-skin distance, (2) quality of radiation expressed as half-value layer in copper, and (3) area of field or percentage depth dose has been investigated. In the light of this analygis, published data have been corrected to standard radiation conditions, and mean values obtained. Tables have been drawn up giving the percentage depth dose for qualities of radiation from 1.0 to 5.0 mm copper half-value layer at depths $1020 \mathrm{~cm}$ for field areas up to $400 \mathrm{~cm}^{2}$ and focal-skin distances from 40 to 100 cm. A less comprehensive survey has been made of radiam tions up to $17 \mathrm{~mm}$ copper hali-value layer, the results of which are embodied in curves and tables. Mean values of percentage surface backscatter have been derived from the literature for field areas up to $400 \mathrm{~cm}^{2}$ for qualities up to $14 \mathrm{~mm}$ copper half-value layer. Information is also given whereby one may derive half-value layers in copper from the radiation conditions. (BJR)

\section{5}

Moos, Walter S. EFTECT OF SLOW CATHODE RAXS ON MICROORGANISMS. Nucleonics $\mathbb{a}_{\mathfrak{a}}$ No. 1, 50-3(1951).
The influence of very slow electrons ( 1 to 8 kev) on Pseudomonas aeruginosa has been studied. Results show a maximum effect of the elcetrons at a voltage that gives them a penetration in depth corresponding to the cell diameter. (NSA)

\section{6}

Skaggs, L. S. DEPTH DOSE OF ELECTRONS FROM

THE BETATRON. Radiology 53, 868-73(1949).

By means of a water phantom the author measured the depth dosage due to a collimated beam of electrons from a betatron. Depth dose curves, normalized to peak ionization at $100 \%$, are given for electron energies of $16.4,14.3$, and 12.2 Mev. They show a rise of ionization from the surface of the phantom to a point a few cm deep, the effect becoming less pronounced at higher energies. A short distance beyond the point of maximum ionization, the dose falls rapidly to a small value, and finally reaches a value of about $1.2 \%$ due to $\mathrm{x}-\mathrm{ray}$ background. The extrapolated ranges obtained from the experimental depth dose curver agree to within $3 \%$ with the theoretical ranges. Finally, 167 isodose curves are given for $16.4-\mathrm{Mev}$ electrons. (NSA)

Tullis, John L., Francis W. Chambers, Jr., J. F. Morgan, and John $\mathrm{Pr}_{\mathrm{P}}$ Zeller. MORTALITY IN SWIME AND DOSE DISTRIBUTION STUDIES IN PRANTOMS PXPOSED TO SUPERVOLTAGE I RADIATION. Am. J. Roentgenol. Radium Therapy 67, 620-7(1952) (NP-1887)

Lethal dose curves established for 110- 10 160-pound swine exposed to 2000-kvp total-body $x$ radiation at $2 \mathrm{~m}$ indicate that bilateral (one-half the total dose to each lateral aspect) irradiation is more lethal than unilateral (total dose to one lateral aspect) ir radaition. The $2000-\mathrm{kvp}$ bilateral 8 radiation is also more lethal than 1000 -kvp bilateral $\mathrm{x}$ radiation. The $\mathrm{LD}_{60 / 30}$ values for each technique at $2 \mathrm{~m}$ are 350 to $400 \mathrm{r}$ for 2000 -kvp bilateral, 500 r for 2000 -kwp unilateral, and $510 \mathrm{r}$ for 1000-kvp bilateral exposure. Depth doge values taken throughout a 28 -cm phantom show that the internal distribution of incident radiation is more uniform when it is divided and administered bilaterally. In the case of 1000-kvp bilateral exposure, while the per cent air dose at the periphery is comparable with that in 2000 -kvp bilatteral exposure, the fall off in the mid-portion of the phantom is considerably more. The dependence of dose distribution on method of $x$ radiation and wavelength of the source is demonstrated. The importane of uniform dose distribution and the difficulties in obtaining the same in a subject $28 \mathrm{~cm}$ thick are discussed. (NSA)

\section{Refects of Deta Rays}

\section{8}

Campbell, Berry and Rosalind Novick. ETTECS OF BETA RAYS ON CENTRAL NERVOUS TISSUES. Proc. Soc. Expt1. Biol. Med. 72, 34-8(1949).

The gross morphological changes evolved in the neutrons and glial cells of the nervous system of cats by high doses of lonizing $\beta$ radiations are described here. The use of local ir radiation by means of a radon applicator was made in order to study a sharp gradient of tissue reaction in the the cercbral cortex. Trephining of the calvarium over the parietal region of the cerebral corter was performed under anesthesia, and the region was exposed to the radiations for $5-32$ minutes, the calculated activity of the applicators being $30-78$ mc; the dura was then returned to place, and after the animal was killed, the irradiated region was dissected out fresh or after perfugion with formalin. Restricted legions were lound, complete necrosis being observed in some cases only a few mm. from normal tsaue. 
Nerve cells showed two types of destruction: lysis of the cytoplasmic st ructures and degeneration into a small aggregation of basophilic granules; and, chromophilic alteration of the cells, the pyxamidal cells of layers 2,3 , and 5 evidencing special susceptibility. The astrocytes were the most susceptible of the cerebral cortex cells, undergoing lysis in the middle of the lesions; the mesoglial cells, 169 oligodendroglîa, and microglia were highly resistant.

(NSA)

Carvalho, S. de. APPLICATION OF BETA RADIATION FROM RADIOACTIVE SUBSTANCES ON THF CEREBRAL CORTEX IN THE CAT. Portugaliae acta biol. ser. 1A 175-84(1946).

The cerebral cortices of 17 normal cats were exposed to filtered $1 \mathrm{~s}$ days from Radium $\mathrm{E}$. Only the more penetrating $\beta$-rays, which passed Al filters of $0.10-0.15 \mathrm{~mm}$ gave rise to evident histologic lesions. (BA)

Cupp, M. N., H. I. Rohn, and G. E. Stapleton. THE EFPECT OF LOCAL BETA IRRADIATION FROM AN EXTERNAL SOURCE UPON THE GROWTH OF THE BONES AND SOME OTHER TISSUES OT RATS. Oak Ridge National Lab. July 22,1948 . Decl. Aug. 16, 1948. 10p. (AECD-2219)

One-day-old rats were subjected to one-hal (anterior or posterior) body irradiation by the beta rays of $\mathbb{P}^{92}$. The glkin exposure on the body was about 1,700-2,000 rep. The skin exposure on the tail, hands, feet, forearm, and leg was somewhat less than twice this. The animals were sacrificed at 200 days of age. No cutaneous tumor's occurred. The irradiated animals weighed $5-10 \%$ less than the controls. Males and females reacted alike. Changes were observed only in the part directly is radiated. The "anterior" animals had significantly smaller ears, skulls, and forelimbs; the "posterior" animals had smaller tails, hindlimbs, and testes. Sireletal measurements are given. No effect was observed on the stomach, kidneys, liver, or heart. (NSA)

171

Douglas, D. M., W. R. Chent, and S. Rowlands. ATROPHY OF THE GASTRC GLANDS PRODUCED BY BETA RAYS. HISTOLOGICAL FINDINGS IN ANIMALS. Lancet 258, $1035-8(1850)$.

The stomachs of dogs were exposed to $\beta$-rays from an intragastric balloon having a layer of latex containing $\mathbb{P}^{22}$. Biopsy apecimens taken from 39 to 74 days after irradiation showed atrophy of the gastric glands. The gastric muscularis and submucosa appeared histologically normal. (CA)

Forse, R. Armour, J. B. R. MeKendry, and D. R . Webster. THE EFFEC OT RADLATION FROM RADIOACTIVE TODINE ON GASTRIC SRCRETION. Surgical Forum American College of Surgeons 54-8(1951).

A limited but careinly controlled study of the effect of radiation from radioactive lodine on gastric secretion indicated that if is possible to reduce gastric secretion by radiating the gastric mucosa with gadioactive iodine. The reduction in gastric aecretion produced in one dog by radiating a gastric pouch with 30 me of IsI $^{15}$ for 5 hours has perwisted for 6 months. The reduction in gastric secretion produced in another dog by radiating a gastric pouch with $30 \mathrm{me}$ of $\mathrm{I}^{13 \mathrm{i}}$ for 9.5 hours has started to return to normal 40 days after radiation. This may indicate an individual resistance or ausceptibilily of the gastric mucosa to radiation Irom I's1. If radiation from radioactive jodine is to become of value in the trealment of peptic ulcer, larger doses than 30 me for 9.5 hours will be required and the ghort period of hypersecretion due to stimulation of the gastric mucoa will have lo be controlled. This was done using subsutaneous banthine on the first dog. This dog weighed approsimately $12 \mathrm{lg}$ and it was possible to reduce his postradiation daily secretion from over 100 ce to less than 10 cc with $15 \mathrm{mg}$ of subcutaneous banthine. This parenteral means of administration is extremely effective and may be of value during this period. (NSA) 1.73

Hedin, Raymond F, Winston R. Millex, and Demetrius G. Jelatis. EFTECTS OF BETA IRRADIATION ON GASTRIC ACDITY. Arch. Surg. 61, 748-57(1950).

The stomachs of 10 dogs were exposed to various doses of $\beta$-irradiation from $\mathrm{p}^{92}$ to determine the effect on gastric acidity. The capacity of the dog stomach was determined and a balloon filled with radioactive $P$ solution allowed to come in contact with the ironed out gartric mucosa for various times. There was a strikng correlation between the changes in gastric acidity and histologic changes in the gastric mucosa after various doses of $\mathrm{P}^{32}$. A dosage in the order of 10,000 rep produces marked reduction in acidity for a prolonged time without complete achlorhydria. (CA) 174.

Henshaw, Paul S., R. S. Snider, and $\mathrm{E}$. R. Riley. ABERRANT TISSUE DEVIELOPMENTS IN RATS EXPOSED TO BETA RAYS. THE LATE EFTECTS OF P PETA RAIS. Radiology 52, 401-15(1949).

Phosphorus 32 rays coming from $P$-impregnated plastic were applied to Sprague-Dawley rats single massive doses obtained with an enclosure-type treatment device or by reneated amall daily doses $(6 \times /$ week) obtained by passing the animals on a conveyer belt through a tunnel lined with the radionctive plastic. Tumors of practically every type that can arise from skin and conmective tissue appeared at 10 months to $1 \mathrm{gr}$ after treatment. Tumors arose in animals irrespective of whether they were receiving single or repeated treatments. Epithelial tumor were almost absent in control animals, both males and females, but were present in large numbers in certain experimental groups. Subcutaneous tumors occurred in more than hal the female controls but in control males only rarely. Treatment increased the incidence in females by only a few per cent, but in males many fold. The optimal single dose treatment for tumor induction appeared to be in the range of 4,000 to $6,000 \mathrm{rep}$. In the case of repeated treatments, the $50 \mathrm{rep} /$ day treatments were most effective, however, since the dosage levels were widely separated, this cannot necessarily be regarded a optimal. The tumors in many instances ahowed a high degree of differenciation, with a tendency for the epilhelium to form aquamous-cell and basal-cell carcinomas, as well as adenomas, and for connective tissue to form tendon, cartilage, and even bone. Nonmaligant abnormalities were also seen. (auth)

175

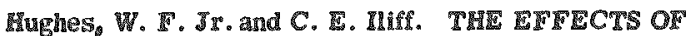
BETA IRRADIATION ON THE RABBIT'S EYE. AM.J. Roentgenol. Radium Therapy 56, 502-12(1846).

The effect of $\beta$ irsadiation from Rn on the normal rabbit' eye are described. A contact glass holder for the $\beta$ applicator is described. Low doses of $\beta$ irradiation affect primarily the epithelium. Larger doses can affect all layers of the conjunctiva and cornea, but the iris and lens are not damaged. Strong exposures to $\beta$ rays are followed by a latent period free of clinical or histologic changes, the length of this period varying inversely with the dosage. Changes thereafter include disorganization of the corneal epithelium, especially loss of cytoplasm, variation in size and shape of the cells, and alteration in the chromatin; loss of stromal cells; decouamation of corneal endothelium with edema of the stroma; relatively little inflammatory cell reaction; Becondary iritis; and late regenerative changes. The cumulative effects of repeated doses of beta radiation is de (auth) 
176

llughes, W. F $J \mathrm{~J}$. and $\mathrm{C}$. $\mathbb{E}$. Iliff. BETA IRRADIATION OF THE EYE. AM, J. Ophthalmol. 32, 351-60(1949).

Beta particles of radon represent a concent rated source of irradiation which is largely absorbed within the furst $2 \mathrm{~mm}$ of tissue and is therefore useful in the treatment of superficial conditions of the lid, the conjunctıva, cornea, and selera. The effective use of $\beta$-irradiation in ophthal mology depends upon and exact knowledge of the differential between the sensitivity of normal tissues and the pathologic tissues to be destroyed. In general, lymphoid tissues (the foldicles in vernal ronjuctuvits) epithelial growths (papillomas), and vascular endothelium (in corneal vascularization) form the most sensitive tissues which can be destroyed by beta-irradiation without undue injury to the normal ocular structures. Because of the great sensitivity of the corneal epithelium and stoma cells, direct irradiation over the connea should be used with caution. Corneal vascularization is best obliterated by irradiation over the scleral portion of the limbus. An ocular condition associated with great cellular destruction or pyogenic infection may respond poorly to beta-irradiation. (NSA)

177

This abstract has been intentionally omitted. 178

Knowlton, Norman P., Jr., Edgar Leifer, John R. Hogness, Louls H. Rempelman, Loren F. Blaney, Dan C. Gill, William R. Oakes, and Charles L. Shafer. BETA RAY BURNS OF HUMAN SKIN. J. Am. Med. Assoc. 141, 239-46 (1949).

Four cases of $\beta$-ray burns of the hands are reported, and the methods of treatment are presented. Estimates indicate that the men received $3,000-16,000$ rep (roentgen equivalent physical) to the outer surface of the skin. The $\beta-5 a y$ dosages were approximately $300-1,600$ rep at $3 \mathrm{~mm}$ below the surface and 30-160 rep at a depth of $6 \mathrm{~mm}$. Since there was little evidence that whole body irradiation exceeded $15 \mathrm{~g}$ of $\gamma$ rays in any case, it is assumed that the whole body exposure had little effect on the radiation effects on the hands. The clinical response to the irradiation consisted of four phases: a tingling and itching at the time of exposure, followed closely by a slight erythema and edema, lasting two to three days; a latent, asymptomatic period of three to Iive days; a secondary erythema, soon followed by vesicle and bulla formation, the vesicles drying and desquamating whin three weeks, leaving a new layer of epthelium; and, a chronic phase characterized by atrophic epithelium and loss of secondary epidermal structure. The only gigniflcant hematologic findings were a neutrophilia, an increased sedimentation rate, and increase in the number of refractive granules in the cytoplasm and a low grade of reticulocytosis. The treatment was local and consisted of roustine burn therapy with accessory chilling, surgical debridement and skin grafting. Rutin, a high protein diet, multigle vitamins and parenteral penicillin were also given. 179 The results are incomplete at this time (NSA)

Lasnitzki, Ilse. THE ETECT OF BETA RAYS ON CELLS CULTIVATED IN VITRO. Brit. J. Radiol. 21, 265ิ-\$(1948).

Tissue cultures of avian fibroblasts were exposed to a dose of $1000 \mathrm{x}$ of pure $\beta$-rays from uranium UX-1. Alteration in mitosis and the production of abnormal mitotic and degenerate cells were examined quantitatively for a period or 24 hours following irradiation. A steep fall in mitosis, the appearance of abnormal mitotic figures, and degenerate cells were observed shortly after exposure. This was followed by a return of mitotic activity accompanied by more abnormal mitosis and cell degeneration. Mitotic recovery was half the normal value at the end of the observation period. A comparison with changes seen in tissue cultures treated with the sane dose of $X$-rays shows that the effect is qualitatively similar, and that the total damage produced is of the same order in both cases, but the time relation differs maxhedly in the two experiments. $\beta$-irradiated cultures show the greatest effect shortly after exposure and an early recovery, while after $X$-rays the radiation effect is delayed and recovery is slow. (NSA) 180

MeKendry, J. B. R. INTRACAVITY VISCERAL RADIATION: IPFECT ON GASTRIC ACID SECRETION. PTOC. Soc. Expt1. Biol. Med. 75, 25-7(1950).

A double-walled balloon apparatus is described suitable for intracavity irradiation of hollow organs accessible to intubation. A solution of a radioactive substance is introduced into the space between the two walls of the balloon after it is in place. In preliminary experiments achlorhydria was induced in dog stomachs by the radiation from Ist. (CA)

181

Parker, H. MOME PHYSICAL ASPECTS OF TRE EFTECTS OF BETA RADIATION ON TISSUE. [Univ. of Chicago Metaliurgical Lab.] Sept. 10, 1243. Decl. June 20, 1950. 55p. (AECD-2859)

This report is concerned with the determination of the depth in tissue at which damage by beta radiation can occur, and the calculation and measurement of beta radiation doses in the superficial tissue arising from expowure to various distributions of beta emilter. Part lig devoted to the calculation of dosages and Part II deals with the expperimental methods and techniques used in beta radiation dogage determinations. (NSA) 182

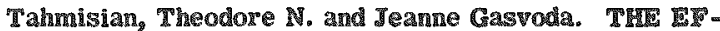
FECT OF BETA IRRADIATION ON THE RESPIRATION AND MORPHOLOGY OF MELANOPLUS DIFIRENTLAL IS EMSRYOS, p.154-80 of BIOLOGICAL AND MEDICAL DVISION; OUARTERLY REPORT, AUGUST, SEPTEMBER, OCTOBER, 1948. Argonne National Lab. Nov. 1, 1048. 27p. (ANL-A227 (p.154-80))

Low diosages of $\beta$ radiation produced extensive damage in the embroyonic cells of grasshoppers. The respiration of grasshopper embroys was increased during irradiation; immediately after irradiation there was inhibition. By the use of Fleming's triple stain it was shown that $\beta$ irradiaton caused pylnotic chromatin to remain as if it were in the metaphase condition. There is evidence that injury sustained by the chromosomes may be direct and indirect. $\beta$ irradiation caused the appearance of mome osmophlic material associated with the chromosomes. The nature of this material is as yet unknown. (auth)

183

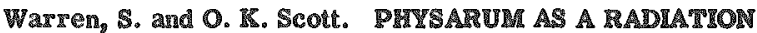
TEST ORIECT. Proc. Soc. Exptl. Biol. Med. 52, 149-51 (1943).

P. polycephalum is a moderately sensitive object for $\beta$ radiation reacting by cessation of growth in the fleld of radiation and sometimes reversal of protoplasmle streaming. (BA)

184

Zixkel, Raymond E., ed. BIOLOGICAL IFTCISY OF EXTERNAI BETA RADIATON. NEW TOR, MCGrawMII Book Co., Inc. 1951. 259p. (National Nuclear Energy Series, Division IV - Plutonium Project Record, Volume 22E) 
The volume presents in book form the first studies of pure $\beta$ ir radiation of considerable tissue areas and of the whole body of laboratory animals. Explanations, with suitable diag rams, are provided for the methods employed using radio- $\mathbf{P}$ placques and impregnated plastic exposure chambers. The first experiments described are on restricted areas of skin exposed to $\beta$ dose of 1000-30,000 r. Damage at lower doses is not greatly different from thermal effects, but at $15,000 \mathrm{r}$ and above, failure of healing may occur and there may be separation of the outer layers of skin. Acute lethal effects caused by total-surface ixradnation were studied. MLD-values were found for baby rats at $2200 \mathrm{r}$; mice, $4700 \mathrm{r}$; rats, $7500 \mathrm{r}$; guinea pigs $7750 \mathrm{r}$; rabbits, about $17,500 \mathrm{r}$. Gross responses and the histopathological changes are described. Of the several species of laboratory animals treated, mive and rats showed significant tumor induction. The optimum dose range for production of tumors was $4000-5000 \mathrm{r}$ of total body-surface radiation, and practically every type of epithelial tumor was produced. Pexiodic total-surface $\beta$ radiation at $625 \mathrm{r} /$ day gave an expanded time-scale as compared with single esposures, so that $50 \%$ mortality occurred in mice at 89 days with a total dose of $43,750 \mathrm{r}$ and in rats at 90 days with an accumulation of $46.250 \mathrm{r}$. Mice and rats exposed to $50 \mathrm{r} /$ day for one year yielded turnor production in the rats and occasional ulceration and some precancerous changes in the mice. Lower daily doses of 5 r were essentially ineffective. In rate exposures of $4000-5000 \mathrm{r}$ or $50 \mathrm{r} /$ day appeared to be optimal for production of malignancies. The book closes with a review of the effects of agents which can produce skin abnormalities. (TIS) 185

This abstract has been intentionally omitted.

\section{Thfects of Neutrons}

\section{6}

Abelson, Philin H. CONFERENCE ON RADIATION CATARACTS AND NEUTRON EFFECTS. Division of Medical sciences, NRC. Feb. 17, 1950. 100p. (NP-1826)

A report is given of the above conference, which comprised two parts, (1) a discussion of the production and detection of neutrons and of experimental neutron-irradi ation of animals and (2) a discussion of various studies made on the lens of the eye and the effects of radiation thereon. 181 references. (NSA)

187

Abelson, Phillip H. and P. G. Kruger. CYCLOTRONINDUCED RADIATION CATARACTS. Science 110, 655-7 (1249).

This is a preliminary report of a survey, carried out under the ausplces of the Division of Medical sciences of the Natlonal Research Council, concerning cyclotroninduced radiation cataracts. A summary is given of the results of an examination carried out at the Wilmer Ophthalmological Institute, Johns Hopkins School of Medicine, by Drs. Alan Woods, Jonas Friedenberg, and Algernon Reese, on 10 scientists, who in connection with their work on high energy machines had been exposed to harmful radiation. Furthermore, information is given as to the probable dates of exposure, the estimated total exposure to neutrons, and the duration of the exposure, for all $10 \mathrm{men}$. It seems probable that the cataracts were caused by chronic exposure to neutrons in the energy range from $0-20$ Mev. (NSA)

\section{8}

Aebersold, P. C. and G. A. Anslow. FAST NEUTRON ABSORPTION IN GASES, WALLS, AND TISSUE. Phys. Mev. 69, 1-21(1946).
The ionization produced by a collimated beam of fast neut rons, fillered for 2 rays, resulting from neut ron collisions un varous hydrogenous and nonhydrogenous gases at pressures ranging from $3 \mathrm{~mm}$ to 3 atm or resulting from collisions with various hydrogenous and nonhydrogenuls wall materals has been measured in unazation chambers of various types. In large wire - defined gas-walled chambers the ioniLing particles are the recolling gas nuelei. The long-range protons of hydrogenous gases expend part of their energy in the wall of the containe at ordindry pressures, hence their ioni/atiun-pressure curves are quasiparabolic and become linear at higher pressures in arcordance with theoretical predictions. The ionization-pressure curves for nonhydrogenous gases are linear except at low pressures. The limiling pressures at which linearity sets in lead to max. values of the range and energy of the recolling muelei and indicate that in the beam of the $37-\mathrm{in}$. Berbeley cyclotron 5 - Mev neut rons predominate. The slopes of the linear portions of the $i-p$ curves permit the calculation of the rate, $\mathbb{F}_{i}$, at which energy is transferged from the neutron beam to nuelear constituents of the gas. The component of the ionization resulting from $\gamma$-rays, produced in the target and in the walls of the collimator and chamber, was found less than $1 \%$ in hydrogenous gases and only $2-6 \%$ in other gases. In thimble chambers with $1-\mathrm{cm}$, and in cylindrical chambers with 2-mm, wall separations, the ionization results in large part from the recolling wall nuelei. Ranges and energies of the heavier recoiling wall neclei are indicated by the limiting pressures revealed in $i-p$ curves. The $\gamma-$ ray percentage is greater than in largevol. chambers. Neutron responses from most nonhydrogenous walls is propurtional to proton content. From the $E_{1}$ values measured in large chambers the energies absorbed per $g$ of various biological substances and of hydrogenous and nonhydrogenous wall materials have been calculated, and (1) predict the respective responses measured in thimble chambers and (2) indicate that the energy absorption per $g$ of tissue must be similar to that for a wall material line amber. Finally analyses of the relative $\mathrm{x}-\mathrm{ray}$ and neutron energy absorptions in such materials yields a factor $\mathrm{k}_{\mathrm{n}}$ greater than 2 , which must be applied to reduce neutron exposures measured in certain hydrogenous-walled chambers to tissue doses. (CA)

\section{9}

Aebersold, P.C. and J. H. Lawrence. THE PHXSIOLOGICAL ETFECTS OF NEUTRON RAYS. AMn. Rev. Physiol. $4,25-48(1942)$.

A review covering production, properties, and measure ment of neutron rays as well as their biological effects and possible therapeutic uses. Thirty-one references appended. (CA)

\section{0}

Biram, M. B. THE CALCULATION OF THE ENERGY DEPOSITION IN SOTT TISSUE BY FAST NEUTRONS EMERGING IROM A NON-CAPTURING MEDIUM. Atomic Energy Research Establishment, Harwell, Berlss. (England). Nov. 21, 1049. 10p. (AERE-T/R-443)

The dose in rep is calculated at interior points of a half space of soft tissue, for the case of monochromatic fast neutrons entering the lissue from a noncapturing isot ropically scattering medium. The variation of mean free path with energy is neglected, and, with this approximation, the biological effects can be shown to be proportional to the energy flux. This is then expressed as a function of depth of tissue, using the Spherical Harmonics $P_{7}$ approximation (MDDC-236). (NSA) 
191

Catsch, A., IX. G. Zimmer, and O. Peter. RADIO-BIOLOGICAL RESEARCH WITH FAST NEUTRONS. Z Naturforsch.2, 1-5(1947).

Radiation effects of fast neut rons are characterized by damage to the leukocytes whereas the erythrocytes are to a large extent resistant to radiation damage. The effect of radiation on the blood forming organs damages cells in the process of dividing as well as immature cells. As a result of the damage to the blood forming organs, secondary changes in the blood appear long after the irradiation. The importance of these results in determining the tolerance close lies in the fact that with neutrons, fhanges in the blood picture are apparent even with very small doses. This is quite different from X-rays. Apparently too in the case of neut rons there is no correlation with radiation intensity nor is there a quick recovery as with X-rays. It is important therefore to study the effects of neutrons, especially the correlation between radiation intensily and damage. It is important too to study the effect on other organs in order to properly protect personnel engaged in nuclear research. (NSA)

192

Cogan, David G., John L. Goff, and Elizabeth Graves. EXPERIMENTAL RADIATION CATARACTS: II. CATARACTS IN THE RABBIT FOLLOWNG SNGLE EXPOSURE TO FAST NEUTRONS. [Los Alamos Scientific Lab.] [nd] 23p. (ALCU-1743)

The production of radiation cataracts and other ocular effects in rabbits has been studied using single exposures to relatively pure neutron sources of $14 \mathrm{Mev}$ and fission energy. The course of events following neut ron radiation is basically similar to that following $\mathrm{x}-\mathrm{ray}$ exposures, and comparable effectivity 28 regards cataracts is obtained with $5 \times 10^{10}$ neutrons, $\mathrm{cm}^{2}$ (14 Mev) or with $9 \times 10^{10}$ neut rons/ $\mathrm{cm}^{2}$ (fission energy) as was obtained with 500 (1.2 Mev). While the morphologic changes in the lenses with neutrong and $x$ rays showed the same differences when heavy and light doses were used, neutron radiation with $14 \mathrm{Mev}$ produced more of the transient vacuoles than did the $\mathrm{g}-\mathrm{ray}$ radiation or the fission neut ron radiation. The threshold dose for cataract production by neutrons is less than $2 \times 10^{9}$ 193 neutrons $/ \mathrm{cm}^{2}$ incident on the lens. (NSA)

Curtis, H. J. and J. D. Teresi. ACTIVITY IN TISSULS INDUCED BY SLOW NEUTRON BOMBARDMENT. [Clinton Labs.] Feb. 25, 1946. 21p. (AECD-2872)

When tissue is exposed to slow neutrong, a nuclear reaction takes place between the neutrons and the varions atoms of the tissue which wlll produce radioactive isotopes. It can be shown theoretically that phosphorus and sodium are the most dangerous elements in this regard, and this prediction has been borne out in experiments on mice. How ever, it is shown that this radiation produced only about 19 of the biological effect produced by a slow neutron exposure; the rest being due to radiations emitted at the time of the exposure. Trom an analysis of the urine for radioactive elements it is possible to estimate the magnitude of a slow neutron exposure. Either phosphorus or sodium analysis should be used depending upon the circurngtances of the exposure and the analysis; but in general it may be said that sodium analysis should be used for short exposures and phosphorus for chronic exposures. A tolerance dose of slow meutrons has been computed as an 8 -hour exposure in a flux of $9 \times 10^{3} / \mathrm{n} / \mathrm{cm}^{2} / \mathrm{sec}$. Using this value it can be shown that under most cireumstances it would be possible to detect an uver-exposure by a urine analysis, but this is not recommended as a routine procedure. (NSA)

\section{4}

Davison, B. PRELIMINARY ESTIMATE OF THE ENERGY DEPOSITION IN SOFT TISSUE DUE TO A IST NEUTRON FLUX. Atomic Energy Research Establishment, Harwell, Berks, (England). 【nd】 34p. (AERE-T/M-13; H.S. 1467)

The energy deposition in soft tissue due to a fast-neutron flux is examined for the following three extreme models: Model $\mathbb{A}$ assumes that after a hydrogen collision the mean free path of a neutron reduces to zero, i.e., all the further collisions take place at the same spot as the first collision with hydrogen. Model B assumes that the mean free path for all energies is the same as for the initial energy, and in all collisions neutrons are scattered isotropically. Model $C$ assumes that on a collision with hydrogen neutron either loses all its energy or continues to move wilh the same energy and in the same direction as before the collision. It is shown that the correct answer should be between the limits set by these three models. For each model the spatial distribution of energy deposition is determined for the case when the human body is replaced by a hall space consisting of soft tissue with neutrons from outside either falling at right angles to the exposed surface, or coming from an isotropic plane source. The total amount of energy deposition in the body, the density of energy deposition at the exposed surface, and the mean depth of energy penetration are fabulated for the three models mentioned above for the initial neutron energy range from 0.5 to 5.0 Mev. (NSA)

Eschenbrenner, A. B., T. Wang, and Eliza Miller. PRELIMINARY REPORT; THE VALUT OF MOUSE TES TES FOR BIOLOGICAL DOSIMITRY OF IONZING RADIATIONS, p.39-41 Of BIOLOGICAL AND MEDICAL DVISIORS QUARTERLY REPORT FHRUART, MARCH, APRIL, 1050. Argonne National Lab. [nd] 3p. (ANL-A51 (p.39-41))

Some preliminary observalions have been made on the pattern of damage and recovery of the testes of mice exposed to neutron irradiation, and the data have been compared with $\mathrm{x}$-irradiation damage and recovery findings from earlier studies. Mice, confined in plastic cages, were exposed to neutrons of an average energy of 4 to $5 \mathrm{Mev}$. The cages were located about 60 crn from the target and the masimum $\gamma$ background of the cyclotron at this distance from the cages was estimated to be about $30 \%$ of the total dose. The animals received a total of $29 \mathrm{~N}$ at about $3 \mathrm{~N} / \mathrm{min}$. Irradiated and nonir radiated mice we re sacrificed and the testes removed at week intervals after exposure. The close similarily of the $x$-ray and neutron data strongly suggested the same biologic response. This is also true for mice erposed to $x$ rays from a 50-Mev betatron. It appears that the response of the mouse testis to irradiation may prove to be a valuable tool for future studies on the relation between biologic effect and physical dose. (NSA)

\section{6}

Evans, Titus C. FFFECTS OF SMALL DALY DOSES OF

FAST NEUTRONS ON MICE. Radiology 50, 811-34(1048).

Dally doses of 0.014 " $\mathbb{N}$ " of fast neutrons $\left(0.07\right.$ " $\mathrm{N}^{\mathrm{N}}$ " per week) equivalent to 0.1 per day of X-rays (on the basis of relative effects of single exposures), had no definite elfect on mice treated for approximately 87 weeks. Some effects (though still not pronounced) were observed in animals exposed dwily to $0.07{ }^{~ "} \mathrm{~N}^{n}$ with at total dose of approximately

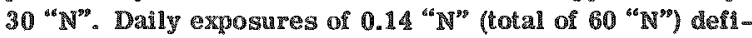
nitely reduced survival time slightly, reduced growth activity of hemopoietic organs and gonads, and slightly increased the frequency of eataracts. Doses of $1.4{ }^{46} \mathrm{~N}^{20} \mathrm{ger}$ day resulted in death of over $50 \%$ of the animals by about the 29 th week (total " $\mathbb{N}^{\text {p }}$ - approximately 200 ). This irradi- 
ation caused pronounced changes within a few weeks in blood counts, activity of hemopoietic organs and gonads. In addi tion, extreme damage to the lens was evident by the time hali of the original number of animals had died. Daily exposures of 1.4 "N" were more effective than those of $11 \mathrm{r}$ (equivalents on the basis of relative effects of single exposures). Some effects, based on total dose at lime of appearance, fndicated a ratio of $12-13 \mathrm{r}-1$ " $\mathrm{N}$ ". The effects of $1.4{ }^{\text {"N}} \mathrm{N}$ " per day on gonads and lens were more pronounced than even this ratio, $12 \mathrm{x}=1$ " $\mathrm{N}$ " would indicate. Considering the results generally, the most important finding was that the lens was especially sensitive to the neutron radiation. (NSA)

197

Evans, Titus C. THE FAST NEUTRON RAZARD.

Nucleonics $4,2-8(1249)$ Mar.

Recent experiments have been made to obtain information on the biological effects of fast neutrons (1-4 Mev) using mice as test objects. The clief purpose of the investigations was to determine whether distribution of the exposure in time would alter the relative effectiveness of neutrons (ca. $4 \mathrm{Mev}$ ) as compared to $\mathrm{X}$-rays (185 kv). The effects of the two types of radiations were determined under three conditions of exposure: (1) single acute, (2) multiple subacute, and (3) multiple very small or chronic. Data on the number of surviwors and mean survival time are shown in a number of curves. An equivalence of $1 \mathrm{n}=10 \mathrm{r}$ has been accepted as a temporary working basis for permissible exposures; however, the difficulty in converting data of the type obtained into data applicable to humans, is noted. (NSA) 198

Gray, L. H., J. C. Mottram, John Read, and F. G. Spear. SOME EXPERIMENTS UPON THE BIOLOCICAL EFECTS OF IST NEUTRONS. Brit. J. Radio. 13, 371-88(1940).

Methods of measuring neutron dosage in biological experiments are not well standardized. Details of a measuring method based on ionization and of one based upon the radlo-activation of $\mathrm{P}$ were discussed. The relation of dose to Inhibition of mitosis in tissue cultures of chick embryo fibroblast: and in roots of broad beans was determined and compared quantitatively with corresponding data obtained with $\gamma$ rayg. In both cases the neutron effect curve was exponential, while the $\gamma$ ray curve was sigmoidal. No intengity dependence was observed in the neutron experiments. The data indicate that the "sensitive volume" must, If apherical, have a radius of aboul $2 \mu$ for the chick fibroblasta and $3 \mu$ for the bean roots. In each case, the same number of lons have to be produced. The differences in the chapes and magnitudes of the mitosig-dose curves indicate that with $y$ rays the ionization is produced by several hundred recoll electrons, and in the neutron case by a single recoil proton. The literature of quantitative effects of fast neutrons is reviewed. A hypothesis is advanced to account for the large relative efficiency of neutrons as compared 199 with $\gamma$ rays. (BA)

Henshaw, D. \$. EFECTS OF PERIODIC DOSES OF SLOW NEUTRONS. Clinton Labs. Aug. 27, 1946. Decl. Feb. 28, 1847. 12p. (MDDC-754; MonH-118)

Periodic doses of a radiation designated as "slow neutron" were given to two groups of $100 \mathrm{CF}-1$ female mice. Each arbitrary unit of dose, expressed in a, was composed of slow neutrons considerably contaminated with fast neutrons. The mice were exposed in a manner similar to that used for fast neutron exposures, but in the cart designed specifically for "slow neutron" irradiation. The result $\mathrm{s}$ are very simllar to those observed as a congequence of periodic Last neutron treatments. A comparison of the shortening of Lfe Induced by each radiation indicates that 1 is is as effec- tive as 7.5 a, a ratio roughly equivalent to that observed after single doses. A dose of 10 a per day depressed the leukocyte level to a range between 2000 and 3000 cells eu $\mathrm{mm}$ more pronounced blood changes probably would have been noted had other strains been used. The terminal pathological condition of the experimental mice was essentially like that observed after periodic fast neutron or gamma ray irradiation. (ADD)

200

Hoffman, Joseph G. IESTIMATION OF SLOW NLUTRON DOSAGE IN WHOLE BODY IRRADIATION. LOS Alamo: Scientific Lab. May 24, 1948. Decl. July 1\%, 1948. 22p. (AECD-2114; LADC-527)

Approximation formulae are used to compute theoretically the integral doses arising from capture gammas frum the reaction $H^{1}(n, \gamma) H^{2}$ in the human body. For an integrated slow neutron density of $4 \cdot 10^{10}$ neutrons gm. tissue the whole boty dose is 0.95 megm-R. This includes the $\mathbb{N}^{1 / 4}(\mathrm{n}, \mathrm{p}) \mathrm{C}^{1 / 2}$ contribution to the dosf. Experiments on a mock-up of the human body illed with $\mathrm{Na}^{24}$ dissolved in water, are described which indicate the validity of the reciprocity theorem between source and absorber. The physical parameters and data from Mayneord's tables on integral doses are shown to apply in the case of the $\mathrm{Na}^{24}$ gamma rays. The data are used to approximate the $\mathrm{H}^{1}$ capture gamma dose which is found to agree with the theoretically computed dose. The use of induced activities in $\mathrm{Na}^{24}$ and $\mathrm{Cl}^{38}$ is discussed with reference to the assessment of tolerance doses of slow neutrons. The gamma activities permit the measurement of slow neutron doses which are 201 35 to 100 times above tolerance levels. (NSA)

Larkin, J. C. SOME PHYSIOLOGICAL CHANGES IN THE SKIN PRODUCED BY NEUTRONS. Am. J. Roentgenol. Radium Therapy $4 \%$, 733-9(1942).

Vasodilation and vasoconstriction of the capillaries, changes in their sensitivity produced by ir radiation with neutrons, changes produced by injecting chemical substances into the shin itself, and skin temperatures were studied with the aid of the 60-in. Berkeley cyclotron. In skin showing an erythematous reaction to the irradiation, a scratch test shows a pergistent white reaction, indicating increased sensitivity of the endothellal tubules. No wheal formation is observed, indicating that the erythematous skin is refractory to histamine action. There is no elevation in skin temperature during the latent period of several days between the Irradiation and the appearance of the exythema, but a marked rise (as much as $3^{\circ} \mathrm{C}$.) after it appears, indicating vasodilation. Even in these areas, the injection of adrenalin, a vasoconsirictant, produces blanching. The nervous mechanism controlling flare formation following the scrateh test is not influenced by Irradiation. (BA)

\section{2}

Lawrence, John $\mathrm{Yr}$. and Ernest O. Lawrence. THR BIOLOGICAL ACTION OF NEUTRON RAYS. proc. Natl. Acad. Sel. U. S. 22, 124-33(1936).

By bombarding beryllium with neutrons in the cyclot ron or magnetic resonance accelerator, an intense source of neutrons, adeçuate for biological investigation, is obtained. After whole body neutron irradiation of white rats, there results a leukopenia, the lymphocytes being more affected than the polymorphonuclears. Per unit of ionization, neutrons are much more effective biologically than are $\mathrm{X}$-rays in producing a lymphopenia in white rats. The ionization produced in tissue by neutrons is many times more dense than that produced by X-rays or gamma rays, so these greater biological effects after neutron irradiation are not surprising. (BA) 
203

Lesitch, James L., Duuglas M. Gdy, and Geurge -A. Neville. EFFECTS OF REPEATED LOW DOSES OF NEUTRONS ON THE ESTROUS CYCLE OF THE WHTE RAT. Am. J. Roentgenol. Radium Theraby 61, 530-3(1949).

Further investigations of the effects of neutrons on white rats with particular reference to the relationships between tumor production and the possible appearance of an irregular estrous cycle are presented. Investigations of the effects of low neutron doses on the estruns cycle of the white rat as determined by vaginal smears are presented. A daily dose of $3 \mathrm{n}$ was given to 23 rats six days a week uniil $111 \mathrm{n}$ had been delivered. Hematological studies indicated a lowering of the white blood cell count to a minimum of $6,000-7,000 \mathrm{cu}$ mm after 16 doses, followed by recovery. A slight luss of weight as well as a retardation of the growth of curred. The main effect was a disruption of the cst rous cycle. Swellings appeared in the mammary 204 clands, and are under investigation. (NSA)

MCDonald, ${ }^{2} . £$ d. NEUTRON EFFECTS ON ANIMALS. Staff of the Biochemical Research Foundation. Baltimore. Willams and Wilkins Company. 1947.

When atomic energy is applied industrially, one of the problems is the protection of workers againgt the chier by product "neutron bombardment". The various types of electro-magnetic and subatomic-particulate radiation are reviewed and the techniques involved in the production of neutrons and the exposure of animals to these are described. The specific effects of ncutruns on rats, rabbits, dogs, and various bacteria and other micro-organisms are described. The method of measuring the dosages is somewhat similar to that for measurement of $\mathrm{X}$-rays in that Victoreen ionization chambers are used to measure radiation intensity. The units are expressed, however, as neut ron radiation Intensity, abbreviated as " $n$ " to distinguish this unit from the roentgen. In these and other studies the biological effect of an $n$-unit of neutrons is different from that of an $x$-unit of X-rays even though both give the same reading when measured with a Victoreen 100-r chamber. In rats the medium lethal dose for neutrons lay between $60-\mathrm{n}$ and $120-\mathrm{n}$. Neutron doses above $180-$ n caused death of rats in from sim to eight days accompanied by extreme loss of weight and maximum leukopenia. A high percentage of the rats surviving for more than 150 days developed malignant tumors of various types. Attention was called to the fact that no ovantan tumors were observed in the neutron reated rats whereas $F$ urth has reported a consistent induction of ovarian tumors in mice with $50 \mathrm{-g}$ of $\mathrm{x}$-radiation. The authors raise the yuestion of whether or not this indicates a difference between the action of $\mathrm{Y}$-rays and neut rons. Furth's animals developed ovarian tumors approximately one year after radiation. The data given for the rats in this book do not appear to extend over more than 150 days. Numerous other specific effects of neutrons are deseribed and in general, testicular changes, blood changes, and other physiolugical effects were similar to those which follow treatment with X-rays and gamma radiation. Since the $\gamma$ radiation was sereened out by three inches of lead. these effects could not have been due to radiation of this type. The authors seem to have been impressed, however, by the fact that neutrons have a greater biological effect than and $\mathrm{x}$-radiation. (GPR) 205

Mitchell, J.S. PROVISIONAL CALCULATION OF THE TOL RANCE IUX OF THERMAL NEUTRONS. Bril. J. Radiol. 20, 79-82(1947).

This paper deals with the derivation of a theoretical tolerance of neutrons in the absence of the availability of any experimental determinations on the subject. Doses are considered in relation to (1) the surface of soft tissues, (2) the $n-\gamma$ reaction in $\mathbb{H}^{1},(3)$ total surface dose, (4) dose in bone. (5) diffusion reflection of thermal neutrons at the surface of the body, and (6) safety factors. Assuming the value of $0.1 \mathrm{r}$ per day recommended by the National Bureau of Standards, Washington (1938), for the tolerance dose rate for total body ? irradiation, the author concludes that the tolerance flux of thermal neutrons should not exceed 1250 thermal neutrons per $\mathrm{cm}^{2}$ per second during a period of eight hours in any one day. (E. G.) 206

Mitchell, J. S. PROVISIONAL CALCULATION OF THE TOLERANCE FLUX OF FAST NEUTRONS. Brit. J. Radiol. $20,177-20(1947)$.

The flus of ast neutrons of energy $\mathbb{E}$, which will give the same energy absorption in an element of volume near the surface of the body as the gamma ray tolerance dose rate of $0.1 x$ in eight hours, is calculated with the aid of Placek'3 expression for the mean energy loss per elastic collision in the slowing down of fast neutrons without capture. If $\mathrm{E}_{0}$ is the energy of a neutron before elastic collision with a nucleus of atomic mass $M$, and $\mathrm{z}$ its energy after collision, then the average value of

$$
\left(\log \frac{\mathbb{E}_{0}}{E}\right)_{A v}=1-\frac{(M-1)^{2}}{2 M} \log \left(\frac{M+1}{M-1}\right)
$$

A useful table is given of the collision cross sections for elements of importance in human tissue and a range of neutron energies, the number of atoms of each element per cc of tissue, the average energy loss of a neutron per collision, and other data used in the calculation. The biological efficiency of ionization due to recoil protons relative to that produced by rays is discussed and the most lukely value for each energy is tabulated. About 80 neutrons per $\mathrm{cm}^{2}$, sec, having energhes in the range 2 to $20 \mathrm{Mev}$, produce energy absorption biologically equivalent to the gamma ray tolerance dose rate, but this number rises to 120 neutrons of $1 \mathrm{MeV}$ and 165 of $0.5 \mathrm{Mev}$. In vlew of the uncertainty in the values chosen for the relative biological efficiencies, and of proposals to reduce the tolerance dose to $0.05 \mathrm{r}$ yer eight-hour day, it is finally suggested that a flux of 20 neutrons per $\mathrm{cm}^{2}$ 'sec be taken as the provisional tolerance flux of neutrons of energy in the range 2 to $20 \mathrm{Mev}$, which can be doubled for neutrons of $0.5 \mathrm{Mev}$ energy. (GM) 207

Mitchell, J. \$. FXPRRMENTS ON TUL MECHANISM OI THE BIOLOGICAL ACTION OF FAST NEUTRONS USING THE SUMMATION METHOD FOR LXTEAL EFPECTS IN MICE (WITH A SECTION OF DOSIMETRY OF FAST NEUTRONS). Rrit. J. of Radiol. 20, 368-80(1247).

Adult male mice were individually exposed either to $\gamma$ radiation from sadium or to a beryllium-polonium neutron source which gave in tissue a dose due to fast neutrons 21 times as great as that due to the associated $\gamma$ rays. It is conchuded that the "mixed" radiations applied consecutively are less lethal than comparable doses of either radiation alone, and that this indicates a difference in the mechanism of the biological action of last neutrons and gamma rays. When the overall time of exposure is 48 hours the relative biological efficlency of neutrons compared with $\gamma$ rays, is 32.0 \pm 8.6 . It is auggested that the lethal effects of $y$ radiations on normal cells are produced, at least in part, by certain types of chromosome interchanges resulting from an intensity-dependent "two hit" process. Possibly, whereas proliferating cells are more sensitive to $\mathrm{g}$ radiation during a short interval in the prophase stage of mitosis, cells exhibit a much more uniform sensitivity to neutrons through- 
out both the resting stage and mitusis, because of the greater efficiency of the neutron radiation. A section deals with the measurement of neutron dose by comparison of the ionization produced in a graphite ionization chamber and a carbon-polystyrene chamber, and the deduction of the cor responding tissue dose. Tables give values of the collision cross sections of $\mathrm{H}, \mathrm{C}, \mathrm{N}$, and $\mathrm{O}$ for neutrons of energy 2.0 to $6.0 \mathrm{Mev}$ and of the fractional energy absurption per unit 208

volume in different media, including water and tissue. [NSA|

Nishina, Y., Y. Sinoto, and O. Sato. EFFECTS OF FAST NEUTRONS UPON PLANTS. III. CYTOPLASML CHANGES IN SPIROGYRA. Cytologia 11,311-18(1940).

The elasticity of cytoplasm in spirogyra, measured by means of the centrifuge method, decreased when exposed to neutron rays. Change of the elasticity induced by the neutron bombardment may be attributed chielly to alteration in the condition of the peripheral cytoplasm especially of Rinnenplasma which connects chloroplasts to the cell membrane. The methods of plasmolysis was also employed, but the 209 results were inconclusive. (Authors' Summary)

Robinson, John N. and Farl T. Engle. EFTECT OF NEUTRON RADLATION ON THE HUMAN TESTES: A CASE REPORT. J. Urol. 61, 781-4(1949).

A. case showing the radiation injury effects from a neutron bombardment inwolving the human testes is presented. The accurate radiation dosage is not known, but the exposure time to the bombardment was only a fraction of a second. Three weeks after injury there was no evidence of spermatozoa, and loss of facial and head hair had occurred. The results of two bilateral testes biopsies are summarized. The first biopsy revealed normal tissue with no tubular fibrosis, but few spermatogonia were present. The second blopsy showed some residual damage to one testis, and marked improvement in the amounts and stages of spermatogenewis in the other testis. The spermatogenesis had begun to refurn between the 12 th and 15 th month following injury. (NSA)

Sato, Keijl and Ioshio Nishina. FFFCTS OF IAST NEUTRONS UPON FOREST TREE SEEDS. II. RELATIONS BETWEEN THE INTEMSITIES OF IRRADIATIONS AND THE GERMINATIONS OF SEEDS, THE GROWTH OF SEEDLINGS OF PLNUS DWNSIFLORA. Sci. Bull. Faculty Agr. Kyushu Univ. 13, 238-42(1951).

Seeds of Pinus densiflora were exposed for 5 to $120 \mathrm{~min}$ to neutrons produced by bombarding Be with 2.8 - to 3 -Mev denterons from the Ingtitute of Physical and Chemical Research cyclotron (this work was carried out in 1243 ). Neutron Intensities are expressed in arbitrary units. The percentages of germinations, the survival rates, and the growth of seedlinges were found to be better in the classes exposed at certain doses than in the controls. Exceeding these limits, the percentages of germinations, the survival rates, and the growth of seedlings were inversely proportional to the doses of radiations. Pine seeds soaked in tap water for 1 to 3 days were more susceptible to neut ron-irradiation 21 damage than were dry seeds. (NSA)

Snell, G. D. and D. C. Aerbersold. THE PLODUCTION OF STTERILTT IN MALE MCE BY IRRADIATION WTTY NEUTRONS. Proc. Nati. Acad. Sci. U. S. 23, 374-8 (1937).

Twelve male mice were irradiated wilh doses ranghng from 110 to $215 \mathrm{r}$ of neulrong and mated to untreated females. Male receiving the larger doses fanled to give ferthle matings. The other males gave fertile matings for about three weeks, after which there followed a sterile period of abuut nine weeks. Production of sperm probably ceases within a few days following treat ment. The initual ferthe period lasts only until the mature spermatosua already in the sperm tract at the time of treatment have become too inartive to effect fertilization. Litter size durnge the initial fertile period was $3.1 \neq 0.5$. For a contrul stoup, the litter size was $8.4,0.6$. Neutron rays, as measured by the amount of jonization produced in a Bakelite-walled thimble chamber are about 5-6 times as potent as $X$-rays in producing dominant lethal changes in the mature sperm of mice. (BA)

212

Snider, R. S. HISTOPATHOLOGICAL STUDHES OF MCE EXPOSLD TO FAST NEUTRON RADIATIONS. Unuv. of Chicago Metallurgical Lab. Dec. 5, 1946. Dec1. Sept. 8, 1947. 34p. (MDDC-1278; CH-3730)

Histological obst revations were made on the followng structures of $\mathrm{CF}-1$ male and female mice after exposure to $65 \mathrm{n}$, and $117 \mathrm{n}$ units of fast neut rons: spleen, liver, pancreas, thymus, bone, cartilape, mesenterir lymph node. heart, Jung, kidney, bone marrow, adrenal, testis, ovary, stomach, duodenum, ileum, colon, peripheral nerve, autonomic ganglia, and skeletal muscle. The bone marrow, thymus, lymph node, and spleen sufered severe damage, and the intestinal epithelium was damaged to a lesser extent. All these organs had completely recovered $b y 30$ days aiter the two lower doses. The testis, which was also badly damaged, showed a few signs of repair 30 days after 65 n

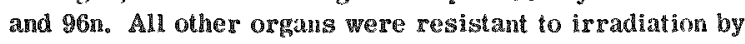
fast neutrons at these doses. Damage was more severe after the larger doses, but no alterations were character. istie of one dose and not of another. In general, the changes observed following fast neut ron irradiation were similar to those deseribed by all workers as following irradiation. A bibliography is appended. (ADD)

213

Snider, R. ACUTE HISTOPATHOLOGICAL FPFTS OF SINGLY DOSES OF SLOW NEUTRONS ON MCE. Univ. of Chicago Metallurgical Lab. Feb. 5, 1947. Decl. Sept. 15, 1348. 17p. (AECD-2326; CM-3766)

Histological observations were made on the following structures of C 1 - 1 female mice (after exposure to 400 arbitrary units of slow neutrons): spleen, liver, pancreas, thymus, bone, cartilage, mesenteric lymph node, heart, lung, kidney, bone marrow, adrenal, ovary, fallopian tube, stomach, duodenum, ileum, colon, peripheral nerve, autonomic ganglia, and skeletal muscle. The spleen, lymph node, bone marrow and thymus were the organs most severely damaged by the treatment. All of these organs had returned to the appearance of those in the control animals by 30 days post -treatment time. No damage was noted in the bone, cartilage, skeletal muscle, peripheral nerve, autonomic ganglia, liver, kidney, panereas, heart, lung, adrenal, stomach, fallopian tube, or colon. There was minor damage in the ovary and duodenum. It is pointed out that the fissue response following slow neutron irradiation is similar to that following X-ray treatment, as reponted in the Herature. (NSA)

21 年 Spear, F.G. TRE ACTION OF NEUTRONS ON BACTERIA. Brit. J. Radiol. 17, 348-51(1944).

Ibxperiments are described which were made to determine the lethal action of a neutron beam on colon bacilli and on spores of Bacillus mesentoricus when they were exposed to gradually increasing doses of radiation and to compare the results with those obtained with other types of radiation. The worls was done with Larence's 37 -inch cyclot ron at the Radiation Laboratory, Herkeley, Caluforma. A diagram is gaven showng the pusition of the bacterial suspensions 7.0 
cm. from the target of the cyclotron and two tables, the first showing the complete details of a single experiment and the second a summary of all the experimental results. An $n$-unit was used for measuring the neutron radiation; it is the neutron dose given when the Victoreen dose meter, (alibrated for $x$-rays records roentgen. Survival curves are given for the cultures of barteria which are exponential in $f, r m$. A tentative comparison between the effects of roentgen, $\gamma$ and neutron irradiation may be obtained by determining the dose required to produce a $5 \%$ lethal action and expressing the result as a $x$ n ratio, $r$ being the measurement of $x$ or i radiation in roentgens and $n$ that of the neutron dose in the author's unit. The $r$ 'n ratio for colon bacilli is 3.2 and that for Dacillus mesentericus 5.3 . The $r$ n ratio shows wide variation according to the material irradiated. It varies from 1 to 10 with a value of 5 to 6 in most esperiments. Until the dosimetry of neutrons has been studied more completely, however, the values of the ratio must be considered provisional. It must not be assumed, however, that for equal energy absorption neutruns produce a greater effect than roentgen or $\gamma$ irradiation. It seems probable that the energy absorbed per unit volume of tissue exposed to 1 in of neutrons is about the 215 same as that from $2.5 \mathrm{r}$ of $\gamma$ irradiation. (AJR)

Speax, F. G., L. H. Gray, and John Read. BLOLOGICAL EFPECTS OF FAST NEUTRONS. Nature 142, 1074-5 (1938).

Chick fibroblasts were irradiated in vitro by $2.4 \mathrm{Mev}$ neutrons produced by bombarding "heavy wax" with a $\sim 700$ microamp. of $300 \mathrm{kv}$. deuterium ions. Doses averaged 3.75 E.S.U. $c c$ of air for 49 minutes in one experiment and 10.5 E.S. U. ce for 42 minutes in another. The biological effect was measured by counting the mitotic cells in the culture and calculating the percentage of the corresponding control counts. Results averaged $81 \%$ and $54 \%$ respectively, in the two experiments. The relation between dose and effect is more nearly exponentially than the sigmoid relation found for $\gamma$-radiation. The exponential relation is that to be expected from a situation in which inhibition of mitosis results from the production of about 1500 ion palrs in a volume of about $3 \mu$ in diameter. (V.E.)

216

Spear, F. G. and IRatharine Tansley. THE ACTION OF NEUTRONS ON THE DEVELOPING RAT RETINA. Brit. J. Radiol. 17, 374-9(1844).

It has been found that degeneration in tissues irradiated with roentgen and radium rays depends on mitotic activily. The experiments here described were made to determine whether neutron irradiation of doses which permit ultimate recovery of the tissues has the same biological effect. The irradiations were given with the cyclotron on immature rat retinas. The technique is described. A table is given showing the mitotic phase and degenerate cell counts after each dose of neutron and a similar table is given for radium irradiation. Degenerate cells appeared after one to three hours. In all the experiments this initial decrease was followed by a renewal of mitosis and with doses above $5 \mathrm{n}$ this return of mitosis was characterized by a marked distortion of the phase ratio. (Doses from $2.5 \mathrm{n}$ to $60 \mathrm{n}$ were used.) As the dose is increased to $60 \mathrm{n}$ the initial decrease of mitosis increases and recovery is more delayed. Some recovery follows the initial fall of mitotic rate caused by neutron irradiation but the increase to a supernormal level seen with roentgen and radium irpadiation does not occur after a dose of more than $2.5 \mathrm{n}$. Up to $1.5 \mathrm{n}$ there is an increase in the prophase count but not in the metaphase and telaphase counts. It seems that the mitotic curves after neutron irradiation do not differ materlally from those after roentgen and radium irradiation but the effect on cell degeneration is much more marked. The degenerate cell count on neutron irradiation is made up partly of cells that are injured and die later when they attempt mitosis. The curve of cell degeneration which accompanies increased dosage occurs much sooner and is markedly steeper than with radium. The curves given show that roughly neutrons are 2.6 times as effective as radium rays per unit energy and 6.5 times as active per $n$ unit. $(\mathbb{R})$

217

Stapleton, G. E. and H. J. Curtis. THE EFECTS OF I AST NEUTRONS ON THE ABILITY OF MICE TO TAKE FORCED EXERCISE. Oak Ridge National Lab. Aug. 22, 1946. Decl. Feb. 26, 1948. 18p. (MDDC-696)

In an attempt to gain some insight into the physiological condition of mice surviving massive doses of radiation, a study of their vitality was undertaken. It was assumed that the ability of these animals to do foreed work would be a measure of their vitality. Stimulating exposed animals to run in exercise wheels to their fullest capacity, has resulted in a demonstration of a striking vitality loss during a post-irradiative period, when no other effects are demonstrable by gross examination. Mice subjected to medianlethal dose of fast neutrons show a continuous vitality loss over a 300 day period following exposure. Control animals show no appreciable loss of vitality up to about elght months of age, after which a gradual loss can be noted, probably due to a normal gereologic process. (ADD) 218

Tait, J. H. THE CALCULATION OF THE TOLERANCE FLUX OF FAST NEUTRONS. Atomic Energy Research Establishment, Harwell, Berks (England). Feb. A, 1949. 13p. (AERE-T/R-273)

The dose in roentgen is calculated at interior points of a semi infinite space consisting of human tissue, for the case when a monochromatic collimated beam of neutrons falls perpendicularly upon the free surface. The variation of the mean free path with energy is neglected, and with this approximation the biological effects can be shown to be proportional to the energy flux. This latter quantity is then determined as a function of the distance into the tissue using the Spherical Harmonics method $\mathbb{P}_{q}$ approximation (ARRE-T/R-231). (NSA)

219

Tait, J. H. THE CALCULATION OF THE ENERGY DEPOSITION IN SOFT TISSUE BY TMERMAL NEUTRONS. Atomic Energy Research Establishment, Larwell, Berlss (England). Oct. 1949. 20p. (AERE-T/R-416)

The amount of energy is calculated, which would have to be deposited by $\gamma$ rays in al semi-infinite medium contalning tissue, to obtain the same biological effect as a unit flux of neutrons entering normally to the free suritace. Two alternative values for the tolerance flux of thermal neutrons are obtained. One is obtained by comparing the doge received in the epidermis, from a given neutron flux over an 8-hour period, with the maximum permissible dose from is rays, and the dose received in the connecting tissue with the maximum permissible dose from $\gamma$ rays. This gives a tolerance flux of $1800 \mathrm{n} / \mathrm{cm}^{2} / \mathrm{sec}$. If, however, it is specified that the maximum dose in the tissue is not to exceed the maximum permissible dose from $\gamma$ rays in an 8 -hour 220 period, the tolerance flux is $1200 \mathrm{n} / \mathrm{cm}^{2} / \mathrm{sec}$. (NSA)

Tait, J. H. CALCULATION OF THE ENERGY DEPOSITHON BY FAST NEUTRONS IN SOFT TISSUE. BRI. $J$. Radiol. 23, 282-6(1950).

The dose in roentgens (rep) is calculated at interior points of a semi-infinite space consisting of human tissue for the case when a broad monochromatic collimated beam 
of neutrons falls perpendicularly upon the free surface. The variation of the mean free path with energy is neglected, and with this approximation the physical effects can be shown to be proportional to the neutron energy flux. This latter chuantity is then determined as a function of the distance into the tissue, using the spherical harmonics $\mathbf{P}_{7}$ azproximation. (NSA)

221

Wilson, A. A. BODY TISSUE IONIZATION DUE TO NEUTRONS. Univ. of Chicago Metallurgical Lab. July 26, 1943. Decl. May 29, 1947. 8p. (MDDC-1025; CK-821)

A calculation is outlined for determining ionization due to slow neutrons. If appears that the number of 2 rays per $\mathrm{cm}^{2}$ per second at the suriace of tissue is approximately equal to the number of slow neutrons entering the tissue per $\mathrm{cm}^{2}$ per second. The ionization in body tissue due to neutrons from an aperture in a pile shield is also calculated. (ADD)

222

Zah, P. A. and F. S. Cooper. LOCALIZATION OF LITHIUM IN TUMOR TISSUE AS A BASIS FOR SLOW NEUTRON THERARY. Science 93, 64(1941).

By injection of the wi salts of dyes into tumor bearing mice, an increase of about $43 \%$ in the radiation dosage of the tumor over that of other tissues was obtained. (BA) 223

Zahl, P. A., F. Cooper, and J.R. Dunning. SOME IN WVO ETECTS OF LOCALIZED NUCLEAR DISINTEGRATMN PRODUCTS ON A TRANSPLANTABLE MOUSE

5rAROCOMA. Proc. Natl. Acad. Sci. U. S. 26, 589-98(1940).

Transplantable mouse sarcomas were injected with Farlous forms of slow-neutron-capturing materials. When the injected tumor-animal was irradiated with slow neutrons, a significant increase in tumor regression was observed. The increase is attributed to the localized ionization seaulting from the nuclear disintegration products of the capture process. (BA)

\section{Efrects of Pile and Fission Radiations}

224

Henshaw, Paul S. and George E. Stapleton. THE BIOLOGIC FFFECTS OF PILE RADLATONS. Radiology 49, 349-60 (1947).

Experiments have been carried out with mice to determine the late effects of perlodic and single exposures of fast neutrons, slow neutrons, $\gamma$-rays, and $\beta$-rays (limited to the sikin). With penetrating radiations these effects are generalized atrophy and neoplasia of hemopoietic organs, attended by shortened life, loss of weight, increase in tumor incidence, etc. No fundamental differences are found in responses of different sexes. The degree of acute damage varies with dose and intensity. The accumulated evidence indicates that the degree of biological effect thus varies with dose and density of ionization produced. (CA) 225

Tobias, C. A., P. P. Weymouth, L.R. Wasserman, and G. S. Stapleton. SOME BIOLOGICAL EFFECTS DUE TO NUCLIAA FISSION. Science 107, 115-18(1948).

Colloidal uranium oxide, enriched with radioactive $U^{235}$, was injected into mice. Three days later the mice were bombarded with a sublethal dose of slow neutrons. All the bombarded mice dled within three weeks; some within eight days. Fur was ruffled, backs were arched, and diarrhea occurred in most of the mice from 2 to 14 days after the bombardment. Neutron bombardment without $U$ killed no control mice. Neither did $U$ that had not been subjected to fission by neutron bombardment. The mice lived even when roentgen rays were substituted for neutrons. Li and $\mathrm{B}$ also undergo nuclear disintegration. Whenever any fissionable substance is bombarded with slow neutrons, the action of the fission products and of induced radioactivity is super imposed on that of the neutrons. The specific effectiveness of fission products may be important in understanding the mechanism of radiation effects. Fission has possible appli cation in radiation therapy, if fissionable element can be put into compounds that localize at specific areas. This knowledge is also of interest from the standpoint of radiation health protection in atomic energy plants. (BA)

\section{Chronice Whole-Body Irradiation}

226

Boche, R. D. ORSERVATIONS ON POPULATIONS OF

ANIMALS EXPOSED TO CRRONIC ROENTGEN RADIA TION. [Univ of Rochester] [nd] Decl. Aug. 17, 1946. 56p. (MDDC-204)

This report discusses the effects of chronic roentgen radiation on the basis of survival of animals, and the effects on their reproductive systems. Dogs, rabbits, mice, rats, and monkeys were used as the experimental animals in this study. (ADD) 227

Brues, A. Mo, G. Sacher, and H. O. France. EFTECT OF TOTAL BODY X-RAY ON WEIGHTS OF ORGANS IN THE RAT. Univ. of Chicago Metallurgical Lab. June 1946. Decl. Aug. 7, 1947. 15p. (MDDC-1197; CH-3835)

Weights and water content of organs were investigated in a group of growing male rats that had recelved $1498 \mathrm{r}$ at $13.82 \mathrm{x} /$ day average. The weights of the majority of the visceral organs were affected less than body weight as a whole; weights of muscle and femur were affected in the same ratio as body weight; and spleen, thymus, gonads, and gonadal fat were reduced out of proportion to body weight. Adrenal weights were normal. Water content per gram of tissue in muscle and bone of irradiated animals was $4.3 \%$ greater than in the control animals. Thus the retardation In growth is similar to that seen in inantion except for the relatively greater effect on organs known to be radiosensitive. Organ weights and total body weights of rats that had received single doses of $\mathrm{X}$-ray up to $100 \mathrm{r}$ several months previously, showed no devlations from the normal. (ADD) 228

Dowdy, A. H., R. D. Boche, and F. W. Bishop. OBSERVATIONS ON ANIMALS EXPOSED TO WHOLE BODY ROENTGEN RADIATION IN DVDDE DOSES OVER LONG PRRIODS. Univ. of Rochester. [nd] Decl. Aug. 21, 1946. 22p. (MDDC-254)

This report discusses only the routhe and the techniques that were used in radiating laboratory animals over a long period of time in order to determine whether the accepted tolerance levels for exposure of persons to roentgen and $\gamma$ radiation are adequate to insure a reasonable degree of gafety. The general objective of the experiments described was to determine whether there exists any exposure level to which animals may be subjected without the manifestation of any deleterious effects, and if so what this level of radiation exposure is, and which organ system is the most sensitive to chronic radiation injury. Actual results are reported elsewhere. (ADD)

229

Eschenbrenner, Allen B. BIOLOGIC EFECTS OF LONGCONTINUED WHOLE BODY IRRADLATION WITH GAMMA RAYS ON MICE, GUINEA PIGS, AND RABBITS. Argonne National Lab. Sept.17, 1946. Decl. Jan. 24, 1947. 46p. (MDDC-642) 
Higtologic observations in animals exposed to long contimued ir radiation with y rays were the following. Mice: The lymphoid tissue was only affected in the $2.2 \mathrm{r}, 4.4 \mathrm{r}$, and $8.8 \mathrm{r}$ levels but changes observed initially did not progress with increasing total dose. Malignant lymphoma (leukemia), which was associated in only a small number of cases with a lymphomatosis in the circulating blood, involved all lymphoid tissues frequently with local invasiveness and metastases to many organs. The interstitial tissue of the testes appeared unaffected for the highest accumulated doses (approx. $5000 \mathrm{r}$ ). Damage to the testes was restricted to the tubules and the various spermatogenic elements, but even at the highest total doses occasional spermatogonia or spermatocytes, but no apermatozoa, were found. Damage to testes is dependent upon dose rate rather than total dose. Damage to the ovaries is dependent upon total dose and is irreversible. It consists of diminution in the number of follicles and final disappearance of these structures followed by tubular downgrowth of the germinal epithelium, and finally the development of several types of ovarian tuxors. In a few cases a sarcoma at the site of the mammary gland was associated with the ovarian tumors. Guinea pigs: The most important changes were observed in the blood forming organs. All animals of the $8.8 \mathrm{x}$ and most of the animals of the $4.4 \mathrm{r}$ and $2.2 \times$ levels died of anemia and thrombocytopenia. Bone marrow in these animals was aplastic to a varying degree. The testes were as radiosensitive as those of mice and showed a similar picture of damage. Ovaries were not affected and showed numerous developing follicles even for total doses as high as $4000 \mathrm{r}$. Lung tumors were observed in two animals that were exposed to total doses of $4000 \mathrm{r}$. These tumors were of alveolar origin. (ADD)

230

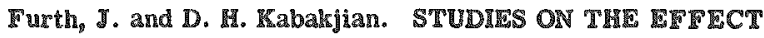
OF CONTINUOUS EXPOSURE OF MICE TO GAMMA RATS OF RADUU. Am. J. Roentgenol. Radium Therapy 32, $227-34$ (1934).

Mice were exposed continuously to $y$ rays emanating from 0.003 to $5 \mathrm{mg}$. of Ra. Rays from 1 to $5 \mathrm{mg}$. of Ra shortened the life of the animals, the average length of life after exposure being 66 and 106 days. The leulrocyte count of mice esposed continuously to $5 \mathrm{mg}$. of Ra dropped much below normal within a week, yet the mice lived for $2-3$ months in spite of the uninterrupted exposure to the same quantity of rays. Continuous exposure to $0.3 \mathrm{mg}$. and to smaller amounts of Ra did not prevent the development of spontaneous neoplasm (lymphoid and myeloid leukemia, tumors of the lung, heart, and ovary). Continuous exposure of mice with transmitted lymphomatosis to $1-5 \mathrm{mg}$. of Ra did not prevent the falal termination of the disease but often prolonged life. These rays increased musceptbility to trans 231 mitted lymphomatosis. (BA)

Lorenz, Egon. BIOLOGIC EFFETS OF LONG-CONTINUED WHOLI BODY IRRADIATION WITH GAMMA RAYS ON MICE, CUTNEA PICS, AND RABBITS, PART II. Argonne National Lab. Oct. 29, 1046. 16p. (MDDC-654; C. -3653 )

Experimental arrangements are described for the simultaneous irradiation of considerable numbers of animals with $\gamma$ rays. Three experimental setups were avallable. In the first a source of approximately 1 g. of radium element was used, with four sets of shelves forming part of a sphere surrounding the source. Dally doses at the different ghelves were $8.8 \mathrm{r}, 4.4 \mathrm{r}, 2.2 \mathrm{r}$, and $1.1 \mathrm{r}$ in eight hours. In the gecond setup a source of $1000 \mathrm{mg}$. radium element in a lead box with a hinged lid was employed to irradiate one set of shelves. The daily dose at this set of shelves was 0.11
In in eight hours. In the third getup a mource of $250 \mathrm{mg}$. element, also housed in a lead box, was used with six sets of shelves. Here the exposure was continuous, the daily doses amounting to $8.2 \mathrm{x}, 4.4 \mathrm{r}, 2.2 \mathrm{x}, 1.1 \mathrm{r}, 0.55 \mathrm{~s}$, and 0.11 in 24 hours. Dose measurements with callbrated 0.25 $x$, Victoreen lonization chambers, were made in every one of the animal cages which were placed on the different shelves. Errors are discussed which arise malny from the dimensions of the cages. They are for mouse and guinea pig cages approximately $\pm 15 \%$, and for rabbit cages $\pm 25 \%$ The precantions taken to reduce the residual dose to a minimurn are described. (ADD)

LOY En, EgOn. BIOLOGIC EIIECTS OF LONG CONTINUED WMOLI BODY IRRADITION WTTH CAMMA RAYS ON MICE, GUINEA PIGS, AND RABBITS, PART 111. Argome National Lab. Nov. 14, 1046. Decl. Feb. 5, 194\%. 118p. (MDDC-65. C

Experimental arrangements and amounts of radiation used are given in the previous abstract. On all daily dose levels, including the 0.11 Ievel, there was a decrease in the life span of mice. The decrease in life span was pronounced in guinea pige exposed to $8.8 \mathrm{I}, 4.4 \mathrm{~F}$, and $2.2 \mathrm{~F}$ per day. The life span of rabbits was decreased in the animals exposed to $8.8 \mathrm{r}$ and $4.4 \mathrm{~m}$. An increase in weight was found in irradiated animals on the lower exposure levels mounting, in male mice exposed to $1.1 \mathrm{r}$, to $50 \%$ of that of the control animals. The hemopoietic system of rabbits is extremely radio resiatant, and changes in the blood picture were found only in the animals exposed 108.8 r per day. The hemopoietic system of mice is also radioregistant; the main exfect of irgadiation consisting in a depression of the lymphocyte count. No damage to bone marrow was observed (nax. accumulated dose 5800 r). The hemopoietic system of guinea pigs is very radiosenst tive. Anemia and thrombocylopenia, were observed in all animals exposed to $8.8 \mathrm{r}$ and $\mathrm{k}$ most animals esposed to $4.4 \mathrm{r}$ and $2.2 \mathrm{~g}$ per day. Bone mayrow damage of moderate to severe degree was associated with the anemia. The carclnogenic action of irradiaton was mainly observed in mice, but some irradtated guinea pigs and rabbits also developed tunors after 3 years of irradiation. The observed tumors are described in detall. (ADD)

\section{3}

LOE Enz, EgOn. SOME BIOLOCICAL PFIECTS OF LONGCONTINUED IRRADIATON. ARn. J. Roentgenol. Radium Therapy 63, 176-85(1050).

The data presented permit the lollowing conelustons: Chronic irradiation will reduce the life gpan of esperimental animals. This effect is a iunetion of many factors some of which are unknown. The effects of irradiation are seen relative to a certain degree. Our experimentr do not give a clear-cut answer to the question whether or not a 0.1 roentgen (the so-called pormisgble dose for man) also will cause a reduction in life gpan. Data on higher dosage rates make such a result likely but in any case the reduction at this dose level will be small. The determination of the extent of the reduction of 1fe apan for this important dose level reguires the collection of experimental data on a large group of animals. Carcinogenis plays the most important role in the reduction of the life mpan in mice. The carchogente action of chronic irsadimtion has been observed lin three apecles, one of which, the guinea pig lo considered one of the least aurceptblo animals to gpontaneous tumos formation. Iit seens that we are justified In assuming that this carcinogenic action of chronic irgadatlon will hold lor other gpeelos as well ar man. (Author's Summary) 
234

Lorenz, Egon, Allen B. Eschenbrenner, Walter $\mathbb{E}_{\text {. Heston, }}$ and Delta Uphof?. MAMMARY-TUMOR INCLDENCE IN FEMALE C 3 HD MICE FOLLOWING LONG CONTINUED GAMMA IRRADIATION. J. NaAl. Cancer InSt. 11, 947-61 (1951).

Virgin $\mathrm{C}_{3} \mathrm{Hb}$ mice were chronically irradiated over total body with $8.8 \mathrm{rally}(8 \mathrm{hr})$ of $\gamma$ radiation from a $\mathrm{RaSO}_{4}$ gource beginning at 1 month of age and continuing throughout their entire 1he mpan. Mean survival time of irradiated animals was shortened by $36 \%$ in comparison to nonirradiated controls. The injurious action of the irradiation was evidenced by a considerable reduction in weight after 1 ys of age. Of the animals dry developed tumors of the mammary gland, $88 \%$ ovarian tumors, and $36 \%$ lymphoid tumors. Pulmonary tumor and hepatoma incidences were also increased in experimental animals in comparison to non irradiated controls. Physlological and hormonal factors 235 involved are discuøsed. (NSA)

Lorenz, Bgon and W. E. Heston. BIOLOGIC EFITCTS OF LONG-CONTINUED WTOLE BODY IRRADIATION WITY GAMMA RAYS ON MICTI, GUINEA PIGS, AND RABRITS, PART I. Argome National Lak. Oct. 29, 1946. Dec. 6 . 6, 194\%. 12p. (MDDC-653)

Male and female mice of the C3L gtrain were conturously irradiated with $4.4 \mathrm{r}, 1.1 \mathrm{r}, 0.11 \mathrm{r}$, and $0.044 \mathrm{x}$ per day. No sigmulicant damage to the hematopoietic system as evidenced by blood counts was found although the highest total dose was over $2000 \times$. Mmmary tumor Incidence was not changed significantly in the animals exposed on the different dose levels. Histologlcally only the gonad showed Jadiation damage and mainly in the animals exposed to 4.4 r per day. The damage consisted in the testes in diminished apermatogemegis and was reverable. Irradiation damage to the ovarles was irreverglble and progressive and resulted in some cases in tubular downgrowths of the germ!mal epithelium which progressed to early tumor formation. Breeding experiments indicated that C3H females were permanently sterilized with total doses of 465 g given at the rate of $4.4 \mathrm{~s}$ per day. Subsequent generations reared and malntaned dr exposure plelds of $1.1 \mathrm{r}$ and 0.11 per day showed no damage to chromosomes as ovidenced by raising of 5 to 6 generations with normal litter gize and 29 arently normal life span. (ADD)

Lorena, Egon, Leon 0. Jacobson and Harriet Sutton.

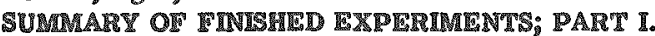
SULVIVAL AND BLOOD PUCTURE OF HYBRE GUINEA PIGS RXPOSED TO LONG-CONTINUED TOTAL-BODY GAMMA RADIATION, p.38-52 Of BIOLOGICAL AND MEDICAL DIVISIONS; QUARTERLY REPORT, NOVEMBER, DFCEMBER, JANUARY, 1949-50. Argonne National Lab. [nd] 15p. (ANL-4401(p.38-52))

Groupe of hybrid guinea pigs were chronically exposed to $\gamma$ radiation at $8.8,4.4,2.2,1.1$, or $0.11 \mathrm{~s}, 8 \mathrm{hr} / \mathrm{day}, 7$ days/week, begtnning in Tebruary 1043. The last curvivor died in May 1949. In the animals exposed to 2.2,4.4, and r.8 Ithere was a pronounced tnerease in mortality. All the animals in the 8.8 group died of pancytopenta, which aleo caused most of the deaths at the other two levelw. There were no deaths from pancytopenia in the $1.1 \mathrm{r}$ group. In guinea pigs anpears that death from radiation-induced pancytopenta is a functlos of dose rate rather than total doce, and there is no linear relationshtp between mean survival the and mean lethal total dose. (NSA) 237

Loterna, Egon, Leon O. Jacobson, Earretet Sutton, and R. SchweIstha. SUMMARY OF FINISPYD EXPYRMMPITS; PART II. RRCOVRRY FROM ANEMAIINDUCED IN
GUMIEA PIGS BY A LIMITED EXPOSURE TO CHRONIC TOTAL-BODY GAMMA RADLATION, p.53-71 of BIOLOGICAL AND MEDICAL DIVISIONS; QUARTERLY REPORT, NOVEMBER, DECEMBER, JANUARY, 1949-50. Argonne National Lab. [nd] 19p. (ANL-4401(p.53-71))

Inbred guinea pige were given limited chronic exposure to $\mathrm{g}$ radiation. The smallest accumulated dosage was 757 I (86 days) and the largest, 1804 (205 days). The animals were removed from the field when the red blood counts reached about 2.5 millon. Three different responses followed removal from exposure: (1) some animals died withIn a few weekg; (2) some showed partial recovery lasting about 25 weeks at which lime there was a lethal recurrent anemia: and (3) in come there was complete recovery. Blood data are given for each of these groupings. Pancylopenta was the cause of death in guinea pigs esposed to 8.8 ro (8 hr/day, 7 days/week). There was guch an abrupt onset of anernia that a hemolytic factor was suggested. (NSA) 238

Lorenz, Egon, Delta Uphor, harriet Sutton, and $\mathbb{R}$. Schweisthal. PROGRESS REPORT: THE 30-DAY LD FOR HYBRID GUINEA PICS, P.50-2 of BIOLOGICAL AND MEDICAL DIVISIONS; QUARTERLY REIPORT F'DRUARY, MARCH, APRI, 1950. Argonne National Lab. [nd] 3p. (ANL-4451(p.50-2))

In previous experiments designed to determine the 30 day $L_{00}$ for hybrid guinea pige, groups of animals were fiven single total-body esposures to 220,260 , and $310 \mathrm{x}$ of 8 radiation. This study has been coneluded with two addi tonal exposure groups of 360 and $420 \mathrm{~g}$. In the flrst three groups there was mo ses difference in gurvival, but in the last two groups, the females seemed more radiosensitive than the males. However, this sinding is probably due to chance since the experiments were spread over a year and hybrid stock was used. The 30-day $\mathrm{LD}_{50}$ ds calculated to be $409 \mathrm{x}$ with 2 lisnits of 307 and $543 \mathrm{r}$. This value is at variance with previously publighed data of other investigatorg, and it is suggested that the low Ifgures reported by these investigators may have been cauged by irradiating diseased guinea pigs. (NSA)

239

Metcalf, Roger C. and Frank A. Inda. PATHOLOGY IN ANIMALS SUBJECTED TO REPEATED DAILY EXPOSURE TO RORNTGEN RAXS. Atomic Rnergy Project, Univ. of Rochester. July 5,1851 . $52 p$. (UR-88)

Detalled observations and conclustone are presented on thasue Injuries observed in rats exposed to $0.1,0.5,1.0$, or $10 \mathrm{x} / \mathrm{day}$ of $\mathrm{x}$ irradiation for $2 \mathrm{yr}$, in rabbits exposed to the arue daly doses for $1 \mathrm{yx}$, and in doge exposed to $0.1,0.5$, $1.0,3.0,6.0$, and 10.0 is for either 500 or 622 days. In rats, the testes are geverely injured with administration of 10 $\mathrm{r} / \mathrm{day}$, but not spectically injured at $0.1,0.5$, or $1.0 \mathrm{x} / \mathrm{day}$. There is evidence to suggest that the incidence of leukemia In all treated rats and mammaxy fibroadenomata in females ta increased by chronic $x$ radiation. There is no histologic evidence of $\mathrm{x}$-radiation injury in other organs. The histologic changes are not influenced by the voliage used in this experiment. In rabbits, the only consigtent lesion attributable to 8 radiation 1 s testicular injury in the 10-r/day group. Injury to teates, appearance of bone marrow hypoplasta, decrease of megalaryocyter, local bowel hemorrhages, and lymph node hemorrhage in the dogs are related to the chronic $x$ Irradlation. 18 figures. (NSA) 240

Pape, Rudolph. RIOLOGICAL IFFECTS OF VERY SMALL X-RAY DOSES ADMINISTERED DAILY FOR ONE FRAR. Strahlentherapie 84, 245-54(2951).

It is posstble to stimulate blood-forming tissues by means of microloses. One rat was Irradiated for 3 month and two rats for $1 \mathrm{yr}$ with dally doses of $0.25 \mathrm{~g}$. Reactions 
were noted in the reticulo-endothelium of the spleen and liver, the resistance of the spleen follicles was lound to be increased, and a considerable lymphocytosis was observed. (NSA)

241

Prosser, C. L. and M. C. Monore. THF CLINICAL PHYSIOLOGY OF DOGS EXPOSED TO DALY TOTALBODY DOSES OF X-RAYS. Univ. of Chicago Metalluggical Lab. Feb. 6, 124\%. Decl. Sept. 9, 194\%. 109p. (MDDC-1271; CRT-3658)

Dogs that received $50 \mathrm{~F}$ and $40 \mathrm{~F}$ and one dog that received $25 \mathrm{x}$ dally, dled after accumulating an average of $380 \mathrm{r}$. Clinically, these animals resembled dogs that received single, acute, lethal doses of $\mathrm{z}-\mathrm{g}$ radiation. They developed a severe loucopenia, showed terminal revereal of heterophil lymphocyte jatio, and had a mid anemia accompanied by increased plasma volume. They differed from single dowe dogs in ahowing earleg diminutions in food and water intake and earlier weight loss. Dogs that received 12.3 $\mathrm{x}$, and a young dog that recelved $25 \mathrm{I}$ dally, accumulated an average of $1262 \mathrm{z}$ and clincally showed a Bubacute picture in which aplastic anemia was prominent. Durdng the first two months of treatment, leucopenia and anemla developed slowly. There was no change ln food and water balance or in body weight unt1 $65-30 \%$ of the total dose had been accumulated. The destruction of red cells was accelerated during the last month of life, terminal hemoglobins averaged 3.4 as compared whth $7.8 \mathrm{~g} / 100 \mathrm{ml}$. at the higher doses, and mean corpuscular volumes increased. (ADD)

242

Sacher, George A. PRELIMINARY RPPORT: DAILY DOSE IETHALITY DATA FOR RABBITS, p. 128-35 of BIOLOGICAL AND MEDICAL DIVISIONS, OUARTERLY REPORT, AUGUST, SEMTEMRR, OCTORER 1350. Argonne National Lab. Oct. 1950. 7p. (ANL-4531(p.12835))

Forty-eight rablits of the Swift Birain were exposed to dally doses of $200 \mathrm{kv}$. $\mathrm{x}$-rays at rates ranging from 40 to 325 I per day, for 6 days per week. Stx animals were irradlated at 11 If per minute, and the remainder at $3.5 \mathrm{~F}$ per minute. The derived Impulge lethalily function of the rabbit shows pealts at 12, 25, and 70 day to slngle doses show maximum xate of mortality at 10 days, with a secondary peas at 6 days. It was concluded that the cumulant lethality function of the rabloit anut conIIft of a guccesslon of waves. The mouse ghows a momeWhat similar behavior in that its impulse lethality function show peals of inyry at certan times. However, 1 barely reaches nornally or ollghtly exceeds it in recovery, whlle the rabbit ghows translent states of marked supernormalsty. (V.E.)

242

Vogel, Howard H., Jr. THE EIFCT OF IR IRLADIATION ON FIGITING BEAMVIOR IN MALIS MCE. Anat. Record 108. $547(1050)$.

This paner is one abstract and is reproduced here in its entirety.

These experiments were carried out to test the effects of chronic $x$ irradiation upon fighting behavior. Male mice (5 weeks old) of three strains, C-57 black, C-3II agouti, and $C F-1$, were hept in isolated cages and were traned to fight in a multiple-escape pen. Six males were traned to become argressive, whereas, under the condit ions of the experiment, Thhting was inhibited in their daily opponens. Aler the frained fighters had defeated a dozen other males of their strain, they were irradiated daily with $50 \mathrm{r}$ of $\mathrm{x}$ radiation, 5 days a week, and fighting was continued daily. The body weights of the irradiated fighting mice were compared with those of nonirgadiated controls over a period of approximately 15 weeks. The accumulated $\mathrm{x}-\mathrm{ray}$ dosage given to each of the 6 experimental mice at the time all fighting ceased and at the time of death are given. The effects of $\mathrm{x}$ irradiation upon fight ing behavior in male mice were cumulative. Tach fightimg mouse retained it characteristic method of attack up to the day when all fighting ceased. There was a definite threshold after which no fighting occurred, but before this threshold the irradiated fighters defeated their daily opponents. The aggressiveness of the attacks decreased as the cumulative $\mathrm{x}-\mathrm{ray}$ dose increased. The condintoning that the mice had received was gtrong, and it look a large dosage (1200 to more than $2000 \mathrm{r}$ ) to eliminate it completely. All signs of fighting were not suppressed until shortly before the death of the irradiated animal.

\section{X- and Gamma Regional Irradiation}

244

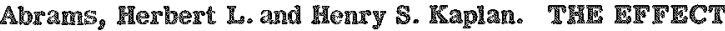
OF SHIRELDNG ON MORTALITY FOLLOWNG IRRADIATION. Stanford Med. Bull. $9,165-6(1951)$.

Lead shelds placed over the head, chest, abdomen, or thighs duxing Irradiation conferred a glgnifleant degree of protection on mice of all tour groups as compared wh unshielded controls. Protection was most gtrinding when the abdonen was shelded. (INSA)

285

Arle, Irving M. TRI DOSES OF ROENTGEN RADIATION UPON THE LIVIER. Radology 5\%, 561-75(1251).

The acute morphologheal changes in the rabbit's liver collowing slrgle massive doses of $x$ radiation to that organ were studled. Single doses of 300 to $100,000 \mathrm{r}$ at $00 \mathrm{kz}$ were given and the animals were gacrifleed or succumbed at varlous interval thereafter. Filema, hyperemia, and lenkocytic infilfration were produced in the vascular gys-

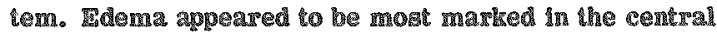
porthon of the lobule and polymornhonuclear leukoryt $1 \mathrm{c}$ Infurration in the portal areas. IIver cello were damaged or destroyed in proportion to ine dosage, vogy high doses caundng complete necrosls, while the lower dosages groduced only slight swolling of the cells. In the lowar dosage rams, focal destruction of the liver colls was noduced, Indicating apparently a variable bensitivity to radiathon. Repalr was rapid when possible; otherwise death occursed. depending apon the dosage and extent of the damage. (NSA) 240

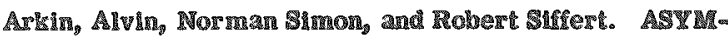
METRCAL SUPRRESSION OF VRRTRERAL GROWTIR WIFH IONIZING RADLATON. Broc. Soc. FEptI. Biol. Med. 69, 1\%1-3(1048).

The lumbar ypines of 30 young rabbits were unilaterally Irrediated with radon aeeds and X-rays. Twenty-one anhnals ded of gastrolntest zadiatlon. Five of the nin gurvivors chowed wedgIng of che vertebral bodles whin the marrowing of the bodies on the wore heavily Irradilated side. Dogege estimates indlcate the growh of the oplphyses of the vertebral bodies of balby rabbits may be sugpressed whth 700 to 1000 is to the

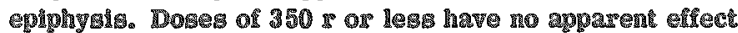
249 OB Uhe Frovth. (NSA)

Raca, $2 . M$, T. Lecomte, and A. HErve. THE ACTION OF IONIEING RADIATONS ON STRRATED IFROG MUSCLE.

Arch. Intern. physiol. 57, 162-59 (1040).

Experimenti are reported on the rectus abdominus

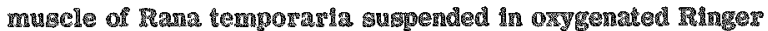


solution and excited at regular intervals by $5 \% \mathrm{KCl}$. The $\beta$ rays from $\mathrm{P}^{32}$ were employed as one test of lonizing radiation effects; the $\mathrm{P}^{22}$ was added in increasing amounts to a bath containing the frog muscle. The $\mathrm{K}-\mathrm{ray}$ experiments were of several types: $\mathrm{x}-\mathrm{radiation}$ at $190 \mathrm{kv}$ with a $5 \mathrm{~mm} \mathrm{Al} \mathrm{filter} \mathrm{and} \mathrm{at} \mathrm{a} \mathrm{target} \mathrm{distance} \mathrm{of} 40 \mathrm{~cm}$. was employed to deliver doses at the rate of $200 \mathrm{r} / \mathrm{min}$. to previously drted muscle; contact X-ray therapy at $50 \mathrm{kv}$ and with no filtration was also used on the dried muscle; x-Irradiation of the frog muscle in Ringer's solution was used; and, Irradiation of the Ringer's solution itself with $\$, 000-8,000$ r was also attempled and the $\mathrm{H}_{2} \mathrm{O}_{2}$ content determined. Fit was found that both the $\mathrm{X}$-rays and $\beta \mathrm{radi}-$ atlons determined the appearance of the Lundsgaard effect (contractlon of gtriated muscle after work). This is conaldered proof by the author's of muscular glucidic metabolifm; the lradiations are enzyme polsons. The calculaHons of the amounts of peroxides formed in the solution during difierent stages of the irradiatlons made possible 248 accurate observations. (NSA)

Baidins, A., L. Claesson, and A. Westman. THE EFPECT OF X-RAY IRRADIATION ON THF PITUITART BODY OF WNE ANTIL RATS TREATED WITH ALPHA-ESTRADIOL MONOBENZOATE. Acta Rndocrinol. 1, 133-40(1948).

Fourtean Iemale rats were treated with a total of $160 \mathrm{\mu g}$ of a-estradiol benzoate subcutaneously, over a perlod of elght days and then irradiated with $40 \mathrm{I}$ in a slingle exposure over the pitutary region with the rest of the body Bhlelded with lead; seven rats were simllarly treated with estradiol and lour other's served as untreated controls; all the animals ware observed for 50 days after treatment. It If concluded that under the inluence of estrogen, the pltuitary of the infantlle rat is gensitive to radiation doses (1 248 to 1,000 r) which fail to affect normal infantle rats. (NSA)

Bender, A. E. ERPERTMENTAL I-IRRADIATON OF THE RAT THERODD. Brit. J. Radiol. 21, 244-8(1048).

Treatment of the thyroid of rat with doses of 8 -rays ranging from 50-5000 1 produces no change in theis osygen consumption, nor in the histological appearance of the glands in (a) mature male rats; (b) young growing rats, (c) mature animals in which the gland had been rendered hyperplastle by oral administration of thlouracll. Doses greater than $5000 \mathrm{I}$ invariably resuited in death of the animals, 9-10 days later, which could be delayed $o$. prevented by the aubcutaneous adminlatration of glucoseBaline. Although death was apparently due to ingufficient Intalse of Iluld, no cause for this was found. (NSA) 250

BLlings, Marta S., A. Ko. Dowdy, Louise Burlingame, and Juanita Lamport. STSTEMIC ERTECTS OF IRRADIATON OF THE FXTERIORIZ D SMALL INTESTINL IN RABEITS. Atomic Energy Project, University of California at Los Angeles. Oct. 26, 1951. 30p. (UCLA-152)

The object of this work was to study the systemic effects of intestinal drradiation in rabbits. In order to elminate irradiation of other Intra-abdominal organs and structures, as well as side scatter effects to bone marrow, the irradiation was given directly to the small intestine aiter surg cal exterlortzation. Doses up to $1800 \mathrm{x}$ did not cause any significant morbidsty. Doses from 1800 to $6000 \mathrm{~s}$ causes Illness after a latent period of 5 days terminating with $100 \%$ lethalitses in the fourth weels. At no thme did the animals exhabit diarshea. Irradiation of the exteriorized gmall Integtine had no immedate specufic eliect on the cheulating blood cells. The eventually developing granulocytopenis and increase in red blood cells after intertinal oxposure to dose of 2000 I or more appear to be the result of Inantion and dehydration which follow local intestinal radiation injury. Leukopenia and anemia developing after total-body irradiation were not modified by lead shlelding of the exteriorized small bowel. Lead protection of the exteriorized small intestine prevented initial shock and carly death of rabbits after total-body ir adation. Lethal 251 doses were slightly higher in the shielded group. (NSA)

Bischof, F., H. J. Ullmann, and L. P. Ingraham. THE INFLUENCE OF IRRADIATION OF THE OVARES UPON ESTRUS AND NEOPLASTIC DEVILLOPMENT IN MARCHBUFFALO MICE. Radiology $43,55-8(1944)$.

Regnectlve groups of $40 \mathrm{Margh}-$ Buffalo mice each received doses of 200 and $400 \mathrm{r}$ of roentgen rays (HVL 1.15 mm Cu) over the ovaries following the establishment of vaginal canalisation. Equal numbers of litter mates served as controls. Immediately after irradiation, the lower dose reduced estrus entirely. For the higher dose, the extent of estrus established for the controls was never attalned throughout 213 month period. The lower dose produced an increase in estrua the sth month followed by a 6 -month period in which the mean estrus did not deviate markedly from that of the controls. The irradiation of the ovaries with $200 \mathrm{~s}$ increased the incidence of adenocarcinoma of the breast gignifleantly, but did not increase the incldence of lymphoid tumosis. Irgadiation with $400 \mathrm{r}$, while reducing estrus markedly, was without influence upon the cumulative incidence of cances of the breast, but increased the incidence of lymphoid tumors. (BA)

Bond, V. P., Marguerite N. Swift, A. C. Allen, and M. C. Fishler. SENSITIVITX OF ABDOMEN OF RAT TO XIREADIATION. Am. J. Physiol. 161, 323-30(1950).

Rat were irradiated either whith the abdomen shielded or with only the abdomen exposed. The shlelds were deslgned to permit equal gram-roentgen exposures with both types, at any given dose level. A compardson of mortality, sur vival time, body welght change, and gross aymptomatology between the 2 group revealed distinct differences, sug. gesting different mechanisms of death referable to the 253 reglon irradiated. (CA)

Braasch, Nancy I. and Margaret J. Nichson. A STUDY OF THE HANDS OF RADIOLOGISTS. Radiology 51, 710. 27(1949).

A group of radiologists was studied for evidences of radiation damage to the akin of their hands. The technique used consisted of (1) examination of wax impressions of the finger ridges, and (2) examination of photograghs of the vessels and papillae in the nail fold. of the 323 radiologitsts examned by the first method $48 \%$ were lound to have ridge abnormalities. The incidence of abnormalty increased with years of esposure to radiation up to $100 \%$ after 35 years. The irregular use of lead gloves does not give adequate protection against damage of the ridges by radiations. Photographs of the nail fold of 152 ardiologlsts were gtudied and compared to simlar photographs of 101 laboratory workers and of 41 perogns over 33 years of age. Some of the abnormalities occurged In all three groups. All of the changes which were found among the radlologlsts were found also in the aged. The Incidence of abnormality among the radiologista lncreased with years of exposure to radation and exceeded the incidence of abnormality in the aged. No radiologist had normal findings aiter 30 years of exposure. Tt is concluded that abnormalities of the vesaels and of the papillae in the nall fold are induced earlier in radologhts than in persons not exposed to radiation. (ADD) 254

Burstone, M. S. THE ETECT OF X-RAY IRRADIATION 
ON THF TEETH AND SUPPORTING STRUCTURES OF

THE MOUSE. J. Dental Research 29, 220-31(1950).

Fourty-lous Swiss and $\mathrm{C}-5 \mathrm{f}$ black mice, 2 to 28 days of age, were irradiated in the area of the mandibular joint and the molar teeth; littermates and littermate controls were used whenever possible. The $X$-rays were delivered by a Philps Metalix contact X-ray machine operating at $50 \mathrm{kv}$ with no f1ltration, at a target distance of $20 \mathrm{~mm}$ and at the output rate of $5,000 \mathrm{r} / \mathrm{m}$ mute; the three dosages which were used were $1,500,3,000$, and $5,000 x$. Following the ir radlation, 32 of the mice which survived were sacriflced at intervals from 5 to 37 days; control animals were also sacriflced, and the weights and sex were recorded; the heads of the mice were examined by serial sectioning for changes in the dental structures. If was found that the degree of radiation damage to the teeth and jaw of mice is dependent upon the age at which the animalg are liradiated 255

and the stage of histogenesis of the individual teeth. (NSA)

Cogan, David G. and David D. Donaldson, EXPERTMENTAL RADIATION CATARACTS. Areh, Ophthalmol. (Chicago) 45, 508-22(1951).

The eyes of rabbit of ages ranging from 3 weeks to 3 years exposed to 200 and $1200 \mathrm{kv}$ \& rays showed epilation occurring in 15 to 35 days with doses of $1500 x$ or greater. Eplation was increased the larger the dose and the less the energy of the x rays. Little variation in susceptibullty was found whth age. The same generalizations held for kerato conjunctivitis. Cataracts occurred with a latent period that was on Inverse function of the dose; the threshold dose for 10-week old rabbits was $250 \mathrm{r}$ or less, and the latent period for the development of radiation cataracts was much less the younger the animal. With $\mathrm{x}-\mathrm{ray}$ doses (1200 kv) of less than $1000 \mathrm{r}$, cataracts were the only abnormality produced. Gross and cytological changes due to $\mathrm{X}$-1r radiation of the eye are discussed in detal. (NSA)

Davidoff, L. M., C. G. Dyke, C. A. Isloberg, and I. M. Tarlov. THE EFP IFCT OF RADIATION APPLIED DIRECTLY TO TIE BRAIN AND SPINAL CORD. I. TEXPERIMENTAL INVESTIGATIONS ON MACACUS RHESUS MONKEYS. Radiology $31,451-63(1838)$.

Exposed bratn and spinal cord of Macacus rhesug monkeys were given single doses of 1,000 to $5,000 \mathrm{~F}$. Three were given two doses totalling $4,800,5,400$, and 7,2008 . Four-thousand $\mathrm{r}$ to the cerebrum is followed almost $\mathrm{Im}$ mediately by paralysis of the contralateral limbs. Twothousand to $3,000 \mathrm{r}$ results in hemiporesis after several monthe. Cerebellar digturbances do not appear until 3 to 5 months after 1 rradiation of the cerebrum. Five-thousand I to the mid-thoracic spinal cord produces immediate paraplegla, and after 4,000 paraplegla develops after 5-1/2 months. There are marked histological changes in the central nervous system after treatment. The contralateral as well as the homolateral hemisphere may be involved after irradiation of one cerebral hemisphere. Smilar histological changes are observed in the cerebrum, and the spinal cord. Chromatolysis of ganglion cells of the cerebellum was found after lrradlation of the cerebrum and vice versa. There are marked changes in the glla and nerve tissues and only slight vascular alterations. Myelin sheaths appear to be affected more than axis cylinders. (BA)

257

Dobbs, W. H. H. A STATISTICAL STUDY OF THE EFFECT OF ROENTGEN RAYS ON WOUND HEALING. AM. J. Roentgenol. Radium Therapy $41,625-32(1939)$.

In this study, the determination of the tensile strength of tissue is used as a measurement of the effect of roentgen
Irradiation on wound healing. In addition, histological sections of control and irradiated wounds were talken at Intervals of $4,5,6,8,10$, 20, and 50 days after operation. The results of the effect of exposure of wounds to $300 \mathrm{r}$, $1020 \mathrm{r}$, and $1800 \mathrm{x}$ immediately after operation; effect of exposure of wounds to $1800 \mathrm{r} 24$ hours after operation; effect of exposure of skin to $1800 \times 1$ and 3 weeks before operation are described. Adult, male, albino rats were 258 used. (S.V.S.)

Dobbs, W. H. H. A STATISTCAL STUDY OF THE LATE IEFFECTS OF HEAVY ROENTGEN IRRADLATION ON HEALING OF SKIN WOUNDS. Am. J. Roentgenol. Radium Therapy $45,107-8(1941)$.

Thirty-nine adult, male albino rafs were given roentgen irradiation to a $4 \mathrm{x} 1 \mathrm{~cm}$ ventral slan fleld to a total dosage of $4,500 \mathrm{r}$ in weekly fractions of 1,500 $\mathrm{r}$ each. Roentgen rays were produced by a full wave, mechantcally rectified generatos, operated at $95 \mathrm{kv}$ with a Coolidge tube uging 4 Ina at $25 \mathrm{~cm}$ distance, the oufput being $152 \mathrm{I}$ per min. Sixty days following irradiation, a $4 \mathrm{~cm}$ linear incision was made in the treated gkin and a gimilar incision in nomal gkin and the wounds were closed with black stlk gutures. At interwals of $10,20,30$, and 50 days following operation, the exptl. wouhds were excised and tested for tensile strength. The wounds recelving Irradiation showed a conglderable decrease in tenslle strength as compared to normal wounds, confirming the feeling of surgeons and roentgenologists generally that wounds in heavily irradiated akin heal but that the process is slow and the scar 1s not so strong as normal scar tissue. (BA) 259

Eck-Vermande, van G. J. and J. Freud. STRUCTURE AND FUNCTION OF MOUSE OVARIES AFTTR X-RATING. Areh. intern. pharmacodynamie $78,49-62(1949)$.

A total of 230 mice (in groups of 3 to 5 animals) under light ether anesthesia were $x$-rayed from the back whout fliter, at a focal distance of $78 \mathrm{~mm}$, in 100 or $200 \mathrm{sec}$, wh th total doses of 333 or $666 \mathrm{r}$; ovaries were gerlally cut; tubes, uteri, and vaginas were extensively investigated at intervals ranging from one day to 3,7 , and 14 weeks after irradiation. The histologreal findings are reported in detall; one hour after irradiation, maxised destructive changeg were found in the ovaries, followed by hyperemia and violent follicular atresia with destruction of granulosa cells and fragmentation of the ovocyte during the firgt days; other results are discussed. (NSA)

260

Engelstad, R. B. THF EFEFT OF ROENTGEN RAYS ON THE STOMACR IN RABBITS. AM. J. Roentgenol. Radium Theray 40, 243-62(1938).

Irradiation of the gtomach whth roentgen rays was carried out on 59 rabloits, with doses ranging from 125 to $4,500 \mathrm{r}$. After the heavy doses of $2,000 \mathrm{x}$ or more the mortality was very high; but even after smaller doses $(1,500$ r) a number of the andmals died. After Irradistion there was a mild Initial reaction durdng the flrot 24 hours whth degeneration of the lymph follicles and hyperemia; therearter a pertod of latency, followed by the onset of the main reaction about seven days after irradation, whth degenerative and inflammatory changes. (Author's Summary)

261

Essenberg, J. M. THI RESPONSE OF GERM CELLS TO GONADOTROPIC HORMONES IN X-RAY INJURED OVARIES OF YOUNG WHIT MICE. Western J. Surg. Obstet. Gynecol. 5\%, 01-6(1949).

Most of the ovardes in 34 young mice, 3 to 4 weols of age, $X$-rayed with 244 to 300 remaned sterle from 3 to 
6 montha, the length of the experimental period. Most of the X-rayed ovaries, treated with gonadotropic hormones, either in the form of injection of gonadophysins or the implantation of the pituitary gland, regenerated germ cells and follicles of all sizes except fully formed or graafian lollicles. Degeneration of germ cells and lollicles occurs Gimultaneously with germ cells and follicle formation. The cells of degenerated follicles are segregated by comective tissue into alveoli-like structures, which are found in the medulla of hormone-treated ovarles and the entire ovary of $X-r a y$ controls. The germinal epthelium was very active in hormone lreated ovaries; germ cells were proliferated and passed through developmental stages before the fully formed stage was reached. Undifferentiated germ cells of germinal epichelium origin may become ova, follicle cells, and probably, interstitial cells. There is great variabilly;' a 1ew $\mathrm{X}$-ray controls may prollferafe germ cells gpontaneously and some gonadotropin treated ovaries may fall to form germ cells. This study supports the conclusion that in the white mouse, gexm cells originate from the germinal epithelium. (NSA) 262

Farr, Richard S, and Peter P. H. DeBruyn. THE EFECT OF STLECTIS SHIELDING OF RABBIT INTESTINE DURING TOTAL BODI IRRADIATON. Argonne National Lab. Oct. 1951. 11p. (AZCU-1820; UAC-459)

Rabbits Hawe been exposed to lir radiation with $x$ ways While most of the colon or small intestine was surgtcally exteriorized Into a lead chamber. The results indicate that the selective ghielding of the rabbit intestine during other Wire total-body irradiation does not decrease the lethal effects of 8 rays. It is auggegted that the protection conterged on animals when the entire abdonen is shelded during otherwise total-body irradiation can best be attributed to a summation of several "ogsgan effects" ${ }^{\text {ss }}$ rather than 263 to one specilic organ. (NSA)

Friedman, N. B. and S. Warren. THR FVOUUTON OF EXPRRIMENTAL RADIATION ULCERS OF THE INTESTWNE. Arch. Pathol. 33, 326-32(1942).

Fvolution of intertinal yadiation ulcers in Irabbit was produced by roentgen ray treatment and observations made ug to a persod of six months following treasment. Irradiation of the integtine resulted in an altered and damaged mucous membrane which was especlaly susceptible to ulcerat lon. Ulceration, though focal, was not localized by vascular occlumion. It seemed rather the chance rifect of zuechanlcal trauma and subsequent Infection acting on a mucosa and bowol wall structurally and functionally 204 damaged by radiation and imperiectly regenerated. (BA)

Friedman, W. B. CELIUULAR DYNAMCS IN TIYT IN-

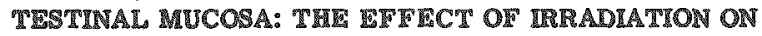

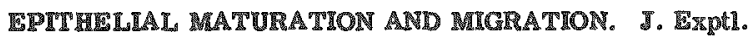
Med. $81,553-8(1245)$.

Rat were Irradiated In groupg of 6 whth 1,000 s given in 30 min. ast $140 \mathrm{kw}$, or with 1,000 g given in 8 to $20 \mathrm{~min}$. at $186 \mathrm{kv}$. All but a $5 \mathrm{~cm}$ band across the middle of the abdomen was ghielded with $\mathrm{Pb}$. The rats were ginled at segular Intervals duraing a 96 in interval after 14 radiation and the so-called "post. irradiation mucous change" in relation to atrophy and regeneration of the Intestinal epithelinm were gtudied. Detalled description ô these changes is given. If is coneluded that although irrediation of the duodenum of rats linhibits mitosis in the crypts and halts the normal passage of cels up the vil, the maturation of goblet cells is not affected. The ripening of mucous elemente while arrested in the crypts, where they form, Instead of durgng then migration along the vill, results in the so-called mucus change, which has hitherto been considered a form of degeneration. During the phase of recovery, the reestablishment of normal migration and descquamation is marked by the appearance of atrata of fully formed goblet cells at successive levels out along the villi. It is suggested that some gast rointestinal disturbances known to occur in spontaneous and experimental vthamin deficiency might be explained in terms of aberratons in the cellular replacement of the intestimal mucosa. (TIS)

265

Gastaldi, Gild. MORPHOLOGICAL ASPECTS OF THE RTGENERATION OF NERVES SECTIONED AND SUBJECTED TO THE ACTON OF X-RAYS. Riv. sper, freniat. 73. $5-41(1949)$.

The repeated irradiatton of the peripheral nerves by zx-rays offered in the present studies an approach to the general problem of the action of $x$-radiation on traumatic lesions of the nerves. The research was conducted on 30 rabbis and on guinea pigs, half of each group serving as controls. The local irradiation $(80 \mathrm{r} / \mathrm{session}$, for a total dose of $160-720 \mathrm{I}$, will a rutration of $0.5 \mathrm{~mm}$ Cu plus 2 mam Al, and $170 \mathrm{kv}$ and $30 \mathrm{~cm}$ target distance) began immediately after the hemisection of the sclatic nerve and continued on the following days; the aninals were sacrificed in pairs in atages from the second to the $19 \mathrm{th}$ day after the nerve sectioning. In 14 of the 30 rabbits, a wital coloration state (by successive injections of a $5 \%$ solution of trypan blue intrawenously) was produced; the animals were created each day before the rectoning and continued to recelve imjections of the dye for variable periods afterwards. Several observations are reported: an intilal state of active hyperemia whin caphary diltation and a focal miliary diapedesis occurred on the second or third day after sectiondig; a glight prolif aration of blood vessel walls occured on the sixth d2y; other phenomena much a accentuated flbrotic metamorphosis of granulosa tissue, iraumatic degeneration, accentuated lewcocyte migration, accentuated phagocytic activity, and prolonged disorientaton of young rerve fibers also occurred; there was, however, no apprechable difference in the contentr of the cell of Schwann. 1\% references. (N\$A) 266

Gershon-Coher, J., M. B. Hermel, and J. Q. Grifrith, Jr. THE VALUE OF SMALI LEAD SFIELDS AGAINST THE INJURIOUS GITECT OF TOTAL-BODY IRRADIATION. Science 114, 157-9(1951).

The effect of shlelding of the liver, right lung, or right lower abdomen of young white rats during whole-body isradiation has been Investigated. The survival rotes of all the partally ghielded raty were higher than those exposed to whole-body irgadiation. Of 20 rats which recelved whole-body 1rmadation, gurvived. There were 10 survivals of the 12 mats protected over the liver, 7 survivals of rats protected over the right lung, and aurvivals of - rats protected over the right lower abdomen. Survival times, waights, and blood counts are recorded in 7 tablea. (NSA) 267

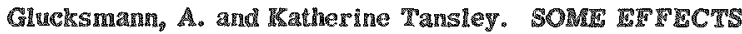
ON GAMMA RADIATON ON THE DEVELOPING RAT RETWNA. Bric. J. Ophihalmol. 20, $497-509(1936)$.

A method of exposing the eyes of young rats lo $y$ radlation is given. With such radiation the undifferentiated cells of the retina degenerate, the anoun depending on the dosage and the age of the rat. The size of the eye is reduce, and a new forber layer is formed dividing the Inner nuclear layer into two under certaln condituns. Rosettes may aløo be produced. The formation of the new 1 iber 
layer and the rosettes is secondary to the degeneration and 268

not a direct effect of radiation. (BA)

Harvey, R. A. EF FET OF ACUTE AND CHRONIC DOSES

OF ROENTGEN RAYS ON MONKEY FINGER RIDGE DE-

TAIL. [Umy, of Rnehester] [nd] becl. Aug. 12, 1946.

16p. (MDDC-215)

Acute roentgen expowures up to $800 \mathrm{r}$ cause no detectable gross changes in finger ridge details of monkeys. Acute doses of $800 \times$ to $1,100 \times$ result in temporary atrophy of ridges. Acute doses above the level cause more severe atrophy, slower regeneration, less complete regeneration and some tendency to secondary weakening of detall. Chronic roentgen exposures of $10 \mathrm{I}^{\circ}$ per day for 50 treatments cause no change in ridge detall. Chronic exposures of $20 \mathrm{r}$ or more per exposure for 50 exposures caube detectable weakening which like that following acute exposures is more severe with increasing dosage, shows a slower tendency to regenerate, less complete regeneration and 268

Harvey, R.A.THE ERTCT OF SHORT WAVELRCTH RADIATIONS ON HUMAN FINGER RIDGE DETAIL. [Univ. of Rochester] [nd] Decl. Aug. 1\%, 1046. 33p. (MDDC-218)

Atrophy of rdiges of the finger point area were detected in $68 \%$ of radiolog $5 t 5,55 \%$ of orthopedists who use a fluorogcope, $40 \%$ of dents sts who hold flims during exposure to $x$-rays and $1.8 \mathrm{~s}$ of the controls. The find Ings in profegslonal groups were aubclinical in a high percentage of cases, and they should not cause alarm if preaent standards of protection are appreciated. Personnel of the Manhatan Project were surveyed in this respect, and steps to be 270

taken for employee malety are proposed. (ADD)

Heald, A. Ho, C. Beard, and W.R. Lyons, TPRE IFITCTS OF ROENTGEN RADIATION AND PROLAN UPON THE TESTES OF IMMA TURE RATS. Am. J. Roentgenol. Radium Theragy 1, 448-52(1039).

Direct Irradiation $(1,152$ to $4,608 \mathrm{r})$ of immature rats tegtas caused degeneration of the seminterous epithellum, but left the Leydig tissue capable of responding to gonadstmulating hormone (prolan). Judging by the wefight: and histologic appearance of the prostates, the otimulated Leydig tissue formed male sex hormone. For the duration of the experiment (12 days) the harmful action of the roentgen rays upon the gemi-Irradlated lemtes was ghargly 271

Hinkel, C. L. THE EFPECT OF IRRADIATION UPON TRE COMPOSETION ANO VASCULARTIY OT GROWING RAT BONES. Am. J. Roentgenol. Radium Therapy 50, 516-26(1943).

Roentgen 1rradiation of the femora of young growing rats is followed by an almost immedhate increase in bone agh and fat content, associated with a drop In matris and water. The mechanism of augmented mineral deposition is not clear. If is associated whth a compensatory decrease in matrix. Several days after irradiation there followg a diminution in the number and calber of the metaphyseal blood vessels. The resultant relative avagcularity may Well be a basic factor in the production of the high ash and low water contents observed. In the months after isradiation of the lntensity used in these experments there is a gradual return toward normal content of ash matrix and fat. Alhough there is considerable regeneraton of vassel some degree of hypovasculartly pergigts throughout the pertod of observation. (BA)
272

Tueper, W. C. and J. de Carvajal-Forero. THE EFFECTS OF REPEATED IRRADIATION OF THE GASTRIC REGION WITH SMALL DOSES OF ROENTGEN RAYS UPON THE STOMACK AND BLOOD OF DOGS. Am. J. Roentgenol. Radium Therapy 52, 529-34(1944).

This gtudy was undertaken to determine the posalble action of roentgen therapy upon the erythropoletic astivity of the bone marrow and upon the blood through a posstble effect upon the production of the so-called "intringis factor $^{\text {s9 }}$ of Castle. It was found that roentgen rays glven in doses of 15 to $120 \mathrm{x}$ up to a total of $4,875 \mathrm{x}$ within 25 weeks over the gastrlc reglon of dogs caused only minor anatomic changes in the gastric mucosa and a transitory and nooderate anemia of the secondary type followed by an erythrocyts phase after the cessation of the actinic treatment. Roentgen rays administered in doses of $300 \mathrm{x}$, up to a total of $6,000 \mathrm{r}$ wthin four weeks, produced a considerable loss In body weight, a moderate to gevere secondary anemia, and perforating gagtrle ulcers at the end of this period. In neither Instance was there evidence of anema of the pernicloug variety, which would have resulted if the treatment given had intertered wth the production of the ${ }^{64}$ intrinsic 1actor" generated by the gastric mucosa, according to Castle. The chief cells of the gastric mucosa are, according to the observations made, delintely more senøstive to the action of the roentgen rays than any other cellum lar olement of the gatric mucosa. The histologic changes found in the organs of the dogs which were treated whth larger doses over a shorter period of the are described In detall. (R)

273

Hursh, John B., Priscilla A. Van Valkenburg, and John B. Moheny. EFETCT OF ROENTGEN RADIATION ON THEROID FUNCTION IN RATS. Radiology $56,111-18$ (1051).

Rat were Irgadlated by 5 says applled locally to the thyrold area at total dose levels of 3000,4500 , and $6000 \mathrm{x}$. Thyrold function was tested by injecting ${ }^{132}$ gt varlous intervals after Irradiation, gacrifichng the animals four hour after Injection, and analyzing the excised thysold gland for uptake of $I^{192}$, as well as the percentage of $I^{131}$ bound in an undialyzable complex. Hintologle sectlons of the thy roids were prepared and examined. In the rats receiving 3000 s. and 6000 is the iodime uptake was greater than in the control ratg, and the snerease was greater the higher the $\mathrm{x}-\mathrm{ray}$ close and the longer the post-irradation interval withtn the limits Investigated. In splte of the increased lodine uptare, no morphological changes of the thyrolds could be lound at these dose levels on the basts of exanuation of rascom gectons. The increased uptake is tentatively regarded as an indirect consequence of a aytemic regponge to radiathon. The level of local II Iradiation necessary to produce destruction of thyrold tisue ia greater than the highest dose (about $5000 \mathrm{r}$ delvered as a single dose) which can be 274 adminlstered to a rat whthout causing its death. (NSA)

Jacobson, Leon O., Edna R. Marks, Melba Robson, and $R$. E. Zirkle. THE EFIECT OF \$PLEN PROTECTION ON MORTALITY FOLLOWING X-IREAOIATION. J. Lab. Cln. Med. 34, 1538-43(1949) (ALCU-535)

Survival data on groups of mice exposed to almgle dosages of $600,700,000,975,1050$, and 1200 \& of total-body $\mathrm{x}-$ radiation (250 kv) with or without lead protection of the gurgicaly moblized apleen are pregented in this paper. These data show that the LD-50 for total-body x-1 sadiation, excluas of the lead protected anleen, is nearly twice as great as the LD-50 tor total-body $\mathrm{x}-1 \mathrm{rg}$ adlation 
inclusive of the spleen. The mechanism whereby lead prolection of the gpleen o significantly increases survival from irradiation is yet to be determined. (NSA) 275

Janzen, A. H. and S. Warren. FFFECT OF ROENTGEN RAYS ON THE PERIPHERAL NERVE OF THE RAT.

Radiology 38, 333-7(1942).

Exposure of the peripheral nerves of the pat to roentgen rays in doses of $4,000,6,000$, and $10,000 \mathrm{r}$ generated at 200 kv produced no demonstrable histologtcal ox physlological changes. Complete degeneration of nerve and some evldence of degeneration of the sensory neurones resulted from exposure to $1,200-1,600$ millicurle hours of $\gamma$ gad m ation. Nerve tissue is extremely resistant to radation. 276 (Authors' Summary)

Jolles, Benjamin. X-RAY SKIN REACTIONS AND THE PROTECTVE ROLE OF NORMAL TLSSUES. Brit. J. Radiol. 14, 110-12(1841).

The skin of the rat has been Irsadiated under constant physical conditions, but with Ilelds of varlous sizes. The stze of the fteld was found to influence quite profoundly the dggree of reaction of the skin; the bigger the area Irradiated, the more severe the damage done, even though the dose of $\mathrm{x}$-rays given per unt area was kept constant. More experiments have shown that the reaction becomes a minimum when the normal tissues can exert thelr maxlmum protective role; this has been arganged experimentally by surrounding the X-rayed area by as much normal thsue as possible. (Author's Summary)

277

Jolles, Benjamin. THE RECHPROCAL VICINITY ETTECT OF MRRADIATED TISSUES ON A DIFUSISLE SURETANCE IN IRRADIATED THSUES. Brit. J. Radiol. 23, 18-23 (1950).

A dose of X-rays was given to two mall areas of human okin separated by protected skin, and the razction produced was aigniflcantly more marked than that over a single area of thin at a symmetrical site exposed to the came dose of sadiation. When the sepasation between the two paired areas was greater than a certan minimum value, the reaction was equal to that on a single area. A diffustble aubstance is tentatively suggested, and the protective role of normal surrounding tigsues is discuøsed. (CA) 278

Jolles, B. and R. G. Mitchell. OPTIMAL SKIN TOLER ANCE DOSE LEVELS. Brit. J. Radiol. 20, 405-20(1947).

The problem of optimal skin tolerance dose levels ls approached on novel lines and a tolerance dose formula postulated, taklng into consideration the perimeter treated area ratio. Figures are given for treatment under stated conditions for treatront perods of $1-6$ weeks. (Authors" Summaxy)

279

Leach, J. E. and K. Suguira. LATR FFIFCTS OF MIGH VOLTAGE ROENTGEN RAYS ON TRE HRART OT ADULT

RATS. Am. J. Roentgenol. Madium Therapy $48,1-7$

(1842).

Late effects of roentgen irradiation on skln; subcutaneous tasue, muscle, and bone are well known. Stmlas effects were looked for in heart muscle, some months after drect heavy Irradiation to the organ. There was no demonstrable effect $2-14$ months after Irreadiation with 29 angle doaes up to and including of,500 roontgens. (BA)

Lecomte, J., A. Herve, and 2. M. Baca. ACTION OF I RAYS AND CYANDOES ON FROG STRIATED MUSCLES. Compt. rend. soc. blol. 144술, 708-9(1950).
The authors have observed (Herve et al, Compt. rend. goc. biol. $143,881(1949)$ ) that an injection of NaCN or of cystelne immediately before a total $x$ lrradiation of mice Feduces considerably the lethality tate due to the radiation. The queation arose whether or not these substances would protect isolated striated nuscles of irog agalngt a pecullas contraction called the Lundsgaard effect, that follows an irgadation and is related to the organ' resplration disturbances; it was thought that the reducing efrect of the cyanide or cygteine might inhibit the oxidizing action of x rays on enzymes. Fxperimental results did not confirm 281 the hypotheris. (NSA)

Lindenbaum, B. THE RADIOSENSITIVITY OF THE PIRIPIIRAL NRRVOUS SISTEM. Fortsch. Cebiete Ro̊ntgenstrahlen $71,088-93(1049)$.

Atter geveral treatments with deep $\mathrm{s}$-rays, senstivity changes occurred; applicationg of the rays to the tongue disturbed taste. These changes vere mostly reveralble, but only after long perlods of tme (monthg). (NSA) 282

Ma, W. C. and C. I. Hsu. THE EFTET OF ROENTGEN RADIATION ON SPINAL GANGLIA OF ALBINO RATS.

Am. J. Cancer $40,335-42(1940)$.

In a parallel cytologlcal study of direct and indirect effects of roentgen radiation on the apinal ganglla of ratts, using gpecial stalns, 1 was found that the extent of cellular change varied directly with the dosage. Prfects were: chrtnikage and Irrogularty of cell-outhines, decrease In Bize and gtaining qualty of mele , decrease or increase In mitochondria, which were granular and stalned poorly, loas of Niasl substance, and reduction of Golgl apparatug. (BA)

283

MacComb, W. S. and R. H. Quimby. THE RATE OF RE-

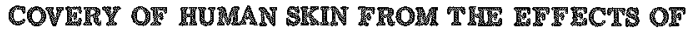
MARD OR SOTT ROENTGEN RAYS OR GAMMAM RAYS. Radiology 27, 196-207(1936).

Following the techndque of Dufy, Arnegon and Vole (Radiology, 23: 480, 103 1) the amount of $200 \mathrm{kv}$ \& radiation necessary to produce the threshold eliect, when given in two equal doses, at intervals of from to $98 \mathrm{hr}$, has been sourd, also the amount for 3 dally and 5 dally doses. From these data have been calculated the recuperation from the IIs lound to aet in promptly, for even aiter $\mathrm{hr}^{3}, 30 \%$ of the eflect has been lost, and after $12 \mathrm{hr}, 50 \%$. prt proceeds more and more glowly with larger intervals. For woft 8 rays and for $y$ zays, the factor for the 24-hy recovery has been found when radiation is admintered in 2 equal doses.

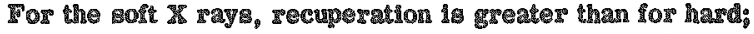
los $y$ rays, it is deluntely less. (Authors' Summary) 284

Myrakawa Tadah4

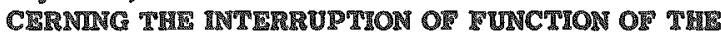

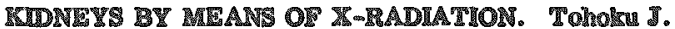
Expt!. Med. 40, 169-88(1041).

The kidney of 66 2-lrg healthy male mobuts were rodiated. Group A recelved a total dose of $3000 \mathrm{r}$; grour $\mathrm{B}$, $6000 \mathrm{~s} ; \mathrm{group}$ C, $9000 \mathrm{r} ;$ and group D, $0000 \mathrm{~s}$ in divded dosage. Urine, blocd, and P.S.P. studes ware made perst odically from 24 hr to 30 wh, following radiation. Macro. gcoplc and microseopic gtudies of the kidneys pere mued. In the first 24 hx. the urine output decreased and a trace of protein, sev erythrocytes and leukocytes, and mome opthelial cells were present in the urine. There were ollght changes in the hemoglobin and erythrocyte count of the blood, and abrupt decrease of the leucocyte blood level occurred which returnod only slowly to normal. A dellnite 
eosinophilia and lymphopenta were noted, which returned to normal incompletely. Petechial henor rhages were noted on the kidney surface. Collapse of renal parenchyma with a relative increase of the interstitial supporting tissue with a round-cell infiltration was noted. Considerable degtruction of tubular epithelium was noted. Regeneration in group $\mathbf{A}$ occurred in $20 \mathrm{wk}$; in group $B$, in 3 wk; in group $C$, in $24 \mathrm{hr}$; and in group $\mathrm{D}$, in 9 wh. The glomerull were quite unchanged with but slight capillary atrophy. Cortical leslons were slight. The P.S.P. excretion was not delayed. The earllest changes were noted in the tubules, and only repeated dosages of radiation caused atrophy of the glomer285 ular vessels, and these effects were slight. (BA)

Neumayr, Anton and Bruno Thurnher. IERTCT OF LOCAL ROENTGEN ITRADLATIONS ON TYF PERMEABILITY OF CAPILLARIES IN MAN. Strahlentherapie 84, 297-305 (1951).

Increased permeability of the caplllaries after $\mathbb{x}$-ray treatments was observed in man, with a threshold effect varying Irom 3 to $100 \mathrm{x}$, depending on the individual sengitivlty of the capllaries, which also determined the duratIon of the morbld state; above the threshold no proportionalty between the increase of the protetn loss and the dose was observed. These facts prove both the Inadequacy of permeablity measurements as a way of establishing biological doge units and their utility in the study of the comparative sensitivity of capllary membranes. Twelve-day observalions shoved that after the healing no changes in the permeability reappeared; therefore, in all probability, the reaction is a one-phase process. (NSA) 286

Nickson, M. REPORT OF HAND STUDY. Argonne National Lab. Aug. 1, 1945. Decl. Aug. 29, 1247. 3\%p.

(MDDC-1270; CN-3368)

Observations have been made of the hands of 973 laboratory and office perconnel. Deviationg from normal have been noted and photograph taken. Finger sidge impressions have been made of $1,28 \%$ individuals of whom $15 \%$ showed some abnormality. Capllary counts have been done on 838 individuals. The number of capllaries in a given area of nall fold shows some correlation with possible exporure to radioactive materials. Capillary photographs have been taken of 93 individuals. There 18 a rough correlation between the morphology of the capllarles and the exposure of the hands of young individuals. Normal aging tends to produce the samo or simllar changes. Further caplllary shdies are necesaary to confirm or deny the apparent relationghip between hand exposure and increased or prematurely aged terminal eapllarles. Photographs 289 and 2 bibllography of 119 references are included. (ADD)

Nickson, M. FFISCT OF SINGLE DOSE X-RAY TO THE NAIL FOLD AREA OF TUMAN SUBJ National Lab. June 20, 1047. Decl. Sept. 11, 1847. 6p. (MODC-1269: CK-3833)

The mall fold area of the left fourth finger of 15 subjects was obaerved before and after a single radiation exposure. The total dose ranged from 200 r to 600 I. From this brief and incomplete study it appearos that radation exposures of 600 r or less when given as a olngle dose under the conditions deseribed, produce no constant microscopic or macroscople reaction. The transtlory efrects were most often microscopic and consisted of hyperemia of the small terminal vessels and dilation of vesselg visible lin this area before and after radition. (ADD)

\section{8}

Nickson, M. and L. Warner. \$ECOND REPORT OT MAND STUDY PROGRAM. Argonne Nalional Lab. Jan. 1, $194 \%$. Decl. Aug. 26, 104\%. 23p. (MDDC-1217; CM-3753)
Methods to determine early radiation damage or damage which has occurred without the individuals"s being conscious of any change fall into two categories, macroscopic and microscoplc. Two methods were used in the laboratory for determining macroscopic changes: (1) direct inspection of the hands. (2) The hands were photographed, the photographs enlarged and the enlarged photographs were studied. There are two macroscopte changes in the gkin after acute and chronic radiat lon, namely erythema following acute radiation, and atrophy after chronic radiation. Unfortunately, both radiation erythema and/or atrophy will also result from stimulation other than radiation. Microscopic examinations of the vessels at the base of the nail were made. Of the 503 Individuals studied for radiation damage to finger ridges, $18 \%$ had some abnormality. All of these abnormalthies were minimal. Revershlole changes due to mechanical flattening of the rfdges were found among machinists, carpenters, and others dolng manual labor. Such findings which may be coniuging after a single examination can be excluded by repeated studies after time intervals to allow for regeneration. Capllary counts were found to ranage between 18 and 22 in over hali of the individuals studted and between 18 and 27 in 80\%. Persons wilh dark skin pigment had slightly lower counts whereas persons over 50 years of age had counts in the high portion of the average range. Sex had no effect on the count. Radiation exporure at levels received lin the laboratory had no effect on the count during the period of time covered by this gtudy. This report is a continuation of studses deserfbed in MDDC-1270. (ADD)

289

Novick, Rosalind. TMM COURSE OF CHANGLS IN SENSORY CELLS OF TRIGEMINAL GANGLION OF THE IRABBIT FOLLOWING IRRADIATION. Proc. Soc. Rrptl. Biol. Med. 61, 355 -8(1246).

In a Beries of rabbits Irradiated in the head with a single dose of 3,000 roentgen units, cxamination of the Nigs! stained trigeminal ganglion cells reveals injury which may be separable, by analysis of the time courae, into three phases. Initially in animals killed immediately following 1rradiation, chromatolysis 18 short-lived, so that as early as three hours after the end of the Irradiation period maxked Improvement can be noted, and at 18 hours, the ganglion cells appear normal. The normal phase persists untll 43 hours at which time there is clearly observable the beginning of and wave of chromatolysts which slowly becomes increasingly widespread in amoun and severe in degree (BA).

290

Pendergrass, E. P., P. J. Modes, and J. Q. Griphth. ET FICT OF ROENTGEN RAYS ON THI MNUTE VESSELS OT TLE SRIN IN MAN. AM. J. Roentgenol. Radium Therapy 52, 123-7(1844).

A method of following changes in visible cutaneous capillaries depends on relating the number vidble to the total rumber present. The total number is obtained by counting the area with a capillary microscope after pricklng 1:1000 histamine. Changes in relative count following adm. of about $300 \mathrm{r}$ of $200 \mathrm{kv}$ X-rays in $3 / \mathrm{min}, 7.2 \mathrm{~min}$, and 30.8 min., respectively, were essentially the same, an increase at the end of hours, which was almost or entirely gone after 24 hours. The mame doge of $50 \mathrm{kv}$ x-Irays gesulted in a mild effect at 6 hours, which was much more pronounced at 24. (BA)

291

Raab, W. and A. B. Soule. ERTCT OT ROENTGEN IRRADIATION ON THE HORMONE CONTENT AND SECRETION OF THIS ADRENAL MEDULLA. Radiology 19 $56-60(1943)$.

Rata, anesthetized whin nembutal, were irradled with 
roentgen rays generated at $200 \mathrm{kv}, 20 \mathrm{ma}$, fultration: 0.5 min Cu and 2.0 mm Al. The cone was focused on the back over the adrenal area. Detn. of hormone content was made, using a modification of Shaw" colorimetric detn. of "Adrenalin". No significant change in the medullary hormone content of the glands was found within 68 hours after $4 \mathrm{r}$ radiation. The doses $100 \mathrm{x}$ to $1000 \mathrm{x}$ were given in a stugle session. After 68 days, however, there was indication of a diminished secretory activity following a dose of $100 \mathrm{r}$, a slmilar decrease combined with slightly dininished hor-mone production was observed after irradiation with 1000 I. (BA)

292

Rigdon, R. H. and H. Curl. EFFECT OF ROERTGEN IRTADIATION ON CAPILLARY PERMEABILITY AND INELAMMATION IN THE SKIN OF TIEL RABBIT. AM. J. Roentgenol. Radium Therapy 49, 250-7(1943).

Trypan blue injected intravenously localizes and concentrates in areas of inflammation in the skin during a spectic interval following inditation. Th has been suggested that this 18 due to a change in the pexweability of the cells because of the injury. Irradiation with $\mathrm{x}$-rays results in changes in cell perrueability. Small shaven areas on the aldes and back of adult rabbits were ir radiated with 6802720 of $36 \mathrm{kv}$ uniflered $\mathrm{x}$-rays. Changes in the glain in these areas occurred immediately, as shown by localla tion and conc. of trypan blue therein, for a brief time after the injection. Areas Irradiated $1 \mathrm{hr}$ or less prior to the administration of the dye, become blue. Areas irradiated 3 hours or more before the dye is injected do not show any more color than untreated areas. Polymorplonuclear leukocytes are pregent in the corium of the irradiated skin aiter aprox. 60 min. Their number increases for several houz:. There is no correlation between the time in which trypan blue localizes and concentrates in areas of irradiated gkin and the time in which polymorphonuclear leukocytes inflitrate the corsum ln the same area. It Is ag gested that both inilammation and irradiation disturb the Ca-K- $\mathrm{P}$ balance in cells, and bring about increase in 293 capllary permeablity lor this reason. (BA)

Russell, Dorothy \$., C. W. Wilson, and Ixatharine Tansley. LXPITIMENTAL RADIO-NECROSIS OF THE BRAIN IN RABBITS. J. Neurol. Reurosurg. Psychist. 12, 187-95 (1949).

If was found that a single surface dose of $2,850 \mathrm{at} 200 \mathrm{kw}$ $X$ rays will produce delayed irradiation necrosis in the rabbit's brain after a latent perlod of appromimately 100 days. The latent period was lengthened by reducing the dose $10 \mathrm{2}, 440 \mathrm{~s}$. Flela mize Was $3 \times 4 \mathrm{~cm}$; surface doserate $154.5 \mathrm{I} / \mathrm{min}$; focus-slan distance $25.5 \mathrm{~cm}$. The appromimate depth doses were: $1 \mathrm{~cm}$ depth, 859 ; $2 \mathrm{~cm}$ depth, 70\%; 3 exn depth, 57\%. The initial higtologle changes consisted of minute focl of hemorrhage and necrosis which are inimately related to the pertorating vessels and, in particular, caphllariea. Increase of capillary pormeablity accompanied the established lealon but was not demonatrated at earler gtages. The lesions, when once intiated, appeared to extend rapidly and to progress to non-impadiated parts of the braln. They were characterlzed, in later gtages, by pronouneed glioses, and by progressive degeneration and sclerogis of the adjacent vessels. The neurones were only focally and secondarily affected. The meninges and their blood vesels were unaffected, and the muperficial parts of the cerebrum escaped, even though the cerebellax cortex might be extengively damaged. (R) 298

Sallman, Ludwig von. EXPRRMMPTAC STUDIES ON EARLT LIENS CRANGES AFTER RONNTGEN IREADIATION.
1. MORPHOLOGICAL AND CYTOCHEMICAL CHANGES. Arch. Ophthal mol. (Chicago) $45,149-64(1951)$.

Cataractous changes were observed in 53 of 56 rabbit eyes 40 weeks after irradiation with 2,000 r of penetrating X-rays. Histological changes are described. Two weeks after irradiation, individual nuelel in a given area contained less desoxy ribonuclelc acid of high polymeri zation than did the nuclef of a comparable area of the control lens. (CA)

295

Sallman, Ludwig von. and Beatrice Locke. IBXPERIMENTAL STUDIES ON EARLY LENS CHANGES AFTER RÖOTGEN IRRADIATION: II. PXCHANGE AND PENETRATION OF RADIOACTIVE INDICATORS $\left(\mathbb{N a}^{24}, \mathrm{~K}^{42}, \mathrm{I}^{131}, \mathrm{P}^{32}\right)$ IN NORMAL AND IRRADIATED LENSES OF RABBITS. Arch. Ophthalmol. (Chicago) $45,431-44$ (1951).

Experiments with $\mathrm{Na}^{24}$ showed increased permeablity of the blood-aqueous barrier up to 6 weeks after irradiation. This phenomenon did not play an Integral part in patho-

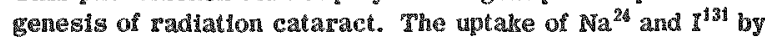
lenses with radiation cataract was greater than the uptake by control lenses and increased with the progress of the cataractous process. In the normal lens $\mathrm{P}^{32}$ accumulated principally at the equator and in the surface layers of the cortex due to the high metabolic rate of these parts. This difference between superficial cortical and deeper portions vanished with total radiation cataract. The uptake of $\mathrm{K}^{82}$ by normal lenses was very rapid. The uptake of $\mathrm{K}^{12}$ was slightly decreased by radiation cataract. (CA) 296

Saxon, R. P. THE EITECT OF X-RAYS ON THP OVARIES AND OESTROUS CYCLE OF THE GOLDEN HAMSTER (CRICETUS AURATUS WATERHOUSE). J. TennesSee Acad. \$ci. $21,128-34(1946)$.

Ovaries of adult virgin female hamsters were subjected to $900 \mathrm{~g}$ in two equal doses one week apart and atudied at intervals of $1,7,15,30$, and 45 days after ir radtation. Degeneration of the follcles was not observed the first day after Ir radiation. Seven days after irradiation, the suall follicles were the flrst to exhibit signs of degeneration. The small follicles appeared to be more susceptible to $\%$-rays than did the large follicles. Groups examined 15 and 30 days after irradiatlon were characterized by the preaence of many amall and large degenerating follicles. Hemorrhagle areas occasionaly were present and a relathely large number of mitolic ligures and multinucleate ova were observed in the large degenerating follicles in contrast to the number found among the controls. Fourty INe days after irradiation, primary follicles were absent and only a few large follicles were observed, most of which were in ame atage of degeneration. Estrous cycles of normal duration, as determined from vaghal smears, recured shythmically after isradiation in 20 to 25 cases. (BA)

297

Stuxkie, P. D.g J. A. Plno, J. L. Weatherwas, A. J. Donnelly, and G.M. Dor rance. $\mathrm{EFFECT}$ OF X-RAYS ON FERTILITE IN WHTE LEGHORN MLLE CHICKENS TREATED BEFORE PUBERTY. Radiology 52, $112-17$ (1949).

Male whtte Leghorn chick 4-6 weeks of age were ir radiated over an area of the body including gonads, adrenals, and parts of the kidiney. Doses: 1200, 1600, 2100 , and $2800 \mathrm{z}$ glven in equal doses of 4 weekly treatment Within 3 weeks. Ten animals were exposed in each group, 2-4 of which were killed about 4 weeks after the last exposure for histologic examination. The rest were mated, All of the anmals receiving more than 1600 r were gterile. Irradiation had no effect on comb size or mating habit. The 
testis of the irradiated animals were reduced in size, but the histological state of the testes was found not always a 298 reliable criterion of fertility. (BA)

TOOSy, M. H. HISTOLOGICAL AND CHEMICAL ALTERATIONS PRODUCED IN MOUSE EPIDERMIS BY SOFT $X-$

RADIATIONS. Cancer Research 11, 361-5(1951).

Areas of epidermis of the backs of adult, female Swiss Albino mice, approximately 4 to 5 months old, were exposed to soft x-radiation from a Phillips Contact-Cavity therayy machine at a distance of $20 \mathrm{~mm}$, the source of radiation being $50 \mathrm{kv}, 2 \mathrm{ma}, 100 \mathrm{r}$ per second without a filter. HItological and chemical examinations of the IFradiated hyperplastic epidermis were made, and the data so obtained were compared to those relating to normal and methylcholanthrene-treated epidermis.

Hiftological Iradings, Including the results of cell type determination, reveled only minor difference between Irradlated and methylcholanthrene-treated epidermis. however, total calcium of irradiated epidermis was about $30 \%$ less than normal, while that of methylcholanthrenetreated epidermis was about 50\% lesa than normal. No difference was noted in the lodine mumber of lipids of both, as compared to normal epidermis. The total lipids of the firradiated as well as of methylcholanthrene-ireated epldermis were nearly the game in both, belng significanty less than normal. A polarographic study indleated no difference in a reducible substance from the lipids of irradiated egidermis (between hal -wave potentials of unbuffered solution and in buffered solutlons at pN 4.40 and at 6.38) compared to those of normal and methylcholanthrene-induced hyperplastic eptdermis. (NSA) 299

VIeten, T. THE EIIECT OF RADIATIONS ON THE SYMPATRETIC NERVOUS STSTEM. SIrahlentherapie 79 , 13-58(1949).

The article is a survey of the actlon of radiations on the sympathetic nervous aystem and on mome bsochemical

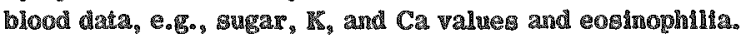
No experiments are reported, but material gcatered in 101 pasers has been collected and 1 d discussed. The aympathetic nervous system seems quite radio-resistant when histologicaly Investigated; possible ganglla cells are more Bengitive than other parts, while functional disturbances appear at therapeutic doses. When irradialion is applied in a tate of normal tonus a vagotonia occurs. Irradiation In a state of increased tonug leads to a reverston towards the mormal tonus. $C_{3}, \mathbb{K}$, and ฮugar values, which in cartaln pathologic states are too high or too low, become normalized through Irradiation. In many Ingtances, such irradiation effects can be shown to take a biphasle or periodic courge. Many exannghes are discussed, c.g., the girln reaction. Maxima and minima and latent perlod, as well as other detalls of the curves are discussed. The author concludes whth some speculatione on the mechanism of the action on the sympathetic nerwous sygtem. The primary effect is probably on the synaptic ganglion cell nuclei. He presumes that the action on the cells with large nucleus is atronger and more rapd than on those 300 with a mall nuseleus. (Fid)

Vogel, Howard H. Ir. CATARACTY FORMATION AND REPINAL DAMAGE FOLLOWING X IRRADIATON OF THE HEADS OF NEWBORN MIC. Anat. Record 11, $497(1951)$.

Data are pregented on the percentage of radiation cataracts developed in newhorn mice following $\mathrm{z}$-Irmadiation of the head, and the apprormate latent perlods before theae opacities of the lenses were observed. (NSA)
301

Wattenwyl, H. von. ACTION OF ROENTGEN RAYS ON

RAT TESTCLES. Schweiz. med. Wochschr. 72, 785-6 (1942).

The work described here was undertaken with the Idea of injuring the tegticles of rats and studying the influence of various hormones on their regeneration. Control studies of regeneration without hormone administration are reported in this paper. Doses of from 60-2400 is (factors not given) were administered and the teaticles were examined 4 to 50 days later. The findings conirm those previously reported by Regaud and by schinz and slotopolsky. (R) 302

Wilde, W. S. and C. W. Sheppard. ELCCTROLPTES IN MUSCLE OF RAT FORELIMB AFTER INTPENSE LOCAL X IRRADLATON. Oak Ridge National Lab. [Md] 1p. (AECU-1796)

Whatar adult male rat under nembutal were secured under a 13 -mm lead-plate shleld containing a window for exposure of the musclea of one forelmb to $x$ rays (single doses, $250 \mathrm{kvp}, 10$ to $73 \mathrm{r} / \mathrm{mm}, 30 \mathrm{~mm}$ irom shutter and 1htered through 3 mm Al; seatter to other parts of andmal $0.11 \%$ of dosage). Companton muscle from the other shielded forelimb cerved as control agahnst Indirect Influences which might arifer from whole-body effects of scattered radiation. For many hourg after exposure, the animal continued to use the limb freely. The rayed muscle, sampled 22 hr after exposure began, Bhowed swelling but mo other viblble evidence of damage and remalned functional. All analyges were mpressed per $250 \mathrm{dry}$ welght of muscle completely cleaned of fat and membranous and tendinous connective tissue. Changes would otherwise be masked by swelling. Total water of the exposed muscle increased whth dosage to exceed controls by $25 \%$. Potasglum content remained unchanged. Simultaneous Inulin and $\mathrm{Na}^{24}$ gpaces In a separate grous of $\mathrm{Hath}^{2}$, nephrectomized a 22 hr to prevent orehary loss of hull water gatn was entirely interstitial. Gath in total water and gatn in $\mathrm{Na}^{24}$ and inuln space were an chree equal. Shielded control muscles irom an rats collectively showed mo changes correlated with total-body Irradiation mach as might occur from scatter. Thus water, $\mathrm{K}$, and Na ware uncharged in exposed muscle flbers, but edema oceurred

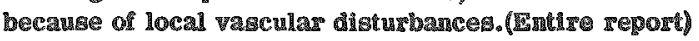

\section{Radiosensitivity}

\section{3}

Abderhalden, R. CONTRIDUTION TO THE PROLLEM OF

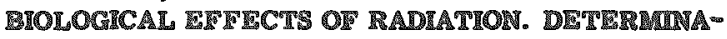

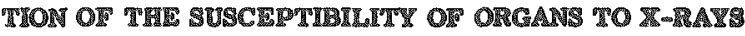
IN VIVO BY MEANS OI A BROCHEMCAL MITHOD. Strahlentherapie 68, 17-29(1040).

The anthor gindied the gusceptibility of a mumber of organs to roentgen rays in rabbit:. He found certain fermento in the urine which are capable of breaking down the proteng of the irradiated organs but not those of non-1ryadiated organs. If doses high enough to produce injury were applied to the hypophysis disturbances in the entire endocrine syeteas could be noticed. With this blochemical method, which is described in the article, the author found that the lower Imit of radiosenitivity of the liver is aroumd $390 \mathrm{r}$, for the brain around $24 \% .5 \mathrm{I}$, and for the hypophysis around $165 \mathrm{I}$. (R)

304

Abrams, Herberl L. INSLUENCE OR ACE, BODY WEICHET, AND SES ON SUSCEPTIBIIIYY OF MCD TO

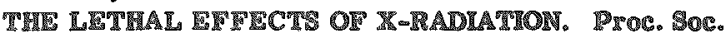

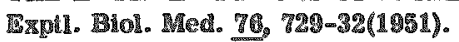

The age at which mice are hrradiated profoumly alter 
theis resistance to a single exposure of whole-budy irratiation. Mortalify 15 low after irradiation at 1 and 15 days of age, very high at 30 days, and decreases rapldy with increasing age thereafter. The possible mechanisms of this effect are discussed. The response of mice to whole-body inxadiation is not related to body weight excent insofar as weight reflects a particular age range. In general, sex appeass to exert no significant intuence on the capacity of 305 mice to withstand whole-body irradiation. (NSA)

Anderson, Fubert S. and H. Turkowilz. TRE EXPERI-

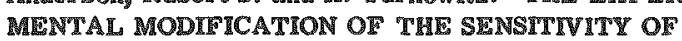
YIAST TO RORNTGEN RAYS. Am. J. Roentgenol. Radium Therapy 46, 537-41(1941).

Cullures of Torula cremoris were ir radiated in the presence or in the absence of almospheric $\mathrm{O}_{2}$, with $190 \mathrm{kv} \mathrm{x}-$ rays (uniltered) at an intensity of $190 \mathrm{x} / \mathrm{min}$. In some cases the air was removed by de-aerating the culture, in others, by 110 wing the culture to grow until it was 50 crowded that an $\mathrm{O}_{2}$-lack was produced. In the latter case the controls were arated by shaking vigorously. In either cage, the cells irradiated in the presence of $\mathrm{O}_{2}$ were more 3nsidive to the x-rays than those which lacked it. (BA)

Back, A. and L. Halberstaedtes. IN LUENCE OF BIOL OCICAL TACTORS ON THE TORM OR ROENTGEN-RAY SURVIVAL CURVES, FWPERIMENTS ON PARAMECIUM CAUDATUM. Am. J. Roentgenol. Radium Therapy 54 , $290-5(1945)$.

Experiments were carried out with 1 . caudatum to determine the difference in roengen-ray response between group of immediate descendants of a single parent (family) and xemote descendants (clone). Using $35 \mathrm{kv}$, a Cu target, and an $\mathrm{Al}$ foll window in the roentgen-ray tube, an intengity of $90,000 \mathrm{r} /$ min, was obtained at the position of the test object. The immediate lethal dose was obtained for specimens irradiated in amall drops of water under thin mica cover glips. This is the dose which produces complete cessation of motility within $10-15$ minutes after inradiation. For famil tes this dose varied between 200,000 and $700,000 \mathrm{r}$, but for any garticular family, all individuals died of the same dose. For clones the variation was the same, but the in dividuals within the clone died at different doses. Both montality curves were migmoid. The difference in behavior pattern between members of a family and of a clone can only be explained on the basis of biologic variability, the variability being greater between members of a clone than 907 between nember of a family. (BA)

Bemison, B. E. and C. Robert Coatney. MNACTIVATION OI MALARLAL PARASTTES BY ZIRAYS. PUb. HEaLh Repes. (10. S. 00, 127-32(1945).

Sporozoite and trophozoite guspensions of Plasmodium gallinaceun were exposed to graded doses of $x-$ ray. Infectivity of the irxadiated suspensions were tested by inom culation into young chicks (Galus domestious). "Trophozo10s were inactivated by 20,000 I but not by 10,$000 ;$ sporom zoites, by 8,000 i but not by 6,000 . Trophozoite guapensmon exposed to 2,000 to $0,000 \mathrm{r}$ produced infections characterized by lengthened prepatent periods and lengthened sur vival times. Therapeutic ir radiation of infected chicks had no significant effect on the outcome of the infection. A sample of human blood parasitized by $\mathrm{P}$. malarie and exposed to $5,000 \mathrm{r}$ 直alled to produce an infection in a susceptible 308 0ationt. (BA)

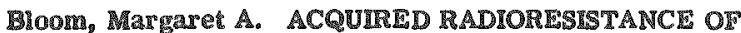
THE CRYPT EPTTELIUM OT TIE DUODENUM.

Radiology 55, 104-15(1950). (ADCU-531)
Repeated total-budy exposures of mice to 60 r $x$-radiamtiun day resulted an lessened sensitivity of the roypt epithelum of the duodenum. Debras in these cells $3 \mathrm{hr}$ af ter the last aradiation passed on successive days through a maximum and then diminished desple cont nuxd treatment. An attempt has been made to measure the deyree of this acquired radionesistance in terms af decrease in dobris and persistence of mitosis. The duodenal cypt cells showed mild damage after $60 \mathrm{~s}$ per day, reahng a pak m miro autopsied 3 hr after 6 to 9 daily exposures. Recovery was complete in mice autopsied 24 hr after the final irradi ation. With contimued treatment (10 or more axposures) these cells became resistant to this daily dosage (as indicated by negligible amounts of debris and persistence of miture activity), even when the lotal dosage had accumulated to $2,100 \mathrm{r}$. Mitusis was not inter muled and was rarely dimmished in this series of low-dosage reneated uradiations. Thas arqured radiug sistance was not sufficant to prevent degeneration of the basal crypt cells or a cessation of mitusis when $600 \mathrm{r}$ ur 1200 was grven subsequently in single dusage. When the sungle dosage followInp the daily expusures was $200 \mathrm{r}$, and an interval of only une day or one week intervened, there was part tion aganst the 200 exposure: the amuunt of debris was not dimimshed, but there were many more mitosis in the crypt cells of mica which has been previously irraduated than in thuse which had not. The enhanced radioresistance present at one day and one week was lost when the interval between the terrination of the small daily dosages and the 200 g dosage was extended to two or four weeks. (NSA) 309

Burnett, W. T., JI.. G. E. Stapleton, M. L. Morke, and Alerander Hollander. REDUCTION OF X-RAY SENSTTVITY OF ESCYERTCLA COL BY SULFHYDRYL COMPOUNDS, ALCOHOLS, GLYCOLS, AND SODIUM DITHONITE. Proc. Soc. Expti. Biol Med. 77, 636-8(1951).

The $x$-ray sensit ivaty of the organisms is reduced if they are ir radiated in the presence of low runcentrations of 2,3 dimercaptopropanol (BAL), cysteine, mereaptosuccinc acid, mercaptopyruvic acid, $2-(2-$ merceptuethusy) ethanol, $\mathrm{Na}_{2} \mathrm{~S}_{2} \mathrm{O}_{4}$, MeOH, $\mathrm{E} 1 \mathrm{OH}$, iso-1P $-\mathrm{OH}, \mathrm{BuOH}, 2-$ methyl-1propanol, 2-methyl-2 propanul, 1,2, 3,-propanetriol, and 1,2-propanediol. (CA)

310

Clark, A. M. and F.M. KEIY. DIPFERENTAL RADIOSENSTIVITY OF HAPLOT AND DIPLOLD PREPUPAE AND PUPAE OF MABRORRACON. Cancer Resear 10 , $348-52(1950)$.

Habrobracon prepupace and pupae were emposed to 5 radiation, and the lethal effects of the adiation were gitied. relosion atios show that $x-$ rays have a greator lothal effect upon haploid males than upon diploid males or diploid females. Diploid males and diploid females are equally susceptible to the lathal ffects of $\mathrm{x}$ - radiation. Prepupae are more sensitive than pupae. No significant difference in eclosion rat 108 wa obtained for adults given the same total doge but at diferent intensitieg. The response, therefore, seems to be independent of intensity. Comparison of adults

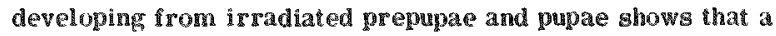
yeater rumber of haploid males show structural mallor mations than do duploids urrespective of sex and that the haploids shuw these malformations to a greater degree. Comparison of individuals unable to emerge from cocouns shows that the duploids are in general more advanced in their development than are the haploids. The data show that diploids are nogresigtant than haploids to the lethal action of $x$-rays, suggesting that the number of chromosume sets is a factor in determining the radiosensitivity of cells. (1) 
311

Clark, A. M. and C.J. Mitchell. RADIOSENSITIVITY OF MAPLOLD AND DIPLOLDS OF HABLOBRACON. Genelic's $36,547(1951)$.

Haplunds and dipluds of Habrobracon were expesed to \&-rays during various stages of pupal development. The abulity it cmerge from the cocuon was taken as the craterion of viabihty. Dusage-eclosion curves show that diploids are more resistant than haplonds at all stages of pupal development. During the earlier stages, diploids are about three times as radioresistant as comparable haploids, while durng the later stages, the differential radiosensi tivity is not as great. For haploid pupae the increase in resistance is expmential with age. Younger diploid pupae increase un resistance at a rate identical to haploids, but older diploid pupae do not inc rease in resistance as rapidly sis that the "survival" curves fing haploids and diploids tend to approach each ather un older pupae. Investigation of egg and adult stages is being done. These data indeate that a correlatum exists between the genome number and ratiosensityity. They suggest that the deleterious action of radiatron upon somatic tissues may be due to mjury to the genetic mechansm. (RG)

312

Evans, T. C. and H. D. Ker. MODIFICATYON OF RADIOSENSITIVITY OF THE SKIN. VI. EFFECTS OF COMPRESSION AND LOW TEMPERATURE ON THE ROENTGEN IRRADIATION REACTION OF HUMAN SKIN. Am. J. Ruentgenol. Radium Therapy 50, 629-35(1943).

It was prevuusly shown (Kvans, T.C.. Effects of Roentgen Irradatun at Low T'emperatures on the St:m of Young Rats, Am. J. Roentgenol., 45; 888-894(1941)) that radioresistance of the slom of newborn rats was greatly inc reased by chilling the anmal to or near $0^{\circ} \mathrm{C}$ during the irradiation. The use of culd and compression during radiotherapy in humans was tested on a series of patients undergong treatment for cancer of the uterine cervis. Pressure was applied by the cone defining the $x$-ray bean; circulation of cold water through the tip of this cone provided cooling, bat not the drast 10 chillugg applied to the rats. Some decrease in radiation reaction was observed, but it was slight and variable. (BA)

313

Priedewald, W. F. and R. S . Anderson. FACTORS INFLUENCING THE INACTIVATION OF TRE RABBIT PAPLLLOMA VIRUS BY X-RAYS. Proc. Suc. Exptl. Biol. Med. $15,713-15(1940)$.

The amount of uradiation requined to completely inact $\mathrm{i}$ vate the rabbit papilloma virus 15 influenced by the virus concn., as also by the extraneous material present along whth it in the irradiated suspensuns. Berkefeld filtrates, wheh contained the virus an large amount, were rendered non-infectious upon exposure to $2-4$ million $r$ of irradiation; only 400,000 to $800,000 \mathrm{r}$ was requared when the virus had been partially purified by repeated differential centrifuga tions. Normal rabbit serum or the non-mfectious supernatant fluids removed from virus filtrates after centrifugalion at $30,000 \mathrm{rpm}$ for $1 \mathrm{hr}$. protected purified virus suspended therein instead of in saline, these suspensions requiring about half as much arradiation to abolish their in fectury as the orminal virus flitrates. (BA)

314

Friedewald, W. . and R. S. Anderion. INFLUENCE OF EXTRANEOUS PROTEIN AND VIRUS CONCENTRATION ON THE INACTVATION OF THE RABBIT PADILLOMA VIRUS RY X-RAYS. J. Expt1. Med. 74, 463-87(1941).

2-4 milhom we we required to inact 1 vate crude papilloma. tastracts (Shope) prepared for the present work; $100,000 \mathrm{r}$ ur less was enough to inactivate comparable concns. of virus after ext ranevus matter had been excluded by repeated differential centrafugations. Addition of normal rabbit serum or crystalline egg albumin to purified suspensions of virus was found to increase greatly the amount of irradiation required to inactivate the virus. The \% destruction of virus in purified suspensions by a given amount of irradiation increased as the concen. was decreased by dilution. As litthe as $3000 \mathrm{r}$ will inactivate much of the virus in very dilute suspensions. The factors of concen. and ext raneous protein also influence the inactivation of the serum antiviral antibody which specifically fixes complement in mixture with the papulloma virus. Evidently, under certain conditions of purity and concen. most of the radiation does not act by direct hits on virus or antibody particles, but indirectly by ionizing or exciting some other molecules present in the exposed suspension, probably those of water, which then reart with the virus or antibody molecule. (BA) 315

Froier, $\mathrm{K}$. and A. Gustafsson. THE INHLUENCE OT SEED SIZE AND HULLS ON X-RAY SUSCEPTIRILITY IN CRREALS. Hereditas 30, 583-9(1944).

Examination of seed properties with respect to their possible gignificance in $x-r a y$ induction of mutations indicated (1) that an increase in seed size - and accordingly also of cmbryo size - causes better germination and more vigor ous growth after treatment, except for the highest doge used, when the average leaf length decreased, and (2) that the hull covering in barley and oats makes the seeds less susceptible to irradiation. The latter result was obtained after a comparison of covered and naked vars. in barley, as 310 well as of normal and hull-less seeds in Victory osts. (BA)

Malberstaedter, L. and A. Back, INE LUENCE OF COLCHICINE ALONE AND COMBINED WITH X-RAYS ON PARAMECIUM. Nature 152, 275-6(1943).

Paramecium caudatum were maintained for 48 hour' 3 in alutions of colchicine, transferred to normal culture media, and irradiated. The irrodiation was carried out in a hanging drop through a mica cover glass by means of a demountable $x-r a y$ tube which was operated at a tension of $18.5 \mathrm{kV}$ and $15 \mathrm{~mA}$ using a copper anticathode. The $\mathrm{X}-\mathrm{ray}$ intensity at the distance of the ir radiated object was 80,000 $\mathrm{r} / \mathrm{m}$. The experiments showed that the immediate lethal dose in considerably smaller - about 50\% - for Paramecia which received prior treatment with colchicine. (S.V.S.) 317

fraley, T. J. and Harriette Harris. THE RESPONSE OF THE GUNEA PIG TO 200 I ACUTE WHOLF BODY XIRRADLATION. ScIence 111, 88-90(1950). (AECU-357)

It is concluded that the guinea pig responds similarly to other animals subjected to $\mathrm{X}$-irradiation and that for many pur poses is much more suitable for such studies than the mouse or rat. Extremely valid result may be obtained using the guinea pig because its size and general temperament are guitable for studies employing large numbers of animals. However, all hematological studies should be reported in both relative and absolute terms to avoid a misinterpretation of the results observed. (NSA)

318

Iall, B. Vincent. GROWTH OF TUMOR TRAGMENTS IIRRADIATED IN VITRO FOLLOWING PRETREATMENT WITH CXSTEINE. Argonne National Lab. Aug. 1951. 24p. (AECU-1866)

The data presented on the survival and growth of mouse tumor fragments following s irradiation in vitro with and without prior treatment with 0.008 M eysteine clearly demonstrate the prevention and alleviation of radiation in- 
jury of mammalian tumor enlls by cysteine. The radiation dose that damaged untreated implants to the extent that only $50 \%$ grew when transplanted into host mice, had to be increased by rear dy $20 \%$ or $569 \mathrm{x}$, to effect equivalent injury of eysteine-treated implants. Delay in initiation of growth by tumos fragments following implatation was increaged by radiation effects, and the effect was found to bear roughly an exponential relation to the adiation dose. Intra- and in tercellular cysteine rather than the cysteine of the mbient medium was found to be the effective afrent in aeducings the radiosensitivity of tumos fragrents. It is suggested that the intracellular cysteine is most effective in preventing and alleviating the cellulax response lo jonizung radiation. 319 31 references. (NSA)

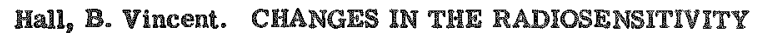

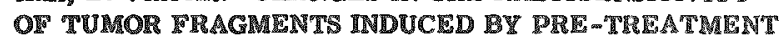
IN VITEO WITY CYSTEINE, METEYLENL BLUE, AND SODLM CWANIDI. Cancer Resear $11,254(1951)$.

resh tumor fragments of a vigorously growing, ant plastic mouse carcinoma were pretreated for $20 \mathrm{~min}$. with cysteme, methylens blue, os NaCN colutoms, and then $1 \mathrm{r}-$ radiated an culture dishe under standard condithons. Solutions of 1.25 mg cysteinc par cubic entimeter enabled $59 \%$ of the implarts to survive $4000 \mathrm{I}_{2}$, whereas none of the control tumor fragments, irradiated ir $0.85 \%$ NaCl, survived. The growth nonirgadiated tumors was not influenced by cysteine at this concentration. Pretreatment of tumor fragments with 0.01 or 0.001 NaCN or methylene blue, on the other hand, greatly decraned the numbes of surviving implants. Treatmen of nonir radiated tumors with $\mathrm{NaCN}$, or methylens blue rolutions of this concentration had little effect upos the mumber of subsequent thes, but the mone concentrated solutions terided to tetard the intial growth of the implants. The deereased tumor radios ensitivity af forded by pretreatment in vitro whth cysteine paralleled cifects observed in vivo. The other agents, which under certain circumstances were protective in vivo, rendered the tumor more aensitive, auggesting elher that they act through diferent mechanisms or that a surnmation of injurise is INwoIved. (NSA) 320

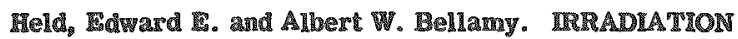

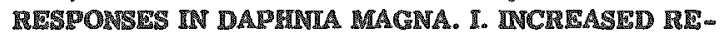
\$ISTANCE TO Y-LRADLATON UNDER LOW ONIGLN TINSION. Atomic Fnergy Project University of Califoria LoS Angeles. ApE, 20, 1950. 10p. (UCLA-69)

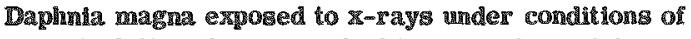
oxygen denletion mhow 2 marked increase in Iesistance to the irrodiation, The inereased resistance is evident both con survival curves and productivity records. (NSA) 32

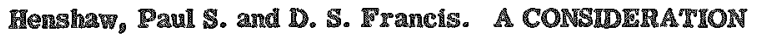

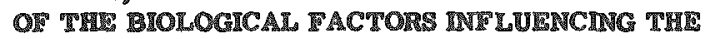

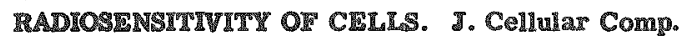

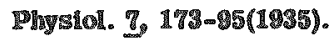

The inter-relationship of radiosensthwity and a number of dufferent biological conditions in Triticum vulgare were

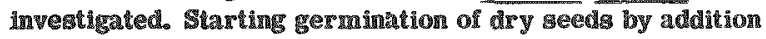
o mois:

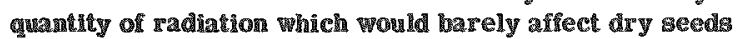
mould rard growth 10 about $50 \%$ in corm wheh had umdergone 18 lin of germanchon. Shopping growth in germunted forms by lower.ng temperature did not increase resintance to radiathon appeciably. Stopping growth by arymg increased the resichance consideraby. STopping growh by aรnhy

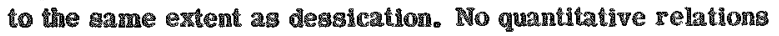

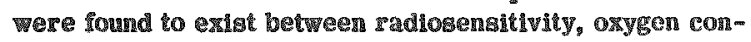

sumption, water absorption, and milosis. In 2 experiments an abundance of water was supplied to dry seods for rognination purposes but development was prevented either by indequate aeration by low temperature. It was fund that Alhough water was absorbed by the orgentsms, the susceptibility did not increase appreciably as is the case when not mal development takes place. (TIS) 322

Hewât, H. B. and John Read. SHAECH FOR AN ETECT OF OXYGEN ON TIIE DURECI" X-RAY INACTIVATION OF"

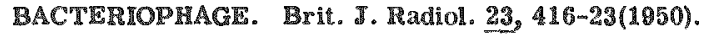

An E. coll phage has been ir radiated with $x-r$ rys in broth which contained sufficien protein to suppress the indirect effert due to ionization in the water. One phage suspension contained 10 to $15 \mathrm{ml} / \mathrm{h}$. of dissolved $\mathrm{O}_{2,}$, and a second zero. The activily of enel suspension diminished exponent-

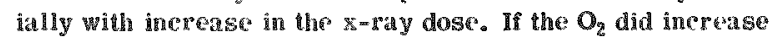
the sensitivity of the phage at all it was unlikely to have been by more than $10 \%$. The mean difameter of the phase particle, deduced from the $37 \%$ inactivation dose and Lea's theury, was 54 min. Other methods have given values of 45 and 75 me for the same phage. Prohn, without phase, con taning the norma amount of dissolve $\mathrm{O}_{2}$, was given the maximum dose used previously, and then the phage particles were added ammediately after the end of the iradiation. No inactivation was detected. (CA)

322

Hollaender, Alexander. PUYSICAL AND CIEMCAL SC-

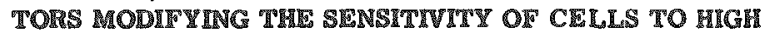
ENEDGY AND ULTEAVIOLET RADIATION. OAK ITIGE National Lab. Nov. 7, 1950, 22p. (ORNL-884)

The effect on the sensitivity of various organisms (bac4eria, fungi, alsae, etc.) to radiation death or chromosomal rearrangenent: of esposure before or after irradintron to hat or to visible, ultraviolet, or infrared light, of water content, and of osygen content is discussed. It may be pos-

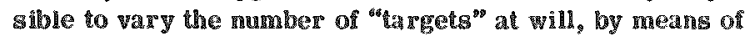
adjustment of growth conditions or phyeical conditions duringg irréciation. (NSA) \$24

Hollacnder, Alezander. PHISICAL AND CEKMICAL

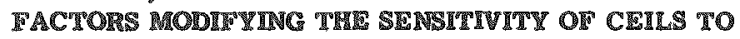
WUCH

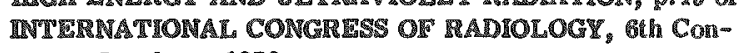
gress, London. 1950.

This maper deals with changes in the radiosensitivity to X-1 at the cellular level by additonal lreament with in $\mathrm{ra}-\mathrm{red}$ radiation, heat, light (photoreactivation), and allitasonics; and by alteration in relative water content, ag of cells, oxyger tension and chemical composition. Fy themselves, these additional trealments produce no recomnisble afects but stringing modifications in radiosengitivity are observed when they are combinco with S-ray exposure. The results of secondwry freatment with inirr-zed radiation and heat Will ba demonetrated by work done with Tradescantia and ริungi: photoreactivation, by work

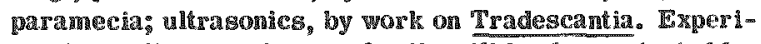
ments in alleration in age of cells will be demonstrated by

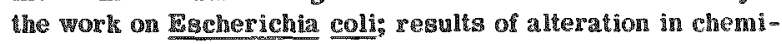
cal composition and ozygen tengion, by work on bacteria, fung, cells in tissue culture, and paramecia. 22

Holacnder, Alewander, W. K. Baker, and I: Y. Anderson.

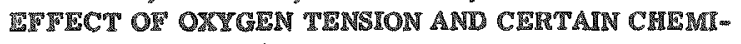

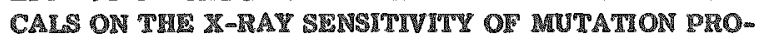
DUCFION AND SURVIVA. Cold Spring Marbor Bymposia Guar. Bul. 16. 315-26(1951).

Recent atrdies of the effert of ongen tersion on $x-$ may 
sensitivity as measured by the lethal effects, chromusome

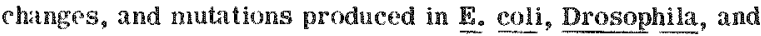
Tradescantia are reviewed. Results of studies of the effects of chemicals, and the mechanisms which may be involved in the action of these chemicals in regard to $x$-ray sensitivity, are discussed. Compounds which were found to afford urotection against radiation damage included strong reduring compounds, especially $\mathrm{Na}_{2} \mathrm{~S}_{2} \mathrm{O}_{4}$; glycerin, propylene glycol, triethylene glycol, and alcohols like methanol, ethanol, and propanol; carboxylic acids, and sulfhydryl326 containing compounds. (NSA)

Hollaender, Alexander, George E. Stapleton, and W.T. Burnett, JE. CHEMICAL ASPECTS OF RADIATION SENSITIVITY ON LIVING CELLS. Oak Ridge National Lab. [nd] 2p. (AECU-1699)

The reptrt is reproduced here in its entirety.

Remarkable decreases in many $\mathrm{x}-\mathrm{ray}$-induced cellular phenomena by reduction of oxygen coneent ration during urradiation have been reported including alteration of inactivatuon rates, frequencies of lethal mutations and chromosomal aberrations, as wall as the duration of milotic in hibiton. As an extension of these studies an investigation has been made concernng the ability of chemical substances to modrfy the cell sensitivity to the damaging effect of $\mathrm{x}$ rays. The discussion will deal mainly with the results of $x-$ ray lethal effects obtained with bacteria, but will include also studnes with other urganisms as far as the data are available. The following groups of chemical compounds have been found to redure significantly the $x$-ray lethal ef fuct on bicterial cells: sulfhydryl compounds (cysteine, BAL, mercapto succinic acid, and others), reducing compounds (sodium hydrosulfite), respiratory inhibitors (sodium cyanide), alcohols and glycols, carboxylic acids (formic, succinic, etc.), and sugars (glucose). It is interesting that compounds of group one and two will protert at either 2 or $37^{\circ} \mathrm{C}$. High concent rations $(10$ to $20 \%)$ of the alcohols are required to give significant protection at low temperature $\left(2^{\circ} \mathrm{C}\right)$ while much lower concentrations $(<1 \%)$ are effective if the cells are incubated with them for a short period (30 min) at $37^{\circ} \mathrm{C}$ prior to ir radiation. The carboxylic acids and glucose are effective only if the bacterial cells are incubated with them prior to $x-r a y$ exposure. Several of these compounds have been tested with interesting results for protective action on mice. The interpretation of results on the basis of removal of radiodecomposition products of water will be given. All of the chemical compounds used yield similar curves relating protective action to concon tration, indicating a saturation phenomenon, the plateau level being different for the different types of compounds used. If two compounda are used sinultaneously at the plateas level, additional protection is obtained, indicating perhaps diferent protective mechanisms. The possible role of metabolism, reductive capacity as well as synthetic processes, especially nucleic acid synthesis in the protective action of the carbosylic acids, amino acids, and glucuse will be indicated. The significance of these findings on the chemistry of radiation damage in general will be discussed. (Paper presented at the American Chemical 327 ociety, New York City, Sept. 3-7, 1951).

Hollaender, Alexander, G. E. Stapleton, and $\mathbb{F}$. L. Martin. X-RAY SENSITYVITY OF E. COLI AS MODIPIED BY OXYGEN TENSION. Nature 167, 103 4 (1951) (ORNL-832)

The influence of oxygen tension on sensitivity of $\mathbb{E}$. coli to $\mathrm{x}-\mathrm{irradiation}$ damage and the iniluence of cultural condiliuns to sensitivity were studied. It was shown that lowering the 0 tension of bacterial suspensions results in striking decrense of radiosensitivity of the cells, as com pared with oxygenated suspensions. The order as well as the slope of the survival curves is different under low and high 0 tension. To set apart radiation effects produced through the intermediate aqueous suspension and those concerned with the bacterial cells as such, various culture conditions were studied which might alter recognizable effect on the cell. Results emphasize the importance of 0 tension, state of oxidation or reduction inside the organisms during exposure to $x$ irradiation and the possible modifying effect of the type of metabolism car ried out by the organism before irradiation. Most of the data are consistent with the idea that radiodecomposition products of water are contributing toxic agents, e.g., $\mathrm{H}, \mathrm{OH}, \mathrm{HO}_{2}, \mathrm{H}_{2} \mathrm{O}_{2}$, some of which are produced only in the presence of 0 irradiation. However, it is thought that not all the findings in regard to 0 concentration can be explained on this basis. (NSA) 328

Holicroft, Joanne Weikel, Egon Loren,, and Marion Matthews. FACTORS MODFYING THE EFF'CT OF X IRRADITION ON REGRESSION OF A TRANSPLANTED LYMPROSARCOMA. Argonne National Lab. Oct. 25, 1951. 27p. (AECU-1818; UAC-458)

A synergistic effect on regression of a transplanted mouse lymphosarcoma occurred after simultaneous gystemic and local tumor irradiation and was absent when the blood supply of the tumor was clamped of during systemic irradiation. The synergistic effect disappeared rapidly when the time interval between systemic and local tumor irradiation was increased from 0 minutes to four hours. Starving the animals for 20 hours before and during local irradiation of the lumor was found to increase tumor regression, while starvation following ir radiation had no ef fect. $\mathrm{H}_{2} \mathrm{O}_{2}$ administered intravenously to the tumos-bearing animals immediately before tumor irradiation increased the effect on tumor regresgion in comparison with noninjected, irradiated controls, but hydrogen peroxide administered after irradiation did not. $\mathrm{H}_{2} \mathrm{O}_{2}$ injected five minutes before irradiation did not significantly increase tumor damage. Irradiation of the tumor of mice kept in a high-orygen at mosphere produced a greater regression and irradiation of the tumors of mice in a low-osygen atmosphere produced less regression than irradiation of the tumors of mice breathing air. Clamping of the blood supply to the tumor during local irradiation of the fumor had no effect on tumor regression. (NSA) 328

Jacobson, L. O. STUDIES OF RADIOSENSITITITY OF CELLS. Science 107, 248-50(1948).

It has been commonly believed that the sensitivity of mammalian cells to ionizing radiatione is proportional to cell activity in terms of mitotic proliferation - the more active the proliferation, the higher the gensitivity. An increased erythropoiesis can be initiated in the laboratory animal by phlebotomy or by the hemolytic action of acetyl phenylhydrazine on the circulating erythrocytes. The ef fect of $800 x$ whole-body $x-1$ rradiation upon animals that have a previougly induced regenerative anemia was compared with the effect of this same dose on normal an mals of comparable age and weifght. The normal irradiated animals developed an memia that reached a maximum at 14 days. The animals in which a regenerative anemia had been produced prior to irsadiation with $800 \mathrm{r}$ develoged no fur ther anemia. The recovery time in both groups was comparable (23 days). The mean reticulocyte value of the phenylhydrazine-injected, $\mathrm{X}$-rayed animals was reduced below the normal control value on the sth post-irradiation day only. The phlebotomized group on animals responded to irradiation in a manner comparable to the animals 
treated with phenylhydrazine and $\mathrm{X}$-ray. The histologic studies made on appropriately sacrificed animals revealed that erythropoietic tissue in the bone marrow and the spleen was essentially completely destroyed by three days in normal animals that received $800 \mathrm{r}$. A relatively slow recovery ensued begining on the 7th to the sth day. On the ofher hand, the erythropoietic tissue in the bone marrow and gpleen was only partially destroyed in animals that were irradiated with $800 \mathrm{r}$ after a regenerative anemia was prom duced by phenylhydrazine or phlebotomy. These data would tend to indicate that erythroblast vulnerability to ir radiation injury is not enhanced by increased mitotic activity and proliferation. In fact, the hyperplastic erythroid fissue sustained less histologic injury than the normal, and the $\$ 30$ production of erythrocytes was maintained. (ADD)

Jennings, . Lamont. EFIECT OF PROTEIN DEPCHTION UPON SUSCEPTIBILITY OF RATS TO TOTAL BODY IRRADIATION. Proc. Soc. Exptl. Biol. Med. 72, 487-91 (1049).

The results of the firgt of a series of experiments undertaken to evaluate the role of protein in radiation damage are presented. Male albino rats of Sprague-Dawley strain were kept on a low-protein diet until they lost about $25 \%$ of their weight (10 weeks) and then were irradiated with total body $\mathrm{x}-\mathrm{gradiation}$; the irradiation was delivered by a $200 \mathrm{kv}$ machine through $0.5 \mathrm{~mm}$ Cu and $3 \mathrm{~mm}$ bakelite filters at a target distance of $71 \mathrm{~cm}$. and a dosage rate of $17-18 \mathrm{r} / \mathrm{min}$; the depleted rats, the weight controls, and the age controls received 300-800 I doses. During the 30-day observation period following the irradiation, all the animals were maintained on the same diets as before. It was lound that the protein depletion materially increase the rat susceptibility to total body irradiation and its effects; the age and weights of the controls, however, had little effect on the suscepti311 bility to radiation. (NSA)

Joyet-Lavergne, P. A NEW CONCEPT ON TYRE REA TION BETWEEN CELL METABOLISM AND SENSTTIVITY TO IRRADIATION. Arch. phys. biol. 15, 84-5(1938).

Discussion. Intengity of cell metabolic activity and sensitivity of the cell to irradiation with X-rays and gamma rays are both in some way dependent on the condition of the mitochondria. (CA)

Kaplan, Henry \$. INFLUENCE OF AGE ON SUSCEPTIBILITY OF MCE TO TIE DEVELOPMENT OF LYMPHOT TUMORS AFTER IRRADLATION. J. Natl. Cancer Inst. $g$, $55-6(1248)$.

Black mice, strain $\mathrm{C}-57$, were divided into age groups of two weeks, one, two, three, and four months, and given fractional doeages totalling $1,000 \mathrm{r}$ of $\mathrm{x}$-radiation administered in 12 consecutive daily doses at $125 \mathrm{kv}, 5 \mathrm{milli}-$ amperes, $30 \mathrm{~cm}$. mouse-to-target distance, $3 \mathrm{~mm}$ Al added ilitration, output $42 \mathrm{x} /$ minute, and a daily dose of $84 \mathrm{x}$. However, the dosage for the 2 -week group had to be reduced to $750 \mathrm{I}$ because of the high death rate following irradiation at $1,000 \mathrm{r}$. The animals were autopsied and examined histologicaly. The tumors were all lymphatic leukemias and Iymphosarcomas. The maximum susceptibility was found to be at one month or earlier and it is associated with a 333

Rimball, R. F. MODIFICATION OF THE EFFECTS OF X RAYS ON STOCK 20 OF VAPIETY 1 OF PARAMECTUM AURELIA. Ok Ridge National Lab. 1p. (AECU-1689)

(The report is reproduced here in its entirety.)

Various conditions have been used to attempt to modify the effect of $x$ rays as measured by death and number of divisions in the first day after ir radiation and by reduced vigor and death after autogamy. The postautogamous effects were less severe when the animals wore irradiated under low oxygen tension, or in $0.6 \mathrm{M}$ ethyl alcohol. No notceable effect was exerted by starvation before irraiation, temperature during the day after irradiation 19 to $31^{\circ} \mathrm{C}$ ), or presence of $0.001 \mathrm{M}$ sodium hydrosulfite ( $\mathrm{Na}_{2} \mathrm{~S}_{2} \mathrm{O}_{\mathrm{H}}$ ), 0.0009 M British anti-Lewisite (BAL), or 0.01 M sodium formate. The last three compounds and alcohol were tried because they had been found by others in this laboratory to protect bacteria against inactivation by $x \mathrm{~g}$ rays. The oxygen effect is well known for a variety of organisms as a realt of work in this and other laboratories. The negative results with hydrosulfie and BAL are not too significant since very low concent ations had to be used. Quite different results were obained in the investigations of desth and division delay in the firgtay. Irow oxygen tension, BAL, and foxmate cause inc mased death and delay in division. All the reatruents have a cigtinctly injurious effect upon the control animals, and it seasns probable that this injurious effect combined with that of the radiation acconnt for the rosults. It is surprising that low orygen tension which chearly proteds against postautogamous effects iails to do So for the immediate effects. Alcohol and hydrosulfite both decrease division delay despite the fact that they are clearly infurions to unirradiated anmals. However, in some experiments, there is more death following irradiation in the presence of these agents than in their absence, suggesting again a combination of injurious effects. Finally, both division delay and death are markedly greater when the animals are ir radiated in phosphate bufer with $3 \%$ of culture Iluid added than when irradiated in undiluted culture fluid, suggesting a protective action of one or more of the component of the culture fluid. One can conclude that it is possible to protect paramecia against both the genetic and nongenetic flects of rays, but it is not necessarily true that an agent which protects againgt he one will protect against the other. Theprs likely that this ha due, at least in part, to the indepsnden injurious action of the st rays and of the substance adding together to produce a total effect greater than for either alone. (Paver presented at Paramecium Genetics Symposium, September 1951, Bloomington, Indiana).

334

Yimbal, R. and Nenita Gaither. MODLICATON OF TEE ACTYON OF X RATS UPON PARAMECIUM AURELIA. Cenetics $36,558(1251)$.

The following is an abstract and is reproduced here in its entirety.

A. number of agents which previously have been shown to modify one or more effects of $x$ ixradiation have been tested on garamecium aurelia. The mutagenic action of $x$ ray in doses of 1 to $6 \mathrm{~kg}$ was found to be reduced when the irradiation was performed in an atmosphere of $\mathbb{N}_{2}$ or in the presence of 0.7 M thyl alcohol. On the other hand, 0.001 M sodium hydrosulfite, a reducing agent, had no influence on mutagenesis. No effect of the metabolic state was found wince iradiation of either fed or strived paramecia, with or without incubation with $0.01 \mathrm{M}$ sodium formate, produced identical amounts of mutation. Likewise, the rapidity of multiplication of the cells after irradiation, as influenced by temperature, was withoul infuence. Two nongenetic actions, division retardation and death with 24 hr., were gtudied using doses of 100 to $300 \mathrm{kr}$. Both the amount of division retardation and of death were decreased by $0.7 \mathrm{M}$ ethyl alcohol present during irradiation. Sodium hydrosulfite $(0.001$ M) clearly protected againgt the lethal action but had no intuence upon division delay or, pertaps, enhanced it. Neither stavation nor incubation with formate was 
found to have any effect. Thus there is at least one agent which affects a variety of different kinds of radiation dam age in paramecium. It may be suggested that sume step which is common to the various effects is involved. How ever, it is possible that sume agents influence the steps leading to certain kinds of damage only or else act more 335

Timeldorf, D. J. D. C. Jones, and M. C. Fishler. THE EFFECT OF EXERCISE UPON THE LETHALUTY OF ROENTGEN RAYS FOR IRATS. Scienc 112, 175-6(1950).

As a means of determmnng the effect of metabolic activity or radiation sensitivity, equal dosages of 3 -rays (total body irradiation) were guven as the test exercise, all animals were subjected to the treatment for 10 trials prior to inactivation and thereafter at daily intervals 5 times per week. It was found that the exercised animals had a higher mortality and a much shorter post-ixradiation survival 336 time. (FM)

Latarjet, R. CELL CONSTITUENTS IN RELATION TO RADIOSENSITIVITY, P.49-50 Of INTERNATIONAL CONGRESS OF RADIOLOGY, 3th Congress, London. 1950.

At the stage of the primary reactions, the following factors are considered and illustrated by the results of various experimental investigations: - Size of the cellular volume involved. Multiplicity of the cellular units (targets) which must be altered in order to produce the lesion, e.g., enzymes. Experimental results obtained by studying the loss of virulence in bacteria infected with $1,2,3 . \ldots$ n virus particles. Genetic structure: comparative study of a bacterium and of its "radioresistant mutant" as regards lethal effect and mutations induced by radiations. Structure of the chromosomes: comparative study of a diploid yeast and of its haploid mutant. Chemical factors in the cell increasing its radiosensitivity, e.g., oxygen. Chemical lactors in the cell increasing its radioresistance, e.g., peroxydases, oxy dation buffers, etc. During the course of the obscure reactions, cell radiosensitivity may be decreased by several processes of recovery. In this connection convideration is given to polyploidy, which seems to favour certain types of gpontaneous recovery.

337

Latarjet, R. and E. Epharati. PROTECTIV ACTION OF CERTAIN SUBSTANCES AGAINST INACTIVATION OF A BACTERIOPHAGE BY X-RAYS. Compt. rend. soc. biol. 142, 497-9(1948)

Phage $\mathrm{T}-2$ active on $\underline{\mathbb{E}}$. coll, was used in aqueous suspension. Thioglycolic acid, tryptophan, glutathione, cystine, and cysteine had marked protective action, decreasing in the order named, and glucose, sucrose, ascorbic acid, phenylalanine, alanine, and histidine had gonewhat protective action. In all cases the protective ufect was less marked if dissolved 0 was removed from the medium before $x$-ray irradiation. (CA) 338

Latarajet, $\mathbb{R}_{\text {. and }}$ B. Ehrussi. SURVIVAL CURVES OF HAPLOT AND DIPLOID YEASTS SUBMITTED TO $\mathrm{X}$ -

RAYS. Compt. rend. 229, 306-8(1949).

Several investigators have noted the gigmoidal shape of the survival curve of yeasts submitted to ionizing radiations. One of the present authors has obtained guch a curve and interpreted it as due to a "wo-hit" process (Ann. Inst. Pasteur 70, 277(1944); ;ubsequently, by using highly ionizing radiations, he has shown that in these two hits two distinct targets were involved (F rilley and Latarjet, Corapt. rend. $218,480(1944)$ ). It seemed probable that these results might be a consequence of a diploid nature of the yeasts amployed. To test this hypothesis, haploid and diploid cultures, derived from the same strain of Saccharomices cerevisiae, received various doses of $\mathrm{x}-\mathrm{rays}$, at $33 \mathrm{kv}$, the intensity at the level of the preparation being $4,000-8,000 \mathrm{r} / \mathrm{min}$. The lesions were observed and counted microacopically after 20 hour incubation at $25^{\circ} \mathrm{C}$. The lesions of haploid yeasta are all irrevergible, while those of the diploids, besides irreversible cases, show frequent cases of restoration and of further reproduction of colonies. The corresponding survival eurves, viz., a sigmoid graph for the diploid organisms and a simple exponential curve for the haploids, confirm well the above stated hypothesis. From the slopes of the curves, the targets could be determined as apheres of 33-38 m radius. The real significance of these facts is unknown; death, due to chromosomal lesions, of a diploid cell seems fo be related to the appearance wither of two recessive lethal mutations, or of a mingle dominant one; the sigmoid curve may result as a combination of these two procegses. (NSA) 339

Lea, D. F. INACTVATION OF VIRUS RY RADIATION.

Brit. J. Radiol. 19, 205-12(1946).

The paper is a brief review of experiments on the inactivation of viruses by radiations. It is claimed that when the virus is irradiated dry or in an aqueous solution of high solid content, the inactivation of the virug is direct and tue to a single ionization. There is correlation between the dose and the size of the virus. Departure from the theoretical curve of the inactivation dose indicates that the virus is composed of radio-sensitive and insensitive structures. The size of the sensitive region was calculated for the vaccinia virus. The result which the author arrived at greatly differs from that of Bonét-Maury and Perault (1941). (EM) 340

Mottram, J. C. ON THE ALTERATION IN THE SENST"YVITY OF CELLS TOWARDS RADIATION PRODUCED BY COLD AND BY ANAEROBIOSIS. Brit. J. Radiol. 8,32 (1935).

Two sets of experiments were carried out: expogures in the cold and exposures in $N$. In the experiments to test the effect of cold, the roots were exposed under water, 6 roots in ice-water and gis in water at $24^{\circ} \mathrm{C}$. The experiments showed that the application of cold outside the period of radiation had no effect on the growth and that even with short exposures the cellig of the bean root are more sensitive when cold than when warm. Anaerobiosis was obtained by placing the beans in a closed chamber and then displacing the air by passing in commercial nitrogen. Bean roots were exposed to the $\mathbb{N}$ and to air, the $\mathbb{N}$ exposure from 120 to 200 min, the air from 100 to 170 minutes. The beans under anaerobic conditlons were much less gensitive to the $\gamma$ radiation than when oxygen was preant, 100 minutes 6 posure in air being equivalent to 170 in N. Anaerobiosis greatly decreased the sensitivity of the roots. The conclusion of Crabtree and Cramer that cold render cells more, and anaerobiosis less, sensitive to radiation is confirmed; and is ohown to apply to the plant as well as the animal cell.

341

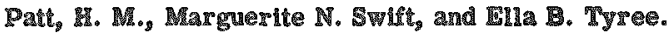
OXYGEN COMSUMPTION AND WATER BALANCE IN TILE X-IBRADLATD FROG. Federation Proc. 124(1249). (ARCU-507)

(This document is in abstract form and is reproduced here in its entirety.)

As a part of a study of the radiation response of frogs and of factors contributing to their radiogenaitivity, we have investigated 1) oxygen consumption after $\mathbb{x}$-1rradiation and 2) sate of water uptake in dehydrated irradiated rrogr. Oxygen consumed by individual unfed Autumn frogs, sedated 
with nembutal, was measured volumetrically at a mospheric pressure and $23^{\circ} \mathrm{C}$. Results of a pilot study in which determinations were made during a cont rul period of several days and at frequent intervals after $\mathrm{x}-\mathrm{i}$ radiation with $300 \mathrm{r}$ (completely letha, mean survival time 26 days), slight early decrease in orygen consumption with masimal depression (34\%) 15 days after exposure. This is lollowed by return to control levels $(0.086+0.002 \mathrm{cc} / \mathrm{gm} / \mathrm{hr})$ around 25 days after irradiation. Although there is evidence that ionzing radiation accelerates catabolim in mammals, especially terminally, we failed to observe any increase in orygen consumption in these frogs. Budy weight of unfod irradiated frogs also did not change appreciably. Frogs kept in dry individual container at $23^{\circ} \mathrm{C}$ for 3 days lose 35-40\% of their water. After irradiation with 3000 and immediate return to water, such partially denyorated irradiated frogs regain water at the mane rate as non-1radiated controls. In both grups, recovery is assentially complete by 4 hourg. There is no change in water content of nondeinyolrated irradiated frogs over this period. Emperiments are in progress to evaluate the infuence of water content at the time of exposuse on radiosensitivity in the frog.

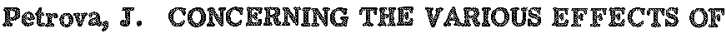
ALPLA RATS ON TRT NUCLITUS AND PLASMA OF THE

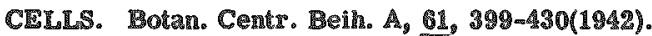

When agar cultures of the alga $\overline{2}$ ygmema were subjected to Po alpha-rays, the extent of the radiation to various parts of the celli was controlled by use of filter of varying strength. When the entire cell (nucleus plus cytoplasm) was fully iradiated, the injurious cfects were the game as aiter beta and gamman radiation: cessation of cell division for averal days, abnormal starch storag, longitudinal Frowth, occurrence of modifications and death of the cell ater several months. If only the cytoplasm was in radiated, the uฌual dose had no effec, while several hundred times this amoun caused cell death within 24 hours, without the other effects mentioned above. The cell nucleus appear 700 times more sensitive than the cytoplasm to radiation, and alpha- 5ems abou 10 times more active than betaradiation. (CA)

343

Quaster, Menry. STUDTES ON ROENTGLN DEATE DN

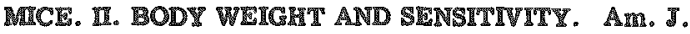

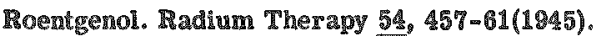

The survival time, after administration of congtant doses, is proportional to the weight, provided the survival time is between 11.25 and 3.3 days. The two plateaus, at 11.25 and 3.3 days, occur in adult mice of all weight classes. The 3 relations, between age and censitivity, between age and weight and between weight and senstivity, are concordant in adult mice. Infant mice survite longer than adult mice after aplication of the same dome. wimple mathematcal formulae for the relations between dosage, weight, and gur344 vival time are given. (auth)

Rea, John. THE INITIUENCE OF OXIGEN THSISTON ON

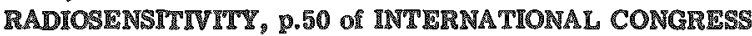
OF RADIOLOGY, Gh Congress, London, 1950.

Previous work concerned with the influence of osygen on the radiosensitivity of normal and tumour tissue is $\mathrm{xe}$ viewed. Experimenta on Vicia faba roots are degcribed which support the following conclusions: $-X$ - adation of roots in the presence of oxygen causes much greater growt reduction than the same dose given in the absence of oxygen. The orygen augments the fifect of all $\mathrm{x}-\mathrm{gay}$ doses in the same proportion, by a facton which increases with concentration in airmoid fachion to a maximum of 3 . With alpha-rays the factor is $1 \cdot 3$. The numbers of abnormal anaphases produced by the two radations are affected in the same way. The hotulagheal picture af rout los arradiated with and whthut oxygen seem qualitatively simulur but in the former case the quant itative change is nuch greater, particularly when judiged by the inhibiton of m-

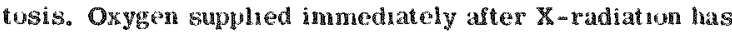
nu observable effect. When the oxygen cuncentration in a root is suddenly changed, the radiosensitivity readjusts it self with a lag (if any) not greater than one minute. The $X_{\text {- }}$ ray sensitivity is the sume whether anaerubiosis is pruduced by bubling nitrogen, argon, or hydrugen through the water in which the roots are immersed. Reduct ion in srowth after immersion in nitrogen mustard solution is similar to that after X-Fys, but anaerobiosis during, and for 30 minutes after immersion dos not dimum the offect. The resuits sugrese that radiosensitivity is altered by a change in the metabolic state, or that oxygen reacts with the immediate products of ion 1 zation to increase the yield of nome effective torin, subsequent biological changes being unaffected by the oxygen it self.

3.45

Rebou, J. and J. M. Maynard. REMARES ON SEVERAL CASES OF CUTANIOUS IYPER-RADIOSENSITIVTTY.

J. xadiol. electrol. $30,732-3(1949)$.

Four cases of reaction to radiotherapy are given in detail: one received sis doses of $250 \mathrm{~m} /$ dose delivered at 200 liv thromgh a 1 iter of $1 \mathrm{~mm}$ Cu and $1 \mathrm{~mm} \mathrm{Al}$ at a distance of $50 \mathrm{~cm}$. and rate of $12 \mathrm{r} / \mathrm{min}$. resulting in a radioder matilis; the second received five doses of 250 ; dose at 200 fw through the game filter and distance at a ate of 14 s/ min., regulting in radiodermatitis; the third received the three doses with radiation delivered as in the wecond case axcept that he focal distance was $40 \mathrm{~cm}$, resulting in peeling, and, after a second cousge of irrediation of 15 doses at $200 \mathrm{r} /$ dose delivered at $220 \mathrm{kv}$ through a silter of $1 \mathrm{~mm} \mathrm{Cu}$, resulting in dermatitis; the last received thee doses of 250 r/dose delivered at 180 grv through $1 \mathrm{~mm}$ Cu and $1 \mathrm{~mm} \mathrm{Al}$ at a distance of $40 \mathrm{~cm}$, resulting in an exudative radiodermatitis four days after the last dose. In these cases all technical imperfections of the therapeutic course were eliminated, and similar courses on other individuals did not caus any reaction. The authors conclude that certain individuals are hypersensitive to these doses and discuss the possibility that percentage plots of the population reacting against the dose may show a signoid population digtribution for aensitivity. (NSA)

34

Sallman, Ludwig von. FURTHER EFTORTS TO IN FU. ENCE X-RAY CATARACT BY CREMCAL AGENTS.

Trans. Am. Ophthalmol. Soc. 69, 391-418(1951).

Intravenously administered cysteine had a markedly protective action againgt radiation damage to the lens of rabbits when the treatment preceded the exposure of the eye to $1500 x$. This protective iniluence has persisted for at least one year. When cysteine was injected subconunctivally twice weekly for three weeks following the irradiation of cysteine-pretreated rabbits, the fine cortical opacities were not reduced by the additional therapy. Subconjunctival injection of $1.0 \mathrm{ce}$ of an 8 percent solution of cysteine prior to irradiation was moderately beneficial in preventing the damage to the lens induced by local exposure of the eye to $1500 \mathrm{x}$. Analysis of microscopically visible changes in the lens, which develog in the firgt two or three weels after sray irradiation with $1500 \mathrm{r}$, showed that almost all lesions were less pronounced in the cysteine pretreated eyes than in the eyes rayed before the use of cysteine. $\mathrm{X}$-ray-induced inhibition of cell division, recovery, and compensatory increase of mitotic activity of the lens epithelium differed very little in cygteine-pretreated animals and nongremed- 
cated controls. Under the conditions of the experment, glutathione and thourea were less effective than cysteine in ine reasing the radioresistance of sensitive elements of the lens. Sodum thioglycollate and BAL were tutally ineffuctive. Pret reatment with other reducing chemical agents (dihydroxyacetone and tocopherol), with the citrovorum factor, and with cyanide did not reduce the injurious effect of $1500 \mathrm{r}$ applied locally to the rabit' $\mathrm{s}$ eye. (auth)

347

Schrek, Robert. STUDIES IN VITRO ON TRE PHXSIOLOGX OF CELLS. FACTORS AF FECTING TEL DELAYLD CYTOCIDAL ACTION OF X-RAYS. J. Cellular Comp. Physiol. 28, 277-304(1946).

By the method of unstained cell counts, the number of viable cells were determined in suspensions subjected to irradiation $(1000 \mathrm{r})$ and incubation under varying condi tions. Itradiation of suspended thymic cells of the rabbit produced no observable immediate changes, but after 5 hours of incubation at $37^{\circ} \mathrm{C}$ many of the cells developed irregularity of shape or edema of the cytoplasm. After 17 hours of incubation nearly all of the cells were dead, as indicated by stain ability with eosin. Lymphocytes were susceptible to the delayed cytocidal action of $x$-rays in vitro but granulocytes were resistant. Thymic cells of the rabbit and kitten were equally susceptible to radiation although the non-ir radiated cells of the kitten survived about twice ass long as those of the rabbit. Varying the temperature $10^{\circ}$ and $\left.25^{\circ} \mathrm{C}\right)$ during the radiation period did not alter the radiosengitivity of thymic cells. Varying the temp. $\left(2-45^{\circ} \mathrm{C}\right)$ during incubation showed that the maxinum radiogensitivity was at appros. $37^{\circ} \mathrm{C}$ and the minimum at $17^{\circ}$ and $45^{\circ}$. The nonirradiated survived longer at lower temps. with a temp. coefficient of 3 . The addition of acid to the suspension after radiation decreased the radiogensitivity of rabbit thymic cells. The survival of non-irradiated cells was not appreciably affected by pH's varying 6.0 to 8.4. The cells 248 were rapidly killed at pH 5.9 and 9.5 . (BA)

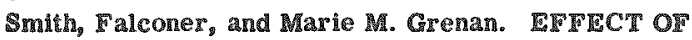
WIBERIATION UPON SURVIVAL TIME TOLLOWINC

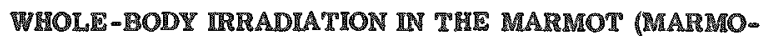
TA MONAM. ScLence 113, 686-8(1951).

raperiments have been pertormed to find whether sensitivity to radiation is lower in the hibernatos than in the nonhibernator. One group of marmots was allowed ho hiberate in a constant-temperature room held at 3.5 to $40.5^{\circ} \mathrm{C}$ and was irrediated 3 weeks after the onset of deep hiberration. A sinilar group was mantained at room temperatute and served as nonhibernating, irgatiated controls. Results in dicate that a delay in the rate of devenoment of he lethal prosesses following ir adiation accompanies lowered body temperature and reduced metaholic rate in the mamot. The fact that irradiated marmots died in hibernation arter only slinhty higher doses (one $650 \mathrm{r}$, one 800 r) than that found for most nonhibermator (550 g) indicates that mo great decrease in sensintivity to radiation is attendant upon the change 0 the poikilothermic state. (NSA)

39

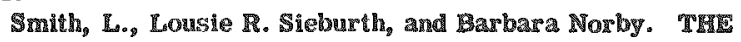
RELATION OF OXYGEN CONCISITATION WN BARLEY

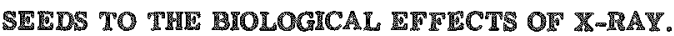

Genetics $35,992(1950)$.

In recent years a maber of workders (ct. Whaden and Smith, Genetes 34: 26-43, 1949) have shown that the atrosphere $\left(\mathrm{O}_{2}, \mathrm{CO}_{2}, \mathrm{~N}_{2}\right.$, vacuum) found affect on the biologichl effecta of ingahasion. "Therefore an attempt was made to try to test the effect of incheasing and decreasing the oxyger content inside gerrunating aeds prior 10 iriadation. This war accomplished by pre-goaking seeds for 24 hours in: 1) cooled boIlled distilled water (reduced supply of oxygen); 2) untreated distilled watex (normal supply of oxygen); 3) distilled water to which oxygen was added (increased supply of oxygen). Preliminary observetions indicate that no cell divisions have orcuryed at the end of the 24-hour period. At the end of pretreatments, the seeds were $\mathrm{x}$-rayed (usually 1,500 g) under identical conditiong in air. The veeds which were provided with additional oxygen were injured markedly more than those which had had normal or reduced amount of oxygen. Seeds with the normal amount of oxygen were injured alighty more than those goaked in boiled water. Injury was measured in terno of survival and rate of growth (haight) of seedingm. A summary of four experiments totaling 400 seeds for each treat ment gave the following re gults. For the control, wolled MOH, untrested HOH, and oxygen-added treatments, the gurvival values were respectively, $1.9,1.6,1.3$, and 1 , and the heights ware $3.4,2.2,2$, and 1. Cytoganetic data are being obtained to supplement the observations on ingury 350

Sparrow, A. H. CLANGES IN SEISITIVITY OH CNROMOSOMES TO X-RA BREARAGI DURIG MCROSPOROGENESHS. Geneties 32, 106-7(1047).

It has previously been established thas chromosomes of Trillum undergo changes in zadiosensitivity during meiosis. This investigation has been continued and a sairly com-

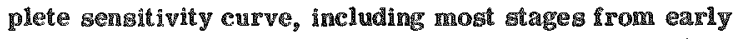
meiotic prophase through pollen rain regting gtage, has been constructed. A gtandard dosage of 50 r was given at all gtages. Sensitivity is determined by counting the mum ber of fragments per 100 PMC. Meiotic prophase glages mo to and inclading diplotene were scored at AI. All later stages were scored at microspore metaphase. The data ghow gradual increase of sensitivity foom early meiotic prophase up to metarhase T. The curve then drops down again to a low at the letrad (or very early reshimg) stage and warts up agam during the resting gtage. The resulto are interesting in that (1) the range of sensitwiticis ls very large (about 1 to 27), and (2) that high and low geng-

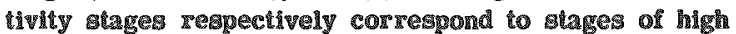
and low concentrathons of desorymibose nucleic aed in the chromosomes. (RG) 31

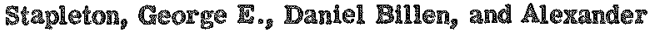

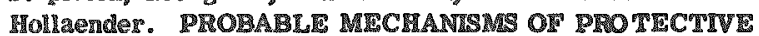

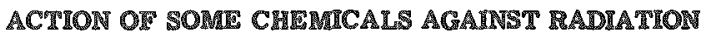
DAMACE. Oak RIRge National Lab. 1P. (ATCU-1690) The repor is reproduced here in în matirety.

The protective action againse $\mathrm{x}-\mathrm{ray}$ damage of a wide variety of chemical aubstances on $\mathrm{F}_{\text {. }}$ eoli $\mathrm{B} / \mathrm{r}$ has been reported (Burnet ot a. Federation Proc. 10, 2 (1051)). Some of these compound have been further invertigated in an atempt to elucidate the mechanim of protection of bacm terial cells. At low temperatures some of the commonly

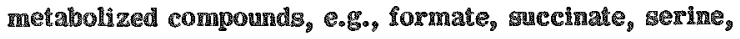

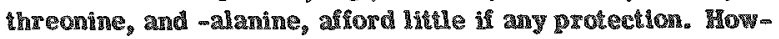
ever, if cells were incubated at $37^{\circ} \mathrm{C}$ in the preaence of these compownd, prior to ir iradiation, a ruaked increase in thell protective capacisy was noted. Marallel atudien on respiration, utilizing Bome of these compounds as anostrate, indicate that the protedive apacisy can be cor related with the rate at whlch these substances are or dized by the bacterial cella. This augrests that prethcubation of cells with these compounds may rasule in 1 rom

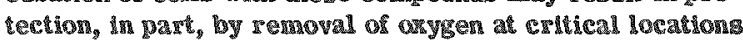
within the cell, thereby inhibiting the formator of mome oxidiring radiodecomoodion products of weter. Further

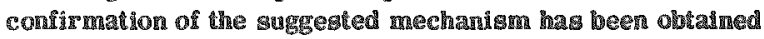


by use of respixatory inhibitors such as sodium cyanide. The inhibition of oxygen uptake by NaCN on several substrates used (amino and carboxylic acids) resulted in loss of protective action by these compounds. Some organic and inorganic sulfur-containing conpounds which show protective action have been investigated in a similar manner and the results will be discussed in relation to the proposed mechanism. (Paper presented at Botanical Society of America, Physiological Section, September 1951, Mimeapolis.)

32

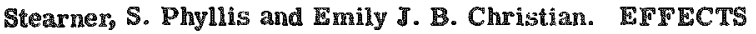
OT I-IRRADIATION IN NEWBORN RATS. J. ENDt1. Z001. 118,495-509(1951). (AECU-1509; UAC-404)

Newborn rats were m irradiated shortly after bi nth $1 / 2$ to $5 \mathrm{hr}$ ) and at 1 or 2 days of age. Radiation doses ranged from 400 to $1000 \mathrm{r}$, and exposures were made at 6,40 , and $80 \mathrm{r} / \mathrm{min}$. Feffects of 8 irradiation upon survival indicated that the newborn rat was nore radiosensitive than the adult; but resistance increased rapidly after birth, and at 2 days the sensitivity was only slighty less than that reported for adults. A higher and earlier death rate followed expogure of $1 / 2-$ to $5-h r$ old rats to $600 \mathrm{x}$ at $80 \mathrm{r} / \mathrm{min}$ than that observed in group exposed at $6 \mathrm{r} / \mathrm{min}$. No effect of variation in dose rate was observed after exposure to 400 and 800 I. Exposure to $1000 \mathrm{I}$, however, produced a shorter mean suxvival time when delivered at $6 \mathrm{~g} / \mathrm{min}$ than when delivered higher rate. Animals exposed at 24 hrs after birth showed no dosem rate effects upon survival and gross pathology with any anount of exposure. Generalized edema, hyperemia, and renal hemorrhage were prominent autopsy findings at 1 to 2 days after irradiation in animals expused within 5 hr after birth. Later gross changes were lypical of those that accompany acute radiation deaths. Hemosrhages developed in the cerebrum within the firgt day and 3. in the cerebellum and medulla after 5 to 7 days. (NSA)

Thompson, T.L., Moy B. Mefferd, Jr., and Orvlle Wyss. THE PROTECTION OF DACTIRIA BY PYRUVAT AGAINST RADATION FETETS. J. Bacteriol. 62, $39-43(1951)$.

The pregence of pyruvate in a bacterial sugpension protects the cellogainst the lethal and the mutagenic action of ultraviolet and $\mathrm{x}$ rays and againgt the lethal action of hydrogen peroxide. Upon prolonged exposure to ultraviolet, pysuvate solutions bscome toxic and mutagenic. The addition of the pyruvate after exposure of the cells or its presence in the plating medium does not modify the biological response to the radiations. Tr organisns lacking in aynthetic ability a rich recovery medium may give higher 354 counts of celis injured by ultraviolet. (NSA)

Tul1s, John L. RADIORESSTANT CELLS IN CERTAN RADIOSENSTIVE TISSUES OF SWIFE FXPOSED TO ATOMIC BOMG RADLATON. AICh. Pathol. 48, 171-7 (1049).

A report is presented on the morphological changes noted in the radiosensitive lissues of swine following irtadiation. These radiosengitive tisues are the lymph nodes, the bone marrow, the intertinal epithelium, the gonads, the spleen, the thymus, the tonsils, lymphoid aggregations in the bowel wall, and lymphocytes in the peripheral blood stream. Aft (2) Irradiatlon, the lynohocytes undergo a prompt seducthon, roid lysis in some cases and slow degeneration in others; increased erythrophagocytosig also may occur. In the bone marow the blast calls are easily destroyed, leaving only the reticular cella intact after heavy Ir radiation. Complete destruction of gections of the bowel epithelum may oceu at random locations after gadiation expo- sure; the fibrorytes and the plasmacytes in the lamina propria are relatively uninjured. After injury to the gonaus, ro sperm cells are apparent, and few spermatagonia and spermatocytes remain; the cytoplasm of the fubular cells becomes granular and vacuulated, but the mofferent cells which line the tubules next to the basement membrane show no alterations. The developing ova in the ovary show vacuolation of the cytoplasm of the follicular cells and other changes, but the primordial ova are more resistant. It $1 \mathrm{~s}$ noted that in these organs digcussed, the so-called stem cells (reticular cells, primordial wa, etc.) of the highly radiosensitive organ are comparatively radiu - resistant; 355 the reason for this is not apparent morphologic ally. (NSA)

Windhol, F。 REACTIONS OF CONNECTIVE TISSUES AFTEX PROTRACTED FRACTIONATED IRRADIATION OT LARYNGEAL CARCWNOMA. Radiology 48, 148-56 (194).

Microscopic studies of the laryn of patrents ir radiated with the protracted fractionated method for malignant tumors revealed that, contrary to the general belief, there are profound and extensive changes in the connective tissue. The following 3 stages of connective tissue effect of X-rays may be observed. The first is a bionegative retrogressive period characterized by exudation of fibrin, loss of nuclear staining and frequent changes in the mic roc hernical reaction of aubepithelial collagenous fibres. This period is coincidental with fibrinous radiomucostitis and disintegration of the epithelial cells and the mucous membrane. The 2nd period might be called a bopositive regenerative phase. Connective lisue fills out the spaces remaining aftar disintegration of the tumor. The retrogressively changed fibrinoid necrotic collagen of the lamina propria is replaced by hyalne sclerotic layers. Myogenous giant cells indicate the regenerative tendencics of these lissues. Inflammatory reactions of the regenerated connective tissue age markedly reduced as specific effect of radiation on this structure. In some ingtances (over exposure or par ticular sensitivity of the connective tissue) a thitod retrogressive phase may be observed. The newy formed connective tissue becomes necrotic, the mucous membrane shows ulcerations, and secondary bacterial infections develop and progress into the deev layer of the irradiated 35 laryin. (BA)

Windholz, FAORLEMS OT ACQUTRED RADIORRSISTANCE OF CANCER: ADAPTATON OF TUMOR CELLS. Radiology $48,398-404$ (1947).

The article yummarizes the various theories so far ado vanced for the explanation of acquired radioresistance of malgmant tumors. The histologic and radiobiologic behavion of the mucous membrane of the lazynz as observed by the author forms the basis for his explanation of this phenomenon. The author explains acquired radioresigtance as due to adaptation of cancer cells to the environment, changed by irradiation. Tumor cells which are able to adapt themselves to this changed environment maintain their newly acquired properties in sucessive cell generations. Thus, gradualy the author believes the entire tumos becomes transforned into a growth, the radiobiologic properties of which are adapted to the biologic conditiong prevailing during and after ir tradiation. In these new sur roundings, the tumor either disintegrates or its radioresistance is increased. (BA) 357

Zirkle, maymond ITY BV MEANS OF MEADLY PENETRATING ACIDS AND BASES. Am.J. Roentgenol. Radium Therapy 35, $230-7(1936)$. 
Using the spore of tho fern Pteris longifulia and also Paramecium, it was noted that an increase of the acids carbon dioxide and hydrogen sulfude up to a certain concentration increased the radiosensitivity and beyond that point, a decrease, while the base ammonia acted in the reverse manner. These chemicals produced no detectable effects witheut the irradiation. Climcal application of the above results depends upon whether mammalian tissues will respond in the same way, both to the chemicals and to irradiation, and also whether such response will be without harm to normal tissue. (R)

358

Zirkle, Raymond E. THE INFLUENCE OF INTRACELLULAR ACIOITY ON THE RADIOSENSTTIVITY OF VARIOUS ORGANISMS. J. Cellular Comp. Physiol. 16, 301-11(1940).

Drosophila eggs of two ages (2 hours and 4.5 hours) were expused during $\mathrm{x}$-irradiation to various concentration of a penetrating acid, $\mathrm{CO}_{2}$. The radiosensitivity of the acidified eggs was deduced from their percentage hatching and the survival curve for normal eggs. When radiosensitivity is plotted against $\mathrm{CO}_{2}$ concentration in $\mathrm{mm}$ of $\mathrm{Hg}$, the resulting curve has maxima at 80,200 , and $350 \mathrm{~mm} \mathrm{Hg}$ and minima at 150 and $250 \mathrm{~mm}$ (temp. $25^{\circ}$ ). This finding holds for both 2 -hour and 4.5 hour eggs. However, the heights of the maxima and minma are widely different for the two age groups. In general, the radiosensitivity of the 2 -hour eggs varies uver a much greater range. The radiosensitivity curves for the eggs resemble those for fern spores in the vecur rence of maxima and minima but these critical points occur at different $\mathrm{CO}_{2}$ concentrations. Reasons are given for considering the shape of the radiosensitivity-acidity curves to be due to several radiochemical reactions, each of which has a maximum at a characteristic pH. The experimental results are discussed in relation to the radiochemical behavior of proteins at various pH values and to the influence of intracellular acidity on the production of 359 chromosome abnormalities by s-rays. (CA)

Zirkle, Raymond E. COMBINED INFLUENCE OF X-RAY INTENSITY AND INTRACELLULAR ACWITY ON RADIOSENSITIVITY. J. Cellular Comp. Physiol. 17, 65-70(1941).

When germinating fern spores are exposed to $X$-rays in the presence of graded concentrations of $\mathrm{CO}_{2}$ and ammonia, their radiosensifivity has maximal and minimal values at definite concentrations of the penetrating acid and base. The heights of the maxima and minima are modified when the intensity of the X-ray beam is varied, and the quantitative change in height is different for the various maxima. The concentrations of acid and base at which the maxima and minima occur are not altered by the $\mathrm{X}$-ray intensity. These results are consistent with the hypothesis that the total radiosensitivity is due to the added effects of several radiochemical reactions which have maximal yields at dif 360 ferent $\mathrm{pH}$ values. (BA)

Zirkle, Raymond E., E. Anderson, E. F. Riley and H. J. Curtis. THE ETECT OF AGE ON TER RADIOSENSITIVITY OF MCE. Clinton Labs. [nd] Decl. Oct. 29, 1946. 6p. (MDDC-418; MONH-89)

Mice of different ages ranging from 1.5 to 12 months were exposed to gamma rays in an effort to determine the change in radiosensitivity with age. The dose necessary to cause $50 \%$ killing was determined for each age group and sex. No change in sensitivity with age was observed with either sex, although the experimental error in the case of the males was quite large. The females were found to be more resigtant than the males. (ADD)
Comparative Efiects

361

Bishop, F. W. COMPARISON OF X-AND BETA RADIATION EFFECTS IN RABBITS. Univ, of Rochester. [nd] Decl. Aug. 17, 1946. 12p. (MDDC-203)

The minimal dosage necessary to produce an erythematous reaction in the ear of the rabbit with $30 \mathrm{kv} X-\mathrm{Xays}$ is in the neighborhood of $1,000 \mathrm{r}$. A somewhat smaller dose (900 r) was able to cause epilation without erythema. Generally the phenomenon of epilation either appears at a lower dosage level or a higher percentage of the animals are epilated than show erythema for a given dosage. As might be expected, the erythema appears sooner and generally lasts longer at the higher dosage levels and epilation also occurs earlier under these circumstances. Scaliness of the areas treated was a usual accompaniment of the erythema but its appearance time and duration was erratic. The lowest dose of beta rays capable of causing an erythema in the rabbit's ear is near the level of 3,000 rep. Epilation was not observed until a dose of $3,500 \mathrm{rep}$ had been administered and the percentage epilated was less for a particular dose than that developing erythema. Generally the appearance time of the epilation was considerably longer than that of the erythema. Scaliness without any other sequela was noted at low dosage levels but was as variable as in the case of the $\mathrm{x}$-rayed rabits. These few experiments indicate that from three to five times the dosage is necessary in the case of beta rays to produce a given effect; e.g., it required approzimately three times the dose of beta rays as compared with low voltage $\mathrm{X}$-rays to produce a minimal erythema, about four times the dose to cause the firgt signs of epilation and about four and onehalf times the dose to produce epilation in all the animals treated. (ADD)

362

Bonet-Maury, P. and F. Patti. LETHAL IRFADIATION OF MCE WITH HIGI DOSES OF ROENTGEN AND GAMMA RAYS. Radiology 57, 19-23(1951).

Comparison of data was made on mice irradiated over a wide dosage range, under identical conditions, with $x$ and $\gamma$ radiation. $\mathbb{X}$ - and $\gamma$-ray doses measured by a graphitebakelite ionization chamber corresponded fairly well to the energy actually absorbed by the mice and are directly comparable. Though the irradiated animals showed identical symptons of poisoning, a greater amount of $y$ energy than of $x-$ ray energy was required in the tisgues lor similar biological effects. This result was attributed to different primary radiochemical reactions resulting from unequal ion distribution along the electronic path of the fwo radiations. (NSA)

363

Czech, H., R. Czech, and I. J. Schmermund. A COMPARATVE STUDY OF THZ ACTION OF IAST ELECTRONS AND K-RATS ON THE CORNEA OF SALAMANDER LARVAE. Strahlentherapie 81, 187-92(1050).

The cornea of the salamander larva was used for a comparative study of the radioblological action of last electrons and $x$-rays. The phenomenon observed was the so-called primary effect of a radiation on the mitotic division of cella. If a cell is irradiated while undergoing division, the effect produced by the irradiation is a primary effect, as disinguished irorn a secondary one produced by irradiating a cell in the resting stage. No qualitative differences in the distrubances produced by the two kinds of rays were observed. However, the frequency of pathologic changes in the mitosis due to the action of electrons was, per unit 
dose, lower than that corresponding to the action of $x-$ rays. These findings are in agreement with the requirements of the "event" or "larget" theory of the action of energetic 364 radiations on biological structures. (NSA)

Devils, Finn. THE TOLERANCE OF RIDERMAL CELLS OF MICE TO HEAVI DOSES OF EXTERRAL ALPHAIRRADIA TON. Acta Pathol.Microbiol. Scand. Suppl. 91, A5-7(1951).

The histological and cytological effects of known quantities of external a irradiation on the gkin of nice are compared with the effects of local s irradiation. If was concluded that the epidermis contains a number of cells which will survive doses of the order of $100,000 x$ of a rays. In the histological sections, 8 irradiation was seen to produce more pronounced changes than were produced by $a$ ray doses of 1 to 2 orders of magnitude above the $x$-ir xadia365 tion dose. (NSA)

Dieckmann, C. and W. Dittrieh. IRLLING OF BACTRRIUM COLI WITM FAST ELCCTRONS OF A 6-MEV BETATRON. Strahlentherapie 81, 215,22(1950).

An agar $-{ }_{n}$ late technique was devised for the investigation of the rate of deterioration of Bacterium coli commune by fast electrons and $x$-rays. In agreement with the "event" or "his" theory of radiobiological processes, 3-Mev electrons were found to be more effective for the above reaction than equal doses of $\mathrm{x}-\mathrm{rays}$ of $180 \mathrm{kv}, 6 \mathrm{ma}, 0.27 \mathrm{~mm}$ half-value layer and $500 \mathrm{~s} / \mathrm{min}$ dose rate; the dose range was 500 to $4000 \mathrm{r}$. The radiobiological effect produced by the electrons is interpreted as a saturation process, in the sense of the above theory; the radio-sensitive volume is composed 366 of about 60 elementary ields of 21 mue average width. (auth)

Dobson, R. LOWry. LETHAL RADIATON EFIECTS OF X RAYS, DEUTERONS, AND ALPHA PARTCLES ON THE BACTERIUM SCMRRICHA COLI. Radiation Laboratory, Univ. of Calif., Derkeley. Feb. 28, 1051. 4p. (UCRL1180)

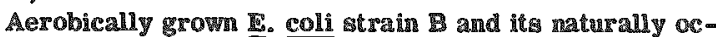
curring radiation resistant mutant strali $\mathrm{B} / \mathrm{P}$ were irradiated in air with $\mathrm{x}$ rays, deuterons, and $a$ particles. In all cases typical exponential survival curves were obtained. In this regpect the bacteria behaved as would be expected ac. cording to simple target theory. $B / x$ was in all cases more resiatant than B. Contraxy to expectations based on target theory both straing were killed more efficiently by lowenergy deuterons than by high-energy deuterons. This gane reversal was found in the case of of rays, lowmengy particles being more effective than high-energy particleg. When in irradiatton was carried out in a $\mathbb{N}_{2}$ atmosphere, gurvival was increased over that in al ${ }^{2}$. Such a protection was also demongtrated for argon in the case of strain $B$ bombarded with particles. A modified target hypothesisthat the "target" may be inactivated not only by an lonizathon occurring within its volume, but also by energy transferred from the track of the ionizing particle through a finite distance in the cell, and further, that the probability of this latter mechanimm regulting in target inactivation at a given digtance increages withi Ion dengily along the track 36 ? is proposed. (NSA)

Evans, R. D. TOXICIY OF ALPRA AND PAST NEUTRON MADLTION. Nature 146, 621(1940).

The author has deøeribed the regults of experiments on mentron tolerance In animals in a paper presented at the amual meetine of the U. S. Nat. Acad. of Sci. on April 2223. He find that the reฮnlta cannot be accurately extra- polated to man because the relative radation stensitivities are entirely unknown for small doses. Biological effects of neutrons are due to the secondary recoll rays which they produce in the budy by collision with hydrogen, carbon, nitrogen, oxygen, and other atoms. The average specific ionization, and hence the ionic effectiveness, of the neut ron recoll rays is closer to that of alpha rays than to any other radiation which has been the subject of extensive biological investigations. Data are available from studies of chronic radium poisoning, and of lung cancer produced by radon inhalation, concerning the effects of alpha rays on human tissue. With these data, it is shown that a dosage of 0.01 s per day of fast neutrons may be unsale if the exposure continues over a period of about 10 years. (\$.V.\$.)

368

Rvans, T. C. and I. H. Quimby. STUDIES ON THE ETFICTS OF RADIOACTIVE SODUMM AND OF ROENTGEN RAYS ON NORMAL AND LEUTERMC MCE. AM. J.

Roentgenol. Radium Therapy 55, 55-6(1946).

The effects of radioactive $\mathrm{Na}$ and of whole body roentgen irradlation on white mice have been compared. Results in reduction of white and red blood counts and in shortening of life are similar for the two types of radiation. Mice with enlarged nodes and extremely high leukocyte counts were found egpecially sensitive to the radiosodium. They were not lested with roentgen rays. The marked response was apparently due to radiosensitivity of the abnormal leukocytes rather than to any gelective concentration of the material in lymph nodes. (BA) 369

Gray, L. T. COMPARATIV STUDIRS OF THE RIOLOGICAL EFELCTS OF I-RAYS, NEUTRONS, AND OTHER IONIZNG RADRATIONS. Brit. Med. Bull. 4, 11-18(1946).

The anthor has studied the measurement of neutron energy absorhed in living cells; comparizon of the effects of X-rays, gamma rays, neutrong and other loniaing radiationg on enzymes, colloids, and numerous plant and animal tisaues, both normal and malignant. His experiments are dim rected toward the treatment of cancer. In this article he discusces: (1) Linear ion denaity, the distinguighing feature of an lonizing radiation, from the biological glandpoint. (2) Ion density produced by dinferent ionizing particles. Figure 1 shows the aeparation of lon clusters in relation to the size of a virus particle $2 \%$ mp in diameter. (3) The iniluence of ion densišy on radiochemical yield. (4) Ion density in relation to the inactivation of elementary biologic units. Figure 2 shows the relative efficiency of loniming radiationg for the intactivation of viruaes (6 types). (5) The structural changes induced in chnomosomes by different types of ionizing radiation (Figure 3). (6) Comparative studies with othes blologic materlal (Drosophila egga, root-tips of Vicla faba. animal embryontc tisgue, tumor tiswe). (7) Neutron therapy. (BA) 370

Gray, 1. H. IEPERMISNTAL STUDIES OF THE INITUENCE OF RADITION QUALITY, DOSF RATE, AND FRACTIONATION ON THE DIRECI INJURY OF CEILS BY IONISING RADIATIONG. Acta Unio Intern. contra Cancrum 6, 704-819049).

The biological effects of equal doses of different lonimg

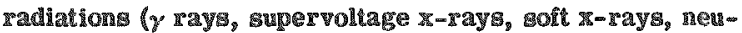
hron, and particles were studied and correlated with the density of ionization along the tracks of ionizing particles, which varies in the extreme from 104,000 ions/ $/ \mu^{\prime}$ the more densely ionizing radiations were found to be either more or less effective in produchngene mutations and in bringing about enzyme inactivation in dilute agueous solution and, in genera, more effective in producing chromo- 
some structural changes and in damaging or inhibiting the growh of many tissues including tumor tissue. Experimental data will be presented for a particular tigsue (root tip meristems) belonging to this latter class. It was found experimentally, as is commonly observed in radiotherapeutic practice, that the inhibition of growth is markedly dependent on dose rate and fractionation when the radiation employed is $\mathrm{X}$ or $\gamma$ radiation. This is not in general the case with the more densely ionizing radiations; no dose-rate dependence was observed with a radiation. It is concluded that the close interdependence of the effects of varying dose rate and quality can readily be understood by neference to the tyoes of chromosome structural change observed to be produced in the irradiated cells. (NSA)

31

Gray, L. M. and J. Read. COMPARLON OF TRE LETHAL EIFCT OF NEUTRONS AND GAMMA RAIS ON MOUSE

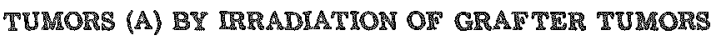

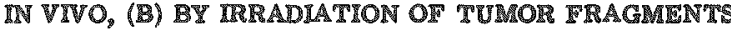
TN VTTRO. Brit. J. Radiol. 21, $5=10(1848)$.

The mean lethal doges of neutron and gamma trays on mouse tumours have been compared (a) by ixradiation of grafted tumour in vivo, (b) by liradiation of tumoug frage ments in vitro. (a) Small pieces of carcinoma 2146 were inoculated into the thigh of a mised strain of mice showing little concomitant immunity. When the tumour reached about $10 \mathrm{~mm}^{2}$ in area they were irradiated either by 2.8 Mev (D-D) neutrons or by gamma rays from 500 mg radium plaque filtered by $0.5 \mathrm{~mm} \mathrm{Pt}$ and $1 \mathrm{~mm}$ brasg. The two sources of radiation were mo ar ranged that they gave roughy the same dose distribution throwghou a moume, ano anean lethal dose in about the same time. Ilght diferent neutron doses were given to a total of 32 mice. The mean lethal dose was 65 energy units, standard deviation $12 \mathrm{en-}$ ergy units. The unit 10 defined as that moun of neutron radiation which releases the same energy per ce in water a. 1 roentgen of gamma xadiation. Nine different doses of gamma rays were given to 90 mice. The mean letha dose was 1500 roentgens, standard deviation 64 roentgens. (b) Eight tumour fragments in phyglological aline were retained, in the central hole of annula parafin wax digcs 2.5 mm thick, by the waxed paper. Six such cells were stacked in glas specinen tube so that their divtances from the target of the neutron generator gave the desired range of doses. The mean lethal dose was 154 energy units, standard deviation 17 energy units. A similar arrangement ued for the fama ir celving four different dobes. The mean lethal dose was 1460 romtgens, standard deviation 62 roentgen. The efficiency ratio of meutrong to gamma rays was $24+6.7$ for the in vivo irradiation and 9.5 . 2.3 for the in vitro ix ra diations. The author discuss these results in arsoriation with thel earlier publighed work on the lethal efiect of lonizIng zadiations on the brond bean aot. They support the idea that the tumour regresses by the death of the cells which have sustained chromosome or chromatid breass sesulting in chromosome aber rations. (EM)

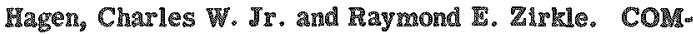
PARATIVE BIOLOGICAL ACTIONS OF CYCLOTRON IAST NEUTRONS AND X RAYS; L. LITYIII ACTION ON MCE AND RABDITS. UnIversity of Chlcago Metallurgical Lab. Nov. 1950. 260. (CM-3903)

X-irydiated rabbits exhibited three digtnct periods of mortality: (1) "one-day" mortality, the iraction of the total number of animals that died in the firgt 3 days following irsadiation; (2) "ten-day" moxtality, the fracton of those rabbits alive at the end of 3 days which died by the 20 th day; (3) "late" mortality, the fraction of those rabbis alve at the 20 th day which died on subsequent days. In the firgt period, weight and sex had considerable effects on survival. The heavier animals were more resigtant than lighter ones. Females were more resistant than males during the "oneday" period but less resistant during the "ten-day" period. Following exposure to fast meutrons, the heavier rabbits again showed less mortality, but there were no signi ifart sex differences. After either $\mathrm{x}$ or neutron ir radiation, the majority of deaths oceuraed in the 4 - to $20-$ day period. A distinct peak of deaths was evident 10 days after $\bar{x}$ irradia tion, but after neutron exposures montality during the 4 - to 20-day period was divided into wo phases with modes at about 8 and 12 days. For rabbits, the 30 -day $\mathrm{LD}_{\mathrm{g} 0}$ was 825 $r$ of $\mathrm{x}$ rays or $145 \mathrm{n}$ units of neutron; the $\mathrm{x} / \mathrm{n}$ ratio was accordingly 5.7. For mean surwiwal times the ratio was 6.3. Mice died in greatest mumber during the fil at 10 days after exposure to elther neutrons or $\mathrm{x}$ rays, but the average survival after neutron exposure was about 4 day ghorter than after $\mathrm{X}$ ir 378 $\mathrm{z} / \mathrm{n}$ ratio accordirghy being 10. (NSA)

Heidenthal, Gertrude and Leonard Clar:. RATES OI WABROBRACON EGGS TREATED IN IIRST

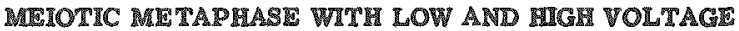
X-RADIATION. Genetics $36,554(1951)$.

studies were made on the aurvival of haplold eggs treated in firgt meiotic metaphase with low voltage (124 kv) and with high voltage (50 Mev) $\mathrm{x}-\mathrm{mad}$ ation. Dokage ranged Irom $100 \mathrm{r}$ to $1750 \mathrm{r}$. Standard techniques previously developed by A. R. Whithg were used hroughout. Since females were not mated in the first serieg of experissenta, partheroge. netic development occurred among the muving eggs up to laxval tages when the observaton were discontinued. The curves for low and high voltage pre pretically identical, ตนrvival following treatment with $400 \mathrm{x}$ was $54 \%$ for high voltage and $51 \%$ for 10 ; $;$ at $1500 \mathrm{r}^{\circ}$ the viablity was about $5 \%$ for both types of radation. The curve for low voltage is essentially a conirmation of that found by A. R. Whitmg. Other whases were made of eggs treated with low volage but laid by females which were mated following irradiation. Under these conditons it would be axpect that if Iecas alve lethals were present, viability should increase. Since the curves fos ogs from mated and from unmated femasos are so very nearly alike, it is tentatively concluded that 374

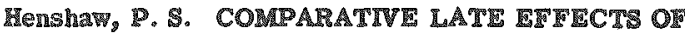

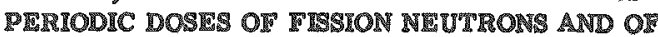
GAMMA RAPS. Clinton Labs. Jun 1948. Decl. Apr. 21, 194\%. 900. (MDDC-1253; MOnK-115)

Small daly doses of fat meutrons $(0.115$ to 13.0 n) and $\gamma$ rays (1. to 17.2 r) were administered times weelly to the whole bodies of treated animals. Most of the animals died either in a state of atrophy or wh lewkemin (mediastinal Iymphomatostg). The time of followed a regular patern and furnished a means of quan titatively comparing the effectveness of the different treatments and methods of exposure. The fart neutron doses

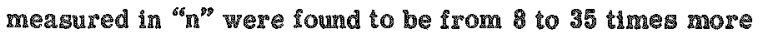
eflective than $\gamma$ rays measured in " $y^{s s}$. The threshold value for this radiation was fomd to be below $0.115 \mathrm{n}$ of trons per day and about $1 \mathrm{~g}$ of $\gamma$ meys. In CF-1 mice about 10 times a meh radation per day was required to produce a threshold change as was required to shorten the life gen detectably. Some diferences were moted in the ranonses of different gtrans of animals to radation but these were mor a matter of degree than of type; no furdanental dis-

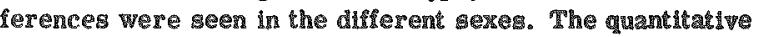
$r$ sults based on survival tme indicate that the degree of 
biologic effect varies not only with dose, but also the density of ionization produced. Although the incidence of leukemia was significantly increased in $\mathrm{CF}-1$ animals, the occurrence of lung tumors was only slightly, if any, modified by the treatments given. Only questionable evidence of increased lung tumor incidence was chserved in animals which normally show a high spontaneous lung tumor inc i 375 dence. (ADD)

Menshaw, P. S., et al. COMPARATIVE LATE EFFCTS OF SINGL DOSES OF IPISION NEUTRONS AND OF GAMMA RAYS. Clinton Labs:. June 1946. Decl. Sept. 8, 1847. 51p. (MDDC-1254; MOnH-116)

Having obtained information about the late efferts of small daily doses of fast neutrons and gamma rays (MDDC1253), the authors now present the results of a study of the late effects caused by the same radiations when single large doses are administered. Lxperiments we re carried out using $C F-1$ and $A B C$ mice. As a rule more than 100 mice were used for each experimental sample. Single neutron doses of $26,50,78$, and $90 \mathrm{n}$ and single gamma ray doses of 500 and $700 \mathrm{r}$ were given. Other special experiments to investigate the influence of dosage-rate and of massive doses were also carried out. Acute illness consisting of emaciation and weight loss, lymphopenia and histologic damage to germinal tissues occurred at four days to two weeks. Recovery was rapid in the animals that sur vived and at three weeks it was difficult to distinguish the experimental from cont rol animals. Such treated animals nevertheless died prematurely more or less in accord with the amount of radiation delivered. Evidence was obtained suggesting that the length of life varied exponentially with dose. It has been noted that $1 \mathrm{n}$ is consistently more effective than $1 \mathrm{r}$ in causing biological ingury; the superiority of a fast neutron unit is greater when judged by late effects than when judged by acute lethal action. The incidence of leukemia (mediastinal lymphomatosis) was greatly in creased-from $15 \%$ in the controls to more than $60 \%$ in the case of gamma rays (incomplete results). The findings further substantiate the idea that the higher the concentration of ions the lower the dose of radiation required to produce a particular effect. Other evidence was obtained indicating that a single application of irradiation exposure is 376 surficient to induce malignant disease. (ADD)

Horn, Edward C. RELATVV BIOLOGIC ERTECTIVENESS OF ROENTGEN RAYS AND NEUTRONS ON REGENERATION OF THE FOREIMB OF AMBLYSTOMA LARVAE. Am. J. Roentgenol. Radium Therapy 46, 727-31(1041).

Report on the comparative effectiveness of neutrons and roentgen rays in retarding and preventing tissue regeneration. Regeneration of an amphibian limb depends primarily on proliferation and cellular differentiation of a mass of undifferentiated cells, the regeneration blastema. This regeneration blastema provides favorable material for a study of the effects of neutrons and roentgen radiation on growth and differentiation. Sufficient doses of neutrons prevent differentiation and hence regeneration of the forelimb of Amblystoma larvae. Neutrons are more effective per unit of ionization than are roentgen rays in preventing regeneration. Roentgen dosage 13-14 times neutron dosage, as measured in terms of ionization in a Victoreen $100 \mathrm{r}$ 377 chamber, is necessary to prevent regeneration. (YBM)

Jacobson, $\mathbb{L}$. O. and E. K. Marks. COMPARATIYE ACTION OF CYCLOTRON FAST NEUTRONS AND X-RAYS. I. HEMATOLOGICAL EF FETS PRODUCED IN THE RABBIT BY FAST NEUTRONS. Univ. of Chicago Metallur gical Lab. June 1946. Decl. Oct. 8, 1951. 35p. (MDDC1372; CIII-3839)
Rabbits, weighing approximately 2000 each, wert exposed to cyclotron fast neutrons. Hematological studies were conducted on the animals after exposure and at frequent intervals thereafter through 37 days. The range of dosiags employed was from 9 to 178 fast neutron unts. Detailed information is given on mortality and effects on the hemopoietic system following various duses of $\mathrm{n}$ radiation. A comparison of the relative effectiveness of cyclotron fast neutrons and $x$ radiation made on the basis of the degree of heterophil or lymphoryte depression at arbitrarily chosen times after exposure of animals to the se gadiations gave variable results. The $X / \mathrm{n}$ ratios thus derived varied from 6 to 22 . The $X / \mathrm{n}$ ratios derived from a statistical study of the lymphocyte-heterophl response in rabbits exposed to fast neutrons and $X$ rays $a r e$ for the lymphocytes 6.4 and for the heterophils 6.3 \% 0.1 . (ADD)

378

Depp, Richard Kurt. THE EFIECT OF FAST ELECTRONS IFROM A 6 MEV-BETATRON ON HUMAN STKN, D.165 of INTERIA TIONAL CONGRESS OF RADIOLOGX, 6th CONgress, London. 1950.

Curcular areas, $24 \mathrm{~mm}$. diameter, on the thigh were grven a single irradiation with electrons of $3 \mathrm{Mev}$. The dose was measured with a thin-walled ionization chamber, graduated in $r$ units. The ratio between any given dose and the number of ion pairs produced was recurded phutometric ally. Doses between $600 \times$ to $1,300 \mathrm{r}$ at intensities of $100 \mathrm{r} /$ minute to $1,600 \mathrm{r} /$ minute were delivered. Doses piven at the high intensity produced greater reaction in all cases. By comparing fields which showed the same degree of reaction, although irradiated under different conditions, the effect of protraction was studied and found to be of greater significance with clectrons than with $\mathrm{X}-\mathrm{rays}$. An cxplanation of this difference may be the time-sequence of mdividual "hits". It would seem, therefore, that in order to avoid serious skin damage, the smallest intensity pussible of any irradiation should be used.

379

Kepp, Richard Kurt. ACTION OF EAST ELECTMONS ON HUMAN SIIN. Strahlentherapie 81, 201-14(1950).

A comparative study of the effects produced on human skin by hard $x$-rays and by fast electrons from the betatron of the Siemens-Reinger plant is described. Fields on the thigh were irradiated with equal surface doses of the two radiations. Unlike the reaction produced by $x-$ rays, that due to electrons is characterized by a rapid development, a high intensity, and a fast healing. Although $x-r a y s$ produced no exudative reactions, the latter were often quite strong after fast-electron irradrations, and ended with a formation of thick crusts. Even in the case of very strong reactions, the healing took place within three weeks after the beginning of the exudative process. The intensity of reactions produced by electronic radations grew with the dose, with the energy of the electrons, and whth the duse rate (ratio $\mathrm{r} / \mathrm{min}$ ). This dependence of the skin reaction on the dose rate may prove very significant in the therapeutic applications of fast-electron beams. The considerably stronger effect of the latter on the skin, as compared with the action of $x$-rays, is explained by surmising a gradual increase of the dose under the surface of the shin, which concentrates the main effect in thuse tissue layers that are responsible for the shin reaction. (NSA) 380

Kepp, Richard Kurt, Kunt Müler, and Herbert Reich. ROLE OF THE TIME ELEMENT IN FAST-ELECTRON IRRADIATONS OF HUMAN SKIN. Strahlentherapie 84, $225-44(1951)$.

Test ir radiations of human shin with $3-\mathrm{Mev}$ electrons from a betatron showed that, contrary to what is ubserved with 8 rays, in the action of last electrons the time fartor 
plays a notable part even in the dose-effictency range 300 to $1800 \mathrm{r} / \mathrm{mun}$. This dufference 1 s perhaps due to a much less dense ionizat ion in the case of electrons, permitting a partial recovery of the tissue elements if sufficient time intervals between individual hits are given; this recovery effect cannot occur amid the dense ionizations produced by $x$ rays. However, the possible role of the highly fraction ated radiation from the betatron also shruld be taken into account. These results show that, in view of the danger to to the skin, fast electrons must be used only in low dose eficiencies. (NSA)

\section{1}

Lampe, Isadore and $F$. J. Hodges. DIFFERENTIAL TISSUE RESPONSE TO NEUTRON AND ROENTGEN RADIATIONS. Radiology 41, 344-9(1943).

In 1927, Fer roux and Regaud demonst rated that it is impossible to produce permanent sterilization of the rabbits test is with a single dose of $\gamma$ rays without severely damaging the sorolal skin. These experiments were repeated by the author's with $X$-rays and compared with the action of neutrons. The results of the rench authors were confirmed. Doses of more than 2,100 r were necessary to produce complete aspermatogenesis and these doses produced at the same time non-healing reactions of the sorotal skin. The neutron irradiation was carried out by usmg the collimated beam method as deseribed by Aebersold (Physic. Rev. 56: $714,1939)$. Measurements were made in aribtrary nunts (1) is the amount of fast neutron radiation necessary to discharge the Victoreen instrument to the same extent, as does $1 \mathrm{r}$ of $\mathrm{X}-\mathrm{rays})$. Twenty animals were treated with doses ranking from $356-800 \mathrm{n}$. With doses of $700 \mathrm{n}$ or more the reaction was that of complete aspermatogenesis and severe cutaneous damage. In the range 513-650 n there was complete aspermatogenesis without permanent skin damage in about half the cases. Although the difference in selective tissue effect between neutrons and $\mathrm{X}$-rays may be slight, the direction of that difference indicates that neutron radiation may hold an advantage over roentgen radiation in the treatment of human neoplasms. (BA)

382

Lawrence, J. H., P. C. Aebersold, and E. O. Lawrence. TRE COMPARATIVE EFFECTS OF NEUTRONS AND XRAYS ON NORMAL AND NEOPLASTIC TISSUE. Occas. Publ. Am. Assoc. Adv. Sci. 4, (Suppl. Science 85 ): 215-19 (1937); Proc. Natl. Acad. Sci. U. S. 22 (9), 543-57(1936).

In a previous paper the marked dest ructive effects of neutrons on the white blood cells of white rats was demonstrated. In this present work whole mice were irradiated with various duses of $200 \mathrm{kV} X$-rays and neutrong. Quali tatively, the lethal effects of neutrons were similar to those of $\mathrm{X}$-rays, causing destruction of hemopoietic and lymphoid tissues, and of the epithelium of the small intestine, also Sarcoma 80 was irradiated in vitro with various doses of $200 \mathrm{kv} \mathrm{X}$-rays and neut rons; neutrons are definitely destructive to this neoplastic tissue. The possibility of an 383

Lawrence, John $\mathrm{H}_{\text {, }}$ and Robert Tennant. COMPARATIVE EFFECTS OF NEUTRONS AND $\mathrm{X}$-RAYS ON THE WHOLE BODY. J. Exptl. Med. 66, 667-87(1937).

Irradiation of the whole bodies of mice with neutron rays in sufficient quantities leads to a clinical, bacteriological and anatomical picture similar to that following roentgen irradiation. Asıde from any possible delayed effects upon exposure to small doses over a long period of time, concermng whel there is no information, these changes after relatively large doses make it imperative that workers in laburaturies generat nag neutrons protect themselves from exposure by screennig. For the same amount of ionization measured by a small bakelite-walled thimble chamber, neutrons are more biologically destructive than $\mathrm{X}$-rays. The average daily dose to those working with neutrons should not exreed 0.25 of the tolerated dose accepted for $X-r a y s$. Also if neutrone are tried the rapeutically normal 384 tissue must be protected from undue irradiation. (CA)

LEa, D. E. THE SPATIAL DISTRIBUTION OF IONIZATION IN IRRADIATED TISSUE, AND ITS RELATION TO BIOLOGICAL EFFECTS. Brit. J. Radiol. 16, 338-9 (1943).

In producing breaks in chromosomes, neutrons are much more effective per ionizing particle than medium wavelength $X$-rays. The author explains this by the fact that the ionization path of the proton ejected by the neutron is much more dense than that of the electron ejected by the $X-r a y$, except very near the end of the electron path. It is assumed that only the dense and part can produce the chromosome break. The relation between length of dense part of path and $X-r a y$ wave length is used to predict relative biological effects for these wave lengths. Experiments confirm the theory. (BA)

385

Marshak, A. CHROMOSOME ABNORMALITIES PRODUCED IN INTERPLASE NUCLEI WITH X-RAYS AND NISUTRONS. cenetics $26,161(1941)$.

Previous experiments showed the frequency of chromosome abnormalities observed in anaphase at three hours after treatment with $\mathrm{x}-\mathrm{xay}$ s or neutrons to be an exponential function of the dose. Abnormalities observed in $\mathrm{V}$. faba root tips at 12 and at 24 hours after neutron bombardment likewise vary exponentially with dose. The glopes of the curves for the 3,12 and 24 hour intervals are $070, .051$, .036 , respectively. The slope for the Vicia $\mathrm{x}$-ray curve at 24 hours is .0025. The ratio of the neutron to 3 -ray slopes $(\mathrm{n} / \mathrm{x})$ at 24 hours is 14.3 as compared with the comparable ratio of 6.6 at 3 hours. Thus chromosomes of the same organism at different stages of the nuelcar cycle give different $\mathrm{n} / \mathrm{x}$ although a constant ratio has been obtained with chromosomes of very different species at the same stage of the nuclear cycle. Pisum and Allum also give exponential curves at 24 hours, but data are not yet sufficient for obtaining $\mathrm{n} / \mathrm{x}$. However the ratio of about 16 obtained by GLLS for chromatid dicentrics in Tradescantia microspores is sufficiently close to the 14.3 for Vicia to suggest that a constancy comparable to the one observed for abm normalities induced at the onsel of prophase may be found for similar parts of the interphase in different species. (RG)

386

Mateyko, G. M., A. Edelmann, P. A. Charipger, and A. S. Gordon. CFECTS OF DIRECT CATHODE RAY PARTICLE IRRADIATION OF THE MYPOPHYSIS AND WMOLE BODY X-IRRADIATION ON THE ADRENOCORTICOTROPUIN, CONADOTROPIINS, AND THYTOTROPYIN OF THE PITUITARY GLAND OF THE RAT. Am. I. Physiol. 167, 808-9(1951) (AECU 1513)

Cathode ray particle irradiation (I-Van de Graafi; $2.23 \%$ $10^{-3} \mu$ a sec) confined to the volume of the pituitary gland, or whole body $x$-irradiation (II- $250 \mathrm{kvp}, 30 \mathrm{ma}, 0.25 \mathrm{~mm}$ $\mathrm{Cu}, 1.0 \mathrm{~mm} \mathrm{Al} ; 1,000 \mathrm{r})$ was given to rats and the adrenocorticotrophic, gonadof rophic, and thyrotnophic titers of the hypophyses were determined by bioassays at 1,6 , and 24 hr after exposure. Following $I$, the adrenocorticot rophin was elevated at $1 \mathrm{hr}$ and remained at high levels 6 and 24 hr post irradiation. Following II, there was a similar rise at $1 \mathrm{hr}$, the level starting to fall by $6 \mathrm{hr}$, and declining to subnormal values within $24 \mathrm{hr}$. In both groups the gonadotrophins had fallen by $1 \mathrm{hr}$ and remained at subnormal titers 
throughout the $24 \mathrm{hr}$ period. Fullowing I, the level of thyrutrophin was elevated at 1 and $6 \mathrm{hr}$, and fell below normal $\mathrm{n}$ $24 \mathrm{hr}$. A similar, but less marked respense, was observed after II. The data suggest that the noxious stimuli of radiation cause an acceleration of adrenocorticotrophic synthesis initially at the expense of the gonadotruphins, and ultimately, of the thyrotrophin. A response was obtained on animals receiving whole body $\mathrm{x}$-irradiation but with their heads shielded. "This might indicate an "indireet" effect on the pituitary as contrasted to "direct" effects as seen after I. (NSA)

$38 \%$

Mottram, J. C. and L. H. Gray. RESPONSE OF THE STIN OF MICE TO X-RADIATION AND CAMMA IRADIATION. Brit. J. Radiol. 13, 31-34(1940).

Skin of the tails of inbred mice was exposed to $\mathrm{X}-\mathrm{rays}$ $(2,500-7,000 \mathrm{r})$ and to $\gamma$-rays $(4,000$ to $8,000 \mathrm{r})$. It was found that a larger dose is requi red to induce skin reaction by $\gamma$-radiation than by $\mathrm{X}-\mathrm{rays}$. The ratio of effectiveness is $1: 3$ for erythema and desquamation, and $1: 6$ for epilation and esudation. The difference may be partly attributed to the different absorption of photo-electrons by $\mathrm{X}-$ and $\gamma$ 388 rays, otherwise it remains unexplained. (EM)

Paul, Wolfgang and Gerhard Schubert. PHYSICAL AND BIOLOGICAL PHENOMENA PRODUCED BY IRRADIA TIONS WITI FAST ELECTRONS. Naturwissenschaften 37, 156-63(1950).

A systematic comparative study of the action of hard $\mathrm{x}$ rays (200 kv) and of fast electrons (from a $6-\mathrm{Mev}$ betatron) is being made at the author's laboratory in Gottingen, Germany. In a general survey of the work done, quantitative differences in the absorption of the two radiations by matter, specific qualitative aspects of their biological action, and the present-day outlook for therapeutic application of (3)

Paul, Wolfgang and Gerhard Schubert. BIOLOGICAL ACTION OF IAST ELCCTRONS TROM A G-MEV BETA-

TRON. 2. Naturfor $5 \mathrm{ch} .5 \mathrm{~b}, 390-4(1950)$.

The action of fast electrons (up to 6 Mev) on various biological objects was compared with that of hard $\mathrm{x}$ rays $(200$ kv). In the majority of cases observed (growth inhibition in barley sprouts, mitosis-ireguency changes in root-end cells of barley and horse beans, killing of Drosophila eggs and pupae) the effect per dose unit is smaller when produced by electrons. On the other hand, no difference is observed in the radiation-induced development of lethal factors in mature or immature male cells of Drosophila or in the simpler forms of chromosome breakings produced by radiations in the same insect. Only in the case of the killing of Bacterium coli do electrons show the greater effect per unit dose. These results can be interpreted in the light 390 of the "hit" theory. (NSA)

grosser, C. Ladd and Marguerite N. Swift. AN INTERSPICIES COMPARISON OF THE RADIOTONICITIES OF X RAY, $\mathrm{Sr}^{82}, \mathrm{AND} \mathrm{Pu}^{288}$. Universily of Chicago Metallur gical Lab. June 1940. Decl. Apr. 18, 1950. 10p.

(ACCD-2828)

The 30-day $L D_{53}$ for $\$ 5^{89}$ on the basis of injected dose decreases with increasing animal gize as shown by $7.8 \mu \mathrm{c} / \mathrm{g}$ for the mouse, through ral and rabbit, to $0.8 \mu \mathrm{c} / \mathrm{g}$ for the $\mathrm{dog}$, and then increases to $1.3 \mathrm{\mu c} / \mathrm{g}$ for the goat. The series is similar when computed on the basis of the dose retained at time of death. Radiation lost outside the body is greater in small than in large mammals, but measurements in mice show such loss to be not more than $3 \%$ of total emitted radiation from $\mathrm{sr}^{3}$. The radiation sensitivity as indicated

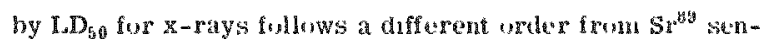
sitivity, the $\mathrm{LD}_{50}$ beums highest in the rabul, least an the don, and internedate in the mouse and rat. Calculation of $\mathrm{Sx}^{89} \mathrm{LD}_{50}$ on the basis of sliceletal weunt unereases the specues differences. When the $30-$ day $\mathrm{LD}_{50}$ of $\mathrm{Sr}^{89}$ is cour reeted for retention and relative radosensitivity (usmgs the radusensitivity of the mouse as a unit), the follownopseres is oblained in $\mu \mathrm{g} / \mathrm{g}$ : mouse 3.5 , rat 2.48 , rallbit 0.57 , dogr 0.57 , and goat 0.98. By extrapolation from thes serles, the 30-

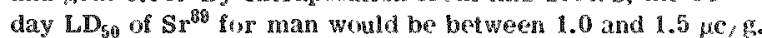
The strontium is deposited in bone and has a range $(50 \%$ of the $f$ rays absorbed) of $0.72 \mathrm{~mm}$ in suft 115 sue. The syscies differences in $I_{s} \mathrm{D}_{50}$ are interpreted on the assumption that some volume of marrow needs to be irradiated to cause death. The speries sernes for pu $^{232}$, the purticles of which have a range of only abrut 40 p in soft tissue, shows little variation in toxicity with size. The 30 -day $\mathrm{LD}_{50}$ on the basis of retained dose in,$c$, and currects for rela tive radiosensilivity is: mouse 0.0412 , rat 0.0399 , and dog 0.0375 . If the relative radiogensitivity is known, it $1 \mathrm{~s}$ jus sible to extrapolate from one species to ancther for $\alpha$ particle dosage, but not for lethal $\beta$-particle dosage. (NSA) 391

Sacher, George A. and N. Pearlman. COMPARATIVE ACTION OF CYCLOTRON NEUTRONS AND X-RAYS. PART II. STATISTICAL ANALYSIS OF BLOOD DATA. UIBiversity of Chicago Metallurglcal Lab. June 1946. Deol. Sept. 16, 1247. 29p. (MDDC-1337)

The heterophil and lymphocyle counts of 67 rabbits exposed to fast neut rons, and those of 123 rabits exposed to $200 \mathrm{kv} \mathrm{X}$-rays, were analyzed. Distributions of eunts were nurmalized in conversion to lograrithms. Respunses of buth blood systems to the two types of radiation were qualdatively similar. The ratio of $\mathrm{X}$-ray and neutron doses for equatl ef fect on heterophil counts was determined by restesstons between $\mathrm{X}$-ray and neut ron responses at like times after exposure, for various pairs of $\mathrm{X}$-ray and neutron dises. The average of 29 estimates for $\mathrm{X} / \mathrm{n}$ using thas methud, with its standard error, is $6.3+0.10$. The above method could not be used in the case of lymphocytes, but an alternative method gives 6.20 as the value for $\mathrm{X} / \mathrm{m}$. The authors conclude that the heterophils and lymphoeytes do not differ significantly in the $\mathrm{X} / \mathrm{n}$ ratios, and that the numerical value of the ratio may be talten to be $\mathrm{K} / \mathrm{n}$ 6.3 10.10 . (ADD) 392

Schaefer, Hermann J. FURTHER EVALUATION OF PRESENT DAY KNOWLEDGE OF COSMC RADIATON IN TERMS OF THE HAZARD TO HEALTH. Naval School of Aviation Medicine, Pensacola. Aug. 15, 1951. 23 p. (NP-3564; U19329)

Data pertaining mainly to the heavy-nuclei component of the primary cosmic radiation and appenrung in the literature between Jan. and Dec. 1950 are summarized. There are evidences that abut $20 \%$ of the incommg primaries are atom nuclei of an atome no. -1 . Changes in the energy spectrum resulting from the greomagnetic ficlds at different latitudes are not accumpanied by changes in the shape of the mass spectrum. The cut-of mechanism of the geomagnetic field wheh sets the lower limit of the energy spectrum of the primaries in dependence on the latitudes is discussed. The ionization peak and thin-down phenumena anpear to be limited to the polar cap (geomagnetic latitudes of $55^{\circ}$ and higher). The values of the specific ionization for the different components of the heavy spectrun cover a range from about 1000 ion pairs per $10 \mu$ of living tissue to more than $1,000,000$. The conversion factor for natural a rays $(25,000$ ion pairs per $10 \mu)$ is 10 ; that for fission products (2.8 million ion paids per $10 \mu$ ) is 27 . Although no accurate interpolation for the different components of the 


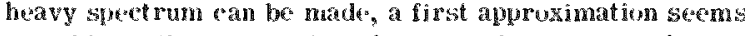
possibl on the assuruption of a keneral convession factor of 10. The cexposure of humans to the primstry cosme roti-

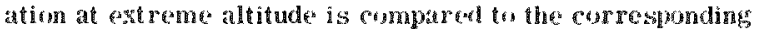

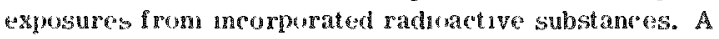

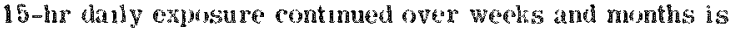
expeced to produce marled symptons of radiat ion anjury. A danly copsusure of $1.3 \mathrm{hr}$ is estimated tu be abut equal to the officlal tolerance limit of 0.1 , Tat in the systom. (NHS 393 abst.) (NSA)

Schmidt, John W. and E. Fo Folk. COMPARATIVE ER FECTS OF TIERMAL NEUTEON IRMAOLATION OF MALZ POLLEN AND MATZE SEDD ON SUBSEQUENT SEEDLING GROWTI. J. Heredity $42,173-7(1951)$.

On the basis of $x$-ryy comparisons, thermal neutrons Wore $\sim 6$ to 12 tumes as afectuve on naze sed as on maize pollen in redug ing subsequent seding helght. Evesn if al -

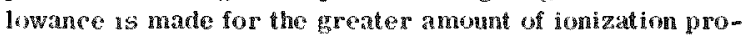
duced in sed than in pollen by equal dosess of thermal neutrons, the is still a lares aiference in the efficiency of these particles on the two plant parts (on the basis of $x-$ ray compra lasmis). It is, the refore, sugreseted that ionizat ion alone may nut account for all of the biolorical effects of thermal newerons and the transmutation of $\mathrm{N}$ lo $\mathrm{C}$ and or $H$ to Li may alsu contrabute to the bulwgical effects. The susceptiblity of mart pollen and maize seed to the amal neutrons was determined throuph the effects on the subse.. quent secding development. (NSA)

\section{4}

Schubert, Gerhard. EFPECTS OF FAST ELECTRORS OF A 6 MEV-BETATRON ON BIOLOGICAL SUBJECTS, D.164 Of INTERNATIONAL CONGRESS ON RADIOLOGY, 6h Congress, London. 1950.

The influence of fast electrons on biolosical substances has been analysed by usmig the Gouttingen-betatron of $6 \mathrm{MeV}$ (Siemens-Reinger-Werke, Erlangen). Physictlly equal doses of fast electrons (2 to $6 \mathrm{MeV}$ and X-rays (200 liv.) cause different quantitative fects in biolugical tissue. The reactions most instinces show a less effect with fast electrons per duse-und than with X-rays. Wath some lypess of biolugical response the same effect follows each kind of radiation, oceasionally fast electrons are mure fifeient than $\mathrm{X}$-rays. The results whaned with bact. coli, Hordeum, Vicia falba, Drosophnla melanosaster, Salamandra maculosa, chick fibroblasts (heart), pat erythroblasts and Ehrlich's carcinoma are summarned and the mode of action of radiation discussed. The observed quant it ative differences between the action of fast fulctrons and $\mathrm{X}$-rays can be explained by variations in sperific ionisation and ion cluster formation. "The consequencts are consudered theoretically in ferms of "concentration", "saturation" and "accurnulation", and the observed qualitative differences of action between the two kands of Fadration explained on this basis. Difference between the biological effect of fast electrons and high energy $\mathrm{X}-\mathrm{rays}$ (quantum-entrgy 2-6 MeV.) are, on 395 this theory, not to be expected.

Scott, K. G. A COMPARISON OF TWO LEVELS OF ROENTGEN AND NEUTRON IRRADIATION ON NORMAL AND LYMPHOMATOUS MICE, USING RADIOPHOSPHORUS AS AN INDICATOR OF CELLULAR ACTIVITY. Radiology $46,173-5(1946)$

Neutron and roentgen rays depress the phosphurus up-

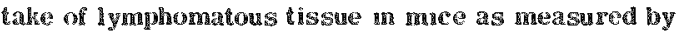
radionhosphorus. By the use of this technic another method is avalable for the study of the effects of various radiations on normal and neoplastic tissues. (BA)
396

Stapleton, G. E. and R. F. Zirkle. COMPARATIVE EFFECTIVENESS AND ADDIVITVY OF FISSION NEUTRONS GAMMA RAYS, AND BETA RAYS ON DROSOPHILA EGGS.

Clinton Labs. Fob. 1946. 31p. (MDDC-584)

Some investiggations have been made to ascertain the smilarity of the mechanism of action of radiations which are different in their specific ionization. A suggested approach was to determme if the energies absorbed from the different types of radiation are additive in their lethat of fects on biological object.s. In an attempt to determine the additivity of ionizings radiations various dose combinations of fast neutrons, gamma rays and beta rays were administered to Drosophila eggs. These test objects were chosen because the ir dimensions are such that they tend to absorb these three radiations uniformly throughout their volume. Although the three radiations were found to vary in the ir biological effectiveness, per unit energy absorbed, all three were shown to be completely additive in their lethal effect on these organisms. (ADD) 397

Stone, Robert S. NEUTRON THERAPY AND SPECIIC IONIZATYON. Am. J. Roentgenol. Radium Therapy 5 S $771-85(1948)$.

Neutrons generated by $16 \mathrm{mev}$ and roentgen rays generated by $220 \mathrm{kv}$ show that $1 \mathrm{n}$ (neutron unit) equals $2.5 \mathrm{res}$ (roentgen equivalent physical). Gross and microscopic studies of mammalian tissues show little or no qual itative difference in the reartions produced by neutron and roentgen ray radiations. Judging by the amount of energy absorbed from the beam in producing a given reaction, the relative biological effectiveness of different radiations vary greatly from 1 test object to another, and from 1 reaction to another in the same tissue. The relative biological effectivenr'ss of radiations on mammalian tissue inereased as the specific tonization increased. Neutrons were more effective than roentgen rays per unit of energy absorbed by tissue. The relative biologic effectiveness of neutrons is greater compared to rocntgen rays for its late effects rather than carly effects. This latter is very important in cunsidering biological effects in treatment of human patients, as the late effects with certain radiations are considexably more marked as compared with $\mathrm{X}-\mathrm{radiation}$ than the carly effects would lead one to expect. (BA) 398

Tobias, C. A., H. O. Hoanger, and J. H. Lawrence. IRADIOLOGICAL USE OF FAST PROTON, DEUTERON, AND ALIPLA PARTICLIE BEAMS, P.51-2 of INTERNATIONAL CONGRESS OY RADIOLOGY, Gh COngress, London. 1950.

A number of radiological expeximents were gerformed using the Berseley $184^{\prime \prime}$ cyclot ron buill by $\mathbb{E}$. O. Lawrence and his assuciates. The $190 \mathrm{MeV}$ deuterons may be brought out into air as a stream of well collimated parallel particles of homogeneous energy. The particles have a well defined range, approwimately $15 \mathrm{~cm}$. in soft tissue and $19.1 \mathrm{~g} / \mathrm{cm}^{2}$ in A1. Corresponding to their Bragg ionization curve, the peak ionization in tissue is reached about .7 $\mathrm{cm}$. belore tho end of the range and has about four times the ionization at the point of entry. This beam is thus suitable for selective ir radiation of deep lying tissues without overdosing the glkin. Using multiple yort irradiation the depth close may be made thrity times the skin dose. Isodose curves will be presented. The 50 per cent lethal dose of high energy deuteron rays on mice is the same as that of $200 \mathrm{kV}$ X-rays, if delivered at the same dose-rute. The 25 per cent of strong mannary carcinoma tumours in "A" mice regressed completely when the beam was directed to the tumourg hrough the body of the mice. All unirradiated tumour controls died. The gre- 
cific effect of different energy portions of the deuteron beam was tested using mice and shin effects as criteria. In experiments carried out in association with $\mathrm{V}$; bond a narrow deuteron beam was passed through different body regions of white rats and the acute lethal affect in function of the dosage was measured. In assoc tation with $\mathbf{R}$. E. Zirkle experiments were made to study the mechanism of radiation effect on micro-organisms as a function of specific ionization.

389

Wachsmann, F. DIFFERENCES IN THE BIOLOGICAL ACTION OF ORDINARY AND ULTRA-HARD RADIATIONS. Strablentherapie $81,273-80(1950)$.

The biological effects of the new radiations furnished by the betatron (ultra-hard $\mathrm{x}$-rays and fast electruns) must differ from those produced by ordinary $x$-rays, owing both to the more favorable dose distribution in space and to the lower ionization density of the new rays. Furthermore, the differences in the biological action are not just quantitatuve. Since the ratio of the responses to irradiations of both kinds of rays varies with different irradiated objects, it is permissible to expect a certain "selectivity." A few examples from the author's studies illustrate the pont. It can be concluded from the shape of the survival curves of ir radiated tadpoles that the biological structures attacked are not the same for the two kinds of radiation. Again, the different ways in which the reticulocyte rate in mice is affected by the radiations point to differences in the mechanism of attack. Finally, tumors inoculated in mice and rats, when treated with 5-Mev electrons, show more satısfactory regression pictures and less deterioration of healthy tissues 400

than in the case of a $200-\mathrm{kV}$ X-ray treatment. (NSA)

Zirkle, Raymond E. THE RADIOBIOLOGICAL IMPORTANCE OF THE ENERGY DISTRIBUTION ALONG IONIZATION TRACKS. J. Cellular Comp. Physiol. 16, 221-35 (1940).

The role of the linear energy absorption along the tracks of ionizing particles was investigated by irradiating 3 species of small cells. Different values of linear energy absorption $E_{\mathrm{L}}$ were investigated by using various linear portions of a particle tracks, and by comparing the action of $\mathrm{X}$-rays (low $\mathrm{E}_{\mathrm{L}}$ ) to that of $\alpha$ rays (high $\mathrm{E}_{\mathrm{L}}$ ). On $\mathrm{Asper}$ gillus spores the effectiveness of the radiation per unit dose (energy absorbed per cubic micron) increased sharply as the linear energy absorption increased. On vegetative cells of Saccharomyces cerevisiae the effectiveness increased only slightly with inerease in $\mathbb{E}_{\mathrm{l}}$. Accordingly the radiobiological influence of the linear energy absorption depends both in magnitude and in algebraic sign upon the species of cell irradiated. (BA)

Zirkle, R. THE RADIOBIOLOGICAL IMPORTANCE OF SPRCIFIC IONIZATION. [University of Chicago] Metallur gical Lab. [nd] Decl. Oct. 9, 1946. 30p. (MDDC-444; CH-946)

II is shown that, in the production of injurious effects in both the higher plants and the higher animals, the amount of injury produced per unit of absorbed energy increases with the specific ionization of the radiation. In particular, the injurious action of fast neutrons is greater than that of X-rays or gamma rays by factors ranging up to 20 , and the effectiveness ratios are calculated in such a way as to nake them conservatively low. Although most of the effects on mammalian tissues show effectiveness ratios ranging from 2 to 3.5 , it must be pointed out that to date comparatively few neutron investigations have been carried out on mammalian tissues in vivo. It is shown that different effects on the same biolugical object may exhibit considerably differ'nt effectiveness ratios, therefore a distunct possibility exists that further observation will disclose important injurusus effects on mammals which have neutron/X-ray effectrvenuss ratios ranging up to 10 . From the protection standpoint, only one such biological effect, though at present unknuwn, is a threat. Until extensive and numerous investifrations allay this threat by failure to reveal injurious effects extremely sensitive to specific ionization, special precautions seem to be indicated when setting tolerance duses in situations where fast neutrons constitute a sizeable fraction of the total radiation present. These considerations are especially pertinent in case $\mathrm{X}$-ray thimble chambers are used in measuring the total radiation. If uncorrected Victoreen measurements are used in comparing the effectiveness of fast neutrons and X-rays, the apparent effectiveness ratios for the known injurious effects on mammalian tissues range up to more than eight. Carbun-walled chambers are even more misleading. (ADD) 402

Zirkle, Raymond E. RADIOBIOLOGICAL ADDITIVITY OF VARIOUS IONIZING RADIATIONS. Am. J. Roentgenol. Radium Therapy 63, 170-5(1950).

A discussion is presented on the problem of additivity of various types of ionizang radiations. A summary is moluded which outlines the degree of additivity found in varuus experiments by the author and others through combunations of $\alpha, \beta, \gamma$, fast-neutron and $X-$ rays on the mouse, bean routs, Drosophila eggs, Drosophila pupea and the human skin. It is concluded from the analysis of these studies that ancomplete additivity of two types of radiation indicates some dufferenc ${ }^{*}$ in the mechanism of action of the radiations. Complete additivity indicates that the mechanisms of action of the radiations are identical in then most essental feature, the promotion of the same determinative events fone of a succession of unknown relevant events leading to the production of the known brological effect) but are not necessarily alite otherwise. It is concluded that it is probably wise to assume that additivity of fast neutrons and $\gamma$ pays is the complete type. (NSA)

403

Zirkle, R. E. and P. C. Aebersold. RELATIVE EFFECTIVENESS OF X-RAYS AND FAST NEUTRONS IN RETARDING GROWTH. proc. Nat1. Acad. Sci. U. S. 22, $134-8(1936)$.

Since the biological effectiveness per ion increases markedly with closer spacing in the paths, it seems geasunable to expect that a given number of cons produced by noutrons would be considerably more effective than the same number produced by $X$-rays. This expectation has been realized in both these experiments (on wheat seedlings) and in the experiments by Lawrence and Lawrence on the rat (Proc. Nat. Acad. Sci., 22: 124(1936)). The authors used wheat seedlings for their experiments, handling them in a manner similar to that described by Failla and Henshaw (Radiology, 17: 1-43, (1931)). (5. V. S.) 404

This abstract has been intentionally omutted. 405

Zirkle, R.E., P. C. Aebersold, and Everett R. Dempster. THE RELATVE EPFECTVENESS OF FAST NEUTRONS AND X-RAYS UPON DHFERENT ORGANISMS. AM.J. Cancer 29, 556-62(1937).

The relative effectiveness of fast neutrons and X-rays, per unit of ionization, was determined for Drosophila eggs, wheat seedlings, and fern spores. The neutron-X-ray ratios of effectiveness were, respectively, $2.1,5$, and 2.5. Therefore, the relative susceptibility of various types of cells to 
neutrons may differ greatly from their relative suscepti106

bility to $\mathrm{X}$-rays. (BA)

Zirkle, R. E. and I. Lampe. DIF FRENCES IN THE RELATIVE ACTION OF NEUTRONS AND ROENTGEN RAYS ON CLOSELY RELATED TISSUES. Am. J.

Roentgenol. Radium Therapy 39,613-27(1938).

The authurs compared the actions of neutron rays (from the University of Michigan cyclotron) and $200 \mathrm{kv} x-\mathrm{rays}$ on the routs and shouts of wheat seedlings, and on three age rroups of Drosophila eggs. It is amposssble to measure the two radrations in terms of the same unit, or to compare them directly. The relative effects of the two on any one tissue were expressed by an $\mathrm{X}, \mathrm{N}$ ratio, where $\mathrm{X}$ denotes the number of roentgens of $x$-rays producing a given effect, and $\mathrm{N}$ the number of arbitrary neutron units producing the same efiect. If the $X, N$ ratios for any two tissues were not equal, then the relative action of the two radiations was different for those two tissues. For Drosophila eggs of average age $1-1 / 2,4$, and 6 hours, the ratios were respectively, 2,3.2, and 2.8. For wheat seedlings the root ratio varied from 6.2 to 11.8 , whlt that fur the shoot varied from 5.9 to 9 , the former being always greater than the latter an any single experment. The authors conclude that the relative action of neutrons on closely related tissues 15 different from that of $X$-rays. (BA)

407

Zirkle, Raymond E., Dorothy F. Marchbank, and Katherine D. Kuck. RXPONENTIAL AND SIGMOD SURVIVAL CURVES RESULTING FROM ALPHA AND X IRRADIATION OF ASPERGILLUS SPORES. Univ. of Chicago and Argonne National Lab. Mar. 1951. 14p. (ANL-4610)

After samples of air-dry spores of Asperillus terreus had been given graded doses of polonum a particles and of 200-kv $x$ rays, survival fractions were determined, the criterion of a surviving spore being the formation of a germ tube. Whon survival fraction was plotted agannst dose, the $a-$ pay curves were exponential, but the s-ray curves were sigrnoid, and the experimental points could be fitted farly well by "twu-hit" theoretical curves. It is concluded that, in this particular radrobiological action, the number of entities which must be altered in each cell is quite small, and that, whereas all of them (one or more) are altered by one suitably lucated $a$ track, two (possibly more) electron tracks are required to produce the same alteration (s). The results also indicat that the decisive events of the radiobiological action occur very early, i.e., before each relevant lonzation track loses its original configuration by diffusion of the initually activated molecules or their chemical derivatives. (NSA)

\section{8}

Zirkle, Raymond E. and Mary Ellen Parrish. COMPARATIV LETHAL ACTION OF X RAYS AND CYCLOTRON NEUTRONS ON DROSOPHILA EGGS. Univ. of Chicago Metallurgical Lab. Dec. 1950. 6p. (CH-3904)

The $\mathrm{x}$ rays were generated at $200 \mathrm{kv}$ and $5 \mathrm{ma}$ and were filtered by $0.5 \mathrm{~mm}$ of Cu and $1 \mathrm{~mm}$ of Al. The target-toobject distance was adjusted to give a dose rate of about $10 \mathrm{r} / \mathrm{min}$. The fast neutrons were generated by 8 -Mev deuterons impinging on a Be probe in the University of Chicago cyclotron. The eggs were exposed in a lead-walled chamber which was so const ructed that radiation directly from the grobe was filtered through $4 \mathrm{in}$. of $\mathrm{pb}$, while scattered radiation from other directions was filtered through at least 2 in. Various lots of eggs were given graded doses of $\mathrm{x}$ rays, of fast neutrons, and of mixed neut rons and $y$ radiation. The $\mathrm{LD}_{50}$ of $\mathrm{x}$ rays is $170 \mathrm{r}$, that of last neutrons is 31 $\mathrm{n}$, and that of the mized cyclotron emission is 4 s Victoreen scale units. Accordingly the $\mathrm{x} / \mathrm{n}$ dosage ratio is $170 / 31$ or
5.5, whereas the apparent $\mathrm{x} / \mathrm{n}$ ratio, from data obtained when ? 7 rays were present, is $170 / 43$ or 4.0 . (NSA)

Zirkle, R. E., J.R. Raper, E. F. Riley, and G. F. Stapleton. ADDITIVITY OF FAST NEUTRONS AND GAMMA RAYS IN THEIR ACUTE LETHAL ACTION ON MCE. Clinton Labs. June 5, 1945. Decl. Sept. 17, 1948. 19p. (AECD2328; Cr-2571)

By exposing various groups of mice to graded doses, the median lethal dose (LD-50) of each of the following radiations was determined: (a) fast neutrons from the Clinton pile; (b) Radiation $G$, consisting of gamma rays contami s nated with fast neutrons; (c) Radiation M, a mixture of fast neutrons and Radiation $G$. The LD-50 of Radiation M was found to consist of 0.52 times the LD-50 of fast neutrons plus 0.55 times the LD-50 of Radiation G. Theoretically, the sum of these two fractions should be unity if fast neutrons and Radiation $\mathrm{G}$ are radiobiologically additive. Since the experimental deviation from unity $(7 \%)$ is within the error of the measurements, it is concluded that complete additivity of these two radiations has been demonstrated. It is assumed that this conclusion concerning additivity of fast neutrons and Iadiation $\mathrm{G}$ may be extended to additivity of fast neutrons and pure gamma rays. The LD-50 of fast neutrons, directly determined, was $91 \mathrm{n}$. The LD-50 of pure gamma rays, indirectly determined from the experimental data, was $812 \mathrm{x}$. In another experiment, performed later for a different purpose, a corresponding value of 840 $r$ was obtained. (NSA)

\section{Pathology}

10

Barrow, Jack and John L. Tullis. THE SEQUENCF OF CELLULAR RESPONSE TO INJURY IN MICE EXPOSED TO 1100 Y TOTAL BODY X-RADLATION. Naval Medical Research Inst. July 6, 1949. 23p. (NP-1411; NM-007039)

Following a single lethal dose (100\% in approximately 96 hours) of $1100 \mathrm{r}$ total body $\mathrm{x}-\mathrm{radiation}, 880$ mice were killed 10 at a thme at hourly intervals. Microscopic observations of spleen, mesenteric lymph nodes, bone maxrow, intestines, and testis were made to determine the sequence of cellular response to the injury. Fragmentation and lysis of lymphoid elements were well established one hour after exposure. The extent and rapidity of progression of destruction vere of equal intensity in the lymphoid elements in the spleen mesenteric lymph nodes, and lymphoid aggregations in the intestinal wall. The erythropoietic elemente also were almost as radiosensitive as the lymphoid tissues. Slightly less radiosensitive were the myelopoietic and megakaryocytic elements in that order. The reticuloendothelial cells remained morphologically intact. In the testis the spermatogonia disappeared by 66 hours, but the spermatocyles and particularly the spermia and Sertoli cells were more radioresistant. The crypt epithelium of the small intestine and to a lesser extent the basal portions of the glands of the large intestine were damaged early, but reoair of these organs was accomplished by the 85th hour. (NSA)

411

Bigelow, R. R., J. Furth, M. C. Woods, and R. H. Storey. FNDOT YIELIAL DAMAGE BY X-RAYS DISCLOSED BY LEMPH ISTULA STUDIES. Proc. Soc. Espt1. Blol. Med. 76, $734-6(1951)$.

The degree of capillary permeability was assayed in dogs and rats by studying the appearance of components of the blood in the lymph. Marked capilary permeability was indicated in dogs and rats exposed to approximately $\mathrm{LD}_{60}$ 
doses of $x$ rays by the appearance of large numbers of erythrocyles in the lymph. The data indicate that the reduced red-cell counts after irradiation are due to the flooding of the lymph compartments and tissue spaces by erythrocytes. The massive erythrophagocytosis and hemosiderosis in sinuses of lymph nodes, if irradiated hosts, indicate that many of the erythrocytes leaving the blood capillaries are destroyed. Loss of exylhroeyles caused by capillary damage and death of aging erythrocytes with in hibition of erythrogenesis are the main causes of radiation ankmia. (NSA)

412

Borak, J. RADIATION EFELCTS ON BLOOD VESSELS. Radiology $38,481-92,607-17,718-27(1042)$.

This review considers various effects of radiations and their gelation to blood vessels. The discussion is centered around the author's own theory that the observations on capillaries are to be explained as direct effects of the radiation on the epithelial cells. The matcrial discussed is divided in three parts: Part I deals with gkin crythema and edema. In Part I, inflammation produced by irradiation, degeneration, suppression of growth capacity of the capillaries, retrugression of blood vessels and radionecrosis are discussed. Part II deals with the etiology of telangi13

Campbell, Berry, Stanley C. Peterson, and Rosalud Novick. WARLY CHANGES TNDUCED IN PUREINIF CELLS OF IABBIT BY SINGLE MASSIVE DOSES OF ROENTGISN IFAYS. Proc. Soc. Frxpll. Biol Med. 61, 353-5 (1946).

Immediate and severe changes were geen in Purkinje cells of cerebellum immediately after exposure to doses of 1000-4000 $x$. Chromatolysis, vacuolization, and the appearance of a clear marginal zone were seen immediately following irradiation. These changes were transient and were replaced in 24 hours by pylknosis and neuronophagia. (BA)

414

Carter, Robert I. SPLENC CHANGES IN CII IPMAL MCE OVER A FORTY -ONE DAY PETIOD POST XIRRADLATON. LOS Alamos Scientific Lab. [nd] $9 \mathrm{p}$. (A.EU-709)

This study was undertaken to determine if, at any lime in the immediate post erposure period, weight changes in the goleen or white pulp atrophy of CF1 female mice coulu be related to the amount of $x$-irradiation the animals received. Splenic weight changes and whhte pulp-red pulp ratio changes over a 4-day period post irradiation are presented. Initial studies of gplenic weight changes indicate that between three and eight days post irradiation the decrease in splenic weinght may serve as a remarlably accurate indication of the radiation received. The changes in the pulp ratios of the spleen were not deemed of sufficient promise to war rant surther investigation. Possible applications of this phenomenon to the field of radiation and biology are men술15 toned. (NSSA)

Denniston, Mollin Heary, II. THE INELUENCE OF ROENTGEN-RAY TREATMENTS OT TUYE MNPOPHXSIS ON RTPRODUCTON REL AND RAT. J. Rxpt1. ZOOL. 11, 23\%-63(1042).

Armual-breeding ground squirrels Citellus tridecemlineatus, were 8 -rayed on the pituitary at various times during the annal cycle with doses ranging from of to 4000 roentgen units. Awnt and immature rat pituitaries were also rayed. No evidence for a "stimulating dose" of $x$-ray was found in gross or cytological studies of ground squirrel tissues fol- lowing pituitary $z$-radiathon in the yearly quiescent phase. Gonads and pituitaries of quiescent animals proved resisiant $t o$ massive $x$-radiation. Gonadot rophr hormone sec ration of the inactive pituitary did not effect recovery or rayed testis within 40 days. Age-correlated elfects follow $x$-radiation of rat pituitaries: $1000 \mathrm{r}$ reduce the growth rate of young rats $50 \%$ : $2000 \mathrm{x}$ inhibí spermatogenešs in same age animals; 6000 are necessary to affert gametogenesis in aduits. "Signet-ring castration cells" appear in these adult pituitaries at lower does levels than those necessary to produce effects on the germ line. (BA) 416

Devilk, Finn. HISTOLOGICAL AND CYTOLOGICAL CKANGES PLODUCED BY ALPRA-PARTICLES IN THE SHIN OF MICE. Acta Radiol. 35, 149-64(1951).

The histological and cytological changes in the skin of a hairless strain of mice following external an uradiation are described. It is found that the epidermis contains cells that are able to survive doses of the order of $100,000 \mathrm{r}$. The effects of the $\alpha$ rays, which mainly are localazed in the epidermis, have been compared to the effects caused by roentgen rays in the skin. This comparison stresses the great importance of the indirect effects brought about by the stroma-vascular bed, when the latter also is irradiated. 18 references. (NSA)

417

ELInge, F. RESPONSE OF TPE LIV TR TO IRRADIATION. Radiology 44, 241-54(1045).

The author discusses the response of the liver to irradiation. If is considered that the liver cells are radiosensitive, but are less so than those of the spleen. An attempt is made lo analyze the experimental results of liver irradiation in various animals. Morphological changes are difficult to assess because of the rapid recuperative capacity of liver cells. All animal species show a derease in glycogen and an increase in sudanophil fat content, the former occurring early after exposure, the latter usually appearing at a later date. (BEMP)

418

Engelstad, R. B. and O. Torgersen. IFPERIMENTAL INVESTIGATONS ON THP RAYS ON THIE SUPRARENAL GLANDS IN RABBTTS. Acta Radiol. 16, 671-87(1937).

The author have examined the suprarenal glands of 41 rabbits four hourg to six months affer roentgen radiation with doses of $2,200 \mathrm{r}-2,500 \mathrm{r}$ administered in one seanoe, and of 17 mormal control animals. These doses produce marked degeneration in the cortes, hyperaenia and in some cases inflammatory infiltration. The radio-sensitivity of the suprarenal glanas shows a very good accord with the shin tolerance in rabbits. The degeneration is most constant and most pronounced in the zona fasciculata and zono. reticularis. In the medullary substane no sure degeneration is proved. The reaction curve shows an initial hyperaemia beginning within the first 10 hours after the padiation and receding during the course of the first $2-3$ days. A pronounced dugeneratuon (the main react ron) is first found on the sixth day after the radiation; it is accompanied by hypereamia and, in a number of cases, slight lymphocytic infiltration. The changes seem to be greatest and most constant three months after the radiation, but also sis months after a clear degeneration can be geen. The lipoid distribution seems to be very ir regular in the irradiated suprarenal glands. (auth)

419

English, James A, and John L. Tullis. ORAL MANHFETATIONS OF IONILING RADIATON. PART I. ORAL LESIONS AND EFFECT ON DEV 
POSED TO 2000 KV TOTAL BOUY X-RADIATION. Naval Medical Research Inst. Bethesda. Dec. 29, 1950. 10p. (NP-3162; U17518; NM-006-012.04.34)

Oral tissues, salivary glands and developing teeth of 41 young swine were studed grossly and histologically to observe pathologic changes resulting from 8 -radiation. These swine were sclected from 120 animals which were exposed to $2000 \mathrm{kv}$ total body $\mathrm{X}$-radiation in doses of about 250 to 800 roentgens in air. Undecalcified teeth and jaws were cut in thm sertions by high speed, water cooled hard rubber dises or steel carcular saws. Gross changes in the oral mucosa consisted of petechial and ecchymotic hemorrhages in $40 \mathrm{per}$ cent of the summals and marked ulceration in 9.8 per fent. Histologre study revealed irregular erosions of the murosat about 30 per cent of the animals and enther petechial or massive hemorrhage around developing teeth of 70 per rent of the swne. Regional lymph nodes showed a hagh degree of sensifuvity to $\mathrm{X}$-radiation by exhibiting homor rhage and depletuon of lymphoid cells. Salivary glands were among the less senst ive tissues, with regard to murphologyc change. However, a definite decrease of basif staning granules was observed in acute stages of raduation sickness, with complete recovery in 30-day survivers. A characteristic land of hypoplasia of the enamel of devtopmig molar teeth was observed among swine wheh referved nore than $400 \mathrm{r}$. Ameloblasts actively produc me thamel matris apueared to be sensitive to ifradiation. Other portions of the teeth showed minimal effects. (auth)

420

Eschenbrenner, Allen B. FURTHER STUDHES OF THE EFFECT OF TOTAL-BODY IRRADLATION ON TERE TESTLS OF MICE, p.28-35 Of BIOLOGICAL AND MEDICAL DIVISIONS, QUARTERL REPORT, AUGUST, SEPTEMBER, OCTOBER, 1948. Argonne National Lab. Nov. 1, 1948. 7p. (ANL-4227(p.28-35))

Author reports the course of injury and recovery of the lestes follown total -body radazton, as reflected in testes we1ghts. It is noted that the minimum wayhts of testes observed is defendent upon dosages-also that the time requred to effert the munum weights as well as the lime required for the weyghts to return to normal are functions of dosage. The author also reports olsservations on the recovery pattern of the testes from a second arute exposure followng recovery from the first. The results of microscopir examnation of the testes of mice exposed to $400 \mathrm{r}$ are summaxized in a table. The se data indicate that the der rease of testes weights followng acute ir radiation is primarily due to marked retardation an rate of production (multiplication of spermatogona for a period of approximately two werks). In prevous studies (ANL-4184) it was centatuvely concluded that the over-all decrease in spermatogenic elements was prumarily due to retardation in the rate of multiplication of spermatogonia. The present studnes support this conclusion. (S.V.S.) 421

Eschenbrenner, A.B. and E. Miller. QUANTITATIVE HETOLOGIC ANALYSIS OF THLE EFIECT OF X-RADLATION ON TUE INTERSTITIAL TISSUE OF THE TESTES

OF LAE-1 MICE. J. Natl. Cancer Inst. 6 , (6) 343-8(1946).

The exposure of mire to doses of $\mathrm{X}$-radiation sufficient to produce severe damage of spermatogenic elements resulted in an apparent increase of interstitial tissuce.

Chalkley's method for the quantitat ave morphologic analysis of tussues was used in a study of the testes of normal and irradrated mice. It was found that the apparent increase of interstitul tissue in the testes of irradiated mice is relatwe and not absolute. (BA)
Eschenbrenner, Allen B. and Eliza Miller. QUANTIATVE HISTOLOGIC ANALYSIS OF' THE TESTES OF MICE FOLLOWING A SINGLE TOTAL-BODY EXPOSURE TO X RAYS, p.42-6 of BIOLOGICAL AND MEDICAL DIVISIONS QUARTLRLY REPORT FEBRUARY, MARCH, APRL, 1950. Argonne National Lab. [nd] 5p. (ANL-4451(p.42-6))

Groups of $L A F_{1}$ male mice were given single total-body exposures to $400 \mathrm{r}$ of radiation. Ir radiated and nonirradiated mice were macrificed and the testes were removed at weekly intervals for 12 weeks after $x$ irradiatıon. Chalkley's method (J. Natl. Cancer Inst. 4, 47-53(1943)) was used for quantitative analysis of the testes and the various cellular elements were identified according to his criteria. There appeared to be no evidence of morphologic cell death even when the testes' weights were reduced to $40 \%$ of normal. The werght decrease was due to a decrease in quantily of the spermatogenic elements, the interstitial tissue and supporting stroma remaining normal in total amounts. This depopulation of spermatogenic elements was due to the radiosensitivity of the spermatogonia. The depopulation was not a result of the destruction of spermatogonia but of the inhibition of mitosis which lasted for a period ranging between 2 and 3 weeks. Mitotic activity of spermatogonia in which they reproduce their own kind is considered to be similar to mitotic activity in somatic cells. If this is true, a similar irradiation effect should be seen in other yradiated somatic cells. Studies are under way to determine this. (NSA) 423

Eschenbrenner, Allen B. and Lliza Miller. EFFECT OF ROENTGEN RAYS ON TIRE TRSTIS. QUANTITATIV HISTOLOGICAL ANALYSIS FOLLOWING WHOLE-BODY EXPOSURE OF MUCE. Arch. Pathol. 50, 736-49(1950).

LAF mice were exposed to $50,100,200,300$, and $400 \mathrm{r}$ of $186-\mathrm{k} v$ x rays directed to the whole body at 3 months of age. The course of damage and recovery of the testes was studied. The damage and recovery pattern can be explained on the basis of a selective effect of radiation on a single stage of spermatogenesis, the spermatogonia. The course of damage and recovery following exposure to a second dose of radiation is the same as that of damage and recovery following an nitial exposure. The pattern of loss and recovery of weights of the testes of mice exposed to 10-Mev $\mathrm{x}$ rays is the same as that following exposure to $186-\mathrm{kV}$ rays. (NSA) 424

Eschenbrenner, Allen B., Fliza Miller, and Egon Lorenz. QUANTITATIV HISTOLOGIC ANALYSIS OF THE FFTECT OF CHEONC WHOLE-BODY IRRADIATION WITH GAMMA RAXS ON TIE SPERMATOGENC ELPMENTS AND THE INTERSTITIAL TISSUE OF THE TESTES OF MICE.

J. Nat1. Cancer Inst. 2, 133-47(1948).

LAF-1 male mice were exposed to $8.8 \mathrm{r}, 4.4 \mathrm{r}$, on $1.1 \mathrm{~g}$, respectively, whole-body,- radiation given in $8 \mathrm{hr}$ dally begnning at 2 months of age. Four mice on each dose level and 4 nonirradiated mice of the same age were killed at intervals of 2 months after 2 to 16 months of ixradiation. Ony the findungs an the testes of these mice are reported. In mice exposed to $4.4 \mathrm{r}$ and $8.8 \mathrm{r}$ daily there was a sharp decrease of testes weights after 2 and 4 months of irradiation. Thereafter the weight curves leveled off at different values for the inferent dose rates. The relative amount of interstitial lissue increased in proportion to decrease of testes weights. Quantitative histologic analysis showed this relative incerase was not an absolute one, the quantity of anterstitial tissue per test is remaining the same as that of the testes of nomirradiated mice. Analysis of the mor- 
phology of the kineys revealed no evidence of measurable decrease of homone outpul of the testes of irradiated mice. Despite marked docrease of total quantity of spenmatomenic elements in the testes of irradiated mice, the proportion of these cells 14 different stages of spermaturents was normal in mee exposed to $1.1 \mathrm{r}$ and $4.4 \mathrm{r}$ daily for as long as 16 months. In mice exposed to $4.8 \mathrm{r}$ daly, di was nor mal for four months, whle after sizur more months of istadiation, there was failure of completion of spermatogenesis, and this was associated with degeneration of Sertoli cells. Some thooretical implications of the dat are discussed, and the practical value of the mouse testis as a test object in quantitative esperiments on the biologic a 425 fect of radiation is indicaled. (NSA)

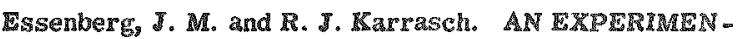
TAL STUDY OF TWE EFFCTS ON ROFNTCIEN RAYS ON THE CONADS OF TIL SEXUALLY MATURE DOMRSTIC FOWL. Radiology 34, 358-65(1940).

A total of 71 animals was used, 10 of which served as controls. Initial injury to the ovarian follicle was protuced by $400 \mathrm{r}$, follicular disintegration by $800 \mathrm{r}$ and total destruction of the follicles by 1800 to $2200 x$. Initial injury to the germinal epithelium occurred with $400 \mathrm{r}$, tolal destruction with $1200 \mathrm{r}$. Acclimatization to the affects of cumulative doses of $\mathrm{X}$-rays was noted in both testes and ovaries of treated birds. The gonads of birds appear to be more resistant to the effects of X-rays than the gonads of mammals. (BA)

Fogg, Lloyd C. and Russell I". Coming. THE CHANGES TN CELI MORPHOLOGY AND HSTOCHEMSTRY OF TEL TESTIS FOLLOWING IRRADIATION AND THELR RELATION TO OTHER WNDUCED TESTICULAR CHANGES. I. QUANTITATIE RANDOM SAMPLING OF GRRMINAL CILLS AT INIERVALS FOLLOWING DLRECT IRRADLATION. Cancer Research 11, 23-\$(1951).

Testes of pure line C57 black mice $18-25 \mathrm{~g}$ were subjected to a single dose of $300 \mathrm{I}$. The effect produced on the germinal cells by $\mathrm{z}$-radiation produced at $100 \mathrm{kvp}$ having a half-value-layer of $2.6 \mathrm{~mm}$. Al, dosage of $187 \mathrm{~m} / \mathrm{min}$ in air at $20 \mathrm{~cm}$. target-\$kin-distance was investigated. Evaluation was based on quantitative random sampling of the germinal elements of the testis, made with respect to frequency of cell population types plotted againgt time after radiation. A small percentage of all germinal elements perasts after a dose of $300 \mathrm{r}$. The period of least number of spermatogonia is from 5 to 7 days post-radiation, that of least frequency of spermatocytes is around 14 days; of spermatids and sperm 28 days. It takes 21 day $10{ }^{\circ}$ svermatogonia to develop into spermatozon. The slopes of the curves in terms of time and progressive loss of cach of the germinal elements are roughy the same. This vนggest that each of the germinal elements are af facted at the same degree but at different intervals. The period of greatent biological variation is from 10 to 28 days for ach of the 4 germinal elements. A single dose of $300 \mathrm{r}$ does not cause the disappearance of Sertoli cells or interstitial cells, nor is connective lissue markedly aflected. (auth)

Togg, Lloyd C. and Russell Towing. THE CHANGLS IN CELL MORPROLOGY AND RTSTOCHEMISTRY OF THE TESTIS TOLLOWING IRRADIATION AND TUEIR RELATION TO OTHER INDUCED TESTICULAR CHANGES; COMPARISON OF EFECTS OF DOSES OF 1,40 AND 5,050 x WHT $300 x$. Cancer mesearch 11, 81-6(1951).

Evidence is presented to show that a dose of $1440 \mathrm{r}$ does not destroy all the germinal elements at any time; there is a sequence for sadiation geaction from gonium to sperm, and there is an eventual reduction of production. In conparison to $300 x$, it is indicated that a single dose of 1440 further reduces the frequency of the appearance of the germinal cells and delays the time of recovery for spermatogonia and spermatocytes. There is also less evidence of numerical variation in the spermatogonia cund spermatocytea and less marked peaks and depressions in the figures. An acute dose of $5050 \mathrm{r}$ destroys all the germinal elements, but all these elements do not disappear at the same time. The gonia disappear first, followed by the spermatocytes, spermalds, and sperm. Once each of the types of germinal elements starts to disappeax, the rate is apprommately the same, regardiess of the dose. The degree of injury is Felative to the magnitude of the dose. No marked pathological changes were noted in cells or tissus other than in the rug germinal Cella. (NSA)

Frederic, J. HISTOLOGICAL AND MISTOCHEMICAL STUDTES OF THE SKTN OF GUTEA PICS TREATED WTTE X-IAYS. Compt. rend. soc. biol. 142, 850-3(1948).

Eleven guinea pigs peceived radiation of 3,000 s, filtered through 1 mm $A 1$, and 10 others received $1,500 \mathrm{r}$ and 1,000 coses. Ipidermal morphology was followed in all animals for 30 days for appearance of lesions, various cytological abnormalities, and restitution. The presence of sulfhydri. groups in the regenerating reas indjcates, the author concludes, that those play a role in the restitution of cells which have been damaged by zadiation. (NSA)

429

Frederic, J. STUDY ON REGENERATION AND ON -SY GROURS IN X-RAXED GUTNEA PIG SKIN, INCLUDWN CHANGES SEN IN THSSUES OUTSTOE TME FIELD OF DIRECI RADIATION, 39 OF INTERNATONAI CONGRESS OF RAIOLOGY, 6h Congress, London. 1950.

A histological study of the guinea pig skin after X-radiation has been made, including a study of - SH groups in ixradiated epithelium and in uirradiated epidermis of the same animal. Doses of $3,000,2,000$ and 1,500 r. were given to the skin of the hind legs, the rest of the animal being protected by thick lead sheets. After a dose of 3,000 r. the apidermis is destroyed but regenerates very actively, becoming much thicker than the oritinal and presenting a very anormal appearance. After 2,000 $\mathrm{d}$. the epidermis is not completely destroyed and regeneration begins carlier but proceeds more slowly. After 1,500 1. the regenerated epithelium is thicker than before but structurally normal. X-ray do not appear to have a direct influence on keratinisation. During the period when the destructive action of radiation on the epinhelium is most marked, -51 groups of the gtzatum granulosum do not appear to be modified as long as this layer is digtinguishable; in the stratum germinativum, on the other hand, -\$H groups fade out progresgively; it seems that the metabolism of the cells in this layer in affected by $\mathrm{X}$ - Iays. There is also a diminution of -SH groups in this skin layer in unirradiuted and protected epithelium of the same animal, indicating a distant effect in addition to the local lesion. In this respect, the epidermis appears to react "as a whole". During the period of regeneration the concentration of $-\$ \mathrm{H}$ groups increases progressively to a hifher level than normal. In unir radiated epithelum, -5H groups increase more slowly and never exceed the normal.

430

Freed, John H., Edmond J. Farris, Douglas P. Murphy, and $\mathrm{Egene}$ Pendergrass. EFF ICT OF LOW DOSAGE ROENTGEN RADIATON ON THE GONADOTROPIC FUNC. TION OF THE YYPOPHYSIS OF THE MATURE AND IM- 
MATURE FEMALE ALBINO RAT. J. Clin. Endocrinol. 8 , 461-81(1948).

Five serally mature female Wistar albino rats given single doses varying from 100 to $200 \mathrm{r}$ on the third day of their oestrus eycles did not appear to be affected. A second group of 57 rats given single or multiple doses varying from 5 to $300 \mathrm{r}$ on the second day of their oestrus cycles showed no change in running activity or periodicity of the oestrus cycle. Gross anatomical studies and weight determination made of the pitutaries, ovaries, and uteri of siz of the two-day treated rats and seven controls showed the pitutitary weights of the treated rats to be significantly greater, the utexine weights only slightly greater, and the ovarian weights similar. Sixteen immature rats given single or five successive daily treatments of 5 to $100 \mathrm{~g}$ per treatment matured sexually within the normal age limits. In gross appearance and weight their pituitary, ovaran, and uterine organs differed in no way from their control. It is concluded that low dosage radiation produces only a transient effect on the normal pituitary function and 431 has no evident harmful effect. (NSA)

Geist, S. H., J. A. Gaines, and G. C. Esehor. VAGINAL ESTRUS IN IRRADIATED MICE. RELATIONSHIP OF VAGINAL ESTRUS TO OVARIAN CHANGES AND PRODUCTION OF' BIOLOGICALLY ACTIVE OVARIAN TUMORS ATTER ROENTGEN IRRADIATION. Endocrinology 29, 59-63(1941).

The effects of roentgen irradiation upon the estrous cycle of female mice were studied in 23 animals by daily smears over a period of $14 \frac{1}{2}$ months. The changes noted within the vagina reflect those within ovaries and are described in correlation with them. The destructive effects of X-ray upon the ovarian follicular system of female in mice in the first $4-8$ weeks after irradiation are accompanied by a temporary abolition of estrus during that time. The luten changes within the redidual ovarian parenchyma which then develop result in the elaboration of sufficient est rogentc hormone to reestablish vaginal estrus in $100 \%$ of the animals. The estrous cycle is found to be totally irregular, with maxked variations in the duration of continuous estrous period ( 1 to 222 days). Individual aifferences are apparently related to the degree and duration of functional activity whthin the luteinized ovarian parenchyma. From six months to one year after ir radiation, surface epithelial proliferation and downgrowth replace a good portion of the ovarian cortex, with diminution of luteinized tissue and increased evidence of degenerative change. During this time, the extent of vaginal stimulation is found to be decreased and the number of mice showing acyclical estrus is reduced from 100\% to $37 \%$. After one year following irradiation, a prolonged absence of est rus may be correlated with senescent changes within the ovary. Those mice, however, which develop biologically active, luteinized granulosa or theca cell tumors once more exhibit increased estrogenic secrecion ezpressed in irregular prolonged estrous periods. (BA)

432

Cersch Manfred and Wilfried Mobius. BOLOGICAL EFTECTS OF SHORT WAVE RADIATIONS. INVESTIGATIONS ON INVERTERBATES. Surahlentherapie $85,565-$ 80(1951).

Radiation experiments were carried out on Enchytraeae (oligochaete worms) and the gnat larva Corethra. The oligochaetes were very resistant and showed no radiation injuries after administration of $\$ 0,000$ r three times in succession. Corethra showed injuries to intestinal cells after being submitted to radiations of medium strength; higher doses resulted, in addition, in degeneration of pericardial cells (20 to 30 days after radiation) and of some thoracic cell complexes. Irradiation with $25,000 \mathrm{r}$ caused great mortality and physiological injury to muscles, tracheal pigment cells, and the hypodermis. The cytological effects of radiation on the cells are described. It seems possible to use the pericardial cells as test cells whereby the action of short-wave radiations on the whole organism can be judged. (NSA) 433

Halberstaedter, L. and M. Ickowicz. THE FECTS OF X-RAYS ON THE OVARIES OF THE RAT. Radiology $48,369-73$ (1947).

The authors have set out to determine whether changes can be demonstrated in the ovary of the rat as early as fou hour's after ixradiation by X-rays. Doses varying from 50 to 2,000 roentgen units were used, on some rats to the abdomen only, on others to the head and thorax only, while others were totally ixradiated. There were four nonirradiated controls. Serial sections of the ovaries were examined, the animal in each case having been killed four hours after irradiation. In the follicles of the non-irradiated ovaries some pyknosig of the granular layer cells can asually be seen, but this is confined to follicles in which the ovum is in a state of division with the nucleus showing mitosis. Those with resting ova show very few pyknotic cells. In the irradiated series no difference was noted in the rats receiving 50 to 200 roentgen units. After a dose of 400 units and more, however, numerous pylnotic cells were observed in all follicles, both among the follicular cells and those in the granular layer. This appears to happen irrespective of the state of the ova. These findings applied only to the animals receiving direct abdominal ir radiation or total inradiation. No abnormality was noted where the abdornen had been protected and the thorax only irradiated. Degenerative changes in the ovaries as early as four hours after irradiation, with doses exceeding 400 roentgen units, are thus demonstrated. Photomicrographs are given. (AWM)

434

Henshaw, P. S. FXPERIMENTAL ROENTGEN INJURY. 1. EF TECTS ON THE TUSSUES AND BLOOD OF C. $\mathrm{MLCE}$ PRODUCED WITH SINGLE SMALL WHOLE-BODY EX-

POSURES. J. Natl. Cancer Inst. 4, 477-84(1944).

Tissue and blood changes were followed in $C_{8} \mathbb{R}$ mice after the application of a single acute $50 \mathrm{r}$ dose of $\mathrm{x}$-rays to the whole body. A slight leukocytosis developed in 2 to 4 hours, followed within 6 to 12 hours by a mild or persisting leucopenia which was found to consist mainly or entirely of lymphopenia. A small amount of nuclear debris was seen in the lymph nodes at 204 hours, which disagpeared and a mild hyperplasia developed in 8 to 12 hours. Seminiferous tubules of the testis showed a reduction in spermatogonia and primary spermatocytes at one week and of secondary spermatocytes at two. The tubules appeared normal again in 4 to 6 weeks. (BA)

435

Menshaw, P. S. FEPERIMENTAL ROENTGEN INJURY, II. CRANGES PRODUCED WHT INTERMEDATIS-RANGE DOSES AND A COMPARISON OF THE RELATIVE SUSCEPTIDILITY OF DIFIERENT IINDS OF ANIMALS. J. Natl. Cancer Inst. 485-501(1944).

Doses of $50,100,200$, and 400 r were applied to the whole bodies of $\mathrm{C}_{\mathrm{g}} \mathrm{II}$ mice, LAF mice, and to guinea pigs. The hematologic and histopathologic changes following exposure were studied; the histologic studies pertained to the lymphoid organs, bone marrow, and testis for the most part. In a general way tissue responses consisted of a parenchymatous-cell loss, followed by a slow return to normal if the damage was not too severe. Plegeneration started from 
primitive cell forms that had survived irxadiation fatal to other cells. Leukocyte reserves appeared to prevent the peripheral blood picture from reflecting the condition of the hematopoietic tigsue when the damage is slight. The problems of the organism after moderate doses of radiation appear to depend largely upon injury to the stem cells 436 of regenerative tissues. (BA)

Henshaw, P. S. EXPERIMENTAL ROENTGENOLOGICAL INJURY. III. TISSUE AND CELLULAR CHANGES BROUGHT ABOUT WITH SINGLE MASSIVI DOSES OF

RADIATION. J. Nati. Cancer Inst. 4, 503-12(1944).

Application of $50,000 \mathrm{r}$ doses of $\mathrm{X}-\mathrm{rays}$ to the whole bodies of guinea pigs, rabbils, and mice was followed by death in a few hours. Guinea pigs were dead at the end of treatment (three hours), rabbits within three to six hours afier treatment, and mice within $24-48$ hours. Death appeared to result from distinctive type of shock, symptoms of which were generalized toxemia, hyperthermia, hyperesthesia with intermittent geizures, and cyanosis. Hemoconcentration was not seen in the acute cases. Widespread tissue destruction occurred during these short intervals. Tissue sections showed extensive nuclear and cytoplasmic degeneration followed by extensive parenchymatous-cell 10\%5. (BA)

437

Henshaw, S. EXPERIMENTAL ROENTGEN INJURY: IV. EIF FECTS OF REPEATED SMALL DOSES OF X-RAYS ON BLOOD PICTURE, TISSUE MORPHOLOGY AND LIE SPAN OF MICE. I. Natl. Cancer Inst. 4, 513-22(1944).

Small daily doses of $5,10,15,20$, and $25 \mathrm{r} /$ day were applied to the whole bodies of $\mathrm{C}$ mice. There was a fall in leukocyte level, owing manny to lymphocyte loss. The amount of fall varied progressively as treatments proceeded and directly with the size of the daily dose. Fxtensive parenchymatous tissue damage was seen in lymph nodes, spleen, bone maryow, and testis; the degree of which varied with sige of daily dose. The life span was shortened, the amount varying directly with daily dose. Leukemia devel438 oped in some of the mice receiving 15 and 20 daily. (BA)

Penshav, P. S. and R. S. Snider. HISTOPATHOLOGICAL EHIECT. CORRELATION OF TISSUE RESPONSES FOLLOWING EXPOSURE TO PENETRATING RADIATIONS. Clinton Labs. July 1946. Decl. Dec. 16, 1946. 19p. (MDDC-569; Mon 14 -144)

A review has been made of the available information concerning tumor formation and the accompanying changes in mammalian forms that take place following exposures to penetrating radiations. The tissues in the order of increasing resistance to radiation may be listed as follows: lymph nodes (small lymphocytes), germinal epithelium of the testis, erythrocytes and myeloblasts, gast rointestinal epithelium, ovary, skin, connective tissue, bone, liver, pancreas, kidney, nerve, brain, and muscle. It was pointed out that the most radiosensitive tissues are of the generative type - $\hat{1}_{\text {.e. }}$, those which in life are continually proliferating new cells which undergo maturation, serve a useful function for a limited period and then die. The skin, testis, hemopoietic organs, gastrointestinal epithelium and perhaps other tissue series show a parallel type of differentiation with these features in common. In all cases, certain parent cells (but not the most primitive tymes), were found to be the most gusceptible to radiation. It is plain that partial damage to a particular tiswue series results in a limited period during which mature cells are being formed, whereas complete damage resulta in sterilization. Stress was laid on the fact that recovery to an apparently normal con- dition takes place even when the acute histologe destruction has been almost complete. (ADD) 439

Henshaw, P. S. and R. S. Snider. THE GENERAL PIC TURE OF IRRADIATION DAMAGE TO TISSUES, PART I. SUPERTICIAL RADIATION (BETA RAYS); PART II. PENETRATING RADITIONS (NEUTRONS, GAMMA RAYS). Clinton Labs. July 8, 1947. Decl. July 18, 1950. 29p. (AECD-3009; MonH-288)

Bart T. Radiation absorbed in the skin causes damage primarily in the germinal layer. When the damage to this layer is partial, the effect is a skin failure and repair takes place from the floor of the damaged area from living germinal cells that remain. When damage to the germinal layer is complete the effect is skin sterilization and healing must be from the edge.

Part 1. The most radiosensitive tissues are of the generative type, that is, those which are continually proliferating replacement cells. In all cases (with the possible exception of lymphocytes), certain parent cells (but not necessarily the most primitive types), were found to be the most susceptible to radiation. Partial damagre to a particular tissue series results in a limited period during which no mature cells are being formed. Recovery to an apparently normal condition talses place even when the acute histological destruction has been almost complete. Subliminal damage in the case of daily exposure may hold an animal nearer to a state of exhaustion so that he is less able to cope with a crisis. When penetrating radiations are applied generally to the whole body, leukemia is the predominant tumor type obtained. (NSA) 440

Jadassohn, W., E. Bujard, R. Paillard, P. Wenger, and P. Gaudin. THE ERFCT OF ROENTGEN, GAMMA AND BETA RAYS ON THE EPIDERMAL CELL. EXPERIMENTS ON THE 'NIPPLE TEST' ASSOCIATED WITH TME DUSTIN REACTION. Acta Radiol. 34, 469-87(1250).

The combined action of radiation therapy and treatment by estrogenic hormones on the nipple of the male guinea pig has been studied and controlled by Dustin's colchicinic reaction. Roentgen, $\gamma$, and $b$ rays administered in sufficiently large doses inhibit mitosis of the epidermal cells of the nipple. This action is also maintained if estrogenic hormones are administered after irradiation. This effect of irradiation is more evident when the irradiation is combined with the application of an estrogen, as the control nipple presents more mitoses. A dose of $200 x$ has no appreciable effect; on the other hand, $400 x$ or still larger doses are effective. A single dose $2400 \mathrm{x}$ given under varying physical conditions and consequently for varying lengths of time (from 4 min 26 sec to 153 min 27 sec) is effective regardless of the type of rays used. The effect of $2400 \mathrm{r}$ decreases if this total dose is applied in fractional doses of $200 \mathrm{r}$ per day over 12 days. Irradiation has the same effect on the nipple showing acanthosis produced by the administration of estrogenic hornones prior to irradiation. Estrogenic hormones cause a thickening of the pidermis of the nipple, i.e., acanthosis, through mitosis and a mild degree of simple hypertrophy of the cells. A few mitotic figures are also seen if estrogenic hormones are administered after irradiation. It is evident that uradiation therapy does not totally destroy the capacity of the epidermal cells to divide. However, the number of mitotic figures is too small to explain the thickening of the epidermis after irradiation. The action of estrogenic hormones administered after irradiation produces a simple hypertrophy involving similarly the nuclei and the cytoplasm, and a certain degree of imbibition of the epidermis, 
the combination of which we call pseudoacanthosis. (NSA)

441

Jolles, B. STUDY OF CONNECTIVE-TISSUE FEACTION OF RADLATION. THE SIEVE OR CHESS METHOD. Brit. J. Cuncer 3, 27-31(1949).

The author describes a new technic for the study of connective-lissue reaction to radiation, consisting in the ir radiation of accessible tumors (breast, skin) through a lead sieve with circular or square apertures of diameters 1.0 to $2.0 \mathrm{~cm}$. On the basis of 9 cases studied thus far, the following preliminary conelusions are reached: The "sieve-chess" technic is a workable one for the study of direct and indirect effects of irradiation. The connectivetissue reaction takes place not only in the irradiated areas, but also in the neighborhood protected from the direct irradiation. There are visible effects on rells not directly exposed to the radiation. "The pattern of connect Ive-tissue destruction and regeneration can be studied. Ass a general inference, it can be sad that the irradiation affects the connective utissue cells and induces a kind of "overdifferentiation." This consists chiefly in the deposition of an excessive amount of collagenous fibrils, which are organized into solid, coarse bundes in the exposed parts where the fine connective-tissue network as grossly altered. Lymphatic channels and blood capilaries are greatly affected, compressed, and blocked. This has a considerable effect on tumor nourishment, the lack of which enhances tumor lissue degeneration. (R)

442

Knowlton, Norman P., $\mathbb{X}$., Louis H. Hempelmann, and Joseph C. Hof man. THE EPECTS OF X-RAYS ON THE MTOTIC ACTVITY OF MOUSE EPIDERMIS. Science $107,625(1948)$.

The mitotic index of the skin of the mouse has been studied as a means of measuring the biological effect of X-rays at doses of 35 and $325 \mathrm{r}$. As little as $35 \mathrm{ro}$ of $250 \mathrm{kv}$ X-mays produce a drop in the mitotic index to zero within one to two hours after radiation. The number of mitoses returned to normal within a fow hours after radiation. The time required for the number of mitoses to return to normal increases greatly with increased radiation dosage. It is believed that the time required for the mitolic index to return to normal will provide an excellent basis for rating the biological effectiveness of radiation of different types and different energies. With $325 \mathrm{r}$ of $\mathrm{X}$-rays the mitotic index required six days to return lo normal and continued to increase to more than twice normal. This is bolieved to be an over compensation phenomena which may also be used as an index of radiation damage. (NSA)

Krebs, A. T. and I. I. Gierlach. VITAL STAINING WITH THE FLUOROCHROME ACRIDINE ORANGE AND IFS APPLICATION TO RADIOBIOLOGY I. ALPHA-RAY EF -

FECTS. Am. J. Roentgenol. Radium Therapy 65, 93(1951).

Using the acridine orange vital staining technique, the results of earlier preliminary investigations have been confirmed and extended. The findings are (1) cells and cell groups inactivated before staining and also irradiated stained cells demonstrate the radiation effect by a color change in the emilted fluorescent light as they became non-viable. When normal, the cells lave a green fluorescence. When completely destroyed, the cells have a light red fluorescence; (2) intermediate stages or degrees of radiation damage are characterized by shades of yellow or orange fluorescence; (3) These changes occurring in cells under alpha radiation have been fixcd on color film; (4) the amount of irradiation necessary to kill cells can be readily quantitated. By this means it is possible to study some of the details of radiation damage. The results reported together with the photomicrographs amply justify the use of the acridine orange vital staining method in radiobiology. (auth)

444

Lamson, Baldwin G. and John L. Tullis. THE PROGRESSION OF MORPHOLOGIC LESIONS IN SWISS MICE EXPOSED TO $625 \mathrm{x}$, 2000 RVP, TOTAL BODY X-RADIATrON. Naval Medical Research Inst. Bethesda. July 31, 1951. 27p. (NP-3667; $\mathbb{R - 6 0 8 9 ;}$ NM-006 -012.04 .37 )

One thousand Swiss male mice were exposed to $625 \mathrm{r}$, $2000 \mathrm{kw}$ total body $\mathrm{x}$-radiation. Nine hundred ninety of these mice were sacrificed serially, ten mice each hour for the succeeding 99 hours. Microscopic studies of the spleen, lymphoid tissue, bone marrow, intestines, liver and testes were made to observe the tissue response to this injury. Damage to lymphocytes, immature erythropoietic tissue and selected epithelial cells of the small bowel mucosa was observed one hour after exposure. Reduction of cellularity of myeloid tissue oceurred more gradually. Damage to lymphoid tissue reached a peak between 7 and 12 hours, and regeneration was generally evin dent by 60 hours. Frythropoletic tissue could not be found after 14 hours, yet regeneration was seen sporadically between 66 and 99 hours. Peals injury to small bowel mucosa extended over the 8 to 32 hour period, whereas repair was completed by 60 hours after radiation exposure. Complete repair of lymphoid, erythropoietic and myeloid tissue did not occur during the 99 hours observation period. Mitotic activity was reduced for a period following inradiation in all organs studied with the exception of the testes. Selective damage to spermatogonia of the testes was not. seen during the period of this study. (auth)

Lasnitzk, I. A QUANTITATVE ANALYSIS OF TYWE DIRECT AND INDIRECT ACTION OF X-RADIATION ON MALIGNANT CELLS. Brit. J. Radiol. 20, 240-\%(1947).

This research determined quantitatively the direct and indirect action of $x$ radiation in vitro and in vivo following a dose large enough to involve the circulatory gystem. Mouse adeno-carcinoma 63 was grown in the right thigh of 2 to 3-month-old hybrid mice, and the tumors were isradiated through a hole in a $P b$ shield about 12 days after inoculation, when they had reached the size of a small cherry. Following the exposure the irradiated tumors were excised for histological examination at various intervals fron $80 \mathrm{~min}$. to 10 days. Tissue cultures were prepared from small fragments taken from the viable twnor edge, grown by the hanging drop method, irradiated $24 \mathrm{hr}$ after explantation, and returned to the incubator for periods ranging from $80 \mathrm{~min}$. to 10 days before fixation. An 8 -ray dose of $2,000 \times$ was given in 20 mink, both to the tumors and to the lissue cultures, generated at $160 \mathrm{kw}$ with $12 \mathrm{~mm}$ Al filtration. The effect of the radiation was assessed by cell counts. Several fields near the skin surface of the tumors in the young growing parts of the periphery only, and in the zone of $g$ rowth of the lissue cultures, were selected, and all of the cells present were counted and classified as dividing, resting or degenerate cells. In each turnor and cullure $300-500$ cells were counted, and each observation represented the average of at least three tumors and four to six tissue cullures. The curative dose for carcinoma 63 was $2,500 \mathrm{r}_{3} ; 2,000 \mathrm{x}$ caused femporary retardation of growh. A slight dilatation of blood vewsels in the tumor and adjoining skin was seen almost immediately: the reaction was marked after 24 hours, increased for four days, and was still present 10 days after the irradiation. Mitosis was absent in both tumors and tissue cultures 80 min. after the exposure. Il reappeared in vivo after 3 hours, 
but not before two days in vitro, most of the cell divisions in both cases being abnormal - multipolar mitoses, clumping, fragmentation of chromosomes, and chromosome bridges. Increase in cell size occurred in both cases, but was much more marked and of longer persistence in the cultures. Degenerate cells appeared shortly after exposure in both cases, being qualitatively similar to each other and to irradiated normal tissue-hyperchromatosis and pylknosis of nuclei followed by disintegration of the cytoplasm and chromatolysis. Degeneration sometimes began in the resting nucleus. The in vivo and in vitro results were qualitatively the same, but in vivo a large group of degenerate cells appeared between 2 and 7 days after in adiation, which did not occur in vitro. Mitosis in vitro was much less affected than in vivo, suggesting that the degenerate cells seen in the tumor replaced the cells seen in mitosis in the cultures. The big group of degenerate cells in the tumor was coincident with the appearance of vascular changes. It is assumed that these cells broke down on reaching division because of the interference with their blood supply. On the other hand it is concluded that the changes seen on the first day were due to the direct action of the radiation on the cell and the early mitotic recovery in the tumor was due to the still intact circulation. (EM)

446

Loren, Egon and Thelma B. Dunn. OCULAR LESIONS WNUCED BY ACUTY EXPOSURE OF THE WHOLE BODY OF NE WBORN MLE TO ROENTGEN RADLATON. Arch. Ophthalmol. 43, 742-9(1950).

Twenty-four male and 32 female mice of strain A were given at birth a single whole-body dose of $400 \mathrm{x}$ delivered by a $186-\mathrm{kv}$ machine at $50 \mathrm{~cm}$ target distance. The ersperiment was terminated 12 months later. Seventeen males and 22 females survived; all showed lesions in one or both eyes. These lesions consisted of opacity of the lens, partial opacity and vascularization of the cornea, and atrophy of the retina, which was only a small fraction of its normal thickness. The difference in radiosensitivity of the eye of young 47 and that of old animals is discussed. (NSA)

Mason, I. E. and S. L. Shaver. THE DEGENERATIVE CHANGES IN THE TISTES OF THE ADULT RAT AFTER X-IRRADLATIONS, p.16-28 Of QUARTERL TECHNICAL REPORT, JULY 1, 1950 TIROUGH SEPTRMBRR 30,1950 . Atomic Energy Project, University of Rochester. Nov. 1, 1950. 13p. (UR-142(p.16-28))

The report deals mainly with (a) a study of the degenerative changes in the testes of the adult rat after 8 irgadiation of 500 r total body, (b) the influence of vitamins $\mathbb{A}, \mathbb{D}$, and $\mathbb{E}$ upon the sensitivity of rats and their genital organs to irradiation, (c) the morphological stages of degeneration in the festes of the ral after the administration of $1005 \mathrm{x}$ locally, (d) the study of the sindirect' factor and its relation to testicular degeneration, and (e) a survey of testicular damage in different strains of rats. It is concluded from the work performed upon this series of animals that with the dose of $500 \mathrm{r}$ administered total body there are no strain differences in the three strains of rats used and that there is yery little or no secondary effect upon the testes of these animals. Yet when animals were irradiated locally in this laboratory with the dosage of $500 \mathrm{x}$, no morphological degeneration was observed in routine histological gections. Therefore the gossibility exists that the degeneration of the testes in the rat after $500 \mathrm{r}$ total body may be due to a combined ef the direct effect of the irradiation, neither of which will produce an observable degeneration alone. (NSA) 48

Metcals, R. G. R. J. Blandau and T. B. Barnett. Trop PATHOLOGICAL CHANGES EXMHBTTED BY ANMALS IX-
POSED TO SINGLE DOSES OF X-RADIATION. Atomic Energy Project, University of Rochester. July 17, 1950. 44p. (UR-123)

Albino rats were exposed to a single dose of $550 \mathrm{~g}$ of whole-body $x$ radiation and were sacrificed at intervals from 15 min to 42 days after exposure. Sections of selected organs were examined microscopically, and studies were done on the peripheral blood and bone marrow and on sperm from the vas deferens. 33 figures. 28 references. (NSA)

448

Montag, C. ON THE EFIECT OF METHYLTHOURACH AND X-ILAYS ON THE THYROID GLAND. Strahlentherapie 81, 1-38(1950).

An investigation of the effect of $x-$ rays on the thyroid glands of rats was carried out with the use of methylihiouracil, a chemical substance which results in a pronounced histological change and makes it possible to influence the functioning of the thyroid gland in a measurable manner. Upon irradiation of the thyroid gland with approximately $1500 \mathrm{r}$, degenerative changes were found in the nervous terminal apparatus (preterminal plexus and nervous terminal reticulum) and in the nuclei of follicular cells. An effect on the protoplasm and the surface areas of the follicular cells is probable. The nuclear changes had previously been unobserved because they become apparent only during cell division. They are made visible by accelerating the mitosis by the administration of methylthiouracil. (NSA) 450

Murray, Raymond, Mila Pierce, and Leon O. Jacobson. THE HISTOLOGICAL EFIECTS OF X-RAYS ON CHICKENS WITI SPECLAL REFERENCE TO THE PERIPHERAL BLOOD AND EEMOPOIETIC ORGANS. Univ. Of Chicago Metallurgical Lab. Mar. 2, 1948. 81p. (AECD-2303; CH-3873)

Chickens, 3 to 11 weeks old, were treated with doses of $200 \mathrm{kv} x$-rays, ranging from $2 \mathrm{r}$ to $1,200 \mathrm{r}$, The median lethal-dose for 3 -week-old chickens appeared to lie between $400 \mathrm{r}$ and $800 \mathrm{r}$. Histopathologrc changes in the 11-week chickens paralleled those reported for mammals of comparable maturity, with the exception of damage to neurilemma sheath cells in the chickens. The 3 -week-old chickens suffered additional damage to immature structures, but the threshold of observable damage to the lymphatic tissue $(25 \mathrm{r})$ was comparable to that reported for mammals. By application of a slimht modification of Syes $^{8}$ method for peripheral blood counting of fowl, dramatic decrease in blood constituents, paralleling those reported elsewhere for mammals, were seen in 3 -week-old and 5 week-old chickens after treatment with $600 \mathrm{r}$ and $800 \mathrm{r}$, respectively. Numerous degenerating cells were observed in dry smears made during the first week after irradiation, in contrast to the scarcity of such findings in smears of mammalian blood at the same period. Although the normal values for leukocytes of chicken blood were found to be several times those of mammalian blood, this difference could account only in part for the greater degeneration observed in the chickens. (NSA) 451

Nettleship, A. TISSUE CHANGES PRODUCED IN C C $_{3}$ MCE BY 50 r WHOLE BODY EXPOSURE. Radiology 42, $64-70(1944)$.

Young adult male mice of an inbred $\mathrm{C}_{3} \mathrm{H}$ colony were given whole body exposures of 50 (measured in air) each of filtered $200 \mathrm{kv}$ X-rays. Blood counts were made and tissues fixed at $1,2,4,8,12$, and 24 hours, $2,4,7,10$, and 14 days, three animals being examined for each count and each tissue fization. Curves showing white blood counts indicate an abrupt rise followed by a sharp fall in both lymphocytes and neutrophils to below normal in a few hours 
and reaching a minimum on the fourth or fifth day. Lymphocytes failed to recover before the fourteenth day, but neutrophils quickly returned to normal or even slightly above normal. Earliest discernible tissue changes were eosinophilia, cloudy swelling of cytoplasm, and poor staining of nucle1, observed in all tissues within one hour, and disappearing at various limes up to 24 hours. In lymph nodes and spleen, multiple flecks of necrosis appeared in germunal follicles within four hours and persisted until 8 to 12 hours. Multi-nucleated giant-cells were seen in from four hours to three days. No clear milotic wave was found. Changes in bone marrow were less pronounced and occurred later. At 12 hours there were abundant megakaryocytes and an increase in myeloblasts. Increased alkalinity lasted two to four days. In the ileum pronounced oedema appeared within two to four hours with return to normal at eight to twelve hours. The testes showed general swelling, with ragged and pyknotic germinal cells in some tubules. However, even at the height of the reaction the majority of the tubules showed normal spermatogenesis. By the end of three weeks the tubules had returned to normal. No signficant changes were seen in any other tissues. None of the changes described could be found in an adequate control material. (EM)

452

Pape, R. PISTOLOGICAL FINDINGS AFTER VEIR Y SMALL DOSES OF IRIADIATION, 16.162-3 Of INTERNA TIONAL CONGRESS OF RADIOLOGY, bth COngress, London. 1950.

After exposure of lymphoid tissue of the rat spleen to single doses of a few roentgens (1-20) given as total body irradiation, loosely arranged cells are seen with a bubbly structure in the nuclear protoplasm. After repeated doses the "Seemann syndrome of the stimulated spleen" is frequently observed, often others associated with a lymphocytosis. After daily doses of 0.25 rextending over one year, there is an increase of lymphocytes up to three times the normal value. The best histological results are obtained when the initial irradiation consists of very small doses followed some days later by doses of the order of $300-600 \mathrm{r}$. Under these circumstances the follicles of the spleen are better preserved and richer in cellular elements including mitotic figures. Similarly, after an inilial irradiation, the spermatogonia of the testis show mitotic activity following a further exposure of $600 \mathrm{r}$.

Inflammatory processes in the granulation tissue of healing wounds, recovery processes from artificial pneumonia caused by staphylococei and from intraperiloneal infections with tubercle bacilli, all show, after small preliminary doses of radiation, a reduction in the amount of exudate which is characteristic of the response to the usual $X$-ray doses. There is also an increase in the perivascular cells, an acceleration of fibroblast formation and of the process of healing and a localisation of the lesion. In tubercle infection there is a diminished tendency to caseation. To sum up, small doses of irradiation have a protertive effect upon cells, increasing their resistance to radiation and enabling mitotic activity to be maintained. 453

Pape, R. NEW ORSERVATIONS AND EXPERIMENTAL FINDINGS ON THE EFTET OF VERY SMALL ROENTGEN DOSES Radiol. Austriaca 4, 35-51(1951).

Following single or repeated ixradiations with small doses (1-20 no) changes are seen in the nucleus a few hours later. The nucleus is larger and may have a foamy appearance. Epithelioid cells are seen surrounding the malphihian follicles in the spleen. In rats irradiated with $312 \times 0.25$ r the number of leucocytes was increased 3 times. By combined ixradiation the spermatogenesis in rats did not decrease but rather increased. The period between the irradiation with small doses and a large dose can be extended 4 weeks. The smaller dose can be either $1 \mathrm{r}$ or $5 \times 20 \mathrm{r}$. Increased growth and decreased exudation is seen in granular tissue, but with doses over $150 \mathrm{r}$ the exact reverse is seen. In th in guinea pigs proliferative alteration is seen but not exudative alterations. The effect of the irradiation with small doses is not only activation of the reticulo-endothelial system, but also decrease of the exudation and increase of the cell resistance. One hour after the irradiation the number of leucocytes decreases slightly ('hour reaction'). The decrease does not depend on volume dose, but on the area of treatment and its gensitivity. The effect is not specific, but depends on neurohumoral reactions. In experiments with growing plants even doses short of 14 accomplished effects on growth and protection. By variation of dosage 3 different levels were established: (1) cell destruction with $20 x$ and above; (2) neurohumoral reflex reactions down to $3 r$; (3) activation of cell partition and resistance with $1 \mathrm{~g}$ and below. (FM) 454

Pape, R. and N. Jellinke. IMPORTANT VARIATIONS IN TEL ORGANIC FINDNGS AITISR TOTAL AND LOCAL IRRADIATON AND THE PARTICULAR EFIECTS OF SMALL SCALR DOSAGES. THE SPLEEN AS TEST FOR VARIOUS RADIATION ERTETS. Radiol. Austriaca 3 , 43-62(1950).

The response of the spleen (rat) to X-rays was investigated under various conditions. After local application of $100 \times$ (or more) definite signs of destruction were observed, but after $30 \mathrm{r}$ (or less) no destructive changes were observed. However, 3 hours after the application of $2-5 \mathrm{r}$ lymphoblasts showed some departure from normal nuclear structure. With larger doses, the histological appearance of the syleen showed distinct differences after total and after local irradiation. After local exposure recovery occurs rapidly from the influs of mature lymphocytes from undarnaged depots through blood vessels, whereas after total irradiation, true regeneration is extended over several weeks or even months. Most interesting results were obtained with preliminary irradiation of 3 to $20 \mathrm{x}$ daily and subsequent exposure to $300 \mathrm{r}$. Aiter a single exposure to $300 \mathrm{r}$, destruction was always found, but it failed to appear in all cases when preliminary small doses had been given. Apparently the preliminary small dose exercises some sort of protection against further radiation damage. This is aseribed to acceleration of cell regeneration. The fundamentals of G. Rickers' theories are referred to in relation to the results revorted. (EM)

\section{5}

Pohle, E. A. G. Ritchle, and W.W.Moir. STUDIES OF THE FFECTS OF ROENTGEN RAYS ON HEALING OF WOUNDS. II. HISTOLOGICAL CHANGTS IN STIN WOUNDS IN RATS FOLLOWINC POSTOPRRATIVE IRRAOLATON WITI VERY SMALL AND MODERATE DOSES. Radiology $52,707-13(1849)$.

The effect of roentgen rays in doses of $10,25,150,300$, 350 , and $700 \mathrm{x}$ on the healing of skin incisions was studied macroscopically in 18 and macroscopically and micromcopically in 122 white rats. The radiation doses were administered by a 100-kv machine, through a $2 \mathrm{~mm} \mathbb{A}$ filter, at a target distance of $30 \mathrm{~cm}$ and at the rate of $45 \mathrm{~g} / \mathrm{min}$. Although all wounds were examed daily, no consistent difference in the healing time between the irradiated and the non-irradiated portions of the wound could be detected macroscopically. Histologically, no true gtimulative effect on the healing - even with the lowest doses used-could be found. Any slight difference in the healing process in favor of the irradiated portion (gmall 
doses only) was considered to be due to a reduction in the amount of exudate, which in turn decreased the amoun of fibrin replaced by the acar tissue. (NSA) 456

Raynaud, Albert and Marcel Frilley. THE HISTOLOGICAL STRUCTURE OF THE TESTTCLES OF FETAL MICE ARTER DISTRUCTION OF THE HY POPHYSTS BY ZRADIATONS. Compt. rend. soc. biol. 143, 954-8(1949).

A total of Ive fetuses, with their hypophyses destroyed by means of elevated doses of $\mathrm{X}$-rays (up to $200,000 \mathrm{r}$ ), were examined to determine the effects on the histological structure of the testicles, The x-radiations were delivered in one dose of $100,000 \mathrm{I}$ at the age of 12 days and an additional 100,000 $\mathrm{r}$ dose at 13 days, localized in an area with $2.5 \mathrm{~mm}$ diameter; the feluses were sacrificed at the age of 18 days and examined histologically. It was found that the testicles were differentiated sexually; the rolative growth and dimensions of the testicular lubules were not preceptibly modifled, however. The interstitial gland of the testicle was relatively well developed. The studies indicate that if the destruction of the hypophyseal line occurs rapidly and completely the testicular growth and the development of interstitial gland may then be accomplished in a 457 nearly normal fashion. (NSA)

Raynaud, Albert and Marcel Frilley. THE HISTOLOGICAL STRUCTURE OF THE OVARIES OF FETAL MCE AFTER THE DESTRUCTION OF THE HYYPOPXYSIS BY X-IRAYS. Compt. rend. soc. biol. 143, 959-63(1949).

The hypophyseal line was destroyed in five female mice by irradiating the base of the cranium at the age of 12 or 13 days with a narrow localized beam of $\mathrm{X}-\mathrm{mays}$ for a total dose of 150,000-200,000 $\mathrm{s}$; the irradiated and control animals were sacrificed when 18 days old. It was found that after the hypophyseal destruction the general growth of the ovaries was not affected noticeably; the germinal cells were entering the miotic prophase. It is concluded that if the destruction of the hypophysis in each animal can be assumed to be done completely and guichly upon irradiation, the ovarian growth must be independent of fetal hypophyseal secretions. (NSA)

Schrek, Robert. STUDIES IN VITRO ON CELLULAR PKYSIOLOGX, THE EFFECT OF X-RAYS ON THE SURVIVAL OF CELLS. Radiology $46,395-410(1946)$.

Cellular suspensions were prepared from thymus, spleen, bone morrow and testis of rabbils and from leucocytes of normal and leukemic blood of men. The suspensions were ir radiated with doses of X-rays varying between 20 and $5000 \mathrm{r} / \mathrm{air}$ (Factors: $250 \mathrm{kv}, 10 \mathrm{ma}, 50 \mathrm{~cm}$. distance, $1 \mathrm{~mm}$ Al tulter, $120 \mathrm{r} / \mathrm{min}$ HVL $0.7 \mathrm{~mm}$. Cu). After exposure the suspensions were incubated at $37^{\circ} \mathrm{C}$ for $1-7$ days. The irradiated and non-irradiated control suspensions were examined at periodic intervals by the method of unstained cell counts (resistance to eosine) and by stained smears. Exposure of thymic and splenic suspensions to $1000 \mathrm{r}$ caused no perceptible change in the number of eosine resistant (i.e., viable) cells in the first 3 hours of incubation but produced a relatively rapid decrease in the unstained cell counts after a short latent period. Irradiated leucocytes from normal blood and from lymphatic leukemic blood had shorter survival geriods than non-irradiated cells. Irradiation and incubation of suspensions from bone marrow from myeologenous leukemic blood and from the teaticle had no effect on the number of eosine resistant of viable cells whon compared to non-1rradiated suspensions. (BA)
459

Schrek, Robert. CYTOLOGIC CHANGES IN TEYMIC GLANDS EXPOSED IN VIVO TO X-RAXS. Am. J. Pathol. $24,1055-64(1948)$.

Intranuclear, acidophilic vacuoles were observed in many cells of thymic tissue of the rat and rabbit, the hours after ir radiation with 1,000 r of $X-r a y s$. On carkfield examination of the suspension of the ir adiated thymic tissue, many of the cells had single or multiple primary vacuoles with or without vacuolar walls. Thymic lissue, five hou's after irradiation, had, on histologic samination, rany pyknotic and fragmented nuelex. Suspenstons of this tissue had many cells with secondary vacuoles and many fragments of cells. Nonirgaliated thymus also had a few colls with infmuclear vacuoles and pylnotic nuclei. The spontaneous and $\mathrm{X}$-ray induced degeneration of lymphocytes was assocked with the formation of intranuclear vacuoles, pyknosis, and fragmentation. Primary intranuclear vacuoles are seen in higher percentage of cells by dark-field 40 methods than by mouthe histulogic technique. (NSA)

Shaver Samuel L. and $\mathrm{Karl}$. Mason, SELCCTVE TESTL CULAR DAMAGE TN RATS DUE TO X-RAYS. UnVERIIY of Rochester. [nd] 1p. (ALCU-747)

The document is in abstract form; it is reptoduced below in its entrety.

A dosage of 500 n total-body 3 -ireadration, adminislesed in approximately $8 \mathrm{~min}$, has proved most sutabla for producing minimal but selective effects upon the testis of the rat.

In 52 rats grven this dose there was negligible cell damage in the germinal epitheliun when testis smears were studied supravitally and by fixation and applacation of histochemical methods for glycogen, lipids, and mitochondrin. However, in histological sections of lestes taken at frequent intervals during the first 2 weeks after uradiation, the occurs a progressive diminution in, and ultmately almost a complete loss of spermatogonia; yet spermatogenesis continues normally. During the following weeks, primary spermatocytes gradually diminish, and cells in advane phases of spe malogenesis predominate. Approxmately 10 days later there is evidence of early regengation in the basal layers.

The infrement occurence of netrosis or pycnosis in spermatogona and other germ cells, the abundance of matuse sperm usually uncontaminated with sloughed germ cells in the epididymis, and the fact that the time required for hese phenomena approxinates the duration of the spermatogenic cycle (about 26 days) suggest a specific but temporary inhinition of the somatc natotic divisions in the spexmatogonis. This is further supported by observations on prepubertal rats given the same dosage and on adull ats showing similas degree of testis injury after $1,005 x$ irradiation applied locally to the testis. (NSA) 461

Smith, Christianna and Lois 4 . Lowenthat. ATUDX OF

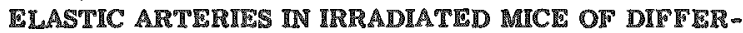

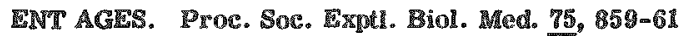
(1950).

Changes companable to those of physiological aring were induced by $x$ radation in the tunica media of elastic arleries of mice of different ares. In the elastic arteries of 20-to-30-day-and 80-10-100-day-old irradiated mice there were inc ${ }^{2}$ eased anounts of ground substance, increased numbers of inter lamellar fibers, and premature ragmelness and fraying of the elastic membranes. No changes attributable to ir adiation were seen in the arteries of the old mica. (NSA) 
462

Spargo, B., J. R. Bloonfield, D. J. Glotzer, E. Leiter Gordon, and O. Nichols. HISTOLOGICAL EFTETS OF LONG-CONTINUED WHOLE-BODY GAMMA-IRRADIATION OF MICE. J. Natl. Cancer Inst. 12, 615-56(1951).

Chronic effects of repeated low doses of external y radiation on mice sacrified at bimonthy intervals from 210 16 mo are reported.

463

Strelin, G. S. HISTOLOGICAL CHANGES PRODUCED IN THE EPITHELIUM OF THE FROG'S CORNEA BY TOTAL AND PARTLLL IRRADIATIONS WTTH X-RAYS. Doklady Akad. Nauk S.S.S.R. 73, 1283-6(1950).

The influence of irradiated portions of tissues on the neighboring nomrradiated ones, and vice versa, was illustrated by observing the depressing action of $x$ rays on the mitotic division in the cornea epithelium of fross; partial irradiations were obtained by usme a lead grid $0.5 \mathrm{~mm}$ thick as a filter. The doses used varied betwern 450 and $7200 \mathrm{r}(350 \mathrm{r} / \mathrm{min})$; the histological observations were made on materal fored at various intervals after the irradiation (up to 10 days). The results showed that partial irradrations with $3600 \mathrm{r}$ produced the same effert als total irradiations with $900 \mathrm{r}$, and that at lower doses the healing effect of protected strups on the uradiated ones was very apparent, as was also the reverse (depressive) action at higher doses. These influences should be ascribed to chemical meractions between cells rather than to cell migrations. The foregoing observations point 10 possible applacations of grid-shaned filters for the protection of the skin an depth therapy, as has been recommended by several authors. (NSA)

464

Tansley, K., L. H. Grays and F. C. Spear. A PRELIMINARY NOTE ON SOME BLOLOGICAL ERECTE OF ALPHA RADIATION ON THE FROG TADPOLE. Brit. J. Radiol. 21, 567-70(1948).

Mitotic and degenerate cell counts were made on the eye and brain of frog taupoles exposed to three diferent doses of - rays and the results compared with those already known for a small dose of - rays. Whereas with the lowest a-ray dose the relation between mitosis and degeneration does not differ from that which would be expeeted to follow a relatively small dose of radiation, after the wo larger $\alpha$-ray doses degeneration far exceeds this amount. It would seem that $\alpha$-rays cause the degeneration of potential dividing cells in considerable numbers. (NSA) 465

Tullis, John L. THE SEQUENCE OF PATROLOGIC CHANGES IN SWINE EXPOSED TO THE LD100/30 OF TOTAL BODY SUPER-VOLTAGE X-RADIATION. Naval Medical Research Inst. Bethesda. Aug. 24, 1951. 17p. (NP-3600; NM-006-012.04.38)

Eighteen 140-pound barrows five to seven months old were subjected to $600 \mathrm{r}$ total body $\mathrm{X}$-radiation administered $300 \mathrm{r}$ to each lateral aspect of the animal at two meters target-skin distance from the $2000 \mathrm{KVP}$ source. The swine were killed at intervals from 50 minutes 10164 hours (seven days) after irradiation and their timsues were studied grossly and histologically from morphologic X-ray-induced changes. Lymphoid cells in the several lymphoid organs of the body had undergone degenerative changes in the swine examined 50 minutes after irradiation. The degenerative processes increased for a while, then ceased. The debris was removed by phagorytosis after which the lymphoid organs appeared quiescent and depleted of lymphocytes. After 36 hours, regeneration and gradual repopulation took place. Immature erythroid elements in the marrow reacted early and progressed to almost complete depopulation with no evidence of regeneration in the seven-day observation period. Inmature myeloid elements lagged behind the erythroid elements, but with a sumilar end result. Mature blood cells and megalaryocytes were more radioresistant. Lesions in the small intestine were limited to early necrosis with rapid regeneration of the epithelium in the deep glands of the mucosa, depletion of the lymphocytes in the lamina propria and solitary nodules, and the congestion of blood vessels. Other lesions in the gast ro-intestinal tract included hemorrhages in two swine after 128 hours and shallow ulcerations in the rectal mucosa in the swine at 92 hours. In a few instances endothelium of the arterioles appeared swollen and distorted. Other tissues examined were not remarkable. (auth)

\section{Carcinogenesis}

466

Boyland, E. CHEMSTRY OF NEOPLASTIC TISSUE. Ann. Rev. Biochem. 18, 217-42(1949).

This article 1 s a review of recent work in the field based on a thorough compilation of the liferature. Included are the treatment of cancer by radioisotopes $\left(\mathrm{P}^{32}\right.$ and $\left.\mathrm{I}^{130}\right)$, the cancer producing properties of radioactive fission products (platonium and $\mathrm{y}^{92}$ ), the inhibition of skin cancer due to radiation by the use of chemicals, and the effect of cosmic radiations on carcinogenesis. 170 references. (NSA) 467

Brent, Robert L. and H. Charles Jordan. POSSIBLE LATE TUMOR DEVELOPMENT IN RAT EMBRYOS IRRADIATED WITH $100 \times$ OF X-RADIATION ON THE 9TH DAY OF GESTATION. University of Rochester. Jan. 12, 1951. 1p. (UR - 150)

The report is reproduced here in its entirety.

Rat embryos were isolated in utero and given $100 \mathrm{~F}$ of \& radiation. In one series sacrificed at intervals up to and including 15 days gestation, groups of tumor cells apparenty originating from neutral tissue were observed in the cephalic portion. These tumors vary in size, differentiation, and position; some are associated with brain tissue, other with the skin, and many are distributed in the intervening mesenchymal tissue.

A second series of embryos were subjected to the same procedure and sacrificed at intervals from 15 days gestation until term. Gross observations have revealed tissue masses of such size as to prevent closure of the midsagittal suture. The largest of these growths were observed at 15, 16, and 17 days, while growths observed at term are uniformly smaller. This apparent failure of embryos bearing the larger growths to survive may account for the sharply increased incidence of resorption seen at term as compared with 16 days. Histologic studies are in progress to determine whether these masses are identical with the above-mentioned types of tumor or are distortions of the brain wall. (NSA) 468

Brues, Austin M. CARCMNOGISTC ETPETTS OT RADATION, p.171-91 of ADVANCES IN BIOLOGICAL AND MEDCAL PHYSICS, John R. Lawrence and Joseph G. Mamilton, eds. Volume 2. New York, Academic Press min. 1951.

A review of the carcinogenic effects of ionizing radiation including sections on (1) carcinogenic effects of totalbody irradiation; (2) careinogenesis by locally directed irradiation; (3) carcinogenesis by absorbed radioelements; and (4) mechanism of radiation carcinogenesis. $106 \mathrm{re}-$ ferences. (S.V.S.) 
469

Brues, Austin M, H. Lisco, and M. P. Finkel. CARCINOGENIC ACTION OF SOME SUDSTANCES WHICH MAY BE A PROBLEM IN CERTAIN FUTURE INDUSTRIES. Cancer Research 7, 48(1947).

An investigation is under way to test the late or chronic effects of exposure to plutonium and products of U fission on rats, mice, rabbits, and dogs. Effects of Ra were also studied for comparison. $\mathrm{Sr}^{80}{ }_{\text {, }}$ with $\mathrm{a}$ 55-day half life and a tendency to become concentrated in bone, produced bone tumors, slightly increased the incidence of lymphoma, and did not affect mammary tumor incidence. Ra produced bone tumors and heavy calcification in the media of the larger arteries and certain other sites. $\mathrm{Ce}^{1 \mathrm{Ad}}-\mathrm{Pr}^{\mathrm{BSO}}$ caused bone sarcoma and atrophy of the liver except in the marginal areas where ionization was less and regeneration could proceed. $\mathrm{Y}^{21}$, fed by stomach-tube, was virtually unabsorbed; 0.1 of the acute lethal dose was fed daily for three months with no measurable detriment, but animals surviving this treatment as well as those surviving a single acute dose of $20-30 \mathrm{mc} / \mathrm{kg}$. showed after several months a variety of intestinal lesions with obstruction. Plutonium $\left(\mathrm{Pu}^{238}\right)$, injected intravenously, became most concentrated at first in liver and spleen, was later translocated to bone. Acute plutonium was similar to acute total body radiation sickness, with the addition of splenic atrophy and gross liver changes. Chronic plutonism involved hair graying, progressive liver damage, and bone sarcoma. After subcutaneous injection of $1 \gamma$ of $P^{239}$, local fibro-sarcomas appeared within one year, and local epilations, ulceration, keratoses, and spontaneous amputation also occurred. (GPR)

480

Brues, Austin M., George A. Sacher, Miriam P. Finkel and H. Lisco. COMPARATIVE CARCINOGENIC IFIIISTS BY X-RADIATION AND ${ }^{82}$. Cancer Research 9, 545(1949).

This paper is an abstract and is presented here in its entirety.

Production of lymphoma has been studied in CFI female mice, using total body $x-$ ray and $\mathrm{p}^{22}$. Control mice show an exponentially increasing susceptibility to the spontaneous disease, with doubling of the tumor expectancy about every 75 days, until 700 days of age. Beyond this lime, the rate fails to increase further. Using different patterns of x-ray treatment, it is found that $400 \mathrm{r}$ total body x-ray is more effective if divided over a 10-day period than if it is either fiven as a single dose or divided over 40 days. Increasing doses are more effective until a saturation value is reached, which appears to be at a probability of approximately 0.01 lymphomas per mouse per day. This is about the same as the saturation value reached in old age (after 700 days). There is also evidence for a threshold dose or dose rate below which early tumors are not induced. $\mathrm{P}^{\$ 2}$ is a comparable carcinogenic stimulus and, again, a $_{9}$ threshold dose or dose rate is encountered. The rate of tumor production with both agents is roughly proportional to the respective lethal doses. $P^{82}$ is effective, whether given in a single dose or in monthly divided doses. 471

Burrows, H. and W. V. Mayneord. A NOTE ON TUMORS IN MICE. Am. J. Cancer 31, 484-5(1937).

Spindle cell sarcoma were produced in mice injected subcutaneously with cholesterol which had been irradiated with w-rays in doses of $2000-110,000$ roentgens. (CA) 472

Butterworth, J. S. OBSERVATIONS ON THE HISTOGENE SIS OF OVARIAN TUMORS PRODUCED IN MRCE BY XRAYS. Am. J. Cancer 31, 85-99(1937).
Ninety-one female mice were irradiated over the entire body in groups of 15 to 25 . The irradiation factors were as follows: $190 \mathrm{kv}, 30 \mathrm{ma}, 50 \mathrm{~cm}$ distance, $0.5 \mathrm{~mm} \mathrm{Cu}+1 \mathrm{~mm}$ Al. The ovaries of these mice and of control mice were studied at various ages from 5 weeks to 25 months to determine the histogenesis of tubular adenomata, granulosacell tumors, and lutcomata. In irradiated mice, tubular adenomata are formed by down-growths of the germinal epithelium of the ovary. The earliest down-growths appear about six months after irradiation. Granulosa-cell tumors are derived from nests of follicle cells which form at the periphery of the ovary after irradiation and in senile mice proliferate to form tumors. These nests of cells are probably derived from normal follicles following degeneration of the ova. Luteomata are usually formed indirectly by luteinization of granulosa-cell tumors, but it is possibie that they are occasionally formed directly by division and proliferation of lutein cells. Granulosa-rell tumors and luteomata of mice are similar to those of women and it is unnecessary to postulate embryonic rests of granulosa cells to account for the histogenesis of granulosi-cell 472 tumors. (S.V.S.)

Cloudman, Arthur M., S. Phyllis Stearner, IKatherine Klamilton, John Sackis, and Dorothy Vinimg. PROGRESS REPORT (HISTOPATHOLOGY), P.102 15 of BIOLOGICAL AND MEDICAL DIVISIONS QUARTERLY REPORT, NOVEMBER 1948 TO FEBRUARY 1949. Argonne National Lab. Feb. 1, 1949. 13p. (ANL -4253(p.102-15))

The irst section of this progress report is a bioassay of the combined effects of the simultaneous exposure to methylcholanthrene (MC) and a beta emitter. CF-1 mice were used. One series received $\mathbb{P}^{32}$ and $M C$ on the shin surface. A second series received subcutaneous exposure to cholesterol pellets containing $\mathrm{X}^{91}$ and/or MC. Observed tumors were grouped according to distribution at the Ireatment site, immediately adjoining treatment site, and in other body regions. Data were tabulated at 10 -week intervals for the first 50 weeks. The number of tumorous mice and the frequency per animal are given. There is a strong indication of a synergistic effect in the production of certain types of tumors. In chichs, the lethal effects of exposure to 2 given dosage of $x$-radiation has been shown to depend on the age of the animal as well as on the rate at which the exposure is made. (ACD) 474

Elson, L. A. and L. F. Lamerton. THE INELUENCE OF THE PROTEIN CONTENT OR THE DIET ON THE RESPONSE OF WALIER RAT CARCINOMA 256 TO $\%$ RADATION. Bril. J. Cancer 3, 414-26(1949).

After whole-body radiation (100 r/day for 8 days beginning 1 day alter tumor implantation) or localized ir radiation of an established tumor (400 $x$ total in dose of $250 \mathrm{r}$ daily beginning 6 days after tumor implantation) tumor inhibition was greater in rats on a $5 \%$ protein diet than in those on a $20 \%$ protein diet. No complete regression of the tumor was obtained. With localized irradiation of the tumor when $4000 \mathrm{r}$ was applied in large daily doses complete regression of tumors was obtained in $90 \%$ of the rats on the $20 \%$ protein diet but in only $15 \%$ of those on the low protein diet. (CA) 475

Figge, Irank H. J. COSMIC RADLATION AND CANCER. Science 105, 323-5(1947).

An experiment is described which inwolved the attempt to intensify cosmic radiation effects by means of various combinations of lead plates and the comparison of the rate of carcinogenesis in mice receiving the intensified cosmic radiation with the rate of carcinogenesis in mice re- 
ceiving non-intensified (normal) cosmic radiation. The rate of induction of cancer in mice kept under the lead plates was found to be consistently and definitely higher than that in the controls. All the mee had been injected with methylcholanthrene. It is suggested that the cosmic radiation is intensified by production of shower's or bursts of ionizing radiations occurring when the rays pass through the thin metal plates, and that the methylcholanthene induces cancer by converting some of the energy of the 476 cosmic raduation into careinogenic stimuli. (BA)

Figge, Frank H. J. STUDIES ON THE INELUENCE OF PENETRATING RADLATYNS ON CARCINOGENESIS. ACta Unio Intern. contra Cancrum 6,782-6(1949).

In order to test the possible influence of cosmic radiation of carcinogenesis, lead plates were placed $5-8 \mathrm{~cm}$ above male mice mjected with $0.5 \mathrm{~g}$ of methylcholanthrene; the frequency of showers of cosmic radiation was thereby increased. The latent period for the induction of tumors was decreased for this group as compared with controls; repetition of this experiment using a different strain of nice gave essentially similar results; the possibility of lead toxicity as a contributing factor was tested and ruled out. Mice were also placed in coal mines at various levels; one group at $300 \mathrm{it}$, one at $600 \mathrm{ft}$, and other groups at $1,035 \mathrm{ft}$ underground. It was found that whle the cosmic radiation decreased as the depth in the mine shaft in creased, in this mine at least, the radiation increased; a group of 40 mice at the 1,035 ft level were placed in al lead cage to partially eliminate the g radiation factor. From the results of this and related experiments there was some evidence that the cosmic particles and photons of $\gamma$ radiation are synergistic and additive but not equivalent as cocarcinogenic agents. The mice shielded only in wire cages at various levels in the mine required longer latent periods for the development of the cancer in the order given: 300 , 600 , and $1,035 \mathrm{ft}$. It is concluded that the potency of such powerful careinogens as methylcholanthrene may be altered by slight variations in the intensily of penetrating 477 envirommental radiations. (NSA)

Furth, J. FACTORS OF INDUCTION OF OVARLAN TUMORS BY X-RAYS: TYPES, CHARACTER AND HISTOGENESIS OF THESE GROWTHS. Acta Unio Intern. contra Cancrum $6,785-6(1949)$.

Following the irradiation of four to six weeks old mice with $87 \mathrm{r}, 175 \mathrm{r}$, or $350 \mathrm{r}$, ovarian tumors began to appear when the mice were about 11 months of age. The frequency of these neoplasms inc reased with time and almost every mouse that lived 17 months developed a unilateral or bilateral ovarian growth ir respective of the dose of irradiation; these growths were transplantable and metastasizing and usually complex: granulosa, luteoma, glandular, and other types. The granulosa tumor caused estrogenization and hypervolemia, while the neoplastic lutein cell masses masculinized (caused atrophy of adrenal cortex and often obesity). It is stated that the histogenesis of these and other tumors induced by $\mathrm{x}$-rays will be discussed. (NSA)

Furth, J. RELATION OF PREGNANCIES TO INDUCTION OF OVARIAN TUMORS BY W-RAYS. Proc. Soc. Exptl. Biol. Med. 71, 274-7(1949).

It was found that mice exposed to $50-200 \mathrm{r}$ of $\mathrm{X}$-rays can have one or several normal pregnancies and still be liable to the development of ovarian tumors in a stock in which this neoplasm is extremely rare. Irradiation at 1-3 days of life with $150 \mathrm{r}$ sterilized only one-third of the mice, while ovarian neoplasms appeared in about $76 \%$. Ir radia- thon at 1.3 days did not hasten the onset of ovarian neoplasms as compared to irradiation at $4-10$ weeks. In both groups the tumors developed in middle-aged and old mice. The author postulates the existence of a specific delayed x-ray effect coupled with a hormonal imbalance provolsed by $X$-rays leading to the development of ovarian tumors. (EM)

479

Furth, J. and O. B. Furth. NEOPLASTIC DISEASES PRODUCED IN MICE BY GENET AL IRRADLATON WITE X-RAYS. I. INCIDENCE AND TYPES OF NEOPLASMA. Am. J. Cancer 28, 54-65(1936).

Mice were exposed to a single dose or repeated doses of X-rays at the age of approximately 5-12 weeks; 775 mice were irradiated, and 1,290 were kept as controls. Irradiation caused an increase of ovarian tumors to 15 times as many as in the controls of myelosis to 8 times, of mediam stinal lymphosarcoma of 7 times. There was a slight but definite rise in the incidence of several other neoplasms, but those of the breast decreased by half. The occurrence of breast cancer appears to be conditioned by ovarian hormonal activity, which is decreased by irradiation but apparently increased with the development of ovarian new growths, in irradiated mice. The high incidence of breast cancer with ovarian neoplasms and the associated endometrial hyperplasia suggest that some ovarian tumors produce hormone. The $\mathrm{X}$-ray induced tumors are indistinguishable from those occurring spontaneously and appear at a somewhat earlier age than the similar tumors of unixradiated mice. It is suggested that $\mathrm{x}$-rays so alter the constitution of certain cells that they or some of their offspring undergo malignant transformation several months

480 after ir radiation. (BA)

Gardner, W. V. OVARIAN AND LYMPHOND TUMORS IN FEMALE MCE SUDSEQUENT TO ROENTGEN-RAT IRRADIATION AND FORMONS TREATMENT. Proc. Soc. Expt1. Biol. Med. $75,434-6(1950)$.

Mice irradiated with $280-380 \mathrm{~g}$ and then given weekly injections of 16.67 estradiol benzoate did not have ovarian tumors whereas those irradiated and given $1.25 \mathrm{mg}$ testosterone propionate weelly or plain sesame oil injections did. Many of the mice developed lymphomas, the incidence was lower in the group given testosterone propionate than in the other 2 groups. (CA) 481

George, E. B., M. George, J. Booth, and 1. S. Borning. INFEUENCE OF COSMIC RADIATION ON INDUCED CARCINOGENIESIS IN MICE. Nature 164, 1044-35(1040).

The influence of cosmic radiation on carcinogenesis in mice was studied at Jung raujoch. Mice receiving $0.25 \mathrm{mg}$ of 20 -methylcholanthrene were divided into three equal groups; the controls in a tunel under $60 \mathrm{~m}$ of rock, the second group in the cosmic ray cabin and the third under a $2 \mathrm{~cm}$ leat screen in this cabin. The results were not conclusive and the repeated experiments on the roof of the London School of Hygiene and Tropical Medicine gave a zandom variation and differed from Irigge's results. (BM)

482

FERShaw, D.S. LEUKRMA IN MCE TOLLOWING EEPOSURE TO XX-RAYS. Radiology 43, 279-\$5(1944).

C-57 black mice received single doses of $200 \mathrm{~m} / \mathrm{air}$ of X-rays over the whole body, repeated at four week intervals as long as this could be done without too severe an effect on the health of the animals. The radiation factors were: $200 \mathrm{kv}, 20 \mathrm{ma}, 0.5 \mathrm{~mm} \mathrm{Cu}$ and $1.0 \mathrm{~mm}$ Al fillration, distance $38 \mathrm{~cm}$. During exposure the mice were kept in a shallow light-weight celluloid tray. Following treatment, 
they were krept under constant observation (blood counts) until death, at which time they were examined grossly and histologically for leukemia. Cont inuous blood records showed a general depression of leucocyte level during ureatment, followed by a slow return to normal after ireatments were discont inued. In some animals, the recovery extended into marked leucocytosis, and these animals at death were invariably found to have leucemic infections. Hyperplasia of hematopoietic organs was seen in all animals recorded as positive, but the distribution of 483 such conditions varied from animal to animal. (BA)

Jenshaw, Paul S. and James W. Mawhins. INCWOLNCE OF LIUUKEMA IN PHYSICIANS. J. Nat1. Cancer Inst. 4 , $339-46(1944)$.

A comparison was made of the incidence of leuremia in physieians and in the general population. Data were obtained from the death list of the J. A. M. A. from the mortality reports of the U.S. Bur. of the Census, and an unpublished compilation of the U. \$. Public Health Service. Comparisons were made on the basis of (1) the ratio of deaths from leukemia to deaths from cancer, (2) ratio of deaths from leukemia to total death rates, and (3) death rates from leukemia. Leukemia was recognized approx. 1.7 times more frequently among physicians than among white males in the general population. Possible discrepancies are discussed. While these observations furmish no direct proof that radiation acts to incite leukemia in human beings, they are in accord with the findings on experimental animals in which exposure to $\mathrm{X}$-rays has

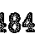
been found to incrense the incidence of leukemia. (BA)

Menshaw, P.S., F. F. Riley, and G. F. Stapleton. TPYE CARCINOGENIC EFIECT OP PILE RADIATIONS. (AM. Assoc. Adv. Scỉ, Section on Chem., Gibson Island Research Conference on Cancer, 1946), Cancer Research 7 , 48(1947).

Mice and rats exposed to penetrating radiations (fast and slow neutrons, $\gamma-\mathrm{rays}$ ) from the $U$ chain reaction in a pile reactor developed neoplasms (mainly mediastinal Iymphomatoses, the incidence of which was less than $15 \%$ in the controls, more than $60 \%$ in some of the experimental groups), lowered leukocyte level, emaciation, and loss of weight and died prematurely. When the animals were exposed to nonpenetrating radiations $(\beta-$ rays) from the pile, the effects were limited mainly to skin abnormalities of all types, appearing several months after single massive doses; in strains that rarely showed skin lesions of any type, the incidence of carcinoma was raised to $100 \%$, and loci per animal were great as 50-100. Fast neutrons were more active than y-rays in producing the late effects. Drily threshold levels for shortening life were in the range of $1 \mathrm{r}$ of $\gamma-\mathrm{rays}$, and something well less than $0.1 \mathrm{r}$ for fast neutrons?). Length of life varied inversely with the amount of exposure, and the amount of effect varied directly with the concentration of ions in space and time. (CA) 485

Henshaw, Paul S. and R. S. Snider. REVIEW OF INFORMATION BEARING ON THE TUMOR INDUCTION ACTION OF SUPERFICIAL RADIATIONS, 19.229-37 Of BIOLOGICAL EFECTS OF EXTERNAL BETA RADIATON. NeW York McGraw - Hill Book Company, Inc., 1951. (NNES Volume 22: Chapter 16)

The pertinent facts associated at this time with induction of skinn lumors with superficial radiations may be indicated as follows: It is important to remember that skin consists of layers of cells with the germinal layer at the base, and that the germinal layer proliferated other germinal cells and cells which bzcame differentiated first to form an intermediate layer and finally the dead corneal layer. In the slin there must continually be a residuum of active germinal cells and a process of maturation to death of cells in order to have normal skin function. Slain abnormalities consist of three types: (1) failures manifested as drying, scaling, fissuring, lessened resillency, and reduced regenerative capacily, (2) anomalies such as badly formed nails, poor quality of hair, and poorly developed teeth; and (3) excesses involving overgrowth and overfunction as in neoplasia. Radiation absorbed in the skin causes damage primarily in the germinal layer. When the damage to this layer is partial, the effect is a skin failure; the germinal layer is unable to proliferate enough cells to maintain the integument with the result that there is drying, sealing, fissuring, possible loss of hair, etc. Repair talkes place from the floor of the damaged area, however, from living germinal cells that remain. When clamage to the germinal layer is complete the effect is skin sterilization; not only is there drying and scaling, but also there is exudation, sloughing, and ulcer formation. In this case healing must be from the edges as no germinal elements remain. Skin tumors often arise out of such wounds that have become refractory. In the case of superficial radiations skin tumors (of practically every type) have been observed to appear in animals that display little or no gross evidence of skin break down. Single applications of beta rays have been observed to be carcinogenic, as many as 50 to 100 loci of tumor growth being produced on rats that ordinarily show no slin lesions. (ADD)

486

Woch-Ligeti, Cornelia. EFF ECTS OF REPEATED XRADIATION OF THE WHOLE BODY ON THE DEVELOPMENT OF TUMORS IN RATS DUE TO FEEDING P-DIMETHYLAMINOAZOBENZENE. Brit. J. Cancer 3, 562-9 (1849).

Forty male and 10 female Wistar rato were fed ad lib a sem- - 3ynthetic diet, containing $0.07 \%$ p-dimethylaminoazobenzene, and 20 of the male rats were given soft $\mathrm{x}$-radiations, while 10 male and 10 female rats received penetrating $\mathrm{x}$-radiations. The frradiations were begun on the same day as the feeding of the carcinogenic compound and simlar techniques of application were utilized. The penetrating radiations were delivered by a 180 -kv machine with a 0.75 mm Cu and $1 \mathrm{~mm}$ Al filtration at a focal distance of $30 \mathrm{~cm}$, while the soft radiations were delivered at $45 \mathrm{kv}$ with no added filtration and at a focal distance of $20 \mathrm{~cm}$. At the beginning of the esperiment, each rat received $10 \mathrm{r}$ once weekly, delivered to the liver as a $10 \mathrm{r}$ dose in the case of the penetrating radiations (with a dorsal skin dose of $13 \mathrm{r})$, and as a dose of 0.3 I to the liver for the soft radiations. Fach rat was irradiated individually and the intervals between ir radiations were extended to two weeks and occaslonally three to lour weeks. The rats treated with hard radiations received a total of $310 \mathrm{r}$ in the liver, while the soft radiation group received a total of $10 \mathrm{~g}$. All nonir zadiated rats receiving $p$-dimethylaminoazobenzene devoloped hepatic tumors, but the development of tumors was prevented, or their rate of growth retarted, in rats treated with soft radiation. The growth of hepatic tumors was alightly retarded in rats treated with penetrating rays and skin tumors developed in two of these rats. The adrenals of rats receiving solt $\mathrm{X}$-rays were significantly enlarged. (NSA)

487

Iaplan, H. S. OBSERVATIONS ON RADIATION-INDUCED LYMPHOHD TUMORS OF MCE. Cancer Research I, $141-7(1947)$.

Strain $A$ mice in groups of various ages yielded a maximum incidence of lymphoid tumors in animals irradiated at one month of age with a sharp decrease al two months 
and later. Thymectomy of strain A mice irradiated at two to three months of age resulted in a considerable increase in the latent period and a very slight docrease in the incidence of lymphomas as compared with the low incidence in intact irradiated control mice of the same age. In a parallel experiment, splenectony was followed by a slight decrease in incidence without change in latent period. The tumors are lymphorytic lymphomas which, in most instances, appear to rise in the thymus, to disseminate to the spleen, lungs, liver, kidneys, and lymph nodes, and to cause leukemia in a considerable percentage of animals. Incidental observations on radiation-induced ovarian tumors and on inhibition of spontaneous mammary tumors 488 following $\mathrm{X}$-ray treatment are presented. (anth)

Kaplan, henry S. PRELIMINARY STUDIES OF THR EPFECTIVENESS OF LOCAL IRIADIATION IN TRE INDUCTION OF LYMPHOLD TUMORS IN MCE. J. Nat1.

Cancer Inst. 10, 267-70(1949).

In order to test the theory that incident radiation acts locally and duectly upon the cell or tissue to produce alterations in the cellular behavior manifested as tumorous growth, the three experiments described here were nerformed. In the first experiment, strain $A$ mice were immobilized and irradiated over one of four dorsal regions through a circular portal of $1 \mathrm{~cm}$. in diameter; the radiations were $800-750 \mathrm{r}$. The second experiment consisted of the irradiation of mice of the $C-57$ strain by a single dose of $1000 \mathrm{r}$, delivered to a Irensverse body section $4 \mathrm{~mm}$. in thickness. In the third experiment, $1000 \mathrm{x}$ was delivered in five dally treatments in two consecutive weeks to mice of the strain C-57; some of the mice were treated over the entire upper hall of the body, and others over the entire lower half. The results show that the in duction of the lymphoid fumors occurred wilhout significant frequency and indicate that their production is, the authors state, probably not solely the result of a simple direct action of radiation upon the susceptible cell or tissue of origm. (NSA)

489

Kaplan, Henry S. LOCAL IRRADIATION AND TRE INDUCTION OF ITMPHOT TUMORS IN MICE. Cancer Research 9, 621(1949).

This paper is an abstract and is presented here in its entirety.

Whole-body exposure of mice to roentgen rays in adequate dosage has long been known to yield lymphoid tumors. Strain $\mathbb{A}$ mice, which are moderately susceptible, and strain C5T black mice, which are highly susceptible to whole -body isradiation, have been studied following local exposure. Strain C57 black mice one month of age were treated over the anterior or posterior half of the body wilh daily doses of $100 \mathrm{r}$ for 10 days. After 17 months, only 2 of 55 mice $(4 \%)$ ir radiated over the upper hall, and 1 of 55 mice (2\%) ixradiated over the lower hall of the body have developed lymphoid tumors. In previous experiments the incidence of such tumors following whole body esposure of young mice of this strain to the same total dose has averaged about $65 \%$ at 10 months. No exact data are available for half of this total dose delivered to the whole body, but extrapolation indicates that a significantly higher incidence might be expected than was observed after half body irradiation. Larger doses sharply localized to the refion of the mediastinum or upper abdomen have produced no lymphoid tumors in either grain, and mice of strain A have also been completely refractory to local treatment over the skull or lower abdomen. These resulks guggest that ahielding one half or more of the body from roentgen rays confers a considerable degree of protection against the induction of lymphoid tumors in mice. 490

Kalpan, HenY S. INELUENCE OF OVARIAN FUNCTION ON INCIDENCE OF RADIATION-INDUCED OVARIAN TUMORS IN MCE. J. NaAl. Cancer Inst. 11, 125-32(1950).

Irradiated ovaries implanted intramuscularly into ir radiated and nonirradiated groups of spayed LAF hybrid mice gave rise to many granulosa-cell tumor , luteomas, and related neoplasms. No such tumors occurred when in radiated ovaries were implanted into noniradiated, nonovariectomized mice. It is concluded that intact ovarian endocrine function inhibits the development of fumors in irradiated ovarian grafts, confirming the reported observations of Lick, Mirschbaum, and Mixer. (Cancer Research, 9: 532-536(1949)) (NSA)

491

Kaplan Hemy S. and Mary B. Brown. INHIBITION BY TESTOSTRRONE OF RADIATION-INDUCED LYMPHOID TUMOR DEVELOPMENT IN INTACT AND CASTRATE MALE MIC2. Cancer Research 11, 262(1951).

Strain C57 black male mice were distributed as litter mates among four groups of about 50 mice each. Two groups were maintained intact, the other two castrated at 2 months of age. Whole-body irradiation was started 3 to 4 days later; all groups received a total of $675 \mathrm{x}$ in 4 equal fractions at $4-$ day intervals. One castrated and one intact group received intranuscular injections of testosterone starting on the first day of irradiation, while the other two groups were injected with the aluminum phosphate suspension (placebo) alone. The injection volume was 0.02 ce of either material given lwice weokly for 10 weeks. To date, lymphoid tumors have been observed in only 3 of 49 intact, testosterone-treated animals $(6 \%)$, and in 4 of 50 castrated, testosterone-treated animals $(8 \%)$, as contrasted with an incidence of 71 and $83 \%$ among intact and castrated placebo-treated mice, respectively. Thus, testosterone exerts a striking inhibitory effect on the development of lymphoid tumors in irradiated male mice. Under the conditions of the present experiment, castration of mature male mice did not significantly modify lymphoid tumor incidence. (NSA) 492

Kaplan, Renry S. and Mary B. Brown. EFIECT ON LYMPMOND TUMOR INCIOENCE OF CHANGES IN TOTAL DOSE, FRACTIONATION, AND PEIODICITY OF WHOLEBODY ROENTGEN IRRADIATION. Cancer Research 11, 262(1951).

(This paper is an abstract and is presented here in its entirety.)

A total of 1733 black mice of both sexes was assigned by randomization of litters to 32 groups, all of which were inradiated al 33 is days of age. Successive groups received yystematic increments of tolal dose, administered either as simgle treatment or in 2,4, or 8 equal daily fractions. Equivalent total doses were also given in fractionated treatments at intervals of 4,8 , and 18 days. Lymphoma incidence roughy maralleled total dose, within the range investigated, for either a single exposure or fractionated exposures at daily intervals. Response to equal total doses was not modified by the degree of fractionation. For a given total dose, however, there was a striking increase in incidence when an interwal of 4 to 8 days occurred between treatments. Response was no greater than for daily treat ment when the interval was increased to 16 days. In contrast, mice exhibited a pronounced ability to recover from the lethal effect of a given total dowe with ather increasing fractionation or, in particular, the interposition of an in 
creasing interval between treatments. The difference in dose-response characteristics indicates that roentgen radiation operates through different mechanisms in oliciting lethal and leukemogenic responses in mice. (NSA)

1998

Raplan, Henry 5. and Mary B. Brown. FURTMER OBSERVATIONS ON INHIBITION OF LYMPHOID TUMOR DEVELOPMENT BY SHIELDING AND PARTIAL-BODY IRRADIATION OF MCE. J. Natl. Cancer Inst. 12, 42735(1951).

Previous evidence that local irradiation is ineffective in the induction of mouse lymphoid tumors and that an indirect systemic mechanism must therefore be implicated was confirmed by partial-body irradiation and mediastinal shielding experiments. There was no evidence of an effect attributable to shielding or transplanted ovaries, while shielding of an indifferent area of the body such as the thigh significantly inhibies the genesis of lymphoid tumor. (NSA)

Kaplan, Henry S., Mary B. Brown, and Sumner N. Marder. ADRENAL CORTYCAL FUNCTION AND LYMPHOID TUMOR INCDENCE IN IRRADIATE MICE. Cancer Research 11, 262-3(1951).

Experiment $I_{0}$ - Groups of $C 57$ black mice received local or whole-body irradiation and/or cortisone in various combinations. In the dose administered, whole-body irradiation yielded lymphomas in 41 of 50 mice. When cortisone injections were started simultaneously with ir radiation, only 18 of 48 mice developed these tumors, and when cortisone treatment was deferred until 6 weeks after ixradiation only of 23 animals responded. Cortisone alone yielded no tumors. Ir radiation over the mediastinum alone, or over the rest of the body with the mediastinum shielded, failed to elicit lymphomas whether or not cortisone was also given. Experiment II. - Two groups of $\mathrm{C5} 7$ black mice were adrenalectomized and a third subjected to sham operations. Group I received no further treatment; the other adrenalectomized group and the sham-operated group received fractionated whole-body irradiation in four treatments at 2 -week intervals. Initial mortality was 20,67 , and $3 \%$, respectively. Lymphoid tumor's occurred among 0 of 24,13 of 20 , and 12 of 59 surviving mice, respectively, indicating a significant increase in susceptibility to lymphoma induction among adrenalectomized mice. (NSA) 495

Kaplan, Fenry S. and Edwin D. Murphy. THE EFIFET OF RADLATION ON THE BIOLOGICAL BELAVIOR OF A MOUSE MAMMARY CARCINOMA. Acta Unio Intern. contra Cancrum 6, 810-11(1049).

In four separate experiments, a transplantable mouse mammary adenocarcinoma (tumor 755 ) was inorulated in the hind legs of groups of $40-50$ mice of $C 57$ black strain. When the tumors reached the diameter of about $1 \mathrm{~cm}$, hall of the animals were treated locally with doses of $400-1,000$ $r$ with the remainder of the body carefully shielded. Animals of both the irradiated and untreated groups were sacrificed simultaneously and pulmonary metastases were found in 27 of 62 mice bearing irradiated tumors, and in live of 52 controls. Collaterally, irradiated and control tumors were removed from their hosts and inoculated subcutaneously into several alien strains of mice and into the anterior ocular chamber of guinea pigs. It was found that the irradiated tumors grew progressively in 7 of 77 alien mice and that the untreated tumors grew in 2 of 108 mice, no progressive growth was observed in guinea pigs. It is suggested that roentgen irradiation in moderate doses may alter the behavior of a tumor in the direction of increased autonomy. (NSA)

496

Kirschbaum, A.g J. R. Shapiro, and W. H. Mixter. SYNERGISTIC ACTION OF IESTROGENIC HORMONE ANU $X-$ RAYS IN INDUCING THYMIC LYMPHOSARCOMA OF

MCE. Proc. Soc. Expti. Biol. Med. 72, 632-4(1949).

Synergistic lymphogenic effects were obtained when estrogenir hormone and $X$-rays were administered simultaneously to mice of the Bagg albino and CBA strains. Thymic lymphoid tissue appeared to be more senstive than other lymphoid tissue. This was also true when $\mathrm{X}$-rays and methylcholanthrene acted synergastically in the dba 497 stock. (BA)

Koletsky, Simon, Frederick J. Bonte, and Hymer L. Triedell. PRODUCTION OF MALIGNANT TUMORS IN RATS WITH RADIOACTIVE PFOSPRORUS. Cancer Research 10, 129-38(1950).

Adult white male rats of the Wistar strain, weighing about $150 \mathrm{~g}$, were used in experiments in which the incidence of neoplasms was observed following enther the injection of a $\mathrm{LD}-50$ dose of $\mathrm{P}^{32}(4.5 \mu \mathrm{c} / \mathrm{g}$ of body weight $)$ or repeated injections of $\mathrm{P}^{32}(1.5 \mu \mathrm{c} / \mathrm{g}$ body weight every three weelss) for a total dose of 9 or $12 \mu \mathrm{c} / \mathrm{g}$; spontaneous bone tumors have not been observed among control animals of this strain. Complete autopsies were performed on all rats which died or were sacrificed, and in almost all the animals routine microscopic sections of the femur and vertebrae were taken. of the 19 animals in the first group, mne developed malignant neoplasms but were otherwise in good condition, except for the last few weeks of life. Of 15 rats in the second group, 4 were discarded, and of the remainmg 11. 4 developed neoplasms. Of the total of 13 neoplasms, 3 were squamous-cell carcinoma involving the face and were associated with exophthalmos; 10 were osteogenic sarcomas, 4 of which were located in the jaw, 2 in the spine, 2 in the tibia, and 1 each in the femur and pelvis; 4 of these latter tumor's metastasized to the lungs. The incidence of tumor development was about $40 \%$. A typical proliferation of bone was usually found in the rats and the lesions, it is concluded, may have merved as a precursor to malignant change. (NSA)

498

Lick, Louis, Arthur Kirschbaum, and Harry Mixer. MECHANSM OF INDUCTION OF OVARIAN TUMORS BI X-RAYS. Cancex Research 9, 532-6(1940).

The results of experiments to determine whether $\mathrm{X}$-rays induce tumors in mice in the presence of normal ovarian function are discussed. The Bagg albino stock of mice was used. The ovaries were irradiated with a Phillips Contact Therapy Machine at $45 \mathrm{kv}$ through $1 \mathrm{~mm}$. Al filtration at a $19 \mathrm{~mm}$ target distance and a rate of $1,500 \mathrm{r}$ per minute. Practically all the females receiving 200 s whole body irradiation at 8-10 weeks of age developed bilateral ovarian tumos's if they survived 16 months after the ir radiation; mice receiving $200 \mathrm{r}$ irradiation to one ovary after the extirpation of the other ovary demonstrated $100 \%$ tumor development; mice receiving $200 \mathrm{x}$ locally to one ovary with the other protected and intact developed no tumors in the irradiated ovary. However, a granulosa cell tumor did develop spontaneously in a non-irradiated ovary. Larger doses of $x$-radiation produced results similar to the $200 \mathrm{r}$ irradiation. (NSA) 499

Lorenz, E., W. E. Reston, M. Deringer, and A. B. E. Eshenbrenner. INCREASE IN INCIDENCE OF PULMONARY TUMORS IN STRAIN-A MICE FOLLOWING LONG- 
CONTINUED IRRADIATION WITH GAMMA-RAYS. CanceE Research 6, 485(1946); J. Natl. Cancer Inst. 6, 349-53(1946).

Sixty mice of strain A ( 35 males and 25 females) were exposed daily for eight hours to $\gamma$ radiation from a $1-\mathrm{g}$ Ra source filtered by $0.5 \mathrm{~mm}$ of Pt. The daily dose was $8.8 \pm 10 \% \mathrm{r}$. The same number of controls were kept. The animals were 1-2 months old at the beginning of the experiment. Exposure was terminated after 287 days when the experimental animals had received a total dose of $2500 \pm 10 \%$ $x$. The incidence of lung tumors in the experimental animals was $77 \%$ and in the controls $47 \%$, a statistically signif500 icant difference. (NSA)

March, H. C. LEUREMA IN RADIOLOGISTS. Radiology 43., $275-8(1944)$.

For some years there has been an impression that exposure to radiation might cause leukemia. Twenty-three cases of leukemia following irradiation have been reported in the world literature but in some of these details were lacking and the causal relationship was not established. The author was inpressed by the death of three radiologists from leukemia during 1943. He therefore made a study of the death of radiologists reported in the Am. J. Roentgenol. and in Radiology for the past 15 years and found that among 175 deaths, the cause of which was reported in 173 , eight deaths had been caused by leukemia. This gives an incidence of $4.57 \%$ for all types of leukemia in radiologists in the past 15 years. The incidence of deaths from leukemia among physicians not practicing radiology during this same period was $0.44 \%$. The incidence of leukemia therefore among radiologists was more than 10 times that among physicians not practicing radiology. A statistical analysis show that there is only one chance in a million of getting eight cases of a condition by chance alone in a group of 175 cases, when the standard rate of incidence is $0.441 \%$. The author suggests that the deaths and major lllnesses of all radiologists be recorded by some central ageney so that the relationship between prolonged irradiation and various pathological conditions may be established. (A.JR) 501

March, Herman C. LEUTEMAA IN RADIOLOGISTS IN A 20-YRAR PERIOD. AM. J. Med. Sc1. 220, 282-6(1050).

The study of the incidence of leukemia in radiologiste has been extended to cover a 20 -year period. It has been mown that during this time leukemia has occur red more than nine times as frequently in radiologists as in nonradiological physicians. This difference has statistical significance. It is suggested that the increased incidence of leukemia in physicians as a whole (compared with the adult male white population) might be due to the same factor of exposure to lonizing radiation. The experimental work showing an increased incidence of leukemia in mice exposed to roentgen irradiation has been reviewed. (NSA)

\section{2}

Martland, Harrison S. THE OCCURRERCE OF MALIGNANCY IN RADIOACTIVE PEHSONS. Am. J. Cancer 15, 2435-2516(1931).

There have been 5 death from osteogenic sarcoma in a total of 18 originating in occupational poisoning by radioactive substances in former dial painters from the New Jersey plant, an incidence of $27 \%$. As the incidence of the ordinary variety of primary osteogenic sarcoma is only about $0.07 \%$, this is overwhelming evidence that the radioactivity of these dial painters is the true cause of the sarcoma. Symptoms and histopathological developments occurred during the various stages of osteogenic sareoma are described to some detail. Attention is called for the first time to the difference in biological effects on the human bone marrow of external penetrative irradiation and internal $\alpha$ radiation as seen in these cases. In this respect attention is called to the fact that anemias developing from undue exposure to external radiation are considered by most authorities as of the aplastic type, whereas anemias caused by internal $\alpha$ radiation are of the regenerative, hyperplastic, or megablastic type. That radioactivity in the human body may play an important part in the production of other forms of malignancy in no way connected with Ra dial painting is suggested; the high incidence of primary carcinoma of the lungs in the Co miners of Schneeberg and in the pitchblende mines of Joachimsthal being noted. The multiplicity of lesions and diseases produced by dradiation frow external exposure to $\mathrm{Ra}$ and $\mathrm{X}$-rays, and padiation from in tern. bombardment by the ourticle is noted. Such a variety of lesions produced by a single agent might indicate that many of the lesions are closely inter related and are only manifestations of various degrees of injury 10, or stimulation of exhaugtion of, certain normal body tissues. (S.V.S.)

\section{9}

Mayneord, W. V. and L. Dorothy Parsons. THE IFIFCT

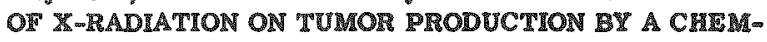
ICAL COMPOUND IN MCE AND TIE ASSOCIATED BLOOD

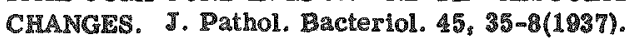

Irradiation of mice either previous to or during the injection of Na $1,2,5,6$-dibenzanthracene- 8,10 - endo- $\alpha, \beta-$ succinate accelerates tumor formation and gives $x$ ise to a greater number of sarcomas. All irradiated mice show a constant blood change immediately following irradiation, i.e., an initial fall in total white count with a subsequent rise, an absolute fall in lymphocytes and a corresponding rise in polymorphs. (CA) 504

Mendeleeff, P. THE ADDITVE ACTION OF METHYLCHOLANTKREPE AND X-RAYS ON THE GUNEA PIG. Compt. rend. soc. blol. 144, 301-2(1950).

Previous attempts by the writer to induce cancer in guinea pige with methylcholanthrene were unsuccessiul. Massive dowes of X-rays or radium alone would only produce radiodermatitis which occasionally would be transformed into a malignant tumor. The author injected four guinea pigs subcutaneously with $30 \mathrm{mg}$. methylcholanthrene and exposed them five to six days later to $400 \mathrm{r}$ at $85 \mathrm{kv}$ at the place of injection. Three of the animals exhibited tumors, two of which were cancerous. These tumors 505 occurred with prior radiodermatitis. (NSA)

M.s. Mashan Jack. ON TRE INCDERCE OF CANCER IN OAK RLDGE, TENNESSER. Office of the Medical Adviser (ORO, AEC). July 2, 1848. 28p. (TD-77)

Since the sull nature of the extent of operations at Oak Ridge was reached after the dropping of the firgt atomic

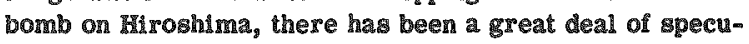
lation concerning the hazards of employment, or residence, in the "Atomic City". To many, radioactive chemicals suggest cancer and one of the hitherto unanswered questions was "are Oak Ridge employees, or residents, more musceptible to cancer than is the general population?" In order to provide a factual, statistical anwwer to these questions the Medical Advisor"s Office of the ORDO of the U. S.A.E.C. conducted an exhaurtive, statistical study of this problem at oak Ridge. Conclustong follow: The age standardized incidence rate of cancer among the Oak Ridge employees and their resident dependents is 123 case an- 
nually per 100,000. This is significantly lower than the accepted national rate of 230 per 100,000 annually. The proportion of employees who have possibly been exposed to radionctive chemicals and who have developed cancer is approximately equal to their proportion in the entire population indicating that there is no reason to believe that they are more susceptable to cancer. The low cancer sate is in harmony with the low death rate in Oak Ridge from most diseases and within the accepted tolerance limits of the ratio of cases of cancer to cancer-caused deaths. The relatve proportion by primary gites of oceurence among white females with cancer is not significanily different from the nationally based expected values. Among white males a gignificantly higher proportion of cancer of the respiratory systen was found than might be expected by chance from the 1238 national averages. A tentative explanation on the basis of a long-established upward trend in cancer of the respiratory system among males was offered. Reference was made to other authorities who offer evidence of the relationship between one's socio-economic group and his susceptiblity to cancer. (ACD)

Mottram, J. C. PRODUCTON OF EPITHELIAL TUMORS BI IRRADIATION OF A PRECANCEROUS SKIN LESION. Am. J. Cancer 30, 746-8(1937).

Application of $\gamma$ - radiation to a precancerous slzin lesion in doses of about $1,000 \mathrm{x}$, or insufficient to destroy the epidermis is followed by a greater number of epithelial tumors than would have occurred had no radiation been applied. This is a corollary to Cramer's finding that doses destructive to the epidermis inhibited epithelial tumors under similar conditions. The similiarity between these experimental findings and the results of a stimulating wose occasionally seen clinically is discussed. The danger of treating precancerous lesions with radiation unless a destructive dose can be given to the whole area, is stressed. The importance of these findings with regard to the mutation hypothesis is considered. (auth)

507

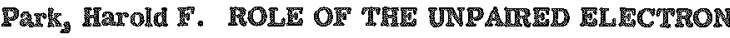
IN CARCINOGENESIS. J. Phys. \& Colloid Chem. 54, 1383$4(1950)$.

Azo dyes, complex hydrocarbons, organic amines and their derivatives, actinic radiation, $\mathrm{x}-\mathrm{rays}$, and radiations from radioactive materials are capable of initiating neo plastic tisaue transformations and also of forming free radicals. Free radicals can be destroyed only as the rem gult of an electron painging reaction. Substances which initiate cancer are identical or similar to the substances which alleviate it. Thus cancer seens to be the result of a ree 508 radical reaction. (CA)

Rajewsky, B. ON THE MUTATON THEORY OF THE ORIGIN OF CANCER. Z. Krebstorsch. 56, 274-84(1949).

The cancerogenic properties of ionizing radiations were examined, in the light of the cell-mutation theory, on white mice submitted to the action of $\mathrm{x}$ rays and particles. The latter were introduced into the animals" bodies by adding radon, in various concentrations, to the air of the cage. The main reault was the discovery of the strong tumorproducing effect of very small doses of a rays. With increasing time of exposure to feeble a radiations, a whole geries of tumos' Irom benign adenoma lo adenocarcinoma and bronchial cancer was obtained. (NSA) 508

ROSS, JOAn M. THE CARCHNOGENIC ACTION OY RADIUM IN TYY RABBIT: THE ETPECT OF PROLONGED IRRADIATION WITH SCREINED RADIUM. J. Pathol. Bacteriol. $43,207-70(1936)$.
Radum-filled platinum fules of $1.0 \mathrm{~cm}$. overall lengt h, filtration 0.55 platinum and content 0.1 me radium clement, were implanted into the tissues of 9 rabbits: 7 of the rabbits developed malignant new growths in mmediate relation to the tubes. Five of the tumors were sarcoma and 2 were squamous cell carcinoma. If was conoluded that ? radiation of wat intensity but prolonged duration (97167 weeks) was the carcinogrenic factor in these cases and that the type of growth produced depends on the nature of the tissue in which the tube is embedded. (auth) 510

Vesin, M. 5. PULMONAIY CANCER PRODUCED BY RADIOACTIV LMANATIONS. Arch. maladies profess. méd. travail et sécurité sociale $9,280-3(1948)$.

A discussion is presented on the efferts of radioactive emanations on the lungs of mine workers in Jachynow. It is clamed that other predisposing factors toward the development of carcmomet of the luxgs in miners are the lack of oxygen, the excess of carbon dioxide, and, possubly, silicosis and arsence dust. Prophylaxis requres better ventilation of the mines; work in the mines, 1 is concluded, should be restricted to 5 to 10 years per person. (NSA) 511

Willams, George Z., Nelson F. Young, and Jeffries P. Moore. THE EFEET OF IRRADIATION ON AZO DYE NEOPLASIA IN THE RAT LIVER. I. INFLUENCE OF XRAY AND RADIO-GOLD ON THE INCIDENCE OF HEPATOMA AND THE MISTOLOGX OF THE LIVER IN IRATS FED DMMETHYLAMNOAZOBENZENE. Cancer Research $11,289(1951)$.

Sprague-Dawley albino rats were maintained 24 weoks on a synthetic diet containing $0.04 \% \mathrm{~m}$-methylaminoazobenzene. Controls fed the basal diet and the azo dye only yielded nne tumors in 38 rats (24\%), which survived 6 months or longer. The diet plus $\mathrm{m}^{\prime}$ meDMB and $200 \mathrm{x}$ rays 3 times weekly for 6 weeks, with a total dose of 3,600 , resulted in 20 liver tumors in 37 animals $(55 \%) ; 20 \mathrm{x} x$ rays 3 times per week for only 3 weeks (total of $1,600 \mathrm{r}$ ), followed by intrasplenic injection of radioactive gold colloid the fourth week of azo dye diet, caused 10 tumor $s$ in 15 rats which survived 6 months or longer (66\%). In animals receiving 8c rays or radio-gold, the tumorg were larger, occurred earlier, and were multicentric. An unusual cytologic pattern found in many irradiated livers is described. It is concluded that sa $^{-}$ray or Au ${ }^{198}$ (chiefly $\beta$ ) irradiation produces a nonspecific liver-cell damage which increases the incidence of tumors produced by $\mathrm{m}^{\prime}$-methyl-dimethylaminoazobenzene. (NSA)

512

Wilson, Jarnes G., Robert L. Brent, and h. Charles Jordan. NEOPLASLA INDUCED IN RAT FMBRYOS BY ROENTGEN IRRADIATION. University of Rochester. Sept. 5, 1951. 30p. (UR-183)

Rat embryos $\mathrm{R}$-irradiated on the 9 th day of gestation developed discrete tumor-like growths in and around the brain. The incidence of such tumors was directly related to the dosage of $x$ rays. They first appeared on the 2nd day after irradiation and thereafter exhibited varying capacities for growth and differentiation. Some grew for 1 or 2 days and then disappeared as a result of dispersal of the colls, others grew rapidly until the 5th and $6 \mathrm{th}$ postirradation day and then became atrophic, and still others continued to grow slowly until the "fh or 8 th day and then became static or underwent atrophic regression. Few remained in term anmals and these were small and gave no evidence of proliferative activity. Although the tumor-bearing animals had a somewhat higher mortality than the nonirradiated controls, neither prenatal nor postnatal death could be attributed to the presence of the tumor's. (NSA) 
Yount, Nelson F, and George Z. Williams. THE EFTECTS OF IRRADIATION ON AZO DYE NEOPLASIA IN THE RAT LIVER. II. CATALASE, ALKALINE PHOSPHATASE, ARGINASE, AND RIBOFLAVIN LEVELS OF THE LIVER FOLLOWING X-RAY AND RADIO-GOLD IRRADIATION OF RATS FED DIMETHYLAMINOAZOBENZENE. Cancex Research 11, 291(1951).

Spraçue-Dawley albino rats were maintained for 24 werks on at synthet ir diet contaning $0.04 \% \mathrm{~m}^{\circ}$-methyl dimethylaminoazobrnzene. Groups were treated with (a) $250 \mathrm{r}$ ( $\mathrm{s}$ ray) at $250 \mathrm{kw} 3$ times weekly to $3,600 \mathrm{r}$, (b) intrasplenic inject ions of $2.0 \mathrm{mc}$ or $0.6 \mathrm{me}$. radioactive gold colloid, and $(c)$ combunation of $1,600 \mathrm{r} \times$ rays and $2.0 \mathrm{mc}$ of int rasplente radioartive Au colloid. Alkaline phosphatase ranged between 40 and 100 Bodansky units with diet and dye only. In livers contaming tumors but without irradation, it ranged from 75 to 110 ( 1 at 378 ), and in the livers contaning tumors and subjected to irradiation, levels were from 75 to 180 with 3 above 325 . Catalase was low durng periods on the ruboflavin-defucient diet but rose to normal levels after the anmals were returned to stock d1et. Ir radation produced no significant changes, and the levels were generally higher an the livers containing tumors. The riboflavm content of the livers was low (10 to $12,(g)$ with basal rboflavin-deficient diet and azo dye, and returned to normal levels of 20 to $30 \mu \mathrm{g}$ on stock diet. The majorty of livers of arradiated and tumorbearng anmals contaned less ruboflavin than normal appearme livers whout irradiation. Irradiation produced no changes in argmase levels. It is concluded that ultimate dietary changes in catalase and riboflavm levels are not influenced by irradiation in the doses applied, and the possible significance of the ehanges in alkalme phosphatase is discussed. (NSA)

\section{Effects on Immunity of Organisms}

\section{4}

Becker, Robert M. SUPPRESSION OF LOCAL TISSUES REACTVTTY (SHWARTZMAN PLIENOMENON) BI NTTROGEN MUSYARD, BENZOL, AND X-RAY IRRADIATION. Proc. Soc. Exptl. Biol. Med. 69, 247-50(1948).

Male albino rabbits were intradermally injected with 0.3 cc. of a solution of avirulent type I meningococcus filtrate or endotoxin in three areas, the epigastrium and the right and left lower quadrants. From 20 to 26 hours later, 2 cc. of undiluted meningrococcus endotoxin/rabbit or $2 \mathrm{cc}$. of the undiluted meningococcus filtrate/kg. body weight was in jected intravenususly through the ear vein. The effects of various agrents were studied to determine their suppressive action on the Sibwartzman phenomenon produced by the meningococrus. When 13 rabbits were exposed once to $800 \mathrm{x}$ of total body $\mathrm{x}-\mathrm{irradiation} 3$ to 7 days before the meningococcal injection there was almost a complete auppression of the reaction in 7 of the rabbits and complete suppression in the other 6 ; one animal which recelved 300 a three days before injection showed no suppression and two animals which received $800 \mathrm{r}$ of the total body irradia tion immediately before the injection also demonstrated no suppression. Benadryl, urethane, crude penicillin extract, penicillin $\mathrm{G}$, atreptomycin, mapharsen, $B A L$, vitamin $C_{3} \alpha$ tocopherol, thyroidectomy, and partial exsanguination had no suppressing effect, but pretreatment with nitrogen mustard or benzol caused complete suppression of the reaction. It is suggested that this study may serve as a basis for a new therapeutic concept in the treatment of diseases involving tissue and vascular reactivity. (NSA)
515

Bennett, L. R., Vera Collier, Marion Kresage, and Paul ${ }_{2}$ Rekers. A STUDY OF BLOOD AND SALIVARY LYSOZY ME IN THE X-IRRADIATED DOG. Alomic Energy Project, University of Rochester. Nov. 1, 1948. 13p. (UR-46)

Blood and salivary lysozyme activity has been studied in dogs receiving 350 r lotal body, singre dose, roentgen irradiation. The depression of blood lysozyme activity paral lels the depression of the white blood cell count postirradiation and reaches near zero levels by the 12 th day in animals that did not survive. The depression in leulocyte values and lysozyme activity is less pronouneed in surviving animals. The salivary lysozyme shows no significant change following ir radiation. (NSA)

516

Bisgard, J. D., H. B. Hunt, and R. H. Dickinson. OF X-RAY IRIADIATION UPON BACTERIAL TOXEMIA IN RABBITS, Radiology 43 , 330-2(1244).

Irradiation causes the production or claboration of a nonspecific antitoxin, produced by the tissues (but not by plasma or whole blood), irrespective of the position of the body ir radiated. The minimal dose is $20 \mathrm{r}$ to produce an $51 \%$ effective quantity of antitoxin in rabbits. (CA)

Bond, V. P., I. L. Schechmeister, M. N. Swift, and M. C. Fishler. THE IEFECTS OF X-IRRADIATION ON A NATURALLY OCCURRING ENDEMC INIECTION. NaVal Radiological Defense Lab. Nov. 29, 1950. 17p. (AD-273(B))

The effects of $x$ irradiation on animals from a laboratory colony in which a pulmonary infection was endemic were studied. The results indicate that irradiation increases the rate of spread of the disease, or "activates" infection that otherwise might remain latent in unirradiated animals, and that the $\mathrm{LD}_{50}$ for rats showing gross evidence of a disease that in itself does not kill immediately is reduced considerably below that for apparently normal animals. The necessity of maintaining a healthy experimental animal colony, particularly where investigations may extend over several weeks and involve a debilitating agent such ac xay, is emphasized. (NSA)

518

Borowskaja, D. EFFET OF ROENTGEN TRRADATION ON THE SERUM CONTISII OF HARMAGGLUTININS IN HUMAN BLOOD. Nature 158, 269(1946).

The author studied the possible influence of radiological stimulation of the reticulomendothelial system on the titre of anti-sheep agglutinins. Thirty-two persons were observed who received roentgentherapy for various causes (cancer, leukemia, inflammatory states, ete.). Dosages varied from 50 r to $6,000 \mathrm{r}$. In no case did the titre of sheep agglutinins after irradiation rise above the dilution of 1:10. Since the standard titre in normal individuals is generally equtvalent to the dilutions $1: 4-1: 8$ of the serum, the author concludes that the titre of gheep agglutinins after irradation remained normal. These observations show that $X$-rays do not influence the behavior of normal anti-sheep agglutining; hence the rise of the titre in some conditions probably does not depend on the stimulation of the reticulo-endothelial system, but on active immunization. (S.V.S.)

510

Bukantz, S. C., I. J. Diszor, G. J. Dammin, and D. W. Talmage. MPTABOLISM OF ANTIGENIC I" PROTEINS: ETFECT OF ANTIGRN AND HOST ALTRRATION. Washington Univ., St. Louis, School of Medicine. [nd] 2p. (ARCU-1239)

This report is reproduced here in its entirety. 
The mechanisms involved in the metabolism of antivens have been investigated (1) in normal rabbits with tracer labeled I* bovine sexum albumin, T* bovine gamma globulin (I*-BGG), and with I - BGG altered chemically by azo conjugation or iodination to saturation; and (2) with $-B G G$ injected into animals after whole-body irradiation, active immunization, passive immunization, and in a specific anamnestic response. I*-BGG disappeared from the circulation in three phases ( $75 \%$ in $24 \mathrm{hr}, 92 \%$ in $96 \mathrm{hr}$, and virtually $100 \%$ in 9 days) and was never retained in $0 r^{2}$ concentrated by tissues. Complement fixing antibody apperred by the 7th day, was maximal at the $10 \mathrm{~h}$, and disappeared by the 20 th day. By contrast, $R-s a l t-a z 0-b i p h e n y l-a z o$ I* BGG and P-amino bemzolc acid-azo-1 - BGG disappeared from circulation precipitously $190 \%$ loss in $6 \mathrm{hr}$ and $99 \%$ loss in $24 \mathrm{hr}$ ) and was retamed in the IRE macrophages of several organs, notably spleen, liver, and lymph nodes, will an approwinate tissue half-life of 3 to 7 days. The Isaturated antigen left the circulation as rapidly as azo antigens, with less tissue retention. Urine excretion of ${ }^{131}$. containing protein breakdown products reflected the blood and tissue alimination of antigen. $X$ rays (300 or more r) did not effect the nonimmune rate of elimination of antigen, although the immune response was suppressed. The intravenous injection of antibody to IF-BGG produced a prompt decrease in circulating antigen in proportion to the amount of antibody injected. Finally, in a specific anamnestic response, anigen disappearance during the finst 2 days was at the usual nonimmune rates, and a typical immume rate 520 began at the $3 r d$ day. (NSA)

Burrows, William. ESTECT OR X-IRRADIATON ON FNTERIC CHOLERA AND COPROANTIBODY RESPONSII IN THE GUINEA PIG, D.131 Of BOLOGCAL AND MEDICAL DTVISTONS, QUARTERL REPORT, $\triangle$ UGUST, SEPTEMBRR, OCTOBER 1949, Austin M. Brues and Herman Lisco, eds. Argonne National Isab. [nd] 1p. (ANH-4360(p.131)) (5)

This report superceded by the paper abstracted as 522 .

Burows, Whllam, Norma G. Deupree, and Dorothy Moore. FIPERTMENTAL ENTERIC CHOLENA IN THE X-IRRADIATED GUINEA PIG, p.110 OI BIOLOCICAL AND MLDICAL DIVISIONS QUARTERLY REPORT, FIBEUARY, MARCP, APRL 1950, Austin M. Brues, ed. Agonne National Lab. [nd] $1 \mathrm{~g} .(\mathrm{ANL}-4451(\mathrm{p} .110))$

This report is superceded by the paper reviewed in the following abstract. 522

Burrows, WIIIam, Norma G. Deupree, End Dorotly E. Moore. THE FTE MENTAL ENTIEIC CHOLERA IN TME GUWEA PIG.

J. Intections Diseases 87, 158-69(1950).

The effect of $\mathrm{x}$-ixradiation in doses of $100-200 \mathrm{r}$ on enteric cholera in the guinea pis was assayed by two methods, autopsy culture of animals sacrificed periodically, and as numbers of vibrios and titer of antibody excreted in the feces. The period of deaths produced by doses of $150 \mathrm{~F}$ was associated with generalized bacterial infection in the majority of animals examined, coincidence consistent with the belief that a bacteremia of intestinal origin is part of radiation sickness. Of the animals examined at autopsy $6 \%$ were so infected, and of these $44 \%$ were infected with the cholera vibrio, and the remainder with hemolytic streptococci and staphylococci. Whole body ir radiation with $x-$ ray in this dosage, then, resulted in a generalized tissue invasion by the cholera vibuio from a focus in the bowel which is not observed in the infected nonirradiated animal. The course of the infection was also slgnificantly alterod by $\mathrm{x}$-irradiation. With doses of 150 and $200 \mathrm{a}$ the intersity of the infection was redured in the first 3 days following inoculation concurrent with a stimulus of coproantibody response, but subsequently the ant body titer drolined and the intensity of the infertion ancreased in the period during which tissue invasion orcurred. While the infection was appreciably reduced in its intensity in its early stages by doses of $100 \mathrm{r}$, coproantibody response was not effectively inhibifed and both intensity and duration of the infection were reduced over that in the nonn radiated animal to give an over-all the gaputic effet of $x-1$ ay in this dose. The observed inverse relation betwen coproantibody on the one hand, and the intensity and tendeney for Eeneralization of the enteric infection on the other, provide additional support for the hypothesis that effective immunity to enteric infection is in part anction of coproantibody. If it be assumed that natural immunity to bacteria of the intestinal flora is also related to coproantibody response, the inhibition of this immune responss by lethal dosess of $x$-ray may account for baeteremiat of intestinal origin in radiation sucknes. (Authors' Summary) 523

Burpows, W., Norma G. Deupree, and Dorothy E. Moore. THE TEFECT OF X-IRRADIATION ON FECAL AND URINARY ANTIBODY RESPONSE. J. Infectious Digeases 87, $169-83(1950)$.

The ffect of pre-and postimmunastion irradiation with s.ray in doses ramping from 50 to $200 \mathrm{r}$ on the immure $\mathrm{re}$ sponse of guinea pirs to cholera 0 vaccine was assayed as serum, fecal, and urinary antibody (agglutinin). Irradiation followink immunization dad not affect serum antibody titer appreciably, but in doses of 100 and $150 \mathrm{r}$ partially or complety inhloited the excrotion of fecal and urinetry antiludy, and the excretion could be partially restored by booster inotulation. Preimmunization irradation resulted in shaply aduced serum antibody titc $x$, but produced a transitory stimulus of fecal and urinary antibody, apparent when immunization was begun at one day, but not 3 days, 228 ater irradiation. (Authors" Summary)

Clemmes? I. THE INE LUENCE OF ROENTGEN RADIATION ON IMMUNTTY TO SHOPE FIBROMA VIRUS. AM. T. Cancer $35,378-85(1939)$.

Shope fibroma virus injectod into rabbits treated with a general roentgen irradiation produces results similar to those described by Andrewes and others in rabbits mected with tas", namoly prolonged growth of the resultant funvo mata, prolonged resorption, and peneralized fibromatosis after intravenous inoculation. The development of inmunity to repeated inoculations with this virus is relayed in $x$-rayed rabbits. The effects of a general roentgen ixradiation on transplantation of tumor and nor nasal calls, inoculation of ibroma virus, and artificel carecinosenessis, have their parallels in the effects of injections of tar and of trypan blue. All these procedures probahy act through the retituloendothelial system. (BA) 525

Clemmesen, Johannes, and Else Krag Andersen. INELUENCE OF IRACTIONED ROENTGEN DADIATION ON BACTIRLAL AGGLUTINATION TITRE. Acta Pathol. Microbiol. Scand. 25, 611-14(1948).

Rabbits were given tolal body irradiation in effective doses of 120 rive times in this investigation of the possibilities offered by $X-r a y$ iradiation as a means of lowering the resistance of experimental animals 10 inoculations of various dinds. Immunization was carnicd out by a single intravenous injection of $0.1 \mathrm{cr}$-Antigen of 3 . paratyphus B (formolized broth-culture). From the agglutination tifer curves and their goners axperinee the athos conclude: (1) Fractioned dosis are more ffective for lowering of 
resistance than singlo doses. (2) It seems possible to prevent development of immunity without serious damage to the greneral health of the animals. (3) A dose of rays which if given before immunisation will delay the development of immunity does not show the same effect in applied after immunization. (4) If an insufficient part of the radiation has been applied before immunization, the remaining dose given after the latter will be more effective than the total dose ziven at the same period. Even an immunity otherwise apparently refractory to radiation may in this way be prevented from developing. (5) The effect may be visualized as a blockage of the reticuloendothelial system which must be established at the appearance of antigen and must be repeatedly renewed in the presence of the latter. 3 tibles and 6 references. (NSA)

526

Cohen, Sheldon G., Lloyd D. Mayer, and Leo H. Criep. THE EFFECT OF X-RADIATION ON EXPERIMENTALLY PRODUCED CUTANEOUS SENSITIVITY. J. Invest.

Dermatol. 16, 91-6(1951).

Albino guinea pigs were sensitized by the daily injection for 7 days of $0.1 \mathrm{ml}$. $0.1 \% \mathrm{p}$-phenylenediamine into the skin. Of these test animals 19 were then exposed to 175 roentgens of total body irradiation filtered through $0.5 \mathrm{~mm}$ $\mathrm{Cu}$ and $1 \mathrm{~mm} \mathrm{Al}$, at a focal distance of $64 \mathrm{~cm}$, and after 16-24 hours again tested for skin censitivity to the reagent; 5 animais exhibited a positive reaction, 11 an attenuated response, and 5 a negative skin reaction, while of 18 sensitized but not irradiated animals, 14 showed a marked positive reaction and 4 and attenuated reaction. The modification of the degree of severity of cutaneous hypersenstivity is accompanied by a destruction of lymphoid cellular elements. (CA)

\section{7}

Craddock, Charles G. Jr. and John S. Lawrence. THE EFFECT OF ROENTGISN IRRADIATION ON ANTIBODY FORMATION IN RABBITS. J. Immunol. 60, 241-54(1948).

The new importance placed on the role of lymphoid tissue and circulating lymphocytes in ant ibody production, and the well-known detrmental efferts of $X$-rays on lymphoid tissur morphologically led to this study in which the attempt is made to correlate the influence of roentgen ir radiation on antibody production with that on lymphoid tissue. Ralbits were exposed to $250 \mathrm{r}$ whole-body irradations and given pre-or post-irradiation injections of antigen. Antibody production was markedly decreased when injections were made following irradiation. This is of a transient nature and the animals regained their ability to respond normally to antigen injection 40 days after exposure to $\mathrm{X}$-ray. Irradiation, however, after the onset of antibody production does not impair ability to produce circulatory antibodies. The reason for this remarkable difference is not apparent, but it is suggested that reserve sourees of tissue must be capable of carrying out antibody formation once it has begrun even though the major portion of lymphoid tissue has been damaged. (NSA) 528

Craddock, Charles G., Jr., William N. Valentine, and John S. Lawrence. THE EFTECT OF MASSIVE WHOLE BODY X-RAY ON THE ANTIBODY CONTENT OF LYMPE, p.166-9 of THE LYMPHOCYTE STUDIES ON ITS RELATIONSHIP TO IMMUNOLOGIC PROCESSES IN THE CAT. J. Lab. Clin. Med. 34, 158-77(1949).

Lymph specimens were collerted every two hours for eight hours following irradiation by a single total body dose of $1,500 \mathrm{r}$. In spite of widespread destruction of iymphoid cells, no appreciable alteration in antibody concentration of the lymph was detected. No consistent correlation was found to exist between the height of the serum antibody liter and that of the lymph. Thus, in several instances no antibody could be detected in the lymph, in spite of a significant serum antibody titer. The concentration of serum antibody at the end of the experiment was higher than that 529 of the lymph in all cases except one. (NSA)

Criep, Leo H., Lloyd D. Mayer, and Sheldon G. Cohen. EFFECT OF X-RAY RADIATION ON HYPEISENSITIVENESS. \$. Allergy 21, 269(1950).

This paper deals with the author's' experience in the evaluation of the effect of $x$-ray radiation on the development of diffuse vascular disease (collagen disease), on the Arthus phenomenon, on the Schultz-Dale reaction, and on the production of precipitins in sensitized animals. The experiment indicates that interference with the function of the reticulo-endothelial system depresses precipitin titers, completely abolishes the Arthus phenomenon, and interfers with the production of diffuse vascular changes so obviously present as a result of sensitization in untreated animals. (NSA) 530

Dizon, F. J., S. C. Bukantz, and G. J. Dammin. THE EF FECT OF SZNSTTIZATION AND IS-RADIATON ON THE METABOLISM OI IODINE 131-LABELED PROTELNS. Science 113, 274-6(1951).

Rabbits immunized by an intramuscular injection $325 \mathrm{mg}$ bovine $\gamma$-globulin (BGG) eliminate injected $\mathrm{I}^{134}-\mathrm{BGG}$ much more rapidly than controls although $\mathrm{z}-\mathrm{radiation}$ interferes with the development of an immune state. Fifty $\%$ of the injected activity is found in the urine of mmunized rabbits by the third day, of the control group by the fourth day, and of the x-radiated group by the sixth day. In fatal anaphy laxis there is rapid loss of activity from the blood and rapid accumulation in the lungs. (CA) 531

Dixon, F. J., D. W. Talmage, and S. C. Bukantz. RADIOSENSITIVE AND RADIORESISTANT PIASES IN ANTIDOOY PRODUCIION. Washington Univ., St. Louis, School of Medicine. [nd] 2p. (ARCU-1241)

The report is reproduced here in its entirety.

The radiosensitivity of the antibody response is well known, but the time relationships of radiation, antigen administration, and antibody suppression or response have not been completely explored. The following observations were made in rabbils using $1^{131}$-labeled bovine gamma globulin as antigen. Antibody was determined by complement fixation, employing four $50 \%$ units. (1) Antibody re sponse to initial antigen exposure is completely suppressed by $300 \mathrm{r}$ or more whole -body radiation given $40 \mathrm{hr}$ before injection of antigen. With $500 \mathrm{r}$ this suppression is evident for 6 weeks. (2) However, no suppression of initial antibody response is noted following $500 \mathrm{r}$ given simullaneously with antigen, and only partial antibody suppression follows $500 \mathrm{r}$ given $2 \frac{1}{\mathrm{~s}} \mathrm{hr}$ before the antigen. (3) The specific anamnestic response, unlike the initial antibody response, is not prevented by $500 \mathrm{r} 40 \mathrm{hr}$ before the second injection of antigen. It is apparent that within a matter of hour after 300 to $500 \mathrm{r}$ radiation an animal loses a substance necessary for the production of antibody. However, since simultaneous radiation and antigen administration does not diminish antibody production in spite of the fact that the necessary radiosensitive substance can persist for only a matter of hours, it would appear that the radiosensitive portion of the antibody response lasts only a few hours. The remainder of the antibody response, during which the antibody protein is actually produced, lasts several weeks and can be carried on undiminished in spite of radiation. The complete radioresistance of the specific anamnestic response indicates a lasting orientation of antibody - 
producing sites to the specific antigen, so that on second antigen administration the radiosensive initiation phase of antibody production is unecessary. (NSA) 532

Mphat, Erela. MECHANISM OF THE EFIECT OI XDAYS ON BACTERIAL TOXINS. Biochem. $7.42,383-9$ (1948).

Staph. hemolysin is much more sensitive to destruction by $x-r a y s$ than tetanus toxin, and purification of the toxin seatly increases its sensitivity. In either case antigenicity and toxicity are destroyed simulaneously. The effect of x-Iays is indirect, through ionizalion, leading either to oxtcation or reduction. Th the case of toxng all the facts indicate that an oxidation leserensible for the destruction. Various proteins, reducing amino acids, glutathione, thioglycolic and ascorbic acids are all affective as protective agents. Okidizing substances we inef fective. (CA) 533

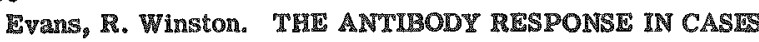
OF RADATON LYMPHOPTNA, ANO IN TYZ RETHCULOSES. T. Pathol. Bacteriol. 60, 123-30(1948).

The antibody response was tested in a series of 30 patents exhbiting a radiation-induced lymohopenia and in a control group of 17 individuals with a normal bloodlymphocyte level. A suspersion of Bact. paratyphosum A(H) was used as antigen. A lower level of antibody production was mecoxded in patients showing a low lymphocyte count. The significance of this finding is nelated to the im munological function of the lymphocyte. A series of reticulo-endothelloses comprising cases of myelocytic and lymphatic leukaemia, Hodgkin's disease, Iymphosarcoma and ymphofollicular reticulosis was also tested for agFlutinin response, which was found to be very poor except in the myelocytic leukaemias. This poor response is attributed to the degree of disorganization of the lymph ghands in these conditions. (NSA)

534

Wischel, Fdward M., Marjorie LsMay, and EIvin A. Kabat. THE EIFECT OF ADREIOCORTICOTHOPHC HORMONE AND X-RAY ON TIIE AMOUNT OF CIRCUIATMNG ANTI-

BOD盗. T. Trmmunol. 61, 80-93(1249).

Experiments initiated to determine whether $X-r a y s$ and the adrenocorticotrophic hormone are able to cause an anamnestic or secondary response in chrculating rabbit anthodies are described. One group of rabbits recesved 25 mg. of $75 \%$ potent $A C T H$ subcutaneously, while the other group recelved $50 \mathrm{r}$, spray radiathon with $134 \mathrm{kw}$ and 25 mas a distance of $35.25 \mathrm{~cm}$. wilh $2 \mathrm{~mm}$ Cu and $1 \mathrm{~mm}$ Al filters. Blesding times and white cell counts were obtained at 6,12 , and 48 hour after the administration of the ACTI and the $\mathrm{x}_{\mathrm{H}}$-rays. The results are presented in two tables. A fall of more then $50 \%$ in the total number of circulating Iymphocytes occurred after the treatments for a corresponding change in the total white count either in the same of lif the opposite disection. The serum antiovalbumin content was elher the same or diminished in all of the rabbits. Therefore, the expected anamnestic change was not demonstrable by the quantitative precipitin-method used. (NSA)

35

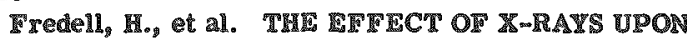
ANTIDODTES (A STUDY OF HEMOLYSIR TITIRATIONS

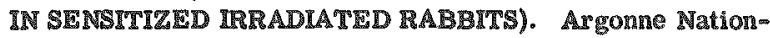
2L Lab. Aug, 1, 1947. Decl. May $26,1948.172 \mathrm{p}$. (AECD-2024)

rmperiments were conducted using the rabbit as a lest animal and measuring the antibody production in terms of hemolysins produced by sheep cell antigen. A group of 16 rabbits was sub-divided into four groups of four animals each. The first group served as control; the second group was is radiated at the peak of the antibody response, approximately 10 days after antigen administration; the third group was irradiated on the day of antigen administration; and the fourth group was irradiated on the day of antigen administration and reirradiated at the peak of antibody re. sponse. All irradialed animals recetved about $550 \mathrm{z}$ at dosage rates of 16 to $17.7 \mathrm{x} / \mathrm{minute}$, using $0.5 \mathrm{~mm} \mathrm{Cu}$ and $1.0 \mathrm{~mm}$ Al filters. In addition, the animals in group four received a second dose of 550 r. A single dose of 550 r total body $\mathrm{x}$-radiation depressed sheep cell antibody production in rabits. When $550 \mathrm{~g}$ was administered at the time of sensitization, antibody production 10 days latar was, for all practical purposes, completely inhibited within the period of study. (MSA) 536

Glenn, Tohn C.Tr. FURTYER STUDIES ON THE INELUENCE OF X-RAYS ON THE PHAGOCYTIC INDICES OF IIEACTIV RABBITS. J. Immunol. 53, 95-100(1946).

A preliminary study with 18 rabbita showed that the phagocytic indices of healthy rabbits teated with staphy lococcus aureus increased significantly 48 to $96 \mathrm{hr}$ follow 1ng radiation by 100 I (measured in air) delvered at $140 \mathrm{kv}$ over a small area of normal shin. In a second experiment using 35 rabbits, 211 treated animals received radiation generated at $140 \mathrm{kv}$ through a $6 \times 6$-cru port over the inner surface of the unshaven and unclipped right hind thigh of from $50 \mathrm{x}$ up to $1,000 \mathrm{r}$, given either in single or in tepeated doses. It was found that the phasocytic index of the white sabbit can be most effectively increased by a dose of $100 \mathrm{r}$. The maximal increase occurs $48 \mathrm{hr}$ after treatment. Repetition of the optimal dose produces an increase in the phagocytic index which is only moderately higher than that obtalned with a single dose, but the index may be maintained at a high level for a shor time. There is a definite tolerance of the animalg to $\mathrm{x}$ rays for producing an fil crease in the phagocytic index, beyond which point a depresision occurs. Previousiy irradiated animals show a return of the phagocytic index to normal varying lengths of time following treatment, so that subsequent treatments following this event again lnstitute a zolse in the phagocytic index which Is identical with that observed in untreated animals. TIS

537

Goldman M. O., Leon O. Jacobson and Melba J. Robson. PRELIMINARY REPORT; THE EFTECT OF SPLELN AND APPENDTX PROTECTION ON THE SHWARTZMAN TEACTION, $1.32 \mathrm{~m}$ OF BOLOCTCAL AND MPDICAL

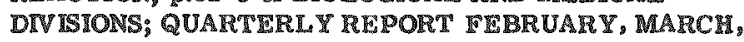
APRIL, 1950. Argonne National Lab. Ind] \&p. (ANL$44510.32-5))$

In rabbits the administration of an intradermal injection of meningococcus endotoxin followed 24 hr later by arin in travenous injection of the same material results in a severe hamor rhagic necrotic reaction at the intradermal gite within 2 to $6 \mathrm{hr}$ after the secorth injection. This phenome-

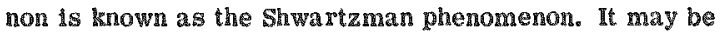
guppressed by such ogents as nitrogen mustard, rays $_{3}$ and benzol if these are administered in large doses prior to intradermal injection. Cxperiments are in progress to investigate the possibilities of altering his suppressive action with the view of finding explanations for the Shwartzman phemomenon. It was found that local ir Iadiation (800 r) administered to the skin of rabbits did not suppress the reaction. Data indlcate that suppression of this phenomenon by total-body $\mathrm{x}$ ir radiation is dependant upon some generallaed change throughout the body, that $\mathrm{Pb}$ pro- 
tection as such does not alter this condition, but that trauma to the abdominal viscera at the time of irradiation

do

Gowen, John W. MODIFYING DISEASE RESISTANCE THROUGH RADIATION, p.55-8 of CONFERENCE ON BIOLOGICAL APPLICATIONS OF NUCLEAR PHYSICS.

Brookhaven National Lab. July 1948. 3p. (BNL-C-4)

Author discusses host variability toward resistance or susceptibility, bacterial variation in disease, heriditary variation in acquired immunity, phenotypic mechanism by which resistance is accomplished, and $\mathrm{x}$-ray effects on resistance. (S.V.S.)

539

Gowen, John W. RADIATION EFTECTS ON MICE AS RELATED TO SURVIVAL. Genetics $35,112(1950)$.

The effects of acute irradiations on the subsequent lengths of life of the exposed individuals have evolutionary, as well as current, interest. Genetic variations exist in different hosts; genetic diversity is found in the organisms intiating a disease; and the envirommental setting in which the disease is to occur is subject to variation. The type and character of the radiation to which elther the hosts or the pathogens are exposed may be considered enwironmental variations. In examining the interactions of these factors, the discussion will be limited largely to the mouse and its typhoid disease caused by Salmonella typhimurium. Six inbred mouse strains of characteristically different typhoid susceptibility ( 8 to $90 \%$ ) and leucocyte counts were exposed to $X$-rays in doses from 0 to 700 roentgens/ $/ \mathrm{cm}^{2}$ of body surface. All six strains reacted alike. Radiation throughout decreased resistance. The decrease in resistance was an exponential function of the dosage of X-rays to which the mouse was exposed. The data, while quite ir regular due to limited numbers, show the same rate of decrease in restatance for each of the strains. Resistance at $600 \mathrm{r}$ exposure was decreased to about $43 \%$ of that expected with no radiation. These results are explained by the exponential destruction of the phagocytic cells by the acute Irradiation. Mice were blood counted, 1rradiated, and the cell counts taken again at one and two weeks after treatment. The results show a corresponding decrease in leucocyte numbers for each of the strains. The decrease, like that for survival to typhoid, is exponential. (RG) 540

Rollingsworth, J. W. ERFECTS OF X-IRRADIATION ON PASSIVELY TRANSFERRED ANTIBODY. PrOC. SOC.

Exptl. Biol. Med. T5, $477-9(1950)$.

$\mathrm{X}$ irradiation in dosage of $300 \mathrm{r}$ had no effect on the rate of destruction of passively transferred homologous or heterologous antisera given intravenously to rabbits $24 \mathrm{hr}$ after irradiation. This X-ray dosage was suffictent, how-

ever, to decrease, but not totally prevent, the formation of
antibody to the heterologous serun proteins. (NSA) 541

Jacobson, Leon O., Melba J. Robson, and Ldna K. Marks. THE EFTECT OF $\%$-RADIATION ON ANTIBODY FORMATION. Proc. Soc. Expt1. Biol. Med. 75, 145-52(1950).

Experiments in which rabbits have been exposed to $800 \mathrm{r}$ of $\mathrm{x}$ radiation have confirmed previous findings (Hektoen, J. Infect. Dis.s 17: 415(1915)) that antibody formation is suppressed by total-body x radiation. It has been demonstrated, in addition, that if the spleen or appendis of the rabbit is protected by lead shielding during total-body ll radiation, the capacity to produce antilbodies to an injected particulate antigen is retained to a marked degree even though lymphatic tissue elsewhere in the body is temporarily destroyed. (NSA)
542

Jacobson, L. O., M. E. Robson, E. IK. Marks, and M. C. Goldman. THE EFIECTS OF X-IRRADIATION ON ANTIBODY FORMATION. J. Lab. Clin. Med. 34, 1612-13 (1949).

This communication describes an attempt to study the capacily of the rabbit to form antibodies during a period in which all lymphatic tissue of the body, with the exreeption of the spleen or appendix, is essentially destroyed. Young rabbits were given $800 \mathrm{r}$ total body $\mathrm{g}$-irradation (exclusive of the lead-protected spleen or appendix) at $250 \mathrm{kw} 24$ hours prior to an intravenous immunization with sheep cells (1 ce. of a $2 \%$ suspension). Memolysin titer were determined on serum obtained before immunization and on samples taken $7,14,21$, and 28 days after immunization. Normal titers were 1 to 512014 days after the injection; in the irradiated animals titers of 1 to 5120 were not observed until the 21 st day after the injection. It is concluded that these experiments corroborate Helktoen's findings that antibody formation is suppressed by total body irradiation and also show that the capacity to produce antibodies to an injected particulate antigen is not lost when some part of the body such as the spleen or the appendix is protected from radiations 543 which degtroy lymphatic tissue. (NSA)

Jude, A., P. Bonet-Maury, and P. Servant. ACTION OF IONIZING RAYS UPON ANTIGEN VI OF SALMONELLA TYPHY (INAGGLUTINABLE O AND AGCLUTINABLE Vi).

Compt. rend. soc. biol. 144, 214-16(1950).

In this note the action of the particles of Rn upon antigen Vi of Salmonella typhi is recorded. Antigen Vi prem served its serological characteristics even at elevated doses of radiation. Radiation dosages up to $40 \mu \mathrm{cd} / \mathrm{cm}^{\$}$, nearly 3 times the sterilizing dose, were used. Neither inagglutinable antigen $O$ nor agglutinable antigen $V i$ were destroyed. (NSA)

544

Kohn, Menry 1. TRE EFIECT OF TOTAL-BODY X-IRTA DIATION UPON THE HUMOLXSIN RESPONST OT THE RAT TO SHIEP ERYTHIROCYTIS. J. Immunol. $66,525-$ 33(1851) (ORNL-391)

$X$-rays were administered to rats at various times during the week before and the five days after a single injection of sheep erythrocytes. When given at any time dur ing this interval, $300 \mathrm{r}$ (CD-50,725 $\mathrm{r}$ ) depressed the hemolysin titer, which is defined as the reciprocal of the dilution of plasma causing $50 \%$ hemolysis in a $0.7 \%$ suspension of sheep erythrocytes. A given dose of $\mathrm{X}$-rays (175-850 r) became progressively less effective as the time of its administration was delayed after the injection of the antigen. The data show that $x-r$ rays given either before or after immunization can inhibit antibody production. The effect of the X-rays upon antibody production always involved a decrease in the peak titer; and under certain conditions, the development of the depressed peals was delayed. Therefore in certain instances, the liter in the irradiated animal, at first below the control level, exceeded the control when the latter subsequently declined. (NSA)

545

Lecomte, J. and P. Fischer. CHRCULATING ANTMODIRS AND TEE ALARM SYNDROME BROUGHT ON BY W-IRRADIATION. Compt. renil. soc. biol. 143, 878-2(1949).

Studieg were carried out to determine whether the dem struction of lymphocyles by $\mathbb{X}$-irradiation is followed, in the sensitized rabbit, by an Increase in the amount of cir culating antibodies. The authors point out the importance of the lymphatic ganglia and the lymphocytes in the producton and accumulation of antibodies: he injection of any 
antiycen is followed swiftly by the appearances of antibodies in the lymphatic ganglia draining the regaon. Four rablits (the serum of which had been rendered greatly precipitant with respect to horse serum) we ae irradiated with $200 \mathrm{r}$ on their hind legs, after the withdrawal of a control sample of blood; samples were also taken three hours after, and four days after the irradiation. If was discovered that the destruction of the lymphocytes which accompanied the alarm syndrome caused by the administration of the $\mathrm{X}$-rays was not followed by a simultaneous increase in the amount of 546 circulating antibodies. (NSA)

Richards, 0 . W. TEAST GROWTH: A POSSIBLE TEST ORGANISM FOR X-RADIATION. Radiology 34, 317-26 (1940).

Populations of a pure strain of Saccharomyces cerevisiae were radiated with from 9500 to $28,500 \mathrm{r}$ at $70 \mathrm{kv}$, and with from 200 to $38,000 \mathrm{r}$ at $200 \mathrm{kv}$ under carefully controlled, established conditions for yeast growth. The increase in numbers of cells per unit volume is retarded by the $\mathrm{x}$ rays. Increased optical densities of the populations were observed with a nephelometer for dosages up to $15,000 \mathrm{p}$, and decreased density for greater dosages. Recovery takes place gradually, and the irradiated cultures reach about the same equilibrium level as the controls by the end of the growth cycles. Percentages of buds and of cells straining with methylene blue are given. While a single criterion of growth may be useful, the analysis of the effect of $\mathrm{x}$ radiation on yeast growth requires the information gained from the simultaneous use of several different criteria and methods for the measurement of the growth of yeast popum lations. (BA)

547

Sarian, Jiraix N. EFFECT OF PHYSICAL AND CHEMICAL FACTORS IN PHAGOCYTOSIS IN THE PR ESENCE OF IRRADIATED HUMAN BLOOD AND PLASMA. Am. J. Roentgenol. Radium Therapy 65, 465-71(1951).

Anticoagulation measures, particularly citration, handicap phagocytosis in the presence of both normal and irradiated blood and plasma. Normal blood and plasma irradiated with 50 and $100 \mathrm{r}$, respectively, did not lose their increased phagocytosis-enhaneing properties after storing at $0^{\circ} \mathrm{C}$ for 48 hours. Nonirradiated samples showed a slipht increase in their phagocytic powers after storing. Heating for 15 min. at $65^{\circ}$ did not destroy the opsonins and agglutinins brought out by small-dose $x-r a y$ irradiations of blood and plasma. Softer ray seened to be more potent in producing such thermostable substances. Diluting the $\mathrm{x}-\mathrm{ray}$ dose optimal for phagocytosis does not cause a proportional de548

Sarian, Jirair N. IRRADIATED HUMAN PLASMA AND PHAGOCYTOSIS. Am. J. Roentgenol. Radium Therapy 65, 940-6(1951).

In an attempt to understand the mechanism of the affects of roentgen rays on inflammations, the author briefly reviews the general theories and then describes his own experimental findings. Small doses of roentgen rays, 15 to $100 \mathrm{r}$, cause a significant rise in the opsonic indices of normal human blood. The injurious effect of $30 \mathrm{r}$ on leucocytes, although small enough to fall within the range of experimental error, is yet demonstrable funetionally. Ir radiating plasma previously separated from normal human blood enhanced phagocytosis to a degree slightly less than that attained by using plasma derived from whole blood irradiated with the same dose. Comparison as to phatrocytosis of Escherichia coli and Staphylococcus aurcus indicates that irradiation either produces, or more probably activates, the specific opsonins of the normal plasma. Thus convalescent or immune sera rould be used (both therapeutically and prophylactirally) more advantageously by irradiating them with optimal doses of roentyen rays. Such irradiated sperific sera could be of particular value in the control of certain virus diseases, for example, neurotropic 549 polionyelitis. 55 references. (NSA)

Schwab, Louis, Frederick C. Moll, Thomas Hall, Henry Brean, Marjorie Kirls, Clinton Van Zandt Hawn, and Charles A. Janeway. EXPERIMENTAL HYPERSENSITIV ITY IN THE RABBIT. J. Exptl. Med. 21, 505-26(1950).

The experiments described here involve the investigation of the inhibition of antibody formation by $\mathrm{x}$-radiation, the relationship between complement and serologic sequences, and the relationship between complement, serologic se-m quence, and histologic changes. In the first experiments several rabbits were subjected to 150 to $400 \mathrm{r}$ per dose of $\mathrm{x}-\mathrm{rays}$, delivered 5 to 8 limes at interval of several days, both before and after the intravenous injection of whole bovine serum or bovine serum $\gamma$ globulin. Tests for the presence of antigen and antibody werc made 9 to 28 days later. Sirmilar experiments were performed with nitrogen mustard instead of the x-radiations. Serum complement determinations, as well as tests for the presence of antigen and antibody, were made in subsequent experiments on rabbits. Other experiments are also de'seribed. It was found that $x$-radiation and nitrogen mustard inhibit the formation of precipitins for whole bovine serum and bovinf serum, globulin in the rabbit. Other data presented are interpreted as evidence in favor of the concept that the lesions seen are due to a reaction between antigen fixed un or on tissue cells and circulating antibody. The possible significance of serum complement in the pathogenesis of anaphylactic tissue lesions is discussed. 68 referencas. (NSA)

550

Shechmeister, I. L. and V. P. Bond. THE RESPONSE OF MICE TO INOCULATION WITH CERTAIN AVIRULENT BACTERIA AFTER EXPOSURE TO SUBLETHAL TOTAL BODY X-IRRADIATION. Proc. SOc. Emptl. Biol. Med. 77, $77-80(1951) \quad(A D-233(B))$

Mice exposed to sublethal doses of $\mathrm{x}$ irradiation and subsequently inoculated with live avirulent, or even dead, organisms generically different from the bacteria normally present in the intestines of these animals exhibited a hiph incidence of bacteremia and death. When avirulent live bacteria were injected, the organisms recovered by sacrifice and culture were either of the strain inoculater or were apparently autogenous bacteria. When heat -killed bacteria were introduced, the recovered organisms were of autopenous origin only. (NSA) 551

Shechmeister, I. L., V. P. Bond, and M. N. Swift. THE SUSCEPTIBILITY OF IRRADIATED MICE TO INFECTION AS A FUNCTION OF POST-IRRADIATION TIME. Naval Radiological Defense Lab. Dec. 12, 1950. 22p. (AD-279(B))

The susceptibility of mice to airborne Streptococeus zoo-epidemicus was determined over a 50mday period fol -

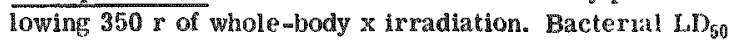
values were determined for normal and irradiated mice on 12 different postirradiation days during this period, and the changes in susceptibility in each case were expressed as the ratio of the two. The resulting curve relating susceptibility to postirradiation time indicated that there was a linear increase in susceptibility over the first 15 days, which aeached a peak five limes that of controls. This was 
followed by an exponential drop which approached the control level shortly after 30 days. The possible relationship of this phenomenon to other radiation effects is discussed. 552 (NSA)

Taliaferco, William H. and Lucy Graves Taliaferro. EFFECT OF X-IRRADIATION ON HEMOLYSIN DECLINE. J. Infectious Diseases 87, No. 3, 201-9(1950).

$X$ irradiation in 2 doses of $400 x$ to the entire body of rablits during the decline of hemolysin 2 to 4 weeks after immunization with one or a series of intravenous injections of sheep red cells did not alter the downwa and trend of the titcr as compared to cont rols not irradiated. Three intravenus injections of ovalbumin $9 \%, 11$, and 12 weeks after immunization only alicited a possible heterologous 553 anamnestic response to red cells in 1 of 7 rabbits. (NSA)

Taluaferro, William H. and Lucy Graves Taliaferro. EFFECT OF X-RAYS ON IMMUNITY: A REVIEW. J. Immunul. $66,181-212(1951)$.

Small amounts of $x$ rays, often administered locally, stmetimes enhance antibody formation and the immunity of exporrmental laboratory animals to nonliving antigens and certain infections. This conclusion has also been reached with respect to a wide range of infections in man, but may not in all cases be justified because of inadequate controls. The mechanism involved in an enhanced immunity by $x$ rays has been variously esplained, but has not been adequately clarufied. Large amounts of $x$ rays have occasionally been reported to produce beneficial effects in the host, but fur ther work on this aspect of the subject is needed. A wellestablished instance orcurs in trichinosis. In this infection, treatment of the host with large doses of $x$ rays directly inhibits early embryonic development of the worms. $X$ rays approaching lothal amounts whern administered to the ent ire body ofter dererease host immunity to specific infections and lower antibody formation. The mechanism whereby immunity is reduced noeds further experimental analysis but is, in large part, reasonably explained by the pronounced radiosensitivily of lymphocytes (including various "Blasts"). There is no evidence that $\mathrm{x}$ rays enhance the virulence of the parasites or affect their antigenicity. The differential effertiveness of the same dose of $x$ rays in different infections and at different periods of the same infection may be partly correlated with the degres of immunity present at the time of radiation. 201 references. (NSA)

554

Taliaferro, William K., Lucy Graves Taliaferro, and E. L. Simmons. INCREASED PARASITEMIA IN CHICKEN MALARIA (PLASMODIUM GALLINACEUM AND PLASMODIUM LOPHURAE) FOLLOWING IS-IRRADIATION.

\section{J. Infectious Diseases 77, 158-76(1945).}

Chickens with sporozoite-or blood-induced infections of p. Gallinareum or with blood-induced infections of $\mathbb{P}_{\text {. }}$ lophurae were irradiated with 500 or $600 \mathrm{r}$ of $\mathrm{x}-\mathrm{rays}$ at intervals beginning on the day of infection and with smaller doses $(100-400 \mathrm{r})$ of $x$ rays about 40 days after the ir intlal infection. Irradiation induced increased parasitemia in both avian malarias but affected different periods of the two infections. Thus, in blood-or sporozoite-induced P. gallinareum infections, irradiation on the day of infection produced a higher parasitemia and a statistically simnificant decrease in survival time of the host; during the acute rise and $c$ risis of unfections trated with quinine to reolue their severity, it was followed by acute fatal inferlions; and thereafter led to malarial pelapses of varying degrees. On the other hand, in blood-indured P. lophurae infections, irradiation on the day of infection produced severe, often fatal, infections and only slight relapses thereafter. The effectiveness of a given dose of $x$-rays varied with the age and susceptibility of the chicken, the species of parasite, the pariod of the malarial infection and the intensity of the infection prior to and at the time of irradiation, all of which modily or are expressions of immunity. Also, as far as tested during the developed infection of $\mathbf{P}$. gallinaceum, single doses of from 300 to $600 \mathrm{r}$ were increasingly effective with those of 100 or $200 \mathrm{r}$ being practically ineffective. In general, two doses totaling $500 \mathrm{r}$ or more within about a week of each other had a cumulative effect, but in general, were not equal to single doses of the same magnitude. The foregoing results of $\mathrm{x}$-irradiation are discussed in relation to natural and acquired immunity and to the destruction of lymphocytes which would not only deplete an important mesenchymal reserve from which macrophages are mobilized but also, possibly, one source of antibodies. (Authors) 555

Wald, B. and V. Varteresz. EXPERIMENTS RELATING THE ANTHPLLOGISTS EFTECT OF X-RAYS. Radiol. Clin. 17, 348-62(1948).

In the experiments described, sodium chloride swellings were produced in humans irradiated with doses of 100 to 6 , $6,500 \mathrm{r}$ in order to determine the functional changes brought about in the capullary endothelium by $\mathrm{x}$-radiations. It was found that the anti-inflammation effect of $x$-rays was due to a reversible temporary paralysis of the capillary endothelium, thereby preventing exudation. X-ray therapy of most allergic inflammations and of some cases of acute nonallergic inflammations is suggested: the irradiation technique in such cases should consist of irradiation at 5to 6-day intervals, since the $x$-ray effects on the endothelium recede in about that length of time. (NSA)

\section{Injurious Bffects of Radiation Therapy}

556

Amory, Harold 1. and Irving B. Brick. IRRADIATION DAMAGE OF THE INTESTINES FOLLOWING 1,000-IEV. ROENTGEN THERAPY. EVALUATION OF TOLERANCE DOSE. Radiology 36, 49-37(1951).

A study of the effects of $1000-k v$ irradlation on the small and large intestine in a group of 20 patients is reported. As contrasted to previous similar reports, the present study is unique in that the patients were male, the disease for which they were being treated (testicular tumor with or without retroperitoneal lymph-node metastases) does not affect the intestine, and only external $1000 \mathrm{kv}$ radiation was used. Damage of varying degree to the small and large intestine occurred in some patients recelving large doses of such radiation into the abdomen. Correlation of roentgenologic findings and autopsy results is presented. Though great individual variation is shown in a study of the cases, it is belleved that the small and large integtine of the adult male can tolerate $4500 \mathrm{r}$ of 1000 -lww irradlation administered in 54 days without deleterious results. (NSA) 557

Bassett, R. C. and R. Lowenberg-Scharenberg. AMYLOID DEGENERATION OF THE HUMAN BRAIN FOLLOWING X-RAY THERAPY. J. Neuropathol. Exptl. Neurol. 9 , $93-102(1950)$.

Two cases of the degeneration of the normal human brain following an overdose of $\mathrm{X}$-rays are presented. The first patient received a total dose of $6,000 \mathrm{x}$ delivered to the left temple region in 10 treatments within 12 days, $600 \mathrm{r}$ being applied dally at $150 \mathrm{kv}$ without filtration; the slze of the field was $57 \times 41 \mathrm{~mm}$. Twenty-two months later she received a second course of radiation therapy, consisting of a total dose of $3,600 \mathrm{r}$ delivered at $180 \mathrm{kv}$ in 84 sessions of 
$150 \mathrm{r} /$ treatment; field size and filtration are not reported. Approximately, 11 months after the first course the patient became nervous, complained of general malaise and headaches and experienced speech difficulties and impaired vision. Upon operation, the skull and dura showed no significant changes, but in a region including the area of Broca, the brain tissue was purple-gray; upon escision an uneventful recovery was made. About 40 months following irradiathon of the right parietal region with about $3,500 \mathrm{x}$ at $130 \mathrm{kv}$ without a filter and $110 \mathrm{kv}$ with a filter, the second patient suffered convulsive seizures and upon operation a similax brain lesion was discovered. The dominant histological IInding in the two cases was the formation of the amorphous substance in the areas of the brain exposed to excessive irradiation. It is concluded that the degeneration noted in these areas is similar to that observed in the spleen and is 55 termed "amylotd" in nature. (NSA)

Bruce, Karl W. and Edward C. Stafne. THIE EFIECT OF IRRADIATION ON THE DENTAL SYSTEM AS DEMONSTRATED BI THE ROENTGENOGRAM. J. Am. Dental. Assoc. 41, 684-9(1550).

Five cases in which irradiation affected the dental system are reported. Each patient received inradiation therapy in infancy for a pathologic process occurring in proximity to the developing dentition. Either $x$ ray, surface radium packs, or radon seed implantation were used as the source of irradiation. Dental examination and roentgenograms were made at a time when the complaunts of the patient centered attention on the dentition. From the evidence revealed by the roentgenograms, it is assumed that Irradiation (1) can injure a tooth germ to the extent that a tooth will not form, (2) causes dwarfing of permanent teeth, (3) effects dwarfing of the roots of permanent teeth that are undergoing development during irradiation, (4) effects premature completion of calcification of permanent teeth, and (5) may cause early eruption of permanent teeth. (NSA) 558

Cathie, 1. A. B. ULCERATION OF TULE SMALL INTESTINE FOLLOWWG IREADIATON OF THE PELVIS. REPORT OF TWO CASES. Am. J. Roentgenol. Radium Therapy 39, 895-8(1938).

In a series of nearly 400 cases treated for carcinoma of cervix uteri, two patients developed intractable diarrhea and died, although the roentgen dosage wag well within the limits of normal tolerance. Since the lesions in the intesthe were practically limited to the small intestine beyond the irradlated areas, the author believes that the changes produced were an example of severe radiation sickness. He enphasises the fact that all transient diarrheas should 560 be regarded with the gravest concern. (R)

Cogan, David G. LESIONS OF THE IEYE FIOM RADIANT

ENERGY. J. Am. MEA. Assoc. 142, 145-51 11950).

This report discusses the sources and types of radiation, the transmission and absorption of radiant energy in the ocular media and the clinical characteristics of the lesions of the eye caused by radiant energy. Radiant energy is diwided into two broad types: the electromagnetic type represented as waves of energy, the range of which covers the gpectrum and includes roentgen rays, grenz rays, radiowaves and light waves: the second type consists of corpuscular radiation consisting of subatomic particles, such as electrons ard neutrons. With the potentals commonly employed in roentgen treatment (100-200 kv), $90 \%$ of the energy inctdent on the anterior surface of the cornea raay be expected to reach the retina; keratitis of the cornea may occur from isradlation with $4,000-6,000 \mathrm{~s}$ in several weeks? tIme, and cataracts are known to be caused by doses of
$500-800$ ir at $100-200 \mathrm{kv}$; roentgen xays are tentatively classed as inactive on the retina. Radiation burns, their chan 961

Dean, A. L. and D. D. Slaughter. BLADDER INJURY SUBSEQUENT TO IRRADIATION OF THE UTERUS. J. Urol. $46,917-24(1941)$.

The bladder not infrequently is injured by irradiation applied to the uterus. While uterine diseases commonly are freated with several types of irradiation the greatest bladder damage follows radium applications. Interstitial fibrosis occurs in the bladder wall and obliterative en darteritis occludes nutrient arteries. Because these changes usually are slow, patients are likely to have no bladder distress for a number of years after the uterus has been treated. For this reason these bladder injuries occur alnost exclusively in patients cured of the primary uterine disease. The onset of symptoms is sudden. The patients suffer aculely with frequency, dysuria and hematuria. Cystocopic examinations usually reveal single areas of ulceration surrounded by acute inflammation. Both the mode of onset and the cystoscopic picture suggest carcinoma. The authors have found that drugs of the sulfonamide group, appropriately chosen on the basis of cultures of the bladder urine, give prompt relief. This is in marked contrast to the unsatisfactory results of previous therapy. It has been interesting to note that the symptoms subside as infection is eliminated. Women with sterile urine can feel quite comfortable even though ulcerated areas of consider able size persist. (S.V.S.)

\section{2}

Drescher, $\mathrm{H}$. CONCERNING LOCAL INJURIES THROUGH IRRADIATION AS A CONSEQUENCE OF THE TRATMENT OF COLLUM CARCWOMA. Strahlentherapie 80, 92-106(1949).

Of 1,039 patients with collum carcinoma treated by radiathon therapy, 26 (2.5\%) developed Injuries of the bladder and of the rectum; three of these patients showed injuries of both areas; four more patients developed radiogenous rectovaginal fistulae of the bladder. No systemalic connection between dosage and injury could be established. After the usually tolerated dosage of $7,000 x$ for the bladder was increased to $8,500 \mathrm{~g}$ in order to oblain higher healing ef fects, neither $\mathrm{X}$-ray ulcers nor carcinoma, nor spontaneous Iractures of the femur neck were observed. Radiogenous shrinkages in the parametrium and subsequent stenosis of the urethra are mentioned. (NSA) 583

Engelstad, R. B. TRE RADLATION REACTION TN THE LUNGS OF TRP MUMAN. Acta Radiol. 18, 32-44(1937).

After a ghort review of experimental pulmonary changes produced in rabbits by roentgen radiation, the author discusses 36 cases of pulmonary lesions in man: 21 casen of cancer of the breast, 10 cases of cancer of the esophagus, 4 cases of pulmonary metastasis of extra-pulmonary tumors and one case of cancer of the lung. The frequency of the radiation reaction in the lungs was $5.4 \%$ in cancer of the breast and $20.4 \%$ in cancer of the oesophagus. Very large doses of roentgen or radium rays had been used in almost all cases where a radiation reaction was demongtrated in the lungs. When an epidermicidal dose is given in radiation of the thorass and particularly when several flelds are irradiated, there is a possibility that the lungs may be injured. The radiosensibility of the human lung seems to correspond rather well with that of the rabbit. The histological findings also seem to be similar to thoge in rabbits. The subjective, physical, and roentgenological symptoms are very little characteristic and a prolonged period of observation is necessary in order to make the 
diagnosis. Fatal lung injuries due to radiation have not 564

Engelstad, R. B. PULMONARY LESIONS AFTER

ROENTGEN AND RADIUM IRRADITION. Am. I. Roentgenol. Radium Therapy 13 , 676-81(1940).

Author reports having found 36 cases of pulmonary leo sions due to roentgen or radium irradiations. Cites five case histories demonstrating lung reactions. (S.V.S.) 565

Friedman, A. B. SPONTANEOUS RIB FRACTURES FOLLOWING IRRADIATION FOR CANCER OF' TRE BREAST. Am. J. Roentgenol. Radium Therapy 50, $797-800(1943)$.

A rase of adenocarcinoma of the breast with axillary and supraclavicular and local skin metastases has been heavily Irradiated and the disease has remained arrested over a 7-year period. As a sequel to the irradiation there are late degenerative changes in the brachial plexus, ribs, and skin with benign pathological fractures of six ribs and motor and sensory disturbances of the arm. (Author's Summary) 566

Gauwerky, Friedrich. COMPLICATIONS DUE TO RADIUM TREATMENT OF COLLUM CARCINOMA AND THER SIGNIICANCE FOR SUCCESSFUL TREATMENT. Strahl lentherapie $80,51-70(1949)$.

A total of 590 cases of collum carcinoma were analyzed for complications which might have developed as a result of radium treatment. 耼 was found that 378 cases (64\%) were free from complications; 87 patients (14.8\%) had developed such radiological complications as exosive proctitis, radiological ulcers of the rectum and radiological lesions and fistulae; 125 cases (21.2\%) had developed such inflammatory complications as cystitis, pyelitis, parametritis, peritonitis, etc. Many cases from the liferature and the author's own series were analyzed, particularly for injuries of the rectum and of the bladder and for formation of fistulae as a result of an overdose of radium. Where inflammatory processes developed, the chances for successful treatment were greatly reduced, even in otherwise mild 567 cases. (NSA)

Gratzel, F. R., E. G. Holmstrom, and L. G. Migler, POST IRRADIATION BONE CHANGES. Am. J. Roentgenol. Radium Therapy 53, 62-76(1945).

Therapeutic administration of $X$-rays in infancy or childhood may result in retardation of bone growth. In adults, it may be followed by spontaneous fracture or aseptic necrosis. These changes occur in a very small percentage of all cases treated; these are not always the ones that have received the heaviest doses of radiation. Dietary deficiency and other factors are discussed but no satisfactory explanation is reached as to why a few palient\& develop these changes whic the majority do not. (BA) 568

Greenield, Maurice M. and Frederick M. Stark. POSTIRRADIATION NEURORATMI. Am. J. Roentgenol. Radium Therapy 60, 617-22(1248).

In a series of 180 cases of carcinoma of the testis treated by intensive million volt $X-r a y$ therapy, three patients developed a pecular neurologic disturbance. The principal feature in each case was a flaccid paralysis of the lower extremilles without sensory loss. The authors have called this syndrome "post-hyphenate irradiation neuropathy" and, to their knowledge, it has not been previously described. The chief site of nerve injury was believed to be the anterior horn cells in the lumbosacral segments of the spinal cord. The three case reports are given. (NSA)
568

Matcher, C. $\mathrm{K}$. DEVELOPMENT OF SARCOMA IN BONE SUBJECTED TO ROENTGEN OR RADIUM IRRADLATION. J. Bone and Joint Surg. 27, 179-95(1945).

The experimental background of bone sarcoma following irradiation and the clinical literature are reviewed. Reports of 24 cases of bone sarcoma following ir radiation were found. Curiously, in 17 of these cases roentgen therapy had been given for tuberculous arthritis, and in one for acute arthritis. The remaining six patients were exposed to radium; evidently these patients ingested or received injections of radionetive elements. Three case reports are presented. Case IIs that of a male with a diagnosis of giant-cell tumor of the proximal epiphysis of the tibia, which was excised, with extensive post aperative $\mathrm{X}$-ray therapy. The microscopic diagnosis was benigh chondroblastoma. Six year, later a mass in the ame location was diagnosed as primary sarcoma in the proximal fibula and on amputation was found to be chondrosarcoma. Case II is that of a female who had received an unknown amount of radum and $\mathrm{X}$-ray treatment for a giant cell tumor of the distal sight radius, followed in three years by surgical removal of the distal radius and operative correction of the deformity, seven years after the original diagnosis. No evidence of malignancy was found. Eleven years after the original diagnosis the distal una showed a growth which was excised and found to be a fibrosarcoma with tumor cartilage. In case III a mass developed at the anterior end of the right 'th sib, 12 years after a right mastectomy followed by irradiation. Excision proved the mass to be a chondrosarcoma. In discussing the cases, the author admits the possiblity of recurrence or metastasis, but is firmly convinced that the malignant changes were attributable to irradiation. Surgery is absorbed, although st also was used in all cases. (Much can be caid against the possibility of irradiation as the cause of the malignant change in all these cases. (R) 570

Helma, Roderich. CONCERNING INDIVIDUAL DIFIYR ENCES IN THE X-RAY SENSITVITY OF HUMAN SRIN, FXXAMNED BY THE APREARANCE OF AN ERYTHEMA。 Strahlenther apie 80, 585-90(1940).

Pifty men ard fifty women were treated through 10 lilelds on the back with $X$-ray dosages from 200-400 $\mathrm{r}^{2}$ increasing by $25 \mathrm{r}$ from fleld to field. Special attention was paid to the appearance of the early $X$-ray erythema and the main X-ray erythema. The eaxly erythema field was the one which, while showing a well-defined red field, had received the smallest $x^{\circ}$-dose. The main erythema field was determined 15 days after irradiation. For the appearance of the early erythema there was an individual variation range of $100 \mathrm{r}$ in men and women; for the main erythema, the 571 variation range was $50 \mathrm{r}$ in men and $75 \mathrm{r}$ in women. (NSA)

HAIIs, T. H. and R. W. \$tanford. THE PROBLEM OR EXCESSIV RADLATION DURING ROUTINE INVTSTIGATIONS OF THE EHART. Brit. Heart J. 12, 45-53(1950).

An account of the types of measurements made durms routine cases of angiocardiography and cardiac catherization is given. Detalls are provided concerning the possible amounts of excess radiation to which the patient and the technical personnel may be exposed during the routine investigations: suggestions are made as to mean whereby the excess exposure to radiations can be avolded or reduced to a safe level. Distance, lead and rubber aprons and gloves, and other protective devices and measures are discussed as close proximity to the patient. Accurate monitoring and calculation of the radiation exposure expertenced by the patient are also mentioned as safeguards. (NSA) 
Lobbs, Arthur A., JIי. FETAL TOLLRANCE TO ROENTGEN RAYS. A CASI REPORT. Radiology 54, 242-6(1950).

A pregnant woman, found to have adenocarcinoma of the breast, and operated upon, was later shown to have an osteolytic lesion in the sacrum and right ilium. Upon refusal to allow surgical interruption of the pregnancy, she was irradiated with a total dose of $1,500 x$ measured in air, the beams being directed dorsoventrally and ventrodorsally, so as to produce a calculated dose of $900 \mathrm{x}$ at the midpelvic region; additional radiation was later directed to the dorsal field, adding $375 \mathrm{x}$ to the dose at the center of the pelvis. Pregnancy was terminated with delivery a month later and soon thereaster the osteolytic lesion healed, but the patient died about six months later of liver metastases. The child has appeared to be normal ever since birth. It is stated that the circumstances and irradiation factors in this case parallel the requirements listed by Harris and Mayer for the accomplishment of spontaneous abortion, and no adequate explanation can be offered for the noneffectiveness of the $\mathrm{x}$-irradiation, but it is concluded that the fetal age 573 was apparently a modifying factor. (NSA)

Hubert, Walther. ON DELAYCD RADIATION REACTIONS IN THE BLADDER AND RECTEM AND THEIR TREATMENT. Strahlentherapie $79,113-26(1949)$.

Gynecological radiation therapy often produces harmful after-effects in the bladder and rectum, especially in patients of advanced ages. It was found that these effects could be successfully treated with cod liver oil, 20 to 50 cm. of which was injected daily into the bladder, and every second day into the rectum, alternating with an injection of camomile tea. In five tables the following information derived from the treatment of 27 patients is summarized: distribution of harmful after-effects according to their 10 cation and the kind of Irradiated uterine or portio carcinoma: age distribution of the patients suffering from vardous after-effects; time of first occurrence of the after effects; and the results of the cod liver oil treatment. (NSA)

574

Tones, Arthur. CLINICAL REACTIONS AND INJURIES IN SUPERVOLTAGE TMERAPY. Proc. Roy. Soc. Med. 41 , 703-9(1948).

This brief account of the clinical effects of supervoltage radiation and of the injuries which have resulted during the treatment of maltgnant disease with the milion-volt apparatus includes the following: (1) injuries to the skin and subcutaneous tissue, (2) injuries to connective tissue, (3) effects on respiratory tract, esophagus, bladder, bone, eye, and liver, and (4) occurrence of muscle librosis. (NSA) 575

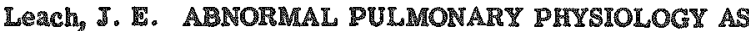
A RESULT OF CHRONIC IRRADIATION PLIEUROPULMONITIS. Am. J. Roentgenol. Radium Therapy 50, 772-8 (1943).

The purpose of this report is to point out some of the striking deviations from the normal pulmonary phy alology that may occur in patients who sustain pulmonary fibrosis following deep roentgen therapy to lumors on or within the thorax. The most disabling feature of irradiation fibrosis of the lung is the marked dyspnea that may occur as a result of ordinary excereise. It is pointed out that chronic irradiation pleuropulmonitis is not merely a pleuropul monary disorder, but also includes changes in the thoracic parietes, as well as the diaphragm and mediastinum. (S.V.S.)
Lukens, R. M. COMPLICATIONS FOLLOWIG IRRADIATION OF THES THYROID GLAND. Ann, Otol. Rhinol, and Laryngol. $57,633-42(1948)$.

Although X-ray irradiation of the thy roid gland for exophthalmic goiter, hyperthyroidism, etc. is considered to be definitely indicated, the five case histories presented in this paper show the damage that can be caused to the blood vessels, overlying tissues, and underlying larynx and trachea when such inradiation is indiscriminate or excessive. The symptoms appearing in these cases were: hoarseness, sensation of lump in the throat, inspiratory dyspnea, wheezing, dy sphagia, cough, expectoration, loss of weight, and chest pain. The physical findings were: congestion of the laryngeal mucosa, telangiectasis and scarring of the neck, stenosis of the larynx and trachea, inflammation of the laryngeal mucosa, telangiectasis of the vocal cords, granulomatous lesions in the tracheal walls, and destruction of the cillary action of the epthellum resulting in viscid adherent tracheal secretions and crusting. The time between termination of irradiation and appearance of the first symptorns was from one to seven years and the patients have been under continuous treatment for relief one year ( 1 case), three years ( 1 case), nine years ( 1 case), ten years ( 1 case), and eleven years ( 1 case). Tracheotomy was required in three cases. (NSA) $57 \%$

Maas, Jerome M. INTESTINAL EFTECTS OF PELVIC IRRADIATION. Modern Med. 16, 47-8(1948).

It is estimated that from $50 \%$ to $75 \%$ of all women treated by gynecologic radiation have permanent rectal sequelae though perhaps unrecognizable. They include extengive scarring, fistual, hemorrhage, or complete occlusion. Radium implants have been found more harmful than $\mathrm{X}$ rays, and injury was greatest when they were combined for cervical or fundal tumor. The treatment of resulting conm ditions of proctitis, stenosis, bleeding, and rectovaginal fistula is discussed. (NSA)

578

Nadal, R, and J. Piequet. POST-RADIOTHERAPEUTIC ESOPHAGEAL STENOSES. J. Radiol. Flectrol. 20, 296-7(1948).

Three cases of stenosis occurring after $\mathrm{x}$-irradiation which Included the esophageal region as a part of the fleld are presented in detail. Irradiation was delivered at 180 to $200 \mathrm{kv}$, through a $1 \mathrm{~mm} \mathrm{Cu}$ and $2-3 \mathrm{~mm} \mathrm{Al} \mathrm{filter,} \mathrm{at} \mathrm{a} \mathrm{focal}$ distance of 40 to $50 \mathrm{~cm}$., giving single doses of 200 to $250 \mathrm{r}$ at varying intervals, with a total dose between $3,000 \mathrm{x}$ and $12,000 \mathrm{~s}$. The stenoses, involving skin area fibroid formation, developed progressively after variable periods; in these cases all other causes of the reaction are eliminated. (NSA)

579

Pennybacker, Joe and Dorothy S. Russell. NECROS1S OF THE BRANN DUE TO RADAATION THERAPY. J. NeurOl. Neurosurg. Psychat. 11, 183-98(1248).

The authors report the clinical and pathological features of five cases of necrosis of the brain following radiation therapy by accepted techniques and dosages. The clinical effects may come on suddeniy, progress to a point, and become arrested; or they may come on gradually and lead to death. In each case there was a latent perdod between the radiation treatment and the onset clinical signs of necrosis. The ghortest interval was nine months, the longest five years. The pathology of the necrosis appears to be related to reactions in the smaller blood vessels in which collagenous thickening, fibrinois necrosis, and thrombosis are con- 
spicuous. As it seems impossible to predict in which cases necrosis will occux, it is suggested that radiation should be reserved for inoperable tumors and those cases in which no 580 further surgical procedures are contemplated. (NSA)

Quimby, Edith H. and W. S. MacComb. FURTHER STUDIES ON THE RATE OF RECOVERT OF HUMAN SIIIN FROM THE EFTECTS OF ROENTGEN OR GAMMA-RAY IRRADIATION. Radiology 29, 305-12(1937).

The authors present curves and a table from which the accumulated dose in the slin can be calculated for any day during the period of treatment. If the total dose and total lreatment time are the same within certain limits, the number of fractions into which the radiation is divided is not particularly important. But this refers only to the skin, and the clinical applicability of this information can be determined only by observing the tumor regression, systemic effect, etc. in a series of cages trealed by each scheme. Within certain limits it appears that if the total dose and total treatment period are the same, the rate at which the radiation is administered within that period is not important; it may be contmuoug or fractionated at will. The lerm "saturation method" applied to the administration of a fairly large dose followed by a number of mall ones, assuming a constant rate of recovery and a constant rate of making up for this, is not accurate. Since the amount of recovery decreases from day to day, it is necessary to know the correct recovery factor for each day. A method is outhined for calculating a true saturation scheme of itc radiation. (YBR)

581

Quimby, Edith H. and \$ldney C. Werner. LATE RADIATION ETFECTS IN ROENTGEN THERAPY TOR HYPERTHYROTOISM; THETR POSSIBLE DEARING ON THE USE OF RADIOACTIVE IODINE. J. AR. Med. Assoc. 140, 1046-7(1949).

Long-term follow-ups on patlents recelving I's! for hyperthyroid condittons are necessary before it can be defintely gtated whether or not this form of therapy may result in late degenerative malignancy in treated areas. Three definte cases out of 10 cases of further malignaney were found in records of 101 radiologists and thyroid experts; thege had all recelved heavy radiation doges. Since the dose of $\mathrm{X}$-ray is 3,000 to $3,500 \mathrm{r}$ delivered to the whole gland, and the dose of ${ }^{131}$ is 5,000 to 6,000 egurvalent $x$ delivered to the whole gland, but concentrated in guch a way In specific parts that it is thought that the dose in some parts may be as high as $30,000 \mathrm{r}$, the possibulity of gubse. guent malignancy is probably far greater with I' ${ }^{194}$ than with X-say theragy. (NSA)

582

Ricketts, Wulam $\mathbb{E}_{.,}$Josegh B. Kirsmer, Eleanor M. fumphreys, and Walter Lincoln Palmer. Castroenterology 11. 18-32(1048).

Moderate roentgen lrradiation (1100 to $2500 \mathrm{P}_{2}$ applled in divided doses) of the fundus and body of the stomach in man produces an acute transitory symptomless gastritts that 13 characterized gastroscopically by hyperemia, hemorrhage, edema, and adherent exudate, and histologlcally by degenerative changes in the epithelial cells and lymph follicles, increased cellular infiltration, and relatively minor changes in the blood vessels. Atrophy of the gastric mucosa often ensues; it is unioformly present in patients with prolonged post-radiation anacidity. (NSA)

583

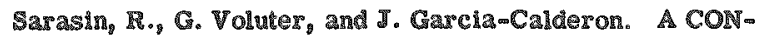
TRIBUTION TO THE STUDY OF TRANSTENT PLEUROPULMONARY MODIFICATIONS OCCURRING AFTER
RADIOTHERAPY OF BREAST CARCINOMA. \#. RaULOL. Electrol. 29, $445-52(1948)$.

Nine case histories of pulmonary complications develoging after the use of radiation up to $12,000 \mathrm{r}$ on breast cancer are given with radiographs of the arfected area. Exudation whth atelectasis is always a part of the symptomological picture: irritation of various types is reported and the histopathology of the mechanisms is discusaed. These phenomena may be accompanied by a sibrold sormation bul are always transient in nature, appearing at times some months after irradiation and rogressing after varlable periods of time. It is mentioned that the radiological picture may resemble a pulmonary tumor. (NSA)

584

Scheie, Rarold G., Richard R. Dennis, Rtchard C. Ryple, Larry $\mathbb{L}$. Calkins, and John A. Buesseler. THE ERTET OF LOW-VOLTAGE ROENTGEN RATS ON THE NORMAL AND VASCULARIZED CORNEA OF THE RABRIT.A PRELIMINARY REPORT ON TKR PRHEIPS MACHINE.

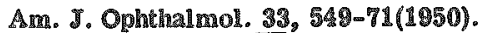

In order to determine the beneflctal and injurious effects of low-voltage $\mathrm{x}-\mathrm{s}$-y when applied to the eye under conirolled conditions and to determine whether or not $\mathrm{x}-\mathrm{r}$-2y might be a satisfactory subgtitute for $\beta$ irradation, the sudies deacribed were periormed. A Philps contact ray machine was operated at 44 kv and delivered varylng total doses elther to the normal rabbit eye or the rabblt cornea. It was found that exposure of the rabbit eye of up to 2,000 I weekly for as long as 5 weeks could be performed without apparent damage to the cornea or lens curng the followng 3.5 month\%. Exposures as gnall as $500 \mathrm{x}$ and limited to the cornea, when given weekly for 21 weeks, resulted in transient corneal opacty. Dosage of $5,000 I_{2}$ given in the same manner, caused permanemt corneal opactiy and ixradiation cataract, flrst observed 18 months following the fir gt exposure. Vagcularization induced by an intracorneal sodium hydroxide snjection could be markedly fnhibited by the low-woltage z-rays; daly exposure was more effective In inhubitng corneal vascularization than expowures given at longer intervals, and small dosages of $300 \mathrm{r}$ were as efLective as larger ones up to $1,000 \mathrm{z}$. Exposures of $400 \mathrm{r}$ and 500 r repeated 3 and 4 times at approxinately 1 -week intervals were used chincally in several hundred gatients without evidence of calaract formation; these patients have been observed for as long as 2.5 years following treatment. (NSA)

585

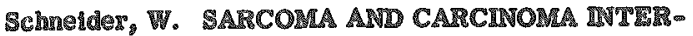
RELATIONSETP TN X-FAY ITEFATED HUPUS VULGARE. strahentherapie 10, 335-60(1940).

Nine cases of sarcoma occurring after s-ray therapy of lupus vulgaris are clinically and histologically described $\mathrm{M}$ detall. Though in all cases a carchnoma developed first, the co-ertitence of a sarcoma which had develoned after the application of high $\mathrm{X}$-ray dogages was histologlcally established in six cases. The sarcomas originated from the chrontc granulation tsssue, not through metaptasiat of epithelial cell elements, and were the collision tumor-type, not the carcino-lype. The fact that high X-ray dosages were applied and that the altes of the turnors were places of sharply expressed $\mathbb{X}$-ray atrophy leads to the concluston that these tumorg, particularly the sarcoma, orighated from severe $\mathrm{X}-\mathrm{ray}$ injuries. (NSA)

596

Teloh, H. A. M. I. Mason, and M. C. Wheelock. A HUSTOPATHOLOGIC STUDY OF RADLATION INYURIES OF THE SKIN. Surg. Gynecol. Obstet. B0, 335-48(1050).

The hatory of irradiation injuries of the skin is briefly 
summarized and clinical aspects of irradiation dermatitis, acute and chronic, are reviewed. of 121 patients with radi ation dermatitis, as a result of the use of $\mathrm{X}$-rays, radium, and radon, $28.1 \%$ showed definite evidence of rarcinoma, usually localized by the stromal barrier. Such epidermal changes as atrophy of epithelium with the consequent loss of rete pegs, alternating with focal areas of hypertrophy, characterized by marked acanthosis, hyperkeratosis, and rarely parakeratosis, were seen. The vascular picture consisted of marked endothelial proliferation with narrow ing or occlusion of the lumen of the artery fibrosiss and hyalinization of the media, fibrosis of the adventitia and occaslonally perivasive edema and lymphocytic infiltration. Stromal changes, and changes in hair follicles, sebaceous glands and sweat glands are also described. It is noted that in the 121 cases studied vascular changes were the most constant, the earliest and the most pronounced of the changes observed in the skin. Evidence is presented on the direct carcinogenic effect of irradiation on the epidermal portion of the skin. It is concluded that the therapy of a benign cutaneous or subcutaneous lesion by means of ir radiation, with the potential production of a much more severe irradiation dermatitis, is not justified unless it can be carried out by a roentgenologist trained in the limitations and potentialities of such therapy. (NSA) 587

Vogt, Alfred. DELAYED INJURIES OF THE CRANIUM AFTER X-RAT THERAPY OF INTRACEREBRAL TUMORS. Strahlentherapie 80, 165-74(1949).

Two cases are described where injuxies of the cranium occurred five and six years after $X$-ray treatment. One patient with a tumor of the hypophysis, and the other with acromegaly had been treated with large dosages of deep $\mathrm{X}$-rays $(10,500$ to $13,200 \mathrm{r})$, delivered in doses of $300 \mathrm{r}$, to three fields over a period of two months to about two years; the radiations were administered by a $185 \mathrm{kv}$ machne, with a filter of $0.5 \mathrm{~mm}$ Cu and at a target distance of $40 \mathrm{~cm}$. At three or four places in the cranium, radiogenous ribroses developed. The author warns that fibroses resulting from pressure through growing intracranal tumors and other factors have to be carefully distinguished from radiogenous fibrosis in the interest of further treatment of a patient. Since both patients showed relief from their symptoms and could work, the possibility of producing fibrosis should not be a contraindication to radiation therapy of the brain where it is otherwise indicaled. (NSA)

Waiter, J. EPITHELIOMA AND PAPILLOMA ARISING ON RECENTLY IRRADIATED SRIN; REPORT OF THEEL CASES. Brit. Med. J. 4648, 273-4(1950).

A series of three cases of epithelioma and papilloma developing a few months after X-ray reactions of only moderate severity is presented and the cases are discussed in detail. In the first case, the total treatment time was 31 days and the estimated tumor dose was $4,600 \mathrm{x}$; the skin reaction proceeded to a deep erythema with dry desquamaton and a few small and moist patches. Healing was rapid and uneventful but about 14 weeks after the end of the treatments, a nodule developed on the irradiation site which was excised and concluded to be a paplloma; the patsent is well to date. About 11 weeks after the delivery of a skin dose of $3,900 \mathrm{r}$ to the second patient, with consequent exythema of moderate depth, a nodule identified as squamous epithe lloma developed and one year atter excision the patient died from the effects of the primary lesion (carcinoma of the hypopharynx). A total dose of about $3,500 \mathrm{x}$ was delivered to the third patient over a period of 23 days with a moderate erythema developing: seven weeks after the end of the treatments a nodule developed in the irradiation site which was identified as being squamous epithelioma: ir radiation for a total of $3,500 \mathrm{r}$ was then delivered in 23 days, and the patient is well to date. Several explanations of the occurrence of these secondary lesions are presented. (NSA)

589

Windholz, FATE CHANGES IN MUCOUS MEMBRANE OF THE IRRADIATED LARYNX. Radiology $48,274-81$ (1947).

Microscopic study of the mucous membrane of the rancerous larynx after protracted fractionated ir radiation reveals that the primary effect of irradiation is cell destruction. At the time fibrinous reaction occurs, the mucous membrane is entirely destroyed. Later, regeneration of epithelium takes place. The regenerated epithelial struetures which often replace the columnar epithelium are also metaplastic, revealing excessive cornification. This metaplasia has also been produced by irradiation in animal eg periments. The structure of the regenerated epithelium varies according to the post-ir radiation condition of the subepithelial connective tissue. In two cases out of ten the subepithelial connective tissue of the larynx was transferred into hyaline selerotic tissue with large anounts of interstitial collagen, few cells, and obliteration of blood vessels. In these two cases, the epithelium was markedly atrophic and the tumor of the larynx has disappeared. Clinically, strong fibrin reaction was observed. In two other cases, displaying large residual or recurrent tumors, the superficial epithelium exhibited definite tendencies toward atypical growth after irradiation. The connective tissues were remarkably well preserved in these cases and clinically no fibrin reaction was observed. These studies indicate that, while the primary epithelicidal radiation ef fects are fading out, the changing generations of metaplastic epithelium adapt the growth and differentiation properties of their structures to the post-irradiation condition of the subepithelial connective tissue. In cases in which the tumor has disappeared, advanced diffuse atrophy (fibrosclerosis) of the connective tissue and epithelium is 590 observed. ( $\mathbb{R}$ )

Woodburne, Arthur R. and Osgoode S. Philpott. RADIATION EFPFECTS ON TRE SKIN AND THEIR TREATMENT. J. Michigan State Med. Soc. 48, 461-79(1949).

A detailed review of studies on 100 patients showing evidence of skin damage due to ixradiation is given. Such general effects as erythema, edema, hyperpigmentation, and dry atrophic, and depigmented skin are observed. Severe cases involving large areas of vessels in an oblitexative vasculitis and consequent ulceration are cited. Dosages of $5,000-6,000 \mathrm{r}$ over areas of $10 \mathrm{~cm}$. diameter or more produce these serious results. Smaller doses of 1,000-3,000 $\mathrm{x}$ produce milder symptoms. The pathological changes, involving the vessel walls, collagen bundles, and nuclel are described in detail. Surgery is urged as soon as feasible in order to relieve suffering, permit rehabilitation, and prevent carcinoma. (NSA)

\section{Effects on Blood}

591

Brown, Crawford S., Esther Hardenbergh, and John L. Tullis. THE BIOCHEMICAL, CELLULAR, AND BACTERIOLOGIC CHANGES IN THORACIC DUCT LTMPH OF DOGS EXPOSED TO TOTAL BODY IRRADIATION. Am. J. Physiol. 163, 668-75(1950). (NP-1762)

A study of the biochemical, cellular, and bacteriologic changes in lymph produced by $500 \mathrm{r}$ of total-body irradia- 
t1on in dogs was undertalen in order to compare lingm with changes which have beon found the ocur un blowt. Forty dogrs were diviled int 9 groups, each group gexpresenting a different tume interval through $132 \mathrm{hr}$ after ir adiation. Dugss were shesthotized with nembut 1 , the thoracic ducts were cannulated wh sllconed glass tabing, and the lymph fistulae allowed 10 drain for 6 to $10 \mathrm{hr}$. Lymph samples were taken cvory 1 to $2 \mathrm{hr}$. Buyd samples were lalsen at longer mitervals. Changes, with time after irodiation, of protein lavel, creatine, urib acid, sugar, and other components are discussed. (NSA)

592

Chanuth, Alfed and Stephan Ludewig. IFIFCT OF WHOLE BODY X-IRRADIATION ON SERUM IRON CONCENTRATION OT RATS. Am. J. Physîn. 166, 380-3 (1951).

Serum 1 ron concent rotichs were determined in malo and female rats exposed to lotal body irradiation with $\mathrm{x}$-rays in doses varyng from 10 to $1500 \mathrm{t}$. The average values are signiffeantly increased in buth groups duning the firgt day following irtadiation whth doses of 100 ar greater. Subsequently, the serum ron concentrations remained clevated for about a werk in the 500- and 600-r groups. Male ani-

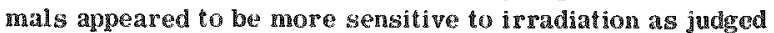
593 by the increased values for serum iron. (auth) (NSA)

Gjessing: Eriand C. and Alfred Chanutin. STUDIES ON THE RROTEINS AND LIPIDISS OF DLASMA FIRACIONS OF X-RAY-IEEADIATED RATS. Arch. BIOChem.27, 191-7(1950).

The nitrogen and lipid contents of plasma fractions are presented for pots exposed to monlethal and lethal doses of $x-$ rays. Pronounced increases in the nitrogen conents of the $a_{1}$-globulin-rich I raction $1 V-4$ and decreases in the allumin-rich risction $V$ are seen. The most pronounced changess in the total lipid and cholesterol content are noted in raction $1 \mathrm{~V}-4$. The percentrge dist ribution of the components of the electrophorotic patterns of the 4 raetions do not deviate significantly from the controls excent in the

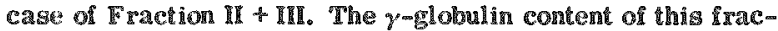
\$\$4 tion is consistently diminighed. (auth) (NSA)

Goldschnide, Leontine, Robert L. Hosenthal, Victor P. Bond, and Maurice C. Pishler. ALTERATONS IN THERMAL PRACILITV OI RAT ERTTIROCYTIS FOLLOWNG TOTAL BODY X-IREADITION. Naval Radiological Defente Lab. Feb. 14, 1950. 12p. (AD$192(8))$

A review of the litereture haw revealed indirect evidence that gadation affets the integrity of the circulating red cell, but that no direct chamge has been demoristrated. A spectrophotometic method for the guatitativ measurement of red cell hemolysis produced by an levated temperature $\left(53^{\circ} \mathrm{C}\right)$ has been developed. By this method, a telinite increase in red cell fagility was demonstrated in rats within 4 to 12 hours following theis exposure to toral body \$-irradiation at 500 and 200 r. The possible mechansm of these red cell changes and their significance in radiation Butckness are diseussed. (NSA 595

Palberstaedter, L. and T. Leibowitz. THMOLFSIS AND

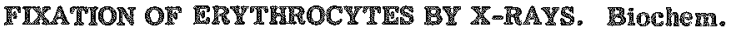
7. 41. $295-9(1947)$.

Suspensions of aythrogtes down to a concentration of about $0.5 \%$ respond to irradiation with an effective $x-$ ray dose by hemolygin indepenenty of the nature of the medium in which they are sugpended. A cell concentratons below $0.5 \%$, the respone of erythrogytes to larediation operds on the medium in which they are suspended: (a) In

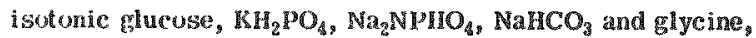
the response to an effetive $x$-ray dose $1 \mathrm{~s}$ lemolysis. (b) In isotonic $\mathrm{NaCl}, \mathrm{KCl}, \mathrm{CaCl}_{2}, \mathrm{NaNO}_{3},\left(\mathrm{NH}_{4}\right)_{2} \mathrm{SO}_{4}, \mathrm{MgSO}_{\mathrm{f}}$ and

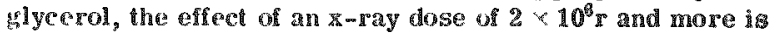
not hemolysis but cell fixation, the lotal loss of the ability 10 hemolyze. Additin to media listed in 2 (b) of serum, phosphalc, or bicarbonate prevents coll fixation. The fination opposing activity of phosphate is considerably below that of serum or bicarbonate. The biearbonate content of Serum accounts for its high activity in preventing fixation. The relevane of the nature of the medium and of the cell concentration of the eryhrocyte suspension for the orcur rance of the lixation affect point lo sune "indirect action" 596

Mennessy, Thomas C. and Rex L. Hufi. DEPRYSSTON

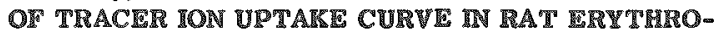
CYTES FOLLOWHG TOTAL BODY X-LREADIATON.

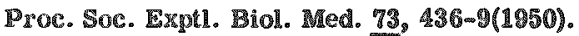

Groups consisting of five adult female Curits Dumning nats weighing approsinately 200 m. were given total-body

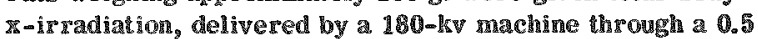
$\mathrm{mm} \mathrm{Cu}$ and $\mathrm{mm}$ Al filtration; the animals were individu ally irradiated in lucite contaners. Four series were irradiated: series A 2 nd B consisted of fous grouns which received 5, 25, 125, and 250 of the whole-body irtadiation: geries consisted of three froups that received 5,10 , and $25 \mathrm{Ir}^{*}$ : intsavenously by êl vein a labeling dose of $\mathrm{F}^{53}$ in $0.5 \mathrm{cc}$. of $\mathrm{PeCl}^{\Im}$ solution containing $2 y$ total ixon and agprosimately

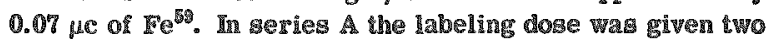

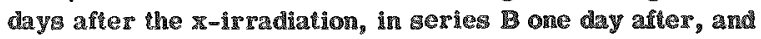
in series $C$ within four hours ater the ixradiatson. F'o lowing the administration of the labeling dose of $\mathrm{Fe}^{\mathrm{m}}$, 0.5 ce. samples of blood were drawn by means of cardac puncture, heparinized and centrifuged. The hematocrit was recorded, and the samples ware tested lor Iradioactivity. Graphs of the red cell $e^{50}$ untake curve in the rats at the various dosage level of total-body $x-1 \mathrm{rgadigtion}$ are given. The results obtained in these studies show that the radioactive iron red cell uptare curve is a wersitive indigeator of x-ir radiation damage to the bone marrow; the histological

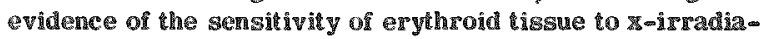
tion 2150 Was confirmed. (NSA) 597

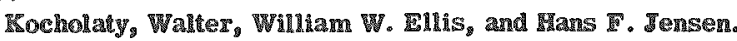

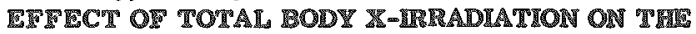

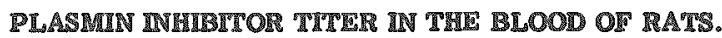

Army Medical Rescarch Lab. Dec. 15, 1951. Pp. (AMRL-71)

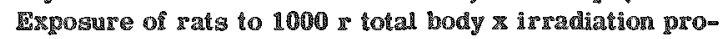
duced a fall in the plasmin inhibitor ther of plasma, beginming at the sccond day aftar ixradiation and thon gradualy increasing. Since starvation was found to extert a wimilar effect, no definite conclusiong can be made 25 to whether ir iodiation per se caused a lowering in the titer. Total

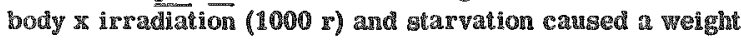
los3 of neariy idemical magmitude. (NSA) 598

Tohn, H. I. CHANGES TN COMPOSTION OR BLOOD

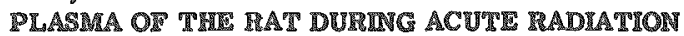

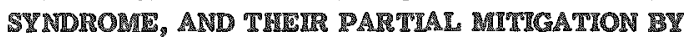

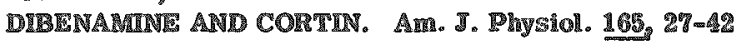
(1951).

Adul xats were given gingle total body cxporure to 250 peallowed for about 30 days. The changes in the glammac curred in 2 phases, unaffected by zex, but comewhar dependent on the strain of rat. The magnitude of the changes

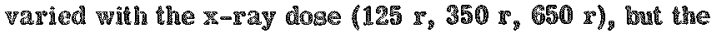


timing and sequence did not. The changes in $\mathrm{Cl}$ and cholesterol were prevented by the daily administration of desoxycorticosterone acetate or lipoadrenal extract. Dibenamine or 2-dibenzylaminoethanol prevented the change in cholesterol alone. Injection of sheep erythrocytes $1 \mathrm{wk}$. before irradiation prevented the rise in albumin-globulin ratio. The greatest rate of change in the composition of the plasma occurred during days 3 and 4 , the days on which the 1 st deaths occur following a lethal exposure. It is proposed that radiation effects be classified as primary when occurring within the cells directly injured, and secondary when oc590

curring in other cells. 55 references. (CA)

Lindemann, Bruno. RADIATION HEMOLXSIS, A CONTRIBUTION TO OUR IENOWLIEGE OF BIOLOGICAL EFIPCTS OF IONIRING RADIATION. Fortschr. Gebiete Röntgenstrahlen 75, 523-59(1951).

Electron-optical analyses were made of the structure of \&-ir Eadiated erythrocytes. Nunerous illugtrations of normal erythrocytes and erythrocytes in the process of hemolysis are given and the mechanism of the effect of $x$ radiation causing hemolysis of erythrocytes is discussed. It is concluded that $\mathrm{x}$ radiation caused osmotical hemolysis of erythrocytes by alteration of the permeability of the erythrocyte membrane due to primary denaturation. Three factors of speed regulate the process of $x$-ray hemolysis, the reaction between hemolyticum and cell membrane, the penetration of the salt, and the disintegration of the cellular contents into a diffusible mass, the latter playing a decisive part. Morphologically analogows alterations of the membrane are caused by acid and $\mathrm{X}-\mathrm{ray}$ denaturation. Degree of hemolysis depends on presence and amount of blood plasme in the suspension; it geems, therefore, that denaturation of the erythrocyte membrane is caused by indirect effects of $\mathrm{z}$ rays. The age of the individual erythrocytes and a protective effect by aggregation of cells influence the physiological degree of scatter in the reaction by an irradiated suspengion of erythrocytes. (NSA) 000

Meyndel, Gaston and Suzanne Bazn. CHANGES IN THF

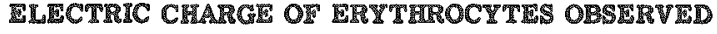
IN IRRADIATED ANIMALS. Compt. rend soc. biol. 144, $1463-5(1950)$,

The effect of ionizing radations on the electric charge of blood corpuscles was examined by totally irradiating guinea pigs with doses of $x$ rays slightly inferior to the threshold lethal dose. An electrophoretic study of erythrocytes showed a $25 \%$ diminution of their surface charge, appearing on the 7th day after the irradiation. since the phenomenon is observed only in the presence of the serum of the animals; it is concluded that it is directly correlated with al601 terations in the proteinic fraction of the blood gerum. (NSA)

Neve, $\mathbb{R}$. A. and Cecil Entenman. CMANGES IN THR PLASMA PHOSPHOLIPIOS IN RATS FOLLOWING WOLE BODY X-IRRADIATION. Naval Radiological Defense Lab. Agr. 10, 1951. 17p. (AD-307(B))

Changes in the plasma phospholipid phosphorus (PLP ${ }^{31}$ ) of fasted female rats have been determined following wholebody, 250-kv, $x-1$ rradiation, with doses ranging from 750 to 5000 r. Ir radiation causes marked increases in the plasma level of PLP31. After $750 \mathrm{r}$ the plagma level of PLP $\mathrm{P}^{31}$ reaches a maximum at the second to third post-irradiation day. The plasma level of PLP $\mathbb{P}^{31}$ after doses of 750,1500 , 2500,3500 and $5000 \mathrm{r}$ was, respectively, $6.47,7.10,7.78$, 8.63, and 9.92 $\mathrm{mg} \%, 2$ days after irradiation. Plasma protein levels remained constant over two days after irradiation. Plasma volume, determined in some of the experi- ments, showed little or no change over an interval of 5 days after irradiation. (auth) (NSA)

602

Sheppard, C. W. and Certrude E. Beyl. CATION EXCMANGE IN MAMMALIAN DRYTHROCYTES. III. THE PROLYTIC EFTECT OF X-RAYS ON HUMAN CELLS.

J. Gen. Physiol. 34, 691-704(1951).

Freshly drawn heparinized human whole blood was exposed $10 \mathrm{x}$ rays in amounts up to $54,000 \mathrm{x}$ in vitro and then equilibrated under a cont rolled atmosphere at 24 or $38^{\circ} \mathrm{C}$. For as long as $26 \mathrm{hr}^{*}$ following exposure $\mathrm{IS}$ was progresw sively lost from the cells and quantitatively replaced with Na with little, if any, osmotic disturbance. The mean rate of loss at $20,000 \mathrm{r}$ and $24^{\circ} \mathrm{C}$ is about $0.4 \%$ of the initial cell $\mathrm{K}$ per hr and approximately doubles for a $20,000 \mathrm{r}$ increase. It is accentuated if blood is stored at low temperature $\left(5^{\circ} \mathrm{C}\right)$ following radiation exposure. Isotope experiments show that the rate of entrance of $K$ into the cells is practically unaltered, the principal effect being an acceleration of the sate from cells to plasma. This suggest s that radiation may have interfered with a mechanism of selective $\mathbb{I r}$ accumulation based on preferential retention of the element. The Na which enters the cells following irradiation contributes to the rapidly changing portion of the cellular $\mathrm{Na}$, sug603 gesting that this fraction is ionic Na. (NSA)

Sheppard, C. W. and M. Stewart. LACK OF AN ONYGEN ERTECT IN THE DISTURBANCE BY X-RAYS OF SELECTIVE POTASSIUM ACCUMULATION IN HUMAN ERYTHROCYTES IN VITRO. Federation Proc. 10, 125(1951).

Abstract reproduced here in its entirety.

When heparinized human whole blood is exposed to Xrays $(30,000 \mathrm{r})$ and subsequently equilibrated in vivo under guasiphysiological conditions, potassium progressively leaves the cell and sodium penetrates in essentially equimolal amounts (Sheppard, Biol. Bull. 93, 310). Experiments were performed in which blood was equilibrated with an atmosphere containing $5 \% \mathrm{CO}_{2}$ and $95 \% \mathrm{He}$ or $\mathrm{O}_{2}$. The completeness of the reduction on oxygenation of the blood was checked by spectrophotometry of the hemoglobin. After $x-$ ray exposure the potassium leakage rate was determined by serial flame spectrophotometry of plasma samples. No dir ference could be detected between the leakage rate in manples irradiated under He or under $\mathrm{O}_{2}$. This finding is in distinct contrast to the wide spread depression of $\$$ - ray ef fects in many other tissues when inradiated under reduced oxygen tension. 604

Storey, R. He, L. Whish, and J. Furth. CHANGES IN CELI AND PLASMA VOLUMES PRODUCED BY TOTAL BODY X-RADIATION. Proc. SOC. FrpL. Biol. Med. 74, 242 (1950) (ORNL-687)

Following irradiation of rabits by $1000 x$ of $x$-rays there was a large drop in erythrocyte volume as measured by

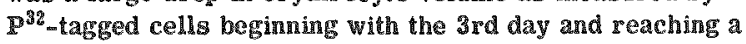
depth at about the 17 th day affer isradiation, followed by a slow recovery. There was at first a slight concomitant plasma-volume drop followed by a rise on about the 10th day that elevated the lowered total volume but did not bring it up to normal in 23 days. Similar changes occurred in mice that were exposed to $575 r$. 11 references. (NSA) 605

Supplee, Helen, James D. Hauschild, and Cecil Entenman. PLASMA PROTEINS AND PLASMA VOLUMES IN RATS FOLLOWING TOTAL DODY X-IRRADIATION. Naval Radiological Defense Lab. May 17, 1951. 21p. $(A D-318(B))$ 
The effects of alterations in plasna volume on the plasma protein concentration after $x$ irradiation were studied. Simultaneous measurements were made of plasma protein concentrations, plasma volumes, and total bloud volume in rats for as long as 20 days after exposure to 400 , 600 , and $750 \mathrm{r}$ total-body $\mathrm{x}$ radiation. In rats that received $750 \mathrm{x}$, plasma volumes decreased to values $20 \%$ below the control by the 3rd day; no significant decrease occurred in animals exposed to 400 or $600 \mathrm{r}$. Beginning with the Ath day after all exposures, an increase in the plasma volume was apparent and persisted throughout the experimental period. Plasma protein concentration was depressed by the $3 \mathrm{r}$ d or 4 th day in all groups althourh the decrease was not statistically significant after exposure to $400 \mathrm{x}$. Recovery was incomplete even after the 20th post irradiation day. Comparison with starved control animals indicated that the initial loss of plasma protein after irradiation could be attributed only partially to impaired food intake. (2 tables, 4 charts) (NSA) 606

Ting, T. P. and Raymond E. Zixkle. THE NATURE AND CAUSE OF THE HEMOLYSIS PRODUCED BY X-RAYS.

J. Cellular Comp. Physiol. 16, 189-95(1240).

In defibrinated beef or human blood, exposed to $33,000 \mathrm{r}$ of 8 -rays, radiation hemolysis sets in about 15 or $20 \mathrm{hr}$. after irradiation. It is preceded by a swelling of the red cells which can be demonstrated by the hematoerit method. If this swelling be prevented by adding 1 vol. of isotonic sucrose to 9 vol. of blood, no hemolysis occurs before the end of $36 \mathrm{hr}$. Swelling is therefore the chief cause of the radiation hemolysis. Since irradiated cells swell as fast in control serum as in irradiated serum, the effect of the radiation is on the cells themselves. Vapor pressure measurements show that the osmotic pressure of the cell contents is slightly raised, but this effect can account for only a few $\%$ of the gwelling observed. The membrane is ac cordingly the more probable site of the important changes. The cations of $\mathrm{Na}$ and $\mathrm{K}$ readily penetrate red cells suspended in appropriate isosmotic salt solns. This, along with the fact that swelling in serum is prevented by suitable addition of isotonic non-alectrolyte soln. (sucrose), indicates that the swelling, and therefore the radiation hemol607 ysis, is due to an increased permeability to salts. (BA)

Volkin, Elliot and Henry I. IRohn. A FACTOR IN TRE PLASMA OF THE IRRADIATED RAT WHCH CHANGES THE A/G RATIO. Arch. Biochem. 30, 326-32(1951). (ORNL-734)

Total-body $x$-irradiation (250 kvp) of the rat caused a rise in the serum or plasma albumin-globulin $(\mathbb{A} / \mathrm{G})$ ratio. The effect was maximal 4 days after exposure to about 700 r. The rise was lound when the $A / G$ ratio was determined by sodium sulfate fractionation or by the analytical ultracentrifuge, but not by electrophoresis. Ether extraction of the plasma just prior to analysis returned the elevated value of $\mathrm{A} / \mathrm{G}$ to normal. A substance was obtained from alcohol-ether extracts of plasma or serum which raised the $A, G$ ratio of normal rat serum or plasma in vitro. 12 ref erences. (NSA)

608

Zacek, Jan, Ul. Klimecky, and D. Motacek. TRE PROBLEM OF RADIOTOKINS. I. Spisy Lekarste Fak. Masaryk. Univ. 22, 8(1948).

Rat exythrocytes were irradiated with $6,030 \mathrm{x}$ and then were injected subcutaneously into healthy animals as 5 cc. suspensions, in order to determine the severity and nature of radiotoxic effects; the leucopenic effects were checked at 24 hour intervals. It was found that the radiochemical changes could not be traced to any particular chemical constituent of the cells; radiotoxing were formed only in the intact, live erythrocytes, probably by complex chain reacthons. Hemolysis of the cells prior to irradiation prevented the formation of toxing, while hemolysis immediately after the irradiation stopped the chain reactions and minimized the concentration of toxic material. (NSA) 609

Zacek, Jan and R. Rosenberg. THE ACTION OF XRAYS ON THE MEMBRANE OF RED CORPUSCLES.

Compt. rend. soc. biol. 144, 462-4(1950).

Blood, drawn by veinous puncture, received an $\$$-ray dose of $2 \%, 000 \mathrm{r}$ at $80 \mathrm{lv}$ after which it was hemolyzed and dried, and then examined under an electron microseope. The size and shape of the cell membrane did not change (as compared with nonir radiated cells). However, the ordinary granular gurface was replaced in gome gpots by a fine fibrous protein-like structure. The authors believe this change is due to the chemical decomposition of the lipo-

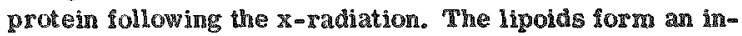
terstitial network which diffuse out during hemolysis and พaรhning. (NSA)

\section{Hemopoiesís - Humans}

610

Bourret, J. THE BLOOD CRANGIS IN ROENTGEN PERSONNTE. Deut. med. Wochschr. 74, 4477(1949).

For giss years at the Civil Hospital at Lyon, a semiannual blood checkup has been pursued in order to determine the amount of radiation exposure the roentgen persomel have suffered from roentgen rays and radioactive materials. Furthermore, a clever device, such as that worn by American Atomic worlsers is worn by the pergonnel, a film badge which indicates an exposure to the rays. However, it has been found that the weelsly exposuse of the personnel is between $1 / 4$ and $1 / 100 \mathrm{r}$, well within the $1 \mathrm{r}$ weekly tolerance limit. Workers specializing in roentgen diagnosis of the digestive tratet encountered the largest amount of exposure. Even here, the systematic blood control system showed no serious damage from ray exposure. (NSA)

BOWning, Ethel. BLOOD CPANGES IN LUMNIERTS USING RADIOACTIVI MATERIAL. Brif́, Med. J. 1, 428-31(1040)

In a 7-year study of 2,737 workers who used radioactive material (and 100 control workers), cormplete blood analyses were done while they were working, and, of these, 366 were followed up. Txposure was limited to anall amounts by various precautionary methods and occurred from external is radiation by a particles, $\beta$ and $\gamma$ rays, radon inhalation, ingestion from hand contamination, and inhalation of radioactive dust. Trangient lymphocytosis, the presence of Turk cells, premyelocytes, and premature large mononuclear cells were found, as well as a rare occurrence of anemia. The author discusses the findings and concludes that the blood changes are due to a slight temporary hyper612 stimulation of the reficuloendothelial gystem. (NSA)

Cantril, \$. T., L. Jacobson, and J. J. Nickson. TRE IFFITS OF IREADIATION ON TRE BLOOD AND BLOOD FORMING TISSUES. Argonne NatLonal Lab. Jan. 11, 1943. Decl. Apr. 1946. 33p. (MDDC-991)

A review is given of the effects of external whole body and local ir radiation in man and experimental animals. In ternal irradiation by radioactive gubstances in man and experimental animals is considered. The lymphocyte and its predecessors are the most sensitive cells in the blood system. The characteristic response is a diminution in the number of circulating cells or an increase in the number of 
lymphocytes. The red blood cells are relatively insensitive to the effects of irradiation except for long continued exposure. Platelets are a fairly sensitive index of irradiation damage to the blood system in the acute exposure of whole body irradiation. Monocytes are of importance only in evaluating the more estreme degrees of irradiation change. When complete monocytosis occurs radiation damage is 613 severe. (ADD)

Cronkite, Eugene $\mathbb{P}$. IONIRING RADLATON INJUNE.

T. Am. Med. Assoc. 139, 366-\$(1049).

A single excessive serious exposure to ionization radiation is evidenced by marked lymphocytopenia followed by a characteristic sequence of events in the other leutrocyte members. The diagnosis of repeated exposures to lesser amounts of lonizing radiation requires blood studies and differential counts of radiation in urine, fecal, and namal secretions. The principal protection from ionizing radiation is physical control of radiation intensilies by establishing monitoring procedures and prevention of contaminathon of personnel by radioactive materials. (N)

\section{4}

Denstad, T. THE RADIOSENSITIVTY OF THE BONE MARROW. Acta Radiol. 22, 347-58(1941).

The erythropoietic as well as the myelopoietic cells of bone marrow are very sensitive to the action of rays, especially the first mentioned ones. The immature forms are the first to disappear. The regeneration capacity, however, is good, even if large doses are administered. It is assumed that granulo-cytopenia (and most likely also lymphopenia) following radiotherapy are both due to a long distance action of the rays, an inhibiting action on myelopoiesis (and lymphopoiesis). The conclusions which might be drawn from this supposition with regard to the action of radiotherapy on leukemia and polyeytosis are discussed. (auth)

615

Dickie, A. and L. H. Hemplemann. MORPHOLOGIC CRANGES IN THE ISMPHOCYTHS OF PERSONS RXPOSED TO IONIZIG RADHTION. J. Lab. CHin. Med. 32. 1045-59(1947).

Persons chronically exposed to ionizing radiation and toric chemicals showed a significant decrease in total leucocyte count, while no significant change in the absolute number or in the percentage of lymphocytes in the differential count was found. Morphologic study of supravital preparations of blood cells of persons chronically and acutely exposed to fonizing radiation or in persons working with toric chemicals showed a striking increase in the number of refractive neutral red bodies in the cytoplasm of the circulating lymphocytes. The ne neutral red bodies have high density and are considered to be granules. They were not identified in fised preparations. (GA)

616

Diethelm, Lother and Wolfgang Hulscher. DANGRE FIOM SADIATION AND TPRE BLOOD PICTURE. Roentgen BLàt. 2, 145-63(1949).

An extengive statistical treatment was given to blood data on average people and on radiologists subject to radiation exposure of up to 10 years, conirming previous findings. Leucocytes, reticulocytes, and hemoghobin were sengitive but eosinophils, monocytes, and erythrocytes were not affected. The normal daily fluctuation of reticulocytes was found to reach a minimum at night and was correlated with the action of sleep. (NSA)

617

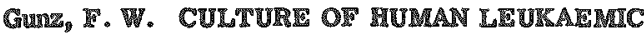
BLOOD CELLS IN VITRO; SOME FTELTS OF X-RAYS. Brif. J. Cances 3, 330-44.4(1949).
This paper presents the pesults of two groups oxporimonts wn the action of x-ays on human leukemic blowd cells; in the first group of experiments, the leucocytes were directly irradiated in vitro; in the second, they are ex. planted after the therapentic ir radiation of the patient. Sarmples of the venous blood of eight patients with chronic myeloid leukemia were incubated in a culture for $15 \mathrm{~mm}$ hen irrudiated with either $10,50,100,1,000$, or 10,000 : by a $20-k v$ apparatus at a target distance of either 21 or 81 cm and at a dosage rate of 622 or $62 \mathrm{r} / \mathrm{min}$, respectivily. It was found that mitosis was temporarily immbited by a dose of $100 \mathrm{r}$, and apparently permanenty inhibited by $1,000 \mathrm{r}$; as a result the number of immature leucocytes declined in the cultures. No increase in mitotic alonormali thas occur od expept when special ar tongements were made, in which case a lage number of abnormal, degenerating cells appeared, probably derived from braking-down prophases. There was mo sign that the $x$-rays acted by direct destruction of the resting cells. In the second series of cxperiments the leukemic leweorytes taken and cultured ater the therapeutic radiation of the patients showed a diminished proliferative act livity in vitro. (NSA)

618

Helde, Mats. THE CONN2 CTON BITWENN ROENTGEN RAY RESS FOR WORKERS AND CMANGES IN THEIR BLOOD PICTURES. Acta Radiol. 2\%, 308-15 $(1946)$.

"This is an investigation of various blood changes as related to probable amount of exposure to roentgen rays. Hy persegmentation, granulocytopenia, pathologic lymphocytes, and shift to the left appers to be well correlated with amount of exposure. Anemia, leukocytopenia, granulocytosí: leukneytosis and basophilia are not wall correlated 619 with exposure. (CPR)

Ingram, M. THE OCCVIRENCE OF WITI BLLOBED NUCLEI IN CFCLOTRON PERSONNEL, 19.59-64 of OUARTIRLI TISCINICAL REPORT, OCTODER 1, 1350 THLO DECEMBER 31, 1950. Alomic Pnergy Project, Univ. of Rochester. Tel. 1, 1951. 6p. $(\mathrm{UR}-152(1.59-64))$

"line incidence of lymphocytes with bilobed muclei in the blood of cyclot ron workers is reviewed for the period excending from July 1948 through October 1050. An ircrease in the incidence of the unusual lymphocytes in the group as a whole occured during the early periods of cyelotron oparation. The construction of a protective earth and concrete dike betwen the cyclotron building and the cyclotron laboratory พas followed by a return of the incidence of lymphocyes with bilobed nuclei to aygroxingately mormal levels. The siggnificance of the relationship between an inereased incidence of lymphocytes with bilobed nuclei and exposure to very small doses of radiation from the cyclotron is discusged briefly. (NSA)

\section{0}

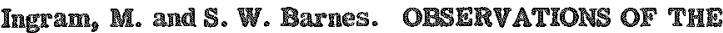
BLOOD OF CYCLOTRON WORERTS. PhysIOL. Rev. 175, $1765-6(1949)$.

This document is reproduced below in ifs entirety. Altention is called to the finding of two types of unisual cella in the blood of pergonnel of the 130 in. cyclot ron at Rochestor, New York. These are, respectively, vesy enrly mononuclear cells and cells which apreas to be lymohocytes with bilobed or double mucled. These cells, particular the lat ter, oceur in very small numbers, and it has been meces sary to inspect severd thousand leukocytes from each por son in order to be assured of the finding. "Ihe "early monomuclear cells" 2 re large, peroxtdase negative, have deeply 
basophilic cytoplasm and no nucleoli. The " ${ }^{\text {atymphocytes }}$ with bilobed nuclei" tend to be somewhat larger than typical large lymphoeytes. The nuclei stain slightly lighter than the nuclei of the other lymphocytes but are similar in consistency and have no nucleoli. The cytoplasm is clear, basom philic, peroxidase negative, and commonly contains a few typical azurophilic granules. Both kinds of cells have beer found in individuals not associated with the cyclotron who have abnormal blood smears due to infections, specifically in several cases of infectious mononucleosis and in one case of acute pharyngitis due to Hemolytic H. Infuenza. There is, however, no indication that the findings in cyclotrun personnel are due to infections. Early mononuclear cells are occasionally found in routine films of "normal blood," however, lymphorytes with billobed nuclei have not, to cur knowledge, been described previously, and it is felt that they represent a true departure from the usual or normal blood picture. Although it is not posaible to draw any conclusions relative to the signipicance of these cells as indicators of radiation damage, the findings may conceivably have such significance, and are presented at this lime so that they may come to the attention of responsible individuals associated with cyclotrons in other institutions. (NSA) 621

Ingram, M. and $\$$. W. Barnes. PISPRRMENTAL CONPIRMATION OF A PREVIOUSLI REPORTE UNUSUAL TINDING IN THE BLOOD OF CYCLOTRON WORRERS. Sclence 113, 32-4(1951) (UR-137)

The relationships between the increased incidence of lymphocytes with bilobed nuclei and exyosure to radiation from the cyclotron has been confirmed experimentally. Three dogs were exposed on three different occasions to doses of radiation which were believed to be well below the tolerance dose. After each exposure the re was a marked increase in the incidence of blood smears containing one or more of the abnormal lymphocytes. The frequency of accurrence of such smears was maximal during an approximately two-week period after each exposure. (NSA) 628

Jacobson, Leon O. and Edna Kx. Marks. THE HEMATOLOGICAL EFFECTS OF IONIZING RADLA TIONS IN TRE TOLERANCE RANGE. Radiology 49, 286-98(1947).

Lymphocyte xeduction in the peripheral blood is the most sensitive indicator of acute or subacute exposure to externally originating or internally deposited radioisotopas. No hematological change is detected in mice, rabbits, or guinea pigs with a daily wholebody exposure to 0.1 r of $\gamma$ radiation extending over three years. Changes may be observed for doses above $1.1 \times$ per day, with guinea pigs most aensitive, followed by mice and rabbits. Human being subjected to Wholebody $\mathrm{z}-\mathrm{ray}$ exposure totaling $300 \mathrm{r}$ given in divided daily doses of 5 to $20 \mathrm{r}$ showed a degree of sensitivity of the hemopoietic system roughly compatable to that of the 623 guinea pig and the dog. (CA)

Kikuchi, T., al. REMATOLOGTCAL OBSERVATION IN THE ATOM BOMB SUPFERER IN MROSPHMA CITY ONE YHAR AYTER THE BOMBING (2ND REPORT).

ISyoto Imperial University. [nd] 11p. (NP-305)

Henatological observations were made of atonic bomb sufferers (510 cases) one year after the bombing and of healthy Japanese (173 cases) as controls in Hiroshima city, with results as follows: Red blood cell counts were less than 4 million in $71.7 \%$ of the sufierers and in $61.8 \%$ of the controls. Color index over 1.21 was found in $50.6 \%$ of the sufferers and in $8.8 \%$ of the controls. In white blood cell count, there was no case under 3,000 in $1 \mathrm{cmm}$ of blood in the controls; and the counts under 8,000 were found in $14.2 \%$ of the sufferers (counts under 3,000 in $2.1 \%$ ) and in $3.5 \%$ of the controls. In difierential count of leuliocytes feosinophils, neutrophils, lymphocytes, monocytes, and plasma cells) and Sugiyama's mean nuclear lobes of neutrophil leukocytes, there were no remarkable differences in both cases; but the curve of neutrophil leukocyte count in the sufferers shifted to lower percentage than that of controls and in the curve of lymphocyte count, this relation was contrary. Blood platelet count was under 90,000 in $28.9 \%$ of the sufferers and in $14.9 \%$ of the controls. Bleeding time was almost normal in both cases, but the cases with bleeding time over five minutes were found in $4.9 \%$ of the sufferers and in $5.2 \%$ of the controls. The data are tabulated. (NSA) 624

Knowiton, Norman P., Jx. THE VALUE OF BLOOD COUNTS IN INDIVDUUAS EXPOSED TO IONIZING RADIATION. Los Alamos Scientific Lab. [nd] 25p. (AECU-307; LADC-682)

A study of the blood counts of 10 men exposed to about $0.2 \mathrm{x}$ of $\gamma$ rays per week for 117 weeks showed a small but significant decrease in the white blood cell, absolute neutrophil, and absolute lymphoeyte counts. A control group of 117 individuals showed no such change while a group of 46 people exposed to 0.01 to $0.1 \mathrm{~g}$ per week showed a slight but not very significant decrease in these blood elements. It was concluded that, although the routine blood count is a poor detector of radiation injury, it is the best clinical test available. (NSA)

625

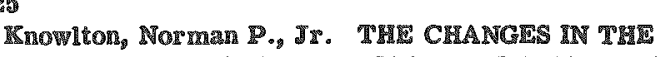
BLOOD OI HUMANS CLRONICALLY EXPOSED TO LOW LRVEL GAMMA RADLATION. [nd] DeCl. Dec. 21, 1948. 16g. (AECU-1021; LADC-587; LAMS-16Rev.) $(0.76-82))$

A group of 10 individuals exposed to $0.2 \mathrm{E}$ of gamma radiation per week showed a significant fall in the total white blood count and the absolute neutrophil and lymphocyte counts during a 77 -week period. The degree of fall would not have been predicted from experimental ir radiation in animals, and other unknown factors might also have been causal agents. It is suggesed that hematological and exposure data in other laboratories by analyzed statiatically to confirm or dimprove the effects of such low dosages of ionizing radiation on humans. (NSA) 626

Low-Beer, B. V.A. and Paul M. Aggeler. NRMATO LOGICAL EIFECTS OF IONIZING RADIATIONS. VI. PROGRESS REPORT APRIL 1, 1240-TEBRUARY 28, 1950.

Radiation Laboratory, Univ. of Calif. [nd] 87p. (UCRL -862$)$

The progreas made in the study of hematological effects of total-body irradiation during the period from $\mathrm{Apr}$. 1 , 1949 , to $\mathrm{Feb}$. 28,1950 is reported. The purpose of this investigation is the observation of morphological chasges which become evident in the peripheral blood of patients collowing exposure to therapentic doses of lonizing radiations. Patients who have been sollowed hematologically include one group treated with 100-, 200-, or 1000-kv 8-rays who had been expozed to total accumulated body doses of 100 to $390 \mathrm{~g}$ as measured on the skin; a groun treated with radiophosphorus, $\mathrm{P}^{32}$, for advaxced generalized arthritis who had received intravenously total doses of 3600 to 8000 $\mu c$; and a group who had been orally administered radiolodine, $I^{198}$, in amounts varying from a few hundred to $100,000 \mu \mathrm{c}$ for Grave's disease. Dose-effect relations are analyzed. (NSA)

$62 \%$

Marks, Edna IR. FFFECTS OF RADLTION ZMPOSUR

ON THE CELLS OF TME PERTPRERAL BLOOD.

Argonne National Lab. Mar. 1947. Decl. Max. 10,

104\%. 3p. (MDDC-912) 
A reduction in the erythrocyte values may be expected following acute and chronic total body irradiation. The anemia following chronic total body irradiation may be normocytic, hyperchromic, or macrocytic depending upon the size of dose and the sensitivity of the subject. The total number of reticulated erythrocytes is reduced following radiation exposure, but no changes have been found in the morphological character of the reticulum. Along with the reduction of platelet values after irradiation, giant thrombocytes are frequently found, occasionally larger than normocytes. The hyalomere is extremely abundant and the granules in the chromomere are usually prominent and large. The most sensitive cellular constituent of the peripheral blood appears to be the lymphocyte, and the reduction in its values closely parallels that of the total number of leukocytes. With increasing amounts of radiation exposure, the number of lymphocytes with abnormal morpho628 logical characteristics increases. (ADD)

Marks, Edna I., George Sacher, and Leon O. Jacobson. EFFECT OF EXERCISE ON LEUCOPENIA; A STATISTICAL ANALYSIS, P.456-66 of INDUSTRIAL MEDICINE ON THE PLUTONIUM PROJECT, Robert S. Stone, ed. New Tork, McGraw-Hill Book Company, Inc. 1951. (National Nuelear Energy Series, Division IV, Volume 20, Part B, Paper 6) (AECD-2133; ANL-HDY -466)

Leucocyte and differential leucocyte counts were made on 35 subjects immediately before, immediately after, and 15 minutes after the subjects performed the "standing running exercise." These individuals were male and pemale employees of the Metallurgical Laboratory. One group was selected from those who worked in that part of the laboratory where exposure to lonizing radiations up to the tolerance arnount was possible, and the other had no known exposure to significant amounts of such radiations. Certain individuals in the exposed group had leucopenias; radiation exposure was suspected in the etiology of these leucopenias. The effect of exercise on the leucocyte response of the leucopenic and non-leucopenic individuals in the exposure group was compared to the response of the control unexposed group. Upon analysis no difference in response of these two groups was noted; all responded with an increase in leucocyte values immediately after completion of the exercise. The increase was comparable in the exposed and non-exposed individuals. Furthermore, no change in the stage of maturation of the polymorphonuclear neutrophils or morphological change in the lymphocytes was noted in either group. The evidence suggest that the response to the exercige test is due to a transient alteration of the distribution of mature cells in the vascular system and lymphatics as a result of increased blood flow. The test could thus be of little value in detecting a decreased response potentiality of the leucoporitic tissues as a result of expo280 sure to radiations. (NSA)

Moses, Campbell and Madeline Platt. CHANGES IN THE TOTAL CIRCULATING EOSNOPHILE COUNT IN CYCLOTRON WORKERS. Science 113, 676(1951).

Eosinophile counts were made on three workers who received an indeterminate exposure while sanding the Cu dees of a cyclotron. Two of the three workers demongtrated a marked increase in the number of total circulating eosinophiles over a period of 12 weelss. All counts have since returned to normal and have remained normal for 6 months. It is suggested that the total circulating eoginophile count may be a useful indication of exposure to radiation in individuals employed in $x$-ray, cyclotron, and other laboratories with radiation hazards. (NSA)

\section{0}

Moshnan, Jack. MEDICAL RECORDS PILOT STUDY. Office of Research and Medicine, ORE, AEC. [nd] 44p. (ORO-13)

A pilot study has been completed of the medical records of operating contractors of the $\mathrm{AEC}$ in which it was designed to indicate any statistically significant changes in an individual's laboratory and clinical examination which might be due to chronic exposure to low-level irradiation. 1050 individual cases are considered from both the medical and statistical aspects. The study consisted of a detailed investigation of five specific hematological factors, namely, the red blood cell count, hemoglobin, white blood cell count, pexcentage of lynphocytes, and the percentage of polymor631 phonuclear neutrophils. (NSA)

Mossberg, Harry. RADIATION THROMBOCYTOPENIA. Acta Radiol. 28, 110-14(1947).

The author has recently published in the same journal an article entitled "Two cases of allergy-like reaction with hemorrhagic diathesis following roentgen treatment." Both cases died of a hemor rhagic diathesis following relatively big doses. It occurred to the author that there might exist a similar though perhaps lass pronounced effect with "ordinary" roentgen treatment. He therefore investigated 40 patiens, in two-thirds of whom he found a definite reduction in the number of platelets. Five patients showed not only thrombocytopenia but also clinical manifestations of hemor rhagic diathesis. Thromboc ytopenia is especially liable to occur when radiation is applied over the thorax, particularly over the sternum. The importance of following the thrombocyte count during and after roentgen treatment is referred to. (EM)

632

Mossberg, Marry. THE BEHAvIOR OR THE THROMBOCYTES DURING AND AFTER LOCAL RADIOTMERAPY. Acta Radiol. 29, 277(1948).

The aim of this investigation was to give a contribution to the knowledge of the number of thrombocytes in the circulating blood during and after local radiotherapy, with doses of a size generally used in, for instance, treatment of malignant tumors. The literature on radiation effects on the hemoiopoetic system is reviewed selectively beginning 1903. Different theories roncerning the radiation effect on the blood and the cells of the bone marrow are quoted and discussed.

633

Mossberg, Harry. PLATELET VALUES IN PERSONNEL ENGAGED IN RORNTGEN DIAGNOSTIC WORE. Acta Radiol. 34, 186-92(1950).

Among personnel occupied in roentgen diagnostic work the author has found a definite decrease in the platelet count in about one-third of the cases. On account of these findings the author is of the opinion that the platelet count should be used as an integral part of the routine blood control of personnel engaged in radiologic work. (NSA) 634

Nickson, J. J. BLOOD CRANGES IN HUMAN BEINGS FOLLOWING TOTAL-BODY IRRADIATION, p.308-37 of INDUSTRIAL MEDICHE ON THE PLUTONUM PROJECT, Robert S. Stone, ed. New York, McGraw-Hill Publishing Company, Inc. 1951. 511p. (National Nuclear Inergy Series, Division IV, Volume 20, Part B, Paper 2) (AECD-2432; CH-3858)

Results of examinations of elements of the peripheral blood in 14 individuals following exposure to total body radiation are presented. The individual s are divided into three groups: Group I consists of eight individuals who 
were irradiated with X-rays at one sitting; Group II cunsists of three individuals who were irradiated with X-rays in divided doses; and Group III consists of three normal individuals who were irradiated with $\mathrm{X}$-rays by three doses of $7 \mathrm{r}$ each. In Group I the most persistent abnormality noted was a diminution in the number of lymphocytes shortly after the completion of the treatment. In Group II depression in the lymphocytic count was also the most marked single change. In Group III no alterations were noted in the ele635 ments of the peripheral blood which were studied. (NSA)

\section{Nordenson, Nils G. BLOOD CRANGDS IN RADIOLOGIC}

WORK. Acta Radiol. 27, 416-32(1846).

Material obtained from bluod investigations on $1,566 \mathrm{in}-$ dividuals working with roentgen rays or radium is presented in comparison with similar gtudies on 450 individuals not su engaged. Blood changes, including leukopenia, granuloeytopenia, inereased "shift to the left," hypersegmentation and pathologic lymphocytes, eosinuphilia and basophlia, tendency toward leukocytosis, polycythemia, and anemia occurred in $52 \%$ of the radiologic personnel. Correlation between the degree of protection and the incidence of blood changes was good when the variation in individual resistance was considered. Significant injuries were observed in about $6 \%$ of the group. These the author designates as persistent granulocytopenia and leulopenia, combined and pronounced changes in an individual study, hemoglobun of less than $62 \%$, a red cell count of less than three million, a whate count of less than 3,000 , and a lymphocytosis of more than $60 \%$. No serious case of anemia was 836 seen. (R)

Parcher, F. and B. Kanee. REACTIONS OF THP HEMOPOIETIC SYSTEM TO AGENTS USED IN THE TREATMENT OF DERMATOSES. EFFECTS OF LOW VOLTAGE ROENTGEN RAY THERAPT. Arch. Dermatol. and Syphilol. 53, 1-5(1946).

This study was made over a period of four years. patients with generalized dermatoses of various types were selected. Low voltage roentgen rays were administered. As a rule, three treatments were given each week for a maximum of eight weeks, and approximately sis fields were ex posed at the tme of each treatment. Seventy-five unfiltered rays were administered to each irradiated field at a focusskin distance of $16 \mathrm{~cm} \times \mathrm{m}$. a. $\times 100 \mathrm{kv}$ for 30 meconds. Fll tered rays were given at a focus-skin distance of $25 \mathrm{~cm} . x$ $5 \mathrm{~m} . \mathrm{a} . \times 137 \mathrm{kv}$ for 30 seconds filtered through 1 or $3 \mathrm{~mm}$ of aluminum. Blood counts were made before treatment was started, repeated at intervals during the course and again after therapy was discontinued. This low voltage irradiated was followed by leukopenia or by leukopenia and hypochromia anemia - -quantitative changes similar to those following high voltage irradiation. It is believed that individual variations in radiosensitivity and dosage are the primary factors in the development of these changes. (BA) 637

Price, C. H. G. OBSERVATIONS UPON THE LFMPHOPENIA OF X-RAY IRRADIATION. Brit. J. Radiol. 21. 481-93(1948).

Obse rvations made upon 16 women treated for carcinoma with deep $\mathrm{X}-\mathrm{r}$ ay irradiation by a formal technigue show that the reaction of the lymphucyte cuunt follows a characteristic pattern. The major change in the total lymphocyte count appears to take place during the early part of a course of treatment, and is mainly shown in the lymphocyte/integral dose curves by the values of component $B$ (an indirect effect, which for small doses may be due to the effect of substances elaborated in the suprarenal cortex and having a regressive effect upon lymphwid tissue). It is suggested that this component may represent the result of the direct action of the radiation (component $A$ ), and the drop in the total lymphocyte count due to this is large and relatively rapid. The further drop in the total lymphocyte count which takes place in addition to component $\mathbb{A}$ may then be due to the indirect action of the radiation, which effect tends to diminish as the dose of radiation increases. Eventually, a stage is reached at which further irradiation, at the rates of dosage here considered, causes only a very small change in the total lymphocyte count. (NSA)

638

Schwart:, S., et al. . STUDIES OF THE HMOLYTIC ERFECT OF RADUATION. Univ. of Chicago Metallurgical Lab. July 10, 1846. 510. (MODC-1342)

Evidence is presented indicating that both acute and chronic xadiation anemia are due in part to a hemolytic reaction, and not solely to an inhibition of erythropoietic activity as has been agsumed by most investigator. The hemolytic concept is based on the following evidence taken from a survey of the literature as well as from the authors" experimental studies. (1) The red cell count in human and animal subjects may fall at a rate that is Iaster than can be accounted for by assuming even $100 \%$ inhibition of erythropolesis. (2) The red cell count may decline at a time when the reticulocyte count is actualy elevated. (3) Histological evidence of increased red cell destruction has been demonstrated repeatedly. (4) The bone mar row of humans that have anemia due to chronic radium poisoning is typically hyperplastic, not aplastic. (5) Increaged bile pigment excretion has been ghown to occur following administration of large doses of $\mathbb{P}^{32}$ or total body $\mathrm{X}$-ray. Excessive irradiation appears to destroy both mature and immature cella in increased amounts. More studies of human subjects are required to evaluate the importance of this hemolytic factor. Special attention in these studies should be given to bile pigment excretion and reticulocyte measurements. A bibliograxhy of 65 references is ineluded. (ADD) 639

Snell, Fred M., James V. Neel, and IR. Ishbash. MEMATOLOGIC \$TUDIES IN MUROSWMA AND A CONTROL CITY TWO YEARS AITER THE ATOMIC BOMBING.

Arch. Antern. med. exp. 84, 509-604(1949).

Nine hundred and twenty-four relatively heavily irradiated subjects were examined in Wiroshms to determine their peripheral hematological condition $20-33$ months after irradiation; they were chosen on the basis of degree of epilation. The results of erythrocyle and leukocyte counts, differential blood counts, hemoglobin concentrations, hematocrit readings, plasma reading, and reticulocyte counts were compared with those of a normal group of gubjects from another city. It was observed that: the erythrocyte count, hemoglobin concentrations and hematocrit readings were slightly, but gignificantly, depressed for the irradiam tion subjects; the irradiation subjects showed a slight relative depression of 1 ymphocytes and a slight elevation of eosinophilg; plasma protein values possibly were higher in the Hiroshima subjects; and, there was a gignificantly greater variability in the regults in Miroshima than in the normal group, which cannot be attributed to a differential response on the part of any particular age or sex group. The individual subjects who absorbed greater amounts of radiation tended to ghow the most pronounced depression in the erythrocyte and lymphocyte counts and the greatest alevation in the eosimophil counts. The authors advise againgt attributing the slight differences observed here entirely to 640 atomic radiation. (NSA)

Tripodo Cesare and Guseppe del Buono. PIEMOPOIITTC FUNCTION AND IRRADIATION OE TRY PILORTC 
DUODENAL TEACT. PART I. BEHAVIOR OF TRYTHROPENLA AND OF DIGLSTVE LEUCOCITOSIS IN NORMAL PIRSONS \$UBJECTED TO IDRADIATION OF TYY PYLORE-DUODENAC TRACT. Haematologica 31, 693-710 (1888).

A total of 11 normal persons was irradiated in the region of the pyloric-duodenal tract with three doses of $200 \mathrm{x}$, delivered by a 160-kV $\mathrm{z}$-ray machine at a target distance of $30 \mathrm{~cm}$ and through an aluminum filter. The irradiation was spaced at intervals of 3 days for a total dose of $600 \mathrm{x}$. No distinet changes in the number of erythrocytes, in the leucocyte picture, in the number of platelets, in the sedimentation rate of the exythrocytes, or in the behavior of the leulrocyte digeative reaction to the administration of a meat diet were noted; alecrease in the normal erythropenic digestive reaction, however, was noted. Confirmation that digestive erythropenia represents the initial mechanism that in normal conditions detexmines and regulates physiological erythropoiesis has been obtained. (NSA)

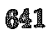

Warren, \$. BLOOD FINDINGS IN CXCLOTRON WORRRRS. Radiology 39, 104-91942).

The blood counts and hemoglobin levels of 85 persons more or less exposed to slight radiation from cyclotrons and temporarily radioactive isotopes have been followed for perlods warying from 3 to 30 months. Most persons under these conditions show no significant changes that are not explained by intercurrent disease. Four showed minor variations in white cell count. As with exposure to $\mathrm{x}-\mathrm{ray}$ or radium radiation, transient depression in white cell count is followed by elevation. This change occurs more rapidly in lymphocytes than in granulocytes. Some persons have an unstable bone marrow as shown by marked fluctuations in White cell count when subjected to only minor exposure to radiation. Such persons should not work where they are exposed to radiation. The precautions for the safety of these two groups of eyclotron workers appear adequate. (auth) 642

Whitby, Lionel. THE EFFECYS OF ATOMC HNERGY ON TYIE BLOOD. Prensa med. argent. (1947).

The deleterious eifects upon the blood of atomic energy and high voltage $x-r a y$ brings up the necessity for a thorough study of the problems and of the more practical method of immunization. The penetrating and pernicions effects of the radiations are similar to those produced by the gamma rays of radium. There are three such effects; (1) those produced by a sudden and intense irradiation, as observed among the victims of atomic bomb explogion; (2) chose produced by repeated small, well-tolerated doses, as observed in workers with radioactive substances and in investigators of nuclear physics; and (3) the effects secondary to continuous intermal irradiation given of by radioactive substances accumulated in the marrow. The inhabitants of Hiroshima and Nagasaki subjected to interse and penetrating acute irradiation, showed, firgt a latent period; then gramulocytopenia, which oceurred during the first three weeks; three to five weeks later, thrombocytopenia, with hemorrhagic diathesis, made its appearance; and from five to seven weelss after the explosion, cases of aplastic anemia developed. The gternal marrow in these cases, was Lound aplastic, physiologically if nol always anatomically. Bones absorb radiun thorium, and radioactive calcium and stronthum. The bone marrow is subjected io a continuous bombardment of destructive alpha rays, as observed in workers in the manuiacture of luminous watches. Aplastic anemia with an aplastic marrow, or with a hyperplastic impotent mara row, develops as a result of this internal irradiation, and the dematologic picture ig aimilar to that observed in the victimas of atomic bomb explosion. (GPR)
643

Zimmer, E. A. CHANGES PRODUCED BY X-RAXS IN BLOOD CORPUSCLES. PART 4. Strahlentherapie 82, 261$80(1950)$.

Results are discussed of an experimental investigation of typical changes produced in the numbers of blood corpuscles by $\mathrm{x}$-irradiations of various organs. Small doses on the bone marrow reduce the number of erythrocytes, while large doses increase it (consequently, the known failures in the radiotherapy of polyeythemia are due to excegsive doses); small doses on the spleen increase the erythrocyte count. The number of thrombocytes increases when bone marrow is irradiated with $400 \mathrm{x}$; it decreases when the spleen is treated with small doges. The leucocyte count decreases after amall-dose irradiation of the liver, or after a large-dose irradiation of the bone marrow; it increases when small doses are applied on the bone marrow. Other observations dealt with irradiations of cancerous patients (atypical blood changes), of patients with hyperthyreosis, of aplenectomized persons (atypical changes), and with irram diations of the skull (decrease of the erythrocyte count). (Extensive bibliography.) (NSA)

Memopoiesis - Animals

64

Adams, W. S. and J. S. Lawrence. THE PRODUCTION OF LEUKOP ABDOMINAL VISCERA OR THE CAT. Univ. of Rochester. Apr. 30, 1948. Decl. May 24, 1948. 14p. (AECD-2006)

eight normal cats were irradiated in the following man ner: daily doses (except Sundays) of 100 r each were delivered to the abdomen, the remainder of the cat being prom tected by a lead shield. A total dose of $3,200 \mathrm{r}$ was given. There was 2 uniform drop in the leukocyte count, polymor phonuclear leukocytes and lymphocytes (a fall of 64,57 and 76 per cent, respectively). This depression may be explained on the basis of any one, or a combination of the following factorg: (1) radiation of a larger segment of the bone marrow than had been suspected, (ionization chamber tests for " "catter" radiation showed that only very small amounts were affecting the part of the cat beneath the lead shield). (2) alterations occurring in the liver and spleen which increased the rate of utilization, destruction, or production of leukocytes. (3) alterations occurring in the intestinal wall which resulted in leukopenia possibly because of (a) increased demands for leukocytes, (b) increased loss of leukocytes through the camaged epithelial surface and/or (c) decreased production of white blood cells because of failure to absorb or elaborate certain gubstances necessary for normal hemopoiesis. (4) damage to an area rich in lymphoid tissue thereby producing lymphopenia. The authorg believe that factors 3 and 4 are probably the most 1 mpor. tant in the production of the observed leukopenia. (ADD) 645

Allen, Bennet M., Ole Arne Schjeide, and Ronald Piccirillo. THE INFLUENCE OF ANONIA UPON DESTRUCTION AND MTOSIS OF THE HEMATOPOIETTC CELLS OF XIRRADLATED TADPOLES. Atomic Energy Project, Univ. of Calif., at Los Angeles. Aug. 14, 1951. 13p. (UCLA-149)

The influence of anoxia on the initial (24-hx) effect of 500 Ir of $\mathrm{x}$ irradiation of $10 \mu \mathrm{y}$ of colchicine upon the hematopoietic cells of tadpoles of the bullirog, Rana catesbiana, has been determined. Anoxia markedly reduces the cell destruction resulting from the $x$ irradiation and in like percentage reduces the colchicine effect-pycnosis and arrested mitosis - during this period. Anozia applied after either of these treatments gave almost as great a ${ }^{6}$ protec - 
tion" against these effects. It is probable that this "prom tection" observed at the end of $24 \mathrm{hr}$ is wholly or in part to be interpreted as a delay of mitosis. Four equally spaced 1-hr exposures to anoxia proved much more effective than two, but there was marled destruction in spite of either amount of anoxia. The closely comparable degree of "protective" effect of anosia upon both the $x-i r r a d i a t e d$ and colchicine-treated groups and the fact that colchicine is an inhibitor of mitosis add further support to the view that destruction of hematopoictic cells takes place at or mear mitosis. (NSA)

646

Anderson, Elimabeth. CHANGES IN PRRIPHIRAL BLOOD AFTER SINGLE DOSES OF EXTERNAL BETA RADLATION, p.185-92 of FTFECTS OF EXTERNAL BETA RADLATON, Raymond E. Zirkle, ed. New York, McGraw-Mill Book Company, Ine. 1951. 242p. (National Nuclear Energy Series Division IV, Volume 22E, Papex ID) (MDDC-534)

In an effort to discover whether beta ray burng can be correlated with thermal burnw, a series of measurements of hematocrit and specific gravity of bloud plasma were taken on two rats which had received total body irsadiation with 7,500 rep of $\beta$ ray. Techniques were selected which gave accurate readings with small samples of blood. The copner sulfate method was used for measuring specific gravisy of the plasma. No gignificant change in the hematocrit was recorded, but specific gravity readings were below the normal range for rats on the $12 \mathrm{th}, 14 \mathrm{th}$, and 17 th days after exposure. These results suggest (1) that the plasma lost due to the $\beta$ ray burns is lost at such a low rate that the blood volume remains unchanged but (2) that the lost plasma proteins cannot be replaced quite fast enough to keep their concentration normal. Since death attributed to thermal burns is grimarily due to a fall of blood volume, it would appear that the physiological changes taking place are quite 647 erent for the two types of burns. (ADD)

Arvy, Lucie, Jacques-Andre Bolifard, and Manired Gabe. INCREASE IN TPRE NUMBER OF LABROCYTIES IN THE HEMATOPOIETIC TISSUES OF MCE FOLLOWING X IRRADIATION. Compt. zend. soc. biol. 232, 1022-4(1951).

The important increase in the number of labrocytes (heparinocytes, tissular basophils) found in the medulla of lymphatic ganglia and bone marrow of male white mice following whole-body exposure to $2000 \mathrm{z}$ of $\mathrm{x}$ gadiation is detailed. The thymic medulla, normally deprived of labrocytes, contains a large number following irradiation. Relation of this phenomenon to the general adaptat ion syndrome is discusad. (NSA)

648

Barnes, W. A. and O. B. Furth. STUDIES ON THE INDIRECT IEFTECT OF ROENTGEN RAXS IN STNGLI AND PARABIOTIC MICL. Am. I. Roentgenol. Radium Theray $49_{8} 62-81(1943)$

In addition to direct effects of $x$-rays on living tissue, there has been much discussion of indirect local and general effects, based on little definite experimental evidence. In the experiments reported, 2 of 4 mice in each group were joined in parabiosis. Two to four weeks later, one mingle mouse and one twin were irradiated over the entire body with $800-6,000$ r of $200 \mathrm{kv}$ lightly filtered $\mathrm{s}-$ rays; the other twin and the other single mouse being completely shielded. Slight leukopenia occurred in the shielded parabiont after exposure of its twin to $800-1,000 x$. In the irradiated parabiont the leukopenia was less severe than in the irradiated single animal. It is suggested that one or both of the follow ing occur: (a) Following the fall in number of leukocytes in the irradiated twin, leukocytes migrate from the non-irga- diated to the irradiated animal, resulting in the mild leutom penia of the former. (b) The blood of the irradiated animal is so altered that when a leukocyle level of the latter, but being diluted in the blood of the non-irradiated animal, the effect produced is small. (BA)

649

Bets, H. and J. Lecomte. HEMTOPOLETC REACWONS FOLLOWING REMOTE IRRADLATION OF RABBITS.

Compt. rend. soc. biol. 144, 303 $15(1950)$.

The rear paws of 12 rabbits were exposed to $200 \mathrm{r}_{2}$, the remainder of the body being protected by a lead shield. The animals were sacrifieed from 3 hr to 14 days following irradiation. Blood and histologic (spleen, cervical ganglious, bone marrow) changes are described. (NSA) 650

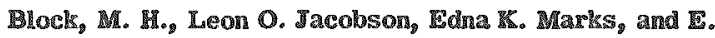
Gaston. THE EFIECT OF TOTAI-BODY X-IREADLATION ON A PRE-FISTING TNDUCED ANEMIA IN RABBITS. III. HISTOPATYOLOGICAL STUDUES. Univ. of Chicago Matallurgical Lab. June 194\%. 45 p. (AECU222: C脰-3890)

Exposure of normal rabbits $10800 \mathrm{I}$ total-body Xaradiation produces an intense destruction and atrophy of lymphatic and nnycloid tissue that is manifested in the peripheral blood by a leucopenia, thromboeytopenia, and anemia. In rabbils with as anemia, reticulocytosis, and with a hyperplaskia and left shiff in erythrocytopoiesis in the spleen and bone marrow, exposure to $800 \mathrm{I}$ total-body $\mathrm{x}$-radiation results in: (a) the usual destruetive and atrophic sequelae in the hematopoletic tissues but with gignilicantly less destruction of erythroblasts and significantly less inhibition of mitotic proliferation of erythroblasts so that comparatively large numbers of immature erythroblasts survive in the marrow and spleen; (b) a continued reliculocytosis which is cerived from these islands of viable exythroblasts during What should, in normal animals, be a much more atrophic period; and (c) a marked reticulocytosis in the regenerative period which is in part explained by a quicker regeneration of normal erythrocytopoiesis in the spleen. These results challenge the commonly held belief that cells that are more inmature and ravidly dividing are more suscentible to irradiation. (NSA)

651

Bloom, M. A. and W. Bloom. THE RADIOSENSTWWTYY

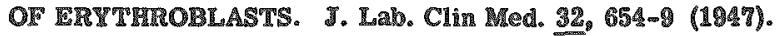

Mammalian bone marrow exposed to total-body irradiaton with x-ray and fast and slow neutrons revealed geverely depressed hematopoiesis. The degree of injury varies whith the dose. Changes in marrow cell anter radoactive lsom tope injection are much the same. The erythropoietic are more auceptible than myelocytopoietic cells and the arythropoletic in turn more susceptible than megakaryoeytes. Similar findings were demonstrated in chickens. Degenerating exythroblasts are not to be compared with degenerating lymphocytes. (BA)

652

Bloom, W. and Leon O. Jacoloson. SOME HIMATOLOGIC 2EIECTS OF IRRADIATION. BLood 30, 586-92(1948).

Studies with acute and chronic application of externally originating ionizing radiations and internally deposited Fadioisotopes fail to reveal evidence in the blood forming tissues and peripheral blood of a primary stimulation of hematopoiesis. Leulnocytosis during the firgt 24 hr after acute exposure is considered to be a reaction to injury. (BA) 653

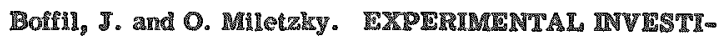
GATIONS ON TWE DAMAGE PRODUCED BY X-RAYS

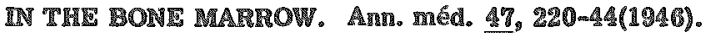


The authors have applied total irradiation to dogs and guinea pigs, subtotal (with protected stermm in dogs, and protected foot or head in guinea pigs), or local radiation, followed by the removal of marrow by puncture or biopsy. Radiation with an apparatus of constant tension, 180 or 200 $\mathrm{kv}$; filter $0.5 \mathrm{Cu}, \mathrm{DF}=1 \mathrm{~m} 50$ for the dogs, $0.50 \mathrm{~m}$ for the guinea pigs. Dose of $400,500, \mathrm{r}^{\circ} 500 \mathrm{r}^{\circ}$ in one sitting. Eleven experiments: three on dogs, eight on guinea pigs. (EM)

Brecher, G. and E. P. Cronkite. POST - RADIATION PARABIOSIS AND SURVIVAC IN RATS. Proc. Soc. Exptl. Biol. Med. 77, 292-4(1951).

Rats can survive an otherwise catal dose of radiation if joined after radiation to nonirradiated lifter mates. Such rats show accelerated bone marrow regeneration when compared with single controls. 论 is suggested that post radiation parabiosis is primarily of benefit because it accelerates recovery of myelopoiesis by the transfer of some cellular or non-cellular material from the nonirradiated partner. (auth) (NSA)

\section{5}

Brecher, G., K. M. Endicot, H. Gump, and H. P. Brawner. EFPECTS OF X-RAY ON LYMPROM AND HEMOPOIETIC TISSUES OF ALBINO MICE. Blood 3, 1259-74 (1948).

In order to gather details of the selectivity of radiation injury produced by whole body irradiation of $400 \mathrm{rr}(186 \mathrm{kV}$ 20 milliamps, at $50 \mathrm{~cm}$ with $0.25 \mathrm{~mm}$ copper and $0.55 \mathrm{~mm}$ aluminum filters, 6.24 minutes), quantitative myelograms, peripheral blood counts, and the histologic appearance of lymphoid tissues were studied in albino mice of the CrW strain during aeriod of four weeks. Measurements were made of the initial damage to all blood forming tissues and the reticuloendothelial system, the extent and duration of the suppression of their activity, and the rate of their rem covery. Nucleated red blood cells disappeared almost completely from the marrow within 24 hours after irradiation. Regeneration of the erythroid series began about the 7 th day. Only mild to moderate anemia developed, presumably due to the longevity of red blood cells. Suppression of mitotic activity and diminution of the myeloid marrow were marked duxing the first weels after irradiation. Granulocy. topenia in the pexipheral blood was severe for a very short period only. In contrast, early damage to the lymphoid tissue was more pronounced and lymphopenia was of longer duration than granulocytopenia in spite of early regeneration of the lymphoid tissues. Differences in the relative amount of damage to lymphoid and myeloid tissues and in their rates of regeneration were not of sufficient magnitude to indicate a truly selective damage to the lymphoid tissues under the conditions of the experiments. No morphologic evidence of damage to the reticulo-endothelial system was found. The possible use of irradiated aninals for functional 656 studies of the lymphoid apparatus is dimcussed. (NSA)

Brues, Austin M. and Leola Reitz. ACUTE MEMTOLOGIC RADIATION RESPONSE OF THE GUINEA PIG AS A FUNCTION OF LETUALITY, p.183-7 of BIOLOGICAL AND MEDICAL DIVISIONS; QUARTERLY REPORT, AUGUST, SEPTEMBER, OCTOBER, 1948. Argone National Lab. Nov. 1, 1248. 5p. (ANL-4227(p.183-7))

Three groups of guinea pigs were uged: four were irradiated with $150 \mathrm{x}$; four were irradiated with $200 \mathrm{r}$ total body I-ray; and four were non-irradiated controls. The lymphocytes respond similarly to those of various other species Fiven equivalent dosages, whereas the response of the heterophils is greater than in any other species on which data are available. Both marked leucopenia and terminal anemia are prominent in the guinea pirg after $200 \mathrm{r}$. Lymphocyte response is a function of $x$-ray dosage, whereas the effect of $x$-ray on the absolute heterophil count appears to bo closely related to the LD-50. (S.V.S.)

657

Cronkite, E. P., W. Ullrieh, D. C. Ellzholtz, C. R. Sipe, and P. K. Schork. THE RESPONSE OF THE PERIPHERAL BLOOD OF SWINE TO WHOLE BODY X-RAY RADLATION IN THIS LETHAL RANGE, Naval Medical Research Institute. Apr. 7, 1949. 23p. (NP-1411; $\mathbb{N M}=007-039 ; \mathbb{N M}-006-012.08 .26$ )

Four groups of swine containing $4,4,12$, and 4 animals were exposed to $200,300,400$, and $600 \mathrm{r}$, respectively, delivered bilaterally from a $1000 \mathrm{kv}$ industrial $\mathrm{x}-\mathrm{ray}$ tube. The mortality in the groups was as follows: $200 \mathrm{~g}, 12.5 \%$ $300 \mathrm{x}, 87.5 \% ; 400 \mathrm{r}, 100.0 \% ; 600 \mathrm{r}, 100.0 \%$. The expected leuropenia appeared with cell levels of the order of 1 to $3000 / \mathrm{cmm}$ after sis to seven days, the initial levels being about $17,000 / \mathrm{cmm}$. The response of the peripheral leukocytes in the $200 \mathrm{r}$ group showed a less rapid downard trend than that of the other groups. A reticulocytosis, in general, preceded recovery and could be used as a fair but not invariable sign of recovery. (NSA)

658

Davis, R. Wendell, N. Dole, M. J. Izzo, and I. I. Young MEMOLYTIC EFIECT OF RADIATION, J. Lab. Clin. Med. 35, 528-37(1950).

Four renal bile fistula dogs were exposed to total-body radiation in single doses of $150 \mathrm{r}$ (one dog) and $250 \mathrm{r}$ ( 3 dogs). The excretion of bilirubin in the urine by each of the 4 dogs inereased significantly during the first or second week following exposure. Possible sources of the increments in bilirubin output are discussed and it is considered that accelerated destruction of mature erythrocytes could be neither established nor disproved by these experiments. It is considered that some bilirubin is derived from destruction of partly hemoglobinized normoblast in the marrow. It is also suggested that somewhat more than the normal portion of the total bilirubin excretion might stem from hemoglobin precursors if such molecules should be less auccessfully incorporated into erythroid cells aster radia tion. The morphology of the red cells did not appear to be altered in any of the radiated dogs and serial measurement of osmotic and mechanical iragility of the erythrocyte revealed no significant alterations after radiation in the 2 dogs tested. Reticulocytosis and erythrophagocytosis could not be detected in smears of venous blood prepared after 658

De Bruyn, P. P. H. THE ERTECT OT K-RAYS ON THE LYMPRATIC NODULE, WITH REFERENCE TO THE DOSE AND RELATIVE \$ENSTTVITTES OF DIFFERENT SPECIES. Anat. Record 101, 373 405 (1948).

Guinea pigs, rabbits, and rats were ir gadiated with $\mathrm{x}-$ rays, $200 \mathrm{kvp}$, filtered with $0.5 \mathrm{~mm}$ of copper and $1.0 \mathrm{~mm}$ of aluminum in order to study the relationship between changes in the lymphatic nodules and dose of administration, and to learn whether there is a relationship between the histologic effects of radiation with $x-$ rays and their lethal action. The dose rate varied from $11 \mathrm{r} / \mathrm{min}$. to 16 $\mathrm{x} / \mathrm{min}$. The amount of $\mathrm{x}$-rays which killed $50 \%$ of the animals with 30 days (LD-50/30 days) (ured as the measure of lethal sensitivity) is $800 \mathrm{r}$ for rabits, $600 \mathrm{r}$ for rats, and $175 \mathrm{x}$ for guinea pigg. The animals were kulled in pairs after irradiation at intervals ranging from 30 minutes to 4 months. The mesenteric lymph nodes, patches of Peyer, 
and in the rabbit, the appendix also were used after being fixed in Zenkerformol, embedded in celloidin, and stained with hematoxylin-eusinazure II. Doses of $800 \mathrm{r}$ and $600 \mathrm{I}^{\circ}$ destroyed complately the great majority of the lymph nodules in rabbits. The nodules began to regenerate 21 days and 14 days, respectively, after treatment. A majority of nodules were only partially destroyed after $400 \mathrm{r}$. These regenerated rapidly and were normal in appearance after five days. A dose of $100 \mathrm{r}$ did not produce a marked change in the majority of the nodules, and $50 \mathrm{r}$ was the lowest dose producing histologically detectable changes. Six hundred roentgens and $400 x$ doses in rats produced changes identical to those in rabbits. However, doses of $175 \mathrm{x}$ in guinea pigs produced changes comparable to those in rabbits and rats after irradiation with $400 \mathrm{r}$ or lower. This suggests, considering the LD-50/30 days value of the three animals, that the intensity of damage to the lymph node is related to absolute dosage and is independent at the $\mathrm{LD}-50 / 30$ days of these species. (NSA)

660

De Bruyn, P.P. I. and R. C. Robertson. EFFCTS OF $X-R A Y$ AND NITROGEN MUSTARD ON LYMPHATIC NODULE OF THE RABBIT. Proc. Soc. Expti. Biol. Med. $72,717-18(1949)$

The histological effects on the lymphatic nodules of the rabbit of 8 -rays and the nitrogen mustard methyl bis (Bchloroethyl)anine hydrochloride are presented; the LD-50 (50\% lethal) dose of these is $800 \mathrm{r}$ for the $\mathrm{x}-\mathrm{rays}$ and 2 $\mathrm{mg} . / \mathrm{kg}$ for the nitrogen mustard; it was found that there is no quantitative histological difference in the response of the lymphatic nodules in rabbits to the $\mathrm{LD}-50$ doses of these two agents. The difference is minor in the mesenteric lymph node, where there is a slightly earlier regeneration after nitrogen mustard; the difference is greater in the appendix, ince the histologic changes after nitrogen mustaro administration at the $\mathrm{LD}-50$ level are comparable to those changes observed after half of the LD-50 dose of $\mathrm{x}-\mathrm{ray}$. (NSA)

661

Dixon, Frank J. RADIATION-INDUCED HEMORRRAGIC DISEASE IN CHICIEENS. Proc. Soc. Exptl. Biol. Med. 68, 505-7(1948).

Young chicks wer given repeated subcutaneous injections of $\mathbb{P}^{32}$ in order to produce severe radiation injury for a study of hemorrhagic disease resulting from radialion. The red blood cell, granulocyte, and platelet counts and blood coagulation time were charted during a 17-day observation period in which $25 \%$ of the birds died. Thrombocytopenia and agranulocytosis developed quickly. Although the character of the blood clot changed markedly, the re was no gignilicant increase in the coagulation 1 me as reported for animals (Allen and Jäcobson, Science, 105, 388, 1947), and the hemorrhage was much less severe than in animals. There was no increase in the number of mast cells such as occurs in people injured by atomic bomb radiation. The chicken appears relatively resistant to radiation when compared with mammals studied (Warren and Whipple, Journal of 682

Experimental Medicine, 38, 741, 1923). (NSA)

Dougherty, T. F. and A. White. PITUITARY-ADRENAL CORTICAL CONTROL OF LIMPHOCYTE STRUCTURE AND FUNCTION AS REVEALED BY EXPERIMENTAL X-RADZATION. Endocrinology 39, 370(1946).

CBA nice, 60 to 80 days old, received total body -radiation. A large dose of $\mathrm{x}-\mathrm{rays}, 200 \mathrm{~g}$, produced within three hours a lymphopenia, tissue lymphocyte degeneration, and total serum protein and gamma globulin increases. These changes also occurred in one day postoperative adrenal- ectomized mice receiving $200 \mathrm{r}$. This dose gave an anamnestic response in previously immunized mice in the absence of the adrenals. It has been demonstrated that 10 r produced the same physiological alterations, including the anamnestic response, in normal, but not in adrenalectomized, mice. Therefore, $10 \mathrm{r}$ influenced lymphoid tissue function by augmenting pituitary-adrenal cortical secretion. Large doses of $x-$ radiation may produce lymphocyte degeneration without adrenal mediation and thus increase circulating gamma globulin and antibodies. This is further proof that adremal cortical steroids produce hyperglobulinemia by their degenerative effects on lymphocytes, an end cell of adrenal cortical hormone action. It is suggested that $\mathrm{x}$-rays exert both a direct and an indirect effect on lymphocytes. The direct action may be manifegted even in the absence of the pituitary or adrenals, whereas the indirect action of $\mathrm{x}$ radiation on lymphocytes is mediated via the pituitaryadrenal cortical mechanism. (auth) 863

Downey, Hal. CYTOLOGY OF RABBIT THYMUS AND REGENERATION OF ITS TIYMOCYTES AFTER IRRADIATION; WITH SOME NOTES ON THE HUMAN THYMUS.

Blood 3, 1315-41(1948).

Newly born rabbits and young normal rabbits, four to five months of age, were irradiated for this gtudy of regeneration of lymphocytes in the thymus. A detailed study of the lymphocytes and of cells from the epithelial and mesenchymatous reticulum, and of the granulocytes was made on nonirradiated animals. The following conclusions were reached. Thymocytes are genuine lymphocytes. The largest lymphocytes of lymph nodes do not occur in the thymus; the smallest ones with pacyenromatic nuclei and scanty cytoplasm are more numerous in the thymus. Some lymphoblasts similar to myeloblasts occur in the rabbit thymus. All three types of granulocytes develop from lymphocytes and to some extent from the mesenchymatous reticulum in the rabbit. In the rabbit the heterophil and eosinophil granules are acidophilic when first formed, while in the human thy mus many have a basophilic quota as in the marrow. The reticulum is largely of epithelial origin and retains its epithelial characteristics in cytoplasm and nucleus. In the rabbit some mesenchymatous tissue is blended with the reticulum and may give origin to lymphocytes and granulocytes. The epithelium may form macrophages but does not differentiate to lymphocytes and other types of cells. In imprints from the rabbit the epithelial cells usually can be distinguished from those of the mesenchymatous reticulum. During regeneration following irradiation the latter tissue can be seen to form some lymphocytes locally; other lymphocytes enter the organ through the lymph vessels. Imprints stained with May-Grunwald and Giemwa are of great value for detailed cytology of the thymus and for determining cell relationships. Sections of thymus sised in Plelly's fluid, stained with methyl green and pyronin and dehydrated in dioxan are excellent for lymphocytes and the transitional stages resulting from their development from fixed mesenchymatous tissue during regeneration of the organ following 664 expogure to $X$-rays. (NSA)

Ducuing, J.g. Marques, and O. Miletrky. ACTON OF X-RAYS UPON THE BLOOD AND THE HPMATOPOIETIC ORGANS ATTER REPEATED TOTAL-BODY IRRADIATIONS (EXPERIMENTAL STUDY). J. radiol. electrol. $\$ 1,666-71(1950)$.

Total body irradiation was given to one dog $(25 \mathrm{r}$ each time, $775 \mathrm{r}$ per series; 4 series with one year's interval between 2 series) and to 3 guinea pigs: no. $1,2 \times 400 \mathrm{r}$ with 52 days' interval; no. 2, $300 \mathrm{r}$ and $25 \mathrm{r}$ after 90 days; no. 3, 
$2 \times 500 \mathrm{x}$ with 80 days' interval). There was always a sudden fall in leucocytes, platelets and erythrocytes after each series, as well as a prolonged bleeding time. Recovery followed, which each successive time was progressively less complete. Alter the 4th series the dog died, while the 3 guinea pigs died rapidly after their second series (no. 2 after a 2 nd series of only $25 \mathrm{r}$ ). The clinical aspect of these deaths was that of acute leukaemia, the bone marrow of the dog and guinea pigs no. 1 and 2 showed signs of true aplasia (lack of all formation of any blood cells), while in the bone marrow of no. 3 the red blood cells totally failed to appear and the few white blood cells geen consisted only of imma663 ture or pathological forms (medullary aplasia). (EM)

Furth, Jacob, G. A. Andrews, R. R. Storey, and Leon Wish. THE EFIECT OF X IRRADIATION ON ERY TYROGENESIS, PLASMA, AND CELL VOLUMES. Southern Med. J. 44, 85-92(1951) (ORNL-878)

The complex pathophysiological disturbances causing postirradiation anemia cannot be investigated by using methods commonly practiced to evaluate the red-cell picture, since simultaneous changes in plasma volume mask the magnitude of drop in erythrocyte mass. The investigations on blood tissues of dogs, rabbita, and mice after massive irradiation reported here include determinations of red-cell and plasma volumes, reticulocyte count, number of newly formed erythrocytes (those that had taken wp intrizvenougly injected radioactive $\mathrm{Fe}$ ), disappearance of various labeled substances from the blood and location of their retention in the body. These lead to the following conclusior.s: After massive irradiation, erythrogenesis ceases almost immediately and is not resumed until after 7 to 14 days. The plasma volume at first drops, and later riges. Heightaned permeability of capillaries permits escape of blood cells and other substances from circulation. Phagocytic $x$ eticuloendothelial function is not marledly altered. The hypothesis in advanced that continuous leakage of erythrocytes through damaged endothelium to tissue spaces and Iymaph channels is a major factor in the causation of post irradiation shock and anemia. (NSA)

\section{6}

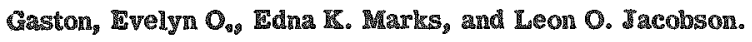

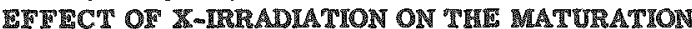
OF THE CIRCULATING ERTCULOCWTS. Uni\%, of Cheago Metallurgical Lab. May 10, 1048. Decl. July 22, 1848. 37p. (AECD-2132; CH-3970)

The time required for the disappearance of the reticulocytes from the blood of normal and $\mathrm{x}$-ir radiated rabbits with and without phenglhydrazine or bleeding-induced anemia was studied in vivo and in witro. In viltro atudies were also made on the rate of disanpearance of the normal human reticuloeyte and the reticulocyte of humans with various di geases. When studied in witro, reticulocytes in blood drawn from normal rabbits disapgear over a pertod of 24-48 hours. Reticulocytes from normal rabbits and from rabbit: with phenylhydrazine or bleeding-induced anemia have a comparable maturation rate. A dose of $800 x$ total-body -radiation does not alter the rate of maturation in anmples of blood drawn three hours after treatment; the rate is delayed in wample drawn 24 hour after irradiation. Blood from rabbits having induced anemia gives comparable reaults. In vivo atudies also ghow comparable results. The reticulocyte maturation time in the blood of normal. human aubjects ranger between 24.48 hour when gtudied in vitro. Nitrogen mugtard does not alter the maturthtion rate in patients having polycythemia vera. (This study was made one day anter the administration of mitrogen mustard.) (NSA)
667

Henshaw, P. S., J. W. Thompson, and H. I. Meyer. EXPERIMENTAL ROENTGEN INJUEY. V. EPTECTS ON HEMATOPOIETIC RESERVES AND REGENERATIV FE

CAPACITY. J. Natl. Cancer Inst. 5, 233-47(1945)

Experiments were carried out to determine the additive effects of doses of $x$-rays (controls) $12^{1} / 2,25,50,100,200$, $400 \mathrm{r}$ ) applied to the whole bodies of $\mathrm{C}_{3} \mathrm{H}$ mice; $4,000 \mathrm{lym}-$ phocytes per cu mm of whole blood as the lower limit of normal. Histologic examination showed mild injury in the hematopoietic organs after repeated small exposures and acute extengive destruction after the large doses. Remarkable regenerative capacities were observed. Similar experiments were made with guinea pigs, by using 25,50 , and $100 \mathrm{r}$ doses, and 3,500 lymphocytes per cu mm of whole blood as the lower limit of normal. Generally the same ef fects were seen as in mice although the guinea pigs showed a greater general susceptibility to radiation. Only mild changes in erythrocyle level were observed in either type of animal at any time. The fact that small doses, that singly elicit no detectable change in lymphocyte level, produce leucopenia after repeated application indicates that subliminal effects are produced and are additive; the destruction of lymphocytes occurs with each small dose and the rate of destruction must go beyond the reserve of $1 \mathrm{ym}-$ phocytes-acellularity in the hematopoietic organs was noticeable in the animals that received the larger coses. The pergi sting lymphopenia might be regarded as the beginning of a state of exhaustion. (BA)

668

MSU, C. L. and W. C. Ma. DIRECT AND INDIRECT EFFECTS OT ROENTGIN RADLTION ON THIP BLOODFORMING ORGANS OF FATS. Am. J. Cancer 39, 319-33 (1940).

Normal albino male rats, 6 to months, $350-450 \mathrm{~g}$. weight received daily doses of $125 \mathrm{v}$ in 10 minutes on the shin of the posterior suriace of the right hind leg to maxi ma of $1,000,2,500$, and 5,000 units, the factors being 100 $\mathrm{kv}, 4 \mathrm{ma}$ through $4 \mathrm{~mm} \mathrm{Al}$, at $30 \mathrm{~cm}$, and were killed by ether ca. 24 hours after completing treatment, for examination of the spleen, submaxillary lymph nodes, the femoral marrw. Results were: decrease of bone marrow cells in proportion to dosage in both femurs, and of lymphoid cells in the lymph nodes and spleen. (BA) 662

Ingram, M. and W. B. Mason. THE FFYCTS OF CHRONIC IESOSURE TO ROENTGEN RADIATION ON THI PERTPHERAL BLOOO OF IXPERIMENTAL ANIMALS. Atomic Energy Project, Univ. of Rochester. July 17, 1050. 23p. (UR-121)

Routine hematological examinations have been done repeatedly on dogs, rabbits, rats, and monkeys which have been exposed to daily doses of whole-body roentgen radiation in anounts near the tolerance range, i.e., doses varying from 0.1 to $10.0 \mathrm{r} / \mathrm{day}$. These dosages have been administered for a continuous period of not more than $2 \mathrm{yr}$. Both 250- and 1000-kvp machines were used. During the experiments the rats and monkeys acquired extraneous pashology of such a degree that it was necessary to exclude these animals. In dogs and Irabbits, changes in the hemograns which followed chronic expowure we re detected only by statistical methods. It would seen unlikely that routine hematological examination would provide a reliable mean. of detecting minimal radiation damage in any one animal. Lymphocytes anpear to be the most aensitive hematological indicators of the damage which results from chronic exposure to roentgen radiat ton. The least sensitive element in the peripheral blood appears to be the mature erythrocyte. 
The effectivenegs of small daily doses of roentgen radiation in produring hematological changes is dependent, in part, on the duration of such exposure. In some instances, changes were first noted during the second year of daily exposure. The data demonstrate a marked difference in the hematological responses of dogs and rabbits exposed daily to the ame duses of radiation. The reticulocyte depression, relative to that of the lymphocyte, is less pronounced in the present experiments than in the case of exposure to gingle large doses of whole-body roentgen radiation. Chronic exposure did not elicie the marked reticulocytosis which eharacteriged the recovery response to acute exposure. No consistent difference was observed in the hematological changes which collowed exposure to like quantities of roentgen radiation from the 250- and the 1000-bvp machines. (NSA) 670

Ingram, M. and W. B. Mason. THE IFTWCTS OF ACUTE EXPOSURE TO ROENTGEN RADLATIN ON THE PERIPHERAL BLOOD OF EXPERIMENTAL ANIMALS. Atomic Energy Project, Univ, of Rochester. July 17, 1950. 36p. $(\mathrm{UR}-122)$

Rats, dogs, and monkeys have been exposed to gingle whole-body doses of roentgen radiation. The dosage for rats varied from 5 to $1500 \mathrm{r}$ and that for monkeys from 300 to $2000 \mathrm{r}$. Dogs recelved $300 \mathrm{r}$, a dose appromimating $\mathrm{LD}_{50}$. Hemograms of all animals were determined at regular intervals for 25 to 60 days following ar radiation. Bone mar row examinationg were included in the studies utilizing doses approsimating $\mathrm{LD}_{50}$. The dafa pertaining to monkeys have been excluded from the present report because of extraneous infections which invalidate attempts to relate the observed changes to the radiation received. The major findings may be summarized as follows: (1) Regults of the present experiments indicate that routine hematological examinations are inadequate for detecting glight radiation damage in any one individual. Changes which occur following exposure to small doses can be demonstrated only by statistical methods. (2) The results of routine hematological examinations appear to be unreliable as criteria for determining the prognosis following isolated instances of whole-body exposure to roentgen radiation. No consistent or convincing difference between leukocyte counts of survivors and nonsurvivors could be demonstrated in the various experiments. (3) Lymphocytes and reticulocytes are strular with regard to the promptness and magnitude of their depression following single exposures to whole-body radiation. In gencral, depression of these cells is more marked and occurs earlier than that of other elements of the peripheral blood. (4) The depression of neutrophils occurs slightly later than that of lymphocytes and reticulocytes. (5) Significant depression of erythrocytes and hemoglobin is the last to occur following exposure, and the dosage required to groduce guch depression is much larger than that required to produce other changes. The recovery phase of the erythrocyte trends is characteristically preceded by a marked reliculocytosis. (6) With routine techniques, examination of bone narrow appears to offer no particular advantage in indicating radiation damage since changes are equally prompt in both marrow and peripheral blood cells. (7) In general, the magnitude of peripheral blood cell changes is roughly proportional to the dose for exposures up to approzimately $L_{50}$. Doses greater than $\mathrm{LD}_{50}$ tend to induce the maximum response and, if sufficiently large, prove latal inea relatively short time. (NSA) 671

Jacobson, L. O., et al. THE EFFET OF TOTAL BODY X-IREADIATION AND $\mathrm{g}^{\text {S2 }}$ ON TIIE PERIPRIERA BLOOD
OF NORALL RATS AND RATS WTTH COBALT-INDUCED POLYCYTHEMLA. Argonne National Lab. Mar. 1248. Decl. Apr. 20, 1948. 2p. (ALCD-2014)

A polycythemia was produced in Sprague-Dawley rats by the subcutaneous injection of cobaltous chloride five times weelly. The polycythemia was fully established in 90 days. The effect of $\mathrm{X}$-irradiation or $\mathrm{P}^{32}$ on the blood and bloodforming tigsue of normal animals and those with induced polycythenia was studied. Six groups of seven rats each were treated in the following manner: Group 1. Control-No treatment was given this group. Group 1I. Control-cobalt The animals were injected with cobaltous chloride inve times weekly. Growp III. X-Ray + cobalt-The animals were injected with cobaltoug chloride live times weekly. When polycythemia was fully established, 300 r whole-body $\mathrm{X}-\mathrm{ray}$ was administered in a gingle dose. The cobalt injections were continued after $\mathrm{x}$-irradiation. Group $\mathrm{IV} . \mathrm{P}^{32}+$ cobalt The animals were injected with cobaltous chloride ive times weekly. When polycythemia was Iully established a dose of radiophosphorus ( $1 \mu \mathrm{c} / \mathrm{g}$. body weight) was administered intraperitoneally. The cobalt injections were continued following the administration of $\mathbb{P}^{32}$. Group $V$. X X rayNormal animal were given one single exposure of $300 \mathrm{~m}$ whole-body $\mathrm{X}$-radiation. Group VI. $\mathbf{P}^{32}$-Normal animal were given one single injection of radiophosphorus ( $1 \mu \mathrm{c} / \mathrm{g}$. body weight). A comparable depression of the leucocytes of the peripheral blood was produced in normal and polycythemic animals after radiophosphorus injection. The dosage of radiophosphorus $(1.0 \mu \mathrm{c} / \mathrm{g}$. $)$, used in this experiment, produced no significant effect on hemoglobin, erythrocyte or hematocrit values in normal or polyeythemic animals. A comparable reduction in hemomlobin, erythrocyte, hematocrit and leucocyte values of the peripheral blood oceurred in normal and polycythemic animals exposed to $300 \mathrm{r}$ wholebody s-radiation. Recovery was essentially the same in the two groups. Histological studies of appropriately maerificed animals in these various groups have not been completed. On the basis of these data no apparent difference in the sensitivity of the hemopoietic system to a single dose of $\mathrm{P}^{32}$ $(1.0 \mu \mathrm{c} / \mathrm{g})$ or to $\mathrm{x}$-irradiation $(300 \mathrm{r})$ is evident between normal and polycythemic rats as judged by the pattern of response of certain hematologic constifuents of the peripheral bloud. (ADD)

672

Jacobson, Leon O., Edna K. Marks, L. O. Gaston, ․ L. SIMMmOns, and M. H. Block. STUDHES ON RADIO-

SENSITIVITY OF CELLS. Sctence 107, 248-50(1948).

The effect of $800 \mathrm{r}$ whole body $\mathrm{x}$-radiation upon animals with a previously induced regenerative anemia was compared with the effect of the same dose on normal comparable animals. Determinations were made of the erythrocytes and reticulocytes/cu $\mathrm{mm}$ and $\mathrm{Kb}$ in $\mathrm{g} / 100 \mathrm{ml}$. Normal irradiated animals develoged an anemia that reached the masimum in 14 days. Animals with a regenerative anemia prior to irradiation developed no further anemia. The recovery time was gimilar for both groups. The mean reticulocyte value of a group of phenylhydrazine-injected $\mathrm{x}$ rayed animals was decreased below the normal control value on the fth postirradiation day only. phebotomiged groug of animals responded to lrradiation in a manner comparable to the phenylhydrazine-treated group. Histologic studies showed that erythropoietic tissue in bone marrow and spleen were wholly destroyed in three days in normal animals receiving 800 roentgens. A slow recovery began on the 7th to 9 th day. The erythropoietic tissue was only par tially destroyed in animals irradiated with 800 is after treatment with phenylhydrazine or phlobotony. The data 
indicate that erythroblast vulnerability to irradiation injury is not increased by increased mitotic activity and proliferathon. (BA)

673

Jacobson, L. O., E. K. Marlis, and E. Lorenz. THE HE MATOLOGICAL ERIECTS OF IONIZING RADITIONS. Radiology 52, 371-95(1949) (A.CD-2085; CH-3864)

The objectives of the Health Division of the Plutonium Project were essentially: (1) to study the fundamental and comparative biological action of external radiations and internally administered radioactive materials, (2) to apply the findings toward the protection of individuals who worked in the Project, and (3) to use the findings generally for the protection of the public which was potentially in danger of exposure to these physical hazards. The hematologic studies conducted were divided into four main categories: (1) the effects of acute whole body exposure to externally administered penetrating radiations, (2) the effects of chronic whole body exposure to exte mally administered penetrating radiations, (3) acute toxicity of parenterally and enterally administered radioactive elements, and (4) chronic toxicity of parenterally and enterally administered radioactive isotopes. In order properly to correlate studies on the peripheral blood effects with those occurring in the hemopoietic tissue, the animals in the came or parallel experiments were sacrificed at appropriate intervals for histological study. All experiments included an adequate number of controls, and the frequency of sampling depended upon the chronic nature of the experiment. (NSA) 674

Jacobson, Leon O., Edna K. Marks, and E. L. Simmons. THE IFTECT OF TOTAL BODY $\triangle$-IRRADI ATION ON A PRE. 32, $341(1047)$.

A regenerative anemia was produced in zabbit by the subcutaneous administration of phenylhydrazine hydrochloride. The hematologic effects of $800 x$ total body $\mathrm{x}-\mathrm{irradia}-$ thon on such anemic animals were compared to effects on animals given only phenylhydrazine and allowed to recover spontaneously and to a group of animals given $800 \mathrm{r}$ alone. A group of normal untreated animals was studied simultaneously. The characteristic rise and subsequent reduction in heterophils and immediate precipitous fall in the lymphocyte value followed the administration of $800 \mathrm{r}$ in the group in which a regenerative anemia had been produced with phenylhydrazine as well as in normal control animals. The time of recovery of those elements to normal limits in the two groups was likewise comparable. An "abortive rise" in the leucoeyte values of both of these groups was noted between the fifth and eleventh days after the irradiation. The group of animals which received $\mathrm{x}$-ixyadiation alone developed an anemia with its maximum at 14 days and with secovery by 23 days. The animals in which a regenerative anemia was produced by phenylhydrazine and which were irradiated at or near the point of maximum anemia developed no further anemia, values returning to normal limits by 23 days. The erythrocyte and hemoglobin values of the group in which an anemia was produced by phenylhydrazine and which were allowed to recover gpontaneously, however, returned to normal within 14 days. The reticulocyte values of animals which had received phenylhydrazine had reached approximately 21 per cent when the x-irradiation was given and were reduced to a minimum value of 1.0 per cent within two days after the $x$ irradiation. Recovery to normal limits occusred by days in this group. The reticulocyte values of nomal control animal which were given $800 \mathrm{~g}$ fell maximally within two days, reached a minimum of less than 0.1 per cent, and recovered glowly but completely by 23 days.
The fallure of $800 \times$ total body $\mathrm{x}-1 \mathrm{i}$ radiation to produce fur ther anemia in animals with a previously induced regenerative anemia or to materially interfere with recovery is dependent in part on the number of surviving erythroblasts in the erythropoietic tissue. Histologic studies made on animals prepared in a comparable manex and sacrificed at varying appropriate intervals indicate that erythropoietic tissue was completely destroyed by 72 hours in the normal rabbits which received $800 \mathrm{r}$ and recovery proceeded slowly. On the other hand, although massive destruction of erythropoietic tissue occurred after exposure to $800 \mathrm{r}$ in animals in whom a regenerative anemia had been produced with phenylhydrazine, this destruction was delayed beyond 72 hours. This appears to indicate that a latency to $x-$ ray elfect was produced in erythropoietic tissue which had pre675

Jacobson, Leon O., Edna K. Marks, 2. L. Simmons, C. W. Hagen, and Raymond E. Zixkle. EFFECTS OF XWAYS ON RABBITS. PART II. THE HEMATOLOGICAL EFTECTS OF TOTAL BODY X-IRRADIATION IN THE RABPIT. Univ of Chicago Metallurgical Lab. July 1946. Decl. July 31, 1047. 11p. (MDDC-1174; CH-3796)

The hematological constituents of the peripheral blood have been studied in Swift snufle-free rabbits betore and after the administration of acute total body 8 -irradiation. Doses of $25 \mathrm{~g}$ to $800 \mathrm{~g}$ were administered and studies made through 26 days in many experiments. From the observatons made, the conclusions derived are: A significant normochromic anemia develops in groups of rabbits exposed to 500 and 800 x. A significant anemia develops only in individual animals within a group with doses below $500 x$. Reticulocyte reduction is significant with doses of $100 \mathrm{r}$ and above. An initial leucocytosis, significantly greater than controls, occurs within the first 24 hours after doses of $500 \mathrm{r}$. After $500 \mathrm{r}$ and $800 \mathrm{r}$, a sharp rise in leucocytes occurs by 12 hours, followed by a decline and another pealk of leucocytes at 24 hours. The leucocytosis during this period is due exclusively to an increase in heterophils. Lymphocytes decrease significantly within 24 hours after exposure to $25 \mathrm{r}$ and above. Reduction is apparent three hours after Irradiation with $100 \mathrm{x}$ and above, with a minimum value at from 24 to 48 hours. Recovery proceeds slowly. Supravital gtaining (Janus green and neutral red) reveals an increase in neutral red granules after $200 \mathrm{I}$ and above. Mitochrondrial changes are variable. Monocytes decreage indtally but return to nor mal limits or are increased by lour to six day after $200 \mathrm{r}$ and above. Meterophil values increase initially after 500 and above. No significant reduction was observed with coses of 300 and below. Doses of $500 \mathrm{x}$ to $800 \mathrm{x}$ inclusive produce a significant depression within 96 hours, with recovery by 23 days or less. Platelet values are reduced in a manner paralleling the granulocytes. An "abortive rise" in lymphocytes and heterophils between the 4 th and 11 th day after x-irradiation characteristically presedes recovery with doses of $200 \mathrm{r}$ and above, respectively. Morphological changes in erythrocytes, leucocytes, and platelets occur after doses of $100 \mathrm{r}$ and above. The lymphocyte values of the peripheral blood are the most sensitive known indicator of effect of acute total body $\mathrm{x}$ irradiation. A bibliography of 59 references is included. (ADD 670

Langendor $1 \hat{f}_{\text {, }}$. THE SUPPRESSION OF THE RETICULOCYTE COUNTS OF PYIE WIITE MOUST ATTER ROEHTGEN RADIATION. II. COMMUNICATION (G).

Strahientherapie $52,304-14$ (1938).

The effects of single doses and protracted fractional doses were compared. The intensily was $0.5-8 \mathrm{r} / \mathrm{min}$. for the 
vingle dose and $1 \mathrm{r} / \mathrm{min}$. for the second method. The total dose applied varied from 100-1,000 $\mathrm{x}$. The effect of the fractional method without protraction was much more pronounced both on the reticulocytes as well as on the organism as a whole than for the protracted fractional dose. The reaction following "long time" irradiation took a longer course in most instances than for the other method; likewise the same total dose was tolerated better with the protracted $67 \%$

fractional dose than with simple fractionation. (BA)

Latta, J. S. and O. C. Ehlers. THE EFIFECTS ON THE BLOOD AND HEMOPORETIC ORGANS OR REPEATED SHORT EXPOSURES TO X-RAYS. Am. J. Anat. 47, $447-74(1931)$

Twelve albino rats were subjected to a series of short $\mathrm{X}$ ray exposures, in most cases totaling $4-1 / 3$ hours; three were shcrificed during this treatment, six at or near the end of the experiment and three after different periods of recovery. Alter complete exposure, a very pronounced leukopenia (less than 1,000 leucocytes) and anemia (slightly over $2,000,000$ erythrocytes) were invariably present. The hemopoietic organs were correspondingly aplastic, with pictures of extreme degeneration. Recovery as evidenced by blood counts and histological conditions in the hemopoietic organs was variable, rapid, but not complete at the end of 28 days. A study is presented of the changing conditions of the hemopoietic organs, particularly in aplastic and regenerating conditions. Evidence is presented pointing to exiravascular exythropoiesis, with the absence of intersinusoidal capillaries, hemoblasts serving as the cells of 778. origin. (BA)

Lawrence, John S., Andrew H. Dowdy, and Wullam N. Valentine. ERTPCTS OF RADIATION ON REMOPOIESIS. Radiology 51, 400-13(1948).

The types of radiant energy under discussion are either electromagnetic energy waves, such as roentgen and $\gamma$ rays, or particulate matter having mass, such as $\alpha$ and $\beta$ particles, protons, and neutrons. The effects following radia tion administered as: (1) single dowe to the body as a whole, (2) repeated small doses to the body as a whole, (3) single or repeated doses to one arez, and (4) internal radiation (probably $\beta$ and $\gamma$ mostly) were considered. The greater part of the observations referring to the irradiation with a single dose to the body as a whole have been performed on animals. The hemopoietic picture is decidedly affected in any post-irradiation period for the duration of the life and by the speed of utilization of the different morphological elements in the blood. For example, after a dose of $500 \mathrm{r}$, the rapid decrease in lymphocytes reflects their short life span, for the first decrease was observed in 15 minutes. The granulocytes did not decreage appreciably in numbers until the end of 24 to 36 hours. The platelets began to disappear at the end of 72 to 120 hours, and the erythrocytes between 120 and 168 hours. After doses of 200 x or less very few or no changes in the erythrocytes or platelets were observed. When the dose was legs than $100 \mathrm{r}, \mathrm{no}$ large reductions in the reticulocytes were noted. However, a dose of 25 caused a decrease in the formation of $1 \mathrm{ym}-$ phocytes at the end of 24 hours. The results were less well defined in studies of the effect of small repeated doses; but In all the animals atudied, distinguishable changes in some of the hemopoietic elements were observed after a year of daily exposure to $10 \mathrm{r}$. In spite of the fact that changes could not always be denonstrated following small repeated doses, no doses were so gmall that they did not affect the average survival of large groups of animals. From this it is apparent that the total dose is important and that the small daily exposures to $x-$ rays are cumulative. The incidence of leukemia clearly increased in the animals exposed repeatedly to small doses. clearly that local irradiation does not produce direct effect upon the hemopoietic tissues that are not directly in the expused region. Since the larger part of human exposure probably take the form of small repeated doges, the most important deductions from these studies are concerned with the cumulative effect and the total dose in the production of pathological changes. There is the possibility of leukemia and related illnesses to be considered whenever there is exposure to a considerable quantity of $\mathrm{x}-\mathrm{ray}$. And finally an apparent recovery from radiation effects may be followed by the sudden appearance of severe anemia or other blood dyscrasies. (NSA)

678

Lawrence, John S., Willam N. Valentine, and Andrew DOwdy. THE IFECT OF RADIATION ON HAMOPOIRSIS; IS THERE ANY INDIRECT EFPECT. Blocd 3, 593$609(1848)$.

The general aim of the investigations has been to outaln evidence for or against "indirect" radiation effects. To this end, 26 sucessiul cros ctreulation experiments (carotid to carotid anastomosis) have been periormed between nor mal cats and radiated cats. Cross circulation was established in most instances at some specified time interval after the radiation of one partner. All intervals up to 82 hours after radiation of one partner were covered. In seven experiments cross circulation was established and then one animal radiated while the other was shielded. These were considered the most critical experiments of the group. Detailed data on leukocyte and lymphocyte count in the normal animals obtained during an approximately 28 day period of follow-up are presented. These data are not considered to support the thesis of indirect effects peculiar to radiation. A trend toward slightly lowe red absolute lymohocyte counts in normal animals after cross circulation was not considered significant, and in no instance did leukopenia develop in the normal animal. The literature is reviewed and discussed. (NSA) 680

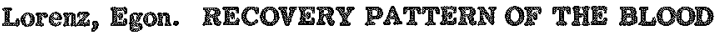
PICTURE IN GUINEA PIGS FOLLOWHG A LIMITIED TOTAL BODY EXPOSURE TO CMRONC GAMMA RADIATON. J. chm. phys. 48, 264-74(1951).

The recovery pattern of chronically $\gamma$-radiation exposed guinea pigra is presented in detail. Inbred gunea pigg were given $8.8 \mathrm{r}$ daily until the number of erythrocyter in the peripheral blood decreaged to approximately 2.8 million cells per mm (normal $5.7 \div 1.2$ million). Data on guinea pigs indicate a sensitivity of the hematopolettc system comparable to that of man. Complete data on the blood picture and bone marrow studies are presented. (NSA) 681

Lutwak Mam, C. and F. W. Gunz. EFIECT OF BODY EXPOSURE TO X-RAYS UPON BONE MARROW. MIOChER. J. 44, II of Proc, Biochem, Soc. (1949).

Rabbit irradiated with a gingle 1,000 r 8 -ray dowe, and rats irradiated with a aingle $500 \mathrm{r}$ dose were observed for two week following irradiation. Weight of the animals, gross and histological changes in bone marrow, bone marrow glycolysis and respiration, adenomine triphosphate, nucleic acid $P$ determinations, and bone marrow mitotic ilgures were lollowed. Partal recovery was observed in hemoglobin content, mitotic figures, gly colysis, regpiration and nucleic acid P. (NSA)

682

Pearce, M. L., M. A. Greenteld, and W. N. Valentme. 
STUDIES ON THE RADIOSE NSFTTVITY OF BONE MARROW; II. THE DYNAMICS OF ERYTHROCTYE REGRNERA TON ATTER MEMORRTAGE AND IRRADIAWION. Atomic Energy Project, Univ. of Califormia, Los Angeles. Apr. 19, 1951. 15p. (UCLA-125)

A method for the calculation of "birth rate" of erythrocytes in the face of a changing blood count is presented in terms of life-span data. By setting up the dynamics of erythrocyte regenexation in terms of a two-compartment system, an equation is derived to predict expected red-cell coun at any time after hemorrhage. The regeneration of erythrocytes after hemorrhage in irradiated and nonirra683 diated cats is compared with predicted values. (NSA)

Price, C. H. G. OBSERVATIONS UPON THE LYMPHOPENA OF X-RAY IRRADIATION; PART II. Brit.

J. Radiol. 24, 556-67(1951).

In experiments in which three groups of albino rats were exposed to fractional doses of $95 \mathrm{kv} X$ rays it has been confirmed that the total lymphocyte count is related to the total dose of radiation administered. It has also been confirmed that the lymphocyte survival/dose curve is of a double exponential form. On comparison of the mean total lymphocyte survival/dose curves of the three groups of animals it could be shown: (a) That the degree of the biological change was more readily affected by an alteration in the volume of tissue irradiated than by a change in the rate of dosage of radiation. (b) That with suitable arrangement the regenerative effect of formative tissues could be demonstrated. (c) That on analysis of the total lymphocyte survival/dose curves into two separate components, the major effect was best explained as the direct result of the ionizing radiation, which effect can be regarded as a single hil target phenomenon. On a group basis there was evidence to show that the integral dose and concomitant change could be correlated with the observed group tolerance of the animals. This was not, however, apparent on consideration of 684 the individual animal. (NSA)

Rekers, P., P. Salisbury, J. Miller, N. Marti, and N. Papageorge' $\mathrm{s}$, Jr. CROSS CIRCULATION STUDHES ON THE IRRADIATED DOG. Atomic Energy Project, Univ. of Rochester. Mar. 30, 1950. 12p. (UR-159)

Cross-circulation studies between dogs that had received a single $\mathrm{x}-\mathrm{radiation}$ dose of $450 \mathrm{~K}, \mathrm{LD}_{95}, 6$ to 10 days previously and normal dogs have been carried out by use of direct carotid to carotid artery anastomosis and a crosstransfusion apparatus. With each technique elevation of the leukocyte and erythrocyte levels was noted during the period of cross circulation. At the termination of the carotid type of cross circulation, there was a drop in the leukocyte count to pre-circulation levels within $20 \mathrm{hr}$, while with the cross atransfusion apparatus the leukocyte count continued to rise for $8 \mathrm{hr}$ or more after cross circulation and returned to the marked leukopenic level $48 \mathrm{hr}$ after the teprinination of the cross-circulation procedure. Data on thrombocyte deflections are not sufficiently definite. (NSA) 685

Roberts, E., L. E. Card, and G. L. Clark. EFFECTS OF X-RAYS ON THE BLOOD CHLLS OF THE CHICK. Biodynamica 6, 165-76(1948).

This work was begun in an investigation of the effect of $X$-ray treatment on the resistance of chicks to pullorum disease caused by Salmonella pullorum. Because the disease is a septicemia, the effects of the $\mathrm{x}$-rays on all the types of blood cells of the classification were studied. This set of data is "apparentiy the first complete account to be published on this subject." Day-old White Leghorn chicks were irradiated with a total dosage of $360 \mathrm{r}$ units over periuds of 120 minutes or 30 minutes. Another series were given a dosage of $720 \mathrm{r}$ units over periods of 120,30 , or 6 minutes. Counts were made on all the blood cells (erythrocytes, heterophils, lymphocytes, monocytes, eosinophils, and basophils) at intervals after treatment ranging from 150 minutes to 48 hours. The lymphorytes were considerably reduced following irradiation. This was followed by an immediate decrease in the resistance of the baby chicks to the pullorum disease. The re was also a slight reduction in the number of basophils, but the effects on the other cells did not appear significant. In both series of experiments the reduction in number of lymphocytes increased as the exposure time inc reased although the dosage remained constant. (NSA)

686

Rosenthal, R. L., B. I. Pickering, and L. Goldschmidt. A SEMI-QUANTITATIVE STUDY OF BONE MAREOW IN RATS FOLLOWHG TOTAL BODY X-IRRADIATION. Blood 6,600-13(1951).

A method is presented for the quantitative enumeration of the bone marrow cells by adjusting the differential count for the degree of cellularity, which is derived from imprint preparations of femoral marrow. This method has been employed in a study of bone marrow changes at various intervals following $700 \mathrm{r}$ of total body $\mathrm{x}$-irradiation in the rat. The findings have been correlated with the peripheral blood counts. The erythroid cells of the bone marrow show rapid decreases nn number because of the ir marked destruction. The myeloid cells chiefly undergo accelerated maturation. The decreases in megakaryocytes, mast cells and lymphocytes appear to be related to their life spans and the absence of further production. The reticulum cells and plasma cells show neither an absolute increase nor decrease. Formation of new cells, except possibly for plasma cells, from the reticulum cells is completely inhibited for the first nine days following total budy irradiation of rats with $700 \mathrm{r}$. Regeneration is first apparent at 12 days after $x-r a y$ as evidenced by areas of erythropoiesis, and, to a lesser extent, Myelopoiesis. Megakaryocytes reappear in small numbers at 15 days. Although this regeneration is apparent in only $50 \%$ of the animals studied at 12 and 15 days following irradiation, all these animals showed evidence of transition of reticulum cells to mast cells. (NSA) 687

Schack, J. A. and R. C. MACDuffee. INCREASED RADIORESISTANCE OF RED BONE MARROW AFTER ANOXIA. Science $110,259-60(1949)$.

One hundred and forty female mice were randomly distributed into two groups, one maintained at sea level as a control unit and the other exposed to a simulated altitude of 15,000 feet for 10-14 hours a day. Both groups were kept at $25-30^{\circ} \mathrm{C}$.; animals were sacrificed frequently and the marrow from the femurs was examined for erythroblasts and normoblasts which were counted logether as an erythroid percentage of the total marrow cells; this percentage was $10.6 \%$ in the normal animals and $18.6 \%$ in the anoxic animals. Both groups were then exposed to $500 \mathrm{r}$ of total-body $\mathrm{x}$-irradiation from a $200 \mathrm{k} \mathrm{k}$ machine and maintained at sea level; the erythroid percentage was rapidly reduced in the first two days, followed by a rise from the fourth to the sixth day, and a fall until the ninth day for normal anmals; the experimental animals were identical in their reaction except for the absence of the last stage. It is indicated that the hyperplasia of the red marrow produced by exposure to low axygen tension increases the resistance of the erythroid elements of the mouse to subsequent exposure to $\mathrm{x}$-radiations. (NSA) 688

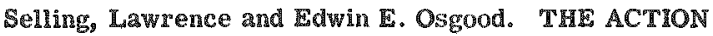


OF BENZOL, ROENTGRN RAYS, AND RADIOACTIVE SUBSTANCES ON THE BLOOD AND BLOOD-FORMNG TISSUES, p.2689-802 of MANDBOOK OF HEMATOLOGY H. Downey, ed., Chapter 39, Volume IV. New York, Paul B. Hoeber, Inc. 3136p. 1938.

A general review of the action of radioactive substances and roentgen rays on body tissues. Includes sections on the effects of $\alpha, \beta$, and $\gamma$ radiation on the blood and tissues in vitro; $\gamma$ ray effects; o ray effects; and a bibliography. (S.V.S.)

\section{9}

SIMa, I. THE ACTION OF BLOOD AFTER EXPOSURE" TO X-RAYS OR ULTRAVIOLET LIGHT. Jap. J.Med. Sci. IV. Pharmacol. 12, 157-65(1940).

Injected into the lymph sac, blood from pigs but not from cattle, rabbits, or toads increased the thickness of pigment layers. After exposure to z-rays or ultraviolet light, the action of pig blood was increased; catthe and rabbit blood showed a positive response, but not toad blood. This action was attributed to decomposition of erythrocytes, with the liberation of histamine which acted on the adrenals of the injected frogs, to increase adrenaline liberation into the circulation. Ir radiation in the presence of trypaflavine, methylene blue or erythrosin did not alter the nature of action of the blood specimens tested. (CA) 690

Stearner, S.P. THE EFFET OF A SECOND ACUTE X-RAY EXPOSURE ON THE RECOVERY OF THE CIRCULATING ERYTHROCYTES IN IRRADIATED RATS. Argonne National Lab. Aug. 1, 1947. 19p. (AECD 2024-D)

Experiments on the rat exposed to acute doses of $x$-rays showed that although recovery was largely complete at. 35 days, a small but significant difference in the erythrocyte count (between control and irradiated groups) persisted for about three months. It was expected that a second exponsure to the anme dose at this interval would produce more severe damage than did the first exposure. White rats, about 2 months old at the start of the experiment were given $600 \mathrm{r}$ at 0 and at 35 days or $600 \mathrm{~g}$ at 35 days only. The data suggest that the anemia following a second irradiation may be more severe than that following the first. Although the red count did not fall lower after the second exposure than after the first, the cont rol count was higher during the latter half of the experiment. In addition those irradiated at 35 days only, showed an anemia that was less severe than that which developed in the group irradiated twice. However, recovery did not appear to be more retarded after the second acute exposure, but in all instances was not complete at 35 days. Comparison of erythrocyte and hemoglobin values of the irradiated groups showed that the recovery of the number of circulating erythrocytes was less rapid than the recovery of the hemoglobin values. This was especially apparent in the first group, but was seen only to a small extent in the group irradiated at 35 days only. This may be accounted for by the macrocytosis that was present at the last sampling (70 days). The mean erythrocyte diameter $\$$ were: Control $-6.85 \mu$; group $600 \times$ at 0 and 35 days) -7.25 H: and group $600 \mathrm{r}$ at 35 days only $-8.17 \mu$. (NSA) 691

Stearner, S. Phyllis, E. L. Simmons, and Leon 0. Jacobson. THE AFFICTS OF TOTAL BODY X-IRRADIATION ON THE PERIPHERAL BLOOD AND BLOODFORMING TSSUES OF THE RAT. Argone National Lab. Apr. 7, 194\%. 42p. (ANL-4001; MDDC-1319)

Following exposure to single doses of s00 $r$ and $600 \mathrm{r}$, a maximum anemia developed after 14-16 days. Recovery was complete at 30 days following $300 \mathrm{r}$, but a significant difference persisted until 24 days between control animals and those exposed to $600 \mathrm{r}$. Detailed histologic study of the bone marrow and blood elements was continued for 35 days after irradiation. Exposure to daily x-ray doses of 33.5 r resulted in a total of $3,080 \mathrm{r}$ and a maximum survival of 3 months. This dose rate produced only a slight depression in hemoglobin and erythrocyte values and a small decreage in number of eirculating heterophils. However, lymphocytes were reduced after 1 week of treatment and fell continualiy until the death of the animals. A dose rate of $7.7 \mathrm{r}$ per day was not shown to shorten the life span of the rats and had a doubtful effect on the circulating erythrocytes. The number of circulating heterophils was not decreased, but lymphocytes were reduced after 1 month of daily exposure and remained at a low level. Exposure to 7.7 ir for over 8 months could not be shown to have produced any change in the blood forming organs. The response of the peripheral blood cells to daily doses of $\mathrm{x}$-rays indicated that the effects upon lymphopoletic tissue were much greater than upon the elements formed in the bone marrow. Response of rats and rabbits were very similar. (TIS)

692

Stearner, S. P., E. L. Simmons, and Leon O. Jacobson.

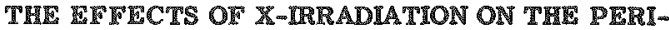
PHERAL BLOOD AND BLOOD-FORMING TISSUPS OF SPLENECTOMRED RATS. Argonne National Lab. Oct. 1, 194\%. Deel. Apr. 23, 1948. 18p. (AECD-2022; ANL -4100)

White rats were splenectomized and after five days, exposed to 600 Ir and 100 r $x-$ radiation. Although a leucocylosis was frequently seen after splenectomy, in some cases no significant change in the peripheral leucocyte count was observed. The lymphopenia and granulopenia that followed exposure to radiation were as aevere in splenectomized rats as in intact rats. Histological studies indicated that splenectomy did not produce a significant hyperplasia of lymphopoietic or granulopoietic tissue in the rat. Therefore, there was no opportunity to compare the effects of radiations on normal and hyperplastic lewcocyte-forming tissues. The functions of the spleen are not clearly understood, but appear to be complex. It is possible that splenectomy may produce numerous physiological changes which complicate the interpretation of radium effects on the bloodforming organs. Although the reason is not clear, the results make it apparent that splenectomy is not a satisfactory technique for producing a hyperplasia of lymphopoietic or gramulopoietic tissue. In Bartonella-infected rats, splenectony resulted in a severe anemia. In animals that survived, irradiation with $600 \mathrm{r}$ did not cause a more severe anemia, but markedly retarded the recovery. The bone marrow of these animals was shown to contain a hyperplasia of erythroblastic elements. A small percentage of erythroblasts persisted during the early stages following irradiation of splenectomized rats, although none were seen at this time in intact irradiated animals. Later, however, damage appeared to be equally severe in both irradiated groups. (ADD)

698

Stearner, S. Phyllis, R. L. Simmons, and Leon O. Jacobson. THE EFFECTS OF STML DOSE TOTALBODY X-IRRADIATION ON THE PEIRPHERAL BLOOD OF THE RAT. Anat. Record 98, 576-7(1947).

Following exposure to single doses of 300 and $600 \mathrm{r}$, a maximum anemia developed after 14-16 days. Recovery was complete at 30 days following $300 x$, but a significant difference persisted until 14 days between control animals and those exposed to $600 \mathrm{r}$. The relatively long life of the red cell (20-30 days) may explain the delayed appearance of the effects of irradiation on the circulating erythrocytes. A macrocytic anemia was demonstrated by the preaence of an 
increased mean corpuscular volume and mean erythrocyte diameter. The white blood elements showed a maximum depression three days after $\mathrm{x}-\mathrm{irradiation.} \mathrm{Recovery} \mathrm{began} 10$ days after exposure to 300 and $600 \mathrm{r}$, and was essentially complete at 30 days. After $25 \mathrm{r}$ there was a very small decrease in number of circulating lewecytes and recovery was complete after 14 days. Exposure to 300 and $600 \mathrm{x}$ produced drastic effects on heterophils as well as lymphocytes. A comparison of the effects of $x$-ixradiation on the peripheral blood of rats and rabbita ghowed that the pattern of response was gimilar in the two species and that only minor differences were present. Recovery of the lymphocyte count to control values was more rapid in the gat than in the rabbit following exposure to median lethal doses to both groups. The heterophil count fell to a lower level in the rat, but recovered at about the same time as did that of the rabbit. $(\mathbf{A R})$

\section{4}

Suter, G. M. RESPONSE OF HEMATOPOIETC \$YSTHM

TO X-RAYS. University of Rochester. Mar. 22, 1947.

Decl. Apr. 7, 1947. 8p. (MDDC-824)

The peripheral blood, constantly replenishing itzelf from its bone marrow and lymphopoietic precursors, is very sensitive to both ganma and neutron radiation. In the study of peripheral blood, numerous laboratory animals wers used. Studies of the effects on the peripheral blood can be divided into two types: that of animals acutely exposed and chronically exposed to whole body radiation. Attempts were made to compare species sensitivity of the various laboratory animals on various bases in order that some conclusions could be made about the effects on humans. On the basis of mortality studies the most sensitive species studied appeared to be the dog, with monkey, rat, and rabbit correspondingly less sensitive. It was found difficult to draw such conclusions from the study of the blood of these animals. The site of injury with $\mathrm{x}-\mathrm{radiation}$ and neutron radiation appeared to be directly in the blood-forming organs, the bone marrow, lymph nodes, and spleen. The peripheral blood showed only slight changes when isolated and radiated directly. Chronic studies were made for purposes of establishing tolerance both to $\mathrm{X}$-ray and neutron radiation. Experimental results are summarized in ten graphs 695

T'Ang, \%. H. THE IXPRIMENTAL BASES OF TOTAL AND SUBTOTAL RADIOTHERAPY. J. belge radiol. 28, $207-99(1939)$.

We have attempted through animal experimentation to establish the scientific bases for total and subtotal radiotherapy. As to hematology, we have studied very careiully the blood changes following total or abtotal irradiation over a wide range of doses. We have also studied various other hematologic tests. We can conclude that doses of 100 to $150 \mathrm{r}$ administered to the whole body of the animal at the rate of 10-25 $\mathrm{r} / \mathrm{day}$ are without danger. This dose increases leucocytosis and stimulates red cell formation. Only when doses of $200 \mathrm{r}$ or more are reached, can the beginning of a mild leucopenia be seen. The dangeroug dose is about $500 \mathrm{r}$ at one time or 5000 to $6000 \mathrm{r}$ at the rate of $10-25 \mathrm{r} / \mathrm{day}$ in whole-body irradiation. The observation of reticulocytosis is not an adequate test in radiotherapy: we believe that a distinct leucopenia with a slight state of anemia constitutes an absolute indication for the cessation of treatment. Vitamin $C$ appeares to decrease the radiosensitivity of an organ to a lethal dose of radiation. The perowide of diformaldehyde increases this radiosensitivity. Vitamins $\mathbb{B}_{1}, \mathbb{B}_{2}$, and $\mathbb{B}_{6}$ are without effect. Some schedules of treatment by gubtotal radiation in human therapy are given, as well as some re- sults by way of example. The domain of application of this therapy is very large, not only in anti-neoplastic therapy, but above all in the domain of inflammatory, endoc rine, 696 allergie and tonic radiotherapy. (auth)

Valentine, W. N., C. G. Craddock, and J. S. Lawrence. THE EFPECT OF BOENTGEN RADIATION ON THE PRODUCTION OF THORACIC DUCT LYMPHOCYTES. Univ. of Rochester. Max. 3, 1948. Decl. Apr. 23, 1948. 5p. (AECD-2023)

A study was made of the effect of 1,500 ro whole body irradiation on the numbers of thoracic duct lymphocytes and rate of flow of thoracic duct lymph in cats. Since the proeedure of thoracic duct cannulation itself results in some reduction in lymphocyte numbers and lymph flow, it was necessary to compare irradiated animals with an untreated control group. Nine control antmals were employed while 11 animals were uged in the irradiated group. In each animal lymph was collected in hourly or two-hourly volumes and studies were made to ascertain the volume, total lymphocytes, and lymphocytes per cu $\mathrm{mm}$ of each specimen. In each case the values for the first two hours flow were expressed as $100 \%$ and the values of each subsequent specimen computed as a percentage of this baseline. Animals were irradiated after a normal baseline was oblained. The total number of $1 \mathrm{ym}$ phocytes in thoracic duct lymph falls rapidly after heavy whole body irradiation as does the lymphocyte count per cu mm of lymph. There is only slight diference in rate of lymph flow in control and irradiated groups. Data are pregented on these points. The data suggest that from the time irradiation was begun the total production potential of the lymphoid tissues drained by the thoracic duct did not exceed about four hours normal supply. In terms of the peripheral blood, this amounts to ability to replace via thoracic duct lymph about 50-60\% of the circulating lymphocytes before total exhaustion ensues. (ADD) 697

Valentine, Willam N. and Morton Lee Pearce. STUDI\&S ON THE RADIOSENSITIVITY OF BONE MARROW, I. THE RELATIVE SENSITIVITY OF ERYTHROID AND MYLOD LIEMENTS. Univ. of Calif., LOs Angeles. Apr. 4, 1951. 29p. (UCLA-123)

A comparison has been made of (a) the regeneratuve capacity of erythroid tissue in irradiated and nonirradiated cats, and (b) the relative regenerative capacity of myelopoietic and erythropoietic tissue in the same animals after the ame ipradiation injury, by irradiating animals immediately after a large acute hemorrhage of $40 \%$ of the circulating red cell mass. Dosage employed was $200 \mathrm{r}$ to the whole body. Recovery from standard anemia was only slightly glower in the irradiated than in the control group. However, myelopoleais was severely depressed for more than 30 days. The data suggest that on the basis of functional impairment, erythropoietic tissue is significantly less gensitive to radiation injury than is the myelopoietic tissue of the same animal. (NSA) 698

Valentine, Villian N., Morton Lee Pearce, and John S. Lawrence. STUDIES ON THE RADIOSENSITVITY OF BONE MARROW; II. THE EFECT OF LARGE, REPEATED WHOLE BODY IRRADIATION EXPOSURE ON MEMATOPOIESIS. Univ. of Calif., Los Angeles.

Apr. 16, 1851. 15p. (UCLA-124)

Six cats were subjected to four whole-body irradiation exposures over a perlod of $1 \frac{1}{2} \mathrm{yr}$. Irradiation dosage was 200 $x$ to the whole body. Irradiation in each case was immediately preceded by a large phlebotomy, wo that functional impairment of erythropoiesis resulting from irradiation could 
be assessed in terms of a defective regeneration of erythrocytes. Little evidence of permanent functional damage to erythropoietic tissue by repeated exposures under the conditions of this experiment were observed. Hemoglobin regeneration was slightly slower after the fourth and last irradiation. Whether this represents encroachment on marrow reserve by multiple irtadiations or whether it was an artifact resulting from iron deficiency cannot be said with certainty. Multiple exposures exerted little apparent effect 699

on the leukocyte regeneration curves. (NSA)

Van Dyke, D. C. and R. L. Huff. LIFE SPAN OF WHTT BLOOD CELLS ASS MEASURED IN IRRADRATED PARABIOTC RATS. Am. J. Physiol. 165, 341-7(1951).

Inbred female rats of the Slonaler strain were used to form the parabiotic pairs. Liftermates were united at 20 30 days of age, and 2 months later one member of each pair was irradiated. Controls were single rats of the same sex and weight which had received the same treatment. $950 \mathrm{r}$ of $200 \mathrm{kv}$ X-rays was delivered to one member, the so-called non-ipradiated members receiving $28 \mathrm{r}$. Total white cell counts were made daily on the pairs and on controls. As the leucopoietic tissues of the irradiated members were destroyed, the white cell count was a measure of the leucocytes introduced into the eirculation from the circulation of the normal member. The relationship between the number of leucoeytes introduced into the circulation and the level maintained in the circulation allows calculation of the average life span of the leucocyte. These studies indicated the life span of the mononuclear lymphocytes in the blood of the irradiated rat to be 170 minutes, and that of the polymorphonuclear leucocytes to be 23 minutes. (V.E.)

\section{Clotting Mechanisms - Humans}

700

Amaniera, G. and B. Bertiglia. PROTHROMBIN STUDY

IN RADIOTHERAPY -TREATRD PATIENTS WITH

NEOPLASMS. Giorn. clin. med. (Parma) 31, 312-24

(1950).

A noticeable increase, no variation, and a slight decrease in prothrombin content was found in 75,21 , and $4 \%$, respectively, of the cases. (CA) 701

Beller, F. STRATING X-RAY AND Ra DAMAGE IN PERSONNIEI BY OBSERVATION OF THE PROTHROMBN LEVEL IN THE BLOOD. Fortschr. Gebiete Roentgenstrahlen t5, 95-8(1951).

Through observations on the personnel of a radiological institute, it is established that slight radiation injuries cannot be demonstrated by the decrease of the prothrombin level. It appears that, in a general way, $x$ rays do not affect the clotting potential of the blood. This conclusion is confirmed by the fact that various authors give discordant information on changes produced by radiations in the differential blood picture and in the coagulation time. (NSA) 102

Iraúmann, J. THE COAGULOGRAM AS A CRITICAL INDICATOR OF IFRADLATION IFECT. Am. J. Roentgenol. Radium Therapy 55, 464-72(1946).

Complete blood studies were made repeatedily on the enrployed personnel of the department of radiology of a large general hospital. These are analyzed as erythrogram, leukogram, and coagulogram. The latter, consisting of platelet count, bleeding time, clot retraction, prothrombin time, fibrin time, and complete coagulation, appears to be most important in revealing early changes due to irradation.
Fibrin, prothrombin and coagulation times are all lengthened before changes appear in red or white cell count. The lengthening of prothrombin time from a masimum normal of 30 seconds -45 geconds warrants a caution to the individual should be temporarily removed from any exposure area, and the deficiency neutralized by every possible means including vitamin K therapy. If the condition does not improve, the individual should seels other occupation, away from the possibility of exposure to radiation. (BA) 703

Masin, Vincenzo. EFFECT OF IREADIATION ON THP COAGULATION TIME OF THE BLOOD IN NORMAL INDIVIDUALS. Radiologia (Buenos Aires) 2, 393-403(1945).

The author reports the effect of $200 \mathrm{r}$ administered over the spleen, liver, lower abdomen or buttocks of normal individuals upon the coagulation time of the blood. As compared with the coagulation time taken before irradiation, such treatment produces a decrease in the coagulation time with littie variation in the fibrinogen and in the plateletg. (R)

704

Ostro, M. and D. I. Macht. A PHARMACOLOGICAL AND RADIOCOGICAL STUDY OF HEMOPRILIA. Southeru Med. J. 39, 860-7(1946).

Evidence is presented to show that upon exposure of blood samples of normal animals and humans, including hemophiliac subjects to therapeutic doses of $x-1$ rays, a definite shortening in clotting time will occur. The effect, in vitro, is best evident when the dosage ranges from $63-105$. The ame thromboplastic effects of $\mathrm{x}$-rays can be obtained by exposing the subjects themselves to small doses of deeply penetrating $x$-rays. Irradiation in the region of the spleen of two hemophiliacs produced a shortening of the clotting time which was evident several days affer initial exposure. On the basis of these findings the authors propose a radiologic approach to the therapeutic treatment of hemophilia. (BA)

705

Segre, Mario. CHANGES OF COAGULATION TMM, BLEEDING TIME, AND MORPHOLOGICAL BLOOD COMPOSITION ATTER IRRADLATION WITH X-RAYS OF THE SPLEEN AND HYPOPHYSIS. Arch. sel. med. 65, 255-302(1938).

The coagulation time and bleeding time were shortened in both cases, bleeding time more in spleen irradiation. The thrombocytes decreased in number, the spleen having a larger effect. An erythropenia after 6 to 12 hours amounted to $20 \%$ in irradiation of the spleen, to $9 \%$ in irradiation of the pituitary. The later effect on erythrocytes was variable. Spleen treatment caused a sustained increase of leucocytes for 15 days; treatment of the pituitary showed a temporary decreage. Details of the various blood elements are discussed. (CA) 706

Soylrova-Pachnerova, 2 . and O. Soyka. CHANGES IN THE PROTHROMNIN LEVEL IN WOMEN WITH CANCER TREATED RF IRPADIATION. Casopís Lékàru Českých 88, $169-71(1949)$.

Checking up on reports in the literature of prothrombinlevel changes after $\mathrm{X}-\mathrm{ray}$ and radium therapy, the author:s examined 65 women with ovarian and uterine cancer by the Quick test at the first treatment, three months after it, and at the gecond treatment. They found the prothrombin-level to be less affected by the treatment than by the patients general condition. A prothrombin-level which does not change during treatment is claimed to be indicative of a favorable prognosis, whereas in cases where there is a change, the bigger the change the worse the prognomis; 
whether the change is upward or downward is claimed to be unimportant. (NSA)

\section{Cloteing Mechanisms - Animals}

797

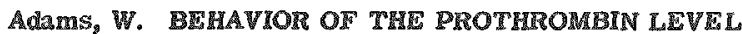

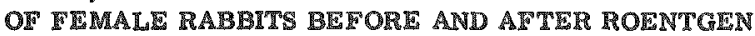
IRPADIATION, Strahentheraple 71, 114-20(1942).

One or repeated doses of 60 up to 600 a caused no essential change in the prothrombin content. Only if the coagulation times before irradiation were relatively prolonged, did there appear after ispadiation a reduction in the cloting time which corresponded to a protlinombin increase. The known efect of roentgen rays on the clotting time can. therefore, not be explained by changes in the prothrombin 708 picture. (CA)

Allen, J. Garrot, Burton J. Grossman, Richard M. Elghammer, Peter V. Moulder, Charles Is. MoReen, Leon 0. Jacobson, Mila Pierce, Trylor R. Smith, and James M. Crosbie. ABNORMAL BLIEDING. J. Am. Med. Assoc. 139, $1251-4(1949)$.

Data on six cases of hemon by $\mathrm{P}^{32}$ include reports on clotting time, protamine titration, exyhthocyt, leucocyte and thrombocyte counts, prothrombin activity and clot retraction, and fibrinolysin. Protamine sulfate and toluidine blue were given with good results in al cases. When the bleeding was uncomplicated, protamine sulate $(50 \mathrm{mg}$ in $5 \mathrm{cc}$ of water intramuscularly every 4 to 6 hours, and $150 \mathrm{mg}$ in saline glowly inturenously) is pre ferred to toluidine blue; isotonic maline solutions of 6 to mg./kg. body weight are given slowly intravenougly once a day for three days and then hal of this dose for three lollowing days. "The author conclude that abnormal bleeding may result from the presence of heparinoid substances in the blood accompanied by increased protamine titration, requently a prolonged cloting time and in some case פevere thrombopenia she bleeding was controlled by toluidine blue and/or protamine sulfate, either of which is capable of reacting with several endogenous substances both like and unlike heparin; thrombopenia did not respond to wher, and in cases where it was accompanied by an in creased protamine titration, the response was insuticient to liminate aplenectomy, since the two conditions are probably independent: th is concluded that the random use of T190 the two compounds will lead to random reauls. (NSA)

Allen, J. Garrott and Leon O. Jacobson. HYPERHEPARIIIERIA: CAUSE OF TIIE HISMORR

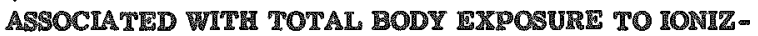
ING RADIATION. Sclence 105, 388-9(1047).

The hemorthagic disease, characterized by a thrombocyo topenia, prolonged bleeding and clotting times, and impaired clot retraction, has been thought to result from the associated thrombocytopenia, but the researches of the authors have lead them to the conclusion that increased free heparin in the blood is of the greategt gignificance, the thrombocytopenia playing only a secondary role. Tvidence for this viewpoint is cited. (V.E.)

\section{0}

Alleri, J. Garrott, Inatherine Lathrop, and Margaret M.

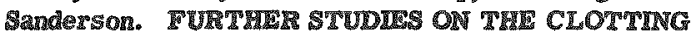
DISTURBANCE IN THE DOG RADIATION. Argorme National Lab. [nd] 12p. (AMCU -496 \% UAC- -95 )

This report is primarily concerned with the disturbance in the clotting time (plasma factors) incident to a radiation exposure. It has been previously reported that an antico- agulant similar to heparin can be demonstrated in the blow of dogs ater lethal $x$-irradiation. Alhough the chemucal identity of this material is not known, it is characterized by cerfain reations which suggest that it mafure is heparinoid. These studies have been extended, and data aro presented which further suggegt the possible heyarinoid nature of the ant icoagulant found in the blood after ingatiation. Other nonheparin anticoagulants may be liberated, but thus far none has been found in the irradiated dog. Thrombocytopenia and capillary damage are also present and may contribute to the hemorohagic gtate. (NSA) 711

Allen, J. Garrot, Peter V. Moulder, D. M. Wnerson, K. A. Lathrop, and M. H. Sanderson. SUMMARY OF TRRADIATION FIMCTS OI THE TLOOD COAGULATION MECHANSM, p.148-67 of PROGRESS REPORT;

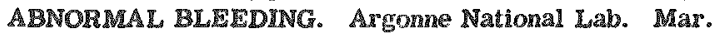
1950. 20p. (ANL-4474(p.148-67))

The: hemostatre mechanism in more complicated than can currenty be explained, and the damages produced by totalbody $x$ ir adiation are so far-reaching that most, if not all, of the clotting mechanism may be directly or indirectly affected. In addition, here is evidence of widespread vascular damage about which lithle is known. However, certain features of the pathogenesis of this syndrome are established and limited therapeutic aid is available. A summary discussion on certain of the phenomena described in the previous parts is presented in this stetion. Some recent data are included to augment the discussion. The whole-blood clotting time, a cloting inhibitor, thrombocytopenia, and capillary injury are discussed. 15 references. (ARSA)

\section{2}

Allen, J. Garrot, M. Sanderson, and Leon O. Jacobson.

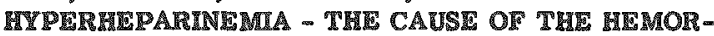
RMACTC DESTESE PRODUCED BV TOTAL RODY FX-

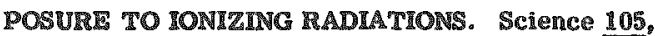
$388(1947)$. (MDDC -836$)$

The hemorthage disease in dogs given a single lethal total body expowure to $x-3 a y(450$ r) is chareterized by a prolonged clotting time. No defeat of the known elements of clotting was demonstrable except for a reduction in the platel number and the appearance of an anticosgulant not normaly present in detectable quantities. The thrombocytopenia per se does not appear to be the important factor responsible fos the hemor ing ritay ocear before the platelets are reduced and transiustons of fresh cifrated whole blood did not prevent or stop hemon'hage. Moreover, the factors that specifically inhibit the anticoagulant, restored the clotting time to normal, but did not prevent throulbocytopenia or clevate the platelet count. The anticoagulant reacts like heparin in all respects tested. Cts activity was destroyed by apecific antineparin gubstances ach as protanine, toludine blue and other dyes of he thionin series. "Holuidine blue prevented hemorrhage

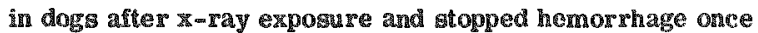
it arpeared. If was possible to isolate from the blood of dogr in the state of hemom fhe basis of unit potency per rag. of weight, was as active as the atandard sodium acid salt of heparin. The adminisfration of large quantitios of vitamin $\mathrm{K}$ and vitamin $\mathrm{C}$ were ineffective in preventing or stopping hemon hage. Toluidine blue was ineffective in preventing or curing the hemoxthagic disease produced in mormal dogs given dicumarol. Thrombocytopenia, neutropenia, and anemia were produced in mormal dogs by the administration of a nitrogen mustard. The whole blood clotting time was mot prolonged and hemorthage did not occur. The homosshse disease associaled with over exposure to $\mathrm{z}-\mathrm{rry}$ was closely correlated with infec- 
tion. This suggestion was made that the anticoagulant was bound to the immune proteins and was reluased when the antibodies were exhausted or destroyed. The administration of high titre anti-sera and antitoxins prevented hemorrhagge and prolonged the lives of the two dogs in which they 713 were used. (ADD)

Allen, J. Garrott, M. Sanderson, M. Milham, A. Irirschon, and Leon O. Jacobson. HEPARINEMIA: AN ANTICOAG. ULANT IN THE BLOOD OF DOGS WITH HEMORRHAGIC TENDENCY AFTER TOTAL BODY LYPOSURE TO ROENTGEN RAYS. J. FXpt. Med. 87, 71-85(1948).

In 25 dogs irradiated with $240 \times$ over the whole body the clottung tume became prolonged about the sixth day, hemorrhages occurred towards the end of the second week, and the dogs died two or three days later. The blood calcium level was unchanged, the prothrombin time fell only terminally, and the fall in the platelets was unrelated to the bleeding or the prolonged clotting time. The clotting time was not shortened by the addition of thromboplast in but was shortened when tryssin or thrombin was added. By addition of the plasma to plasma from a normal dog, anticoagulant was demonstrated in the abnormal plasma. As heparin can be inactivated by some basic dyes and proteins, toluidine blue and protamine sulfate were added and found to neutralize the antucuagulant both in vitro and in vivo, although they did not shorten the bleeding time. Vitamin $\mathrm{K}$ did not prevent hemorrhages in these dogs, and toludine blue did not arrest the hemor rhage due to dicoumarol. A heparin-like substance was isolated from the abnormal blood. (AWM) 714

Allen, J. Garrot, L. J. Savage, poter V. Moulder, and D. M. Enerson. CLOTTING TIMIE CHANGES AFTTER TOTAL-BODY X IRRADIATION, p.19-23 of PROGRESS REPORT; ABNORMAL BLEEDING. Argonne National Lab. Mar. 1950. 5p. (ANL-4474(p.19-23))

This report is an analysis of the clotting times of $79 \mathrm{ir}-$ radiated dogs studied consecutively from July 1,1948 to February 1, 1950. The clotting time measurements were made by the same personnel, and the data obtained are illustrated. The platelet counts made daily on this same series of animals are also presented. It is indicated beyond doubt that the evidence pointing to inereased elotting time after 1rradiation is not attributable to chance alone. (NSA) 715

AMbrosi, F. ACTION OF X-RAYS ON BLOOD PROTHROMBIN CONTENT. Boll. Soc. ital. biol. sper. 16, 178-80(1.941).

Irradiation of blood in vitro with moderate doses of $\mathrm{X}$ rays did not alter the prothrombin content as determined by Quick's method. (CA)

716

Barnes, W. A. PROTHROMBN FORMATION FOLLOWING INJURY OF BONE MAREOW BY ROENTGEN RAYS. Am. J. Roentgenol. Radium Therapy 46, 356-61(1941).

Mice, rats, rabbits, and a dog were subjected to large doses (up to $6,400 \mathrm{x}$ ) of $200 \mathrm{kv} x$-rays and the course of the prothrombin level was followed. No signiflcant alteration was found during periods of 1-6 days after single large doses, although the bone marrows became almost aplastic. After long-continued irradiation, the numbers of platelets and the leucocyte counts were conspicuously lowered, but. 717 no change in the prothrombin level was found. (CA)

Cohn, Stanton H. EFFECTS OF TOTAL BODY SY-IRRADLATION ON BLOOD COAGULATION IN THE RAT. Naval Radiological Defense Lab. Mar. 2, 1951. 23 p. $(A D-298(B))$
The hemorrhagic syndrome following exposure of rats to 400 of $x$ irradiation was studied, and a blood coagulation defect was found. Although the Lee-White clotting time was determined to be normal following irradiation, the clotting defect was quantitatively reflected by an increased heparin clotting time and by alterations in blood resistance measurements. The thrombocytopenia following exposure of rats to radiation appears to be a major factor in the defective clotting and consequent hemorrhage. The heparin clotting time measurements and electrical resistance measurements presented in this paper provide guantitative baselines for the clotting reactions of normal and irradiated animals. These values may be used as standards in evaluation of various methods of prevention and treatment of the spontaneous bleeding. (NSA)

718

Cronkite, R. P., B. Halpern, D. D. Jackson, and George V. LEROY. A STUDY OF THR HRMORRIAGIC STATE IN DOGS AFTER A LETRAL DOSE OF TWO MLLION VOLT X-RAYS. J. Lab. Clin. Med. 36, 14(1950).

Ten dogs were given a simgle doge of $000 \mathrm{r}$ (300 $\mathrm{r}$ to each side) of $2,000,000$-volt $x$ rays. The following studies of blood clotting were performed serially: platelet count; clotting tirme of whole blood; clot retraction and lysia; onestage prothrombin time; protamine-heparin titration of whole blood (Allen); test for circulating anticoagulants (Conley); heparin-protamine titration of oxalated plasma (LeRoy); and determination of fibrin N. All animals died between the sth and 13 th day after irsadiation. There was viঞible evidence of spontaneous hemorthage in every one for at least $48 \mathrm{hr}$ betore death. The occurrence of purpura bore a very close relation to the onset of elevation of temperature. Difiuse, extensive hemorrhage was found in every animal at autopsy. During the periormance of the autopsies the blood cloted firmly. The mean clotting time of whole blood was aignificantly prolonged after the bth day. The mean platelet count was less than 50,000 after the 8 th day. There was no evidence that heparinemia was responsible for either the prolonged clotting time or the hemorrhagic state. In every dog whose clotting time was longer than $1 \mathrm{hr}$ it was possible, at some time, to reduce it to less than 1 hr when protamine sulfate was added in the courge of the protamine-heparin titration (Allen). In every animal the one-stage prothrombin time was within normal limits. Conley's test for circulating anticoagulants was positive in two doggs, and was questionably positive in four dogs at some time after irradiation. However, in every animal Conley's test was negative at some time after irmadiation when the cloting time of whole blood was longer than $1 \mathrm{hr}$. Fibrinogen was present in increased amounts in all dogs from the Fh day after irradiation. It appears that the hemorrhagic state that occurs in animals that have received lethal doses of $x$ rayg is not identical with any of the hemorrhagic diseases encountered in clinical practice. In addition to the thrombocytopenia, there is reason to suspect the occurrence of a circulating anticoagulant, the nature of which is not known. (NSA)

718

Dixol, Frank J. RADLATION-INDUCED HEMORRRAGIC DISEASE IN CHICIENS. Proc. Soc. IXpti. BIol. Med. 88, 505-7(1248).

in order to study the hemorrhagic disease resulting from radiation, severe radiation injury was produced by repeated subcutaneous injections of $\mathrm{P}^{32}$ into young chicks. Agranulocytosis and thrombocytopenia developed rapldiy. Blood coagulation time was not altered but the blood clots were jelly-like and completely devold of ability to retract. The hemorthagic disease which developed was much less se- 
Vere than that seen in mammals. The increased number of mast cells associated with atomic bomb radiation injugy in 720

Enerson, D. M., B. J. Grossman, and . G. Allen. PLASMA ANTITITOMBIN IN IRRADLATD AND MEDARINIZED DOGS; A PRELIMINARY STUDY, $1.88-88$ of PROGRESS REPORT: ABNORMAL DLEEDING. ArgOME National Lab. Mar. 1950. 11p. (ANL 4474 (P.88-98))

The blood of 4 normal ologs given intravenous injections of heparin ( $0.5 \mathrm{mg} / \mathrm{mg}$ of body weigh) displayed a prom nounced increase in the whole-blood clotting time with only alight increase in antithrombin titer. Fifleen of $17 \mathrm{dogs}$ fiven total-body $\mathrm{x}$ ix radiation developed mild to moderate increases in the whole blood clotting times wath pronounced 0. 1 increase in the antithrombin titer. 11 references. (NSA)

Calluz: Mrouldes, and J. Garrot Allen, PHYSICAL PHENOMENA IN BLOOD COAGULATION, PORT; ABNORMAL BLEEDTM. Aggonne National Lab. Mar. 1950. 13p. (AML-4.4\% $4(0.99-111))$

An incrase in plasma surtace tonsion has been observed following total-body $\mathrm{x}$ irradiation in the dog. This increase can be explained in part on the basis of changes in both the concentation and the state of particulate aggeregation of plasma proteins. The in vivo changer a re greatly out of proportion to he measuable in vitro changes of gurface tension with variations in protein concentration. (NSA) 7.

Weld, A. and H. Hubach. THE FIBRONOGEN CONTENT OP TIV BLOOD UNDER THE WELUENCF OF ROLNI GEN TRRADITION AND TME PRTMARY RADIFION. Strahentherapie 51, 664(1934).

It has been known that the coagulation time of the blood has decreased following exposure to roentgen rays. The authos found in their experiment that this is probably due to an increase of the fibrinogen. The reticulo-endothelial rystem is undoubtedly involved in the reaction. The drop in the fibrinogen content of the blood which lollows immediately after the mposure to roentgen rays is explained as a di723 rect effect of radiation. (R)

Eolden, Willam D., Jack W. Cole, A. B. Pormann, and *. Stor 2asli. MIPOTHPOMOPLASTINEMIA FOL

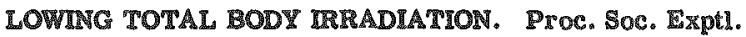
51ol. Med. 70, 553-6(1949).

Determinations of the hemoglobin, erythrocyte count, leucocyte count, platelet count (blood mear $3,2.5 \%$ sodium cirsate), clotting time, protamine titration where indicated, and thromboplagtin assay of dogs subjected to total body irzadiation were made prior to, and on successive days following, lradiation. Healthy mongrel dogs were irradiated with 450 I ( $\mathrm{LD}-100 \%)$ from a 220 inp machine at $15 \mathrm{ma}$ through a 0.5 mm copper filter and a $1.0 \mathrm{~mm}$ aluminum filtex, at a rate of $6-75$ per minute. The resuls obtained are given in tabular and groph form. A hemornhagic syndrone w2. produced which was manifested by muliple areas of hemorrhage in the pulmonary, gastrointestinal, and urinary systems, anemia, leucopenia, thrombocytopenda, prolonga724 tion of clot retaction, and hypoturomboplantinemia. (NSA)

Jackson, D. P., E. Cronkile, G. T. Jacobs, and C. T. BeMrens. PROTMROMDN UTLLIZATON TN TATAL

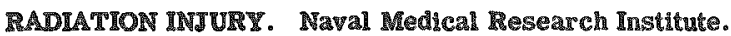

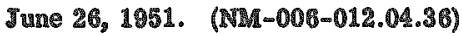

Fight dogs were given $600 \mathrm{x}, 2,000 \mathrm{~K}$.V.P., $1.5 \mathrm{ma}$, 1. V.L. 4.3 mm. Pb, 15.0 (-10.15) r per minute, whole body radiation by the bilateral exposure technique. The follow- ing studies were performed serially: hematocrit, whte blood cell count, platelet count, prothrombin concentration (Ware and Seegers), whole blood clotting time, and prothrombin utilization (Buclwalter, Blythe, and Brinkhous). All eight dogs died between the ninth and fifteenth postizradiation day. Clinically and at autopsy, all of the dogs exhibited evidence of extensive diffuse hemorrhage. The typical pancytopenia was demonstrated in all eight dogs. A slight terminal decrease in the concentration of prothrom bin occurred in four of the dogs. The results indicate that prothrombin utilization after this anount of radiation apMroaches zero from the minth post-nradiation day to drath. The whole blowd cloting time became prolonged only after the utilization of yrothrombin approached zero and thas plat det level had fallen below $50,000 \mathrm{per} \mathrm{mm}^{3}$. At autopsy, the bloot clotted promptly after the vessels were cut, and the blond was allowed fo escape into the tissues, In the opunon of the authors, it does not seem necessary to introduce the concept of the $\mathrm{Hresence}^{\circ}$ of anticoagulant in or der to explain the hemorrhagic syndrome of adiation in jury. The occurrence of a profound pancylopenia, severe infections, increased capillary frasility with possibly othes vascular factors, and the changes attributable to these conditions would appear adequate to explain this synorome. However, the restults do not rule out the oxistence of a weak and, as yet, uncharacterized anticoagulant. (auth)

\section{5}

Jacobson, L. O., R. IR. Marks, E. Gaston, J. G. Allon,

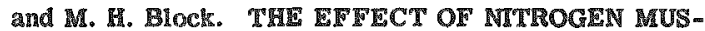
TARD AND I-IRADLATOV ON SLOOD COAGULATION. J. Lab. Clin. Med. 33, 1566-77(1948).

A prolonged clotting time occurs in rabbits after a $3 \mathrm{mg} / \mathrm{kg}$ intravenous dose of nitrogen mustard within 24 hours after administration and persists for approximately 12 days. Spontaneous recovery occurs in surviving animals by the $12 \mathrm{th}$ to 1 thth dry. A maximum reduction in blood platelets occurs by the 4 th day in rabbits receiving $3 \mathrm{mg} / \mathrm{kg}$ nitrogen mustard, with zecovery by the 12th to 14th day. Thus, the clotting time is prolonged prior to significant platelet reduction. However, the period of platelet seduction (4th through 12th day after nitrogen mustard administration) roughly parallels the peroid in which masimum incease in the cloting time exista. Spontaneous recovery of the clot. tung defect and the xeturn of platelet values to normal leyels in the peripheral blood occur at a comparable time (circa 14 days). Animals which developed a prolonged clotting time had nomal calcum and prothrombin values. The prolonged clotting time and decreased "heparin tolerance" are reversible with antheparin substances. The anticongulant preaent in the blood is probably heparin or a heparinlike gubstance. The clotting defect produced by nitrogen mustard resembles that produced by whole-body $x-1$ radiation (800 r) in rabbits and is also revergible with antheparin mubstances. It is aggested that the potentially serious elotting defect may be produced by other agents producing tosic sfects directly or indirectly on the blood-forming tis gue. This shculd be borne in mind since a method of treatment ivalable (antiheparin substances) which may make it possible to pass the critical period of potential hemor rhage until. spontaneous recovery ensues. (NSA) 726

Lathrop, Fatherine and J. Carrot ALlen. STUDIES ON ULTRA-VIOLET ABSORPTION OF DOG PLASML, P.130. 5 OR BIOLOGICAL AND MULICAL DVISIONS; QUARTERET TEPORT, AUGUST, SETTEMBER, OCTOBER, 1948. Argonne National Lab. Nov. 1, 1948. 5p. $(A N L-4227(1.190-5))$

Authors report on an investigation of the ultra-violet ab. sotpition of plasma from doge under various experimental 
conditions to determine if any information can be obtained to further clarify the nature of changes taking place which alter the clotting mechanism. Determinations were made of the ultra-violet absorption curve of oxalated plasma from (1) normal dogs, (2) irradiated dogs (450 r total-body $\mathrm{x}$ irradiation), and (3) normal plasma plus heparin in vitro. Data are presented which may indicate that changes in the ultra-violet absorption pattern in dog plasma following totalbody $\mathrm{x}$-irradiation are due to heparin-like compounds. (S.V.S.)

727

Lathrop, K. A., R. F. Gilmore, and J. G. Allen. IN VIVO CHANGES IN TIIE ULTRAVIOLET ABSORPTION SPECTRUM OF DOG PLASMA FOLLOWING EXPOSURE TO X IRRADLATION, p.123-7 of PROGRESS REPORT; ABNORMAL BLEEDING. Argonne National Lab. Mar. 1950. 5p. (ANL -4474 (p.123-7))

A study was undertaken of the ult raviolet absorption spectrum of plasma of dogs following exposure to $450 \mathrm{r}$ total-body $x$ ir radiation. Simultaneous plasma protein and albumin-globulin assays have been made on a large number of the animals studied. The ultraviolet absorption at 278 mu closely parallels the protein level, irrespective of quantitative changes in the albumins and globulins. (NSA) 728

Moulder, D. V., K. A. Lathrop, B. J. Grossman, N. J. Galluzz, M. H. Šnderson, Allen. CORRELATED STUDIES ON THE HRRADLATED DOG, p.128-47 of PROGRESS REPORT; ABNORMAL BLEEDING. Argonne National Lab. Mar. 1950. 20p. (ANL-4474 (0.128-47))

A number of blood and plasma abnormalities in the irradiated dog have been presented in previous sections. In an attempt to correlate these abnormalities, three dogs were given $450 \mathrm{r}$ total-body $\mathrm{x}$ Irradiation and the following studies were made: (1) complete hemocytology; (2) coagulation survey; (3) plasma proteing; (4) surface tension and viscosity measurements; (5) plasma ultraviolet light abmorption; and (6) protein and proteose sulfhydryl polarographice curves. Data were collected from the three animals, and large quantities of blood had to be drawn each day. The results, however, correspond with the findings in other igram diated dogs in which the effect of exposure upon each of these analyses was made. One of the reamons why the ase 'studies were made was the hope that some of thege functions might serve as an index of the severity of $x$-ray exposure. No group of changes could be demonstrated shortly after irradiation that could be used to indicate the lethality 729 of the dose. (NSA)

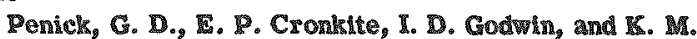
Brinkhaus. PLASMA ANTIHEMOPHLIC ACTIVTTY FOLLOWING TOTAL BODY IRPADLATON. Proc. SOC. Exptl. Biol. Med. 78, 732(1951).

Plasma antihemophilic activity of dogs subjected to total body irradiation remained at normal levels. Whole hemophilic blood with a full complement of platelets accelerated the clotting of irradiated doge blood, while platelet-poor hemophilic plasma did not. (auth)

\section{0}

Rosenthal, Robert L. and Agnes L. Benedek. DLOOD COAGULATION AND HEMORRHAGE FOLLOWING TOTAL BODY M-LRRADLATION IN THE RABDIT. Am.

J. Physiol. 161, 505-14(1850).

Rabbit: exposed to single dowes of total body $\mathrm{x}$-irradiation from 200-1000 $\mathrm{r}$ showed a blood coagulation impariment characterized by a thrombocytopenia, diminished clot retraction, increased clotting time of recalcified plasma, and increased anticoagulant effect of both added heparin and toluidine blue on the clotting time. These changes can be attributed to the diminished platelet count and function fol lowing $x-i$ irradiation. No free excess heparin was demonstrated in the blood following radiation. The rabbit, in contrast to man, dog, and guinea pig, shows an incongpicuous hemorrhagic responge following $\mathrm{x}$-ray exposure. (CA) 731

Scheraga, H. A. and L. F. Nirms. THE ETECT OF XRAYS ON FIBRINOGEN. Cornell University and

Brookhaven National Lab. [nd] 1p. (AFCU-368)

This document is reproduced here in ts entirety.

High doses of two-million-volt $x$ rays produce a marised increase in the viscosity of alutions of bovine fibrinogen. Examination of thege solutions in the ultracentringge indicates the presence of significant amounts of an additional component of higher sedimentation congtant than that of the original material. Simultaneous studies of the thrombininduced clottability of these solutions geems to indicate that the clotting activity is not gignificantly decreased even though there are these changes in the structure of the native fibrinogen. The character of the clots does change, however, the clots formed from solutions irradiated with the higher doses compacting and breaking up upon slight mechanical disturbance; thoge clots formed from the original material and also those from solutions irradiated with the amaller doses exhibit normal syneretic propereles at the pH gtudied. (NSA) 732

Seheraga, A. And L. T. NIms. THE ACTRON OF Y RAYS ON FIBRINOGEN SOLUTIONS. Broothaven National Lab. [nd] 21p. (AECU-1628)

Irradiation of fibrinogen solutions leads to fragmentation as well as polymerization of the original fibrinogen molecule. Irradiated solutions exhibit increased viscosity and, at doses greater than $300 \mathrm{kr}$, gel spontaneously. The magnitude of the changes can be decreased by the addition of protecting agents. The kinetics of the Irradiation reactions are in accord with the hypothesis that the action of the 8 rays Inwolves free radical resulting from the son mation of water molecules. Despite the marked changes in the structure of fibrinogen produced by 8 rays, all of the material is recoverable as fibrin upon addition of thrombin even when only $10 \%$ of the native fibrinogen is lett. It seems evident, therefore, that the gplit fragments resulting from the $\mathrm{x}$ irradiation contain the groups necessary for the clotting reaction and that these are not those easlly oxidized by $\mathrm{OH}$ 733 radicalm. (NSA)

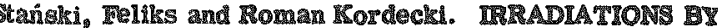

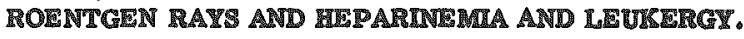
Med. Weterynax. (Poland) 6, 453-5(1950).

IExperiments were conducted on dogs divided into two groups. In the first group, the dogs were irradiated with roentgen rays in various doses, and leulergy and coagulathon time were examined. In the second sroup, the exgertmental doges received heparin intravenously in various doses, and leukergy and coagulation time were examined. The results of the experiments in the first group are ligted. It appears that small doses of roentgen rays cause a mow prolongation of the coagulation time, and the leukergy phenomenon appears only when the coagulation time is considerably prolonged (5x). The regults of irradiations by larger doses of roentgen rays over a period of one month are also listed. The marked prolongation of the coagulation tire wass accompanied by a marked positive leukergy reaction. Postmortem and histological findinge are reponted as follows: local depilation of alkin on the Irradiated area, parenchymatous (cloudy swelling) degeneration of the liver, small focal hemorrhage and perivacular mall-roukd-cell in- 
filtrations in the kidneys, and proliferation of the histiocytary cells, especially in the liver. The results of experiments with heparin injected intravenously (vena saphena externa) in an 8 -mg dose are shown. The coagulation time increased slowly, and leukergy appeared after $2 \mathrm{hr}$, culminating in $8 \mathrm{hr}$, when the coagulation time dropped almost to mormal. The results of the iniluence of a large dose of heparin (12 mg) on the coagulation time and leukergy are shown. The coagulation time was very prolonged, and leukergy appeared after $2 \mathrm{hr}$, deaching its highest peak after $3 \mathrm{hr}$. Postmortem and histological findings were negative. It may be concluded that roentgen rays, causing an increase of heparin in the blood, produce thus a positive leukergy reaction. (NSA)

Sutherland, J. M. and §. G. Allen. FURTHER STUDIES ON TTHE ISOLATION OF HEPARIN FROM BLOOD OF IRRADIATED DOGS, p.112-22 of PROGRESS REPORT; ABNORMAL BLEDDING. Argonne National Lab. Mar. 1950. 11p. (ANL -4474 (p. 112-22))

4 method is presented for the extraction of heparin from blood by which crystals of the barium salt of heparin can be prepared and identified from blood to which heparin has been added in amounts of $10 \mathrm{mg} \%$ or larger. The amount of heparin necessary to cause prolonged clotting time in irradiated dogs is 4 to $28 \mu \mathrm{g} \%$, or less than $5 \%$ of the leagt amount detectable by the method employed. By this procedure, no anticoagulant could be extracted from the blood of irradiated dogs. 17 references. (NSA)

\section{Miscellaneous}

\section{5}

BLair, Renry A. RADIUM TOXICITY AND EXTLRNAL RADIATION - BIOLOGICAL IEFFECTS, p.6-45 of QUARTERLY TECHNCAL REPORT JULY 1,1951 , THRU SEPTIMMRR 30, 1951. University of Rochester. Oct. 25, 1951. 40p. (UR-182(p.6-45))

Radium analygis of municipal water supplies of 33 cities, comprising about $20 \%$ of the population of the United States, is presented in tabular form. Results of a study of the differential leukocyte count of an Indian elephant presented in table form and discussed briefly. Serum, sodium, potassium, chloride, and carbon dioside content were determined serially on dogs receiving single massive whole-body exposures to 8 rays. Sodium, potassium, and chloride were also studied by alance technique. Regults give riøe to preliminary conclusions that the glight, but probably real, electrolyt changes in dogs following single massive wholebody exposures to $\mathrm{x}$ rays are in keeping with changes known to occur as a result of dehydration and starvation and are probably attributable to these factors. Preliminary observations of the effects of radiation on the metabolic process in balser's yeast confirm previously published data showing complete inhibition of colony formation without inhibition of over-all metaboliam following irradiation with $20,000-\mathrm{F}$ 250-kv 8 rays. Using $90,000-\mathrm{F}$ air dose, of $250-\mathrm{kv} \times$ rays, a small but constant stimulation of both oxygen uptake and anaerobic $\mathrm{CO}_{2}$ production was observed. Rate of uptake of glucose by the cells was not glowed up by an 7 -ray dose almost five times that sufificient to produce complete inhlbition of colony formation. Results are presented by graphs and tables, accompanied by a brief discussion. A feasible method for the degradation of porphyrins of the protoporphyrin 1X type is described. Urinary and serum fibrinolytic activity was measured in dogs after 450 to $700 \mathrm{I}^{\circ}$ of whole-body radiation. Three or four days before death both the ugine and serum fibrinolytic activity increased gignificantly, reaching maximum values just before death. The relation between the enhanced fibrinolytic enzyme activity and the hemorrhagic lesions noted at autopsy is discussed. (NSA)
736

Bless, A. A. BIOELECTRIC POTENTIALS AS A MEASURE OF RADIATION INJURY. Proc. Nat1. Acad. Sci. U. S. 36, 617-23(1950).

potentials measured from the tip of the coleoptile to the seed coat have been compared with coleoptile length in wheat secdlings irradiated with up to $6000 \mathrm{r}$ of $\mathrm{x}$ irradiation at various ages. Coleoptile lengths in a given group are more homogeneous in value than the corresponding potenlial measurements. On the other hand, bioelectric potentials are very sensitive to temporary changes in the condition or environment of the organism. It is reasonably safe to say that the average value of the potential of specimens is as good a measure of radiation injury as any other parameter, provided an adequate number of specimens are used. It is possible that a series of readings on one specimen may give as good an indication of radiation injury as a single reading of many specimens. (NSA) 737

BIOLOCY AND MEDICINE, p.131-62 Of QUARTERLY PROGRESS REPORT; JULY 1 - SEPTEMBER 30, 1951. Brookhaven National Lab. [nd] 32p. (BNL-132(p.131-62))

Progress is reported on a number of biological problems. Preliminary observations of cytological and morphological changes induced in plants by chronic $\gamma$ irradiation are reported lor plants grown in the 200 -curie $\gamma$ field. Data on weight changes and water consumption and metaboliam of rats following $x$ irradiation, histological changes in the pituitary of rats following irradiation, and the kinetics of phosphate interchange in tissues are included. Studies of uptake and distribution of $\mathrm{Ba}, \mathrm{La}, \mathrm{Cu}$, and $\mathrm{P}$ by Drosophila are reported. Further studies of the effects of radiation upon nucleic acids in plants and animals and metabolism of nucleic acids are discussed. A decontamination unit for use in event of heavy contamination of personnel is described. $\mathrm{Cl}^{38}$ was found to reduce ascites in three patients with metastatic growths in the peritoneal cavity. The mechanism of this reaction is under investigation. Effects of radiation upon antibody production are being studied and preliminary data are included. (NSA)

738

Brues, Austin M. RADIATION EFTECT, p.21-40, 56, $58-9,91-8,184-70,177-86,192$ of BIOLOGICAL AND MEDICAL OIVISIONS, QUARTERLY REPORT, FEBRUART, MARCH, APRIL, 1951. Argonne National Lab. [nd] 44p. (ANL-4625) (p.21-40, 56, 58-9, 91-8, 164-70, $177-86,122)$

Survival data are presented for two strains of mice, $\mathrm{C}_{3} \mathrm{HL}$ (without the milk agent) and the hairless strain, given a single whole-body $400-\mathrm{r} \times$ irradiation within $12 \mathrm{hr}$ after birth or chronle $\gamma$ irradiation of 4.4 or $8.8 \times(8 \mathrm{hr})$ beginning at age 1 month. Survival data on mice (progeny of crosses between $\mathrm{C}_{3} \mathrm{H}$ and $\mathrm{C}-57$ black strains) exposed 6 to $12 \mathrm{hr}$ after birth to $x$ irradiation of the head alone indicate a 20 day $L D_{60}$ of 1000 to $1100 \mathrm{r}$; observations on rate of hair growth, age at which eyes opened, and formation of cataracts are reported; examination of the eyes by opthalmoscope is recommended for early recognition and study of cataracts. Some preliminary data on the plasma electrolyte balance in dogs receiving a single total-body $x$-ray exposure of 450 x are given. The binding of organic ions by bovine serum albumin $\times$ radiated with 1000 to $120,000 \times$ has been investigated; the binding energy decreases with increasing $\mathrm{x}-\mathrm{ray}$ dosage above $10,000 \mathrm{r}$. The dosage rate required to reduce mitotic frequency below $50 \%$ in regenerating rat liver and Hall mouse carcinoma is given as a function of distance from 2 to $200 \mathrm{w} \mathrm{Sr}^{30}-\mathrm{Y}^{30}$ beads; effects of the radiation on tumor cells are illustrated. Incidence and microscopic appearance of tumors induced in female Sprague-Dawley rats by radiation on the dorsal skin from point and diffuse sources of $\mathbf{S r}^{20}$ are shown. The comparative and additive 
physiological and genetic effects of $\mathrm{x}$ radiation and nitrogen mustards on Paramecium aurelia, and the action of infrared, visible, ult raviolet, and $\mathrm{x}$ rays on the mating reaction in Paramecium bursaria, are being investigated. Some data are given on the effect of $x$ rays on the metabolism of haploid and diploid yeasts, and on the oxidation of dihydrodiphosphopyridine nucleotide (DPNH $)$ by $\mathrm{x}$ rays. (NSA) 739

Brues, Austin Meg ed. CHRONIC EXTERNAL IRRADLATION; NEUTRON TOXICITY, AND HEMATOLOGY, p.947 of DIVISION OF BIOLOGICAL AND MEDICAL RESEARCH; QUARTERLY REPORT; AUGUST, SEPTEMBER, OCTOBER, 1951. Argonne National Lab. [nd] 39p. (ANL $-4713(0.9-47)$ )

Data on the effects of homologous and heterologous bone marrow injections on survival in mice following $\mathrm{x}$ irradiation are presented in tabular form. The addition of $0.025 \mathrm{M}$ K citrate to distilled water suspensions of Rous sarcoma virus gave a high degree of protection against an $X-r a y$ dose of 75,000 r. Studies on the mechanism of citrate protection are in progress. Data on the pattern of loss and recovery of weight of the testes of mice exposed to a single dose of $x$ radiation and a single intravenous injection of $\mathrm{Rn}$ in equilibrium with its short-lived decay products are presented in talbular form. Results indicate that the $a$ particle does not manifest as high a factor of biological effectiveness relative to $x$ radiation as has been assumed. A summary is included of visible lens opacities 6 to 7 months after various doses of $x$ radiation to the head of newborn mice. Histologic examination showed progressive destruction of the cells of the retina following $1000 \mathrm{r} x$ irradiation, with obvious signs within $3 \mathrm{hr}$ after irradiation. The effects of shielding of liver, intestine, or spleen, and of spleen shielding followed by splenectomy at 5 min and at $24 \mathrm{hr}$ upon the hematocrit values, reticulocyte and leukocyte counts of mice exposed to $1025 \mathrm{r} \times$ irradiation are presented graphically. Data indicate that cysteine injected up to 4 days before sacrifice does not significantly alter the 24 hr uptake of $\mathbf{p}^{32}$ by rat and mouse tissues. Studies of the effecta of spleen shielding and subsequent splenectomy upon antibody formation in rats receiving total-body $x$ irradiation confirm previous observations concerning the protective effects of 740 spleen shielding on antibody formation. (NSA)

Brues, Austin M., ed. PHYSIOLOGY, RADIUM TOXICITY, MSTOPATHOLOGY, AND RADIOCHEMISTRY, 0.48-73 of DIVISION OF BIOLOGICAL AND MEDICAL RESEARCH; QUARTERLY REPORT, AUGUST, SEPTEMBER, OCTOBER, 1951. Argone National Lab. [nd] 26p. (ANL-4713(p.48-73))

Data from a study of the effects of eysteine and hypoxia on the survival of rabbit thymocytes irradiated in vitro with 200-r \& rays, and data on the effects of chilling on the protection afforded $x$-irradiated thymic cells by cysteine are presented in tabular form. From results of the gtudy it is concluded that the effect of cysteine on ir radiated thymiccell-gerum guspensions can not be ascribed to a direct competition for the short-lived free radicals presumably formed in the aqueous phase, and also that the protection is not due simply to the removal of $O$ and conseguent decrease in the toxic breakdown products of water. Preliminary results suggest that cysteine binds $O$ rapidly in the presence of thymic cells sugpended in serum, and the effect of chilling suggests that other events, presumably related to the binding of $O$, must take place. Further work on this problem is in progress. Changes in body weight, food intake, and water intake were found to be related to $\mathrm{x}-\mathrm{ray}$ dosage in rats, and cysteine pretreatment and a force-fed, semiliquid diet greatly decreased the characteristic postirradiation drop in body weight. A method of measurement of the $\gamma$ activity in humans due to the nonemanating iraction of $\mathrm{Ra}$ present in the body is reported and comparizon is made of counting results in patients whose Ra burden was established by the absolute method of measurement, and with Rn exhaled in the breath by these patients. In the clinical study of persons exposed to radium, one 68-year-old male given radium chloride intramuscularly 25 years ago was found to have an estimated total-body radium of $13.9 \mu \mathrm{g}$, however, complete radiography indicates only minimal skeletal changes. 1 dosage of $800-\mathrm{r}$. radiation delivered to the tenotomized legs of rats did not appear to greatly disturb tendon regeneration although the regenerative process took a longer time in irradiated than in control rats. (NSA)

T실 1

Brues, Austin M., ed. SPECLAL PROBLEMS, p.74-86 of BIOLOGICAL AND MEDICAL DIVISIONS, QUARTERLY REPORT, AUGUST, SEPTEMBER, OCTOBER, 1951. Argonne National Lab. [nd] 19p. (ANL-4713(p.74-86))

A method Is described whereby data on tumor incidences may be put into such a form that comparison can be made according to standard statistical procedures for handling rare discrete events. In a study of the effects of pile-irradiated cholesterol on mice it was concluded that Lonizing radiation does not produce appreciable carcinogenic activity in cholesterol and that direct action of ionizing radiation on cholesterol is not responsible for radiation carcinogenesis. Data on the mortality of cysteine-treated chicks following $x$ irradiation are presented in tabular form. Preliminary data indicate that larger cysteine doses are reguired to protect against radiation toxicity in newly-hatched than in older chicks. Dog plasma was found to afford protection against radiation injuries to mice when administered intravenously just prior to exposure to $800-\mathrm{r} \times$ radiation. The tozicity of normal dog plasma to mice was found to be lost after $5 \mathrm{hr}$ incubation or perfusion through the dog spleen. White's medium, a gynthetic nutrient containing salts, amino acids, and vitamins, did not aford protection to mice after perfusion through dog spleen, but did give active protection if given intravenously after periusion through the spleen that had been lead-protected during irradiation of the dog. Neither agent was protective when administered before $x$ irradiation. (NSA)

\section{2}

Brues, Austin M., ed. INVTRTERBRATR ZOOLOGY, PHYTORADIOBIOLOGY, AND MICROBIOLOGY, p.87-100 Of DIVISION OF BOLOGICAL AND MEICAL RESEARCH: QUARTERLY REPORT, AUGUST, SEPTEMBER, OCTOBER, 1951. Argone National Lab. [nd] 14p. (ANL$4713(p .87-100))$

Win the grasshopper egg as a test object, a study was made of the effect of $\mathrm{X}$ Irradiation on enzymogenesid. Data on the effect of $\mathrm{x}$ irradiation on the acetylcholine content of postdiapause grasshopper egg are preaented graphically. Preliminary experiments on the radiosensitivity of the native enzyme complez involved in the conversion of tryotophan to auxin in bean seedlings are gummaxized. In an at̂tempt to correlate knowledge concerning the degradation of nitrogen mustards in solution with loss of biological effect with time, groups of $\mathrm{P}_{\text {. }}$ aurelia were exposed for $1 \mathrm{hr}$ to the solution at various times after its preparation. Results indicate that the mean half life of the initially active muatard component was about 20 min. Paramecia were irradiated th the lettuce medinm in which they were grown, in salt solution into which they had been put just prior to exposure, in calt zolution into which they had been put $14 \mathrm{hr}$ prior to esposure, and in a fresh salt golution after having cemained in a salt solution $14 \mathrm{hr}$ prior to exposure. 5uppreseion of fission and preautogamous death were markedly inereased immediately in animals that were placed in the salt solution 
just before irradiation. Further investigations of the radiogensitivity of the mating reaction of $\underline{P}$. bursaria has regulted in the hypothesis that release of the mating substance is increased by illumination while its production is inhibited by illumination. It is thought that a flavin acts as the initial 743 photoreceptor in the early reactivity period. (NSA)

Coon, Julus M. QUARTERLY PROGRESS REPORT ON RADIOBIOLOGY, REPORT NO. 4. TORIcity Lab., Univ. of Chicago. Jan. 15, 1950. 88p. (TID-364)

This report is composed of sections entitled as follows: Continuous Exposure of Animals to $\gamma$ Radiation; The Toxicity of $\gamma$ Radiation to Mice Exposed at Varying Dose Rates; Changes in the Distribution of Phosphorus in Irradiated Rabbit Bone Marrow; Studies on the Metaboliam and Toxicity of Radioactive Metals; Studies on the Mechanism of Acute Beryllum Poisoning. III-The 1effects of Beryllium on Phogphatases and on Intermediary Carbohydrate Metabo118m; Studies on the Carbohydrate Metabolism of the Rabbit Ileum; The Effects of Beryllium Chloride in Locke-Ringer Solution upon the Irolated Rabbit lleum and Heart; Rffect of X-Radiation on Intestinal Cathepsins; cifect of Vitamin $B$ 12 on X-Ray Toxicity to Mice; Further Studies on the Acthon of Beryllium Chloride; Some Notes on the Sampling of 744 Aerosols by Impaction Methods. (NSA)

Coldhaber, G. and B. Feldman-Munsam. IMMEDIATE

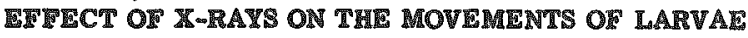
AND PUPAE OF MOSQUTOES. Nature 153, 528(1944).

Cisperiment is degeribed which demonstrates the acceleration of the movements of larvae and pupae of the mosquito Aedes aegyoti immediately aiter the onset of irradiation. When the irradiation is interrupted, they return at 44: once to normal behavior. (S.V.S.)

Jones, M. W., B. Tivel, and A. A, Bless. THE BIOELFCTRIC POTENTIAL OF SEDDS AS A FUNCTION OF GROWTH AND OF X-RAY DOSAGE. Plant Physiol. 26, 19-29(1051).

It was found that $x$ rays, in general, decrease the bioelectric potential of plant seeds and lower their vitality. High-yielding seeds were found to have higher potentials than those of low-yielding varieties. The bioelectric potential is not a function of the weight of the seed; it is zero at dormancy and rises glowly during the first few hours in a humid environment, then it rises much more rapidly as coleoptlle elongation beging. (NSA)

746

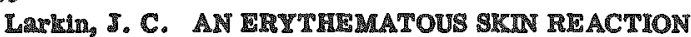
PRODUCED BI AN ALPRA-PARTCLE BEAM. Am.J. Toentgenol. Radiun Therapy 1 s. $_{3}$ 109(1041).

While works with the cyelotron, a graduate student inadvertently put his hand in a beam of $32,000,000$ volt alpha particles. He degeribed the aensation as one of extreme hear and withdrew his hand as rapidly as possible so that the time of exposure was estimated at about one second. In lour hours, the area was erythematous, two gmall blister appeared, but the origginal senation of pain did not persist. The next day the araa became painiul and remained so for lous days during which the erythema regressed. By the 10th day there was desquamation of the stratum corneum, marked thickening of the skin, and the area was insengitive to touch. On the next day, the area was again becoming -rythematous, quite swollen and very sore. After 18 days the erythema began to regress, but the thickening and roughening of the lkin continued to progress even after Iour months. E'ven aster four months there was some thickening of the dermis. This is the first case of a visible erythematous reaction caused by a beam of alpha particles. (BA)

749

QUARTERLY PROGRESS AND WORK ACCOMPLISHMENT REPORT FOR QUARTER ENDING SEPTEMBER 30, 1951. Tennessee Univ. [nd] 33p. (ORO-54)

Progress is reported in the gtudy of the fission-products metabolism in domestic animals and the dependence of their behavior upon normal mineral metabolism. Bloodvolume values for swine were determined using the $\mathbf{P}^{32}$ labeled red-cell method. Swine with average body weight of $10 \mathrm{lb}$ were found to average $7.4 \mathrm{ml}$ of blood per $100 \mathrm{~g}$ body weight, while those averaging $344-1 \mathrm{~b}$ body weight had $4.6 \mathrm{ml}$ of blood per" $100 \mathrm{~g}$ body weight. Values for intermediate body-weight groups are also included. Blood values for the gouthwestern burro (Equus asinus asinus) were determined and are presented in tabular form. The multicurie irradiation field for large animals was loaded, calibrated, and placed in operation. Normal blood values for the healthy burro have been established to serve as a basis for following the changes caused by irradiation. Particular attention is invited to the results of a preliminary experiment on the effects of whole-body irradiation upon the subsequent fertility of the male rabbit. These data emphasize the fact that observations on the sperm themselves or even on the initial fertilization do not suffice to give a true measure of ipradiation fiects on fertility; it is necessary, therefore, to use the number of viable young produced as the criterion. As an indication of results, it was shown that while control rabbit in 34 matings produced an average of 6.56 viable young per mating, the values for males exposed to 100,200 , and $300 \mathrm{x}$ were 3.4 in 15 matings, 4.5 in 26 matings, and 3.2 in 16 matings, respectively. (NSA)

\section{IFECTS OF INTERNAL RADIATION AND ME- TABOLISM AND TOXICOLOGY OF INTER- NALLY DEPOSITED RADIO-ELEMENTS}

\section{General Dosage Studies}

748

Abrams, R. PRODUCTION AND ANALYSIS OF RADIOACTIV AEROSOLS. [Univ. of Chicago Metallurgical Lab.] July 2, 1946. Decl. Mar. 11, 1947. 80p. (MDDC-795; CH-3629)

The biological program calling for exposure of animals to air-borne radioactive materials made it necessary to develop procedures for producing aerosols, exposing animals to the aerosols, and measuring particle size and concentration. Since carrier-free materials were used, techniques were devised for the remote control handling of material with negligible weights, but with high radioactivity so as to obtain maximum deposition in the lungs of the experimental animals. Of a number of methods of producing aerosols that were investigated, three were extensively used. Atomization of aqueous solutions in a right angle jet atomizer was useful for tracer. But because of the great dilution with air it was not possible to obtain high efficlency. Burning a aalt in a D.C. carbon arc was adequate for obtaining high intensity fission product smokes, but falled with Pu where it was necessary to use larger masses of material. High concentration Pu smokes were obtained by wrapping Pu metal in thin aluminum foll and discharging a high energy condenser (1000 joules) through the foll. To maximize lung deposition and to avoid akin contamination and ingestion anesthetized rats which 
breathed from a small chamber through glass cannulae were used. This chamber consisted of a 2.8 liter brass cylinder from which 32 rats breathed while lying on a surrounding bakelite superstructure. In the case of the exploding foil it was necessary to use a 4.1 liter cylinder with a partition which protected the rats from the violence of the explosion. A number of methods were investigated for collecting smoke samples for analysis. The most satisfactory was the thermal precipitator which makes use of the dust-free zone around hot bodies to precipitate all the particles in a sample of air on to a Parlodion-coated microscope cover slip. Concentrations were estimated by doing direct $\gamma$ and $\beta$ eounts on these cover slips. Later the Parlodion film was stripped off the glass and mounted for particle size observation in the electron microseope. Mean particle size with the methods used in the experiments averaged 0.1 to $0.2 \mu$. (ADD)

\section{9}

AMERICAN ASSOCLATION OF ANATOMISTS, SIXTY SECOND ANNUAL SESSION AT TEMPLE UNIV., PHL ADELPHIA, PA., APRIL 1949. Anat. Record 103, 417. $595(1949)$.

Papers reviewed are the following: Deposition of radioactive strontium and the rare earth promethium in the skeletal tissues of the rat, C. W. Asling, D. A. Heller, D. H. Copp, and J. G. Damilton. Detection of the entry of radiophosphate in bones with the help of a new technique, the "inverted autograph," Leonard F. Belanger. Deposition of $\mathrm{C}^{14}$ in the metaphysis of long bones of young rats, William Bloom. The effect of 8 -ray ipradiation upon the lumbo-sacral region of the axolotl (Siredon mexicanum), V. V. Brunst. Effects of $\mathrm{P}^{32}$ on regenerating amphibian tissues, J. N. Dent. Steady state of the iodine in the thyroid gland of the male rat, J. Dougherty. The use of radioactive phosphorus in studies of fetal circulation, Newton Everett and Robert J. Johnson. The development of roentgenographic methods to determine depth of penetration of liquid jeta into living human subjects, Frank H. J. Figge. The localization of physiological amounts of thyroxine labeled with radioiodine in the tissues of the rat, J. Gross. Radioautographic reactions in tissues other than bones and teeth after injection of radio-phosphorus, C. L. Stevens. The following papers were read by title: A radioautographic atudy of the localigation of radionctive europium in certain soft tissues of the rat, C. D. Van Cleave. Studies on the effects of $x$-radiation on morphogenesis of Amblystoma punctaturn, s. W. Haniord. The effect of intraperitoneally injected radium chloride upon the peripheral blood of

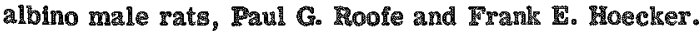
Roentgenology as a means of instruction in anatomy in North American medical schools, Wayne A. Simril, A. Jack Stacy, and Sydney 1. Thomas. Detection of radio-elements by various radio-autographic techniques, C. P. Leblond, L. F. Belanger, R. Bogoroch, C. E. Stevens, J. Gross, and G. Wilkinson. Abstracts of the papers are presented. (NSA) 750

Brues, Austin M. BIOLOGICAL IIZARDS AND TOXICITY OF RADIOACTIE ISOTOPES. J. Cln. InYest. 28, 1286-28(1049).

There is no evidence that radiation dosages corresponding to standard permissive dosages will result in increased incidence of mallgnant disease detectable in a population of moderate size. 32 references. (CA)

\section{1}

Brues, Austin M., ed. TOXICITY, p.55, T2-84, 00-124, 142-51, 187-91, 193-4, 209-21 of QUARTERLI REPORT, FERUARY, MARCR APRL, 1951; DTVISION OF BIOLOGICAL AND MEDICAL RESEARCH. Argone National
Lab. [nd] 70p. (ANL $=4625$ (p.55, 72-84, 99-124, 142-51, 187-91, 193-4, 209-21))

Radioautographic studies of the distribution of $\mathrm{Ra}$ in trabecular and cortical bone of the tibia and fibula of a human $25 \mathrm{yr}$ following $\mathrm{Ra}$ ingestion, distribution of $\mathrm{Ca}^{245}$ in rat bone, and distribution of $\mathrm{Ra}$ and $\mathrm{Pu}^{298}$ in dog bone are pre sented. Studies of the fate of $C^{14}$ absorbed by mice and rats during a 30 - to $60-\mathrm{min}$ period of exposure to $\mathrm{c}^{16} \mathrm{O}_{2}$ have been made. The calculated rates of passage of alveolar $\mathrm{CO}_{2}$ into the blood were $23 \mu$ moles/min in the mouse and $160 \mu$ moles/min in the rat. Uptake of $\mathrm{C}^{\text {14 }}$ was essentially unaffected by the $\mathrm{CO}_{2}$ concentration of inhaled aix. Passage of $\mathrm{C}^{\text {IS }}$ from the bicarbonate compartments into inner compartment proceeded at a somewhat slower rate. Rates of uptake of $\mathrm{C}^{\text {th }}$ from $\mathrm{C}^{14} \mathrm{O}_{2}$ by the livers of adult and newborn mice are compared, and the bone carbonate turnovers of adult mice and rats are discussed. The toxicity ratio between $\mathrm{Ra}$ and Pu has been evaluated from the data of a large number of experiments. The best available $\mathrm{Ra} / \mathrm{Pu}$ ratios, in terms of injected $\mu \mathrm{c} / \mathrm{kg}$ are: acute toxicity to small animals, 15; chronic survival, 10 ; formation of bone tumors in rats and mice, 15; formation of bone tumors in rabbits, 8; and bone fractures in rats and rabbits, about 10. A maximum permissible retained dose of $0.04 \mu \mathrm{c}$ of $\mathbb{P} u$ in man is the best value available from present biological information. The median lethal dosage to rats of $\mathrm{H}^{3} \mathrm{O}_{2}$ administered continuously over a 2 -month period correspondes to between 57 and $77 \mathrm{rep} /$ day on the basis of dosage rate in body water. The mean accumulated dosages to death are between $\$ 000$ and 3700 Iep, being consistently higher at 77 than at $96 \mathrm{rep} / \mathrm{day}$. The effectiveness in protecting againet Be poisoning of various dyestuffe having structural similarity to aurin tricarboxylic acid was tested with negative results. The acute toxicities of members of the mesothorium chain $\left(\mathrm{Ra}^{234}, \mathrm{Th}^{238}, \mathrm{Ra}^{228}-\mathrm{Ra}^{238}-\mathrm{Po}^{210}\right.$ equilibrium mixture, and $\mathbb{R a}^{284}-\mathrm{Th}^{228}$ equilibrium mixture) have been studied in CF-1 female mice. The 20- to 30-day $\mathrm{LD}_{60}$ 's for all the elements except $\mathrm{Ra}^{326}$ fall between 36 and $58 \mu \mathrm{c} / \mathrm{kg}$. The $\mathrm{Ra}^{228}$ values range from 230 to $1100 \mu \mathrm{c} / \mathrm{hg}$. (NSA) 752

Brues, Austin M., ed. ON ESTIMATION OF TOTAL RADLATION DOSAGE FROM THE METABOLIZED OR

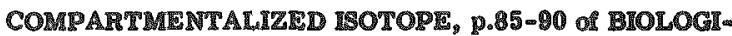
CAL AND MEDICAL DIVISIONS, QUARTERLI REPORT, FLBRUARY, MARCR, AND APRIL, 1051. AFgonne

National Lab. [nd] Cp. (ANL-4625(p.95-00))

Despite the complexity of time-retention curves in a steady-state system of many homogeneous compartments, the integrated dosage from an isotoge entering guch a system under known external conditions is wholly predict. able. If can be computed on the astumption that all the exchangeable isotope in any volume maintains a spechlc activity $x$ time equal to that in the external compartwent. A deviation from this indicates the presence of an ifreverable process or deviation from the steady gtate, or possibly a situation where a diffusion gradient is important. To the extent that a metabolic aystem satimiles the reguirements indicated, the use of "mean retention time" lo wald. (2Nas)

\section{8}

Brues, Austin M. COMPARATIVI CRRONIC TORTCRTE OF RADTUM AND PLUTONTUM. AFGome Nationgl Lab. Apr. 1051. 20p. (ANL -4790)

A preliminary quantitative gurvey if presented of the survival and gross pathologic changes in animals given Ro and Pu, and a discussion of the significance of the Indings and their relation to the human toxlcfty problem is outlined. The torlcity ratlo between Ra and Pu is evaluated from tho data of a large number of emgerinentm. (NSA) 
754

Buchanan, Donald L. AN INTERRELATIONSHIP BETWEEN TIME-RETENTION CURVES IN SHORT VS. PROLONGED ISOTOPE EXPOSURES. Argone National Lab. Nov, 1950. 13p. (AECU-1183; UAC-329)

A concept is presented which relates isotopic retention of an isotope in animals subjected to a "single dose" to the level of retention found experimentally after prolonged constant intake of the same isotopic compound. The mean retention time, which is easily obtained from experiments of the latter type, specifies the integral of the time-retention curve obtained in experiments of the former type and by so doing rigorously sets an upper limit to the total radiation dose that the animal may receive from the intake of a known amount of a given isotopic compound. The concept is extended to allow calculation in localized arbitrary volume: of tissue. (NSA)

755

Bush, F. THE INTEGRAL DOSE RECEIVED IROM A UNIFORMLY DISTRIBUTED RADIOACTIVE ISOTOPE. Brit. J. Radiol. 22, 96-105(1949).

Methods of calculation of the integral dose received in simple geometrical bodies containing a uniformly distributed $\gamma$-ray emitting radioactive isotope are described, and a general relation between this dose and the volume, and the elongation of the body, deduced. These calculations have been extended to the case of a "patient," composed of eylinders and elliptical cylinders. A table is given, showing the integral dosage-rates in patients of different heights and weights from a uniformly distributed $\gamma$-ray emitter. The effect of variation in the quantum energy of the emitted radiation on the integral dose is examined. An expression is found which gives the fraction of the energy due to $\beta$ particle emission which is lost from the surface of the patient or animal. This loss may be important in the case of small animals. (NSA)

756

Waldo, Cohn E. TOXICITY OF INHALED OR INGESTED RADIOACTIV PRODUCTS. Nucleonics 3, 21-6(1948) July

A general formulation is presented which relates the concentration of a radiosubstance in air, water, or food to the total dose or daily intensity of radiation received by a given tissue as a result of the type and extent of intake of the polluted source. This relation is expressed in terms of biological constants (degree of absorption, deposition pattern, excretion rate) and physical constants (half-life, radiation energy) of the contaminating substance. Use of the derived formula is demonstrated by a number of examples. (CA)

757

Copp, D. H., D. J. Axelrod, and J. G. Hamilton. THE DEPOSITION OF RADIOACTIVE METALS IN BONE AS A POTENTIAL HEALTH HAZARD. Am. J. Roentgenol. Radium Therapy 58, 10-16(1947).

Radio-Sr follows the path of Ca metabolism and is deposited only in the bone salts, whereas radionctive $\mathrm{Pu}, \mathrm{Y}$, and Ce are laid down in the uncalcified organic matrix of bone in contrast to the accepted view with regard to behavior of other heavy metals which deposit in the skeleton. Radio-Sr is eliminated more readily with a biological hal life of three to four months. The other elements mentioned are excreted very slowly with a biological half life of one to

Craver, B. N. RADIOACTIE EMANATIONS: THEIR NATURE, MECHANISM OF ACTION, BIOLOGICAL EPFECTS AND TOLERANCE LIMTS. I. Ind. Hyg. and Toxicol. 29, 196-200(1947).
The characteristies of the various common types of radiation produced by atomic disruption have been described. The nature of the biological changes that they produce have been discussed as well as the mechanism of their production. The curie and the roentgen have been defined and human tolerance-limits in terms of those units. (Author's Summary) 759

Evans, R. D. TISSUE DOSAGE IN RADIO-ISOTOPE THIERAPY. Am. J. Roentgenol. Radium Therapy $58,754-$ 6(1947).

The biological action of roentgen rays is due to the effects on living cells of high-speed secondary electrons produced by the primary roentgen rays. The beta rays from a radioative isotope are also high speed electrons. These beta rays can therefore be expected to produce tiasue effects similar to those produced by roentgen rays. The beta rays have a maximum range of only a few millimeters of tissue actually containing the radioactive isotope, and the dosage rate at any time is proportional to the concentration of the isotope in the tissue. It might be desirable to express the radiation doses produced by the beta rays in some fundamental energy units, such as ergs per gram of tissue. Ordinarily, however, the doses are converted into equivalent roentgens, with air as the standard substance. A discussion is given of dosage rate and total dosage delivered by radioactive isotopes. (GPR)

760

Mert, K. E. DEPOSITION OF RADIOACTIVITY IN RATS DRINIRENG PILE EFLLUENT WATER. Hanford Worls. Jan. 24, 1951. 13p. (HW-20102)

Two groups of rats drank ad libitum only effluent nuclear reactor cooling water adjusted to activity density levels of approximately $3 \times 10^{-4}$ and $3 \times 10^{-8} \mu \mathrm{c} / \mathrm{ml}$ for periods of 200 to 500 days. Growth rate of the experimental rats was not affected by the radioactivity, and no histological changes were observed. Both effluent-drinking groups showed $\beta$ radiation levels significantly higher than the controls. In the group receiving the greater concentration, blood and kidneys usually had the highest activity density with an accumulation of $\mathrm{Na}^{28}$ and $\mathrm{P}^{32}$ approximating $2 \times 10^{-6}$ $\mu \mathrm{c} / \mathrm{g}$. This study suggests that the hazard to mammals drinking Columbia River water containing greatly diluted effluent is negligible if the diet provides all the essential elements. (NSA)

761

Lanz, H., K. G. Scott, J. Growley, and J. G. Hamilton. THE METABOLISM OF THORIUM, PROTACTINIUM, AND NEPTUNIUM IN THE RAT. [Radiation Lab. Univ. of Calif.] Augs. 23, 1946. 18p. (MDDC-648)

The distribution of carrier-free thorium, protactinium and neptunium in the rat following intramuscular and oral administration has been gtudied. Oral absorption for all three is negligible. Following parenteral administration, the skcleton is the organ showing the highest degree of prolonged and selective localization. (ADD)

\section{2}

Lisco, Hermann. DOSAGE LEVELS IN ADMINISTRATION OF ISOTOPES TO ANIMALS AND MAN. U. \$. Naval Med. Bull. Supplement, $48,161-3(1948)$.

Radioactive isotopes have injurious effects upon animals and man. These effects are functions of a number of factors, two of which are the hall-life and the distribution of the isotope in the body. Since the distribution varies from isotope to isotope, depending upon metabolic function, turnover, and excretion rates, different manifestations of radiotoxicity can be expected. Laboratory studies have provided information on the ranges of dose levels in animals at which acute and chronic injurious effects can be 
expected. Isotope siudies include $\mathrm{Sr}{ }^{39}, \mathrm{x}^{81}, \mathrm{Ra}, \mathrm{Ce}^{144}, \mathrm{Pu}^{239}$. Although this list does not include isotopes commonly used for tracer studies, certain conclusions may be drawn from the data which have been obtained. Studies of $\mathrm{P}^{32}$ and $\mathrm{C}^{14}$ are now being carried out. Virtually nothing is known at present about the distribution of $\mathrm{C}^{14}$ in the mammalian body and its retention in and toxicity to the organism. Some preliminary data suggest, however, that some retention does occur in bone and this fact should be borne in mind by investigators using $\mathrm{C}^{14}$. There is information on dose levels of $\mathrm{P}^{32}$ and $\mathrm{I}^{131}$ presently used in man for treatment of disease, and the blood picture (lymphocytes, platelets) is a fairly accurate and reliable indicator of incipient radiation damage. However, it will be exceedingly difficult to detect minute quantities of radioactive isotopes lodged in discrete areas in the body and producing localized irradiation over a long period of time without concomitant damage to the bone marrow. It has been tacilly assumed that tracer levels of radioactive isotopes have no effects upon the systems under investigation. Although this is probably true in most instances, it might be worth considering 2 number of ad hoc experiments, so that definite information may become available on tolerance-levels as applied to 763 animal experiments of this type. (NSA)

\section{Marinelli, L. D. DOSAGE DETERMINATION WITH}

RADIOACTIVE ISOTOPES. Am. J. Roentgenol. Radium Therapy 47, 210-6(1942).

With the inc reasing employment of radioactive isotopes in chem., biol., and phys. research, it becomes increasingly important to have an accurate method of dosimetry. By the use of a suitable method of specifying energy absorption, the question can be closely related to $x$-ray and radum dosimetry. On this basis, the radiation from these substances can be specified in "equivalent roentgens." Formulas are derived which permit the estm. of the radiation delivered when the conen. of the isotope in the material is known. In biol. or clinical use of the substances, the dosage in addn. to initial tissue uptake, depends also upon elimination; the importance of this depends partly on the half life of the particular isotope. The main difficulties in this dosimetry are of biol. nature; accurate detn. of isotope concn. in different tissues requires either actual radioactive measurements on tissue fragments obtained from living individuals, or on calcns. based on thorough quant. knowledge of tissue metabolism which is not available at present. (CA)

764

Marinelli, L. D. DOSAGE DETERMINATION IN THE USE OF RADIOACIVIE ISOTOPES. J. Clin. Invest. 28, 1271-80(1949).

In this extensive paper, formulas expressing the relationship between radiation dose and isotope concentrations are presented and their clinical applications are discussed by the use of tables containing a considerable amount of information. Examples of the application of the formulas to such specific isotopes as $\mathrm{P}^{32}, \mathrm{Sr}^{63}, \mathrm{Ca}^{46}, \mathrm{Na}^{24}, \mathrm{Na}^{22}, \mathrm{~K}^{22}$, $I^{134}, I^{130}$ are included along with definite problems. In the two specific tables presented, for $\beta$ and $\gamma$ rays, respectively, the half-life, radiation average energy, fraction disintegrating per day, and specific dosage data are contained. References for those isotopes which are not included in the body of the article or for supplementary information are presented in a special section in the bibliography. (NSA) 765

MarineLli, L. D., E. H. Quimby and G. H. Kine. DOSAGE DETERMINATIONS WITH RADIOACTIVE ISOTOPES. II. PRACTICAL CONSIDERATIONS IN THERAPY AND PRO-
TECTION. Am. J. Roentgenol. Radium Therapy 59, 260$78(1948)$.

When radioactive isotopes are employed either as tracers or in therapy, it is important to be able to determine the radiation dosage. This camot in general, be measured, but when the half life, radiation energy, and biological uptake and excretion are known, it can be calculated. The paper is divided into two parts, based on clinical and physical aspects of the problem. In the physical part (Part II) are given mathematical developments of formulae for dosage rates and total doses for beta and gamma-ray emitting isotopes, together with subsidiary formulae for safe concentration, weight per me, etc. In the clinical part (Part) these formulae are accepted, and doses considered for apecific cases, for a number of iøotopes of common interest. Particular consideration is given to the determination of safe tracer doses. Two extensive tables are presented, for beta and gamma rays respectively, giving half life, radiation average energy, fraction disintegrating per day, and specific dosage data, including the safe tracer concentration, for some 38 isotope elements. An extensive bibliography is included. (GPR)

766

Maynoerd, W. V., J. H. Martin, and D. A. Layne. PRODUCTION OF RADIOACTIVITY IN ANIMAL TISSUES BY HIGH-RERERY X-RAYS. Nature 164, 728-30(1949).

Based on the fact that photo-disintegration of atomic nuclei by high-energy $\mathrm{X}$-rays occurs, induced activity in tissues is observed when rat tissues are exposed to beams of $x$-rays. The decay curves, plotted semilogarithmically, reveal two half-lives of 2.1 and $20 \mathrm{~min}$, respectively, agreeing with the known half-lives of oxygen ${ }^{15}$ and carbon ${ }^{11}$. Decay curves for ice and water coincide with the $2.1 \mathrm{~min}$ curves of the tissues. Calculation of the rate of energy absorption from the $x$-ray beam results in a value of 0.93 ergs/g sec, whereas the rate of absorption of energy due to the disintegration from $\mathrm{O}_{3}$, by assuming a mean energy per disintegration of $0.7 \mathrm{mev}$, is $0.4 \times 10^{-6} \mathrm{ergs} / \mathrm{cc} \mathrm{sec}$. Even if $C$ disintegration is included, the rate of absorption of energy during irradiation due to the activation is only of the order of $0.01 \%$ of that due to the more usual mechanisms of absorption, namely, electron recoil and pair absorption. (CA)

767

Morgan, $\mathbb{K}$. Z. TOLERANCE CONCENTRATIONS OF RADIOACTIVE SUBSTANCES. J. Phys. and Colloid Chem. 51, 984-1003(1947).

Reviews some of the elementary equations and procedures involved in determining tolerance concentrations of radioactive substances when taken into the human system. (GPR)

768

Morgan, $K$. Z. SYMPOSIUM ON PREPARATION AND MEA SUREMENT OF ISOTOPES FOR USE IN BIOCHEMISTRY. HAZARDS PRESENTED BY RADIOACTIVE MATERLALS AND HOW TO COPE WITH THEM. U. S. Naval Med. Bull. Supplement, 48, 142-60(1848).

Equations are given for calculating the tolerance concns. of radioactive material in the body. The tolerance values of gome of the radioactive elements are summarized. (CA)

769

Norris, Wulliam P. and Walter Kisieleski. COMPARAIIVE METABOLISM OF RADIUM, STRONTIUM, AND CALCIUM. Cold Spring Harbor Symposia Quant. Biol. 13, $164-72(1948)$. 
Sprague-Dawley rats, kept on a constant diet, received $\mathrm{Ca}^{45}$ or $\mathrm{Sr}^{89}, 80$ intravenously, $40 \mu \mathrm{c}$ of $\mathrm{Ca}, 115 \mu \mathrm{c}$ of $\mathrm{Sr}$, and $25 \mu \mathrm{c}$ of Ra were included in the injections, respectively. Time determinations on the blood, feces, urine and skeletal levels of the elements were then performed; the results of which are tabulated and discussed. Other similar experimental courses were $\mathrm{zun}, 0.02-0.91 \mu \mathrm{c} / \mathrm{g}$ of body weight of radium ger animal being administered intraperitoneally to 100 Sprague-Dawley rats; the retention was measured as before. 38 references. (NSA)

T70

Oddie, T. H. DOSAGE FROM RADIOISOTOPES UNIFORMLY DISTRIBUTED WITMIN A SPHERE. Brit. J. Radiology 24, 333-6(1951).

Formulas are developed for a geometric factor to allow for variations of dosage rate at points in tissue both inside and outside a sphere containing a uniformly distributed radioactive material. Values of this factor are tabulated. The results are applied to find the order of variations of dosage rate that are to be expected with a spotty distribution of $\mathbb{1}^{131}$ in a thyroid gland. It is shown that the dosage rate may quite easily change by a factor of more than 100 in different parts of the tissue mass. (NSA)

771

Pearson, I. A., J. M. Hammer, R. E. Corrigan, and H. S. Hayden. STUDIES ON THE DERAVIOR OF FUNGI IN THE PRESENCE OF RADIOACTIVE ISOTOPES. J. Bacteriol. 56, 397-402(1948).

The behavio of 43 pathogenic and nonpathogenic fungi in the presence of radioactive phosphorus, iodine, and high voltage $x$-ray was studied, and a technique for handling cultures containing radioactive substances was developed. The amounts of $\mathbb{P}^{32}, \mathbb{I}^{131}$, and $\mathrm{x}$-ray were not lethal, and exposure to them did not cause any marked changes in the morphology or pathogenieity of numerous fungi studied. It was found that solutions of radioactive phosphorus and iodine have no sell-sterilizing properties. Autoradiographs of fungi were made showing that the fungi themselves were Iadioactive. It was impossible to obtain autoradiographs of single spores or mycelia since considerable scattering was present; the technique is being studied further and developed. (NSA)

772

Posin, Daniel Q. INVESTIGATIONS WITH RADIOACTIVE SR, P, AND FE. Proc. Montana Acad. Sci. 3 and 4 , 10-15(1942-43).

Four days before giving bisth to young, a pregnant rat was given an injection of radioactive p. One of the young, less than a day old, showed considerable body and skeleton radiation. After about two months the radioactive $p$ was about equally distributed in density in the young and the mother. A hen injected with radioactive $\mathrm{Sr}$ laid eight radioactive eggs. The first egg was boiled for 20 minutes and it was found that some activity was gresent in the white, practically none in the yolk, and the shell was very active. When the hen stopped producings $\$ \mathbf{r}$-radioactive eggs, radioactive Fe was injected and the eggs laid for several days thereafter were found to be radioactive. The activity was exclusively in the yolk. Saline washings of E. coli grown on a medium containing radioactive $\rho$ were radioactive. This activity was most marked in the first washing and was almost negligible in the fourth. The sedimented mass was very radioactive. When a suspension of this mass was injected in a rat the bacteria hovered principally near the point of injection. A radioautograph of a rat injected with $\mathrm{Sr}^{89}$ showed the leg-bone to be more active than the marrow and the skull more active than the brain. The relative distribution of radioactive $\rho$ and $\mathrm{Sx}$ in rats is shown by other radioautographs. (CA)
773

Prusser, C. Ladd, C. W. Hagen, Jr., and W. Grundhauser. THE LETHAL ACTION OF X-RADIATION, STABLE ISOTOPES OF PISSION ELEMENTS, SR ${ }^{80}$, AND (BA - LA) ${ }^{140}$ UPON GOLDFISH. Argonne National Lab. July 1947. 21p. (MODC-1480; ANL-4017)

Solutions of stable barium at 1 ppm killed $50 \%$ of goldfish within 30 days. Cerium was toxie at $10 \mathrm{ppm}$, and bromine, at $1,000 \mathrm{ppm}$. Solutions of strontium and iodine were nontoxic, at least up to $1,000 \mathrm{ppm}$. Single doses of $850 \mathrm{r} \times$ radiation killed $50 \%$ of goldfish within 30 days. Half a group of goldfish receiving $100 \mathrm{x}$ daily were dead at 26 days; half a group at $50 \mathrm{r}$ daily were dead at 33 days; whereas half the controls and those receiving $25 \mathrm{r}$ daily were dead at 40 days. Calculations of radiation dosage show that in solutions containing fission products at 1 $\mu \mathrm{c} / \mathrm{ml}$, the dose due to external radiation is a small fraction in comparison with that due to internal radiation. In solutions of $\mathrm{Sr}^{88}$ at an initial concentration of $0.1 \mu \mathrm{c} / \mathrm{ml}$, deaths were no more frequent than among controls. At an initial concentration of $0.4 \mu \mathrm{c} / \mathrm{ml}$, however, a significant number of deaths occurred. On the basis of measurements of accumulated $\mathrm{Sr}^{\mathrm{B}}$, the median total accumulated dose of internal beta radiation varied from 1,580 to 27,550 rep at daily (24 hour) rates of 50 to 680 rep. Lethal doses for goldish in aquaria in which $\mathrm{Sr}^{28}$ was replenished monthly were similar to those given in a single dosage. This similarity was also observed for lethal doses in single experi ment with $(\mathrm{Ba}-\mathrm{La})^{1 / 0}$. (ADD)

774

Prosser, C. Ladd, William Pervinsek, J. Arnold, G.

Svihla, and P. C. Tompkins. ACCUMULATION AND DISTRIBUTION OF RADIOACTIVE STRONTIUM, BARIUM - LANTHANUM, FISSION MLXTURE AND SODIUM IN GOLDFISH. Univ. of Chicago Metallurgical Lab. Feb. 15, 1945. Decl. Nov. 18, 1946. 40p. (MDDC-496)

Small nun-feeding golditsh accumulated radioactive strontium, barium-lanthanum, fission mixture elements and sodium from solution in which they were immersed. Accumulation was rapid initially and continued at a rate greater than the loss of radioactivity in the rnedium by decay and adsorption. Hence, the ratio of the concentration of active material in the fish to that in the solution inereased with time. After a few days this concentration ratio was usually between 10 and 100. The concentration ratio was independ ent of dose over the range investigated $(0.01 \mu \mathrm{c}$ to 1.0 $\mu \mathrm{c} / \mathrm{ml})$. Calcareous tissues, such as skeleton and scales, accumulated about $75 \%$ of the radio-strontium and radiobarium in the whole fish. Lanthanum and a mixture of cerium, yttrium and zirconium-columbium appeared to be accumulated in visceral tissues, especially the intestine, and to be more actively adsorbed on feces and glass than were the alkaline earths. The comparable distribution, particularly of bone-seekers, on immersion and injection indicated that these elements were taken up through gill and oral membranes and were distributed by the blood. Radio-sodium was present in greater proportion in the gills at six hours than later. Fission mixtures shifted in abundance from soft parts to bony portions of the gills during the first few days. Injection experiments indicated that there was no fecal excretion of strontium but that there was a small amount of fecal excretion of $\mathrm{Ba}^{140}-\mathrm{La}^{1 \mathrm{AO}}$ and of fission mixture. Some fission mixture components appeared to be taken out of the medium, either from feces or water, and precipitated along the intestinal folds. The yolk of eggs in ripe ovaries accumulated some components of fission mixture but not strontium or barium. Young growing or regenerating bone accumulated more radio- 
active material than did older bone; cartilage accumulated none of the bone-seekers. Large mature goldfish aceumulated radioactive material more slowly than did small fish. This may have been in part related to their calcium turnover. Uptake of strontium ${ }^{39}$ and uptake of fission mixture were shown to be greater when the sur rounding pond water was low in calcium (1.8 p.p.m.) than when it was high in calcium ( 36 p.p.m.). Inactive strontium also retarded the uptake of radio-strontium. When the fish were transferred from active to inactive pond water they lost some Ba-La and fission mixture radioactivity from soft tissues during the first few hours but they did not lose the major part of their radioactivity any faster than by decay alone. The rate of loss of radio-strontium appeared to be unaffected by the calcium content of the water. Coldish were unable to desorb any appreciable quantities of fission ele775

ments from suspended clay. (ADD)

Richards, Paul I. and Benjamin A. Rubin. IRRADIATION OF SMALL VOLUMES BY CONTAINED RADIOISOTOPES. Nucleonics 6 , No. $6,42-2(1950)$.

This paper treats the problem of radiation dosage where the average path length of the emitted $\beta$ particle is a significant fraction of the dimensions of the volume being intermally irradiated. A calculation of this type is particularly useful when considering the decay of radioative substances within small organisms. The fraction of ionizing energy escaping from a slab is calculated for lowenergy radiation (nonuniform energy loss in slab) and for very energetic radiation (uniform energy loss) and is plotted as a function of $S / R$, where $S$ is the slab thickness and $\mathrm{R}$ the range. Other special cases are considered, i.e., strips long compared to $R$ with width $b<R$ and $S \& b$; the correction to this for smaller strips; thin circular disk of thickness $\mathrm{S}$ 《 (radius) $\mathrm{r}<\mathrm{R}$; the case where one dimension of the body is larger compared to $R$ but the of her two are $R$ or less; the case where all dimensions are small, but the deviations from sphericity is an elongation rather than a flattening. 11 references. (NSA)

\section{6}

Rossi, Harald H. and R. Hobart ELis. DISTRIBUTED BETA SOURCES IN UNIFORMLY ABSORBING MEDIA.

I. Nucleonics 7, No. 1, 18-25(1950).

Values of dose and flux may be estimated if a belaemitting isotope is uniformly distributed in a limited region of a biological nedium, and if such a "distributed source" has one of a number of simple geometric shapes. In this introduction to the subject, theoretical consideration is given to doses and flux received from point sources, half spaces, slabs, planes, and spheres. The concluding portion of this article, Part II, in Nucleonics (1950) Aug. will be devoted to practical applications of the method. (NSA) 777

Salerno, Paul R., H. L. Friedell, James H. Chrisie, and Marvin Berg. THE SYNERGISTIC LETHAL ACTION OF CERTAIN RADIOISOTOPES IN RATS. Western Reserve Univ. School of Medicine. Oct. 22, 1951. 15p. (NYO1624)

Synergingtic lethal action has been clearly established with a number of internally distributed radioactive isotopes. The synergism appears to be a function of the simultaneous radiation injury of two separate systems. It would appear that the reticulo-endothelial system and the hematopoietic systems are closely related and the effects are much more profound when both are injured, than when the injury is confined to either one. An attempt to clarify the relationship between these two systems will be made by further experiments. It is to be noted also that when two different isotopes are restricted to the same organ system by virtue of their distribution, the effects appear to be due to simple summation. (Authors)

778

Schubert, Jack. ESTIMATING RADIOLLEMENTS IN IXPOSED INDIVDUALS -II. RADLATION DOSAGE AND PERMISSEBLE LEVELS. Nucleonics 8 , No. $3,66-78$ (1951).

The practical side of judging the amounts and fates of radioactive substances that enter a worker's body involves consideration of arbitrary standards. These standards interrelate physiological data on "standard man" and the properties of the radioelements most harmful to him. 38 $7 \%$ references. (NSA)

Wahlberg, T. A METHOD FOR THE DETERMINATION OF THE RADIATION DOSE PRODUCED BY ARTIFICLAL RADIOACTIVE SURSTANCES IN TISSUE. Acta Radiol. 30, 291(1948).

A method is described for the determination of the radiation dose and activity by means of Lonization measurements when radioactive substances are distributed if the body. The relationship of equivalent $r$ and me units expressed in the equation $1 \mathrm{mc} / \mathrm{g}=7.1 \times 10^{-7} \mathrm{~V} \beta$ e.r $/ \mathrm{sec}$, where $V_{\beta}$ is the mean energy of the $\beta$-particle in ev, and e.r. is the equivalent roentgen defined as the energy absorbed per gram of aix irradiated with a radiation quantity of one $r$, can be directly measured and tested by this apparatus. The radiation dose at a point within the body is measured by the ionization of an air-filled cavity around the point. The ionization is in turn defined in terms of electrostatic units/cm. The method of measurement is based on definitions and facts inchuded in the article. Measurements show that the magnitude of the dose, within a margin of error, is the same whether the radioactive substance is distributed in water, soft tissue, or bone tissue. (NSA)

Arsenic

780

Ducoff, Howard. PROGRESS REPORT: PRODUCTION OF ARSENIC 76 AND IN VYTRO METABOLISM OF ARSENIC, P.162-8 of BIOLOGICAL AND MEDTCAL DIVSTONS QUARTERLY REPORT, NOVTHERR 1948 TO FEBRUARY 1949. Argonne National Lab. Feb. 1, 1949. 6p. (ANL-4253(p.162-8))

The rate of entry of $\mathrm{As}^{\mathrm{B}}{ }^{\mathrm{C}}$ as arsenite into rabbit erythrocytes in whole blood, in suspension in plasma, and in suspension in saline was measured at $37^{\circ}$ and at $5^{\circ}$. The concentration of radioarsenic within the cell fluid was found to exceed that in the suspension medium; the effect was more marked at $5^{\circ}$ than at $37^{\circ}$. Decline of activity in $\mathrm{As}^{7}$-tagged rabbit erythrocytes resuspended in plasma and in saline was measured at 37\%. The substitution of arsenate for phosphate in some steps of the glycolytic cycle is discussed as a possible factor influencing uptake of As. (ACD) 781

Ducoff, Howard S., William B. Neal, Robert L. Strabe, Leon O. Jacobson and Austín M. Brues. BLOLOGICAL STUDIES WITH ARSENIC ${ }^{\text {"6 }}$. II. EXCLETION AND TISSUE LOCALIZATION. Proc. Soc. Exptl. Biol. Med. 69, 5ิ4854(1948).

Arsenic excretion was studied in man, rats, and rabbits. Less than $10 \%$ of the excreted arsenic is found in feces in any of these species; rats have by fax the slowest rate of excretion. Data are given for arsenic distribution in various organs in man, the rat, the rabbit, and 2 strains of mouse. The degree of individual variation within each 
species was very great; in contrast $10 \mathrm{man}$ ard to other animals studied, the rat retains most of the injected dose in the blood for a considemble length of time. "The ratio of arsenic concentration in kidney, liver, and splecn of healthy inbred mice was foum to be fairly constant for a given ifme after administration, and this ratio is suggested as a criterion for effects of various types of treatment. Using this ratio as exiterion, it wa found that arsenic distribution is altered by the presence of transplanted tumors. Fastors changing arsenic distribution are dis cussed in selation to effects on levels of sulfhydrylcontaining substaneces. (NSA) 782

Lanz, Henry, Ji., Patricha C. Wallace, and Jostph G. Jamilton. METAEOLISM OY ARSENIC IN LABORATORY ANIMALS USING ARSENIC 74 AS A TRACER. URIV. Calif. (Berkeley) Publ. Pharmacol. 2, 263-82(1949).

The motabolism of As was studied following intramuscu

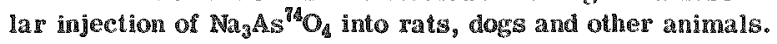
(12. D. B.)

\section{Bariนun-1 - santhanum}

783

Finkle, R. D. R. R. Snyoles, and Walter I Tisielegki. ACUTE RADIOTOXICITY OP $(B A-L A)^{140}$ IN RATS AND MUCIS. PART IV. LETHAL ACTYON AND CLINICAL SYMPTOMS. Univ. of Chicago Metallurgical Lab. [nd] Decl. Aug. 12, 194\%. 61. (MODC-1207; CH-3825)

In the $(\mathrm{Ba}-\mathrm{La})^{140}$ experiments, rats and mice wore injected intraperitoneally with toxic doses of radiobarium. The dose of (Ba-La) necessary to kill $50 \%$ of the injected mice in one month was about 3 to $5 \mu \mathrm{c} / \mathrm{g}$. The dose required to kill half of the injected rats in 90 days was about. 4 uc/g. The mice that died and some of those that gecovered showed outward symptoms of injury. They were lethargic, snd there was usually some loss of hair. Berore death, the eyes became watery and the lids encrusted, and findly, the eyes were sunken and neary closed. The eye symptoms and lethargy noticed in the mice were also apparent in the rats. The anmals were usully nervous and irribuble when disturbed. Lange quantities of inert barium chloride were injected subeutaneously into mice. $31 \mathrm{mila}$ experiment with inert lantharum was performed. The cyperiment designed to test chemical toxicity of barium and to test another route of administration was unsatisfectory. (ADD)

764

Smyder, R. R. George A. Sacher, and J. D. Tores:

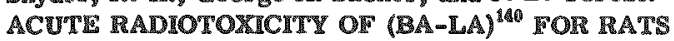
AND MTE. PAPT II. EIFECTS ON WETCHT AND HOOD

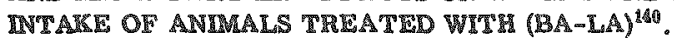
Univ. of Chicago Metallurgical Lab. Tune 24, $194 \%$ Decl. Sept. 15, 1947. 23p. (MDDC-1343; CL-3832)

In the (Ba-La) ${ }^{150}$ experiments mice and rats were injected intrageritoneally with toxic doses of radiobaxiun. The weight curves indicate that hore was definite retadation of growth in mice at $2.25 \mu \mathrm{c} / \mathrm{g}$ and in rats at $1.96 \mu \mathrm{c} / \mathrm{g}$. The threshold for weight loss was, therefore, about half of the midlethal dose for each species. Fhere was a permanent, roughly constant deficit of growth after injection, which increased in magnitude with the size of the dose at at rate of 2.72 . 0.25 per cont per $\mu \mathrm{c} / \mathrm{g}$. Graphs and charts ase included. (ADD)

\section{5}

Snyoder, R. M., W. K. Kisielesini, D. S. Anthony, and R. D. Finkle. ACUTE PADIOTOKICITK OF $(B A-L A)^{140}$ IN IATS AND MICE. PART II. METABOLISM OY (BA-LA) ${ }^{140}$.
Unที. of Chicago Motalurgical Lab. June 1986. 17p. (MDDC-1279; CH-3826)

In these experiments mice and rats were injected int ra peritoneally with toxic doses of $(\mathrm{Ba}-\mathrm{La})^{1 \mathrm{NO}}$. Absorption was pelatively gapid and excetion was conside rable during the first day. Mice usually excreted $25^{\prime \prime}$ of the injerted dose by 12 hours, and rats rocreted a comparable amount by 50 hours. This excretion rate was comparable to thet repoxted by Hamilton who found for rats a lotal excretion. in four days, of about $46 \% \mathrm{Br}^{133}$ and $25 \% \mathrm{Ba}^{1 \mathrm{AO}}$. Wxchotion doclined rapidly so that after six days, mice exceted $0.4 \%$ and rals excreted $0.5 \%$ of the injected dose per day. In rats, a little more than twice as much $3 a^{\text {isto }}$ was excretrd in the feces as in the urine. In mice, about $45 \%$ of the injected dose of $\mathrm{Ba}^{\text {Ho }}$ was aeposited in bone, and in rats, about $60 \%$ of the dose was deposited there. In both spectes. these values represcnt practically all of the retaned $\mathrm{Ba}^{\mathrm{B} 0}$. Absorption and decay curves on soft tissues, however, show that most of the ingerted La ${ }^{140}$ was present in the spleen, liver, and lidney immediately âter ingection. Limited data indicated that pratieally none of the Lat ${ }^{260}$ formed none by $\mathrm{Ba}^{140}$ decay left the bone ${ }^{140}$ the soft tigsues. It can be concluded that the expetion and distribution of radiobarium, and the distribution of radiolanthanum the toxic levels are comparable to those at the traces levels. No significant variations of bariun metabulsm with the size of dose were noted in mice. With rats, however, large: (lethal) doses were retaind more completely by the skeleton than were the lowe doses. It is likely that wis affect of dose represents a "llowding" of the excretion apparatus and, therefore, is dependent on the amount of inactive

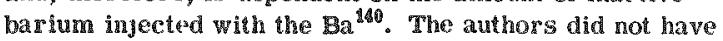
the oportunity to confirm both he observation and expla nation with a larger number of anirnals. (ADD)

\section{Beryllum}

786

Crowley, Josephine $\mathbb{F}$. Joseph G. Hamiltum, and Kenmeth G. Sroth. THE METADOLISM OF CARRIER-IRE E Br" IN THE RAT. J. Biol. Chem. 177, 975-84(1949) (AECD-2854; UCRL $\rightarrow 148)$

Recent studies have shown that the soluble Be compounds aro toxic, and the toxicity is a function of both $\mathrm{B}$ and the anionic part of the molecule. Acute beryllum poisoning manfest itself in at least three organ $3 y$ stems of the body, midzonal necrosis of the liver, necrosis of the distal third of the proximal convoluted fubule of the bidney, and poisoning of the hemopoetie system leading fo anema and leucosytosis. Owing to the necessity of using at least micrograms of material when studies are made will stable isotopes of berylium, it is difficut to measure the normal metabolism of beryllium because of its toxicity. When a radioactive isotope of this element is used the actual num ber of beryllium atoms which ean be fraced is reduced by a factor of at least $10^{\circ}$. For this reason it is possible to ascertain the manere in whe the normal chnimil handles extremely minute amounts of beryllium which are far below the possible lovels of chemical toxicily. The metabolism of boryllium in the at has been investigated with tracer anounts of carrita free Be ${ }^{7}$. About one half microcurie of $\mathrm{Be}^{7}$ in anistonic solution was administered to each rat wian int armuscular ingection. Within 24 hours $40 \%$ of the Be had been absorbed from the injection site and $53 \%$ of the absorted $\mathrm{Be}^{7}$ had been eliminated from the body, mainly in the urine. By the 64th day after injection, a total of $80 \%$ had been absorbed from the injection site and $70 \%$ had been climinated from the body. Of the absorbed Be which re- 
mained in the body, the major portion was taken up by bones. Within 24 hours after injection, the bone had arcumulated $29.4 \%$ of the absorbed Be $^{7}$ and maintained this level to the 64 th day, when the bone contained $26.9 \%$ of the absorbed material. Many of the soft tissurs of the body demonst rated a small initial uptake followed by a ten fold decrease in $\mathrm{Be}^{\mathrm{B}}$ content by the 64th day. The radio-beryllium. content of the spleen and muscle remained relatively consiant throughoul the interval of the experiments. Lest than $0.1 \%$ of the $B e^{8}$ administered orally was absorbed from the intestinal tract. (NSA)

787

Sentt, J. K., W. F. Neuman, and Roberta Allen. We FECT OF" ADDED CARRIER ON THE DISTRIBUTION AND EXCRETION OF SOLUBLE BERYLLUM ?. Biol. Chem. 182, 291-8(1950).

When Be only $\left(9.3 \times 10^{-11} \mathrm{~g} / \mathrm{kg}\right.$ for rats, $4.4,10^{-11} \mathrm{~g} / \mathrm{kg}$ for rabbuts) was injected intravenously, most of the $B e^{8}$ was excreted in the urine and deposited in the bone. When carrier plus $B e^{7}$ was injected, Be was found in the liver, spleen and bone martow also. The clearance of Be from the blood was much faster when Be only was injected than when $B$ e plus $\mathbb{B e}^{7}$ was injected. Significant amounts of $\mathbb{B} e$ were exereted in feres of rats when Be plus Be ${ }^{7}$ was injected. (CA)

\section{Calcium}

788

Copp, D. Haxold, Juseph G. Hamilon, D. C. Jones, D. M. Thumson, and C. Cramer. THE EFFECT OF ACE AND LOW PHOSPHORUS RICKETS ON CALCIPICATION AND THE DEPOSITION OF CERTAIN RADIOACTIVE METALS IN BONE. Radration Lab., Univ. of Calif. Sept. 1951. 31p. (UCRL, -1464)

The effect of age and low-P rickets on the retention of

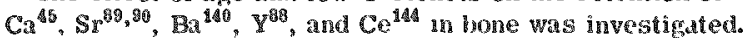
The radiouctive metals were injected into young growing rats in which there was active growth of new bone, young rats with low-P rickets in which new osteold matrix was forming but deposition of bone salt did not take place, and mature adult rats in whe h bone growth had practically cersed. Urine and fecess were collected separately and the animals were sacrificed ater 8 days. Crereta and tissues were analyzed for radioisotope and radiographs prepared from thin undecalcified sections of bone. Data indicate the metabolism of $\mathrm{Ca}_{\mathrm{S}} \mathrm{SP}$, and Bat was markedly alffected by age and low-P rickets, while metabolism of other heavy metals such as $Y$ and $C e$ was not affected. Radioautographs of rachitic femur show that $\mathrm{Sr}^{29}$, 80 and $\mathrm{Ca}^{35}$ werce deposited only in the presence of bone salt, while $\mathrm{x}^{93}$ and Ce $\mathrm{e}^{\mathrm{AS}}$ and other heavy metals were also laid down in uncaleified osteoid matrix. Kinetie atudies of skeletal uptake and excretion of madiocalcium and radiostrontium showed considerable mineral exchange, even in adult rats. Skeletal uplake was more rapid and greater in growing animals, and the radioactive isotopes remained fixed in the new bone salt. In rachitic rats the initial uptake by bone was rapud, but $\mathrm{Ca}^{65}$ or $\mathrm{Sr}^{83}$, 20 was then rapidly lost from the skeleton and exereted in urine. When mormal animals were injected with $\mathrm{Ca}^{46}$ or $\mathrm{Sr}^{30,90}$, and then fed a diet low in $\mathrm{P}$, the excre lion of the radioactive isotopes in urine was increased, and removal from the skeleton was accelerated. (NSA)

789

Dudley, R. A. and B. M. Dubyns. THE USE OF AUTOTADIOGRAPHS IN TIRE OUANTITATIVE DETLRMINATION OF RADLATON DOSAGES IROM CA 45 IN BONE. Sciance 109, 327-8(1943).
An autoradiographic technique capable of quantitatively measuring local dosage rates in tissue, currently being applied to the study of radioactive $\mathrm{Ca}^{\mathrm{B}}$ dosages in bone, if described. The technique is similar to that deseribed by Axel rod and Tamilton (Am. J. Path., 23: 389(1947)) in their radionetive sulfur and arsenic studies of nustard gas and lewisite concentration in the shin and eye lissues. It consists of making an autoradiograph of the tissue being studied, producing calibration exposures from radioactive sources of known activity on the same film, developing the tissue and calibration exposures simulaneously, and comparing their blackening mic rophotometrically. The application of the technique to studies on the $\mathrm{Ca}^{\mathrm{BS}}$ bone-uptake in the dog is discussed and autoradiographs and a microphotometer regord obtained in such experiments are given. It is noted that, if the same dilution that exists in dogs also exists in humans, the maximum local dowage rate would be a permissible 0.034 reps/day. (NSA) 780

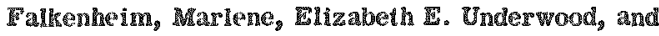
Harold C. Prodge. CALCIUM EXCHANGE AS THY MECHANSM FOR ADSORPTION OF THE RADIOACTIVE ISOTOPE BY BONE: J. Biol. Chem. 188, 805-17(1951) (ARCU-95: UR-53)

The exchangeable bone calcium represents at the most less than $20 \%$ of the total bone calcium; this fraction is of the same order of magnitude as that previously reported for the exchangeable bone phosphate. This reaction is reversible to the same "quasi equilibrium" point. (Author's abstract)

791

L'Pleurew, Maurice $V_{0}$, Wilbur R. Tweedy, and Rlinor

M. ZOR. EXCRETION OF CALCIUM ${ }^{35}$ RI NORMAL

RATS. Proc. Soc. Expll. Biol. Med. 71, 729-32(1949).

Graded amounts of labeled Ca $(0.07-14.0 \mathrm{mg})$ were in jected subcutaneously and the relative proportions excreted 92

in the wrine and feces were determined. (CA)

Liente, R. I., George Cullen, and Wallace D. Armstrong. STUDIES ON THE EXCRETION AND DISTIRIDUTION OP RADIOACTIE CALCIUM. Trans. Con. Metabolic Interrelat., 1st Cone. 73-6(1949).

The fate of $\mathrm{Ca}^{35}$ was followed during and after the contimuous int raperitoneal injection of $\mathrm{Ca}^{\mathrm{SE}} \mathrm{Cl}_{2}$ in rats. Absorption from the peritoneal cavity was incomplete. \$ignificant amounts of $\mathrm{Ca}^{\mathrm{A}}{ }^{\mathrm{f}}$ was excreted in the feces. Of the tissues, the gleleton retained by far the largest amoun of $\mathrm{Ca}^{55}$. (CA)

793

Norris, Willam P. METABOLISM OF BONE-SEERTE

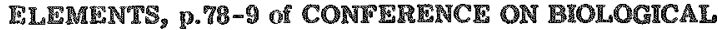
APPLICATIONS OF NUCLEAR PHYSICS. Brookhaven National Lab. 1948. 1p。 (BNL-C-4(p.78-9))

Discussion of the metabolism of bone-seeking elementa: $\mathrm{Ca}^{\mathrm{A5}}, \mathrm{Sr}^{39}$, or Ra in rats; intravenous injections. (S.V.S.) 794

Nor ris, William P. and Walter Kisieleski. THE METABOLISM OF INTTAVENOUSLY INJECTED CALCIUM p.134-9 of MBDICAL AND BIOLOGICA D DVHSIONS, QUARTEILI IEE PORT, NOVEMBER, DECEMBRR, TAN UARY, 1948-1949. Argome National Lab. Feb. 1, 1949. 5p. (ANL-4253(p.134-OB))

As part of a study of the metabolism of the alkaline earths, rats were injected int ravenously with calcium 45 (1.5 mg. Ca and 45 uc calcium 45 per ral) and its metakolism was studied. The animals were sachificed at intervals of 1,5 , and 20 minutes at $1,6,24$, and 72 hours, and at 10 , 30, 70,124 , and 200 days after ingection. Frager samples 
were collected and each rat was analyzed in 15 parts that included several of the soft tissues, bone, blood, pelt, muscle, and residual carcass. The metabolism of intravenously-injected calcium was found to proceed in a fashion that was qualitatively similar to that geported for $\mathrm{Sr}$ and Ra. The bones were observed to take Ca from the blood at a rapid rate with the retention at 10 days being $90 \%$ of the injected dose and the maximum content being reached at about 400 minutes. The biological half-time in bone was approwimated to be in the order of 50 to 500 years. Ca in the soft tissues was found to be in rapid equilibrium with blood Ca. Within six hours after injection the specific activity (per cent injected dose per mg. $\mathrm{Ca}^{40}$ ) in the blood, soft tissues (kidneys, lungs, liver, gastrointestinal tract, testes, muscle, pelt), urine, and feces became almost identical and continued so for the duration of the experiment. (ACD)

795

Singer, Leon and W. D. Armstrong. RETENTION AND TURNOVER OF RADIOCALCIUM BY THE SKELETON OF LARGE RATS. Proc. Soc. Exptl. Biol. Med. 76, 229-33 (1951).

The Ca specific activities of the femoral epiphyses, femoral diaphyses, humeri, lumbar vertebrae, and the remainder of the skeleton of large rats differ soon after administration of $\mathrm{Ca}^{45}$ but decrease to a common value after 52 days, following which the specific activities decline at equal but much reduced rates. The retained fraction of the dose of $\mathrm{Ca}^{45}$ declines to $42-45 \%$ on the 52nd day, after which excretion is slow. These results indicate the existence in large rats of two kinds of skeletal Ca which differ markedly in their rate of turnover and in the length of time over which they fix injected Ca. Some data on the $\mathrm{Ca}^{45}$ activity of the urine and teeth are also given. (CA)

\section{Carbon}

\section{6}

Armstrong, Wallace D. and S. H. Zbarsky. EXCRETION AND DISTRIBUTION OF CARBON 14 DURING AND FOLLOWING CONTINUOUS INTRAPERITONEAL IN JECTION OI CARBON 14 AS SODIUM CARBONATE.

Trans. Conf. Metabolic Interrelat. 1st Conf. 67-71(1949).

The distribution of carbon 14 in continuous injection is similar to that after a single intraperitoneal injection, although a somewhat larger fraction is taken up by the tissues. The device used for continuous injection of rats is described briefly. (CA)

\section{7}

Bloom, William. DEPOSTTION OF $C^{14}$ IN THE METAPHYSIS OF LONG BONES OF YOUNG RATS. Anat. Record $103,425(1949)$.

The following is an abstract and is reproduced here in its entirety.

In 1947 , Bloom, Curtis and Me Lean reported that in rats examined three days to 4 months after injections of $\mathrm{NaHC}^{1 \mathrm{~A}} \mathrm{O}_{3}$ little or none of the isotope was demonst rable in autoradiographs of the metaphysis of long bones. The study of more animals and earlier intervals has shown that the above statement is true but that it is not correct for the first two days after injection. Seven newly weaned rats were examined within two days after intraperitoneal injection of $2 \mu \mathrm{c}$ of $\mathrm{C}^{14}$ per gram. Autoradiographs show that the isotope is deposited immediately in large amounts in the metaphysis. However, the relatively weak beta particles emitted apparently do not interfere with the growth of the bone, for by 48 hours the "old" metaphysis with $\mathrm{C}^{18}$ has been almost entirely resorbed. The "new" metaphysis which has formed is devoid of radioactivity except for the tips of the trabeculae of the secondary spongiosa. This is in contrast to those bone-seeking isotopes emitting alpha or strong beta particles which, in the dosages we used 798 previously, interfere with growth at the metaphysis.

Bloom, W., H. I. Curtis, and F. C. MeLean. A NOTE ON THE DEPOSTTION OF $C^{14}$ IN BONE. Seience 105, 45 (MDDC-516)

The autoradiographs of the bones of rats injected with carbon 14 show a markedly different picture from those of the strontium 89 rats. Those from rats killed three days after injection of carbon 14 show the shat of the boness as black lines. The bone in the epiphysis is a faint grey, while the metaphysis of the growing end of the bone is negative or practically so, leaving a gap of about $2 \mathrm{~mm}$ between epiphysis and diaphysis. The non-growng end of the bone is completely outlined in grey. The marrow cavity is so pale that it is probably negative. The autoradiographs of the $2,4,8$, and 16 week specimens show essentially the same picture as those after three days, except that the bones have grown in length and width. With the growth in length, the unblackened zone at the metaphysis increased to $3.4 \mathrm{~mm}$ at two weeks, $4 \mathrm{~mm}$ at four weeks, $10 \mathrm{~mm}$ at eight weeks and $12-17 \mathrm{~mm}$ at 16 weeks. Since the blackened lines representing the lateral extent of the diaphysis at the time of injection are still present after 16 weeks, it would seem that the marrow cavity did not increase much in diameter at the site of deposition of radioactive carbon. However, new bone was deposited externally so that the bone as a whole increased in thickness. Sections of the liver and kidney gave fairly intense autoradiographs at the 3-day and 2 -week stages, but were negative after the longer intervals. Since the films were exposed for the same length of time. it would appear that there was approximately as much carbon 14 in the bones after 16 weeks as after three days, while the re was a great decrease in the carbon 14 content of the soft tissues. From the autoradiographs of bones of these few rats it is concluded that carbon 14 injected as carbonate appears primarily in those areas occupled by pre-existing bone. It does not appear in appreciable quantities in the areas of most recently deposited bone salt. This observation demands further study on the carbon metabolism of bone. Since the carbon 14 content of the bones did not decrease apprechably in four months, it is believed that the health hazards involved in working with this isotope must be studied. Particular attention should be 799 paid to the possible development of bone tumors. (ADD)

Brues, Austin M. and Agnes N. Stroud. RETENTION OF $C^{1 / 3}$ ADMINISTERED AS BICARBONATE TO ADULT AND EMBRYONIC MICE. Argonne National Lab. Nov. 1950. 19p. (AECU-1091; UAC-326)

Mice were xposed to $\mathrm{Na}_{2} \mathrm{C}^{14} \mathrm{O}_{3}$ by injection, followed by a 10-hr period of rebreathing their expired air. Obsfrva tions have been made on tissue $\mathbb{C}^{16}$ concentration to 10 months after exposure in adults and 20 months in mice exposed as embryos. Eventual loss rates in xposed cmbryos were parallel to those in exposed adults. The retention in various organs follows roughly a logarithmic function with concentration decreasing as the square root of time. This relationship holds, within a factor of 2 , from 1 day to 10 months after exposure. It is possible that this curve may represent a series of exponential decay rates, and certainly no conclusions can be drawn as to the fate of $\mathrm{C}^{\mathrm{A}}$ beyond the period of investigation. Bone carbonate $\mathrm{C}^{18}$ disappears somewhat more slowly than organic $\mathrm{C}^{14}$. Consideration is given to the further data required to determine 
Safe human exposure values to $\mathrm{C}^{14} \mathrm{O}_{2}$ and $\mathrm{Na}_{2} \mathrm{C}^{14} \mathrm{O}_{3}$. It is obvious that present values are much more conservative 800

than necessary. 12 references. (NSA)

Buchanan, Donald L. ELIMINATION OF INTRAVENOUS CARBON $^{14}$ BICARBONATE BY MAN. J. Clin. Invest. 30, 630-1(1951) (AECU-1262; UAC-359)

A body of data has been obtained concerning the excretion rates and tissue retention of inorganic $\mathbb{C}^{1 / 6}$ administered to rats and mice. The present study compares the human excretion of injected $\mathrm{NaHIC}^{14} \mathrm{O}_{3}$, to rates observed in smaller animals in order to provide a basis for the computation of permissible human doses of this isotopic compound. In this work $10 \mu \mathrm{e}$ of material was injected intravenousiy into healthy human volunteers and all expired $\mathrm{CO}_{2}$ was collected in fractions during the following $3 \mathrm{hr}$. With the subjert at rest, approximately $75 \%$ of the radioactivity is exhaled as $\mathrm{CO}_{2}$ durintg the 1 st hour; $90 \%$ is gone in $2 \mathrm{hr}$ : and $95 \%$ has been blown off at the end of the $3 \mathrm{rd} \mathrm{hr}$. Within the error of the methods used the rate of isotope excretion by different-sized animals is proportional to the metabolic rate per unit mass of animal. When published datat of others are included, this relation seems valid for animals as large as the cow (Kleiber) and as small as the mouse. On this basis the extrapolation to man of information obtained in long-term experiments with rats and mice indicates that an untravenous injection of 20 to $40 \mathrm{me}$ of $\mathrm{NaHC}^{14} \mathrm{O}_{3}$ or the inhalation of a comparable amount of $\mathrm{C}^{16} \mathrm{O}_{2}$ would be required to give a $70 \mathrm{kgg}$ man a total radiation dose of 1 roentgen equalent over his entire life span. 801 (NSA)

Buchanan, Donald L. UPTAKE AND RETENTION OF FIXED CARBON IN ADULT MICE. J. Gen. Physiol. 34, $737-59(1951)$.

Mice were continuously exposed to air containing $\mathrm{C}^{18} \mathrm{O}_{2}$. The specifir radioactivities of urea $\mathrm{C}$, total fecal $\mathrm{C}$, and numerous components 3 of tissue $C$ were compared as at function of the duration of exposure with the radioactivity of the air $\mathrm{CO}_{2}$. The data indicate that the total $\mathrm{CO}_{2}$ fixed from the air is proportional to its concentration in the air. The rates of uptake at the beginmyg of the exposure and the rates of loss at the termination of the exposure differed strikingly among the tissues studied. However, the ultimate ratio of fixed air $\cdots \mathrm{CO}_{2} \mathrm{C}$ to total $\mathrm{C}$ in these tissues seemed to be approaching limiting values which would not vary by more than a factor of 3 from one another. It appears that of the total organic $C$ in an adult mouse, approximately $0.01^{\prime \prime}$ may orignate directly from the $\mathrm{CO}_{2}$ of the air when the anmal respires in air having a $\mathrm{CO}_{2}$ concentration of $0.03^{\prime \prime}$, and that $1.8^{\prime \prime}$ or more of the total $\mathrm{C}$ may originate from $\mathrm{CO}_{2}$ within the animal. Data are presented which indicate the life span of the mouse erytlurocyte to be 49 days. The cont inuous permissible level for mice is given as $31 \mu \mathrm{c} / \mathrm{m}^{3}$. 28 references. (NSA)

802

Govaerts, J. RATE OF ELIMINATION OF $C^{14}$ ADMINISTERED AS BAC ${ }^{16} \mathrm{O}_{3}$. Seience 111, 467-9(1950).

After a brief review of previous work, experiments are described in which nine mice received int ravenous injections of a fine suspension of $\mathrm{BaC}^{14} \mathrm{O}_{3}$ in isotonie glucose in order to deposit the barium carbonate in the lungs in a time determined by preliminary experiments. The animals were placed in metabolic cages for the duration of the experiments. Lxpirated $\mathrm{CO}_{2}$, urine, and feces were collected and analyzed for counts. After the animals were sacrificed, the lung,s, kidney, liver, and spleen were removed and prepared for assay using the Van Slyke-Foche method. The data are presented graphically and discussed; the author concludes that most of the $C^{16}$ absorbed by the organism as barium carbonate is expired by the lung as $\mathrm{C}^{18} \mathrm{O}_{2}$, and that the dangers to those who work with $\mathrm{C}^{18}$ are not so great as expected. (NSA)

903

Skipper, Howard E., Martelia J. Bell, and Juanita $B$. Chapman. THE HAZARD INVOLVED IN THE USE OF CARBON 14. II. THE EFFECT OF A SINGLE DOSE OF CARBON 14 LABELED SODIUM BICARBONATE ON THE PATTERN OF DEATHS FROM SPONTANEOUS LEUKEMA IN AKM MICE. Cancer Research 10, 362-3(1950).

As a portion of a study of the danger involved in research with $\mathrm{C}^{14}$, the effect was studied of this isotope, as $\mathrm{C}^{14} \mathrm{O}_{2}$, on a neoplastic disease (leukemia) known to be influenced by radiation. Injection of $\mathrm{NaHC}^{14} \mathrm{O}_{3}$ at the rate of $18 \mu \mathrm{c} / \mathrm{mouse}$ had no fect on the pattern of deaths from spontaneous leukemit in a leukemia-susceptible strain of mice. (CA) 804

Skapper, Howard E., Constance Nolan, and Linda Simpson. STUDIES OF TRPE HAZARD INVOLVED IN USE OF C ${ }^{18}$; III. LONG TERM RETENTION IN BONE. J. Biol. Chem. $189,159-66(1951)$.

As judged from autoradiographs made from long bones of mice injected with $\mathrm{NaHC}^{21} \mathrm{O}_{3}$, the rate of turnover of carbonate carbon by various areas in the bone cross section may be listed as follows: marrow and epiphyseal cartilage metaphysis of the growing end - epiphysis and the nongrowing end bone shaft (diaphysis). After a period of 4 months the greatest part of the injected $C^{1 / 4}$ can be found in the bone. The approximate per cent of the injected dose retained in the skeleton was 0.25 at the end of $24 \mathrm{hr}, 0.03$ at the end of 1 month, 0.02 at the end of 6 months, and 0.01 at the end of $1 \mathrm{yr}$. The average skeletal radiation received by mice injected wilh $18 \mu \mathrm{c}$ of $\mathrm{NaKC}^{14} \mathrm{O}_{3}$ was calculated to be approximately $0.07 \mathrm{rep} / \mathrm{day}$ for the perod 0 to $24 \mathrm{hr}$, 0.008 between 2 and 4 weeks, about 0.003 from 5 to 6 months, and 0.002 for the interval 10.8 to 12.7 months. The "active" volume of the bone shaft (as estimated from autoradiographs) was receiving approximately $0.16 \mathrm{rep} / \mathrm{day}$ from 1 to 2 weeks after injection of $18 \mu \mathrm{C}$ of $\mathrm{NaHC}^{18} \mathrm{O}_{3}$ and about $0.04 \mathrm{rep}^{\prime}$ day at from 5 to 6 months. A single injection of $18 \mu \mathrm{c} \mathrm{NaHC}{ }^{14} \mathrm{O}_{3}$ (a 50 -me man-equivalent) has resulted in radiation to certain bone-shaft cells ("active" volume) of mice which is greater than the maximum allowable radiation to tissue for $\operatorname{man}(0.04 \mathrm{rep} / \mathrm{day})$. 5 figures. (NSA)

805

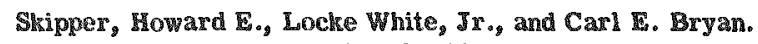
BODY LETENTION OF CARBON 14 FROM LABELED SODIUM BICARBONATE. Science 110, 306-7(1949).

Twelve mice were injected int raperitoneally wilh a solution containing $2.5 \mathrm{mg}$ of $\mathrm{NaHC}^{14} \mathrm{O}_{2}$ and a total activity of $18 \mu \mathrm{c}$. The expired $\mathrm{CO}_{2}$ from all animals was collected over varyng intervals and two mice were sacrificed for organ and tissue analysíg at $24 \mathrm{hr}, 48 \mathrm{hr}, 1,2,4$, and $12 \mathrm{w}$ week periods. Procedures used in determining expired $\mathrm{CO}_{2}$ and excreta have been reported. No outstanding selective accumulation of $\mathrm{C}^{14}$ from a gross anatomical standpoint was observed. The rate of uptake and loss by the jejunem was sugnficantly greater than for other tissues studied. Similar toses would be considerably lower than human plerance limits. Studies on long-term $\mathrm{C}^{14}$ retention from insoluble carbonates and organic substance are necessary before any final $C^{14}$ hazard conclusions can be drawn. Data are given. (CA)

806

Skipper, Howard E., Locke White, Jr., and Carl E. Bryan. STUDIES ON THE HAZARD INVOLVED IN USE OF $\mathrm{C}^{\mathrm{AS}}$. 


\section{REMTENTION OF CARBON FROM LABELED SODIUM} BICARBONATE. J. BIOl. Chem. 180, 1187-95(1949).

Following injn. of $\mathrm{NaHC}^{1 \mathrm{O}}$ in mice, greater than $00 \%$ of the total activity injected was lost through the gespixatory route in one houx. After one day most of the blood $C^{84}$ was cixed in a noncarbonate form. No profownd localization of $\mathrm{C}^{14}$ was observed although uptake and turnover were more rapid in the jejunum. Exptw. with pregnant nice indicated that the embryos were able to fix $\mathbb{C}^{14}$ more rapidly than the parene tissue. Data are given on the retention of $\mathrm{C}^{4}$ in 9 tissues, bone, and the lotal body of mice at various intervals after intraperiton, injns. (BA)

\section{Cerisum}

807

Anthony, D. S. and X. A. Lathep. ACUTE RADTOTOKI-

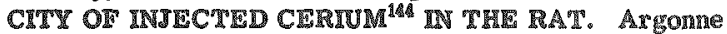
National Lab. Jwne 26, 1947. Deel. Sept. 19, 194\%. 25p. (MDDC-1326; CH-3824)

Groups of 10 or more sats were given doses of $10,7,5$, 3, and $2 \mu c$ of $(\mathrm{Ce}-\mathrm{Pr})^{14}$ per gram of body weight by intravenous injection. The metrabolism of the Co was studied and the efiect of this radioactive material on the general clnical condition, survival time, and weight was observed. At no time was the exeretion of corium rapid, and in fact, it required approximately 35 days for the rats to excrete half of the injected dose. The rate of excretion of $\mathrm{Ce}^{144}$ in the urine fell continuously with time, while the rate of excretion in the leces rose to a masimum at about one week and then dropped with time. The ratio of the amount excreted in the urine to that in the feces fell continuously with time. The early retention of $\mathrm{Ce}^{144}$ was largely in the liver but this dropped rapidiy with time. Apparently, some of the radiocerium released by the liver was talken up by the skeleton since the activity of the bones rose to a maximum during the period when the liver was rapidly losing its cerium. As the liver became exhausted of $\mathrm{Ce}$, the activity in the skeleton dropped with time. The rats that died within the first 30 to, 40 days showed an almost conplete lack of gross lesions which are usually noted in radiotoxicity atudies. In 4 of the animals that died between 70 and 120 days aiter injection, there was abundant visible evidence of radiation injury to the liver. In a few animals dying at still later times, osteogenic sarcomas were found. The 30-day LD-50 for intravenously injected Ce $e^{\text {lin }}$ in the rat was found to be about $\mu c / \mathrm{g}$ of body weight. By 120 days, the LD-50 was down to between 2 and $3 \mu \mathrm{c} / \mathrm{g}$. At all dose levils there was a dimmution of the growth rate as measured by increase in body weight. (ADD)

\section{8}

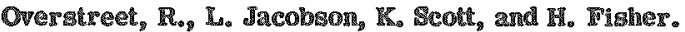

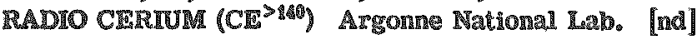
15. (MDDC-1142-18; CH-554; $A-678)$

Preparmation without Carrier - Radio cerium has been prepared from a uranium fission mixture without the use of Ce carrier. "Ihorium was used as a carrier and the thorium and cerium finally geparated from one another by iodate preelpitation. The identily of the purifled radio cerium without carriar was demongtrated by the following procem dure: (1) Chemical Identiflcation with the use of iner Ce as 2 carrter upon an aliquot fraction of the final purified solution. (2) Confirmation of the presence of short-lived gragewdynum daughter which was described by Spedding. (3) Beta-ray absorption curves were cound to be comparable to those publighed in various reports of the chemistry group. Traces Studies-A large group of animals have been injected with Ce $^{\text {sto }}$ (including some unknown activities) by intraperitoneal and intramuscular injection whout carrier. A sufficient number have been injerted so that they may be sacrificed in groups of three extending from one day period to the 64 day period. In addition some animals have received $C e^{>140}$ by stomach tube. Preliminary results, which include, the one, four, eight, and sixteen day period, reveal that $\mathrm{Ce}^{\mathrm{B}} \mathrm{A}$ is handled in a manner very similar to that observed with La ${ }^{160}$. No significant absorpthon oceurs by way of the digestive tract. Considerable retention by the lungs was noted when the radio cerium was administered directly into this organ. A full report will follow. (ADD)

809

Seibert, R. C. and R. Abrams. ACUTE TOXCITY OF INPALED 275-DAY CE ${ }^{145}$. Argonne National Lab. July 25, 1946. Decl. Sent. 1946. 28p. (MDDC-329; CH-3539)

In an attempt to estimate the acute toxicity of insoluble, radioactive malcrial in the lungs, rats were exposed to smokes containing 275 day $\mathrm{Ce}^{144}$. Groups of $200-g$ rats received ihe collowing doses: $3.2,14,39,50$, and 200 $\mu \mathrm{c} / \mathrm{mat}$. Weight loss was significant only in those exposed to 200 uc. These had a median survival time of 39 days and were all dead within 120 days. The 39 ue rats had a medran survival time of 282 days, while $90 \%$ of the $14 \mu \mathrm{c}$ animals were stull alive after 300 days. As has been demonstrated in tracer experiments, absorption from the lungs was slow, and liver and skeleton were the primary sites of deposition of the absorbed material. It was not possible to show any definite effect of dose on distribution. Radiation doses to the various lissues were calculated, but, in general, it was not possible to correlate death with the dose to any aingle tissue. Preliminary histological examination revealed sume tendency for the bronchial epithelium to undergo a squamous proliferation. (ADD)

\section{Cobalt}

810

Braude, R. TRE DSTRIBUTION OF RADIOACTVE

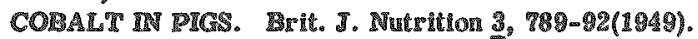

Two female pigs were fed diets containing a mixture of $\mathrm{Co}^{56}, \mathrm{Co}^{58}$, and $\mathrm{Co}^{58}$, for 43 days. The relative amounts of yadionctive Co found (values tabulated) in most organs were similar to those found by Comar and Davis, except that the values for the pancreas, liver, and bile were lower, 11 11 and those for cartilage and long bones were higher. (CA)

Comar, C. I. and George R. Davis. COBALT MPIBOLISM STUDIES. IV. THSEUR DISTRIBUTONO OT RADIOACTVWE CORALT ADMTMISTERED TO IRABBITS, SWINE, AND YOUNG CALVES. J. Biol. Chem. 170, $377-89(1947)$.

The fissue distribution of radioactive $C O$ when given either by vein or oraly was determined and the concentrations in most tissues were tabulated for rabbits, swine, and young calves. No species difference of Co metabolism or distribution was noted. Toung calves absorbed more from the intestimal traet than did older animals but distribution was the same. About $95 \%$ of intravenous Co was removed from the blood in a few minutes, that which remained being almost entil rely in the plasma. About $65 \%$ is ultimately excreted in the urine and about $30 \%$ in the feces. After 17 hours the blood contained $0.035 \gamma$ and the liver $3.56 \% / 100 \mathrm{~g}$. The Co roneentration in the pancreas was about $4 / 4$ that in the liver and fwice that in the spleen. The concentration in red bone marrow was about $1 / 2$ that in the spleen and 3 times that of white bone maxrow. (CA) 
812

Conar, C. L. and George R. Ddvis. COBALT METABOLISM STUDIES. III. EXCRETION AND TISSUE DISTIE BUTION OR RADIOACTIVE COBALT' AS ADMLNISTERED TO CATTLE. Arch. Biochem. 12, 257-66(1947).

A dipping type of Ceiger-Muller counter was used for the determmation of radioactive $\mathrm{Co}$ in cattle tissue and $\mathrm{ex}-$ creta. Alpprox. 18\% of the orally administered Co appeared in the feces, but only traces in the urine. When Co was injected, about $62 \%$, appeared in the urine and about $33 \%$ in the feces. Significant quantities of $\mathrm{Co}$ were found in the urine $40 \mathrm{~min}$. after injection. The estrmated amount of Co eliminated by daily bilisry secretion was 5-15\% of the injected $\mathrm{CO}_{\text {. }} \mathrm{Co}$ injected into animals which were defictent in Co was distributed in practically every tissue and organ of the animal with the higher concentrations of $\mathrm{Co}$ in the glandular tissues, particularly the adrenals, thyrold, liver, thymus, intestinal lymph glands, and the pancreas. Co accumulation in musele tissue was small, and there was no particular accumulation in the spleen or red bone marrow. Significant quantities of Co are transrifted from the dam to the retus. (CA)

813

Comax, C. L., George R. Dawis, and Ruth IF. Taylor. COBALT METABOLISM STUDIES. 1. RADIOACTIVI COBALT PROCEDURES WITH RATS AND CATRLIR. Arch. Biochem. 9, 149-58(1946).

A procedure is described for the determination of $\mathrm{Co}$ in metabolism studies. Radioative $C o$ is administered orally or intraperitrneally to the experimental animal, and the Co in the $\mathrm{YCl}$ solution of the ash from the lissue being exam ined is recovered by an clectroplating meration in an allaline solution (6 $\times 10^{-10} \mathrm{~g}$ of Co can be detected). It was concluded from the results obtained with the piebald rat that the requirement of the rat for $\mathrm{CO}$, if there is such, must be very amall. With the rat, only the liver showed a small accumulation of $\mathrm{Co}$, but it was not retained. Eighty per cent of $\mathrm{Co}$ is excreted in the feces and $10 \%$ in the urine in $24 \mathrm{hrs}$. Only $0.25 \%$ of the orally administered Co was retained by Co-deficlent cattle, but $5 \%$ of the intravenously administered $\mathrm{Co}$ was retained with the bulk of the Co being retained in the adrenal glands. A agniflcant amount of the 814. injected $C o$ was found in the abonasum contents. (CA)

Comar, C. L., George K. Davis, Ruth F. Taylor, C. P. Huffman, and Ray E. Ely. COBALT METABOLISM STUDIES. II. PARTITION OF IRADIOACTIV COBALT BY A RUMEN FISTULA COW. J. Nutrition 32, 61-8(19A6).

When $174 \gamma$ labeled $C o$ was injected into the jugular vein of a rumen fistula cow about $6 \%$ of the dose was present in the blood after several hours with the value falling to $10 \%$ less after 10-15 days. None was found in the rumen con tents, about $7 \%$ appeared in the feces, and about $65 \%$ was found in the urine with large amounts being rapidly elimi nated by this path. Very small amounts were found in the milk and saliva. When $174 \gamma$ labeled Co was introduced directy into the rumen, none was atected in the blood. About $82 \%$ of the dose was present in the rumen contents after 4 hrs with the amount decreasing regularly to less than $1 \%$ after 7 days. Over $65 \%$ was accounted lor in the feces and only extremely small anounts in the urine. No Co was detected in the milk or saliva. (CA)

815

Culbertson, W. J., Audrey A. Free, and Doreen M. Thornton. DISTRIBUTYON OF RADIOACTVE COBALT IN THE RAT. Brit. J. Nutrition $4,42-8(1950)$.

When a diet containing $0.01-0.06,0, \mathrm{~g}_{0} \mathrm{~m} . \mathrm{Co}^{60} \mathrm{Was}$ fed to pats for 14 days $5-7 \%$ was relained. The concentrations of
$\mathrm{Co}^{60}$ in the organs of the rats maintained on low $\mathrm{Co}^{60}$ diets for 14-90 days are given. The highest concentrations were found in the liver, kidneys, spleen, and pancreas. (CA)

\section{Turopium}

816

Van Cleave, C. D. A RADIOAUTOGRAPHIC STUDY OF THE LOCALIZATION OF RADIOACTTVE IUROPIUM WN CERTAIN SOFT TISSUES OT TIIE RAT. Anat. Record 103, 514- $15(1949)$.

The rare earth curopium is one of the many fission products whose metabolism is being studied under the direction of Dr. J. G. Hamilton at Crocker Radiation Laboratory, University of Callorna, where the work here reported was done in collaboration with $\mathrm{Dr}$. Dorothy Heller. One series of rats was given an intravenous injection of 50 p of europium per animal; another series, the same amount intramuscularly. The animals were sacrificed after 1,4 , and 16 days and samples of liver, kidney and spleen fined in alcohol and prepared as usual for parafin sectioning. Radioautographs were prepared using s-ray film; the tissue sections were later stained. Camera lucida drawings of the stained section, mag. $30 x$, were made; the radioautograph substituted and orlented to conform with the landmarks of the original tracing; areas of activity indicated in the autograph were marked in the tracings. PreIiminary examination of the material seems consistent with the interpretation that following intravenous injection, radioeuropium is rapidly removed from the blood by the reticulo-endothelial system and at the same time excreted by the kidneys. Activity in the liver is strongest in central and hepatie collecting veins; absent in portal canal struclures. Pick-up from sites of intramuscular injections is too scanty to produce autographs in spleen and kidney but not in liver. Activity in bone was not investigated.

\section{Fission Producti and Heavy Metals}

\section{7}

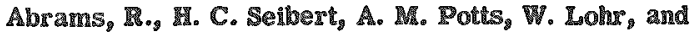
5. POStel. METABOLISM OF INUALDD TISSON PRODUCT Am ROSOLS. Univ. of Chicago Metalurgical Lab. Apr. 16, 1966. Decl. Aug. 28, 1946. 114p. (MUDC-248: CHA3485)

This paper deals with the watabolism, in rats, of airborne issaton products. Four different isotopes were used, selected for their long lives and as representatives of the various chemical specles occurring in fission. There were $\mathrm{Sr}^{29}$ (55 days), $\mathrm{Zr}^{25}$ (65 days), $\mathrm{Y}^{21}$ (57 days), and $\mathrm{Ce}^{144}$ (275 days). Aerosols of these materials were produced by atomizing agueons solutsons and by burning dried realdues in a carbon arc. Strontium differs from the other elements investigated in the ease with which it is absorbed from the lungs. Over $50 \%$ left the lungs within a matter of minuter, and $95 \%$ was gone within one hour. Or the $\mathrm{Sr}^{20}$ originally in the lung, $60 \%$ was degosited in the skeleton in less than four hours, and after 12 hours the total radiation dose to the skeleton exceeded that to the lungs. These results indicated that Inhalation was as effective a means of administration as intravenoug injection, and that lung damage as a result of inhalation of $\mathbf{S r}^{\text {Bin }}$ would be of less importance than bone marrow darnage. The other thee elements, $T_{,}, \mathbb{Z}_{\text {, }}$ and $\mathrm{Ce}$, may be grouped together insoiar as they differ from Sr. They were all eliminated from the lung much more slowly than was $\mathrm{Sr}$. Thus the thmes required for $00 \%$ ellmimation were: $\mathrm{Y}^{91}, 12$ days; $\mathrm{Z}^{95}, 25$ days; and $\mathrm{Ce}^{148}, 45$ days. In all cases the skeleton was the major site of deposition 
and eventually contained between 15 and $30 \%$ of the initial lung deposit. In no case, in periods ranging up to 200 days, did the total radiation dose to the bone raceed that to the lung, it did within 12 hours with Sr. With all three elements the probability of radiation damage to the lung is great. Very little deposition occurred in the soft tissues. The kidney contained about $0.5 \%$ of the dose with all the elements. Concentration in the liver was lower than in the kidney with all isotopes except $\mathrm{Ce}^{144}$. This element showed a very high initial liver deposition, but this was gradually eliminated so that after two months the liver concentration fell below that in the kidney. (ADD)

81

Cochran, Ienneth W., John Doull, Maxcella Mazur, and Senneth P. DuBois. ACUTL TOKCITY OI ZLCONTUM, COLUM IUM, STRONTUM, LANTIANUM, CESIUI, TANTALUM, AND YTTRIUM. Axch. Ind. TYYg. and

Occupational Med. 1, 637-5011950).

Aqueous solutions or suspensions of compounds of $2 \mathrm{x}$, $\mathrm{Cb}, \mathrm{Sr}, \mathrm{La}, \mathrm{Cs}, \mathrm{Ta}$, and $\mathrm{Y}$ were administered in single doses by intraperitoneal injection or orally by stomach tube to male and female rats. Observation was made for 10 days, since the animals either recovered or succumbed during this period. The LD values are given for the various compounds used. Differences in these values are pointed out for the several compounds of each element studied, and the dependence of these values on the route of administration (oral or injection) 1 is discussed. 28 81 references. (NSA)

Greenberg, D. M. PROPOSED PROCEDURE FOR STUDY OF IEMOVAL OF LONG LIFE FISSION PRODUCTS FROM THE BODY. Argonne National Lab. [nd] 35. (MDDC - 1143-

A group of proposed procedures for the gtudy of removal of long Ife fission products from the body has been compiled. Measures which have been tried and have a considerable measure of success are: acid or allsall administration associated with a low calcium diet; parathyroid hormone administration; high potency vitamin $\mathrm{D}$; citric acid administration; displacement with non-radioactive 8. isotopes. $(\mathrm{ADD})$

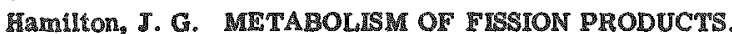
Iniw. of Chicago Metallugical Lab. Ang. 9, 1943. Decl. May 1\%, 104\%. 300. (MDDC-1000; CH-848)

Ce ${ }^{140}$ was prepared whout carrier from uranium ission material. The identity and purity was confirmed by suitable chemical and physical studies which included isolation and Identification of the 18 minute Radio-Praseodymium daughter. A large group of animals received a solution of $\mathrm{Ce}^{\mathrm{Ad}}$ as $\mathrm{CeClg}$ by different routes of administration. Its metabolic behavior in nost respects qualitatively resem. bles those of yturium and lanthanum although it is more poorly mobilized when administered parenterally or directy into the lungs. In this respect it more closely regembles gircontum than the rare earths. Radio-strontium wthou carrier was found to be chefly deposited in the skeleton whether given either orally or by intraperitoneal injection. Approximately $15 \%$ was absorbed when given by mouth and $70 \%$ retalned when injected. The rate of elimiration of the assimilated strontim is slow and is less than the rate of radioactive decay of $\mathrm{Sr}^{30}$. The simultaneou. administration of inert $\mathrm{S} r$ with $\mathrm{Sr}^{85}$ diminishes the skeletal deposition by as much as $50 \%$ following intraperitoneal injection. Other measures, which included the administration of $\mathrm{NH}_{\mathrm{g}} \mathrm{Cl}$, sodium citrate, the parathyroid bormone, and massive doses of vitamin were without significant effect. (ADD)
821

Uamilton, J.G. TECRNRCAL PROCRESS REPORT ON THE METABOLIC STUDIES OF IISSION PRODUCIS. Argonne National Lab. May 2, 1944. Decl. June 25, 1947. 2p. (MDDC-1061; CH-1572; A-2240)

Incomplete tracer studies with plutonium indicate that less than $0.05 \%$ of this element is absorbed from the digestive tract in any of the three valence states. The behavior of plus 4 plutonimm following intramuscular and intrapulmonary administration is very similar to that of 2irconium. The metabolic properties of plus 3 and plus 6 plutonium are very similar to those of ytrum and plus 3 uranium, (ADD) 822

Elamilton, J.G. METABOLTSM OF ISSION PRODUCTS. Argone National Lab. Apr. 22, 1944. Decl. May 14, 1947. 27p. (MDDC-1001; CK-1659; A-2270)

A progress report emphasizing the following: (a) prepa-

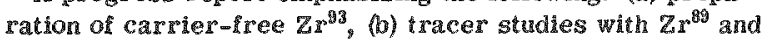
$\mathrm{Zr}^{33}$, (c) intramuscular and intrapulmonary tracer studieg with carrier-free $\mathrm{r}^{\text {wa }}$, (d) intramuscular and intrapulmonary tracci studies with carrier-fres $\mathrm{Ce}^{\text {tho }}$, (e) decontamination studies of radio-strontum, radio-yturum, and radiocerium. (ADD)

\section{3}

Hamilon, $\pi . G$. TRACER STUDLS ON FISSION PRO

DUCT METABOLISM. Radiation Lab.g Univ of Calif. Feb. 2, 1945. Decl. June 24, 194\%. 80. (MODC-1062; CH 2673 )

Radioactive isotopes, free from inert carrier, of $\mathrm{Sr}, \mathrm{X}_{\text {, }}$ $\mathrm{Zr}, \mathrm{Cb}, \mathrm{Ru}, \mathrm{Te}, \mathrm{Cs}, \mathrm{Ba}, \mathrm{La}, \mathrm{Px}, \mathrm{Ce}$, and Np were administered to ats as chlorides in isotonic solutions of $\mathrm{NaCl}$ at pH ranging from 2.3 to 3.5. Iodine was given as $\mathrm{Na}$, and Ke was given by imhalation in an oxygen-gas mixturg. Un separated fission products, free from iner carrer and uranium, after 90 days cooling were administered ln an isotonic solution of $\mathrm{NaCl}$ at pH 2.5. Adult rats received the various radioactive preparations in solution by oral, parenteral, and intrapulmonary administration. In the earlier expeniments the parenteral administration was by way of the intraperitoneal route. Due to the difficulty of absorption of some of the radioactive isotopes, considerable retention of activily on the surfaces of the intraabdominal organs took place. In order to gain a more accurate picture of the true deposition of these radioactive isotopes in the intrabbdominal organs it became necessary to employ the intramuscular route of administration. The animals were sacrificed in groups of three for each route of administration and at varying intervals rangimg from 1 to 64 days. Sixteen to 18 representative tissues were removed from each animal and their activities individually meas. ured. The most significant conclusions that can be derived in the survey of all of the work done with the long life fission products can be summanized in the following manner: the alkaline earths, rare earths, $2 \mathrm{r}, \mathrm{Np}$, and $\mathrm{Cb}$ when introduced into the body are deposited primaxily in the skeleton, where the average degree of zetention of the absorbed radioelement is appromimately $50 \%$ of the inital absorbed quantly. The retained fractions of these radioelements are eliminated at rates which are less than their rates of adioactive decay with the exception of the 340 day Ce and possibly $\mathrm{Sr}^{20}$. The remaining five long-lived radioclements, Ru, Te, $\mathrm{Xe}$, $\mathrm{Cs}$ do not show any significant degree of localization in the skeleton and in addition there is no very striking deposition in any other of the tissues with the exception of deposition of I in the thrroid. The rates of elimination for all of these four radioelements are much greater than their rates of radioactive decay, with the exception of I accumulated in the thyroid. In this particulas 
case the release of accumulated I in the thyroid is many times slower than its rate of radioactive decay. The members of the long-lived fission product group which are absorbed by way of the digestive tract include the alkaline earths, tellurium, iodine, and cesium. A high degree of retention by the lungs has been observed following direct intrapulmonary administration of the rare earths, $\mathrm{Zr}, \mathrm{Cb}$, $\mathrm{Ru}$, and $\mathrm{Np}$. The pulmonary retention observed for the alkaline earths, $\mathrm{Te}, \mathrm{I}$, and $\mathrm{Cs}$ is negligible under the con924 ditions of the experiment. (ADD)

Hamilton, J. G. THE MEAVIEST ELEMENTS AND FISSION PRODUCTS. Radiation Lab., Univ. of Calif. [nd] Decl. Nov. 18, 1946. 2p. (MDDC -490)

A study has been made of the metabolism in the rat of the heaviest clements and of fission products. The heavy elements studied include thorium, protactnimm, neptunium, plutonium, and americium, and the fission products investigated were radioactive isotopes of $\mathrm{Sr}, \mathrm{T}, \mathrm{Zr}, \mathrm{Cb}, \mathrm{Ru}, \mathrm{Te}, \mathbb{I}_{9}$ Xe, $\mathrm{Cg}, \mathrm{Ba}, \mathrm{La}, \mathrm{Ce}$, and $\mathrm{Mv}$. In addition, a rather detailed investigation of the metabolism of an unseparated mixture of uranium ission products was undertaken. The reault of these studies indicated that these heavy elements are absorbed to a very small degree by way of the digestive tract, deposited primarily in the skeleton following parenteral administration, and climinated very slowly. The metabolism of plutonim in two human aubjects was investigated and found to be comparable to the behavior of this radio-element in the rat. The most significant conclugions that can be derived in the survey of the work done with the long-lived fission products can be summarized in the following manner: $\mathrm{Sr}, \mathrm{Ba}, \mathrm{Y}, \mathrm{La}, \mathrm{Ce}, \mathrm{Pr}, \mathrm{Zr}$, and $\mathrm{Cb}_{8}$ when introduced into the body, are deposited primarily in the skeleton where the average degree of retention of the absorbed radio-elements is approximately $50 \%$ of the initially absorbed quantity. The retained fractions of these radio-elements are eliminated at rates which are less than thelr rates of radioactive decay with the exception of the 340-day $\mathrm{Ce}$ and possibly S $\mathrm{x}^{\circ 0}$. The remaining five longlived radio-elements, Ru, $\mathrm{Te}, \mathrm{Xe}, \mathrm{I}$, and $\mathrm{Cg}$ do not show any significant degree of localization in the skeleton and in addition, there is no very atrilking depowition in any other of the tissues, with the exception of the deposition of lodine in the thyroid. The rates of elimination for all of these five radio-elements are much greater than their rates of radioactive decay, with the exception of iodine accumulated in the thyroid. In this particular case, the release of accumulated lodine in the thyrold is many times slower than it rate of radioactive decay. The members of the long-lived fission product group which are absorbed by way of the digest tract include strontium, barium, tellurium, iodine, and cesium. Radioautographic studies of the deposition of $\mathrm{Y}_{3} \mathrm{Zr}, \mathrm{Ce}, \mathrm{U}, \mathrm{Pu}$, and $\mathrm{Am}$ in bone indicate that these materials are selectively accumulated in the osteoid matrix. (ADD)

825

Hamilon, J. G. SUMMARY OF METABOLISM OF CARRIER-FRE ISESION PRODUCTS IN THE RAT. Radiation Lab., Univ, of Calif. May 14, 1947. 3p. (MDDC-1002; CK-775)

The following carrer-free fission products were studied to determine their effect upon the metabolsm of the rat: $\mathrm{Sr}^{85}, \mathrm{Y}^{88}, \mathrm{Zr}^{83}, \mathrm{Cb}^{93}, \mathrm{Ru}^{406}, \mathrm{I}^{131}, \mathrm{Xe}^{129}, \mathrm{Ba}^{133}, \mathrm{La}^{140}, \mathrm{Ce}^{190}$, and a raw fission mixture. The results are presented in 826 chart form. (ADD)

Hamilton, J.G. THE METABOLISM OF THE ISISION PRODUCTS AND THE IIEAVIEST ELEMENTS (PLUTO-
NIUM PROJECT SYMPOSIUM) Radiology 49, 325-43 (1947).

All radioactive elements that accumulate in bone with the exception of $\mathbf{S r}$ are not deposited in the mineral structure of bone, but are localized in and adjacent to the osteoid matrix. The large fraction of $\mathrm{La}, \mathrm{Ce}, \mathrm{Pr}, 61, \mathrm{Am}$, and $\mathrm{Cm}$, deposited in the liver was an unexpected finding, and it may be predicted that the same will be true for $\mathbb{N d}$, Sm, and $\mathrm{Eu}$. (CA)

827

Hamilion, J. G. THE METABOLIC PROPRRTEES OF THE FHSION PRODUCTS AND ACTINDE TELCMENTS. Revs. Mod. Phys. 20, 718-28(1948).

An investigation of the assimilation, distribution, retention, and excretion of the fission products and actnide elements in the rat auministered by mouth and by injection has been conducted. The fission products studied included the carrier-free radioactive isotopes of $\mathrm{Sr}_{\mathrm{r}} \mathrm{Y}, \mathrm{Zr}, \mathrm{Nh}, \mathbb{R u}$, $\mathrm{Te}, \mathrm{I}, \mathrm{Xe}, \mathrm{Cs}, \mathrm{Ba}, \mathrm{La}, \mathrm{Ce}, \mathrm{Pr}_{\mathrm{r}}$ and $\mathrm{Pm}$. The actinides studied were $\mathbf{A c}, \mathrm{Th}, \mathbb{P a}, \mathrm{U}, \mathrm{Ng}, \mathrm{Pu}, \mathrm{Am}$, and Cu, The fission products $\mathrm{Sx}_{\mathrm{x}} \mathrm{Ba}, \mathrm{Y}, \mathrm{La}, \mathrm{Ce}, \mathrm{Pr}, \mathrm{Pm}, \mathrm{Zr}$, and $\mathrm{Nb}$ and all of the actinides are absorbed to a very small degree by way of the digestive tract, deposted primaxlly in the gkeleton following parenteral administration, and eliminated very slowly from that organ. From $25 \%$ to $70 \%$ of the material absorbed following parenteral fnjection is ladd down in the skeleton and is eliminated from that organ at rates less than the rates of radioactive decay, with the exception of

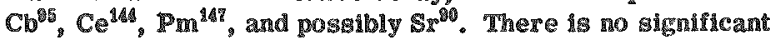
degree of localization of the remaining 5 elements in the skeleton or in any other tissue with the exceptlon of the accumulation of $I$ in the thyroid gland. The rate of elminathon for all of these 5 radioelements is much greater than their rate of radioactive decay with the exeegtion of I, the release of which accumulated in the thyrold is much slower than its rate of radioactive decay. The members of the long-lived îssion product group which are absorbed by way of the digestive tract include $\mathbf{S r}, \mathrm{Ba}, \mathrm{Te}, \mathbb{I}$, and $\mathrm{Cs}$. With the exception of $U$, limination from bone occurred very slowly. Radioautographic studies indicated that $\mathrm{Sr}$, and presumably $\mathrm{Ba}$, are deposited primarily in the mineral structure of the bone. The other fisston products deposited in this organ are laid down in the region of the inner and outer coverings of the bone and about the trabeculae, but do not apparently enter into the mineral atructure of this organ to a degree comparable to $\mathrm{Sr}$. In the case of the actinide elements, a similar accumulation in the region of the non-mineralized portion of the bone has been observed with $\mathrm{Ac}, \mathrm{Tl}_{2}, \mathrm{Pu}_{\mathrm{u}} \mathrm{Am}$, and $\mathrm{Cu}$. The curious property of the members of the fission products and the majority of the actinide elements to be laid down in this portion of the skeleton and to be retained there for prolonged intervals, renders them of considerable concern from the point of view of the possible medical hazards to be oncountered should these materials be accidentally introduced into the body. (TIS)

828

Hamilton, J. G. PART I. PROGRESS REPORTED ON TRACER STUDIES CONCERNED WTTH METABOLISM OF $\mathrm{ND}^{147}, \mathrm{PR}^{143}, \mathrm{SR}^{163}, \mathrm{AND} \mathrm{GA}^{B 8}$. PART II. BLOLOGICAL STUDIES OF RADLATON EFFETS, MEDICAL AND HEALTI DIVISIONS QUARTERLI REPORT, JANUARI, FEBRUARE, MARCY 1040. Radiation Lab., Univ. of Calif. [nd] 88p. (UCRE-332)

Results of tissue distribution studies are tabulated for $\mathrm{Nd}^{\mathrm{BS}}, \mathrm{Sn}^{\mathrm{IIS}}$, and $\mathrm{Ga}^{67}$, well as data on metabolic eflects of $\mathrm{Zr}^{85}$ and bone uptalke of $\mathrm{Sr}^{8090}$. An extensive study on blood coagulation is reported. The heparin clotting time 
test and the clot retraction rate test, a quantitative measure of clot retraction obtained by electric resistance measurements were talken on numerous human subjects and on patient3 with chronic leukemia, polycythemia and other hematopoletic diseases. Coagulation defects found in patients with these diseases are reported in greater detail and the possible relations of platelets and heparin to vartous hemorrhagle and thrombotic conditions is discussed. Preliminary observations indicate that radioactive isotope therapy in leukemia and polycythemia has boneficial effects upon altered blood coagulation. 104 references. Preliminary reports are included on hematolog cal effects of total body irradiation and $\mathbb{I}^{31}$ therapy. (TIS) 229

Hamilton, Joseph G. TRE METABOLISM OF THE RADOACTIVE ELEMENTS CREATRD BY NUCLEAR IISSION. New Engl. J. Med. 240, 863-70(1949).

A review Is made of part of the medical research activities within the atomic-energy program and covering the biological properties and metabolism of the radloactive dements created by nuclear fission. summary of the metabolism of the principal members of the long-lived fisslon products (also neptunium and plutonium), obtained after parenteral and oral administration in the sat, is contalned in one table. Abeorption of the products through the digestive tract and the lungs, and retention of the products in the skeletal system and other parts of the body are discussed. The length of retention of some of these products In the sheletal system creates a great radiation hazard. Dractical progress in studies developing methods of releasing these products from the bone are discouraging. In conclusion, it is stated that there is no new major mystery surrounding the problem of protection agalnst the radioactsve products associated with the release of atomic 330 energy. (NSS)

Memilton, J. G., C. W. Asling, W. M. Garrison, X. G. Scott, and D. Arelrod-Heller. DESTRUCTIV ACTION

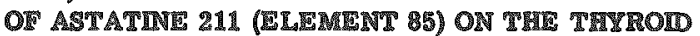
GLAND OF THER RAT. Proc. Soc. Expll. Biol. Med. 73. $51-3(1950)$.

Astatine 211, prepared by alpha particle bombardment of

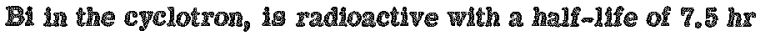
but unlike most artificial radioelements it emits alpha parteles rather than electrons and gamma rays. It also

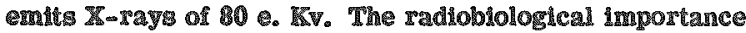
of the emission of alpha particles is that each of there melear particles will disalpate as initial kinetic energy of 6 mev in about $50 \mu$ of a soft tissue. The beta rays from the 8-day I Ist posgers a mardmum energy of 0.6 mev with a range of nearly 2000 of of tissue. When $A t^{213}$ chloride is injected into rats the At accumulates in the thyrold and produces an ertreme degree of radiation injury of the thyroid เ1็สนe without apparent involvenent of the adjolning para931 thyroldes. (CA)

Irawin, Bergene, D. . 3TUDIES OF THE MPTABOLISM OF CERTAN FISSION PRODUCTS AND ELUTONIUM. Radiation Lab., Univ. of Call. Aug. \%, 1050. 120p. (UCRL-812)

The distribution and excretion of radiolsotopes of cerinm, njobium, plutonium, strontium, yttrilum, and zIrcoมนm has been studied in adult femal sats of the LongZvans straln. The metabolism of Pu(VI) was unaltered by general treatment with the auhihydryl compounds $2,3-$ dincreaptonropanol (DAL) and cysteine. Tha gugrests that the metabolism of Pu is not related to that of sulfinydryl growp. Theme compounds do not appear to have use as therapeatic agents lor plutonium poironing. Cystelne ap- peared to increase the amount of Pu retained at the site of Intramuscular injection. Parathormone had no significant effects on the distribution and excretion of esther $\mathrm{Pu}$ or $\mathrm{Y}$. The resorption of bone by parathormone did not appear to labilize these bone-deposited elements, nor make them more susceptible to the positive effects of zirconium citrate. Massive doses of rirconium citrate appeared to have some positive effects on the distribution and excretion of $\mathrm{Ce}, \mathrm{Nb}, \mathrm{Pu}_{3}$ and $\mathrm{Y}$. Sodium citrate had relatively little effect on the metabolism of these elements, with the exception of $\%$. Both citrates were without significant effect on the metabolism of radiostrontium. Zirconium cilrate may alter the metabolism of the several radioisotopes throush a "carrier" action as a chelated radiocolloidal citrate complex. The possible influence of this complex on the uptake and excretion of the several radiolsotopes during the Pirst hour following their adminigtration has been discussed. (NSA) 832

Lan, E. C. and J. D. Teresi. THE METABOLISM OF SHORT-LIVED AIR-BORNE FISSION PRODUCTS.

Argonne National Lab. May 1945. Decl. May 14, $184 \%$. 39p. (MDDC-934; CH-2844)

A flssion recoil apparatus has been constructed in which uranium foil is subjected to neutron bombardment in the gile. Gas passing over the foll picks up the fission recoll atoms and carries them to animal exposure chambers and measuring equipment located outstde the pile shield. The time taken for an atom to go from the foll to the exposure chamber (hold-up time) varied from 1.8 to 30 seconds. A special ionization chamber was developed to measure the activity, which varled from 17 to $0.5 \mu \mathrm{c} / \mathrm{cc}$. It has been Ghown that this gas contalns not only the noble gas fission products but also all fission products which normally exist in the solid state. Rats and mice exposed to this gas in an exposure chamber guffer damage and die, but no more 30 than would be expected from the amount of external gamma radiation which they recelved whlle in the exposure chamber. A speial apparatus was constructed which would allow rats to breathe the gas without receiving external radiation. Rats receiving exposures of eight hours yer" day for live consecutive days recelved burns of the mouth and upper respiratory tract. Some of these animals lived but most of them died, apparently from starvation because of Iallure to eat. Metabolim experiments have shown that of the material that is inhaled, about $25 \%$ goes to the skeleton and is excreted very slowly: about g\% gtays in the lungs and is excreted slowly; about 6\% goes to the liver and is slowly excreted; and the remainder if escreted with moderate rapidity. The highest spectic activity is found in the inyroid. A hiotological study of tissues of animals gacrificed at various times following exposure has whown that the most severe treatment possible wth this apparatur has failed to produce any detectable tissue danage which could be atributed to internal depostuon of radioactive materials. Radioautographs ghowed that the activity was deposited uniformly over the bronchial and alveolar suriaces. It was concluded that for short-lived alr-borne fission products the hazard if very much greater from external radiations than from internally deposited radoactive materialw. (ADD) 833

Overstreet, R., L. Jacobson, H. Fisher, and R. Scott. PROGRESS IREORT ON METSOLISM ON FISSION PRODUCTS FOR PERIOD ENDING OCIOBIR 15, 104. Argonne National Lab. [nd] 40p. (MODC-1011; A-1441; CH-1089)

Unseparated Ission product have been fsolated from 18 pounds of neutron bombarded $\mathrm{VO}_{2}\left(\mathrm{NO}_{3}\right)_{2} \cdot 6 \mathrm{H}_{2} \mathrm{O}$ iree from carrier and containing less than 0.03 g of U/pe of activity. 
The final solution was assayed for its content of $\mathrm{Sr}, \mathrm{Y}, \mathrm{Pr}$, $\mathrm{Zr}, \mathrm{Nb}, \mathrm{Ru}, \mathrm{Te}, \mathrm{Cs}, \mathrm{Ba}, \mathrm{Ce}$, and $\mathrm{UX}_{\mathrm{y}}$. Tracer studies were done on a large group of rats which received the prepared solution by intramuscular, intraperitoneal, intrapulmonary, and oral routes of administration. The tme intervals ranged from 1 to 64 days. The skeleton was the chief point of deposition for the absorbed radioactive mixture for all 4 routes of administration. Considerable and prolonged retention occurred in the lungs following intrapulmonary administration. From 3 to $5 \%$ was absorbed from the digestive tract. Ixcretion was slow and the digestive iract was the chief channel of elimination. Approximately $50 \%$ of the activity was excreted over the 64 day period following intraperitoneal and intramuscular injection. Tissue distribution studies are reported to some detall for $\mathbb{R}^{105}$ and $\mathrm{Np}^{238}{ }_{2}^{239}$. Photomicrographs of representative lung sections and their corresponding radio-autographs are included in the report. No signufficant deposition was noted in the bronchi, lymphoid sissue, and blood vessels, while the radioactivity was distributed widely throughout the alveolar structure of the lungs. Frequently a tendency for the alveolar pattern of the section to be duplicated on the radio-autograph was observed. (TIS)

\section{4}

Overstreet, R., L. Jacobson, and I. Scott. UNSEPARATRD FISSION PRODUCTS. Argonne National Lab. [nd] (MDDC-1143-C)

A procedure has been devised for the separation of raw fission products without carriers from neutron-irradiated uranyl nitrate. An analysis of the final separated materal which had been completely freed from uranium gave a distribution which was quite similar to the values noted by Coryell and Seaborg. The intraperitoneal injection of this material in small group of rats sacrificed at 4 and 16 days after injection revealed the skeleton to be the single most important organ for retention of this material. At four days approximately one-third of the administered dose was in the bones and this value was found to be slightly higher at the end of 16 days. High values for retention were observed in the liver and spleen of the four day interval. By the 16 th day, however, these levels fell considerably. The over all pichure of excretion indicated that the rate of elimination was rather slow although somewhat more rapid than in the cass of yttrium. Four days after oral administration only $1.6 \%$ of the administered dose was absorbed and almost all of this was found in the skeleton. This observation suggests the possiblity that a very large proportion of the absorbed fission material was strontium. (ADD) 895

Scot, $\mathrm{R}$. G., et a. THE METABOLSM OF CARRIER FRER FISSION PRODUCTS IN THE RAT. Radiation Lab., Univ. of Call. [nd] Decl. Aug. 26, 194\%. 209p. (MDDC-1275)

The alkaline earths, rars earths, zirconium, and columbium when introduced into the body are deposited primarily In the sireleton, where the average degree of rentention of the absorbed radioelements is approximately $50 \%$ of the Initially absorbed quantity. The retained fractiong of these radioelements are eliminated at rates which are less than their rates of radioactive decay with the exceptlon of the 340 day cerium and possibly $3 r^{80}$. The remaining five longlived radioelements, ruthenium, tellurium, senon, lodine and cesium do not show any signiflcant degree of localization in the sleeleton and in addithon, there is no very strilsing deposithon in any other of the tissues with the exception of denosition of iodine in the thyroid. The rates of elmmination sor all of these sous radioelements are much greater than their rates of radionetive decay, with the sxception of Iodine accumulated in the thyrold. In this particular case, the release of accumulated lodine in the thyrold is many times slower than its rate of radioactive decay. The mem bers of the long-lived fission product group which are absorbed by way of the digestive tract include the alkallne 836 earths, tellurium, lodine, and cesium. (ADD)

Scot, K. G., et al. STUDIES ON THE INHALATION OF FISSIONABLE MATERIALS AND FISSION PRODUCTS AND TIEIR SUBSEQUENT IATE IN RATS AND MAN. Argonne National Lab. Dec. 31, 1845. Decl. Aug. 28, 1947. 166p. (MDDC-1276; CH-3590)

The puxpose of these experiments was to ascertain the possible hazard resulting from inhalation of issionable materials and fission products. Aerosols of plutonium, uranium plus fission products, protactinium, and shortlived fission products obtained from the cyclotron and the Clinton Plie were administered to rats. $\mathbf{A} \mathrm{zr}^{38}$ aerosol was administered to one human subject and to rats. Aerosols of the above elements were almost totally retaned by the head and lungs immediately after exposure. After four days the lungs contained the largest percentage of these lements. The elements deposited in the head were quickly eliminated via the gastrointestinal tract. The same avenue of elimination was used by the lungs, but at a slower rate. The mmall percentage absorbed into the body was primarily deposted in the skeleton after conditions of equilibrium had been established. Radioautographic studies indicate that the site of devosition in the lungs of these materials is in the bronchial passage and the alveolar structures. They are rapidly removed from the bronehal tree, presumably by clliary action and are very slowly released from the alveoli. No accumulation of any of the radioelements was observed in either blood vessels or lymph nodes. Charts, diagrams, and photographs are included, and a bibllography is appended. (ADD)

837

Scot, Kenneth G., D. Plarald Copp, Dorothy I. Aselrod,

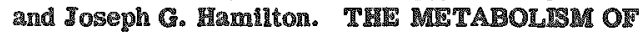
AMERICUUM IN THE RAT. J. Biol. Chem. 175, 601-703 (1948).

An Investigation of the metabolism of americhun in the rat has been conducted, following its administration by intramuscular injection and by stomach tube. No appreciable absorption took place from the digestive tract. WithIn 24 hours after parenteral administration, $55 \%$ of the total amount of americium absorbed from the site of linjection was deposited in the $11 v e r$ and $20 \%$ accumulated by the skeleton. Exxcretion occurred primarily by way of the difestive tract and most of the merichun eliminated anpeared to come from the liver. There was no algnifleant change in the content of anerichum in the olseleton through out the interval of the experiment. The distribution of americium in bone was studied by the radioautographle technique with thin, undecalcified sectlons of the cemur. Amertcinm was heavily derosited in the rerron of trabecum lar bone below the epiphygis, at the endosteal and periosteal surfaces of the shaxt, and throughout the cortlcal bone in the region of sman blood vessels. (NSA)

\section{Gallum}

838

Brucer, Marshall. DUSTRUUTION OF CALLTUM IN MAN: AUTOPSY EVIDISACE. 3. Phar Therap. 101, 1-5(1051).

This paper is reproduced here in its entirety.

One 14-yr-old patient with osteogenic sarcoma (primary removed) metastatic to the lungs was given 50 me of $\mathrm{Ca}^{\text {T2 }}$

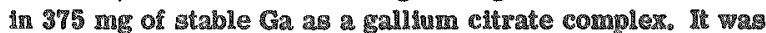


administered intravenously in divided doses of 5 to $15 \mathrm{mc}$ at A-day intervals. At autopsy the distribution in 18 types of tissue was found relatively uniform. Only in kidney, 11ver, and bone was there increased retention of $\mathrm{Ga}^{72}$. All metastatic nodules sampled showed greater activity per gram than did any normal organ. One 68-yg-old patient whin multiple nyeloma received intravenously $178 \mathrm{mc}$ of $\mathrm{Ga}^{72}$ in $681 \mathrm{mg}$ of stable $\mathrm{Ga}$ in the chtrate complex. The doses of 4-day intervals ranged from 20 to $50 \mathrm{me}$ each. On autopas, the marrow, cortical bone, liver, kidney, and gpleen showed the rreatest retention of $\mathrm{Ga}^{72}$. There was no normal bone, but the greater relative distribution in areas of increasing myeloma activity was shown by autoradiograms. (NSA)

조오ำ

Dudley, 1. C., C. W. Tmirie, J I., and I. T. Istock, DE. POSITON OW RAOLOGALLIUM (CA ${ }^{\text {T2 }}$ IN PROLIFERATIIJG THSUES. Radiology 55, 571-8(1950).

Radlogallum, $\mathrm{Ga}^{2}$, has been shown to concentrate at areas of ostcogenic activity, 1.e., the epiphysenl junction and the callus of healng fractures in rabbits, and in osteogenic sarcoma of the mouse. In certain flbroadenomas, stbrosarcomas, and the mixed mammary tumor of animals, no signiflcant localization of $\mathrm{Ga}^{12}$ occurged. Autoradiographic studies of the degosicion of $\mathrm{Ga}^{22} \mathrm{in}$ bone, by $1 \mathrm{~m}-$ pregnation of bone sections with liquald photographic emulglon, has made posstble mose datalled localization of this element. It is suggested that radogallum citrate is worthy of elinical trials in tracer anounts for diagnoatic purposes and at higher levels for possible therapeutic effect. (NSA) 80

This abstract has been intentionally omitted.

81

Dudley, R. C. and G. E. Maddow. DEPOSHTON OF TAOIO GALLIUM (GA ${ }^{2}$ ) IN SRY Pharmacol. and ${ }^{2 x y}$ th. Therap. 26, 224-7(1949).

The results of a radioautographic study of the distribution of $\mathrm{Ga}$ in the skeletal tissues of the rabbit and dog fol lowimg injections of non-toxtc doses of radio-gallium (Ga') are presented. The radionglum was Injected as the lactate of citrate into the animals at a dosage level of 0.5 me/kg of body welght: the antmal were sacrikiced 12 hours after the injections, and the bones were removed, cleaned of adhering t1sure, rnounted in dental mtone, gectoned, and placed on emulsion $\mathrm{X}-\mathrm{ray} \mathbf{f H m}$. The radioautographs ghow that in yourg animals the Ga is alectively derostied in the areas of greatest osteogenic activity (he epiphyseal line); In adult animals there is also a depositson in this general area, but tit takes place in more diffuse manner throughout the trabecular bone. The most significant Inding is the 34: rapidity of deposition of the Ga. (NSA)

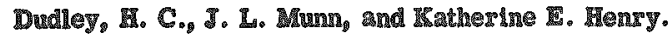

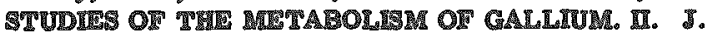
Pharmacol. and Pspth. Therap. 28, 103-10(1950).

Radiogallium (Ge with a geclilc activity of $0.11-0.12$ $\mathrm{mc} / \mathrm{mg}$ was Injected subcukancously as gallum citrate 20 mog amounts into a series of 12 rats In order to determine the distribution of the gallum. Sinuar experiments were gertormed with rabbits and dogs: In rabbits the excretion of the leotope was aleo invertigated. It was found that in the zat, gabbit, and dog only bone and the kdaneys receive large amounts of the lement. Following the gubcutaneous injection of the element as citrate, the rabbit excretes 30-55\% of the total dose in the uxtne within 16 hours, while the osteoid struetures absorb an additional $30-48$ \% within this tme. Definte amounts are almo excreted into the gtosach and intestines by the gastrointeatinal anucosa; the contents of the tract contain 1-2\% of the subcutaneous dose within 12-18 hours after injection. (NSA)

849

English, J. A, and H. C. Dudley. DISTRIBUTION OF RADIOACTIVE GALLIUM IN THE TEETH AND JAWS OF EXPRRMENTAL ANIMALS. Dental Research 29, 93 100(1950) 14p. (Naval Med. Research Inst. Report NM-008-009(Report i))

Radiogallium in the form of soluble Ga lactate $(\mathrm{pH} 7.0-$ 7. 1) was injected mubcutaneously into rats and rabbits. The dosage was 0.2 to $0.4 \mathrm{mc}^{\circ}$ of $\mathrm{Ga}^{22} \mathrm{per} \mathrm{kg}$ of body weight (6 $\mathrm{mg} / \mathrm{kgg})$, which is well below the toxic level. The animals were isilled six hours after injection and thin sections $(50-$ $100 \mu)$ were made of the bone and teeth by cutting with high speed abrasive discs and grinding to the desired thickness against an alorasive wheel. These thin sections were placed nest to special $x-x a y$ film for 24 to 48 hours. The rays emanating from the radioactive isotope affected the film Jugt as do the rays from an 3 -ray tube. The developed negatives were compared with transillumination photographs which were subseguently made from the ground sections. By this means di was determined that radion gallium is predominantly deposited in ondosteum, periosteum, tooth pulp, and perlodontum. There appear to be a moderate deposition in cancellous bone. Cortical bone and the dentin of the root portion of teeth take a barely perceptible amount of radiogallum, whereas coronal dentin and enamel give no indication that any radiogallium is deposited. The main advantages of the autoradiographe method over methods using the electroscope or Gelger counter for determining the radioactivity are that Iractional portions of teeth and bone are not required, and the visual localization is rapid and effective. The relative distribution of the radioactive substance in exch anatomic area is apparent, however, quantitative measurements were not attempted in this study. (NSA) 정션

King, 2. Te., Jesse D. Perkinson, Jr., H. D. Bruner and TOe Gray. RICRTION OF GALLIUM BY THR RAT AND BY MAN. Science 113, 555-7(1951).

A detalled comparison is made between excretion of Ga in the rat following intravenous administration of $\mathrm{GaCl}_{3}$, a rapidily lonizing salt, and the slowly lonizing complex gallium citrate. The rats received 200 to $300 \mu \mathrm{c}$ of $\mathrm{Ga}^{72}$ in 1.5 to $2 \mathrm{mg}$ of stable Ga. About $79 \%$ of the Ga given as the citrate was excereted in the urine by the fourth day compared with $58 \%$ for the chloride salt. The recal erceretion of the $\mathrm{GaCl}_{3}$ was about twice that of the citrate. $\mathrm{At} 96 \mathrm{hr}$ the urdne/feces ratio was $\sim 8$ for the chloride salt and 19.3 for the citrate complex. A second comparison is made between excretion by the at and by man of tracer and larger intravenous doses of gallium chrate. In man the chief route of excretion is also the urinary tract. In the rat studies the effective half life at the dose level used was 8.33 hr. For the larger doses in man it was $\sim 12.5 \mathrm{hr}$. (NSA)

\section{Gold}

845

Burgtone, M. S. STUDIES ON THE EFECT OF RADIOACTIVI COLLOIDAL GOLD ON THE DEVELOPMEN T OF THE ORAL STRUCTURES OF THE MOUSE. ArCh. Pathol. 50, 410-20(1950).

The injection of colloidal radioactive gold, $\mathrm{Au}^{198}$, adjacent to the jaw of the immature mouse will result in abnormalities of osteogenesis and odontogenesis, including fallure of root formation. The changes are interpreted as being the result of local ir radiation, since the side of the 
head farther from the Injected area exhitoits relatively normal development of tooth and bone structure. These changes are similar to those observed in immature mice receiving gystemic dosages of radioactive phosphorus. The serous-type glands manifest a greater radiosensitivity than the mucous type. The duct elements are relatively radio86 resistant. (NSA)

Desaive, P. and J. Closon. RADIOACTIVE GOLD AU 193 AND ARTICULATIONS. J. belge radiol. 24, $438-44$ (1251).

Colloidal $\mathrm{Au}^{198}$ in $3-$ to $12.8-$ mc doses was injected intravenously, intraperitoneally, or intraarticularly into rabbits. Subsequent distribution and apparent radiationinduced changes in synovial and adjacent tissues are illus847 trated. (NSA)

Fredell, Hymer L. MONTHLT PROGRESS REPORT, NOVIRBRR 1251. Atomic I nergy Project, Western Reserve Uni. [nd] 6p. (NYO-1639)

Distribution studies in normal and sub-total hepatectomized ratz injected intravenously with colloidal Au ${ }^{198}$ showed a marked alteration of deposition and hematological response in the hepatectomized animals. These changes are discussed. Rais receiving colloidal $\mathrm{Au}^{196}$ prior to 8 irradiation showed approximately one-third of the $\mathrm{x}-\mathrm{ray}$ effect after receiving pretreaiment with cysteine. (NSA)

848

Mahn, P. Marvin A. Jackson, and Horace Coldie. LIVER CIRRYOSIS WITI ASCITES, INDUCED IN DOCS BY CHRONIC MASSTE MEPATC IRRADLATON WTH FADIOACTIVE COLLOIDAL GOLD. Science 14,303-5 (1951).

A report is made of lesions in the liver of 7 -week old doges following the intravenows administration of from 59 to $102 \mathrm{mc}$ of radioactive colloidal Au given in three doses over a period of approximately six weeks. Dosages were estimated at 67,000 and 65,000 equivalent $\beta$-roentgen to the liver. Approximately weeks after the last injection of the colloid, increasing amounts of ascitic fluid accumulating in the abdominal cavity were noted. A marired retardation in growth and general appearance was also noted. Tracer guantitieg of radioactive Au colloid administered by vein showed a marked lag in the time of disappearance from the circulatory system, suggesting an impaired ability for phagocytosis by the reticuloendothelial system. Both dogs died from the severe damage induced by the radiation in the liver. At autopsy about $1,000 \mathrm{ml}$ of clear, slightly yellow fluid was withdrawn from the peritoneal cavity of one dog and about $1,800 \mathrm{ml}$ from another. The most obvious effects of irradiation in both dogs were disappearance of endothelium and marked increase in connective lissue sur rounding the blood vessels of the liver. Pathological changes in the liver induced by the massive chronic irradiation resembled a combination of Chiaris syndrome (obliteration of the hepatic vein) and of central fibrosis. Considerable damage to both spleen and kidneys was also noted. (NSA)

849

Sheppard, C. W., J. P. G. Goodell, and P. F. Hahn. COLLOIDAL GOLD CONTAINING THP RADIOACTIVE ISOTOPE AU ${ }^{193}$ IN THE SELECTVY INTERNAL RADLATION THERAPY OF DISEASES OF THI LYMPHOT SYSTEM. J. Lab. Clin. Med. 32, 1437-41(1947).

Colloidal gold containing $\mathrm{Au}^{128}$ was obtained from the chain-reacting pile at Oak Ridge and used in therapy of lymphoid diseases. This isotope has an ideal half-life and good radioactive characteristics. Very stable colloids can be prepared. It is easily administered and the cost of such therapy is low. (BA)

850

Wheeler, Brownell, Marvin A. Jackson, and Paul . IRann. HEMATOLOGY OF THE DOG FOLLOWUNG IVTRAVENOUS ADMNISTRATION OF RADIOACTIV COLLOLAL COLD. Am. J. PhysLiol. 166, 323-7(1051).

Fourteen dogs were injected with $1 \mathrm{mc} / \mathrm{kg}$ of $\mathrm{Au}^{198}$ by vein. All the dogs showed a definite decrease in white count, but only one was thrown into a marked leukopenia. In 12 out of 14 dogs sedimentation rate increased following radiation, although this response was not as clear cut as the decrease in white count. Hemoglobin and hematocg it decreased in a slow and progressive manner over a period of several' weeks Liver function tests lalled to reveal any marked degree of hepatic impairment, but a few dogs howed changeg indicative of mild liver damage. Some of the possible mechanisms for these changes are discussed briefly. The results show that intravenous administration of radioactive colloidal gold at dosage levels similar to those employed therapeutically in the treatment of leukemia and cancer is relatively well tolerated by normal dogs. It seems probable, therefore, that, insofar as hematological changes and liver functions are concerned, thege dosage levels pregent little immediate danger to patients who receive them. (NSA)

\section{Iodine}

851

SYMPOSIUM ON TADIOIODINE. Brookhaven National Lab. July $28-30,1248$. 118p. (AECU-51; BNL-C-5)

The lollowing papers were preaented: Radioactive Iodine: Its Use in Studying Certain Functions of Thyrold Tumors, Rulon W. Rawson; The Blological Effect of II radiation by Radioactive Iodine, Bengt N. Skanse; The Mechanism of Action of Anti-thyroid Drugs, Malcolm M. Stanley; Turnover Rates as Measured by Radiolodine, William T. Salter, MacAllister Johnston, and John Gemmel; Standardization of Radioactive Iodine, Serget Feitelberg; Calculation of Dosage in Radiolodine Therapy, Edith H. Quimby; Uptake and Excretion Measurement: and Their Significance, Eleanor Oshry and Charlotte Schmidt; The Radiation Treatment of Hyperthyroidism, M. Chapman; Graves Disease. Treatment with Radiolodine $\mathbb{I}^{131}$, Mayo H. Soley, EarI R. Miller, and Nadine Foreman; Indicator of Thyroid Function in Man, Widney C. Werner, Edith H. Quimby, and Char lotte Schmidt; Histologic Types of Thyroid Carchoma and Their Respective Abilities to Store Radioactive Iodine ${ }^{131}$ as Demonstrated by Radioautograms, Parrick J. Fitzgerald; Radioautographs: Methods and Uses, Titus C. Evans; Studies on Dosage in Cancer Therapy, L. D. Marinell and Ruth F. IIIl; Clinical. Methods and Resultr of Radiolodine Therapy in Metastatle Thyrotd Cancer, S. M. Seidlin; ${ }^{131}$ as 2 Therapeutic Agent in Cancer of the Thyroid, J. B. Trunnel1. (NSA)

852

Chapman . M. TREATMENT OF GRAVISE DISEASE

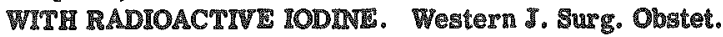
Gynecol. 58, 1-64(1048).

Radioactive lodine is prepared by the nuclear bombardment of tellurium with deuterons in the cyclotron. The principal isotopes produced are I ${ }^{130}$ (half-life of twelve hours) and I'191 (half-life of eight days). From observationg in treating 45 patients with diffuse goiters and hyperthyr oidism, Chapman belleves that sadioactive jodine with 2 half-11fe of twelve hours is an effective single thera- 
peutic agent. Like other methods of therapy it has certain limitations. Its possible late toxic effects will have to be observed with the passage of time. Contraindications to its use seem to be pregnancy beyond the fir st month, and dis853 ease of the kidneys. (NSA)

Feller, D. D., I. L. Chalkof, Alvin Taurog, and Rardin B. Jones. THE CHANGES INDUCED IN IODINE METABOLISM OF THE RAT BY INTERNAL RADIATION OF ITS THYROID WTTH TODINE 131. Endocrinology 45 , 464-79(1949).

Adult male rats were injected intraperitoneally with esther $24,30,300$, or $875 \mu \mathrm{c}$. of carrier-free $I^{131}$ and macriflced at $1,2,3,6,8$, or 10 days thereafter. A method for estimating the dose in rep is presented. Criteria used as indicators of metabolic changes in the thyroid gland were: (1) concentration of protein-bound I in plasma, (2) chemical iodine content of the gland, (3) retention of the initial $I^{131}$ by the gland, and (4) capacity of the gland to concentrate a gecond injection of $\mathrm{I}^{131}$. The earliest indicator was the concentration protein-bound I in plasma. After $875 \mu \mathrm{c}$ of $\mathrm{I}^{131}$ a triphasic curve of response was observed. At 24 hr post injection a decrease occurred followed by a sharp rise, with highest values on the third post-injection day. The plasma I then fell to thyroidectomy levels. Injection of $300 \mu$ of $1^{131}$ decreased the concentration of protein-bound I in plasma, and was not followed by a rise. Changes in $\mathrm{I}^{127}$ of the thyroid gland appeared two days after 300 or $857 \mu \mathrm{c}$ of $1^{121}$ and the gland was depleted by the sth day. The percentage of initial $\mathbb{I}^{131}$ one day post-injection was the same regardless of dose, but at the end of 2 days the glands receiving larger doses of $1^{131}$ were losing it rapidly. Rats treated with $300 \mu \mathrm{c}$ and given a second dose 3 days later collected only about $1 / 10$ as much as controlig given an initial dose. Thyroid function did not appear disturbed in the rat up to 10 days after 24 or $30 \mu \mathrm{c}$ of $\mathrm{I}^{131}$. (CA)

Findlay, D. and C. P. Leblond. PARTLL DESTRUCTION OF RAT THYROID BY LARGE DOSES OF RADIOIODINE Am. J. Roentgenol. Radium Therapy 59, 387-\$5(1948).

Two adult female albino rats, kept on a low lodine diet Lor ten weeks, were given single doses of 78.5 and 90.8 microcuries of carrier-tree radiolodine, $I^{191}$, respectively. Thyroid function was estimated by basal metabolic rate and heart rate determination. After six days the animals were sacrificed and the thyroid histology studied by means of stained sections and autographs. It was calculated that about 20,000 equivalent roentgens were receiwed by the thyrold glands of the animals. Such doses of radio-iodine inapais the function of the thyroid, as suggested by some decrease in oxygen consumption and heart rate of the asimals. The higtological study of the thyroid glands of these animals shows destructive lesions of the follicles especially in the central areas of the gland. Many peripheral follicles retain normal appenrance. Autographic studies show that only the normal looksing follieles with smooth colloid have the ablity to retain radiolodine. Although the thy roid gland is resistant to the action of radiations, it can be destroyed by the isotope $I^{131}$. The damage is favored by the retention of radioiodine inside the thyroid follicle until they are marlediy damaged. (NBA)

Furth J. and W. T. Burnets, J" HORMONE-BECRETMV FRANSPLANTABLE MEOPLAMIS OF TIV PITUITART INDUCED BI ISI. Proc. Soc. Repti. Biol. Med. 76, 2푼
Pituitary growths induced by thyroid destructive doses of $1^{131}$ are readily transplantable in mice whose thyroid glands had been similarly destroyed, but not in normal mice. These growths discharge gonadotropic hormones which in turn stimulate the production of gonadal hormones. Discharge of thy rotropic hormones by the se tumors is assumed but remains to be demonstrated. On the basis of observations made it is postulated that the growths of the pituitary induced by thyroid-destroying quantities of $1^{131}$ are conditioned neoplasms formed by cells which are driven to proliferation through the gtimulus created by the absence of $\mathrm{TH}$; the same chromophobe pituitary cell can discharge both gonadotropic and thyrotropic hormones. 856

Godwin, John T., B. J. Duffy, Jr., Patrick J. Fitzgerald, J. B. Trumell, and Rulon W. Rawson. PATHOLOGLCAL EFFECTS AND RADIOAUTOGRAPHIC LOCALIZATION OF ISI IN HUMANS. Cancer $4,936-51$ (1951).

Radioautographic localization and pathological findings in postmortem studies of nine patients who were given carrier-free $1^{131}$ in varying doeses and intervals prior to death are reported. Radioautographs were negative on all organs except the thyroid, neoplastic thyrold lealons, the corpora amylacea of the prostate in one case, and the submaxillary-gland ducts and spleen in another. The degree of $I^{131}$ pickup and histological effects varied widely in lesions of similar histological pattern in which localiation would be anticipated. No effects directly attributable to $1^{131}$ were seen in tissues other than thyroid carcinoma, metastatic deposits, and marrow in one instance. (NSA) 857

Goldberg, R. C. and I. L. Chaikoff. THE CYTOLOGICAL CHANGES THAT OCCUR IN THE ANTERIOR PITUITARY GLANDS OF RATS INJECTED WITH VARIOUS DOSES OF $7^{191}$ AND THEIT SIGNITCANCE IN THE ESTIMATION OF THYROID FUNCTION. Endocrinology $46,91-104$ (1950).

A cytological study of the pituitaries of rats injected with $18,300,525$, and $875 \mu \mathrm{c}$ of $\mathbb{I}^{131}$ is presented. The injection of $18 \mu \mathrm{c}$ of $1^{131}$ reaulted in no cytological changes in the anterior pituitary and is concluded to be a safe tracer dose. Evidence is presented to show that the changes occurring in the anterior pituitaries of rats atter the injection of large amounts of $1^{131}$ are not the result of radiation damage to the pituitary ifself but solely the consequence of thyroid destruction. The injection of 300

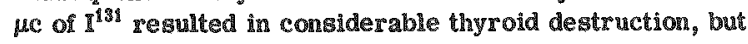
5 to 8 months later, normal glandular function was regained. From a study of the reaction in the anterior pitultary, it was shown that the atypical thyroids present 6 to 8 months aiter single injections of $525 \mu \mathrm{c}$ of $1^{13 i}$ synthesize negligible amounts of thyroxine or none at all. The cytological changes occurring in the anterior pituitary after the injection of $875 \mu \mathrm{H}$ of $\mathrm{I}^{131}$ are concluded to bo identical with those occurring after total surgical thyroidectomy. (NSA)

858

Goldberg, R.C. and I. L. Chankoff, DEVELOPMENT OF THYROM INEOPLASS IN THE RAT IOLLOWNG A SINCLE INJECTION OT RADIOACTIV IODINE. Proc.

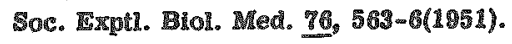

Tyamination of 10 rats sacrificed 18 months after a Bingle injection of $400 \mu \mathrm{c}$ of radioactive I revealed the following changes in the thyroid gland: (1) Hitrthe-like elements (previously reported by the authors in thyroids of rat macrificed 8 months after a gingle ${ }^{131}$ injection) were 
evident in the parenchyma of all glands. (2) Mutiple adenomata, apparently benign in nature, were evident in the parenchyma of all glands. (2) Multiple adenomata, apparently benign in nature, were found in the thyroids of 2 rats. (3) Two types of anaplastic, nonencapsulated neoplasms, one of which was invading adjacent thyroid tissue, were also found in the thyroids of these 2 rats. (NSA) 859

Goldberg, R. C., I. L. Chaikot, Stuart Lindsay, and D. D. Feller. MISTOPATHOLOGICAL CHANGES INDUCED IN THE NORMAL THYROID AND OTIER TISSUES OF THE RAT BY INTERNAL RADIATION WLTH VARIOUS DOSES OF RADIOACTIVE IODINE. EROcrinology $46,72-90(1850)$.

Rats of the Long-Evans strain were given single intraperitoneal injections of $18,300,525$, or $875 \mu \mathrm{C}$ of $\mathrm{I}^{\mathrm{f} 31}$. Autopsies were performed at intervals of three days, and $1,5,6$, and 8 months after the injection. No evidence of damage to any tissue was observed as early as 12 to 24 hours after injection in the thyroid glands of animals receiving 300 or $525 \mu \mathrm{c}$ of the isotope. The process consisted of five stages: degeneration and necrosis of epithelial cells with follicular dissuption; vascular degen eration and thrombosis; acute and chronic inflammatory changes: fibrous organization; and, epithelial regeneration. The thyroids of rats subjected to 875 je of the isotope responded in a similar fashion, except that no epithelial regeneration occurred; regeneration to normal thyroid Btructure was found in the animals which had received 300 He of I $^{131}$, but atypical thyrold cells (resembling Hurthle cells) were present 5 to 8 months after the $525 \mu \mathrm{c}$ administration. Viable-appearing parathyroid glands were demonstrated in all the injected animals, with some 11brosis apgearing only in the higher sotope concentration groups. Evidence of tracheal damage, and slight reversible changes in the lidneys were noted; normal livers were demonstrated. (NSA)

860

Gorbma, Aubrey. EFPECTE OF RADIOTOXC DOSAGES OF I' ${ }^{131}$ UPON THYROID AND CONTIGUOUS TISSUES IN MCE. Proc. Soc. Expt. Blol Med. 66, 212-13(184\%).

Radloactive I ( I $^{131}$ as $\mathrm{NaI}$ ) was given in a single subcutaneous injection in doses ranging from $3-50$ millicur ies/lgg. Higher doses produced complete thyroid destruction within a few days. Lower doses permitted survival of some thyroidal epithelium in the ishmus and cranial apes of the gland for as long as 120 days after injection. Lesions were noted in the tracheal epithelium with all doses, and in the recurrent laryngeal nerve with higher dogages. (BA) 861

Gorbman, Aubrey. TUMOROUS GROWTHS IN THE PITUITARY AND TRACREA FOLLOWING RADIOTOAIC DOSAGES OF I" Proc. Soc. Expt!. Biol. Med. 71, 237-40(1949).

Following doses of radioactive lodine sufficient to cauge destruction of most or all of the thyrofd, tumorous enlargements were found in the pituitary glands and tracheas of mice. Hypophyseal growths as large as $240 \mathrm{mg}$ (control, $2.1 \mathrm{mg}$ ) occurred. Less than 10\% of animals sacrificed mose than 250 days after I $^{131}$ administration had pituitaries weighing less than 10 mg. Tracheal tumor of the fibrous tunica propria, obstructing the tracheal lumen, were of lower incidence (4\%) but apreared earlier. Fibrous thickenings of the tunica propria and metaplastic regeneration of tracheal epithelium were more common. This study is based on 158 mice observed for a period of time surfictent to show the metaplastic and atypical growth changes, as well as 34 untreated controls. (R)
862

Gorbman, A. DEGENERATIVE AND REGENERATIE CHANGES IN THE THYROLD GLAND FOLLOWWG HZGGR DOSAGES OF RADIOACTIVE IODINE. Trans. N. T. Acad. Sei. 2, 201-3 (1949).

The results of experimental studies to determine the degree of thyroidal and other tissue injury per given amount of radioiodine are given. Mice of C57-CFW-strain, whose thysold glands had an average weight of $5 \mathrm{mg}$, were uged. They were injected subcutaneously with $100,200,400$, or 1000 mc doses. The "biological half-life" of the iodine was 2 days. By calculation, it was decided that the doses delivered were 26,000 and 75,000 equivalent $x$. The mice were sacrificed at intervals of $2,3,7,24,120$, and 300 or more days after the injections. The severity of injury to the thyroid after two or three days varied from minimal to complete destruction within the 100-1000 me range of dosages. Under speciffed circumstances, $200 \mu \mathrm{c}$ or more of $1^{131}$ cause complete thyroidal destruction in mice. Doses of $100 \mu \mathrm{e}$ permit survival of amall bits of atypical thyroidal tissue, which even after 400 days show no tendency toward regeneration. (NSA) 863

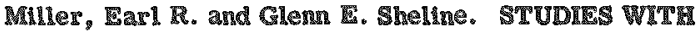
RADIOIODINE. III. PROBL TREATMENT OF HYPERTHYOIDM. Radiology 57 $720-8(1951)$.

Attempts to predetermine radiation dose of radiolodine, expressed in roentgens of radiation to the thyroid gland, in the reatment of hyperthyroidism are reviewed. Digcrepancies in data obtained from test and therapeutic doses are discussed. Results of treatment of hyperthysoid patients with $I^{131}$ are presented in tabular form. (NSA) 94.

Rugh, Roberts. THE ETIECT ON THE NURSHC MOUS OF THIRAPEUTIC DOSES OF I'194 P.168-9 of INTEY NATIONAL CONGRESS OF RADIOLOCY, 64h COngress, London. 1050.

gighty per cent of radioactive iodine ${ }^{181}$ injected subcutaneouly into the adult mouse, is ercreted within 24 hours. Most of the remaining iodine is concentrated in the very small thyroid gland. If the mouse is mursing, the young begin to remove lodine in the milk within 8 minutes of the mother being injected, and at a rate guch that within 4 hours the content of iodine in the litter may exceed that retained by the mother. This indicates that the milk functions as an accessory excretory channel, so that the thyxold of the nursing mouse recelves relatively less iodme than does the thysoid of the non-nuraing mouse. The ratio of iodine concentration in the major tissues of the nursing as oposed to the non nursing mouse 4 hourg after injection is approximately a follows: mammaxy $3 / 1$; thyyodd $1 / 7$; stomach $1 / 8$; and carcass 1/4. It is quite obvious therefore that the distribution of radio-iodine is drastically altered by the mere fact of lactation. Mouse thyroids examined histologically at intervals following a single injection in doses ranging from 3,10 to $20 \mu \mathrm{c} / \mathrm{gr}$. body weight, show no inmediate damage. Whithin one month, however, the thyroids of both the mothers and the nursing young were severdy damaged, though the irradiation damage to the thyrolds of the young was sonewhat less than that aeen in the thysoid of the mothex. This may be due to the lower concentration of the radioiodine rather than to any differential radiosensitivity of the two age group thyroids. Controls consigted of mice treated with the same concentration of lodme which was not radio-active. Damage to the thyroid begins at the centre of the gland and progresses outward; the peripheral follicles are the least affected and the lagt to be damaged. 
At the higher dose level a fibro-necrosis develops beginning in the centre of the gland, which involves particularly the interfollicular tissue but also damages the contiguous follicles. This differential effect is due to the fact that homogeneously distributed beta says have an accumulative effect, causing maximum damage at the centre of the gland. The maximum range of the beta rays is 2 to $3 \mathrm{~mm}$. and the concentration range is from 0.5 to $1.0 \mathrm{~mm}$. in tissue, so that the mouse thyroid is particularly suited to demonstrate this beta ray effect since its thickness is only slightly greater than the effective beta range in tissue. The $0.1 \mathrm{~mm}$. thick thyroid band which joins the two lobes posterior shows no such additional effect, since îts thickness is much less than the beta ray concentration range. The parathyroid glands are not directly affected, but gince the surrounding tissue becomes necrotic and the blood supply to the parathyroid is affected these paired glands are secondarily damaged. There is no radio-autographic evidence of any 865 adio-iodine concentration in the parathyroids.

Rugh, Roberts. RADIOIODINE AND HISTOPATHOLOGLCAL EFTECTS. J. Morphol. 89, 457-99(1951).

Histopathological effects on the thyroid glands of nursing adult mice and their suckling young, following the injection into the mother of $\mathrm{NaI}^{131}$ in doses ranging from 3 to $20 \mu \mathrm{c}$ pex gram of total body weight, at a time when the litter young were but three days of age, are discussed in detall. The thyroid gland of the mothers and their young were studied histologically at two months and at 10 weeks, follow386 ing a single treatment with $\mathbb{I}^{199}$. 55 references. (NSA)

Watts, Wulliam E. and Don R. Matheson. STUDIES ON LYMPHOCYTES FROM PERSONS TREATED WTY RADIOACTIVE IODINE. I. Lab. Clin. Med. $35,885-9$ (1950).

A study was made to determine whether the number and frequency of refractile cytoplasmic granules lnown to be pregent in $34 \%$ of the normal lymphocytes increase on exposure of the host to ionized radiation. Samples of blood were obtained from the capillaries of two patients with cancer of the thyroid and from eight patients with exophthalmic goiter before the administration of therapeutic doses of $I^{131}$ and at varying intervals thereafter. Further control studies were made on blood from normal persons and from patients with other diseases. Samples were also taken from three technicians who had been exposed to ir radiation for 8 to 27 years and from four patients who were receiving massive doses of $x$-rays in the treatment of malignant diseases. The number of refractile granules in each sample of blood were counted. A significant change in the number of frequency of the refractile granules in lymphocytes from persons exposed to ionized irradiation was not found. A search was made for other concise or suecific qualitative cytologlc effects of ixxadiation, but none was found. The refractile granules were found to be best studied in sresh, unstained preparations by phase microscopy. (NSA)

Iron

867

Stewart, W. B., R. T. Snowman, C. L. Yuile, and G. H. Whipple. RADIOLON EXCRETION BY THE SKUN AND KDDNEX OF DOGS. Proc. Soc. Wxpt. Blol. Med. 73, 473$5(1950)$.

After intravenous or oral administration of single doses of $\mathrm{Fe}^{55}$ to dogs, little or no $\mathrm{Fe} \mathrm{e}^{55}$ could be washed from the skin and hair; this fact indicates that no significant amount was excreted through the skin in 1-7 days. A small amount was found in the urine. (CA)

\section{Lanthanum}

868

Overstreet, $\mathbb{R}$., L. Jacolson, K. Scott, and $\mathbb{H}$. Fisher. RADIO IANTSANUM (LAA9). Argonne National Lab. [nd] 12p. (MDDC-1142-A)

Preparation of Radio Lanthanum without Carrier $-\mathbb{A}$ satisfactory method for the preparation of $\mathrm{La}^{140}$ has been successfully developed. The twelve day $\mathrm{Ba}^{1 / 4}$ has beer separated from fission products with the use of inert $\mathrm{Ba}$ as a carricr. Following the preparation and purification of the $\mathrm{Ba}^{140}$ the radio lanthanum was separated from the former using ferric hydroxide as carrier. The iron was subsequently removed by extraction with isompopyl ether. Decay curves have been measured on $\mathrm{La}^{180}$ samples thus separated and the half-life found to be 40.2 hours. No detectable long-lived tail was observed in samples followed over as long an interval as seven half-lives. This indicates that there was present less than 1 th of any longlife impurities such as radio barium or radioactive rare earths. The half value thickness for the beta-rays using Al absorbers was found to be 86 milligrams per square centimeter. Tracer Studies - The distribution of La ${ }^{140}$ following intraperitoneal and intramuscular injection was followed for a period of eight days. A significant proportion of the injected materlal remained unabsorbed by both routes of administration. The liver and skeleton were found to show the highest accumulation of this element. The uptake per gram of tissue in the liver was considerably higher than in the case of $\mathrm{X}^{86}$. The uptake by the bone par gram of weight was significantly less in the case of La ${ }^{140}$. However, the $\mathrm{La}^{140}$ was accumulated in the bone and was firmly fixed in this organ. No significant absorption took place by way of the digestive tract. When La ${ }^{180}$ was introduced directly into the lungs it was found that a large proportion was firmly fixed in this structure. (ADD)

\section{Phogphorus}

869

Abels, J. C., J. M. Kenney, L. Craver, L. D. Marinell, and C. P. Rhoads. POSTIRRADIATION CHANGLS IN THE LEVTLS OF ORGANIC PMOSPHORUS IN THE BLOOD OF PATIENTS WITL LEUKEMA. Cancer Research. 1, 771-5(1941).

The ingestion of a subtherapeutic dose of radioactive 8 (123) by 5 leulsemic patients was in each instance followed by a rapid and maxked change in the conc. of the organic acid soluble phosphorus of the leukocytes and erythrocytes. All detns. were made by the method of Fiske and Subbarrow. No such alterations were observed after the ingestion of a comparable amount of non-radioactive $P$ $\left(P^{31}\right)$. That these changes were due solely to the radiation delivered by the radioactive isotope was confirmed by observing similar changes in the organic acid soluble $p$ fraction in the blood cells of these same patients after the adm. of either $3 x$ (air) whole-body $\mathrm{x}-\mathrm{ray}$, or $20 \mathrm{r}$ (air) s-ray to the precordial area. These observations indicate that the radiation delivered by "tracer doses" of radioactive isotopes, used to study metabolism, may, in some instances, disturb normal cellular function, in which case the study would reflect radiation rather than normal 870 metabolian. (BA)

Anthony, D. S. and R. H. Snyder. ACUTE RADIOTOXICITY OF INJECTED P ${ }^{32}$ IN MICE. PART I. METABO- 
LISM AND SURVIVAL. Argonne National Lab. Feb. 11, 1247. 16p. (MODC-881)

The results of pilot experiments on the metabolism and radioloxicity of radioactive phosphorus are reported in the paper. Data were obtained on the toxicity of a well -known radioactive element in the same strains of animals and under the same experimental conditions as data obtaned with less familiar radioactive elements. Determinations of the relative radiotoxicity of different isotopes could therefore be made without reference to work done under. different conditions. Since the effects of radioactive phosphorus on man has been studied, comparisun of these effects with those on laboratory animals provided a basis for extrapolation with other radioactive elements. The experiments with radiuactive phosphorus greally facilltated planning and interpretation of later exueriments on the toxicity of other heretofore rare radioactive elements. (ADD)

871

Blume, James Mog C. E . Hagen, and Ruth W. Mackie. RADIATION INJURY TO PLANTS GROWN IN NUTIIENT SOLUTIONS CONTAINING $\mathrm{P}^{32}$. Soll Sci. 70, 415-26(1950).

Experiments are described in which young barley plants were grown in nutrient solutions containing various amounts of $\mathrm{P}^{32}$. The lengths of leaves and the dry weights of tops and roots were used as the criteria of injury caused by the radioactive material. Meristematic regions of the plants were examined histologically, and photomicrographs of normal and of injured root tips and apical buds are shown. It was found that damage to the tops was more severe than damage to the roots, and that the injury was due primarily to radiation from the $\mathbb{P}^{32}$ accumulated within the plant, the $\mathrm{P}^{32}$ which remained in the nutrient solution having relatively little effect. There was no evidence of damage to cells other than those which were in zones of active division. Absorption of phosphate from solution was not affected by radiation injury. The lowest level at which damage was produced under the experimental conditions employed corresponds to $5.6 \mathrm{me}$ of $\mathrm{g}^{32}$ per gram of $\mathrm{P}_{2} \mathrm{O}_{5}$. (NSA)

872

Bould, C., D. J. D. Nicholas, and W. D. 空. Thomas. RADLATION EPFECTS IN PLANT NUTRITION EXPERMENTS WITH PHOSPHORUS 32. Nature 167, 140-2 (1951).

Concentrations of $\mathrm{P}^{32}$ far greater than is normally used in tracer work and with much higher specific activities, i.e., ranging from 12 to 29,760 microcuries $\mathrm{p}^{32} / \mathrm{g}$. $\mathrm{P}^{31}$ produced very small radiation effects which are not of sufficient magnitude to preclude the use of $\mathrm{P}^{32}$ in plant 873 nutrition experiments. (CA)

Burstone, M. S. STUDTES ON TPE ALTERATION ON DEVELOPMENT OF THE TEETR AND ADJACENT STRUCTURES FOLLOWING LOCAI ADMINISTRATION OF CMROMIC PHOSPHATE CONTAINING PHOSPHORUS ${ }^{32}$. J. Am. Dental Assoc. 42, 418-21(1951).

The suspension of $\mathrm{Cr}$ phosphate had an approximate activity of 1 millicurie per ce; 0.05 cc. was injected subcutaneously into each of 30 mice, adjacent to the left mandible; examination was made 8-60 days later and revealed changes in both histodifferentiation and morphodifferentiation. The alterations in development included distinct hemiatrophy of the face on the injected side and occlusal imbalance of the incisors, and were also similar to those produced by systemic injection of solutions containing soluble radioactive $\mathrm{P}$ compounds. (CA)
874

Cornatzer, W. E., George T. Harrell, Jr., David Cayer, and Camillo Artom. SUBACUTE TOXICITY OF RADIOACTIVE PHOSPHORUS AS RELATED TO THE COMPOSITION OF THE DIET. Proc. Soc. Bxptl. Biol. Med. 73, $492-5(1950)$.

In the present investigation the subacute toxicity of $\mathrm{P}^{32}$ given parenterally to mice (in one dose in the form of $\mathrm{Na}_{2} \mathrm{HPO}_{4}$ ) was studied in relation to the possibility that changes in the composition of the diec might modify the action. It was found that in mice maintained on a $10 \%$ casein, $32 \%$ fat diet, the $\mathrm{LD}_{50}$ dose at the 21 st day was 4 to $6 \mu \mathrm{c}$ of $\mathrm{P}^{32} / \mathrm{g}$ of body weight; neither the fatty infiltration of the liver or its grevention (by supplementing the diet with choline) changed the survival figures. The survival time of the mice was highest when they were maintained on a diet low in fat and protein and was significantly decreased when the level of either the fat or the protein or both was increased in the diet. Enrichment of the diets with inorganic phosphate seems to afford a moderate degree of protection against the injurious action of $\mathrm{p}^{32}$. (NSA)

875

This abstract has been intentionally omitted. 876

DEN, J. N. LMMB REGENERATION IN TRITURUS VIRIDESCENS AS AFTECTED BY LETHAL DOSES OF RADIOACTIVE PKOSPHORUS. Anat. Record 105, 325-35(1949).

Phosphorus 32 injected intraperitoneally into adult male specimens of $T$. viridescens was lethal in single doses of 33,66 , and 166 microcuries per $g$ of body wh. The average lengths of time which the animals of the 3 dosage groups lived after injn. were respectively, 36,30 , and 25 days. Right forelinbs excised in the carpal region and reamputated 23 days later for histological study gave evidence of inhibited or greatly retarded regeneration. Distal tips of these limb stumps showed a scarcity of mitotic figures and distortion of epithelial cells. Blastemata were not present on the limb stumps from animals which had received 166 microcuries per $g$ and were either lacking or rudimentary on the ones from animals injd. with the lower dosages. (BA) $8 \%$

Deysine, A. and B. Bonet-Maury. BOLOGICAL EFFRCTS OF TRE BETA RADIATION OF $\mathrm{P}^{32}$ ON B. COLI Compt. rend. soc. biol. 145, 1091-\$(1951).

B. coli in nutrient media containing 0.1 me of $\mathbb{P}^{32} / \mathrm{ml}$ was carried through seven generations. The decrease in number of living cells was observed in each generation for 150 days. Development of radioresistance in the later generations was noted. Increasing the $\mathrm{p}^{32}$ concentration to $1 \mathrm{mc} /$ ml caused a much more rapid decrease in viablity. No distinction in respiration rates could be found by the Warburg method between proliferating and inactivated cultures. (NSA)

878

Down, C. A., B. I. Wiseman, C. S. Wright, J. Re. Geger, W. Myers, and J. W. Myers. SIX-YEAR CLINICAL EVALUATION ON RADIOPMOSPHORUS $\mathrm{p}^{32}$ (IN DISEASE). J. Lab. Cln. Med. 32, 943-69(1947).

A eritical review of 100 cases treated with $\mathrm{P}^{32}$ is given. The main success of $\mathrm{P}^{32}$ has been in polycythaemia sera, in which the clinical and haematologic features were well controlled. In leukaemias, $\mathbb{P}^{32}$ was sometimes a valuable adjunct to other therapy, but in Hodgkin's disease it was without value. Deep bone pain and primitus may be controlled in some cages. (NSA) 
870

Erf, L. A. A NOTE ON THE EIIECT OF DERMAL AND ENTERAL IRRADLATION BY RADIO-PHOSPHORUS ON THE BLOOD LEVLLS OF DOCS. Am. J. Med SA. 202, $650-4(1941)$.

Beta-radiation (emitted by radio-phosphorus) applied dermally to dogs was followed by a slight in the absolute number of circulating lymphocytes; applied enterally, by significant decreases. (BA)

800

Finkel, Miriam P. THE RETENTION OF $\mathrm{p}^{32}$ BY YOUNG ADULT CF-1 IEMALE MICE. Argonne National Lab. Aug. 1, 1947. 172p. (ADCD-2024-I)

The retention of radiophosphorus by the animals comprising the singie dose chronic experiment was studied one month after injection. The external counts of those animals which recelved $0.1-0.5 \mu \mathrm{c} / \mathrm{g}$ ware too low to permit conversion to microcuries. The $10.0-0.5 \mu \mathrm{c} / \mathrm{g}$ groups gave adequate external counts. At these levels it was found that the absolute retention ranged from 1.2 to 1.7 per cent. (NSA)

881

F riedell, Hymer L. and James H. Christie. THE SYNER GISTIC EFTECT OF PHOSPHORUS ${ }^{32}$ AND COLLOHAL GOLO ${ }^{18}$ ON SURVIVAL IN MALIE ALBINO RATS. PYOC. Soe. Expt1. BLol. Mel. 76, 207-10(1950) (NYO-1609)

Data are presented which indicate that colloidal $\mathrm{Au}^{198}$ and $\mathrm{P}^{32}$ adwinistered as $\mathrm{Na}_{2} \mathrm{HP}^{32} \mathrm{O}_{4}$ are synergistic in their killing effect on the male albino rat. A discussion of the distribution of $\mathrm{P}^{32}$ and colloidal A $\mathrm{u}^{198}$ and possible mecha382

Cellen, Abraham, Robert Loevinger, and Bernard S.

FOLF. SURT ACE ACTIVITY FOLLOWING ADMINISTRA WION OF RADIOACTVE PHOSPEORUS. Radlology 56, 857-68(1951).

Thirty patients with various malignant diseases were atudied, by means of an end-window Geiger-Muller counter, for $\beta$-particle surface activity following oral or intravenous administration of radioactive $\mathbb{P}$. Surface activity appear at once, reaches a maximum between 3 and 50 min. after intravenou administration, and decrease apgroximately exponentially after 24 hours. Schematic surface activity curves for vartous areas are preanted. The extreme variation in aurface activity between sym. normal areas 1. about 15\%, while the activty over the calvarium and the nipples is characteristically about $100 \%$ higher than over other areas. Over ulcerated and nonulcerated cutaneous malignant tissue, the surtace activity may be 2 to 5 times that in. normal areas. This differential appears whthin a few minute after aduinistration. Sur ace activity over Iymph node metastases was in some patients higher than, and in other patients not appreciably different from, the control areas. Surface activity of the cervix was 3 to 4 times that of the thifh in normal patients, but was at least 6 times that of the thish in 3 cases of carcinoma of the cerwis. This ratio was reduced ater dadiotherapy. The meatrement of ฮuriace $p$ activity following adminitration of inorgante $\mathbb{P}^{2}$ does not appear to be necesmary for the dagnosis of cutaneous maligmant lesions, nor reliable for the diagnowis of gubeutanens ledions or metastatie lymph 383 nodes. (CA)

Grad, and C. E. Stevens. HITOLOGICAL CIANGES

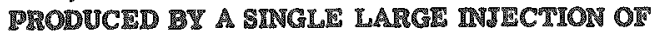
TADIOACTIV PHOSPRORUS (P') IN ALBNO RAT AND IN C3A MCE. Cancer Rasearch 10, 239-96(1850).

Twenty-two normal young male and female albino rat and eight aduit female C3r mice received a single large dose of radiophosphorus, $\mathbb{P}^{32}(0.5$ to $4.0 \mathrm{mc})$, and were sacrificed from two hours to nine days later. The cellular degtructions consisted mostly of pyknosis and karyor rhexis of mitotic nuclei. The cytoplasm of damaged cells showed vacuolation and reduced staining ability. The destructive cffects of $\mathrm{P}^{32}$ were most marked in the lymphatic organs and bone marrow, the small intestine, granulosa of ovarian follicles, and mammary tumors. In the crypts of the small intestine, massive degeneration and pyknosis of dividing cells resulted in thinning of the epithelim of crypts and villi, probably because no new cells were supplied from the crypts to balance the continual loss of cells by extrusion normally occur ring at villi tips. The mammary tumors showed many scattered pylnotic cells at 24 hours after injection; at 48 hour 8 , large blood lakes had formed without much tissue damage; at nine days, most of the fumor was necrotic and liquefied. Lesser changes were noted in the stomach and colon, in the seminiferous tubules of the testis, and in the hair follicles; little or no damage was observed in the salivary glands, pancreas, liver, genitourinary system, endocrine glands (excent the ovary), brain, 884 and muscular and connective tissue. (NSA)

Graff, W. S., 1K. G. Scott, and J. H. Lawrence. HISTOLOCICAL EFTECTS OF RADIOPHOSPHORUS ON NORMAL AND LYMPHOMATOUS MCE, Am. J. Roentgenol. Radium Therapy 55, 44-54(1946).

Mice were injected intravenously with $1.2 \times 10^{7}$ lymphoma cells and were given varying amounts of $\mathrm{P}^{32}$ in isotonic sodium phosphate solutions 7 or 13 days after injection. The results ghowed an initial depressing action of $\mathrm{p}^{32}$ on the lymphocyte series in the peripheral blood stream. After four days, when the granulocytes begin to drop off markedly, there already was an increasing deposition of $\mathrm{P}^{33}$ in bone. The retention of $\mathbb{P}^{82}$ in bone is of much longer duration than in lymphatic or any other tissue. There is no direct evidence that the neoplastic cells are more radiogensitive than other cells. It was found that, at corresponding stages in the lymphomatous process, the treated animals had less infiltration of organs by leukemic cells than the non-treated groups. In both the normal and leuRemic anmals, the greatest drop in white blood cells occurred during the first four days after administration of $\mathrm{p}^{22}$. The chief cell lype to be affected is the lymphocyte and the lymphoma or leuke mic cell. Duxing the following five days the rate of drop diminishes, but during this period the greatest decrease in granulocytes occurs. Studies of the exchange of $\mathrm{P}^{32}$ in leukremic tissues of mice revealed a higher uptake than in other soft tissue. This greater uptake in enoplastic tissue is of interest. since these cells receive relatively more radiation. The predominant effects of $\mathrm{P}^{32}$ on leukemic as well as normal elements of the marrow constitute its limiting factor in its use in the therapy of leukemia and make it of doubtrul value in 285 lymphosarcoma and allied diseases.

Mershey, A. D., M. D. Famen, J. W. Kenmedy, and H. Gest. THE MORTALITY OF BACTERIOPHAGE CONTAINTC ASSIMLA TED RAOIOACTIVE PROSPRORUS. J. Gen. Physiol. 34, 305-19(1951).

Bacteriophage T4 containing assimilated $\mathrm{P}^{32}$ is inactvated at a rate proportional to the specific radioactivity of the constituent $\mathrm{Z}$. The $\mathrm{P}$ radiation from the $\mathrm{P}$ makes a neglighle contribution to this effect. The inactivation is ther atore a direct consequence of the nuclear reaction, which kills the phage will an efficiency of about $1 / 12$. Several phages related to T4 behave similarly. When radioactive phage is grown from a seed of nonradioactive phage, all the phage progeny are subject to killing by 
radioactive decay. The phage is killed by is radiation from $\mathrm{p}^{32}$ with an efficiency of about $1 / 100$ per ionization within the particle volume. Bacteriophage $T 4$ and its relatives contain about 500,000 atoms of $P$ per infective particle. Virtually all this $\mathbf{P}$ is adsorbed to bacteria with the specificily characteristic of the infective particles, and none of it can be removed from the particles by the enzyme desoxyribonuclease. The $\mathrm{P}$ content per particle, together with the published data on analytical composition indicates a particle diameter close to $110 \mathrm{~m} \mu$ for the varieties of 866 phage studied. (NSA)

Holler oft, Joanne Weikel and Egon Lorenz. THE 30DAY LD OF $_{60}$ TWO RADIATIONS OF DIF FERENT ION DENSITY. J. Nat. Cancer Inst. 12, 533-44(1951).

The 30-day $L D_{50} \times$ radiation was compared with that of the a particles emanating from shorl-lived decay products of radon injected intravenously in strain A male mice. The integral dose of $x$ radiation necessary to produce $50 \%$ death was found to be 1.42 times that of particles. Regression curves are given. The distribution of short-lived decay products of radon was followed for the $2-\mathrm{hr}$ period following infection. The distribution of the dose was calculated. (NSA)

887

Iu, T. N., S. G. Holmes, C. M. Pomerat, C. S. Livingood, and R. P. McConnell. A METHOD I OR STUDYING THE EFFCT OF $\mathrm{P}^{22}$ ON LIVING ADULT HUMAN ISTOERMAL CELLS IN A PERTUSION C and Med. 9, 738-48(1951).

Experiments are reported on the effect of $\mathbb{P}^{32}$ on living adult human epidermal cells when introduced in a nutrient fluid within a perfusion chamber designed to permit stilland motion-picture photographic recordings of cellular structure. This preliminary study is designed to illustrate the feasibility of the method with respect to observations 888 upon adult human tissues. (NSA)

Jones, H. B., C. J. Wrobel, W. R. Lyons. A METHOD OF DISTRIBUTWG BETA-RADITION TO THE RETICULO-ENDOTHELIAL SYSTEM AND ADJACENT

TISSUES. J. Cun. Invest. $23,783-8$ (1944).

Chromic phosphate is precipitated from $\mathrm{Na}_{2} \mathrm{HPO}_{4}$ and $\mathrm{Cr}\left(\mathrm{NO}_{3}\right)_{3}$ solns. buffered by NaAc. The washed, dried precipitate is heated to $600^{\circ} \mathrm{C}$. The heat drives of water of crystallization and converts the soluble precipitated $\mathrm{CrPO}_{4}$ into an inert ansorphous powder. This powder is ground to a colloidal state in isotonic saline by a glass-bead mill. The suspension (avg. particle size, $1 \mu$ ) is lnert and nontoxic. As much as one $\mathrm{mg} / \mathrm{g}$ body wt. is well tolerated by mice when given intravenously. Chromic phosphate can be prepared with radio-active $P$. Intraven. administered this material is taken up and retained by the reticulo-endothelal system; the $\beta$ radiation of $p^{32}$ is distributed to the endothelial system and adiacent tisaues. The material accumulates in the Hver, gpleen, and lungs. In the dog $90 \%$ goes to the liver, $6 \%$ to the spleen, and $2 \%$ to the lungs. In the mouse the bulk goes to the liver, but the apleen and lungs acquire more than the same organs in the dog. The $\mathrm{CrPO}_{4}$ is retained in the endothelial cells of mice sor at least a year without detected decrement. Radioactive doses which delete the gpleen do not aftect the liver. Radioactive doses of the order of 80,000 in the liver may induce hepatoma. The absorption of $\beta$ radiation in tissues is discussed with special reference to tissues less than $0.6 \mathrm{~cm}$ thick. (BA)

889

Kisieleski, Walter E. and William P. Norris. THE TEFECT OF BAL ON THE DISTRIBUTION AND MEPABO-
LISM OF P ${ }^{32}$ AND SR ${ }^{20}$. Proc. Soc. Exptl. Biol. Med. T7, 694-6(1951) 7p. (ALCU-1368)

The effectiveness of 2,3-dimercapto-1-propanol (British antilewisite, BAL) in increasing the elimination of injected $\mathrm{P}^{32}$ and $\mathrm{Sr}^{30}$ in mice was investigated. It is concluded that BAL is essentially without effect although, in the case of $\mathrm{p}^{32}$, the rate of uptake in bone appears to be reduced during the first hour. No such difference could be demonstrated after $24 \mathrm{hr}$. (NSA)

890

ISligerman, Morton M. THE RFFECT OF RADIOACTIVE PHOSPRORUS ON THE GROWTH OF THE ALBHO RAT. Am. J. Roentgenol. Radium Therapy 63, 380-95 (1250).

Five groups of 21 -day old Wistar Institute white male rats received $\mathrm{P}^{32}$ on the following basta: Group $\mathrm{A}(24 \mathrm{ratg})$ $44 \mu \mathrm{c} / \mathrm{kg}$ body weight; Group B (24 rats) $132 \mu \mathrm{c} / \mathrm{kg}$ body weight; Group C (24 rats) 400 ue/Rg body weight; Group D (24 rats) $1,200 \mu \mathrm{c} / \mathrm{kg}$ body welght; and Group $\mathrm{E}$ (20 rats) $3,600 \mu \mathrm{c} / \mathrm{ks}$ body weight. Five more grouss of rats, 42 days old, received corresponding doses. Other detalls of the experimental procedures are included. It was found that doses as small as $44 \mu \mathrm{c} / \mathrm{kg}$ of $\mathbb{P}^{32}$ retarded normal weight gain in the 21 -day old rat;; doses of $132 \mu \mathrm{c}$ retarded the growth of bone (iemux) and larger doses produced a greater effect: $4 \mu \mathrm{c} / \mathrm{kg}$ seemed to accelerate the growth. The effect of $\mathrm{p}^{32}$ on $\mathrm{grow}_{\mathrm{s}}$ (wetght and long bones) ceased when its concentration fell below a threshold dose; this level was reached within 42 day after the last injection. The effect of the $\mathrm{P}^{32}$ was found to be temporary, treated rats equaling normal rats in weight, stze, and appearance when 300 days old. However, when $1,200-3,600$ $\mu \mathrm{c} / \mathrm{kg}$ was given, the physical appearance and activity of the rats were affected, and half of the 42 -day old sats receiving 3,600 we/ $/ \mathrm{kg}$ died before reaching 112 days of age; the 21-day old rats showed depression of all the cellular elements with prolound changes in the white blood count. An increase in the densely calcitied area on the metaphyseal side of the epiphyseal line algo appeared. (NSA)

891

roletsky, SImon, and James I. Christie. BOLOGIC TH FECTS OF RADIOACTNE PHOSPHORUS PONONMG IN RATS. Am. J. Pathol. 2\%, 175-84(1951).

Lethal doses of $\mathrm{P}^{32}$ produced extensive morphologic lesions in xats. These were simllar to previously de scribed ffects of radiation, from either external or internal souree, in both animals and man. Similar changes result also from the nitrogen mustards. By virtue of its distribution, $\mathbb{P}^{32}$ acted as a powerful hemopoietic poison producing aplasia of the bone marrow and hypoplasia of the lymphoid sissues of the body, meluding spleen and thymus. This was reflected in the peripheral blood by rapidly developing leulropena, thrombocytopenia, and anemia. In addition, the intestinal tract, especially the small bowel, and the gonads were sensitive to radiation. Organs such as lver, pancreas, adrenals, kidney, heart, and brain were radiosesistant. Destruction of tissue occurred rapidly, being well developed from 6 to $12 \mathrm{hr}$ after injection of the $\mathrm{P}^{32}$. The principal raethod of cell degeruction was by karyorrhexis. Most of the resulting debris was removed by the ind or 3rd day. Involution of the hemopotetic organs was marked at the end of $84 \mathrm{hr}$ and was then progressive over the next aeveral days. During the period of necrosis and involution the animals usually were in good condition and asymptomallc. However, this latent interval was followed by progressive inasithon, debility, and weight loss, terminating in death within 3 weeks after the administration of the $\mathbb{P}^{32}$. At the time of 
death there were aplasia of bone marrow, atrophy of lymphoid tissues, and persistent leukopenia, but the hemopoietic often revealed beginning on even fairly gubstantial regeneration; the intestinal damage was largely repaired, whereas the injury to testes was progressive. In animals that recovered there was usually good repair of bone marrow and lymph nodes after a few months. Regeneration was less effective in spleen and thymus, and in the testis generally ranged from slight to negligible. One of the late effects of $\mathrm{p}^{32}$ poisoning was the development of malignant neoplasms. The mechanism of radiation death is not established. With massive doses of $\mathbf{P}^{32}$, after which death takes place within a few days, the outcome is usually attributed to a general toxemia resulting from extensive destruction of tisene. With $\mathrm{LD}_{50}$ doses of $\mathrm{P}^{32}$, aiter which death occurs within a few weeks, the almost constant presence of infectious lesions suggests that bacterial 392 togemia may be significant. 30 references. (NSA)

Lawrence, John H., B. V. A. Low-Beer, and James W. J. Carpender. CFRONC LYMPRATIC LEUKRMA; A STUDY OI 100 PATISNTS TREATPD WITI RADIOACTIV PHOSPMORUS. J. Am. Med. Assoc. 140, 585-8 (1949).

A gtudy of 100 cases of chronic lymphatic leuhemia treated with phosphorus-32 alone or in conjunction with roentgen rays is presented; the doses were usually $1-2$ me/week for $4-8$ weels, the treatment gertes being repeated if mecessary (but not to exceed about $50 \mathrm{mc}$ for the combined series). Comfortable life appears to have been prolonged as compared with the average life span after other methods of treatment. At the time of writing, $33 \%$ of the group have lived 5 or more years after onset and $10 \% 8$ or more yearg; 24 of the patienta are still living. When compared with 5-year end results in other types of neoplastic disease, such as carchoma of the breasts, prostate, lung, stomach, and egophagus, these results are concluded to be relatively good and do not encourage the prewalent hopeless attitude taken by many doctors confronted with the problem of treating chronic leukemia. (NSA)

Lisco, M., Miriam D. Fukel and G. Husch. PROGRESS REPORT ON THE CMRONIC $\mathrm{P}^{\text {I2 }}$ MOUSE IXXPRIMENT. Argone Natlonal Lab. Nov. 1, 194\%. 180p. (ACCD2000단)

The data that are being collected on the $\mathrm{CF}-1$ female mice that either received a single intraperitoneal infection or are receiving monthy injections of radiophosphorus are beginning to reach proportions which permit tentative analysis. At 290 days after the initial injection in each series (single and repeat doses), $20 \%$ of the fotal of 937 animals had died or had been sacrificed in a moribund condition. Although the sinal complete diagnosis of many animals must await histological study of one or more tisøues, 政 is posmible at this time to note some radiation efects. The tentative conclugions at this interval in the experiment (290 days) are that: (1) The incidence of Iymphoma is increased in the groups which received $10.0-5.0 \mu \mathrm{c} / \mathrm{g}$ and 1.0-0.5 (and probably 0.1 ) $\mu \mathrm{c} / \mathrm{g} / \mathrm{mo}$ of $\mathrm{P}^{92}$. There is no evidence at 290 days that animals receiving Low doses of radiophosphorus have a decreased probabisty of developing lymphoma. (2) The incidence of mammary tumors among $\mathrm{CF}-1$ female mice which received 10.0-0.01 $\mu \mathrm{c} / \mathrm{g}$ of $\mathrm{P}^{32}$ or which are receiving 1.0-0.01 $\mathrm{Mc} / \mathrm{g} / \mathrm{mo}$ is not diferent frow the spontaneous occurrence of mammaxy tumors in this gtrain. (3) The incidence of lung tumors is increased by $10.0-5.0 \mu \mathrm{c} / \mathrm{g}$ and $1.0-0.1$ $\mu \mathrm{c} / \mathrm{g} / \mathrm{mo}$ of $\mathrm{F}^{32}$. (4) Growth is retarded by $1.0 \mathrm{~m} 0.5 \mu \mathrm{c} / \mathrm{g} / \mathrm{mo}$ OR $\mathbb{2}^{2 *}$. (ADD)
894

Low-Beer, Bertran V. A. ETTERNAL THERAPEUTIC USE OF RADIOACTIVE PHOSPHORUS. I. ERYTHEMA STUDIFS. Radiology 47, 213-22(1946).

For the study of skin effects of radioactive $\mathrm{P}, 69 \mathrm{ex}-$ posures were made on the forearm and arm of 12 healthy subjects. The radioactive $\mathbf{P}$ was applied by using disks of blotting paper $2.5 \mathrm{~cm}$ in diam. and $0.4 \mathrm{~mm}$ thick, soaked with $\mathrm{Na}_{2} \mathrm{HPO}$, soln. Radioactive $P$ applied to skin in this manner produces erythemata showing a monophasic course. The intensity of the erythema depends on the amount of exposure. "Threshold erythema" was produced by an exposure to 34 microcuxie hours $/ \mathrm{sq} . \mathrm{cm}$. Dry, scale epidermite was produced by an exposure to 2,000 microcurie hours $/ \mathrm{cm}^{2}$; and bullous epidermite resulted from an exposure to 4,400 microcuric hours $/ \mathrm{cm}^{2}$. The depth of discernible biologic effect from externally applied radioactive $\mathbb{P}$ is approx. $5 \mathrm{~mm}$ for erythema producing doses. (BA) 895

LOW-Beer, Bertram V. A. RADIOACTIVE PHOSPHORUS AS AN EXTERNAL TIERAPEUTIC AGENT IN BASAL CELL CARCINOMA, WARTS, AND HEMANGIOMA. AM.

J. Roentgenol. Radium Therapy 58, $4-9(1947)$.

Superficial skin diseases were successfully treated with direct application of radioactive phosphorus $\left(P^{32}\right)$ to the area of the lesion. Office type blotting paper, $0.4 \mathrm{~mm}$ thick and weighing 21 mgn per square centimeter, is cut to the size of the lesion with a $0.3-1.0 \mathrm{~cm}$ margin and backed with adhesive tape and dried. It is then soaked in active $\mathrm{Na}_{2} \mathrm{HPO}_{8}$ solution, dried and secured to the area of the lesion with adhesive tape. The threshold erythema dose was found to be 34 we per $8 q \mathrm{~cm}$; mild erythema was produced by 620 uc per sq $\mathrm{cm}$ and severe epidermitis by $4.400 \mu \mathrm{c}$ per sq. cm. Treatment doses for basal cell carcinoma, hyperikeratosis, verruca, plantor wart, sub-

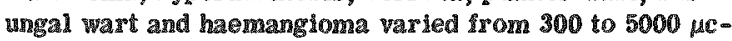
hr per sq cm depending upon the size and type of lesion. of the lesions treated, 88.6 to $100 \mathrm{ger}$ cent disappeared without recurrence. (FM)

8อง

Low-Beer, Bertram V. A. ISOTOPES: RADIOACTVE; RADIOPHOSPLORUS AND RADIOSODHUM P.45A-60 of MISDICA I PHYSICS, Vol. 2. Otto Glasser, ed. Chicago, Year Sook Publ., Ine. 1950. 122\%p.

$\mathbb{P}^{32}$ uptake and excretion are discussed for different routes; exchange problems, detection and measurement and therapeutic applications are considered. Clinical studies on uptake and excretion of $\mathrm{Na}^{24}$ show that equilibr îm is reached in three hours following administration but that exeretion is very slow. (R.D.B.) 8907

Martin, A. TME IFITCTS OF RADIOACTIVI PIOSPHORUS ON CRICETUS AUPATUS. Cenetics 35,679 (1950).

This paper is reproduced here in its entirety.

Males of the golden hamster, Cricetus auratus, have been given intraperitoneal injections of various dosages of radioactive phosphorus and subsequently mated to untreated ferales. Histological and cytological examination of the tissues of the treated animais is in progress, and progeny are under observation. The maximum sublethal dose lies between 6 and $9 \mu \mathrm{c} / \mathrm{g}$ of body weight. A few animals sur vive a dosage of $8 \mu c$, and recover from radiation slchess in about 11 days. Three microcurie induce permanent stenility in 30 days or less. Dosages of 3 or more $\mu \mathrm{c}$ result in the degeneration of Sertoli cells and the filling of the seminiferous tubules with fibrous connective tissue. One microcurie has no effect on aperms in the tubules in $24 \mathrm{hr}$, but in 15 days the tubules are devoid of sperms. In 20 days, however, the teates again appear normal, and 
the tubules contain apparently normal sperms. Following dosages of 1 and $3 \mu c$, the white blood cell count is much reduced. No statement is as yet available on effects on other tissues. The available progeny to date bear out the histological and cytological findings, in that none has been produced by males treated with 3 or more uc. When not completely destroyed, the germ plasm seems to be remarkably stable, in that progeny show no phenotypic abnormalities, litter size is unaffected, and ofispring are of normal viability. (NSA)

\section{8}

Muix, R. M. FFECT OF RADIATION FROM RADIOACTIVE ISOTOPES ON THE PROTOPLASM OF SPIROGYRA. J. Cellular Comp. Physiol. 19, 244-7(1042\%.

Beta radiation from radioactive $\mathbb{P}\left(\mathbf{P}^{*}\right)$ increased the viscosity of protoplasm in Spirogyra when the radioactive strength of the solution was 4.0 millicuries/1iter of 17.0 millicuries/liter but had no effect when the radioactive strength was 2.1 millicuries/liter. The total concentration of $P$ fluctuated in filaments immersed in solutions of $P$, but remained relatively constant for $0.001 \mathrm{M}$ and $0.005 \mathbb{M}$ solutions of the nonradioactive $\mathbb{P}$ and showed an accumulation for the $0.01 \mathrm{M}$ solution. Maxima occurred after periods of treatment which were shorter for the solutions which had freater radioactivity. Changes in total $\mathbb{P}$ content may result from permeability effects of beta radiation from $P *$ (CA)

O'Nell, J. B., J.R. Jowsey, C. C. Lee, and J. W. T. SpInks. DISTREUTION OF $\mathrm{P}^{22}$ IN CHICK EMBRYOS. Can . J. Research, D, 27, 223-32(1940).

By feeding a hen repeated daily doses of $\mathrm{NaH}_{2} \mathrm{PO}_{6}$ of equal $\mathrm{P}^{32}$ activity, eggs with high and nearly uniform $\mathrm{P}^{32}$ content were obtained for use in hatching expts. Eleven eggs were incubated. The embryos and chicks that died during or after incubation were dissected and analyzed for total and specific activities in the various fractions. Two of the chicks, both females, are still living. They are being raised so that observations may be made on their ability to reproduce and the effect of the radioac000 tivily, if any, on theis of mpring. (BA)

Pal, R. K. and B. N. Mitra. EFPITCT OF RADIOPHOSPEORUS IN MMNUTE DOSES ON BLOOD AND SOME IMPORTANT ORGANS. Indian $J$. Physiol. and Allied Sci. $4,42-9(1950)$.

Rabbits were injected with a preparation of radiophosphorus $\left(\mathrm{P}^{32}\right)$ in an alkaline $\mathrm{K}_{3} \mathrm{PO}_{4}$ solution. The animals did not change weight within 18 days. The entire hematopoietic function of the red bone marrow, however, was deleteriously affected by $\beta$ emanations. A mreat rise in the $\mathrm{Ca}$ content of the blood, through action of the tracer dose as an economizer and mobilizer of reserve supplies was found. There was no harmful action on the bone structure but adverse effects upon the thyroid gland, kidneys, and liver, were indicated. (CA)

Platt, R. ERIECTS OF RADIOACTVE $P^{32}$ ON NORMAL TISSUES. Arch. Pathol. 43, 1-14(1947).

Radioactive $\mathrm{p}^{32}$, administered as a dibasic alt to a weries of human patlents, produced characteristic changes in many tissues, varying in intensity but similar to those produced by other types of radiation. Prominent features of the changes were abnormal mitoses, producing giant irregular nuclel, fibrosis, hyalinization of collagen, thickening and hyalinization of small blood vessel and cellular death. Selective locationization of large quantities of radioactive $\mathbf{P}$ and greater injury was observed in tissues most often involved by neoplastic cells of malignant lymphomatoses and myelomatoses, i.e. bone marrow, liver, spleen, and lymph nodes. Changes were demonstrated also in the lungs, kidneys, gastrointestinal tract, ovaries, and testes. (EM)

902

Powers, E. L., Jr.o, Deborah Sheiner, M. J. Richard, and R. Sato. LETHAL ETIECTS OF $\mathrm{P}^{32}$ ON PARAMECIUM AURELIA. Argonne National Lab. Aug. 1, 1947. $17 \mathrm{p}$. (AECD-2024-P; ANL-4078)

Further results on the response of $\mathrm{P}$. aurelia to exposure to $\mathrm{P}^{32}, \mathrm{Sr}^{80,00}$ and $\mathrm{Y}^{80}$ have been obtained, and a partial summary statement of observations to date is presented. There is observed increasing death with increasing $\mathbb{P}^{32}$ radionctivity. Death following exposure to the Srolution increases with increasing activity. Comparison of gurvival following phosphorus exposure to that following strontum treatment at the same activity level, reveals that in all ingtances surwival of the P-treated animals is the same or significantly lower than survival of Sr-treated animals. From these data it appears that the P-treated animals are injured to a greater extent than the Sr-treated animals. (NSA)

903

Rubin, B. A. THE PATTERN AND SIGNIICANCE OF DELAYED PHENOTYPIC EXPRESION OF MUTATIONS WDUCED IN E. COLI BY ABSORBDD E: Genetics 35 $133(1050)$.

Btologically absorbed radioactive phosphorus $\left(\mathbb{P}^{32}\right)$ has been shown to induce gignificant increases in the proportion of streptomycin resistant cells among growing populations of Escherichia coli (strains $\mathrm{B}$ and $\mathrm{B} / \mathrm{x}$ ). Great diferences in the absorption of $\mathrm{g}^{32}$ by the bacteria have been produced by varying car pier phosphorus. Calculations show that large increases in concentration of $\mathrm{P}^{32}$, in a cell of bacterial dimensions, produce insignificant changes in specific lonization. The increase in mutation rate, however, is disproportionately high. When bacteria were grown in solutions of $\mathrm{P}^{32}$ (up to $8 \mathrm{me}$ per mi) the mutation rate increased with dose. But in all cases the relative size of the mutant population changed upon further cultivation. The pattern of change (diverse rates of increase, sometimes followed by decrease) varled with the concentration of $\mathbb{P}^{32}$ and with the amount of absorption, as controlled by carroler phosphorus levels. The complete phenotypic expresston of the mutations produced seemed also to depend upon the number of bacterial divisions, since similar changes dld not occus in resting cultures exposed in the same way, for similar periods. Thege patterns indicated a quantitative, as well as qualitative difference in distribution of mutant types produced by an effect which can not be accounted for by lonization alone. Calculations indicate that this effect may be attributed to the transmutation of $\mathrm{P}^{32}$ to $\mathrm{S}^{32}$. (G) 904

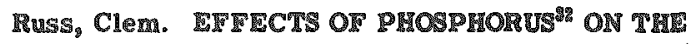
IIAMSTER. Proc. Soc. Exptl. Blol. Med. 74, 729-31(1250).

Effects of untraperstoneal injection of the hangter with radiophosphorus, $\mathbb{P}^{32}$, are described. Animals treated with $10 \mu \mathrm{c} / \mathrm{g}$ of body weight died in 11 days. There were no apparent changes in the teates in this time. Toxic effects were observed in animals injected with as little as $6 \mathrm{~m} / \mathrm{g}$. Damage to the testes of animals ireated with 6 and $8 \mu \mathrm{c}$ respectively, was extensive, consisting of marked decrease in glze, absence of spermatogenic cells in tubules, and actual damage to the tubules themselves, suggesting permanent sterility. No changes in the testes of animals treated with $1 \mu \mathrm{c} / \mathrm{g}$ were apparent in 24 hour. However, some damage was observed 30 cays after treatment with 
$1 \mu r$. Spermatogenic cells were more sensitive than Sentoli cells. Some interstitial cells appeared to be afreated. (NSA)

905

Russell, R. S., S. N. Adams, and R. P. Martin. RADIA-

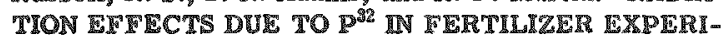
ME'STS. Nature $164,093-5(1949)$.

Barley was fertilized with the equiv. of $8 \mathrm{lh} \mathrm{p} / \mathrm{acg}$; this included from 0 to $4 \mu \mathrm{c} \mathrm{g}^{32} / \mathrm{pot}$, or $0-460 \mu \mathrm{c} \mathrm{p}^{32} / \mathrm{g} \mathrm{p}$. There were no significant fifects on ory wh. of shoot or root after 39 days; but at 4 He/pot there was a def rease in absorption of fertilizer $\mathrm{P}$, and oven at $0.8 \mu \mathrm{\mu l} / \mathrm{pot}$, a significant decrease in ratio of fertizer $P$ to soll $P$ absorbed. Increase of this ratio for shoots at 70 days (when activity had decayed to $3.4 \%$ of inisial) may have been correlated with root regeneration in the surface layers of soil, as more short adventitions roots were found. (BA)

906

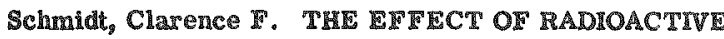
PLOSP YOUS UPON $\triangle$ SUSPENSION OF ISCMERICHIA COLI. T. Bacteriol. 55, 705-9(1948).

Radioactive phosphorus $\left(\mathrm{g}^{32}\right)$ added to a suspension of Echerichia coli in phosphate buffer has a definitely lethal effect upon the cells that in a general way is related to the initial concentration. Complet sterilization of the suspansion was not obtained in the highest concentration teated. The survivor curve indicated a more rapid rate of killing during the first time interval, and the existence of a small percentage of the cells that were more resistant and survived to the and of the experiment. The present experiment does not auggest in any way the possibility of the application of radiogctive materials to the sterilization of 907 food products. (NSA)

Scot, ${ }^{2}$. G. and S. T. Cook. WHE EFECT OF RADIOACTIE THOSPMORUS ON TEL BLOOD OF THE GROWING CEICIR. Proc. Nath. Acad. Sci. U. \$. 23, 265-72

(1937).

The administration of radioative $\mathrm{H}_{3} \mathrm{PO}_{4}$ (birds were used axperimental animals) gives xise to effects upon the constituents of the blood that are not obtained by $x$-rays. The lymphocytes which are sensitive to $x$-ray radiation were not garticulary affected. The administration of radioactive $\mathrm{P}\left({ }_{15} \mathrm{P}^{32}\right)$ (I) brought about a great decrease in the number of polymorphonuclear leucocytes. This decrease 18 atcributed to the selective deposition of 1 in the bones, which 2low bombardment of the bone marrow with $\beta$-rays. The basophiles and monocytes were not appreciably affected, although an increase in eosinophiles was noted. Bixds treated in this manner grew normaly and any permanent 11 effects from the administration of I were not observed. (CA)

908

Scot, IK. C. and J. T. LaW

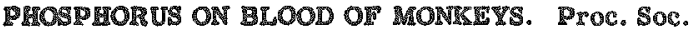
Expt. B10L. Med. 48, $155-8(1941)$.

The administration of radio-p to monkeys lowers the absolute numbers of red calls, lynphocytas, and granulocyte in the blood. Lywphocytes and granulocytes are affected to about the rame degree. Red cells are not as gadio-gensiln ve as the former. Monkeys tolerats doses of 0.76 and 0.71 millscurles of $P^{32}$ per pound of body weight. 1.04 millicuries of $\mathrm{P}^{32}$ per pound of body weight was fatal. (3A)

90

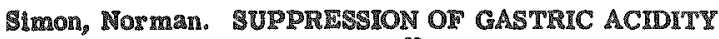

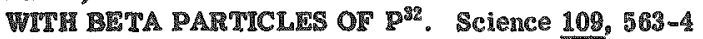
(19) 919$)$.
Irradiation of the gastric mucosa by $x-$ radiations suppresses gastric acidity, but may also damage more sensitive adjacent organs, such as the liver and small intestinc. In order to restrict the radiations to the gastric lining, applications of $\mathrm{P}^{32}$ were made, for the f-particle emission range for this isotope is only about $8 \mathrm{~mm}$. The $\mathrm{p}^{32}$ was applied topically to the nucosal lining of the Heidenhan stomach pouches in dogs. A deseription of the appliestus is included, and it was prepared to hold as much as $25 \mathrm{mc}$ of $\mathrm{P}^{32}$ absorbed to its surface. The balloon was inserted into the stomach and held there for $2-6$ hours. "The radiation was followed after a few days by a decrease in total secretion as well as a decrease in the quantity and concentration of $1 \mathrm{Cl}$ in the pouches of all five dogs studitad. Two of these doss had anacid pouches and three had livporcid pouches. Calculated doses of $1,800,12,000$, and 25,000 equivalent roentgens in the first min of mucosa were given to one animal, while the rest recaived loses of $20,000-$ 25,000 equivalcnt roenterses nthe lst of mucosa. The longest period of observation after irradiation of any animals was 125 days. There were no changes in blowd count, weight, or general condition of the animals which could be attributed to the radiations. One dog returned to normal acidity after a period of three months, and an autopsy showed an uleer of the stomach pouch. (NSA) 910

Stevens, C. L. IRADIO-AUTOCRAPHC REACTIONS IN TISSUES OTHER THAN BONES AND TEETH AFTER INJECTION OF IRAOLO-PHOSPIORUS. Anat. Record $103,509(1949)$.

The following is an abstract and is reproduced here an its entirety.

After administration of radio-phosphorus to rats (as carrier-fiee phosphate), fairly large amounts of it may be detected by the Geiger counter in most organs and tissues. An attempt was made to locate this radiophosphorus histologically by the autographic method after parafin embedding. While some phosphorus containing compounds are removed by the fat solventa (dioxane, paraffin) used in the technque, it was found that several others are still retained in the tissues. However, gute different results are obtained according to the type of firation. After fixation with formalin most of the radioactive matexial is removable with $\mathrm{r}$ ibonuclease and is presumed to be ribonucleic acid. Some labeled desosyribonucleic acid is present in cells which have recently divided by mitosis. Small amounts of other radioactive compounds (phosphoprotein, adenosine di-phosphate?) are also found. After fixation with bichromate containing fluids (Orth), much nore intense autographic reactions are obtained in most organs and tissues. It is probable that bichromate retains, besides the above-mentioned phosphorus compounds, such substances as simple organic phosphates or even phospholipids which would normally be extractable with water or fat solvents. 911

Warran, S. and R. F. Cowing. DISTIRUUTION OH LARGE DOSES OF RADIOACTIVE PHOSPMORUS IN RATS. Axch. Pathol. 39, 730(1940).

Radioactive phosphorus, prepared by the department of physics of Han ward University by neutron bombardment of red phosphorus, was injected, in the form of dibasic sodium phosphate, intrapenitoneally in ats. The dose given ranged from 16 to 30 microcuries per animal. The content in the blood dropped in the first 48 hours and the content in the ure fine fll sharuly also. Apurowmately 6 of the amoun injected was axcieted in the first 3 hours. Measurements were made with a Laur itsen lype electroscope. The experimental aror is pronaly $15 \%$ The 
lissues containing most phosphorus were: liver, voluntary muscle, bone, spleen, and kidneys. The histological observations will be reported. (AP)

912

Warten, S. and R. F. Cowing. THE DISTRIBUTION OF DOSES OF RADIOACTVE PMOSPMORUS IN RODE NTS.

J. Lab. Clin. Med. 26, 1014-16(1941).

Radivactive was injered intravenously or intretperitoneally in mice, ats, and rabbits as an aqueous solution of $\mathrm{Na}_{2} \mathrm{HPO}_{3}$. The dosages of active material injected ranged from 5 to 17.5 microcuries in the mouse, from 30 to 233 in the rat and from 50 to 84 in the raboit.

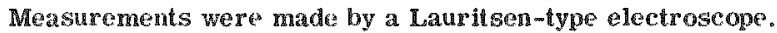
The per entage partition between the various organs varied in the diffirent species, but the spleen, liver, kidney and bone of all showed a material degree of absorption and retention. The urinany oxcretion of the active material was rapid; about ${ }^{1}$, the dose was exereted within 48 hours. There wore minor valiations in the percentage partition in various issuas among individual animals of the same suters. (CA)

913

Warren, Shields, Margaret W. Holt, and Sheldon C. Sommers. SOME IARLY NUCLEAR IFIECIS OF IONIRING RADIATIOH. Proc. SJoc. Expt1. Biol. Med. 77, 288-91(1951).

Irozen-dricd, dry-mounted, Feulgen-staned preparations of tissues of rats and mice int raperitoneally injected with large doses of $\mathrm{p}^{32}$ were mployed to study early cyloloric changes after ionizing adiation. Fine nuclert details are preserved by the freezing-drying method. Lethal radiation effect observed in radiosensi tive cells include formation of large intranuelear vacuoles with stretchine of nuclear membranes. Nonlethal changes in raduoresistant tissues include small intranuclear vacuoles, docreaces Feulgen stain of nuclear chromatin and sap, and loss of egular sharp staining of nuclear menubranes. Whu changes in liver alls mav contribute to the acute adiation syndrome. (NSA)

914

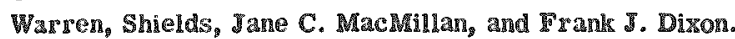

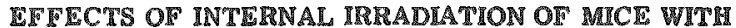

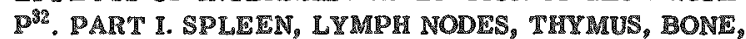
AND BONE MARROW. Radiology 55, 375-89(1950).

A histologic survey of the effects of continuous internal irradiation of mes with varying dosages of $\mathrm{P}^{32}$ is presented. The tissue showing radiation injury earliest is the thymus, but the lhymus also appear's to possess a "radioresilency" in that 1 thens to normal most rapaly and completely. Splenic issue and bone maryow apear about tho same in respect 10 sensifivity $10 \mathrm{P}^{32}$, but bone marrow is more nearly completely destroyed than sulenic tissue by an equal tose. Repair and return of myelopolesis can be seen in both tissues at 10 to 15 days following injection. The sulew becumes hyperolastie by 20 days, but the bone marow does not become hyperplastic unil some time later. Lymph nodes are the last lissue to show morphologic recovery from effects of radiation. In the group of animals given the sinimal lose an overgrowth effect wh observed in sone of the tissues after a promininary depressive effect. Marled lymphopoiesis, myclopoiesis, and hyperulasia orcur, with pesultant increase in the size of the spleen, thymus, and amount of bone narow but not in the size of the lymph modes, which appear permancenty damaged and shrunken by this small dose. Aso of interst is the histologic observation of rean and recovery in the lymphoid tissues and bone mag row. Hesturaticn to almost normal was accomplished in some anmals dying because of radiation injury. (NSA)
915

Warren, Shields, Jane C. MacMillan, and Frank $\mathbb{J}$. Dixon. FFFCTS OF INTERNAL IRIRADIATION OF MICE WITI P.2 $^{32}$. PART II. GONADS, KIDNEYS, ADRENAI, GLANDS, DIGESTIVE TRACT, SPINAL CORD, LUNGS, AND

LIVER. Radiology 55, 557-70(1050).

A study has been made of the histologic damage suffered by a series of mice injected with $25 \mu \mathrm{c}$, and $2 \mathrm{mc}$ of $\mathrm{p}^{32}$. The most characteristic and consistent change in the mouse ovary following injection of $\mathrm{p}^{32}$ is a reduction in total amount of ovarian tissue. An absolute sterilizing dose of $\mathbf{P}^{32}$ short of a lethal dose has not been found for mice. The $250 \mu c$ series of mice affords the best opportunity for $^{\circ}$ study of progressive irradiation changes in the testes. Radiation injuries first the least mature of the spermatogenic cells, as evidenced by nearly complete loss of spermatogonia aiter 9 dayg of irradiation. The kidneys of the animals injected with $25 \mu$ of $P^{32}$ showed no morphologic changes. At 10 days, in the kidneys of the animals injected with $250 \mu$ c, there was occasional shrinking of the epithelial cells lining the renal tubules. The adrenals showed no specific radiation effects but slight variable congestion was observed, which was not correlated with the amount of radiation received. With doses of 25 and 250 $\mu c^{*}$ the esophagus and stomach of almost all the experimental amimals were apparently unaffected morphologically. In a few instances, small petechial estravasations of blocd were noted in the ventral horns of the spinal cord of the mice receiving $250 \mu \mathrm{e}$. In addition, at least two large central hematomas extended vertically in the cord. Hixcept for variable degrees of congestion, there were no specific radiation changes in the lungs of any of the animals. The maximum radiation effect on the liver, found about 20 to 50 days after injection, was a sendency toward regeneration of liver tissue shown by numerous mitotic figures in the liver cells. 39 ilustrations. (NSA)

\section{Plutonium}

916

Alorams, R., et al. METABOLISM ANO DISTRIBUTION OF INRALLD PU IN RATS. Univ. of Chicago Metallurgical Lab. Oct. 29, 1946. 46p. (MDDC-67\%: CH-3655)

Several different techniques were used to introduce tracer quantities of $\mathrm{Pu}$ into the lungs of rats. When aerosols were produced by atonizing aqueous solutions of the nitrates it was found that approximately $20 \%$ of the dose was absorbed and deposited in the skeleton with tetra, hexa, and trivalent plutoniurs. Thus with these compounds, the lungs represent a more dangerous portal of entry than either oral $(0.05 \%)$ or intramuscular (13\%) administration. Tetravalen $\mathrm{Pu}$ ws eliminated most slowly from the lung, the average half-time during the first month being 15 days. But when citrate was added to complex the $\mathrm{Pu}$, over $70 \%$ was eliminated within one day. This was accompanied by a deposition of over $30 \%$ in the sireleton and over $20 \%$ in the liver. Presumably citrate breaks up the insoluble colloid in which Pu exists in the lunge. Complexing with cupiergon which, unlike cirstate, produces a "fat soluble" compound, greatly diminished the extent of absorption from the lungg. Plutonium oxide smokes which were produced at high temperatures were eliminated more slowly from the lungs (average half time of 30 days during the first month) and were deposited in the skeleton to a much lesser extent (6.2\%) Uhan was the case with the nitrates. (ADD) 817

Arnold, J. S. RADIOAUTOGRAPRY. Argone Natlonal Lab. Jan. 1951. Decl. June 5, 1951. 4p. (AECD-3191) 
In an effort to demonstrate the migration of Pu from its initial site of deposition 14 rats were given subtoxic amounts of Pu citrate intravenously and were sacrificed at weekly intervals. By radioautographic means it has been definitely shown that the Pu migrated from its initial endosteal site of deposition into the cortical bone by the process of having new nonisotope-containing bone laid down over it. Further, it seemed that the Pu that had migrated into the cortex was concentrated on the surface of the laminae of bone or in the cement substance betweess laminae. In a dog that had been given Pu 2 years before sacrifice, the relained $\mathrm{Pu}$ was distributed in a rather spotty manner in both trabecular and cortical bone. $U^{233}$, which had been administered intravenously as the nitrate, was found to appear in aggregates in the macrophayges of the spleen of mice. A photograph of this phenomenon is reproduced in the report. The kidney accumulation of in jected $\mathrm{U}^{239}$ appeared to be in the proximal convoluted tubes in the form of a cast deposition. The results of tests with NTA and NTB liquid muclear track emulsions show that there is definite advantage in their use for localization studies. (NSA)

918

Barron, I. S. G., R. Abrams, R. D. Finkle, and R. P.

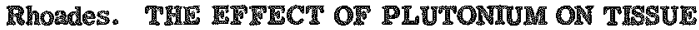
METABOLISM OF RATS. Argonne National Lab. June 1046. 54p. (MDDC-1653; C CH-384)

Detailed information is given on metabolic changes in the various body tissues aiter introduction of $\mathrm{Pu}$ into the body by injection, after intratracheal instillation of Pu or after injection. Iffects on the $\mathrm{QO}_{2}, \mathrm{QO}_{2}$ succinate, anaerobic glycolysis, $\mathrm{QO}_{2}$ pyruvate, $\mathrm{Q}$ pyruvate, $\mathrm{Q}$ and $\mathrm{QO}_{2}$ butyrate, acetoacetate formation, phospholipid synthesis and removal are discussed. The various effected organs and tissues as kidmey, liver, thymus glands, adrenals, submasillaries, blood and lungs and the small intest ine are treated specifi19

Bloom, Willian and Margaret Bloom. SOME LATE HISTOLOGICAL CRANGES IN MCR AFTIR LOW DOSES OF PLUTONIUM AND RADIUM, P.174-84 of BIOLOGICAL AND MPDICAL DIVISTONS; QUARTRRLY REPORT, MOVEMBER, DECERIBER, JANUARY, 1949-50.

Axgonne National Lab. [nd] 11p. (ANL-4401(p.174-84))

Mice were injected intravenously with plutonium nitrate $(0.03$ or $0.003 \mu \mathrm{c} / \mathrm{g})$ or intraper itoneally with $1 \mathrm{da}(0.3 \mathrm{or}$ $0.03 \mu \mathrm{c} / \mathrm{g}$ ) and sacrificed at intervals of 2 months to a year thereafter. Results of histological examination of the spleen, lymph node, thymus, liver, testis, lung, skin, gastro-intesthal tract, and pancreas are given. The red pulp of the spleen was greatly increased after the higher doses of Pu and Ra and moderately increased after the low dose of Ra. The increase in hematopoiesis in the apleen was undoubtedly compensatory for the continued and progressive depression of the blood-forming mechanism of the bone marrow of these mice. Even where hematopoiesis In the marrow pergisted, it was predominanty myelocytopoietic rather than erythropoietic. (NSA) 020

Brues, Austin M. FF"FCTS OF PLUTONIUM IN THE LIVER. Trans Conf. Liver Injury, Th Conf. 59-61(1948).

It has been determined that the early distribution of plutonium is in the liver and spleen and that it is gradually translocated to the bone. The effect of injecting a large dose ( 1 r per in the rat) was, as in total body radiation, to produce death. However, rats and mice given a smaller dose may aurvive for several months, although they show clinical evidence of liver disease, and severe cell damage and atrophy of the liver. Although liver carcinomas did not develop, bone carcinomas frequently occurred nearly a year later. Hypertrophy of liver tissue around the margin of atrophic nodules has been attributed to the tendency of cerium-144 to concentrate in the liver. (NSA) 921

Finkel, Muiam P. SOME FURTHER NOTES ON THE MCE TREATED WIII PLUTONIUM BETORF WEANING. Argonne National Lab. Aug, 1, 1947. (AECD2024- - II)

In August 1945 an experiment was begun to investigate the late effects of plutonium upon mice which were treated in utero. It was discovered that young mice obtaned additional plutonium from the breast milk of their treated mothers. Therefore it was necessary to run another series of experiments which measured the $\mathbb{P}$ absorbed by suckling mice as well as the retention of the material by young animals. This information has made it possible to assign definite dose levels to the mice of the original experiment and to analyze the data which have been accumulating since the birth of these animals. Topics discussed are the dose levels, the relative contributions of plutonium from the placenta and the milk, the retention of plutonium by mice treated before weaning, and the effects of low levels of plutonium on growth. (NSA) 922

Finkle, R. D.g et al. THE TOXICITY AND METABOLISM OF PLUTONIUM IN LABORATORY ANIMALS. Argonne National Lab. Aug. 1846. Decl. July 25, 194\%. 144p. (MDDC-1140; CH-3793)

Methods used in tissue analysis after intracardial, in travenous, or intramuscular injection of $P u$ are discussed and new methods used are deseribed. No species, strain, or sex differences were found in hematological effects of Pu. Doses of 0.0003 or $0.0006 \mu \mathrm{c} / \mathrm{g}$ of Pu caused no detectable hematological alteration in the peripheral blood of mice, rats, and rabbits during 300 days of observation. A dose in the range of $0.003 \mu \mathrm{c} / \mathrm{gg}$ produced a minimum to moderate depression of the leucocytes and platelets in all 3 species within the firgt 100 days. A dose of $0.0063 \mu \mathrm{c} / \mathrm{g}$ produced a moderate to severe and sustained leucopenia in all species studied and caused an anemia and early death In mice and rats (135 days). Doses of 0.0156 to $0.125 \mu \mathrm{c} / \mathrm{g}$ produced correspondingly more severe and sustained anemia, leucopenia, reticulocyte reduction, and platelet deficiency; and early death in mice and rats. Pu intoxication produced morphological changes in the nucleated cells of the peripheral blood comparable to those which occur after exposure to external radiations. A macrocytic anemia developed in rats following the parenteral administration of Pu In doses between 0.0063 and $0.063 \mu \mathrm{c} / \mathrm{g}$. This effect was less significant in mice and rabbits after comparable 223 doses. (TTS)

Mamilton, Joseph G., D. J. Copp., D. M. Greenberg, M. J. Chace, L. Van Middlesworth, and $\mathrm{E}$. M. Cuthbertson. THE DEPOSITION OF PLUTONIUM AND CERTAIN FISSION PRODUCIS IN BONE AS A DECONTAMINATION PROBLEM. [Radiation Lab., Univ. of Calif.] May 15, 1946. 281. (AECD-2483; CH-35ఖ1)

Bone deposition of $P u$ and the ission products of radioactive $\mathrm{S} r, \mathrm{~T}$, and $\mathrm{C}$ was studied in view of possible decontamination. Although most treatments had little influence on the absorption of $\mathbf{S r}$ from the gut, both age and dietary Ca did have a profound effect, and growing rats, on a diet low in Ca, absorbed over 25 times as much of a given dose as did adult rats geceiving ample $\mathrm{Ca}$ in the diet. There was also 4 -fold difference in the retention of injected radio active $\mathrm{Br}$ but no appreciable effect on the retention of radioactive $\mathrm{Y}, \mathrm{Ce}$, or $\mathrm{Pu}$. The chronic elimination by the rat of 
radioactive Sg, $\mathrm{T}, \mathrm{Ce}$, and $\mathrm{Pu}$ was followed for a period of several months. No appreciable decontamination was obtained by various prolonged treatments. Experiments on healing bone fractures showed that the peak uptake of radioactive $\mathrm{Pu}$ and $\mathrm{Y}$ by the callus occurted several days before the commencement of active calcification and deposition of radioactive $\mathbf{S r}$. There was a $4 \mathrm{x}$ reduction in the retention of radioactive Sr by animals suffering from a severe dietary $P$ deficiency but no significance in the retention of $\mathrm{Pu}, \mathrm{Y}$, and $\mathrm{Ce}$. It was found that the effects of severe $P$ deficiency, including resorption of bone salts, could be produced by adding $\mathrm{Al}\left(\mathrm{SO}_{4}\right)_{3}$ or freshly preeipitated $\mathrm{A} 1 \mathrm{O}(\mathrm{OH})$ to a normal diet. Some demineralization of bone could be produced even in adult animals. Radioautographs of undecalcilled bone sections from such animals showed that the radioactive $\mathrm{Sr}$ was deposited only in the presence of bone salts while radioactive $\mathrm{Pu}, \mathrm{Y}, \mathrm{Ce}$, and $\mathbb{Z r}$ were laid down in the uncalcified organic matrix. These, and the preceding metabolic studies, indicate that while Sr follows the path of $\mathrm{Ca}$ and is deposited in the mineral of bone, the metabolism of the other elements is unrelated to that of Ca and they may be laid down in the organic bone matrix. Radioautographs or undecalcified bone sections of adult rat demonstrated the superficial deposition of $\mathrm{Pu}$, and the relative immobility of these deposits. By this technique, it was shown that these $P_{u}$ deposits in the slreleton may be overlayed with newly formed non-radioactive bone, thus shielding the sensitive cells of bone marrow and bone from the short range $a$ particles. This has been suggested as a possible means 824 of reducing the chronic toxicity of $\mathrm{Pu}$ in the gkeleton. (TIS)

Katz, J., C. W. Delong, M. F. Getzendaner, A. C. Case, and Esther S. MMler. ABSORPTION OF PLUTONIUM FED CHRONCALLY TO RATS; I. BRYTCT OF \$EX ON FRACTION ABSORBED AITRR IIGR DOSAGE LEVELS. Hanford Works. Mar. 13, 1051. 11p. (WW-20011(pt. 1))

Preliminary investigationg undertaken to determine the percentage absorption from the rat's gastrointestinal tract of Pu administered chronically and to find whether the sex of the animal plays a role in the rate of absorption are described. Five male and five female rats each received perorally an average of $5.9 \mu \mathrm{g}$ of $\mathrm{Pu}^{239}(+4)$ as the nitrate over a period of 20 days. Radiochemical analyses indicated that an average of $0.0095 \%$ of the administered dose was deposited in the total carcass. "the pelt continued negligible amounts, but the gastrointestinal tract and contents retained considerable quantities of $P u$ even after a fast of 3 to 5 days. There was no evidence of any sex difference in $P$ r retention. (NSA)

925

Kawin, Bergene and D. Harold Copp. A STUDY OF TH1: FIFECT OF 2, 3-DMMERCAPTOPROPANOL (BAL) UPON THE METEBOLISM OF PLTONUM. Radiation Lab. Univ, of Calif. Tan. 23, 1950. Decl. Apr. 5, 1950. 8p. (AECD-2814)

The effects of 2,3-dimercaptongopanol (BAL) upon the metabolism of intramuscularly injected Pu(VI) in rats have been investigated. The use of BAL produced no significant alterations in the distribution and excretion of plutonium as compared to the pattern shown by control animals. The metabolism of plutonium does not appear to be related to sulhyydryl groups. On the basis of its ineffectiveness in changing the metabolism of piutonium, BAL does not appear to be an efective therapeutic treatment for plutonium poisoning. 10 references. (NSA)
926

Kisieleski, Walter : and L. WoodruR. STUDIES ON THE DISTRIBUTION OF PLUTONIUM IN THE RAT. Argonne National Lab. [nd] 18p. (AFCD-2009-F)

The citrate and nitrate salts of plutoniug in the 6 valence states were injected intramuscularly into male rats at a dose of $0.1 \mu \mathrm{g} / \mathrm{g}$. Animals were macrificed at intervals from 7 to 420 days, and the plutonium content of the various tissues was determined by the standard $\mathrm{LaF}_{3}$ method for plutonium determination. When plutonium is injected intramuscularly as the citrate salt, the presence of the citrate as compared to nitrate seems to increase absorption of the plutonium from the site of injection. After 10 days $51 \%$ of the injected dose was found in the injected leg of the nitrate animals where only $30 \%$ was found in the citrate group. At the termination of the experiment (420 days) $20 \%$ was found after injecting the nitrate as compared to $12 \%$ with the citrate. The skeleton was the principal site of deposition regardless of the form in which the plutonim was injected. Liver was next in order, containing $2 \%$ of the injected dose at 10 days in the nitrate animals and $5 \%$ in the citrate group. The spleen contained only about $0.1 \%$ of the injected dose in both the citrate and nitrate amimals, though in specific activity ( $\mu g / g$ of wet weight) it was as high as liver or bone. Most of the other soft tissues contained insignificant amounts of plutonium activily and it was noted that this concentration decreased with time, the gonads being the exception, where a reverse trend was observed. There was no evidence to show transhocation of plutonium from the goft tissues into bone. All of the tissues in the citrate groups showed a higher intial uptake than can be seen in the nitrate groupa. The specific concentration of the various tissues gives a better picture of the increased initial uptake by the citrate group as compared to the nitrate. At seven days the specific activity in $\mu \mathrm{g} / \mathrm{g}$ of wet weight of liver in the nitrate Group was $0.039 \mathrm{\mu g} / \mathrm{g}$, while the citrate was $0.214 \mu \mathrm{g} / \mathrm{g}$. The specific activtty of the spleen was as high as lyver having $0.043 \mu \mathrm{g} / \mathrm{c}$ at seven days in the nitrate group and $0.123 \mu \mathrm{g} / \mathrm{g}$ in the citrate group. Bone in the citrate group at sever days was $0.533 \mu \mathrm{g} / \mathrm{g}$, while it was $0.398 \mu \mathrm{g} / \mathrm{m}$ in the nitrate group. A significant diference was noted in the apecikic concentration in thymus in the two grousps. The concentration was only $0.008 \mu \mathrm{gg} / \mathrm{g}$ in the nitrate group at seven days, while it was $0.046 \mu g / g$ in the citrate group. (ADD)

287

Langhan, W., at. METABOLISM OF PLETONIUM.

J. Blol Chem, 171, 273-83(1947) (MDDC-719)

The purpose of the experiments conducted at this laboratory was to study the ef valence state, and various other factors on the body distribution and excretion of plutonium folsowing its intravenous administration to the rat. The first day following intravenous injection of $\mathrm{PuO}_{2}\left(\mathrm{NO}_{3}\right)_{2}$ the urinary excretion of plutonium was $7.5 \%$ of the close as compared to 0.33 , 0.57 and $0.71 \%$ when administered as $\mathrm{PuCl}_{3}, \mathrm{Pu}\left(\mathbb{N O}_{2}\right)_{4}$, and $\mathrm{Pu}^{\text {t4 }}$ cirrate complex respectively. Fecal excretion durings the first day was correspondingly lower following injection of $\mathrm{PuO}_{2}\left(\mathrm{NO}_{3}\right)_{2}$. On the 13 th day following intravenous injection there were no significant differences in eilher urinary or fecal excretion of plutonim admingtered as $\mathrm{PuCl}_{3}, \mathrm{Pu}\left(\mathrm{NO}_{3}\right)_{4}, \mathrm{Pu}^{2-4}$ citrate complex, and $\mathrm{PuO}_{2}\left(\mathrm{NO}_{3}\right)_{5}$. At this time the average urinary excretion was $0.014 \%$ of the injected dose and the average fecal excretion was $0.22 \%$. 
The average ratio of fecal to urinary exeretion was 16,1 . The sheleton was a major site of deposition regardless of the form in which the plutonium was injected. Four days following injection of plutonium as $\mathrm{PuCl}_{3}, \mathrm{Pu}\left(\mathrm{NO}_{3}\right)_{4}, \mathrm{Pu}^{+4}$ citrate complex, and $\mathrm{PuO}_{2}\left(\mathrm{NO}_{3}\right)_{2}$ skeletal deposition was $44.9,29.3,56.9$, and $56.5 \%$ of the injected dose respet tively. Deposition in the liver under the above conditions was $22.9,39.7,9.6$, and $9.1 \%$ of the injected dose resper tuvely. Deposition of plutonium in kidney, spleen, and un "balance" was not greatly affected by the form in which the plutonium was administered. The size of the injected dose of $\mathrm{Pu}^{4}$ eitrate complex did not affect the per cent of the dose excreted in the feces and urine. likewise, the size of dose did not alter the per cent of injected material present in the various tissues six days following injection. Absorytion of plutonium from the gastro-intestinal tract was quite low $-0,01 \%$ of the administered dose. When puts was administered orally in $5 \%$ sodium citrate solution, the presence of the citrate seemed to increase absorption of the plutonium from the gastrointestinal tract. Absorption was still quite low - only $0.3 \%$ of the administered dose.

Seventy-nine per cent of the glutonim absorbed from the gastrointestinal tract was deposited in the skeleton and $7.3 \%$ in the liver. Plutonium absorbed slowly via this route seems to give a higher deposition in the skeleton than it does when administered intravenously as $\mathrm{PuCl}_{3}, \mathrm{Pu}\left(\mathrm{NO}_{3}\right)_{4}$, $\mathrm{Pu}^{\text {th }}$ cit rate complex or $\mathrm{PuO}_{2}\left(\mathrm{NO}_{3}\right)_{2}$. Resulis are tabulated 928 and graphed. (ADD)

MELICAL AND MEA LTH PHYSICS QUARTIRLI RE PORT, JANUARY, FEBRUARY, AND MARCH, 1950. Radiation Lab. Univ. of Calif. May 2, 1950. 105p. (AECD-2901)

The localization of $\mathrm{Ca}^{45}, \mathrm{Sr}^{96}, \mathrm{Pm}^{147}, \mathrm{Am}_{\text {, and }} \mathrm{Pu}$ in the skeletal tissue of rats has been investigated by radioautography of the costochrondral junction. The distribution of Iracer Ca and Sr coincides exactly with that of bone sall and of Ca deposited in cartilage. $\mathrm{Pm}$ and $\mathrm{Am}$ is deposited at calcified surfaces and in potentially osteogenetic tissues found free from osseous components, $\mathrm{Pm}$ is found in the same areas and also in spotty deposits in the vascular or perivascular regions within bone. $P_{0}$ may have a transitory localization adjacent to Ca salts (1 hr after injection) and localizes mainly în actively osteogenetic tissues, periosteal extension and inner and outer side of bone. Young rachitic rats show the same rapid initial uptake of $\mathrm{Sg}^{63,90}$ as do young normal animals and the maximum uptakse is as great, but $\mathbf{S x}^{89,00}$ does not memain fired in the skeleton and is lost rather rapidly. The failure of the $\mathrm{Sr}^{89,90}$ to remain fixed in the akeleton may be a result of the low $\mathbb{P}$ level and failure of actual new bone-salt deposition in the rachitic rats. The rapid removal of $\mathrm{Sp}$ in these rachitic animals suggests the possibility of im portant decontamination of $\mathrm{Sr}^{8 \mathrm{~g}, 30}$ deposited in the skeleton if similar conditions can be reproduced in normal animals. Results are tabulated of tissue distribution studies of $\mathrm{Ta}^{102}$ after intravenous or intramuscular administration and after inhalation. The intraperitoneal injertion of in organic phosphate, even in low concentrations, into young rats lowers the blood phosphatase level. The value of massive doses of $Z$ reitrate as a decontaminant for $\mathbb{N}^{96}$ is demonstrated. The initial rapid drop in blood $\mathrm{Nb}^{35}$ level and the high urinary excretion rate are maintained by the 2. citrate action. Data on the effectiveness of $\mathrm{Ca}_{3}$ vergenate (ethylenediam inotetracetate) in removing $Y$ already deposited in the body are presented, and the toxicity of versene acid and Ca versenate to rats is determined. At has a highly selective destructive affinity for thyroid gland lissues. Photomicrographs of the lhy roid tissues are griven and hematological studies are repurted. Improved methods are given for the production of me amounts of $A t^{214}$ and for the reerovery and analysis of $A t$ in tissues. The preparatuon of earrer-1ree $R e^{103}, 184$ from

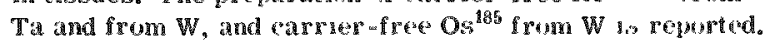
(TIS)

929

Schuber, Jack. A STUDY OF THE THEATMENT OF PLUTONIUM AND RADIOYTTIIUM POISONING BY ZIRCONIUM CTRATE. Argronne National Lab. Sept. 1, 1948. 23p. ( $\mathrm{ECD}-2358 ;$ ANL-NDY -592$)$

A study was made of the effect of a single dose of z1rconium citrate on the excretion and distribution of plu tonium and radioyttrium in rats and a growing dug. It was found that the admunistration of zirconium entrate is rats increased the excetion of pu from $1 \%$ to $50 \%$ and that of Y from $25 \%$ to $60^{\circ} \%$ of the injected dose during a 24 -hour period. The amount of $P u$ and $Y$ deposited in the sheleton was reduced by faltors of 6 and 2.6 respectively. No signfirant changes in the $P u$ or $Y$ contents of the feces, liver, spleen, or kdneys were found. The adminstration of zirconium citrate to a dog five months after exposure to Pu was sollowed by a wustained increase in the urinary $\mathrm{ex}$ cretion of Pu. The extent of the increase was proportional to the dose of zirconium citrate. Two and one-half years after exposure to $\mathrm{Pu}$ the infection of a small amount of zirconium citrate was followed by a twofold iness ase an the urinary excretion of Pu. A discussion is given of the chemical concepts involved in applying metal displatement principles to the removal of radioactive metals from the 930 ody. (NSA)

Schubert, Jack. REFCT OF ZIRCONIUM AND SODIUM CITRATE ON THE DIETRIBUTION AND RXCRETION OF YTTRUUM AND PLUTONIUM. J. Lab. Clin. Med. 34, 313-25(1949) (ANL-4206)

In a previous report it has been shown that zireonum, when administered as the cutrate salt, uncrases the ex-

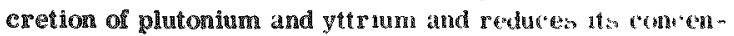
tration in the bone (Schubert, Sc1ence, 105: 389, 1947). These studies have been extended and jrelmmary data art briefly described in this repurt. (NSA) 931

Schuber, Jack, Mariam P. Finkel, Marica R. White and Gertrude M. Hursch. PLUTONIUM AND XTTRIUM CONTENT OF THE BLOOD, LIVER, AND SKELETON

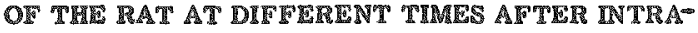
VISOUS ADMHISTRATION. J. Biol. Chem. 182, $635-42$ (1950).

Eighteen female Sprague-Dawley rats weighing between 236 and $254 \mathrm{~g}$ received an injection of $0.4 \mathrm{ce} 1 \mathrm{~K} / \mathrm{Na}$ cit rate solution containing both $\mathrm{Pu}^{239}$ and $\mathrm{F}^{91}$ into one of the lateral tail veins. The animals were sacrificed in pairs at 5,15 , $30,60 \mathrm{~min}$ and at $2,6,12,24$, and 48 hr and blood, bone, and liver samples wero analyzed by ashing and determinng the alphatradiations of $P u$ and beta-emissions of $\mathrm{Y}$. Five min after intravenous injection about $50 \%$ of the $\mathbb{P u}$ and $80 \%$ of the $Y$ had disappeared from the blood. After this sharp initial drop Pu disappeared more slowly from the bloodstream; 12 hr after administration aboul $20 \%$ of the injected dose was still being circulated. Within $6 \mathrm{ht}^{\circ}$, al most all the $Y$ had disappeared from the blood, $48 \mathrm{hr}$ were reguired for removal of most of the $\mathrm{Pu}$. The Pu content of the akeleton increased regularly unt il a maximum value of about $45 \%$ of the injected dose was reached in $24 \mathrm{hr}$; in $6 \mathrm{hr}$ the concentration was near its peak of $45 \%$. After an initial rapid increase of $\mathrm{P} u$ and $Y$ concentration in the liver, the level of $\mathrm{Bu}$ continued to rise at a diminished rate until it reached $24 \%$ of the injected dose at $24 \mathrm{hr}$. The $\mathrm{Y}$ 
level decreased very slowly after the 2 nd hr at which time the maximum value of close to 10\% was attained. During the first $24 \mathrm{hr}$ following injection the rat excretes about $3 \%$ of the Pu and $25 \%$ of the $Y$; after this period the body content of Pu and $Y$ remains nearly constant. Interpretation of the data on the basis of a diffusion-controlled mechanism is discusised. (CA)

932

Schubert, Jack and Marica $R$. White. THE EFFCT OF DIFTERENT DOSE LEVISL ON ZIRCONIUM ON THE EXCRETION AND DISTRIBUTION OF PLUTONUM AND YTTIUUM. J. Biol. Chem. 184, 191-6(1950).

Early administration of zirconum citrate to rats previously injected with tracer levels of plutonium, $\mathrm{Pu}^{239}{ }^{230}$ and yttrium, $X^{9 t}$, results in a marked increase in the urinary excretion and a considerable decrease in the bone content of the injected radioelements. Relatively large amounts of ziroonium, about $200 \mathrm{mg} / \mathrm{k}$, were uged in these exper:ments. In order to clarify the mechanisms involved, graded dose levels ranging from 12.3 to $1336 \mathrm{mg} / \mathrm{k}$ as $\mathrm{zr}$ have been given. (NSA)

293

Scoti, K., el al. METABOLISM OF PLUTONIUM LN RATS.

J. Biol. Chem. 176, 283-93(1948) (MODC-1018)

The results of detalled metabolic gtudies of plutoninm followng or 1 , intramusculas, intravenous, subcutanecus, and intrapulmonary administration are presented. Plutonium is not absorbed from the digestive tract. The skeleton is the chief organ of deposition for plutonium absorbed by the body following parenteral administration. The digestive tract is the principle channel of elimination and the degree of retention is very great. The rate of excretion is very slow and the half-period of retention of plutonum by the skeleton is estimated to creeed sizk months. No sugnificant differences in the metabolic propert 1 ses of plutonum absorbed by the loody were observed for at is thre ved lence states. Considerable retentuon of plutonum by the lungs following un redpul monary administration was observed and was found to be highest for the 4 state, less for +3 plutonium and least for +6 plutonium. A significant degree of absorption of plutonium from the lumgs with subsequent deposition in the skeleton took place following the intrapulmonary administration of solutions of this clement in ifs three valence states. (ADD) 934

Scot, Ienneth G., Dorothy Axelrod, Tosephine Crowley, and Joseph G. Familton. DEPOSITION AND I A ${ }^{\circ}$ OF PLUTONIUM, URANIUM, AND TRERT FISTON PRODUCTS IRLALED AS ARROSOLS. Areh. Pathol. A8, 31544(1949).

The purpose of the experiments described was to ascerfain the possible harat ds resulting from the inhalation of Tissionable materials and fission products. Wath the exception of one study on a human aubject, young rats were uwed for all of the experiments, and after anesthetigation, were given $\mathbb{P u}, \mathbb{U}$, and mixtures of $119310 n$ products con-

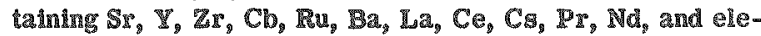
ment 61 in the form of smokes and gprays. The rats were given: plutonium oxide as smoke, plutonium nitrate as a gpray: 1 ission products, containing $\mathrm{Pu}$ and $\mathrm{U}_{8}$ as aerosols: carrier-free fission products aerosols; aerosols of carrier - free wr or zirconium oxides and aerosols of $\mathrm{Pa}$ as protactinlum oxide. "The preparation of the aerosols is mpiven in detail. The gltconim aerosol was administered to both a hurnar subject and the rats. If was shown that the aerosols were almost totally retained by the head and lungs innmediately aiter exposure; after four days the lungs contained the largest percentage of these elements. The ele- monts deposited in the head and the bronchial tree were quickly elininated through the gastrointestinal tract, while the nonciliated portions of the lungs removed the gubstances at at slower rate. The main pulmonary gite of deposition of the substances was in the bronchial passages and alveoli; a small amount was absorbed and appeared in the sikeletal structure, but mone was found in the blood vessels or in the lymph nodes. (NSA)

035

Van Middlesworth, L. STUDY OF PLUTONUM MISABOLISM IN BONE. Univ. of Callif. [nd] 99p. Deel. June 6. 194\%. (MDDC-1022: MP-69)

A method is described whereby $\mathrm{Pu}^{239}, \mathrm{Sr}^{26}$, and $\mathrm{Y}^{38}$ may all be distinguished in the same animal by differences in their radiations. The chronic excretion of $\mathrm{Du}^{232}, \mathrm{se}^{88}$, and $\mathrm{Y}^{83}$ was followed for 11 weeks in rats injected simulaneously with these three elements. The very low rate of elimination of all three arter the first monh emphasizes the danger of chronic toxicity. Radioautographs of the tibia ghow radioactive strontium deposited throughout the bone with a heavy concentration in the region of new bone growth and active calcification beneath the epiphysis. Plutonium was shown to localize superficially in the endoateal and periosteal surfaces of the bone with no apparent coneentration in the zone of bone growth and calcification. Severe phosphate deficlency was found to diminish greasty the retention of radioxctive strontium in the glreleton. The retention of plutoniun at the site of intramuscular injection was reduced in the phosphate deficient rats, but the distribution of plutonium in the body was otherwise unchanged. The maximum uptake of $\mathrm{Pu}^{238}$ and $\mathrm{Y}^{38}$ in the fracture callus occurred on the second or third day following fracture, before calcification had commenced. On the of her hand, active $S x^{\text {bi }}$ deposition did not begin until the 4-5 day, and reached a peak at 8-12 days when calcifica tion was most active. Although plutoniurn and yttriun appear 10 deposit in uncalcified bone matris, scurvy, which produces changes in connective tissue and osteoid matrix has no significant effect on the distribution of these elements, aside from a moderate decrease in the amount retained at the site of injection. A period of three weeks on a protein free diet did not alter the distribution of plutonum in the rat, alhough it did decrease somewhat the retention at the injection site. Following intramuscular ing fection, mox'e Ru(IV) was retained at the injection gite than Pu(VI), but the distribution in the body was otherwige similar. Following intravenous in ection, Pu(VI) disappear from the blood stream much more rapidly than does Pu(IV). There was also a much Iarger proporton of Pu(IV) deposited in the liver. These resulls indicate that the Pu(TV) may be in a colloidal atate. Methionine supplements re sulted in a moderate decrease in the plutonium in the skeleton. When large doses of cysteine were injected one hous prior to the intramuscular infection of $\mathrm{Pu}(\mathrm{VI})$, the absorption from the injection sile and deposition tn the skeleton was reduced. There was no effect when the Pu(VI) was administered intrayenously. (ADD)

\section{Poloninm}

230

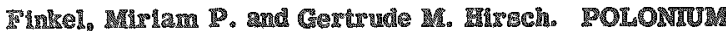

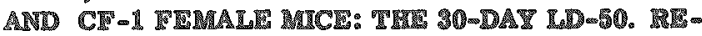
IENTION, DISTRIDUTION, AND COLCENIRATON,

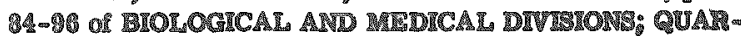

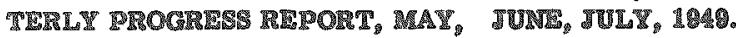

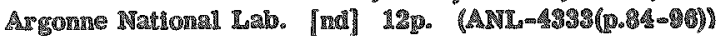

Poung aoin CF-1 temale mice were Injected hniravenously with polondum ${ }^{210}$ chloride. Tt was found that the 
30-day L.D-50 lies between 0.04 and $0.03 \mu \mathrm{c} / \mathrm{g}$. The per cent retention was independent of the dose within the range used $(0.1$ to $0.005 \mu \mathrm{c} / \mathrm{g})$. The biological half-period was 26 days. Seventy-two per cent of the injected material was present in the mouse 10 days after injection, $90 \%$ was present at 100 days. Three days after injection $20 \%$ of the Po in the body was present in the liver, $12 \%$ in the kidneys, and $2 \%$ in the spleen. The following order of decreasing magnitude of specific activity ( $\mu \mathrm{c} / \mathrm{g}$ ) was found: ovary, kidney, spleen, liver, mesenteric lymph node, lung, femur, gastrocnemin muscle. At 38 day concentration was greatest in the spleen. Doses higher than the 30-day LD-50 resulted in rapid loss of weight: 0.025 and $0.01 \mathrm{\mu c} / \mathrm{g}$ prevented weight increase during the la-day period of observation, while $0.005 \mu \mathrm{c} / \mathrm{g}$ had little effect on weight increase. (S. Y. S.)

$98 \%$

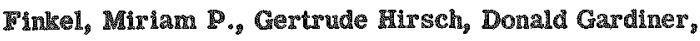
and Ruth McNun:s. PHOGRESS OR TLE LORG-TERM POLONUM-MOUSE IEPIRIMENT, p.78-95 of BTOLOCICAL AND MEDICA DVISIONS; QUARTERL

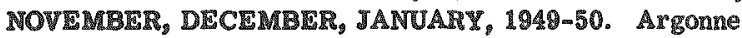
National Lab. [nd] 80. (ANL-4401(2.78-92))

An analysis has been made of a portion of the data that are being collected on the long-term toxicty of intravenougly administered polonium chloride. The experiment involves $720 \mathrm{CF}-1$ female mice (including controls). At 280 days the unquestionable deleterious fifects of 0.027 to $0.008 \mu \mathrm{c} / \mathrm{g}$ and possible adverse effects of $0.004 \mu \mathrm{c} / \mathrm{g}$ on survival, body wight, intestinal infection, spleen weight, and white blood-cell count were evident. Changes In the x-ray appearance of the long bones appeared in animals that received as liftle as $0.0009 \mu \mathrm{e} / \mathrm{g}$, and osteogenic sacomar occurred in two animals that were given only $0.00046 \mathrm{c} / \mathrm{g}$. (NISA)

\section{8}

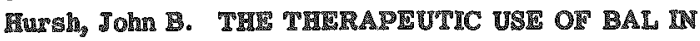

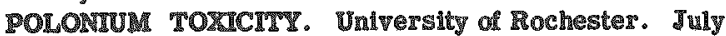

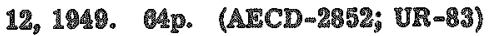

Britsh ant -lewiste (BAL) and related dithiol compounds have been tested for thelr effects on the toxicity of roloniur In rats. When polonum is ingected Intravenously, $\mathrm{BAL}$, the mose effective of the dithiols tested, approximately doubles the 10 -day excretion of polonimm. Where polonimm is in jected subcutaneously, BAL, Is less ffective than BAL acid (NDR 17) or the urea derivative of BAL (NDR 399). The tissue-distribution results show that the BAL rats at 10 days have consistenty higher polonimm concentration in muscle thas do the control rats. H BAL is given as a single intramescular injection one hour after intravenous polonium administzation, the polonium concentration in the whole blood is very sharply decreased. Tissue distribucion of polonim three hour postmaninistsation is given for BAL and control 1ats. These data show that BAL mobilizes polonium from the blood into he muscle, intestine, and kidney. Twenty rats were injected with a dose of polonium (36 $\mathrm{Mc} / \mathrm{hg}$ ) which was Known to be associated with a median survival time of about 25 days. Ten rats ware treated with intramuscular BAL. records were kept of survival thme, daily weight, and periodic blood-sample 1tndings. The troated sats had a median suzplval tume of 8 days, as contrasted with a median aurvival tme of 21 days for the untreated rats. The data appear fo Indicate that the therapeutic effect of BAL is related to both the increased sacretion of polonim and to the ghif of polonimin to less radiosensitive tisaues. (NSA)

\section{8}

IRegberg, Lelv and Fim Devik. ORSERVATIONS ON THY WISW REACTION TO THE DUECUTANEOUS AP-
PLICATION OF METALLIC POLONIUM IN MICE. Acta Radiol. 35, 356-62(1951).

Anickel plate covered by polonium was introduced under the skin in a series of mice. Some of the polonium was dissolved in the tissue fluid and caused general and local radiation damage. The local changes are described in some detail, and the mechanism of the very marked hyperplasia is discussed. The evidence in favor of the existence of an indirect effect through the connective vascular system is stressed. (NSA)

940

Watts, N. P. and K. P. McConnell. FFFCT OF POLONIUM $^{210}$ AND SELENIUM"5 IN EXPERIMENTAL SCHISTOSOMA MLANSONI INFECTION IN MCE. AM. J. Hyg. 53, 11-6(1951).

Neither $\alpha$ nor $\gamma$ radiation, as supplied internally by $\mathrm{Po}^{210}$ and $\mathrm{Se}^{75}$, have been observed to be damaging to the form of Schistosoma mansoni in the experimental infection in mice. (NSA)

\section{Radiurn}

941

Bloom, Margaret A. and William Bloom. LATE EFECTS OF RADUUM AND PLUTONUM ON BONE. Arch. Pathol. 47, 484-511(1949) (AECD-2053; ANL-HDY-496)

Radium injected into mice intraperitoneally at dose levels of 0.3 and $0.03 \mu \mathrm{c} / \mathrm{g}$, and plutonium, 0.03 and $0.003 \mu \mathrm{c} / \mathrm{g}$ given intravenously, produce an overgrowth of bone in the femurs and vertebrae. In the femur this overgrowth begins in the distal metaphysis and is more extensive there than in epiphysis or shaft. This atypical bone, surrounded by gelatinous marrow, fills the metaphysis and encroaches on the marrow of the shaf, which is also invaded by new bone from the endosteum, and in the extreme cases, the two processes combine in some areas to obliterate the marrow. The bone formed after radium treatment is atypical and fibrous, and much of it becomes devitalized. The cortical bone of the shaft also contains many empty lacunae. After plutonium treatment the newest bone is also fibrous, but the greatly thickened trabeculae of spongy bone becomes progressively more compact, although the extremely ir regular arrangement of lamellae and osteocytes distinguishes them from normal bone. Empty lacunae are fewer than after radium. Early changes of an atypical proliferative nature were seen in a 3 -month specimen after radium and in a 7 -month specimen after plutonium, and may represent early sarcoma or a predisposing atate. Resorption of the necrotic bone does not seem to have occurred. In raction of the marrow was seen in two of the radium- and one of the plutonium-injected animals. The changes in the vertebrae 92 are essentially like those in the femurs. (NSA)

Brownmg, IhI. BLOOD CPANGES IN LUMUNIZES USUNG RADIOACTIVE MATERIAL. Brit. Med. J.1, $428-31$ (128영)

In a 7 -year study of 2,737 workers who used radioactive material (and 100 control workers), complete blood analyses were done while they were working, and, of these, 366 were followed up. Exposure was limited to small amounts by various precautionary methods and occurred from external irradiation by a particles, $\beta$ and $\gamma$ rays, radon inhalation, ingestion from hand contamination, and inhalation of radioachive dust. Transient lymphocytosis, the presence of Türk cells, premyelocytes, and premature large mononuclear cells were found, as well as a rare occurrence of anemia. The author discusses the findings and concludes that the blood changes are due to a slight temporary hyper stimulation of the reticuloendothelial system. (NSA) 
943

Evans, R. D. RADUM POISONING: A REVIEW OF DRESENT KNOWLEDGE. Am. J. Pub. Health 23, 1017-23 (1933).

Radium poisoning results when a few micrograms of radium or other $\alpha$-ray emitting solid radioactive substance become fixed in the system.

Its action is principally to destroy the blood producing centers, and to weaken the bones. Calcium therapy, following Aub's and Flinn's work seems the only hope of removing radium from a victim's system. Modes of entrance of radium include the ingestion or breathing of radioactive substances by watch dial painters, chemists, and miners, drinking radium water nostrums, and IV and other injections of radiur, by physicians. From 60 to $98 \%$ of the radium taken into the system is eliminated principally in the leces $(90 \%)$ and urine (10\%). Some radon is lost in the expired air; no radium is lost in perspiration, Necrosis of the jaw, osteogenic sarcoma, and regenerative anemia are among the most common symptoms of radium poisoning. At least six unambiguous physical laboratory tests are avallable for icentifying radium poisoning. (Author's Sum4 술 mary)

Tvans, R. D , E. S. Harris, and J. W. M. Bunker. RADIUM METABOLISM IN RATS, AND TYIE PRODUCTION OF OSTEOGRNIC SARCOMA BY RXPERIMENTAL RADIUM POISONING. Am. J. Roentgenol. Radium Therapy 52, $353-71(1844)$.

The oral or intradermal administration of $25-100 \mathrm{\gamma}$ of RA in young adult of Wistar rats always resulted in chronic Ra poisoning and in a high percentage of cases in the development of primary osteogenic sarcoma. Many of the classic symptoms of $\mathbb{R}$ a poisoning in humans were exhibited by the animals. There was hypercalcification at the ends of the long bones, which become rragile but healed satisfactorily, with marked cc ncentration of the Ra at the site of repais. The tumors were usually primary in the vertebrae, with metastases in lungs and other organs, and were transplantable. At death $1-7 \%$ of the Ra administered orally remained in the body: whyreas about $50 \%$ of that injected intradermally was retained. A terminal retention of $1 \mathrm{\gamma}$ was sufficient to produce osteogenic sarcoma. The incldence of anemia was low and there was no correlation with the Ra content. It was shown that the rat cannot be used for obtaining an estimate of the toxic dose of Ra in humans; its 945 tolerance/ $\mathrm{kgg}$ of body weight is much higher. (BA)

F'ajerman, Henr, Pascaline Daudel, and Roland Muxart. METABOLISM OF RADIUM FIXED ON RONY TISSUE. Compt. rend. 2as, 1255-7(1946).

A procedure is given for the study of the retention of radioactivity in bones of white rats given gubcutaneous injections of $\mathrm{RaCl}_{2}$. When radioactivated bones are decalcified in vitro wilh the aid of various acids, a notable portion of the radioactivity is removed with the Ca salts without destroying the bone structure; this suggests the possibility of treating radium poisoning in vivo. (CPR) 246

Fishman, Harold C. THORIUM-X INVESTIGATIONS: FISTOLOGICAL STUDY OF THORMUM-X APPLICATION ON RABBIT SKIN. J. Mnestigative Dermatol. 15, T1-4 (1950).

The acceptance of thorium $\mathrm{X}\left(\mathrm{Ra}^{226}\right)$ application in the treatment of skin disorders has been slow because of the possible radiation danger and the lack of histological studies on effects of the application in andmals. The histological changes in the skin of rabbits was therefore investigated. The rium $X$ in alcoholic solution (1000 electro- static units/ce) was applied to the barium sulfide-eplated backs of five adult white rabbits, and biopsy specimens were taken weekly for sis weeks for histologic comparison with normals. The development of hyperiseratosis, vaculolization of the cytoplasm of the priclsle cells, and acanthosis is detailed. The unique skin structure makes rabbits not the best choice as experimental animals for this type of investigation. (NSA)

947

Roecker, Frank I. and Paul C. Roofe. \$TUDIFs OF RADIUM IN HUMAN BONE. Radiology 56, 88-98(1051).

Portions of several different bones from two cases of osteogenic sarcoma attributed to radioactive substances in the bones have been examined microscopically and autoradiographically. The radioactive substances (chiefly Ra and its disintegration products) are found to be concentrated in numerous microseopic localizations. The maximum density of Ra in these localizations appear to vary by a factor of only about 1.5 from one type of bone to another, but the rrequency of occurrence of these localizations may vary by a factor of nearly 25. Certain regions of some bones may be entirely devoid of Ra. The mineral residue of the garcoma of one case contained only a negliglble amount of Ra. This is believed to indicate that Ra was not mobilized from other portions of the body, although this apparently did occur in the case of $\mathrm{Ca}$. This is interpreted as one line of evidence to indicate that Ra is not 048 biochemically similar to $\mathrm{Ca}$ in all respects. (NSA)

Rursh, John B. and Allan A. Gates. BODY RADIU CONYENT OF INDIVIDUALS WTTH NO KMOWN OCCUPATIONAL PEPOSURE. Nueleones I, No. 1, 46-50(1950).

The radium content of the body of 25 adult individuals with no known occupational expowure to radiun has been measured. The average value is believed to be $1.18 \times 10^{-10}$ g radiun per whole body. This agrees qualitatively with predictions based on the radium content of the water supply and known constant ror radium metabolism by the body. The radium content of the body, as derived from measurements reported in this paper, is about $1 / 100$ great as analogows igures previously existing in the diterature and about $1 / 60,000$ of the radium content known to produce neoplastic bone changes. It 1s, therefore, concluded that to the extent to which the present measur ments are representative, it is unilisely that irradation of the bone by body-radium will be an important lactor in the production of osteogenie arreoma in individuals not occupationally exposed to radium. (NSA) 248

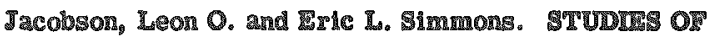
TUE METABOLISM AND TOXC ACTION OF INSECTED

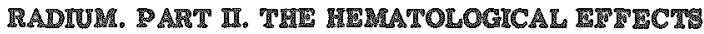

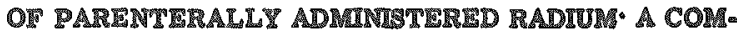

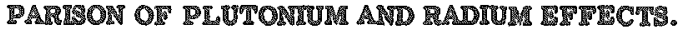

Untw. of Chicago Metalurgical Lab. June 1846. 104p. (AECD-2372: CR-3884)

The effects of the parenteral administration of radium chloride on the henatological constiments of the perdpheral blood of ratB, mice, and rabbits have been atuded. Intraperttoneal and intravenous doses of from 0.005 to 0.84 $\mu \mathrm{c} / \mathrm{g}$ have been given to rats; intraperitoneal doses of from 0.000066 to $0.85 \mu \mathrm{c} / \mathrm{g}_{\text {s }}$ to mice; and intraperitoneal or intracardial doeses of from 0.0016 to $0.1 \mu \mathrm{c} / \mathrm{g}$, to rabbits. There appeared to be mo signilcant specles duference in the sensitivity of the hemopoictic systems of the rat and mouse to radum, but rabbits appeared to be glighth more resistant than the other two species. Also, CF-1 female mice appeared to be momewhat more reatitant than $A B C$ male mice, but whether thic was a gtrasn or ser diference 
has not been ascertained. In the nat, nouse, or rabbit no siggnificant hematological alteration occur ged after an in jection (by any route) of $0.01 \mu \mathrm{\mu} / \mathrm{g}$ or less of radium chloride. A dose of $0.02 \mu \mathrm{k} / \mathrm{g}$ produced an anemia and sustained leucocyle reduction in rats. A dose of $0.03 \mu \mathrm{C} / \mathrm{r}$ resulted in a severe recurent anemia in ABC male mice (but not in C $\mathrm{C}-1$ fembles), comparable changes in heterophil levels in both strdin, and in an initjal moderate reduction in lymphocytes in lhe $\mathrm{ABC}$ male mice. Doses high as $0.1 \mu \mathrm{c} / \mathrm{g}$, however, falled lo produce an anemia in the rabbit, but did camse a mild, early, persist cht reducthon in leucocytes. A dose of $0.94 \mu \mathrm{c} / \mathrm{g}$ in the rat resulted in an initial reduetion in teticulocyles wh subseguent owerproduction and In a maged reduction in platelets with only moderate necovery. Morphological changes were seen in the nucleated cells of the peripheral blood atter radum administration. These were largoly comparable to those previously described in anmals following the axternal application of radiations such as $x-\mathrm{m} 2 y 3, \gamma-y^{3} \mathrm{y} s$, and forst meutrons, or interna 1 radiation in andmals follow" Ing the parenteral administration of plutonium. As evidenced by wn mcreased erythrocyt diamster and increased mear corpucula: hemoglobin, macrocytosis became apparent in rats, mice, and rabbits after the parenteral ad ministration of doses of radium between 0.1 and $0.2 \mu c / g$. (WRA)

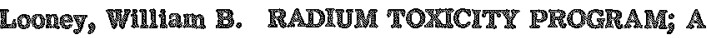

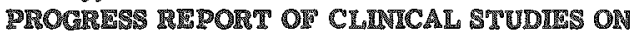

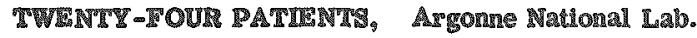

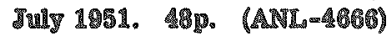

Clindes received radum chlonida $20 \mathrm{yr}$ previonshy. Clinical data

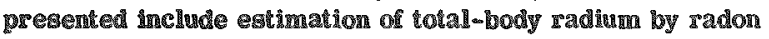
breath and $y$-ray body measurements; clnical history;

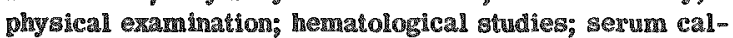
chrm, phomphorus, and alkaline phosphatase deteminations;

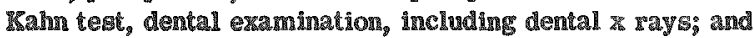
roentgenographic examination of the gkeleton. The amoun of sadium present in the body was determined as the surn of the enanating and monemanat in: fractins obtained by

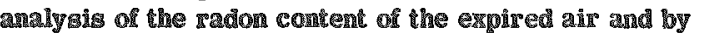

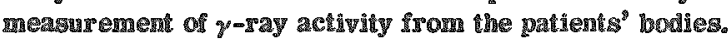
Regults of complete roentgenographic examitition of the

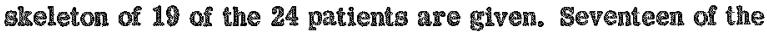
19 pathents had changes in bone density, and, in 8 of the 10

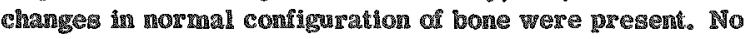

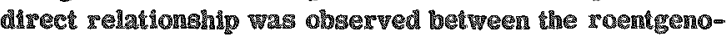
craphic changes or gymplonatology and the amonn of

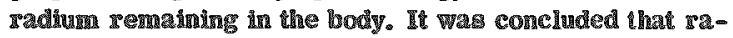

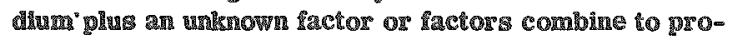
cuce bone degeneration. (NSA) 装

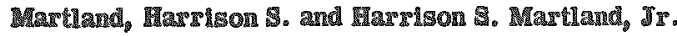

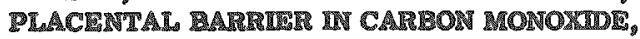

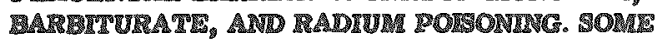

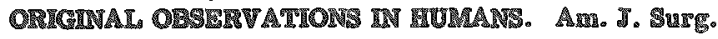
20. $270-9(1950)$.

Seventeen chldiren of 10 of the original New Jersey

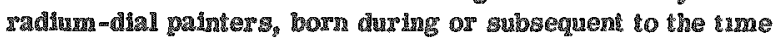
their raothers had ingegted radoactue substances, have

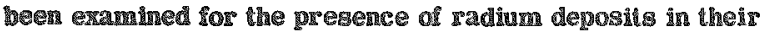

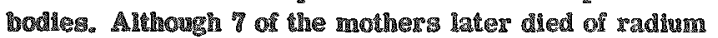

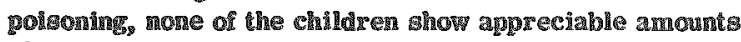

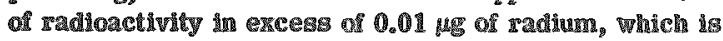

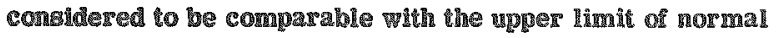

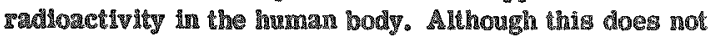
prove that the gasous amanations constanty present in

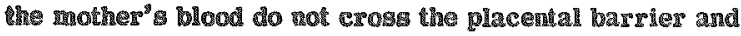

render the fotus radioactive, should they do so, radiuctive deposits in the children would be too small ever to produce hasminl results in the children or their descondants. The effect on the fetus of $\mathrm{CO}$ and barbituate poisoning of the 952 mother has also been studied. (NSA)

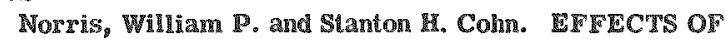
INJECTED RADUUM ON ALKALINE PKOSPLATASE ACTIVITY OF BONE ANO THSULS. Argonne National Lab. Oct. 1951. 22p. (ACCU-1703; UAC-451)

The influence of radium as an internal a emitter on the phosphatase activity of bone and issue was buded in a number of ats injected with varying amounts of padium Chorido. The results indicate that: radium deposiled un bone causes significant reduction in the allaline phosphatase content of bone; at loast a part of the serum phosphatase is derived from bone; the phosphatase conlent of the sof tissues analyzed is largely independent of that of bone and is not affected by ingection of radium; radiation dosages, from deposited radium, of the order of 1 to $10 \mathrm{gep} / \mathrm{he}$ will produce significart reduction in the alkaline phosphatase content of bone within 24 hr: in viteo studies w1th $x$ - rays and with radium added to serum and bone homogerates produced no change in phosphatase activity up to 10,000 \& therefore, the chect of deposited radum as reflocted by the reduchon in alkuline phosphatase activity is almost entirely on the phosphatasefosming elements of bone). (NSA)

25.

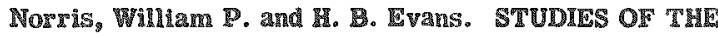

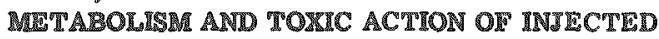

RADIUM. Tniv. of Chicg go Metallurgical Lab. Ine 1946. 115. (ADCD-1965: CII -3652)

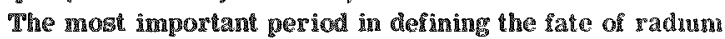
An the animal body is that closely followimg administration, pyoviding she material is geadily available to the buty 17uids. This period as demonstrated in ras by the saptct disappearance of radium from the blood afles intravenous injection and by the araid uptake of fadum from the peritoneal cavily as indicated by blood concentration curves following intraperitoneal admainistat ion. The excretion of intragsitonealy administered radium in rats and mice was very rapid and reached such a low malue

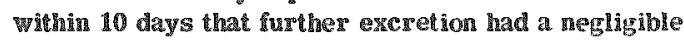
function in determining the final jetention. There exists a linear logarithmic dependence of per cent injected dose excreted pes day (log L) on cime (log T). More of the axcreted radum was present in the feces than in the

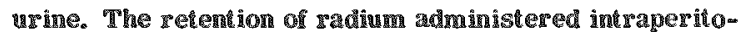
neally 10 rats, win dose lovel. There azsts a linear logarithmic dependence of per cent retention (log R) on lose level (log 1 ). Three possible explanations for this fiect are offered. In ratr and mice, in cound to cause calcifucation of some of ho boft tissues. The effect was moted high inciderce in the aortas of both 3pecies. In rats, calcareons sites were also found in the pylores, liver, periloneal wall, the region of the upper nediastinum, and, oceasionaly, th the testicles. In mice, the only other observed sile of calcification was on the

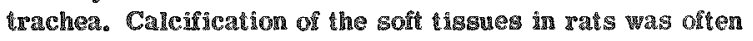
accompanded by mathologic lesions throughout the thodomen and $\mathrm{a}$ high radium content in the viscera. The evaluation of

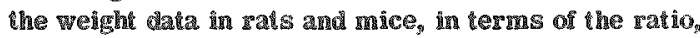
Q(ï, of , the injected 10 control animals shows simele

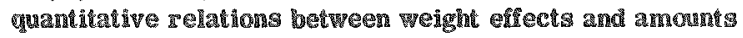

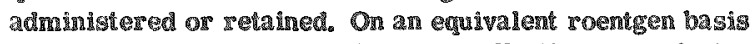
冰-rays appes to be sornewhat move effective in producing

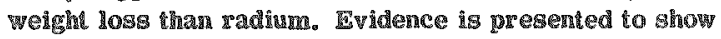


that there existis a linear dependence of mortality (log mi on retaned mactocures of radium (log $R$ ). (ADD) 954

Read, J. RADIUM POISONING. Brit. J. Radiol. 12, 632$7(1939)$.

The damser uf radium poisoning has recently become at matter of mportance because of the possubility of the scatterug of Ra elenent by bombing. The literature on the subject has been surveyed and the pesults summarized. From a consuderation of cases of Ra poisoning both expermental and accidental it is postible to obtain sone idea the distribution of the element in the various ussues when absorbed in different ways. Typical cases of punsonng are described, and particular attention is paid to the question of the getention of inhaled dust. The general conclusson is that about $100 \mathrm{\mu gm}$ by mouth or $50 \mathrm{\mu gm}$ inhaled would probably produce a permanent deposit of the order of 1 or $2 \mu \mathrm{gm}$, a quant ity which may be lethal. These quantitues may be much less in the case of $\mathrm{RaSO}_{4}$ which has a low water solubility and this sall is therefore probably much more dangerous. A very comprehensive 955 boblioger raphy is attached. (BA)

Roofe, Paul G., Mal Bingham, Ralph Comer, and Martha Madison. FETECT OF RADIATION FROM INTRAPERITONEALLY INJECTED RADIUM CHLORIOE UPON MEGARARYOCYTE AND BLOOD PLATELET PRODUCTION IN ALBNO IATS. Anat. Record 108, 537(1950).

Thri puper is an abstract and is reproduced here in 1 its entirety.

Twenty-five rats were divided into groups of five, cach group receving respeetively $10,20,30,40$, and $50 \mu$ of $\mathrm{AaCl}_{2}$ nitraperitoneally. One rat from each group was sacrificed every 3 rd day starting 3 days postinjection. Using a camera lucida, quantitative megalkaryocyte counts were made from slides prepared from intact bone marrow of the femur. The platelet counts were made using a hemocytometer and a $2 \%$ sodium citrate diluting luid. Megakaryocytes decrease progressively with time through the oth day after injection of $\mathrm{RaCl}_{2}$. From this period 10 the 14th day, a trend loward regeneration was noted. The lowest number of megakaryocytes was observed in animals receiving $20 \mu \mathrm{g}$ independent of time of sacrifice. Regenerat son appeared earlier in the higher dosages. Following an initual increase of platelets on the 3 rd day, there was a progressive decrease up to and including the 14th day. Regardless of time, higher dosages also produced a decrease from the normal. The initial destruction of the megalraryocytes is reflected on the $3 r d$ day by an increase in platelets. As the megakaryocyies regenerate it appears that platelet production does not correæpond guantitatively. presumably because young megakaryocytes do not fragment.

858

Roore, Panl G. and Frank Roeker. BTUDIES OF APPARENT SUBMICROSCOPIC CIANGES IN BONE MATRW AND PRIRPHRRAL BLOOD CMANGES ASSOCTATED WITH MIPABOLIZED RADUM. Anat. Record 103, 500(1940).

The following is an abstract and is reproduced here in its entirety.

Apparent submicroscopic structural changes in the bone matrix associated with metabolized radium are presented by photomicrographic technics. A new lype of microtome enables us to cut serially thin sections of bone which are immediately exposed to NTA plates without further treat ment. The radium distribution has been determined by means of tracts in the NTA emulaions produced by alpha particles. Exact regitry between the microscopic bone structure and the alpha-particle tracts is made possible by new autoradiographic technics. Ionizing radiation from radium intraperitoneally injected depressed the red cell count only slaghtly. White cells were depressed considerably. Two unjections, $10 \mu \mathrm{MLCl}$ each, produced a much slower rate of whole body growth.

95 \%

Roofe, Paul G. and $\mathbb{F}$. $\mathrm{E}$. Hoecker. THE EFECT OF INTRARPRITONEALLY INJECTED RADUUM CHLORIDE UYON THE PERIPHERAL BLOOD OF ALINO MALE RATS. Anat. Record 103, 555-6(1048).

The following is an abstract and is presented here in its enturety.

Twenty-five albino rats were injected intraperitoneally with radiurn chloride in groups of 5 , each separate group receiving the following dosages: $10,20,30,40,50 \mu \mathrm{g}$. Twelve animals served as controls. Complete blood counts and fraglity tests were done prior to $R$ aCl treatment. One animal from each group was sacsificed at 3 -day intervals up to 15 days. Two controls were also sacrificed on those days. All tests were done on day of sacrifice. Results are grouped in two ways: those which received same dosage but. at different time intervals; those which were ancriftced on same day but dosage varied. Normal red counts on 165 samples averaged 7,320,000 per cubic milimeter. Ony a very slight drop in red cells was noticed after RaCl, treat $^{-}$ ment. Normal whites were 20,250 per cubic millimeter. Both increased time interval and inereased dosage showed progressive arop in white cells. No great chasnge occurred in the differential count. Platelet counts dropped from a normal of 852,000 to 142,000 per cubic millimeter, in those rats with $50 \mathrm{\mu g}$. Fragillty tests zemained unchanged.

Sabin, IR. R. C. Doan, and C. E. Forknex, Ther PRODUCTION OF OSTEOGRIIC SARCOMATA AND THE EFFECTS ON LYMPH NODES AISD BONE MARROW OF INTRAVENOUS INJPCTIONS OF RADIUM CRLORDD AND MESOTHORTUM IN RABBITS. J. Expt. Med. 56, 267-828(1032).

Authors began study of the effects of radioactive substances on the cells of the blood and connective tissues in rabbits in 1926. Radium chloride and mesothor jum were used: these compounds were put up in sealed ampoules of such a strength that 2 ce of normal salt golution contained about 5 micrograms of the active material. In Jamary 1928, the strength of this material was tested and was found to be for an ampoule of radium chloride the equivalent of 5.1 micrograms of radium and for one ampoule of mesothorium, 7.7 micrograms of radium. The dose of the mesothorium was also greater because it is known that it gives of 5 alpha particles to 4 from radium chloride. Nine rabbits were used for the experiment, five of which received radiun chlor ide and four mesothor sum. The injections were given intravenourly once a month. The blood cells were counted once a weels. The differential counts were made with the supsavilal technique. Each animal was killed when signs of some damage became evident, such as a fracture or marked weakness. protocol for each rabbit is given. The observations in this work suggest that with certain doses of radioactive material, the fundamental damage in the lymphoid lissues is to the stem cell and that the damage is to the chromatin of the nuclei of these cells. The erythrold tissues are apparently less susceptible to radioactive material than the lymphoid tissues but an origimal anemia of secondary type from peripheral destruction may eventually be changed to one of primary type through decreased maturation of primitive calls in the marsow. The darnage of dymph modes and bone marrow leads to atrophy of these organs. The cells of the liver and 
thymus suffer nuclear damage of the same general character as is seen in the dymph nodes, and there is an atrophy of these organs. The storage of the radioactive material in the bones gave rise to osteogenic sarcomata in 2 out of 7 rabbits surviving from 11 to 19 months. A repetition of the experiment has been undertaken with more intensive studies to test the validity of the findings. (S.V.S.)

950

S1Lerstein, Hannah L. RADIUM POISONING; A SURVEY OF THE LITERA TURE DEALING WTTH THE TOXICITY AND METABOLISM OF ABSORBED RAOIUM. University of Rochester. May 26, 1945. 41p. (AECD-2122; M-1695)

This report is based upon a detailed survey of literature concerning radium poisoning from 1908 through 1944. Of 17 human fatalities following ingestion of radium mixed usually with meso-thorium degcribed in the literature post-mortem activities were determined in nine; activities ranged from 1.2 to 126 micrograms radium equivalents. Only two were due definitely to radium alone. The actual intake was known in only one case, namely 2,800 micrograms of radium taken over a period of pive years, leading to death about a year later: 73.66 micrograms were retained at death. All showed advanced regeneration type anemia, acute leucopenia, and varying degrees of bone destruction. Sir deaths ascribed in the literature to inhalation of radioactive dusts occurred 5 to 15 yengs after begimning exposure. Dyspnea and lung hibrosis were the prodominant gigns, but bronchial carcinoma was also present in one case. There were only two early reports dealing whth acute levels of dosage. One dog received 75 micrograms of radium per kilo body weight, in a single intravenous injection, and died at 14 days. Four groups of experiments with prolonged or intermittent dosage resulted in chronic poisoning. In four longstanding chronic human cases the coefficients of elimination were 0.002 to $0.005 \%$ per day, at which rate it would take 45 years to get rid of half the fixped radium in the body. The amount of radon lost through the lungs depends on the degree of fixation of radium in skeletal tissues and relative case with which the radon produced can escape into the circulation. Deposition and distribution of radium in the body is analogous to that of lead and calcium. One early experiment has been said to indicate that at $3 / 2$ months after intravenous injection it is still widely distributed throughout the soft tissues, the highest concentrations being in bone marrow and liver, moderately high in lung. Deposition in bone probably continues to take place as long as any radium remains in soft tissues and in the chrculation, going especially to reglons associated with the most rapid uptalse or turnover of calcium. Seven human eases are known to have carried more than 0.02 and less than 0.5 micrograms of radum in their system for 7 to 25 years without showing any clinical signs of poisoning, yet as little as $1.2 \mathrm{microm}$ grams has been found in a fatal case. Therefore, the Bureau of Standards in 1941 get 0.1 microgram fixed in the body as the maximum level of radium content. (NSA) 960

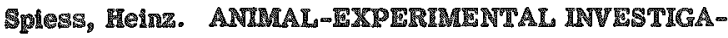

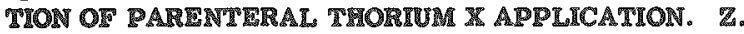
ges. expti. Med. 117, 567-86(1951).

The clinical effects on rabbits of intravenous injection of ThX (R224) in single or divided doses up to 1000 esu has been studied. The changes in the blood picture are reported, including effects on blood sugars, serum proteins, and serum $\mathrm{Ca}$ and $\mathrm{P}$ contents. Effects on the kidneys, reproductive system, and metabolism are noted. $\mathrm{X}-\mathrm{ray}$ photographs and autoradiographs illustrate the localization and damage caused by ThX in bone and cartilage growth and fracture healing. (NSA)
961

Spiess, H. H. Poppe, and O. Wuinsche. TFECT OF THORIUM \& ON DY UPTAKE BY CELLS. Z. ges. expt1. Med. 116, $\$ 44-53(1950)$.

Injection of high doses (5 doses of 150 electrostatic units in 2.5 weeks) of ThX into rabbits, followed by $\mathrm{ml}$ of $1 \%$ trypan blue, gave a higher serum and urine dye level in a period of 72 hours, than in animals not receiving ThX. Interposing 10 days between the last ThX injection and the dye injection maintained this difference. Smaller doses (15 doses of 25 units in 8 weeks) caused a reversal, in that serum and urine dye levels were lower than those of con982 trol animals not receiving Thx. (CA)

Welkel, J. and Egon Lorenz. RXCRETION OF RADIUM FROM THE MOUSE. Radiology 51, 865-70(1948).

The possibility exists that radium will form an organic protein compeund and that its excretion might be influenced by BAL (2,3-dimercaptopropanol). Three groups of $\mathrm{C}_{3} \mathrm{H}$ male mice, 50 in each group were injected intraperitoneally with 10 micrograms of radium element as bromide. One group of animals was injected with $5 \mathrm{mg}$ of BAL daily for the first 20 days; the second group was given the same amount of $\mathrm{BAL}$ beginning at the 20 th day for 20 days after injection of the radium. The exeretion was measured by determining the amount of radium remaining in the animals. For this purpose a multiple Geiger Mueller counter was developed, in the inside of which the animals were placed. The number is described and the excretion data given. It was found that BAL does not influence the excretion of radium either in the acute or in the chronic stage of radium poisoning. (ADD) 963

Wilson, C. W. and J. R. Greening. GAMMA-RAY PROTECTION IN RADUM THERAPY. Brit. J. Radiol. 21, 211-20(1948).

It is suggested that if the $\gamma$-ray dosage received by radium the rapists during their radium work could be assessed (even if only roughly), more speciflc opinion might be formed as to what levels of exposure have proved to be safe. Calculations and measurements of local dosage and integral dose received by radium mould technicians and radium therapists are made in order to make each assessment. On the basis of these results the implications of the various proposed standards of protection are examined and set out in terms of actual working conditions. For this purpose protection is considered in the categories in which it seems the subject should be divided, viz., local exposure of the hands, local exposure of the gene material and integral dose throughout the whole body. In relation to medical worls ît is concluded (1) that many busy radium personnel may receive local exposure to the hands in excess of that now recommended in the U. S. A. as a safe amount (1 $\mathrm{r}^{\mathrm{p}}$ per day), (2) that if circumstances dictate is ghould be practicable, by the use of suitable screenage, to reduce the local dose received by the genitals of mould technicians and therapists to the low figure recently suggested by sievert 10.01 per day), and (3) that this low figure may be impossible of realization for the integral dose received by personnel handling radium. It is suggested that in respect of integral dose, past experience indicates that a figure of $0.1 \mathrm{x}$ per day may be gafe. It is, however, desirable to achieve $0.01 \mathrm{r}$ per day, particularly for personnel continually receiving radiation at a low but approximately uniform doge-rate over the whole bodythis is because injury to the gene material is the foremost consideration. Suggestions are made for the future collection of data which will assist in placing protection standards on a aurer foundation. (NSA) 
964

Witten, Victor H., Milton S. Ross, Eleanor Oshry, Arthur B. Hyman, and Vera Holnström. STUDHES OF THORUM X APPLIED TO HUMAN SKIN; I. ROUTES AND DEGREE OF PENETRATION AND SITES OF DEPOSITON OF THORIUM X APPLIED IN SELECTED VRHCLES. NEW York Unie, Hospital and New York Univ. [nd] $22 \mathrm{p}$. (AECU-1470)

A basic problem which has remained unsolved in using thorium $\mathrm{X}\left(\mathrm{Ra}^{224}\right)$ for therapy in dermatological conditions is whether Th $\mathrm{X}$ is confined to the surface or penetrates the skin in any way. A report is presented of an investigalion of the routes and degree of penetration and sites of deposition, using nine pieces of tissue from eight different

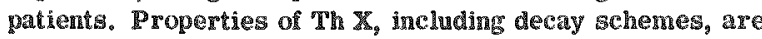
discussed. Th $\mathrm{X}$ (adsorbed on $\mathrm{CaCl}$ in alcoholic solution) was applied to a small (about $5 \mathrm{~mm}^{2}$ ) area of skin, previously cleansed with alcohol and walled of with rubber on Duco cement. The amount applied varied from 0.001 to $0.015 \mathrm{~cm}^{3}$ and the strength from 0.06 to $3.0 \mu \mathrm{c}$. The area was covered with collodion and allowed to dry. One to five days later the collodion was stripped of , and the treated area excised. Radioautograms prepared from frozen sections of the tissue show the isotope penetrates the epidermis and is distributed through the prickle cell and basal layers, flows down the hair follicle and penetrates the follicular wall, and passes down the sweat duct and into the sweat gland. Radioautograms showing the deposition of TI $X$ in human skin are included. (NSA)

\section{Radon}

\section{5}

Arkin, Alvin M. and Norman Simon. RADLATON SCOLIOSIS: AN RXPERIMENTAL STUDY. J. Bone and JoLn: Surg. 32A, 396-401(1950).

Examples are presented concerning the production of scolioses in rabbits by the direct action of radiation upon the spinal epiphyseal plates. A radon seed, containing 0.6 me of radon, was placed adjacent to an intervertebral space in the lumbar spine of a rabbit in one case, and the radiation caused a suppression of the growth of the epiphy sis on the side nearer the seed; a few months later, growth of the spine manifested asymmetry by a wedging of the vertebrae adjacent to the source of radiation. When radon seeds of $0.6 \mathrm{mc}$ were placed opposite the middle of a vertebral body, or when $0.2-\mathrm{me}$ seeds were placed near one epiphyseal plate, no discernible wedging was noted. Growh suppression of the vertebral epiphyseal plate near the 0.6 -me seed was induced by a dose of about $2,700 \mathrm{r}$; the epiphysis nert above the seed grew normally although it received a dose of $300 \mathrm{x}$. Similar asymmetrical growth arrest was effected by the use of an $x-r a y$ beam directed to one side of the spine; the radiations were delivered by a $140-\mathrm{kg}$ machine through a filtration of $0.25 \mathrm{~mm}$ of Cu and $1 \mathrm{~mm}$ of Al at a target distance of $30 \mathrm{~cm}$ for an epiphyseal dose of 1,000 5 ; wedging appeared 7 weeks later. It 18 con cluded that a single dose of $1,000 \mathrm{~g}$ is required to produce definite wedging in the spines of young rabbits. (NSA) 966

Cornitield, Jerome. RETENTION OF RADON BY TUL MOUSE. II. METPOD OF FETIMATING BIODECAY FROM EXPERIMENTAL DATA. NuCleonics 5 , No. 4, $68-75(1949)$.

This paper describes the method of estimating radon disintegration from the experimental data; the exper'fmental data referged to is that which was presented by Molleroft and Lorene (Nucleonics, 5: 63, 1949). A formula is derived for wD, the root mean square error, where $D$ is the number of radon disintegrations. The author suggests how the error formula can be applied to the planing of ex967 periments. (NSA)

Kolleroft, Joanne Weikel and Igon Lorenz. RETENTION OF RADON BY THE MOUSE. 1. IXPRRIMENTAL DETERMINATION OF BIODECAY AND ENERGY ABSORRED. Nucleonics $5, \mathrm{No} .3,63-71(1949)$.

This report describes the elimination of radon from mice following the administration of an aqueous radon solution: biodecay and the determination of the energy absorbed by the animal from the disintegration of the radon and its subsequent short-lived decay products are discussed. The radon used in the experiments was obtained by the bombardment of RaBr; $75-80 \%$ of the total radon prepared was obtained in solution. Two methods were used to study the elimination of the radon: in the first, groups of mice were sacrificed by cervical dislocation $5,10,30,60,90,120$, 180 , and 240 minutes after the radon administration, and were gealed quickly in glass tubes for subsequent measurement; in the second, after the administration of the radon, the live animal was placed in a small vessel, and by means of an air transfer system and activated charconl collection bulbs, the radon was collected from the exhalations and measured at intervals. Prelminary studies were also made on the modes of administration of the radon; an intraperitoneal injection of $0.25-0.5 \mathrm{me}$ of radon was given and the amount in the mosse was determined $5,10,15$, and 30 minutes later; the rate of elimination after oral administration was alko gtudied. The realts showed that the elimination rate, at least for the first hour, differed according to the route of administration used and was generally higher for the intravenous than for the other methods. The amount of fat present in the animal influenced the radon retention only after intraperitoneal injec968 tons. (NSA)

Lange, T. and R. D. Evans. ABSORPTION OF RADON THROUGR THE SKIN AND ITS EXHACATION THROUGH THE LUNGS. Radiology 48, 514-16(1947).

The authors followed the fate of radon originally contained in an ointment ( 44.4 microcuries of radon per ce of lanolin) to determine to what extent it enters the tissues. The experiments were carried out on normal intact skin, as well as on the surface of large postphebitic leg ulcers. In the firgt group of experiments, samples of exhaled air were taken after 20 minutes, $2 / 3$ hours, and $4 / 2$ hours. Approximately $2.4 \%$ of the radon applied was exhaled within the first $4 \frac{1}{2}$ hours when the ointment was applied to the leg ulcer while the amounts exhaled from cases of normal skin were only $0.13 \%$ and $0.08 \%$ for the rame period. In the second experiment, the patient with the ointment on the intact skin exhaled during the first four hours approxlmately 1.2 microcuries or $0.4 \%$ of the amount applied, and during 23 hours $4.5 \%$. The patient with the leg ulcer exhaled during the first four hours approsimately 6.7 microcuries or $1.9 \%$ of the amount applied, and during 23 hours 10\%. All calculations are based on the assumption that the subject exhaled 5 liters of air per minute. Precautions were taken in both experiments to prevent inhalation of radon a stated that appreciable amounts of radon applied in ofntments are absorbed through the intact skin, and gtill nore through open wounds. The radon exhaled must therefore have been earried to the patien" circulating blood or lymph. (S.V.5.) 269

MeClellan, Walter S. and Carl R. Comstock. CUTANE OUS ABSORPTION OI RADONT FROM NATURALIS 
CARBONATED MENLRAL WATER AND PLAIN WATER BATHS. Arch. Plyys. Med. 30, 29-36(1949).

Two subjects were studied under various conditions in baths of 30 min duration. The extent of Ra absorption cluring and after the bath was determined. The concentration of Rn in the expired air was greatest $(2.74-3.53 \times$ $10^{-10}$ curies/1 air) when the subject bathed in $\mathrm{CO}_{2}$ water containing $R n(0.107 \mathrm{uc} / 1)$ and breathed air over the surface of the tub. About the same amount of Ru was excreted by the subjects in a $\mathrm{CO}_{2}$ bath containing $\mathbb{R n}$ but breathing out side air through a mask $\left(1.77-2.89 \times 10^{-11} \mathrm{cur} r \mathrm{es} / 1 \mathrm{a} \mathrm{a} \mathrm{r}^{\circ}\right)$ as when he bathed in plain water containing Rn and breathing air over the isb $\left(2.08-9.34 \times 10^{-11}\right.$ curies $/ 1$ air $)$. When the patient was in a bath of plain water and Rn but breathed outside air, he excreted $0.17-0.62 \times 10^{-12}$ curies $/ 1$ air. The Rn was eliminated almost completely in 3 hours. Rn is absorbed through the skin in greater amounts when the water contains $\mathrm{CO}_{2} \cdot(\mathrm{CA})$

\section{\$ुodนum}

\section{0}

Evans, T. C., M. Lenz, C. P. Donlan, and M. J. LeMay. FETETS OF RADIOACTIE SODUM ON LEUKEMA AND ALLIED DISLASES, PRELIMINARY REPORT. Am. 7. Toentgenol. Radium Therayy 59, 469-81(1948).

Radiosodium, taken by mouth, in a suitable quantity and at approperiate intervals, is effective in reducing symptoms of chronic myelogenous leukemia, chronic lymphatic leullemia and polycythemia vera. The rate of response to each treatment appear to be intermediate between that of roentgen therapy and treatment with radiophosphorus. The contratndications are similar to those for other forms of radiation therayy. 18 references. (NSA)

\section{1}

Finkle, INJECVED N2 ${ }^{24}$ FOR MCE AND RATS. PART I. METABOLISM AND GENTERAL TOXICTTY. UnIv. of Chicago Metallurgical Lab. June 1946. Decl. May 24, 1948. 29p. (ARCD-2011; C 1 -3856)

Introductory work on the toricity and metabolism of radioactive subatances derived from uranium fission was gtarsed indirectly by using cyclotron bombarded sodim ${ }^{24}$, an arificial radiogetive isotope with a half-life of 14.8 hours. The isotope decays by emission of $\beta$-particles (1.4 Mev) and $\gamma$ rays $(1,4$ to $3,6 \mathrm{Mev})$ to stable magnesium. Mice and rats were administered doses of radioactive sodium $\left(\mathrm{Na}^{24}\right)$ ganging 1 rom 12 to 150 microcuries per gram of body weight. It is apparent that the LD-50 lies bstween 20 and $40 \mu \mathrm{c} / \mathrm{g}$ with the possibility that females may be more senßstive than males. Immediately after injection of $33 \mu \mathrm{c} / \mathrm{g}$ or more, there was a drop in weight with the rate and magnitude of the loss varying with the dose. At the lowest dose level, $12 \mu \mathrm{c} / \mathrm{g}$, there was no significant effect on growth. Clinically, aside from loss of halp, encrugting of the eyelids, and hunched position of the animals at death, no characteristic lesions were noted. Rats that received $90 \mathrm{~mm}$ by intraperitoneal injection excreted $19 \%$ of the radioactive sodium in the first day and another $9 \%$ on the second day. Rats given smaller doses excreted an average of $15 \%$ on the first day and $12 \%$ on the second. The ratio of the fecal sodium content to the urinary content was 0.05 or less. In mice, the sodium turnover was somewhat faster than in rats. Mixed excreta measurements showed $33 \%$ of the sodium excreted by 24 hours and $50 \%$ by 72 houra. Analytical recovery of the injected sodium was between $80 \%$ and $100 \%$ in most groups. Discounting the systematic errors and the uncertainty for X-ray comparison, it might be aad that the ratio of beta ray affectiveness to roentgen ray effectiveness is approximately one. (ADD)
972

Jacobson, Leon O. and E. L. Simmons. THE ACUTE RADIOTOXICITY OF INJECTED Na" FOR MICE AND RATS. PART II. THE EFFECT OF Na ${ }^{24}$ ON TRE LEUCOCYTES OF THE PERTPHERAL BLOOD OF MCE. Univ. of Chicago Metallurgical Lab. June 1946. Decl. June 9, 1948. 11p. (AECD-2036; CH-3857)

Groups of male mire of the $A B C$ and $C F-1$ st rains were injected intraperitoneally with $\mathrm{Na}^{24}$ as the chloride in doses of $12,23,47,65$ and $90, \mu \mathrm{c} / \mathrm{g}$. Leucory te counts and differential blood smears were made on all animals before injection of the radioactive isotope and at intervals thereafter; through 10 days in one experiment and thrugh 15 days in another. A moderate to severe leucopenia followed the injection of all the above doses except the $12 \mu \mathrm{c}^{\circ} \mathrm{k}$ Death of the animals in the groups which received 47,65 , or $90 \mu \mathrm{c} / \mathrm{g}$ occurred before the eighth day. The number of neutrophils per cubic millimeter rose slightly for thee days in the group which received $90 \mu \mathrm{c} / \mathrm{g}$. No further sampling was possible in this sroup beeause of the death of all the animals. No reduction of neut rophils followed the injection of $23 \mu \mathrm{c} / \mathrm{g}$ in one experment; 23,47 , and $65 \mu \mathrm{c} / \mathrm{g}$ produced a marked neutropenia in another experiment. Lymphopenia occurred in all groups of animals which re ceived $23,47,65$, or $90 \mu \mathrm{c} / \mathrm{g}$ and continued until sampling was discontinued or until death of the animals. (NSA) 973

Jacox, harold W. THROMBOCYTOPLNIC PURPURA FOLLOWING THERADEUTIC ADMRISTRATION OF RADIOACTIV SODIUM. Radiology 51, 860-1(1948).

Thrombocytopenia purpura developed following therapeutic administration of radioactive sodium in a case of metastic sympathicoblastoma. During a 5 -month period a total of 202 millicuries of radiosodium (285 5 ) wass administered to a 21-year old male. Pain was alleviated and repeated blood counts showed no abnormalities until after the last treatment when hemor rhagic manifestations appeared. The number of platelets fell rapidly but all the other blood elements continued to be normal. Death was attributed to aplastic anemia. An autopsy revealed punctate hemorrhages everywhere, clotted blood in the pelvis of the left kidney, and hemosiderosis in the spleen and bone marrow. In this case the purpura was caused by the radiosodium and not the disease itself. Although other patients have received much more radiosodium without a similar reaction, this patient seemed to become more susceptible 974

SInyder, R. H. and W. E. Kisieleski. TME RHLATRE BIOLOGICAL IFFECTVISNESS OF BETA AND RONTGEN RADIATION AS SHOWN BY THE RADIOTOXICITY OF SODIUM" FOR MTCE. Radiology 54, 743(1950).

Mice were injected intraperitoneally with doses of radioactive Na ${ }^{24}$ ranging from 12 to $150 \mu \mathrm{c} / \mathrm{g}$ of body weight. The 20 -day $\mathrm{LD}_{50}$ (lethal dose for $50 \%$ ) was found to lie between 20 and $40 \mu \mathrm{c} / \mathrm{g}$, the final value arrived at by interpolation being $30 \mu \mathrm{c} / \mathrm{g}$. The calculated radiation dosage corresponding to this $20-$ day $\mathbb{L D}_{56}$, considering excretion and decay, was 680 rep. The ratio of $x-r a y$ to beta-ray effectiveness is approximately $1.4: 1$. (CA)

\section{Strontium}

975

Anthony, D., K. Lathrop, and R. Finkle. RADIOTOXICITY OF INJECTED SR $^{80}$ FOR RATS, MICE, AND RABBIT, PART II. METABOLISM AND ORGAN DISTRIBUTION. Univ. of Chicago Metallurgical Lab. June L, 1946. Dech. Oct. 9, 194\%. 36ip. (MDDC-1363; CM-3846) 
Muce, rats, and rabbuts excreted $\mathrm{Sr}^{89} \mathrm{SWr}^{80}$ very rapidly following intraperitoneal or int ravenous administration. The greatest part of the excretion trok place durug the fir st three days, and by 10-15 days virtually no more of the retained struntium was exreted. Average total excretion values were $54 \%$ of the inject ${ }^{2}$ dose for the mouse, $40 \%$ for the rat, and about $70 \%$ for the rabbit. Average total retention values were $45 \%$ of the injected dose for the monse, $59 \%$ for the rat, and $21 \%$ for the rabbit. After three days almost all of the retained $\mathrm{Sr}^{28}{ }_{-} \mathrm{Sr}^{30}$ was found in the slealeton. The concentration in bone was more than 100 times that in soft tissue. There does not appear to be any significant variation in retention of $\mathrm{Sr}^{\text {82 }}$ on a per gram basis between different types of bone. The logarithm of the retention, the rate of axeretion, and the blood concentration of radioactive strontium in each case was found to be a straight-line func976 tion of time. (ADD)

Anthony, D., R. Lathrop, and R. Snyder. RADLOTOXICITY OF INJECTED SR' TOR RATS, MCE, ANO RABBITS. PART III. LETMAL ACTION AND CLINICAL CHANGES. Univ. of Chicago Metallurgeal Lab. June 1946. Decl. Oct. 17, 1947. 20p. (MDDC-1364; CH-3845)

The LD-50 in 30 days was found to be between 7 and 8 $\mu \mathrm{C} / \mathrm{g}$ of $\mathrm{Sr}^{2}-\mathrm{Sr}^{20}$ when administered intraperitoneally to mice: for rats it was slightly less than 5 je gand for rabbits it was also slightly less than $5 \mu \mathrm{c} / \mathrm{g}$. The food intake and gain in weight of rats and mice given doses of $2 \mu \mathrm{c} / \mathrm{g}$ or more, but showing no apparent symptoms of radiation sick97 ness, were less than those of the control animals. (ADD)

Asling, C.W., D. A. Hellex, D. I. Copn, and J. G.

Hamilton. DEPOSITION OF RADIOACTIVE STRONTIUM AND THE RARE TARTY PROMRTHUUM IN THE SRELE-

TAL TISSUES OF TRE RAT. Anal. Recosd 103,420 (1249).

The following is an abstract and is reproduced here in its entirety.

Radioautographs of sections through the costochondral junction of young normal and phosphorus-deticient rats were compared with underalcified silver-gtained sections to determine any differenees which might exist in deposithon of carrier -free radio-st rontism $\left(\mathrm{Sr}^{29}\right)$ in bone, osteoid and hyaline cartilage (calcified and noncalcified). The rats were sak rificed one and 72 hours after intraperitoneal ad. ministration of the radio-element. Radiom gt rontium was found in the cortical bone of both normal and deficient rats as well as in the spongy bone of normal controls, but not in the osteold of the deficient animals. The digtribution and degree of depostion was comparable at one and 72 hours. In both control and deficient rats radio-gtrontium was demongtrated in the caleified cartilage but not in the noncalcified cartilage in the chondral segment of the rib. The degree of deposition ayproached that of the cortical bone of the same sections. The distribution of gronnethium (element 61) in normal adult wats 96 hours after injection dilfered remarkably from that of strontium. The promethim was observed in the region of periosteum, endosteum, and perichondrium and not within the cortical bone, calcified cartilage, os non-calcified cartilage.

978

Buchanan, D. L., B. Gasvoda, and E. S. Guzman Barron. ERTECTS OF A SINGLE LETHAL INTRAVENOUS DOSE OF \$TRONTUM CILORIDE ON THE PLASMA OF A GOAT. Argonne National Lab. June 1, 1247. $178 \mathrm{p}$. (MDDC-1418-15)

rlectrophoretic patterns of the plasma of a goat besore and after the intravenous injection of a lethal doae of radiom active strontium are presented. Changes seen are a mod erate decrease in albumin, $\alpha_{2}$ glohulin, and $\gamma$ globulin. (ADD)
979

Chaikof, I. I., M. C. Fishler, and C. Entenmann. RADIO STRONTIUM (SR85). Argone National Lab. June 1, 1947. 1p. (MDDC-1142-D).

Tracer studies-A continuation of thig work with more conaplete data inolicates that the retention of $\mathrm{Sm}^{85}$ by the skeleton was essentially as has been described in a previ ous report. The uptake of this material in the bones of the animals which received it by mouth was lound to rise by almost a factor of two between the 4th and 10th days. Otherwise, there were no mignificant differences to be observed between the present more complete informat ion and that which was given previously. The rate of excretion of $\mathbf{S} \mathbf{r}^{\text {is }}$ has now been followed for a period of 30 days, and after the 15th day tis excretion is apparently lew than the rate of 980 decay of that which is retained in the body. (ADD)

Chaikrof, I. I.. M. C. Fishler, and C. Entenmann. RADIO STRONTIURY $\left(\mathrm{SR}^{\mathrm{BS}}\right)$ ). Argonne National Lab. [nd] $8 \mathrm{p}$. (MDDC - 1483 - B)

Inconplete experiments with $\mathrm{Sr}^{85}$ indicate that the intraperitoneal injection of this element is followed by the prompt deposition of approsimately $75 \%$ of the dose in the bones. The soft tissues contain negligible quantities of $\mathbf{S t}^{25}$ as compared to the skeleton. The oral administration of $5 \mathrm{r}^{\text {g5 }}$ was followed by an uptake of approximately $10 \%$ of the administered dose at the end of 24 hours. The relative dismtribution of that which was absorbed was ahnost identical 981 to the values observed after intraperitoneal injection. (ADD)

Cowan, Frederick $\mathbb{P}$. Lawrence B. Farabe, and Robert A. Love. HEALTH PHYSICS AND MEDICAL ASPRCWS OF A STRONTIUM-90 INHALATION INCIDIRTE. Brookhaven National Lab. [nd] 12p. (AECU-1169)

A number of health physics, medical, and chemical aspects of the management of an incident in which an individual became contaminated from activity released in the labogatory curimg the prepartion of a $5^{20}$ source are dis cussed. The results of the analysig of the uxinary elimination of $\mathrm{SP}^{00} \mathrm{X}^{80}$ for the period following the inhalation are pregented. (NSA)

\section{2}

Curtis, H. J. THE EFIECT OF CLAY ON TME INTESTINAL ABSORPTION OF 5RRONTIUM. MOnsanto Chemical Co. Jan. 24, 1046. 8p. JMDC $=410 ;$ MONH -4 (D)

Rats have been fed active strontium which was adsorbed on clay, while their controls were fed the ame amount of active strontium in aqueous volution. There was no sifmillcant difference in the amoun deposited in the glkeleton bem tween the two groups, and it is concluded that if long are adsoribed on inert material, they will be eluted dur Ing their pasage through the gastrointestinal tract. (ADD)

Finkel, Mirlam P. TPE TRANESMSSION OF RADIOSTRONTUM AND PLUTONUM FROM MOTYIER TO OTFSPREN IN LABORATORY ANIMALS. PhySIOL. 2000. 20, $405-21(1947)$.

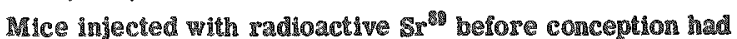
fewer litter than the controls. Injection of radoactive gtrontivan or of plutonium increased the number of gtinborm litters and the rumber of atuborn young in the 1 tteres that Iived. Pregnant mice treated with either $\mathrm{Sr}^{\mathrm{BO}}$ or $\mathrm{Pu}$ lived longer than simularly treated nonpregnant iemale mice. Treated pregnant mice also gufiered legs rađiatun damage. After delivery, total Sr retamed by mother was the anme as that of virgin females at alnilar time intervalo after injection. The proportion of St or Fus transmitted to the young at birth varded with the injection - delivery perlod was within four days, the SI activly per gram body welght of 
the newborn mouse exceeded that of the mother. The initial excretion of radioactive $\$ \mathbf{S}$ by very young mice was considerably lower than that of adult mice. Long bone malformaton, anemia, retardation of growth, and osteogenic sarcoma occurred before weaning in animals that were treated in utero with $5 x^{8}$. No pathologic conditions developed in the animals treated with $\mathrm{Pu}$. No difference in sensitivity to $\mathrm{Pu}$ 984

or $\mathrm{Sr}^{\mathrm{E}}$ was found in young and acult tissues. (N)

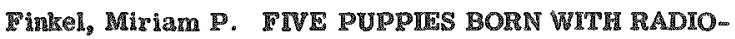
\$TRONTIUM. A PRELIMTNARY NOTE, p.71-22 of BIOLOGICAL AND MEDICAL DIVISIONS QUARTERLY REPORT, $A U G U S T$ TO NOVEMBER 1948. Argonne National Lab. Nov. 1, 1948. 12p. (ANL-4227(p.71-82))

On May 4 , 1946, a pregnant dog of unknown ancestry was Injected subcutaneously with me of radiostrontiun (1.C $\mu \mathrm{c} / \mathrm{g}$ ). Sis days later she delivered five apparently healthy pups. The last of these died October 30, 1948. The mother died 1. days after receiving the isotope (12 days after delivery) with typical irradiation purpura. There was no grosis evidence of infection. The results show clearly what damage a bone-meeing $\beta$ emitter can do to the skeletal system when administered very early in the life of the animal. (\$.V.W.)

Greenberg, D. M. and N. Raplan. A PRELIMINARY STUDY OF THE REMOVAL OF RADTO STRONTIUM (\$E $E^{\text {ES }}$ FROM THE BODY. Argonne National Lab.

Tune 1, 194\%. 5ू. (MDDC-1142-R)

Preliminary experiments on removal of $\mathrm{Sr}^{85}$ from rats has indicated that sodium citrate, parathysold hormone, ammonimm chloride, and strontium chloride all apparently slightly exhance the removal of $\mathrm{Sr}^{20}$. Magaive doces of irradlated argosterol has mo guch effect. This study must be repeated upon a much larger group of animals at varying periods arter $\mathrm{Sr}^{25}$ admaini mprisal as to the relative effect of the we diferent agents 280 may be made. (ADD)

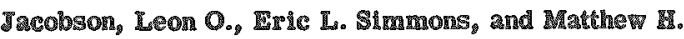
BLock. THE IEPTECT OF SPLENECTOMN ON THE TOXCITY OF SR TO TME MEMATOPORTIC SYSTEM OF MCE. J. Lab. Clin. Med. 34, 1040-35(1949)

In order to test the hypothesis that anemia fals to develop in CF - 1 mice following the administration of large doses of $\mathrm{sm}^{\text {so }}$ due to the rapid development of an intense cctoplc erythrocytopolesis in the gpleens of the animals, the experiments degeribed were periormed. Three grougs of CF-I Lemale mice were provided: the mice in the first groun were mplenectomized; those in the gecond Froup recetved a simgle intraperitoneal injection of $2.0 \mu \mathrm{He}$ of $\mathrm{Sr} / \mathrm{g}$ of body welght; and the mice in the third group were first ablenectomized and 84 days later treated with the same dose of $\mathrm{Sr}^{\mathrm{W}}$ as ured in the second group. A second injection of $2.0 \mu \mathrm{c}$ of $\mathrm{Sr}^{\mathrm{m}}$ was given to a representative number of animals from the gecond and thrd groups. Distribution, oxcretion, hematologic and hiatologic gtudies were made on the animals at intervals following the injections. A persiatent leucopenta but no anernia of giguificance was produced in the peripheral blood of the mice; the game dose of $\mathrm{sr}^{\text {ge }}$ prodnced both a lewcopenia and an anemia in the gplenectomired mice. Rematopolesis was markedly reduced in the bone marrow of all irradiated mice within three days; recovery Irom the hematopolesil and the anemia occurred uฮn:ly by the 119th day. Fetopic erythrocytopolesis and megakaryocytopolesis were greatly increased in the apleen throe days after the inotopic injection and lagted throughout the 118-day geriod of obes:vation: splenic lymphocytopolesis Foe reduced in three days and recovery was not complete
116 days later, while the process was essentially not affected in other tissues. Ectopic granulocytopoiesis in creased more slowly in the suleen of the irradiated animals than normally. It is concluded that the rapid development and the persigtence of ectopic erythrocytopoiesis in the spleen prevent anemia in the mice irradiated with $2.0 \mu \mathrm{c}$ of $\mathrm{Sr}^{83}$. (NSA)

$98 \%$

Jones, D. C. and D. Harold Copp. STUDTES ON BONE METABOLISM WITI THE AID OF RADIOACTIVE

STRONTIUM. Radiation Lab., Univ. of Calif. Jan. 16, 1948. 14p. (AECD-1962; UCRL-60)

The kinetics of skeletal uptake and urinary excretion of radio-strontium were studied during the critical first hour following intraperitoneal injection of a carrier-free dose. These experiments not only provided valuable data concerning the metabolism and fixation of this important fission product, but, because of the close similarity of gtrontium and calcium, also gave basic information on the process of calcification. Three grougs of rats were compared: normal mature adults, young growing normal animals, and young rachitic rats. In all group, the blood radioactive strontium rose to a maximum in $10-15$ minutes, and then declined as the strontium continued to deposit in the skeleton or to be excreted in the urine. Muscle and gln strontium curves followed those of blood quite closely, suggesting a rapid equilibrimm between their extracellular fluid and blood plasma. In all groups there was a continuous uptake of strontium by the sheleton. In the adult, this was almost constant, suggegting uptale by absorption and exchange with the calciun of the bone mineral. In the young rats, both normal and rachitic, the very rapid initial rate of uptake of radio-strontium tapered off ghargly with time, indicating that some of this radioelement was coming back from the bone into the blood stream. This suggested a rapid labile combination of strontium in these animals, which, since it also occurred in the rachitic group in which normal calci ifration is inhibited, probably associated with the protein octeoid matris pregent in both. A hypothetical calcification mechanism was suggested by these findings. Urinary excretion and renal clearance of gtrontium was constant in all three froups, but the clearance was almost ten times as great in the rachitic as in the normal young and adult rats. This would seem to indicate a direct effect of rickets on the renal mechanism of radio-strontium (and calcium) excretion. ( $A D D$ )

\section{8}

Jones, D. C. and D. Harold Copp. THE METABOLISM OF RADIOACTIVI STRONTIM $\mathbb{E}$ ADULT, YOUNG, AND RACHTIC RATS. J. Biol. Chem. 106, 509-14(1951)

The metabolism of carrier-iree radio-\$r was studied in adult, young, and rachitic rats during the critical firgt 24 hris. after intraper Itoneal injection. Radio-\$r is removed from the plasma much more slowly in the adult animals than in the other 2 group. The uptake by adult bone is continuous for the firgt 2 hrs., and reaches a maximum within 4 hro. Skeletal uptale of radio-\$r 1 much more rapid in the young animals, reaching a maximum within 30 minutes; very little was lost in the urine. The deposited latope remaing fised in the bone. In rachitic rats, the rapid initial uptalse was gimilar to that in the nomal young animals, but was followed by active loss from the skeleton; only $1 / 3$ was left after 24 hours. A labile combination with bone is indicated. A large part of the dose of radio-Sr was excreted by the rachicic rats within the first 24 hrs., and the plasma clearance was 10-15 times as grat as in the normal animals. Rickets may have a disect effect on excretion by the kidney. (CA) 
989

Nelson, J. Eugene, John G. Gibson, 2nd, Bert L. Vallee, and Marvin A. Van Dilla. THE EFFECT OF RADIOAC. TIVE STRONTIUM ON THE PERIPLERAL BLOOD

PICTURE OF THE NORMAL DOG. Acta Unio Intern. conira Cancrum. 619-24(1949).

A mixture of $\mathrm{SI}^{-8}$ and $\mathrm{Sr}^{90}$ was given intravenously as the nitrate to two normal male dogs and one castrated dog (2 me, $7 \mathrm{mc}$, and $20 \mathrm{mc}$, respectively). The mixture consisted of $88 \%$ of $\mathrm{Sr}^{89}$ (half-life 55 days; 1.5 Mev maximum $\beta$ radiation) and $12 \% \mathrm{Sr}^{80}$ (25-year half-life; $0.6 \mathrm{Mev}$ maximum $\beta$ radiation). $\mathrm{Sr}^{90}$ is followed by a daughter $\mathrm{Y}^{90}$ (64-hour's hall-1ife; 2.5 Mev maximum radiation); $\mathrm{Y}^{30}$ and $5 \mathrm{~s}^{20}$ come into equilibrium in four to five days, accounting for $22 \%$ of total radiation thereaftes. Due to the interval between ex periments the ratio of radiation due to $\mathrm{Sr}^{20}$ and $\mathrm{Sr}^{89}$ was greater in the dog receiving 20 me than in the other two. All three animals whowed a decline in the white count of venous (jugular) blood which reached a maximum 21 days after injection. It was found that the fall in count in dogs 1-5 (7 me injected) and 2-5 (2 me injected) was due entirely to a recuction in the number of granulocytes, there being no gignificant change in the absolute number of lymphocytes or monocytes. The venous hematocrit fell in all three animals by more than could be accounted for by red cells removed in sampling: the fall in per cent of pre-injection levels was proportional to dosage; the low points were reached at approsimately the same time as the lowest white count: 21-25 days. In the dogs which survived the period of most intense leukopenia (1-\$ and 2-\$) the hematocrit rose but not to preinjection levels; these two animals gradually declined, lost weight, refired lood and became apathetic. There was a terminal rise in rectal temperature to 41 to $42^{\circ} \mathrm{C}$. and death was preceded by generalized convuls long; at postmortem there were no signg of acute infection other than early bronchopneumonia of the left hobes. Sections of bone maxrow from the upper portion of the femur of dog \$-3 showed evidence of marged diminution of erythropoleris and myelopolesis. The localization and distribution of $\mathbf{s r}^{24-\rightarrow 0}$, based on radioactivity measuraments in the organs and slreleton and the approximate radiation to which the bone marrow 980 was exposed will be discusaed. (NSA)

Norris, W. R. and W. E. Kisieleski. THE EARLY DHSTRIBUTION OF SR $^{80 \cdot 90}$ IN RATS. Argone National Lab. [nd] 12p. (ABCD-2009-A)

Twenty white sprague-Dawley rats (ca $200 \mathrm{~g}$ each) were infected int ravenously (tail vein) with $0.5 \mathrm{ml}$ of isotonic sodiun chloride, pH 6 , containing 115 we of a mixture of

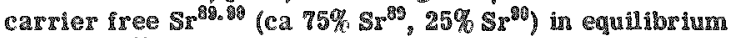
with the $Y^{80}$ daughter of $5 r^{20}$. Animals were acrificed by decapitation at intervals of 1 minute, 2 minutes, 5 minutes, 20 minutes, 1 hour, 6 hours, 24 hours, 3 days, 10 days, and 30 days after injection. The intravenous administration of sf into the tail vein was attended by a retention of $\mathbf{S}$ s in the tall which is not identical, in the first $1 / 2$ hours, to the metabolic picture observed in the other bones of the rat. The consistency of the resulf from geveral rats indicates that the initial high concentration of $\$$ result of variable injection technique, but rather a regult of a consigtent loss into lisgue or some other physico-chemical process occurring around the injection sile at the time of administration. Dusing the period Immediately after injection, there occurs a rapid upiake of $\$ 5$ by the bones which continues for $1 / 2$ to 2 hours. This is shown raphically for the femur and scapula. The rate of uptake by the femur was almost exactly that for the acapula. A second quantitative difference ia demonstrated by the lact that, whereas the femur has acquired only 11 of of its masimum activity dux- ing the first minute after injection, the gcapula, in the same period, has reached $25.5 \%$ of its maximum value. The time-content curves for all the bones, femur, scapula, tail, and careass (the $\mathrm{Sr}$ content of which is almost entirely in the bones after three days), indicate that the maximum bone Sr content, reached at about 90-120 minutes after injection, is maintained for about six days. In a preliminary experiment to determine whether $x$-irradiation can influence the

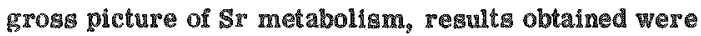
identical, qualitatively and quantitatively, with that observed 981 in the non-irradiated rat described. (ADD)

Sacher, George A. THE EXCRETION, WTERTION, DISTRIBUTION, AND CLINICAL EFIECTS OF STRONTWUM" ${ }^{80}$ WN THE DOG. PART II. STATISTICAL ANALYSIS OF EXCRETION AND RETENTION OT STRONTIUMI" FOR INDIVIDUAL DOGS. Univ. of Chicago Metallurgical Lab. June 19A6. 21p. (AECD-2108; CR-3866)

Excretion data of 17 dog@ injected with $\$ x^{80}$ were analyzed. Exscretion was assumed to conform to the sunction $\log \mathbb{E}_{t}=\log \mathbb{E}_{1}+\mathrm{b} \log t$, where $\mathbb{E}_{t}$ is the excretion per day at time $t, E_{1}$ is the instantaneous excretion at the end of the first day, and $b$ is the slope of the $\log$ I verbus $\log t$ diagram. Values of $\mathbb{E}_{\mathbf{l}}$ and $\mathrm{b}$ were estimated for urinary and fecal excretion of individual dogs. Exrors of extimate were also compused. A negative correlation $(\mathrm{r}=-0.575)$ was found between the urinary and fecal excretion slopes of adult doge. Slopes and intercepts of urinary and fecal excretion lines of beagle pups were lower than those for adult dogs. In the urine-leces correlation and in dependence of excretion parameter 8 on age, the $\mathrm{Ss}^{20}$ results paralled the result with plutonium. The total elimination, $00^{\infty}$, was dem fined and estimated for indiwidual adult dogs. It was found that on the average more than $90 \%$ of the injected $3 x^{80}$ conld be acconnted for by extrapolation of the exeretion line baced on observed ereretion in the first few weeks after infection. The calculated total elimination of pups, based on the observed arcretion behavior, showed at leagt $40 \%$ of the in jected $5^{3}$ unaccounted lor, presumably because of the in crease of total -body strontium concomitant with bodily growth. (NSA)

פ2

Simmons, Eyic L. and Leon O. Jacobson. RADIOTORC ITY OF INJECTED \$R BITS. PART IV. THE HEMATOLOGICAL FFIECTS OF

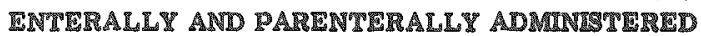
STIONTIUM IN MAMMALS. Univ. of Chcago Metallur. gical Lab. Aug. 1946. 101p. (MODC-138\%; CR-3797)

Fematological studies of the peripheral blood of rabbits, rats, and snice, after the intraperitoneal or atomach tube administration of $\mathrm{Sr}^{30}$ in doges irom 0.015 to $14.5 \mu \mathrm{c} / \mathrm{B}$ lor varying persods up to 100 weeks, are serorted. Whth the techniques employed in this experiment, a reduction in the heterophil values was the most senstive indicatos of acute and aubacute effects. Moderate but angulicant reduction In

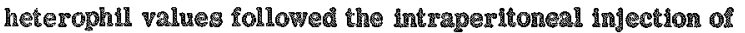

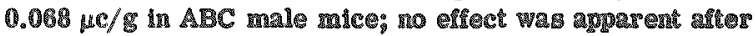
the administration of $0.015 \mu \mathrm{c} / \mathrm{s}^{\mathrm{s}}$ by the ame route in two strains (ABC males and CF-1 serales). Progrewively more ginnificant and sustained seghetion in hetorophl and lymphosyte values followed higher doses. No anema oe curred in mice with doses of $0.034 \mu \mathrm{c} / \mathrm{B}$ or lower, buit moderate and sustained anemias were apparent wh does of approximately 2.0 uc/S. The minimal exerting a mesn detectable reduction in heterophil values in rat was botwen 0.22 and $0.25 \mu \mathrm{c} / \mathrm{g}$. Doses above this level produced \& progressively more severe heterophl depression and lymphopenta. Stomach tube adminiatration of the Imotope was studled in rats cor comparison whth the intraperstoneal goute. 
Approwimately twice the intraperitoneal dose is required by stomach tube to produce a comparable effect on the hematological constituent of the peripheral blood. An int raperi w toneal dose of $1.0 \mu \mathrm{\mu} / \mathrm{g}$ produced a zeduction of heterophil values of gignificance in the rabbit. Followh an intraperitoneal dose of $3.0 \mu \mathrm{c} / \mathrm{g}$, a significant reduction in heanoglobin and exythroeyte values oceurred with a rapid recovery. A gustaned heterophil and lymphocyle reduction of significance was also apparent after this dose. $5 \mathrm{r}^{2}$ produced morphological changeg in the cell of the peripheral blood in these three species comparable to those observed following the adminiatration of plutonium, radium, or total body II $^{-1}$ rradiation. Anemias that developed in these three

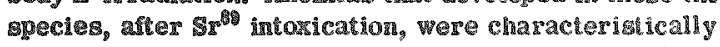
normocytic, normochromic, but cceasionally hypochromic, and macrocytic anemias were seen. Although dragtic te pletion of hemopoiesis in the bone marrow tollows srotin towication, anemias falled to develop in mice with doses of $0.34 \mu \mathrm{c} / \mathrm{g}$, due apparently to the rapid development of exif ramedullary erythropoiesis h the gpleen and liver particularly. A dose of $2.0 \mu \mathrm{c} / \mathrm{g}$, however, produced a moderately 993 severe anemia in this species. (ADD)

(3พ CRETION, RETENTION, DISTRIBUTION, AND CLINICAL EMTECTE OF STRONTIUM IN THE DOG. PATT I. REPORT ON TXPLRIMENTAL WORK. Univ. Of.ChLCago Metallurgical Lab. July 24, 1847. 93p. (MDDC-1388; CR-394:

Three groups of doges (adults, younes adults, and puppiem) were given intramugcular injectlon of $\mathrm{ss}^{20}$ in doges of 0.1 . 3.13 $\mu \mathrm{c} / \mathrm{T}_{\mathrm{G}}$. three days, with Iecal content $50 \%$ higher than that in the uxine. Both excretion gatterns were exponential with an 2verage slope of 1.8 on log -log plot. Blood concentrations declined similarly. Survival the ahow some, but not close, correlation with dosage. Symptoms in non-surviwors were Liver, lethargy, anorexia, los in weight, cutaneous henorrhages, ulcerated areas on the body or in the mouth, rapid decline in levcocyte count, and frequentiy eciemm of race, neck, or estremilies. Surviwor's ghowed gome or all of thege aymptoms in addition to lower red cownts (ur 10 460 dergean) ater three to gis weeks. Analyses were made of the caxcasses of some of the amimals for $5 \mathrm{r}^{30}$ con tent. Autopsies on gone of the animals ghowed extensive hemor rohages. $\left(\mathbb{E}_{0} \mathbf{C}_{0}\right)$

(28)

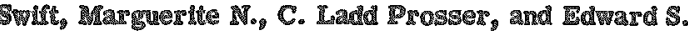

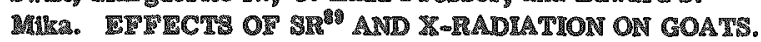
Univ. of Chlcago, Metallurgical Lab. Ђune 1846. 16p. (ARCU-108: CE-38B)

It geemed advisahle to determine the torictiy and other

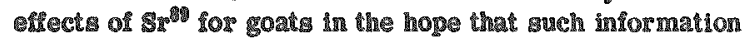
would provide a rational basis for extrapolation to man; such studies have already been made for mice, rats, gabbits, and doge. The gtudy of स-radiacion effects was inchuded for corman:

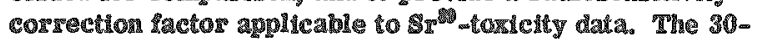

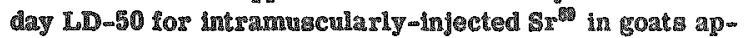
pears to lie between 1.0 and $1.5 \mu \mathrm{c} / \mathrm{g}$. The rate of excretion of $5^{3} \mathrm{~s}^{3}$ in urine and leces and the per cent retaned varies considerably, bat over a range gimilar to that reported for th dog. Toxkicty on the basis of body weight in thus the wame or less in the goat than in the dog (30-day LD-50, 1.0 He/gh, and if the goat be assumed representative of the largar marnas relating animal size to $\$ r^{89}$ toxicity, does change in the range $30-60 \mathrm{~kg}$, and toxicity is clearly not a simple function of body weight. The clinical course and gross pathology following sublethal and lethal doses are in general similar to those observed in the og. The pattern of hematological change is similar in goats and dogs, but the decrease in leucocyte count is noticeably less in nonsurvivors as well as in survivors in the former species. The 30-day LD-50 for total-body $\mathrm{x}$-irradiation in goats is about $350 \mathrm{r}$, a figure which approximates that obtained for the dog. The post 8 . irradiation syndrome is grossly indistinguishable from that following injection of $\mathrm{Sr}^{6}$. There is some indication of a Greater depression of the leucocyte count following $\mathrm{x}$-irradiation. Blood coagulation times after $\mathrm{z}$-ixradiation were erratic and failed to show the definite rise characteristic of the $\mathrm{s}$-irradiation, $\mathrm{Sr}^{80}$, and $\mathrm{Pu}^{2{ }^{2}}$ syndrome in dogs. (NSA)

\section{Tellurium}

995

Jacohson, Lours, Roy Overstreet, I. Chaikof, and ASSOCLALS. METABOLISM OF IFISTION PRODUCTSRADIO TELLURIUM. Radiation Lab., Univ, of Calif. Oct. 1944. Decl. June 5, 1947. 13p. (MDDC-1005; CH-2258)

Methods of preyaring $\mathrm{Te}^{\mathrm{t28}}$ and $\mathrm{Te}^{128}$ without carrier are described. The results of oral and intramuscular experimenta using tellurious tellurim $(+4)$ and telluric tellurium (+6) are given. Tellurium, whether administered either in the tellurious or telluric gtate, is aborbed by way of the digestive tract. It seems anute possible that in these experiments the +6 tellurim was not as readily talsen up by way of the digestive fract and that its assimilation takes place by it first beimg converted to tellurious tellurium which is then absorbed. The rates of elimination of absorbed tellurism in both valence states and by both oral and intramuscular administration are rapid and essentially com parable. The rates of excretion during the tim intervals studied indicate an approximate half time of retention in the body of 16 days. This is substantially less than the halflives of $\mathrm{Te}^{\mathrm{IIT}}$ and $\mathrm{Te} \mathrm{e}^{\mathrm{I2O}}$ which are 32 and 90 days, respectively. This observation places tellurium in the gmall list of the long life figsion products whose rates of elimination are greater than their rates of radioactive decay. The other

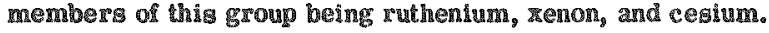
The principle organs of deposition for absorbed tellurium are blood, liver, and kidney. On the per gram basis the lidney tlase is from 2 to 5 thnes more active than any other of the tissues. In all of the intramuscular studies whth telluxious and telluxic tellurim the blood was more active per gram than the liver. The blood showed the highest per or o gan content of tellurium and it is of interest to note that these selative levels were maintaned even up to the $32 n d$ day interval. The gmall but constant setention of activity in the skeleton, which showed no Bignticant change throughout the entre 32 day interval, together with the fact that the relative proportion of activity in the alkeleton as compared to other lissues was somewhat lower in the oral experiments as conpared to the corregnonding intramuscular experi ments anggests that there may have been 1 rom 2 to $5 \%$ of radioactive impurities in the tellurium preparations employed guch as alkatine earths, rare earths, zirconium, and columbium. It does not appear that the tellurum shows any gignificant tendency to elther deposition or retention by the gleleton. (ADD) 
Thallium

996

Thyresson, Nils. EXPERMMENTAL INVESTIGATION ON THALLIUM POISONING IN THE RAT. DISTRIBUTION OF THALLIUM, ESPECIALLY IN THE SRIN, AND EXCRETION OF THALLIUM UNDER DIF FERENT EXPERIMENTAL CONDITIONS. A STUDY WITI THE USE OF THE RADIOACTIVE ISOTOPE THALLIUM 204. ACta Dermato-Venereol. 31, 3-27(1951).

After 24 hours the subcutaneous injections to rats of 40 , $g$ budy wenght TINO, labeled with Thed, gave decreasing amuunt Tl in the order: kidneys, intestinal tract, thysoid, testes, pancreas, skin, bone, adrenals, muscle, apleen, bran, spinal marrow, liver, fatty tissue. The whole blood, plasma, and red cells contained $2.4,2.1 \mathrm{\gamma} / \mathrm{ml}$ and $2.7 \mathrm{~F} / \mathrm{g}$ wet werght, respectively. After injection of $20 \gamma \mathrm{THNO}_{3} 4.4$, 36.7 , and $61.2 \%$ (feces 42.2, umne 19.0) of the dose was exreted un 24 hours, 1 and 4 weeks, respectively. Oral $6 y_{-}-$ tme nereased the excretion in the urine, but nut in the foces after repeated doseg; however, after a single doge, cystine as well as $\mathrm{BAL}, \mathrm{Na}_{2} \mathrm{~S}_{2} \mathrm{O}_{3}$, and methionine did not affect the excretion. The amount of $T$ in the glsin 24 hours after an myection of $40 y$ varied with the age of the rat; it increased to a maximum with the 20-30-day-old and reached another masimum with the $44-5-d a y-o l d$, corresponding to the growth of the 1st and 2nd coats of hair. Radioautographs indicate Tl uptake by the har folliclea. (CA)

\section{Thorium}

997

Ackerman, 萝., R.P. Allen, G. Bonner, W. L. Downs, H. C. Modge, E. A. Maynard,W. Neuman, J. K. Scot, A. Sparks, and R. E. Stokinger. DRELIMINARY STUDIRS OF THE TOXICITY OF THORIUM. University of Rochester. Feb. 26, 1948. Decl. Sept. 15, 1248. 45p. (ARCD2283; UR-13)

$\mathrm{LD}_{50}$ values of $\mathrm{Th}\left(\mathrm{NO}_{3}\right)_{4} \cdot 3 \mathrm{H}_{2} \mathrm{O}$ injected intraperiloneally $\mathrm{bl}$ adult albino rats are $\sim 1,770 \mathrm{mg} / \mathrm{kg}$, while with intratracheal administration the $\mathrm{LD}_{50}$ is $\sim 85 \mathrm{mg} / \mathrm{kg}$. With the latter test $<1000 \mathrm{mg} / \mathrm{kg}$ of $\mathrm{ThO}_{2}$ was tolerated. No consigtent leaions which could be attributed to the Th compounds were found in any organs other than the lung. Th(NOH. . $_{3} 3 \mathrm{~K}_{2} \mathrm{O}$ provoked 2 peculiar focal coalition of monocytes in the alveolar sacs. $\mathrm{ThO}_{2}$ provoked minimal or no response. Weanlings fed $20 \%$ $\mathrm{Th}\left(\mathrm{NO}_{3}\right)_{4} .3 \mathrm{H}_{2} \mathrm{O}$ died promptly. The weanlingg fed $10 \%$ grew very little but survived for 11 month. A small-scale pared feeding study demongtrated clearly that the weight depression was only partly due to the decreased food consumption. Labeled Th with UX, given intravenously to rats, rabbits, and gunea pigs shows that Th is logt very slowly from the blood and the major portion appears in the liver. After 14 days one-fourth has been excreted in the feces. When administered by other routes, the Th tends to stay where placed. (TSS)

988

Cassel, Chester, Julian M. Ruffin, and Robert J. Reeves.

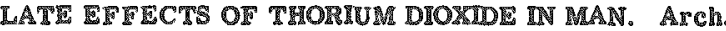
Internal Med. 83, $42-50(1951)$.

Three patients were studied, 17,16 , and 13 years, reapectively, ater $\mathrm{ThO}_{2}$ administration. While the presence of $\mathrm{ThO}_{2}$ could still be demonstrated no effect on hemopolesis, hepatic sinnction, or general health was diselosed. (CA) 820

Christensen, William $\mathbb{R}$. and Sheldon $\mathbb{C}$. Sommers. CFTOLOGL CHANGES FOLLOWING INTRAYENOUS IN. JECTION OF THORIUM DIOXIDE. Cancer Research Inst., New England Deaconess Hospital, Boston. [nd] 7p. (ARCU-1535)
In view of reports in the literature of the toric and posaim ble carcinogenic effects of thorim dionde when used as a contrast medium for radiological diagnosis, an attempt was made to induce malignant changes in the reticuloendothelial systems of four rats following infravenous injection of colloidal thoriun dioside (Thorotrast - 20\% thorium dioxide by weight). After 15 months no gross changes of reticuloendothelial gystems were observed by s-ray examination. Microscopic studies showed the livers of two rats to exhibit marked hyperplasia of reticulo-endothelial cells, and anaplastic variation in size, shape, and chromatin arrangement of the affected cells. No invasion was fond, but the areas were considered of precancerous gignificance. (NAA) 1000

Easton, Thomas W. RETENTION OF WJIETED COLLOMAL THORUM DIOKADE IN THY BONY OF THE

LABORATORY MOUSE. Anat. Record 108, 515(1950).

This paper is reproduced here in is entirety.

Mice were injected intravenously through the tall vein with colloidal $\mathrm{ThO}_{2}$ (Thorotrast), $0.2 \mathrm{ce} /$ day on alsernate days for three doses, a total of 0.6 cc. Success of injections was checked loy roentgenogram, a heavy ghadow at base of tail indicating poor injection. Such mice were not used. Thorotrast in the body was determined by aghing the entire mouse after dividing into various Bampl es, and measuring the radioactivity with a Geiger counter. Thorotrast was determined in the whole body, in liver and apleen alone, and in the remainder of the body by conparing meas. ured activity with that of mearured amounts of the material injected. Values obtained were as follows: one day after last injection, mice retained from 0.4 to 0.59 ec of the origt mal 0.6 cc. Of this the liver represented 25 to $55 \%$; the spleen, 14 to $28 \%$ and the remander of the body, 17 to $51 \%$. Ten days after last injection the total was $0.4 \mathrm{cc}$, whin vard ations up to $0.1 \mathrm{cc}$. Or this, the liver held 55 to $70 \%$; the spleen, 25 to $30 \%$; and the remainder of the body, 5 to $20 \%$ This level was maintained for 130 days, at least. Most of the variations observed in amount of thorotrat retained appear to be due to variationes in liver content. Neither splenectomy nor fasting begun prior to injection appear to have any marlsed effect on retention or loss of $\mathrm{ThO}_{2}$. 1001

Engels, Alfred, Werner Maurer, and Annemarie Nirilas.

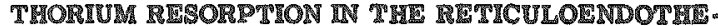

LIAL SYSTEM. Z. ges. expt1. Med. 115, 221-8(1949).

Tables give the distribution of radioactivity $24 \mathrm{hr}$ after intravenous injection of $\mathbb{T h}\left(\mathrm{NO}_{3}\right)(5,2$, and $0.001 \mathrm{mg}$, or $10^{-11} \mathrm{G} \mathrm{Th}$ ) containing $\mathrm{Th}^{2 \mathrm{2}}$ into rabbits, rats, and pigeons. The liver and goleen contain the largest amount; moderate amounts are found in bone marrow, lymph nodes, and adrenals. Sensitizing rabbits with horge serum causes an 1 crease in the radioactivity of the liver and spleen hr after injection. Treating ratr with a near lethal dose of trypan blue in an attempt to block the reticuloendothelial system did not afiect the distribution of radioactivity. (CA)

1002

Engels, A., W. Maurer, and A. Niklas. BEHAVIOR OF THORIUM IN GRAVID AND NON-GRAVID RATV. Z. IES expti. Med. 115, No. 5, 525-39(1950).

In a previous paper (Deut. med. Rundachau 3 , 261(1040)) the authog's have shown that hogiun injected into animals was accumulated mainly in the liver, the gpleen, and the bones. In the preaent work the ame techndque (intravenous

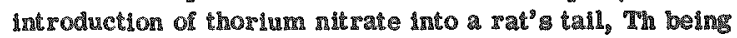
labeled with $\mathrm{UX}_{1}$ ) was appled to a atudy of dilierences in the distrilbution of Th in gravid and nongravid ratd. It was cound that the above mentioned organs were chelly dnvolved in both cages; however, in gravid animals the Th concontrations were lower, and the decrease of th content in blood 
proceeded more slowly than in nongravid individuals. It was also establighed that the volume of blood contained in the adrenals of a gravid rat is twice that found in those of a nongravid animal, the body weight being only silghtly increased in the former case. (NSA)

1002

Mall, R. R. INEALATION TOXICETY OF THORHUM ORALATE, $0.72 \% 5$ OF QUARTERLY TECINCAL REPORT; JULY 1, 1950, TRRU SEPTEMBRER 30, 1950. University of Rechester. Nov. 1, 1050. 4p. (UR-142) (p.72-5)

As a meang toward evaluating the nature and extent of this hazard, two experinents have been completed in which dogs, rabbits, and rats were exposed to thorium oxalate dust by inhalation. The gole evidences of toxicity following in halation of thorium omalate cust were decreases in the werum Ca concentrations of the dogs and decreases in the number of clrculating red blood cells with a concomitant increase of mean corpuscular volume, resembling the developaent of a macrocytic anemia in the dogr and in one 100. 2abbit. (NSA)

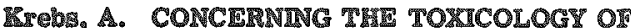
THORIUM RMANATION (G). Naturwissenschaften 28 , พ66-7(1940).

The difect of Th on mice was gtudied. The tolerance dose of Th in aip is less than 70 mst per ce (55-day test), the gresence of $1,750 \mathrm{mgt}$ per ce cause death in five days. 1005

Relatively Th id legs active than Rn. (CA)

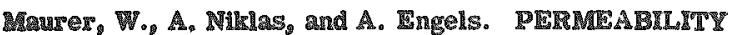
OF RAT PLACENTA FOR THORIUM IONS WITH VARY ING PREGNANCY PERIODS. Z. ges, expt. Med. 11 , 510-2.

Afer intravenoug injection of $\mathrm{Th}\left(\mathrm{NO}_{3}\right)_{4}$ labeled with $\mathrm{Th}^{234}$ Into pregnant rats, the mpecilic activity of the felus drops Irom $0.1 \% / \mathrm{g}$ setur at the 14 th day of pregnancy to $0.001 \% / \mathrm{g}$ on the ald day. The permeability of the placents decreases 1006 Iagidis. (CA)

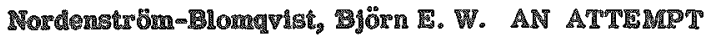

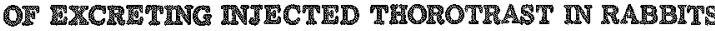
BY 2, -DMEERCAPTOPROPANOL (BAL). Acta Radiol. 34. $583-45(1050)$.

An emperimental attenpt was periormed to excrete intra vonously infected thorotragt in rabbits by $2,3-d i m e r c a p t o-$ propanol (BAL). No exeretion of Th was found in urine. The radloactivity in urine deponds upon disintegration products

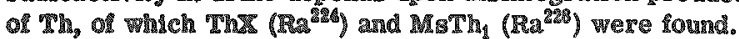

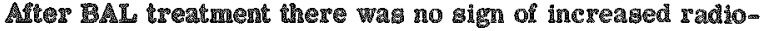
actiwly in urane or aignificant decrease of the radioactivity In liver, spleen, kidney, or yellow bone marrow. The radioactivity of the goleen way 1our times as high as that of the liver, masured on the $y$ intensity per gram. The radioactiwity of the kidney was low in comparison with that of $100 \%$ the liver and spleen. (NSA)

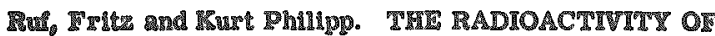

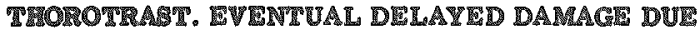

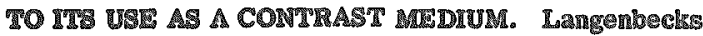
Arch. k11m. Chir. 263, 573-87(1940-50).

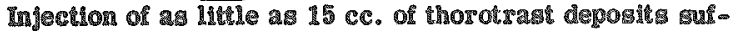
Ifesent Th in organe cuch as the liver and apleen to expose aach of organ to $0.5 \mathrm{5}$./day or twice the tolerance dose. Bnce the Th is not exereted, the dose of irmadition can esceed 1000 rodrtgen in 10 years. In most cares larger quarteties of thorotragt aro injected, leading to grenter

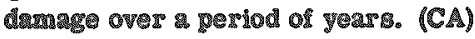

1008

Salerno, Paul R. and Paul A. Mattis. ABSORPTION AND DISTRIBUTION OF THORIUM NITRATE IN THE RAT.

J. Pharmacol. Expt1. Therap. 101, 31-2(1951).

This paper is reproduced here in its entirety.

$\mathrm{Th}\left(\mathrm{NO}_{3}\right)_{4}$ was administered to adult male rats by several routes, and the tigsue distribution was studied by means of $\mathrm{UX}_{1}\left(\mathrm{Th}^{234}\right)$ as a radioactive indicator. Studies on $\mathrm{xats}$ given $30 \mathrm{mg} / \mathrm{kg}$ by stomach tube revealed that maximal absorpthon had occurred within 4 days and that more than $90 \%$ of the absorbed dose was deposited in the skeleton. After 32 days, $100 \%$ of the retained Th, which represented $0.06 \%$ of the administered dose, was found in the skeleton. When larg er doses of $\mathrm{Th}(700$ to $800 \mathrm{mg} / \mathrm{kg}$ ) were administered by oral intubation, no absorption could be measured, which inn dicates that less than $0.001 \%$ is absorbed at thi dosage level. Absorption following subcutaneous administration was found to be progressive in that 0.03 and $0.07 \%$ of the injected dose was found in the skeleton at 16 and 30 days, respectively. Following the intraperitoneal injection of 60 $\mathrm{mg} / \mathrm{kg}$ of $\mathrm{Th}, 1 \%$ of the dose was deposited in the slreleton after 30 days. Th( $\left(\mathrm{NO}_{3}\right)_{4}$ administered intravacularly by intracardiac injection was retained to the extent of 95,89 , and $83 \%$ at 3,8 , and 14 days, respectively. Tissue distribution in terms of per cent of injected dose was $16 \%$ to the skeleton, $55 \%$ to the liver, and $5 \%$ to the gpleen after 14 days. Two hours after intracardiac injection, $50 \%$ of the Th is removed rom the blood, and $7.6 \%$ is found in the gkeleton. Less than $1 \%$ is found in the blood after $18 \mathrm{hr}$. Most of the $\mathrm{Th}$ which is exereted is found in the feces and apparently is trangponted from the liver via the bile. (NSA) 1009

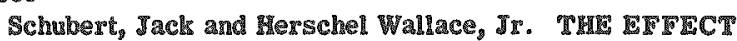
OF ZIRCONIUM AND SODHUM CHTRATE ON THE DISTRIBUTION AND IEXCRETION ON SMMULTANEOUSLY INJECTED TIORIUM AND RADIOSTRONTIUM. J. BLI. Chem. 183, 157-68(1950). (ADCD-2621)

The distribution and daly excretion of tracer levels of injected thorium and strontium were studied in rats receiving zirconium or sodium citrate intraperitoneally $1 / 2$ hour after the adminigtration of the radio-elements ( ${ }^{68}$ early" treated) or three days later ("1ate" treated). One group of ratr received no other treatment. In contrast to previous studies on plutonum and yttrium, zirconium citrate had no specific effect on $\mathrm{Th}$ or \$r metabolim other than that as goclated with the citrate part of the molecule. The early administration of zirconium or sodium citrate resulted in about a 3-fold increase in the urinary arcretion of Th and a 2 -10ld increase in the urinary excretion of Se during the following 24 hours. No effect on the fecal excretion was found. The Th concentration in the Liver, mensenteric Iymph node, pancreas, and gpleen were markedly reduced as a reault of early aodum citrate administration. No singnificant charges in the metaboligm of Th or $3 \mathrm{r}$ were found in the late treated gran. The deposition of $\mathrm{Th} / \mathrm{g}$ was highest in the soft tissues, particularly in the liver; while \$ro accumulated mainly in the skeleton. The results indicate that cationic radioelementr are IIsed in bone by at least three different mechanisma. An interpretation of the results in terms of lon exchange, burace absorption, and complex lon formation is preacented. (NSA)

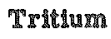

1010

Drues, Austin M., Agnes N. Stroud, and Leola Rietz.

TOSICITY OF TRMIUM OXIOE TO MICE. Proc. Soc.

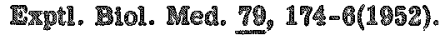


The 30-day median lethal dose of $\mathrm{H}_{2}^{3} \mathrm{O}$ for CF No. 1 female mice is about 1 me per $g$ body weight when given in a single injection. The estimated radiation dosage is about $800 \mathrm{rep}$ in the first 3 days and about 1000 rep in the first 12 days after injection. It is concluded that no unexpected factor of biological effectiveness exists for tritium $\beta$ rays in 1011 relation to $\mathrm{X}$ or $\gamma$ rays. (Author's' Summary)

Pinson, Ernest A. and Ernest C. Anderson. THE ABSORPTION, DISTRIBUTION, AND EXCRETION OF TRITIUM IN MEN AND ANIMALS. LOS Alamos Scientiiic Lab. [nd] $4 \mathrm{p}$. (AECU -937 ; LADC-867)

The tritum absorbed by rats from inspired air containing HT or HTO is proportional to the tritium activity in the inspired air. When present as HTO a very high percentage of the tritium activity inspired is taken into the body fluids indicating rapid exchange and absorption of activity across respiratory membranes. If present as IIT in inspired aix only about $0.05 \%$ of the inspired activity appears in the body as HTO. Apparently, biological catalyzation of HT to HTO takes place in the body. The activities studied were not high enough for the absorption of HT per se in body fluids to be detected. Three millicuries of HTO ingested by man in 200 ce of water was completely absorbed in about 45 minutes. Absorption is linear with time. Absorbed tritum dilutes with 57 to $68 \%$ of the body weight suggesting that body water is primarily involved. Tritium elimination from the body in both rats and man is an exponential function of total water turnover. The reservolr calculated from the rate constant is again 57 to $68 \%$ of the body weight. The biological hal life of $14 \%$ in nine human subjects varied from 9 to 14 days on ad libitum water intalse and was reduced to $2 / 2$ days in one subject on high water intake. The biological half-life in rat sanged from 4 to 5 days on ad libitum water intake. The tritium activity in excreted sweat, insensible perspiration, expired water vapor, sputum, and urine is essentially the rame as that in the blood. (NSA) 1012

Powers, E. L., Jr. and Deborah Shelner. TRITIUM INDUCED EFTECTS IN PARAMECTUM AURELIA. Proc. Soc. Expth. Biol. Med. 78, 493-7(1951). (AECU-1485; UAC-408)

Isolated specimens of Paramechum aurelia, kept during 2 days in culture fluid containing 1 to $100 \mathrm{mc}$ of $\mathrm{T}$ per $\mathrm{ml}$, calculated to emit a radiation dogage of 630 to $64,450 \mathrm{rep} /$ day, divided 6 to 8 times during that period. No effects were observed in the animals, washed after exposure, until autogamy occurred. Increasing death rates $(1.2$ to $98.1 \%$ after autogamy were obmerved with increasing radioactivity of the medium. The observed difference in the efficiency be tween $\mathrm{T}$ and $\mathrm{S} \mathrm{r}^{83}{ }^{30} \mathrm{Y}^{80}$ in inducing the effects observed $1 \mathrm{~s}$ consintent with the observations of other concerning the dependence of the mutation constant upon rate of energy losg by densely ionizing particles. (NSA)

\section{3}

Stroud, Agnes N. and Austin M. Brues. THP IFFCT OF TRITIUM $\left(H^{3}\right)$ ON CELLS CULTIVATED IN VITRO, 0.11117 OF BIOLOGICAL AND MEDICAL DIVISIONS, QUARTERLY FE PORT FEBRUARY, MARCH, APRIL, 1050.

Argonne National Lab. [nd] 7p. (ANL-4451(p.111-17))

A series of cultures of chick embryo murcle was treated with $\mathrm{H}_{2}^{3} \mathrm{O}$ at dosage rates of 11.5 to $3000 \mathrm{rep} / \mathrm{hr}$, ug to lotal dosages of 72,000 rep. Under continuous irradiation, completion of the mitotic cycle becomes very much delayed even after the accumulation of a few hundred rep. Not all mitoses are equally delayed at a given gtage, and the delay appear to result from a varlety of processes, perhaps related to abnormal nucleic acid metabolism. Some cells eventually divide unequally, without evidence of apindle or plate formation. Delay may occur in any stage of mitosis, perhaps excepting prophase. Cell degeneration occurs only in dividing cells at the lower dosages; after 20,000 rep and more, degeneration occurs auddenly in regting cells, beginning with an "explosion" followed by shrinkage of the nucleus. Others degenerate without exploding. Preliminary data suggest that at a given dose rate, the onset of mitosis is suppressed partially, an equilibrium rate of cell division being attained. Due to concomitant delays in completion of the cycle it is impossible to measure directly partial inhibition of onget. The cytoplasmic behavior of continuougly irradiated cells is described. (NSA)

1014

Thompson, R. C. THE GROSS RETENTION AND METABOLISM OF TRTTUM OXIOE WN THE MOUSE.

Hanford Works. Jan. 23, 1081. 13p. (HW-20002)

For evaluation of radiation hazard in handing of tritium, $0.5 \mathrm{ml}$ of a $0.86 \%$ aline colution, containing $120 \mu \mathrm{c}$ tritiun per ml, was injected into mice intraperitoneally dally for 13 days. Tritium was determined in body water and in the ground combusted remains of the dried carcass. The inital biological half life for tritum oride in body water was about 1.1 days; for tissue-bound tritium about 3 days. Thirty-two days after the final injection $90 \%$ of the tritium in the animal was in the bound state, being lost with a biological half life of approximately 30 days. (NSA)

1015

Thompson, R. C. RETENTION BY MCE OF TIITIUM ADMINISTERED AS THE OXIDE. Hanford Worlss. June 19, 1051. 14p. (HW-21494)

Tritium oxide was administered to mice by intrapericoneal injection. The retention of trithum activity in the body water and in the dry carcase was determined. The biologleal hall life of body water in the mouse is appromimately 1.1 days. The curve of tritiun loss from the tissue-bound state can be falrly well resolved into components of D-day and 90-day half lives. These components are thought to represent, sespectively, groups of relatively dynamic body constituente, and of relatively gtable body congtituents. They are of comparable tritium binding capacity and under chronic exposure to trithum would accumoulate about 17 9 as much activity as would be present in the body water. (NSA)

\section{Un}

1016

Arnold, James 3. EXIIIIIT-RADIOAUTOGRAPRS OF $0^{229}$ IN BONE AND SOFT THSSUE. Argonne Natlonal Lab. Mar. 1051. Decl. June 6, 1951. 17p. (AECD-3176)

A serles of radioautographs is preanted rhown'n depost tion of $\mathrm{U}^{293}$ and $\mathrm{Pu}$ in the bone and soft tisgues of animalø, sacrificed at periods varying from 24 hr to $4 \mathrm{gr}$ follow injection. (NSA)

1017

Bauer, Frederick C., Jr., Grant C. Johnson, Lym

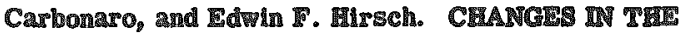
\$ERUM LIPIDS OF RABBITS WTIR ACUTE URANIL NITRAT POISONING. Arch. Pathol. 51, A414-5(1251)

Uranyl nitrate poisoning in rabbits was as aoctated with transient arotemia and lpemia of 2 to 3 weeks duration. All lipids participated in this increase, but the greatest incroaso was in the neutral fat fraction. The nonprotein $\mathrm{N}$ returned to near the control level several days belorethe Mpids. The Hignifeance of these result with other formo of nephrith is discussed. (NSA)

1018

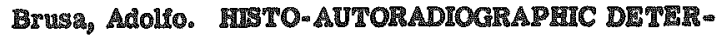
MINATIONS OF RADIOACWIV IUBSTANCES IN THE BODT. A FUDF ON URANIUIR. Boll. soc. ltal. biol. sper. 26, 673-5(1050). 
Nonlethal doses of urany I acetate were injected aubcutaneously in gumea pigs, and aections from various organs were examined autoradiographically 1 to 6 days following the injection. The principal concentrations of 0 occur in the kidneys, being localized there in the cortical substance. In other organs the following main localizations are obserwed: the extreme portions of the bronchial ramifications of the lungs, the mucosa of the integtine, and the parenchyma of the lobuli of the liver. (NSA)

1019

Hodge, Harold C. PHARMACOLOGIC TOOLS IN THE STUDY OF THE MECRANISMS OF URANIUM POISONING. Asch. Ind. MYg. Occupational Med. 2, 300-11(1950).

Agplication of fluorophotometric analysis for $U$, ultrafiltration of $\mathrm{H}_{2} \mathrm{CO}_{3}$ comples, protein precigitation, exchange adsorption atudies of bone with radioisotopes, radioautographs of hidney, renal clearance studies, histopathology, anzynic activity in the urine, and cell physiology, U was Iound to enter the body as the uranyl ion, to be carried as a nondiffusible protein complex and diffusible $\mathrm{H}_{2} \mathrm{CO}_{3}$ complex, to be partly degosited in bone, and to be mostly excreted in 1020

the urine after damaging the tubules of the kidneys. (CA)

Fodge, N. C., E. A. Maynard, and W. L. Downs. ANTIDOTAL ACTON OF POLYPHOSPIATES IN URANIUM POISONING. J. Pharmacol. Pxpt. Therap. 101, 17-10 (1951).

This paper is reprohed here in its enthety.

In previous gtนdies it has been establighed that uranhm nitrate hexahydrate fed at $2 \%$ of the diet or given intraxeritoneally in a single dose of $2.5 \mathrm{mg} / \mathrm{kg}$ to adult albino rats produce approximately $50 \%$ mortality during the ensulng 21 days. Previous attempts to provide an antidote for acute U poifondag led to the muggeston of bicarbonate becauge this ion surnishes a diffusible comple with the uranyl son. Following the recent demonstrations of the comples egtabHshed between uranyl lon and bone surface (Neuman), between the uranyl ion and the surfaces of yeast celng (in both cases probably with phosphate groups), and between the uranyl ion and various polyphosphates (Rothstein), tests were carried out to gee whether polyphosphates could be used in the treatment of acute experimental U poisoning. The excretion of $\mathrm{Ca}$ alts of polyphosphates in urine fGosBelin) promgted the trial of these compounds both in feeding atudies and after intraperitoneal injections of $U$. The polyphosphate was administered gometimes as a single dome and mometimes in multiple injection intrapertonealy. There was considerable evidence that a marked reduction in guortality can be achieved. (WSA) 1021

Murwit: LCON. A ININETIC STUDI OF NORMAL AND URANIUM MNIBIY Universily of Rochester. May 3, 1950. Decl. June 15, 1950. 108p. (ABCD-2857; UR-117)

The induxtรial use of uranium in recent tumes has led to meveral toricological problems. One of the most important of these is the deleteriour effect of uranium on normal rena? function. In order to gain some undermtandias of itø poimonous action in the kidney, several investigator have fund it valuable to determine the effect of uxanum on cei". taboligm. Yeast cells being much asisg to work w th than Idiney cells, they have been considered preferabre for the grtนdie.

Rinetically, glmeose appears to form a loose comples whth the active call groups involved in aerobic and anaer. able metabolim, leaving the glucose mogtly in the rree state. The $\mathbb{K}_{\text {a }}$ (dissociation constant) for aerobic metabo- lism is $3.6 \times 10^{-3}$ and for anaerobic metabolism $4.4 \times 10^{-3}$ Fructose was also found to form a looze complex with the active cell groups under anaerobic conditions. Its $\mathrm{K}_{\mathrm{g}}$ is $1.2 x$ $10^{-2}$. The maximum velocities of anaeroble glucose and fructose metabolism are equal. The value is approximately $28 \mu \mathrm{CO} \mathrm{CO}_{2}$ per milligram yeast per hour at $25^{6} \mathrm{C}$ and pH 3.5 . This may indicate that a single enzyme system or that equal concentrations of two different enzymes dominate the ratelimiting step in the metabolic scheme. The rate-limiting step in the aerobic metabolism of glucose is glower than in the anaerobic metabolism of glucose. It velocity is 13.30 $\mu 1$ per milligram yeast per hour at $25^{\circ} \mathrm{C}$ and pH 3.5. It has been ahown that the anaerobic production of $\mathrm{CO}_{2}$ by yeast is proportional to the anaerobic utilization of glucose. Evidence was presented to show that the rate-limiting process on the yeast cell gurface is an enzymatic reaction thus lending support to the theory of active transfer of material across the cell membrane. Anaerobically, there exists for the most part a noncompetitive inhibition of glucose metabolism by uranium. However, some degree of competition can be found in both aerobic and anaerobic glucose metabolism. In the fermentation of fructose, competition is apparently due to a uranium-mubstrate combination. The temperature characteristics of aerobic glucose metabolism is about $12,700 \mathrm{cal} / \mathrm{mole}$ between $18^{\circ} \mathrm{C}$ and $30^{\circ} \mathrm{C}$ and $20,850 \mathrm{cal} / \mathrm{mole}$ between $18^{\circ} \mathrm{C}$ and $10^{\circ} \mathrm{C}$. When uranium is added the temperature characteristic becomes $23,400 \mathrm{cal} / \mathrm{mole}$ throughout the whole temperature range measured with no break at $18^{\circ} \mathrm{C}$. In the anaerobic metabolism of both glucose and fructose, the termperature characteristics are about the same. They increase as temperature decreases whether uranium is present or not. In the control data, average $(\mu)$ values are about 21,800 cal/mole between 20 and $25^{\circ} \mathrm{C}$, and $10,700 \mathrm{cal} /$ mole between 25 and $30^{\circ} \mathrm{C}$. In the presence of uranium, the anaerobic metabolism of sxuctose and giucose has an average temperature characteristic of $17,000 \mathrm{cal} /$ mole between 20 and $25^{\circ} \mathrm{C}$ and $23,000 \mathrm{cal} /$ mole batween 25 and $30^{\circ} \mathrm{C}$. The increage in aerobic $(\mu)$ values when uranium is added amounts in effect to a revergal of inhibition 25 the temperature is raised between 18 and $30^{\circ} \mathrm{C}$. This also holds true for anaerobic glucose and fructose metabolism between 25 and $30^{\circ} \mathrm{C}$. Uraniun forms a complex in a $1 / 1$ ratio with active groups in the anaerobic glycolytic system. The Hinial steps in the respiration and fermentation of glucose are accomplished by different aystems, each possessing widely dufferent characteristleg. It has been postulated that either the aerobic, anaerobic, or both systems associated with glucose metabolism may be the composite of a number of dissimilar processes. 33 references. (NSA) 1022

Kisteleski, W. E., W. G. Wraghan, and W. P. Norris. THE RETENTION AND DISTRIDUTION OF U ${ }^{23}$ IN MYCF. Axgonne National Lab. Nov, 1050. Decl. Feb. 2, 1051. 2p. (ASCD-3057)

A study of the metabolism and organ disteilbution of $\mathrm{U}^{289}$ injected intravenously in mice is reported. The major portion of the injected material was rapidly excercter; about $50 \%$ was retained at 3 to 4 days and only about $20 \%$ at 6 days. The bone was the prineipal site of depostton. (NGA) 1023

LaFrance, LEO J. A PARTLL BIBLIOGRAPHY ON URANIUM AND IIS COMPOUNDS WIIE ISSPECIAL RETIRENCE TO RHARMACOLOGY AND TOXCOLOGY. University of Rochester. May 11, 1951. 32p. (UR-166)

This annotated bibllography contains 114 references to - apers dealing with pharmacological, toxicological, and physiological effects of U and its compounds on antmal. 
No references to the effects of $U$ un plants are included. (NSA)

1024

MacNider, W. deB. INABILITY OF 2-3 DITHIOPROPANOL (BAL) TO PROTECT THE LIVER AGAINST TOXIC ACTION OF URANIUM NITRATE. PrOC. SOC. Exptl. Biol. Med. 68, 160(1948).

The author has shown that doses of BAL (10\%, benzyl benzoate $20 \%$, in peanut oil) equivalent to $15 \mathrm{mg} / \mathrm{kg}$, when administered subcutaneously to dogs for 8 days, were ineffective against the hepatotoxic action of $4 \mathrm{mg} / \mathrm{kg}$ of uran um nitrate; instead, it tended to increase the towic effects. The livers showed diffuse necrosis with advanced fatty degeneration. (NSA)

1025

Masturzo, Aldo. PERIPHERAL-BLOOD AND BONEMARROW TESTS DURING EXPERMENTAL WTOOICATION WTTH URANIUM. FOLia med. (Naples) 33, GA3$71(1050)$.

In continuation of observations on gradual intoxication of guinea pigs and rabbits with uxanium chloride (Folia Med. (Naples) 33, 535(1950)), an examination was made of the changes in the peripheral blood and the bone marrow; samples of marrow were obtained from the femur of living animals by means of a surgical operation. The alterations of the blood pointed to an anemia of a normochromic type, with a hyperchromic tendency in rare cases; the bone max row exhibited hyperplasias, the hyperactivity extending 1028 mainly to the less mature elements. (NSA)

Masturzo, Aldo. BLOOD STUDUES IN INTOKMCATION EXPERIMENTS WITH URANIUM CRLORDE. Folia med. (Naples) 33, 748-50(1950).

Weekly blood tests of rabbits intosicated by repeated doses of uranium choride revealed notable modisications in the coagulation characteriatics of the blood (lengthening of the prothrombin and coagulation times). (MSA) $102 \%$

Neuman, W.. M. W. Neuman, and B. J. Mulryan.

FAILURE OF NONISOTOPIC URANIUM TO INHIBIS RESORPTVE PROCESSES IN THE RAT FRMUR. J.

Biol. Chem. 182, 151-2(1950).

The previously described Inhibition of the resorptive processes after injection of radioactive $\mathrm{UO}_{2}\left(\mathrm{NO}_{3}\right)_{3}$ was ghown to be caused by radiation rather than by any speciple chemical elfect of $\mathrm{U}$ itself. (CA) 1028

Tannenbaum, Abert, ed. TOXICOLOGT OF URANUM. McGraw- HIII Book Company, Inc., New York. 1951. 333 p. (National Nuclear Energy Series, Division IV, Volume 23)

This volume contains report of investigations on the toxicology of uranium compounds together with agsociated studieส. The report represents experiments performed for the Manhattan Project by the Metallurgical Laboratory of the University of Chicago and the Medical Revearch Institute of Michael Reese Hospital. Included are chapters on the nature of uranium poisoning, factors affecting uranium poisoning, distribution and excretion of uranium, biochemical effects of uranium poisoning, mechanism of action of uranium and the transport of uranium to the tissues, and the relation of experimental studies to uranium poisoning in man. Also included are sixteen paper in the collected papers section on the various aspects of the toxicology of uranium compounds. (S.V.S.) 1029

Voegtlin, Carl, and Harold C. Hodges, eds. THE PIARMACOLOGY AND TORICOLOGY OF URANIUM COMPOUNDS.
MeCraw-Rill Book Company, Inc., New York. PARTS I, II, 1949. 1084p. PARTS IT, IV, 1953. 2466p. (National Nuclear Energy Series, Division VI, Volume1)

This is one of the firgt avalable volumes of the Natlonal Nuclear Energy series, Manhattan Project Technical Section. If ments on the toxicity of uramium compounds and the mechanism of uranium polsoning. Txperiments on the toxic qualim thes of uranium compounds were made preceding various conditions, such as arposure by inhalation of dust or fumes, by skin and eye application, and by oral and parenteral administration. Large acale acute tegts were made of the dem gree of air contamination by uranium compounds. The problems of aerosol production, control, and measurement are described; details are given on such aspects as the relationship of particle gize to toxicity and the adaptation of the cascade impactor for routine cust measurements. phasis is placed on the mechanism of action of uranium. The physico-chentcal rules which govern the formation of uranium-protein complexes and bicarbonate complexes are applied to the trangpors of uranium in the body and to its excretion through the kidney. The characteristic uranium in the kidney is described from histopathological and from function studies. The role of citrate in tolerance is also covered. Many of the methods and technques are given in detail, such ag the messurement of catalase in urine and the standardization of toxicological procedures for large scalle investigation. New uranium methods presented are: alkn and eye toxicity, the analytical methou, inhalation toxicity, distribution and excretion, theory tolerance, enzyme and protein effects and the catalase method. (NSA)

\section{Yttrolum}

1030

Anthony, D. S. ACUTE RADIOTOXICITY OF INJECTED

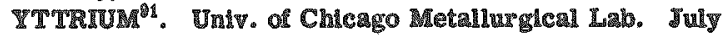
3, 104\%. Deel. sept. 10, 194\%. 10p. (MDDC-1240; C(M-3838)

Male rats in groups conaisting of five animals exch were given intracardial injectione of $\mathbb{X}^{11}$ at dose levels of $1,3,5$, 7 , and $10 \mu \mathrm{c} / \mathrm{g}$ of body watght. A control groug of hive antmals was given isotonic aaline. The exseretion, retention, and distribution of the yttrum was measured, and lts effect on survival and growth was observed. The average total ercretion of $\mathrm{Y}^{\mathrm{P} 1}$ to the time of death (Irom 76 hours to 24 days) was a little leas than $30 \%$ of the injected dose. Abont

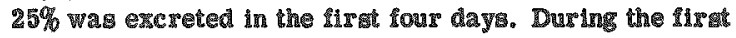
four days, nearly $75 \%$ of the excretion was via the urine; whlle between the fourth and the ninth days, nearly $00 \%$ of the excretion was wa the feces. The average total retention at death (average time, 8.5 days) in 15 rats was 68 㛭 of tho injected dose. This retention was distributed grosaly as follow: about $55 \%$ in the carcass, $1 \%$ in the mise viscera, and $2.5 \%$ in the remur. The highegt total retention in the viscera was found in the liver (6\%) while the highest retontion/gram of arof tis we was found In the kddney (1.7\%). The concentration in the femur was three times higher than the highest concentration in the aft tigsues. The 30 day LD-50 was between 1 and $3 \mu \mathrm{c} / \mathrm{g}$, indicating a toxicity nemrly twice aิ great 23 that observed with other siston product. The explanation of the high toxlcity of $Y^{31}$ is not aprarent 1 rom the data in this experiment. Although ther wrer no derth in the period between 4 weels and 6 months, all fours of the remaining rats that had been given $1 \mu \mathrm{c} / \mathrm{g}$ ded betwern 6 and 8 months. Three of these deathe were due to tumors. The growth rate, as measured by lnerease in body weifght, was depressed approsimately $10 \%$ even by the lowest ( 1 $\mu \mathrm{C} / \mathrm{g})$ dose of $\mathrm{Y}^{\mathrm{a}} \mathrm{E} \cdot(\mathrm{ADD})$ 
1031

Brues, Austin M. and Leola Rietz. RESPONSES OF MTOTIC CELLS IN LIVER AND CARCMNOMA TO CONTLWUOUS INTERNAL IRPADIATION. Cancer Research 11. 240(1051).

This paper is reproduced here in itg entirety.

Regenerating rat liver was exposed to internal $\beta$ irradiation by insertion of glass beads containing $\mathrm{Y}^{01}$ or $\mathrm{Ce}^{146}-\mathrm{Pr}^{146}$ The distribution of the absorbed energy around these point sources was such that concentric spheres of the tissue recelved graded dosagea varying between zero and amounts suficient to cause early necrosis. The frequency of mitotic igures was reduced more or less uniformly at a given dosage rate between 8 and $48 \mathrm{hr}$ after insertion, and followed an exponential depression with increasing dosage. Mitotic Mgures decreased in number in the heavily irxadiated areas within $1 \mathrm{hr}$ after ingertion of the radioactive source, and virtually disappeared between the 4th and 8th hour. A few residual figures probably represent prolongation of a few aitoses under continuous ir radiation. Exposure of the Hall mouse carcinoma under the same conditions resulted in a similar picture. In addition to this, large cells appeared in the region of depressed mitotic activity. It appears probable that this results from continued nuclear and cytoplasmic growth where mitosi is inhibited. (NSA) 1082

Dobson, E. L., et a. STUDIES WTTH COLLOIDS CONTAINING RADIONSOTOPIS OF ITTRIUM, ZIRCONUUM, COLUMBIUM, AND LANTIANUM, PART II. THE CONTROLLED SELECTIVE LOCALIZATION OF RADIOISOTOPES OF YTTRIUM, ZIRCONIUM, AND COLUMBIUM TN THE BONE MARROW, LIVER, AND SPLEEN. ग. Lab. Clin. Med. 34, 305-12(1949) (AECD-2055; UCRL-92)

Studies with several colloids containing radioisotopes of zirconium, columbium, and yttrium have shown that the biological distribution of such materials depends upon the nature of the colloldal particles. Colloids of zirconium of relatively large particle size show rapid disappearance from the blood atream (half-life $=30$ seconds to 1 minute), and are deposited primarily in the liver and spleen. Collolds of intermediate particle size, both of zirconium and yttrium, disappear much more slowly from the bload stream (hif -1 ife $=30$ minutes to 80 minutes) and are de posited primarily in the bone marrow and spleen, the liver acquiring only $1 / \%$ the specifle activity of marrow. Both typer of colloids once deposited show no signilicant change in distribution pattern, at least over 2 to 4 week period, and only glow excretion from the body. (NSA)

Foreman, Ilarxy and Joseph G. Hamilton. THE USE OT CHELATING AGENTS TOR ACCELERATMG EXCRETION OF RADIOLLEMIENS. Radiation Lab., Univ. of Calif. June 14, 1951. Decl. Sept. 21, 1951. 18p. (ALCD-3247)

The chelating action of (ethylenediamine)tetraacetic acid (ㅁDTA) was investigated as a means of accelerating the excrethon of radioelements which have become fired in the amimal skeleton. Rats injected with $Y^{11}$ lollowed by the adninistration of Ca $5 D T A$ showed excretion of $\mathrm{Y}^{21}$ greatly increased when compared with controls which received $Y^{81}$ Mlone. Rat recelving both $\mathrm{Ce}^{\mathrm{HW}}$ and $\mathrm{Pu}^{253}$, followed by $\mathrm{Ca}$ EDTA showed an increase in urinary excretion of Ce when fiven duxing the firgt 24 hr following injection of the isotope. Ca EDTA enhanced urinary excretion of $P u$ to levels which were approzimately 10 times greater than that of control animals. The effect lasted for several days after the cessation of treatment. Fecal excretion of Pu was not affected by the chelating agent except when $\mathrm{Ca}$ CDTA was adminigtered during the fir $24 \mathrm{hr}$, at which time there was anarked rise in Pu excretion. (NSA)
1034

Jacobson, Leon O. and Eric L. Simmons, THE EFFCTS OF INJECTED YTTRIUM ${ }^{01}$, PART VIII. HEMATOLOGHCAL EFEECTS. Univ, of Chicago Metallurgical Lab.

June 1946. 21p. (AECD-2037; CH-3859)

Radioactive yttrium $\left(\mathrm{Y}^{11}\right)$ was administered by gavage to rats in single acute doses of 5,10 , and $20 \mu \mathrm{c} / \mathrm{g}$. Daily doses of from 0.3 to $2.0 \mu \mathrm{c} / \mathrm{g}$ were administered to other rats, and at some dose levels treatment was continued up to 178 days. A single dose of $20 \mu \mathrm{c} / \mathrm{g}$ produced a marked initial elevation in erythrocytes and hemoglobin, followed by an erratic anemia due to bleeding. A $75 \%$ reduction in lymphocytes occurred within three days, but these elements reached a normal value by 42 days. The heterophil values after this dose were extremely variable. A dose of $10 \mu \mathrm{c} / \mathrm{g}$ produced a temporary lymphopenia and an erratic heterophila only. No hematological effect was observed after single doses of less than $10 \mu \mathrm{c} / \mathrm{g}$. The daily gavage of $\mathrm{Y}^{81}$ in amounts from 0.3 to $2.0 \mu \mathrm{c} / \mathrm{g}$ produced no anemia. A sustained heterophila was present in all groups receiving daily treatment. In one experiment a moder ate Iymphopenia, which reached its maximum at 60 days, was produced by the daily treatment of 0.3 and $1.0 \mu \mathrm{c} / \mathrm{g}$ but the 1 ymphocytes gradualiy recovered to normal values by 90 days even though yttrium administration continued through 178 days. In another experiment, daily ingestion of $0.3,1.0 \mu \mathrm{c} / \mathrm{g}$ produced a lymphopenia which reached its maximum at 98 days. By 14 days after the discontinuation of $\mathrm{Y}^{81}$ gavage (at 95 days) the lymphocyte values had returned to normal levels. It is suggested that the Iymphopenia described above results from the local effect of $\beta$-radiation on the lymphatic tissue of the gastrointestinal tract. No significant morphological change in the nucleated cells or exythrocytes occurred after singly or chronically administered doses of ingested $\mathbf{T}^{\text {9! }}$. (NSA)

1035

KIdman, Barbara, Margaret Tutt, and Janet Vaughan. EXCRETION OF YTTRIUM-91 IN RABBITS. Nature 167, $858(1951)$.

The effect of age on excretion and retention of $Y$ in rabbits has been demonstrated following injections of $Y^{\text {?iI }}$. The mean retention in three weanling rabbits 5 to 6 weeks old, on a medium calcium diet, killed 9 days after an injection was $0.63 \% / g$ of bone, while in three 7 -month old rabbits it was $0.33 \%$ of the injected dose/g of bone. The mean excretion in the urine in weanlings was $12.0 \%$ of the injected dose, whereas in adults it was $24.0 \%$ of the injected dose. These findings confirm that the decay-curve method for estimating the excretion of $\mathrm{X}^{80}$ has validity. (NSA)

1036

risleleski, W. E., B. E. Kline, and R. P. Rusch. A PRELIMINARY STUDY ON THE EFFECTS OF INJECTED YTTRIUM" ON THE RIBOFLAVIN CONTENT OF RAT LIVERS, p. 172-6 Of BIOLOGICAL AND MEDICAL DIVISIONS, QUARTERL REPORT, FEBRUARY, MARCH, APRIL, 1949. Argonne National Lab. [nd] 4p. (ANL$4291(\mathrm{p} .172-6))$

Data on the effects of injected yttrium ${ }^{21}$ on the riboflavin content of rat livers have been presented. Preliminary indications note a fall in ribollavin; however, no histological or morphological changes were noted, and this fall may be a sign of minimal radiation damage or, more likely, a part of a more general toxic picture that is manifested by loss in body weight. Calculations of the radiation dose to the livers and a method for riboflavin determination have been pregented. (Authors' Summary)

1037

Lisco, H., Austin M. Brues, Miriam P. Finkel, and W. Grundhauser. CARCINOMA OF THE COLON IN RATS 
FOLLOWING THE FEEDNG OF RADIOACTIVE YTTRIUM. Cancer Research 7, 721(1947) (MODC-1418-C)

Ytrium ${ }^{91}$, one of the common radioactive fission products obtained in a chain-reacting pile, is a pure $\beta$-emitter with an energy of $1.5 \mathrm{Mev}$ and a half life of 57 days. It is essentially not absorbed and, since the material remains longer in the colon than in any other portion of the intestinal tract, most of the damage occurred in this region. One group of rats received a single feeding by stomach tube of from 1.0 to 6.0 millicuries of $Y^{31}$. Of the 33 animals in this group, four died with adenocarcinoma of the colon. The earliest tumor was seen at $\mathbf{1 3 5}$ days and the latest, at 506 days. Additional animals died with acute and chronic ulceration of the colon accompained by benign and atypical hyperplasia of the mucosa. A second group of rats was given 78 feedings of $0.46,0.20$, or 0.06 millicuries of $Y^{91}$ per feeding over a period of three months. The total accumulated doses were $31.20,15.60$, and 4.68 millicuries, respectively. Clinically all animals appeared well during the feeding period and growth was not impared. Six of the eight animals at the two higher levels died with carcinoma of the colon from 304 to 548 days after the first feeding. No malignancies were observed at the lowest level. However, many of these animals died with superficial ulcerative lesions of the colon. (ADD)

1038

Scott, I. RADIO FTTRIUM $\left(\mathrm{Y}^{89}\right)$ Univ. of Chicago

Metallurgical Lab. [nd] (MODC-1143-A)

Extended experiments coverimg a period of 64 days revealed that approximately $50 \%$ of $\mathrm{Y}^{83}$ introduced into the peritoneal cavity 1 deposited in the bones. No significant decrease of this deposition was observed between the imst and sixty-fourth day. The rate of elimination of $\mathrm{Y}^{38}$ was extremely slow after the firgt few days of administration. The dally rate of ellmination after the fir st weels is negligible as compared to the rate of radioactive decay of this radio element. The oral administration of $\mathrm{Y}^{8}$ indicates that the total absorption of this element from the digestive tract is less than $0.1 \%$. When $\mathrm{x}^{88}$ is introduced as a solution of $\mathrm{yCl}_{3}$ directly into the lungs apparentiy a large proportion of the administered material remains unabsorbed in this organ at four days. The proportion that underwent absorption was distributed in the tissues in a maner gimilar to the locallzation after intraperitoneal injection. (ADD)

1030

Walkex, Leonard A. LOCALIZATION OF RADIOACTVE COLLOTOS IN LYMPY NODES. J. Lab. Clin. Med. 36, $440-9(1950)$.

The purpose was to gtudy the uptake by the lymph nodes of radioactive colloids injected into lymphatic channels. Unguccessul attempts were made to cause colloids injected subcutaneously to enter lymphatic vessels and localize in regional nodes. A method for intralymphatic injections was developed and atudies were made on the localization of yttrium 90 colloids. One type gave a concentration in the most active node of 284 times that of the most active tismue; a second colloid gave 7900. (CA)

\section{Zirconium}

1040

Overstreet, Roy, et al. RADIO ZIRCONIUM (ZR/9) Univ. of Chicago Metallurgical Lab. [nd] 5p. (MDDC$1143-D)$

A satigfactory method has been developed for the preparation in the pure state of $\mathrm{Zr}$ as $\mathrm{ZrOCl}_{2}$ without the presence of carrier. When administered by intraperitoneal and subcutaneous injection approximately $80 \%$ at two and sis day intervals appear to remain at the site of injection. No marked degree of specific localization was noted in any of the tissues examined. (ADD)

\section{RADIATION SICIKNESS}

Syndrome in Lumang-General 1041

RADIATION \$ICKCNESS. Brit. Med. J. 4585, 909-10(1848).

The cause of radiation sickmess can probably be found in changes in the metabolism of cells which are sensitive to radiation. There is no general agreement on what the changes are, however. Breakdown of blood cells and release of substances, release of histamine, achlorhydric stomach, and change in endocrine function are all mentloned as posstble causes. The following remedies are listed as having given good results in the hands of various workers: histaminase, "benadryl, " hydrochlorte acid, sodium chloride, magnesium chloride, high carbohydrate diet, large amounts of fluid, vitamin $C$, vitamin $B$, amphetamine, pancreatin, and pyridoxin. (NSA)

1042

Bean, William B., Tom D. Spies, and R. W. Vilter. A NOT: ON IRRADIATION SICINESS. Am. J. Med. SEL. 208, 46-54(1944).

Nausea, vomiting, headache, cramps, and diarrhea comprise the syndrome of irradiation sickness often complicating the course of therapeutic irradiation. From previous successful attempts at treatment of this Hiness with nicotinic acid, and with the discovery that the behavior of urinary pigments and the cohydrogenases I and II following irradiation over the spleen resembled that in severely ill pellagrins, it was planned to make a comprehensive examination of the effects of a standard dose of radiation. Normal well-fed subjects, those given a vitamin-deficient diet with and without some of the vitamins, pellagrins and those with poor diet had resulted in ulness without speclfle deficiency stigmata were investigated. The irradiation factors were: $200 \mathrm{kv}$, 2 Thoraeus fulter, 20 ma, $20 \mathrm{~cm}$ distance, $33 \mathrm{r} / \mathrm{min}$. The dose was $400 \mathrm{r}$ over the upper abdomen and spleen. Five normal subjects consuming a good diet or varlous vitamin I supplements showed no III effects. Irradiation sickness occurred in varying degrees of severity in the rest of those studied, including the normal subjects who had subsisted on a vitamin $B$ deficient diet for sis weeks. In all cases there was a rough correlation between the severity of the reaction and the degree of vitamin depletion as gauged clinically. Once the Iliness was established, large doses of thiamine or nicotinic meid were of relatively ittle value. Irradiation sickness could be prevented or reduced in severity if the vitamim aupplements were given for a few days before therapy was started. A patient with moyomata and carcinoma of the uterus suffered from severe radiation sickness because of repeated doses of radiation. She was not given supplements of vitamins and subsequently pellagra and beriberi developed. It would appear Irom these studies that the optimum time for vitamin therapy is before and not after Irradiation sickmess develops. The fact that patients meeding radiotherapy often have a deranged nutrition makes careful dietary and vitamin therapy logical, 1 if only on emplric grounds. (R)

\section{3}

Brown, W. Court. THE VALUE OF PLATELET COUNTS IN RADIOTHERAPY. BRit. J. Radiol. 21, $221-5(1848)$. 
The effect of wide-field irradiation on the blood platelet level is described, and it is suggested that the blood platelet level is a more reluble indicator of the effect of radiation on the bone marrow than the total white cell count. By using the blood platelet level in this manner it is possible to avoid altogether the production of partial or permanent marrow fallure. The use of the platelet level in the planning of treatment is described. The relationship between the development of petechal haemorrhages and radiation-induced thrombocytopenia is discussed. (INA) 1044

Brues, Austin M. RADLATION SICKNNESS, P.778-71 of MPDICAL PEYSICS, VOLUME 2, Oto Glassen, ed. Chicago, Tearbook Publ., Inc. 1950. $1227 \mathrm{p}$.

Symopsis of the clinical picture, basis of acute radiation gickness, effects of sublethal doses, alom bomb ininuy, diagnosis and therapy of radiation eroosure. (M. 1045

Bryan, Fed A. PROBLEMS REATIV TO POSSIDLE PHYSTOLOGICAL EFTISTS CAUSED BI RADIATION. Indust. Med. 17, 367-71(1948).

Some of the problems occurring in the use and manuLacture of rodioactive materials are alscussed from the viewpoints of possibility of exposure, the physiological effects on personnel resulting from exposure, and the maintenance of safe practices and good health in personnel. The rreatest hazard of all can be the lack of knowledge concerning the physiological and tossicological effects of radioactive materials by the people using them. It is pointed out, however, that if all safety procedures are well morked out and diligently applied, use of atomic energy or its by-products is as safe as any other type of manufac1046 turing. (NSA)

Butler, Nell and James . Crenshaw. POSITIVE CEPA-

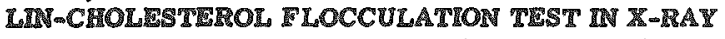
WORKRES. Am. J. Med. Technol. 14, 255-8(1948).

Carefully controlled experiments are described, testing cephalin-cholesterol serum floceulation in $28 \mathrm{x}-\mathrm{ray}$ workers who had been exposed for periods ranging between 4 months and 20 years. The dats are presented in a table which correlates exposure time, age, sex, gastrointestinal symptoms, jaundice, liver tenderness, liver eniargement, and splenic enlargement with the results of $24-h r$ cephalin and $48-\mathrm{hr}$ cholesterol flocculation tests. The author conclude that at least $22 \%$ show some positive tests and suggest that this may be a measure of slight changes in liver 1057 function due to roentgen irradiation. (NSA)

Cronkite, rugene P. TRE DLAGNOSIS OF IONIZMG RADLATON INTERY BY PHYSICAL FICAMWALION AND CLINICAL LABORATORY PROCEDURES. Naval MedIcal Research Inst., Bethesda. Apr. 8, 1848. 15p. (Report No. 8)

The syzaptomatology and diagnosis of acute and chronic radiation lliness Is discussed. Characteristic symptoms of the acute Illness are nausea, vomiting, diarthea, hemorrhagic manifestations, agranuloeytosis followed by nonpurulent necrotiding infections, and eplation. The blood picture is the most sensitive biologic index of exposure to lonizing radiation. Lymphocytopenta appears promptly after the gingle intense exposure. The symptomatology and blood pleture of chronic exposure is protean. In order to evaluate the blood changes of chronic exposure, rigid control data must be established. The most significant blood change following chronle exposure is a depression of the mean leukocyte count of the exposed groups below the contral groups. Dosumetry, the measurement of radiation intensities, must remain the basis of all protective measures. (NSA)

1048

Hempelmann, L. H. THE ACUTE RADIATION SYNDROME. Los Alamos Seientific Lab. [nd] 6p.

(AECU-379)

The acute radiation syndrome is an acute generalized ilness lasting only a matter of weeks and terminating either in death or in complete recovery of the patient. "The syndrome differs in this respect from the chronic form of radiation injury caused by repeated or prolonged exposure of the body to radiation. Factoris determining the severity of the injury are the dose rate, the type of radiation, and variation in the response of the individual. The clinical response of putients suffering with acute radiation syndrome are discussed in four phases occurring in the following chnountoglogich order: the initial reartion (prostration, nausea, and vomihing; a period of relative well-being; the gradual development of fever (toxemia) assoctated with diarrhea, bleeding, infection, and loss of hair; death or prolonged convalescence. Besides the easily discernible bodily reactions there are a number of equally and perhaps more fundamental responses of persons with the acute radiation syndrome which can be detected only by use of laboratory techniques. These are: changes in the 100 cells and in the male reproductive organs, distortion of the normal function of some of the internal organs of the body and cextain kinds of cellular danage that are responsble for tissue injury and for the clinical response of the individual. It is concluded that there is no practical way of preventing the death of cells or tissues fatally injured by radiation. However, if the body can be helped to survive the acute state of the ilness, even the most radiosensitive tissues, such as the bone marrow, may regenerate to the point where they can carry on their nomal functions. Means by which the body can be helped through the acute state of the illness are: blood transfusions to replace temporarily the red and white blood cells; penicillin to prevent infection; certain materials (toluidine blue) to diminish the clotting time of the blood, and mantenance of the water and salt content of the body. (TIS)

1048

Fenshaw, Paul S. FURTHER PROBLEMS IN X-RAY PROTECTION, II. IRRADIATION INJURY AND THE TOLFRANCE DOSE. Radiology 44, 569-80(1945).

The problem of tolerance dose and $\mathrm{X}$-ray protection has been reviewed. It has been recognized that various effects such as skin ulceration, sterility, anemia, and even death may result from exposure to lonizing radiation in amounts not greatly exceeding those which accumulate in comparatively short periods of time in varlous laboratorles, shops and clinics; that for such effects there are threshold levels of exposure; that other changes, such as premature aging and neoplasm may appear long after exposure; that for still other effects, irradiation induced mutations, there appears to be no threshold level of exposure, and that there is roughly a factor of 100 difference between the accepted permissible dose of $0.1 \mathrm{r} / \mathrm{day}$ and cosmic and earth radation to which everyone is continuously exposed. (BA)

1050

Nutta1, John R. THE EFTECTS OF OCCUPATIONAL EXPOSURES TO I -RAYS AND RADIOACTIVE SUB . STANCES. Clin. J. (London) 72, 181-5(1943).

A review of the effects of $\mathrm{x}$-rays and radioactive substances, with particular reference to effects upon reproductive system and upon the skin, to radiation tiredness, and to blood changes. (CA) 
1051

Nuttal, John R., J. C. Mottram, S. Russ, and J. M. Vaughan. DISCUSSION ON THE EFIECTS OF OCCUPATIONAL EXPOSURE TO X-RAYS AND RADIOACTIVE SUBSTANCES. Proc. Roy. Soc. Med. 35, 717-22(1942).

A review of the dangers confronting workers in the field of $X$-ray and radioactive substances and the means of combating them; mutations produced by radiation in relation to mankind; hat miful effects of radioactive substances; effect of oceupational exposure to $\mathrm{z}$-rays and radioactive substances upon haemopoiesis. (S.V.S.)

1052

Paintex, E. E. and A. M. Brues. THE RADIATION

SYNDROME. New Engl. J. Med. 240, 871-6(1949).

A discussion is presented on the theoretical aspects and the clinical manifestations of the radiation syndrome. The initial shock, the acute, the subacute, and the chronic periods are described. The therapy for radiation damage is noted as not being adequate at the present time. It is ubserved that no single clinical reaction is peculiarly specific for the radiation damage, and many of the delayed effects of zadiation can be duplicated by various toxic materials. In conclusion, it is stated, that damage to the radiosensitive cells will explain a great part of the picture, but much further understanding of the syndrome is required in the interest of prevention and therapy. (NSA) 1053

\section{WHAT EVERT MEDICAL OFICER SHOULD WNOW} ABOUT THE ATOMC BOMBS. PART IV. EVALUATION OF THE FIVE ATOMIC EXPLOSIONS. BUL. U. S. Army Med. Dept. 8, 350(1948).

Lethal effects will be produced by air-burst atomic bombs over an area of 2 square miles and measurable effect over an area of 7 square miles as a result of the gamma radiation emitted at the time of detonation. There is little importance in the residual radioactivity except in the area close to the center of a low-altitude explosion. In an underwater detonation, radioactive fission products and unissioned material will be spread by the cloud and base surge over a large area. The gamma radiation from these materials will be lethal to exposed personnel more than two miles downwind, and sorious contamination will result at much greater distances. A serious hazard will be provided by this detonation for an indefinite period. At the time of the detonation prompt evasive action will permit the reduction of casualties. The pathologic effects of radiation are presented by outlining the early and late changes in tissue cells, organ systems, total body irradation, and internal radiation by radioactive materials introduced into the body either accidentally or therapeutically. The sensttivity of various body tissues has been established and has been expressed largely as the relation of one tissue to another. (NSA)

\section{4}

WHAT EVERY MEDICAL OFTICER SHOULD FINOW ABOUT THE ATOMC BOMB. PART V. FUNDAMENTAIS OF RADIATION PATHOLOGT. Bull. U. \$. Army Med. Dept. $8,357-62(1048)$.

The pathologic effects of radiation are presented by outlining the early and late changes in issue cells, organ systems, total body irradiation and internal radiation of radioactive materials introduced into the body either accidentally or therapeutically. A table is given which shows the relative radiosensitivities of various body tissues listed in decreasing order. (NSA)

1055

WLAT EVERY MEDICAL OFFICER SHOULD IRNOW ABOUT THE ATOMIC BOMB. PART VI. PATHOLOGIC
ANATOMX OF RADIATION FFECTS OF ATOMC EXPLOSION. Bull. U. S. Army Med. Dept. 8, 422-8(1948).

The radiation effects of an atomic explosion on the skin, pituitary, adrenals, heart, lungs, genitourinary system, gastrointestinal tract, spleen, lymph nodes, bone marrow and the reliculo-endothelial system are reviewed. The case history of a man at a distance of 0.7 knlometers from the explosion center is given. (NSA) 1056

WHAT EVERY MEOICAL OFFICER SHOULD ISNOW ABOUT TEE ATOMC BOMB. PART VI. DETYCTION OF OVEREXPOSURE TO IONIZIVG RADATION. Bull. U. S. Axmy Med. Dept. 8, 428-31(1948).

The effects of overexposure may be acute or chronic. The exposures may result from any type of radiation applied externally or internally. The clinical picture will depend on the anount, rate of delivery and depth of the dose. The signs and symptoms such as thermal burns, diarthea, nusea, vomiting, headache, anuria, purpura, blood changes, fingernall brittleness, pigmentation, etc., are discussed. A procedure to be followed in atudying a person suspected of being overexposed is glven. (NSA) 1057

Waren, Shields. THE PATHOGENESH OF IRPADIATION SICKNESS. Chicago Med. SOc. Dull. $51,957-0$ (1049).

The syndrome of radiation sickness is discussed as if applies to mass radiation by atomic weapons. The cells of the body which have a short life-span are more effected by the radiation than those with long life-spass, and rapidly dividing cells, or those in mitosis, are more afiected than cells in the resting phase. A dose of $400 \mathrm{x}$ is usually fatal to about $1 / 2$ of the human population present. There is gseat variabillty in the sensitivity of different cells and organismas in general. It is belleved that the heaviest types of doses cause disruption in the enzyme systems and vital functions of most of the body cells and death occurs promptly without much evidence of morphological change. slides are presented which indicate specific and individual reactions to the radiation doses. These include multiple leukopenic absesses and cellulitis of the aeck. The dif ferent stages of development of the symptoms are pointed out. If the radiation dose is more than $500-600 \mathrm{x}$, the chances of survival are small. Future problems connected with mass effective medical care of radiation victins age discussed. Prevention rather than cure is suggested as the mosf promising method of handing the radiation sickness problem. Lecture with slides. (NSA)

1058

Warren, Shields and John 2 . Bowers. TWE ACUTE RADATION IYNDROME IN MAN. Anמ. Internal MEd. $32,207-16(1950)$.

ine pathogenesis, histopathology, symptomatology, clinical course, laboratory findings, complications, sequelae, therayy and prognosis of the acute radiation symdrone in man are discussed. Tables are included which demonstrate the variation in the sensitivity of the tissues of the human body to lontging radiations and variations in sensitivity between species; such concepts of the mechanism of the effects of radiation on the body as the coll hydration theory and the target theory are outlned. Cellular, stromal and vascular effects of the radiation are noted, and the prominent mandestations of the syndrome (asthenia, diarrhea, nausea and vomiting, mucosal ulcerations, epilation, fever and tachycardia are correlated with three types of radiatton illness seen: the fulminating form (most severe), the hemorrhagic form, and the pancytopenic form; hematological responses and bacterial infections are 
discussed along with such sequelae as possible mutation effects and neoplasia. It is stated that the treatment of Individuals exposed to damaging amounts of ionizing radiation is at this data supportive and nonspecific, there being no known specific agent which will amellorate the injury. Such agents as folic acid, toluidine blue, rutin, protamine, adrenal cortical extract and parenteral therapy (for the correction of aberrations in blood electrolytes) are mentioned. Patients who develop the fulminating course of the radiation syndrome are concluded to have lethal amounts of radiation. However, those patients exhibiting the hemorrhagic and pancytopenic forms, with consequent delayed radiation effects, are said to have received either lethal amounts or sublethal amounts and a certain proportion can be expected to survive. (NSA)

1059

Wolf, B. \$. MEDICAL ASPECTS OF RADLATON SATETY. Nucleonics 3 , No. 4, 25-9(1948).

This brief discussion emphasizes what the author calls psychomatic aspects. He takes up the following five items in connection with radiation which seem most worrisome to people and attempts to place these special hazards in their proper perspective. They are (1) the inability to detect exposure through the five senses, (2) the penetrating power of radiation, (3) the delayed effects of overexposure, (4) the sterilizing effect, and (5) the genetic effect. (NSA)

Syndrome in Humans - Hiroshima and Nagasak:

1060

CLINICAL AND PATHOLOGICAL OBSERVATIONS ON THE EFTCTS OF THE ATOMC BOMB. Atomic Bomb Investigating Groups, Manhattan District. [nd] $\$ 2 p$. (NP-1202)

Part I. Skin Burns. Nine cases are discussed demonstrating the pathology of shin burns caused by the atomic bomb explosion at Nagasaki. The nine victims were Japanese prisoners of war who were interned in "Camp No. 14 " which was just outside the area of extreme atomic bomb destruction; the burns were thus caused by the exploston of a bomb well over a mile away.

Part II. Atomic Bomb Disease. Among the survivors of the atomic bombing of Nagasaki there developed a disease, often fatal, which was apparently independent of mechanical or thermal injuries. A clinical and pathological study of the disease showed it to be entirely comparable in all respects to the syndrome caused by a large generalized dose of gamma radiation. The mortality of the disease would have been much lower provided that adequate medical care had beèn given. (NSA)

1061

Beck, J. \$. P. and W. A. MeISSner. RADIATION ETFECTE OF THE ATOMC BOMB AMONG THE NATIVES OF MAGASARI, IETUSGU. Am. J. Clin. Pathol. 16, 58602(1846).

Some observatlons are reported on the radiation effects of atomic bombing among the inhabitants of Nagasali, Ixyuhu, during the sixth and seventh weeks after the bombing. Among 20 patients whose blood or bone marrow was atudied, there was anemia, neutropenia, and thrombocytopenta, which were due to depression of the marrow. In 7 postmortem examinations there was confirmatory evidence of damage to the bone marrow. IRvidence of recovery of bone marrow was found both clinically and post mortem. mintologic evidence of Ipid depletion of cells of the adrenal cortex was found. Aspermatogenesis was noted in some instances. The mortality rate of "atom bomb disease" could have been lowered by adequate medical care. (BA)
1062

Benkwith, K. B. RETINAL HAEMORRHAGE AS SEEN IN AN ATOMIC-BOMB CASUALTY. Am.J. Ophthalmol. 29, $799-800(1946)$.

A fourteen-year-old Japanese girl who at the time of the atomic-bomb explosion at Nagasaki, Japan, was about one mile from the accepted hypocentre of the explosion has suffered from malaise, headache, fever and nausea ever since. Early in September she was admitted to the Ophthalmic Institute of the Kyushu Imperial University at Fakuoka suffering from a disturbance of vision. On examination massive pre-retinal haemorrhages were found and, in addition, superficial retinal haemorrhages and small "fluffy" spots of exudation close to the disc and to the retinal vessels. Petechiae in the skin, bleeding from the gums, and mild epilation were also noted by the patient. Although little treatment was attempted and she remained an ambulatory patient, the ill effects of the radioactivity subsided slowly, the vitreous remained clear, and by December 1945 the only pathological changes visible in the eye were number of white discolored areas about the posterior pole. Initial laboratory tests showed the presence of a leucopenia with a W.B.C. of 2,200; and anemia with an R.B.C. of 2,200,000; a decrease in the number of blood platelets; and a prolongation of the bleeding time. There never was any evidence of blast wounds. (EM) 1063

Bernier, J. L. THE EFECTS OF ATOMC RADIATION ON THE ORAL AND PHARYNGEAL MUCOSA. J. Am. Dental Assoc. 39, 647-57(1949).

This report is concerned chiefly with the changes observed in patients who received both lathal and sublethal doses of $\gamma$ ionizing radiation during the atomic explosions at Hiroshima and Nagasaki. The changes in the blood and the histopathologic changes are described in detail for the three significant stages (lethal irradiation, severe but sublethal irradiation, and mild irradiation). A very early depletion of the hematopoietic system occurred with resulting anemia, agranulocytosis (shown by pharyngeal angina, gingivitis and severe secondary infections) and thrombocytopenia (manifested clinically in a few weeks by the purpuric state); epilation became evident in about 10 days. Profound and early changes of both squamous and glandular types were noted in the epithellum; lesions appeared in the oral cavity and the gastrointestinal tract. It is concluded that further studies are necessary. (NSA) 1064

Bowers, John Z. ACUTE RADIATION SYNDROME. J. Am. Med. Assoc. 145, 63-5(1851).

The pertinent information that has been presented may be summarized as follows: The acute radiation syndrome is caused by sudden exposure to the penetrating radiations liberated by the atomic bomb. The resulting changes depend primarily on the inherent sensitivity of the exposed tissues and the type of radiation and are attributable to damage to the blood and blood-forming organs, the blood vessel endothelium and the bowel. These particular tissues all show tendency toward ultimate regeneration, and it is on this fact that present therapeutic programs are based. The primary manifestations are hemorrhagic diathesis, nausea, vomiting and diarrhea and depression of the formed elements of the body. Treatment is at this date supportive in nature. (auth)

1065

Cogan, D. G., S. F. Martin, and S. J. Kimura. ATOMC BOMB CATARACTS. Science 110, 654-5(1849).

Ten cases of cataract which seem to have been caused by exposure to the atom bomb blasts at Hiroshima and Naga- 
saki are described. Of the 10 subjects, 7 were males and 3 females, ages at time of exposure varied from 13 to 55 years, all were within $550-950 \mathrm{~m}$ of the hypocenter of the explosion, all were in the open or were shielded by wooden structures at time of explosion, all had epilation of the head after a latent period 1-4 weeks with complete baldness lasting $3-7$ months, and all had normal vision at time of explosion. The only ocular complaint of the subjects was failing vision, latent period varying from 6-30 months. The cataracts consisted of opacities in the araal zone of the posterior lenticular capsules over an area of $2-4 \mathrm{sq} . \mathrm{mm}$, with oceasional punctate dots farther toward the periphery; the findings were approx. symmetrical in the 2 eyes. Detalled nature of the cataracts is described. (BA) 1066

DeCoursey, E. HUMAN PATHOLOGIC ANATOMX OF IONIZING RADIATION EFFECTS OF THE ATOMIC BOMB EXPLOSTONS. Military Surgeon 102, 427-3241948).

The effect of ionizing radiation on human anatomic structures was studied by means of necropsy findings conducted in Nagasaki and Hiroshima. Investigations were made on those dying during the early period (first two weeks), intermediate period (3-6 weeks) and late period (6 weeks - 4 months). The outstanding symptoms were nausea and vomiting on the day of the bombing, followed by fever, leukopenia, diarrhea, and after two weeks eplation, purpura and oropharyngeal lesions. The spleen showed gross change as early as the fourth day and there was almost total disappearance of blood forming cells in the bone marrow. All parts of the body in which hemor rhage is posslble were affected. The gastro intestinal tract was among the first locations to present gross lesions. The tests showed prominent microscopic changes, all dying males having almost all their sex cells destroyed. The ovaries, except for hemorthages, presented no distinct change and very little microscopically. About the surest. macroscopic evidence of lonizing radiation and the most prominent external effect was the epilation of the scalp. (NSA)

1067

Fnloe, C. F. SOME EFTECTS OF RADIANT ENERGIES ON THE TEE. Sight Saving Rev. 18, 77-83(1948).

The author reviews the various types of radiant energy, and reports in some detail present-day knowledge concerning the energy of the atomic nucleus and its relation to the eye. He notes that thus far the effects of radiation lave not presented a great problem in the fleld of occupational ophthalmology and that the small amounts of radioactive material which might be found in factories in peacetime produce little direct eficet upon the eye. It is stated that to date no primary damage to the ocular structures has been observed that could be interpreted as a direet result of atomic radiation. The lestons occurring from exposure to the flash during bomb detonation were found to be identical with those resulting from overezposure to inirared. The retinal changes and the four lypes of hemorrhage (tlame, petechial, "Roth"-type and vitreous) observed in people subjected to atomic bombing are felt to be directly related to the defictency in the blood elements and in no way directly related to the action of radiation on the eye. These lesions disappear after the blood returns to normal, and thus far there has been no seport of permanent residual damage (rom the retinal lesions. (NSA)

1068

rowland, J. W. and S. L.Warren. THF EFTECTS OF IRRADRATION FROM THE ATOMC BOME ON THE

JAPANESE. Cancer Research 6, 4420(1946). (MDDC-1301)
A brief description is given of the medical effects following the atomic explosions at Hiroshima and Nagasaki. A discussion of the immediate clinical human pathology resulting from blast and burns is included. The effects of acute whole body irradiation on the human are considered in detall as well as pertinent observations on living individuals observed one year later. The comparative clinical and pathological effects of whole body irradiation on animals are given briefly. The pathological findings on tissues sensitive to irradiation namely lymphatic system, bone marrow, gastrolntestinal canal and gonads are discussed. (ADD) 1069

IVEller, Paul D. A CLINICAL SYMDROME FOLLOWING FXPOSURE. TO ATOMC BOMB EXPLOSIONS. J. Am. Med. Assoc. 131, No. 6, 504-6(1846).

Twenty-one patients who developed a delayed illness following their exposure to atomic bomb explosions in Japan, 20 of whom were indoors at the time, were affected similarly to excessive irradiation of the body with $8-$ rays. There was destruction or suppression of the hemopoietic syster, disturbed liver function, and increased erythrocyte sedimentation rate; evidence of extensive tissue destruc. tion within the body. (BA) 1070

Rikuch, T., et al. MRATOLOGICAL ORSERVATIONS IN THE ATOMC BOMB SUFFERRR IN HIROSHIMA CITY ONE Y PORT). Kyoto Imperial Univ., Japan. [nd] $11 \mathrm{p}$. (NP-305(J-6))

Hematological observations were made of atomic bomb sufferer (510 cases) one year after the bombing and of healthy Japanese (173 cases) as controls in Hiroshima city with regults as follows: Red blood cell counts are less than 4 million in $71.7 \%$ of the sufferers and in $61.8 \%$ of the comtrols. Color index over 1.21 is found in $50.6 \%$ of the suffer ers and in $8.8 \%$ of the controls. In white blood cell count, there is no case under 3,000 in 1 cmm of blood in the con trols and the counts under 4,000 are found in $14.2 \%$ of the sufferers (counts under 3,000 in $2.1 \%$ ) and in $3.5 \%$ of the controls. In differential count of leucocytes (eosinophils, neutrophils, lymphocytes, monocytes, and plasma cells) and Sugiyana's mean nuclear lobes of neutrophil leueocytes, the re are no remarkable differences in both cases, but the curve of neutrophil lencocyte coun in the auferers shifis to lower percentage than that of controls and in the cuxve of lymphocyte count, this relation is contrary. Blood platelets count is under $\$ 0,000$ in $28.8 \%$ of the oufferers and in $14.8 \%$ of the controls. Bleeding time is almost normal in both cases, but the cases with bleeding time over five misules are found in $4.9 \%$ of the sufferers and in $5.2 \%$ of the conm rols. Tabular data show findings. (ACD)

\section{1}

Kikuchi, T., G. Wakiraka, T. Yoda, S. Note, It. Yamada, and K. Miki. REPORT ON HEMATOLOGICAL OBSERVA-

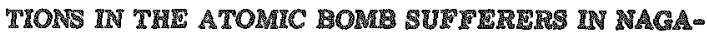
SARI CITY ONE YEAR AND FOUR MONTISS AF"IER THR BOMBING. Ryoto Imperial Univ. Japan. [nd] 12p. (NP-306(T-T))

Conclugions and tables of seaults of hematological exami nations of 172 atomic bomb sutserers and 45 controls (healthy citizens who were not in Nagasaki city at the time of the bombing) are presented. It is concluded that the atomic bomb sufferers in Nagasaki city have returned to normal in the majority of cases, but there are still a sew cases with anemia, leucopenia, thrombopenia and prolongotion of bleeding time. (ACR) 
1072

Larksin, John C. DISTHEUTION OF RADIATON IN THE ATOMIC BOMBING OF NAGASAM. Am. J. Roentgenol. Radium Therapy 55, 525-32(1046).

This study is based on the clinical records of 95 patients in three hospital in Nagasal, who were still hospitalized al weeks after the bombing of the city. Some of the energy released was in the form of radiation, the remainder and sreater part of the energy being released as heat. In order to facilitate this study the patients were grouped according to the zone of the city they happened to be in at the time of the oxplosion. There were lew gurvivors in zone I (within 1,000 meters of the center of the explosion). Zone $2(1,000$ to 1,250 meters) was the zone where survivors showed masimum irradiation affects. Those in zone $3(1,250$ to 1,500 meters) showed slight irradiation effects. In areas beyond 1,250 meters, severe wupericial burns but gractically no irradiation effect were fotand. Protection by walls or roois of buildings played a part in reducing the irradia tion effects. There were nine people in concrete buildings in zone 2, lour of whom had only mild evidence of roentgen rickness and no sign of burns. Twelve people in this zone, with less protection, aufered severe roentgen siclnessprostuse vomiting, hematemesis, melena, and diarrhea. In zone 3 symptoms were much less severe and in the outer zone they were very slight. In no case was a typical rythema attributable to gamma radiation reported. Superfieial radiation effects (i.e., ultraviolet, visible light, and inframred) were by far the most widespread reactions, being found in all zones and as far as 2,500 meters from the radiation source. People in zones $3,4,4$ and 5 fell no heat but noticed burns several seconds after the explosion. Al. mogt any particle of clothing seemed to give them adequate protection. A single layer of clothing night give partial protection, but several layers would not be penetrated. The burned areas were alway facing the radiation mource. These -ffecte were not due to gamma radiation. In all cases healing was rapid and complate. Seventy-gix per cent of per aons in zone 2 and $22 \%$ of those in zone 3 lost part of their hair. Ten out of 11 women in zone 2 whose menses had been regular and 9 out of 12 in zone 3 had amenor rhea after the bombing. In some inatances, this may have bean due to psy. chological factorg. Maxsanum depression of the white blocd count was reached near the asth day. The average count of 11 cases in wone 2 was 280 . In zone 3 the average was 3,750 . There was a mornal count in a patient in zone 2 who was protected by a conerete roof. Ratients who had combined irradiation effects and superficial burns died much earlier than those with pure ligradiation effects. In the latter group the greategt sumber died at the end of the reventh week, denondratms the delayed reaction to irradiation alone. (R)

107

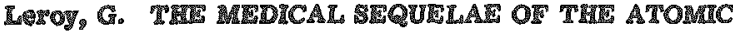
BOMTE EXELOSTON. J. Am. Med, Assoc. 134, 1143-8 (1947).

This paner concerns the investigations by the Army and Navy medical misatons at Hiroahima and Nagasaki. The data was complled before July 1946 as part of a report gubmitted to the Surgeon Ceneral of the U. S. Army. Two mator छubjects are considered: (1) the tremendous toll of casmaltieg and (2) the syndrome of imjury due to large amounis of garnma radiation. Iffects of the blasts on bone marrow, blood cells, Iymphatic organs, epilation, gastrointegtinal disturbances, skin wounds and burns, the gpleen, genisal tisøu, mucous membranes, and mortality are discussed in relation to ages, clothing, positions whinin buildIngs, time of maximum effect $g_{9}$ distance Irom the explogion, attempted therapy, etc. $\mathbf{A}$ kote by $\mathrm{Dr}$. Verze $\mathbb{R}$. Mason at the end of the paper amphasized that the study is not com- plete, but that he expects to find correspondence between developmant of leukopenia in the patient, with the known life span of the white blood cell and between the time of death from aplastic anemia with the known life of the red blood cells. (E. G.) 1074

LEROY, GEORg V. REMATOLOGY OR ATOMC BOMB CASUALTIES. Arch. Internal Med. 86, 691-710(1950).

Data from the detailed hematological studies made on Japanese injured by the atomic-bomb explosions over Hiroshima and Nagasaki indicate that it is possible to describe the syndrome of radiation injury in man in terms of typical clinical symptoms and a typical blood picture. The time Irend of the syndrome is important, and the characteristic elements in the diagnosis become apparent at various times after exposure. In general, the severity of the symptoms paralleled the severity of the leukopenia. Likewise, the important symptoms became evident at about the time the lowest leukocyte count was observed. The changes in the thrombocytes paralleled those in the leukocytes. The onset and severity of a hemorxhagic tendency followed closely the variations in the thrombocyte count. The alteration in the red blood cells was less consistent and less useful in diagnosis or prognosis than was the time trend of the leukopenia. From the atandpoint of prognosis, the Japanese physicians stated that patients with less than 500 leukocytes per $\mathrm{mm}^{3}$ were least likely to recover. The data presented here neither support nor deny this clinical observation. In the event of an emergency, it would be perfectly justifiable to perform only leukocyte counts and determinations of hemoglobin, hematocrit, and protein by the copper sulfate method. This would be an economical procedure and would provide the type of information required to evaluate cases and to aid in control of therapy. (NSA) 1075

Leucutia, T. RADIANT ENERG INJURIRS. Am. J.

Toentgenol. Radium Therapy 59, 890-3(1048).

In this editorial some of the medical aspects of the atomic bombing of Hiroshima and Nagasaki are considered. Most of the biological damage came from the heat and the rodiations, above all from the gamma rays. The author reviews the various types of injuries sustained in the five zones arbittrarily determined by the distance of various sections from the bomb target. The effects of wome of the injuries as they appeared two years after the bombing are mentioned briefly. (NSA)

1076

Liebow, Averill A., Shields Warren, and Elbert DeCoursey. PATHOLOGI OF ATOMC BOMB CASUALTIES. An. J. Pathol. 25, 853-1028(1949).

A description is preasented of the effects of the atomic explosione at Nagasaki and Hiroshima: the human injuries were mechanical, thermal, and ionizing in nature. Direct blast injuries analogous to those inflicted by high explosives were almost unlnown among the survivors; however, damage produced by shattered glass and the like accounted for a high toll of injury. The burns among the survivors were mostly of the lash type, the result of an extremely large quantity of radiant heat acting for an extremely short period of time, and were chaxacterized by sharply outlined areas; the effects of exposure extended up to 4,000 yards from the bomb. Depigmentation occurred in an area close to the bomb, but intense pigmentation occurred at larger distances from the explosion; the depigmentation occurred without deseruction of the aquamous epithelial gkin surface, and was probably the result of the action of certain specific wavelengths. The radiation effects consisted of exrly gymptoms of nausea and vomiting followed by diarrhea and fever, and developing into complications such as aplastic anemia, 
leukopenia, and secondary infections such as pneumonitis or ulcerative enteritis. At necropsy, changes were found in the shin, gastrointestinal traet, gonads, and hematopoietic tissues. The specific radiation effects on these areas are demeribed in detail. The remarkable resistance of the rethculum cell and the tendency of the bone marrow ultimately to regenerate are emphasized, and it is hoped that thege phenomena indicate that successiful defense measures against the radiationg will be devised. Further studies are still in progress to investigate the relationship between life span and irradiation, the incidence of neoplasia among gurvivors, and the radiation effects on growth, sterility, and genetic mutation. (NSA)

\section{7}

Nakaidzumi, Masanori. THE RADIOACTMTTY OF' THE ATOMC BOMB FROM THE MEDICAL PONT OI VIEW. Tokyo Imperial Univ. [nd] 10p. (NP-302)

The radiations which hit the human body were the following: neutrons and gamma rays in the air (including the primary neutrons and gamma rays from atomic bomb and gamma rays produced by the reaction between neutrons and ntrogen nuelei in the air), the radioactivities of the tissues of the human body and the soil resulting Irom the bombardment of neutrons ( $\beta$ and $\gamma$ rays), and the fission fragments which fell on the ground ( $\beta$ and $\gamma$ rays), etc. These all acted together, and the significance of each is complicated. Moreover, the scattering of $\gamma$ rays by the buildings difiered very much according to the type of building and its location. So the total dose received by each pergon may be different even when at the same distance from the esplosion center or in the same building. The radiation intensity does not always decrease according to the inverse aquare law. Therefore, the factors affecting the total dose of radiation on the human body are very complicated and not rasily calculated. In the estimation of total dose by clinical symptoms, epilation is a rather accurate indication. However, the effect of protection by shelter, etc., should be considered and its effect on the appearance of symptoms should be decided statistically. Irom the incidence of epllation the dose which was given to the human body can be estimated. Assuming the head epilation dose to have been $1,000 x$, with $2 \mathrm{~km}$, where $90 \%$ of all patients with epilation were, it is estimated that the dose directly beneath the explosion center was from 10,000 to 15,000 r. This also assumes ezplosion center to be 550 meterg above the ground and the radiant rays to decay by the inverse equare law. Protection from the radiant rays of the atomic bomb afforded by caves, concrete walls, and building structures is discussed. (ACD) 1078

Novy, rederick George, Jr. SURVIVORS OF BOMBNG OF HMROSHIMA TRRE YREARS LATER. Arch. Dermitol. and Syphilol. 61, 379-83(1950).

The results of a survey on the skin condition of gurvivors of the atomic explosion at Hiroshima three year after axposure are presented. Careful examination was made for any lonizing radiation effect such as telangectasla, atrophy, or hyperpigmentation of the skin, but in none of the patients could any of the burms be interpreted as due to radiation injury. It is concluded that there is a remote possibility that one or two instances of chronic cutaneous radiation injury may have occurred at Hiroshima, but it is not probable. (NSA)

\section{9}

Oughterson, Ashley W., George V. LeRoy, Averill A. Liebow, Ee. Cuyler Kammond, Menry L. Barnet, Jack D. Rosenbaum and B. Aubrey Schneider. MBDICAL EFFECTS OF ATOMC BOMBS; THE REPORT OF THE JOINT COMMISSION FOR THE INVRSTIGA TION OF
THE EFECTS OF THE ATOMC BOMB IN JAPAN; Volume 1. Army Inst. of Pathology. Apr. 19, 1951. 246p. (NP-3036; AM-2837\%(Vol. I))

The firgt of five unclassified volumes (NP-3036 through NP-3040) first. reproduces the original orders establishing the Joint Commission, list the personnel, and gives original data on the mean and differential leukocyte counts of resident: of Nagasaki not directly exposed to the atomic explosion but perhaps exposed to residual radioactivity. Copiougly illustrated descriptions of the terrain, buldimgs, populace, and of the raids of Aug. 6 and 9,1945 , and their immediate effects on Hiroshima and Naganaki are followed by eyewitness accounts of the atomic bomb explosions and of their consequences. 64 figures. 2 maps. (NSA)

1080

Oughterson, Ashley W., George V. LeRoy, Averill A. Llebow, E. Cuyler Hammond, Henry L. Barnet, Jack D. Rosenbaun, and B. Aubrey Schneider. MEDICAL

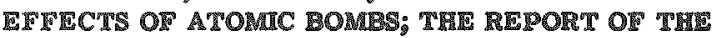
JOINT COMMSSION FOR THE INVESTICATION OF TPY

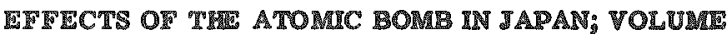
II. Anmy Inst. of pathology. [nd] 345p. (NE-303\%; AM-2837(vol. II))

Clinical records and specimens taken by Japanese physicians following the raids on Hiroshima and Nagasaki and Iater collected by the Joint Commission are reviewed, and résumés of original Japanees reports are given. Clinical observations on the etiologic iactors and conseguences of injury by blast, heat, and ionizing radiation are reported in detail. Photographs of damage to gtructures, "ghadow" effects, and selective destruction of elothing, etc., by heat are shown in addition to those of clinical interest. 129 ilgures. (NSA)

1081

Oughterson, Ashley W., George V. LeRoy, Averill A. Liebow, E. Cuyler Iammond, Henry L. Bamet, Jack D. Rosenbaum, Aubrey sechnedder. MEDCAL ERTECT OF ATOMC BOMES; THE RTPORT OF TME JOINT COMMISSION FOR TYRE INVESTICATHON OF THE EFIECTS OF THL ATOMC ROMB IN JAPAN: VOLUME III. Army Inst. of Rathology. [nd] 212p. (NP-3038; AM-2837(vol. III))

The studies made by Japanese sources and by the Joint Commission on the changes in peripheral blood, bloodclotting mechanimm, and bone marrow of patienty with zadiation injury resulting trom the atomic bomb explowions over Hroshima and Nagasalk are regorted in dotal. (NSA)

1082

Oughterson, Ashley W., George V. LeRoy, Averill A. Lewbow, R. Cuyler Mammond, Henry L. Barnett, Jack D. Rosenbaun, and B. Aubrey Schneider. MDDICAL EISTCTS OF ATOMC BOMBS; THE REPORT OF THE JONN COMMISSION FOR THE INVESTIGATION OF THE RFPECTS OF TR: ATOML BOMB IN JARAN; TOLUME IV. Army Lnst. of Pathology. [nd] 324p. (NP-3030; AM-2837(vol. IV))

The present volume ia devoted to the pathology observed in material obtained at autopey of victims of the atomic bomb explogton over Hiroshima and Nagawaht. Particular attention is paid to the effects of ionizing radiation. Syatematic organ-by $m$ organ degerigtions are given, whth a statistical account of the material available and of the changes observed. 193 igrures. (NSA)

1083

Oughterson, Ashley W., George V. Lertoy, Averll A. Llebow, E. Cuyler Hammond, Henry L. Barmett, Jack D. Rosenbaum, and B. Aubrey Schneíder. MDDCAL FECT OF ATOMC DOMBS; THE REPORT OF TRE 
JONT COMMISSION FOR THE INVIESTGATION OF THE EFECTS OF THE ATOMC BOMB IN JAPAN; VOLUME V. Army Inst. of Pathology。 [nd] 343p. (NP-3040; AM-2B37(vol. V))

A detailed statistical analysis is presented of the medical history of 6882 individuals in Miroshima and 6621 in Nagasaki known to be alive 20 days after the atomic bombings. Burns, mechanical injuries, blast effects, radiation symptoms, hematology, and deaths are related to distance, shielding, age, aex, etc. (NSA)

\section{4}

Pearse, Herman E. and J. Thomas Payne. MECMANICAL AND TEREMAL INJURY FROM THE ATOMIC BOMB.

New Ingl. J. Med. 241, 647-53(1849).

A description is given of the incidence and varieties of traumatic and the rmal injury from the atomic bomb and the results of the clinical production of llash burns. The components of the atomic bomb flash were: a temperature of over $4,000^{\circ} \mathrm{C}$; transmission largely by radiation; a spectrum high in ultraviolet, infrared and in visible light and brillancy; and, duration of less than a second. Shadow burnm ing, the production of kelolds, contracture and ulceration are described and correlated with the distance from the radiation source, the degree of protection afforded by clothing and other ghelter, and the incidence of radiation sickness and traumatic Injuries. The flash produced by magresium was used to produce a flash burn similar in characteximtics to the atowic radiation burng; extremely high intensity of radiation, short duration $(0.1 \mathrm{sec})$ and other criteria were antimied. It was noted that clinically there was an abrupt and diagrammatic demarcation between the burned and normal sisin; the normal, basophilic cells changed on a straight line into acidophilic, burned cellg. In the dermis the demareation was at the burn border in the crypts and hair follicles. The method of healing of this type of burn was unusual; the burn consisted of a coagulative, fired bype of necrosis in the epidermis and dermi, with eschar Lormation and subsequent sequestration, rather than the oryngizat on sen in the noncoagulative, necrotic tissue of the moderate temperature burn. With a ilash burn of mod-rate aeverity the epithelium grew out from normal borders and has Lollicles and the healing was rapid; with widegpread radiation injury and destruction of the epithelium in the crypte and halr follicles, delayed repaix due to the lack of epithelial islands occurred. Prevention of infection and adeduate welcer and diet are gugegested as methods of treatling theBe burng. (NSA)

\section{5}

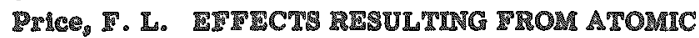
BOM EXPLOSION. J. IHORId Med. Assoc. 34, 651-3 (1048).

The atomic bomb explosions In HIroshima and Nagrakik ereated alast wave that lasted almost a mecond in contrast to the rev millideconds of pressure resulting from an ordimaxy explosion. No estimate of the number of deaths or symatoms caused by blagt pressure conld be made, but the preasure developed on the ground under the explogion was sot sufflcient to kIll more than those people who were very near the center of damage. In HHI Magragl $95 \%$ of Immediate death were cauged by burng, and $20 \%$ of the burns anlered by patients who reached first ald atations were gecond degree. The radiations which cansed infuries were primarly those created within the S13 st recond ater the explosion; and it is estimated that gamum rags and neutrong caused about $7 \%$ of the deaths. The Lrgadaton elferts of nausea and vomithg, Lever, diarshea, puspura, ulcerations of the mucous membrane, and Ieukopenis varied In intensty accordng to the aeverity of irgadiation, Death followed within 10 days of bevere in- jury, and mortality was $50 \%$ for those receiving moderate injury. There was almost complete disappearance of all cells of the myelopoietic and erythropoietic series. Men who had received enough radiation to produce symptoms did not have spermatozoa and most of the spermatogenic tissue was replaced by prolife rating cells of sertoli. The female reproductive system was affected to a much smaller extent. In Nagasali the estimated radiation intensity at a distance of 1,250 meters from the point of explosion was $473 x$. It is believed that the mortality rate for all types of injuries could have been reduced if electrolite solutions, plasma, whole blood, and penicillin had been avallable. (NSA) 1086

Sassa, Ranshi. CLINIC OF THE ATOMIC BOMB PADIATION SICINESS. Tokyo Imperial Univ. [nd] $27 \mathrm{p}$. (NP-303)

This is a summarized report on the chinical observations of radiation sicliness caused by the atomic bomb explostons in Hitroshima and Nagasalki. Over 10,000 patients were examined. Mechanically caused or heat injuries such as wounds and burns are not included. The etiological factors considered are age, sex, distance from center of explosion, and shelter. General symptoms such as fever and fatigue are described. Observations on the digestive, respiratory, and sex organs, the nervous system, the oral cavity, eyes, skin, blood and urine of victims of radiation sickness are discussed. (NSA)

$108 \%$

Schlaegel, T. G., Ix. OCULAR MISTOPATHOLOCY OF SOME NACASART ATOMIC DOMB CASUALTIES. Am. J. Ophthalmol. 30, 127-35(1947).

Sections of eyes of patients who had died from the effects of the Nagasali $A$-bomb irradiation one month after the bombing were studied. Changes seen in the cornea and lens are apparently due to the direct effects of radiation, while the findings in the rest of the eye appear to be zecondary to the systemic condition. The cornea and lens may have been damaged by direct irradiation. Some of the corneas are found to be denuded with evidence of epithelial regeneration. That lenses may be in the early stages of developing radiational cataracts is evinced by several vacuoles in the superficial cortex and a thickening of the postertor capsule. The following changes were due apparently to the patient' $\mathrm{s}$ systemic condition: (1) serous exudation into and from the cillary body; (2) invasion of the eye by bacilli; (3) Beptic chorolditis; (4) nodular cellular infiltration of the retina: (5) fibrin nets on the surface of the retina; (6) and, in one case, atriking distention of all the ocular vessels by white cells, most of which were mononuclears. In general, the damage to the eyes was less than that to other orgass. Conjunctivitis and superficial keratitis were found in many patients, but the esfects disappeared in about a month. Epigcleritis in conjunchion with corneal infiltration was found in gome cases. (S.V.S.) 1088

Takrahara, T., Akikro Mor', and Shigeko Natsumoto. CON* CERNING OTORIINOLARTISGOLOGICAL OBSERVATIONS DLAGNOSED AT THE TME OF THE EXPLOSION OT THIS ATOMIC BOMBS. Osalsa Medical College for Women, Japanese. [nd] 11p. (NP-313)

Research worlt with 83 patients had been reported about one month after the explosion of the atomic bombs. Principally from the view point of otorhinolaryngologlcal investigations, data have been presented regarding the symptoms and the time of their appearance. Observations were made on peripheral blood cells as well as nueleated blood cells in the marrow of the bones, the digtance from the explos ton center in the bombed area, ages, Bex, and injuries by burning, bleeding, falling -ofl of hatr, diarshoea, and other symptoms in general. (ACD) 
1088

Timmes, Joseph J. RADLATION SICINESS IN NACASAK1. PRELIMINARY REPORT. U. S. Naval Med. BuII. A6, 210-24(1246). 3040 .

This report has been superseded by reports NP-3036-

\section{0}

Warren, S. THE PATHOLOGIC EFIRCTS OF AN INSTANTANEOUS DOSE OF RADIATION. Cancer Rem search 6, 449-53(1948).

The distinctive feature of the atomic bomb is the large amount of radiant energy that it produces. While this energy cover a wide range of the electromagnetic spectrum, its chief physiologic and pathologic effects may be divided into two groups: first, the effects of heat, producing primary thermal injury of the flash burn type, and gecondary thermal injuries due to induced fires; 2 nd, the effects of short-wave radiation and neut rons, paralleling closely the effect famultar to us Irom the experimental madies of the biologic effects of $x$-rays. The immediate effects from radiation injur y were marifested as weakruess, malaise, lever and often death, and appeared usually within 40 hours. These presumably present a syrdrome of radiation sickness carried to an extreme degree. The delayed effects from radiation injury consist largely of damage to the hematopoietic tissue with resulting leusopend, granulocytopenta, and anemia. (BA)

1001

Warren, Shields. NAGASAKI SURVIVORS AS BETEN IN 194\%. Miltary Surgeon 102, 98(1848).

The Atomic Cagualty Commigaton is actively cooperating with the Japanese in a long range study of the surviving population of Hiroshima and Nagasalk. The explosions at Hiroghima and at Nagasaki were ald burgt go that little of the ifssion products remained at the site. Instead, they were widely disseminated through the stratosphere. Consecuentiy, all of the bombed area is anfe for habitation and for cultivation. The sear's of flash burns are relatively ifrequent among the survivors. Special attention is being given to laeloid formation in these scars. The ineidence of the keloids appears to be somewhat greater among Japanese than whites, but there is no evidence that here ls any gecific relationship between the occurrence of kelold in scar tissue and exposure to the explosion os an atomic bomb. Diaturbances of the hemopoietic lissue were evident in 1945, but by 1947 most of the survivors had returned approxinately to normal. Up to the present time three cases of leuknma, one prohable case of leukemia, and one of multiple myeloma have been reported among the survivorg. An abnormal prevalance of monocytes and reticular cells suggests that the marrow in Bome may not be entirely normal. No tumors of gonads have been reported among the gurvivors. It will probably be 25 years betore reliable dala can be oltamed on changes in genes. (NSA)

\section{2}

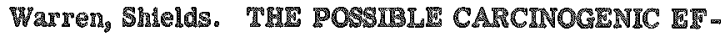
FECTS OF THE ATOMC BOMB AT MIROSEMMA AND NAGASAKI. Acta Unio Intern. contra Cancrum $674-7$ (1840).

The explosion of the atomie bombs in Japan produced injury by alr blast, thermal radiation, and lonizing radiation. Alr blast, both its primary and secondary effects, produced much traums, with extensive scarging and some kelold for mation resulting as the injurieg to the gkin healed. Thermal radiation produced burng of varying meverity which almo healed with extensive scarring and kelold formation; no tumors have yet developed in these scars. If is stated that it is probable that some will ultimately appear, but not to a greater extent than in burn scars from other sources. It is claimed that ionizing radiation in the amount received by survivors may be expected to produce some cases of leukemia and, possibly some ovarian tumors. As yet no significant increase in incidence of leukenia has occursed. (NSA)

1003

Warnen, 5. and R. JURIES PRODUCED BY THE ATOMC BOMBS AT MEROSHIMA AND NAGASATI. U. B. Naval Med. Bull. 슨, $1343-53(1946)$.

Although injuries following the two explosione of atomic bombo in Japan ranged from instant death to delayed sickness, a clear pattern is discernible. To Draeger's classidication of blast injury into that due to air blast, water blast and solid blast the authors now add the term "radiatlon blast injury." In the two Japanese cities water and solid blast inJuries were absent, the casualties being the result of alr and radiation blast. The unique feature here was the liberation of ioniaing $\gamma$-raye and neutrons, with the result that, in addition to injuries from thermal burns, there was also observed the pathological changes which follow over aexpogure to $\mathrm{x}$-rays. In those exposed only to the ionizing rays there was at first no visible effect, but subjective malaise and nausea appeared early. Lymphocytopenta developed in a few hour's in those heavily exposed, and death occurred in a few days. In those who lived longer there was evidence of injury to gonadal tissue and of bone marrow change, lewkopenia, thrombocytopenia with hemorrhage, and anemia appearing in that order. Proof of induced radioactivity and sealdual ra. dioactivity was not encountered. (BA)

\section{Syndrome in Animals}

I094

Bennet, I. R., D. E. Relers, and J. W. Mowland. THE INELUENCE OF INIECTION ON TIYE MERATOLOCICAC EIIECTS AND MORTALIVY FOLLOWING MDOLETIRA X-RADLTION. Radiology 5\%, 99-103(1051). (UR -140)

The caredul andlygiø of blood cultures taken on 55 doge following whole-body 8 ir radiation had demongtrated the relative frequency of blood cultures of all types of organimms. Infection appears to be definitely related to mortality in tho:e animals surwiving the firgt 2 weels of irradiation. This relation is less apparent in animals dying at an earller tume. No direct correlation between the detimite leukopenia of dying animals and infection can be made. The pregence of gram-negative organians within the circulating blood appeares to be definitely related to subsequent death of these animals. As a result of such obaervations preliminary as perimentation has been carried out to determine the effect on morbidity and mortality of dogs and rats following vart ous antibacterial agents. Aureomycin, gtreptomyein, gulia drugs, penfillin, and terramyein have been studied extensively. Results will be reported. (NSA)

\section{5}

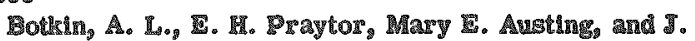

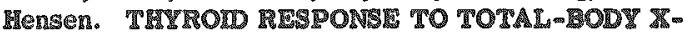
IRRADIATON. Army MLedical Research Laba, Fort Irao: Sept. 29, 1951. 9p. (AMRL-66)

Changes in thyroid and serum I'st content (inorgantc ard organic) of rat, after total-body $\mathrm{x}$ irgadiation at $1000 \mathrm{x}$, indicate a stimulation of thyrold activity by 2 hrofter $1 \mathrm{Ir}^{-\infty}$ gadiation. This increased activity is apparent until one day after Irradiation, from which time until the susth day ther is a jrogressive deerease in activity. These changes in functional activity of the thyroid are probably due to Bys- 
temic damage caused by the radiation and are mediated through the hypophysis. The initial increased thyrotropin teleage from the hypophysis is followed by a shift of pituitary function toward increased adranoconicot sopin alabo1096

Bowers, John 2. and Keneth G. Scot. TIIE MOVEMLIT

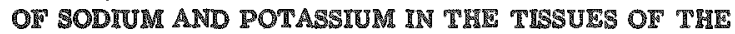
RAT FOLLOWING ACUTE RADLATON TNVURY USING NAME AND 120A3 AS TRACERE. Radiation Lab., Univ. of Calte. Jan. 1951. 20p. (JCEL-1101)

The purpose of the experiments mas to detemine alterations in $\mathbb{N a}$ and $K$ content of various organs and tissues, rnd to check Na and $K$ deiecalon and excetion at various time intervals after total-body i pradiction. Two uc of $\mathrm{N}^{22}$

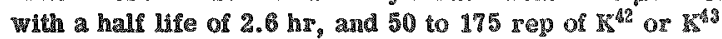
were injected subcutaneously. Radiations of $\mathrm{L}_{50}$ and $\mathrm{L}_{\mathrm{S}} \mathrm{D}_{100}$ were used. Acute radiation injury if chanecriged by heavy and early losses of If from aensitive tiasues (lymph nodes,

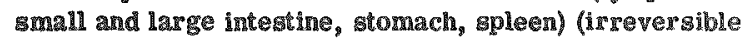
1njuy), and less pronounced losses rom other tissues gonads, k10ney, heart, lung, liver, brain, skin, skelcton, muscle, yes) ( hese is an ealy loss of Na, but gubsequenty a penetration of $\mathrm{Na}$ into damaged colls, as indicated by an ampansion of Na space. These are no characteristic or significant changes in the $\mathrm{Mr}_{\mathrm{g}} \mathrm{O}$ contert of the various organg demonst these experiments. $(6$ tables, 2 charts) (NSA)

\section{7}

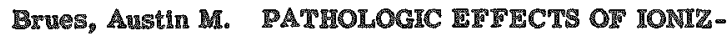

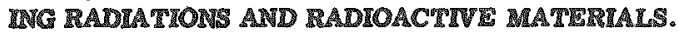

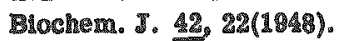

Certain purely chemical êtects of honizing radiations are understood. These include the dissociation of water into $\mathrm{I}$ and OF Iadical and the inhibition of certain enzymes such ar those contalning sulhydryl goups. In addition to these prosinate eficcts, various forms of genetic and cell damage have been extengively wtuded. The relation of these changes to most clinical radiation effects remains largely to be estalolshed. In acute lethal zadiation exposure in mammals, an immediate respona is seen correguonding to clinical $\mathrm{x}$ xay alcknes. Sucentible andmals w11 show hypontersion, vorithing, or entherra of the whin. By the recond day, acute symptoms have largely abated, bat there is a mared decresse in cisculatmg whte calls whin continues unth death. During the enmuing was, andmals geruse food and water and

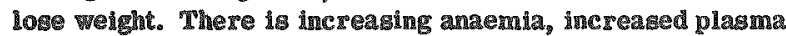
and cracelular volume, accelerated sedimentation rat.,

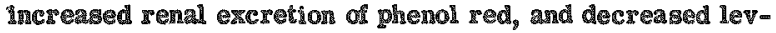
elof blood non-proten nitrogen, area and polypeptdes. IIntologic damage is most marked in the blood ongar, gonads, and intesthal tract. During the second reek, the clothng time is Freaty $^{2}$ increased the to an overabundance of circulating heparin; fever and subcutaneou octernes apyear and death occurs, atended by loss of blood, bleeding into vital aroas, such as the hoar and central ner-

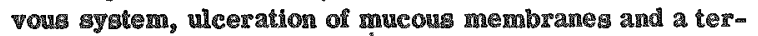

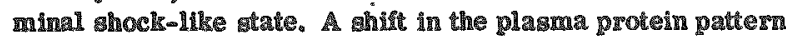
(\{ncreaged beta and grama globuHM) has been gesn in dogs,

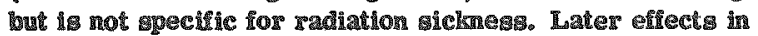

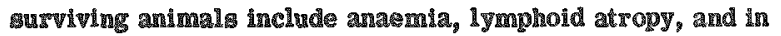
mice, an enhanced tendency to lymphoma. Chronic eifects linclude decrease in life span, greying of hair and an in-

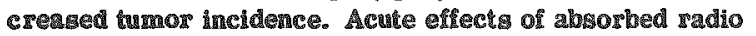
clements generaly resemale those of total body radiation. Ther Iate dif

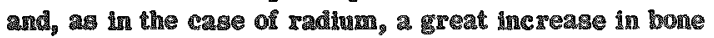
tumor 18 sem after abmorytion of radiostrontium, as well

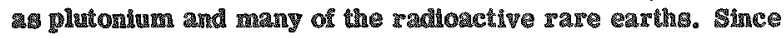

$\mathrm{C}^{1 /}$ has been ghown to deposit in bone (Bloom, Curtis, and Melean, 1947), the question is raised whether it may present a human hazard analogous to radium. An wttempt has been made to consider the human tolerance on the basis of known facts and it is presently thought that a fotal human absorption in excess of 30 microcuries mut be conzidered hazardous unitil further knowledge is gained regarding the retention of carbon administered in various forms. In the case of a hypothetical carbon compound, such as a steroid, which mifht remain localized in a vey small organ, even this figure may need revision. (NSA)

1098

Brues, Austn M. ed. DVISION OF BHOLOGICAL AND MEDICAL RESTARCH; QUARTERLY PH PORT, MAY, FUNE, JULY, 1951. Argonne Nathonal Lab. [nd] $112 \mathrm{p}$. (ANI-A676)

Data ase giver on blood cell count of guinea pigs having received bone-rinarow injections before or after lethal s-ray doses. The prophylactic effect of bone-marow injections against radiation injuries varies with the strain of mice. In 30 aday LOF comparisons of radon and $x$-radiation effects, the $\mathrm{x}$-ray $\mathrm{LD}_{60}$ was 564 with a standard deviation of 23 I $52,400 \mathrm{exgs} / \mathrm{g}$, the radon golution was $0.1415 \pm$ $0.001 \mathrm{mc} / \mathrm{g}$ or $36,800 \mathrm{ergs} / \mathrm{g}$. The nergy absorbed from the z radiation was 1.42 times that of the particle energy. The biologic effects observed in mice injected intravenously with radon are caused $26 \%$ by and $14 \%$ by $\beta$ radiation. The higtological changes in the retina of new-born mice due to 1000 - $\mathrm{x}$ adiation are illustrated data are given on percent age of catarat formation and length of latent periods olapsing before these are visible. Intormation is given on the effects of spleen shidang during 8 radiation on blood cell counts, and effect of injection of embryonic suspensions, maghed placental hissues, and frowen splez implants on sensitivity to radiation. Spleen shelding of rabibs during itradiation with lethal $x-r^{*}$ y doses preserved the capacity of forming antibodies, wen though the spleen was removed

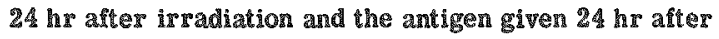
aplenectomy. Data on the effect of marrow sugpensions 1rom nommal and estrogen-treated mice and of $\mathrm{p}-\mathrm{ch}$ - $10 \mathrm{r}$

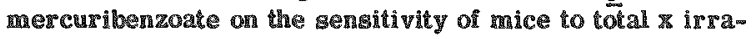
dation are given. Supernatant plamas of blood periraed gpleen of dogs were protective when injected in $1-\mathrm{ml}$ dosage into mice immediately before giving the mice 800 i total-body $x$ irmadiation. The effects of massive doses of cortisone on mortalisy in the initial period following $\mathrm{x}$ lreadiation of young cheks were mmall. In newly hatched chickn, the ahidding of different mal areas of the body duxing 1000 I $x$ irradiation resulted in minilas decreases in mortalsty, indicating that mome lesion were effect of direct radiation. Renal fallure was one of nymerous factors conm syibuting to the deaths. Preparation of $\mathrm{C}^{14}$-laboled dextran by biosynthesis is described. Treffects of dethanolamine alt of maleic hydrazide on kiler clones of Paramecium are reported. Studies with sezm imhibition of respiration of the cells when sposed to large doseg of sadiation, the dose degending on the degres to

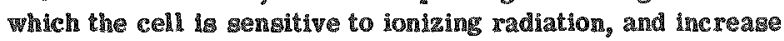

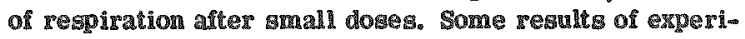
ments of lonizstion in wir from $\beta$-ray point gource atre

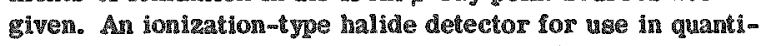
tative measuring has been developed. (NSA) 1090

Brues, AuEt?

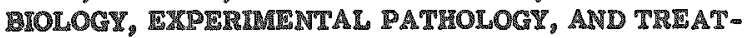

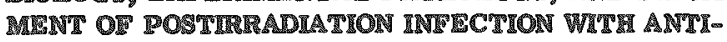

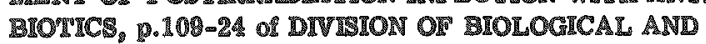

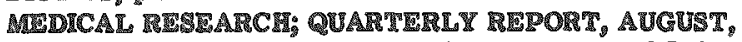

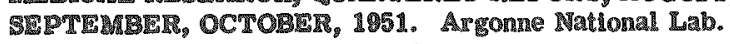

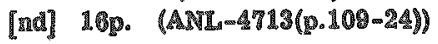


Preliminary data from a study of the pharmacodynamic of compounds derived from ribonucleic acid and the effects of the se compounds upon physiological changes following total-body irradiation showed that the blood pressure of rats is appreciably decreased by small amounts of adenosine and 3-adenylic acid, while other nucleic acid derivatives have much smaller effects. An approsimate formula for the standard error of an absolute white cell count is used to show the dependence of the standard error on the blood count paraneters and sample sizes and to develop formulas for minimum error at fired cost as well as for minimum cost at fired relative error. Data on the survival and ineldence of malignant bone tumors in mice that had received less than $22 \mu \mathrm{c}$ per $\mathrm{kg}$ of $U$ from 210 to 415 days previously, and experimental degign and survival figures at 221 days of mice which had received injections of $\mathrm{Ra}^{228}$ and $\mathrm{Pu}^{238}$ are presented in tabular form. Biometric analygis of data on the effect of various patterns of antibiotic therapy on the survival of mice following a fixed dose of $x$ rays showed streptomycin dosages ranging from about 3000 to 6000 units per day were protertive to the same degree. Penicillin plus atreptomye an appeared to be less protective than streptomycin alone. Aureomycin, chloramphenical, and terramycin, all supplemented with streptomycin, conferred 1100 about equal degrees of protection. (NSA)

Carter, Rovert nan. THE EFFECT OF ACUTE DOSES OF X-IRRADIATRON ON THE SDLENIC AND THYMIC VYRIGIST OF CF-1 FEMALE MICE. Los Alamos scientific Lab. Mar. \%, 1950. 8p. (LA-1075)

A high correlation is obtained between $x$-radiation received during a single exposure and loss of weight of the spleen and thymus of female mice. A 250-kv peak $x-\mathrm{ray}$ machine was used with a $3.9 \mathrm{~mm}$ Cu filter in addition to the inherent filtration of the tube (equivalent to approximately $2.00 \mathrm{rnm}$ of Al). The exposure rate was $14 \mathrm{r} / \mathrm{min}$. Curves of the log of the total dosage (in roentgens) and the per cent weight loss of the thymus and spleen are linear on a semilog graph. The use of splentc and thymic weight change as a means of biologically comparing various lonizing radiations is suggested. 15 references. (NSA)

1010

Chrom, Sv, A. STUDYYS ON THE EFIECT OF ROENT: GEN RAYS UPON THE INTESTINAL EPITHELIUM AND UPON THE RETCULO-ENDOTHELIAL CELLS OF THP LIVER AND STLEEN. Acta Radiol. 16, 641(1935).

Histological and bacteriological studies were made on white mice iryadiated with rays. Univergal radiation with 250-550 $r$ resulted in a general infection with intestinal baceria. Abdominal irradiation with the gpleen and liver protected did not produce the infection characteristic of irradiation of the entire abdomen. The effect of irradiation was evidently damage to the protective functions of the infestinal epithelimm and of the liver and spleen. (BA)

$110 \%$

Conard, Robert A. WETET OF X-IRRADLATION ON DNTESTINAL MOTHLITY OF THE RAT. AM. J. PhysLiol. 165, 375-85(1851).

The effect of 8 irradiation on inteatinal motility of the rat Was studied in vivo. Kymographic studies showed that $\mathrm{x}-\mathrm{ray}$ doses of $100 \mathrm{r}$ and above produced a consistent pattern of intestinal response characterized by a latent period of about $1 \mathrm{~min}$ followed by increased tone and motility, the degree and length of tone rise, in general, increasing with dose. Using doses of $300 \mathrm{x} \times \mathrm{x}$ xy, propulaive motility was found to be accelerated during the firgt hr with a tendency to a decrease below normal from the third hr through the third day and a return to normal after the third day. The most striking feature of both studies was the shortness of the latent period. A study of the influence of various drugs on the response indicated that the action of $\mathrm{x}$ irradiation resulted in a stimulation of the cholinergic nerves. The site of action was found to be largely localized in the intestine with little systemic component, and the chief site of action on the vagus appeared to be at the level of the enteric ganglia. (NSA)

1102

Coulter, M. P., F.W. Furth and I. W. Mowland. Trie INCDENCT OF BACTEREMA IN THE X-IRDLATED

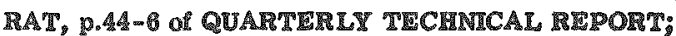
OCTOBRR 1, 1950 TERU DECRMBER 31, 1950. University of Rochester. Teb. 1, 1051. 3p. (UR-152(p.44.6))

A total of 294 rats received $700 \mathrm{r}$ of whole-body radiation and were sacrificed at intervals postradiation for heart blood culture studies. Blood culures were obtained on 55 normal rats for control studies. From 20 to $64 \%$ of the culturea from radiated rat were positive during the aecond week postradiation. The greatest mortality also oceurred during this period. The ormanisms recovered were those normally found in the gastrointestinal tract. (NSA)

1104

Cronkite, Eugrame P. THE CLINICAL MANIFESTATHONE OF ACUTR RADIATION ILLNESS PRODUCDD IN COATS BY EXPOSURE TO AN ATOMC BOMB, TIST ABLE, BLEIN, 1946, WIT COMMENTS ON THERAPY. NAVAI Medical Research Inst., Bethesda. Max. 19, 194. 42p. (NP-574; Renort No. 10)

The clinical manifegtations of acute lonzing radiation illness in the goat at which it progresses are functions of the amount of radiation received. The fully-develoged illness consist of apathy, anorexia, diarmea (orten bloody), purpura, epilation, marked leukopenia, and a high mortality. However, many Iimns may be absent wh overwhelming or gublethal radiation. The therapeutic use of pemicilln and trangingions auggest that these agentr may be of value. (NSA)

\section{5}

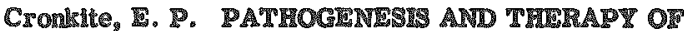
RADLATION ILLNESS. Med. Ann. Dist. Columbia 18, 230 셜 (19A9).

The radiation sickness produced by whole body radiation is discussed from a descriptive and therapeutic standpoint. Whe syndrome is initiated by protoplasmic injury, through the following mechaniams: (1) enzyme inhibition, particularly øulfhydryl enzymes; (2) some alterations in the osmotlc properties and the cell permeablity; (3) generalized protein denaturation and (4) an intoxication by histanninelike compound gupposedly liberated as a result of the radiatons. There is a reciprocal relatonghip between the degres and reveraibility of the ongyme imhibition, the prom tein denaturation, and the alterations in osmotic propertier and collular permeabilisy. As the dosage increases, the degree of revermibility decreases and the mortality riges. A list of varying degrees of susceptibility of different tisues to necrosis is included. Tomic symptoms develon immediately after or during the diffuse radiation. These consist of nausea, vomiting, dias rhea, malaise, etc. and they aubsids within $24-48$ hourg. A latent perhod 10llows in which leukopenia, thrombopenia, and a slowly progreasive leukemia develop. Fhysiopathologic manifegtations involving the adrena function, the adaption syndrome, the intorication by histamine-like substances, and bacterial invasion also take place. I inally, the panleukopenia and hemorshaglc gymptoms develop 4 - weeks after the radiation. Capillary fraglity and clotting defects also occur. Th is agseated that an adeguate method of geparating hopeless cases from the cagualier is exantal to conserve time, permonel, and therageutic 
agents. Among the gpecific the rapeutic methods which may be used are: stifhydryl agents to reverse cnzyme inhibition: anti-histamine compounds to neutralize towins; adrenal hor mones to replace losses; agents to maintain the water and electrolyte metabolimm; prophylaxis treatments of infections in the presence of a granulocytopenia; the prevention and therapy of anemia; the treatment of hemorrhagic tendencies; and means to prevent and remedy the marked loss of tissue and negative nit rogen balanee. The use of penicilin, aureomyein, chloromycetin, rutin, and protamine gulfate are also discussed. (NSA)

1106

Cronkite, Eugene P. THE CLINICAL MANIFESTATIONS OI ACUTS RADIATION ILLNESS IN GOATS. U.

Naval Med. Bull. 49, 108-215(1949).

The goats considered in this senort were exposed to ionlating radiations in amounts ranging from $100 \mathrm{r}$ to more than 10,000 $\mathrm{r}$ at Bilini. They were divided into lour groups based on the severity of their iliness and the depression of their leukoryte counts. In Froup 1, severest radiation illneas, the animals survived leas than sis days, mortality was 100\%, and sudder death occurred. An extremely marked depression of the leukocyte developed. There was no visible evidence of hernorrhage during life and no epilation. The minimals in group 2, gevere radiation illness, survived manfegtatlons with a varlable clotting defect occurred, eplation was prominent, and mortality was 100\%. In group 3. less severe radiation ilmess, survival was in excess of 44 days and two were alive $1 / 2$ years later. The aigns were imilar to group 2 but developed with less rapidity and severify. A moderate leukopenia was preaent, and epllation was minimal. Hemorrhagic phenomena were legs prominent and a clotting defect was not found. In group 4 , mild radis-

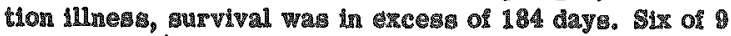
wore aive $1 / 2$ years atter irradiation. Signs were mild and of chort duration. Temorrhage, epilation, and rhinitio were absent. The clinical plcture in goats 1 g gimllar to that of man except for the absence of womithn and the more ravid progremalon of algns. Pentellin and whole blood transfuwon were used therapeutcally. For lack of comparativaly arpowed control animals delinite conclusions cannot be dมพణw about the use geat that they may be of value. (NAA)

1107

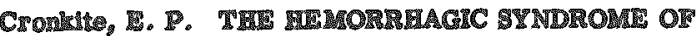

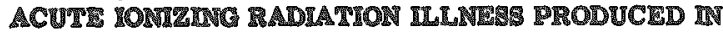

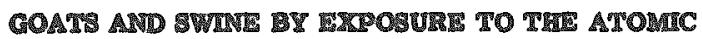
BOM AI BHCW, 1046. BLood 5 , 32-16(1050) (ND-1802)

This prolim July 1948 is concerned wh Ihasic symdrom encountered in goat and swine lollowing

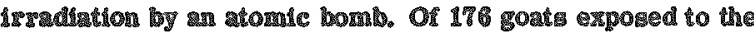
a.rial atomic mplosion, 31 developed detertable of ects of swdiaton: 14 of these whowed purpura, while lour exhibited a molonged sloting tme. A total of 20 swine were exposed to the undorwater atomic exploason; all animals were below decks and it is concludod that most of the Irradiation to which they were abjected was $y$ radiation at the sme of the oxplosion or $y$ emisgion from liabion product ghowered upon the ghip after the explomion; the greater part of thim 1radiation was recelved whthin two days. Red blood cell counts, prothrombin and whol, blood clotting times, hemom Globil deterrinastons, hematocrit readings, white and difSorental blood coll counts and clot retraction tmes vore taken on the wine and gorts before and at intervals after crpomure. The homorrhaglc asndrome is considered to bo predominantly a result of combination of "tnereaged vas cular Iragillity" and thrombopenia. Inisequently, a blood coagulation defect characterized by a prolonged clotting time due to a circulating antibody with heparinilse properties may oceur, but only in fatally irradiated animals. Evidence 13 presented suggesting that serum fibrinolysins may have been activated by the irradiation. It is concluded that a hemorrhagic syndrome can develop in irradiated dogs, goats, swine, rats, chickens, and guinea pigs without the appearance of a prolonged clotting time and without a detectible "heparinemia." The most common phenomena observed in the syndrome, it is stated, appear to be an increased vas108 cular fragility, thromopenia, and ulcerations. (NSA)

Dillard, G. H. L., G. Brecher, and E. P. Cronkite. SEPARATION, CONCENTRATION, AND TTRANSFUSION OF PLATLLTIS. Proc. SOc. Expt1. Biol. Med. 76, 796-8 (1951).

A simple method is pregented for the efficient separation and concentration of platelets from burrow, dog, and guinea pig blood in quantities muficient for transfugion. This method depends upon the use of Sequesterene $\mathrm{Na}_{2}$ as an anticoagulant, which prevents the development of platelet stickiness and elumping in shed blood. Platelets prepared by this method and transfused into the thrombopenic, irradiated dog remain in the circulation, reverse the congulation desect, and prevent hemorrhages. (auth)

\section{0}

Draeger, R. H. And S. Warren. MEDICHNE AT THE CROSSROADS-A REPORT BY THE MEDICAL CORPS OF THE NAVY ON THE STUDY ON ANTMALS USED IN THE BIRIN TRSTS. บ. \$. Naval Med. Bul1. A7, 219-25 (1947).

The biological experimenta conducted during Operations Crossroads are outlined and their reaults summarized. Twenty-two gcientists, 200 goats, 200 pigs, 5,000 rats, 120 mice, and 60 guinea pigs were trangported to Bikini on U..S.3. Burlegon, which aerved as the laboratory. In addition to the mammals, other biological material-insects, bacteria, geeds, sera, homones, etc. - and inorganic substances such as soll and drugs were included. Most of the animals were used in Test Able, where the atomic bomb was exploded above the ground. All were retrieved within two days. It was lound that the majority of early death were due to blast injury, radiation effects being a lesser cause of cesth at this time and llash burns being minimal becase of the furry coat of the animals. The animals which were expoged to appreciable amounts of radiation ceveloped the aame algns and gymptoms as were noted in the human survivors of Hiroshima and Nagasaki. The fir git sin was atropy of lymphoid tissus then appeared a hemorrhagic diathegis and gevere enteritis, often with necrosis of mucosa and submueon. Marked changes in the blood picture occur red and, some weels later, partial deplation. The sequence of the pathologic charges is being studied by meang of sections from the tissues of animals gacrificed at intervals after the exposure. Inomormation obtained by placing swatches of different cloths on fir plywood conflimed the impression gained in Japan that white material protects against Ilash burn better than dark cloth. After "Test Baker, in which the bomb was exploded under water, the test subject could not be recovered for geveral days because of the intense residual radioactivity resulting from the flooding of the target Gys with radioactive "sain. "The animals thus recelved not only a more intenge radiation but also a much longer exposure than those at Tegt Able. The gurvining animals and the inert materials are now boing studied at the Naval Medical Research Inatitute. (BA)

\section{0}

Fdelman, Abrahan and W. J. Eversole. CHANGES L ANTIDIURREIC ACTIVITY OR RAT SERUM AITTER 
X-IRRADIATION. Am. J. Physiol. 103, 709(1950)

(ADCU-1081)

The report is reproduced here in its entirety.

The experiments reported here concern an attempt to determine the physiology involved in the previously reported (Federation Proc. 8, 39(1949)) diuresis occurring within 24

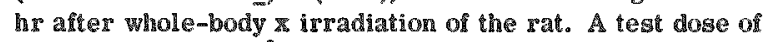
water, $4 \mathrm{ml} / 100 \mathrm{~cm}^{2}$ body surface, was given by stomach tube 30 min or $24 \mathrm{hr}$ after whole-body $x$ irradiation (160 kvp, $10 \mathrm{ma}, 0.2 \mathrm{~mm}$ Cu). Control animals were similarly treated except they were not irradiated. Exposed animals excreted the administered water at a more rapid rate lhan the controls. Serum taken from irradiated rats at various times after exgosure was fested for antiduretic activity by the method of Birnie et al. (Proc. Soc. Exptl. Biol. Med. 70 . 83(1949)). Significant decreaseg in antiduretic activity were found at 12 and $24 \mathrm{hr}$ after irradiation. "These results indicate that a decrease in amoun of circulating antidiuretic substance or substances is actor involved in the increased

111 urine flow after irradiation. (NSA)

Evans, T. C., Grace Clarke and Estelle sobel. INCREASE IN I ${ }^{131}$ UPTAKE OR THY ROID ATTER WHOLT BOOX

ROFNTGEN IRRADIATON. Anat. Fecord 99, 57\%(194\%).

Adult male rats were exposed to $\mathrm{x}$-rays $(500 \mathrm{r}$ and 1000 r) and 2 days later a tracer dose of radioactive lodine was given mubcutaneously. Twenty-four hours after IIsI administration, thyroids were removed and Ielative amount of radioactivity determined. The activity in the thyroids of the irradiated animal wa wach higher than that of the con trols. Animals were tegted at diferent times after irsadiation and the maximum increase in activity (relative to that of the controls) occurred during the third day after the 15 radiation. At this time the leukocyle count was very low. Increased activity of the thyroid in the irradiated animals occurred even when the thyroid itself was not irradiated. In fact, the largest relative activity of the thyroid occurred in an animal receiving irradiation over the abdomen (head and thoras protected with lead). The IsI content of the blood of the irradiated animals was usualy higher than that of the controls. In one experiment, the thyroid of adrenalectomized rats did not retain as much radio-iodine as did those with the gland. Irradiated animals without adrenals ghowed an increase in I $^{131}$ over their controls (without adrenals) in about the ame proportion as if adrenal were present. New born ratis (3 days of age) did not regpond in the game way as did the adults. Irradiation of the babes reaulted in a partial depression of the I'si ugtake of the thyrold. (NSA)

1112

Trance, OWen. CHANGES IN THE WATER CONTERT AND DISTRIBUTION IN RAFS AND MICE AETER CHRONL AND ACUTE TOTAL-BODY X-IRRADIATON. Univ. of Chicago Metallurgical Lab. June 1046. $34 \mathrm{p}$. (ALCU-131; CR-3889)

In order to determine gomething of the nature of the changes in mater metabolism that occur after total-body Irxadiaton, gtudies were made of the relative water contents of muscle, plasma, and the entire carcass of rats and mice. Water digtribution, as measured by the volume of distribution of injected thiocyanate lon and of intravenoualyadministered biue dye, T1824, was used as an estimate of changes in the extracellular and vagcular compartments, respectively. A transtent increase in thiocyanate space occurred in rats at an $\mathrm{x}$-ray dosage that was about $15 \%$ of the LD-50. A similar increase was observed in the total-body water of mice that was about $30 \%$ of the LD-50. No changes occurred in relative muscle or plasma water, or in plasma volume, until the lethal level of $\mathrm{x}$-irradiation were ap proached, at which time increases were observed in all five types of measurements. The increases occurred in at least some cases in the terminal period, and in the preaence of a negative water balance. Chronic ir radiation with $\mathrm{x}-\mathrm{rays}$ and with $\alpha$-rays from injected plutonium resulted in a state of relative hydration. The thiocyanate space, again, was the most sensilive indicator of change in water metabolism, although aome increases in plamma and muscle water did result from the higher dosagen. By means of gtudying the ef fects of pre- and post-irradiation water restriction on the survival time of rats and mice, it was determined that the dehydration realing from this procedure was agparenty helpful in that the irradiated animals so treated lived longer than the $\mathrm{X}$-1rradiated controls. (NSA) 1113

Waley, T. J., Maxgaret R. Andem, Richard $\mathbb{P} . \mathbb{R} 1$ ley, and Idamae Williams. THE PRESERCE AND IDENTITY OF VASOTROPIC SUBSTANCES IN THE BLOOD OF RATS SUBJECTED TO ACUTE WOLE BODY ROENTGEN RAT IRRADITION. UnW. of Calú, LOS Angeles. Oct. 18, 1051. 17p. (UCLA-181)

A. systematic Btudy has been made of the effect of $600 x$ of acute whole and regional $x$ irradiation on the perigheral vagcular gystem of the rat. The effects of guch radiation upon the formed elements of the blood, the intertinal tract, and other body tissues have been correlated with the published data concerning yadiation damage of these tigsues. Is has been demonstrated that peripheral vacular function is not impared by a direct action of the radiation on the precapillary mphineter and terminal arterioles bat is mediated through the release of a vasodepreasor material (VDM) by the liver. This material, which Is present in the circulation wuring the firgt post-irradiation week, has been shown to be the same as that which appears in the blood of animals in ir reversible shock. It fits all the criterla for ferritin. However, the sequence of hts appearance is diforent from that reported for physiological ghock ratea. A vasoexcitor material (VRM) has been found in the blood of irradiated animals during the second post-irradiation weer. This material Itts the criteria establined for the VIM found in the blood of animals in the reveraible phase of hock. (NSA)

1114

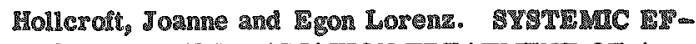
IFECTS IN THIS IRRADLATION TREATMEST OF A TRANSPLANTED MOUSE LYMPROSARCOMA, D. 17\% Of TNTERNATIONAL CONGRESS OF RADIOLOGY, Oth Congress, London. 1950.

The lymphosarcoma used in these experiments originated in an isradiated gtrain A mouse. When gubcutaneously or intramacularly transplanted this tumour grows at the site of inoculation and kill the animal in 30 to 40 days. Whole body irradiations were cargled out with roentgen radiation or intravenously injected radon in equilbrium w1 short-lived decay products. The rador dose in rep equalled the roentgen radiation dose in $x_{\text {. It }}$ (wos cound that 2 whols body irradiation with 400 z or rep of elther goentgen radistion or radon caused regression of only the tumor and a few permanent cures. Roentgen irradiation of the tumour or of the body alone with the amme dome of $400 \mathrm{r}$ had almogt no aifect on tumour regresion. Dores to the tumour alone of $1,300 \mathrm{r}$ had little effect while doses of $4,000 \mathrm{r}$ were approxlmately as effective as a whole body expocure of $400 \mathrm{~g}$. Sinular effects, however, could be obtancd by giving a tumour dose of 1,000 r or more collowed by a whole body exposure to $100 \mathrm{~g}$. This mubrequent whole body oxposure ta 
oxly effective if given within a few hours of the irradiation of the tumour. The experimental findings suggest that a systemic effect is involved in tumour regression induced by radiation.

115

Johnson, C. G., C. F. Vilter, and T. D. Spies. IRRADLATON SICRNESS IN RATS. Am. J. Roentgenol. Radium Therapy 56, 631-9(1048).

Groups of Spsague-Dawley albino rats fed either on a vitanin free diet or a nutritionally complete stoek diet, adjusted in quantity so that no appreciable weight changes occurred from day to day, received single x-may doses in the range $600-800$ roentgens (200 kv excilation, filtration $0.5 \mathrm{~mm}$ Cu plus $1 \mathrm{~mm}$ Al). These doses were chosen 80 that approximately half of the rats died. other groups, simLlaxly treated, recelved dally oral vitamin gupplements, or parenteral medication, commencing three and two days, reapectively, before irradiation and continuing until the end of the experiment. A third treatment was with three wubcutaneous doses of crude liver estract. The protective action of the fe treatments was judged by mortality, postirradiation survival time, weight changes, activity, food consuraption, and autopsy findings. Results are summam rized below:

Diet

Medication

Protective Value

Vitamin-Lifee

Crude liver extract, subcu taneously

Stock

Stock

Stock

Vhtamin-1

Vitamin-free

Vitamin $\mathbf{K}$, intraperitoneally

Pyridorine, intraperitoneally

Thiamine and nicotinamide, intrape ritoneally

Folic acid, subeutanecusly

Dally oral supplements

Plus (?)

Plus (?)

Plus (?)

Plus

$\begin{array}{lllr}\text { Thiamine } & 0.2 \mathrm{mgn} . & \text { Nicotinamide } & 2.5 \mathrm{mgm} \\ \text { Pyridoxine } & 0.2 \mathrm{mgm} . & \text { Calchu pantothenate } & 10.0 \mathrm{mgm} \\ \text { Inositol } & 0.2 \mathrm{mgm} . & \text { Choline hydrochloride } 20.0 \mathrm{mgm} \\ \text { Riboflavin } & 0.2 \mathrm{mgm} . & \begin{array}{c}\text { Cod-liver oil preparation contain - } \\ \text { Iing vitaming A and D }\end{array}\end{array}$

Young rats were more susceptible to severe irradiation glckness than were older rats, and young rats receiving a vitamin-iree diet were markedly more susceptible than rats mainfained on an adequate diet. The daily oral vitamin supplements greatly decreased the incidence of fatal ir radiation sickness. In all, 224 rats wer. used in 20 experiments, usually in groups of 52 . (FM)

1116

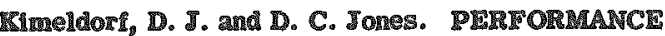
FUUDIS ON ANLALS EXPOSED TO FXTERNAL RADIATION. I. EXIAUETIV IERTERTE TESTS ON RATS FXPOSE TO T-RAYS IN THE LFTHAL DOSE RANGE. Naval Radiologlcal Dofense Lab. Aug. 25, 1950. 23p. (AD $241(1))$

The performance of rat in a bindardized exhaustive periormance test wa depressed following whole-body ir radiation with relatively lethal doses of $\mathrm{x}-\mathrm{rays}$ (700 and 360 I). The extent of per Iormance depression appears to be related to the post-1rradiation gurvival time. The extent and rate of mortality following irradiation, with the doses described, were maricelly increased by repeated periods of ezercise in the post-irradiation survival time. (NSA)

\section{$111 \%$}

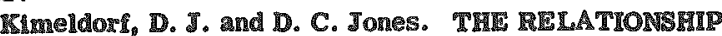
OF RADIATON DOST TO LFTHALITY AMONG EXRCHED ANLALS PXPOSED TO ROENTGLN RAYS. Am J. Physiol. 107, 020-32(1951) (AD-321(B))
The mortality of exercised ir radiated rats was compared with that of nonexercised ir radiated rats following $x$-ray dose ranging from 200 to $1000 \mathrm{x}$. Swimming to exhaustion under standard conditions of testing constituted the exercise. Where exposure to $\mathrm{x}$ rays if followed by daily exhaustive exercise the mortality is significantly greater than with irrabiation alone. For exercised irradiated rats $L D_{50}$ was found to be $510 \mathrm{r}$ and for nonexercised irradiated rats $\mathrm{LD}_{50}$ was $710 \mathrm{r}, 28 \%$ higher. The mortality is not affected by exercise undertaken prior to irradiation. exposing the rats daily to water of different temperatures without exercise had no influence upon the postirradiation mortality of exerciged irradiated animals, showing that the specific environment associated with the method of exercise is not responsible for the mortality increase observed with exercise. (NSA)

1118

Kohn, Henry I. CHANGES IN THE COMPOSITION OF THE BLOOD PLASMA OF THE GUNEA PIG DURING THE ACUTE RADATION SYNDROME. Am. J. Physiol. 162, 703-8(1950). (ORNL-464)

The plasma of the guinea pig was studied during the 8 days following a single total-body exposure to $250 \mathrm{kvp} \mathrm{x}-$ rays. The changes came in wo phases with respect to time, the first phase beginning within 1 day of exposure, the second phase beginning about 3 or 4 days later and cor responding to the time when the first serious symptoms were noted. The changes after $200 \mathrm{r}$, the approsimate $\mathrm{LD}_{50}$, were maller than after $600 \mathrm{~g}$, but the patterns of change were alike. After $200 \mathrm{r}$, the following was observed. Glueose concentration rose $60 \mathrm{mg} \%$ during the first day and gradually returned to normal during the next 5 days. The nonprotein nitrogen was elevated by $10 \mathrm{mg} \%$ for 5 days. The albumin-globulin ratio rose from a normal of 3.1 to 4.5 during the first 2 days and returned to normal during the next 3 days. The rise was due to nonprotein material which could be rendered ineffective by extraction of the plasma with ether. The total protein level fell about $1 \mathrm{~g} \%$ during the 5th to 7th days after exposure. The chloride concentration was elevated by as much as 50 mg $/ 0$ during the $3 d$ to 5th days. Cholesterol was elevated by $20 \mathrm{mg} \%$ during the 4th and 5th days. A peculiar, transient syndrome was also noted during the first hour or two immediately following exposure, especially after $600 \mathrm{r}$, whose signs were almor mal posture, labored regpiration, and lacrimation. 16 ref erences. (NSA)

1119

Lawrason, $F$. Doumlas and $2 . P$. Cronkite. INCLDENTAL FWDING OF MEGALOBLASTIC-LIKE CELLS IN BONE MARROW OF ONE OF TWO SWINE WITH MACROCYT IC ANEMIA AND ACHLORHYDRIA. Tale J. Biol. and Med. 22, 57-6(1049).

Extensive hematological observations were made on two awine (Z) for 17 month after they were exposed to atom bomb ionizing radiation at Bikini. Red blood counts were compared with test swine beyond the range of significant radiation, several surviving heavily irradiated swine, and controls; white blood counts were compared with test swine beyond the range of significant radiation and surviving swine subjected to heavy radiation. The results are dism cussed in terms of nut rition, radiation, age, and previous history of the animals. The authors found in bloud microscopic analyses and marrow smear's evidence for true mac rocytic anemia in both animals $(\mathcal{Z})$ and a hyperplastic megaloblastic-like bone marrow in one. Achlorhydria was found in both animals (Z). (NSA) 1120

Lourau, M. and O. Lartigue. MODNICATION OF THE 
GLYCEMIA PRODUCED BY IRRADIATION OF THE ENTIRE BODY WITH X-RAY. Arch. sci. physiol. A, $197-208(1950)$.

Ir radiation caused guinea pigs to exhibit a hyperglycemia after $15-48$ hour fasting. It also strongly diminished their tolerance to injected glucose. The tolerance curves, differing from diabetic curves, may be the result of modification of intestinal absorption. (CA)

1121

Miller, C. Phillip, Carolyn W. Hammond, and Marianne Tompkins. THE INCIOENCE OF BACTEREMA IN MCE SUBJECTED TO TOTAL BODY X-RADIATION. Science 111,540-1(1950) (AECU-739)

Male, white mice of a strain having an LD-50 (30 days) of approximately $400 \mathrm{r}$ were subjected to total body s- $^{-}$ radiation in a single exposure of 600 or $450 \mathrm{r}$. Cultures of heartg, blood and spleen were made on mice killed at daily intervals post-irradiation. The results showed that bacteremia occurred most frequently during the period of greatest mortality, and its incidence roughly paralleled the dally death rate. Among the mice receiving $600 \mathrm{r}$ the inci dence of bacteremia reached a maximum of $85 \%$ on the 9th day post-irradiation, and among the mice receiving $450 \mathrm{r}$ a maximum of $54 \%$ on the 10 th day. With few exceptions the baeteria were all members of the species normally inhabit122

Miller, C. Philip, Carolyn W. Rammond, and Mariane Tompkins. THE ROLF OT INISCTION IN RADLATON INJURY. J. Lab. Clin. Med. 38, 331-43(1951)

(ARCU-1543)

Male white anice averaging $20 \mathrm{~g}$ in weight were subjected to 600 or $450 \mathrm{r}$ total-body 8 irradiation in a single exposure. In the $600 \mathrm{r}$ series, 288 mice were killed and cultured (at the rate of 20 a day). Of these, 113 ghowed positive cultures from heart's blood and/or gpleen. Righty per cent of the positive cultures occurred during the and week postirradiation, the period of greatest mortality. In the 450-r series, 595 mice were killed and cultured (at the rate of 35 a day). 152 showed positive cultures from heart's blood and/or mpleen. $66 \%$ of the positive cultures occurred during the 2nd week postirradiation. In a majority of the animals the bacteremia was aevere enough to be regarded as an overwhelming aepsis. The bacteremia was caused by microorganisms normally present in the lower bowel of this strain of mice. Control cultures on unirradiated mice were all sterile. It is concluded that septicemia caused by microorganiems of enteric origin is in large measure reaponsible for the death in mice irradiated with these doses of $\mathrm{x}$ rays. (NSA)

1123

Montgomery, Phulp $0^{9} \mathrm{~B}$. and Shelds Warren. THE MECMANSM OF ACUTE HYPOTIENSION FOLLOWHC TOTAL BODY IREADIATON. Proc. Soc. Exptl. BLOL. Med. 77, 803-7(1951) (AECU-1488)

The effect of doses of 1500,2000 , and $2500 \mathrm{x}$ of total body $z$ irradiation upon the blood pregsure, blood volume, and hematocrit were similar regardless of the dose. Blood pressures, hematocritt, and blood volumes of rats irradiated with 1500 r 30 hr after cutting the spinal cord at the level of $C 6$ were the same as found in nonirmadiated controls that had undergone the game operation. Bilaterally adrenalectomized rats after $1500 \mathrm{r}$ total-body irradiation exhibited behavior identical to that shown by normal rat. given the game dosage. It is concluded that the cauge of the immediate hypotension following large doses of total-body irradiation is depresaton of the nervous gyatem. (NSA)
1124

Moon, V. PH., 低. IRornblun, and D. R. Morgan. TRE PATHOLOGIC NATURE OF IRRADIATION SICLNESS: $\triangle$ NEW METHOD FOR PRODUCIIG SHOCR. Arch. Pathol. 29, g2s(1940).

Ir radiation sicknegs was produced in dogs in order to malse observations on the hemo-concentration and on the visceral changes. From 1,400 to 2,800 was given in divided doses over different parts of the abdomen. After an interval of 60 to 72 hours, severe illness developed, and hemoconcentration, ranging from 15 to 50 per cent, appeared. Urination was decreased, and blood was seen in the urine, feces, and vomitus. The illnes progressed rapidly, and death occurred by circulatory failure within 24 hours. The gross and microscopic features were those charactesistic of ghock. They included capillovenous congestion of the viseera, petechial hemorrhages in mucous and serous surfaces, edema of the lumgs and of the mucosae, and parenchymatous degeneration of the liver and kidneys. The results were concordant in 12 animals so treated. An additional feature was degeneration, progressing to necrosis, of the gastrointestimal mucosa. The epithelium liming the crypts and covering the villi showed all gtageg of disintegration. \$ome cypts contained nuelear and cellular delors: others were denuded and empty. Some of the villi retaned their epithelial covering while that in the erypts was necrotIc and disintegrating. Our results corroborate those of Whipple and others: Roentgen radiation of high voltage causes delayed necrosis of the intestinal mucosas, accompanied by shoclilike manifestations. Apparently the latter are due to absorption of material from the damaged mum cosa. The condition is accompanied by progressive hemo concentration, and the visceral changes observed post mor tem are characteriatlcally those of shock. These results provide mperimenter with a new method for induchs shock by causing darnage to the timsues. This method eliminates pain and emotional resuonses, sympathoadrenal hyperactivity, hemorthage and anesthesia, conditions which 1125 are assigned as causes for shock by ana writer: (AP)

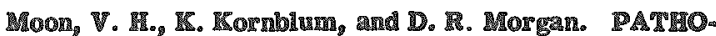
LOGI OF IRRADATION פICKNESE: A NEW METHOD

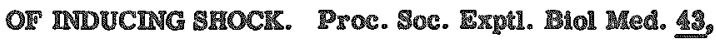
305-6(1080).

Dogs glven abdomanal rosntgen irradiation developed delayed sickness. Vomiting, diarrhea, low blood pregsure, hemoconcentration, prostration and death resulted. Postmortem finding were those of shock: capillovenous congestion, petechial hemorrhages in mucosae and seroøae, edema of sof tissues, and parenchymators degeneration. Injury by X-rays cauged delayed Herrosis of the integtinal mucosa. The syndrome of shock resulted from this infury, independent of anesthesia, hersorrhage, emotional reactions and of sympatho-adrenal hyperactivity. The results gubgtantiate the interpretation that chock may reant from tiasue damage, independent of other confusing iactors. (BA) 1120

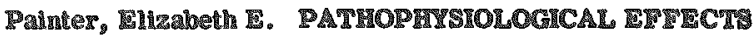
OF TOTAL BODY IRRADLTON. Chemg Med. Bchool Quart. 11, 08-0(1050).

The author deseribes various pathophysiological effects of total-body irradiation. Some of the physiological and biochemical alterations occurring after erposure to lonizhis radiation appear to result from direct damage to the tissa concerned, while others result from indirect eliects by agents carried in the blood from one reglon of the body to another; the signs and symptomg are simulas with every 
kind of ionising radiation. A list is included which covers the single total-body dosage of $\mathrm{z}-\mathrm{ray} 8 \mathrm{needed}$ to ki11 $50 \%$ of the animals involved. The characterigtic phases in the radiation-efiect pattern (initial shock, acute period, subacute period, and chronic period) are discussed under gepaate heading. The total-body dosage either of $25,50,100$, or 200 I is correlated with such specific efects in the guinea pig, dog, rabbits, and rats as lymphopenia, granulocytopenia, recuction in the platelet count, reduction in blood pressure, elevation of basal metabolism, inhibition of intestinal absorgtion of glucose, elevation of sedimentation rate, anemia, absence of reticulocytes, and prolongation of 1127 thing tirne. (NSA )

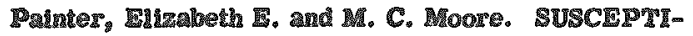

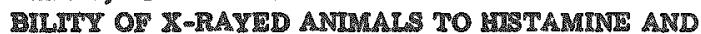
TO ADENOSWII. Federation Proc. I, 00(1848).

The report is reproduced here in its entrety.

A atudy was made to determine the gusceptibility of $\mathrm{x}$ rayed animals to the blood presure lowering effects of tisaue brealdown products, such as histamine and adenosine. The compounds were infured at a congtant rate over 4 -min. periods. The amounts requised to produce a perceptible decrease (5- man 1 Ig) in carotid blood pressure were determined for the controls and for the g-rayed animals dur Ing the firges thour after argosure. All animals were anesthetized with pentobarbital belore carotid cannulation. Rabbits given a ฐingle total body dose of $800 \mathrm{r}$ require only $2-5 \mu g / \mathrm{kg} / \mathrm{min}$. of higtamine base at 1 to 2 hours after radiation to produce a perceptible decreane in blood presaure. At 3as hours, however, the amount required, 3-8 Mg/Ng/min., approaches that for the control animals, 10-15 $\mu \mathrm{Bg} / \mathrm{mm}$. With adenosine this change in susceptibility is not so apparent: $70-100 \mathrm{\mu g} / \mathrm{kg} / \mathrm{min}$. for the X-rayed rabbits during the first hours as compared with $90-130 \mu \mathrm{g} / \mathrm{kg} /$ 2aim. sor the controls. Preliminary experiments on dogs Siven a alngle total body dose of $400 \mathrm{r}$ show a significant change in ausceptiblity to both compounds. These finding may be correlated with the following changes occurring in the anianal body during the early perlod after exposure to 2-radiation sickness: (1) liberation of tissue brealodown products, (2) alteration in the detoxifying powers for such substances, and (3) alteration in the compensatory mechaaimas for redueng the effects of such gubstances. 1128

Panter, E. D., C. Ladd Proserer, and M. C. Moore.

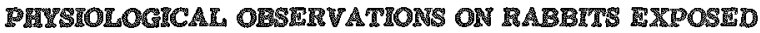
TO आHALL DOSES OF X-RAYS. Unversity of Chicago Metallurgical Lab. [1846] Dec. Mar. 4, 1947. 79p. (MDDC-761; CE-3727)

Wogt of the acute deaths among rabbits after single tolal-

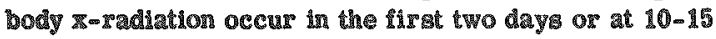
days after irradiation. Signe of clinical depression are seen in wrylvors of aublethal doses during each of these periods, termed the initial shock period and the principal acute radiation death period. The femoral arterial blood pregsure of unanesthetized rabbits declined rapidly from a control of apgromimately $98 \mathrm{~mm}$ IIg to a minimum of about 50 marn $\mathrm{Hg}$ at $1 / 2$ hours after doses of $600 x$ or greater. Proportionately maller reductions in blood presmure occurred after 400 and $200 \mathrm{x}$. The threshold for $10-20 \mathrm{~mm}$ drop was apgroximately 50 I. During the first iev hour the heart rate increased. Survivors of the initial shock period showed partial recovery of blood pressure, but at about 10 days, blood pressure again deelined, partheularly in rabbits which died acutely. Low blood presaures were not observed at this time after doser of 400 or less. Plama protein concentration declined alightly during the acute post-irradiation perlof. Plasma volume a meagured by IFvan blue gpace was not significantly altered in the initial shock period after a dose of $800 \mathrm{r}$. Int radermally injected dye (Evans blue) spread more rapidly in the irradiated than in nonirradiated skin during the first few hums after irradiation. At the same time intravenously injected dye appeared in the interstitial space of a patch of irradiated skin much earlier than in non-irradiated gluin. Recovery in survivors occurs gradually over about a month. Average weight loss is marked (10-15\%) in the first three days; weight remains diminished for about one week, after which some rabbits dic and ollers begin a gradual recovery in weight which is not complete by 30 days. Some individual rabbits show two minima in food and water intake and in weight; one, at 1-2 days, and the second at 10-15 days. Rabbits show a polyuria on the 2nd to Ath day after receiving 800 r. Thereafter urine output decreases slightly below control values but not as much as water intake decreases. Blood removed from rabbits $1 / 2$ hour after $800 \mathrm{r}$ caused an average fall of 26.2 $\mathrm{mm}$ in arterial blood pressure of non irradiated hosts, blood removed 5-12 days after irradiation caused variable but much smaller fall in the pressure of the host. The plasma of non-irradiated rabbits had an average of 0.3 $\mu \mathrm{g} / \mathrm{ml}$, while plasma removed about $1 / 2$ hour after $800 \mathrm{x}$ contained a mean of $0.6 \mu \mathrm{g} / \mathrm{ml}$. The values for whole blood declined due to removal of cells in subsequent samping. (ADD)

1129

Prosser, C. Ladd, Elizabeth R. Painter, and Marguerite N. SwLLt. THE CLINCAL PHYSIOLOGX OF DOGS EXPOSED TO SINGLE TOTAL-BODY DOSES OF M-RAYS. Univ. of Cheago Metalurglcal Lab. Dec. 12, 1946. Decl. Sept. 2, 1947. 18\%p. (MDDC-1272; CF-3738)

The median lethal doee of single exposures of $200 \mathrm{kvp} x$ rays in the dog is approsimately $315 \mathrm{~F}$. During the first several hr after recelving a dose in the lethal range, dogg are lethargic, often have diarthea, and vomit. Blood pressure, heart rate, and temperature remain relatively constant until $10-15$ days after treatment, then heart rate and temperature are levated and blood pressure depressed until death, 3 to 7 days later. Dogs that survive doses of $250 \mathrm{x}$, or above, ugually show a gimilar rise in temperature for a few days during the game acute period. Detailed information is given on changes in the blood picture including count of lymphocytes, lencocytes and erythrocytes at various geriods after irradiation with from 50 to $350 \mathrm{r}$, blood sedimentation rates, clotting times, plasma volume and protein contents. Body weight declined very little during the firgt 7 to 10 days after a midlethal doge but thereafter fell rapidly to a minimum of about $\$ 0 \%$ of control weight in nonsurvîor:. Some loss in weight by survivors of doges of 100 $F$ and above also occurred in the 10 to 15 day period. Food and water consumption were usually alightly diminished for 1 to 3 day after irradiation and were reduced nearly to zero in the acute "depression" period. Metabolic balance data also indicate an increase in catabolism after irradiation. Fecal output corresponded roughly to food intake, but urine output was diminished much less than was water intake. $N$ balance became negative as food consumption was reduced, and in the terminal period $\mathrm{N}$ excretion was relatively undiminished even when $\mathbb{N}$ intake was zero.. Details in metabolic changes are given and the onset of hemorrhages 2130 are described. (TIS)

Quastler, Henry, Elizabeth F. Lanz, Mildred I. and James W. Osborne. ACUTE INTESTINAL RADIATION DEATIP. STUDHES ON RORNGIEN DEATE IN MAC. III. Am. J. Physiol. 164, 546-56(1951).

Mice were subjected to $\mathrm{s}$ irradiation, delivered in one or two exposures to the whole body or to parts, and the the 
elapsing between inradiation and death was recorded. A survival time of $3 \frac{1}{2}$ day is stable over a wide range of doses. It occurs with radiation of different qualitues, in mice of various strains and different weights. Because of Its stability, a survival time of $3 \frac{4}{2}$ days is considered a manifestation of some intrinsic property of mice, and called a characteristic value of the response spectrum. If a dose which will produce death in $3 \frac{1}{2}$ days has been given, additional irradiation does not further reduce the life aspectancy. A mouse can be made to die $3 \frac{1}{2}$ days after a small dose has been given if a supplementing treatment with a large dose is given within a certain time, which depends on the amount of the small dose. In order to produce the reaction which supersedes the $3 \frac{1}{2}$-day process after large doses, it is necessary and sufficient to irradiate the head. In order to elicit the $3 / 2$-day process, it is mecessary and 1131 suficicient to irradiate any portion of the intestine. (NSA)

\section{Rekers, Paul E. INDUCED PREGNANCY IN A MAM-} MALIAN HOST FOLLOWING SEVERE TOTAL BODY X-IRRADLATON. J. Lab. Clin. Med. 37, 331-411951).

Pregnancy was readily and naturally induced in dogs after large doses of total-body irradiation of LD 50 or more. A full-term, viable pup was whelped from the dan receiving the largest dose of irradiation. Asecond dam aborted fol lowing a pulmonary infection. The third dam delivered three living pups, one of which was underdeveloped and succumbed. This dam and the two off spring have remained well. During the gestation period of 50 days or more there were normal gross alimentations, constant weights, and varying degrees of depression of the peripheral blood elem ments without bacterial invasion of the blood atream or in travascular hemolysis. After whelping or aborting there was a rapid decline in the phyaical condition of two of the three dams, gubsequent death, and the findings of sepsis and degeneration including the hemopoietic tissues. From the data presented it does not appear that fetal tissue provided an active hemopoietic center for the irradiated dam. The question, however, of the protective action of embryonic tiæsue for maintaining resiatance to infectiong and of benefit from fetal endocrine or enzyme gyetems is raised. 29 references. (NSA)

1132

Rosenthal, Robert L. OPALESCENCE OF GEUM AFTER TOTAL BODY RADIATION AS A PROGNOSTIC SICN OF DEATK. ScIence $110,43=4(1949)$.

During an invegtigation of the clotting reaction of blood after irradiation with $200 \mathrm{kv} \mathrm{x}-\mathrm{rays}$, the appearance of a marked opalegence was noted in the serum and plasma of rabbits which subsequently died a few days after exposure to a single lethal dowe of total body irradiation.

This opalescence appeared within 24 hours following exposure to radiation. In all cases, it disappared completely 72 hours after exposure. A review of the literature falled to reveal any mention of this phenomenon. An attempt was made to find a correlation between varying intensities of the opalescence and the death rate of the rabbits. At present, there is no clear explanation of the mechanism by Which radiation produces this opalescence. In view of its apgarent relation to death, this phenomenon may not only provide a valuable early measurement of the effect of acute exposure to radiation, but may lead to further knowledge concerning the nature of radiation sickness and its lethal mechanimm. (NSA)

1133

Shacter, Bernard. DFET OF X-RADITION AND OF RADIOMMMETC SUBSTANCES ON THE SULFHYRYL CONPENT OF PLASMA: RELATIONSHT TO ADRENAT
ACTIVITT FOLLOWING STRESS. Cancer Research 11, 277-8(1951).

An abstract of this paper appeared in Cancer Research 11: $277-8(1951)$ and is reproduced here in part.

A study was undertaken on the effects of radiation and of radiomimetic substances on the sulfinydryl content of rat plasma. In addition, because of the possible relationghip between sulfhydryl groups and adrenal activity, the effect of adrenalectomy and of sham operation on plasma aulhydryl levels was also determined. Exposure of ratg to 750 I total body x radiation was followed after several days by a marked decrease in the sulfhydryl content of the plasma. Similar effects were observed following administration of the radiomimetic mubitances, nitrogen mugtard and trisethylene triazine. A decrease in plasma sulfhydryl levels was observed within 24 hr after gurgical treatment of rats. Since the plasma sulfhydryl changes occurred concomitantly with the initiation of active tissue regeneration, it is sug gested that decreased plasma sulfhydryl levels may be indicative of an increaged demand for sulfhydryl groups in tissues during periods of accelerated cellular proliferation, and that the plasma proteins may serve as sources of sulfhydryl grougs for tiøsue requirements. (NSA) 1194

Smith, D. E., G. Svihla, and H. M. Patt. T巽E ETFCTS OF X-RADIATION ON CHCULATION IN THE WING OF THE BAT. Physiol. ZoÖ1. 24, 249-57(1951).

Direct observations and cinemamicrophotographs were made of the blood vessels in the wing of the living bat, Myotis lucifugus, each day following total body irgadiation and following local irradiation of a portion of the wing. No circulatory changes were noted after total-body irradiation with dozages of less than $10,000 x$. Doses over the range of $10,000 \times$ to $60,000 \mathrm{x}$ elicited sticking of leucocytes to blood vessel walls, aggregation of red cells into clumps and stagmation of blood. No other changes were seen. In the areas locally irradiated no alterations in clrculation were noted lollowing dosages of less than $50,000 \mathrm{r}$. After $50,000 \mathrm{r}$ to $5,000,000$ I leukocyte sticking, red cell clumping, white cell clumping, platelet thrombi and stagnation of blood occurred. With the exception of red cell clumping the varlous Intravascular changes were quantitatively related to radiation dosage. The authors discrsged the significance of the several intravascular reactions in relation to the stagna tion of blood. (auth)

\section{5}

Soberman, Robert J., Richard P. Keating, and Roy D. MaWELI. ETSECT OF ACUTH WHOLE-BODY X-IRRADIATION UPON WATIR AND ELECTROLYTL

BALANCE. Am. J. Physiol. 164, 450-6(1951).

Simultaneous repeated measurements of total body water, ertracellular space, godium gace, whole blood, red cell, and plasma volume, and plasma electrolytes were carried out in adult female doge followng 450- to 500-r whole-body s frradiation. Fxcept for an increase in plamma volume and 2. decrease in red-cell volume, no consigtent changes were noted in any of the ceterminations. (NSA) 1136

Tulls, John L. THE RESPONSE OF TISSUE TO TOTAL BODY IRRADIATION. Am. J. Pathol. 25, 829-52(1949).

Two series of experiments involving whole body irradiation of swine are described and the rewults are discugacd. The results of the Bulini atomic bomb experiments were found to be aimilar to experimentr performed meparately at the Naval Medical Reaearch Inotitute (experimenty which consisted of Irradiating swime fron a $100 \mathrm{kv}$ gource whth no filtration, at a distance of $1 \mathrm{~m}$ with whole body $\mathrm{x}$-radiation and at ate of $30 \mathrm{r}$; in the latter axperiments the $50 \%$ 
lethal dose/30 day period was found to be $275 \mathrm{r}$ measured in air. The lesions produced in both series were characterized by hemorrhage, necrosis and secondary infections; lymphoid cells, myeloblasts, germ cells and intestinal epithelium were found to be particularly radiosensitive, injury to the se cells causing anemia and lowering of resistance to infection. Irradiation caused a dilitation of capillaries, and imparment of circulation and tissue anoxia; the pathological state was self-perpetuating. It is concluded that since the most primitive hematopoietic stem cells, the reticular cells, are relatively radio-resistant, the effort to reduce mortality does not appear to be hopeless; management of the effects of total body irradiation should be directed at the prevention of secondary infections, such as pneumonia, which often occur when the organism is in a weakened condition, and the treatment of anemia, impaired circulation and anoxia. (NSA)

1137

Tullis, J. L. and S. Warren. GROSS AUTOPSY OBSERVATIONS IN TIE ANMALS EXPOSED AT BKKINI. A PRELIMINARY REPORT. J. Am. Med. Assoc. 134, $1155-8(1247)$.

Goats and pigs are approximately as sensitive to total body irradiation as man, guinea-pigs are more sensitive, and rats more resistant. Ionizing radiations were responsible for most of the mortality in the first test, and the animals were sheltered from blast in the second. The deaths which were due to the effects of total body irradiation occurred in the acute phase, and no latent or chronic effects had been observed at the time of the report. Almost all the deaths occurred within one month of the test, the mortality reaching a peak within two weeks of the test. Details of the case histories are given for these animals. The conclusions drawn include the following: (1) the flimsiest of shielding seems to afford ample protection from thermal radiation, but heavy armor does not always protect from the ionizing radiations. (2) An underwater atomic bomb explosion is more lethal than an air explosion, since the spray which showers down on the ships in the vicinity carries with it fission products; these contaminate the ships' surfaces, cannot be removed easily, and remain dangerously radioactive for long periods of time. (TIS) 1138

Van Dyke, D. C. and R. L. Huff. EPILATION IN TRE NON-IRRADIATED MEMBEIS OF PARABIOTICALLY UNITED RATS. Proc. Soc. Exptl. Biol. Med. T2, 266-9 (1949).

After X-irradiation of one member of parabiotically united rat pairs both members showed marked hair loss. The non-irradiated member lost its hair earlier and to a greater degree than the irradiated member. An effect such as epilation must result from the transfer of material from one member to the other through their common circulation. Whether the epilation represents the passage of physiologi cally active material from the irradiated to the non-irradiated or is a manifestation of loss of material from the non-irradiated to the irradiated is not demonstrated. (BA) 1139

Wilder, Helenor Campbell and Russell M. Maynard. OCULAR CHANGES PRODUCED BY TOTAL BODY IRRADIATION. Am. J. Pathol. 27, 1-19(1051).

Pathological changes in the eyes of 21 goats and 31 pigs receiving varying amounts of total-body ionizing radiations at Operations Crossroads, Bikini, have been compared with those in the eyes of 24 piggs exposed to 200 to $600 \mathrm{p}$ of totalbody roentgen radiation and of 4 fetuses from $x$-i rradiated sows. Nine control goats and 15 cont rol pigs were exanined. The pathological changes in the bomb-exposed and $\mathrm{X}$ irradiated animals were similar. For the most part, they were regarded as results of bone marrow depression and secondary systemic changes, rather than as direct effects of the rays. The henorrhages were attributed to thronibucytopenia and to a heparin-like substance. Serous exudates were the resulf of increased vascular permeability, possibly secondary to anemia. Septic churoiditis and bacteria in the vessels were manifestations of the septicemia. Vacuoles were present in the lenses of many of the irradiated an1mals but were found also in the controls and therefore were not regarded as a specific manifestation of irradiation. Massive cataract in irradiated animals may have been due to direct radiation, although secondary nut ritional effects associated with anemia and severe hemorrhages could not be ruled out. Immediate changes in the lens specific for irradiation were not appatent in the young or mature animal. Although the secondary effects of ionizing and roentgen radiation were similar in the eyes of both pigs and goats, there were no ocular changes which could be attributed to unequivocally to direct irradiation. 11 figures. (NSA)

\section{Prophylaxis and Therapy-General Reviews}

1140

Allen, J. G., P. V. Moulder, and D. M. Enerson. PATHOGENESIS AND TREATMENT OF THE POSTIRRADIATION SYNDROML'. J. Am. Med. Assoc. 145, 704-11(1951).

The postirradration syndrome is characterized by infection, hemor rhage, anemia, and malnutrition. Progress has been made in the treatment of these important disorders, and there is reason to anticipate even beticr results when several rational therapeutic programs are combined. Anemia can be controlled by blood transfusion, but this does not increase the platelet or leukocyte counts. Transfusion alone did not increase the survival perrod in our dogs in radiated with $450 \mathrm{r}$, although it may appear more benefical in less serious exposures. Transfusion every second day plus daily doses of aureomycin, however, did increase the survival time, and two of 11 animals so treated have survived the critical period of 6 weeks. The general nutritive status of the person can be cared for orally for 1 or 2 weeks after exposure, but there after parenteral feeding may be necessary for several weeks. Frequent transfusion of blood and probably plasma are perhaps the best means to achieve this end. This is a new and serious problem which is largely foreign to previous experiences in burn therapy of nonirradiated persons, for whom extensive oral feedings are generally possible. Irradiation injury is a systemic disease. Wounds, burns, and other injuries must be considered in the light of the entire body economy, for the careful management of local wounds is of little consequence if the subsequent general physiological disorders are not taken into account. (Authors' Summary)

1141

Brues, Austin M. THE NATURE AND THERAPY OF RADIATION SICKNIESS. Chicago Med. School Quart. 11, 105-8(1950).

A description is presented of some of the symptoms present in humans after atomic irradiation. Leucopena, epilation, sterility, and keloids are mentioned in connection with the chronic and acute forms of radiation sickness. Such methods of therapy as blood transfusion, injection of toluidine blue, general supportive measures, and injections of antibiotics are named and outlined. Pretreatment with cysteine and phenylhydrazine is mentioned; the radiosensitivity of tissues and genetic mutation are briefly con sidered. (NSA)

1142

Lacassagne, A. CONCERNING RECENT RESEARCH ON 
PROTECTION AGAINST HIGH-INTENSITY WHOLEBODY EXPOSURE TO PENETRATING RADIATIONS. Fortschr. Gebiete Röntgenstrahlen 75, Suppl., 98-105 (1951).

The author reviews the investigations carried out since 1909 on chemeal prophylaxis and treatment of radiation sucleness and describes an experiment of his own in which adult rats were injected with Loiseleur's solution (glucose, cysteme, thourea, ascorbic acid, and succinic acid) $15 \mathrm{~min}$ before or after x urradiation with 668 to $836 \mathrm{r}$. Histological observations are peported briefly. The lower mortality of the treated animals is apparently caused by survival of a greater number of stem cells in the hematopoietic tissue. 53 rếterences. (NSA)

1143

Langendorf, H. CHEMICAL PROPHYLAXIS OF RADIATION INJURIES. Strahlentherapie 85, 321-400(1951).

When actung on higher organsms, high energy radiations always pruduce a local radiation injury and also a general stress situstion raused by the upset of interaction between the vegetative nervous system and endocrme gland function. The prophylactir action of certain chemicals is explained: cysteme, glutathione, p-chloromercuribenzoate, BAL, $\mathrm{KCN}$, and $\mathrm{HgCl}$ effect cell reactions locally; general atress-damping effects regult from usage of doca (desoxycorticosterone aretate), sutı, pyridoxin (Vitamin $\mathbb{B}_{8}$ ), nicotinamide, d-catechol, and antibiotics. The importance of compounds protecting free SH-groups is stressed. (NSA)

1144

SElle, W. A. ATTEMPTS TO PREVIENT AND COUNTERACT EFTECTS OF IONIZING RADIATIONS BY CHEMI CAL AND PHARMACOLOGICAL MEANS. NEPA DIV., Fairchild Engine and Airplane Corp. Sept. 15, 1249. 30p. (NEPA-1127)

The purpose of this report is to summarize available information concernug attempts to prevent or reduce radiation injury by pharmacological means. The pathogenesis of lethal and sublethal radiation exposures, and the theories and therapies of radiation sickness are discussed. The effects of the following agents on human radiation sickness are summarized in tabular form: desosycorticosterone acetate and pyridoxine; antihstaminics, vitamins, and migcellaneous drugs; antıhistaminics, trangfusions, and ant ihemor rhagic substances; ascorbic acid, ribollavin, thiamin, nicotanamide, and nucleic acid compounds; rutin and related drugs; folic ací; adrenal cortex, desoxycorti costorone, and related drugs; estrogens and other sex hormones; cysteine, cystine, and glutathione; and penicillin and blood transfusions. (NSA)

\section{Anoxia}

\section{5}

Bennett, Leslie R., Sarah M. Chastian, Jerry S. Flint, Robert A. Hansen, and Alvin E. Lewis. LATE EFFETS OF ROENTGEN IRRADIATION; ITUDIES ON MATS IRRADIATED UNDER ANOXIC ANOKA. Univ. Of Calif., Los Angeles. Sept. 7, 1951. 24p. (UCLA-154)

Late effects have been studied to 540 days in rats receiving 600 to $1400 \mathrm{r}$ of whole-body roentgen irradiation under anoxia and surviving the acute post -irradiation period. The findings were marked shortening of life span, high death rate due to pneumonia with a rather specific cellular type in the granuloma, nephrosclerosis and generalized arteriosclerosis, hypertension, blood dyscrasia characterized by thrombocytopenia and anemia (possibly suggesting hypersplenism), cataracts in the animals sur- viving 10 months or more, general cachexia, and late epilation over head and back. (NSA)

1146

Bennett, Leslie R., Robert A. Hansen, and Andrew H. DOwdy. THE EFPECT OF ANOXIA ON THE HEMATOLOGICAL INJURY IN RATS CAUSED BY ROENTGEN IRRADLATION. Univ. of Calif., Los Angeles. Sept. 12, 1951. 18p. (UCLA-156)

Hematograms were made on rats following 500 or $1000 x$ of whole- body $\mathrm{x}$ irradiation administered while the animals were subjected to extreme anoxic anoxia $\left(4 q_{2} \mathrm{O}_{2}\right)$. The results showed that the peripheral blood elements receive a partial degree of protection under these conditions. (NSA) $114 \%$

Dowdy, Andrew H., L. R. Bennett, and Sarah M. Chastian. PROTECTIVE ACTION OF ANOXIC ANOXIA AGAINST TOTAL-BODE ROENTGEN IRRADIATION OF MAMMALS. Radiology 55, 879-85(1950).

The effects of brief extreme anoxic anosia on the $\mathrm{LD}_{\mathrm{ge}}$ and on the radiation syndrome observed in mammals after total-body roentgen irradiation has been investigated. The 30-day $\mathrm{LD}_{50}$ for rats irradiated in $5 \%$ oxygen appears to be between 1200 and $1400 \mathrm{IP}$ as contrasted with an $\mathrm{LD}_{50}$ of slightly higher than $600 \mathrm{r}$ in irradiated control rats. Anosia produced by injection of $\mathrm{NaCN}$ failed to protect rats from roentgen irradiation. These experiments support the theory that radiochemical reactions involving free oxygen play a vital role in radiation injuries. (NSA)

1148

Lacassagne, A. LACK OF SENSITIVIYY TO K-RAYS ON THE PART OF NEWBORN MCE IN A STATE OR ASPHYXIA. Compt. rend. acad. sci. U.R.S..S. 215, 231-2 (1942).

For newborn mice sensitivity to $\mathrm{X}$-rays waw reduced by exposure to a temperature just below $0^{\circ}$, by $N$ asphyria and by $\mathrm{CO}_{2}$ asphysia. (CA)

1149

Limperos, George. ERTCTS OF VARYING OXYGEN TENSION ON MORTALITY OF X-RAYED MCE. J. Franklin Inst. 249, 513-4(1950).

Oxygen tension is reported to play an important part in the biological effects of $x$ radiation. The production of $\mathrm{H}_{2} \mathrm{O}_{2}$ and $\mathrm{HO}_{2}$ and the "release" of the $\mathrm{OH}$ radicals from a back reaction may account for increased mortality of mice $x$ rayed under a higher-oxygen atmosphere. The low-oxygen atmosphere exerts a protective efiect possibly because it decreases the concentration of $\mathrm{OH}$ and $\mathrm{HO}_{2}$ radicals and of $\mathrm{H}_{2} \mathrm{O}_{2}$. (NSA)

\section{0}

Smith, D. E., H. M. Patt, and Ella B. Tyree. PROGRESS REPORT; THE EPECT OF ANONIA UPON THE SURVIVAL OF ADULT FROGS AND DAY-OLD RATS, p.53-6 of BIOLOGICAL AND MPDCAL DIVISIONS, OUARTERLY REPORT TEBRUARY, MARCH, APRIL, 1950. Argonne National Lab. [nd] 4p. (ANL-4A51(p.53-6))

Pairs of male frogss (Rana pipiens) were exposed to total-body $x$ radiation consisting of single doses of 3000 , 6000, or $12,000 \mathrm{r}$. One of each pair was subjected to anoxia in $100 \% \mathrm{~N}_{2}$ during irradiation while the other was irradiated in room air. Daily checks on mortality indicated that subjecting frogs to anoxia during s irradiation does not alter their radiocensitivity as measured by survival. One-day-old Sprague-Dawley rats were given aingle total-body doses of 500 I in $100 \% \mathrm{~N}_{2}$ or in $20 \% \mathrm{O}_{2}-80 \% \mathrm{~N}_{2}$. Sixteen of the 17 rats exposed in $100 \% \mathrm{~N}_{2}$ were surviving at 20 days post-irradiation, while none of 17 irradiated in the $20 \% \mathrm{O}_{2}-80 \% \mathrm{~N}_{2}$ mixture were alive. Death in the con- 
trol group occurred from the 9 th to the 13 th day after $\$$ irradiation. It appears that the \$urvival of one-day rats is greatly increased by anoxia during ir radiation. 1151

Smith, Willie W., Robert Dooley, and Twin C. Thompson. SLMULATED HGH ALTITUE FOLLOWING WMOLE BODT RADATION OF MICE. J. Aviation Med. 19, 227\$7(1248).

Mice exposed to single doses of 400 to 500 x were subjected to simulated high altitudes of 20,000 to 25,000 feet at periods of 1 to days after irradiation while circulating leukocytes were undergoing reduction, and after 10 to 12 days when platelets and erythrocytes were greally reduced. Radiation was given at the rate of $64 \mathrm{~m} / \mathrm{min}$. $(189 \mathrm{kvp}$, 20 rna, and $0.25 \mathrm{~mm}$ of copper and $0.55 \mathrm{~mm}$ of aluminum added filtration). Five litter-mate pair's were lnept in each cage and irradiated simultaneously. One from each pair was taken for the ground group (total 336 ) and one for the altitude group (total 341). Several non-irradiated mice were always subjected to altitude with the exposed groups. The extent of damage was determined by weight loss, deaths, and hematological changes. There was no evidence found that altitude adversely affected the probability of survival nor did it affect the total or differential count of circulating leuksoeytes when they are maximally reduced. The course of diminution and recovery of platelets, erythrocytes, and hemoglobin was not altered by altifude. It is pointed out that the conclusions relative to the absence of altitude effect are based on results with high energy tradiation alone, where no vascular effects were observed such as would result from flash burns occurring after atomic bomb detonations. (NSA)

\section{Antiobioticg}

1152

Arons, Isidore and J. W. Freeman, THE USE OF AUREOMTCIN AS AN ANTIDOTE IN RADIATION SICKNESS. Harlem Rosp. Bull. 3, 123-6(1950-51).

The authors studied the erfect of aureomyein on sive patients with radiation sickness who did not respond to pyridoxine or to the combined uge of penicilin and sulfithalidine. All of the patients reaponded favorably to the treatment. The author conclude that athough there are aeveral therapeutic agents which, when administered prior to therapy, are capable of preventing radiation sichness, aureomyein apparently is the only drug which can bring about regression of all the symptoms once they have become manufest. (S.V.S.)

1153

Felseneld, Oscar, Sachiko Janet Ishihara, Thora Waters, and Jeannette Norsen. IRRADIATON DAMAGE TO MICE. Lab. Digest 14, 18-18(1951).

Experiments were conducted to examine the influence of Bome antibiotics on radiation sickness in mice. The results show that the number of surviving animals after the irradiation, which was an approwmate of LD $_{B 0}$, was significantly increased when given Neomycin, Glucomycin, Neobacin, or Prodiglosin in doses which would be well-tolerated by humans. Thus oral treatment of bacteremia resulting from total-body liradiation is possible. (NSA) 1154

Purth, F. W. and M. P. Coulter. THE EFPCT OF AUREOMYCW ON THE RADIATION SYNDROME IN DoGs. University of Rochester. Dec. 15, 1950. 18p. (UR-146)

Observations are presented on a group of 24 dogs that were exposed to 450 r of $250-k v$ x radiation. One-half of these dogs received aureomycin, $100 \mathrm{mg} / \mathrm{kg}$ daily begiming immediately after irradiation and continuing for 28 days. The remaining dogs served as controls. Dogs treated postradiation with clinical doses of aureomycin have shown decreased mortality as compared with the control animals, The onset of morbidity and mortality was delayed for 5 to 7 days in the treated dogs. Pathological studies revealed severe gastrointestinal hemor rhage and ulcerations of the small intestine in the control dogs and minimal gastrointestinal hemorrhage and absence of small-bowel ulceratons in the treated dogs. Bacteriological atudies showed a greater number of positive blood eultures in the control animals post-radiation than in the trealed animals. Bacteria recovered from autopsy material from the control dogs were sensitive to aureomycin, while those recovered from the treated dogs were resistant to aureomycin. (NSA)

\section{5}

Furth, F. W., M. P. Coultex, and J. W. Howland. FAIL URE OF ALPHA TOCOPHEROL TO PROTECT AGAINST RADIATION INJURY IN THE RAT, P.34\%8 Of QUARTERLY TECHNICAL REPORT; OCTOBER 1, 1950, "THRU DECEMBET 31, 1250. University of Rochester. Feb. 1, 1051. 5p. (UR-152(p.34-8))

The administration of large amounts of alpha tocopherol or aureomycin just previous to exposure to large doses of $x$ radiation tails to alter the lethality of the irradiation in rats. The experimental method is described, with results shown by a graph. (NSA)

1156

Furth, F.W., M. P. Coulter, and J. W. Howland. EF FECT OF CEUDE AND CRYSTALLIZED AUREOMYCIN ON IME WRICHT OF NORMAL AND T-RADLATED RATS, P.39-43 of QUARTERLY TECHINICAL REPORT; OCTODER 1, 1950, THROUGR DECEMBER 31, 1950. University of Rochester. Feb. 1, 1951. 5p. (UR-152(p.39-A3))

Aureormycin administered orally in elther the crude or crystalline form failed to increase the weight gain of nor mal or $x$-irradiated rats, although the incidence of diarThea in the X-irradiated rats was markedly diminished. (NSA)

\section{7}

Gustafson, Gordon E. and Simon reletsky. EFFECT OF ORAL TERRAMYCIN PRIOR TO WHOLE BODY XRADLATION. Proc. Soc. Exptl. Biol. Med. 78, 489-90 (1951).

Terramycin has been shown to be effective in reducing the mortalify in rat when administered for period of only 48 hours prior to 660 of whole body $x$-radiation. (Authors' Summary)

1158

Hammond, Carolyn W. and C. Philip Miller. THE EE FECT OF TERRAMYCIN ON POST-IRRADIAYION INFECTION IN MICE. Ann. N. Y. Acad. Sci. 53, 303-8 (1950). (ALCU-873)

Cultures of heart's blood and spleen of mice subjected to a single dose of $x$-radiation showed a high incidence of bacteremia during the second week, the period of greatest mortality. The gepticemia was caused by microorganisms normally present in the lower bowel of the stock mice. In a majority of animals, the infection was severe enough to be regarded as an overwhelming sepsis. The administ ration of terramycin protected irradiated mice against the septicemia occurring the second week post-irradiation, unless it was caused by torramycin-ingensitive Lacteria. The most common of these was Pseudomonas against which no antibiotic or combination of antibiotics tested 
thus far has been found to be effectrve. Prelimmary experments suggest that the administration of streptomyen together with tersamycin may prove more effective than terramycin alone in controllng infections caused by drugsensitive organsms. (NSA)

\section{9}

Howland, Joe W., Frank Furth, L. R. Bennett, Molly Coulter, and G. M MeDonnel. STUDIES ON ICTORS EFFECTING THE RADIATION STNDROME I THE EFFECT OF AUREOMYCIN AND ANTIBIOTICS ON WHOLE BODY IRRADIATION. Unversity of Rochester. Oct. 14, $194935 \mathrm{p}$ (UR-94)

Results are presented in considerable detanl on the exposure of more than 600 rats and 8 doge to lethal doses (LDQ 100 ) of $250 \mathrm{kwp} \mathrm{x}$-radiation. Observations of the treatment of a proven lymphoma (Hodglsm's type) of liver and spleen are also grven. Chnucal doses of aureomycin given therapeutically to $r$ ats in the diet or drunkmg water can cause almost complete alleviation of the darrheal state followng lethal doses $\left(\mathrm{LD}_{90}\right)$ of $250 \mathrm{kvp} \mathrm{x}-\mathrm{rad}$ ation. As such it is superior, to date, to other antibiotics tested, uncluding chloromycetin, streptomycin, sulfadiazine, and pencullu. Survival of life is prolonged an additional 5 to 7 days. The anumals die of a hemorrhagic death which is not considered to be typical of a radiation death for this species. Anmals may regain pre-radiation weights as early as $\$$ or 4 days following the exposure. Bacter'sological studies andicate a suppression of the normally uccurrung gram negative organisms in the ntestunal tract. Possible causes of the sudden mass mortality are discussed. Dogs treated with chncal doses of aureonyen or combined aureomycin-streptomycm have defmitely shown no evidence of radration snckness and have had bleedng tendencres associated only whth local trauma. Three of $\$ 1 x$ anmals treated in this manner have survived cunulative doses of as much as 750 r an the last 14 months, includng $450 \mathrm{r}$ at the tme of the experiment. Pathological findungs in the late deaths resemble somewhat atypically those noted in the hemorrhagic perrod for the species 14 to 28 days. Bacter1ological studies show depression of sensituve organsms. The case history of one patient with proved lymphoma of lodghm's type is included. From a careful study of the presented data one can state that a favorable step has been made toward the understanding of the rada tion anckness which follows locallzed abdommal urradiation in the human subject under treatment for malignancy of the abdomnal viscera and adjacent areas. Caution is advised in the further treatment of human cases belore a better undergtandng of the nature of the coaguatation of abnormality is determmed. An analysis of the physiological approach necessary in the study of the biological effects of penetratung ionzung radiation 1s given. (NSA)

1160

Howland, Joe W., Frank Furth, and Molly Coulter. THE FIFCT OF AUREOMNCIN AND ANTIBIOTICS ON WHOLE BODY IRRADIATION. Federallon Proc. 9, 334 (1950) (AECU-748)

The document 18 in abstract form; it is reproduced below in 1 ts enturety.

Results are presented on the exposure of more than 1,200 rats and 18 dogs to lethal doses of filtered 250 kwp $x-$ radiation. Clincal doses of aureomycu or other combued antubiotics given to rats after lethal x-radiation results 1 in almost complete allevation of the drarcheal state. Surwival of life is prolonged an addituonal 5 to 7 days. Ammals die of a sudden hemorrhagle death. Preradiation weight may be regamed as early as 4 days after exposurc. Bacterological studies show suppression of normally occurrung gram-negative organisms in the mtestunal tract.
Dogs treated with only clinical doses of aureomycu (equivalent to 50 to $100 \mathrm{mg} / \mathrm{kg}$ ) or combned aureomyemstreptomycin have shown no evidence of radiation s1ckness. Occasional bleedng tendeneres are assocuated only with local trama. Survival of he 18 prolonged from 10 to 14 days. Deaths in control anmals occur between the 10 th to 14th day; in treated anmals, after the 22nd day. Mortality in the dog 18 considerably improved. To date all dying treated anmals have shown sepsis with aureomycum- or streptomycin-resistant organims $48 \mathrm{hr}$ before death, whth the same organsm beng cultured post-mortem from all tissues examned. Pathological fundungs in the treated annmals have not been, un general, typical of radiation disease. Lesions of the gastrointestunal canal have not been observed in the treated anmals. Bacternological observatrons mdicate a general alteration in the bacterial flora of the intestinal canal with a suppression of gram-negative organsms.

1161

Howland, $\mathbb{J}$. F. W. Furth and M. P. Coulter. E FECT OF TERRAMYCIN ON ACUTE RADATION STNDROME IN DOGS. Federation Proc. 10, 67(1851).

Thus report is reproduced here in its entirety.

During the course of invegtigations on the role of infection in the acute radiation syndrome na dogs various antubrotucs have been admunstered to dogs which have received large doses of $\mathrm{X}-\mathrm{radiatuon}$. Previous unvestugations showed that aureomycin reduced the morbidity and mortalaty of the acute radiation syndzome un dogs. Terramycm has a sumlar effect. Twenty-geven adult mongrel dogs were exposed to a sungle dose of $450 \mathrm{I}$ of $\mathrm{x}-\mathrm{radiat}$ an from a $250 \mathrm{kv}$ source. Immediately after Irradiation 14 of the anmals were started on a dose of appros. $100 \mathrm{mg} / \mathrm{kg} / 24$ hours of terramycin admunistered an divided doses every 6 hours for 28 days. The remanng 13 dogs berwed as controls. By the 60th day post-radiation 12 (92\%) of the control dogs had dred whereas 7 (50\%) of the treated dogrs had dred. A delay of $4-5$ days in the onset of mortalnty in the treated group was found. The dogs an the treated group dud not appear as aick as those in the control group. Blood cultures on both groups of dogs showed no agnificant dif ference 1 in the incidence of posituve cultures between the groups. No significant difference in the decrease in peripheral blood elements or in weaght loss was noted in the iwo groups.

1102

IKoletsky, Simon and James H. Christie. FTIRCT OF ANTIBIOTICS ON MORTALITI FOM INTERNAL RADLATION. Proc. Soc. Expt1. Wol. Med. T5, 363-611850). (NYO-1614)

Streptomycn and pentenlin m combunation proved effectwe in reducing the mortality of rats given lethal doses of radioactwe phosphorus. Morbuduy was almo reduced and survival tme prolonged. (NSA)

1163

Lambert, G., J. Maisin, M. Mandart, I. Pluygers, and G Podio. FFFECI OF PERORAL ADMINISTRATION OF AUREOMYCIN ON THE SURVIVAL OF RATS POSED TO LETEAL DOSES OF IX RAYS. Compt. rend. soc b1ol. 144, $1558-60(1950)$.

Severe leukrocytopenia, observed in anmals submitted to lethal x-ray doses, points to infection as a possible cause of death. Rats totally Irradiated with 500 and 600 I Irecerved aureomyen hydrochloride (6 ag twice dally) by mouth until death, or until the 21 in day in cases of lons survival. Although in the $500 \mathrm{~m}$ grovp this treatment produced a notable ncrease in the gurwival rate, no favorable result was observed n the $600-5$ group. (NSA) 
1164

Miller, C. Philip, Carolyn W. Hammond, and Marianne Tompkins. THE TREATMENT OF POST-IRRADIATION INT CTRON. Chicago Univ. [nd] 16p. (AECU-1816)

These experiments seem to demonstrate that generalized infection played a mignificant role in the death of mice exposed to moderate doses of total-body $x$ radiation. Infection was caused by bacteria belonging to the intestinal flora. Treatment with antibiotics reduced the mortality to a significant degree; and, among the antibiotics tried, streptomyein was the most effective. Next in order of eflectiveness were chloroamphenicol, aureomycin, and terramyein, among which there was no demonstrable differace in therapeutic value. (NSA) 1165

Miller, C. Philip, Carolyn W. Hammond, and Mariane TOMplins. REDUCTION OF MORTALITX FROM XRADITION BY TREATMENT WITH ANTTBIOTICS. Science 111, 719-20(1950) (ARCU-738)

Previous experiments (AECU-739) have suggested that infection might be a significant factor in death from radiation injury. An attempt has therefore been made to reduce the mortality from 8 -radiation by controlling the bacteremia by the administration of antibiotics. Treatment with streptomycin alone or combined with penicillin caused a significant reduction in the mortality ( 30 days) of mice following total body s-radiation with $450 \mathrm{r}$. Chloramphenicol although somewhat less effective was the best 11 196 of the other antibiotics tested. (NSA)

Miller, C. Philip, Carolyn W. Hanmond, Mariane Tompkins, Certrude Shorter, George A. Sacher, Sylvanus A. Tyler, and TOan GurLan. TIIE TREATMENT OF POSTIRRADIATION INFECTION WITH ANTIBIOTICS; AN EXIPIRIMENTAL STUDY ON MIC. PART I AND PART I. Insticute of Radiobiology and Biophysics, Uniw. of Chicago. [nd] 65๊p. (AECU-1730)

Young adult mice were subjected to total-body $\mathrm{x}$ radiation in a single exposure, varying firom 450 to $600 \mathrm{r}$. Aiter irradiation the mice were treated daily with antibiotica; one only or several in conbination were used. Streptomycin, given by subcutaneous injection, decreased mortality from $73 \%$ in control animals to $14 \%$. Penicillin given in addition to atreptomycin did not increase its ef fectiveness. Chloramphenicol, aurconycin, and ter ramycin (mixed in food) caused significant reductions in mortality: their mupplementation by daily injections of atreptomycin during the second and third weeks postIrrediation increased the protection. Neomycin was ineffective, polymyxin too toxic. Infections or epidemics by drug-resigtant atrains could not be controlled by the antbiotics used and the importance of infection as a lethal factor in mico exposed to moderate doses of $\mathrm{x}$ radiation is stressed.

Data on the effects of various patterns of antibiotic therapy on the survival of mice following a fixed dose of I rays were subjected to blometric analysis. Streptomycin dosages ranging from about 3000 to 6000 units per day were protective to the game degree. Penicillin plug streptomycin alone. Aureomych, choramphenicol, and terranyein, all supplemented with streptomycin, conferred about equal degrees of protection. The measure of protecthon employed was the difference of probits of mortality for control-therapy cage pairg. This measure was validated by an analysis of the relation between per cent mortaily in cages and mean survival time of decedents. The probit difference was shown to have a small positive regression on level of killing in cages. (N\$A)
Antihistamines

1167

Beeler, John W., Jan M. Tillisch, and Walter C. Popp. A NEW DRUG IN THE TREATMENT OF RADIATION SICKNESS. Proc. Staff Meetings Mayo Clinic 24, 477-83(1949).

A deseription of a new drug, dramamine $(\beta$-dimethylaminoethylbenzohydryl ether 8-chlorotheophyllinate) for the treatment of rediation sickness is presented; the similarity between the symptoms of motion and radiation sickness suggested the use of dramamine for the latter. Excellent results were obtained in $25 \%$ of the 82 cases treated, while good results were shown in $53 \%$; maximal effectiveness was achieved when $100 \mathrm{mg}$ of the drug was given 30-60 minuter before the radiation treatment and repeated 1.5 hours and 4.5 hours after treatment, for a total dosage of $300 \mathrm{mg}$. One or more of the side reactions such as drowsiness, "bad taste", paresthesias, and nausea occurred in about $30 \%$ of the cases. It is concluded that a combination of dramamine and pyroxidine should be of valuable therapeutic aid în the control of radiation sickness, especially in severe cases. (NSA)

1168

Brown, W. M. Court and R. B. Hunter. ANTIHISTAMINE DRUGS AND RADIATION SICKNESS. Brit. Med. J. 116, 984(1948).

It had been claimed by gome that radiation sickness is due to himtamine release and that antihistamine drugs alleviate this condition. The authors experimented with neoantergan in radiation siclness. One group of 17 patients was given 600 to $1,000 \mathrm{mg}$ of the drug daily for five days before radiation, another group, of 15 , placebo tablets, and a third group no medication at all. Irradiation symptoms occurred in $94.1 \%$ of the group treated with neoantergan, actually a higher incidence than in the other groups. Between the 3rd and 5th days following the radiation, five of the neoantergan group developed a rash between the shoulder blades which persisted until the neoantergan was discontinued. The eruption may be due to the toxic action of the rays on tissues which normally would protect themselves by a defense mechanism. In the presence of the antihistaminic drug the defense mechanism may not operate, so that the toxic effect is increased. (RM)

1169

DeFeo, Edward, Paul H. Reitman, and M. Herbert Nathan. RADIATION SICKNESS AND ITS TREATMENT WITH DRAMAMINE. Radiology 56, 420-2(1951).

One hundred patients were given dramamine as soon as Bymptoms of radiation sickmess appeared following radiation 1reatments. In only 46 of these patients was any improvement noted following treatment with dramanine. Onehalf of this number had no recurrence of symptons after administration of the drug for 1 day ( $400 \mathrm{mg}$ ). In the remainder, it had to be given daily throughout the period of irradiation. In the latter group symptoms recurred on an average of 2.1 days after the drug was discontinued. Because of this recurrence of symptoms, it was possible to observe the effect of placebos given at intervals during irradiation in 24 of the 46 patients claiming improvement with dramanaine. The placebo was identical in appearance and taste to the drug. Seventeen of the 24 patients (70\%) did as well with the placebo as with the drug itself, reducing the actual number of patients whose improvement could be eredited to dramamine to 29. These results emphasize the important role of psychogenic factors in radiation sickness, and the care which must be exercised in drawing conclusions as to the effectiveness of any drug in a syndrome in which criteria of improvement are 
mainly subjective. In 11 of the cases dramamine produced undesirable side effects, consisting of intense drowsiness in 6, giddiness in 3 , and a metallic taste in 2 . The drowsiness was of such a degree that the medication had to be discontinued, since this side effect was more debilitating than the radiation sickness. As a result of this experience the authors believe that the effectiveness of dramamine in radiation sickness is proportional to its antihistaminic effect. It is generally known to be one of the less effective of the antihistamines. The problem of radiation sickness will not be solved until there is more general agreement as to its cause. Only then can treatment be directed to the underlying factors and not to the gymptoms alone. (NSA)

1170

Fetzer, A. and E. Werle. X-RAY SICKNESS TREATED WITH ANTISTINE. Strahlentherapie 78, 619-24(1949).

12 patients with a marlsed ireadiation gyndrome (X-ray sickness) after heavy $X$-ray irradiation were treated with antigtine; 11 of them responded very well. It is questionable if the influence of antistine is predominantly histamine-antagonistic. Like the other anihistamine substances, antistine also influences the cholinesterase -acetyl choline system by inhibiting cholinesterase and by decreasing the reaction on the organs affected by acetylcholine. (WM)

1171

Field, John B. and Paul R. Rekers. A PROPHYLACTC APPROACH TO THL ANAPEYLACTIC FACTORS IN IRRADITION DISEASE. Lancet 254, 489(1948)

(MDDC-1658; M-2009)

Both of two dogg each given $25 \mathrm{mg}$ benadryl or gyribenzamine three times daily beginning two weeks prior to exposure with 350 r single dose total body s-irzadiation succumbed with typical severe signs of radiation disease. This compares with a mortality of $64 \%$ (16 of 25$)$ untreated dogs. Five of 10 rats given approximately $20 \mathrm{mg}$ pyxîbenzamine daily succumbed 15 days after exposure to $750 \mathrm{x}$ aingle dose total body irradiation compared with the death of 6 of 10 control rats at 10 days. When ethylene disulphonate in distilled water was injected in various schedules, only 2 of 11 (18\%) dogs given $350 \mathrm{x}$ wingle dowe whole body $\mathrm{X}$-irradiation suceumbed. The treated animals in general had fewer signs of irradiation toxicity. However, when $450 \mathrm{r}$ was the irradiation dose given, of $5(80 \%)$ dogs succumbed compared with 3 of 4 (75\%) of untreated dogs. The injection of ethylene disuphonate into rats and rabbits which were irradiated did not affect the extent of toxic signg or the mortality. (ADD)

1172

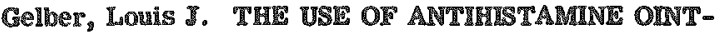
MENT IN CONJUNCTION WITI DEEP I-NAY THERAPY. N. Y. Physician 34, 22, 24, 46, 48(1250).

A zeries of experiments was carried out to determine to what degree anthigtamine substance in ointment form rem duces skin reactions to irradiation and whether or not there is an increase in gkin tolerance to irradiation. A total of 31 cases was gtudied, some with a $1 \%$ solution of Euphorbia pilulifera, some with Rayderm Ointment, and some with both agents; irradiation was carried out at 1,300 to 2,200 Ir in air, the radiation being delivered by a $200-\mathrm{kv}$ machine at a 50-cri target distance through a filtration of 0.5 man Cu and $1 \mathrm{~mm} \mathrm{Al}$. The results of five of the cases are presented in tabular form. It was found that all methods achieved good results, the combined method yielding the most satisfactory results; with it, $2,200 \mathrm{r}$ in air could be delivered with less reaction than 1,300 r without medication. (NSA)
1173

Clenn, J.C. Jr. and R. J. Reeves. TRETATISAT OF RADIATION SICKKNESS WIT ENTLNE-DEREOBARBITAL: PRELIMLNARY RPPORT. Radiology 4\%, 392-6(1946).

To 65 patients æuffering from radiation aickness, trasentine-phenobarbifal tablets were given (three 75 mas tablets four times a day up to totals of $1,000 \mathrm{mg}$ ). Cornplete relief of symutoms was obtained in 39 cases, no 1174 relief in six. (BA)

Haley, T. J. and D. H. Marris. THE DFECT OF SEVPRAL ANTIHISTAMUNIC DRUGS ON MORTALITI DUI TO X-IRRADIAION. Stanford Med. Bull. $b_{3} 1-7$ (1950).

A study has been made of the effect of several antihistaminic drugg on mortality due to x-imradiation in guinea-pigs. The drugs investigated were synergistic with the radiation, increasing its lethality. A significant decrease in the coagulation time was observed after antihistaminie therapy in both the medicated and the medicated irradiated groups. The antihistaminic drugs caused a pronounced leukocytosis in normal guinea-pigs that received the drugy daily for periods of 14-23 days. Gall bladder rupture and enlargement of the common bile duct and gall bladder were observed in guinea pigs chronically medicated with anthistaminic agents. Pyranisamine was the only drug investigated that caused ulceration and performation of the gtomach. The leukocyte count in x-irradiated, medicated or nonmedicated guinea piga follows the pattern ween in other apecies after irradiation. (AWM)

1175

MLESch, WML. CONTRBBUTION TO THE PATROLOCY OF GENERAL X-RAY INJURERES, WIT TI SPICIAL EMPHLASIS ON THE ROLE PLAYED BY HISTAMMTE. Sirahlentherapie 8\%, 255-71(1951).

A test was made of the theory of Reding and Much which ascribes general injurious actions of \& anaphylactic toxic shocks. Since this view is essentially related to another rather widely accepted one of the influence of accumulations of histamine in the body, 20 guinea pigs were mbmitted, after a determination of their histwmine-gensitivity threshold, to a fractionated irradiation leading to the lethal dose. Although the intracutaneous histanine-senaitivity increased 3- to 7-10ld, parallel experiments in which protective injections of antistine were made did not show any effect of the latter on the lethal dose. It was concluded that the histamine accumulation did not play a decisive part in the general injury of the body produced by zx rays. Death was caused by grave lesions of the blood-forming sygtem; no pathologicomanatomical changes pointed to anaphylattc processes. Views ascribing decisive aignificance to the latter and to general intoxicationg in \$-ray effect should be rejected. (NSA)

\section{6}

ISULlander, S. SOME ORSERVATIONE ON RADLATON SICKNESS AND ITS TREATMENT. ACta Radiol.28, $221-4(1847)$.

A definite inswificieney in the production of ICL in the storach occurs during severe radiation sickmess. When PCI is given, the gymptoms of radiation gickmess disappars. Injections of histamine were also effective in alleviating the symptoms of radiation sickness. Thege observations definitely contradict the theory that histamine or Bimilar Bubstances are the etiologic factors In the causalon of ram diation sickness. (BR)

1179

Lar: 


\section{IRRADIATON SICKNESS IN MCE Am. J. Roentgenol} Radum Therapy 62, 547-9(1249).

Lxperments were performed on mice to determune whether the alleviation of the affects of lonmung radations by antuhatamne drugs was due to then ant-histaminic effect ox their slight atropine effect. Albino Swiss female mice were nrradiated in groups of $10 \mathrm{~m}$ a clrcular chamber by a $200 \mathrm{krv}$ machine; $1 \mathrm{~mm}$ Al fulter was used, the distance from the target to the chamber was $30 \mathrm{~cm}$, and the irradiation rate was about $192 \mathrm{r} / \mathrm{mm}$. The mice were $\mathrm{mm}$ jected daily, beginning 24 hourg before urradiation, whth $0.1 \mathrm{ce}$ of $0.43 \times 10^{-3} \mathrm{M}$ solution of atropme sulfate; the injections were continued to the $15 \mathrm{~h}$ day. The typical gigns of uradiation melness were observed in all of the control mice: in the treated groups, the symptoms were not noted as early, but frequently muce which exhbited no aigns of severe urradiation ancluness one day, were found dead the following day. When the radiations exceeded $910 \mathrm{r}$, the treated groups falled to survive, while all of the control groups receiving $740 \mathrm{r}$ perished. The uncrease in gurvival rate wheh was observed in the treated groups is concluded to be greater than chance probabulaty; the average aurvival time for the lethal dose of 1,152 $\mathrm{x}$ was seven days longer for the treated group. It it concluded that: the ionzung radiations must produce multiple injurious reac. tions; and, the particular effect which atropne produces does not involve the most umportant of these reactions since the protection afforded by this drug is incomplete. (NSA)

1178

Lofstrom, J. E. and C. E. Nurnberger. ETIECTS OF RADATION. IRRADIATION SICKNESS: WISTAMIE EFFICT TREATED WITH BENADRYL; A PRELIMINARY REPORT. Arn. J. Roentgenol. Radum Therapy 56, 211-19(1946).

Relief of severe symptom of ir radiation suekness m 19 cases by administration of benadryl (beta-dimethylaminoethyl) benzyhydryl ether hydrochloride is reported by the authors. The complaints of anorexa, naurea, vomitug, weakness and headache were used as index of the efficacy of the drug. For intravenou injection, 5 to 10 ec (50) to $100 \mathrm{mg}$ ) of benadryl was gaven. Patients thus treated experienced a side reaction consisting of drzziness, unsteady motion, weakness, and drowsiness lastug for one to four hours. Later a general feelng of well-being developed. In seven cases benadryl was gren by mouth only, in the form of 50 mag capsules, one every four hours durung the day. These patients showed no marked sude reactions, and satisfactory relief was obtained n most cases. The causes of irradiation are unknown. There is evidence to suggest. that histamine-nlve bodies are developed in the blood of the patuent subjected to roentgen therapy, and the relief afforded by benadryl may be the result of its antahastamme reaction.

1178

Mains, M. P. ANTI-HISTAMNE OINIMENTS FOR SKIN PROTECTION IN RADLATON THERAPY. Radrology 52, 579-81(1049).

Some anti-hrstamine ountments, beneficial in combatug radio-dermatitis caused by the release of histamne and histamine-like substances during tissue damage, are discussed. The author advocates the use of Aquaphor as a base for both Benadryl and Pyribenzamine in $5 \%$ strength. Uang $200 \mathrm{kv}$ at $50 \mathrm{~cm}$ target distance with $0.5 \mathrm{~mm} \mathrm{Cu}$ and $1.0 \mathrm{~mm}$ Allter, 1,800 in anr formerly produced marked skun reactions. Doses as high as $2,400 \mathrm{x}$ in air produce less reaction when the anti-hstamine ountments are applied to the treated skin. Examples are given of the beneficial and protective effects of oral and local anth-histamines in specific cases treated by the author. (NSA)
1180

Panzrom, $p$ E. and E ZIll. HISTAMINE AND ROENT GEN INTOXICATION REPORT ON THE THERAPEUTIC ACTION OF A SYNTHETIC ANTIHISTAMINE PREPARATION: DIMETINA Nuntus Radiol., Belluno, $13 / 3=4$, $167-85(1947)$.

After a brief review of the various theories advanced ance the begunung of the roentgen period to elucidate the roentgen intoxication an extensive exposition is given of the conception of the occur rence of roentgen intorication and its various expressions, whch are generally accepted today; viz that $1 \mathrm{~s}$ to be put down to a toxnc state of the organsm provoked by histamine or histamine-resembling sulbstances. According to the various theories the therapeutuc substances suggested were different, too, unt 1 synthetic antingtamane drugss were prepared in connection with the histamme theory. The favorable results obtaned by these preparalions (the author treated 30 patients with a new gynthetically prepared drug, dmentina, with success in 26 patuents) are thought to substantrate ex juvant lius the hastamme theory of the origin of the roentgen untoxication. (FM)

1181

Salva, J. A and M. Badell. TREATMENT OF RADIATION DISEASE BY A SYNTHETIC ANTIHISTAMINIC DRUG. Presse méd 5\%, 888(1949).

A study is presented concerning the the rapeutic qualities of varuous anthistamines in the treatment of radiation disease. The case studnes are divded into three groups: the prophylactic treatment of the radiation symptoms; the curative treatment of patients c xhibitng intolerance to irradiation; and, the treatment of the sequeliae of irradidtuon. Several drugs are discussed; among them are benadryl, neoantergan, and phenergan. The results undicate that treatment by phenergan seems to be effective in relieving the radiation symptoms, the drug 18 well tolerated and conducive to sleep. Phenergan's mfluence on the capllary changes produced by various agents is also discussed. (NSA)

1182

Salva, J. A, and M Badell. THE CONTRIBUTION ON THERAPEUTICS IN IRRADIATION SICKNESS MEd clin. (Barcelona) 13, 30-3(1349)

Six cases of urradiation gickness caused aiter anteruor urradiation are presented, and the effects of Phenergan on the rellef of the radiation sickness syndrome are noted. The Phenergan was admustered orally once a day un doses of up to 25 mg; complete radation protection was afforded by the Phenergan for the radiation doses noted; it was also found that Phenergan was of greater value in the treatment of urradration ackmess than the classical medications and the other antihstammes. (NSA)

Cyanide Studies

\section{3}

Bacq, 2. M. and A. HErve. CYANLDE AND IX-RAXS. J. physiol. (Paris) 4 1, 124-5A(1949).

In a previous note it was shown that an mjection of $0.1 \mathrm{mg} \mathrm{NaCN}$ made mmedrately before $\mathrm{x}-1 \mathrm{xraduation}$ of 580 reduced to $30 \%$ what would have been $100 \%$ mortaluty in mice. New experiments are reported here on mice which receved larger doses of NaCN and $\mathrm{x}$-irradation of $650 \mathrm{r}$. An injection of $0.1 \mathrm{mg} \mathrm{NaCN}$ mmediately before $\mathrm{ir}-$ radiation reduced the $100 \%$ morkality to $50 \%$ while the game amount of NaCN immediately after Irradiation delayed the 100\% mortaluly for three to four days; if the injection was made 15 munutes after the arradiation, no reduction in the mortality rate occurred. Suliocyanide produced no reduc- 
tion in mortality when injected before irradiation; when ir radiation producing a mortality rate of 10-40\% was ad ministered, a prior injection of $0.1-1 \mathrm{mg}$ of the sulfocyanide augmented the mortality rate. The injection of $1 \mathrm{mg}$ of a tocopherol 40 hours and 24 hours before the irradiation reduced to $30 \%$ a mortality rate of $60 \%$ in the controls. The author's theory of the protective action of these compound is that important chemical reactions take place in the organism which are inluenced by the cyanides; the reac tions are connected with the formation of peroxides by x-irradiation and the action of peroxides on the cells of the organism.(NSA)

1184

Baca, Z. M. and A. Herve. PROTECTION OF MCE AGANST A LETHAL DOSE OF I RAYS BY CYANDE, AZIDE, AND MALONONITRILE. Brit. J. Radiol. 24, $617-21(1951)$.

Cyanides (but not thiocyanate or cyanate), azide $\left(\mathrm{NaN}_{3}\right)$ and malononitrile protect a significant percentage of mice irradiated by a single lethal whole-body dose of $x$ rays; these poisons are ineffective when given after irradiation. The protective effect of previously injected glutathione has been confirmed; the action of cyanide is increased by simultaneous administration of glutathione. (NSA) 1185

Bacq, Z. M., A. Herve, J. Lecomte, and P. Fischer. CYANDE PROTECTYON AGAINST X-IRRADIATION. Science 111, 356-7(1950).

Mice of pure breed (C-57 black or A.X.A.), weighing about $30 \mathrm{~g}$ and 4 to 6 months old, were irradiated by groups of 10 , using $230 \mathrm{kv}, 12 \mathrm{ma}$, copper filter $0.25 \mathrm{~mm}$, focal distance $50 \mathrm{~cm}$, field $100 \mathrm{~cm}^{2}$, meran out put $30 \mathrm{r} / \mathrm{min}$. The 42 cuntrol mice receiving 500 to $600 \mathrm{r}$ all died between the sth and 8th or 9th day. Animals receiving $0.1 \mathrm{mg} \mathrm{NaCN}$ just before similar ir radiation showed 50 to $80 \%$ survival. The same duse of NaCN given immediately after irradiation only delayed mortality. When the cyande wats injected 15 minutes after irradiation, the mortality curve was exactly the same as that of the controls. This protective action is attributed to the $C N$ anion, and the possible modes of action of this protective factor are discussed. (NSA) 1186

Betz, H. MECHANISM OF TRE PROTECTIVE ACTION OF POTASSIUM CYANIDE AGAINST THE LRTHAL ACTION OF X-RAYS IN MICE. Compt. rend soc. biol. 144, 593-5(1950).

In mice exposed to $720 \mathrm{r}$, an immediately previous injn. of $0.1 \mathrm{mg}$ of $\mathrm{KCN}$ intraperitoneally protected against shock and death but did not prevent the cellular lesions in the thymus, lymphatuc ganglions, spleen, and testicles which usually appear $1-7$ days after the irradiation. (CA) 1187

Betz, H. and L. Fuhling. INELUENCE OF POTASSIUM CYANIDE ON THE MORTALITY OF MCE X-IRPADLATED IN FRACTIONAL DOSES. Compt. rend. soc. biol. 144, 595-7(1950).

Mice were exposed to $84 \mathrm{r}$ dally for 12 consecutive days, with intraperitoneal injn. of $1 / 60 \mathrm{mg}$ of $\mathrm{KCN}$ just before each treatment. Instead of protecting, the $\mathrm{KCN}$ increased the mortality. Possibly this was due to accumulation of SCN", which is known to augment the toxic effects of $\mathrm{X}$ 1188 rays. (CA)

Betz, H. and L. Fruhlim. REGENERATION OF REMATOPOIETIC ORGANS IN HEAVILY-IRRADIATED MICE PROTECTED BY ICN INJECTION. Compt. rend. soc. biol. $144,1013-15(1950)$.
Adult C57 mice were irradiated with $700 \mathrm{r}$ of $250 \mathrm{kv} \mathrm{x}$ rays immediately following an intraperitoneal injection of $0.05 \mathrm{mg}$ of $\mathrm{KCN}$. The $75 \%$ of animals gurviving the irradi ation were sacrificed after periods of $24 \mathrm{hr}$ to 10 months. The regeneration of blood, bone marrow, gpleen, thymus, lymphatic nodes, and liver is described in some detail. The delay in regeneration is the game as that observed at much lower radiation doses. In addition, the rate of regeneration exceeds that of normal animals. The present results confirm the view that mortality in irradiated animals is not due to cell destruction but to a more general 1180

Beiz, H. and A. Herve. PROTECTIVE ACTION OF ISCN AGAINST MORTALITY IN MICE IRRADIATED WITH VARIOUS DOSES OT RAIS. Compt. rend soc. biol. 144, 1015-17(1950).

Experiments have been performed to determine whether the protective action of $\mathrm{KCN}$ injected prior to irradiation with the minimal $x-$ ray doge (700 r) persists at higher exposures. Six control lots of 30 to $40 \mathrm{C57}$ adult mice were irradiated with $500,700,900,1200,1800$, and $3000 \mathrm{~g}$ of $250-\mathrm{kv}$ x rays. Five identical lots were ixradiated with the same doses (except 3000 r) following intraperitoneal injection of $0.05 \mathrm{mg}$ of $\mathrm{KCN}$. The 500-1 exposure caused no mortality in either control or injected mice; at $700 \mathrm{r}$ the KCN reduced the death rate from $100 \%$ at 10 days to $75 \%$ and at $900 \times 22 \%$ of the injected mice survived. The mortality curves of control and injected mice were very simLlar at $1200 \mathrm{x}$, the $\mathrm{KCN}$ prolonging the lives of a few animals by only 2 days, and were identical at the $1800-\mathrm{r}$ irradiation. The mechanism of radiation damage causing this reduced effectiveness is discussed. (NSA) 1180

Merve, A. and Z.M. Baca. CYANDDE AND A LETRAL DOSE OF X-RAYS. Compt. rend. soc. biol. 143, 881-3 (1949).

Mice of the black $C-57$ strain were irradiated by a single exposure to $500-600 \mathrm{r}$ of $\mathrm{z}$-radiations, delivered by a 230 $\mathrm{kv}$ machine with a $0.25 \mathrm{~mm}$ Cu filter, at a focal distance of $50 \mathrm{~cm}$ and at the rate of $30 \mathrm{r} / \mathrm{min}$. The technique consisted in dividing the mice into three series: the first Beries received the 8 -radiation alone; the second series received an intraperitoneal injection of $0.1 \mathrm{mg}$ of $\mathrm{NaCN}$ in an aqueous solution; the treatment for the third groug consisted of giving the radiations immediately after the administration of the NaCN and while the animal were gtill in the state of prostration from the injection. The mortalities of the groups were recorded every 24 hours. The regults indicate that the NaCN injection reduced the mortality of the mice, for the radiation dosage given, from $100 \%$ to $30 \%$. (NSA)

1101

Herve, André, z. M. Baco, and R. Betz. CHEMCAI MODIFICATION OF THE LETHAL EFIECT OF XRADIATON AND THE MECLANISM OF TPE ACTON, 10.169-70 Of INTERNATIONAL CONGRESS OF RADIOLOGY, 6th Congress, London. 1950.

A series of experiments to study the protective action of different substances on the mortality of $\mathrm{X}$-irsadiated mice (single dose of $700 \mathrm{x}$ ) has shown that an injection of $0.1 \mathrm{mg}$ of $\mathrm{NaCN}$ given immediately before irradiation reduces the mortality from 100 per cent (control animals) to 30 per cent.

The same amount injected immediately after irradiation retards death by 4 days. If injected 15 minutes after the end of irradiation, the $\mathrm{NaCN}$ has no effect. 
Sodium nitrite gives a similar protective effect.

Histological study of the most sensitive organs from irradiated and "protected" animals shows no difference in the intengity of the radiolesions.

Regarded, on the other hand, from the aspect of "shock," the animals show important differences (suprarenal discharge very slight, and a small excess of fat in the liver among the protected animals). This indicates that death of irradiated mice, unprotected by NaCN, is due to shock rather than to cellular lesions produced by radiation, since among protected animals the mortality is greatly diminished while the cytological lesions are similar to those in mprotected animals.

\section{2}

Herve, A., Z. M. Bach, and H. Betz. PROTECTIVE PROPERTIES OF SODUM CYANHDE AND SODIUM NITRWE AGAINST X RAYS. J. chim. phys. 48, 256-7 (1951).

Injection of $0.1 \mathrm{mg}$ of $\mathrm{NaCN}$ immediately prior to ifradiation reduces by 50 to $80 \%$ the mortality rate of mice totally irradiated by $700 \mathrm{x}$. Administered after the irradiation, the injection is inefective. The above treatment does not protect against higher $\mathrm{s}$-ray doses. Similar effects (40 to $50 \%$ mortality decrease) are obtained with $0.1-\mathrm{mg}$ doses of $\mathrm{Na} / \mathrm{N}$ or $2-\mathrm{mg}$ doses of glutathione; a simultaneous injection of $0.1 \mathrm{mg}$ of $\mathrm{NaCN}$ and $2 \mathrm{mg} g \mathrm{gluta}-$ thione produces additive effects (90 to $\$ 5 \%$ mortality deerease). While no differences are observed in anatomical radiolesions, the injections tend to prevent such characteristic effects of the irradiation as the disappearance of corticosuprarenal lipids and the accumulation of liver fats. (NSA)

\section{Flavonolds}

\section{3}

Arons, I., S. Weintraub, I. Wright, B. Solkolofi, and T. $_{\text {. }}$. Dutcher. THE PROTECTYV ACTION OF FLAVONOMS AGAINST RADIATION, D.170 Of INTERRATIONAL CONGRESS OF RADIOLOGY, th COMgress, London. 1950.

Using a vitamin $P$ compound containing four identieal flavonoid factors, the author demonstrated a decrease in capillary fragility in rats and rabbits. In another weries of experiments 100 rat received 800 r total body X-radiation in a single exposure. 40 of the rats served as controls, while the remaining 60 rats were given vitamin $\mathrm{P}$ conpound isolated from citrus fruit. Mortality rate of the control group was 80 per cent. All rats of this group showed gross haemorrhages of varying gravity and pronounced pathological lesions in the reticular zone of the adrenal corts. The rats which had received 5 milligrams vitamin P daily for of days prior lo expoware and for 23 days after radiation, had a mortality rate of 10 per cent, with much less pronounced hemorrhagic diathesis.

Histolomical investigation of the group of vitamin ireated animals revealed only minor injury to the capillary wall with intercellular pores remaining intact. It appears that vitamin D factors have a specific affinity for the intercellular cement of the capillary wall by strengthening at and giving it congiderable protection againgt a total body, near lethal dose of ionising radiation.

1204

Clark, W. C., R. P. Uncapher, and M. L. Jordan. E. PCT OF LAVONOLS (VITAMIN P) ON MORTALITY FROM TOTAL-BODY RORNTGEN IRRADLATON. Science 108, 829-30(1948).

4. Lawonoid preparation derived from lemons was administered in the drinking water of a group of guinea pigs subjected to $225 \times$ (dose in air) total-body irradiation in a single dose. The experiment was designed to confirm the anti-hemorrhagic effects of vitamin $\mathbb{P}$, and for this purpose the visible manifestation of purpura and anernia were checked at intervals. Of a control group which was similarly irradiated, but not given the flavonoid preparation, $67 \%$ died, whereas of the (protected) group only $35 \%$ died. In additon, the hemor rhagic symptoms of the protected group were considerably less than those of the cont rol series. (TM)

1195

Cronkite, E.P., W. H. Chapman, and F.W. Chambers. FALURE OF A PLAVONOID TO REDUCE RADIATION MORTALITY IN MICE. Proe. Soe. Expt1. Biol. Med. 76, $282-4(1951)$.

A flavonoid (vitamin " ps") $^{\text {) }}$ compound isolated from citrus fruit was given subeutaneously twice daily to white Swiss inbred male mice, beginning 5 days before irradiation with $750,850,950$, or $1050 \mathrm{r}$ of $2000-\mathrm{kwp} x$ rays, and contimuing until the mine died or for 15 days after inradiation. Total daily dose was $0.13 \mathrm{mg} / \mathrm{g}$ of mouse. At the doses of radiation used the compound gave no protection. There was no evidence of any toxicity of the compound in mice at the dose used. It is concluded that a flavonoid compound is of no value in the treatment of radiation injury in the $\mathrm{LD}_{60}$ to Lo $\mathrm{D}_{100}$ I0ange in mice. (NSA)

1196

Cronkite, E. P o D. C. Eltzholtz, C.R. Sipe, W. H. Chapman, and 1 . W. Chambers. FAILURE OF PUTIN TO DECREASE THE MORTALITY OR ACUTE IONIZING RADIATION ILLNESS IN MICE. Proc. SOc. Erptl. Biol. Med. 70, 125-8(1949).

Rutin, a crystalline rhannoglucoside of quercetin, has been found to be beneficial in the hemorrhagic syndrome of acute radiation sickness in dogs. It also accelerates healing of gkin lesions due to radiation in rats. Increased capillary fragillty is in some way influenced by this substance, especially where there is no deficiency of ascorbic acid. An extensive experiment has demonstrated that, in mice, mortalify fron lethal-range doses of $x$-rays is not reduced by rutin administration but is rather increased. Rutin was fed to mice in drinking water (10 to $13 \mathrm{mg} / \mathrm{ml})$ or in food $(20 \mathrm{mg} / 100 \mathrm{~g})$. Treated mice and untreated controls (as many as 400 at a time) were subjected to $\mathrm{x}$ radialion at $1,000 \mathrm{kv}$ and $3 \mathrm{ma}$. The specially constructed cages were arranged in two tiers as segments of a circle of 1 metre diameter. (Full details of the cages and their arrangement are given in a previous paper.) The average output of the tube was 31.2 per minute at the geometric centre of the cages $104.4 \mathrm{~cm}$ from the target. From cage to cage the variation was at most $10.5 \%$. The exposure time was 2.5 mimutes for all mice, the dose being 705 : 11 . All mice were weighed daily before and after irradiation. The clinical signs of radiation illness were identicall in all mice. A few were selected for necropsy and appearances were typical. By the 17 h day the mortality rate in the rutin group was significantly greater than in the control group, although at the end of the 28-day observation period the mortality rates had reached the same level, a Iitle over $40 \%$. There was no significant difference in the weight-loss rates in the two groups, the maximum loss in weight occurring at 12 to 14 days aiter exposure. The authors point out that, of the many factor influencing survival from radiation exposure, capillary iniegrity may not be the critical one. Irradiated animals often die with only minor hemorrhage. It is suggested that similar thvestigations be carried out on guinea pigs, whose requirements for mantenance of caphllary integrity nore nearly resemble those of man. (AWM) 
1197

Field, John B. and Paul E. Rekers. STUDHES OF THE EFFECTS OF FIAVONOIDS ON ROENTGEN IRRADIATION DISEASE. 1. PROTECTIVE INV LUENCE OF RUTIN IN IRRADIATED DOGS. Am. đ. Med. Sci. 218, 1-15(1949).

Sixty per cent of a group of dogg given a single dose of 350 r lotal bondy $x$-irradiation died when left untreated, $36 \%$ of a similar group succumbed when treated with a preparafun derived from lemon peels, and $11 \%$ of a third group died when rutm was given before and after the radiations. When rutin was only given before the radiations, $33 \%$ of the dogs died, and when only given afterwards $60-80 \%$ of the animals succumbed. Hemorrhagic diathesis appeared more frequently in the untreated specimens and those receiving rutin than in the remainder of the animals. Rutin prevented petechiae and caused a reduction in hemorrhagic signs despite a depression in the blood platelet count and a moderate hypowcoagulability; the rate of anemia production and the magnitude of the increased sedimentation mate were reduced while the erythroblast forms and the nucleated red boud cells were increased. Septicemia was present in $62 \%$ of the dead dogs, but it is suggested that the primary cause of death in animals receiving the $350 \mathrm{r}$ was a bleeding tendency assnclated with a vascular dysinnction, as well as a thrombocytopenia. The existence of ${ }^{86}$ vitamin $P$ " is claimed to be supported by this report and it is suggested that this investigational method may be used as part of an assay for this vitamin substance. (NSA) 1198

Field, John B. and Paul E. Rekers. STUDUTS ON THE EFFECTS OF THE TLAVONOIOS IN RÖNTGEN IRRADI TION DISEASE. II. COMPARISON OF THE PROTECTVE INFLUENCE OF SOME FLAVONOIDS AND VITAMIN C IN DOGS. J. Clin. Invest. 28, 747-51(1949).

When normal dogs were exposed to 350 r single dose, total-body $\mathrm{x}$-radiation, $60 \%$ succumbed with a prominent hemorrhagic syndrome. Five flavanone substances appeared to be roughly equal in activity in reducing the hemorrhagic signs when administered continuously, pre- and postirradiation. When rutin was fed, $11 \%$ of the dogs succumbed; with hesperidin 17\%; with epimerized d-catechol 10\%; and with homoeriodictyol and morin none. Other flavonoids had little effect, including quercitin, quercitrin, and naringen. Benzopyrone and coumarin had slight anti-irradiation activity. Ascorbic acid alone had no effect, but when given with quercitin (which alone had no effect) it was markedly protective. Potent hyaluronidase inhibitors such as dopa and $\mathrm{Na}$ gentisate had no inîluence. Hyaluronidase plays liftle or no role in this syndrome. (CA) 1199

Grifith, J. Q. Jr., E. Anthony, E. P. Pendergrass, and R. Perryman. EFFCT OF RUTIN ON RECOVETRY TIME IROM RADIATION INJURY IN RATS. Proc. Soc. Esptl. Biol. Med. 64, 332-3(1947).

All animals were given $2,385 x$ of radiation to one leg (single dose, $200 \mathrm{kv}, 15 \mathrm{ma}, 1 / 2$ mm $\mathrm{Cu}+1$ mm Al filter, $26 \mathrm{~cm}$ skin target distance). Half were used as controls and the other hal were given rutin, $20 \mathrm{mg}$ every third day, implanted subcutaneously as a pellet. The time of onset of reaction and the height of reaction were essentially the same for the two groups but the rats treated with rutin recovered more rapidly. (BA) 1200

Kohn, Henry I., P. W. Robinett, and M. N. Cupp. THE FFECT OF RUTIN UPON THE RESPONSE OF THE RAT TO TOTAL BODY X-IREADLTION. OAR RIIGE National Lab. June 7, 1948. Decl. July 22, 1948. 13p. (A.CD-2176)
Experiments were performed to determine whether rutin can influence the reaction of the rat to $250 \mathrm{kv} / \mathrm{s}-\mathrm{rays}$. The drug is the rhamnoglucoside of $3,5,7,3^{\circ}, 4^{\circ}$, pentahydroxyflavone. In all cases $5 \mathrm{mg}$ per day were given subcutane"usly, beginning either on the day of irradiation or one week before, and continued for at least 18 days. Oral supplements were also given in some experiments. It was found that rutin did not affect the LD-50, the survival following two sublethal exposures, the coagulation time, the thrombocyte count, the total white cell count, and the hemor $r$ hage into the lymph nodes and the gastrointegtinal tracî. Because rutin is sald to affect the hemorthagic tendency in certain conditions, in vitro studies were carried out on normal human and rat blood. The prothrombin time is not affected, nor the coagulation time. It does not prevent the artion of heparin. However, it does antagonize the anti1201 heparin action of toludine blue. (NSA)

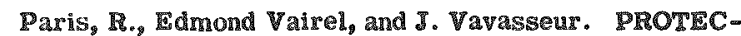
TIVE ERECT OF INAVONE DERIVATIVES ON MORTALITY IN IRRADATION OF THE RABBIT AND MOUSE. Ann. pharm. franç 9, 68-78(1951).

Rutin prevented in the rabbit irradiated with $700 \mathrm{r}$ the increase in capillary fraglity and often, the prolongation of clotting time. It slightly decreased the mortality. At $800 \mathrm{~g}$ no beneficial effect was observed. Alimentation low in green vegetables caused a decrease in resistance, but in these cases the effect of flavone compounds was most marked. Rutin, hesperidin methylchal cone aphtiol, quercitol, and naringoside given orally before irradiation exerted a slightly favorable action on the mortality of mice Irradiated with 600 to 800 r. (CA)

1202

Rekers, P. E. and J. B. Field. CONTROL OF MIEMORRHAGIC SYNDROME AND IEDUCTION IN X-IRRADLATION MORTALITY WITH A FLAVANONE. Science 107, 16-17(1948).

Fifty dogs were exposed to $350 \mathrm{x}$ of total body x $\mathrm{x}$ irradiation. Twenty-live were given 50 mg of rutin, orally, times daily, starting one week prior to exposure and continuing through the test period; of the rutin-treated animals $10 \%$ died, of the untreated animals $64 \%$ died. (CA)

\section{3}

Solroloff, Boris, Walter $\mathrm{Y}$. Eddy, and James B. Redd. TYIE BIOLOGICAL ACTIVITY OF A IFIVONOID (VITA MIN "P") COMPOUND. J. Clin. Invest. $30,395-400$ (1951).

A flavonoid preparation from citrus fruit and contalning four identified factors was tested, experimentally and climically, on its biological activity. In addition to demonstrations of protective action against leukotaxime and bacterial polysaccharide, it was shown that the flavonoid gave a considerable but not complete protection to rata against a total-body, near-lethal dose of $\mathrm{x}$ radiation. Ninety-two human patients, undergoing deep $x-$ ray theray and given the mixed flawonoids, 300 to $600 \mathrm{mg} /$ day orally, for 5 days prior to the exposure and during the whole period of radiotherapy, manifested considerable diminution in radiation crythema of the glkin and in gome cases complete absence of erythema. Nausea and vomiting, however, persisted in some of the treated patients. (NSA)

1204

Solvolof?, Boris, James B. Redd, and Raymond Dutcher. VITAMIN I PROTECTION AGAMST RADUATION. Science 112 112-13(1950).

Rats were fed vitamin $\mathbb{P}$ (CVP compound), isolated from citrus waste, both before and after a total-body radiation of $200 \mathrm{r}$ of $\mathrm{x}$-rays. Considerable protection againgt the near- 
lethal dose was observed, probably due to the prevention, by the flavonoids present, of an increase in capillary fragility. (NSA)

1205

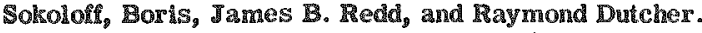
CAPILLARY FRAGILITY AND VTTAMIN 'P" PROTEC-

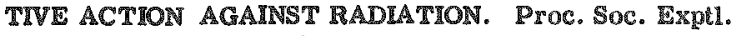
Biol. Med. 75, 6-9(1950).

Administration of leukotaxine, a bacterial polysaccharida, or an exposure to ionizing radiation induces increased capillary Iragillity. Injury to the capillary wall, whether it is caused by ionizing radiation, by levkotaxine, or by a bacterlal polysaccharide, can be prevented to a considerable degree by the administration of a vatamin "g" compound composed of flavonoids naturally present in citrus fruit. (NSA)

\section{Formones}

\section{6}

Bet:, M. FFECT OF ACTH ON TRE MORTALITY OF IRRADIATED MTCE. Compt. rend. soc. biol. 145, 12402(1951).

Adult C57 mice injected daily with $0.5 \mathrm{mg}$ of ACTM for 7 days before irradiation with 700 r of $250 \mathrm{mk} x$ rays had a lower gate of mortality than untreated controls, and there was $20 \%$ survival. However, when the daily injection was started after the irgadiation, the mice died more rapidly than the controls; all succumbed by the 8th day, in contrast. to the 11th day for controls. Mice injected intraperitoneally with $0.05 \mathrm{mg}$ of $\mathrm{KCN}$ immediately before a $700-\mathrm{r} \times$ irradiation had a $75 \%$ survival rate without further treatment, but only a $30 \%$ rate when a daily 0.5 -mg ACrK injection was begun after the irradiation. The ACTY elearly increases activity of the adrenal cortex, which in the irradiated ani mals is already excessively affected by radiation shress. (NSA)

1207

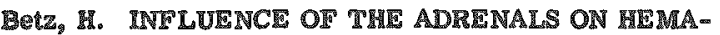
TOPOLE TIC REGENERATION IN THE X-IRRADITED RAT. Compt. rend. soc. biol. 145, 465-7(1951).

Adrenalectomy of rats before $\mathrm{x}$-irradiation prevents formation of erythroblastic and myeloblastic changes in the mpleen. This erfect is overcome by injection of desoxycorticosterone acetate. It thus appears that the adrenals play a gart not only in the regulation of the lymphoid system, but also in that of myeloid tissue. (CA) 1208

Blount, Henry C. Ir. and Wulle W. Smith. THE INILU ENCE OF TYYPON AND TRLURACIL ON MICE EXPOSED TO ROENTGEN RADIATION. Secience 109, 83-A (1049).

Experiments to determine the effects of thyroid and thiouracil feeding, and heat on mice exposed to damaging roentgen radiations are summarized. Radiation doses delivered with an effective voltage of $170 \mathrm{kv}, 20 \mathrm{ma}$, through aluminum and copper fulters, were administered. Mice on thyroid and thiouracil diets for 13 and 8 days belore radiation were examined up to four weeks dater. Mortality rates and temperature experiments indicate that metabolic activity rather than body temperature is the predominant factor in increasing the mortality of mice exposed to the rays. Further experiments are in progress. (INSA) 1209

Craver, B. N. TEFECT OR ADRENAL CORTMAL INJURF ON THE TOIICITY OF ROENTGEN RAYS. AM. 7. Roentgenol. Radium Therapy 50, 404-7(1248).

Two aeries of 25 white male rats (Wistar strain) were exgosed in the following manner to ir radiation by roentgen rays: (1) the "protected" rats were covered over the adre- nal area of the back with a lead strip 1 inch wide maintained in that position by confining the rats in cellophane cones; (2) the "unprotected" rats had two $1 / 2$ inch lead strips shielding them, one over the upper chest and the other over the lower abdomen. Since the 1 inch strip protected the lower chest and upper abdomen, the two $1 / 2$ inch strips were placed as described so that they might protect approximately equivalent amounts of like tissue. The technical factors of the exposure were as follows: $250 \mathrm{kv}, 15 \mathrm{ma}$, 24 in. target skin distance, $660 \mathrm{r}$ total dose, $19.6 \mathrm{r} / \mathrm{min}$. output, an exposure of 33 minutes and 40 seconds. All 50 of the rats survived for 30 days following exposure, except three of the "unprotected" series which died 12,12 , and 16 days after exposure. Twenty-nine days after the first exposure, the animals were exposed again, to $880 \mathrm{r}$. The technical factors of the exposure were as follows: $250 \mathrm{kv}, 15 \mathrm{ma}$, 20 $\mathrm{dm}$. target skin distance, $880 \mathrm{x}$ total dose, $21.3 \mathrm{r} / \mathrm{min}$. output, an exposure of 40 minutes. After this exposure two of the "protected" animals died, one on the fourth and one on the twelfth day after exposure. Seven of the "unproterted" animals died on the $3 \mathrm{rd}, 9 \mathrm{th}, 16 \mathrm{th}, 17 \mathrm{th}, 24 \mathrm{th}, 26 \mathrm{th}$, and 3oth days after this exposure, after which the remaining animals were destroyed. In the "protected" series, three rats were in poor health; in the "ungrotected" series six rats were in poor condition. This evidence suggests that rats whose adrenals are "protected" are less susceptible to the lethal effects of radiation than rats receiving equivalent doses iut not so protected. The possible relation of the adrenal-lymphoid gystem to irradiation sickness and the lethality of roentgen radiation iq discussed.

1210

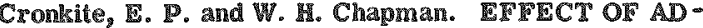
RENALECTOMY ON PADIT TON INDUCED MORTALTY OF THE MOUSE. Proc. Soc. Expll. Biol. Med. 74, $337-40(1950)$.

Adrenalectomized and control mice were exposed at the rate of $15 \mathrm{r} / \mathrm{min}$ to the beam of a $2-\mathrm{Mv} \mathrm{x}-\mathrm{ray}$ tube. The results were: (1) The adrenalectomized or "adrenal insufficient" mouse is more sensitive to irradiation than the normal mouse. (2) The survival time of adrenalectomized mice is shortened. (3) Mice adrenalectomized $48 \mathrm{hr}$ before irradiation were more sensitive than those adrenalectomized 15 days before irradiation. (4) The cause of death in the adrenalectomized irradiated mouse is apparently due to mechan!sms other than infection and hemorrhage. (NSA)

1211

Edelmann, Abraham. THE EFTECT OF ADRENALECTOMY UPON SURVIVAL OI RATS ARTER X-RAOLA-

TION. Brookhaven National Lab. [nd] 3p. (ARCU-325)

Bilaterally adrenalectomized male albino rats (SpragueDawley) were given $650 \mathrm{r}$ of whole body $\mathrm{X}-\mathrm{zradation}$. The average survival of the adrenalectomized irradiated rats was 4.1 days as compared to 7.6 days for the adrenalectomized controls. Subjection of these data to an analysis of variance reveals that the irradiation significantly shortened the lives of the adrenalectomized rats. (NSA)

1212

Rdelmann, Abraham. ADRENAL CORTEX AND SURVIVAL OF RATS AFTER X-IRRADIATION. Federation Proc. 9, 36(1950).

This report is reproduced here in itw entirety.

Protecting the adrenals of male rats by means of lead shields placed about the glands algnificantly increased the survival incidence following $850 \mathrm{x}$ of $\mathrm{x}$-irradiation (160 KVP, $10 \mathrm{ma}, 0.15 \mathrm{~mm}$ Cu). The x-irgadiation $(650 \mathrm{r})$ shortens the lives of adrenalectomized rats from an average of 8 days to 4 days. Substitution therapy in the adrenalectomized rat is efiective inasmuch as $3 \mathrm{cc} /$ day of a whole gland ad- 
renal extract (Upjohn) or $5 \mathrm{mg} / \mathrm{day}$ of desosycorticusterone acetate resulted in 20 and $40 \%$ mortalities respectively at 15 days after $600 \mathrm{r}$, whereas $90 \%$ of the adrenalectomized controls were dead at this time. It is thought that the adrenal cortical response to $x$-ir radiation is similar fo that following any other stress.

1213

Edelmann, Abraham. ADRENAL SHIELDING AND SURVIVAL OF RATS AFTER X-IRRADIATION. Am. J. Physiol. 165, 57-60(1951).

Lead shields placed around the adrenal glands of rats appreciably decreased the number of deaths following 700 or $800 \mathrm{r}$ of whole-body $\mathrm{x}$ radiation. It is thought that the shielding prevents direct radiation damage to the glandular cells so that they retain their ability to respond to nonspecific stress. The gland was extruded from the shield by 3 months, probably because of the formation of connective tissue within the shield. Enucleation with degeneration of the medulla appeared to result. It is thought that available histological and histochemical techniques do not reveal the true radiosensitivity of the adrenal cortes. (NSA)

1214

Edelmann, Abraham. SURVIVAL OF ADRENALECTOMIZED RATS WITH AND WTHOUT REPLACEMENT THERAPY FOLLOWING IIRAADLTION. Am. J. Physiol. 167, 345-8(1951). (AECU-1599)

The average aurvival time of rats whole-body irradiated with $650 \mathrm{x}$ of $\mathrm{x}$ rays 4 days following adrenalectomy was 4.1 days compared to 7.8 days for adrenalectomized, untreated controls. In contrast, $80 \%$ of adrenalectomized, irradiated rats given $3-c c$ daily doses of adrenal extract and $60 \%$ given $5-\mathrm{mg}$ of desoxycorticosterone acetate (DOCA) daily were alive 20 days after irradiation. The length of survival after discontinuance of therapy of the irradiated adrenalectomized rats treated with DOCA or adrenal extract was similar to that of the unirradiated, untreated adrenalectomized rats. (NSA)

1215

Edelmann, Abraham and Seymoux Ratsh. THE SURVTVAL OF RATS ADRENALECTOMIZED AFTER X-IRRADIATION. Federation Proc. 10, 38(1951) (AECU-1137)

This report is reproduced here in its entirety.

Rats were adrenalectomized at various times after a dose of whole-body xirradiation $(600 \mathrm{r}, 160 \mathrm{kvp}, 0.2 \mathrm{~mm}$ $\mathrm{Cu}$, a dose which does not kill normal control rats in this laboratory), and the time of death was noted. It was observed that the life span was reduced when the time interval between irradiation and adrenalectomy was decreased. This relation was not linear, a greater effect being noticed at the shorter intervals. If the irradiation was given 5 or more days before the adrenalectomy, the life span of the irradiated-adrenalectomized rats approached that of the unirradiated-adrenalectomized rats. This may be interpreted to mean that the need of the animal for adrenal hormones to survive is most acute immediately after irram diation and rapidly diminishes after 3 to 5 days. 1216

Ellinger, Friedrth. PROTECTVE ACTION OF DESOXYCORTICOSTERONE ACETATE AGAINST X-RAY INDUCED LIVER CIANGES. Science 104, 502(1946).

Total-body irradiation with $x$-rays was given to white male mice. The doses were 500 and 1,000 $8 /$ air in one exposure or in fractions of $100 \mathrm{x}$ dally. The radiation factors were: $200 \mathrm{kv}, 10 \mathrm{ma}, 0.25 \mathrm{~mm} \mathrm{Cu}+1.0 \mathrm{~mm} \mathrm{Al}$ filters. $50 \mathrm{~cm}$ distance, $20 \times 20 \mathrm{~cm}$ field, $23.4 \mathrm{c} / \mathrm{min}$., HVL $-0.75 \mathrm{~mm} \mathrm{Cu}$. Daily doses of 0.25 to $0.5 \mathrm{mg}$ desoxycorticosterone acelate (in oil) were administered subcutaneously six times weekly to one group of irradiated ani mals. The other group of irradiated animals served as con trols. Tutal doses varying between 2.5 and $8.0 \mathrm{mg}$ were given within 10 to 18 days. The effects of mortality rate, fat content of the liver, and radiation changes in other organs were studied over a period of 40 days. The outstanding observation made was a striking reduction in the amount of sudanophile fat in the irradiated and desoxycorticosterone-treated group of animals as compared with those receiving irradiation only. There was a slight decrease in: the mortality rate produced by the varioua doses of $\mathrm{x}-\mathrm{rays}$ in favor of the desoxycorticosterone-treated group. This protective action of desoxycorticosterone against $x-r a y-$ induced liver changes supports the val ue of degoxycorticosterone in the treatment of radiation illness. The results also seem to support the histamine hypothesid of radiation effect.

1217

Ellinger, Friedrich. EPTCTS OF DEOXYCORTCOSTERONE ON MCE IRRADIATED WITI X-RAYS. PYOC. Soc. Expt1. Biol. Med. 64, 31-5(1247).

Fatty changes in the liver brought on by irradiation of mice with $1 . D-50$ of $\mathrm{X}$-rays caused death. The percentage of mortality and liver damage was reduced to an extent proportional, within limits, to the dose of deosycorticosterone administered. The towic action of $\mathrm{x}-\mathrm{ray}$ is due to the liberation of histamine like substances by tissue breakdown, since some histamine effects are known to be antagonized by deosycorticosterone, while this sulostance has no effect on $x-r a y$ damage to the spleen and bone marrow. (NSA)

1218

Ellinger, Friedrich. INELUENCE OF PHARMACOLOGI CAL AGENTS ON EFIECTS OF IRRADIATION. RadiolOgy $50,234-43(1948)$.

A simple nethod for the evaluation of the action of pharmacologic agents on the effects of irradiation is described. The criteria selected (mortality rate produced in total body irradiation and changes in spleen, bone marrow, and liver) were easily observable and lent themselves to statistical analysis. By means of this method, the following results were obtained. Desoxycorticosterone acetate marledly decreased the lethal effect of various $\mathrm{x}-\mathrm{ray}$ doses in mice and prevented the radiation-induced fatty changes in the liver. The chemically closely related steroid pregnenolone failed to show these effects. Vitamin $B$ complex ghowed a definite reduction of the fatty changes in the liver. Both vitamin $B$ complex and vitamin $C$ failed to produce significant changer of the radiation effects on the spleen. The importance of pharmacologic analysis of radiation enfects for the under standing of their mechanism is discussed. The significance of the demonstrated possibility to decrease the mortality rates of ionizing radiations produced in tolal body irradiation for the imgrovement of radiation therapy of widely spread cancers and the possible utilization of these experiences in the treatment of perions accidentally exposed to lethal doses of radiations generated during nuclear fission are indicated. (BA) 1219

rllinger, Friedrich. THE USE OF ADRENAL CORTCAI HORMONE IN RADIATION SICIKNESS. RAdlology 51, $304-2(1948)$.

In the light of recent studies radiation sickness appears to be caused by a general intoxication brought on by tissue decomposition products of higtamine-like substances, or histamine itself, and adrenal cortical insufficiency. An important accompanying condition is the fact that there are fatty changes in the liver. Since a synthetic hormone of the adrenal cortes, desoxycorticosterone acetate (DCA), is known to counteract mineral losses caused by adrenal cortical insurficiency and to play an important part in the 
inactivation of histamine, it seemed logical to use DCA in the treatment of radiation sickness. In an earlier series of tests (Sclence, 104: 502, 1946; Radiology, 50: 234-242, 1248) the author gave mice varying doses of total body ix radiation and found that DCA protects the liver against radiation-induced fatty changes and that this liverprotecting action is accompanied by a decrease in the mortality rates. Here the author has extended his studies on the influence of DCA on the effect of $X$-rays to include the effects on the spleen, bone marrow, and adrenal cortex. One hundred Swiss white mice (average weight $25 \mathrm{~g}$ ) were exposed to $500 \mathrm{r} / \mathrm{aIr}$ of $\mathrm{X}$-rays (h.v.l. $0.75 \mathrm{~mm}$ Cu) given as total body irradiation in one exposure. Part of the animals were given daily doses of $0.5 \mathrm{mg}$ DCA. They were killed at different intervals and the tissues examined histologically. The irradiation changes in the spleen were not affected by the hormone. However, there were well defined changes in the marrow observable in the DCA groug, and there was a decreased depletion of the sudanophile fat in the adrenal cortex during the first week only. This seens to be explainable by the fact that cortical fat content had returned to mormal. The lack of observable change in the spleen may have been factual, or the result of faulty method. The conclusion is made, taking other atudies into consideration also, that the administration of DCA prevents exhaustion of the adrenal cortex caused by irradiation. Mention is made of a clinical study by the author and others on a group of 50 patients under treatment for radiation sickness where 37 patients were relieved of nausea and/or vomiting upon administration of DCA. (NSA)

1220

Ellinger, Friedrich. SOME EFTECTS OF TESTOSTER ONE PROPIONATE ON MTE IRRADLATED WTI X-

RAYS. Proc. Soc. Exptl. Miol. Med. 74, 616-19(1950).

Administration of testosterone propionate in dally doses of 0.25 and $0.5 \mathrm{mg}$, segpectively, following exposure of mice to the $\mathrm{LD}_{50}$ of $\mathrm{x}-\mathrm{ray}$, up to 18 days, markedly increased the lethal effect of irradiation. No changes in the x-ray induced organ effects were accomplished in all investigated organs with the exception of the liver. Radiation induced accumulation of sudanophile fat in this organ was definitely suppressed with a larger dose of teatosterone. Radiobiological and some clinical implications of these findings are discussed. 15 references. (NSA)

\section{1}

Ellinger, Friedrich, J. Elagg, and W. Brooks. PHARMACOLOCICAL ITUDIES ON MRADLTED ANIMLIS. I. EHTECTS OF CORTISONE ON X-RAY MORTALITY OF

MICE. Proc. Soc. Exptl. Biol. Med. 80, 214-17(1952).

Injection of $0.25 \mathrm{mg} /$ day of cortisone intramuscularly for 14 days after the higher $\mathrm{LD}$ doses of $\mathrm{X}$-ray increased the mortality rate. With the lower LD doses it did not inerease the mortality but it shortened the latent perlod between $1 r^{-}$ radiation and death. Simultaneous treatment with ascorbic ach did not gigniflcantly modify these effects. (CA) 1222

Mllinger, Friedrich B. Rostwlt, and S. M. Glasser. THE TREATMENT OF RADIATION SICKNESS WITY ADRENAL CORTICAL RORMONI (DESOXYCORTICOSTERONE

ACITATE). Am. J. Roentgenol. Radium Therapy 61, 387-90(1949).

The authors analyze the response of 50 patients guffering from radiation siclsness to desoxycorticosterone acetate (DOCA). This clinical investigation was based on the assumption that irradiation of animal tissues liberates some towhe histamine-like substance. One of the authors has previously adduced evidence from animal experiments that DOCA protects the Iiver againgt the laty changes induced by irradiation, and also reduces the lethal effect of irradim ation on mice. The authors claim that the present investigation corroborates his findings. Symptoms of radiation sickness are classified in five groups: (1) general; (2) gastrointestinal; (3) hematologicat; (4) cardiovascular;

(5) psychic. This report deals with the effects of DOCA on groups 1 and 2 only - mainly on nausea and vomiting. Five mg in peanut oil was injected I.M. every eight hours until relief of symptoms (but for not more than five days). All of the patients in this series suffered from nausea and/or vomiting, as well as other symptoms, after irradiation therapy for a variety of benign and malignant lesions. Only three patients failed to respond in any way to DOCA and two of these had advanced brain tumors. Of the remaining 47 cases, 37 were completely relieved of the most distressing symptoms, nausea and/or vomiting and 19 became symptom-free. Of 27 cases in which the liver was included in the irradiated area, 23 patients were relieved of nausea and/or vomiting. In the individual case records "body section irradiated" (that is thorax, abdomen, neck, groin) is cited but no details are shown of actual field sizes and vol umes irradiated. Details of dosage are not given but the correlation of daily and total cumulative dose with onset, frequency and severity of symptoms will be presented at a later date with a larger series of cases. (AWM) 1223

Graham, J. B. and R. M. Graham. THE MODIFICATION OF RESTSTANCE TO IONIZING RADIATION BY IUMORAL AGENTS. Cancer $3,709-17(1950)$.

Methods of modifyng resistance to radiation in animals are reviewed. Experiments on Swiss mice exposed to totalbody 8 -ray radiation are recorded. $A$ number of substances including horse serum, stilbestrol, estradiol, estrone, testosterone, and adrenal cortical extract given either 10 days before or immediately after exposure to $x$-rays were found to increase or decrease the mortality rate. The effect produced was determined not only by the substance but also by the time of its administration. The ability of the hormones to modify radioresistance could not be correlated with their established endocrine activity. The sensitivity of animals to ionizing radiation was reflected in the appearance of their epithelial cells. Characteristic cytological alteration was observed in vulnerable animals after exposure. Experiments here and elsewhere indicate that the adrenal cortes has little to do with resistance to radiation. A variety of substances, including stilbestrol and some steroids, have been found to induce a cellular response in animals closely resembling that produced by radiation. This suggests that these materials and radiation have a similar effect on the organism and may explain the ability of these agents to modify gesistance to radiation. (NSA)

\section{4}

Graham, J. B., R. M. Graharn, and A. J. Graffeo. THE INFLURNCE OF ADRENAL CORTICAL HORMONES ON SENSITVITY OF MCE TO IONIZING RADIATION.

Endocrinology $46,434-40(1950)$.

More than 2000 adult Swiss mice including controls were given cortical extract (ACE), desoxycorticosterone (DCS) and 11-dehydrocorticosterone (HCS) at varying times before and after total body irradiation of 400 to $600 \mathrm{~F}$. The proce. dure is outlimed, and the results are tabulated and discussed. The authors conclude that ACE given 10 days prior to irradiation increases the survival rate in both sexes, when given immediately before irradiation raises the mortality in females, and does not alter the survival rate under any other conditions tested; despite reasonable postulates that the adrenal cortex plays a significant role in the resistance to radiation, the authors did not find any evidence for the mediation of radioresistance by the adrenal cortical hor- 
mones used, and probably not by the cortex itself; they further conclude that the demonstrable effects of adrenal compounds on radioresistance are in the nature of a nonspecific stimulus since they may be duplicated by injection of other material such as foreign protein or estrogen. (NSA)

1225

Gray, John L., Elizabeth J. Moulden, John T. Tew, and 1. Jensen. TYE PROTECTIE EIITECT OF PITRESSIN AND OF EPINEPHRINE AGAINST TOTAL BODY XRADIATION. Proc. Soc. Exptl. Biol. Med. 79, 384 - 7 (1952) (AMRL-64)

The survival of rats, exposed to lethal $\mathrm{x}-\mathrm{ray}$ dosage, was found to be significantly increased after pretreatment with pitressin or epinephrine. The protective effect is attributed to their property of producing a temporary tissue anoxia. (NSA)

1226

Halberstaedtex, L. and M. IClkowicz. THE EFPECTS OF X-RAYS ON THE LIMPHATIC ORGANS OF NORMAL AND ADRENALECTOMIZED RATS. Radiologia Clin. 16, 240-57(1947).

A series of normal and adrenalectomized rats were in radiated with an $\mathrm{X}$-ray dose of $2,000 \mathrm{H}$. Histologic reac tions are reported concerning the pycnotic reaction an thymus, spleen, and mesenterial lymph node within a few hours following the irradiation. It was found that: (a) The greater the volume dose, the stronger the pyenotic reaction. (b) Following irradiation of one part of the body, an indirect influence is demonstrable in the non-irradiated part of the body. (c) Adrenalectomy diminishes the X-ray effect, especially the indirect effect. (d) Thymus, spleen, and mesenteric lymph node do not react to $x-$ ray with equal pycnotic intensity, especially if the effect is an indirect one. The thymus shows the greatest sensitivity. This is in conformity with the finding of Dustin, that the thymus generally exhibits a greater degree of susceptibility to toxic substances than either spleen or lymph node. (NSA) 1227

Haley, Thomas J., Samuel Mann, and Andrew H. Dowdy. A COMPARISON OF THE RESTONSE OF NORMAL AND HYPOTHYROW MCE TO ACUTE WHOLE BODY ROENTGEN RADIATION. Science 112, 233-4(1950). (UCLA-61)

The ability of antithyroid drugs to protect animals against the lethal effects of $\mathrm{X}$-irradiation has been investigated with adult mice. It has been concluded from the results that the hypothyroid state does not protect the mouse from $x$-irradiation damage. The amount of available oxygen and not its rate of utilization determines the extent of such damage. (NSA)

\section{8}

Haley, Thomas J., Samuel Mann, and Andrew H. Dowdy. THE EFECT OF ROENTGEN RAY IRRADIATION ON NORMAL, MYPOTHYROW, AND HYPERTHYROLO RATS. Endocrinology 48 , 365-9(1951). (UCLA-81)

Thyroparathyroidectomy does not significantly increase the survival time nor decrease the tolal mortality of roentgen-ray irradiated rats. Hyperthyroid:am produced by the subcutaneous injection of thyrowine or thyroglobulin into rats causes a bignificant increase in mortality rate as compared with normal and hypothyroid animals even though there are no significant differences in tofal mortality. Thyroxine and thyroglobulin premedication of hypothyroid rats decreases their survival time, as compared to nonmedicated hypothyroid and normal animals, but total mortality was practically unchanged. (NSA)
1229

Haley, Thomas $J .$, Samuel Mann, and Andrew H. Dowdy. THE INABILITY OF THIOUREA TO MODIF X ROENTGEN RAY IRRADIATION MORTALITY IN RATS. Science 114, 153-4(1951). (UCLA-112)

The hypothyroid state induced by feeding $0.1 \%$ thiourea confers no protection on rats subjected to $600 \mathrm{r}$ of acute whole-body roentgen irradiation. Hyperthyroidism produced by thyroxin injections results in a slight increase in the rate of mortality under the same experimental conditions. However, the greatest increase in the rate of mortality was seen in those animals which had been treated with both thiourea and thyroxin. (NSA) 1230

Ickowicz, M. EARLY EFPECTS OF X-RAXS ON OVARIES OF NORMAL AND ADRENALECTOMIED RATS. Proc. Soc. Expt1. Biol. Med. 66, 646-9(1247).

The appearance of pycnotic lesions of the cells in the granular layer of the ovarian follicles of rats four hours after $x-$ ray irradiation was inhibited by previous 1231 adrenalectomy. (BA)

JOE1, C. A. EFECT OF TESTOSTERONE PROPIONATE ON THE REGENERATION OF TESTES INJURED BY X-RAYS. Endokrinologie 24, 310-17(1942).

Adult white rats were irradiated with $360-450$ r. Some of the animals were then treated with testosterone propionate (total dose $30 \mathrm{mg}$ in four weeks) while others served as controls. After 25 days the testes of the treated and untreated animals were about alike but after 50 days, when the reparative processes had already been started, the teates of the treated rats had manisested regeneration to the normal condition, whereas those of the untreated rati showed only beginning regeneration. (CA) 1232

IFatshy Seymour and Abraham Edelmann. TRPE ADRENAL CORTICAL REQUTREMENTS OF ADRENALECTOMLZED, X-IRRADIATED RATS. Federation Proc. 10, 73(1951) (ALCU-1131)

Adrenalectomized male rats were maintained with whole-gland adrenal extract (Upjohn), the amount being adjusted so that the animals had a slight weight gain during the period of observation. The animals were irradiated with $600 \mathrm{gr}$ of $\mathrm{x}-\mathrm{r} 2 \mathrm{ys}(160 \mathrm{kvp}, 10 \mathrm{ma}, 0.2 \mathrm{~mm}$ Cu). It was found that the level of cortical extract had to be increaged several fold for 3 to days after inradiation to inhibit. weight loss. A gradual reduction in extract requirenent then occurred, basal levels being reached after 5 days. This clearly indicatas the need for inereased adrenal horyones following $x$ irradiation.

\section{3}

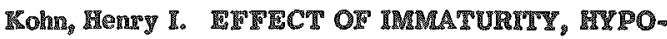
PHYSECTOMX, AND ADRENALECTOMN UPON CRANGES IN BLOOD PLASMA OF RAT DURING ACUTE RADLATION SYMDROM. Am. J. Physiol. $165,33-58(1951)$.

Following whole-body irtradiation of the immature rat, the pattern of change in the plasma was like that in the mature animal, bult the LD $D_{00}$ was about 200 I legs and diarrhea appeared sooner. The effect of hypophysectomy uron the radiation reaction depended upon the age at wheh operation had occurred. In the adrenalectomized mature animal, the glucose and NEN reactions to irradiation were greatly diminished, the chloride reaction was modisied, and the cholesterol, protein, and $\mathbf{A} / \mathrm{G}$ reactions were unchanged or somewhat enhanced. Irradiation of the head and neck alone led to no changes in the plasma. Whatever role the pituitary or thy roid glands played was therefore an in- 
direct one. Some conclusions are drawn about the role of the pituitary-adrenal system. (NSA) 1234

Kohn, Henry I., Nancy Swingley, W.J. Robertson, and Marian Rirslis. EFPECT OF IMMATURTY, HYPOPHYSECTOMY AND ADRENALECTOMY UPON THE CPANGES IN TUE BLOOD PLASMA DURING THE ACUTE RADIATION SYNDROME IN THE RAT. Oak Ridge Nationa Lab. June 7, 1951. 32p. (ORNL-656)

Changes that occur, during the 10 to 14 days following total-body $x$ irradiation of the rat, in the plasma levels of glucose, nonprotein nimogen (NPN), phosphorus, total protein, albumin/globulin ratio $(\mathbb{A} / \mathrm{G})$, chloride, and cholestarol were reported previously (Am. Ja Physiol. 165, 4356(1951)). The effect of immaturity, nypophysectomy, or adrenalectomy on the pattern of these plasma changes has now been investigated using the same experimental conditions. Following irradiation of the immature animal (aged 35 to 40 days) the pattern of change in the plasma was like that in the mature animal (aged 90 days plus), but the $\mathrm{LD}_{50}$ was about $200 x$ less and diarxhea appeared sooner. Hypophysectony in the immature animal prevented the chloride reaction. In the mature animal, it modified the cholesterol reaction. In both, it greatly reduced the glucose and NPN reactions. It had no effect on the protein and $A / G$ reactions in either type. A striking difference due to age also was seen in the unirradiated hypophysectomized animals; in the mature group, fasting had no effect on the level of NPN. Following adrenalectony in the mature animal, the glucose and NPN reactions to irradiation were greatly diminished, the chloride reaction was modified, and the cholesterol, protein, and $\mathrm{A} / \mathrm{G}$ reactions were unchanged or somewhat enhanced. A system is proposed in which radiation effects are classified as primary or secondary, depending on whether they occur within the irradiated cell or elsewhere. It is suggested that the $\mathrm{A} / \mathrm{G}$ reaction might be an indication of a primary injury, whereas the other reactions were more likely to be indications of secondary effects. (NSA) 1235

Lambert, G., J. Maisin, M. Mandart, T. Meersseman, G. Podio, and E. Pluygers. INF LUENCE OF DESOYY CORTICOSTERONE, N-HEXYL BUTYRATE, ALPHANAPHTHYLTHIOUREA, AND ACACLA GUM ON THE SURVIVAL OF RATS RECEIVING A LETHAL DOSE OF T-RAYS. Compt. rend soc. biol. 144, 4A2-52(1950).

The injection of small doses of desorycorticosterone acetate or $\alpha$-naphthylthiourea before and after the ingadiation had no algnificant effect on the survival time. Injections of hexyl butyrate or acacia gum shortened the 1230 survival time. (CA.)

Loiseleur, Jean and Georges Velley. IMMUNITY CONFRRRED BY MYPRRGLYCEMA AGAINST LETRAL DOSTS OF X-RAYS. Compt. rend 231, 182-4(1950).

Fifty-four adult rats were given total body lethal doses of $\mathrm{x}$-rayg (950 a at $180 \mathrm{kv}$ ) 10\% 45 minutes. Forty-înve minutes prior to irradiation, 27 of them were given an intraperitoneal injection of glucose $\left(4 \mathrm{~cm}^{3}\right.$ containing $2 \mathrm{~g}$ glucose per $150 \mathrm{~g}$ body weight). All controls died, between a period of 5 and 23 days following irradiation, with the usual symptoms of radiation exposure accompanying death. Among the glucose treated rats, five died. All the others showed no Ill effects and, in fact, exhibited an average gain of weight of $15.5 \%$. The author discuss the reason for this high Imrunity. (NSA)

$123 \%$

Marder, Sumner N., Henry S. Kaplan, and Egon Lorenz. IACK OF PFFECT OF ADRENALECTOMY ON THE LYMPMOPRIC RESPONST OF C-57 BLACK MCE TO
TOTAL-BODY IRRADIATION AT A LOW DOSAGE LEVEL, p.21-27 of BIOLOGICAL AND MEDICAL DIVISIONS, QUARTERLY REPORT, AUGUST, SEPTEMBER, OCTOBER, 1948. Argonne National Lab. Nov. 1, 1948. 6p. (ANL-4227(p.21-7))

Marked variation in the level of cir culating lymphocytes of $\mathrm{C}-57$ black mice has been noted, and the difficulties thus imposed on the demonstration of minimal acute changes are discussed. Lymphopenia within six hours following total-body irradiation with 30 a has been demonstrated in both splenectomized and nonoperated male mice of this strain. (Authors' Summary)

\section{8}

Patt, M. M. R. L. Straube, E. B. Tyree, M. N. Swift, and D. S. Smith. INFLUENCE OF ESTROGENS ON THE ACUTE XI-RRADIATION SYMDROME. Am. J. Physiol. 159, 269-80(1949) (AECU-524; UAC-92)

The phenomenon of estrogen protection against $x-r a y s$ has been investigated. Several types of experiments were performed. The following results are reported. Pretreatment with estradiol benzoate improves the survival of male mice after lethal $\mathrm{x}$-irradiation has been confirmed. It has been demonstrated further that the estrogen protective ef fect occurs in female mice as well and that benzestrol, but not progesterone and testosterone, is also effective. The time of injection of estrogen relative to the time of irradiation is critical, for estradiol is most effective when it is given 10 days before the exposure. Estrogen injection 5 or 15 days before irradiation also protects but administration at other intervals fails to increase survival and, indeed, may even potentiate toxicity. Estradiol in dosage sufficient to protect against $x$-rays does not influence survival after poisoning with a nitrogen mustard. Estradiol $(0.166 \mathrm{mg} \mathrm{per}$ mouse I.M.) increases adrenal weight and accelerates thymic involution but has no appreciable effect on weight of the spleen, inguinal nodes or kidneys. Although the increase in adrenal weight after $x$-irradiation is somewhat greater in mice pretreated with estrogen, the radiation-induced involution of spleen, thymus, and inguinal nodes is not altered by the treatment. A leukopenia with maximal depression around 10 to 14 days aftex estrogen injection has been observed. The reduction in heterophils is more marked and sonewhat more consistent than the lymphocyte response. Little change is noted in erythrocyte count following the single injection of estradiol. Leukocytes are decreased to the same levels in both the estrogen-treated and control irradiated animals, although maximal depression and recovery occur earlier in the former. "The more rapid recovery of leukocytes in irradiated mice which received a prior injection of estrogen residues in the heterophil component. The anemia of radiation is also less severe in the estrogen-treated animals. Significantly, the maximal estrogen protective effect is observed when mice are irradi ated during their leukopenic period. That there is some correlation between heterophil response and lethal effect is indicated in the finding that a rapid recovery of heterophils but not of lymphocytes occurs in mice treated with estradiol 10 days before $x$-irradiation. (NSA) 1239

Patt, Rarvey M., Marguerite N. Swift, Robert L. Staube, Ella B. Tyree, and Douglas E. Smith. INFLUENCE OF A CONDITIONING INJECTION OF ISTROGEN ON TIE HEMATOLOGIC AND ORGAN WIGIGT RESPONSE TO XIRRADIATION. Federation Proc. 8, 124(1949) (AECU-487; UAC - 38)

Estradiol benzoate $(0.166 \mathrm{mg}$ I.M. in a single injection 5-15 days before exposure) greatly enhances resistance of mice to total-body x-irradiation. Progesterone and testosterone are ineffectual. To elucidate this phenomena we 
have studied the effect of estradiol on organ weights, blood count, and microscopic anatomy in control and irradiated mice. Bstrogen increases adrenal weight by $35-40 \%$ four days after injection and accelerates thymic involution but has no appreciable effect on weight of spleen, inguinal nodes, or kidney. Leukopenia with maximal depression around 10-14 days after injection has also been observed. Decreases are noted in lymphocytes and heterophils. Little change is seen in erythrocytes. Significantly, the maximal estrogenic protective effect is observed when mice are ir radiated during their leukopenic period. Estrogen injection at the time of irradiation or 25-35 days before exposure does not protect. Radiation-induced involution of spleen, thymus, and inguinal nodes is not altered by estradiol. However, the increase in adrenal weight is somewhat greater in these animals. Leukocytes are decreased equally in both estrogen and control (sesame oil injected) irradiated animals, although maximal depression and recovery occur somewhat earlier in the former. Erythrocyte count of irradiated controls is decreased by $45 \%$ at 11 and 14 days after exposure. Estrogen-treated animals manifest a smaller decrease in red count (25\%) seven days after ir radiation and normal levels at 11 and 14 days. The possibility that the protective action of estrogens is mediated by 1240

Patt, H. M., M. N. Swift, E. B. Tyree, and E. S. John. ADRENAL RESPONSE TO TOTAL BODY X-RADIATION. Am. J. Physiol. 150, 480-6(1947).

The changes in adrenal cholesternl content and adrenal weight observed after total body x-radiation at $650 \mathrm{x}$ and $900 \mathrm{r}$ suggest a considerable adrenal response to these dose levels. Aftor $200 \mathrm{r}$, there is little change in adrenal activity as judped by these indices of response. (GPR) 1241

Patt, H. M., M.N. Swift, E. B. Tyree, and R. L. Straube. X-IRRADIATION OF THE FYPOPHYSECTOMIZED RAT. Science 108, 475-6(1948).

In order to determine whether hypophysectomy would prevent adrenal response to $\mathrm{x}$-radiation and to note whether survival and some of the typical changes in organ weights would be altered, 30 white male rats were hypophysectomized one week prior to a single exposure of 750 s $(200 \mathrm{kw}$; dose rate $18 \mathrm{r} / \mathrm{min}$ ). The animals were sacrificed three hours and four days after irradiation and the adrenals, spleen, thymus and left kidney were removed. The per cent changes in organ weights and in adrenal cholesterol were determined. Hypophysectomy appeared to potentiate x-ray toxicity. It was concluded that the initial, intermediate, and terminal adrenal changes regulting from $x$-ray exposure were prevented by the operation, and that under these conditions the extent of the splenic and thymic involution is not altered, although toxicity appears to be enhanced. (NSA)

1242

Smith, Willie W. and Falconer Smith. EFTET OF THYROID HORMONE ON RADLATION LETHALITY. Ans. J. Physiol. 165, 639-50(1951).

The effect of the thyroid hormone in increasing $x$ radiation lethality was evaluated, and the critical period of susceptibility to the thyroid effect delineated. Dinitrophenol was found to increase the proportion of deaths after irradiation. Thiouracil and propylthiouracil falled to protect against radiation lethality. A yeast supplement falled to prevent thyroid from increasing the mortality after irradiation. Weights of adrenal, lymphold tissue, and kidney were determined and blood studies made in thyroid-treated and control irradiated mice. No evidence was found to indi- cate that the thyroid effect is secondary to its action on the adrenal. After sublethal radiation, leucocytes and hemoglobin were more depressed in thyroid-treated than in control mice. (NSA)

1243

Smith, Willie W., Falconer Smith, and E. C. Thompson. FAILURE OF CORTISONE OR ACTH TO REDUCE MORTALITY IN IRRADIATED MICE. Proc. Soc. Exptl. Biol. Med. 73, 529-31(1950).

Intraperitoneal injections of cortisone, or subcutaneous injections of $\mathrm{ACTH}$, failed to increase survival time or 1244 number of irradiated mice surviving. (Author \&' Summary)

Straube, R. L., H. M. Patt, E. B. Tyree, and D. E. Smith. INELUENCE OF THE LEVEL OF ADRENAL CORTICAL STEROIDS ON SENSITIVITY OF MICE TO X-IRRADIATION. Proc. Soc. Exptl. Biol. Med. 71, 539-41(1949)

(AECU-529; UAC-102)

The radiosensitivity of intact mice with and without whole adrenal cortical extract or desoxycorticosterone has been determined. Data on the sensitivity of adrenalectomized mice which were obtained as part of another study have been included for comparison. From these data it if concluded that the survival of $\mathrm{z}$-irradiated mice appears to be independent, at least within certain limits, of the amount of adrenal cortical steroids present. The adrenals are apparently involved only secondarily as part of the organism's buffer response to the stress of irradiation. The rationale of employing adrenal corticoids to alter radiation mortality is therefore open to question. (NSA)

1245

Swift, M. N., H. M. Patt, and E. B. Tyree. THE ETIECg OF ADRENAL CORTICAL FXTRACT ON ADRENAL RESPONSE TO TOTAL BODY X-IRPADIATION. Federa tion Eroc. \%, 121-2(1948).

This paper is an abstract and is reproduced here in its entirety.

The transient $20-50 \%$ decrease in adrenal total cholesterol observed in albino male rats three hours after a midlethal total body exposure to $\mathrm{x}$-radiation (Patt, et al., Am. J. Physiol., 150, 480, 1947) is largely prevented by subcutaneous injection of adrenal cortical extract (Wilson). Total doses of $0.3-1.8 \mathrm{cc} / 200 \mathrm{~g}$ rat were similar in effect, with no graded responge over this range. This is additional evidence that the early cholesterol fall reflects increased adrenal activity. In order to investigate the nature of the marked rise (70-109\%) in adrenal cholesterol observed 3-7 days after midlethal exposure to $x$-radiation, a second similar exposure was administered to rats four days after the first. No fail in adrenal cholesterol occurred by three hours after the second exposure, and the cholesterol level at this time was unaffected by cortical extract dosage sufficient to prevent the early decrease seen axter angle s-may exposure. Flevated adrenal cholesterol is reported to occur only after prolonged gtimulation by adenotrophic hormone, and may in this case also regresent overstimulation in excess of cortical hormone demand. Rats given $0.1 \mathrm{cc}$ of extract three times daily, an amount gufileient to prevent the early cholesterol fall, show adrenal cholesterol changes of slightly lesser degree than do controls over a 15-day post-irradiation period. Survival is not en hanced by this treatment. Larger amounts of extract might possibly prove effective since inereaged survival has been reported after 8 -Irradiation by shelding the adrenals (Craver, Fed. Proc., $6,319,1947$ ).

1246

Torda, Clara and harold G. Wolff. EFECT OF LYMPKOID NECROSIS DUE TO NITROGEN MUSTARD AND 
ROENTGEN IRRADLATION ON NEUROMUSCULAR FUNCTION OF MYPOPHYSECTOMTZED ANIMALS. Am. J. Physiol. 163, 201-8(1950).

Hypophysectomized rats exhibited at marked decrease of the ability of nerve to maintain the amplitude of action potential during repetitive stimulation, and a decrease of the ability of nerve tissue to synthesize acetylcholine. Roent gen irradiation and administration of nitrogen mustard in creased acetylcholine synthesis in hypophysectomized rats about $30 \%$, and increased by about $30 \%$ their ability to maintain the amplitude of action potential during repetilive stimulation. Administration of ACTH restored in hypophy sectomized rat the acetylcholine synthesis and the ability to maintain the amplitude of action potential to normal. The effect of.ACTH in improving the function of nerve is probably dependent only in part on the reduction of the mass of thymus (perhaps also the lymphoid tissue).

\section{7}

Treadwell, A., W. U. Gardner, and J. H. Lawrence. ETFECT OF COMBINING ESTROGEN WITH LETHAL DOSES OF RORNTGEN RAY IN SWISS MICE. Endocxinology 32, 161-4(1943).

Groups of ruice were given 400 to 550 units of 8 -ray. Estrogen was given $1 / 3$ of the mice at the time of irradiation. One-third of the mice had received estrogen nine days before irradiation. At the end of four days the survival of the irradiated mice was $20.6 \%$, of mice given estrogen and $x$-rays at the same time, $11.7 \%$ and estrogen nine days before irradiation, $75 \%$. (BA)

1248

Van Eck-Vermande, G. J. and J. Freud. ACTION OF GONADOTROPHINS AND OF OESTROGENS ON X-RAYED MOUSE OVARIES. Arch. intern. pharmacodynamie 78, $67-78(1949)$.

Mouse ovaries irradiated with $330 \mathrm{x}$ and $660 \mathrm{x}$ are moderately responsive to human placental, mare aeral, and horse hypophyseal gonadotropins. The response of irradiated ovaries is continually simplified in comparison with those of normal mice as the time after irradiation inereases and prospective follicle forming structures diswppear. The final result is eutrophy of interstitial ovariocytes, when hardly anything else remains in the irradiated ovary. The estrus and progestational activity of these ovariocytes is indicated in the genital accessories. The immediate destruction of follicles by $\mathrm{x}$-rays is irreparable. But the "secondar $y^{\text {ss }}$ damage caused by irradiation on follicles that develop in subsequent batches is greatly reduced either by estrogens or by hyphyseal gonadotrophin. After such treatment primary follicules show abundant granulosae, secondary follicules do not fail to develop, luteinigation follows, and corpora lutea can assune the pregnancy type. Poorly enveloped oocytes and anovular follicles are found. The theoretical implications, especially doubt as to the existence of follicle stimulating hormone and luteinizing hormone, concerning the cycle and the alleged inhibition of the hypophysis by moderate doses of estrogens are discussed. (NSA)

\section{9}

Velley, Georges and Jean toiseleur. AUGMBNTATION OF THE CUTANEOUS RADIOSENSITIVITY OF THE RABRIT RESULTING IROM THE ACTION OF INSULIN. Compt. rend. 230, 2132-3(1950).

Rabbits were irradiated with various doses of x-radiation (2250 to $3000 \mathrm{x}$ ) after receiving an injection of insulin $(0.5$ clinical units per kilogram). The onset of epilation at the oxposed area was greatly accelerated by the ingulin injection. The explanation the author offers hor this is as fol- lows: When cellular oxygen becomes activated by $x-r$ ras, its activity is shared by the metabolic molecules, principally glucose, and the cellular components (the cause of the appearance of radiolesions). The insulin hypoglycemia, causing the oxidation of the cellular constituents to be preponderant, results in an increase in the cutaneous radiosensitivity. (NSA)

\section{Sulfhydryl Compounds}

\section{0}

Chapman, W. H. and E. P. Cronkite. SULFHYDYLCONTAINING AGENTS AND THE ETFECTS OF IONIZING FIADATIONS; II. FURTHER STUDIES OF THE BENEFICLAL EFIECT OF GLUTATHIONE ON F-IRRADIATED MCE. Prac. Soc. Mexpll. Biol. Med. 75, 318-22(1950). $(\mathbb{N P}=1833)$

The effects of the subcutaneous injection of glutathone. prior to irradiation, were studied in four consecutive experiments in whrch 2343 male, Swiss Albino mice were exposed to various doses of total-body $x$ radiation. The lethal dose curves for glutathone-injected and nontreated mice were simultaneously determined in each experiment. The 28-day $\mathrm{LD}_{50}$ for the nontreated mice was $740 \mathrm{r}$ : for mice that received $1.6 \mathrm{mg}$ of glutathione per gram of mouse, the 28 -day $\mathrm{LD}_{5}$ was $840 \mathrm{r}$, and for mice that received $4.0 \mathrm{mg}$ of glutathione per gram of mouse, the 28-day LD $D_{50}$ was 950 x. These results indicate a quantitative relationship be tween glutathione dosage and the degree of protection it affords against $x$-ray damage. Weight change and the effect of trauma were studied in the irradiated survivors in an attempt to demonstrate differences in the recovery rates of the glutathione-injected and the nontreated mice. For these studies, all mice were weighed daily throughout the 28-day observation period of each experiment and on the 29th day all surviving animals were subjected to controlled mechanical trauma produced by a Noble-Collip drum. The glutathione-injected mice lost less weught and were more 1251 resistant to trauma than their nontreated controls. (NSA)

Chapman, W. I., C. R. Sipe, D. C. Eltzholtz, E. P. Cronkite, and $F$. W. Chambers, Jr. SULFHYDRYLCONTAINING AGRTIS AND THE EFIECTS OF IONIING RADIATIONS. I. BENE ICIAL ERTECT OF GLUTATHIONE INJECTION ON X-RAY-INDUCED MORTALITY RATL AND WEIGHT LOSS IN MCE Radiology 55, $865-73(1950)$.

Male and female mice were exposed to total-body $x$-ray irradiation, in doses of $800 \mathrm{r}$. Four hundred were given glutathione belore or both before and after irradiation. Two hundred were given control saline injections, and $200 \mathrm{re}-$ ceived no treatment. The survival rates of the glutathionetreated groups were greater $(63.8 \%)$ than the survival rates for the comparable control groups ( 40 to $46 \%$ ). Statistical analysis showed that the differences in survival rates were definitely significant. The surviving glutathione-treated in radiated animals lost less weight and recovered weight more rapidly than the surviving inradiated control anumals. Glutathione-treated survivors, when traumatized, had a lower mortality than the nontreated controls. (NSA) 1252

Cronkite, Eugene P.g Ceorge Brecher, and W. W. Chapman. SULFHYDRYL-CONTAINING AGENTS AND THE EFTECTS OF IONIZING RADIATONS; II. STUDIES ON THE MECLANISM OF TIE PROTECTIVE ACTION OF GLUTATHIONE. Military Surgeon 109, 294-307(1951). (NP-1876)

After injection of reduced glutathione, clearance and tissue distribution studies were performed in mice, dogs, 
and rats. Anmals were ir radated during a period when tissue concentration of reduced glutathone was signif cantly elevated. Following ir radiation of mice whth $820 \mathrm{r}$, granulocytes and lymphorytes were apparently alosent from the blood of the untreated mice for a fow days. During thes same period granulocytes and lymphocytes were seen in small numbers in the blood of glutathione-treated mice. The spleen and thymus of irradiated glutathione-treated mice recover normal werght more rapidy than untreated mice. Histologically hematopoiesis returns earlier and with greater frequency in the treated mice than in the untreated mice. Dogss are also partially protected from radiation by intravenous glutathione, $50 \%$ recovering from a dose of radiation that has so far been unformly fatal in untreated dogs in this laboratory. 15 references. (NSA) 1253

Cronkite, E. P., G. Brecher, and W. H. Chapman. MECkANISM OF PROTECTVE ACTION OF GLUTATHIONE AGAINST WHOLE BODI IRRADIATION. Proc. Soc. Eryul. Biol. Med. 76, 396-8(1951).

White Swiss inbred male mice weighng 20 to $24 \mathrm{~g}$ were irradiated with the radial beam of a $2.0-M e v$ x-ray tube. All mice were given $820 \mathrm{r}$ with the exception of the mice in which the testicles were studied. The latter received $750 \mathrm{r}$. Before irradiation the mice received either $4 \mathrm{mg}$ of glutathione per gram of mouse subcutaneously as a $10 \%$ solution at $\mathrm{pH} 6.5$ or isotonic saline in the same volume. The similarity of the glutathione-treated and untreated irradiated mice in respect to their radiosensitive-organ weights, blood counts, and histologic picture durng the first 3 to 5 days is highly suggestive that cellular destruction of radio. sensitive tissues (bone marrow, thymus, spleen, and testis) is of equal degree in the two groups. It is impossible to prove this conclusively by histologic techniques because a few blasts might survive and be undetected. In contrast to the similarity in the destructive phase the bone marrow, spleen, and thymus regenerated much more rapidly in the glutathione-treated animals as judged by the criteria used. (NSA)

\section{4}

Cronkite, L. P. and W. H. Chapman. EFECT OF ADRENALECTOMY AND GLUTATHIONE ON XR-RAY INDUCED MORTALITY IN MCE. Federation Proc. 9, $329(1850)$.

The 28-day mortality of mice simultaneously exposed to various doses of 2.0 mev $\mathrm{X}$-ray was studied on 3 groups of the same age and sex. Group 1: adrenalectomized 10-15 days before exposure and maintained on 1\% saline; Group 2: normal control mice; Group 3: injected subcutaneously with glutathione $4 \mathrm{mg} / \mathrm{gm}$ mmediately before exposure. It is of interest that adrenalectomy 10 days before reduces the total body and hepatic reduced glutathione concentration significantly. The present data indicate that adrenalectomy increases the sensitivity of mice to radiation, this may be related to sulfhydryl levels of tissue. In addition this data refute in part the statement of Straube, al (Proc. Soc. Exptl. Biol. Med. 71, 539, 1949) "the radiosensitivity of intact or adrenalectomized mice, with or without exogenous adrenal cortical steroids, is similar."

Estimated Percentage Lethal Doses in $\mathrm{a}$ from Mortality Dose Curve

$\begin{array}{lccc} & \begin{array}{c}\text { Adrenalecto- } \\ \text { mized }\end{array} & \text { Control } & \begin{array}{c}\text { Glutathione } \\ \text { treated }\end{array} \\ \mathrm{LD}_{0} & <450 & 550 & 550 \\ \mathrm{LD}_{50} & <620 & 730 & 840 \\ \mathrm{LD}_{100} & <790 & 940 & >1050\end{array}$

1255

Cronkite, E. P., W. H. Chapman, and George Brecher. RELATION OF CLEARANCE AND DISTRIBUTION OF INJ ECTED GLUTATHIONE TO PROTECTION AGAINST RADIATION INJURY. Prue. Soe. Exptl. Biol. Med. 76, 456-9(1951).

It has been demonstrated by experiment that: (1) The clearance of intravenously injected reduced glutathione from the blood of dogs is rapid ( $30 \mathrm{~min}$ ). (2) The disappearance of subcutaneously injected glutathione from the body of the mouse is much slower $(>3 \mathrm{hr})$. (3) The digtribution of injected reduced glutathione is not uniform throughout the body. Of the organs studied it concentrates to a considerable extent in the liver, spleen, and kidneys.

(4) These experiments suggest that concentration of glutathione at sites vital for survival may be the prime factor in glutathione protection of the irradiated mammal. (NSA)

\section{6}

Fischer, P., L. de Landtsheer, and J. Lecomte. REDUCED GLUTATHLNE CONTENT OF THE BLOOD AND TISSUES AFTER TOTAL-BODY IRRADIATION WITH LETHAL DOSES OF X-RAYS. Bull. soe. chim. biol. 32, $1009-11(1950)$.

In mice and guinea pigs fatal $\mathrm{x}$-ray doses did not alter the reduced glutathione content of blood, liver, kidney, heart. or muscie. Parenteral administration of dilute $\mathrm{H}_{2} \mathrm{O}_{2}$ to uninradiated animals caused a decrease in reduced glutathione in the kidney but not elsewhere. (CA) 1257

Forssberg, Arne. POSSIBILITY OF PROTECTING THE LIVING ORGANISM AGANIST RÖNTGEN RAYS BY CHE MICAL MEANS. Acta Radiol. 33, 296(1850).

Altempts to protect living organisms againgt the effects of Roentgen rays are reported. Cysteine, given to bacterial cells immediately before the ir radiation, partly protects the culture from the irradiation injury. Other SH-containing chemicals act similarly, but not related chemicals without. a sulfhydryl group.

Cysteine, injected into the skin of guinea pigs, prevents epilation more or less after doses which without cysteine treatment result in epilation. (CA) 1258

Goldie, Horace, Gadson J. Tarleton, Jr., and Paul T. Mahn. FIIECT OF PRETREATMENT WITH CYSTEINE ON SURVIVAL OF MICE FXPOSED TO EXTERNAL AND INTERRAL IRRADIATION. Proc. Soc. ERptl. Biol. Med. $77,790-4$ (1951).

Mice pretreated with small doses of cysteine before irradiation with lethal doses of $x$ rays showed a high per. centage surviving 10 to 14 days after irradiation. In mice receiving high doses of cysteine the x-ray treatment was followed immediately by death or agony. The condition of mice treated with one factor alone was affected only after several days indicating a potentiation of treatment with cysteine (high doses) and $x$ irradiation. The effect of high doses of cysteine was absent in 8 -ray treated mice possessing copious peritoneal exudate due to the growth of free $\$-37$ cells in the peritoneal fluid. This action was attributed to the inactivation (oxidation) of cysteine by some factor of the peritoneal fluid. Pretreatment with small doses of cysteine had unfavorable effect on survival of mice injected with radioactive colloidal gold. A possible explanation offered is the increased cysteine penetration from the blood stream into tissues after increase of blood vessel permeability due to high doses of irradiation. (NSA) 1259

Hennessy, Thomas G. and Flening B. Folsom. THE INABILITY OF CYSTEINE TO PROTECT ERYTHROPOIETIC BONE MARROW AGAINST IRRADLATION IN- 
JURY AT A DOSE LEVEC OF $800 \mathrm{x}$. Naval Radiological Defense Lab. Nov. 6, 1950. (AD-266(B))

Studies of the radioiron, $F e^{69}$, red-blood cell uptake in rats that had received cysteine previous to $\mathrm{x}$ irradiation were conducted. Control groups of irradiated and nonirradiated rats were also studied. It is coneluded that cysteine show no protective effect against depression of erythropoiesis at the 800-r level of $x$ irradiation. (NSA) 1260

Hennessy, Thomas G., Fleming B. Folsom, and John K. Clover. THE INABILITI OF GLUTATHONE TO PROTECT ERTTYROPOIETIC BONE MARROW AGAINST IR RAOIATION INJURY AT A DOSE LEVEL OF $300 \mathrm{r}$. Naval Radiological Defense Lab. Nov. 6, 1950. $11 \mathrm{p}$. $(\mathrm{AD}-267(\mathbb{B}))$

The uptake of radioiron, $\mathrm{Fe}^{68}$, in the red blood cells of rats that had received subcutaneous injections of glutathione $0.5 \mathrm{hr}$ previous to total-body $\mathrm{x}$ irradiation was studied along with that of control groups of irradiated and nonirradiated rats. It is concluded that glutathione shows no signilicant protective effect against depression of erythropoiesis at the $300-\mathrm{r}$ level of total-body $\mathrm{x}$ irradiation. (NSA)

1261

Limperos, G. and W. A. Mosher. PROTECTION OF MCE AGAINST X-RADIATON BI THIOUREA. Science 112, 86-7(1950).

Varying anounts of thiourea were given to mice by intraperitoneal injection in a single dose five minutes before irsadiation with a $650 \mathrm{r}$ dose of $\mathrm{x}-\mathrm{rayg}$ and by administration in the drinking water in a concentration of $1 \%$ for a period of six days before lrradiation. Mice receiving thiourea had a lower mortality gate than control mice. The authors believe that thiourea and possibly other reducing agents lower the mortality due to $x$-radiation because of the protection afforded to certain vital cellular constituents 1262 such as nucleic acid. (NSA)

Loiselews, Jean and Georges Velley. CURATIVI TREATMENT OF RADIOLESIONS RESULTING FROM THE ADMINISTRATION OF LITHAL DOSES OF X-RAYS. Compt. rend. $231,529-31(1950)$.

From a brief analysis of phenomena taking place in cells collowing lethal doses of $\mathrm{x}$-rays, the hypothesis was made that prompt administration of oxygen acceptors capable of penetreating the cells and reducing the dissolved oxygen would be a curative treatment for radiation injury. Eighteen rabbits were exposed to $950 \mathrm{r}$ of whole-body $\mathrm{x}$-irradiation, and ten of these were immediately injected intraperitoneally with 20 ce of a solution of cysteine and ascorbic acid. Additional 10 ce doses were injected after 30 minutes and 2 hours. Six of the ten survived, are in good health, and have galned weight 2 to 4 months after the irradiations. All the untreated controls died 5 to 28 days after receiving the irradiation. (NSA)

1263

Mole, R. R., J. St. L. Philpot, and G. R. V. Hodges. REDUCTION IN LITISAC EFIECT OF X-RADUTION BY PRE'IRREATMENT WITH THROUREA OR SODIUM ETHANE DITPIOPROSPEONATE. Natwe 166, 515(1950).

A few substances, selected in the hope that they would enter cells without being too toxic or too rapidly metabolized, and would protect against radiation damage by competing for hydroxyl radicals, were administered intraperitoneally to mice a few minutes belore or aster x-irradiation. Saline solution was used as a control. The protective effecte of thiourea and dithiophosphonate were highly sigsificant, those of glycerol barely so. Thiourea given im- mediately after the irradiation was ineffective, and saline was not deleterious. (NSA)

\section{4}

Patt, Harvey M., Margared E. Blackford, Robert L. Straube, and Douglas E. Smith. THE EFECT OF $\mathrm{K}$ RAYS ON THYMOCYTES AND ITS MODIFICATION BY CHEMICAL MEANS. Am. J. Physiol. 167, 815(1051). (AECU-1557; UAC-409)

The report is reproduced here in its entirety.

X radiation decreases the life span of rabbit thymocyles in vitro, the percentage of surviving cells (eosin resistant) decreasing exponentially with increasing radiation dosage. The $\mathrm{LD}_{50}$ at $24 \mathrm{hr}$ is $150 \mathrm{r}$ and definite effects appear with $50 \mathrm{x}$. Sensitivity is decreased by a factor of 2 over the range of 50 to $1500 \mathrm{r}$ when cysteine $(0.02 \mathrm{M})$ is added to the cell suspension before irradiation. The small reduction in toxicity which occurs when cysteine is added after posure may be due in part to persistence of a toxic substance in the medium, as well as to reversal of a chain of radiation action, since ir radiation of only the medium (serum-phosphate buffer) is slightly toxic to cells added subsequently. Anoxic anoxia and cysteine afford comparable protection to thymocytes irradiated with $200 \mathrm{r}$ but they are not additive under these conditions. Packed cells are less radiosensitive than cells suspended in serum and are not protected by cysteine or anoxia. This may be at tributed perhaps to the fact that packed cells are already anoxic since cells exposed to oxygen before centrifugation are as sensitive as cells in suspension. These findings suggest that at least two mechanisms are concerned in the cytocidal action of $x$ rays, one of which is subject to the influence of cysteine and anoxia. It is assumed that these factors act on toxic chemical intermediates that are formed during irradiation, though not necessarily in the same manner. Aspecific metabolic action cannot yet be ruled out, however. (NSA)

1265

Patt, Harvey M., Douglas E. Smith, Margaret E. Blackford, and pobert L. Straube. PROGRESS REPORT; THE EFFECT OF X RAYS ON THE OXIDIZABLE SULFHYDRYL GROUPS OF RATS RECEIVING CYSTEINE, p.75-7 of BIOLOGICAL AND MEDICAL DIVISIONS QUARTERLY RE PORT, NOVEMBER, DECEMBRR, JANUARY, 1949-50. Argonne National Lab. [nd] 3p. (ANL-4401(p.75-7))

The oxidizable sulfhydryl content of tissues obtained from irradiated $(800 \mathrm{x}$, total-body) and nonirradiated rats reeeiving cysteine has been compared. Cysteine 1950 $\mathrm{mg} / \mathrm{kg}$ intravenous) increased the sulfhydryl content of serum, kidney, thymus, small intestine, and liver. Similar increases were noted in cysteine-pretreated $\mathrm{x}$-irradiated rats. X radiation had little effect on the oxidizable sulf hydryls of the control-irradiated animals. The studies suggest that cysteine apparently does not protect against x radiation because it acts as a substrate for oxidation by the free radicals that are presumably formed durng exposure. (NSA)

1266

Patt, Harvey M., Douglas $\mathbb{R}$. Smith, and Eugenia Jackson. THE ETFECT OF CYSTEINE ON TIE PERIPHERAL BLOOD OF THE IRRADIATED RAT. Blood $\underline{5}, 758,-63$ (1950).

A single intraverous injection of cysteine $(950 \mathrm{mg} / \mathrm{kg}$ ) five minutes before tolal-body exposure of rats to $x-r a y s$ $(800 \mathrm{x}$ ) significantly modified the radiation-induced hematologic changes. Depression of the heterophils, Iymphocytes, and erythrocytes was less severe and recovery more rapid. The hematologic observations are consistent with the thesis that cysteine reduces the biological effer tiveness of the radiation. (NSA) 
$126 \%$

Patt, Harvey M., Douglas $\mathrm{E}$. Smith, Ella B. Tyree, and Robert L. Straube. FURTHER STUDIES ON TME MODIFICATION OF SENSITIVITY TO X-RAYS BY CYSTEINE. I. TIME COURSE OF THE PROTECTION AND COMPARISON OF CERTAIN RELATED SUBSTANCES. PrOC. Soc. Exptl. Biol. Med. 73, 18-21(1950). (AECU-599: $\mathbb{U A C}-148$ )

Cysteine $(950 \mathrm{mg} / \mathrm{kg}$, neutralized 1. V.) greatly reduces sensitivity of rats to total-body $\mathrm{x}$-irradiation $(800 \mathrm{r}, 250$ kv), provided that the amino acid is given before the exposure. Comparable protection is obtained when cysteine is administered intravenously immediately or one hour before irradiation (87\% survival of 79 cysteine irradiated; $16 \%$ survival of 82 irradiated controls). Injection immediately after exposure or 6 or 24 hours before is without influence. Oral cysteine $(1,900 \mathrm{mg} / \mathrm{kg})$ affords a significant protection when given $30-60$ minutes prior to $\mathrm{x}$-irradiation. However. oral administration is not as efficient as intravenous. Cystine, unlike iss reduced counterpart, cysteine, is not fective by either route. Glutathione, on the other hand, can reduce $x-$ ray toxicity but only when it is given intravenously before exposure. Oxal glutathione does not protect rats or mice even though the oral dose is equivalent to the effective oral doses of cysteine in sulfhydryl content. Methionine, ascorbic acid, and sodium sulfide do not alter survival after lethal $x$-irradiation. Results with colloidal sulfur are equivocal and iurther work is necessary. These findings suggest fir that the critical reactions leading to morbidity are complete when the irradiation is terminated and that these reactions are apparently not reversible. Secondly, a sulfhydryl group distinguishes the substances which can protect from those which cannot. It is important to bear in mind, however, that all sulfhydryl-contaning materials may not necessarily protect and that other reducing substances which are properly distributed, temporally and spatially, may. (NSA)

1268

Patt, Harvey M., Douglas $\mathrm{E}$. Smith, Margaret E. Blackford, and Pobert L. Straube. NATURE OF CYSTEINE INDUCED RADIORESISTANCE: SULF HYDRY LEVILS AND DISTRIBUTION OF CYSTEINE SULPUK. AM. $J$. Physiol. 163, 740-1(1950).

The report is reproduced here in its entirety.

Cysteine may increase radioresistance by: 1) decreasing (ree radical production, 2) competing directly for oxidizing free radicals, 3) shifting the redox potential of critical cell elements, thus protecting them against oxidation, and 4) altering metabolic pathways. Assuming selective action on sulfhydryl groups by products of irradiated water, less than 1\% of nomprotein-SH (as cysteine) could theoretically be oxidized when a $200-\mathrm{gm}$ rat is exposed to $800 \mathrm{r}$. $\mathrm{X}$-ir radiation had no immediate observable afect on -\$IL levels in control and eysteine rats, although cysteine per se (950 $\mathrm{mg} / \mathrm{kg}$ intravenougly) increased the oxidizable - \$n content of TCA filtrates of serum, kidney, thymus, intestine, and liver. Rather identical retention of $\mathrm{S}^{35}$ was noted in blood of rats receiving labeled cystine, cysteine, or cysteine followed by $\mathrm{X}$-irradiation, $(60 \mathrm{mg}$ cystine or cysteine, $5 \times 10^{5}$ counts/min. intravenously). Uptake of $\mathbb{s}^{35}$ by liver and intestine was more rapid in cysteine injected rats. $\mathrm{X}$-radiation appeared to decrease rate of uptake and retention. The intial concentration of $\mathrm{S}^{35}$ in bone marrow was similar in cystine and cysteine animals but fell sharply during the first hour in the latter. Marrow uptake was diminished by irradiation. No clear relationship between $\mathbb{S}^{35}$ concentration in these tissues and the time course of cysteine protection was evident. Our data do not support the concept of direct or indirect competition by cysteine for oxidizing radicals. If guch a mechanism is operating, oxidation must be localized in specific sites where -\$H or other essential substances are few and their inactivation critical. 1269

Pat, Harvey M.g Ella B. Tyree, Robert L. Straube, and Doumlas E. Smith. CYSTENE PROTBCTION AGAINST X-IRRADIATION. Science 110, 213-14(1049).

Some observations concerning the influence of cysteine and cystine on the gadiosensilivity of rats are reported.

Brief exposures to intense irradiation were employed and the radiation factors were a $250 \mathrm{kv}$ machine delivering radiations through $0.5 \mathrm{~mm} \mathrm{Cu}$ and $3.0 \mathrm{~mm}$ Bakelite filterg at a target distance of $27.5 \mathrm{~cm}$ and at a dowage rate of 210 $\mathrm{r} / \mathrm{min}$. Male and female rats weighing $150-250 \mathrm{~g}$ gecelved 800 of total-body $x$-radiation in a single exposure. Cysteine was injected into the tail vein one hour belore, 5 minutes before, or five minutes after the exposure. Irour different doses of cysteine were compared $(175,350,575$, and $875 \mathrm{mg}$ of cysteine/ $\mathrm{kg}$ body weight), and a $20 \%$ solution was used, the cystense being injected slowly over a period of minutes; ir radiated controls were injected with an equivalent volume of aline solution. The reaults show that pretreatment with cysteine markedly geduced the toxicity from lotal-body $\mathrm{X}$-1 ir radiation oince there was an $82 \%$ survival from the treated group as compared with a $19 \%$ survival from the controls. Cystine, however, did not influence the survival rate. There were variation in the degree of protection per gram of cysteine depending on the pll of the administered solution; the injection of cysteine immediately after exposure was ineffectual. It is concluded that cyøteine, but not cystine, administered to rats prior to 8 irradiation in the nearly completely lethal range, greatly diminishes loxicity. (NSA)

1270

Rosenthal, Robert L., Leontne Goldschmid, and Bryant I. Pichering. HEMATOLOGIC CHANGSS IN RATS RRO-

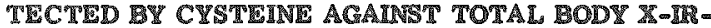

RADIATION. Am. J. Physiol. 166, 15-19(1051).

(AD-215(B))

Jollowing 800 r total body z-irgadiation, cysteineprotected rats showed significantly higher neutrophil valuea in the blood, and more myeloid cells of all stages in the bone marrow, than did aline-injected ratr. The pogm wible aignificance of these indings in relation to mortality and infection is discussed. (NSA)

1271

Smith, D. E., Harvey M. Patt, and EIla B. Tyrea. PROGRESS REPORT: NOTYS ON 'THE IEVALUATON OF' MATERALS TEAT MICHT BE EXPRCTED TO ALTER RADIOSENSITIVITY, $p .72 \sim 4$ of BIOLOGICAL AND MIEDICAL DIVISIONS QUARTLELY REPORT, NOVIMBER, DECEMBER, JANUARY, 1949-50. Argone National

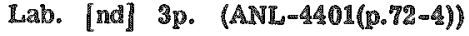

Thirty-day survival, time to $50 \%$ mortality, and average survival time of rats subjected to a single total-body exposure of $1000 \mathrm{~g}$ were taken as criteria of the capacity of materials to alter sensitivity to s radiation. Of materials so tested, BAL and cysteine $+\mathbb{K C N}$ administered prior to ir radiation significantly decreased radiosensitivity; while thiamine-HCl, L-taurine, and tyrosine were withoul elfect. Moreover, cysteine + KCN reduced radiosensitivity to the same degree as the same dose of cysteme alone, indicating that cysteine owes its protective ability to factors other than its reducing powers. (NSA)

\section{2}

Smith, Douglas E., Rarvey M. Pat, Ella B. Tyree, and Robert L. Straube. FURTHER STUDIES ON MOORI 
CATION OF SENSTTIVITY TO X-RAYS BY CYSTEINE. Proc. Soc. Exptl. Blol. Med. 73, 18-21(1950).

Cysteine greatly reduces the sensitivity of rats and mice to lethal amounts of $\mathrm{x}$-rays delivered at either high or low dose rates provided that the anino acid is given before the exposure. Rather comparable protection is obtained when cysteine is injected intravenously in rats immediately or one hour beiore irradiation with $800 \mathrm{r}(90 \%$ reduction in the 28-day mortality). Injection immediately after exposure or 6 or $24 \mathrm{hr}$ before is vithout influence. Cygteine also $\mathrm{hm}$ proves survival significantly when it is given orally 30 to 60 minutes prior to the irradiation. Glutathione (IV but not oral) can also diminish radiation toxicity. Cystine, methionine, ascorbic acid, and sodium sulfide do not alter survival of the irradiated animal. Results with colloidal sulfur are equivocal. The possible significance of these obserwations is discussed. (Author's Summary)

1273

Smith, D. E., H. M. Patt, E. B. Tyree, and R. L. Straube. QUANTITATIVE ASPECTS OF THI PROTECTVV ACFION OF CYSTEWN AGAINST X-RADIATION. Proc. Soc. 嫼xptL. B10l. Med. 73, 198-200(1950).

Three groups of male Sprague-Dawley rats received a $10 \%$ solution of cysteine intravenously and within five minutes after the dose were irradiated by whole body exposure at $250 \mathrm{kv}$ through a filter of $0.5 \mathrm{~mm}$ Cu and $3.0 \mathrm{~mm}$ Balrelite at a target distance of $26.5 \mathrm{~cm}$ with a dose rate of 210 $220 \mathrm{~g} / \mathrm{min}$. Each group had controls and they were divided into groups as follows: $50-950 \mathrm{mg} / \mathrm{kg}$ of cysteine was given in graduated doses to test protection from $800 \mathrm{r}$ and 1,000 19; the protective value of $950 \mathrm{mg} / \mathrm{kg}$ of cysteine was determined for graduated doses from $600-1,800 \mathrm{x}$; a dual injection composed of one dose of $475 \mathrm{gng} / \mathrm{kg}$ followed in $45-$ 60 minutes by a second dose of $950 \mathrm{mg} / \mathrm{kg}$ was given and then the animals were irradiated as belore with a total dose of 1,200 1P. Results are briefly given and discussed and the authors conclude that the protection given by cysteine is roughly proportional to the amount of cysteine given. (NSA)

\section{4}

Straube, Robert L., George Svihla, Margaret I. Blackford, and Harvey M. Patt. IFIFCT OF CESTEINE ON THL RADIOSENSITVITY OF THYMC CELLS IN VITRO. Am. J. Physiol. 167, 829(1051) (AECU-1532; UAC-407)

The report is reproduced here in its entirety.

The effect of cysteine on the radiosensitivity of thymic cells incubated in vitro has been studied cinemaphotographically. Normal thymic cultures and cultures to which cystine has been added, as well as irradiated cultures, are Shown for comparian, both immediately and 20 to 24 hr lollowing preparation. Cysteine (0.02M) does not alter the activity of normal cells. Motility, protoplasmic streaming, pseudopod extension, nuclear granulization, and vacuolization, occur to the same degree in both preparations. Loss of motility and rounding up of the cells to form indisunctly granular spheres, with occasional nuclear vacuolization, is usually preeeded by a flurey of activity. Such gpherical, quiescent cells stain with eosin or safranin. More quiescent cells are found after incubation for 20 to $24 \mathrm{hr}$ in all preparations. Since the mature thymic cells almost never divide, population reduction due to cellular genescence and death is a constant factor. Irradiation of a thymic suspension with $1000 \mathrm{r}$ greatly reduces the number of cells surviving at 20 to $24 \mathrm{hr}$. The majority of surviving cells, anlike those in the cystine 1000-r series, are nonmotile. Thus, on a qualitative basis, control cells and cells to which cystine has been added are essentially similar at both 0 and $24 \mathrm{hr}$. Control irradiated cells and cystine irc- radiated cells are similar at $0 \mathrm{hr}$, but at $24 \mathrm{hr}$ preparations of the latter contain almost as many motile forms as the normal, whereas those of the former show predominantly quiescent forms. (NSA) 1275

Works, Lee, John H. Grotts, and Gordon W. Raleigh. EFFECT OF BAL ON RADIATION MOETALITY RATE IN MICE. Quart. Bull. Northwestern Univ. Med. School 24, 295-6(1950).

A group of 66 mice irradiated with $550 \mathrm{r}$ of 210 -kvp x rays showed a $5 \%$ survival rate at the end of 5 weeks, in contrast to a $39 \%$ survival rate in a group of 61 animals of the same strain, each of which had received a subcutaneous dose of $2.6 \mathrm{mg}$ of BAL (2,3-dimercaptopropanol) $1 \mathrm{hr}$ prior to the ixradiation. A $94 \%$ survival rate was found in mice subjected to the BAL treatment only. (NSA)

\section{Surghcal Treatment}

\section{6}

Brown, James Barreti, Frank MeDowell, and Minot $P$. Iryer. RADIATION BURNS, INCLUDING VOCATIONAL AND ATOMIC EXPOSURES. TREATMENT, AND SURGICAL PREVENTION OF CHRONIC LESIONS. Ann. Surg. $130,593-607(1949)$.

Acute and chronic radiation burns and their treatment are discussed. For the treatment of the acute burns, such as those caused by single, laxge exposures, atomic radiation and the like, rest, sedation, prevention of infection, and alleviation of the severe pain are advised, as well as grafting of open wounds as soon as possible. Chronic burns, commonly seen on the nands of radiologists and due to repeated exposures over a long period of time to small amounts of radiation, are treated by excision and repair of the skin defeets with grafts or flaps; the treatment should be done in the early or the quiescent stages, rather than in the ulcerated stage. The authors conclude that the pathology of atomic radiation burne is probably similar to that of the burns under discussion and the same types of therapy will be beneficial; chronic skin lesions in heavily irradiated areas may be prevented by excision and grafting before the chronic changes (coagulation, atrophy, endarteritis of the small arterioles, telangiectasis and similar phenomena) oceur. (NSA)

\section{$127 \%$}

Brown, James Barrett, Frank McDowell, and Minot P. Fryer. SURGICAL TREATMENT OR RADLTION BURNS. Surg. Gynecol. and Obstet. 88, 609-22(1949).

The treatrnent by excision and grafting of burns due to excessive radiation on normal skin and regions with acne, oczema, and other abnormalities is described. Pathological effects of the radiation, such as loss of appendages of gkin and obliteration of blood vessels, are noted, and specif ic treatment in these cases is detailed. The symptoms of severe burng are excessîve pain, swelling, weeping slsin, and possible sloughing of the epidermis. Treatment for this type of burn and for chronic improvement-resigting burns is, and should be, entirely surgical. Anatomical regions most commonly affected are described separately. In special cases immediate flap closure, delayed pedicle closure, or pedicle grafts are called for instead of the regular procedure. Surgery becomes necessary frequently to prevent the development of carcinoma in the affected areas. (NSA)

1278

Greeley, Paul W. RECONSTRUCTION FOLLOWINC IRRADIATION INJURES. Arch. Surg. 56, 741-9(1948). 
Although present knowledge of $\mathrm{x}-\mathrm{ray}$ and radium therapy is extensive, a surprisingly large number of irradiation injuries still occur. They result from incidental reactions following treatment of deep lesions and mismanagement by untrained or inexperienced operators. It is emphasized that all types of treatment by $\mathrm{x}-\mathrm{rays}$ under disguised names and usually in the hands of lay operators are a serious menace to the public. X-rays produce irreversible reactions that are characterized by severe pain, itching, ulceration, and eventual malignant degeneration. These symptoms can be relieved promptly and permanently by wide surgical excision. The rewulting defects are then closed by one of several different plastic surgical procedures. (NSA)

\section{9}

Huff, R. L., W. F. Bethard, J. F. Garcia, B. M. Roberts, L. O. Jacobson, and J. H. Lawrence. THE $\mathbb{E F F}$ FCT OF SPLEEN PROTECTION ON IRON DISTRIBUTION IN IRRADIATED RATS. J. Lab. Clin. Med. 36, 40-51(1950) (ANL-A401(p.33-4))

Eighty female Curtis-Dunning rats were divided into 4 groups treated as follows: (I) maintained as normal controls, (II) surgical mobilization of the spleen, but no radiation, (III) single total-body egposure to sr radiation, as well as a sham operation in which the spleens were mobilimed but not protected, and (IV) irradiated with the mobilized spleen in a lead chamber. Each of the s-irradiated animals received $500 \mathrm{r}$. Three days thereafter, and at comparable times for controls, each rat received intravenously approximately $10,000 \mathrm{cpm}$ of $1 \mathrm{e}^{50}$. Bone marrow of groups I and II contained $60 \%$ of injected Fe $\mathrm{e}^{59}$ within 3 hr after injection. Similar curves for groups III and IV yielded maximum uptakes of 11 and $16 \%$, respectively. Unespect edly, the bone marrow in group IV animals showed significantly greater function than that in group III. Uptake of $1 \mathrm{e}^{59}$ in liver was greatest in group III $\left(56^{\circ}\right)$. Groups I, II, and IV gave values of 15,15 , and $43 \%$ in the order given. The uptake of $F^{59}$ in spleen was nearly equal $(1 \%)$ for groups I, II, and III. In the irradiated, spleen-protected animals, however, $7 \%$ of the injected tracer was found in the spleen. (NSA)

\section{0}

Jacobson, Leon O., Edna K. Marks, Evelyn Gaston, Melba J. Robson, and Raymond T. Zirkle. THP ROLE OF THE SPLEEN IN RADIATION INJURY. Proc. Soc. Exptl. Biol. Med. 70, 740-2(1949).

A technique different but related to that previously described by Jacobson at anat. Recoral 100, 1948; National Nuclear Energy Series Div. IV Vol. 22B) for the study of the significance of the spleen in recovery from or compensation for radiation injury is presented. The materials and methods are described in detail. Radiation consisted of $600 \mathrm{r}$ whole-body $\mathrm{x}-\mathrm{radiation}$ administered to mice with or without their spleens protected by lead boxes covering all but the pedicles. Hematologic and histopathologic studies were carried out at intervals. The mean hemoglobin, erythrocyle, and hematocrit values of the lead-protected mice were not significantly altered while marked reduction in the umprotected mice was noted. The mean platelet and reticulocyte values of the unprotected group also fell appreciably. These data indicale that severe anemia, leucopenia, and thrombocytopenia develop in mice after a single dose of $600 \mathrm{r}$ whole-lody $\mathrm{x}$-radiation. cytopoiesis, in the lead-protected spleens compensates with such rapidity and so extensively for the destruction and Interruption of this activity in the marrow spaces that no anemia of significance hecomes apparent. Ectopic Franulocytopoiests and megaknryocytopoiesis in the leadprotected spleens compensates significantly but at alower pace and less completely for the bone marrow destruction. A marked and sustained decrease in the amount of lymphatic tissue is produced in the lead-protected spleens. This decrease in lymphatic tissue may perhaps be a result of (a) unsuccessful competition of the lymphatic tissue with the ectopic hematopoiesis for nutritional requirements, $(b)$ actual indirect effect of radiation, and (c) a differential humoral suppression from some unknown site. This lechnique provides a method of studying potential sites and mechanisms of the production of ectopic blood formation, possible secondary effects of radiation as vell as of fering possibililies for determining the potential role of such sites in immune reactions, in preventing or alleviating radiationinduced hemorrhagic phenomena and in the study of survival or recovery from radiation injuxy. (NSA) 1281

Jacobson, Leon O., Jite I. Simmons, W. Bethard,

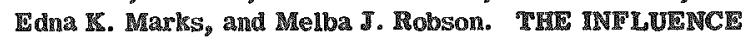
OF THE SPLER ON HEMATOPOIETC RECOVIRY APTER IRRADLATON INJURE. Proc. Soc. Expt. BLlol. Med. 73, 455-9(1950).

Young adult female mice were exposed to 1,025 wholebody 8 -irradiation delivered by a $250 \mathrm{mv}$ source through an 0.25 mm Cu filter. The spleens of these mice were mobilized surgically through an abdominal incision and leadprotected during irradiation in one group and mobilized but not lead-protected during irsadiation in another group. The bone marrow and lymphatic tissues were dostroyed, and no regeneration was apparent on the tenth day in the amimals irradiated without lead protection of the spleen. In the animals with lead protection of the gpleen during irradiation, these tissues were not depleted of free hematopoietic cells, and the bone marrow and the lymph nodes were normal in cellularity eight days after irradiation. Several possible interpretations of the role of the spleen under the conditions of these experiments are ofiacussed. (NSA) 1282

Jacobson, Leon O., Eric Is. Simmons, Zdna J. H. EIdredge. RECONERY HOM RADLITON WJUEY. Science 113, 510-11(1951).

An attempt has been made to clarify some of the factors involved in the greatly increased $\mathrm{LD}_{\mathrm{g}}$ of opleen- or livershielded mice (J. Lab. Clin. Med. 34, 740(1949) and 35, 746(1950)). The results indicate that the factor involved in the shielding or transplant experiments is definitely effective after irradiation, and that the factor responsible for recovery from radiation under these condilions is frobably a substance of a noncellular nature. (NSA) 1239

Jacobson, Leon 0., Eric I. SImmons, Edna R. Mariss, Lvelyn O. Gaston, Melba J. Robson, and J. M. Eldredge. FURTRER STUDIES ON RECOVERY FROM RADIATION INIURY. T. Lab. Clin. Med. 37, 683-97(1951).

The 28 -day $\mathrm{LD}_{50}$ for spleen-8hielded mice is $\sim 1100 \mathrm{x}$ of whole-body x-radiation compared to $\sim 550$ z for mice without spleen shielding. Unlike glutathione, cysteine, $\mathrm{O}_{2}$ deprivation, or cyanide intoxication, the "factor" in spleen shielding which effects a significant increase in survival of mice from whole-body $\mathrm{z}-\mathrm{r} 2 \mathrm{~d}$ iation is unnecessary dusing the actual irradiation process and is definitely offective after irradiation. In these experiments the general assumption is made either that (1) the shielded tissues produce a substance of a noncellular nature which exerts a beneficinl effect on the recovery of the irradiated aninal or (2) that cells migrate from the shielded tissue which enhance recovery of the irradiated tiswes. The evidence appears to be in favor of the hirst assumption. The potential significance of these observations to the therapy of radiation injury and other climical syndromea is discussed briefly. (NSA) 
1284

Jacobson, Leon O., Eric L. Summons, Edna $\mathbb{K}$. Marks, Melba J. Robson, W. B. Bethaxd, and Evelyn O. Gaston THE ROLE OF THE SPLEEN IN RADIATION INJURY AND RECOVERY. J. Lab. Cln. Med. 35, TA6-70(1050).

In a study to compare the effect of $1,025 \mathrm{r}$ on the blood and blood-formmg tissue of mice wheh had had lead protection of the surgucally moblized spleen durmg radiation with the effect of this dose on mice without lead protection, 165 female CF-1 mice aged 10 to 12 weeks were divided 1nto tour groups, including one control group of 40 anmals and three other groups which were prepared by surgucal mobilization of the spleen under nembutal anaesthesia Two of the three groups received X-ray doses with urradiation delivered by a $250-k v$ machine through a $025 \mathrm{~mm}$ Cu illter, with a dose rate of $53.2 \mathrm{r} / \mathrm{min}$ at $55 \mathrm{~cm}$, hematologacal, gross and microscopic tisgue examnations, and determkations of body weyght at various intervals were done The data and results are extensively presented an graphs, Lables, and plates; they are thoroughly diseussed. (NSA) 1285

Lorens, Egon, Delta Uphoff, T. R. Rerd, and Emma Shelton. MODIFICATION OF IRRADIATION INYURY IN MICE AND CUINEA PIGS BY BONE MARROW INJECTIONS. J. Nath. Cancer Inst. 12, 197-201(1951).

Data indicate that bone marrow imected either intravenougly or intraperitoneally posturradiation grves excellent protection to mice and gunea pigs from the acute irradiation syndrome. (NSA) 1218

Mason, Michael L. THE SURGICAL TREATMENT OF IRAADLAION INJURIES. Munois Med. J.' 95, 20-6(1949).

The frve large groups into which irradiation unjuries of the skun and subcutaneous tussues may be grouped are reviewed. Examples are given of nujury treatment which consists in removal of all involved skn and plastic repan of the resultant defect. Additional urritants, particularly $1 r^{-}$ radiation of any sort, must be avosded since they unerease the severity of the process and delay surgical repan (NSA)

$128 \%$

Reler:, Paul. TRANSPLANTATION OF BONE MARROW INTO DOGS THAT HAVE RECFIVED TOTAL BODY SIMGLIZ DOSE RADATION. University of Rochester. [nd] 55. (ARCD-1266; UR-11)

The major results of this paper are condensed in Rekers, Coulter and Warren (Arch. Surg 60, 635-67 (1950)). See abstract 1348

1288

Robins On, Davd W. THE SURGICAL RERAIR OF IRRADIATION DERECTS. J. Kansas Med. 5̃oc. $49,456-61$ (1048).

The types of unjuries caused by urradration in dufferent tismea are brefly descrabed and the surgical repar techniques for different structures and different locations are discussed Cages are classified with respect to etrology as follows: (1) accidental $x$-ray burns of physicians, (2) 1njury from intentional massive dosage for neoplasm, (3) late change from repeated small dosage given for superficial therapy, and (4) burns to patuents from prolonged exposure under the fluoroscope The repar of njuries 19 deseribed in reports of llustrative cases for each etrologic group (NSA)

1289

SImmons, ErIC L. PRELIMINARY REPORT; FURTHER \$TUDIES OF THE PROTECTVIE EFTECTS OF SHIRLDING, D.27 Of DIVISION OF BIOLOGICAL AND MEDICAL
RESEARCH QUARTERLY REPORT, MAY, JUNE, JULY, 1950. Axgonne National Lab. July 1950. 1p. (ANL$4488(p .27))$

A brief report states that data from experuments show that a marked nncrease in survival occurs in both mile and female rats when the inlestune is surgically mobrlized and lead-protected durung total-body $x$ ar radiation Intual studies of survival of the $\mathrm{x}-1 \mathrm{rradated}$, spleen-shelded rat show less posituve results than those obtaned when mice were irydiated with spleen protection preliminary experiments have falled to show any increased capacity of the spleen to ald survival following $x$ arradiation when priming doses of 100,200 , or 300 r were given belore $800 \mathrm{~g}$ However, there was an uncrease in morlality with uncrease in the total dose admumstered (NSA)

1290

Stewenson, Thoms W SURGICAL TREATMENT OF XRAY BURNS. Rocky Mt. Med. J. 46, 198-200(1949).

There are two types of deep ulceration following $\mathbf{a r}-$ radiation In the acute type the mitial erythema progresse rapidly, vesicles appear, and later the skm becomes necrotic The sloughng of of necrotic tissue 18 greatly retarded and may not occur for several months Granulation thssue falls to appear and no sign of marginal epithellalızation 15 seen. The retarded slough and lack of granulation thssue and epithelialization distunguish this lesion from any other form of traumatic wound. Pan is usually a strikng feature, the slightest drying of the surface or pressure of dressung causing extreme discomfort. In the chronic form, ulceration may take place in an area wheh had remaned healed for several vears. This usually results from progressive fibricosis and consequent reduction in local blood supply, which eventually leads to ischeme necrosis. Intense pan is also a feature of this type of ulceration. Complete excision of the unvolved area with skin graftung is the treatment of chore. In the author's experience the source of the largest group of superficial injuries was the treatment of hypertrichosis, fracture reduction, and varıous dermatologie conditions. (RA)

1291

Talbot, John M. BONE MARROW IN THE TREATMENT OF RADLATION INJURY, p.125 Of INTERNATIONAL CONGRESS OF RADIOLOGY, 6th Congress, London. 1850.

An untial group of rats with bone marrows depressed by benzene recelved intrahepatic, intramuscular, and subcutaneous anjections of fresh femoral marrow from anmals of the same stran Healthy marrow growths were found an the livers at $\$ 1 x$, fourteen, and twenty-31x days A second serics recenved acute $\mathrm{LD}_{50}$ doses of whole body $\mathrm{X}$-radiation with trbaae, fibulae, and about one-half of each femus" shrelded by lead Total WBC and RBC showed significantly less depression during the two weeks' observation periods than did the unshelded controls Deaths occurred in both the whelded and unshelded anmals Followng acute $\mathrm{LD}_{50}$ doses of total body X-radiation two serues of rats were grven unt $r$ thepatic and intramuscular mections of approxnmately $005 \mathrm{cc}$ of fresh femoral bone marrow from donors of the same stran Healthy survvral of the mplants was found in anmals sacruficed at seven, and at fourteen days No significant changes were noted in peripheral blood puctures and survival rates Another serses with arute radiatron injury was treated with two or more intramuscular umections of fresh femoral marrow Blood pictures and survival rates in this series did not differ sugnificantly from those of controls 
1292

T'albot, John M. and Ernest A. Pinson. THE TXPERTMFNIAL USE OF BONE MARTOW IN ACUTE RADHATION INUURX. Miltary Surgeon 108, 412-17(1951).

Studies of bone marrow homotransplants in rats whose marrows were depressed by benzene injections and by whole body $x$ ir survive in a reasonably healthy condition for an approximate minimum of 4 weeks. Bone mar row given intramuscularly following acute, whole-body ionizing radiation injury in the lethal and sublethal ranges had no significant effects upon survival, changes in total white blood cell counts, and changes in body weights. Shielding of $1 / 3$ to $3 / 4$ of the hind limbs of rats produced significant differences in Survival and weight logs as compared to the transplanted group. (NSA)

Vitamin Studies

1293

Adams, WILiam S. and John S. Lawrence. THE NGCATVE EIECT OF FOLIC ACID ON IRPADIATION LEUKOPENL IN THE CAT. Am. J. Med. Sc1. 216, 656-60 (1948).

The prophylactic and therapentic administration of folic acid to cats did not alter the occurrence or the magnitude of leukopenia caused by exposure to 200 r whole body irradiation. (NSA)

1294

Ames, Stanley R., J. G. Baster, and T.Q.Griffith, J". PREVENTION BY A - ALPLA TOCOPHEROL OI INCREASPD CAPILLARY FRAGILITY IN RATS FOLLOW ING IRRADITION. Intern. Z. Vitaminiorsch. 22, $401-7$ (1951).

Each of 30 adult rats was given 1 cc of 200 microcuries per cc. Rn ontment intraperitoneally: 10 were controls; each of 10 was Injected daly with 0.11 ce o-tocopherol concentrate containing $34.6 \%$ total tocopherols, of which $61 \%$ was $\alpha$, the remainder $\gamma$ and $\delta$; each of 10 was injected daily with 0.55 ce of a concentrate containing $72.4 \%$ total locopherols which ware $50 \% \gamma$ and $50 \% 6$. Al the end of 15 days, capillary fragility was deternined by the positive pressure method. Both concentrates apparenty of iered significant protection against the increased irgility produced by Rn. However, multiple, apparently sterile, subcutaneous abscesses developed at the point of injection of the concentrate. When adult sats were given $0.5 \mathrm{cc}$ of $80 \mathrm{mi}-$ crocuries per ce Rn ointment intraperitoneally, and $\alpha$ tocopherol in olive oil was administered by mouth at a level of 2.5 or $10 \mathrm{mg}$ daily for 10 days, the incidence of increased capillary fragility was reduced; the loweg dose of tocopherol was more effective than the highes. (CA) 1295

Artom, Camillo, W. E. Cornatzer, and George T. Sarrell, Tr. THE PROTECTIVE ROLF OF PYRIDOXTN AGAINST THE TOXIC EIFECTS OF $\mathrm{P}^{32}$. Bowman Gray school of Medicine, Wake Forest Coll. [nd] 1p. (ARCU-1811)

In a continuation of our previous atudies on the ef ects of internal radiation by $\mathrm{P} 32$ as related to various dietary factors, the role of pyridown has now been investigated. Mice placed on various experimental dists and receiving a mixture of $\mathrm{B}$ vilamins without pyridoxim were injected with a sighle dose of radioactive phosphate (5-6 uc/g). In the groups on low-protein diets (in which the vitamin deficiency Was presumably slight or not yet developed) the administration of pyridoxin did not affect the survival of the animals. On the contrary a marked protection by pyridoxin was observed in experiments in which the vitamin def ciency was made more severe by adding a pyrdoxin analog (desoxypyridoxin) to the low-protein diet, of by increasing the metabolic demand for the vitamin (high-protein diets with added cystine or methonine). Fon inatance, in the groups recelving pyridoxin together with its analog, the day of $50 \%$ deaths was shifted from the 14 th to beyond the 42 mo, and average time of survival from 15 to 33 days, and the proportion of aurvivors at the end of the $3 r d$ week from 12 to $77 \%$. These differences are larger than those which might result from a mimple addivive cfect of internal radi ation and vitamin delicieney. (Abstract of paper for Mllanta Meeting of Southeastern Section of the Society for Experimental Biology and Medicine, Winter, 1952.) (NSA) 1298

Carter, Robent R., Flizabath Busch, and Verda Strang.

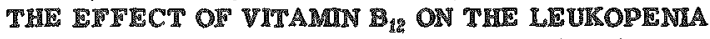
INDUCED BY RAOLATON. BLOO $5,753-7$ (1950).

(AECU-A16; $\mathrm{AADC}-699$ )

Crystalline vitamin $B_{12}$ administered via intramuscular injection, in single and in multiple doses, produced no effect on the leukopenia induced in rats by $400 \mathrm{x}$ of $250 \mathrm{kv}$ X-2ays. (NSA)

\section{7}

Conatzex, W. 哭, Camilo Artom, George T. Marel, Ir,

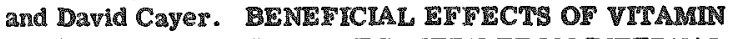
$D_{12}$ AND NOLIC ACID ON RECOVERE FROM ITTEREML RADLTION DY P. Proc. Soc. Mxpt. BIol. Med. T6, $552-4(196)$.

The addition of 5 enerous amounts of folic acid, or folle acid and vitamin $\mathrm{B}_{12}$, to experimental diets had no significant effect on the survival of mice injected with a dose of $\mathrm{P}^{32}$ in the higher range of the $\mathrm{HD}_{50}$ (21) day). On the other hand, when sulfasusidine was added to the dist and the mice were injected with a dose of $\mathrm{P}^{32}$ sighty below the $\mathrm{LD}_{50}$ (218t day), the administration of vitamin $B_{12}$ and folle acid increased significantly the time of $50 \%$ deaths, the average time of survival, and the percentage of survivos at both the 21 at and the 56th day. (NSA) 1298

Cronkite, PJigene P., John L. Tullis, Carl Tessmer, and

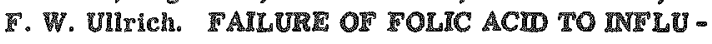

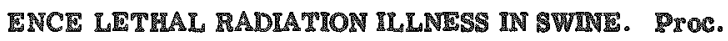
5ొoc. BEgt. Biol. Med. 73, $406-7$ (1950).

Eight swine were exposed to $400 \mathrm{~F}$ total body radiation. Fou were treated with inttamuscular injections of 45 mg of folic acid per day. Fhe clinical and hematological courses were observed and postmortem axaminations were periormed. No differences were observed in the responses of the treated and untreated swime. 11 referanceg. (NSA) 1299

Coldfeder, Annz, Llonel Cohen, Charlotte Muler, and

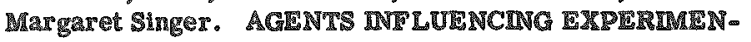
TAL RAOIATON INZURY. WIFECTS OR FOLIC ACHO

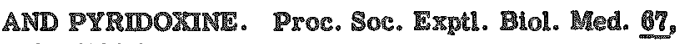
$272-8(1048)$.

White mice, exposed to $x$-radiation, were given a medium dose of $350 \mathrm{r}$ in a single exposure. $70 \mathrm{lic}$ acid and pyrdoxine hydrochloride were used as posslble protective agents. No appreciable effect of hese druge on the hemopoictic ystem was noted. On the other hand, it was found that $15 \mathrm{y}$ of folic act injected daly during a period of seven day grios to radiation and 15 days after radiation prolonged aignificanty the survival time of the irsadited mice. Similar observations were made with pyridoxime hydrochloride. Thus, igradiated mice injected with $50 \%$ of pysidoxine hydrochloside intramuscularly dally seven days prior to gadiation and 13 days post-padiation, extended rignificanty the life of the irradiated mice, as conpared with the lradiated, unmedicated mice. (GPR) 
1300

HEVE, A. CONSIDERATIONS OF RADIATON SICREESS AND ITS THERAPY. Bruxelles med. 29, $445-50$ (1949).

Observations of 210 cases receiving radiation delivered at 151 to $200 \mathrm{kv}$ and 59 to $400 \mathrm{kv}$ show that $34.4 \%$ and $16.9 \%$ developed radiation symptoms at low and high tensions, respectively. The author concludes that higher voltages are better tolerated for the same dose than the lower voltages, but that other physical factors and specific radiation sensitivity must also be talren into consideration. Vitamin B complex therapy gave good or favorable results in 46 out of 62 cases, showing an initial remission of gastrointestinal 1301 symptomas. (NSA)

Herve, A. and \%. LeComte. ACTION OF TRP SIMICARBAZONE OF ADENOCHROME ON PRTECHEA CAUSED BI X-RADIATON. Arch. intern. pharmacodynamie 70, 109-12(1949).

Fenperiments are described which indicate that the ad m ministration of the semi-carbazone of adenochrome (adrenozyl-1) greatly reduces the cutaneous purpura produced by 8 -radiation in mice of the A.R.A. strain although the length of survival-time is not significantly augmented. One hunded and forty mice of the pure straing A.K.A., C and $C_{57}$ received daily, $400 x$ s-ray doses for fous consecutive days. Hal of the experimental animals had previously received 200 Hc of the adrenoxyl by intraperitoneal injection in 0.2 ce volume, whereas the other half, serving as controls, were similarly injected with 0.2 ce of ghysiological gerun. The glkins of the animals, which were acrificed six days after the inradiation was begun, were detacheu. Examination of the internal surfaces of the sluing indicated that while the control animals showed petechiae (purpuric errptions) and hemorrhages, particularly in the sacral resions, the adrenoxyl-injected group did not show any capillary hemorrhage and few purpuric eruptions. It is noted that prelminary experiments have shown that $x$ radiation does not always produce petechiae in mice and that $\mathrm{C}_{57}$ mice anetimes die from irradiation before they show \$igns of petechiac. (NSA)

1302

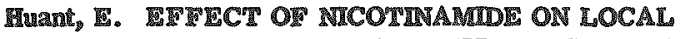
TOLERANCE OF THE SIIN AND DEEP TISSUES TO X.RAYS. RESULTS OF WNCEEASING TRT DOSAGE. GaE. hôpitaus (Paris) $120,313-14(124 \%)$.

This work is babed on observation of 110 cases receiving radiotherapy for various conditions. During perriods of radiation treatment, nicotinamide was admimisterce prel-

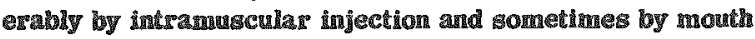
with an average dose of 40 to $60 \mathrm{cg}$. In $70 \%$ of the cases the skin tolerance dose could be increased by 50 to \%5\% and doubled in $20 \%$ of the cages. If hicotinamide should serve to protect the deeper tissues such as nerve, vessels, and bone from harminl offects of gadiation, larger $\mathrm{x}-\mathrm{ray}$ dosed way be uded, especially in the treatment of Lumos's of the cervix, laryns and mediastinum which are in highy Iruervated and vascularized apeas. A case is presented of a gkin ulcer with muco-hemor rhagic exudation following X-ray therapy of a mixed tumor of the parotid with complete healing after 18 days of nicotinamide administered 120 locally and M.M. (EM)

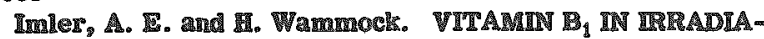
TION SICINESS. Am. J. Roentgenol. Radum Therapy 43 , 243 3 -8 (1910).

The author employed large doses of พitamin $B_{1}$ in 21 cases of Irradiation Blckness with good results. In most. canes the mubcutaneous Injection of 3,000 IU daily gave . rapid and complete relief. In a few cases recurrence of symptoms Hecessitated a temporary, or permanent, increase in dosage. Marked anorexia, nausea, and giddiness could generally be controlled by 3,000 IU daily, given by mouth, but nausea, anorexia, and vomiting required 6,000 IU or more daily. With the oral route also nelief of symptoms did not occur before 24 or 48 hours whereas with subcutaneous injection relief occurred within 1 to 3 hours. Treasment was continued for periods varying from 3 to 20

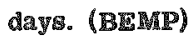

1304

Jacobson, Leon O.g. \$. Phyllis Stearner, Eric L. Simmons, and Mathew Block. THE FIFCC OF FOLIC ACID ON THE RESPONSE OF THE PERIPHERAL BLOOD AND PLOOD-FORMING TISSUE OF THE RAT TO PARENTPRALLY ADMINISTERED STRONTHUM ${ }^{82}$. ARgonne National Lab. Mar. 15, 1948. 6p. (AECD-2013; ANL4125)

Female white rats Irom the Sprague-Dawley farms were injected intraperitoneally with $4.2 \mu \mathrm{c} / \mathrm{g} \mathrm{St}^{80}$ and were treated with daily injections of 15 us folic acid. Hematological studies were made of the pertpheral blood before injection and at 3,6 , and 8 weels after the $\$ \mathbb{F}^{39}$ injection. The experiment was then terminated and all surviving animals autopsied for histological study of the blood-forming tiscues. Folic acid had no observable effect on the macrocytic anemia and leucopenia that developed after the administration of $\mathrm{Sr}^{39}$. When given daily from the start of the experiment, folic acid did not prevent the appearance of the effects of $\mathrm{Sx}^{89}$ on the peripheral blood; when it was given after the appearance of radiation effects, no improvement was observed. Although the reaton is not clear, survival appeared to be poorer in the groups that received folic acid and $\mathrm{Sr}^{32}$ than $\mathrm{in}$ the group that received $\mathrm{Sr}^{82}$ alone. Wistological atudy showed that $\mathrm{Sr}^{\mathrm{B}}{ }^{2}$ produced damage to bone marrow and lymphatic tissue. Very little evidence of regeneration was apparent at the time of autopgy. Bone marrow damage was largely compensated for by increased ectopic myelopoiesis in the spleen. $\mathbf{S r}^{82}$ animals that received folic acid showed no more active regeneration in hemopoictic or lymphatic tissue than rats that had received only $\mathrm{SI}^{83}$. The macrocytic menia observed in the peripheral blood of strontium-injected animals was not accompanied by a megaloblastosis in the blood-forming tissue. (AOD)

\section{5}

Lessa, Z. T. NICORINC ACID IN TREATM NT OF IRRADIATION STCRNYZSS. Rev. paulista med. 26, 135-50 (1945).

The author administered a daily dose of $25-50 \mathrm{mg}$ of nicotimic acid orally to prevent the development of "radiation ickness ${ }^{35}$ in 65 patients receiving roentgen therapy for different conditions. He reports that more than $3 / 4$ of the patients did not show any general effects of radiation. In a lev cases, cutaneous reactions, tenesmus, and diarThea developed as a consequence of the administration of the drug. (R)

1300

MacFarland, M. Lo, M. V. Peters, R. M. Ballantyne, and

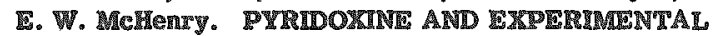
X-RADIATON INJURY IN RATS. Am. J. Physiol. 163, $384-9(1950)$.

To investigate a possible relationship between pyridoxine Insufficlency and $x-r a y$ injury, the effects of total-body sradiation on pyridoxine-deficient rats and on rats rem ceiving this factor were compared. Pyridoxine deprivation was not found to increase the susceptibility of rats to $\mathrm{x}$-ray damage and irradiation did not affect the concentration of 
total vitamin $\mathbb{B}_{B}$ in the livers of either deficient or control animals. (NSA)

1307

Maxfleld, J. R., Jx., A. J. Mcllwain, and J. R. Robertson. TREATMENT OF RADIATION SICENESS WIT P VITAMIN B. (PYRIDOXINE HYDROCHLORIDE). Radiology 41, $383-8(1943)$.

The authors state that the use of vitamin $\mathbb{B}_{6}$ (pyridosine hydrochloride) intravenously has given them the best ingle method of control of radiation sickness to date. The drug is simple to administer, safe to use, and has no contraindications. It should supplement and not replace other methods used for the control of radiation sickness. Reports on three cases of particular interest are given. (\$.V.\$.)

1308

Oppenheim, A. and B. Lih. PYRORAL ADMINISTRATION OF VITAMIN B-6 (PERIDOXINE HYDROCHLORID) IN THE TREATMENT OF RADIATION SICKNESS. Radiology 4. $381-5(1946)$.

A total of 50 patients received $75-100 \mathrm{mg}$ of pyridoxine one-hall hour before meals, 3-4 times a day. In 30 cases $(60 \%)$ the results were excellent, in six cases $(12 \%)$ good, in eight cases (16\%) fair $x$, and no results in six cases (12\%). Symptoms of radiation sickness were aggravated in one patient following therapy. In on patient a scaly and follicular type of eruption developed, which disappeared on withdrawal and reappeared on readministration of the drug. (BA)

1309

Reeves, R. J. TREATMENT OF ROENTGEN SICRNESS WITH ORAL ADMINISTRATION OF PYRIDOXINE

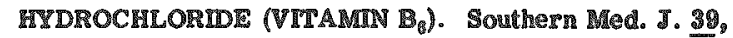
405-7(1846).

A dally dose of $100 \mathrm{mg}$ of pyridoxine hydrochloride was found to be a most effective drug in the treatment of irradiation sickness. Because of its ease of administration and effectiveness, oral administration is advocated with greliance on intravenous injection in those cases which fail to reapond. (BA)

1310

Rovello, and L. Ferri. TREATMENT OF LIECOPENIA DUE TO IRRADLATION WITH VITAMIN B. Haematologica 32, 319-22(1848).

In 10 patients treated with roentgen ray for different types of tumours, the decrease in the number of leucocytes was controlled by the adruinistration of pyridoxine (vitamin $\left.\mathbb{B}_{8}\right), 100 \mathrm{mg}$ daily. (EM)

1311

Scott, G. M. TEIPECT OF EXTRA VITAMMN DIEDING ON RATS EXPOSED TO WEAT CAMMA RADIATION.

Brit. J. Radiol. 18, 329-32(1946).

Ratt were exposed to gamma radiation in doses varying from approximately 60 to $85 \mathrm{x}$ per week for a period of four months. Studies were made on the effect of weight gain and fertitty with and without feeding extra vitamin B. Rats receiving extra vitamin B showed greater weight gain than the controls and thelr coats were in better condition. Rats receiving radiation but no extra vitamin gained less than the controls. Both female and male irradiated rats were mated with unirradiated. The irradiated fenales became pregnant as often as normal, but there were many cases of abortion, some died in parturition, and few of the litters were reared. These effects were not as pronounced in the rati receiving extra vitamin $B$. No offspring resulted from matings with irradiated male rats. (R)
1312

Scot, L. D. and G. J. Tarleton. PYRDOKINE RYDROCHLORIDE (VITAMIN B-6) IN THE CONTROL OF RADIATION SICIKNESS: PRPLIMINARY REPORT. RadLOLOGy 47, 386-91(1946).

In twenty patients irradiated for various forms of malignant tumors who received 25 mo of pysidoxine IV, none developed nausea or vomiting. (BA)

1313

Shorwon, L. M. PYRIDOKINT IN TREATMENT OF RADIATION SICKNESS. Brit. J. Radiol. 19, 369-70(1946).

This article begins with a short exposition of radiation ricliness varying in antensity with the part of the body treated and the area of exposure. The author mopts 24 cases treated by pyridoxine and claims good results in 22 as regards the radiation sickness. The beneicial effect on the leucocytes is also stressed. Young (Am. J. Foentgenol, 35. 681, 1836) strongly recommended injections of liver extract. Pyridoxine is a member of the vitamin $B_{2}$ group occurring principally in liver and yeast, which shorvon finds superior in effect to other remedies. Among the 24 cases reported, a patient aufering from Hodgkin's disense is quoted as much distressed by radiotherapy and benefitted by pyridoxin. In another case, "a female aged 60 with a large abdominal mass and secondaries in lungs from? carelnoma of the tongue previously treated by radiun and $x$-rays also suffered from radiation aickness but was promptly relieved by pyridoxine." (AWM)

1314

Shorwon, L. M. A FURYLER SULVEY OF RADLATION SICISNESS WITH DARTICULAR RETERIENCE TO ITS TREATMEST BI PYRIDOXUNE. Brit. J. Radiol. 22, 49-55(1049).

The theories advanced to explain the causation of radiathon gichness and the remedies recommended are brielly revieved. The results of the use of pyridoxine in 69 cases are analyzed. It is shown to be a zellable, though not infallible, drug in the treatment of radiation sickness. It has also a beneficial effect on $\mathrm{X}-\mathrm{ray}$ leucopenia and the addithonal mertt of conferring on some patients a sense of general wall-being. (NSA)

1315

Stearner, 5. Phylus. EMTECTS OF FOLIC ACD ON THE ANEMA INDUCED BI X-MRADIATION. Proc. Soc. Expt1. Blol. Med. 09, 518-21(1848).

In this experiment, the number of animals observed was not large and the reaults obtained showed only small differences. For these reasons no definite claim can be made concerning the therapeutic effect of folic acid on the damage resulling from $x$-irradiation. The results can only be considered to be suggeative of a positive ef ect. Jacobson, however, stated that in patients treated with irradiations or nitrogen mustards, folic acid has not been shown to pro. duce any but equivocal results. Further worls is necessary to establish more definitely the possible effectiveness of folic acid in alleviating the leucopenia and anemia that collow irradiation. (NSA)

1316

Stearner, Phylls and Leon O. Jacobson. THP EFFICT ON FOLIC ACHO ON TRE RESPONSE TO TUL PERIPEIRAL BLOOD OF THE RAT TO \$TRONTIUM ${ }^{3 \%}$. Argonme National Lab. June 1, 1947. 13p. (MDDC-1418B)

Folic acid had no effect on the macrocytic anemia that followed the administration of $5 r^{29}$. At least in the case of radioactive strontum, the injury is probably exerted di- 
rectly on the bone marrow and is not caused indirectly by any damage to the gastrointestinal tract or to the liver.

The action of folic acid has been reported to be similar to that of liver extract and it therefore should correct any defleiency resulting from radiation damage to liver or stomach and subsequent failure to form an anti-anemic factor. Folic acid was equally ineffective in alleviating the effect of irradiation on the number of circulating leucocytes. This fallure to affect the leucopenia that followed the administration of $\mathrm{Sr}^{89}$ does not substantiate the suggestion by Watson that folic acid may be effective as a treatment for the leucopenia following exposure to radiatons. It is concluded that folic acid is ineffective as a treatment for the hematological effects of internal radiathons and probably by implication, for those of external radiations as well. Since total body irradiation is more llkely to damage viseeral organs, however, the latter question is being investigated. (ADD)

\section{7}

Van Haltern, H. L. THE USE OF PYRTDOWINE HYDROCRLORIDE IN RADIATION SICTRESS. Radiology 4 \% $377-80(1946)$.

A total of 81 patients received $25-50 \mathrm{mg}$ pyridoxine intravenously. Excellent resuls were obtained in 44 cases (54\%), good resulte in 28 cases (35\%) and in 9 cases (11\%) vomiting was not entirely relieved and 2 troublesome degree of nausea remained. (BA)

1318

Wallace, W. \$. STUDIES IN RADIATON SICKNESS: VITAMINS B, AND C AND SMALL TNTESTINAI CRANGE IN RADIA ION SICKNESS. Southern Med. $3.34,170$ 3 (1941).

Following heavy roentgen irradiation of the pelvis, small intestine changes similar to those seen in vitamin deficlency are found. The roentgen changes, in the ileum are segmentation of the barium stream, flattening of the mucosal pattern, diminution in motility, and narrowing of the lumen. Avitaminosis $B$ and $C$ are already present in advanced carcinoma of the uterine cervix. With these facts in mind, ifve patients with previously untreated cervical carcinoma were given daily intramuscular injection of $10 \mathrm{mg}$ thiamin chloride along with routine pelvic irradiation, While ive others received daily doses of $50 \mathrm{mg}$ sodium agcorbutic acid (Lilly's cevalin). The irradiation was in tensive, reaching $600 \mathrm{r}$ daily, with either $400 \mathrm{kv}$ with a HVL of $5 \mathrm{~mm}$ Cu or $200 \mathrm{kw}$ with a HVL of $2 \mathrm{~mm}$ Cu. Each of Bix portals received 2,000 to $2,200 \mathrm{r}$ lotal dosage and eight patients received 2,500 to $5,000 \times$ supplementary intravaginal radiation at $200 \mathrm{kv}$. As a result of this study the author coneludes that vitaming $B$ and $C$ administered durIng heary pelvic lrradiation fails to diminish the occurrence or severity of diarrhea or to alter the radiographic changes in the ileum resulting from the ir radiation. The ileal changes are apparently not due to lack of vitamin $\mathbb{B}$ or C. Severe nausea and vomiting were, however, almost entirely eliminated by the vitamin $B$ and $C$ administered. (a)

1318

Whitmore, W. T. THE PREVENTION OF IRRADIATION SICRNESS. USE OI VITAMIN $B_{1}$ IN ROENTGEN TRERAPY. Am. J. Roentgenol. Radium Therapy 49 , $83-98$ (1043).

On the basis of the similarity of symptoms of vitamin $B_{1}$ deficiency and those of radiation sickness, thiamin chloride was administered to patients undergoing radiation therapy. Radiation sickmess is usually prevented by the administration of 6-9 mg daly during therapy; if symptoms of the wickness develop, the dose of thiamin may be incrased. (BA)
Miscellaneous Treatment

1320

Baclesse, $F$. and $J$. Loiseleur. CHANGE IN CUTANEOUS SENSITIVITY TO X-RAYS DURING EXPERIMENTAL HYPERGLUCEMA IN RABBITS. Compt. rend. soc. biol. $141,743-5(1947)$.

Intramuscular injection of a near-lethal dose of glucose 45 minutes previously to $\mathrm{K}$-ray irradiation reduced the sensitivity of the gkin to s-rays. (NSA)

1321

Bacy, Z. M. and A. Herve. PROTECTIVE ACTION OF METHYLAMINE AGAINST X-IRRADLATION. Nature 168, $1120(1951)$.

Pure-bred C57 black mice 4 to 6 months old were ixradiated with $250 \mathrm{kv} \times$ rays at the rate of $90 \mathrm{r} / \mathrm{min}$ follow ing intraperitoneal injection of $2 \mathrm{mg}$ of methylamine neutralized in water solution. Forty controls received either 700 or $850 \mathrm{~g}$. All the mice irradiated with $700 \mathrm{r}$ died between the 5 th and the 10th day after irradiation; those which had received $850 \mathrm{x}$ died between the 4 th and the 8 th day. In a group of 50 anmais injected and irradiated with $700 \mathrm{~g}$, the mortality (from the 5th to the $12 \mathrm{kn}$ day) was small; $73 \%$ of the animals survived. The mice injected wilh methylamine and ir radiated with $850 \mathrm{r}$ died between the 4 th and 11th day, later generally than the controls; only $7 \%$ survived. An injection of 2 or $2.5 \mathrm{mg}$ of methylamine did not protect any animal against a dose of $x$ rays greater than $850 \mathrm{x}$. 1 dose of $2 \mathrm{mg}$ of methylamine injected 5 or 15 min. after the end of irradiation (700 $\mathrm{r}$ ) shifted the mortality curve slightly to the right. (NSA) 1322

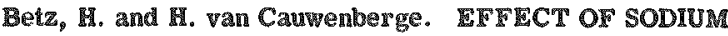
SALICYLATE ON RESISTANCE OF MCE IRRADIATED WITH X RAYS. Compt. rend. soc. blol. 145, 1910-13 (1951).

Adult C57 mice were injected intraperitoneally with 12 daily doses of 400,500 , or $600 \mathrm{mg}$ of Na salicylate/kg, then were irradiated with $700 \mathrm{r}$ of $250 \mathrm{kv} \times \mathrm{x}$ rays. Survival rates were 25,55 , and $50 \%$, respectively, compared to zero for controls. Injection with $500 \mathrm{mg} / \mathrm{kg}$ daily following a $700-\mathrm{r}$ irradiation resulted in much more rapid death than among the controls, all dying within 6 and 12 days, respectively. A stimulating action of $\mathrm{Na}$ salicylate on the adrenal cortex is suggested to explain these results. (NSA) 1328

Blaix, Nenry A. RETENAL RADIATION-TOLERANCE AND THERAPY, p.46-55 Of QUARTERLI TECHNICAL REPORT JULY 1, 1951, THRU SEPTEMBER 30, 1951. Univergity of Rochester. Oct. 25, 1951. Op. (UR-189 (p.46-55))

From an investigation of the effect of spleen shielding on $\mathrm{x}$-irradiation mortality in dogs it is concluded that lead shielding of the exteriorized spleen during $x$ isradiation is an effective means of reducing mortality. Preliminary hematological gtudies based upon peripheral white-bloodcell counts and hematocrits also indicate an early recovery of the hematopoietic tissue in spleen-shielded dogs. A preliminary report of a study of the effect of aureomycin and whole blood, singly and in combination, on $x$-irradiated doggs ghows the group treated with aureomyein and whole blood to have a lower mortality rate than those treated only with blood or aureomycin. An increased incidence of severe pulmonary hemor rhage in the groups receiving blood transfusions appeared to be significant. (NSA) 1324

Boys, Fi. and 1. D. Harris. THE EFPECT OF HEPARINIZATION ON EXPLRIMENTAL POST-IRRADIATION TISSUE CHANGES IN THE LUNG. Am. J. Roentgenol. Radium Therapy 50, 1-8(1943). 
Rabbits were heparimzed to keep the coagulation time above 20 minutes and the entre thorax drradiated to a total of $4,200-4,900 \mathrm{r}$ in two weeks. Lung damage was less than in non-heparmized anmals receiving the same 14 radiatron. (BA)

1325

Brues, Austin M., ed. RADIATION SICKNESS, 14.14-20, $41-54,57,60-3,125-41,152-4$ Of QUARTERLY REPORT, FEBRUARY, MARCH, APRIL, 1951, DIVISION OF BIOLOGICAL AND MEDICAL RESEARCH. Argonne National Lab. [nd] 46p. (ANL-4625(p.14-20, 41-54, 57, 60-3, $125-41,152-4)$ )

Survival was enhanced significantly in groups of LAF, mice injected intravenously or intraperitoneally with bonemar row suspensions within 10 to 15 min following a single total-body exposure to $900 \mathrm{r}$ of $\mathrm{x}$ radiation. Spleen brei injections did not afford any protection. Only a small amount of bone marrow (1.5 mg) grave nearly complete protection. Data on survival and hemopoietic recovery of mice exposed to a $1025-r$ total-body $x$ irradiation with protection of the spleen and other organs and of spleen-shielded mice exposed to repeated 1025-r doses are tabulated. Peritoneal implantation of four spleens from baby mice enhanced survival of previously $1025 \mathrm{~m} \mathrm{x}-\mathrm{irradiated} \mathrm{mice,} \mathrm{whereas} \mathrm{a}$ two-spleen implant had no effect. Leaving a blood vessel at the distal tip of the apleen unsevered during exteriorization and shielding greatly enhanced survival. Intraperitoneal injection of minced mouse embryos following a 1025- $r$ x ir $r$ radiation reaulted in $\sim 35 \%$ survival of mice. Combinations of spleen shielding, cysteine, and estrogen resulted in an enhancement of gurvival greater than the sum of individual protective values. Additional data indicating the failure of postirradiation cysteine and ascorbic acid reatment to influence radiation mortality are given. The beneficial effect of head shielding on survival of dogs exposed to $450 \mathrm{~g}$ of $\mathrm{x}$ radiation are shown. The effect of cyanide in increasing the radiosensitivity of mouse tumor fragments was not oblained in the absence of $\mathrm{O}_{2}$ and was not caused by destruction of tumor cells. Young gats were implanted subcutaneously with the Hall mouse carcinoma 1 to $2 \mathrm{hr}$ after 100 200-, or 300-r total-body $\mathrm{x}$ irradiation; organ weight and histological atudies show that a growing heterologous tumor is able to evole significant hyperplasia of proximal lymph nodes and spleen in counteraction of the effect of Irradiation; in reduction of the thymus, tumor and radiation have an additive effect. Admimistration to 2- to 4 -day chichs of $25 \mathrm{mg} / \mathrm{kr}$ of Benemid immediately prior lo a $1000-\mathrm{s} \times \mathrm{ir}^{\mathrm{P}}$ radiation did not increase survival or alter renal function. (NSA)

\section{6}

Cafiaratti, Enrico. SOME SUPSTANCES WTH REDUCING ACTION (CYSTEINE, THIOGLYCOLIC ACID, ASCORBIC ACD, COMBINED CYSTEINE AND ASCORBIC ACD) AS PROTECTIVE AGENTS IN BIOCHEMCAL XRAY INJURIES. Radioterapia radiobiol. e fis. med. A, $378-84(1951)$.

Mice, weight $20 \mathrm{~g}$, age 30 to 35 days, were injected intravenously with $500 \mathrm{mg}$ of cysteine $/ \mathrm{kg}, 540 \mathrm{mg}$ of thioglycolic acid $/ \mathrm{kg}, 100 \mathrm{mg}$ of ascorbic acid $/ \mathrm{kg}$, or $500 \mathrm{mg}$ of cysteine $+100 \mathrm{mg}$ of ascorbic acid/ $/ \mathrm{kg} 5 \mathrm{~min}$ before irxadiation with 800 r (5-week $\mathrm{LD}_{\mathrm{B} 0}$ ) of 180-kv $\mathrm{x}$ rays. The mutal protective action of the cysteine and ascorbic acid was marked, $94 \%$ of the mice so treated being alive 3 weelss after irsadiation, in contrast to $45,80,50$, and $50 \%$, respectively, of control and cysteine-, thioglycolic acid-, and ascorbic acid-treated mice. (NSA)

1327

Campbell, Ira L. and Mary H. Ross. PROTECTION EXsPERIMENTS AGAINST RADIATION INJURY WITY
LYMPHOCYTES. Oak Ridge National Lab. [nd] 7p. (ORNL-1193)

This experiment was undertaken to determine whether the injection of lymphocytes into irradiated animals would offer protection against the effects of radiation. The experinuents were so designed as to obtain living lymphocytes in their natural medium and introduce them into genetically compatible hosts. The results were essentially negative 328

Cole, L. J., V. P. Bond, and M. C. Fishler. PRE-PROTECTION OF MCE AGAINST IT-IRRADIATION MORTALITY BY SODIUM NITRETE. Naval Radiological Defense Lab. Sept. 21, 1951. 14p. (AD-331(B); Technical Objective AW-6)

The mortalty of mice receiving $600-$ or 750 - single dose whole-body $x$ irradiation was reduced markedly following preirradiation intraperitoneal injection of $\mathrm{NaNO}_{2}$ (100 to $125 \mathrm{mg} / \mathrm{kg})$. Definite, but less marked, protection was also observed with a nitrite dose of $62 \mathrm{mgg} / \mathrm{kgg}$. The decrease in radiosensilivity afforded by $\mathrm{NaNO}_{2}$ administration was reflected in the minimal postir radiation body weight losses observed in the nitrite-treated groups, as compared with the control animals. The possibility that nitrite protection is mediated via its effect on catalase activity is discussed. (NSA)

1328

de Carlos, R. LEUCOPENA DUE TO K-RAYS. PROPHYLAXIS AND THERAPY WITI VIYAMHN C (ASCORBIN). Gazz. sanit. 18, 92-7(194\%).

In view of the reported good effects of vitamin $C$ in leucopenia and benign agranulocytosis the author tried its eflect in 30 patients ( 15 men and 15 women) receiving $x$ ray therapy for cancer of various organs, including cancer of the prostate 3 , of the lung 2 , of the intestine 4 , of the bladder 2, and of the marrow 2; and in the women, cancer of the cervix 8 , of the breast 1 . Irradiation therapy was carried out daly with a dose of 240 r. Vitamin $C$ was given daily in $50 \mathrm{mg}$ doses IM or IV. In six patients, this administration was begun some five days before the -ray therapy, in eight on the same day, and in 16 about a week after the beginning of $x$-ray therapy. In the first group the leucocyte count rose (on an average by $80 \%$ ) before and fell to normal levels during $\mathrm{z}-\mathrm{I}$ ay therapy. The rise was mainly due to a lymphocytosis, which was followed by a lymphopenia. In the second group patients with a slight initial leucopenia showed a rise in the whe; in those with initial leucocytosis the count returned to normal, while normal counts did not vary. In two patients a slight leucopenia was unchanged. There was little change in the differential counts in this group. In the third group an initial leucopenia was followed by a transient rise to normal levels and then by a more lasting leucopenia, mainly due to a rall in lymphocytes. With the Vitamin $C$ the leucocyte count rose to a normal level again and then fell slightly, but remained above the minimal level attained after begiming the $\mathrm{x}$-ray therapy. No effect was observed in three cases. The aus thor concludes that vitamin $C$ has prophylactic and curative value in leucopenia due to $\mathrm{x}-\mathrm{ray}$ treatment. Detalled counta are not given except by graphs for three of the cases. The author does not report any control observations. (AWM) 1330

Fulton, John D., Anna C. Arnold, Roland B. Mitchell. THE ANTIBODV RESPONSE OF ANMALS RXPOSID TO X-RAOTATION. III. THE PROTECTIVE EFIECT OF CHEMOTHERAPEUTTC AGENTS ON A SPECISIC IMMUNE STATUS OF I-RADLATED MCE. USAT School of Aviation Med., Randolph Field, Texas, 15p. (Project No. 21- $17-002$, Report No. 3) 
A study was made to determine if treatment with adrenal cortical extract, thiourea, glutathione, Reticulose, and chloromycetin, either alone or in combination, would increase the survival time of mice immunized against Salmonella typhimurium, $x-r$ adiated, and subsequently infected with virulent $\mathbf{S}$. typhimurium organisms. Under the conditions of the experiment it was observed that pre-radiation treatment with glutathione alone and post-radiation dosages of chloromycetin alone increased the survival time of immunized, $x$-radiated, and infected mice. The combination of pre-radiation glutathione and postradiation chloromycetin increased the survival time considerably more than did either of the agents when administered alone. Administration of adrenal cortical extract, Reticulose, and thiourea immediately prior to $\mathrm{x}$-radiation did not alter significantly the survival time of the infected experimental animals. (Authors)

1381

巽all, B. V. PROTLCTION OF MCE TROM LETRAL EFFECTS OF X-RADIATION WITH INTRAVENOUS INJECTION OF METHYLENE BLUE. Federation Proc. $10,58(1951)$.

The report is reproduced here in its entirety.

In 12 independent experiments, over 600 male and female mice were given varied $\mathrm{x}$-ray doses of $500-900 \mathrm{r}(200 \mathrm{kv}$, hw1 $0.98 \mathrm{~mm} \mathrm{Cu}$, target distance 11 inches, dose rate 104 $\mathrm{r} / \mathrm{min}$.). Half of the mice were injected intravenously $2-8$ min. before irradiation with methylene blue golutions whose dye content varied from 2.5 to $10.0 \mathrm{mg} / \mathrm{cc}$. Dosages of $25,37.5$, and $50 \mu \mathrm{g}$ of dye/gm mouse were used. Varying the amount of dye injected had little effect on protection, yet it appears that the concentration of the dye solution is a factor influencing survival after ir radiation. Fiscellent protection was found consistently in 8 experiments employing solutions of $0.250 .5 \%$, whereas only 1 of $4 \mathrm{ex}-$ periments using $1 \%$ solutions yielded statistically gignificant protection. The maximum protection obtained was $50-60 \%$ differential between the percentage with methylene blue 30-day survival of dye protected and control mice. Mice injected with methylene blue after irradiation were not protected. The percentage of 303 dye-pre-treated mice surviving 30 days after irradiation was nearly double that of 310 dye-free irradiated controls. The methylene blue protection may possibly result from the formation of met hemoglobin which induces hypoxia of the tissues sufficient to ameliorate the lethal action of irradiation, or methylene blue may have more direct action on tissues affording protection. Experiments are being continued which may help answer these questions.

1332

HENIy; J. A. TOTAI X-IRRADIATION OT RATS UNDER URETHANI ANAESTHESIA. Nature 163, 134-5(1949).

Albino rats were injected intraperitoneally with $1 \mathrm{cc}$ of $10 \%$ urethane solution per $100 \mathrm{~g}$ body weight. A part of the group were irradiated with $600 \mathrm{r}$, and a part with $500 \mathrm{r}$, as were two groups of unanesthetized control rats. Irradiation with a 500 I dose killed about $20 \%$ of the animals within 14 days, both controls and anesthetized. The $600 \mathrm{r}$ irradiation, however, killed $50 \%$ of the controls within 14 days, but killed $90 \%$ of the anesthetized rats within the same period. The effect may be one of cumulative action, or of sensitization to $\mathrm{X}$ - rays by the urethane. (V.E.) 1333

Merve, A. SEMICARBAZONE OF ADRENOCHROME AND X-RAYS. Arch. intern. pharmacodynamie 85, 242-4(1951).

The semicarbazone of adrenochrome (adrenoxyl) given by mouth reduces the purpura produced in mice by $\mathrm{x}-\mathrm{ray}$, but does not prolong the æurvival time. (CA)
1334

Herve, A. and Z. M. Bacq. SULFOCYANATE, TOCOPHEROL AND X-RAYS. Compt. rend. soc. biol. 143, 1158-9(1949).

In the present report the authors offer an interpretation of the protective action of cyanide against the effects of strong whole-body irradiation in mice. The protective action was not demonstrated by sodium sulfocyanate, and the authors have searched for other substances which would inhibit the lethal effect of $x$-radiations, but which would be less toxic than the cyanide, an inhibitor of metabolism. The authors suggest that the cyanide acts either by hindering the formation of the peroxides or by diminishing the reaction of formed peroxides with reducing groups guch as the -\$E group. This hypothesis was tested by using vitamin $E$ ( $\alpha$ tocopherol), an excellent inhibitor of peroxide formation by the action of $\mathrm{s}$-rays on lipids. It was found that the mice withstood the dose of $x$-rays administered ( $400 x$ ) much better ( $30 \%$ mortality to $60 \%$ mortality) than mice not treated with two $10-\mathrm{mg}$ intravenous injections of the a tocopherol at 24 -hour intervals before the irradiam lion. It is concluded that the protection by cyanide against the effects of $\mathrm{X}$-rays in mice is due to the action of the CN-ion; the protection is limited in the sense that it is weak if the dose of $\mathrm{x}-\mathrm{rays}$ is increased, and is very weak If the injection is made as late as 15 minutes after irradiation. (NSA)

1335

Herve, A. and Z. M Bacq. PROTECTIVE ACTION OF SODIUM NITRIDE AGAINST LET TIALITY PRODUCED BY X RAYS IN MICE. Compt. rend. soc. biol. 144, $1124-5(1950)$.

It has been reported in former notes that an injection of $0.1 \mathrm{mg}$ of NaCN immediately before a $700-\mathrm{r}$ irradiation of mice reduced the lethality by $70 \%$ (Compt. rend. Soe. biol. 143, 881 (1949); Betz and Herve, ibid. 144, 1015 (1950)). An analogous series of experiments is described here using $\mathrm{Na}_{3} \mathrm{~N}$, whose biochemical properties (imhibilions of enzyme functions) are similar to those of NaCN. An injection of 0.1 to $0.2 \mathrm{mg}$ of $\mathrm{Na}_{3} \mathrm{~N}$ in aqueous solution in mice before the administration of $700 \mathrm{~g}$ of $\mathrm{x}$ rays reduced the lethality by 60\%. (NSA)

1336

Herve, A. and J. Lecomte. ADRENOXYL AND X-RAYS. Arch. intern. pharmacodynamie 79, 109-12(1949).

Mice injected intraperitoneally with adrenoxyl before isradiation do nol show petechial hemorrhagea in the gkin such as are seen in control animals. (CA) 1337

HOhl, IS. APPLICATION OF THE SODIUM SALT OF ALPHA-OXXBENZYLPPIOSPHINIC ACID IN LONG IRRADLATION WTL X-RAYS. Prasis (Bern) 38, 568-9 (1949).

Phoselit (Na salt of $a$-oxybenzylphosphinic acid) inhibited toxic side effects of $x$-ray irradiation; body weight restoration was especially accelerated by this drug. Phoselit increased glycogenesis acting as a donor in the oxidation-reduction process. (NSA) 1338

Holmes, B. E. SERUM CHOLESTEROL AND RRRADIATION SICKNESS. Brit. Med. J. 1, 314(1941).

This article briefly but effectively presents a worthy effort on the part of the autlor to elarify the elusive situation of radiation sickness. The data presented were obtained by serum analysis in 11 cases under 8 - ray therapy. The findings are incomplete but information is available which points to the conclusion that patients who suffer no post-irradiation discomiort have a rise in cholesterol 
serum content and that those who have a typical gastric upset following irradiation fall to show this rise. The natural deduction is that cholesterol administered in susceptible cases will prevent radiation sickmess. This treatment 1339 was effectively tried by Levy-Dom and Bergheim. (R)

Jenkinson, E. L. and W. H. Brown. IRPADIATION SICKNESS. A MYPOTHESIS CONCERNING THE BASIC MECHANISM AND A STUDY OF THE THERAPEUTIC EFFECT OF AMPHETAMINE AND DEXTRODESOXY EPREDRINE. Am. J. Roentgenol. Radium Therapy 51, $496-503(1944)$.

Of 62 cases of irradiation sickness, 21 were well controlled by amphetamine and 34 by dextro-desoxyephedrine. Evaluation of relative effectiveness of the two was not attempted. A hypothesis is suggested to explain the basic mechanism of irradiation sickness as an increased capillary permeability. This results in disturbance of intracellular and extracellular equilibrium of electrolytes and fluids, which is followed by local congestion, edema, and stasis of circulating blood. A vicious cycle is set in motion by reduced blood volume and tissue anoxia. The above drugs apparently act by their ability to maintain peripheral circulating blood volume and prevent visceral stasis. Detailed suggestions are given for the management of irradiation sicknems. (BA)

1340

Kahn, J. B. J". MODIFICATION OF SENSITIVITY TO X RADIATION BY MORPHINE SULFATE. Proc. Soe. Expt1. Biol. Med. 78, 486-9(1951).

Morphine sulfate, injected intramuscularly into $2 \mathrm{BC}$ male mice 30 min before exposure to total-body s radiation, raises the $\mathrm{HD}_{50}$ of the radiation from 609 to $830 \mathrm{r}$. Possible mechanisms of this effect are discussed. (NSA) 1341

Kohn, Henry I. ON THE MODIFICATION OF THE TOXICITY OF X-RAYS BY IMMUNIZATION. Oak Ridge National Lab. Oct. 27, 1949. 9p. (AECU-610; ORNL-386)

Following injection with sheep erythrocytes, the SpragueDawley and Osborn-Mendel strains of rat showed a rise of about 50-75 $x$ in the LD-50\% of $250 \mathrm{kv} \times-r a y s$. The Koltzman strain showed a fall of about $25 \mathrm{r}$. The injection of sheep cells prevented the rise in albumin-globulin ratio which invariably occurs after irradiation in nonimmunized ratg. (NSA)

1342

Lambert, G., J. Maisin, M. Mandart, M. Meersseman, G. Podio, and E. Pluygers. THE INF LUENCE OF CERTAIN METALLIC IONS ON THE SURVIVAL OF RATS RECEIVING A LETHAL DOSE OA X-RAYS. Compt. rend. suc. biol. $144,444-8(1950)$.

Rats received a total body 5 -ray dose of $600 \mathrm{~F}$ (180 kv) for approximately 13 minutes. Subcutaneous injections of barium chloride, lithium lactate, or mercuric chloride were administered to some of them either before or after Ir radiation. The barium and lithium lons hastened death, while mercuric ions seemed to retard it and diminish the percentage of the ratg which guccumbed. (NSA) 1343

Lavilk, Paul, Jack R. Leonards, G. Warren Buckaloo, Charles Heisler, and $\mathrm{Kymer}$ L. Friedell. IN VIVO DLALYSIS OF BLOOD AS A MEANS OF INTLUENCING THE SURVIVAL OF X-IRRADIATED DOGS. NEW York Operations Office, AEC. Dec. 15, 1951. 21p. (NYO1637)

By means of an "artificial kidney," dogs given a lethal dose of $x$ irradiation were subjected to an in vivo dialysis of their blood either during or al some time after the ir- radiation. No beneficial effects of the dialysis procedure were observed in the ir radiated animals, even though the "artificial kidney" was shown to be highly efficient in removing a dialyzable gubstance from the extracellular fluid of dogs. While in vivo dialysis was not detrimental to nonirradiated controls or dogs dialyzed at the time of irradiation, sudden death occurred following dialysis in about $50 \%$ of the animals that had been irradiated 12 or more hours earlier. The latter effect of dialysis can be attributed to the removal of some essential factor(s) from the blood, for it could not be demonstrated in irradiated dogs subjected to a "sham-dialysis" procedure. Peritoneal lavage was without significant effect upon the survival of $\mathrm{x}$-irradiated dogs. (NSA)

13 서소

Mole, R. H. PROTECTION FROM WHOLE-BODX IR RADIATION BY CHEMICAL MEANS. J. chim. phys. 48, 258-61 (1951).

The chemical protection afforded mice against radiation injury, lollowing whole-body x irradiation, by injected doses of thiourea, sodium cthane di-thiophosphate, glycerol, sodium thosulfate, and sodium dithionate was studied. Of these, thiourea was the only chemical giving a definite protective effect (equivalent to a reduction in the effectiveness of radiation by about $10 \mathrm{or} 15 \%$ ) and this only when given 1345 immediately before irradiation. (NSA)

Molomut, Norman, Milton Friedman, Irene Jacob Ryby, and Alvin Harber. EFFECTS OF ANTISPLENIC TISSUE SERUM ON ROENTGEN-RAY DAMAGE (WHOLE BODY IRRADIATION). Am. J. Roentgenol. Radium Therapy 66, 245-7(1951).

The effect of antispleen serum upon recovery of mice from damage to the reticulo-endothelial system caused by whole-body $x$ irradiation was investigated. Under the conditions used, antispleen serum did not affect the increased susceptibility of $\mathrm{x}-\mathrm{irr}$ radiated mice to infection with Rickettsia pom. (NSA)

1346

Parker, James Preston, Paul I. Ewing, and G. A. EmerSOR. LACK OI ANTAGONISM OF MALONONITRILE AND DESOXYRIBONUCLEIC ACD TO TRYPANOSOMIASIS, ALLOXAN DLABETES, AND LETTEAL FTFECTS OF X-IRRADLATION. Temas Repts. Blol. and Med. 257-61(1950).

The authors demonstrate that neither malononitrile nor desoxyribonucleic acid, in massive repeated doses, has appreciable effects on experimental trypanosoniasis, alloxan diabetes, or lethal effects of $\mathrm{z}-\mathrm{irradiation}$ in anice. (NSA)

1347

Paterson, Edith and Joyce J. Matthews. PROTECTIVIE ACTION OF ETHYL ALCOHOL ON IRRADLATED MICE. Nature 168, 1126-\%(1951).

Pure-bred "A" strain mice 6 to 8 weeks old were given two $1-\mathrm{ml}$ doses orally of ethyl alcohol $(10 \%$ by volume in saline) 80 and 20 min before a $700 \mathrm{z}$-irradiation. Mortaity was $63 \%$ compared with $100 \%$ for controls. A rase in the $\mathrm{LD}_{50}$ radiation dose from 554 to $325 \mathrm{I}$ and an extension of the life of nonsurwivors were noted with the alcohol treatment. No protection was oblained when the alcohol was given after the irradiation. The difference in protective valre between 5 and $10 \%$ alcohol was not gignificant. Intravenous cysteine, $20 \mathrm{mg} /$ mouse, gave grater protective effect than the alcohol in the doses used. Protection by alcohol was unrelated to its anesthetic effect. Intravenous nembutal, $1.3 \mathrm{mg} /$ mouse, and intraperitoneal ethyl casbarnate, $20 \mathrm{mg} /$ mouse, did not change the $\mathbf{L D}_{60}$. (NSA) 
196

Rekers, Paul 2., Molly P. Coulter, and Stafford L.

Warren. FIFCE OF TRANSPLANTATION OF BONE MARROW INTO IRRADIATED ANIMALS Arch. Surg. $60,635-67(1050)$.

The results of the transplantation of marrow suspensions, bone and marrow and ribs, are presented; in some cases bone shver's were transplanted to the anterior chamber of the eye, or the liver or spleen, but usually the material removed from a normal dog was transplanted to the same location in an irradiated dog. A single dose of 350 . of whole body irradiation was given to the test dogs before the transplantation; similar experiments were performed on rabbits. It was found that the transplantation of the normal marrow to intramedullary marrow sites was without appreclable bonefit to the 1rxadrated anmals. The transplantation of bone and marrow resulted in the death and replacement of the graft; trangplantation of the normal marrow to normal or irraduated spleen or liver was also without benefit. Other gesults are outhned. (NSA) 1348

Revici, Emanel. INFLUTECE OI VARIOUS LIPIDS DPON THE HEALING PROCESS OF XPERIMENTAL RADIATION BURNS, p.175 OI INTERNATIONAL CONGRESS OF RADIOLOGY, 6th Congress, London. 1950.

The author describes the techngue used in order to obtain comparable radiation burss in rats, using $10 \mathrm{mg}$. radum needles. The lipids studied are divided nto two clasaes, according to the nature of thenr polar group.

The influence exerted seems to be opposite, according to the pregence of a carboxyl or a hydroxyl, as polar group of the lipid. The lipids with carboxyl polar groups admmIstered to the anikals presentung radum burns have appeared to interfere with the healing process, ancreasing the extent and the duration of the uiceration. On the contrary, the lipids with a hydroxyl radical, promote rapid healing.

\section{0}

Salishbury, Peter F., Paul R. Rekers, Joseph H. Muler, and Norman F. Marti. EFFET OF EARLY CROSS TRANSYUSION ON $X-I R R A D I A T I O N$ DISTASE. Science $113,6-7(1951)$.

An examination of possible protective and regulatory factors of cross transfusion in the reduction of $\mathrm{s}-\mathrm{rad}$ tation toxichly is reported A summary of some of the data obtained with the use of a cross-transfusion apparatus (\$al1sbury, Proc. Soc. Exptl. Brol Med. 71, 604(1949); Salıbury and Miller, ibud. 74, 16(1050)) is presented. Twenty-fuve adult mongsel dog were used. The control group consisted of 19 dogs, and the treated group of 6 The latter underwent a aingle cross transfusion for approximately $2 \mathrm{hr}$ within $4 \mathrm{hr}$ after an irradiation of $450 \mathrm{r}$. A quantity of blood equal to the body weight of the recrpient was exchanged between each pair of anmals. Peripheral blood studies ghowed a depression of the total leukocytes to a mean value of 400 cells/ $/ \mathrm{mm}^{3}$ or less in the control group, whereas the cross-transfuged group manifested a depresgion to only 1800 cells/mm $/ \mathrm{mm}^{3}$ during a corresponding period after irradiation. The rehculocyte response in the control group was $0.2 \%$ or less in the enture posturradiation perod, whereas the cross-trangfused dogs showed a reaponse between 1 and $2 \%$ by the 15 th day after 1 rradiation (NSA)

1351

\$mmons, Erie $\mathbb{L}$. Leon 0. Jacobson, N. Pearlman, and C. L. Prosser. THE IECTIVENESS OR DRUGS IN PREVENTING OR ALLEVIATING X-RAY DAMAGE. Argone National Lab. Oct. 1946 68p. (MDDC-1277; CH-3329)
The protective and ameliorative properties of a variety of substances following irradiation were invest agated in rabouts and mice. Four man groups of chemicals were studied: (1) Physiological saline, (2) substances capable of altering the oxidation-reduction equilbrum of the body, (3) hemoposetic stimulants, and (4) enzyme precursors and activators. None of the substances tested prevented the drastic Immedate fall in leucocyle level that resulted in rabbits after $600 \mathrm{r}$ total body $\mathrm{x}$-1rradiation Physiological salme proved to be the most effectuve stumulant followng irradaation Ascorbuc acid was nest in effectiveness, followed by pentnucleotide, although the results with these druge were not as significant. Many substances produced marked temporary elevations in heterophis during the late recovery period, but these were not mantaned and, in fact, were frequently followed by returns to the control level. Lymphocytes, in general, were less responsive to drug stimulation than heterophis In no case had lymphocyte counts returned to their pre-rradiation level of 37 days. (ADD)

1352

Storer, John B. and Julnus M. Coon. PROTECTIVE EFFECT OF PARA-AMINOPROPIOPHENONE AGAINST LETHAL DOSES OF X-RADIATION. Proc Soc. Exptl Brol. Med. 74, 202-4(1950).

Para-ammoproprophenone (PAPR) when injected intraperitoneally un mice produces a maxumum methemoglobunemia in 30 muntes with only a slight decrease nu the methemoglobin level after 60 minutes of 180 mice given a single exposure of $800 \mathrm{r}$ total-body $\mathrm{x}$-radiation, $100 \%$ of 60 controls died withm 30 days pre-breatment of groups of the remaning 120 mice whth PAPP in doses of $20,30,40$, and $50 \mathrm{mg} / \mathrm{kg}$, 30 muntes before $\mathrm{x}$-ray exposure resulted in 30-day gurvival rates of $45,50,60$, and 72.5 , respectrvely. Injectuon of $50 \mathrm{mg} / \mathrm{kg}$ of PAPP after irradiation dio not affect mortality In a prelmmary test pre-treatment with PAPP also reduced the 30-day mortality in rats exposed to $x$-radiation (Authors' Summary) 1353

Swusher, S. N. and F. W. Furth. THE EFFECT OF EARLY SMALL EXCHANGE TRANSFUSION ON THE 8-IRRADIATED DOG Proc. Soc. Exptl. Brol Med. 78, 226-9(1951) (UR-186)

Immeduate removal of $250 \mathrm{cc}$ of blood and replacement with an equal volume of normal compatible whole blood in 20-1b dogs given $550 \mathrm{x}$ whole bodv 1 rradiation (expected $\mathrm{LD}_{90}$ ) results an a marked delay in onset of radration syndrome and mortality. There 15 a suggestuve decrease in ultimate mortality as well No differences in average leucocyte count, platelet count, or hematocrit in transfused dogs and in control dogs were observed, nor were there differences in clmical or postmortem anatomical fundings (NSA)

\section{MECHANISM OF RADIATION EFECTS}

General Theoretical Considerations

$135 \%$

Allen, A. O. EFFETS OF RADIATION ON MATERLALS, 10.207-22 of THE SCIENCE AND ENGINEERING OF NUCLEAR POWER, Chap. 13, Vol. I, Clark Goodman, ed. Adduson-Wesley Press Incorp., Cambridge, Mass. 1049.

Study of changes in chemical constitution and physical properties by high energy radiation is mportant to nuelear engmeering because (a) use of materials around pile structures is limited by radiation damage suffered; (b) the study of chemcal effects of radiation are umportant in the minter- 
pretation of biological effects. The types of radiation and their interactions with matter are summarized. Subjects briefly discussed include effects of radiation on the different types of chemical bonds, simple gases, organic compounds, water and aqueous solutions, metallic corrosion, and solids. (M.E.)

\section{5}

Allsopp, C. B. THEORTES OF THE BIOLOGICAL ACTIONS OF IONIZING RADIA TIONS. Brit. J. Radiol. 21, 72-4 (1948).

The two basic theories of the mode of action of ionizing radiation the target theory and the "poison" theory are examined critically. The present complete ascendancy of the target theory based on the work of Lea and Crowther are challenged. The author believes that the poison theory ghould not be rejected entirely and points to the formation of the free hydroxyl and hydrogen radicals when ionizing radiations pass through aqueous solutions. The possibility exists therefore that there can be a poisoning effect by 0 II radicals by breaking chromosomes chemically. This hypothesis does not entail a rejection of the target theory where the chromosome is destroyed by a "direct hit" by an ionizing particle, but rather may supplement this theory. (NSA)

1358

Atwood, IS. C. and A. Morman. ON THE INTERPRETATION OF MULTI-MIT SURVIVAL CURVES. Proc. Natl. Acad. 5ีc1. U. S. 35, 696(1949).

It is proposed that sigmoid survival curves can be interpreted profitably on a multi-unit, single hit per unit of hypothesis, and some consequences of this hypothesis are examined. An aid to the analysis of experimental data is given and its use is illustrated. Reasons are given for preferring the multi-unit hypothesis to the usual multi-hit hypothesis. Some data on the ultra-violet irradiation of Neurospora crassa conidia are analyzed. (Author's Summary)

$135 \%$

BaCq, 2. M. THE INDIRECY ACTION OF X-AND ULTRAVIOLET RADIATION. Experientia 7, 11-19(1951).

Recent literature and the author's experiments favor strongly the general idea that the action of ultraviolet and x-rays on enzymes, cells, and organisms is not entirely a direct one, that chemical substances (peroxides in the case of ultraviolet rays, oxidizing short-lived free radicals in the case of $x$-rays) resulting mainly from the action of rays on water molecules, are really the responsible agents. The greatest part of the action of ultraviolet or $x$-rays is indirect. It is possible by injection of cyanide, azide, cysteine, glutathione, thiourea, etc., to decrease very significantly the death rate of rats or mice irradiated with $\mathrm{x}$-rays, but only if the substance is injected before irradiation; so far, the biochemical lesion of $\mathrm{x}-1 \mathrm{x} \mathrm{z}^{\mathrm{r}} \mathrm{adiation}$ has been found irreversible. (NSA)

1358

Bojanowsky, Rudolph. QUANTUM PHYSICS AND BROLOGX. Verhandl. naturforsch. Ver. Brün 7\%, Vorträge vili-s (1911).

Subatomic aspects of biology are discussed. The lilling of a population of single-celled organisms (Euglena, Escherichia coli) by means of ultraviolet light, or Röntgen, cathode, or $\alpha-r a d i a t i o n$ has an exponential course which is a sunction of the time of exposure of surviving cells, $n=e^{-1 D t}$, where $n$ is the number of surviving cells, $t$ is the time of exposure, I is the intensity of radiation and $\mathbb{K}$ is a constant. A cell of $\mathbb{E}$ uglena must receive 8,000 quanta before being killed. Apparently a very small vital portion of the cell must be hit, perhaps a single atom or atom group in the mucleug. Direct radiation of the nucleus or of the cytoplasm of fern apores, gave $95 \%$ of the effect only in the nucleus. production of mutation in multi celled organisms follows the same law. The possible number of atoms in a gene is discussed. Poisoning with compounds such as $\mathrm{FgCl}_{2}$ or the action of a homone follows the same law. (CA) 1358

Burton, Milton. ELEMENTARY CHEMCAL PROCESSES IN RADIOBIOLOGICAL REACTIONS. Univ. of Notre Dame. [nd] 28p. (AECU-889)

The elementary chemical processes of radiobiology are the elementary chemical effects of high energy radiation on aqueous solutions containing oxygen, on pure organic matter, and on organic matter suspended in aqueous solution. In the aqueous layer the inportant primary physical process is ionization; in the organic portion both ionization and excitation must be considered. In biological systems the active particles in the aqueous layer are principally $\mathrm{O}$ and $\mathrm{HO}_{2}$. The presence of the latter particle increases the volume of the effective aqueous layer around the biological particle. The effectiveness of a hit in the organic materlal is determined in part by the propertles of the surrounding cage and depends, among other factors, on the size of the biological particle. The effect of a hit may be propagated by ionization transser, by free radical diffusion, by a chain reaction, or by change in local pH. Furthermore, Iree 11 and resultant $\mathrm{HO}_{2}$ are formed in the ambient liquid even when the hit is in the particle itself. In view of these elementary processes, the biological particle cannot be uniformly gensitive to radiation over its entire volume, the target of "target theory" is not to be identified with the biological garticle, a single ionization act is not necessarily lethal, nor is the target dimension simply related to the true zige of the biological particle. 28 references. (NSA) 1360

Claranf, 2. THE BIOLOGICAL ACTION OT RADIATONS IN RELATION WITIE CERTAIN PROBLEMS OF FIPERPMFNTAL PATHOLOGy. Quadern sez. perugina soc. ital. blol. sper. $2,35 p$. (1049).

A clear review, including a critical investigation of the mechanism of action of radiations on elementary biological indicators (virus, bacteria, protozoa) and on higher organisms, as well as an examination of the effects of radiations on cells. The theory of mutations is discussed with aference to the genesis of tumors. (GM)

1361

Dufresene, O. ROENTGENYILERAP OF PAIN. Can. Med. Assoc. J. 60, 227-9(1949).

Several theories have been advanced to explain the sedative action of $\mathrm{x}$-rays. They may be classified in two categoxies: those implicating a dixect action of $\mathrm{x}-\mathrm{ray}$ on the nervous elements, and those producing an indirect action on the nervous elementw, by humoral modih cation. As striking results from direct action of $\mathrm{x}$-rays on the nervous elements, the following may be mentioned: diminution of the ercitability of nerve endings, and modilication of the conductibility of nerves by a photo-electronic effect. Among the theories of the second category is the one advanced by Gilbert that $x-$ rays, by destroying white cells, could produce the liberation of triphosphate of adenostne. He thought also that mucleo-protein where it is lberated should provoke the vaso-dilatation of capillaries, modifying the mutrition of the sensory receptors and consequently mould relieve pain. Whatever the way by which the ${ }^{\prime}$-rays act on the pain, be it direct or indisect action, or by a combination of both. or even by the simple destruction of inflammation compresaing nerves, there are chemical fact that warays relieve pain. (S.V.\$.) 
1362

Dustin, piorre Jr. MTTOTC POISONS AND THE METHOD OF ACTION OF IONIZHG RADIATONS WN BIOLOGY. Euclides (Madrid) 2, 188-95(1949).

A review with 64 references. (CA) 1363

Pllinger, 要 redrick. THE PROBLEM OF RECOVER Y

FROM RADITION EFECTS. Radiology $40,62-71$ (1943).

The problem of recovery from effects of radiation is ditcussed from the point of view of cellular physiology, photochemistry, and clinical apylication. To clarify the problem of recovery, "true recovery" is designated as the connplete morphologic and functional resioration of single cells, while the term "pseudo-recovery" is suggested for the pestoration of organ function arising from sprowth of cells which have escaped xadiation injury. "Pscudo-recovery" therefore, is the expression of the regenerative power of the tissue in question. "Clinical recovery" means restoration of organ structure and function and may include "rue $\mathrm{Pe}-$ covery" as well as "pseudo-recovery." An evaluation of experimental and clinical observations on recovery of single cells, tissues, and organs shows the difficulties in determining "true recovery" and denonstrates that most of the known facts are easily explainable by "pseudo-recovery." (BA)

1364

Fanla, G. SOME ASPECTS OF THE BIOLOGICAL ACTION OF IONIZING RADIATIONS (JANEWAY LECTURE 1939).

Am. J. Roentgenol. Radium Therapy 44, 649-64(1940).

Experiments by Sugiura are analyzed in an attempt to explain some aspects of the effect of tonizing radiation. S. irradiated mouse sarcomata in vivo and subsequently transplanted them into other mice where they were inactivated by about half as much $x$ irradiation than when irradiated in vitro. In addition to the direct effect of the rays on the tumor cells, there is an indirect one exerted by the host and the tumor bed as a whole. In other experiments of S. fragments of sarcomata were irradiated with $1,500 \mathrm{r}$, then kept in distilled water, hypotonic, isothonic or hypertonie Locke-Ringer solutions; hypotonic solution, and distilled water enhanced the radiation effects, the others had no iniluence. All experiments suggest that radiation of lects are effected by shifi in osmotic pressure. Certain considerations in radiation therapy, such as varying radiosensitivities of similar tumors in different locations in the body, the determination of the value of pre-operative irradiation, and the proper interval between such irradiation and surgery, are discussed in the light of these findings. (TIS)

\section{5}

Forssberg, Arne. THE BEARING OF RADIATION CHEMISTRY ON THE MODE OF ACTION OF RADIATION, p.48 Of INTERNATIONAL CONGRESS OF RADIOLOGX, 6th Congress, London. 1950.

Ir radiation of aqueous solutions, especially of enzymes, has shown that a marked inactivation may result from quite moderate doses but that the presence of other substances in the solutions may fundamentally aller the result. If a similar action on high molecular substances, e.g., enzymes, also takes place in vivo, we should expect to observe secondary metabolic effects in the organism. The radioactive trace technique has yielded important results in the study of these problems, leading to the conclusion that even after moderate doses $(<1,000 \mathrm{~g}$.) the turnover of different intracellular substances, including not only nucleic acids but also proteins, fats and carbohydrates, is markedly and rapidly influenced. We are not in a position so far to judge the biological meaning of these metabolic changes; presumably, however, they may be of radiobiological significance. The mechanism of "protecting" an organism against harmful action of $X$-rays by the injection of certain substances has also been studied with this tracer technique.

1366

Friedman, Milton. THE RELATION OF TISSUE RECOVERY AND THE HEALING PROCESS TO THE PERIODICITY OF RADIATION EFFECTS. Radiology 33, 633-43(1939).

The phenomena resuling from protracted fractionated radiation may be divided into two phases, the destructive phase and the healing phase. Each phase has specific char acteristics and is modified to varying degrees by the quallty of the radiation, the size of the daily dose, individual susceptibility, the daily exposure time, and the continuity of the treatment. "The healing process is self does not function at a constant rate. Its fluctuating prowess determines whether destructive or healing phenomena dominate the clinical picture. It is ths cyclic alteration of destructive phase with healing phase that can be termed periodicily. The extent to which the character of the skin and mucosal reactions are reflected in the tumor probably depends upon the latter's degree of differentiation and consequently its morphologic and physiolugic resemblance to the parent tissue. During the healing phase, residual tumor cells have usually been malured by irradiation and they too have adapted themselves to radiation effects. It is, therefore, recommended that during a prolonged course of radiation no treatment be given during the healing phase. (Author's Sunmary)

1367

Gerstner, Herbert B. and Oskar L. Ritter. THE BIOLOG ICAL AND MEDICAL ASPECTS OF IONIZING RADIATION, I. THE EPTECT OF X-RADIATION ON THE POTENGLA DIF FRRENCE ACROSS ISOLATED FROG'S SKIN, USAF

School of Aviation Medicine. 6p. (Report No. 1)

For living membranes or tissue slides an electrode has been developed which enables the determination of resting potential, electrical impedance, permeability to tracer isotopes, and the simultaneous exposure to ionizing radiation. By means of this electrode, isolated frog's skin was exposed to an intensity of $\mathrm{x}$-rays of $10,000 \mathrm{r}$ per minute. Two main observations were made: first, $\mathrm{x}$-radiation affects the skin potential without delay; second, $x$-radiation destroys the processes essential for the maintenance of the potential. The technique employed should prove useful in shadying the mechanics of tissue damage and the effectiveness of protective agents. (auth)

\section{8}

Glucksmann, A. CYTOLOGICAL ASPECTS OF PROTECTION FROM IONIZING RADIATIONS. Brit. J. Radiol. 23, 41-5(1950).

A review is presented concerning the cytological aspects of protection from radiation. The two main concepls of the mechanism of radiation action on cells and the radiation ef fects on germ cells and somatic cells are briefly discussed. The target theory of the biological action of ionizing radiations explains the radiation effect on the assumption that ionization oceurs in or in the immediate neighborhood of certain molecules which constitute a vital target. A hit on this target is assumed to produce changes such as cell death in bacteria and viruses, gene mutations, or simple chromosome breaks. The "intracellular poison" concept holds that radiation effects such as inhibition of mitosis are brought about by radiation chemical changes almost anywhere within the cell. Localization of xadiation effects is secondary and due to a specific sensitivity of some cell constituent to radiation chemical products. The poison concept accounts for effects known to depend on the rate and fractionation of the 
radiation dosage. The mechanisms for the production of genetic effects are gene mutations and chromosome structural changes. The direct and indirect carcinogenic effects of ionizing radiations are described. It is concluded that until more fundamental information is available we can do no more than hazard guesses at the maximum permissible doses of radiation; our knowledge of cytological radiation effects is incomplete and further research is required, particularly with regard to the effects of chronic exposure to ixradiation and to the mechanism of tumor induction by radiation. (NSA) 1369

GTAY, L. H. THE INFLUENCE OF RADIATION QUALITY ON RADIOBIOLOGICAL RESPONSE, P.48-9 Of INTTRRA TONAL CONGRESS OF RADIOLOGY, 6th Congress, London. 1950.

The following types of radiobiological lesion may be distinguished: That in which the damage inflicted on individual cells appears to result from the integrated effect of the ionisation produced by a considerable number of particles. That in which the primary damage is caused by a single ionising particle or even by a single ion pair or cluster. Each class of radiobiological damage will have a character istic dependence on the type of ionising radiation employed, determined by the distribution in both space and time of the 1370 primary acts of ionisation and excitation.

Howard, A. REPORT OF SYMPOSIUM ON MODE OF ACTION OF IONIZING RADIATIONS. Nucleonics ?, 26-30(1950) DeC.

Summary of papers dealing with the mechanism of the biological action of ionizing radiatlons, presented at the Sixth International Congress of Radiology, London, July 1950. (PA)

1371

Latarjel, R. PRIMARY BIOLOGICAL EPFECTS OF RADIATIONS ORSERVIRD IN THE STRUCTURE OF MTCROORGANISMS. Rev. can. biol. 5, 2 47(1946).

The primary effect of radiation on a cell or a mieroorganism is to initiate a chain of reactions leading to an observable injury, and must be considered photochemical, affecting a limited number of absorbing molecules. Two such physically different absorption processes as ionization and moleeular ionization can induce the same chemical changes in the same sensitive cellular zone. This zone uses an equal a mount of energy in both cases, the effect of low energy photons accumulating to give the same effects as ionization. The energy of a single photon (4-5 electronvolts) is sufficient to produce a chemical change in nucleoproteins. A single photon may inactivate bacteriophage. The gone which governs the division of the dysentery bacillus is of a nucleoprotein nature. Pls diameter is greater than or equal to $60 \mathrm{~m} \mu$. In yeast, division is governed by two distinct formations similar to those in bacteria. The bacteriophages contain a radiosensitive nucleoprotein which supports the specific lylic power. The disappearance of lylic power results from the alleration of a single molecule. In some viruses, particles observed with the electron microscope and measured by filtration or centrifugation seem to consist of "minimal units." The organizafion of the viruses seems to become complicated in propor tion to their size. (BA)

\section{2}

Layne, D. A. THE PRODUCTION OF RADIOACTVITY IN ANMMAL TISUES BY MTERE RNERGY X-RAYS, p.180 of INTERNATIONAL CONGRESS OF RADIOLOGY, 6th

Congress, London. 1950.

The main object of the experiments to be described was to investigate the production of radioactivity in tissues by exposing rat tissue to beans of $\mathrm{X}$-rays from the Royal Cancer Hospital 30 Mev synchrotron. Activilies having half-lives less than aboul one minute or more than a few hours would not be detected in our experiments, but within these limits, oxygen and carbon appear to be the elements mainly responsible. Attempts have been made to calibrate the experimental counting equipment and to calculate the number of quanta above threshold energy level falling on the tissue per unit time. From these the cross-sections for the photo-nuclear reactions have been estimated and are of the order of magnitude estimated for other $\gamma-$ n reactions. The biological significance may be calculated by considering the absorption of energy in tissue from the $X-r a y$ beam and from the disintegrations. It seens that, unless there are strong short-lived reactions and high resonances, the absorption of energy in tissues from disintegrations will not contribute significantly to the biological effects of high energy $\mathrm{X}$-rays in them.

\section{3}

Lea, D. E., R. B. Haines, and E. Bretcher. THE BACTERICIDAL ACTION OF X-RAYS, NEUTRONS, AND RADIOACTIV RADIATONS. J. MYg. 41, 1-16(1941).

It is suggested that the lethal effect of these rays on bacteria is due to the ionization of vital areas or sensilive chem. regions in the cells. Perhaps these "targets" are genes. Many ionizing particles could pass through a cell without siriking such a structure. About 100 particles must pass through a colon bacillus to cause death. The effect was independent of the time required for them to pass through. The lethal effect was independent of temperature or the concentration of the ionizing rays. (CA)

\section{4}

Lea, D. E., K. M. Smath, B. Folmes, and R. Markham. DIRECT AND INDIRECT ACTIONS OF RADIATION ON VIRUSES AND ENZYMES. Parasitology 36, 110-18(1944).

The inactivation dose for tobacco mosaic virus of gamma rays is independent of concentration when the virus is irra diated dry or at high concentrations. At low concentrations the inactivation dose reaches a const. but lower value, Over the intermediate range, the inactivation dose increases with concentration. When the virus is irradiated dry or at high concentrations, the inactivation is direct and is due to ionization produced inside of the virus mol. At lower concentrations the inactivation is largely indirect and due to ionization of water. Addition of gelatin to virus solutions protects against the indisect action. Dry, powdered preparations of zibonuclease and adenylpyrophosphatase are inac tivated by radiation with $3.4 \times 10^{7}$ and $5.5 \times 10^{6} \mathrm{r}$, respectively. On the assumption that a single ionization in an enzyme molecule leads to its inactivation, measurement of the inactivation dose gives a method of estimating the molecule weight of the enzyme. There appears to be no fundamental difference in the mechanism of radiation-inactivation of viruses and enzymes. (CA)

\section{5}

Minder, W. ANEW THEORY OF THE MECHANISM OF IRRADLATION ERTECTS. Radiologia Clin. 16, 73-81 (1947).

Continuing his previous work, the author gives mathematical proof of his theory of the effects of radiation based on experiments and the laws of chemical reactions. This theory expresses at least formally all the four types of the action of radiation in relation to dosage, namely: (1) proportional course of the curves of action; (2) "delayed" linear conrse; (3) exponential course; and (4) "delayed" exponential course. The time-factor of the effect of radiation is also consistent with this theory. (BA) 
1978

Minder, W. ON THE EXPONENTIAL LAW OF THE

RADATION EFIPCT. Radiologia Clin. 18, 300-41(1949).

As lis known, the exponential shape of the dose curve is explaned by the "iarget" (or "hit" efect on living cells by considering this effect as due to guanifized events whose probability is expressed by certain volumes, the "target volumes" of the effect under consideration. In the light of recent invertigations of chemical reactions produced under the action of $\mathrm{x}$-rays (oxidation of

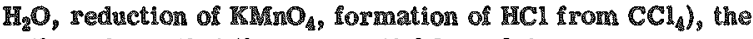
author shows that the exponential law of these processes can easily be explained with the aid of wuch mechanisans as rever sible reactions, secondary reactions, or inbibitory effects of compounds present; the work of these mechanimms is governed by certain probablitiew, each expressed. by a volume, the "reverge action volume"; the latter" absolute dimengions vary within the same limits (100 to 400 A linear dimension) that characterize the "target volume" of the radobloblogical theory (e.g., the inactivation of various bacteriophagle microorganimms by $x-$ ray action). These analogies camot be accidental and should motivate a reshaping of the target theory. That the concepts involved have a deeper physicochemical meaning than that accepted by the biological theory is corroborated by the fact that for many chemical reactions, induced by $\$-r a y$, , there exist diluting upper $11 \mathrm{mits}$ below which a radiochemical reaction doss not depend on the concentration: at thowe limitr the distances between molecules involved reach the same values, 100 to $400 \mathrm{~A}$, mentioned albove. A concept which describes this phenomenor, and which evidenty underlles all the others surveyed above, is that of energy transportation without Loss, performed along a characteristic elementary distance. (NSA)

1 เงตา

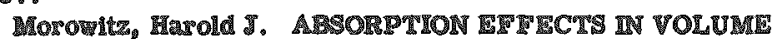
IRRADLTION OF MCROORGANSMS. Science 111, 229$3011950)$.

Irom target theory a relation has been derived which gives the percentage of organism, sugpended in a medium, which survive irradiation of a glven dosage (Bee Pollard and Forro, Science, 100: 374, 1949). This derivation is strictly valid only If the suspending mediun does not absorb the radiation, or if the organisms are spread out in a negigible thickness of the muspending materal (surface irradiation). The author derives a corresponding expression for absorbIng gugpending materials, for the two cases in which the organisms are fired in the guspending material, and for the case in which the organisms are contantly stirred. (NSA) 1878

Ner, Ladislam. THEORETCAL AND IXPIRIMISTAL

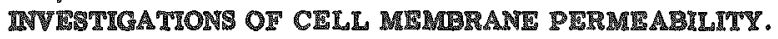
Thtrahlentherapie 79, 141-63(1049).

By agplying the laws of classical mechanics, the author attempts to explain and coordinate certain features of cell membrane permeability, wuch as its periodicity and its change under the influence of Fadiation. It is fourd that the membrane permeability can be considered a phenomenon which is propagated through the organimm in wave form, the phase velocity of the wave being of the order of magnitude of the velocity of conduction of nerve impulses. This leads to the assumption that the medium in which the waves are propagated is the nervous system. Special attention ia paid to the changes of cell membrane permeability after irradiaton with $\mathrm{I}-\mathrm{Fay}$, and to the periodicity of biological radiation effects in general. (NSA)

1379

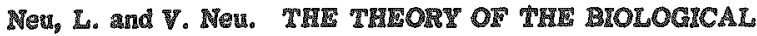

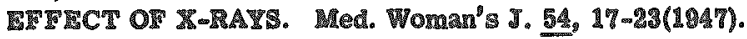

The permeability of cell membranes is increased after X-ray ir radiation; unstable cell membranes have an increased sensitivity to $\mathrm{X}$-rays. A hypothesis is advanced showing a close connection between the periodic behavior of the perneability of cell membranes and the recurring $X_{\text {- }}$ rays effect erythema. The conc. of ions in a cell inereases with irradiation tine; the primary effect of $X$-rays is an irseversible poisoning of the living cell. (NSA)

1380

Opatowski, I. THE PROBABILISTIC APPROACH TO THE PFIECTS OF RADLATIONS AND VARIABILITY OF SENSITVITY. Bull. Math. Biophys. 8, 101-12(1946).

The calculation of the Bize of the "Bensitive volume" or "control center" in biologic effects of radiations is discusged from the viewpoint of the probabilistic theory of these phenomena based on the concept of random "effective events." On the basig of that theory, the resigtivity of a microorganism to radiation is defined as its "mean life" under a radiation of one $\mathrm{r} / \mathrm{min}$. This means is calculated for processes with and without recovery. The case of variable sensitivity, as it occurs, for instance, curing mitosis, is discussed in detail. Methods are given to calculate this variability from survival curves or similar exptl. data. The theory is applied to expts, of A. Zuppinger on irradiation of Ascaris eggs with X-rays. (BA)

1381

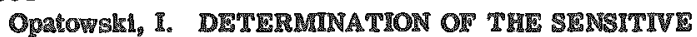
VOLURE BV IRRADLATION AND TKE IONIZA TION DEN-

5ITY. Bul. Math. BLophys. 13, 263-71(1051).

Lea's formula for the determination of size of the sengltive volume is corrected and applied to experiments on inactivation of the tobacco mosaic virus using three different wave lengths of $\mathrm{x}$ rays. The purely geometric concept of the sensitive wolume is not gufficient for explaining the known dependence of the inactivation of this virus on wave length. The concept of trangser of energy around the ionizing par ticles is diseusged in this connection in a manner similar to that suggested by Pollard and Forro for the inactivation of the $\mathrm{T}-1$ phage, and restults similax to those given by these authors are obtained from an analysis of the inactivation data of J. W. Gowen (tobacco mosalc virus). Differ ences of interpretation of these two sets of experiments carried out with different types of radiation are pointed out. (auth)

1302

Opatowski, I. and F. R. Tangherlun. DUHUSION AND RECOMBINATION OF PRODUCTS OF IONIZING RADIATIONS. Zu11. Math. BLophys. 13, 273 -88(1951).

The relative importance of diffusion and recombination of the products of ionizing radiations is examined in several typical cases and methods for the golution of the differential equation of columnar ionization are discussed. It is shown that Jaffés method is not applicable to radiobiological or purely radiochemical processes in which no external electric fields are acting. A method based on a recent suggestion of $\mathrm{K}$ ramerg appears to be particularly good when recombination is strong. (auth) 1383

Osgood, F. E. IS THE ACTION OF MOENTGEN RAYS DIRECT OR INDTRECI, (AN INVISSTMATION OF THIS

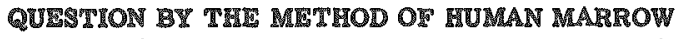
CULTURE). Am. J. Roentgenol. Radum Therapy $48_{8}$ 214-20(1042).

Ten cc of human marrow obtained by sternal puncture was mired with 25 ec of cilrated balanced salt soln. After manipulations to eliminate most of the non-nucleated red cells, the final culture consisted of about $100,000,000 \mathrm{nu}-$ cleated marrow cells muspended in 50 cc of culture medium. With this material, 3 types of irradiation were done, - cella 
and medium, cells alone, and medium alone. When the medium alone was irradiated, and then added to non-ir radiated cells, there was no effect on the cells, whereas increasing cell destruction with increasing amounts of radiation was observed in the specimens in which both cells and medium, or cells alone, were irradiated. (BA) 1384.

Pinney, Edmund. ON A MATHSMATICAL THEORY OF THE REACTION OY CELLS TO II-RAF IRRADIATON. Bull. Math. Biophys. 12, 199-206(1950).

To deacribe the variation of concentration of certala substances in cells subjected to $x-$ rays for a finite length of time, R. M. Sievert (Acta Radiol. 22, 237-51(1941)) has given an equation of mixed diferences involving time-lag and having discontinuous coefficients. In the present paper, Laplace transform methods are applied to solve this equation in terms of a serieg valid for all values of time. In particular, the conditions previously giver that the concentration approaches a constant value are confirmed; otherwise, steady oscillations in concentration may be expected, or oscillations appear whose amplitude steadily increases until the cell is destroyed or the assumed mathematical model otherwise fails to apply. (NSA)

1385

Pollard, 2 . IONIZING RADIATON AS A TEST OF MOLECULAR ORGANIZATION. Am. Sclentist 39, 98(1051).

The nature of the effect in a large molecule of energy release due to ionizing radiation has been atudied. Results ghow that where precise molecular organization is necessary for the action of a molecule such as, for example, in the action of an enzyme on a substrate, the enzyme organization is destroyed over a large region by the action of primary ionization. Rough ligures for molecular weight can be obtained by these methods, and some conclusions about enzyme structure can be drawn. The present study includes the results of many invegtigators. (NSA) 1386

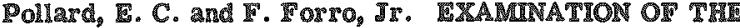
TARGET THEORY BY DEUTERON BOMBARDMIENT OF T-1 PHAGE. Science 109, 3\%4-5(1049).

The authors investigate the target theory of biological action, according to which a localized sensitive volume of definite size exigts and within which there must be considerable liberation of energy for biological action to take place. Although Lea has examined the theory (Brit. d. Radiol, Suppl. 1, p.35 (1947)), according to his views a specimen irradiated in high concentration, and in the presence of a high concentration of large molecules, shousld have a low eficiency for indirect action and the direct, targettype action should predominate. Since there is some question as to whether direct action ever oceurs, the target theory has been investigated in deuteron bombardments in which it should certainly be valid; these were made using T-1 bacteriophage. Tabulated data on the percentage survival as a function of the number of deuterons per unit area are given for beam energies of $1.5,2.5$, and 3.5 Mev. Taba lated data on the temperature inactivation of the same phage are given; these data suggest that Lea's theory should be modified to include the possibility of inactivation caused by secondary radiation reaching the target from an ion path which does not go through the target. This is developed mathematically and the results obtained indicate that the data are consiatent with the following hypotheses: 1) a target of $28 \pm 4 \mathrm{~m} \mu$ exists which is much smaller than the phage itself, and 2) the meaøured target gize is greater than the true target size owing to the fact that radiation from the path of a deuteron which misses the target can inactivato the phage. (NSA)
1387

Read, John. THE LATERAL DISTRIBUTION OF IONS ACROSS THE TRACK OF AN IONIZING PARTICLR. PART 1. THE PRESENT PICTURE AND ITS IMPLICA TONB. Brit. J. Radiol. 22, 368-74(1949).

If Lea"s (Brit. J. Radiol. (Supplement 1), 59, 1947) picture of the lateral diatributions of positive and negative lons across the track of an jonizing particle is correct, then the separation of charge must lead to a radical electric ileld. Expressions are deduced for this field, the corresponding potential differences, and the energy per ion paif stored in the field. Numerical values are obtained for an fon densify of 1,000 lons ger micron in water, hexane, and a chromosome thread. The fidd is of the order of $10^{5} \mathrm{v} / \mathrm{em}$ at relatively large distances from the asis of the track. An approsimate expression is derived for the distribution of the positive and negative ions when in thernal equIIbrim. Small lon densties can be in equillorimn with quite large aegaration of charge, but in the case of lon densities of 1,000 ions per micron tea's distributions are far from exulibrium, and the electrons would return to the positive core

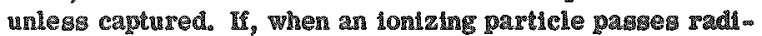
ally across a chromosome, this separation of charge occurs, and the electrons become attached to the rabric of the chromosome, then tersions of the order $10^{6} \mathrm{dynes} / \mathrm{cm}^{2}$ would be produced normal to circles of radius comparable with that of the chromosome. A tension wave would travel in both directions away from the point of intersection of track and chromosome. If the chromomome has weak points, e.g., between genes, break might be produced hers, and the minute delettong might be due to the two tenglon waves each producing a break. Reference is made to the work of Jaffe (Ann. Prys. $12,303,1013$ ). (NSA) 1383

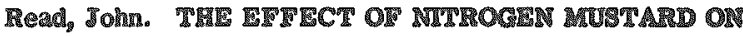

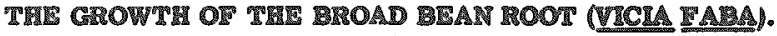
Brit. I. Radlol. 23, 504-5(1050).

Nitrogen mustard is aaid to be radiometric becaume it produces effects apgarenty 1 imilar to gone produced by londzing radiations. IExeriments ghow that oxygen does not enhance the efrect of $\mathrm{N}$ (under condition which greatly augment the effect of z-rays. Alternative hypotheses are (1) oxygen increases radiosengtivity by changing the metabolic gtate, (2) the oxygen reacts with the products of ionization (II atoms) to produce an increase in some toxic product $\left(\mathrm{HO}_{2}, \mathrm{H}_{2} \mathrm{O}_{2}, \mathrm{O}\right)$. This experiment does not prove that hypothesis (2) is correct but is one which might have disproved it and has not. (M.

1382

Read, John. PHYICAL ASPECTS OF TRE BIOLOCHCAL

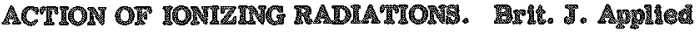
Phy1. 2, 337-45(1951).

The influence of such physical factors as the linese fon density created by different radiations, the presence of dissolved oxygen or certain other molecules, and the dose zate, on the production of blologlcal effects by loniming radiationg is discussed. Certain radiochemical experimentr are outlined which show mimilarites whth radiobiologlcal oxperdments. Hypotheses are guggested which attempt to correlate and explain some of the biological observations. (NEA) 1980

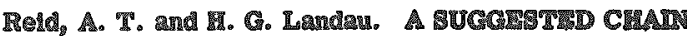

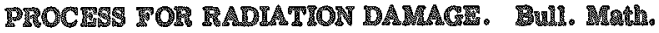
Riophys. 13, 153-61(1051).

A mechanim by which transmiasion of the initial yadia tion damage, causing fonization in a ansttve volume due to absorption of radiation quanta, may talse place, and a finite Markov chain model applicable to this tranamisalon 
are postulated and discussed. This mechanism is assumed to be the depolymerization of exsential chain molecules which are conmected to some "central group" associated with the sensitive volume. The depolymerization of the macromoleculs following a hit in the gensitive volume is postulated to be determined by a chain mechanism, which acts in a manar inverse to the mechanism controlling the polymerization process. A mathematical study of this problem is made, using the theory of Markov chains. The probability of complete degradation of the chain macromolecule and the probability of recombination of the units to give the intact chain were determined, assuming that the probability of accessive steps in the degradation inc reases linearly from the intact state to that of complete breakdown. (NSA)

1391

Ros, W. C. J. BIOLOGCAC ACHON OF X-RAYS,

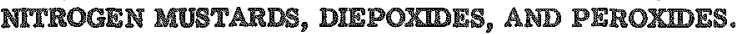

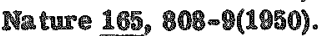

Organic peroxides are mutageric, and induce chromosome abnormalities in Vicia faba. Similarify of the effects produced by $\mathrm{N}$ mustards and dieposides, which react by a carbonim-ion mechanism, and those of $\mathrm{x}$-rays and peroxides, which ract by a free radical mechanism, suggest similarities in their mode of action. The nucleophilic centar of the original sygtem is changed to a highly reactive electrophilic center. The new electrophilic canter could 5eact fruther with a nucleophilic group on the same surice or with a group on a different surface. Mustards, dieporides, and perosides appear to produce breakage of the long chromosomes of 'Vicia laba at apeciflc regions while ion 1242 lang radiations produce more random breakage. (CA)

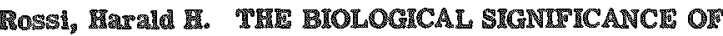

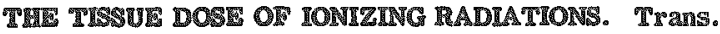

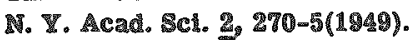

A general discussion of ionizing radiations is given, with agecial reference to the problem of a quatitative measur ment of radiation and the question as to how far the result of

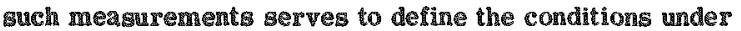
Which a radiobiological experiment in pertormed. 5patial and temporal considerations of dosinetry in connection with ionization and induced electronagnetic inalds are gualitatively discussed; the author zuggests the further collaboration of physicterts with biologist for more exact dosimetry messurements and for discussion of the complex theoratical problems which arise in treatment of biological material. (1)

\section{9}

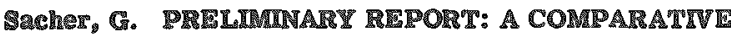

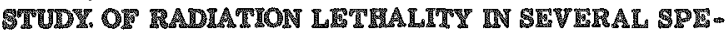

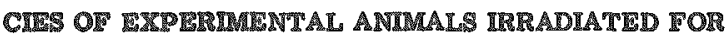
FEIF DVRA TOLOCICAL AND MLDICAL RESEARCH: OUARTIRLY REPIT, May, Jum, July, 1950. Argonne National Lab. T147 1950. 14

An analysis of radiation lethality in metazoam forms has been developed on the basis that (1) many or all aspects of physiological function are affected by radiations, (2) the regponses of phyplological systems lo irradiation, and the interactons between sytems, are linear, (3) a lethal procc) ar: constiment systems, (4) death accurs when this process intersects with a lethal bound of Inyuy, and (5) when aging Is a mignificant factor, the effects of the aging process and the radiation process combine additively. The Iurther ag promimation ir made that the behavior of a population is described by a representative animal, taken to be the mean or median animal. This latter approximation permits the interpretation of data from small groups of animals, which is carried out in the present paper. The data analyzed were mean survival times at various daily exposures. Data for several species were available. By using the above hypothesis, it has been possible to deduce fur each species a cumulant lethality function and an impulse lethality function which describe the course physiological injury would follow conssquent to continuous and to single erposures, respec. tively, if the species behaved according to the hypothesis. No internal test of consisteney is possible, but determina tion can be made as to whether the derived lethality functions are consistent with other hinds of information. At least semiquantitative consistency was obtained for several types of comparison. 20 references. (NSA) 1394

Sanden, Kunt von. TARGET-TMEORETICAC INTERPRETATION OF THE RELATON BRTWEEN THE STISPNESS OF THE DOSAGE ATYON DENSIXY. Z. Natur

A statistical theory is developed which describus the ef lect of a given dosage of ituizing radiation on a set of biological objects distributed at random in the space filled by the radiation. It is assumed that an elementary target area must suffer simultaneous multiple hits to be affected by the radiation. The ionization density enterg fom the beginning into the formulation of the statistical problem, with the sult that the steepness of the dosage-efect curve dec seases with increasing ionization density. (NSA) 1395

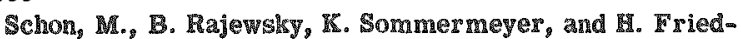
rich-IIESA. QUANTUM PHYSICS AND STATETCS IN BIOLOCY. In FUAT Rewiew of German Sclence, 1939-1940: Blophysics, Part 1, p. 1 -49.

This siew article is primarily a guide to the literature of guantum physics and gtatistics in biology. Approximately 150 relerences are given. Separate sect lons deal with quar tumtheoretical features of biological problems; foundations of the target theory of the biological effect of radiation; funther development of target theoretical concepts; energy migration in molecule complexes and structural units; forees which play a role in the composition of biological structural units. (NSA)

1396

Schubert, G. ANALYSIS BY PADIATION OF BIOLOGICAL LLEMENTARY STRUCTURES. Strahlentherapie 80, 1-16(1949).

This is a review article, written primarily from the gtandpoint of the target theory, on the effect of radiation on elementary biological structures such as genes, bacteriophages and viruses. In particular, the number of hits on a target necessary for biological effect, the role of energy migration in the elementary structure, and the possible size of the elementary target regions as deduced from ir radiation experiments are discussed in come detail. (NSA) 1398

Scott, C. M. SOME QUANTITATIVE ASPECTS OF THE BIOLOGICAL ACTION OI I AND GAMHA RAYS. MEA. Research Council (Brit.) Spec. Rept. Ser. No. 223, 1-99 (1037).

In Part I (pp 1-71) the literature bearing on the mecham nism of action of the rays is briefly and critically surveyed. Part II (pp. 71-99) reports experimental work 100,000 $\mathrm{r}$ of $\mathrm{x}-\mathrm{rays}(80 \mathrm{kv}, 5 \mathrm{ma}$, digtance $12 \mathrm{~cm}$, filter $0.1 \mathrm{~mm} \mathrm{Al}$, intensity $550 \mathrm{r} / \mathrm{min}$ ) produced no change in the rate rhythm or amplitude of contraction of the isolated heart of Rana esculenta; $16,500 \mathrm{~g}$ had no effect upon the heart's respiration or upon it conversion of carbohydrate to lactic acid. The contraction of isolated auricles was unaffected by several days' continuous exposure to the $\beta$ and $\gamma$ gays from 5 
$\mathrm{mg}$ Ra element (distance $2 \mathrm{~mm}$, filter $0.5 \mathrm{~mm}$ soda glass). When the hatching of eggs of Colliphora erythrocephala was inhibited by $\mathrm{X}$-rays $(80 \mathrm{kv}, 5 \mathrm{ma}$, distance $47 \mathrm{~cm}$, filter $0.1 \mathrm{~mm}$ Al) the Bunsen-Roscoe law was valid for intensities ranging from 13 to $100 \mathrm{r} / \mathrm{min}$. The dose required to kill. $50 \%$ depended upon age and ranged from 50 to $340 \mathrm{r}$. The radiosensitivity of the eggs was increased by chloroform, ethyl ether and petroleum ether, but was unaltered by niIrous oxide. When $\mathrm{x}-\mathrm{ray}$ was superimposed upon injury by low temp., the results indicated that the two injuries were unrelated in mechanism but that in general the individual eggs which were sensitive to cold were also sensitive to x-rays. Similar results were obained when asphyxia or $\mathrm{CO}_{2}$ was substituted for low temperatures. (BA)

\section{8}

Sommermeyer, IF. THE THEORY OF RADIO-BIOLOGICAL SATURATION FFFECT. Naturwissenschaiten 30, 104-5 (1942).

In detg. the satn. factor of biol. radiation effects the no. of active ionizations per unit vol. count. The satn. factor is proportional to $\mathrm{pr}^{\prime \prime} \mathrm{z}$ with $\mathrm{p}$ the activity probability, $T$ the vol., L the distance of successive ionizations. It is stressed 1399 that only the no. of active lonizations counts. (CA)

Sommermeyer, K. ON THE EIFECTIVE CROSS SECTION OF PROTONS AND ALPHA-RAYS, AS COMPARED WITI THE RADIATION-SENSITIVE VOLUME, IN ELEMENTARY RADIO-BIOLOGICAL REACTIONS. 2. Naturforsch. $36_{9}$ 57-9(1948).

In a previous work (Naturwissenschaiten $31 ; 172$ (1243)) the author has shown that some radiobiological phenomena cannot be interpreted as due to a saturation fofect if the total cross sertion of the ionizing proton is considered effective. Thus is confirmed by the findings of Lea (Actions of Radiations in Living Cells, Cambridge 1946) who has shown that, for many processes, the saturation hypothesis explains very well the quantitative data if the biologically effective cross sections of protons and $a$-rays are disregarded as against the dinensions of the radio-sensitive volume. However, the author objects to the interpretation of this situation offered by Lea, and suggests, as a mechanism of the elementary biophysical reaction involved, a capture of a secondary electron by a complex organic molecule. (NSA)

\section{0}

Sommermeyer, R. RESULTS AND PROBLEMS OF THE TARGET THEORY IN RADIOBIOLOGY. Naturwissenschaften $38,289-98$ (1951).

The only assumption made by the target theory is that radiation absorption in biology follows the laws of the guantum theory, the aim of which is to find the relationships between the primary reactions of the ionization radiation with the biological target and the ultimate fotal biological effect. Stais stical experiments serve as fundamental application of the theory. Examples given of experiments with high-energy radiation show dependence of target numbers on dosage and ionization distance (from primary reaction to ultimate path in the radiosensilive medium). Fxperiments with primary biological structures such as viruses, phages, genes, and chromosomes are reviewed, dependence of radiation effects on the size of these bodies is shown; radiation effects (inactivation, chromosome aberrations) decrease with bodies of increasing size under the same strength of radiation. The biochemical foundations of these reactions are analyzed in delail. Reviews of target-number compulations for i inazation-radiation effects on higher organisms are guven for bean seedlings and eggs of Drosophila. The relationshp of radiosensintive volume and indirect ionization-radiation reactions, the safety in using exponentiat curves resulting from experiments in target mumber com putations, and the energy distribution of the primary ionizations and their dependence on the energy of the ionized particles are discussed. Zxperiment with the kapazitron investigate the ionization-radiation cifectiveness of particles of various cnorgies on primary biological structures of larger size. (NSA)

1401

Stewart, L. N. and Jean Bouchard. RICENT ADVANCLS IN RKOWLEDGE OF THE BIOLOGIC EISIECT ON RADIATION. J. Can. Assoc. Radiol. 1, 12-15(1950).

Irradiation can induce changes in biological materials through an intermediate product (probably or) derived from the solvent. The stage of prophase remains the reritical period in radiosensitivity of the cell and nuclear changes depend on radiosensilization of a target molecule in the vicinity. Biological reaction is determined by linear în density. (CA)

1402

TimoféeV-Messorskǔi, N.W. and R. G. Zimmer. THE TARGET IRINCIPLE IN BIOLOGY. Hirzel, Berlin. 194.

A mong the subjects covered are: The historical development and formal statement of the larget theory; the target theory with relation to biological variability, analysis of release of gene and chromosome mutation thru radiation, biophysical analysis of bacterieidal effects of radiation; mechanism of energy migration in an ordered system; static ultramic roscopy with fonizing radiations. (N.F.)

1403

Tobias, Cornelius A. MECHANISMS OF BIOLOGICAL ERPFETS OF PENETRATING RADIATION. Federation Proc. 10, 595-601(1951).

Brief descriptions are given of the mechanism of interaction of radiation with matter, various biological effects of breakdown of macromolecules by direct action of ionizing radiation, and examples of recent experimental approaches to indirect action of radiation. The efects of radiations at the cellular level were studich in haplot and diplotd yeast cells. The 5 times greater resistance of diploid cells against radiation injury is explained as due to the presence of paired genes and chromosumes in the diploid cells, controlling the cell mechanisms from a number of mutually inderendent pairs of sites, whereas the one set of chronosomes in haploid cells results in each site being represented only once. Radiation effects on mammals and new modes of therapy of radiation injuries are reviewed. (NSA)

140

Van der Werf, 3. T. FURTIER DEVLLOPMIST OF THE THEOY OF BIOLOGICAL REACTIONS CAUSED BY ROENTGEN-RAYS. Acta radiol. 28, 168-84(1947).

The author maintains that each theory on a biolowic problem should take the existing metabolism into account. In 1942 , he based a mathematical theory of the biologic reactions caussed loy Roentgen-rays upon the principle and in this paper he described a further development of this theory. If some "brogenic" substance, in the steady state before irsadiation, is influenced by $\mathrm{X}$-rays, a change not only in the rate of breaking down bul also of the rate of buildingup of that substance may occur. With the aid of some assumptions it becomes possible to const ruet an "equation of metabolism." In order to test his theory, the author malkes use of $A$. Forssberg's studies on the influence of $X$-rays upon the growtin rate of the fungus-cell Phycomyces blakesleeanus. With the aid of the proposed equation, a full explanation of these experimenis can be given. The theory can also be applicd with equal suceess to the phenomena of axcitation of merves and so covers lwo large flelds of biologie reactions. (BA) 


\section{5}

Warren, S. MECHANISM OI RADIATION EITECTS

AGAINST MALIGNANT TUMORS. J. Am. Med. Assoc. $133,462-3(1047)$.

A brief discussion of current theories on the effect of $x-$ rays and radioactive radiations upon normal and diseased tiswes. (BA)

1406

Watson, J. D. INACTVATING MUTATIONS PRODUCED BY X-RAYS IN BACTERIOPHACES. Genetics $33,633-4$ (1948).

Lur'ia reported in 1947 that reactivation by multiple infeetion, such as he discovered for ultraviolet inactivated bacteriophages, could not be detected after X-ray inactivation. In reinvestigating this problem, we found that $\mathrm{X}$-rays, besides inactivating phage, also suppress the ability of phage to infect bacteria, one "adsorption suppressing" hit occurtring on the average for every three inactivating hits. Because of this, reactivation is difficult to detect by the multiple-infection technique. We tested for it by infecting individual bacteria with one particle $T 2 r^{*}$ inactivated by $\mathrm{X}$-rays and one particle T2r inactivated by ultraviolet. A significant proportion of these bacteria liberate a mixture of active $\mathrm{T}^{\mathrm{t}} \mathrm{x}^{+}$and active $\mathrm{T} 2 \mathrm{x}$, which proves that $\mathrm{X}$-rayed phage can participate in genetic recombination and reactivation. Similar conclusion was reached by infecting bacteria with one active particle $\mathrm{T}^{+} \mathrm{r}^{+}$and one $\mathrm{X}$-rayed particle T2. Finally, reactivation was also detected by mised iniection with one particle each of X-ray inactivated T2 $\mathrm{r}^{*}$ and T2s. Quantitatively, we found that for equal numbers of inactivating hits, the contribution of an X-ray inactivated par ticle to reactivation is lower than that of an ultraviolet inactivated one. The relative contribution of X-ray and ultraviolet inactivated particles is congtant in all types of experiments listed above. This result is considered as evidence that one $\mathrm{X}$-ray hit produces on the average more lethal mutations than one ultraviolet hit, thus giving the fir ext experimental evidence for an actual difference in the spatial domain within which the genetic effects of one hit of each type can be exerted. (G)

\section{$180 \%$}

Watson, James Dewey. THE PROPRRTHES OF X-RAY-

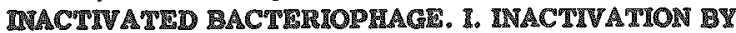
DRECT FFTECT. J. Bacteriol. 60, 697-718(1950).

The inactivation by $x$ rays of bacteriophages of the $T$ group auspended in broth has been investigated, and the propertien of the inactive viruses have been studied. Inacdivation is a simple exponential function of the dose, indl cating a one-hit inactivating mechanism. All the inactive particles are able to adsorb on sensitive bacteria, but only a fraction of them retain the ability to kill bacteria or to exclude anothe phage type. The ability to inhibit lygis or to lywe bacteria from without is not lost when the ability to kill bacteria is deatroyed. Multiplicity reactivation and photogeastivation of x-ray-inactivated ghage are observed in alight anownts. The results have been used to distinguigh two stages in the early period of phage reproduction, the first stage being called "adsorption" the second "invasion." (NSA)

\section{8}

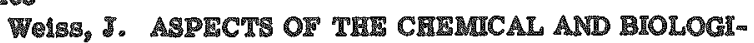
CAL ACTHON OF RADLATONS. Trans. Faraday Soc. 43 114-2싱(1047).

The general principles involved in the chem. and biol. action of radiation ( $\mathrm{z}-\mathrm{rayg}, \gamma$ rays, $\alpha$-particles, neutrong) are eramined. In penetrating radiations the absorgtion of radiation energy is not specific and is approx. proportional to the mass but practically indevendent of the chem. linkage.
The direct and indirect action of radiations, the formation, action, and the implications of free radicals, and the relation to the phys. "hit" theory have been discussed in detail. It is found that the recombination prucess following the primary formation of the radicals is of considerable intportance. If recombination can be neglected, the effects are approx. independent of the nature and wave length of the radiation and depend only on the total dose, while this is no longer the case if the recombination process plays a part. The extension into space of reaction chains can assume significant proportions and may be of considerable importance in the biological effects. (CA) 1408

WijSman, R. A. ON THE INTERPRETATION OF THE DOSE-FREQUENCY CURVE IN RADIOGENETICS.

Genetics 36, 478-87(1951).

Comparison of curves of multiple-event models with the curve for a single-event model supports the theory that a linear relationship between mutation frequency and radiation dose is caused by a single event. An exception has to be made for the possibility that some events may occur with much higher probability than any of the others, in which case a multiplenevent model could give rise to a single-event type curve; this case is physically unlikely. Opatowski's curve (Bull. Math. Biophys. 12, 19-26(1950)) is shown to represent a one-event mechanism, and his conclusion that a many-event theory can give rise to a linear $1 \mathrm{~d} 10$

Zirkle, Raymond 5. RELATIONSHIPS BETWREN CWEMCAL AND BIOLOGICAL IFTECTS OF IONIEING RADIATIONS. Radiology 52, 846-55(1949).

A discussion is presented of the nature of the connecting events occurring in the "latent period" which intervenes between the energy absorption in ionizing radiation conditions and the biological effect 3 produced by these radiations. The awthor assumes that the formation of new combinations which produce the biological effect is brought about by chemical reactions. Two phases of the chemical reactions are discussed, the nature of chemical reactions and the manner in which they lead to the biological effect. Carly relevant reactions may occur in the cell medium, and later ones at the cell surface or within the cell itself, due to the absorption of some chemical product. The possibility of the ionization of water by the radiations and the production of changes of many products in this medium in vivo are dig cussed. Three steps are proposed to atudy relevance of chemical reactions: a wecific radio-chemical reaction must be suspected of relevance to a given biological effect; second, if the reaction is only known to occur in vitro it must be proved to occur in the intact and irradiated cell; third, if the reaction passes the foregoing preliminary tests, it must be connected to the biological effect by a definite procedure. An indirect method of substantiating the third step by correlation of the variations in biological effects with variations in the radiations is outlined. The author concludes by stressing the importance of chemists in the radio-biological field and the need for further knowledge of the mechanisms of radio-biological reactions. (NSA)

Ceneral Blochemical Studies

141

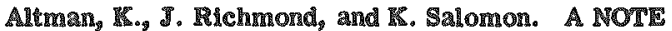
ON THE SYNTHESIS OF FATTY ACDS IN BONE MARPOW HOMOGENATES AS AH FECTED BY X-RADIATION. Biochim. biophys. acta 7, 460-5(1951) (UR-169)

The synthesis of saturated and ungaturated fatty acids in bone marrow homogenates from untreated and $\mathrm{x}$-irradiated 
rabbits has been studied with $\alpha-C^{14}$-acetate as a precursor. Two- and three-fold increases, respectively, over preirradiation levels in safurated and unsaturated fatty acid synthesis were observed immediately after ir radiation. Whereas saturated fatty acid synthesis, which approached control values at $48 \mathrm{hr}$ after ir radiation, again increased $3 / 2$ times at $72 \mathrm{hr}$, unsalurated fatty acid synhesis had fallen to $18 \%$ of the preirradiation value. At $158 \mathrm{hr}$ the relationship was reversed since unsalurated fatty acid synthesis had risen to $283 \%$ and saturated fatty acid synthesis had dropped to $58 \%$ of the preirradiation value. Oxygen uptake and $\mathrm{C}^{14} \mathrm{O}_{2}$ production increased immediately after irradiation, dropping to preirradiation values $48 \mathrm{hr}$ after exposure, $\mathrm{C}^{15} \mathrm{O}_{2}$ evolution decreased steadily throughout the experimental period while oxygen uptake was unchanged 158 hr after exposure. (NSA)

1412

Anderson, C. T., H. Blaschro, J. H. Burn, and R. Mole. THE PRESSOR AMINES IN THE ADRENAC MEDULLA AFTER IRRADIATION. Brit. J. Pharmacol. 6, 348-50 (1251).

Pyyidosine hydrochloride seems to protect againgt radiation sickness and to reduce mortality of mice given total-body $\mathrm{x}$ irradiation. The relation of pyridosine and the enzyme dopa-decarboxylase to the formation of pressor amines (noradrenaline and adrenaline) has been investigated. Beginning $48 \mathrm{hr}$ after lethal total $\mathrm{x}$ irradiation the content of pressor amines in the adrenal gland was progressively reduced. Dopa-decarboxylase activity in the liver, reduced after irradiation to some extent, was restored by adding pyridoxine to the diet; this did not inlluence the fall in pressor amines. Fiffect of insulin injection was investigated. Hypertrophy of adrenal glands 1413 following irradiation was observed. (NSA)

Bancroft, Geo. R. and Victor E. Kinsey. THE EFTECT OF X-RAXS UPON TRE MITABOLISM OF TUMOR TISSUE. Biochem. I. 31, 974-9(1937).

A definite lowering of the R.Q. of rat sarcoma in vivo was produced by 8 -rays. A parallel increase in the aerobic acid production, due mostly to lactic acid, indicated that the lowered R.Q. was not due to incomplete oxidation with the formation of acids other than lactic. Under the same conditions $\mathrm{x}$-rays did not affect the metabolism of rat kidney slices. Since irradiated tumor and kidney slices metabolize pyruvic acid normally, x-rays probably attack the carbohydrate oxidation before the pyruvic acid stage is reached. (CA)

1414

Barron, E. S. G., Veronica Bonzell, Betty Gasvoda, and Louise Seki. THE RESPRATION AND ACETATE OXDATION RATE OF X-IRRADIATED BACRERIA (MYCOBACTERIUM CREATINOVORANS) p.87-8 of BIOLOGICAL AND MEDICAL DIVISIONS; QUARTERLY REPORT, FEBRUARY, MARCS, APRL, 1050. Argone National Lab. [nd] 2p. (ANL-ASAs51(p.97-8))

It was shown previously (ANL-4291) that $\mathrm{x}$ irradiation of Mycobacterim creatinovorans with doses varying from 1000 to $10,000 \mathrm{~m}$, caused an increasing inhibition of reapiration of the bacteria irradiated in the eulture media containing acetate. On irradiation of three-times washed bacteria the residual resplration was unaffectea by 6000 rowile resplration in the presence of acetate was 30\% inhibited. To determine whether permanent changes could be produced in bacteria by continuous irradiation with relatively small doses of $\mathrm{x}$ rays, liquid cultures of M. creatinovorans (in acetate medium) were ir radiated with 1000 to. One-day-old cultures were irradiated in test tuhes with $1000 \mathrm{r}$ (effective irradiation about $800 \mathrm{r}$ ); the cultures were transplanted $8 \mathrm{hr}$ later into another teat tube which was irradiated on the following morning. The total amount of effective radiation was $11,200 \mathrm{r}$. The residual respiration, as well as the oxidation rate of acetate, was depressed in the $\mathrm{x}$-irradiated bacteria. Pigment formation in the x-ixradiated bacteria was also lest than in the control samples. The $\mathrm{x}$-ray dose received daily, $800 \mathrm{r}$, when given in a single dowe had no effect either on multiplication of bacteria or on respiration. (NSSA)

1415

Barron, E. S. G. and Veronica Flood. THE EFFET OF X-IRRADITION ON THE METABOLISM OT THE SMALL INTESTINE OF THE RAT, p.157-61 of BIOLOGICAL AND MEDCAL DTISIONS; QUARTERLY REPORT, NOVEMBER, DECFMBER, JANUARY, 1948-1949. Argonne National Lab. Feb. 1, 1940. 5p. (ANL-4253(p.157-61))

Sprague-Dawley rats, weighing $200 \mathrm{~g}_{\mathrm{g}}$ each, $_{\text {, were given }}$ total-body $\mathrm{x}$-radiation at different dosages ranging from 50 to $500 \mathrm{r}$. The animals were sacrificed at different inter vals and the metabolism of strips of the small integtine were determined. The $\mathrm{O}_{2}$ uptake of the strips of tissue from $x$-irradiated rats was always below normal when measured soon after irradiation. When the rats were irradiated with $50 \mathrm{r}$, regpiration returned to normal values five hours later. Anaerobic glycolysis was not affected when measurements were made soon after irradiation. (ACD)

1410

Barron, E. S. Guzman and Veronica Flood. TTUDHEs ON THE MECIANHSA OF ACTION OF IONIZING RADLATIONS. V. OXIDATION OF THIOLS BY IONIZING RADLATIONS. J. Gen. Physiol. 33, 228-41(1950).

The su groups play a very important role in the proeesses of cell diviaion and of cell growth. It was found prevlously that enzymes requiring SR groups for activity were reversibly inhibited by ionizing radiations, which indicates that the SH groups belong to proteins. In this study various thiols (Glutathione, 2,3-dimercaptopropanol, propane-1, 3-dithiol, and N-phenylaminopropanedithiol) are shown to be easily oxidized in aqueous solution by loniaing radiations (g-rays, $\beta$-rayg, and $\gamma$-rays). The mechanism probably involves decomposition of $\mathrm{H}_{2} \mathrm{O}$ and formation of different oxldizing agents (OR, $\mathrm{O}_{2} \mathrm{H}, \mathrm{O}, \mathrm{H}_{2}-\mathrm{O}_{2}$ ). The lonic yield (number of oxidized molecules divided by the number of lon pairs produced by ionization of $\mathrm{H}_{2} \mathrm{O}$ ) for the oxidatson (2 RSH $=$ RS - SRl) was 3 for 8 -rays or $\beta$-rays at $\mathrm{pH} 7$, and $23 \%$ less for $\gamma-r a y s$, and this increased with a rise in $\mathrm{g}$ value. In the absence of $O_{2}$, the oxidation of thiols by lonizing radiation was only $/ / 3$ that obtained in the presence of dissolved $\mathrm{O}_{2}$. In the presence of dissolved $\mathrm{O}_{5}$, the irradiated thiols solutions were protected $17-27 \%$ irom oxidation by catalase. (CA)

1417

Barron, E. S. Guzman, Betty Gaswoda, and Veronica Flood. TFIECT OF RADIATION ON TIIE RESPIRATION OF SEA URCRIN SERRM, P.148-63 Of BIOLOGICAL AND MEDICAL DIUISIONS; QUARTERLY REPORT, MAT, JUNT, JULY, 1848, Austin M. Brues and Lisco, eds. Argonne National Lab. Aug. 1, 1048. 16p. (ANL-4205(0.148-63))

Authors reinvegtigated problem of ir radiation of angle cells. Data are presented on the effect of $x$-rays on the respiration of sea urchin (Arbacia punctulate) sperm. Es:perimental procedure is described: effect of dilution on respiration of ger urchin sperm and eifect of $\mathrm{x}-\mathrm{ray}$ on respiration of sea urchin sperm. In summary: respiratlon of sea urchin sperm increased with dilutton, up to a dilution of $1: 200$, where maxmum values were found. At thi 
dilution the $\mathrm{QO}_{2}$ value was $19.6+3.3$. Sperm diluted $1: 10$ gave a $\mathrm{QO}_{2}$ value of 1.7 and a dilution of $1: 1,000$ gave a $\mathrm{QO}_{2}$ value of 2. The regpiration of dilute suspensions of sea urchin sperm was inhibited by $x-1$ ispadiation. $A$ dosage of $20,000 \mathrm{r}$ inhibited $60 \% ; 10,000 \mathrm{r}, 35 \% ; 5,000 \mathrm{r}, 25 \%$; $1,000 \mathrm{x}, 14 \%$. There was little difference in inhibition when the dosage was decreased to $100 \mathrm{x}$. (S. V. S.) 1 1418

Barron, E. S. Guzman, Betty Gaswoda, and Veronica Flood. MTUDIES ON TME MECLANISM OF ACTION OF HONIEING RADLATIONS. IV. EPPECT OF X-RAX IRRADLATON ON THE RESPIRATION OF SEA URCMIN SPERM. Biol. Bull. 97, A14연 $0(1949)$.

The results of studies on the gea urehin (Arbacia punctulata) merm resplration rate at various sperm dilutions and after dosages of $\mathrm{x}$-radiations are pregented. The experimental procedures and the technique are described. X-ray dosages of 100-20,000 r were adminitered in meparate experiments at a water dilution of $1: 200$ and with or whout added succinate or acelate. The respiration of the aea urehn aperm increased whin dilution ap to allution of 1:200, where the average maximum value of $19.6 \pm 3.9$ was found. When the dilution was increased to $1: 1,000$ the reapiration dropped sharply to 2.0 , and a dilution of $1: 1,600$ gave no measurable respiration. When a dose of 20,000 r of $\mathrm{X}$-radiation was dellvered to the sperm zolumion an Inhibition of $66 \%$ in the regpiration was seen, which snereased during the second hour: 10,000 r produced a $30 \%$ decrease; $1,000 \mathrm{x}$, a 22\% decrease; $500 \mathrm{x}$, a $14 \mathrm{~m}$ decrease; and, $100 \mathrm{~s}$, a $10 \%$ decrease. It was found that when the gperm was is radiated with 1,000 in, there was uro resplration recovery for sive hours; on addition of auccinate and of acetate to the colution, the oxygen uptake was furher inhibited. (NSA) 14.

Baron, RESPIRATHON, CELL DIVISON, AND IONIZTEG RADIAFIONS. Argonts Natlonal and Untw. of Chesgo. Ort. 1951. 189. (ATCU-1221: UAC-AO2)

On s irradiation of the eggs and apern of Arbacia punchulats there was inhbition of zespigation with relatively large doses, whereas there was an increase with small doses. The dose required to produce an increase of regphrmaton depended on the degree of senfilivity of the cell to the effect of lonizing radiation. Sperm cells were most vensthve; then cane ferhined eggs; unerthized eggs were the leagt genditive. The Inhibiting effect of z rays on cells

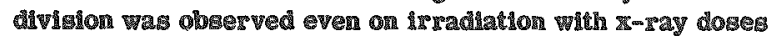
which produced an inerease of respiration. These results are compared to similar effects producet by thlol reagents and are attritouted to oxidatron or the hhol compound in the cell. (NSA)

1 1920

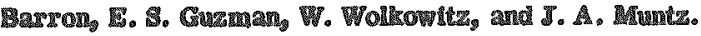
TH:

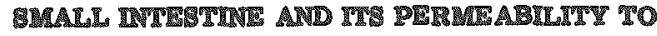

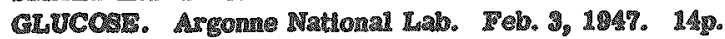

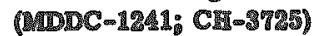

The erperiments presented 1n this parer demonstrate

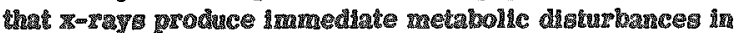
the mall intestine. All the experiments were made on rats which recelved total body Imzadiation, Four hours after iroradiation, there was an abugt drop of resplsalon in the

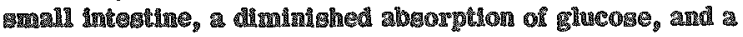
4น

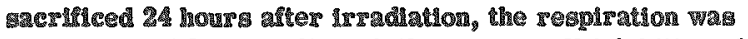
whinis mormal dimits, 2Lhough there was still inhibition of phoswhorylation. Forty-aght hours after trealment there

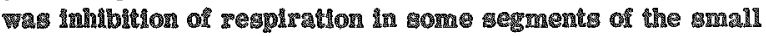

intestine. The inhibition increased 72 hours after treatment; the rate of phosphorylation of fructose was unimpaired. There was some inhibition of respiration 96 hours after treatment. Anaerobic glycolysis was generally normal. There was a linear relationship between the inhibition of glucose absorption and the amount of irradiation. (ADD) 1421

Barrow, Jack, John L. Tullis, and F.W. Chambers, Jr. DFISCT OF X-RADIATION AND ANTIHISTAMINE DRUGS ON THE RETICULO-ENDOTHELIAL SXSTEM MEASURED WITH COLLOUAL RADIOGOLD. Am. J. Physiol. 164, 822-3(1951).

The function of phagocytosis by the reticulo-endothelial system was studied by radiometrically determining the rate of removal of collotdal gold, radioactive in tracer amounts, injected intravenously into normal rabbits, and into rabblts varlously treated with $\mathrm{X}$-rays, thorotrast, antisthe, pyrikenzamine and histamine. Thorotrast temporarily suppresed almost completely the removal of colloidal gold from the circulation. Pretreatment of normal and x-irradiated rabbits with anthigtamine drugs and/or histamine did not alter the rate of phaggocytosis of the gold colloid. (CA)

1422

Baur, H. and M. Ludin. HUSTAMIIE CONCENTRATION AFIER IRRADIATION WITI X-RAYS. RadLA. CLI. 10, 288-76(1950).

X-rays cause an increased blood hitamine concn. in man and animals amounting to 5-6 times the normal values. In vitro irrad. of blood gives no increase. Trgts. indicate that the histamine production is caused by irradiation of the reticulo-endothelial system or the bone marrow. (BA)

1423

Bennett, I. R., V. C. Bennett, and J. Wowland. Fow FET OF ACUTE X-RADATION ON DISTREUTION AID TXCRETION ON RADIOSODUM IN THIR RAT. Federation Proc. 8, 350(1049).

Radiated rats have been shown to have an increased thocyanate grace for 2 weeln following a mid-lethal dose of $\mathrm{x}-\mathrm{Tadiation}$. study of the sodum distribution and excretion of onilar animals using radiogodiux has been made. In rats during the first four days post radiation a marked retention of zadiosodium occurs. Timase distribution studies show ahift into the intestinal tract which may exceed that obaerved in control anmals by as much as $2 \%$ of the total dose adninistered. Smaller ghifts of radiosodium into the kidney and gnleen are noted. There is an increased radiosodium content of the liver which appears to bo defintely related to the increased serum level to wheh it holds a constant relationghip. The serum sodium content increases, the maximum levels (up to $5-8 \mathrm{~m}$ mg) being observed at the end of 3 - d days after lryadiation. Urinary radiogodum excretion may be reduced to one half that observed in control animals. Studles on dehydrated rats indicate that the urinary retention and elevated blood codlum level are not accounted for on the basis of dehydration alone. (Author')

1 14⿻ำ

Bentett, L. Pro, Vera C. Bemett, Alpha shaver, and Theresa Grachus. ABSOREION AND DHTRIBUTION OF VITAMNA A IN X-IZRADIATED RATS. PROC. SOC.

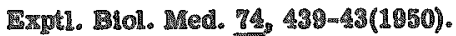

Toung ferale ratg were treated whith $625 \mathrm{x}$ whole-body izradiation. The rate of aborgthon of vitamin A alc. Irom the digestive tract was increased for a few days. Inver wtramin A was decreased and that of the carcass increased by the treatment. (CA) 
1425

Bennett, Leslie R., Sarah M. Chastain, Arthur B. Decker, and James F. Mead. THE FFTECT OF ROENTGEN TR PADIATION UPON PROTEIN ABSORPTION IN THE MOUSE. Proc. Soc. Expt. Biol. Med. 77, 715-18(1951) (UCLA-137)

The rate of protein absorption was measured in normal mice with $\mathrm{I}^{19}$-labeled human serum albumin and was compared with absorption in mice exposed to $600 \mathrm{r}$ of wholebody 3 radiation. No change in the absorption rate after exposure was observed, although a change in tone and motility of the gastrointestinal tract was noted. (NSA) 1426

Berner, F. THE ACTION OF ROENTGEN RAYS ON THE MNERAL METABOLISM OF UNICELLULAR ORGANISMS WITS THE VIEW OF IINDING AN IRRADIATION RHYTHM WHCH DESTROYS THE CULTURES OF UNICELLULAR ORGANISMS IN THE SFORTIST POSSIBLE TIME WITI A SMALL TOTAL QUANTITY OF RATS. Strahlentherapie 71, 1-60(1942).

The alteration of the mineral metabolism of Paramecium caudatum under the action of roentgen irradiation was ob1427

served. A favorable irradiation rhythm was found. (CA)

BLLen, Daniel, G. E. Stapleton, and Alexander Hollaender.

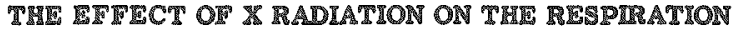
OF BACTERIAL CELLS. Oak Ridge National Lab. [nd] 1p. (ARCU-1801)

Studies with \& rays revesled that one effect was inhibition of the respiratory system of washed suspernsions of EscherIchia coll, although the magnitude of the luhibition warieg with substrate, temperature, and strain of the organism used. It was found that a doge of $60,000 \mathrm{I}$, while decreasing the number of viable cells of a sugpension of $\mathrm{g}_{\text {. }}$ coll $\mathrm{B} / \mathrm{r}$ by more than $99.25 \%$, had no apparent offect on the Inital respiratory rate of the exposed cells. However, the inttal period of normal activity was followed by a marked decline in oxygen consumption rate as compared to the control cells. The period of mormal activity was longer when pyruvate and succhate were uned as substrates as compared to rlueose utilizatlon. A perlod of mormal rem spiratory activity on glucose in contrast to an inmediate inhibition of orldattve activity on pyruvate mas observed with E. coll (Texas). Both exposed and nonexposed cells chowed a resplratory quotient of one on glucose, although the former required a longer perlod of thme to complete the orldation of the substrate. Holding the exposed cells in buffer at $37^{\circ} \mathrm{C}$ for 60 min before adding substrate decreased the pertod of normal activity and caused a sharper decline in respiratory rate, while an exposed sumpension held in buffer at $2^{\circ} \mathrm{C}$ for 3 hr following irradiation was as active as a sugpension tested immediately a A conparison of exposed cells respiring at 37 and $26^{\circ} \mathrm{C}$ in the presence of glucose or pyruvate revealed an increased adverse effect of the radiation-induced inhibition of respiration at the higher temperature. (Entire report) 1198

Blokhin, N. N., B. M. Graevskaya, and R. Ya. IRellna. BIOCHEMCAL PROCESSES IN TIE BRAWN IN DIRECT

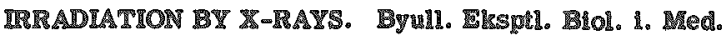
23. $338-42(1247)$.

X-ray irradiation of dog brain, at $160 \mathrm{kv}$, at $23 \mathrm{~mm}$ digtance by using 0.5 Cu-3.0 Al filter, each dog recelving a total of 5 unit skin doses of Irradiation in 4 exposures with alternate Irradiation of the right and the left temple area, was lnwestigated in respect to biochem. effects by detn. of blood sugar (femoral artery and Binus venosus cerebri), spinal luid sugar, blood serum protein, and the albumin-
Globulin fractions of the latter. As the total irradiation increased, the total serum protein rose until the goth day (after the 1st irradiation) is reached $200 \%$ of the initial value. In the same period the abumin fraction rose by only $26 \%$ for arterial and $32 \%$ for venous blood, while the globulin fraction rose 324 and $323 \%$, respectively. The abs. amt. of protein in the gpinal fluid remained within exptl. variatons, but its albumin/globulin ratio gradually changed to 1.0 from 0.56. Arterial blood sugar remained normal, but if fell in the venous blood untl the gugar utilization by the brain at the 90-day period was $37 \%$ of initial. Spinal fluid sugar did not increase. The changes are ascribed to a colloidal swelling of the brain cells, with decreased amount of Intracellular fluids. (CA) 1429

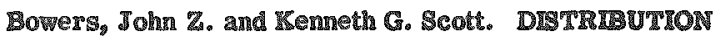

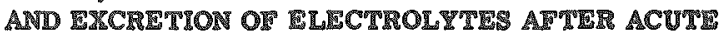

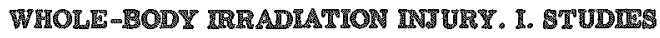
WITE RADIOPOTASSIUIA. Proc. Soc. Fint. Blol. Med. 78, 64.5 $-52(1951)$.

Radosensitive tissues ghow a variety of alterations ln radiosodium concentration after total-body irradiation above an LD. In aeveral timses there 1 a decrease followed by a sharp increase; the decrease occura at the ame the as a decrease in radiopotassum concentration. An increase in percentage water content may occur when there is a fall in radiogodium concentration. Percent loss of dry welght per day 1 in general mont pronoumced In radiosensitive hissues. Urinary excretion of radiosedima after total-body Irradiation at levels approximating an LD $_{\text {Wo }}$ chow a complementary homeostatc decreame In respone to the excessive loss of radiosodium through the gastrointestinal tract. (NSA)

\section{0}

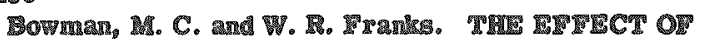

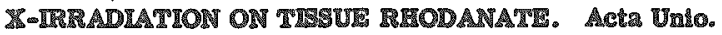
Intern. contra Cancrum $5.765-7(1049)$.

The results of Inveatrgatons to determane whether the degtruction of tissue rhodanate by $\mathrm{x}-1 \mathrm{r}$ radation $1 \mathrm{~s}$ accompanted by digturbances in the Pasteur geacton of the tirsue are reported. The bodles of mice under light Nembutal anesthesla were covered with lead shelds, ondy the head reeeiving X-ray surnace dovages of 10,000 I (bane dowage

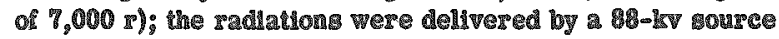
with no Illiration, at a distanee of $10 \mathrm{~cm}$ over a persod of 10 minutes. The irradiated animas were macrificed at varlous Intervals and estimations of the ghodanate activity, respiration, anacrobic and aerobic glycolyais of thelr grey

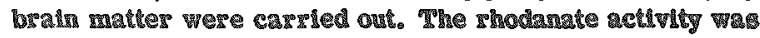
determined by alightly modified Lang method, which is described here. It was found that the Nerabutal did not afect enther the rhodanate actvity or the regriration and slycolys of the mouge bran grey matter; the m-irrediaHon did not inhlbit the rhodamate activity in Iat liver homogeneate in vitro, but was lethal to the mice in 2 to 10 dxy. Suramaxizing, no gigniftcant activity changes in the shodanate, respiration or glycolygs: were detected. (NVI)

1431

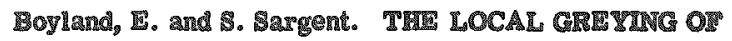
IAR IN MLE TREATED WITH IFAYS ANO RADIOLI-

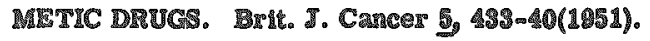

The intradermal injection of nitrogen mustards or multur mustard into colored mice causes a permanent grayimg or bleaching of the hair similar to that occurring after myosm ure to lonising radiations. This graying arnears to be a radiomimetic affect. ฐมm proportion of cases when water is injected but mever followtne the injection of alme. Gramg of har was pro- 
duced by injection of known carcinogens, such as $\beta$ naphthyl-bis ( $\beta$ chloroethyl) is an example of limited toxic action, and so allied to vesication and induction of mutathons and cancer. (NSA)

1432

Buchanan, D.L. and E. S. Guzman Barron. STUDHES ON THE EFFECT OF RADIATION ON PROTEINS. Argonne National Lab. June 1, 1947. 22p. (MDDC-1418-D)

Data are presented showing certain effects of ultraviolet and $\gamma$ rays on the absorption spectra of solutions of erystalline egg albumin. The changes differ with the two types of irradiation, will variation in pH, and questionably with oxygen tension of the solution. The changes do not resemble those seen in heat, acid, or alkali denaturation. In an arbitrary fashion the rate of change in the solutions being irradiated was shown to have the "zero order" type of rate constant, implying the rate of formation of product to be independent of the concentration of solute within the limits of concentration studied, but giving no information as to the actual reaction rate. (ADD)

1433

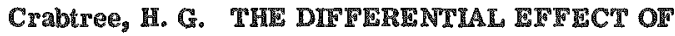
RADIUM RADIATION ON THE CARBOHYDRATE METAB. OLISM OF NORMAL AND TUMOR TISSUES IRRADIATED AT LOW TIMPERATURE, Biochem. J. 29, $2334-43$ (1985).

Ra radiation of tumor-tissue slices at $37^{\circ} 5^{\circ}$ either under aerobic or anaerobic conditions or with $\mathrm{HCN}$ present (which made the tissue more sensitive) caused a selective lowering of respiration while glucolysis remained unaffected. Irradiation or tumor slices at $0-10^{\circ}$ under the same conditions caused a pronounced lowerimg of glucolysis, and the respiration was unafected. The irradiation of normal tissues (spleen, liver, kidney, testes) at $0-10^{\circ}$ under identical conditions produced little or no effect on their metabolism, since their respiration was not accompanied by aerobic glucolysis. This difference in the effect of irradiation of tumor cells at $0-10^{\circ}$ may be of clinical value. (CA)

1434

Davison, R. A., P. Twoets, and W. C. Buzell. EFTECT OF ROENTGENOTHERAPY ON URINARY 1\%-KITOSTERID EXCRE'TON IN ANKYLOSING SPONDYLARTHRITIS. J. Clin. Endocrinol. 9, 79-88(1949).

Urinary 17 -ketosteroid excretion was found to be increased in spondylarthritis of the ankylosing type in both male and female patients. High values continued for long periods of time and approached lower levels only when the patient reached a state of exhaustion. Roentgenotherapy seemed to provide a stimulus for increased excretion followed by a relative decrease. Following the roentgenotherapy the values returned to high Ievels although the symptoms and signs of the disease had abated. (NSA) 1435

Decker, Arthur B. and James F. Mead. THE RELATONSMIP BETWEEN ESSENTIAL F ATTY ACIO DEFICIENCY AND RADLATION DAMAGR IN THE MOUST. Univ. of Calif., Los Angeles. Dec. 1, 1949. 64p. (UCLA-52)

The production of both acute and chronic essential fatty acid deficiency in mice is described, and the importance of the chronic deficiency is discussed. Both chronic and acute essential fatty acid deficiency markedly increase the moro ality rate of mice exposed to $x-r a y s$. The symptoms of essential fatty acid deficiency and radiation damage are similar in many respects, and each condition apparently aggravates the other. 57 references. (NSA)
1436

Deho, G. G. CHANGES IN TIE PROTEIN CONTENT OF BLOOD AS A RESULT OF RRADIATION OF THE SKELETON WITH X-RAYS. Rass. fisiopatol. clin. e terap. (Pisa) 21, 817 28(1049).

In 10 of 14 patients with dorsolumbar arthrosis irradiation with low doses of $x-$ rays caused an increase of the total protein content of blood (mostly from 0.65 to $1.45 \mathrm{~g} \%$ ). Albumins and globulins, especially the latter, increased. Before irradiation, the total protein content was rather high $(6.08-9.29 \mathrm{~g} \%)$. (CA)

1437

Dietz, A. A. and B. Steinberg. CHEMCAL COMPOSI-

TION OF IRRADIATED BONE MAREOW. Arch. Biochem. 23, 222-8(1949).

The right forelegs of rabbits older than 4 months were treated with $3,000 \mathrm{r}$ unfiltered $\mathrm{X}$ rays delivered in a field of $380 \mathrm{sq} \mathrm{cm}$ at the rate of $160 \mathrm{r} / \mathrm{min}$. The rest of the body was covered by $1.5 \mathrm{~mm}$ of lead. The animals were killed 1-32 days after irradiation. Samples of marrow from nonirradiated areas ware used as controls. There was a decrease in water, residue, non-protein sulfur fractions and total lipid and NPN and an increase in lipid. The increased amt. of water in a unit weight of residue in irradiated marrow is consistent with oedlema observed in irradiated tissues. The inorganic phosphate frequently showed a decline during the first 8 days and then an increase over the control marrow. (EM)

1438

DuBois, Kenneth P., Denneth W. Cochran, and John Dounl. INPIBITION OF CITRIC ACD SYNTHESIS IN VIV BY X-IRRADIATION. Proc. Soc. IExptl. Biol. Med. 76, 422T(1951).

The technic of Potter (Proc. Soc. Expti. Biol. Med. 76, 41(1951) ) for measuring the effects of toxic agents on citrate accumulation vivo was applied to studies on $\mathrm{x}$ ir radiation. Lethal doses of $\mathrm{x}$ ray $(800 \mathrm{r})$ markedly inhibited cilrate accumulation in spleen, thymus, ileum, pancreas, and testes of Iluoroacotate-treated rats but exerted no significant effect on brain and heart. Sublethal doses of $\mathrm{x}$ ray markedly inhibited citrate accumulation in spleen and thymus. The extent of inhibition was dependent upon the dose of z ray and was reversible after sublethal doses and irreversible when lethal doses were given. Accumulation of citrate occurred in the livers of irradiated rats following fluoroacetate treatment in contrast to the inability of livers of normal male rats to accumulate citrate following fluoroacetate treatment. (NSA)

$14{ }^{2} 39$

Edelman, A. EFFECTS OF IX-RADIATION ON WATER AND ELECTROLYTE MITABOLISM TN THE RAT. Federation Proc. 8, 39(1949).

The intakes of disthled water, $1 \% \mathrm{NaCl}$ solution, and food and the exeretion of urinary $\mathrm{Na}, \mathrm{K}, \mathrm{Cl}$ and water by male albino rats were determined daily previous to and following exposures to $100,250,500,800$, and $1000 \mathrm{r}$ of x-rays. Polydipgia and polyuria occurred during the first and fifth 24 hour period following radiation. The urinary excretions of $\mathrm{Na}, \mathbb{K}$, and $\mathrm{Cl}$ were increased at these times. Following doses of $500 \mathrm{r}$ or more there is a relative and absolute decrease in intake of the salt solution. This is a monophasic response reaching its minimum at about 3 days. The salt solution intake returns to previous levels at 5 days. After doses of 100 or $250 \mathrm{r}$, the $1 \% \mathrm{NaCl}$ solution intake follows the biphasic curve of water intake. Food intake decreased for 2 days following radiation and 
then returned to normal in about 2 days. Budy woight changes paralleled the food consumption. 1440

Ellinger, Fiedrich. THE MISTAMINE HYPOTHESIS OF THE BIOLOGICAL EFECTS OF RADIATION. Schweiz. med. Wochschr. 81, (2) 55-67(1951).

Correlation of local and systemic (general) effects of radiant energy in the animal and human body is attempted. Experimental and clinical evidence is adduced (56 references) to demonstrate the distribution of pharmacologically active substances which are locally formed or released from cells disintegrating under the impact of irradiation in the exposed area. The importance of histamine, acetylcholine and adenosine compounds for the explanation of radiation effects is discussed in detail. On the base of the available exptl. evidence histamine appears most readily to account for the production of local as well as systemic 1441 radiation effects. (Author)

Feinstein, Robert N. and Carrie L. Butler. EFECT OF WHOLE BODY XI-RADIATION AND OF INTRAPERITONEAL PYDROGEN PEROXIDE ON A NUCLEOPROTEIN-CONTAINING FRACTION OF RAT INTESTINE AND ON WHOLE RAT INTESTINE. USAF School of Aviation Medicine, Nov. 1951. 7p. (NP-\$642; U21021)

Whole body $\mathrm{s}$-radiation of rats decreasea the relative viscosity of alkaline blends of the intestine and decreases the percentage of the whole blend which forms tactoid. $\mathrm{X}-$ radiation decreases the amount of dry matter, nitrogen, and phosphorus found in the tactold. The loss in phosphorus is dexived from the nucleic acid fraction. X-radiation decreases the tactoid ash and tactold ash aluminum to an extent that is of dubious gtatistical significance because of wide variakion in control values. The ash sodium does decrease ๓ignificantly.

In one experiment, intraperitoneal $\mathrm{H}_{2} \mathrm{O}_{2}$ appeared significanty radiomimetic. In a second esperiment this was not found true, despite the "statistical signiflcance" of the firgt indings. Such erratic results of $\mathrm{H}_{2} \mathrm{O}_{2}$ have been noted before on different lots of laboratory animals.

1442

Fink, IX. BLOOD CHEMISTRY STUDY IN DOGS EXPOSED TO CHRONC X-RADITTON. [University of Rochester] [nd] 16p. (MDDC-211)

The purpose of this study was to determine whether some of the biochemical components of the blood were affected in dogs by chronic $x-$ radiation at $0.1,0.5,1.0$, and $10 \mathrm{r}$ per day over a long period of time. The concentrathons of serum proteln, plasma fibrinogen, whole blood NPN, serum cholesterol, serum chloride, and serum acid and alkaline phosphatass were not gigntificantly altered in dogs subjected to chronic $x$-radiation at $0.1,0.5,1.0$, and $10 \mathrm{I}$ per day, six day a week. All the dogg in the $10 \mathrm{x}$ groups and four dogs in the remaining groups guceumbed before the experiment was terminated at the time of 622 exposures. There were no consistent changes even in the doge which died as a result of the radiation. The only obvious changes observed in the dogs given 10 I per day which died were high flbrinogen concentrationg in three of the dogs, two of which had high alkaline phosphatase levels. These changes did not develop until about a week before the animals died, so even had they oceurred in all the dogs which died, they would have been of little practical value as an inder to radiation damage. (ADD)

Frankenthal, L. and A. Back. THE EFTECT OF XRARAS ON THE RESPIRATION OF FOWL ERYTHROCYTES. Bhochem. J. 38, 351-4(1044).
The respiration of fowl erythrocytes is markedly and increasingly stimulated by $x$-ray doses from 500,000 to $2,000,000 \mathrm{r}$. When optimum doses of radiation are used the stimulation may last for several hours (as long as there is no hemolysis). But whereas non-irradiated erythrocytes, incubated at $39-40^{\circ}$ and shalsen, hardly undergo hemolysis, the hemolysig is progressive and proportional to the previoug $x-r a y$ dose in irradiated erythrocytes. (CA)

1444

Goldedex, A. and J. I Fershing. THE IFFECT OF RADIATION ON CELL RESPIRATION. I. RESPIRATION AND ANALROBIC GLYCOLYSIS OF MOUSE MIDNEY IN VITRO FOLLOWING RADIATION. Radiology 31, 81-8 (1938).

E'xcised mouse kidney was irradiated in vitro at $4^{\circ} \mathrm{C}$. with $200 \mathrm{KV}$ so-rays. There was no change in $\mathrm{O}_{2}$ consumption and $R . Q$. measured immediately after treatment with 1,800 to $30,000 x_{8}$ no change in $O_{2}$ consumption or $R_{0} Q_{0}$. after treatruent with $375 \mathrm{mg}$-hars. of radium at $37.5^{\circ} \mathrm{C}$ or at 4 with doses up to $4,164 \mathrm{mg}$. hrs. After treatment with radon ( $B$ plue $\gamma$ rays), overnight doses totalling 1,180 to $6,170 \mathrm{mg}-\mathrm{hrs}$, there is a drop in $\mathrm{O}_{2}$ consumption but not in R. Q. Anaerobic glycolysis is decreased to a greater extent than respiration with equivalent doses. The resulfs contradict the hypothests that Ir radiation affects cell catalysts. B-rays are more effective than $\gamma$ rays and have a direct effect on respiration and anaeroble glycolysis. (BA)

1445

Goldman, Douglas, MeTABOLIC CMANGES OCCUREING AS TRE RESULT OF DEEP ROENTGEN TRERAPY. I

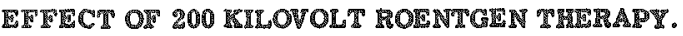
An. J. Roentgenol. Radim Therayy 50, 381-91(1243).

The patients obserwed were on constant, accurately weighed diets, potentially neutral, low in purines and $\mathrm{Ca}_{\text {, }}$ and in most instances minimal in chloride. Changes in the excrelion of total nitrogen and Its components are the most definite deviations from the control values presented by the investigations. There was, in general, an increase in the urdnary output of total nitrogen elther during the periods when radiation was being given or immediately following cessation of the treatment. Nitrogen loss was greater in patients with radiosensitive tumors. Both uric acid, and to a lesser degree, ammonia were found to be involved in this nilrogen increase. The total acid of the urine was definitely higher in most cases durms or anter roentgen therapy, probably the result of greater destructon of body protein. A slight diminution in amino-acid nitrogen excretion took place two to six days aster inradiation and continued to the end of the observations. This geems to indicate a gtorage of nitrogen after the prlmary deatructive effects of lrradiation had worn off. No signiflicant deviation of the Ca balance from control values was observed. Inerease in the urinary output was found during or following roentgen therayy whth deviation of the balance to the negative side. Anall but dellnite inerease in the urinary $\$$ and suliote occurred following irradiation. All of the patientr who became severely ul mowed a dellnite chloride loss during the Irrodiation perlod. The chloride balance became positive shortly betore or gimultaneously with improvement in symptoms. $\mathrm{NaCl}$ by mouth is very frequently an effective therapeutlc agent in Irradistion sickness. In some caves its action was dramatic. There is a derinte auscepthility to roentgen atckness of patients with protracted NaCl starvation. (Ri)

1446

Goldscheidex, G. FFFECT OF WNRA-UTERIN RADTUM THERAPY ON OVARIAN GUNCTION. Lancet 1, 621-3 (1949). 
In an attempt to determine whether the amenorrhea produced by radiation is of ovarian or non-ovarian origin, the uxinary excretion of follicle-stimulation hormones has been determined in 28 women before and after intrauterine treatment with radium for menorthagia. The regults oblained were compared with those obtained for a few patients who had been castrated with $X$-rays or had received a comblned treatment of X-rays and radium. The absence of an increase in follicle-stimulating hormone guggests that an artifical menopause had not been established and that the amenorrhea observed following radiation treatment was of non-ovarian origin. It is guggested that the destruction of the uterine glands could be the cause of the observed amenorrhea. Artificial menopause occurred only in women of menopausal age in whom small doses may influence ovarian function. (NSA)

\section{4 새의}

Gounaris, I. EXPERMMENTS ON TIE ACTION OF XRAYS ON THE RETICUR_O-ENDOTHELIAL SYSTEM, p.17\% of INIERNATIONAL CONGRESS OF RADIOLOGY, 6th Congress, London. 1950.

The fact that alcohol is metabolised by recticulo-endothelnal cells led us to study the action of X-rays on this interesting cellular gystem in respect of alcohol metabolism in dogr and gheer beiore and after a given dose of radiation. The following are some of the advantages of this method:

(1) Observations are made on the same animal so that a separate control, which might show a difierent biological actlon, is not needed.

(2) The technique can be applied to human beings, and

(3) It is possible to collow the collular reaction during the adruinistration of the radiation.

We have reacheo the following conclustong:-

(1) Small doses have an inhibiting action during the first 24. hours following Irradiation, and a stimulating action about 24 hours and 48 hours after irradiation.

(2) Larger doses have an inhibiting action even 48 hours after irradiation. The stmulating action under these conditions appear 4 days after irradiation.

(3) Alcohol metabolism returns almost to normal one month after Ir sadiation.

(4) I It is desised to "reactivate" the reticuloendothelial gytem Iurther Ir radiation must not be given until at least 48 hour have clagsed.

(5) The radiation effect is not a result of increased reatatance of of ordation due to enzymes liberated by cellular autolygí.

I년슬

Gros. Charles and Pau Mandel. VARLATONS IN THI MUNAMU ENDOGENEOUS MTTROGEN FXCRETION AS AN IFIFET OF TOTAL-BODY T-IRRADIATION. COmpt. remp. 251, 6s1-3(1950).

After rats on arotein-free diet had resched the minimun of their endogeneous nitrogen axcretion, they were Irreadiated whth 400 I of 150-kv \& Iays. Determinallons of the total-nitrogen, allantoln, and creatinine excretions belore and 6 or more days after Irradation showed an average increase In the total endogeneous excretion of $36 \%$ with a daaximum of 56\%. The excretion of allantoln, which ts remarirably constant normally, increased by 4 A\% age and $62 \%$ in the limiting case. In contrast, no variation was found in creatinine elimination. Several possible intermetatlons of these reaults are discussed briefly. (NSA)

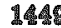

Mauschilt, James D. and Helen Supplee. THE EFFCT OF WHOL BODY X-IRRADIATION UPON THE TOLAL NITROGEN, TUTAL PROTEIN AND REITACTVE INDEX
OF RAT PLASMA; INTERIM REPORT. Naval Radiological Defense Lab. June 13, 1949. 11p. (AD-131(B))

A preliminary investigation has been made upon the changes of plasma refractive index, plasma protein, and plasma nitrogen of rats which had received 650 r total body $x$-irradiation. A marked decrease was found to occur in all cases in the 2 to 4 -day post-irradiation period. Refractive index changes paralleled those of protein and total nitrogen in plasma. (NSA) 1450

Mevesy, Georg von. ENECT OF X-RAYS ON THE RATE OF TURNOVER OF PHOSPHATIOES. Nature 158,268 (1946).

The effect of $x$-rays on the turnover of phosphatides of the cell nuclei and on the total tissue of the Jensen sarcoma and the liver of the rat is tabulated. The gate of turnover of the phosphatides in the nuclei is maxkedly diminished under the effect of $x-r$ rays and that in the cytoplasm is also diminished. In the nuclel of the liver the rate of renewal of phosphatides lags behind the rapid turnover in the cytoplasm. (CA)

1451

Hevesy, Georg von. EFIECT OF X-RAYS ON THE INCORPORATION OF C 14 INTO ANIMAL TISSUE. Nature 164, 269(1949).

Rapddly growing rats irradiated with $x$-rays showed 2 significant increase in the incorporation of $\mathrm{c}^{14}$ in the body tissues. Since the basal metabolism of Irradiated rats is decreased, it is suggested that the regulting higher actiwity level in the organism causes an increased incorporation of $\mathrm{C}^{14}$ into organic processes. (CA)

\section{2}

thevesy, Georg von and K. Zerahn. THE RFTCT OF ROENTGEN RAYS AND U.V. RADIATION ON THE PERME ABILITY OF YEAST. Acta Radiol. 27, 318-27(1946).

Irradiation with a dose of $30,000 \mathrm{r}$, sufficient to kill about $10 \%$ of the yeast cells, influenced the amount of free radio-phosphorus and radio-potassium ( $\mathrm{P}^{22}$ and $\mathrm{K}^{42}$, respectively) migrating daily from the yeast cells into the nutrient solution, and thus the cell permeability, but only to a very slight extent. The amount of migrating $\mathrm{P}^{\$ 2}$, but not however of $\mathbb{K}^{42}$, was greatly increased after ir radiation with a sublethal dose of u.v. radiation. Irradiation does not increase the free phosphate content of yeast cells. The phase boundary is thus influenced by the effect of the radiation. The migration of intracellular $\mathrm{p}^{32}$ into the nutrient solution is not an interchange process, as the same percentage of $p^{32}$ migrates into a phosphate-containing as into a phogphate-free molution. When the yeast cells were shaken with a nutrient solution containing arsenate, the amount of immigrating free $\mathrm{p}^{32}$ and $\mathrm{K}^{42}$ was found to be reduced to about half their normal valuew. (BA) 1453

Hewitt, John E., Thomas L. Hayes, John W. Golman, Hardin B. Jones, and Frank T. Pierce. IFIFCTS OF TOTAL BODY IRRADLATION UPON LIPOPROTEIN METABOLISM. Radiation Lab., Univ. of Calif. [nd] 27p. (UCRL-1549)

An investigation was made of the lipid metabolic derangement produced in rabbits as a result of irradiation. A correlation was found to exist between the high level of total lipoprotein $30 \mathrm{hr}$ after irradiation and subsequent death of the animal. Serum opalescence was associated with low-density lipoprotein only and not with total lipoprotein level. Changes observed in the lipoprotein level after irradiation were found to be consistent with the theory of conversion of low-densily lipoprotein to higher density components. The injection of heparin after irradiation 
hastened the return of lipoprotein levels to normal values. Injection of toluidine blue, protamine sulfate, or quinine produced changes in the lipoprotein pattern similar to those shown after irradiation. (NSA)

1454

Higimbotham, Noe, Edward S. Mika, and Robert B. Withrew. THE EFFECT OF X-RAYS ON UPTAKE AND LOSS OF IONS BY DISCS OF POTATO TUBER TISSUE, p.111-14 of BIOLOGICAL AND MEDICAL DIVISIONS; QUARTERLY REPORT, IFERUARY, MARCH, APRIL, 1948, Austin M. Brues and Hermann Lisco, eds. Argonne National Lab. May 1, 1948. 4p. (ANL-4163(p.111-14))

This report concerns the preliminary results of a study on the effect of ionizing radiations on the capacity of varim ous types of plant tissues to exchange ions with the environment. The present data show the effects of $x-r$ rays on uptake of radionctive rubidium ions from an external solution by discs of potato tuber tissue. Iffect of x-nays on the outward diffusion of ions into distilled water was studied by means of the electrical conductance method. Data are presented in Table 10. Results show that higher doses of $\mathrm{x}$-rays appreciably increase tissue permeability. (S.V.S.)

1455

Holmes, B. E. PHYSICAL, BIOCHIMLAL, AND TLERAPEUTIC ASPECTS OF VOLUME DOSE. HI. BIOCMEMICAL ASPECTS OF VOLUME DOSE. Brit. J. Radiol. 18, 238-40 (1945).

General diseussion by the author on biochemical agpects of volume dose: includes discussion of cholesterol in the blood, liberation of histamine after $\mathrm{x}$-radiation, etc. Read before General Meeting of the British Ingtitute of Radiology, November 16, 1944. (S.V.S.)

1456

Hornykiewytsch, Th. THE INELUENCE OF X-RAYS ON HYDROGEN ION CONCENTR ATION IN TISSUES UNDER VARIOUS EXPERIMENTAL CONDITIONS, $1.166-7$ of INTERNATIONAL CONGRESS OF RADIOLOGY, 6th Congress, London. 1950.

A ghort review of methods of ph mensurement with a glass-needle electrode is given. The following experimental results are reported: - The measurement of $\mathrm{ph}$ in the subcutaneous tissues of the thigh of the rat after a single irradiation with $4,000 \mathrm{r}$. After an initial acidity, there follows an alkalinity, 4 hours after irradiation, which lasts approximately 3 days and then fall in value. This is followed by four further alkaline "waves" at intervals of 5-6 days which, however, have no connection with erythema. Fluctuations in ple of a similarly periodic character occur throughout the body after lrradiation of the thigh. Irradiation of inflamed tissue with 500-1,000 r causes a slight "relative alkalinity" in the tissue, which lasts 2 days. This is preceded by small acid waves which are not periodic but depend on the mumber and the time of the irsadiations. The pH measurements of X-ray ulcers at varying periods of development show a distinct alkaline reaction in the ulcerated area. Changes in pH also occur after Irradiation (200 r) of the whole body of mice. An inttial actd phase is followed by an alkaline phase lasting 3-4 days. The initial (normal) values are reached agaln 7-8 days after irradiation. The physico-chemical changes which may produce pH fluctuations are discussed, and a comparlson is made between the influence of $\mathrm{X}$-rays and ultrasonde treatment on tissue reactions.

\section{7}

Jarvis, J. L. and D. Cayer. RFFECTS OF RADLATON THERAPY ON THE URINARY EXCRETION OF NIACIN AND RIBOFLAVIN IN PATIENTS WITH MALIGNANT
DISEASES. RELATIONSHIP TO RADIATION SICKNESS. Radiology 52, 574-8(124.9).

Experiments to determine whether malnutrition predisposes radiation sickness are presented in which the urinary excretion of niacin and riboflavin in a group of patients with malignant disease was measured before and after $\mathrm{x}-$ ray therapy. Of the 30 patients studied, 21 received tolal radiation doses of $1,800-8,120 \mathrm{x}$. Four patients received radium doses of $3,600-4,800 \mathrm{mg}-\mathrm{hr}$ and five patients received a combination of the two types of therapy. The excretion levels of niacin and riboflavin are given in a table included in this paper. Seven patients who had nausea and vomiting show significantly lower vitamin excretion levela than those without these symptoms. Good results have been obtained by the use of vitaming of the $\mathrm{B}$ complex for radiation disease therapy. (NSA) 1458

Tirschner, Leonard B., C. Ladd Prosser, and Penry Quastler. INCREASRD METABOLIC RATE IN RATS AFTER X-IRRADIATION. Pros. Soc. Rexpt1. Biol. Med.71, $463-7(1949)$.

In order to verify the hypothesis that catabolism is increased by irradiation, the basal metabolism of albino rats was measured after total body exposure to $\mathrm{x}-\mathrm{m} 2 \mathrm{ys}$. The rats were irradiated, two or three at a time, from a 200-kv source with no filter and at a target distance of $55.5 \mathrm{~cm}$, one-half of the dose being delivered from above, and one-half from below the anmals; the average thase dose rate was $40.5 \mathrm{r} / \mathrm{min}$. Twelve rats recelved $800-972$ $r$, siz of which developed severe diarchea, showed ax oxygen congumption elevation of $35 \%$ in 24 hour ${ }_{3}$, logt weight rapidly, and died in $3-5$ days. The remainings rats died 9-10 days after irradiation, and two out of eight irradiated with 648 also died at this time; all howed an oxygen elevation of about $58 \%$. The sis rats which survived the 648 inradiation, showed an average increase in oxygen consumption of $32 \%$ during the firgt seven days, and a lesg pronounced increase over a pertod of 16 days after the 12 radiation. Doses of 54-A32 $\mathrm{x}$ were given to other groupes of rats and cassed an average increase of about $0.5 \%$. In all of the experiments, the increased metabolisu did not coincide with weight loss either in time course or th dose dependence. (NSA)

\section{9}

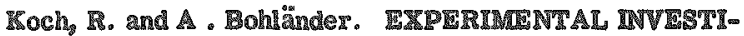
GATION ON PTIECTS OF VITAMINS ON LIVER METABOLISM IN ANIMALS SUTRTERG FROM RADLATON INJURIIS. Strahlenthormple 85, 31-41(1051).

Total-body 8 gadiation in white ralce reanted in a reduction of the liver glycogen to $27 \%$ of 1 tr original value. In homogeneous and heterogeneous aniwal naterial, the distribution of the Iver glycogen corresponds to that of the normal Caussian distribution. No quantitative changes of the total $\mathbf{N}$ content of the liver were found. (NRSA) 1400

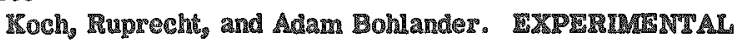

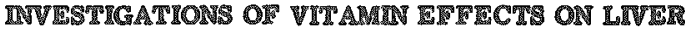

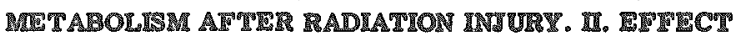
OF LACTOFLAVIN AND PVIRTORIN ON GLYCOGES AND TOTAL NITROGR N OF THE LIVER IN TOTAL BODY IRRADIATED ANIRAIS. Strahlentheragie 2 B 500-605(1251).

Mice were subjected to total-body x Iradiations of 350 10 400 tr each on 5 consecutive days, while 0.5 meg dactoIlawin or 2.5 mg pyridoxin were glven by gubcutameous injection 6 days before the firgt irsadiation and after each irradiation except the last one. The aninals were klled and invegtigated chemically several hours anter the last 
Irradiation. Irradiation did not change the $\mathbb{N}$ content of the liver, and vitamin treatment had no effect. The glycogen content of the liver dropped to $27 \%$ normal after in radiation; lactoflavin treatment decreased the loss of glycogen to $55 \%$ normal, and pyridoxin treatment decreased it to 51\% normal. (NSA)

1461

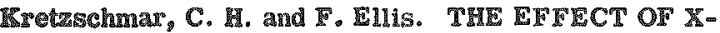
RAYS ON ASCORRIC ACID CONCENTRATION IN PLASMA AND IN TISSUES. Brit. J. Radiol. 20, 94-9(1947).

"Arl adequate dally dose of ascorbic acid can prevent or minimize the fall of white blood eells which follows x-ray exposure. It diminishes the risk of $\mathrm{x}$-ray sickness. There is however no uniformity in the result." Vogt, quoted by the authors, does not believe that exposure to $\mathrm{x}$-rays has any relation to the excretion of ascorbic acid in the urine, but he considers that the marked deficiency of ascorbic acid in malignant patients is purely due to the disease, whether cancer or Iymphadenoma. The authors estimated the ascorbic acid in plasma of patients under $\mathrm{x}-\mathrm{r}$ ay treatment, and in most cases it was found to be below the initial level before exposure. After g-ray exposure of normal rabbits estimation of ascorbic acid was made and a fall in the level was found for some time after exposure. The ascorbic acid content of musele and hidney tissue from rats orposed in vivo to $x-$ rays was also found to be lower than 1062 norma. (AWM)

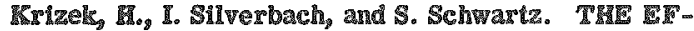
FECT OF LETHAL DOSE OF TOTAL BODY X-RAY ON URIC ACM AND ALIANTOIN RICRETION IN DOGS. Univ, of Chicago. June 15, 1946. Decl. Jan. 15, 1248. 28p. (MODC-1047; CH-3831)

In Ifve dogs given single lethal doses of total body x-ray, urinary excretion of uric acid increased 3 to $6-1 / 2$ days before death; allantoin arereton showed no significant change. Uricolytic indices, therefore, dropped during the terminal period. Uric acid excretion was determined in ane dog given 12.5ิ $\mathrm{I}$ dally total body g-Iay, and in one glven a slowly lethal dose of plutonium, and showed a simIlar terminal increase. These changes are probably due to IIver dygtunetion. In all dogs studed excretion of subgtances giving a color wh the uric acld reagent but not dercroged by incubation whin uricase showed no consistenc change after lrzadiation. Rabbits appeared to be poorly guted for atudy of purine metabolism. (ADD)

1403

Latarjet, R. and J. Loiseleur. FIXATION OF OXYGIN IN

RADIOBIOLOG\%. Compt. rend. soc. phys. hist. nat.

Gentye 5\% 234-5(1042); Compt. rend. soc. bilol. 136, 603(19:32).

Tr-rays Lonze the dissolved 0 and mumerous organic compounds present in the trasues then act as acceptors. (CA)

1 14.

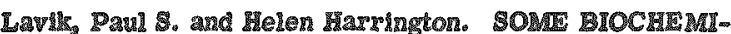
CAL IPIPCTE OF RADIATION ON LYMPRATIC TISSUE. Wegtern Iexerve Untw. School of Medicine. July $13,1051$. 30p. (NYO-1626)

The effect of a toxtc dose of $\mathrm{P}^{22}$ or whole-body $\mathrm{x}$ irradiation on the $\mathrm{P}$ composition and $\mathrm{P}^{\mathrm{s} 2}$ incorporation of thymus, spleen, and normal and segenerating 11 er has been studied in adult rats. The changes observed in chemical composition have been related to the histological irradiation damage. The radiosensitive lymphatic tissues, thy mus and spleen, showed a marked decrease in desoxysibonucleic acid $\mathrm{P}$ as a result of ir radiation, whereas the P composition of normal and regenerating liver, nonlym- phatic tissues, did not change significantly. Radiation caused an inhibition of the $\mathrm{P}^{32}$ incorporation of into the desoxyribonurleic acid fraction of all tissues but normal liver, which had a very low $\mathrm{p}^{32}$ uptak into this fraction in both normal and irradiated rats. Adrenalectomy did not prevent the changes occur oing in thymus after irradiation. The relation of inhibition of desoxyribonucles ac id synthesis to the chemical and histopathological changes which occur is discussed. (NSA)

1465

Lawnence, Geo. H. THE EFFECT OF TOTAL-BODY X-RADIATION ON 17 IEITOSTEMOD EXCRETION IN DOCS. Endocrinology $45,383-8(1949)$.

Fhe urinary excretion of 17 - 5 ctostaroids was followed in 11 dogs after exposure to a minimum lethal dose of total abody $\mathrm{x}$-radiation. Significant increases in steroid excretion occursed in all animals between the 5 h and 12 th day after treatment. The excetion then fell to normal or subnormal levels, then showed a trend toward normal levels by the 30 th to 40 th day. The changes in excretion are interpreted as alteration of adrenal cortlcal and/or testculas activity in these animals, and are gimilax to those seported in adaptation to other stimuli. (CA) 1466

Loofwourow, John R., Mary Elizabeth Englert, and Cecelia MaẼ Dwyer. INCREASED VILD OF NUCLEIC ACDDLIEF SURSTANCES IROM IRRADLATED YAST. NatuSE $148,118-14(1941)$

A comparison of nuclete acid present in lryadiated ant non-1radated yeast (Saccharomyces cerevisiae) showad a higher per cent in the for ${ }^{\circ}$ as well as areater prom liferation oromoting activity. (BA)

1467

Loura, Marguerite and Odette Lartigue. MODIICATIONS OF GHYCEMA UNDER THU INPEUENCE OF TOTAL RAY KADLATON. Compt. rEUd. 230, 1426-8(1950).

In previous work (Experientia 6, 25, 1950) It was shown that total body $\mathrm{x}$ - ixrdiation causes modifications of yclucose metabol sm: this maper 10 aresentaton of the results of exper ments to determine the extent of the

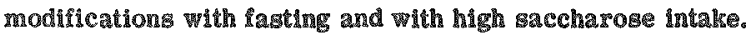
Two rroups composed of 13 munea pigs each, ware irgadated in one dose calculated to deliver 500 r/animal: the blood glucose linels were determined varying Intervals for pertods up to 35 days. It was ghown that the hyperslycerala occuring after the ingeston of saccharose by

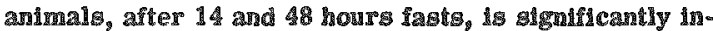
creased by the radiation dose given, and that there 13 a large decrease in ghcose tolerance cue to the isradiaton, ance 1 tos found that the hyperglycemla was much more rrtreme in the animals irradated 12 and 14 day belore dose of $1 \mathrm{~g}$ of saccharose than in nontriadiated animals. The authors conclude that the importance of the two effects 18 not equal alchoush they apparenty arise from the arme phenomena. (NSA)

1468

Lourau Marguenite and Odette Lurthue. MIPATC GLYCOCENESIS IN WHOLE-BODY Y-IRRADIATED ANIMLIS. Compt. rend. 232, 114

Glycogenesis in the guinea pir Ir drminghed following

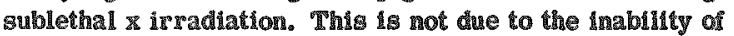
the hepatic call to sythealize rlycogen but to the absence of mubstrate, the ricose in the chrculton probably having been abnor mally metabolized to a mubstance incapable of men: 1469

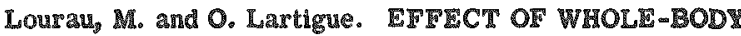
Y-TRRAOLATON OT TKIE GUTEA PTC ON UTLLIZATION 
OF GLUCOSE BY THE LIVER. J. physiol. (P aris) A3, $593-603(1951)$.

Male guinea pigs weighing 400 to $500 \mathrm{~g}$ were irradiated sublethally with $500 \mathrm{~g}$ of $180 \mathrm{kv}$ x radiation. Hepatic glycogenesis was observed to be deficient. This deficiency was manifested less by a diminution in the quantity of glycogen formed in the course of glucose absorption than by the impossibility of preserving the stocks of glycogen during the period following absorption. The authors hypothesize that irradiation does not affect the direct synthesis of glycogen from alimentary glucides, but impedes synthesis from small molecules. (NSA)

1470

Ludewig, Stephan and Alfred Chanutin. EFFECT OF WHOLE BODY X-IRRADIATION ON THE STORAGE OF TRON IN LIVER, SPLEEN, AND IKIDNEY OF RATS.

Am. J. Physiol. 166, 384-6(1951).

The iron contents of the liver, spleen, and lidneys of rats were determined at varying intervals after whole body exposure lo $\mathrm{x}-\mathrm{ray}$ doses of $500 \mathrm{~m}$. The values for the liver and spleen increased steadily during the first two weeks and deereased during the third week following irradiation. Small increases were noted in the kidneys during the second post-radiation week. Unusually high iron values were chserved in the liver and spleen of anemic irradiated rats A derangement in iron metabolism follows $\mathrm{x}$-irradiation and is manifested by the storage of excess iron in tissues. (NSA)

1471

Marinelli, L. D. and J. M. Kenney. THE ABSORPTION OF RADIOPHOSPHORUS IN IRRADIATED AND NON-IRRADIATED MICE. Radiology 37, 691-7(1941).

Expeeiments were undertaken to investigate the effect of poentgen irradiation on gross phosphorus metabolism in some animal tissues. Albino mice more than 12 months of age were uscd. All animals were given tracer doses of $\mathrm{p}^{32}$ intraperitoneally as $\mathrm{Na}_{4} H \mathrm{PO}_{1}$ immediately after irradiation and saerificed at different intervals. Comparative quantitative studies were made of skin, muscle, and bone removed from both the treated and untreated extremity of the same animal. There was no difference in the $\mathbb{P}^{32}$ content per $g$ of tissue in either the muscle or skin of the 2 legs. In the bone, however, the $\mathrm{P}^{32}$ content of the irradiated bone was higher than in the untreated extremily. (BA) 1472

Markees, S., O. Kåser, and R. Lanz. PYRUVATE MRTABOLISM IN NORMAL AND PATEOLOGICAL PREGNANCI IN NARCOSIS, AND X-RAX IRRADIATION.

Schweiz. med. Wochschr. 80, 1079-81(1950).

The following average values in mg/h were obtained for blood pyruvate levels: normal 0.8 ; after the 20 th week of pregnancy 1.3 ; nephropathy and preeclampsia; 1.5 ; eclampsia 3.7 ; $6-8$ hours after narcosis, 2.1. The increase in blood pyruvate concentration after $\mathrm{x}$-ray treatments paralleled the symptoms of radiation sickness. (CA) 1473

Mead, James I", Arthur B. Decher, and Leslie R. Benneth. THE TEFIFCT OT X-IRRADLATION UPON FAT ABSORPTION IN THE MOUSE. J. Nutrition 43, 485-500(1951). (UCLA-90)

Fat absorption in mice was followed using methyl oleate containing $10^{\circ}$ of the spectrophotometrically active methyl octadecadienoate as a tracer. The rate of absorption of this mixture was shown to be nearly constant. Mice which had received 600 to $700 \mathrm{r}$ wholo-body $\mathrm{x}$-irradiation showed a tendency toward increased tone and motility of the gastro-intestinal tract, resulting in retention of oil in the stomach or rapid passage past the absorbing surface. De- spite these changes, fat absorption per se appeared to be about normal. A post-irradiation increase in fecal fat was shown not to be due to lowered absorption since it occurred in animals on a fat-free diet. It is concluded that there is no evidence for post-ir radiation imparment of fat absorption. (NSA)

\section{4}

Milne, John and Abraham White. ERFECT OF AORENAL CORTICAL EXTRACT AND OF X-RAY IRRADIATION ON SERUM PROTEINS OF MCE. Proc. SOc. EXPLI. Biol. Med. 72, 4224-8(1949).

Normal mice and mice bearing a transplantable lymphosarcoma were given single injections of adrenocortical extract or exposed to a single total body $x$ - ray dose of $200 \mathrm{r}$. Examination $3-6$ hr after the $x$ - 5 ay treatment revealed a manked increase in lotal serum proteins, but electrophoretic tests showed no significant alteration in the relailve distribution of the serum proteins. (CA) 1475

Muntz, A. A., E. S. Guzman Barron, and C. L. Prosser. MECIANISM OF ACTION OP IONIZING RADIATIONS. III. PLASMA RROTEIN OF DOGS AFTER X-RAY IRRADIATION. Arch. Biochem. 23, 434-45(1049).

Lethal doses of $x$-rays were given to mongrel dogrs as a lotal body dose. For one week following, the electrophoretic patterns of their plasma proteins showed no change, but from the seventh day wntil death, albumin decreased steadily. Three to 5 days before death, fever ensued, and this was accompanied by a striking increase in $a_{3}+a_{4}$-globulins, with some increase in $\beta$-globulin fibrogen. Extraction of lipids did not decrease the amounts of $\alpha_{3}+a_{4}$-globulins as determined electrophorectically. A simgle injection of bovine albumin equal to one half the total normal plasma albumin caused no effect other than a temporary rise in plasma albumin. In the terminal period, blood volume and total protein inereased. Death occurred 1476 12 to 18 days after exposure. (CA)

NNat, R. E., C. Heusghem, and A. Herve. CHANGSS IN THE ADRENAL CORTEZ ANTER X-RAY IRRADIATION OF A DISTANT PART OF THE BODY OF THE RABBIT. Compt. rend. soc. biol. 143, 876-7(1949).

With the abdominal region shielded, the hind legg were subjected to a single dose of $2000 \mathrm{r}$. The adrenal cortex showed large decreases in total lipides and cholesterol within $3 \mathrm{hr}$. Recovery occurred within 48 hr. Urinary ezcretion of 17-kelosteroids remained within the normal range (125-300 $/ / 24 \mathrm{hr})$. (CA)

$147 \%$

North, N. and L. F. Nims. TIME-DOSE STUDY OF BIOCYEMICAL RESPONSES OF RATS TO X-RADLATON. Federation Proc. 8, 119-20(1949).

A time-dose study of biochemical responses of the adrenal-pituitary system in fasted rats following whole-body x-radiation indicate a pattern of changes comparable to that following other stresses. Male albino rats were subjected to radiation doses of 500 to $2000 \mathrm{~g}$ at a rate of $50 \mathrm{~g}$ per minute and sacrificed at periods of time ranging from 3 to 48 hours following exposure. Analyses of tissue constituents show an immediate and progressive increase in liver glycogen and no change in testis glycogen. There is a simultaneous decrease in the adrenal and liver cholesterol but little change in the testis cholesterol and a late increase in the plasma cholesterol. The adrenal also show an initial decrease in ascorbic acid content with a subsequent return to normal, while lithle variation from the unirradiated controls is seen in pituitary, liver or testis ascorbic acid. There are no evident effects of radiation on 
total body, adrenal, or pituitary weights in fasted animals. (NASA)

1478

ORIOW, S. M. PROSPHORUS-CALCIUM METABOLISM IN EXPERIMENTAL RICKETS AND THE EFTECT OI IREADITION BY X-RAYS. Voprosy Pediat. I ORhrany Materinstva i Detsiva 18, No. 5, 10-14(1950).

Initiation of rickets in young dogs depends not only on deficiency of $\forall$ itamin $D$, but also on severe disproportionate $\mathrm{Ca}$ and $\mathrm{P}$ content in food (such as $\mathrm{Ca} / \mathrm{P}$ of $1 / \mathrm{p}$ ). Reestablishment of nearly normal $\mathrm{Ca} / \mathrm{P}$ ratio in the diet rapidly normalizes the assimilation of these elements, while mere addition of vitamin $\mathrm{D}$ (125,000 units in a days) is insuffi clent. Irradiation of vegelative nervous centers (360-725 r) of the medulla leads to a sharp lemporary improvenent of asmimilation of $\mathrm{Ca}$ from the intestinal tract, as well as a significant improvement of $\mathrm{P}$ assimilation. (CA)

1479

Painter, Elizabeth E. and E. Wo Pullman. WATER AND ELECTROLYTE CHANGES IN RAT INTESTINE AITER TOTAL BODY X-RADIATON. Federation Proc. 9, 97 (1950).

Preliminary experiments carried out on the small intestine of $220 \mathrm{gm}$ male rats before and after a single dose of total body $x-m$ radiation (LD 70) indicate that increases in sodium content can be correlated with decreases in potassium and with increases in chloride and water content in the first fow days after radiation. These increases in sodium content of the intestine have been confirmed by radioactive sodium gtudies reported earlier by us. That increase in sodium is in part related to the exchange of sodium for potassium in gut cells appears to be a valid 1480 conelusion from our results. (Authorg)

Peterson, Ruth D. Clarissa H. Beaty, and Zdward S. West. FEST OF TOTAC BODY X-IRRADIATION ON GLUTATITORE LEVELS IN RATS. PrOc. SOc. Rxpt1. Biol. Med. 7\%, 74\%" (1951).

A study has been made of blood and liver glutathione in sate after 500 r total body $x$ irradiation. The data show, first, that irradiation generally causes a decrease in blood glutathione; and second, in some cases, a decrease in liver glutathione. (NSA)

1481

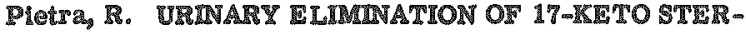
ODS AFTER IRRADIATION OF TIIE HY POPHYSHS. Folia Endocrinol. (PLS) 3, 200-2\$(1050).

Euman beings showed no substantial changes from normal elimination. (CA)

1482

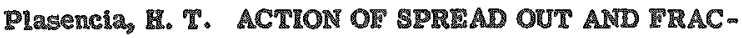
TIONATED RADLATIONS ON SOME CHIMICAL RQULI BRIA OF THE BLOOD. CONTELUETON TO THE STUDY OF THE GEIERAL BIOLOGICAL $\triangle$ CTION OF RADIA-

FION. Acta UnLo. Intern. contra. Cancrum 4 , 333-58(1039).

Due to the destruction of cells there is a hydremia, a decline in the alburin: globulin ratio and a desrease in the amount of chloride in the red cells and in the plasma. The digturbances in the interrelatlong between the endocrine organs and in the nervous regulationg are followed by a dimished alkall reserve, be diverse changes in the propor ton of antons to cations, by an inerease in the amount of acetone bodies and in the circulating glutathione, by a fall In blood sugas, and by hypockolesterinamia. These as well as the climical symptoms depend wnon the Intensity of the dally Irradiation. (BA)
1483

Ragran, C., C. P. Donlan, J. A. Coss, Jro, and A. F. Grubin. EFIECTS OF X-RAY IRRADIATION ON VISCOSTTY OF SYNOVIAL FLUID Proc. SOC. ExpLI. Biol. Med. 66, 170-2(1947).

In vitro reduction in viscosity of synovial fluid when exposed to X-ray irradiation was noted. Reduction in viscosity varied directly with the amount of irradiation. The hyaluronic acid content did not change and the hym aluronic acid was still susceptible to enzymatic hydrolysis after exposure to $\mathrm{X}$-rays. Reduction in viscosity of pure $\mathrm{Na}$ hyaluronate solutions was noted as well but to a lesser degree. It is suggested that the effect of X-rays may be a breaking of secondary valences present in synovial fluid hyaluronic achd without a profound change in the hyaluronic acid molecule. The physiologic implications are discussed in light of the widespread presence of this and similar acid mucopolysacchaxides throughout the connective tissue of the organism. (BA) 1484

PRichnond, Jonas e.g Rurt I. Altman, and Rurt Salomon. THE RELATION OF OXYGEN UPTAIE TO HEMOGLOBIN SYNTHESIS. Science 113, 404-5(1951).

Bone marrows of normal rabbits and of rabbits exposed to $800 \mathrm{r}$ of $\mathrm{x}$ xay were removed at varying time periods after exposure to radiation. Homogenates were prepared from these marrows and incubated at $38^{\circ} \mathrm{C}$ in the presence of $a-C^{14}$-glycine as a hemoglobin precurmor. $O_{2}$ consumption of the homogenates and the amount of hemin and globin synthesized was determined. The results a difference in the time relation between $O_{2}$ consumption and the synthesis of hemin and globin, respectively. Hemin gynthesis as well as $\mathrm{O}_{2}$ consumption increases considerably in homogenates from bone marrows removed immediately after irradiation. Furthermore, $O_{2}$ consumption and hemin aynthesis appear to reach a minimum about 1 week after exposure to radiation at a time when marked degenerative changes prevail in the marrow. Globin synthesis also increases after radiation, but, in contrast to hemin synthesis, it reaches a maximum $48 \mathrm{hr}$ after radiation at a time when $\mathrm{O}_{2}$ consumption already agproaches a minimum value. However, the decay curve of globin resembles in its shape that of $\mathrm{O}_{2}$ conaumption and hemin synthesis. Recovery of all three functions begins 2 to 3 weeks after radiation, which agrees well with histologlcal findings. (NSA)

1485

Richmond, Jonas E., Rurt I. Altman, and Kurt Salomon.

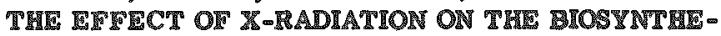
SIS OF MEMOGLOBIN. J. Biol. Chem. 100, 817-25(1951).

Temoglobin gynthes:s was studied in bone marrow and spleen homogenates of rabbits expoged to $200 \mathrm{r}$ of $\mathrm{x}$ radiation at a rate of 26 to $27 \mathrm{~s} / \mathrm{min}$ with $\mathrm{C}^{\mathrm{I} /}$ labeling of specific precursors for hemin and globin. Hemin and globin synthesis are diferently affected by radiation, both evidently going on at differen rates. A new term, W, as an expression of the hemin- and globin-forming capacity of the organ systems is introduced and its value calculated for bone marrow and spleen homogenates. (NSA)

1486

RLey, Richard I and Esther Richter. PAPER CHROMATOGRAPHIC STUDY OF FREE AMINO ACIDS OF TOUK OF DEVILLOPMG NORMAL AND X-IREADIATED CRICR EMBRYOS. Univ. of Calii., Los Amgeles.

Nov. 26, 1951. 8p. (UCLA-171)

The free amino acids of yolk of fertile chick embryos between the 5ొth and 20 th day were examined by paper chro- 
matography after removal of proteins by alcohol precipitation and lipids by petroleum ether and ethyl ether extraclion. $\alpha$-Alanine, arginine, glutamic acid, glutamine, leucine(s), lysine, and tyrosine were found consistently, and cysteic acid, glycine, methionine, proline, and serine, threonine, and valine were also found in most extracts. Appreciable amounts of peptides were absent. $500 \mathrm{r}$ of $\mathrm{x}$ irradiation $\left(\sim \mathrm{LD}_{50}\right)$ administered on the 10 th day did not alter this picture appreciably. (NSA) 1487

Robertson, James E. METABOLIC CHANGES OCCUR RINC AS TME RESULT OF DEEP ROENTCEN THERAPY II. EPFECT OF 1000 KSILOVOLT ROENTGEN THERAPY. Am. J. Roentgenol. Radium Therapy 50, 392-9(1943).

Seven patients were subjected to a careful study of nitrogen and chloride metabolism before, during and after $1,000-\mathrm{kv}$ roentgen therapy. The results proved to be gimilar to the results of the study conducted in the same laboratories on the effect of $200-\mathrm{kv}$ roentyen therapy. Supervoltage roentgen radiation was shown to produce an increased exeretion of nitrogen, uric arid, and chloride in some of the patients, just as does radiation of lower voltage. That the negative $\mathbb{N}$ balance results entirely from tissue destruction is strongly indicated by its restriction to cases in which large tumors disappear under the influence of roentgen radiation. In all cases in which study was sufficiently prolonged, a period of $\mathrm{N}$ storage followed that of $\mathbb{N}$ loss. Chloride metabolism changer in the nature of a negative chloride balance were demonstrated but less frequently in the patients receiving the 1,000-than the $200-\mathrm{kv}$ therapy. Though the chloride change seems to be correlated with the degree of nausea and vomiting, this study suggests that if is a secondary result of a disturbance in water balance rather than the precipitating cause of the roentgen sickness. Roentgen sickness was observed much less frequently under million-volt roentgen therapy than under 200,000 - kv roentgen therapy. No significant differences in changes in metabolism induced by irradiation with $200 \mathrm{kv}$ and $1,000 \mathrm{kv}$ roentgen rays were demonstrated. (R) 1488

Ross, M. H. and J. O Rly. NRUTRON AND ROENTGENRAY EFFECTS ON PROTEIN CONTENT OF RAT INTESTINE. AM. J. Roentgenol. Radium Therapy 62 , $718-22(1049)$.

The present investigations are extensions of previous studies on the effects of penetrating radiations on the chemical constiluents of the cells of the crypts of Lieberkuhn. White rats of the Brooklyn straln were used for the estimation of total protein; 10 ratg received $600 \mathrm{z}$ of roentgen radiation, 9 received 56 n of neutron radiation, 10 recelved 100 n of neutron radiation, and 10 recelved 200 n of neutron radiation. Each irradiated rat was pared with a control rat of the same weight and all the rats were killed 24 hours after the completton of the irradiation; zections of the duodenum of each rat were firsed in alcoholacetic acid, embedded and sectioned, and the sections were photographed with an ultraviolet microscope for tyrosine, tryptophane and arginine determinations. In a second esperiment to determine the effects of irradiation on the nifrogen content of the intestinal cells, two groups of 10 white ratg were irradiated with 200 n of neutron radiation and killed 24 and 48 hours after the treatment; the entire small intestine was extracted and the total dry weight of the mucosa was determined. It was found that there was a signiflcant decrease in the mucosa weight 24 hours after Irradiation and a greater decrease 48 hours after Irradiation; a slight decrease in the percentage of nitrogen was also noted after irradiation. Decreases in the tyrosine and tryptophane contents of crypts were noted after 600 roent- gen irradiation, but not after 56 n total body neutron ir radiation; decreases were also noted after irradiation with 100 and 200 n neutron irradiation; the arginine contents were found to decrease 24 hours after 600 ror $200 \mathrm{n}$ irradiation. (NSA) 1489

Ross, M. R. and I. O. Ely. RADLATION EFIECTS ON LIVER GLYCOGEN IN TRE RAT. J. Cellular Comp. Physiol. 37, 163-71(1951).

A total of 830 rats were irradiated with $x$ rays or neutrons in a study of the $x$ effects of irradiation on the amount of hepatic glycogen in the liver. Glycogen deposits were found after irradiation in the liver cells of rats fasted prior to and after ir radiation. Glycogen deposits were also found in fasted irradiated rats after shielding various portions of the body. From these results it was deduced that the effect of radiation on glycogen deposition is not a primary one. (NSA) 1400

Salomon, K., K. I. Altman, G. W. Casarett, and T.R. NOOnan. EFTECT OF X-RAI RADIATION AND OF STARVATION ON HEMOGLOBIN SYNTHESIS IN THE RAT. Federation Proc.8, 247(1949)。

After whole body radiation and in acute starvation exythropoiesis is depressed. Information concerning the anabolism of the circulating hemoglobin under these conditions seems desirable. Since the alpha-carbon atom of glycine has been shown to be incorporated in hemin and globin, methylene labeled glycine was used in this investigation. Glycine (1 or 2 microcuries) was administered to radiated and to starved rats. All animals were killed 24 hours later. Hemoglobin, hemin and globin were isolated from their blood and the C-14 content of these compounds determined. The C-14 activity ratio of hemin to globin on a gram to gram basis was found to range from $4-13$ in all control animals. The ratio of C-14 activity of hemin to globin isolated from rats injected with glyeine immediately after radiation with $600 \mathrm{r}$ varied from 25 to 32 . Hemin synthesis was increased in all these rats. On the other hand, when glycine was injected 6 days after exposure to $600 \mathrm{r}$ no measurable hemin synthesis occurred, and the activity ratio was found to be zero. The activity ratio after 5 days starvation was 64; hemin synthesis in these rats was increased whereas globin formation was depressed. In 7 days starvation the activity ratio was zero, hemin synthesis was not measurable, clobin formation was depressed. In both radiated and starved animals the ratio of C-14 activity of hemin to that of globin deviates congider. ably from control values. 1491

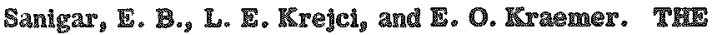
EFIECT OF ULTRAVIOLET RADATION AND SOFT X RAYS ON THE SEDIMENTATION AND LICEYI ABSORPTHON OF PURIFIED IUMAN SERUM ALBUMN, B10chem. J. 33, 1-16(1039).

The sedimentation constant of the unaggregated protels in the ir radiated solutions remained eseentially that of

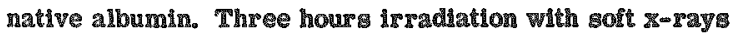
$\left(29,000 \mathrm{r}, u_{0}\right)$ caused no detectable change in the sedimentation behavior or the absorption apectrum. (Past of Authorg' Summary)

\section{2}

Scartozz, Cesare and Glovann SLvestri. ACTLN OF X-IRRAOIATION O THE SPLEEN ON EASILY ERTRACTABLE IRON. Pathol. sper. 36, 65-80(1948).

Three days" irradiation of rabbits at the gpleen with the exciting Irradiation, 3 min with 21 ror 4 min whth $28 \mathrm{r}^{2}$ caused increase in easily isolated Fe of blood, which be- 
came normal again after 15-25 days. After an interval of 140 days the same rabbits were subjected to inhibiting irradiation, $15 \mathrm{~min}$ at $110 \mathrm{r}$, and ater 3 days showed no difference in easily isolated $F c$, but after 8 days diminution of easily isolated Fe from original values of the order of $1.500-1.600 \mathrm{down}$ to $0.560-0.580 \mathrm{mg} / 100 \mathrm{ml}$ blood the values remained low for 45 days and returned to normal only at 65 days. When liver and musele were similarly irradiated, labile $F$ e values were either affected, although a slight rise was noted in the case of inhibiting ir radiation of the livex. (CA)

1499

Shacter, Bernard and M. C. Fishler. DECREASE IN THE SULFHWPY, CONTENT OF RAT PLASMA FOLLOWING X-IRRADIATION. Naval Radiological Defense Lab. Apr. 26, 1950. 10p. (AD-214(B))

Tixposure of rats to $750 \mathrm{r}$ lotal body s-radiation is followed after several days by a marked decrease in the sulfhydryl content of the blood plasma. (NSA) 1 1셩연

Shacter, Bernard, Relen Supplee, and Cecil Entenman. EFFECT OF X-RADIATION, RADIOMTMETIC SUB STANCES AND SURGICAL TRAURA ON THE SULFHYDRYC CONTENT OF PLASMA, FINAL REPORT. Naval Radiological Defense Lab. Nov. 27, 1951. 22p.

(AD-333(B); Technical Objective AW-6; U20402)

Plasma sulfhydryl concentrations (SC) were determined for 3- to 6-month-old rats of both sexes. Determinations were performed by amperometric titration with a rotaing Pt electrode (TIP U16692); SC was estimated on both a protein and unit-volume basis. A Westinghouse xmraytherapy unit was enployed at $250 \mathrm{kvp}$ and $85 \mathrm{r} / \mathrm{min}$. Animals subjected to $700 \mathrm{x}$ total-body $\mathrm{x}$ irradiation in a single exposure showed an SC decrease 2 days following ix radi ation. Lowest values were obtained 6 to 8 days after exposure when SC approximated $60 \%$ of control concentrations. $B y$ the twelfth day, SC values approached normal levels. A similarly delayed decrease, of lesser magnitude, was observed after intraperitoneal administration of $\mathbb{N}$ mustard and irisethylene triazine at a dose rate of $1 \mathrm{mg} / \mathrm{kg}$ body welght. Lowest values were obtained on the fifth day. No well-defined changes in SC were detected during a 6-day starvation period or after a single exposure $10300 \mathrm{r}$ totalbody $x$ irradiation. Surgical trauma induced by laparotomy produced a significant $5 \mathrm{C}$ decrease at $24 \mathrm{hr}$ after injury, followed by a linear return to normal concentrations over a 12-day period. No significant ehanges occurred in the first $12 \mathrm{hr}$ subsequent to laparotomy; no sex differences were observed. It is suggested that decreases in SC following tissue injury may be indicative of a markedly increased rate of unilization of sulfhydryl groups by regenerating tissue. (Presented in part at a meeting of the American Association for Cancer Research, Inc., April 1951.) (NRS abst.) (NSA)

\section{5}

Shacter. Bernard, Helen Supplee, and Cechl Entenman. EIIECT OI X-RADIATION AND LAPAROTOMY ON TRE POLYSACCHARIDE CONTENT OF PLASMA; RELATIONSHIP TO PLASMA SULFRYDRYL CHANGLS, FINAL REPORT. Naval Radiological Defense Lab. Nov. 27, 1951. 11p. (AD-334(B); Teehnical Objective AW -6 ; U20432)

The nonglucosamine polysaccharide content (PC) of rat plasma. was determined colorimetrically by the iryptophane method of Shetlar and others (Proc. Soc. Expti. Biol. Med. 67. 125(1948)). Blood was collected in syringes moistened with $20 \%$ K oxalate. Male rats subjected to 700 -r totalbody $x$ irradiation in a single exposure showed no PC change during the first 5 postirradiation days. PC levels gose significantly on the sixth day, remained elevated for a few days, and returned to normal by the twelfth day. Exposure to 300-r total-body x radiation had no effect on PC. Luparotomy resulted in a significant $P C$ increase in $24 \mathrm{hr}$. Highest values, which were attained 1 to 2 days following laparotomy, returned gradually to nor mal. PC was consistently greater for male control and laparotomized rats than for the corresponding female animals. In general, sulfhydryl changes (TIP U20409) were detected sooner and lasted longer than the polysaccharide changes. If was tentatively concluded that the same conditions produce the 2 changes; however, the polysaccharide elevations may be less detectable by the methods employed. (NRS abst.) (NSA) 1496

Schoenberg, Melvin D., Robert D. Brooks, John J. Hall, and Herman Schneiderman. EFIECT OF X-IRRADIATION ON THE MYALURONDDASE-HYALURONIC ACDD SISTCM. Arch. Biochem. 30, 333-40(1951).

X-irradiation caused a reduction in viscosity of vitreous and umbilical-cord hyaluronate solutions, and a continuing loss in viscosity after cessation of the in radiation, and markedly decreased the average nolecular weight. The half-time values for irradiated hyaluronate were greater than for nonirradiated. There was a decrease in the activity of x-irradiated hyaluronidase solutions. (CA)

\section{7}

Schwartz, Samuel. SOME BIOCHEMTCAL EFFECTS OF IONIZING RADIATIONS. Bull. Univ. Minn. Hosp. Minn. Med. Found. 20, 617-54(1949).

From experimental studies and from a survey of the literature, the following conchusions have been drawn: The most striking biochernical changes found after excessive radiation exposure in humans were (1) an increase in urime "corticosteroid-like substances in all of the five individuals studied. (2) a prolonged increase in the "adenosine triphosphate" phosphorus in the penipheral white blood cells of a patient given total body 8 -ray for the treatment of a metastasizing parotid tumor, (3) pronounced terminal increases in urinary coproporphyrin excretion in three patients who died within a few weeks after heavy exposure to external radiations, and (4) increased excretions of "urorosein fraction" pigments in severely ill patients given large doses of total body $\mathrm{x}$-112radiation. Other changes found included a shift to the left in the Arneth count, and an increase in erythrocyte sedimentation rate. The chief changes found in experimental animals include the following: (1) a drop in urinary coproporphyrin excretion followed by a terminal rise, (2) an increased czcretion of fecal urobilinogen, indicative of increased red cell destruction, (3) an increased terminal exeretion of kynurenie acid, (4) an increased excretion of uric acid and a decreased uricolytic index, and (5) terminal increases in excretion of urinary urobilinogen. More fundamental information is needed on the nature of both early and late biochemical effects of radiation. Studies of physiologically important. oxidation-peduction phenomena, and protein and enzyme effects seem especially promising. (NSA)

\section{8}

Schwartz, Samuel. STUDIES OF LIVER IUUNCTION IN WXPERTMENTAL ANIMALS WITY SPECIAL RETERENCE TO RADIATION AND METAL RXPOSURE. Univ. of Chicago Metalurgè Leal Lab. July 31, 1947. Decl. May 12, 1948. 37p. (ALCD-2020, CH-3855)

Total-body X-Iadiation was administered, with a $200 \mathrm{kv}$ x-ray machine operated at 15 ma. Radiation was deliverod at the rate of aboul $6 \mathrm{x} /$ minute. One-half mm copper and one nm aluminum filters were used. Plutonium and strontiun ${ }^{62}$ were administered intravenously or intramuscularly. The various (nonradioactive) metals studied 
were injected subcutaneously in the thigh. These metals included beryllium, lead, uranium, thorium, mercury, arsenic, and lanthanum. Relutively extensive studies of liver function were made only with beryllium. In addition, the effect of BAL was studied in relation to the use of this substance in the treatment of metal poisoning. The metal galts used were dissolved in water and generally brought to pH 5- 6 by the addition of sodium hydroxide. Chief emphasis was given to the excetion of uxinary urobilinogen. This test, employing Watson's method, was chosen. Three tests for liver function were investigated: the cephalin flocculation test of Hanger, the colloidal gold test of Gray, and the thymol tarbidity test of MacLagen. Most complete studies were made of the firgt of these tests in dogs, rabbits, and rats, as well as in human subjects. Occ.ssional analyses of serum billrubin were also made. Venous blood was used in the dog studies. Cardiac blood was drawn from rabbits and rats. In the present work only urine studies were done routinely in the rabbits; both urinary and fecal exeretion of porphyrin was followed in many of the irradiated doge. It is apparent from the data presented that liver function, as reflected in urinary excretion of urobilinogen, was not profoundly affected, except terminally, in animals exposed to lethal amounts of radiation. Furthermore, any changes in serum proteins produced by radiation were not associated with significant changes in the serum tests of liver function uged. These results are in agreement with 1490

the general consensug of oplnion in the literature. (ADD)

Schwartz, Samuel. STUDIES OF PORPHYRIN METABOLISM. IV. THE IEFTCT OF IRRADIATION ON COPROPORPRYRIN EXCRETION. Univ. of Chicago Metalurgcal Lab. Nov. 25, 1946. Decl. Aug. 8, 1948. 28p. (ADNO-1221; CH-3\%10)

The effect of Irradiation exposure on coproporphysis excretion has been studied in 30 dogs. A diminished excrethon of this porphyrin in urine and feces was lound to follow the administration of lethal or nearly lethal doses of total body $\mathrm{x}-\mathrm{ray}$, strontium ${ }^{82}$, and plutonium. In most instances, a sharp increase in porphyrin excretion occurred during the terminal period. Preliminary studies showed no Blgniflcant change in the ratio of the two coproporphyrin isomers (I and III) following irradiation. (ADD)

\section{0}

Setala, KaI. PRELIMINARY OBSERVATIONS ON THE एFIFCTE OF IRRADATION UPON THE CHYLOMLCRONS IN EUMAN BLOOD. Radiology 50, 803-9(1948).

An attempt was made to determine the effects of roentgern or radtum rays on the mobile visible lipids in human blood following administration of a fatty meal and before the lipids were denosited in the tissues. As a method of isvestigation, ingtead of quantitative chemical determinaHons, which are subject to considerable error, chylomicron counts were obtained at hall-hour intervals. Observations were made on 32 individuals, and every precantion was talsen to avoid the posslbility of error. It was found that Iradiation regularly produced changes in the amount and roetabolsm of the wisible blood lipids. Further it was lound that, to produce changes, the inradiation must be cone whin a riven thme interval atter the ingestion of the iatty lood. It appeared, also, that irsadiation of certain regions of the body, as the abdomen, the pelvis and the bifurcation of the common carotid was followed by a pagteularly marled effect. Whin the histamine theory in mind, the effect of intramucular injections of histamine acid phosphate was alfo Investigated. Such Injections, छuitably timed In relation to the fatty meal, effected changes in the ind metabolism comparable to bhose following irradiation. In persons with symptoms of radiation sheluess, the changes in the blood lipids were particularly severe. It is assumed that the decrease and/or disappearance of the mobile visible lipids observed in these studies in an eflect of hemoconcentration due to increased permeability of the capillaries caused by irradition or histamine adminigh rafion. (NSA)

1501

Setälä, $\mathbb{R}$. and P. Exmala. ON IRRADIATION SICTRMSS. Ann. Chir. Gynaecol. Fenniae 37, Sืuppl. 1, 1-59(1848).

The relationship between irradiation sckness and moblle visible blood lipids is discassed in this report based on 65 cases. The cases uniformly demonstrated a marked decrease in the chylomicron count duxing radiation mickness, which was not present in irradiated cases not developing the sickness; the degree of sickness was proportional to the deggec of depression in the count. Irradiation in excess of $350 \mathrm{r}$ over the abdomen, pelvis, and the bifurcation of the common carotid produced radiation sickness with changes in chylomicron count, while corsesponding irradation over extremities produced no sickness or changes in count. The administration of histamine acid phosphate caused changes in chylomicron counts aimilar to those found in radiation sickness. The author suggest that thels work corroborates that of others who found that irradiation liberates a "histamine-llke substance" (not in all effects similar to histamine, and unresponsive to anthistaninics): paraganglonic elements may be affected either directly by the irradiation or by a toxin resulting from irradition. (NSA)

1502

Smith, D. E., T. B. Tyree, M. M. Patt, and 焉. M. Jackson. THE EFIECT OF WHOLE-BODY X-MRADLITON ON THE METABOLISM OF TME RAT. Proc. SOC. EXpLt. Biol. Med. 78, 774-77(1951) (ABCU-1253; UAC-367)

Determinations have been made of the inluence of whole-body $x$ irradiation upon the rate of weight loss of the gtarving rat and upos the level of bagal $\mathrm{O}_{2}$ consunption of both the starved and the fed rat. Since irradiation was without effect upon these guantules it was concuded that single, whole-body exposures to irradiation do not bring about increased metabolim. The discrepaneies between these and the previous data which gupported the hypothests of a radiation-induced increase in metabollsm are digcusged. 11 references. (NSA) 1503

Smith, Palconer and Wulie W. Smith. EXERCHE FECTS ON TOLERANCE TO RADIATON. Am. I. Physiol. 165, 662 -6(1951).

As a whole, the effect of moderate ererche for 1 hr consecutively a day, or for $12 \mathrm{hr}$ a day th mually gpaced periods of $3 \mathrm{hr}$, was to decrease slightly, if at all, the tolerance of mice to whole-body irradiaton. After a rathe ation dose which was $100 \%$ lethal, to tolerate 3 conseculve days of exercise for $8 \mathrm{hr} / \mathrm{day}$. Those अurviving the exeretse pertod whowed no dherence in survival time from thetr l rradiated controls. Inenobarbital sedation after Irradiation ralled to Iserease the radiation tolerance of mice. It is concluded that sodintion lethality does not parallel the rate of $\mathrm{a}_{\mathrm{a}}$ uthumation umder the conditions of these ermerimente. (INis) 1504

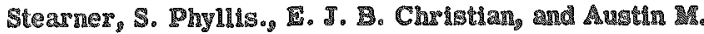
BEues. EYIECTS OF X-IRRADIATION ON RERAL FUNCTION IN NEWLY WATCHID CHICKS. PNOE. BOS. Exptl. BLol. Med. 76, 676-9(1051).

Young chicks were exposed to $1000 \mathrm{~g}$.

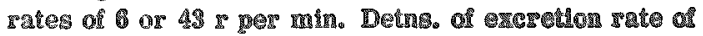
urate and phenol sed and of bleod urats lowels tndiested that death in the initial period (within -24 howre) followlug 
high rate exposures was preceded by renal failure and a rapidly developing azotemia. Individuals that survived 24 hours or more after exposure, whatever the dose rate, continued to excrete at the normal rate. Phenol red getention invariably accompanied failure of urate excretory function. (BA)

1505

Thompson, H. $\mathrm{E}$. and L. T. Steadman. TRE ERECTS OF WHOLE BODY X-IRRADIATION ON THE BLOOD GLUCOSE LEVELS IN RABBITS, $p .13-33$ Of QUARTERLY TECHNICAL REPORT, OCTOBER 1, 1950 THRU DECEMBER 31, 1950. University of Rochester. Feb. 1, 1951. 20p. (UR-152(p.13-33))

Rabbits were ir radiated under light anesthesia with 200 kvp $x$ radiation, half-value layer of $1.6-\mathrm{mm}$ Cu. With doses of $500 \mathrm{z}^{*}$ and greater, temporary elevations în plasma and red blood cell glucose were observed with a maximum increase at 2 to $4 \mathrm{hr}$ from the start of the irradiation and with a return to normal within $24 \mathrm{hr}$. The rise in the glucose level increased with the dose and for $1000 \mathrm{r}$ the maximum elevation in the plasma was about $90 \%$ and in the red cells about $80 \%$. Animals which were irradiated with a second dose of 500 r between 7 and 33 weeks after the irst irradiation showed glucose increase comparable to those receiving a single dose of $1000 \mathrm{r}$. (NSA) 1506

Trudova, R. G. MEASUREMPRT OF ISOELECTRIC POINT OF CHLL COLLOLS IN PLANTS UNDRR THE ACTION OF X-RAYS. Doklady Alkad. Nauk S.S.S.R. 72, 197-9(1950).

Irradiation of oat rootlet tips with $250 \mathrm{x}$. (in $1-\mathrm{min}$. exposure) gave only a temporary growth retardation: at $500 \mathrm{~s}$. the effect was more definite, and at 13000 r. mitosis was essentially stopped. A shift of isoelcetric point of colloids of the plasma and nucleus occurs simultaneously but returns to normal within 24 hrs. even at $13000 \mathrm{r}$. dosage; this maximum shift is to 2.8-3.4 pH for plasma (2.4-2.6 normal) and $3.4-4.2 \mathrm{pH}$ for nucleus (2.8-3.0 normal) taken 15 minutes after $x$-ray exposure. The individual spread of values is greater after irradiation than in controls. (CA)

1507

Uchimura, T. THE FIFET OF ULTRAVIOLFT AND ROENTGEN RAYS ON THE REDOX-POTENTEAL OF THE FROG MUSCLIE IN VIVO. J. Blochem. 25, 207-17 (1937).

The redor-potential of normal frog muscle is nearly $1-230 \mathrm{mv}$ F $\mathrm{h}$. Irradiation with ultraviolet or roentgen gays causes the increase of the reduction-potential, which is highest at the dosage of 10 min. exposure with uv-rays and at $135 \mathrm{r}$ with Roentgen-rays. At larger doses this increase was rather inhibited. With wy-rays the rise of the reduction-potential took place soon after the irradiation, but with Röntgen-rays it happened after certain intervals. This observed change in potential took place only when each component of the reducing system is muscle (dehydrogenase $4 \mathrm{H}_{2}$-donator) are all present. The recovery of the potential to the inilial value took place earlier in the case of uv-rays than in the case of Röntgen rays. There exists an optimal dosage of irradiation for the increase of the reduced glutathione. By applying excess it was rather inhibited. If is proposed that the biological effect of light might be partially sought in the activation of the reducing agents in the tissue. (Author's Summary)

1508

Venters, K. D. and Elizabeth E. Painter. SENSITIVITY OF X-RAYED ANIMALS TO INIPUSION OY HISTAMINE: AOENOSINE, AND TRYPSIN. Federation Proc. 9, 129-30(1950).

Control and $\mathrm{x}$-rayed rabbits and dogs given an $\mathrm{LD}-50$ dosage have been infused in the manner described previously (Fed. Proc. $7: 90,1948$ ) and "threshold values" have been established for exogenous histamine, adenosine, and trypsin. It appears that sensitivity of rabbits to adenosine is not increased after radiation, but sensitivily to histamine increases marlsedly. Maximal changes in sensitivity are directly correlated with other physiological findings occurrimg at the same time after radiation and must be a function of damage us tissue. Marked susceptibility of $x-F$ rayed rabbits to trypsin has made it impossible to record changes with this substance. It is apparent from the table below that dogs show their maximal increase in sensitivity to all 3 , substances (appromimately $50 \%$ change) at $2-2 \frac{1}{2}$ hours after $x$-radiation. These findings are correlated exactly in time with the main clinical sign of vomiting in the dog. Our studies suggest that more than one breakdown product may be involved in the early biochemical dism turbance to account for the symptoms of radiation sickness.

Threnold Values for Maintaining $5-8 \mathrm{~mm} \mathrm{Hg}$ Drop in Blood Pressure

\section{Control Rabbilis}

Histamine 6.7 (14)

Adenosine 111.2 (12)

Trypsils $107.0(10)$

Control Dogs

Histamine $1.2(17)$

Adenosine $120.4(21)$

Trypsin $113.2(16)$

1508

Wattenberg, L. RADIATIONS-INDUCFD CHANGTS IN ULTRAVIOLET ABSORPTION SPECTRA OF URINE: 1 . QUANTITATIVE SPECTROPHOTOMTTRIC STUDIES. Argonme National Lab. Jan. 14, 194\%. 30p. (MDDC-701; CH-3661)

Methods are deseribed for the quantitative estimation of kynurenic acid in dog urine. These are based on analysis of the ultraviolet absorption spectrum either of unpuritied diluted urine or of purified urine axtracts. Urine kynurenic acid excretion has been studied in 13 mongrel dogs administered lethal anounts of $\mathrm{x}$-ray in either single or multiple doses. Twelve of the animals showed an increase in urine kynurenie acid concentration at wome time shortly before death. This increase is best seen in the urine ultraviolet absorption spectrum as a plateau or actual maximum at about 245 mu Because of diminished terminal per dism urine volume in several dogs, the per diem excretion of kynurenic acid rose significantly in only 10 animals, with questionable increases in two of the olhers. Two dogs injected with plutonium were also studied. One, which died after 90 days, showed a terminal increase in urine kynurenic acid excretion. The other, which died 234 days after receiving the plutonitum excreted very lithle kynurenic acid throughout its course. This dog showed the most evidence of liver damage of any of the dogs studied. Data are insufficient to interpret the findings adequately. Some of the factors that may be involved in kymurenic acid excretion are discussed. (ADD) 1510

Wattenberg, L. and Samuel Schwartz. RADIATION-INDUCED CHANGES IN ULTRAVIOLET ABSORPTION ON 
SPRCTRA OF URINE: I. GENERAL EFFECTS. AIFOnnE National Lab. July 25, 1046. 21p. (MDDC-700; CHW 3541)

Several hundred ultraviolet absorption spectra (Beckman spectrophotometer) have been prepared from urine of control and irradiated dogs receiving either external or internal radiation. Unless death occurs too soon (i.e., within less than two weeks) there is a consistent increase in absorption in the regions of 245,310 , and/or $270 \mathrm{~m} \mu$ in acid solution. The 235 and 310 mu increases are shown to be due to kynurenic acid, a metabolic derivative of tryptophane. The 270 mu increase is shown to be due to uric acid. The effect on the spectra of variations in pH are described. (ADD)

1511

Weber, R. P. and F. R. Steggerda. HISTAMINE IN RAT PLASMA: CORRELATION WITH BLOOD PRESSURE CMANGES FOLLOWING X-IRRADIATON. PrOC. SOC. Exptl. Biol. Med. 70, 261-3(1949).

Rats, after s-Irradiation wath mid-lethal dosage (600 5 ) show assayable histamine in concentrations of 1 to $2 \mathrm{y}$ per 2 ce of blood plasma at two hour and five day intervals following the irradiation. Rats irradiated similarly to those uged in the histamine assay experiments show a fall in blood pressure that correlates' with the appearance of hisfamine in blood plasma and strongly suggests that histamine is responsible for the lowered blood pres aure. Rats, fron four to six days after a mid-lethal dosage of incadation, show a lows in agpetite and weight, and a critical fall in blood pressure from which only 50 \% will recover. The rest died within 9 or 10 days after the irradiation was given. (NSA)

1512

Whilden, R. M., Enzmann, $\mathrm{E} . V .$, and C. D. Raskins. THE INFLUENCE OF ROENTGEN RAYS UPON THE NITROGEN FEATION BI AZFTOBACTER. J. Cen. Physiol. 24, 789-95(1041).

N fixation decreases approximately lnearly with increasing doses of $\mathrm{x}$-rays. Respiration is afected indirectly by some inhibition of cell multiplication. Small doses of \$-ray often produce a slght, transient increase in rate of o uptake. (CA)

1513

Woodward, G. R. GLUTATHIONE AND ASCORBIC ACHD IN TYIE TISSUES OF NORMAL AND TUMOR-BE ARING

ALBINO RATS. Biochem. J.29, 2405-12(1935).

Walker No. 250 carcinoma and Philadelpha No. 1 garcoma were found to contain glutathione (I) In amounty comparable to those of other tissues and ascorbic acld-like material (II) In amounts higher than In any tissue except the adrenal. I and II were found only in the growlng part of the Iumors. The ratio of 1 to Re In sarcoma was under 1.4 and in caremoma over 1.6. Corresponding tirsues from normal and tumor-bearing rats showed no differences in the amounts of I and II. Long-continued Injections of ascorbic acld, mannose, glucose or oxldation-reduction dyes carsed no changes in the amounts of 1 or 1 in tumors and the tumor growth was unaffected. Treatment of tumor's with x-rays decreased the values of I, If the z-rays retarded the tumor growh. The value of II was decreased only in the sarcoma by $\mathrm{x}$-ray treatment, $\mathrm{z}$-ray resistant ftmos showed no decrease in the amounts of 1 and II. (CA)

\section{4}

Zahl, Paul A. and Hary G. Albaum. ADEROSIN TRIPLOSPIATE LEVELS IN MOUSE BLOOD AETHE WROLE-BODY IRRADATITON AND DURTVG TUMOR

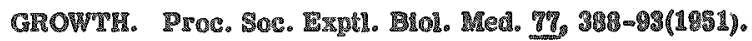

No changes were observed in the blood levels of total nucleotide or of adenylic acid, adenosine diphosphate, or adenosine iriphogphate during the wasting pertod following whole-body s irradiation of mice. Massive injected doses of adenosine triphosphate (ATR) falled to induce overt symptoms of ghock in mice. In mice with implanted turnors there was found a continuous decrease in blood ATP unth about the loth postimplantalion day, followed by a ritee of ATP. The deerease paralleled the increase in turnor weight. No significant change were observed in total mucleotide values for blood during tumor growth, nor wa the adenylic acid or the adenoane friphosphate picture different Irom that for normal mice. (NSA)

Studies on Nucleoprotelns and Nucleic Achds

1515

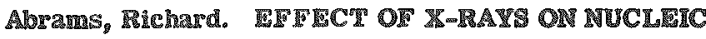

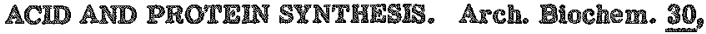
90-21951).

Rats and rabbits were irradiated with 500 re and 800 r. respectively. They were injected intraperitoneally immediately, and at antervals up to 24 hrs wilh isotopically labeled glycine. Purines were fractionated from the in testines of the animals and the adenine and guanine contents measured isotopically. The rates of syminesig of desoxyribonucleic acid and ribonucleic acid was markediy seduced by 5 -rays, but the synthesis of protein was relatively unafiected. (CA) 1516

Ahlstron, Lucie H. Hans vo Ruler, and Ceorg vo Hevesy. ACTION OR X-MAYS ON NUCLIC ACD METAROLISI IN RAT ORGANS. Arliv. Kemi. Mineral. Ceol. A 10,

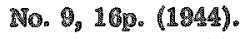

Nucleir acid (I) metabolism in liver, gpleen and intestinal mucosa of adult rats was inhibited $1 / 3$ to $1 / 2$ during the $2 \mathrm{hr}$ immediately after irradiation of the animals with 1,500-3,000 r. This result was similar to that obtained in previous studies on Jensen sarcoma. In the liver and spleen of rats $3 / 2$ days old the percentage of newly formed I mols, was 2 and 10 , resp., which was 20 and 4 times as great as in the corresponding organs of adult mnimals. The daily wh. increase of the liver of rats $31 / 2-4 / 2$ days old was 10.20\%, and studies with radioactive $P$ as an indicator showed that the content of new I nols. was twice as great as that which would parallel the increase in wh. The formation of each 3 new I mols. was accordingly linked with the disappearance of 1 old 1 mol.; this reault was also similar to that obserwed with Jensen sareoxa. The percentage decrease of 1 metabolism in the organs of rats $3-4$ days old under the influence of $x$-rays was about the game as that in the organs of adult rats. If the irradiation dose mentioned above was spread over 2 hx, the effect was more pronounced: during this period, I metabolism ln the liver of rapidly growing rats fell from $2 \%$ to $0.2 \%$, that in the spleen from $10 \%$ to $2 \%$. A considerable portion (\% with liver) of the inhibitory action of z-rays therelore appears to disappear during the first 2 hr after radiation. Dureing IrIadiation of rats $3-4$ days old with 15 s pers min. addul. 15 装 formation of I and Iiver growth practically ceased, (CA)

Ahistrom, Luele H., Goorg v. Hevesy, and R. AYIEMPTE TO IND PRODUCTS BLOCRRA NUCLEIC ACT TORMATION IN TRP嫼 CIRCULATION OI AN MRADI

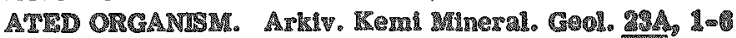
(1047).

Several rabbits four weels old were 1rradiated wh

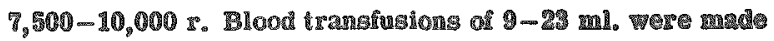


from these animals to paired litter - mates which had not been irradiated. Radioactive phosphate (20 microcuries) was administered subcutaneously to the nonirradiated rabbits and after two hours the specific activity of the desoxyribosenucleic acid phosphorus extended from the kidneys, liver, and intestinal mucosa was compared with the free phosphate extended from the corresponding organisms and from the plasma. Control experiments were carried out on rabbits transfused with blood from nonir radiated litter-mate rabbits. As the result of the transfused, irradiated blood the formation of labeled desoxyribose nucleic acid is definitely slower in the kidney, probably slower in the liver, but unaffected in the intestinal mucosa. (BA)

1318

BuRE: G. C. IEFIFCT OF I-AND GAMMA-RAYS ON AQUPOUS SOLUTLONS OF SODIUM THYMONUCLISATE. Can. J. Research 27B, 972-87(1040).

The changes in viscosity of highly polymerized $\mathrm{Na}$ thymonucleate solutions upon irradiation with $\mathrm{x}$ - and $\gamma$-rays was investigated. It was found impossible to correlate absolute viscosity values with particle size and shape. The relations of change produced to total dosage, to the presence of protective agents, to concentration of bolution and to dosage rate indicat that the radiations act through the mediation of activated water molecules. Direct Ionization, on the other hand, in the nucleate molecules has 1510

Rutler, 3. A. V. and B. E. CONWay. ACTION OF IONIZING RADIATIONS AND OF RADIOMIMIIIC SUBSTANCES ON DESONYRIBONUCL

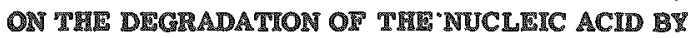
J-RAYS. J. Chem. Soc. 1950, J11-18(1950).

Ipradiation of $0.1 \%$ solutions of thymus nucleic acid at the rate of $206 \mathrm{r} / \mathrm{min}$ gave a fairly large and a complete lose in viscosity after 7,000 and $40,000 \mathrm{r}$, respectively. The logs of viscoatly ceaged at the end of ixradiation of 0 -1ree solutions but continued with 0 -saturated solutions. The after effect was ascribed to a product of molecular $O_{2}$, 15: which, however, was not $\mathrm{H}_{2} \mathrm{O}_{2}$. (CA)

Butler, J. A. Vo, L. A. cilbert, and I. A. Tmith. RADIO WMMETIC ACTION OF \$ULIUR AND NITROGRN MUSTAEOS ON DESOXIRHBONUCLIC ACD. Nature 1B5, 118-8(1050).

Methyl bis-B-chloroethylamine and B-dichloroethylwifide, but not chlorodiethyldimethylamine destroy the atructural viscosity of thymus desoxyribonucleic acid. The original gtructural viscosity, relative viscosity used with 2acreaising ratio of alow, is gradually converted to a viscostly similar to water, independent of pressure, and showling no atreaming birefringence. X-rav irradiation of 22,000 also prodinces an effect in the structural vigcosity of rucleic acid which is quantitatively similar to that of $2 \mathrm{mg}$ of $\mathbb{N}$ mustards. This ratio of effectiveness is wimilar to that observed in rats and mice for total body $\mathrm{x}$-ray irrad1521

Conwey, B. T., L. GIlbert, and I. A. V. Busler. ACTION OF IONTRMC RADATIONS AND ON RADIOMMMETIC

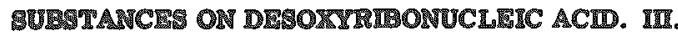

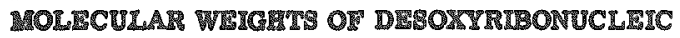
ACID DRGRADED DI Y Y-RAYS AND BY TREATMENT

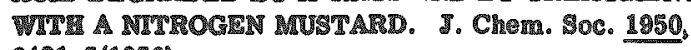
3A21-5(1050).

Trisadiation with $40,000 \mathrm{r}$ (0 prosent) cassed a decrease In the molecular welght of thymus nuclelc acid from approxhmately $1.5 \times 10^{6}$ to $5.3 \times 10^{5}$. Reaction of $10 \mathrm{mg}$
( $\left.\mathrm{ClCH}_{2} \mathrm{CH}_{2}\right)_{2} \mathrm{NCH}_{3}$ for 5 days decreased the molecular weight to $4.5 \times 10^{6}$. The dograded substances showed a considerable degree of polydispersity. (CA) 1522

de Nicola, Marina. EFTECT OF X-RAYS ON THE CELL DURING ACTIV METABOLISM OF NUCLEIC ACIOS. Publ. staz. zool. Napoli 22, 283-93(1950).

The effect of $\mathrm{x}$ rays on nucleic acid metabolism in germinal and secretory cells was studied on the testis of Asellus aquaticus during spermatogenesis. Doses of 250 to $5000 \mathrm{r}$ with intensity $1330 \mathrm{r} / \mathrm{min}$ at a distance of $5 \mathrm{~cm}$ from the ventral side without filters were used. The fundamental effect of $\mathrm{X}$ rays in various doses is always the same: all the desoxyribonucleic acid (I) gradually disappears and is transformed into ribonucleir acid (II) which is expelled from the cell unthl this, completely deprived of nucleic acids, dies. The gonad appears poorer and poorer in germinal and to lower degree in theca cells; after 2 months' treatment the gonad still exists but with a greatly reduced number of cells. It is believed that the cells whose function is essentially to metabolize nucleic acids, for their own and external use, are particularly radiosensıtive. (EM)

1523

de Nicola, Marina. ACTION OF X RAYS ON TRE MFTABOLISM OF NUCLIIC ACIDS IN PROLIFIRATIVE AND SECRETORY CELLS. Experientia 6, 432-3(1950).

The action of $x$ rays on the metabolism of nucleir acids in proliferative and secretory nonproliferative cells has been investigated. X rays cause a stoppage of desoxyribonucleic acid synthesis and a conversion of desoxy ribonucleic acid into ribonucleic acid. All germ cells, in cluding spermatozoa, show this effect, and the same is true for the follicular secretory cells. The germ cells ar blocked at different stages of mitosis or meiosis. On the contraxy, the follicular cells continue their characteristic Becretury cycle, until they are entirely deprived of both nucleic acids and eventually die. The conclusion is draws that the action of $x$ rays is essentially specific for the mucleic acids as such, and not for some intermediate substance of their metabolism, and that stickiness, metaphasic block, and chromosome abnormalicies are probably a secondary effect of this action. (NSA)

1524

HIy, 3. O. and M. R. ROSS. NUCLRTC ACL CONTENT WV INTESTINES OF RATS AFTER X-RADIATION.

Cancer Research 285-284(1048).

The effects of total-body $\mathrm{x}$-radiation $(600 \mathrm{r})$ of rats on the crypts of Lieberkinhn were studied by ultravioletlight-absorption and other methods. It was found that 8-radiation caused a decrease in the amount of ultraviolet light-absorbing material in the crypts of Lieberkiuh of rats at various levels along the intestinal tract; the decrease in the amount was found at various periods of time ranging from 4 hours to 17 days. Involved in the decreased absorption of ultraviolet light were desosyribonucleic acid, ribomucleic acid and the structural framework of the tissue, all of which were found to be reduced. The amount of inor ganic ash also was found to be reduced. (NSA)

1525

Ely, 1.0 and M. R. Ross. NUCLEIC ACID CONTRNT IN INTESTINES OF RATS AITER NEUTRON RADIATON. Cancer Research 8, 607-12(1948).

It was reported in a previous paper (Cancer Research, 2 , 285,1948 ) that whole-body $\mathrm{x}$-radiation of rats resulted in decreased amounts of desoxyribonucleic acid, ribonucleic acid, structural proteins, and non-volatile ash in the cells of the crypts of Lieberkihn in the integtines. Studies on the effects of whole-body neutron irradiation of rats on the 
crypts of Lieberkihn have been made using ultraviolet absorption and chemical staining methods. It was found that neutron radiation caused a decreased ultraviolet light absorption in the crypts of Lieberkiihn in the duodenum of rats; the decrease was found at various periods of time ranging from 4 to 18 hours after irradiation. The effects produced increased with the dosage and the decreased ult raviolet absorption has been linked with ribonucleic acid and desoxyribonucleic acid. It was also found that the amount of inortanic ash was reduced. These effects of neutron radiation are similar to those previously found after whole-body exposure to $\mathrm{x}$-rays. (NSA)

\section{6}

Errera, M. IN VITRO AND IN SITU ACTION OF IONIZING RADIATIONS ON NUCLEO-PROTEINS OF THE CELL NUCL FUS. Cold Spring Harbor Symposia Quant. Biol. 12, $80(1947)$.

Radiation effets on tho nueleoprotein complex of the cell nucleus have been studied. Experiments in vitro showed an indurect action of the radiation while experiments in stu evidenced a direct effect of irradiation. Nu cleoprotein complexes of the nucleus were shown to be strongly affected when irradated in situ. (CA)

1527

Feinstein, Robert N. EFFCI OF IRRADLATION AND OF VARIOUS REAGENTS ON VISCOSTTY OF NUCLTOPROTEIN SOLUTTONS. Proc. Soc. Expt1. Biol. Med. 76, 646-9 (1951).

Cysteine, glutathione, cyanide, and thiocyanate greatly increase the viscosity of alkaline solutions of nueleoprotein (thymus chromosomes), while urea, uracil, thiourea, thiouracil, cystine, and BAL do not do so. Ascorbic acid increases the viscosity only after prolonged incubation. $\mathrm{H}_{2} \mathrm{O}_{2}$ is without effect on the viscosity. If cysteine is added to the alkaline mucleoprotein solution before $x$ ixradiation, the expected reduction in viscosity is largely prevented. If the cysteine is added after $\mathrm{x}$ ir radiation, it is much less offective in this respect. The suggestion is made that these phenomena may be relevant to the question of the mechanism of cysteine and some other agents in increasing registance of organisms to x-ray lethaliry. (NSA)

1528

Harrington, helen and Paul S. Lavik. THE RFFECT OF IONIZINC RADLATON ON THE DESOKYRIBONUCLEC ACD CONTENT OF RAT THYMUS NUCLEI. Westerm Reserve Untversity, School of Medicine. Sept. 27, 1951. 161. (NYO-1627)

The DNA phosphorus content per nucleus has been determined in thymus cells of normal and irradiated rats, and it has been found that iradiation causes no significant change in this value. Since there is a decrease in the DNA phosphorus concentration of thymus issue following irradiation, several mechanisms of radiation action which could cause a decrease in DNA phosphorus concentration while allowing the DNA phosphorus per nucleus to remain unchanged are discussed. It has been suggested that an inhibition of DNA synthesis, resulting in a decreased new cell production, followed by the elimination of old lymphocytes from the thymus could account for both experimental findings. (NSA)

\section{8}

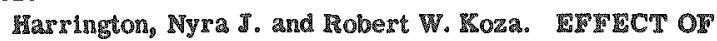
X-RADIATION ON THE DESOXYRIBONUCLEIC ACHD AND ON TME SIEL OI GRASSHOPRIR IMBRYONIC NUCLEI. Biol. Bull. 101, 138-50(1951).

Cytological and cytochemical studies were made on embryonic nuclei of the grasshopper, Chortophaga viridifasciata, atter $x$-ray doses of $4000,10,000$ and $12,500 \mathrm{r}$. The changes induced were photometrically measured by using (1) the Feulgen reaction to determine relative changes in the DNA desoxypentose, and (2) the methyl green stain to indicate the degree of polymerization of the nucleic acid. $X$-radiation was found to cause swelling of the nuclei. When correction was made for this, the Feulgen-stained nuclei showed no significant loss of DNA after irradiation, but the nuclei stained with methyl green disclosed a highly significant loss of stainability. This is interpreted to indicate that $x$ rays do not destroy the DNA but induce depolymerization of the nucleic acid. This study has shown that it is not safe to make quantitative estimates of DNA (stained by Feulgen or methyl green) from microscopic observations and photographs. The visually apparent loss of Feulgenstainability after ir radiation is probably not due to a decrease in the DNA desoxypentose but rather to the increased dispersion of the DNA in nuclei that have undergone $\mathrm{x}-\mathrm{ray}$-induced enlaggement. (NSA)

1530

Hevesy, Georg v. ON THE EFPECT OF ROENTGEN RAYS. ON CELLULAR DIVISION. Revs. Mod. Phys. 17, 102-11 (1945).

While mitotic counts give important data on the effect of Röntgen rays on growing tissue, this method fails in the investigation of behavior of full-grown tissue. The effect of ionizing irradiation on all types of tissue can be studied by detg. the amt. of newly formed desoxyribonucleic acid before and after irradiation. The newly formed nucleic acd is detd. by using radioactive $\mathbb{P}$ as an indicator. Therapeutic and larger doses of Röntgen rays inhibit the formation of desoxyriboncleic acid in the growing and full-grown tissues to about the same extent. A large percentage (about $\%$ of the inhibiting action disappears within $2 \mathrm{hrs}$, after irradiation. The difference between growing and grown tissues is interpreted by assuming that a far longer time elanses for an av. full-grown tissue cell to reach the mitotic stage and thus to have a greater chance to regenerate from the chamaging influence of the irradiation. The problem of direct and indirect action of Röntgen rays on cell division is discussed. (CA)

1531

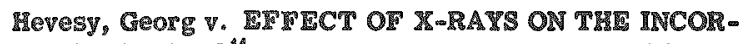
PORAYION OF C $C^{14}$ INTO DESOXYIRONUCUEIC ACID. Nature 163, 869-70(1949).

To determine whether the rate of formation of desoxyribonucleic acid is reduced by the effect of lonizing radiations, $\mathrm{C}^{14}$ was incorporated into the purines of desoxyribonucleic acid in the organs of rapidly growing rats after X-radiations. About $5 \mathrm{me}$ of $\mathrm{C}^{11}$ incorporated into the carbonoxyl group of sodiux acetate were injected gubcatancously in each of 91 one - to three-week old rats. The rats were silled after a lapse of six hours, and the desory ribonucleotides were separated from the proteins by extraction with trichloroacetic acid; the puxines obtaned were precipitated and their radioactivity ascertained. Hal of the rats had been previously radiated whth a dose of $950 \mathrm{x}$. The amount of labelled purines obtaned from the irradiated rats was about half that obtained Irom the controls. The rate of incorporation of $\mathrm{C}^{\mathrm{A}}$ into the protelng was not reduced by irradiation; so it is concluded that the rate of formation of desoxyribonuclese acd is reduced by the radiations. This rate reduction of one of the matn constituents of cell nuclei may help to explain the bloeksing effect of irradiation on cell division and the formation of anomalous mitotic products in the irradiated tiasue. (NSA) 1532

Holmes, Barbara E. TRE INITHITION OF RIEO- AND THYMO-NUCLETC ACD SYNTTESIS IN TUMOR TUSUE BY IRRADIATON WITH X-RAYS. Brit. 3. RadLOL. 20, $450-3(1947)$.

Using P-32 as a tracer, the uptake of this element in the thymonucleic acid fraction in rat tumor tissue (Jensen rat 
sarcoma) is markedly inhibited by $\mathrm{x}$-rays, while the ribonucleic acid fraction is inhibited to a smaller extent. Nembutal as a sedative inhibits the uptake of $\mathrm{P}-32$ in both these fractions and should not be used. There is confirmation of Hevesy's observation that irradiation of one tumor can effect the mucleic acid metabolism of another tunor in the same animal. (BA)

1533

Holmes, Barbara E. THE INDIRECT EFIFECT OF XRAYS ON TYHE SENTEESIS OF NUCLEIC ACD IN VIVO.

Brit. I. Radiol. 22, 487-91(1949).

The synthesis of thymo-and ribo-nucleic acid (in the Jensen at sarcoma) was studied in irpadiated tumors and in other tumors in an irradiated animal which were themselves not receiving irradiation. The directly irradiated iumor's received a dose of $2,000 \mathrm{r}$, which was suffictent 10 produce regression in many cases. Radioactive phosphorus, $\mathbb{P}^{32}$, was introduced intraperitoneally to measure the radiation effect on the nucleic acid synthesis as demonstrated by the inhibition of the uptake of $\mathbb{P}^{32}$. The details and results of the various experiments undertaken are presented. Thymonucleic acid synhesis was reduced to abut $25 \%$ of the normal in the screened tumor; the $x$ ibonucleic. acid renewal was affected similarly, but to a much smaller extent. In animals killed one hour after irradiation, the direct and indirect effects of the radiations were large. When $\mathbb{P}^{32}$ was injected about 2 hours after ir iradiation and the animal killed one hour later, the ribo-nucleic renewal was nearly normal in all tumors; the thymonucleic synthesis of the screened tumor was nearly normal, and in the irradiated tumor was about 60\% of normal. When injected at four hours after irradiation and killed one hour later, the thymonueleic synthesis was found to be proceeding at a normal rate. Counts indicate that the dose of s-radiation used produces a considerable amount of abnormal milosis, while no normal mitosis is taking place five howrs after irradiation. (NSA)

1534

Jungner, Ingmar. DHELECTRIC DETERMINATIONS OF MOLECULAR WEIGHT AND DIPOLE MOMENT OF SODUM THY MONUCLBATE, THE INTLUENCE OF XRAYS. Acta Physiol. Scand. Suppl. 20, 69(1950).

Studies of $\mathrm{x}$-ixradiated aqueous solutions of DNA show that $x$-rays mainly have a depolymerizing effect. Only after very large doses $(100,000 \mathrm{r}$ to 20.05 per cent solution) does the specific polarity all considerably whereas the molecular weight remains relatively constant at about 30,000. The effect of the $\mathrm{X}$-rays on stronger concentrations of DNA $(0.625$ per cent) is smaller but essentially similar.

No after-effect of the x-rays on the dielectric properties can be noted within $12-48$ hours of irradiation. (Author's Summary)

\section{5}

Kamen, M. D. APPLICATION OF RADIOACTIVIISOTOPES TO THE STUDY OF RADIATION EFTECTS IN CELLS. Radiology 49, 223-30(1947).

Metabolizing cells which are not actively synhesizing new protein do not transfer phosphate fron the "mucleoprotein" fraction. Syynthesis of new protein or enzyme is associated with transfer of phosphate from the "rucleoprotein" fraction. Agents which prevent enzyme formation and protein symthesis also stop flow of phosphate from the " cleoprotein" fraction. It would be premature to suggest that disturhances in nucleoprotein metabolism are the central factor in induction of the pathological manifesta tions observed to result from irradiation of cells. There does appear 10 be suficient evidence available to indicate that investigation of cellular responses to a wide variety of chemical agents may clarify many aspects of the radiation problem, particularly in conjunction with isotope studies. (GPR)

1538

Kelly, Lola S. and Hardin B. Jones. EFECTS OF IRRADLATION ON NUCUEIC ACID FORMATION. Proc. Soc. Exptl. Biol. Med. 74, 493-7(1950).

Mice of the A strain bearing bilateral transplants of mammary carcinoma were used to demonstrate an indirecl effect of irradiation on the desoxypentose nucleic acid turnover rate. In two experiments the animals were in radiated by means of radioyt $\mathrm{r}^{\mathrm{i} u m}\left(\mathrm{Y}^{30}\right)$ colloids which localize in the liver when injected intravenously and rem main at the site of injection when given intramuscularly. The nuclete acid turnover rate was measured by giving a trace dose of radioaclive sodium phosphate $\left(\mathbb{1}^{32}\right)$, sacrificnge the animals after two hours, and isolating the desosypentose nucleic acid from their lumors and livers. The spec if ic activily of the nueleic acid phosphorus was measured and constered to be an index of the turnover rate. It was found necessary to purify the nucleie acid much more carcully than is done in standard methods in order to obm tain a consiant specific acilvity upon successive reprecipitalions. The animals whose livers were irradiated with $4.25 \times 10^{5} \mathrm{erge}$ showed only $66 \%$ of the tumor nucleic acid specific tretivity of the control group. The animals whose muscles were in radiated with $4.25 \times 10^{5}$ ergs showed only $84 \%$ of the fumor nucleic acid speciric activity of the control group. Both uhese experiments definitely confirm the existence of an indirect effect of radiation on the desoxypentose nucleic acid turnover rate. One group of animals received 60 r $\left(1.4 \times 10^{5}\right.$ ergs) total body $\mathrm{z}-\mathrm{ir}$ radiation with $180 \mathrm{kw} x-$ rays and both the tumors and livers showed a significant depression in the turnover rate. (NSA) 1537

Lawrence, J. H. and H. B. Jones. THE BIOLOGICAL SFTCTS OR RADATION: PROGRESS REPORT FOR THE PERTOD JANUARY 1947 TO JUNE 1947. University of California. June 30, 1247. 6p. (NP-516)

"Wo growth inhibiting tactors, dibenzanthracene and a folic acid derivalive, were found not to inhibit desoxyribose mucleic acid metabolism as does ionizing radiations. It was possible to inradiate bone marrow cfectively using a aadioactive colloid which is highly concentrated in bone marrow. The depression of bone marrow nucleic acid metabolism did not seem to be as sensitive to irradiation, relative to its general radiosensitivity, as compared to liver. The enzymatic \$ystems responsible for the symhesis of thyroxine were extremely resistant to irradiation in contrast to mucleic acid metabolism. Technicues have been worked out to test the effects of irradiation on various or1538 ganic metabolic processes using radioactive carbon. (NSA)

Limperos, George. ROENTGEN IRLADIATION OF DHSOXYIRIBONUCLIC ACID. III. "THE RELATION OF DOST TO DRCREE OF DEPOLYMPREEATON IN VIVO AND THE INELUENCE OF OXYGEN TENSION. Cancer Research 11, 325-9(1951).

The degree of depolymerization of DNA extracted from rat thymus $24 \mathrm{hr}^{\mathrm{r}}$ after poentgen irradiation in vivo, as measured by the struchural viscosity and streaming birefringence of the solutions, was found to be directly proportional to $\mathbb{x}$-ray doses of 250 to 1000 . A reduced oxygen tension (10\%) was found to inhibit the depolynerization of DNA, whereas an increased oxygen tension (100\%) was found to be relatively ineffective in increasing the depoly. merization. A $10 \%$ oxygen tension was found lo be less erfective than thiourea in preventing the involution of rat thy mus tissue induced by roentgen irradiation. (NSA) 
1539

Limperos, G. and W. A. Mosher. ROENTGEN IRRADIATION OF DESOXYRIBOSENUCLEIC ACID. I. MECHANISM OF WHE ACTION OF IRRADLATION IN AQUEOUS SOLUTION. Am. J. Roentgenol. Radium Therapy 63, 681-90 (1950).

Desoxyribose nucleic acid (DNA) in water solution was irradiated with various doses of $\mathrm{x}$-rays from a $120 \mathrm{~m} k \mathrm{kv}$ source, at the rate of $1700 \mathrm{r} / \mathrm{mm}$; the DNA was placed in petri dishes (10 $\mathrm{ml}$ in each dish) and covered with a sheet of duralumm $0.001 \mathrm{in}$. thick before the inradiation. The DNA was found to be depolymeg ized in aqueous solution by the $x$-rays as well as by chemically generated free radicals; thiourea inhibited depolymarization by both means. These results were interpreted as evidence that the mechanism of action of 8 -rays; on the DNA in water solution is indirect, mediated through the production of free radicals from the solvent; additionat evidence of the indirect action wis obtained by the inability of $x$-rays to depolymerize the DNA in a dry state, in ethylene glycol, or in frozen aquevus solution. The presence of $\mathrm{NaCl}$ and desoxyribosenurelease was found neither to inhibit nor increase the depolymerization. There was evidence of hy1540 drosen bonding in the DNA. 20 references. (NSA)

Limperos, G. and W. A. Mosher. ROENTGEN IRRADIATION OF DESOXYRIBOSENUCLEIC ACD. II. PHYSICOCREMICAL PROPERTIES OT DESOKYRIBOSENUCLEIC ACID FROM IRRADIATED RATS. Am. J. Roentgenol. Radium Therapy 63, 691-700(1950).

Male albino rats were irradiated from a $120 \mathrm{kv}$ source in groups of 10 in a circular copper cange through a 0.75 mm Cu filter, with a total of 1000 to $2000 \mathrm{x}$, with or without prior injection with thiourea. The thymus glands were removed immediately or 24 hr later and extracted for DNA. The irradiation of the DNA in vivo produced swo effects: (1) when the animals were sacrificed immediately after irradiation, the DNA extracted from the thymus was low in N (due to a splitting of of purine bases): (2) when the animals were sacrificed $24 \mathrm{hr}$ after irradiation, the DNA was almost completely depolymerized. Thiourea injected before irradiation did not inhibit the splitting of of the purine bases from the DNA but did inhibit the depolymerization to some extent. Possible mechanisms lor these reactions are suggested. (NSA)

1541

Loofbourow, I. R., A. Oppenheim-Irrera, Dorothea G. Loffbourow, and Constance A. Yeats. RELEASY ON NUCL FOTDDS AND NUCLEOSDD LIVING CELLS. Biochem. J. (London) 41, 122-9(1947).

An investigation was made of the role of nucleotides and nucleosides in the increased proliferation mpomoting ac tivity of cell-free suspension fluids from cells slowly damaged by lethal radiation (I) as compared with similar fluids from undamaged cells (II). The nucleotide $-\mathrm{N}$ and nucleoside- and iree purine-N contents of I were respectively about 34 and 5 times those of $\mathrm{II}$. Nucleotides isolated from I, when combined with amino-acids and B-factors found in 1 , duplicated the prolieration-pronoting effect of 1 . The nucleotide and nucleoside fractions consisted principally of adenine and guanine derivatives. Spectroscopic evidence indicated a synthesis of materials absorbing at $2600 \AA$, within the living, injured cells during the cour ae of injury. (BA)

1542

Mitchell, J. \$. DISTURBANCE OF FUCLEIC ACTD METABOLISM PRODUCED BY TIIERAPEUTC DOSES OF - AND GAMMA RADLATIONS. PART I. METMODS OF INVESTIGATION. PART II. ACCUMUTATTON OI PENTOSE
NUCLEOTDES IN CYTOPLASM AFTER IRRADLATON. PART III. INHIBITION OF SYNTHESTS OF THYMONUC LEIC ACID BY RADLATION. Brit. J. Expt1. Pathol. 23, 285-313(1942).

The experimental methods are described. Ultraviolet phutomicrography was mainly used, bus measurements of ultraviolet absorption and fluorescence spectra, and histochemical tests, were also made. Unirradiated and irradiated fixed, unstained biopsy apecimens were photographed on the same microscopic field, and the degree of blackening at any point was measured photometrically. Maximum "optical staining" was obtained by use of the wave length 2,537 A. From these dat the effective concentration of the nuelero acids was deduced. (CR)

1543

Moses, M. T., R. DuBow, and A. H. Sparrow.THE Trow FECTS OF X-RAYS ON DESOXYPENTOSE NUCLIEC ACID IN STTV; QUANTITATVE CYTOCHEMCAI STUDIES ON TRILLIUM. Brookhaven National Lab. [nd] $1 \mathrm{p}$. (AECU-1162)

$X$ rays are known to reduce the viscosity of solutions of desoxypentose mucleic acid (DNA). This has been interpreted as a depolymerization of the DNA molecule. How ever, this effect has only been observed on extracted DNA, and it is not known that depolymerization actually occurs in the intact cell. Such evidence has been sought cytochemically, utilizing quantitatively the methyl green reaction for polymerized DNA and the Feulgen reaction for total DNA. Moreover, a modification of the latter reaction involving prolonged $1 \mathrm{~N} \mathrm{HCl}$ hydrolysis measures the rate at which acid degradation occurs and yields further information on the state of the molecule. Living buds of Trillium erectum undergoing meiosis were irradiated at 50,200 , and 20,000 $x$, the latter dose being suffeient to markedly reduce viscosity of DNA solutions. Methyl green and Feulgen re. actions were carried out on sections of the Carnoy-fixed anthers. Microphotometric measurements were made of the dye content of pollen mother cell nuclei. In no. case did irradiation produce the anticinated decrease in methyl green content. In one experiment, pachytene nuclei, irradiated at 20,000 r showed a $20 \%$ increase in methyl greer content over controls. The Feulgen reaction (optimal hy drolysis) was not affected, indicating no change in total DNA. However, the degradation of DNA (loss of thymic acid) through prolonged hydrolysis was markedly inhibited after heavy irradiation. If rom the methyl green results it camot properly be said that depolymerization of DNA, such as is caused by treatment with hot water or the enzyme DNase, has been induced by irradiation. Indeed, if would seem that the net result has been to incr case the stability of the DNA molecule rather than to decrease it. 1544

Paolett, Mario. EFITCT OF IX RAYS ON THE METABOLISM OF TIE NUCLIFIC ACIDS. Radioterapia radiobiol.

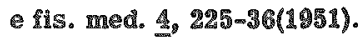

The effect of 100 to $200 \mathrm{I}$ of $\mathrm{x}$ radiation on nuclear atain m ing of epidermal cells of the grinea pig was investigated. A decrease in nucleic acids, particularly thymonucleic and 154 ribonucleic acids, is suggested. (NSA)

Rubin, B. A. THE RELATON OF NUCLEIC ACIO METABOLISM TO TUE GENETIC EFTECT OF RADIA TION IN BACTERIA. Brookhaven National Lab. [nd] 11. (ADCU 1436 )

The report is reproduced here in its entirety.

Levels of continuous radiation ( 8 rays or $\beta$ rays) which rapidly lall resting bacteria ( $\mathrm{E}$. coll $\mathrm{B} / \mathrm{x}$ ) permit the growth of metabolizing organisms. When resting cells are inoculated into a fresh medium the inception of growth 
(lag) is delayed as a direct function of the radiation dose rate; but when multiplication begins, the pate of inerease of cell mass during continuous xadiation is equal to that in unirradiated controls and the same final culture densities are reached. Average cell size increases with the number of generations that have occurred during irradiation, but this accounts for only a fraction of the cell mass, the logarithmic increase in viable count makes up the rest. An examination of the mutant population (resistant to streptomycin) in cultures which have grown from 0 (rest ing) to 14 generations during irradiation indicates that the mutation rate is affected by the radiation dose, but not by the number of genetic reduplications dur ing irradiation. Under the same conditions, changes in nucleic acid metabolism appear which are related to the extent of synthesis that has occurred during the irradiation.

1546

Scholes, George, Gabriel Stein, and Joseph Weiss. ACTION OF II-RAYS ON NUCLIIC ACIOS. Nature 164, 709-10(1949)

The chemical effect of large doses, e.g., 2 to $4 \times 10^{6} \mathrm{r}$ units on Na thymonucleate and Na ribonucleate were studied. The irradiation was conducted on $100 \mathrm{ml}$. solutions containing up to $2 \%$ of $\mathrm{Na}$ thymonucleate or $\mathrm{Na}$ ribonucleate. In every case deamination was caused by the s-rays yildding $\mathrm{NH}_{3}$. The production of $\mathrm{NH}_{3}$ is of the order of 0.1 millimole per $10^{8} \mathrm{~g}-\mathrm{x}$. In the presence of atmospheric oxygen the yield is greater. Van Slyke amino $\mathrm{N}$ determinations indieate a ring opening in the purine or pyrimidine bases. At the ame time there is an increase in the amount of titratable acid as determined by titration with phenolphthalein and alkali; the yield is of the order of $0.3 \mathrm{mg}$ equivalents of titratable acid grouns per dose of $4 \times 10^{8} 3$. For the separation and identification of any lower molecular Iragments formed, x-ray-treated thymonucleate or sibonucleate solutions were electrodialyzed at room temperature for $40-50 \mathrm{hrs}$. An increase in dialyzable phosphate and also in inorganic phosphate was observed in the positive pole section. From the negative pole of the dialyzer bases were obtained which could be precipiated by $\mathrm{AgNO}_{3}$ in acid solution and which were afterwards isolated as a mixture of the picrates. Preliminary asperiments have shown that one of these bases is ademine. A second $\mathrm{Ag}$ salt was obtained in alkaline golution which after hydrolysis gave a positive pentose test and also gave a small amount of the bases in the form of picrates. This evidence indicates the presence of a small amount of mucleosides. The action of OH radicals produced chemically by means of $\mathrm{H}_{4} \mathrm{O}_{2}$ and $\mathrm{Fe}^{\text {tht }}$ on thymonucleate and cibonucleate in aqueous solutions at room tenperature was studied. From this reaction mistures of urea and guandine were isolated from ribonucleate and urea was isolated from thymonucleate. (CA)

\section{7}

Scholes, George and Joseph Weiss, CMMMCAL ACTON

OF IONIZING RADLATONS ON NUCLEIC ACIOS IN

AQUEOUS SYSTRMS. Nature 166, 640-2(1050).

Samples of yeast ribonucleic acid and thymus desoxyribonucleic acid are irradiated with $\mathrm{x}-$ radiation ranging in intensity from 30,000 to $90,000 \mathrm{r}$ units. The yields of ammonia and inorganic phosphate produced during ixradiation are measured with the gample in air, in vacum, and $\mathrm{H}_{2}$. The yields of $\mathrm{NH}_{3}$ decrease from air to vacuum to $\mathrm{H}_{\mathrm{g}}$. It $1 \mathrm{~s}$ concluded that the attack on the bases in the nucleic acid is predoninanty by an oxidative radical, e.g., hydroxyl. Determination of the inorganic phosphate formed under gimilar conditions shows an increase in yield from air to vacuun to H. $_{2}$; this leads to the conclusion that the $\mathrm{T}$ atoms (produced along with OH from $\mathrm{H}_{2} \mathrm{O}$ by the radiation) may also play some part in the degradation of the nucleic acid. (CA)

1548

Skipper, Howard E. and Jack H. Mitchell, Jr. EFECT OF ROENTGEN-RA RADLATION ON THE BIOSYNTHESIS OF NUCLEIC ACIDS AND NUCLEIC ACID PURINES. Cancer 4, 363-6(1951).

Roentgen-ray radiation (950 $\mathrm{r}$ ) has been shown to imhibit the incorporation of radioactive carbon from bxcarbonate and formate into viscera nucleic acids and nucleic acid purines over a period of $6 \mathrm{hr}$. Under similar conditions, firation of cabon from these compounds into viscera homogenate appeared to be increased sumewhat following roentgen-ray radiation. Glutathione had no apparent effect on the radiation-induced mucleic acid inhibition. (NSA)

1549

Sparrow, Arnold H., Montrose J. Moses, and Robert Steele. SENSITIVITY OF CHROMOSOMES TO BREARAGE BY I-RAYS AND ITS RELATIONSHIP TO THE NUCLEIC ACID CICLI IN DIVIDING CELIS. Brookhaven National Lab. [nd] 1p. (ALCU

The document is in abstract form; it is reproduced below in its entirety.

The number of chromosome breaks produced after irradiation by $50 \mathrm{r}$ of $160 \mathrm{kv} \mathrm{K}$-rays at various stages of the miotic nuclear cycles have been studied in Trillium erectum. Sensitivity, as determined by counting the number of chromosome fragments, shows at least a 50 -fold change. It is highest at late prophase and first metaphase and lowest at early postmiotic interphase. Since production of acentric fragments leads to cellular dysfunction and death, it is reasonable to assume that our sensitivity curve is a measure of the ability of the cell to recover from radation damage.

The experimental modification of the sensituvity of dividing cells (e.g., tumor cells) to $x$-ray damage would be facilitated by an understanding of the biochenical systems immediately involved. In hopes of delineating some of the factors which determine eilher high or low sensitivity of the chromosomes to breakage and since metabolism of the nucleic acids has been shown to be easily upset by $\mathrm{X}$ - and ultraviolet irradiation, an attempt was made to correlate changes in RNA and DNA during rmiosis with sensilivity of the chromosomes to brealage. Microchemical analyses of the DNA and PNA content of Trillum pollen mother culls (DMC) at different miotic stages show no significant change in amount of either DNA or PNA per PMC throughout divisions. The significance of these results in terms of changes in nucleic acid concentration, synthesis, "target" size, and phystcal effects will be briefly discussed. 1550

Sparrow, A. H. and Florence M. Rosenfeld. X-RAY INDUCED DEPOLYMERIEATION OF THYMONUCLEOHISTONE AND SODIUM THYMONUCLEATE. Science $104,245-6(1946)$.

Solution of $0.2 \%$ Na thymonucleate in watex and $0.4 \%$ thymonucleohistone in $1 \mathrm{M} \mathrm{NaCl}$ were irradiated with T-rays. Streaming birefringence, present in both solutions before $\mathrm{x}$-raying, was considerably decreased by 120,000 r and the loss was much greater for the nucleate than for the histone. These changes probably represent a degradation or partial depolymerization of high mol. wt. purticles into shorter, more symmetrical chains or segments. These may (1) represent the initial step in the production of gene mutations and chromosomal breals, and (2) be instrumental in causing a breakdown of the normal nucleic acid metaboIIsm of the cell. (BA) 
1551

Taylor, Babette, Jesse P. Greenstein, and Alexander Hollaender. EFIFCTS OF X-RADIATION ON THYMUS NUCLEIC ACID. Seience 105, 263-4(1947).

The effects of $x$-radiation up to $56,000 \mathrm{r}$ on dialyzed solns. of sodium thymus nucleate (TNA) were studied. Two phenomena were observed: (1) a decrease in viscosity of TNA proportional to dosage of $x$-rays and independent of temperature, and (2) a progressive and presumably indirect effect manifest at higher temps. and not detected at $0^{\circ}$. Chem. and enzymatic studies indicated that whatever the way in which the particles had been damaged by $x$-radiation it did not involve a splitting of primary linkages $0 r^{\circ}$ a rearrangement of spatial configurations neces1552 sary for its susceptibility to enzymatic attack. (CA)

Taylor, Babette, Jesse P. Greenstein, and Alexander Prollaender. FFFETS OF X-RADIATION ON SODIUM THYMUS NUCLEATE. Arch Biochem. 16, 19-31(1948).

The effect of $X$-rays on aqueous solutions of sodium thymus nucleate is apparently to break up the long, fibrous particle into shorter fragments of variable dimensions. The passage from a state of monodispersion to a state of wide polydispersion continues, after the actual irradiation has stopped, for several hours unt 1 a new equilibrium is reached. The extent of the change, or the level of that new equilibriurn depends on the dose of roentgens delivered and is independent of the time over which that dose is given. The chemical properties and susceptibility to desoxyribonuclease - $\mathrm{Mg}^{++}$of sodium thymus nucleate are essentially independent of the state of polymerization or dispersion as altered by X-rays. (NSA)

1553

Euler, Hans von and L. Hahn. INILUENCE OF ROENT GEN RAYS ON ISOLATED CELL NUCLE耳. Acta radiol. 27, $268-80(1946)$.

Nuclei carefully isolated from calf thymus at pli $4-6$ were irradiated with 25,000 and $65,000 \mathrm{r}$ to compare the physlological state of isolated cell nuclei with their state in a living tissue. Nucleoproteins were prepared from the irradiated nuclei and characterized by analysis, and by viscosity, dialysis and adsorption measurements. A study of the properties of the mucleoproteins showed no significant changes due to the irradiation. This result is not interpreted as meaning that the nuclei have totally lost their biological activity. (NSA)

1554

Culer, Hans von and ceorg v. Mevesy. IFTCCT OF XRAYS ON THE NUCLEIC ACID EXCHANGE IN JENSEN SARCOMA TISSUE. TKgl. Danske Videnskab. Selskab. Biol. Medd. 17, 3-38(1942).

A radioactive phosphate solution was injected into rats with Jensen sarcomas and after 2 hours the meleic acid of the sarcoma tissue was isolated. In the 2-hour period 2$3 \%$ of the nucleic acid $\mathrm{P}$ had been replaced from the injected material. The sarcoma tissue contained $9 \mathrm{mg} / \mathrm{g}$ of nucleic acid $P$. Irradiation of the sarcomas with $450 \mathrm{x}$ of $X$-rays usually caused a $35-50 \%$ decrease in nucleic acid formation but some sarcomas were irradiation-resistant and showed no decrease. No significant changes in the free phosphate contents of plasma and liver were produced by the irradiation. In necrotic sar coma tissue the nucleic acid exchange was $25-50 \%$ of that in nonnecrotic tissue. The phosphate $\mathrm{P}$ of the sarcoma tissue was exchanged rapidly with that of the blood plasma. X-ray irradiation of $2000 \mathrm{r}$ had no appreciable influence on the rate of renewal of the acid soluble $P$ compounds of the sarcoma. (CA)
1555

Euler, Hans von and Ceorg v. Hevesy. ACTION OF W-RAIS ON NUCLIC ACID METABOLISM IN TENSEN SARCOMA. Arkiv. Kemi Mineral. Geol. 17A, Bop. (1244).

Experiments in which phosphorus 32 is injected tnto rats having Jensen sarcoma show that $\mathrm{x}$ - $\mathrm{xadiation}$ inhibits mitosis and, possibly earlier, decreases production of desoxyribonucleic acid in the growing sarcoma, the effect decreasing when the interval between the irradiation and the injection is increased from a few min. to several days. The production is not appreciably affected by fasting. New production of nucleic acid is accompanied by simultanecus degradation of previously formed nuclelc acid and, in a degenerating sarcoma, degradation counterbalances or more than counterbalances new production. The contents of free $P$ in the liver and plasma of rats are 58 and $6.5 \mathrm{mg} / 2$ regpectively, and the free and total $P$ contents of the $\mathrm{Bar}-$ coma are 49 and $298 \mathrm{mg} \%$ respectively; these values being unaffected by irradiation. (CA)

\section{Enzyme Sudies}

1556

Abderhalden, R. FURTHER STUDIES ON THE APPEAR ANCE OF IKNOWN ORGANIC SPECIRIC PROTRINASES IN URINE AFTER ROENTGEN-RAY TREATMENT.

Termentforschung 16, 215-20(1939).

The specific known proteinases appearing in the urine after $x$-ray irradiation of the brain and liver can be detected $6-6 / 2$ hrs after the x-rays. The enayme possesses not only organ but also species specificily. The magnitude of the dose which causes the appearance of cell proteinases of the irradiated organ in the urine amounts to $330 \mathrm{r}$ for the liver, 247.5 for the brain and $165 \mathrm{x}$ for the hypophysis in the dog. (CA)

1557

Arvy, L., J. A. Boiffard, and MA. Gabe. ERFET OF IAARGE DOSES OF X-RAYS ON THE DISTRIBUTION OF ALIKALINE PHOSPLATASIS IN SOME ORGANS OF TIPE MOUST. Compt. I'end soc. biol. 143, 233-4(1949).

Histological changes are described in adult white mice given whole body radiation of 2,000 r. The Gomori technique showed characteristic shifts in alkaline phosphatase in the cells of the spleen, intestine, and kidney. (NSA) 1558

Baldini, Mario. ACTION OF X-RAYS AND SHORT WAVES ON THE URICASE OF PIG LIVER. Atti accad. fISIOCTI. Siena, Sez. Med.-fis. 18, 202-11(1950).

The activity of pip liver uricase extracts, obtained by shaking powdered pig liver with a Na borate solution at pll 3.3 for $3-4$ houxs, was found got to be substantially influenced by either $\mathrm{x}$-ray or short wave irradiation. (CA)

\section{9}

Barnard, R. D. A NOTE ON THE DEPRESSION OT BLOOD CHOLINEST TRASE LEVIEL FOLLOWING X-RA THERAEY AND ITS BEARING ON THE MECHANISM OF RADIATION SICKMISSS. Med. Record 161, 350-52(1948).

The blood cholinesterase concn. is reduced by $\mathrm{X}$-ray therapy. This change affords an explanation of the cholinergic symptoms of radiation sickness. (CA) 1560

Barron, E. S. Gurman. EFT METABOLISM. Argonne National Lab. Oct. $29,1046$. 63p. (AECD-2316; CF-3654)

The effect of $x$-rays, given in a single dose of total body radiation at doses varying from $900 \mathrm{r}$ to $100 \mathrm{x}$, on the 
metabolism of tissues has been studied. Litter-mate rats of the Sprague-Dawley strain were treated with $\mathrm{x}-\mathrm{rays}$ and sacrisiced at different intervals. The metabolism of the following tissues was studied: spleen, liver, kidney, thymus, adronals, testis, and submaxillary glands. Whenever possible measurements were made of the $\mathrm{O}_{2}$ uptake in the presence of oxidizable substrates requiring sulfhydryl enzymes for oxidation, as these enzymes, were previously found more susceptible to the action of $x$-rays. In general, the respiration of all tissues and the osidation of substrates requiring sulfhydryl enzymes was diminished immediately after irradiation, with thymus as the only exception. Oxidation of pyruvate, of succinate, of d-amino acids and of $1(+)$ glutamate-all requiring sulfhydryl enzymes was inhibited in the different tiasueg tamined. (NSA) 1561

Barron, E. Gurman. THE FHECT OF IONIZING RADLATONS ON THE ACTIVITY OI FNZYMES, p.10-16 of CONTPRENCE ON BIOLOCICAC APPLICATIONS OF IVCLEAR PHYSTCS, JULY 12-27, 1048. Brookhaven National Lab. July 1048. 7p. (BNL-C-4(p.10-16))

Results indicate that the biological action of ionizing radiations is due mainly to the products of the irradiation of water. When irradiation is used in small doses this effect may be limited to the oxidation of the sulfhydryl groups present in the cell, either the fixed-\$H grougs of the protein moiety of enzymes or the soluble - SH groups. Oxidathon of the first will produce inhibition of the sulfhydryl enzymes; oxidation of the second group will produce inhibition of cellular division and cellular growth. When irradation is used in larger doses it will produce protein dematuration by a series of mechanisms still unknown. The first effects are reversible; the last are irreversible and the extent of recovery will be determined by the rate of protein synthesis. (Author's Summary)

1562

Barron, E. S. Guzman and S. Dickman. STUDIES ON THE MECLANISM OF ACTION OI IONIZING RADIATIONS. $\mathbb{I}$. INHUSITION OF SULFYYDRY IENZYMES BY ALPHA-, BSTA-, AND GAMMA-RAYS. J. Gen. Physiol. 32, 595005(1949).

Two crystalline \$r enzymes, phosphoglyceraldehyde dehydrogenase and urease, were used to study inactivation by honizing radiations, Po in citrate buffer served as a gource of $\alpha$-radiation; solution of $\mathrm{Sp}^{80} \mathrm{Cl}_{2}$ was the source of P-radiation; and $\mathbb{R a}$ enclosed in a brass tube was the source of $\gamma$-rays. The \$H enzymes were inhibited by these radiations through the oxidation of - SH groups essental for their activity. When the - SH groups were protected from oxidation by conversion to mercaptides, a usually inhibiting dose of $\gamma$-I:ays was without effect and the enzym. could be completely reactivated with glutathione. The mechanimm of inhibition by ionizing radiation is through $\mathrm{P}_{2} \mathrm{O}_{2}$, and partial protection car be obtained by small amounts of catalase. The oxidation of - Br groupes by $\mathrm{H}_{2} \mathrm{O}_{2}$ which causes inhibition is variause. In $\alpha$-radiation oxidation by $\mathrm{H}_{2} \mathrm{O}_{2}$ contributes $30 \%$ of the inhibition, bus in radiakion by p-rays this proportion-is much greater. Ionizing radiations affect proteins either by oxidation of their - \$H groups, which is a reversible phenomenon, or by denaturation and destruction of molecules, which is an irreversible phenomenon. The firot requires fewer lonizing radiations. (CA)

\section{9}

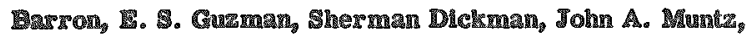
and Thoma. P. SInger. \$TUDIES ON THE MLCRANISM OF $\triangle$ CTION OF IONIIING RADLATIONS. 1. INHIBITION OF (19).
Dilute solutions of sulfhydryl enzymes (phosphoglyceraldehyde dehydrogenase, adenosinetriphosphatase, succinoxidase) showed reduced activity on irradiation by small amounts of X-rays. When the inhibition was partal the enzyme was reactivated on addition of glutathione. When the inhibition was more complete, reactivation was only partial. These observations are interpreted as being duc to oxidation of the -SH groups of the protein by the products of water irradiation, the radicals $O H$ and $O_{2} H$, and $\mathrm{H}_{2} \mathrm{O}_{2}$ and atomic oxygen. The mechanism of the oxidation is best explained by Weiss's theory (Nature, 153, 748, 1944; 157, 584, 1946). The irreversible inhibition which occurs when the dose of $\mathrm{x}$-rays is increased is attributed to protein denaturation. Inhibition of the non-sulfhydryl anzymes trypsin, catalase, and ribonuclease, which required larger amounts of $\mathrm{x}$-rays, is attributed to protein denaturation. These experiments are further evidence that inhibition of enzymes by ionizing radiations is due to the indirect action of the products of irradiated water rather than to direct ionization of the enzyme through collision with ionizing radiation. (NSA)

\section{4}

Barron, E. S. Guzman, Sherman Dickman, Thomas P. Singer, and John A. Munt. THE EFIECT OF X-RAYS ON TRIE ACTIVITY OF ENZYMLES. University of Chicago, Metallurgical Lab. Aug. 1948. A4p. (MDDC1131; CE-3806)

Moderate amounts of $\mathrm{x}$-rays produce inhibition of en-

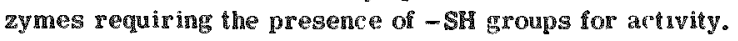
This inhibition is due to oxidation of the -\$Y groups to the inactive disulfide as shown by enzyme reactivation with glutathione. Inhibition and reactivation were performed on phosphoglyceraldehyde dehydrogenase, adenosinetriphosphatase, and succinoxidase. Besides this oxidation of $-\mathrm{SH}$ groups, X-rays have been shown to irreversibly inhibit the activily of enzymes. This inhibition has been attributed to protein denaturation. A number of crystalline enzymestrypsin, catalase, lactic dehydrogenase, ribonuclease-when tested under the conditions of our experiments (with the enzyme, the rate-limiting step in the reaction) were nut inhibited by moderate amounts of $\mathrm{x}-\mathrm{rays}$. However, inhibition was produced on diminishing the amount of enzyme. These inhibitions are produced by nonspecific protein denaturation produced by large amounts of irradiation. (ADD) 1565

Barron, B. \$. Guzman and Veronica Flood. THE EFTECT OF IIREADITTON ON CYTOCHROMR C, p.167 of BIOLOGICAL AND MEDICAL DIVISIONS QUARTERLY

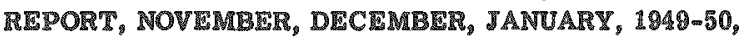
Austin M. Brues, ed. Argonne National Lab. [nd] $1 \mathrm{p}$. (ANL-A셩01(p.167))

Reduced cytochrome $C$ was easily oxidized on irradiation with hard $\mathrm{x}$ rays (200 kw), the oxidation being proportional to the intensity of irradiation. The oxidation produced by $10,000 \mathrm{r}$ was completely reversed on addition of a suilable reducing agent. The ionic yield was 0.55 . It seems that this oxidation is produced only by the of radicals given off on irradiation of water. $\mathrm{H}_{2} \mathrm{O}_{2}$ had no effect at all since the addition of catalage did not diminish the extent of oxidation. $\mathrm{O}_{2} \mathrm{H}$ radicals (presumably formed on reduction of $\mathrm{O}_{2}$ dissolved in water) aeen also to have no effect because the extent of oxidation was not diminished on irradiation in the absence of dissolved $\mathrm{O}_{2}$. On irradiation of ferricytochrome $C$ in neutral, acid, and alkaline solutions with $\mathrm{x}$-ray intensities of 50,000 and $100,000 \mathrm{r}$, it was found that cytochrome dissolved in acid solution was more susceptible to the destructive effect of ionizing raciations. On irradiation with $100,000 \mathrm{r}$ in $0.005 \mathrm{M}$ HCl there was almost complete disappearance of the big soret band of the porphyrin nucleus and of the band at $5300 \mathrm{~A}$ of ferricytochrome. 
1566

Bier, M. and F . F. Nord. THE EFECT OF CERTAN IONS AND OF RADIATION ON CRYSTALLINE TRYPSIN. Arch. Biochem. and Biophys. 31, 335-6(1951).

Ca ions decrease the rate of autodigestion of trypsin; Mn iuns also have a stabilizing effect but other ions are without affect. Electrophoresis showed that the active enzyme had a mobile boundary. Ca had no effect on proleolysis of other protens. The sensitivity of the enzyme to radiation (high intensity alectron bursts of about $\mathbb{1}$ usec from a 3 -mev capacitron) is higher at pH 2.6 than at pH 8. Frozen buf fered solutions of trypsin were less sengihive to radiation than liquid preparations. (CA) 1567

Billen, Daniel and Herman C. Lichstein. THY EFFET OF X RADLATION ON THE ADAPTIVE FORMATION OF FORMIC HYDROGENLYAST IN ESCRERTCHIA COLI. Oak Ridge National Lab. [nd] ip. (ARCU-1705)

The report is reproduced here in its entirety.

During studies on the mechanism of $\mathrm{x}$ radiation effects on mir roorganisms carred out in this laboratory, the adaptive formation of formic hydrogenlyase in resting cell suspensions of Escherichia coli was investigated. The data show that 8 ixradiation of resting cell suspensions of $\mathrm{E}_{0}$ coll, in duses of $60,000 \mathrm{r}$ or greatex, prevents completely the ability to synthesize formic hydrogeniyage, while having no affect on preformed hydrugenlyase activaty. (NSA) 1568

Brandi, C. L., P. J. Freeman, and P. A. Swenson. THF THFECT OF RADIATIONS ON GALACTOZTMASE FORMATION IN YEAST. Science 113, 383-4(1951).

Galactorymase formations in yeast have been subjected to radiation of ultraviolet light, visible light, and $\mathrm{x}$ radiation, resulting in a different reaction to each. Photodynamie action of visible light seems to be conimed to the surface whereas ultraviolet light appears to strike deeper and prevents galactozymase synthesis. $x$ rays appear to prevent division but have no effect on galactozymase formations. (NSA)

1569

Brooks, Robert $\mathrm{F}_{\mathrm{D}}$ and Herman Schneiderman. STWUDES

IN THE HYALURONLDAST-HY ALURONIC ACID SYSTEM.

TI. THE REACTION OF' X-IRRADIATED IIYALURONATE

WITH HYALURONIDASE. Universîty of Califormia

Los Angeles. JuIy 14, 1949. 22p. (ARCU-425; UCLA- \$C)

It has been reported (ALCU-170) that 8 -irsadiation of bovine vitreous humor potassium hyaluronate results in a loss in viscosity due to irradiation, and a continuing loss for about five hours after irradiation. In this report, the interaction of the irradiated hyaluronate with hyaluronidase and the effect of $\mathrm{x}$-irradiation on the enzyme are considered. The previously reported loss in viscosity has been confirmed experimentally with bovine vitrecus humor and human umbilical cord hyaluronate. The viscosity dilution curve for irradiated and nonirradiated hyaluronate is similas in the range of concentrations used. For a similar inicial relative viscosity, the reaction of hyaluronidase with irsadiated hyaluronate is much slower than with nonirrad ated hyaluronate. Suggestions are made as to the mechanisms of these effects and their relation to radiation damage. Results suggest that any damage resulting from x-irradiation of the hyaluronidase-hyaluronic acid system is due more fo the reduction in viscosity of the hyaluronic acid than to the action of 8 -rays on the enzyme. (NSA)

Carter, C. TION ON THE ACTWYTY OF SEVERAL RNZYMATIC SYSTEMS OF RAT IPLEREN. Oak Ridge National Lab. Apr. 26, 1949. 24p. (APCU-231)
Because of the senaltivity of mammalian hematopoletic tissue to damage from ionizing radiation, rat spleen has been assayed for enzymatic activity catalyzing the degradation of benzoyl-L-arginimeamide, glycyl-L-leucine, leucylglycylglycine, and glycyldehydroalanine, substrates for intracellular proteolytic enzymes; the phosphate activated desamidation of glutamine; and the degradation of ribonucleic and desoxyribonucleic acid in spleens from normal aninals and from animals exposed to 8 -radiation. The late effects of whole body -gadiation on the sevrsal enzyme systems of rat spleen are described. of the enzymes studicd, ribonuclease shows the most strilking alferation of activity following radiation, depressions of activity being found 24 hours following exposure to doses as low as 100 r. In rats exposed to $500 \mathrm{~g}$, ribonuclease activity in spleen is severely depressed and no evidence of recovery was found in periods up to 12 days following radiation. (NSA) 1571

Collinson, E. F. Daintor, and B. Holmes. INACTIVATHON OR RIBONUCLEASES IN DLUTE AQUEOUS SOLU TIONS BY RYDROXYL RADICALS. Nature 165, 267-9 (1950).

Hydroxyl radicals (ONI) were prepared in aqueous solu-

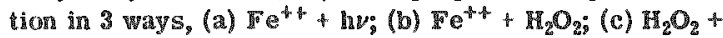
lw. In every case if was shown, with suitable control expts. that (OH) was responsible for the inactivation of ribonuclease. It was also shown that the inactivated enzyme competes effectively with the active enzyme for (OH). These results suggest that the effects produced by absorption of nuclear radiat îns in living tissues may, in sone cases, be due to the action of free radicals. (CA) 1572

Dale, Walter M. THE BIFET OF X-RAXS ON ENZYMES. Biocheris. J. (London) 34, 1367-73(1940).

Irradiation of erystalline carboxypeptidase and of partly purified polyphenoloxidase by $x-$ rays leads to the destruction of a constant anount of enzyme by a given dose of $x$-rays independent of the concentration of the enzyme. In consequence the per cent destruction of the enzyme increases with decreasing concentration. This is shown for a wide range of $\mathrm{x}-\mathrm{r}^{2} \mathrm{y}$ doses and concentration of the enzymes, very small doses of x-rays acting in the wame way as big doses. When carboxypeptidase is irradiated while mixed with its substrate, no destruction occurs. ERzymes apparently play an important part in explaining the effect of radiation on living Iissue. Their concentration and state during Irradiation will determine the biological effect of radiation in those cases where they are a Immiting factor in cell metabolism. (BA) 15793

Dale, W. M. TME ERECT OP X-RAYS ON THE CONJU GATED PROTEYN D-AMINO-ACID ONIDASE. BLChER. J. (London) 36, 80-5(1942).

The enzyme d-amino-acid oxidase can be aplit into alloxazine adeninedinueleotide (prosthetic group) and a highty specific protein. The high chemical activity of these components allows the measurement of the effect of Is-says on them in conens. as lov as they occur in living matter. The radiosensitivity of these 2 substances is greatest when they are Irradiated separately. When Ir sadiated as a muture, however, the inactivation is almost entirely due to the inactivation of the protein since the drucleotde is "protected ${ }^{3 s}$ by the presence of the protein. (BA) 1574

Dale, W. M., J.V. Davies, and W. J. Meredih. FURTH ORSERVATIONS ON TRE PROTICTION FIFIECT IN

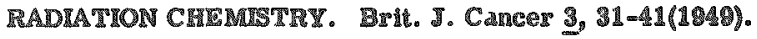

Solutions of carbozypeptidase and of allowazine-adentmo dinucleotide were irradlated with $\mathrm{x}$-rays. The congtaney of 
the lonic yield was verified. The protective power of various substances (e.g. glucose, urea, dimethylurea, thiourea, dimethyl-thiourea, egg albumin, colloidal $\mathrm{S}$, and $\mathrm{Na}_{2} \mathrm{~S}_{2} \mathrm{O}_{3}$ ) was determined. Compounds containing $\mathbf{S}$ had a very high protective power. With certain substances the protective power diminished as the concentration increased. This was 1575

observed only with carboxypeptidase. (CA)

Dale, W. M., L. M. Gray, and W. J. Meredith. THE INACTIVATION OF AN ENZYME (CARBOKYPEPTDASE) BY Z-AND ALPHA-RADIATION. Phil. Trans. Roy. Soc. (London) 242, 33-62(1049).

The inactivation of aqueous solutions of pure crystalline carborypeptidase by $\mathrm{x}$-radiation and $\alpha$-radiation has been studied. It if coneluded that in each case the enzyme molecules are inactivated by collision with a labile product regulting from the ionization of the water. The $\mathrm{x}$-ray lonic yield is approximately constant and equal to about 0.18 enzyme molecules inactivated per ion pair of water at all enzyme concentrations between about $2 \times 10^{-4}$ and $2 \times 10^{-2}$ enzyme/ml. At lower concentrations a smaller x-ray Ionic yield was observed. At an enzyme concentration of $3 \times 10^{-5} \mathrm{~g} / \mathrm{ml}$ the results are not affected by the absence of dissolved orygen. The $\alpha$-ray ionic yield is only about one-twentieth of the $x$-ray ionic yield, that is, 0.01 molecules of enzyme inactivated per ion pair of water, and appears to increase about twofold over the concentration range $5 \times 10^{-6}$ to $5 \times 10^{-3} \mathrm{~g}$ enzyme $/ \mathrm{ml}$. An attempt is made to correlate the $x-r a y$ and $\alpha$-ray ionic yields with what is known concerning the gpatial distribution of the ions. It is concluded that the extremely high concentration of positive ions which forms the core of the $\alpha-$ ray track probably plays a very significant role in the general radiochemistry of $\alpha$-radiation. The small inactivation of carboxypeptidase brought about by exposure to $\alpha$-rays could be ascribed to the secondary electrons ( $\delta$-rays) which travel clear of the $\alpha$-ray track and it is not certain that any inactivation of enzyme is brought about by the primary aray ionization. (NSA)

1576

da Silveria, Marieta and M. Deodata Azevedo. MROTEC. TION OF AN ENZYME ACAINST THIS DESTRUCTIVE ACTION OI NEUTRONS. AEch. portugaises sc. biol. Suppl. 10, 114-18(1951).

The presence of $\mathrm{Na}_{2} \mathrm{SO}_{3}$ prevented inactivation of aqueous fumaric hydratase by a Ra-Be neutron source, whereas the presence of L-cysteine had no protective effect. The $\mathrm{Na}_{2} \mathrm{SO}_{3}$ increased the engymatic power of the hydratase both before and after irradiation. (NSA)

\section{$15 \%$}

Dimning, James S.g 1. Meschan, Cecila I. Kelth, and Paul

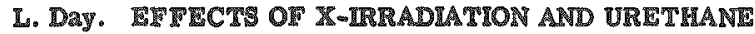

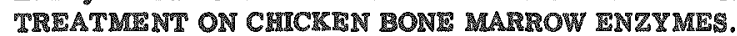
Proc. Soc. Espt1. BLol. Mea. 74, 776-7(1050).

Mature hens were glven a gingle whole-body x-irradiation of $300 \mathrm{~g}$. This treatment markedly reduced the bone marrow endogenous respiration, choline oxidase, and wuccinoxidase. The intraperitoneal injection of urethane also reduced marrow choline oxidase, and reduced succinoxidase but to a lesser degree. Both $\mathrm{k}$-1rradiation and ureI5T: thame treatment rendered the chickens leucopenic. (NSA)

Doherty, DaYia G. PROTECTMN OF ALPHA-CMIMO-

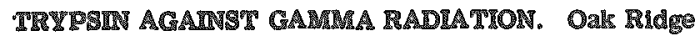
National Lab. [nd] 1p. (ARCU-1794)

Flectrophosetically homogeneous $\alpha$-chymotrypsin was prepared Irom bovine pancreas by the usual procedures, lyophilized, and utilized for all the radiation experiments.
Rnzymatic activity was determined by following the rate of hydrolysis of glycyl-L-tyrosine amide with a potentiometric formol titration. Dilute chymotrypsin solutions were exposed to $\gamma-$ ray dosages from 1000 to $120,000 \mathrm{r}$ from a cobalt source at the rate of $140 \mathrm{r} / \mathrm{min}$; maximum inactivation of $30 \%$ was obtained at the $40,000-r$ level. Dry chymotrypsin inradiated at dosages from 500,000 to $10,000,000$ r gave essentially similar results alhough shifted to a higher dosage level. The $\mathrm{Km}$ remained un changed from all the irradiated enzyme, indicating that there had been no alteration in the active centers of the enzyme molecule. This was substantiated by binding studies with a radioactive tagged substrate by the procedure of Doherty and Vaslow (J. Am. Chem. Soc., (1252) Jan.). Since substrates protect enzymes againgt radiation damage it might be expected that competitive inhibitors would show the sane effert. Adjustment of concentrations might yield conditions which would give protection but very little inhibition. Thus chymotrypsin was irradiated with $20,000 \mathrm{r}$ in the presence of several inhibitors, i.e., acelylL-tyrosine, 4-p-hydroxyphenyl-3-acetamino-butanone-2, p-hydroxyphenyllactic acd, and phenyllactic acid. Inhibition varied from 2 to $50 \%$ while, with the exception of phenyllactic acid, which gave $50 \%$ protection, the other inhibitors afforded complete protection to the enzyme. Dif ferent inhibitors are being invesingated in an effort to gain some insight into the structural part of the enzyme damaged by radiation. (Entire report)

1579

Doyle, Willam $L$. THE DISTRIBUTION OF PHOSPHATASES IN THE RABBIY APPENDIX AFTER X-IRRADIATON. Am. J. Anat. 87, 79-117(1850).

Staining reactions and quantitative determmations of the acid and alkaline phosphatase content of the cells of the rabbit appendix at various levels from the mucosa to the muscularis are presented for the normal animal and for rabbits irradiated with a total-body dose of $625 \mathrm{x}$ of penetrating $\mathrm{x}$-rays, 29 figures. 12 references. (NSA) 1580

Teinstein, Robert N., Carrie L. Butler, and Daniel D. Hendley. EFIECT OF WHOLE-BODY X-IRRADLATION AND INTRAFEITONEAL HEDROGEN PEROXDE ON MOUSE LIVER CATALASE. Science 11, 149-50(1950).

Whole-body $\mathrm{x}$-irradiation $(17 \mathrm{~g} / \mathrm{min}$, total $800 \mathrm{~g})$ reduces liver catalase progressively from $9 \%(2 \mathrm{~min}$ after irradiation) to $82 \%$ (1 week), with no later data available. Twenty milliequivalents $\mathrm{H}_{2} \mathrm{O}_{2}$, injected as $4 \%$ solution, decreases the same activity $32 \%$ after $2 \mathrm{~min}, 41 \%$ after $2 \mathrm{hr}$, showing a slow irregular secovery from the dh day on with $10 \%$ activity decrease at the end of 4 weeks. Agreement with in vitro experimenta is discussed. (CA)

1581

Forssberg, Arne. ACTION ON ROENTGHN RAYS ON THE ENRYME CATALASE. Acta radiol. 27, 281-93(1946).

The mode of action of roentgen rays on living organisms is discussed and the variation in sensitivity of the cells of different organs, thsues, and species is noted in relation to the conditions under which radiation is administered. To gtudy the problem of whether the action of radiation is dixect, the hit theory, or indirect, a "radiochemicall" action, the author has investigated the effect upon the enzyme catalase using a solution of the purified crystalline substance. By varying the catalase concentration of the solution, the intensity of irgadiation, and the gas concentration of the solution, results were obtained which indicate a complicated interaction of the irradiation products formed in the solvent with the enzyme, as well as some direct action upon the enzyme molecules. Results obtained when 
the enzyme solution was frozen seems to indicate that the reaction is predominantly in the immediate vicinity of the enzyme molecule. $(\mathbb{R})$

\section{2}

Forssberg, A. MECHANISM OF THE ACTION OF X-RAYS ON ENZYMES IN WATER-SOLUTION. Nature 159, 308-9 (1947).

Pure crystalline catalase was diluted to $3.7 \times 10^{-8} \mathrm{~g}$ per ml at pH 7.4 and exposed in the absence of oxygen to a dose of $1.24 \times 10^{4} \mathrm{r}$ of $\mathrm{x}$-rays at $275 \mathrm{r} / \mathrm{min}$; $54 \%$ of the enzyme was inactivated. The effect of adding various organic compounds in 0.1 to $0.2 \mathrm{millimol}$ concentration was tested. Unsaturated acids (fumaric and maleic) partly protected the enzyme aganst inactivation by $\mathrm{x}$-rayis. Related saturated acuds (malonic and suceinic) had no effect. Cysteine increased and cystine decreased the percentage of enzyme inactivated; similarly glutathione increased and oxidized glutathione decreased the percentage inactivated. If Weiss's view that the action of $\mathrm{x}$-rays on aqueous solutions is due to free $\mathrm{H}$ atoms and $O H$ radicals is accepted, and it is assumed that the $\mathrm{H}$ atoms inactivate catalase, these results can be explained by involving the reactions 2RSH + $\mathrm{OH}-\mathrm{RSSR}+\mathrm{H}_{2} \mathrm{O}+\mathrm{H} . \mathrm{A} 10^{-5}$ to $10^{-3}$ molar concentration of lodide ions protects catalase against inactivation by $x$-rays. (AWM)

1583

Frederic, J. HISTOLOGICAL AND HSTOCHEMICAL SFUDY OF THE SKIN OF THE GUINEA PIG TREATED WITH X-RAYS. Arch. biol. (Liège) 60, 79-101(1949).

A study was made of morphological changes produced by x-rays in the epidermis, and, in particular, in the histological elements containing substances with - SH groups (sulfhydrules). The skin of the paws of 32 gunea pigs was irradiated with $3,000,2,000$, and $1,500 \mathrm{r}$. The histological and histochemical evolution is shown on nine microphotom graphs. (NSA)

1584

Gierlach, Z. S. and A. T. Krebs. THE TETRAZOLES AS INDICATORS OF RADIATION DAMAGE. ARmy Medical Department. Jan. 3, 1950. 14p. (NP-1119)

Plant seeds Irradiated with $x-$ rays and then treated with tetrazolium salts show that the irradiation effect can be detected by changes in the reducing power of the enzyme systems and can be measured by the amount of colored formazan formed. The relation between dose and irradiation effect on the reducing systems can be written in exponential functions with a constant characteristic for the proper enzyme systems. The environment is of great importance. The radiation effect is altered when seeds are irradiated in the wet or dry states, when different pH values are used, or upon pre- or post-treatment with various chemicals, etc. The tetrazolium salts used were $2,3,5$ triphenyl tetrazolium chloride, 2,3 dipheny 1-5-methyl tetrazolium choride, and neotetrazolium. 29 references. (NSA)

1585

Holle, Fritz. SERUM CHOLINESTERASE ACTVVTYY DURING INE LAMMATION AND X-IIRADIATION, Z.ges. expti. Med. 115, 107-26(1949).

Cholinesterase (I) was determined by titration according to Hall-Lucas. The normal range of total serum I was equivalent to $3.0-4.8 \mathrm{ml} 0.01 \mathrm{~N}$ NaOH. It was constant for an individual. After inflammation induced by bacteria, there was a ghort initial increase in I followed by a prolonged decrease, with a rise to normal values after $8-10$ days. X-irradiation or a combination of irradiation and inflammation resulted in a decrease lasting for $36-48 \mathrm{hr}$.
I was probably bound to the plasma colloids of the albumin fraction. The causes for the variation of I are discussed. (CA)

1586

HOLmE, B. INACTIVATION OF RHBONUCLEASE IN DHLUT AQUEOUS SOLUTIONS. Nature 165, 266-9(1050).

Enzymes in aqueous solution are inactivated by $x-r a y s$. However, certain other materials in this solution can protect the enzymes. Thus very little destruction of the enzyme occurred while sufficient glutathione was present to give a definite nitroprusside test for reduced glutathione at the end of the irradiation. Oxidized glutathione also had some protective action. In the case of ribonuclease and probably of other enzymes not contg. essential SH groups, there is no evidence that dissolved $\mathrm{O}_{2}$ or the formation of $\mathrm{H}_{2} \mathrm{O}_{2}$ plays any essential part in the effects produced by $\mathrm{X}$-rays. (CA)

1587

Jacobsohn, Iurt and Marieta da Siveira. ACTLON OF RADIUM AND NEUTRON-SOURCE RADIATIONS ON ENZYMATIC ACTIVITY. Arch. portugaises sel. biol. Suppl. 10, 105-0(1950).

Fumaric hydratase in liver extract was not inactivated by prolonged Ra or 8 radiations but was completely inactivated by a 5-day exposure to an unmoderated Ra-Be neutron source. Thiaminase in fern-leaf extract was not inactivated by either $\mathrm{Ra}$ or neutron source. (NSA) 1588

Jacobsohn, Iurt and Marieta de Silveira. ACTrON OF NEUTRONS ON ENZYMATIC ACTIVITY. ArCh. portugaises sci. biol. Suppl. 10, 110-11(1950).

Aqueous fumaric hydratase was not inactivated by exposure to a Ra-Be neutron source moderated by $10 \mathrm{~cm}$ of paraffun. It is concluded that only fast neutrons decrease enzymatic activity. Exposure to the unmoderated source slowly inactivated alkaline serum phosphatase and serum cholinesterase. (NSA) 1589

Jacobsohn, Kurt and Marieta da Silveira. INVESTICATION OF TME MECHANISM OF ENZYMATIC INACTIVATION BY NEUTRONS Arch. portugaises sci. biol. Suppl. 10, $112-13(1850)$.

Dry fumaric hydratase, prepared by precipitating liver extract with acetone and ether, was not inactivated by irradiation with fast neutrons for the same period of time that completely inactivated the aqueous enzyme. A secondary inactivation fifect is attributed to the irradiation products of water. (NSA)

1590

Jacobsohn, Kurt $\mathbb{P}$. and Marieta da Silveira. ON THE MECHANSM OF FERMENTATION INACTIVATION BY NEUTRONS. Bull. soc. chim. blol. 33, No, 7, 673-30(1051).

The enzymes fumaric hydratage, urease, alkaline serum phosphatase, serum cholinesterase, and thiaminase, elther dry or in various aqueous substrates, were irsadiated with $\mathrm{X}-\mathrm{ray}, \mathrm{Ra}$, and $\mathrm{Ra}-\mathrm{Be}$ sources. The fumaric hydratase, in particular, undergoes a sudden and cotal inactivation on irradiation by fast neutrons in aqueous fermentative extracts; no effect is noted when dry or when irradiated by slow neutrons, $\gamma$ rays, or $x$ rays. The concluston that the destruction of the fermentation aystem is caused by products of neutron-induced decomposition of water is discussed. (NSA)

1501

LeMay, Marjorie. THE AESENCE OF INUTBITION OF SRI TION, P.22 Of DIVISION OF BOLOGICAL AND MEDICAL 
RESTARCK QUARTERLY RTPORT, MAY, IUNE, JULX, 1950. Argonne National Lab. July 1950. Ip. (ANL4.

An experiment has been designed to determine whether the reduced $\mathrm{O}_{2}$ uptake by rat tissues following irradiation is due to (1) inhibition of - SH enzymes and not to inhibition af other enzymes concerned with complete oxidation of gubstrates, or (2) ialure of the substrate to diffuse as easily in irradiated tissue as in normal tissue. Resulis of this experiment indicate that $\mathrm{z}-\mathrm{ray}$ doses that are high enough to cause hematological changes and death in zats do not cauge measurable effects in the metabollsm of rai kidney slices nor in the enzyme systems studied, including 1592 auccinic dehydrogenase. (NSA)

LeMay, Marjorie. IPECT OF SUCCNOADDASE OF RAT ILIDEX. (19772). Proc. Soc. Exptl. BLI. Med. 7y, 337-9(1951).

The succinoxidase system, ws well as its component enzymes, the -\$I enzyme succinic dehydrogenase, and cytochrome axidase, were determined in rat kidney homogenates within 2 he after rate were $\mathrm{x}$-irradiated with 400 or $800 x$. No inhibition of any of the enzymes was found. The matabolism of kidney slices from rats given the foregoing amounte of 8 radiation was studied manometrically with sodium succinate and lithium pyruvate substrates. No statistically significant difierences were obtained between the oxidlative activities of the kidney slicen from the control and irradiated animals. The lack of correlation between cextain of the in vivo and in vitro elfects of irradiation 1593 enzme systems is discussed. (NSA)

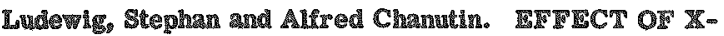
RAY TRADLATON ON THQ ALEALINE PROSPHATAST OF THE PLASMA AND THSUES OT RATS. AM. J. Physiol. 2183, 64-54(1050).

The activity of plasma alkaline phospluatase of rats was determined after single and mulliple doses of $x$ rays at varylng periods ater total-body z Irradiation. Ralatively small decreases in phosphatase activity were found after exposure to $300 \mathrm{I}$. More marked and prolonged decreases were obtalned after 500 - and 600 -1 doces in bohn fed and fated antmals. Multiple daly radiation with mall doses also canses a decrease in plasma exzyme activity. The alkaline phosphatage activity of the thymus is markedly increased during the firgt two days following irradiation. \$mall increases are observed in the spleen, Practically no change in enwyme activity is seen in the livers and kidneys of Irsadlated rata. The changes in the weights of the thy mus, spleen, and liver atter irradiation are presented. (NSA)

1304

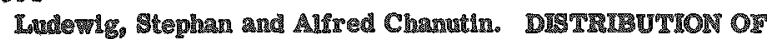

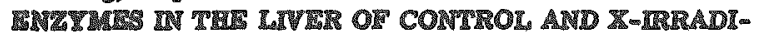

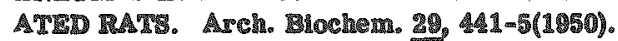

The activisies and percentage distrlbutions of nonmex-

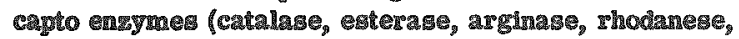
and alkalne phosphatage) in rat liver hornogenate and liver connective t1søus are presented. The alkaline phosphatase In the only myze whose activity is greater in connectre tisme than in liver homogenate. The activites

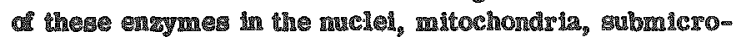
scopic partieles, and supermatant fluid of liver homogenates 2re presented. wothl-body syosure of rats to a dome of $500 \mathrm{r}$ of $\mathrm{z}$ rays has no effect on the activity of nommercapto

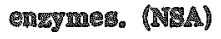

1595

Manoĭlov, S. $\mathrm{E}$. and L. $F$. Semenov. INACTIVATION OF DESOXYRIBONUCLEIC ACID DEPOLYMERASE BY X RAYS. Doklady Akad. Nauk S.S.S.R. 71, 359-60(1950). Irradiation with $250-11,400 \mathrm{r}$ of $\mathbb{X}-$ rays (no specifications of frequency range) of the enzyme preparations (made according to Mac Carthy) in distilled $\mathrm{H}_{2} \mathrm{O}$ and examination of the activity of the product on polymerized nucleic acid, prepared by numerous techniques and used with $0.15 \mathrm{M}$ $\mathrm{MgCl}_{2}$ activator, showed that $1000 \mathrm{r}$ dosage lowers the activity very significantly and total inactivation results with 11,400 s dosage $(93.6 \%)$. No action was noted at 250 r level. (CA) 1596

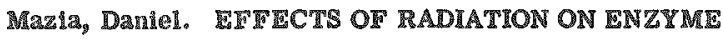
SYSTEMS, $1.7-9$ OA CONFIERENCE ON BIOLOGICAL APPLCATIONS ON NUCLEAR PHYSICS. Brookhaven National Lab. July 1048. 154p. (BNL-C-A $(p .7-9))$

The aim of this investigation was to set up a system adapted to the study, at a biochemical level, of the special problems of chromosomal effects of radiation. The system studied has been an enzyme-substrate gystem incorporated into a monomolecular film. The enzyme-substrate films have an expectedly high sensitivity to radiation. Calculations suggest that in such "solid phase" system the ef fects of a single ionization or ionization product may gpread over a considerable area of the 1 ilm. The sensitivity of an enzyme-substrate system may depend on its physical organization. The thims described are more Bensitive to $x$-rays than comparable solution. The sensitivity varies with the compression of the film. These findings are discussed in relation to the problem of intrinsic sensitivily. (S.V.S.)

$159 \%$

Mazia, Daniel and Gertrude Blumenthal. INACTIVATION OF ENLYME-SUDSTRATE FILMS BY SMALL DOSES OP X-RAYS. Proc. Natl. Acad. Sc. U. 5. 34 , 328-36(1948).

Pepsin-albumin films may be inactivated by small doses of X-rays. Doses of about 100 r produce about 50 per cent inactivation. The sensitivily to radiation depends on the physical configuration of the molecules. It may be varied by surface compression. Calculations suggest that the ef fects of a single gadiation event (ionization or radical production) may be spread to include a large number of enzyme molecules. (NSA) 1598

Mazia, Daniel and Certrude Blumenthal. INACTIVATION OF BNEYME-SUBSTRATE IILM BI SMALL DOSES OF X-RAYS. J. Cellular Comp. Physiol. \$uppl. 1, 35, 171-86 (1950).

It has been shown that one may, using an enzyne-gubstrate film, set up in a blochemical sygtem which has a surficiently high sensitivity to radiation to permit comparisons with more complex biological systems and which is adapted to the study of some of the variables, particularly structural variables, with which radiation biologists are concerned. The radiation sensitivity of the system depends on is structure and may be varied by physical manipulathon so that some of the factors involved in intrinsic sensitivity may be investigated. The evidence that the high radiation Benestivity of this systen may depend on an inter molecular spreading, over considerable distances, of the rfects of a gingle primary radiation event is discussed. (NSA)

\section{9}

Petrakkis, N. I. E. M. Ashler, and R. L. Ferkel. 
MISTOCHEMCAL STUDIES ON THE IFIECT OF TOTAL BODY X-IRRADIATION ON THE ALEALINE PHOSPHATASE, RIBO- AND DESOXYRIBONUCLEIC ACID CONT装NT OF RAT LIVER CRLLS. Naval Radiological Defense Laboratory. June 2, 1949. 18p. (AD-126(B))

The quantitative histochemical changes in alkaline phosphatase, ribo- and desoryribonucleic acid were studied in xat liver epithelium for a six-day period after $600 \mathrm{r}$ and $1,500 \mathrm{x}$ total body $\mathrm{x}$-irradiation With the $600 \mathrm{x}$ exposure, temporary decrease in phosphatase occurged in the nucled and cytoplasm of these cells during the first six hours. This was followed by an increase above normal at the one, two, and six day time intervals studed. A temporary încrease in ribonucleic acid concentration occurred during the sirst 24 hours, followed by a decrease below normal at - the two- and six-day interval. The concentration of melear desoxyribonucleic acid was decreased at the two- and at the six-day intervals. An immediate increase in all three of the above substances was found during the first 24 hours after $1,500 \mathrm{r} \times$-irradiation. All observations indicate that the rat liver epithelium is histochemically sensitive to these levels of $\mathrm{X}$-irxadiation. (NSA)

1600

ROS: M. K. and J. O. IEly. NEITRON ETIIECTS ON ALISAINE PHOSPHATASE OF. RAT INTESTINE. AM. J. Roentgenol. Radun Therayy 62 , 723 -5 (1940).

The effect of neutron irradiation of $200 \mathrm{n}$ on the alkaline phosphatase concentration of the rat duodenum was investigated in 10 albino rats of the Brooklyn strain. An increase in alkalume phosphatase activity at pH 9.0 was found in the nuclel and rucleoli in the cells of the crypts of Lieberkihn Wher $\beta$-glycerophosphate, desoxyriboneleic acid, ribomucleic acid, adenylic acid, guanylic acid, or diphenyl phosphate was used as the source of organe phosphorus. No difference could be observed between the phosphatase activity of the cells in the villi of the duodenum from irradiated and from nonirradiated animals. No difference in phosphatase activity at pH 7.0 was noted in any of the parts of the duodenum of irradiated and nonirradiated animals with any of these substrates. (NSA)

\section{1}

SetLow, R. B. THE INACTVATION OF CATALASE BY DEUTERONS AND MEAT. Arch. Biochem. Biophys. 34, $396-408$ (1951).

The inactivation of beef red-cell catalase by fast deuterons, heal, and a combination of the two has been reported. The data have been analyzed quantitatively in terms of the target theory of ionizing radiation and the theory of absolute reaction rates. Evidence for the existence of at least two forms of stable catalase has been presented. The data are shown to indicate iwo possible alternative molecular weights (about 250,000 and 130,000) for catalase. (NSA) 1602

Sherman, Frederick G. and Herman B. Chase. EFP CTS OF IONIYING RADIATIONS ON RNZIMIE ACTVITIES OF YEAST CELLS. I. RELATION BETWIEMNANARROBIC CARBON DIOLTDE PRODUCTION AND COLONY PRODUCTION AT INTERVALS AITER X-RADIATION. J. Cellular Comp. Physiol. 33, 17-25(139).

Saccharomyces cerevisiae, grown in Reader's solution was ir radiated 30 minutes with $3,000 \mathrm{r}$ per minute. The maximum effect of approximately $30 \%$ inhibition of $\mathrm{CO}_{2}$ production occurred in $2-4$ hours affer starting irradiation and remained constant for 24 hours. At the mame time approximately $85 \%$ of cells were unable to form visible colonies on solid medum. Between 12 and 24 hours after irradiation anaerobic $\mathrm{CO}_{2}$ production on a par cell basig declined progressively. Colony-producing cells which re- mained after irradiation proliferated more rapidly than nonir radiated cells, preaumably under the influence of substances released from damaged cells. (CA)

1608

Sherman, Frederick G. and Herman B. Chase. FEFTS OF IONIZING RADHATIONS ON ENZYME ACTIVITIES OF YEAST CELLS. II. INELUENCE OF DILUTION ON $\mathrm{M}$ RAY-INDUCED INERBITION OF ANAEROBIC CARBON DIOXODE PRODUCTION AND COLONY FORMATION.

J. Cellular Comp. Physiol. 34, 207-18(104).

Yeast grown in a synthetic (I) and a nongynhetic (II) medium was irradiated with $00,000 \mathrm{~g}$ in 30 minutes. Dilution of the cell suspension increased the effect of irradiation on anaerobic $\mathrm{CO}_{2}$ production of yeast grown in I but $\mathrm{CO}_{2}$ production was not affected much in yeast grown in II. Dilution gave only a small increase in the effect of radiation on colony formation in yeast grown in I but did not change the effect of yeast grown in II. When non-irradiated cells were mised with irsadiated bufer or irgadiated cell were mixed with nonimradiated buffer the amount of $\mathrm{CO}_{2}$ was reduced at lower concentrations of cells but the effect of radiation became nil at higher cell concentrations. Inhibition of colony lormation occurged only at low cell concentratlons when irradiated buffer was remixed with nonirradiated cells. X-rays exsert effects on cell division and enzymes associated with anaeroble $\mathrm{CO}_{2}$ production through different mechanisms. (CA)

\section{4}

Somnenblick, B. P. REDUCTION OF NEOTETRAZOLIUM CHLORIDE BI X-RADRATHD ROOT TIP CELIS OF ALLIUM. BLol. Bull. 101, 230(1951).

Teports by several investigators (Antopol, Glaubach, Goldman, Narahaia, Sonnenblick) indicete that the enzyme systerns responsible for the reduction of nectetrazoliun chloride (NT) are present in many living tissues. The faintly yellow salt undergoes a progressive Beries of color changes and is finally converted to an insoluble purpleblack granular compound located character istically in the cytonlasm about the nuclear membrane. Studies on the mechanism of NT Ieduction show that active -\$H groups play a significant role in the process. Since reports explet indicating that moderate amounts of $8-$ rays efect tnhbithon of sulfhydryl enzymes in vivo and in vitro, it was decided to gtudy the reduction of NT by $\mathrm{X}-\mathrm{radiated}$ root tip cells. Dormant bulbs and actively growing roots were irradiated and excised roots gubsequently tosted at various periods following exposure. Roots were given 300,600 , or $900 \mathrm{x}$, while bulbs were exposed to 300,1200 , or $2400 \mathrm{x}$. Tests indicate that celis given treatments causing a moderate frequency of chromosomal aberrations show no inhibition in the speed and intensity of $\mathrm{NT}$ reduction. With dosages causing severe nuclear damage, the reauls are variable, but even after much exposures INT reduction can proceed as well as in control material. To interpret these pesults the lollowing must be considered: (1) the greater sensitivity of dilute aqueous solution of enzymes to irradiation than when accorded the protection of other subtances within cells, (2) the possiblity of unequal inmibition of different sulfhydryl enzymes by $\mathrm{x}-\mathrm{rays}$, and (3) the role played by other than - SM enzymes in NT reduction. Very recently LeMay of Carnbridge Univ. reported no inhbithon of the - SH enzyme succinic dehydrogenase in rat kfdney slices and homogenates sollowing $400 \mathrm{I}$ or $100 \mathrm{r}$ of total body 8 -radiation, a result in vivo contrating markedly to the effect in vitro of $\mathrm{x}$-Ixays on dilute molutions of that enzyrase.

1605

Tahmislan, T. N. and D. M. Adamson. OxwDASE IN- 
CREASE IN MELANOPLUS DTFIRENTIALIS EGGS CAUSED BY X-IRRADLATION. J. Expt1. Zool. 115, 37997(1950). (ANL-4401)

When diapause eggs of Melanoplus differentialis are In-irradiated ( $200 \mathrm{kv}, 15 \mathrm{ma}$ ) with dosages ranging from 25,000 It $10200,000 \mathrm{~g}$, the hydrogumone oxidase activity in the eggs is decreased immediately after the exposure. The enzyme activity increases with time so that the hichest activity is obtained approximately eight days after exposure. Oxidase activity afler irradiation waries in the following sequence: $25,000 \mathrm{r}>50,000 \mathrm{r}>100,000 \mathrm{r} \rightarrow 200,000$ $r>$ control. The ozidase is inhibited by cyanide, carbon monoxide, and heat. Its activity is not afferted by azdde, phenylthiourea, nor diethyldithiocarbanate. The oxidase is specific for hydroquinone, and in addition, il appears to have some effect on p-phenylenediamine. It does not oridize the - \$H group in glutathione nor cysteine. Resorcinol or tyramine hydrochloride are also not oxidized by the enzyme. The nitroprusside test reveals that the native - SII groups are not oxidized as long as 124 hours after $\mathrm{x}$-inadiation. However, by 18 days practically all -5H groups are absent. The increase in lydroquinone oxidase may be due either to the abolition of check mechanisms that normally repress oxidation or to an increase in the oxidase itself resulting from the breakdown of zymogens in the egg. (Authors)

\section{6}

Woodard, Heien Quincey. SOME EFITETS OF RADIATION ON LIVING BONE, p.161-2 OF INPIRRNATIONAL CONGRESS OF IRADIOLOGY, 6th Congress, London. 1950.

Preliminary work indicated that the bones of young adult mice contain surficient alkaline phosphatase for quantitative measurement. As the phosphatase activity diminishes markedly after moderate doses of roentgen rays, the bones are suitable test objects for the quantitative study of the effects of irradiation in vivo. Accordingly about $1.5 \mathrm{~cm}$. of one hind leg of each of a series of male albino mice of about $20 \mathrm{gms}$. body weight was ixradiated, the remainder of the body being shielded. At intervals of 4 to 50 days after irradiation the mice were killed, and the alkaline phosphatase activities of irradiated and control legs were compared. The rnajority of the experiments were percormed with $185 \mathrm{kv}$ roentgen gays with a hal value layer of $0.8 \mathrm{~mm}$. copper and a dosage rate of $160 \mathrm{r} / \mathrm{min}$. With doser of $900 x$ to $1,500 x$ a depression of phosphatase activlty was apparent in the irradiated bones in 4 to 7 days, whth minimum value after 10 to 20 days. This was followed by partial secovery, but, even after a dose of $900 \mathrm{r}$, recovery was not complete in 50 days. In some cases there was indication of a secondary depression beginning at abput 35 days. After doses of $3,000 \times$ phosphatase activity was almowt abolished, and there was no evidence of recovery in per lods up to 45 days. A study was also made af the effects of roentgen rays with half value layers of $6 \mathrm{~mm}$. of aluminium and of $4 \mathrm{~mm}$. of lead.

\section{$\mathrm{H}_{2} \mathrm{O}_{2}$ Problem}

\section{7}

Alper, Tikvah. HIPDROGEN PEROKHDE AMD TEE INDIREC 1 IFE 102, 615-16(1948).

Experiments undertaken on bacterlophage $\mathbf{s} 13$ in vilro apparently have whown that when the phage is in allute colution the hydrogen peroxlde formed by lonizing radiations will inactivate 1t. And in experiments on "indirect effect" of ionizing radiations on this phage the ratio of lonic yield for direct action only to donic yleld in dilute solution is of importance for gaining an insight into the "active radical" mechanism. (ISSA) 1608

Barron, E. S. Guman, Veronica Flood, and Betty Gasvoda. STUDIES ON THE MECHANISM OF ACTION OF IONIZINC RADIATIONS. V. THE EFFECT OF HYDROGEN PEROX IDE AND OF X-RAY IRRADIATED SEA WATER ON THE RESPIRATION OF SEA URCHIN SPERM AND EGGS. Biol. Bu11. 97, 51-5(1949).

The results of experiments on the sea urchin sperm respiration rate after the addition of hydrogen peroxide to the medium and after $\mathrm{x}-1 \mathrm{pr}$ radiation of the medium are presented. The hydrogen peroxide was used at concentrations of $0.01-0.0001 \mathrm{M}$ in a 1:200 sperm solution; x-ray dosages of 50,000-200,000 $\mathrm{x}$ were given. Total inhibition by hydrogen peroxide occurred at $0.001 \mathrm{M}$ concentration; the inhibition decreased with peroxide concen tration and approached $26 r$ at $0.0005 \mathrm{M}$. When sea water was irradiated whis $200,000 \mathrm{r}$, a $60 \%$ sperm inhibition occurred; $50,000 \times$ produced a $10 \%$ inhibition, while the addition of calalase, previous to the sperm addition and after inradiation, had no effect on the inhibition. Since the sea water lnradiated with $200,000 \mathrm{r}$ gave no positive test for hydrogen peroxide it is postulated that inhibition is not due to the action of $\mathrm{H}_{2} \mathrm{O}_{2}$ but to stable organic peroxides. (NSA) 1609

Barron, E. S. Guzman and Betty Gaswoda. PRELMMNARY REPORT: HYDROGEN PEROXIDE FORMATION ON $\mathrm{X}$ IRRADLATION OF WATEIR WITH RARD AND SOFT I RAYS, p.164-6 of BIOLOGICAL AND MEDICAL DIVISIONS; QUARTERLY REPORT, NOVISMBER, DECRMBER,

JANUARY, 1949-50, Austin M. Brues, ed. Argonne National Lab. [nd] 3p. (ANL-4401(p.164-6))

Hydrogen peroxide formation on irradiation of distilled water by hard $x$ rays (200 kv) and by soft $x$ rays ( $50 \mathrm{kv}$, $40 \mathrm{ma}$ ) was measured by colorimetric determination at the end of irradiation. On irradiation with an intensity of 100,000 r there was formation of $4 \mu g / c e$ with hard $x$ rays and of $1.3 \mu \mathrm{g}$ with soft $\mathrm{x}$ rays. There was a small increase on irradiation with soft 8 rays when the water was stirred with a magnetic stirrer. (INSA)

\section{0}

Barron, E. S. Guzman and Betty Redfield. PROGRESS REPORT: STUDIES ON THE MECHANISM OF ACTION OF IRRADLATED WATER ON CELL METABOLISM, p.78-81 of DIVISION OF BIOLOGCAL AND MEDICAL RESRARCH QUARTERLY REPORT, MAY, JUNE, JULY, 1950, Austin M. Brues, ed. Argonne National Lab. [nd] 4p. (ANL-4488(p.\%8-81))

It was shown previously (Barron et al., Biol. Bull. 97, 51-6(1949)) that the respiration of seamurchin sperm was inhibited when the cells were suspended in $\mathrm{x}$-irradiated sea water or artificlal sea water. The mechanism of this inhibition is still unknown. Possibility of $\mathrm{ClOH}$ formation was tested, but none was detected. Consideration of the influence of electrolytes on $\mathrm{H}_{2} \mathrm{O}_{2}$ formation in $\mathrm{x}$-irradiated water and of effects of 8 -irradiated water on sperm respiration gave indeterminate results. (NSA)

\section{1}

Barron, E. S. Cuzman and L. Seik. BLOCARMISTRY AND PHYSIOLOGICAL CMEMISTRY, p.101-5 Of DIVISIONO BIOLOGICAL AND RIEDCAL RESEARCH; QUARTERLY REPORT, AUGUST, SEPTEMERR, OCTOBER, 1951, Austin M. Brues, ed. Argonne National Lab. [nd] 5p. (ANL-A713(p.101-5))

The role of $\mathrm{H}_{2} \mathrm{O}_{2}$ in the biologleal effect of ionizing radiation was investigated by determination of the amount of $\mathrm{H}_{2} \mathrm{O}_{2}$ produced after $x$ irradiation of $\mathrm{H}_{2} \mathrm{O}$, the effect of 
$\mathrm{H}_{2} \mathrm{O}_{2}$ on the metabolism of bacteria and yeast, the respiration of marine eggs and sperm, and the oxidation of glutathione and dihydrodiphosphopyridine nucleotide. From these experiments it was concluded that the role of $\mathrm{H}_{2} \mathrm{O}_{2}$ in the effects of ionizing radiation on the substances and biological processes mentioned is negligible. Specific activity was determined for some of the contents of an aquarium that was seeded with $\mathrm{C}^{14}$-containing Scenedesmus $3 \mathrm{yr}$ ago and sealed. Regults of the analyses appear to anprosimale the true isotope effect, which seems to be about $8 \%$ or less between mised organic and inorganic carbonate. (NSA)

\section{2}

Bonet-Maury, 19. TME FORMATION OF HIDROGEN PEROXIDE IN WATER EXPOSED TO IONIZING RADLATIONS, P. 42 OF INTERNATIONAL CONGRESS OF RADIOLOGY, Gh Congress, London. 1950.

If seems that hydrogen peroxide is formed by different mechanisms under the action of $\mathrm{X}-\mathrm{rays}$ (from $\mathrm{CH}$ radicals and dissolved oxygen) and of a rays (from or radicals). Interpreting these facts in the light of recent theories leads to the consideration of irradiated water as an oxy-reduction system (a solution of $1 \mathrm{H}$ and ON radicals) in which action on the dissolved material degends not only on such physicochemical factors as temperature, concentration of the dissolved material, pH, energy of activation, etc, but athll more on the nature and intensity of the radiation conditioning the initial geometrical distribution of the radicals in the liquid. This geometrical factor constitutes an essential 1613

characteristic of the chemistry of ionizing radiations.

Bonet-Maury, D. and M. LeFort. TME FORMATION OF $\mathrm{F}_{2} \mathrm{O}_{2}$ IN WATER IRRADIATED WITR X-RAYS AND ALPMA RAYS. Compt. Iend. 226, 1363(1948).

Water was irradiated with $x-r a y s$ of wavelength $0.9 \mathrm{~A}$ and with $\alpha$-rays from $\mathrm{Rn}$ and $\mathrm{Po}$. The amoun of $\mathrm{H}_{2} \mathrm{O}_{2}$ formed with $\mathrm{x}-\mathrm{rays}$ at $20^{\circ}$ was greatly dependent on the concentration of dissolved $\mathrm{O}_{2}$ in the water. $\mathrm{No} \mathrm{H}_{2} \mathrm{O}_{2}$ was formed in water completely freed of dissolved $\mathrm{O}_{2}$. With a-rays at $20^{\circ}$ it was obserwed that the yield of $\mathrm{H}_{2} \mathrm{O}_{2}$ was Independent of the amount of dissolved $\mathrm{O}_{2}$ and linearly dependent on the amount of irradiation. (CA)

\section{4}

Dustin, $p_{3} \mathrm{Jr}$. and C. Compel. ACTION OF MYDROGEN PEROXID ON CELL MIOSIS IN THE MOUSE INTISTINE. Compt. rend. soc. biol. 143, 874-5(1949).

Dilute solutions of $\mathrm{H}_{2} \mathrm{O}_{2}$ were injected intraperitoneally in adult mice. A few hr later the intestinal wall showed a decrease in number of normal mitotic figures, and the presence of numerous pycnonecrotic muclel similar to those seen after $\mathbb{X}$ mray irradiation. Similar nuclear lesions appeared in the thymus. (CA)

\section{5}

Wvans, T. C. RETECTS OF HYDROGEN PEROXIDE PRODUCED IN TRE MEOUM BY RADLATON ON SPREMATO2OA OF ARBACIA PUNCTULATA. Biol. Bull. 22, 99-107(1947).

Heavlly irradiated water (over 100,000 s) was found to have deleterious effects on sperm placed therein. These effects were reduction of survival the and delay in that cleavage when such treated sperm were used to ingeminate fertlle eggs. The injury became more pronounced the greater the irradiation dose and the longer the sperra semained in the irradiated water. The chief, fif not the only, agent responstble for these effect of Irradiated water was hydrogen peroxide. This was whown by chemical test, by sumiarty of its action whth that of hydrogen peroside, and by removing the toxicity with catalase extract. The effect of hydrogen peroxide on fertilly and subsequent cleavage time are discussed regarding possible interpretations of similar reactions of the sperm to more direct affects of roentgen radiation. (auth)

\section{6}

Wagner, R. P., C. H. Haddox, R. Fuerst, and W. S. Stone. TIIE EFTCT OF IRRADIATED MEDUM, PEROKDDE AND CY ANDE ON THE MUTATION RATE DN NEUROSPORA. Genetics 35, $137-8(1050)$.

Blochemical mutations have been induced in Neurospora crassa at a rate significantly higher than the control gate by treatng the conidia with nutrient broth which had previously irradiated with ultra-violet light, and also by treating the conidia with hydrogen peroxide and whth gotassinm cyanide. The mutations were detected after having been passed through the aex cycle. The rates Induced by these various agents are not signilicantly different from one another, but are significanty lower than the rate induced by drect irradiation with ullra-wiolet. The rypes of biochemical mutants groduced, as far as they have been idern iffed, do not seem to be any different from those induced by direct irradiation. These results wilh Neurospora fully confirm in all important respects the resuls obtained by Stone et al. $(194 \%, 1948)$ and Wyss at al. (1948) uanthe bacteria, and definitely establish the producton of mutantz by indirect irradiation, as well as by hydrogen peroxide which has been postulated to be one of the mutagenic agents formed as a result of irradiation. (G)

1617

WeISS, JOSEph. ACTION OF IONIEING RADLATIONS ON AQTEOUS SOLUTIONS. J. chim. phys. $46,593-6(1049)$.

In a lecture delivered in Paris, the author outined his Vlews on the action of lonizlng radiation on aqueous solutons. Unilke photochemical agents, for which water is trangarent and the primary action is localized in the dig. solved subatance, the lonizing agents act primarlly won water molecules. Electron ransfers lead to the formation of lons $\mathrm{H}_{2} \mathrm{O}^{+}$and $\mathrm{PL}_{2} \mathrm{O}^{-}$which decompose into ( $\mathrm{H}^{+}+\mathrm{OH}$ ) and $\left(\mathrm{OH}^{-}\right.$+ $\left.\mathrm{H}\right)$ respectively, thus producing neutral sragments OH and $\mathrm{H}$, capable of various interactions with a numbar of dissolved substances. Several examples of such reactions and heir phys co-chemical characterstestcs are glver. The inportance of these studies in the field of radioblology is shown by certaln transformations of organic compounds obtained by the author and esplained according to his views, (irradiation of phenols, amino acids, ete.). (NSA) 1618

Wyss, 0., J. B. Clark, F. PRas, and ROLE OF PEROXIOE IN THE RIOLOGCAL IREADLTED BROTH. J. BaCt. 30, 151-7(1048).

There is a marked similardty between certain biological effects produced by ultraviolet irradiation of nutrient broth and by the addn. of $\mathrm{H}_{2} \mathrm{O}_{2}$ to broth. The ffects of elther can be negated by catalase. Because the direct action of perorfde on the cells in the absence of an org. substrated falled to increase the mutation rate, tis probable that the results are due to the inter? metabolic function. Whether the catalase reacts wh the org. peroxide (or some oxidized mol.) or only with the $\mathrm{Mg}_{2} \mathrm{O}_{2}$ in eguil. With it, is not known. In either case, since the celle atudied contaln active catalase, it 1s necemsary to postulate that aome reactor must occur such as the union of the lablle substance with an anabolic enzyme which protects the mutatng mol. Irom the catalase of the cell. (CA)

\section{Lom Temperature Studien}

1810

Allen, Bemet M., OLe Arme Gchjelde, and Lynotte

FOc. 
THE DESTRUCTION OF X-RAYID HEMATOPOIETIC CELLS OF TADPOLES. J. Cellular Comp. Physiol. 38, 69-82(1951). (UCLA-93)

The object of this work is to determine the influence of temperature upon the destruction of the hematopoletic cells of tadpoles of Rana catesbiana exposed to a dosage of $500 \mathrm{r}$ hard $\mathrm{x}$ rays. Counts in 426 tadpoles were made and the percentage of destroyed cells was determined. Temperatures continuously maintained at different levels from 0 to $11^{\circ} \mathrm{C}$ hold destruction evenly to a very low percentage Which increases rapidly from 13 up to $33.5^{\circ} \mathrm{C}$ - the himhest temperature tolerated. Experiments at temperatures up to $23^{\circ} \mathrm{C}$ are upon a basis of $24 \mathrm{hr}$. The destruction and rapid resorption reaches a maximum at $33.5^{\circ} \mathrm{C}$. Low temperatures applied inmediately after $\mathrm{x}$-ray treatment are as effective as If applied during and after irradiation, and the rate of destruction increases proportionally to subsequent temperature increase. The destruction rises very slowly in $\mathrm{x}$-rayed tadpoles kept at 4.5 and $10^{\circ} \mathrm{C}$ through a period of 10 days. Visible destruction of $\mathrm{x}$-rayed hematopoietic cells of tadpoles is due to destructive reactions subsequent to irradiation conditioned by the temperature at which they are reared. (NSA)

\section{0}

Allen, Bennet M., Ole Arne Schjeide, and Lynette B. HOchwald. IELATION ON LOW TEMPERATURES TO $\mathrm{X}$ RAT INUURY OF REMATOPOIETIC TISSUE OT TAD POLES. Proc. Soc. Fxpll. Biol. Med. 73, 60-2(1950).

The hematopoletic cells of tadpoles occur in the caudal portion of the kidneys and undergo extensive mitotic division. They are destroyed by hard $\mathrm{X}-\mathrm{rays}$ with a dosage of $500 \mathrm{r}$, leaving very few remaining after the lapse of 7 days. 196 tadpoles of Rana catesbiana were used in these expts. A study of cell destruetion in tadpoles refrigerated at $0-5.5^{\circ} \mathrm{C}$ showed that these low lemps. preserve all but a very small per cent during the $24 \mathrm{hr}$ of refrigeration. The ratio of destruction in the refrigerated compared with that at normal temps (averaging $18.9^{\circ} \mathrm{C}$ ) ranged from $1: 6$ to $1: 33$. In one series of 58 , tadpoles refrigerated for 4 days showed extremely little cell destruction, while those removed at the end of 2 days and mainiained for 1 day or 2 days at normal temp. showed a very great amount of destruction. Refrigeration before and during X-xay treatment did not give results appreciably different from those produced by refrigeration after irradiation. Thus, refrigeration prevents the degeneration of $\mathrm{x}$-rayed hematopoietic cells but does not prevent the primary injury that leads to their degeneration at normal temps. (BA) 1221

Bachofer, C. S., C. I. Chret, and S. Mayer. PRPIMI NARY REPORT; FETECT OF TEMPERATURE ON INACTIVATION OF BACTERIOPHAGE BY X RAYS, p.170 Of BIOLOGICAL AND MEDICAI DIVISIONS; QUARTERLY REPORT: NOVEMBER, DECEMBER, JANUART, 194950, Austin M. Brues, ed. Argome National Lab. [nd] 410. (ANL -401(p.170))

Procedures have been established to determine the rate of inactivation by $\mathrm{x}$ xays of T-1 coliphage in broth and $\mathrm{In}$ chemically defined media, for liquid, dried, and lyophilized states. Eguipment has been designed for ir radiation in vacuo at different temperatures with simultaneous registering of temperature. The effect of temperature on $x-1$ ray inactivation has been investigated at various points from 77 to $310^{\circ} \mathrm{K}$. 酐 has been established that lower tempera1822 tures decrease the rate of $\mathrm{x}$-ray inactivation. (NSA)

Cook, 尽velyn Vanderwilt. INILUENCE OF LOW TEMPERATURE ON RECOVERT FROM ROENTGEN RAYS.

Zadiology 32, 289-83(1939).
Eggs of Ascaris equorum (megalocephalia) variety univalens, were irradiated at room temp. with $5,000 \mathrm{x}$ and transferred to $5^{\circ} \mathrm{C}$ and $25^{\circ} \mathrm{C}$. Cleavage is delayed from 2 to $5 \mathrm{hr}$ by the irradiation at $25^{\circ} \mathrm{C}$ but is not completely inhibited. $\mathrm{Al} 5^{\circ} \mathrm{C}$ the delay in cleavage by $\mathrm{z}$-rays is the same as at $25^{\circ} \mathrm{C}$. Eggs irradiated and kept at $25^{\circ} \mathrm{C}$ show $1-2 \%$ normal embryos, but those kept at $5^{\circ} \mathrm{C}$ show a greater $\%$ (maxirnum $45 \%$ ) which increases with increase in length of time of storage at $5^{\circ} \mathrm{C}$. Storage at $5^{\circ} \mathrm{C}$ without irradiation promduces no injurious effects. Recovery from $x$-ray injury may occur in the absence of appreciable metabolism. (BA) 1623

Evans, T. C., J. P. Goodxich, and J. C. Slaughter.

RADIOSENSITIVITY OF SIIN OF NEW-BORN RATS. III. SENSITIVITY AT DIFTERENT TEMPERATURES. Proc. Soc. Exptl. Biol. Med. 47, 434-7(1941).

New born rats were irradiated (2010 $\mathrm{x}$ ) at different temperatures $\left(0-40^{\circ} \mathrm{C}\right.$.) and the skin sensitivity increased as the temperature was raised. The rate of heart-beat and $\mathrm{O}_{2}$ consumption responded to temperature in much the same manner as the radiosensitivity. It is possible that temperature exerts its effect on radiosensitivity through its control of such metabolic processes as these. (BA) 1624

Ivans, T. C., J. P. Goodrich, and J. C. Slaughter. TEMP. ERATURE AND RADIOSENSITIVTTY OF SKIN OF NEWBORN RATS. IV. EFIECTS OF DECREASED CIRCULATION AND BREATTING DURING IRIRADIATION.

Radiology $38,201-6(1942)$.

New-born rats were irradiated under conditions in which the metabolic rate was lowered. In all cases, an increased radio-resistance of the skin toward roentgen ray occurred. (BA)

1625

Evans, T. C., W. A. Robbie, J. P. Goodrich, and J. C. Slaughter. LOW TWMPIRATURE AND RADIOSFNSTTIVIIY OF SKIN OF NEW-BORN RATS. II. RESISTANCE AT DIFFERENT DOSAGES. Proc. Soc. Expt1. Biol. Med. 46 , $662-4(1941)$.

Lowering the temperature $\left(0-8^{\circ} \mathrm{C}\right.$. $)$ of newborn rats during irradiation increased the resistance of the skin to roentgen irradiation. A dosage increase of 1200-1700 r was required to produce an injury comparable to that of animals rayed at room temperature. The ratio of dosage reguired at low temperature to the dosage at room temperature decreases from 5 (at $1500 \mathrm{r}$-cold) to $2.2(3300 \mathrm{r}$ cold). (BA)

1626

Henpeinann, Louis 1. ., Teodore T. Trujillo, and Norman P. ISnowlton, Ir. THE EFFECT OF LETEAL DOSES OF X-RAYS ON CHILLED AND THYIOLDECTOMHED ANIMALS. Los Alamos Scientific Lab. [nd] 1.5p. (AECU-239; LADC-663)

New-born rats chilled to $2-5^{\circ} \mathrm{C}$ belore exposure to lethal amounts of $\mathrm{x}-\mathrm{ray}$ irradiation showed an increased survival rate as compared to unchilled control rats. The lowering of the envirommental temperature of adult mice exposed to $675 \mathrm{r}$ of $\mathrm{x}$-ray decreased the 30 day survival rate. Adult ratฐ and mice showed no significant increase in the 30 -day survival pate when chilled aiter, or before and after irradation. Adult rats were not protected from the lethal effects of $x-$ radiation by thyroidectomy. (NSA) 1627

Kempton, J. H. and Louis R. Maxwell. EPTECT OF TEMPERATURE DURING TRRADIATON ON THE X-RAY SENSITIVITY OF MAIZE SEED. J. AgE. Research 62, $603-18(1841)$.

Repeated expts, show that the $x$-ray sensitivity of airdry ( $8 \%$ moisture) seeds of maize depends upon their temp. 
during irradiation. Temps, used ranged from $-187^{\circ}$ to $66^{\circ} \mathrm{C}$. Maximum sensitivity occurred for the temp. range $0^{\circ} \mathrm{C}$ to room temp. Increases or decreases of seed temp. from these values resulted in a reduction of the $\mathrm{x}-\mathrm{ray}$ sensitivity. (BA)

\section{8}

Lamarque, P. and C. Gros. NEW IEXPERTMENTS ON THE RESTORATION FACTOR OF RADIOBIOLOGY. I. radiol. électrol. $30,539-42(1949)$.

In previous experiments the authors have demonstrated (Acad. Med. 1 (1944) Nov.; J. Radiol. Electrol. 26, 129 (1944-1945)) that the eggs of Bombyx mori exhibil certain restoratve properties after being subjected to irradiation, and that refrigeration suspends this restoration. In the present paper, reports are given of three types af experiments: (1) the eggs were irradiated in single doses and incubated immediately afterwards; (2) the radiation was of the fractionated type, consisting of equal dosages $48 \mathrm{hr}$ apart (the eggs were incubated between the treat ments); and (3) the eggs were irradiated as in experiment 2 but they were placed in a refrigerator between the first and second, and between the second and third irradiations. Total doses ranged from 300 to $3,000 \mathrm{r}$ for all groups of experiments. In group 1 it was seen that the threshold of efficacy lies at about $1350 \mathrm{r}$; in order to prevent $50 \%$ of the eggs from hatching, it was necessary to use $1500 \mathrm{r}$. The eggs of group 2 exhibited more restorative power than those of group 1, probably because they were able to restore themselves partially between irradiations. Results of the third group of experiments were identical with those of group 1. This illustrates that the refrigeration serves to suspend the restoration. (NSA)

\section{9}

Latarjet, Raymond. EFFECT OF COLD ON THE RESTORATION OF A YEAST AND A BACTERIUM DAMAGED BY RADIATION. Compt. rend. 217, 186-8(1943).

Sacoharomyces ellipsoideus, if thept at $5^{\circ} \mathrm{C}$. remains metabolically active and is able to inhibit the damage of x-rays. In Bacillus "paradysenterial $Y^{\prime \prime}$ at $5^{\circ} \mathrm{C}$. the primary lesion remains latent and develops on rise of temperature. This is because the bacteria are unable to repair the primary damage since there is simultaneous stopping of metabolism at $5^{\circ} \mathrm{C}$. Yeast resembles the behavior of Ascaris ova studied by $\mathrm{L}_{0}$, V. Cook (Radiology 32 : 289 (1939)). A table states the exposure to $\mathrm{x}$-rays used and also to ultraviolet light. (BA) 1630

Maxwell, Louis R., J. H. Kempton, and Vernon M. Mosley. EFFECT OF TEMPERATURE ON THE X-RAY SENSTTVITY OF MAIZE SELDS. J. Wash. Acad. Sci. 32, 18-24 (1942).

Air-diry seeds were subject to $35 \mathrm{kr}$ (kilo-roentgens: $1000 \mathrm{r}) \mathrm{x}$-rays at $45 \mathrm{kv}$. The seeds were heated or cooled before, during or after ir radiation. To decrease 8 -ray sensitivity by hot or cold treatments the high or low temperatures must prevall during irradiation. There is a generall but varying amount of recovery with time elapsing between $\mathrm{X}$-raying and planting for all the treatments used. (BA)

1631

Patt, Rarvey M.and Marguerite N. Swit. INFUURNCE OF TEMPXRATURE ON THE RESPONSE OF FROGS TO S-MRRADATION. Am. J. Physiol. 155, 388-93(1948).

The authors found that frog survival was not influenced by altering body temperature during and/or for the first 24 hr after total-body irradiation with 1,000 and $\$, 000 \mathrm{r}^{\circ}$ Survival was greatly enhanced when animals were kept in the cold $\left(5-6^{\circ} \mathrm{C}\right.$.) continuously after exposure. This allered sensitivity was thought to result from decrease in rate of development of radiation damage (prolongation of the latent period) rather than to any appreciable recovery. When animals were removed from the cold after 60-130 days, there was no change in absolute survival or any clear difference in the time course of deaths from that observed in irradiated animals mañtained at $23^{\circ} \mathrm{C}$. (YBR) 1632

Patt, Harvey M. Marguerite N. Swifs, and Ella B. Tyree. INF LUENCE OF TEMPERATURE ON RADIO-SENSITIVITY IN THE IROG (RANA PIPIENS). Federation Proc. 7., 90-1(1948).

Influence of temperature on survival after X-irradiation was studied in frogs. In preliminary experiments animals were given.total body $\mathrm{x}-\mathrm{radiation}$ at $23^{\circ} \mathrm{C}$ and were kept at different temperatures after irradiation. Survival of experimental animals was corrected for the small mortality observed among non-irradiated controls. Results are tabulated. Prolonged survival with low temperature treatment is clearly evident. Some $30-90 \%$ of animals kept at $5^{\circ} \mathrm{C}$ for periods of 3 to 4 months after irradiation have survived a dose which is lethal to animals at $22^{\circ} \mathrm{C}$ within 3 to 6 weeks. Experiments are in progress to determine whether the altered radio-sensitivity is accomplished merely by a decrease in the rate at which degenerative changes oceur or by a more rapid and connplete recovery from the effects of irradiation. (FP) 1633

Smith, Wille W., Benjamin J. Highman, J. R. Míchell, and Henry C. Bloun, Jr. EFIEECT OF ENVIRONMENTAL TEMPERATURE ON THE RESPONSE OF MCE TO WHOLE BODY ROENTGEN RADIATION. Proc. Soc. Exptl. Biol. Med. 71, 489-501(1949).

Young male mice were irradiated in groups of 10 with approximately $500 \mathrm{r}$ delivered by a $170-\mathrm{kw}$ machine at a focal distance of $50 \mathrm{crn}$ and 2 dose rate of $56-64$ r per minute, the radiations being screened through 0.55 mm of

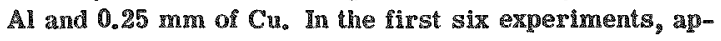
proximately half of the animals were previously acclimatized to the temperature conditions to which they were subjected after the radiations; in the $30^{\circ} \mathrm{C}$. enviromment, $46 \%$ of those acclimatized died compared to $63 \%$ of those not acclimatized, while in the $10^{\circ} \mathrm{C}$. environment, $52 \%$ of those acclimatized died in comparison to $71 \%$ of those not acclimatized. Leucocyte counts made on the first and fourth days after irradiation ghowed no difference attributable to the temperature. In the last three experiments, when the mice were caged singly and thus were unable to huddle logether, $69 \%$ in the $10^{\circ} \mathrm{C}$. room died compared with $39 \%$ in the $30^{\circ} \mathrm{C}$. room. The results show that the net effect of a sufficiently cold environment is to lower the resistance of mice to the lethal action of $\mathrm{x}$-rays; subjection to the environment previous to the irradiation favors survival. It was also noted that mice kept in a $30^{\circ} \mathrm{C}$. environment, which die following irradiation, frequently show fatty changes in the liver. (NSA)

1634

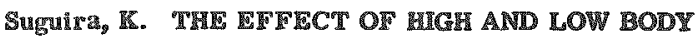
TEMPERATURES UPON TME GROWTH OR MRRADATID MOUSE SARCOMA 180. Radiology 3\%, 85m-93(1941).

X ixradiation of 500,750 , and 100 r was given in vivo to 7 days old (5-8 mm) sarcomas. Within 2 hr after s-ray treatment the animals were exposed to uitra short radio waves at a potential of 17 or 18 volts $10 \mathrm{~s} 5$ to $30 \mathrm{~min}$; the short wave treatment was repeated on 5 for 6 successive days. X-ray treatment of 500 a alone caused $\%$ regressions, when combined with heat $20 \%$ regressions; with $750 \mathrm{r}$ s.trays alone $15 \%$ regresslons and combined with 
heat $45 \%$ regressions resulted; with $1000 \mathrm{~m}$ alone $50 \%$ and in combination with heat $75 \%$. Transplants of sarcoma 180 were made into mice kept for 1 to 3 days in a refrigerator at $3-5^{\circ} \mathrm{C}$, they were then returned to the low temperature environment for 4 weeks. Tumor growth was retarded during the 1 st week but similated that of the controls thereafter. Takes occurred in $100 \%$ of the animals. When tumors were removed from refrigerated animals and transplated to normal mice at 1,2 , and 3 weeks, takes were similar to the controls. Mice bearing 7 oday old sarcoma 180 were refrigerated at $-10^{\circ} \mathrm{C}$. and after death were kept at $3^{\circ} \mathrm{C}$, for $48-72 \mathrm{hr}$. The tumors were then transplanted to normal mice; except for a 1 week delay in growh trans missibility was not affected. Growth capacity of the sarcoma was completely destroyed if the host was sacrificed by a blow or anesthesia and the body kept at $22^{\circ} \mathrm{C}$. for 18 hr. Tumors, 7-day old, were inordinated with dosages of $500 \mathrm{r}, 750 \mathrm{r}$, and $1000 \mathrm{r}$ and within $3 \mathrm{hr}$ the animals were placed in 3 to $5^{\circ} \mathrm{C}$ refrigerators and kept there for 4 weeks. With 500 r no tumor regressions occurred although there were $3 \%$ in the controls. With $750 \mathrm{x}$ alone there were $15 \%$ and with refrigeration, $12 \%$; with $1000 \mathrm{r}$ alone $50 \%$ regressed and with refrigeration only $27 \%$. Small fragments of tumor weighing approx. $6 \mathrm{mg}$ were placed upon filter paper moistened with Locke-Ringer soln. wrapped in paramm and placed in thermos bottles with solid $\mathrm{CO}_{2}$, removed at varying intervals $(1-168 \mathrm{hr})$ thawed to room temperature and inoculated into normal mice. Growth was only slightly inhibited, the takes numbering 80 to $90 \%$. (BA) 1635

Suskind, S. R. RESUSCITATION OF HEAT $\sim$ INACTIVATED SELDS BY X-RADLATION. J. Heredity 41, \$7-102(1950).

Previously reported resuscitation of heat-inactivated seeds by $\mathrm{x}$-radiation was not confirmed. Changes in humidity and subsequent changes in the moisture content of the seeds caused considerable variation in lethal temperature. A refined technique using a modified Abderhalden drying apparatus and constant boiling liquids, proved to be an accurate and versatile method lor the heat treatment of biol. materials. (auth)

\section{6}

Trudova, R. G. EFPECT OF TEMPERATURE ON THE SENSTIVITY OF WHEAT GERMINATION TO X RADIATION. Doklady Akad. Nauk. S.S.S.R. 79, No. 2, 353-6 (1951).

Effects on the mitotic activity of the root-tip meristem of wheat of $500 \mathrm{r}$ of $\mathrm{x}$ radiation prior to exposure at 0,20 , and $36^{\circ} \mathrm{C}$ and of $250,500,1000$, and 2000 r following exposure to these temperatures are presented graphically. (NSA)

Radiation Chemiatry of Aqueous Solutions

1037

Allen, Alexander J., Robert 2. Steiger, Mary A. Magill and Rachel G. Franklin. AMTNO ACIDS, ACYLAMINO ACDOS, DIPEPTIDE, OCYL DIPEPTIES AND DERIVATIVES OF THESE COMPOUNDS. II. EFFECTS OF IRRADIATION WITH CATHODE RAYS AND ULTRAVIOLET LICHT. Biochem. J. 31, 195-204(1937).

Solutions of several amino acids and a large number of derlvatives of amino acids and dipeptides were submitted to the action of cathode rays and ultraviolet light. In every case the compounds underwent a change and $\mathrm{NH}_{3}$ was liberated. The absorption after irradiation was ugually shifted toward the longer wave lengths. Possible reaction mechanimms are given. (CA)
1638

Allen, A. O. RADIATION CHEMITTY OF AQUEOUS

SOLUTIONS. J. Phys. \& Colloid Chem. 52, 479-90(1948).

Water irradiated by 1 mev cathode rays or $x-$ rays decomposes to $\mathrm{H}$ and $\mathrm{H}_{2} \mathrm{O}_{2}$, the latter subsequently decomposing to yield 0 . The decomp. is reversible and comes to a standstill when the $\mathbb{H}$ pressure over the water reaches 1 or $2 \mathrm{~cm}$. Previous claims that water is stable to $\mathrm{x}$-rays resulted from failure to detect the very small concn, of decomp. products present at the steady state. More densely ionizing radiations yield higher steady state II pressures; h-rays from tritium (av. energy $5 \mathrm{kv}$ ) give $10 \mathrm{~cm}$ or more, cyclotron deuterons (8 mev), pile radiation (mixed $\gamma$-rays and fast neutrons) give 1 atm. or more, and $\gamma$ rays apm parently give a still higher value. All types of radiation break up $\mathrm{H}_{2} \mathrm{O}$ to free radicals $\mathrm{HI}$ and $\mathrm{OH}$. The products, mol. $\mathrm{H}$ and $\mathrm{H}_{2} \mathrm{O}_{2}$, form by dimerization of these radicals in regions where they are produced in relatively high concn. Radicals that escape by diffusion into the main body of water react with the products, reducing or oxidizing them back to $\mathrm{H}_{2} \mathrm{O}$ by a chain reaction process analogous to the gas-phase combination of $\mathrm{HI}$ and $\mathrm{Cl}$ mols. The fraction of radicals that escape is smaller the more densely ionizing the radiation; hence the steady-state concn. of If for fastelectron or pile radiation was found to be greatly increased by the presence of solutes that react with solute. Two possible theories are suggested for the regular increase of Initial yleld with ionization. (CA)

1639

Anderson, R. S. and B. Harrison. THE QUANTITATIVE EISTCT OF X-RAYS ON ASCORBIC ACD IN SIMPLE SOLUTION AND IN MLYTURES OF NATURALLY OCCURRING COMPOUNDS. J. Gen. Physiol. 27, 69-75(1943).

Data are presented on the disappearance of ascorbic acid in inorganic soln. under irradiation with $x-$ rays. The presence of serum albumin or plasma does not materially affect the reaction, but in the presence of rat muscle this is considerably smaller. (CA) 1840

Barron, E. S. Guzman and Veronica Flood. TIE OXIDATION OF SULFYDRYL-CONTAINING SUESTANCES BY IONIZING RADIATIONS, p. 116-40 Of MEDICAL AND BIOLOGICAL DIVISIONS QUARTERLY REPORT, FEBRUARY, MARCH, APRI, 1948, Austin M. Brues and Jermann Lisco, eds. Argonne National Lab. May 1, 1948. 25p. (ANL-4163(p.116-140))

The oxidation of the sulfhydryl groups of glutathione, 2,3-dimercaptopropanol, and propane 1,3-dithiol, under the action of ionizing radiations produced by $x$-rays and $\gamma$ rays, has been studied. $X-r a y$ dose varied from 500 $5,000 \mathrm{r}$, and $\gamma$ radiation from 270 to $1,380 \mathrm{r}$. In mildly acid solutions (pH 5 or below) glutathione was not appreciably oxidized by $500 \mathrm{r}$. At pH values above 6 , oxidation was readily measurable. The amount of oxidation, at constant $\mathrm{pH}$ values and constant $-\mathrm{SH}$ content was proportional to the amount of radiation. Oxidized glutathione was not reduced by the ionizing radiations produced by $x$-rays or $\gamma$ rays. A micromethod for the colorinetric determination of sulfhydryl groups is presented. The biological significance of these experiments is discussed. (auth) 1641

Bonet-Maury, $p$. and M. rilley. PRODUCTION OF $\mathrm{H}_{2} \mathrm{O}_{2}$ IN WATER UNDER THE ACTION OF X-RAYS. Compt. rend. 218, 400-2(1944).

An exptl. detn. of the ratio of the quantity of $\mathrm{H}_{2} \mathrm{O}_{2}$ produced under definite conditions to the energy absorbed by the water. The peroxide is titrated photocolorimetrically. 
The energy absorbed is calculated as the no. (N) of pairs of ions per cc in terms of the number of rontgens measured at the surfaces of the water, the absorption coeff. of the water, and the thickness of the water irradiated. The concn. in $\mathrm{H}_{2} \mathrm{O}_{2}$ increases with $\mathrm{N}$. It is independent of the wave length and the time of irradiation. The method is therefore applicable to the detn. of the absorbed energy regardless of the kind of ionizing radiation employed. It is accurate to $5 \%$ (CA) 1642

\section{Burton, Milton. ELEMENTARY PROCESSES IN TIE} RADIATION CHEMISTRY OF WATER AND IMPLICATIONS FOR RADIOBIOLOGY, p.42-3 of INTERNATIONAL CONGRESS OF RADIOLOGY, 6th Congress, London. 1950.

In the radiation chemistry of aqueous systems, primary ionization processes are more important than primary excitation processes. The ionization mechanism can be divided into three stages: ionization, neutralization, decomposition (Eyring, Hirschelder and Taylor). \$low noving "radiations" which produce a high density of ionization (e.g., protons or alpha particles) are more affective than fast-moving ones giving a low density (e.g., beta particles and electrons from absorption of $x-$ or $\gamma$-rays). The heavy particles readily produce $\mathrm{H}_{2} \mathrm{O}_{2}$ and $\mathrm{H}_{2}$ in pure water, but special means are required to demongtrate chemical ef fects from the fast moving radiations. This was achieved by Allen and Ghormley, who also showed that the difference is, in fact, one of veloeity and not of mass, by demonstrating that the slow-moving electrons emitted in the radioactive decay of tritium produce chemical effects typical of high density ionization. The neutralization process has been examined by Magee and Burton. There are two mechanisms: (1) capture of an electron by a positive ion, followed by dissociation of the excited molecule produced; and (2) interaction with neighbouring molecules, from which decomposition does not necessarily result. In water, the electron primarily enitted is slowed down to thermal velocities and ultimately captured in a dissociative process ylelding atomic hydrogen and the hydrated OH-ion. It is the latter which reacts with hydrated H-ion formed in the ionization column and neutralizes it. The chemical effects observed in water and biological systems are related to four elementary phenomena. (1) II and OH radicals primarily produced enter into back reactions involving $\mathrm{H}_{2} \mathrm{O}_{2}$. Allen has explained the behaviour of radio-sensitizers (e.g., Br-or I-ion) on this basis. (2) 1 and $O H$ radicals may react with redurible and oxidizable entities present in biological systems. (3) The mirroscopic effect of production of high local concentrations of Hoion in the column or of rather high concentrations of OH-ion in its periphery. The associated changes of pH might profoundly affect any labile molecule locally present (Franck). (d) When a hit and ensuing ionization occurs deep within an organic molecule the probability of chemical change (mutation) may be less than when the hit occurs near the surface. Per unit volume large targets may be less effective than small targets.

1643

Casati, Annibale. UNIFIED THEORY OF THE MECHANISM OF ACTION OF X RADIATION. "REDUCING POWER" AND "SECONDARY CHEMICAL ACTION." Radiol. med. 37, 1020-4(1951).

The mechanism of decomposition of chemical substances by $x$ rays is discussed solely in relation to the lonizing action of the radiation. The secondary chemical action is considered to be the intermediate formation of unidentifiable and unrecoverable substances which react with less radiosensitive substances to give the observed results. Thus, an aqueous solution is considered to be a mixture of radiosensitive $\mathrm{I}_{2} \mathrm{O}$ and insensitive solute. A diseussion is given of the protective effect of a solute having great chemical afinity for the decomposition products of $\mathrm{H}_{2} \mathrm{O}$ on the stability of the other solutes. (NSA)

\section{4}

Dainton, I.S. ON TPE EXISTENCE OF IREE ATOMS AND KADICALS IN WATER AND AQUEOUS SOLUTYONS SUBJECTED TO IONIZING RADIATION. J. Phys.\& Colloid Chem. 52, 490-517(1948).

From a consideration of the molecular structure of water, electron-impact data on water vapor, and the general mechanism of energy loss of fast charged particles passing through matter, it is concluded that the primary radiochemical act in water vapor is in part ionization, with formation of $\mathrm{H}_{2} \mathrm{O}^{+}$, and $\mathrm{H}^{+}$(and $\mathrm{OH}$ ), and $\mathrm{OH}^{+}$(and $\left.\mathrm{H}\right)$, and in part excitation, probably with dissociation to $1\left({ }^{2} \mathrm{~S}\right)$ and OH (in either the ${ }^{2}$ II or ${ }^{2} \Sigma$ states). Charge neutralization and ionmeutral molecule reactions result in more $\mathrm{H}$ atoms and on radicals baing formed. Effects of radical recombination and wall adsorption on the ionic yleld of water vapor and its dependence on dose rate are discussed. The kinetics of the radiolysis of pure water and of indirect action on solutes are discussed, and it is concluded that in high solute concentrations the lonic yleld should be independent of both solute concentration and dose rate. At low solute concentration under conditions where only a fraction of the avallable radicals react with the solute, $\mathrm{H}_{2}+\mathrm{H}_{2} \mathrm{O}$ are to be expected among the primary products and the lonic yield becomes a function of the dose rate and solute concentration. Very preliminary results are presented on the $X-$ and $y$-ray hinduced and the hydrogen peroside-photosensitized polymerization of acryionitrile and show the OH group to be present. The kinetics of these reactions indicate that 1 atoms and or radicals are formed by the action of $X-$ and $\gamma$-rays on water and that these species are probably not uniformly distributed but exist in high local concentration. (auth)

\section{5}

Dale, W. M. ACTION OF X-RATS ON AQUIOUS SOLUTIONS INCLUDNG THE RELATIONSHTP BETWEN RADIATION EFIECT AND CHEMCAL STRUCTURE, p.43-4 Of INTERNATIONAL CONGRESS OF RADIOLOGY, 6th Congress, London. 1950.

The action of ionizing radiations on substances in aqueous solution is partly direct and partly indirect, the indirect effect being preponderant at the level of concentration which occurs in tissues and cells. In certaln cases the indirect effect can be proved by the fact that the ionic yield is independent of the concentration of the solute over a wide range. Only at extreme dilution is there a falling of in the efficiency of radiation. In other cases this independence does not occur even at higher concentrations, although the radiation action is still mainly indirect. Examples of this type of reaction are the deamination of amino acids and the liberation of sulphur from thiourea. The effective agent in the indirect action of radiation is generally as sumed to be $O H$ radicals and 11 atoms formed from the solvent (water). The presence of a second solute able to react with radicals will reduce the radiation effect on the original one by competing for these radicals. The first solute, therefore, appears to be "protected" by the second solute. The degree of protection afforded can be used as a measure of the radiosensilivity of various solutes of different molecular structure when the size of their molecules and their concentration is taken into account. Substances of low molecular weight with special atomic grouping show wide differences in response to radiation. The simple theory of sharing of radicals by solutes is not guficiclent to explain fully the protection effects observed, 
aince the protectiwe power per unit mass of substance is dependent on the concentration of this substance (phenomenon of the "changing quot lent"). The spectificity of the deamination of amino acids and their derivatives by $\mathrm{X}$-rays again demonstrates the dependence of the radiation effect on the structure from which the amino group is split off. 1640

Dale, W. M. and J. V. Davies. IIBERATION OIF HYDROGEN SULTDE BY K-RADIATION FROM CYSTEINE AND GLUTATHIONE. Biochem. J. 48, 129-32(1951).

The oxldation of the thiol of glutathione or proteins to the disulfide form is considered to be a biologically important step. These experiments reveal that apart from any reversible oxidation there are also irreversible changes produced by $\mathrm{x}-\mathrm{rays}$ leading to the liberation of $\mathrm{H}_{2} \mathrm{~S}$. However, it is not known whether this is an oxidation or reduction process. The discovery of the $\mathrm{H}_{2} \mathrm{~S}$ is due to the fact that the present method of investigation made if possible to determine aceurately the thiol group within a minute fraction of $1 \%$. Although amino actds in general are deaminated by $\mathbf{X}-\mathrm{sadiat}$ ion, will cysteine the radiation energy seems to be used for $\mathrm{H}_{2}$ S production and not for deamination. The instability of glutathione is well known, and these experiments show that glutathione responds to radiation with production of $\mathrm{H}_{2} \mathrm{~S}$, except for a sharp depression at about pH 5.5. (CA)

1647

Dale, W. M., J. V. Davies, and C. Gibert. THL IKINIETICS AND SPICIFICLTHES ON DEAMINATION OF NITROGENOUS COMPOUNDS BY X-IRADATION. Biochem. J. 45, 93(1949).

Cxperiments are described, showing the deamination by x-Trys of some amino acids and their derivatives, polypeptides, and related nitrogenous compounds; the relationship between the lonic yield and the radiation dose had been studled in detall for glyeine. The lonic yleld rose from 0.3 to 2.9 for very dilute to nearly saturated solutions; the ionic yleld for dry glycine was 2.96. An explanation of the high ionic yield with glycine is presented in terms of a hypothests assuming edther a chain reaction or the formation of radicals by excilation in addition to charge neutralization. The continuous rise of the ionic yield is interpreted as being caused by recombination of radicals conpeting with deamination even at the highest concentrations of glycine, due to a low aflinity of glycine to radicals. The explanation offered, although consistent with the experiments described, is concluded to be somewhat unsatisfactory in view of the radiation effects on thiourea. The biological gignificance of the results is discussed. (auth) 1648

Dale, W. M. J. V. Davies and C. Gilbert. THE DEAMINATION OF GLCCLNE BE ALPHA-RADLATION FROM THE DISINTEGRATION OF BORON IN A NUCLEAR PRACTOR. Biochem. J. A5, 543-7(1949).

The deamination of glyeime by the densely lonizang particles from the disintegration of boron by slow neutrons in a nuclear reactor was studied; an account of the necessary technigue is given and the dosimetry is fully discussed. The ionie yields for the liberation of ammonia were 0.17 and 0.56 for the 1 and $20 \%$ solutions of glycine, respectively, being 15 and $19 \%$ of that due to 8 -radiation. The higher efficieney of deamination by this radiation compared with the inactivation of an enzyme by a rays from radon 164 and its products is discussed. (NSA)

Dale, W. M. W. Meredith, and M. C. K. Tweedie. MODE OF ACTION OF TONIZING RADLATIONS ON AQUEOUS SOLUTIONS. Nature 151, 280 1(1943).
Experiments on the $\mathrm{x}$-irradiation of a wide range of concentrations of carboxypegtidase showed that the proportion of the molecules inactivated was determined by the ratio of the dose to the initial concentration and that it inc reased as the ratio increased. Thr may be written $y / y_{0}=f\left(D / y_{0}\right)$, where $y_{6}$ and $y$ are the concentrations before and after receiving a dose of $\mathrm{D}$ roentgens. It is necessary to postulate that the energy received from the radiation forms a quantized intermediate product-perhaps "activated water" which distributes the energy between the active and inactive enzyme molecules, inactivating the former. "Protective" molecules are effective in extremely small concentrations since this transiferred energy is distributed between the two or more molecular species. Protection cannot be due to direct absorption of the $\mathrm{x}$-radiation, since quantities are too small to absorb the energy directly; neither can it be due to compound formation, since there are so many prolecting compounds. Calculations based on the direct-hit theory also fall to account quantitatively for the results obtained. The formula presented explains results obtained 1650

Dunlap, C. E. and $\mathrm{F}$. C. Probbins. THE HFCT OF ROENTGEN RAYS, RADON, AND RADIOACTIVE PLOSPHORUS ON TRIAMN CHLORDDE. Am. J. Roentgenol. Radium Therapy 50, 641-7(1043).

IExposure to radon and to radioactive phosphorus in vitro partially destroyed the activity of aqueous solutions of thiamin chloride as tested on Phycomyees and Phytophthora. Large doses of $\mathrm{x}$-rays had no effect. Erposure of thiamin to large doses of radon did not result in splitting the molecule with anbsequent selective destruction of either the thiazole or pyrimidine intermediate. Heavy If radiation in vivo produced no detectable change in the thiamin content of mouse blood or liver. (BA) 1651

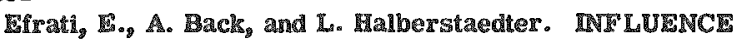
OF THE MEDIUM ON TYE INACTIVATION OF TETANUS TOXIN IT X-RAY IRRADIA TION. Nature 159, 715-6 (1947).

Two types of telanus towin, prepared in different ways, were used to study the influence of the medium on toxin inactivation in vitro by $\mathrm{X}$-rays. The medium was varied by adding a series of proteins and peptones to the toxin samples. The results of the irradiation are given. The addition of different substances to the medium diminished the $\mathrm{x}$-ray effect on the toxin in varying degrees. (S.V.S.) 1652

Farmer, 1 . T., G. Stein, and J. Weiss. CKEMCAL ACTIONS OF IONIZING IRADIATIONS ON AQUEOUS SOLUTIONS. PART I. TNTRODUCTORY RRMARKS AND DLSCRIPTION ON IRPADIATION ARRANGEMENTS. J. Chem. Soc. \$241-5(1949).

The development of the theory of the action of lonizing radiations on aqueous solutions, leading up to the theory of formation of Iree radicals (Weiss, Nature 153: 748 (1944)), is discussed. Apart from the intrinsic interest in the action of the radiations, these investigations are of interest because they allow the reactions of free radicals to be gtudied in the absence of interfering reagents and because the products of reaction are somewhat similar to those obtained in bological systems in which the same molecules are involved. Experimental arrangements for irradiations with $\mathrm{x}-\mathrm{rays}$ ( $\gamma$ rays), neutrons, and $a$ rays, and the radiation dosimetry, are discussed in detail. (NSA) 1653

Fricke, R. Wha B. W. Petersen. THR RELATION OF' CPEMCAL, COLLOTAL, AND BLLOGICAL IFFECTS 
OF ROENTGEN RAYS OF DIFFERENT WAVE-LENGTHS TO THE IONIZATION WHICH THEY PRODUCE IN AR. 1. OXY MEMOGLOBIN IN AQUEOUS SOLUTION. Am. J. Roentgenol. 12adium Therapy 17, 611-20(1927).

X-rays cause the oxidation of oxyhemoglobin to methemoglobin, and is radiating a solution with increasing doses the rate of transformation is proportional to the momentary concentration of oxyhemoglobin. The ratio of the amount of axyhemoglobin transformed to the ionization produced in atmogpheric air is cound indegendent of the wavelength of the rays $(0.25$ to $0.75 \mathrm{~A} . \mathrm{U}$.) when the coefil clents of mass absorption of the oxyhemoglobin solution $\left(\mathrm{u}_{1}\right)$ and of air $\left(\mathrm{u}_{2}\right)$ are equal. When $\mathrm{RCl}$ is added to the oxyhemoglobin solution making $u_{1}>u_{2}$, the ratio is only constant for the longer wavelengths, the chemical actlon of the shorter wavelengths being comparatively too small, which effect is due to the production of Compton-electrons. (BA)

1654

Filley, M. THE RADIOCHEMSTRY OF AQUEOUS SOLUTIONS: A SURVIY OF RECENT FRRNCH RESEARCH. Brit. J. Radiol. Suppl. 1, 50-5(1247).

Work performed on the production of $\mathrm{H}_{2} \mathrm{O}_{2}$ by irxadiation of water is reviewed. The $\mathrm{H}_{2} \mathrm{O}_{2}$ yield is the same for both $\mathrm{X}$-rays and $\alpha$-rays. The role of dissolved orygen in the production of $\mathrm{H}_{2} \mathrm{O}_{2}$ by irradiation of water is described. The $\mathrm{H}_{2} \mathrm{O}_{2}$ yield is increased in the presence of hydroquinone, crysteine ascorbic acid, and aldehydes; organic acids, however, diminish the yield of $\mathrm{H}_{2} \mathrm{O}_{2}$. pH changes and the mechanism of the action of lonizing radiations on aqueous solutions are considered briefily. (NSA)

1655

Gray, L. H. THE PRESUMED DISTRIBUTION OF IONS AND TXCITE GROUPS IN LIQUHDS. J. chim. phys. 48 $17 \%-8(1951)$.

Blological effects are discussed in terms of type of ionization produced by radiation. Dense ionization as produced by a particles results in high local $\mathrm{H}_{2} \mathrm{O}_{2}$ concentratlons. Some blological effects of radiation are enhanced and some are reduced in going from low iomization density to high tonization density. (CA) 1656

Grimmet, L. G. SOME ASPECTS OF TML MECHANISM OF ACTION OF IONIZING RADIATION ON LIVING CELLS. Texas Repts. Biol. and Med. 8, 134-46(1950).

This article discusses some of the theories advanced to account for the effect of radiation on cells and the sensitivity of cells to varying intensities of radiation. The author emphasizes the role radiochemistry can play in explatning the mechanism of cell damage guch as the production of pree radicals from water by radiation. 11 relerences. (NSA)

1657

Iashchenko, L. A. EFIECT OF RADIANT ENERGY ON THE GONADOTROPIC FACTOR OF THE ANTERIOR

LOBE OF THE RYPOPEYSIS. Doklady Akad. Nauk S.S.S.R. 77, No. 1, 157-60(1951).

Anterior pituitary hormone extract was exposed to 30,000 to $1,034,000 \mathrm{~F}$ of $\mathrm{x}$ x'adiation, to 0.022 to $\$ 5.00 \mathrm{med}$ of radium $\beta$ radiation, or to ultraviolet light. Changes in gonadotropic activity are tabulated. Activity changes on up to 90 -days storage following the $100,000-r$ \& ir radiation also are listed. (NSA)

1658

Ifoeng, Virgil L. and J. D. Perrings. PHYSICOCMEMCAL EFPECTS OF RADLATON; 1. FTFECE OF X-RAYS ON FIBRINOGEN AS REVEALED BY TYL ULTRACENTRI FUGE AND VISCOSTYY. LOS Alamos Selentifle Lab. [nd] 28p. (APCU-1725; LADC-102\%)
Bovine fibrinogen irradiated in the dry state and dissolved in $0.1 \mathrm{M} \mathrm{NaCl}$ and $0.1 \mathrm{M} \mathrm{Na}_{2} \mathrm{HPO}_{4}$ has been studied on the ultracentrifuge at various concentrations. SIgnificant differences in the ultracentrifugal behavior were observed for different amounts of radiation. Significant differences in viscosity behavior of irradiated fibrinogen dissolved in $0.1 \mathrm{M} \mathrm{Na}_{2} \mathrm{HPO}_{4}$ from the control unirradiated fibrinogen were observed. Fibrinogen ir radiated in the presence of oxygen, nitrogen, and helium was studied on the ultracentrifuge. Fibrinogen irradiated in the presence of hellum showed greater alteration than in either orygen or nitrogern. Fibrinogen irxadiated when dissolved in $0.1 M$ $\mathrm{Na}_{2} \mathrm{HPO}_{4}$ showed alteration. When the radiation was inereased a heavier second component appeared on the ultracentrifuge diagram. Less radlation is requiged in the dissolved state than in the dry state to produce comparable changes. At least $1 \times 10^{8} \mathrm{r}$ is necessary in the dry gtate to produce a significant change in the fibrinogen. in the dissolved state $5 \times 10^{6}$ is is sufficient to produce a significant change. (NSA)

1659

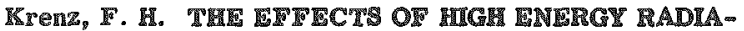
TIONS ON WATER AND AQUPOUS SYSTEMS. CAN. J. Research 8, 26, 647-56(1948).

In order to obtain acter ldea of the effect that irradiated water has when biological systems are exposed to high energy radiation, the properties of the water were investigated. Evidence was obtained for vibrationally excited "polymers" of water which result in long lived activity. It is concluded that the large "indirect effect" of water on biological systems may well be due to the properties of the "polymers." A cross gection of the dilatometer used in measuring the water expangion upon gamma radiation, erpansion curves and curves of lag elfect in water and some aqueous solutions are presented. 35 references. (NSA)

1660

Landau, 1 . G. AROBLEM IN RADIOBLLOGY: DIF FUSION AND RECOMBINATION OF IONS. Bull. Math. Blophys. 12, 27-34(1950).

The problem of the distribution of ions which are formed in the path of an lonizing particle and undergo difuston and recombination is solved by a perturbation method. When diffusion is more important than recombination this treatment gives a more accurate distribution than previous approsimations. (CA)

1661

LOISEleu, J. FLECTRONC EXCHANGES IN WATER ACTEO ON BY II-RAYS. Compt. rend. 214, T(1942).

The amount of $\mathrm{H}_{2} \mathrm{O}_{2}$ formed on irradiation whin $\mathrm{x}-\mathrm{ray}$ was found to increase with decreasing $\mathrm{pH}$; but the pH of pure water was found to decrease on irradiation. From the second lact, it is agreed that the primary step must be disappearance of $\mathrm{OH}^{-}$. The prowosed mechandam is: $2 \mathrm{OH}^{-}+$ $\mathrm{O}_{2}=\mathrm{H}_{2} \mathrm{O}_{2}+\mathrm{O}_{2}^{=}$tollowed by $\mathrm{O}_{2}^{=}+2 \mathrm{H}^{+}=\mathrm{H}_{2} \mathrm{O}_{2}$. (CA) 1662

Loiseleur, J. and C. Crovisier. INCREASID IN VISCOSITY AND REFRACTIPE INDEX OF WATER SUDJECTED TO THE ACTION OF X-RAYS. Compt. rend. soc. biol. 136, 739-40(1942).

Twice-distlied water was exposed to the $\mathrm{K}_{a}$ rays $(x=0.71 \mathrm{~A})$ from a Mo target for long periods. The viscosity and $\lambda$ increased very glightly in all cases. Small quantulies of $\mathrm{H}_{2} \mathrm{O}_{2}$ were formed in water contahning dissolved $\mathrm{O}_{2}$, but not in water freed of $\mathrm{O}_{2}$. (CA)

\section{3}

Lolseleur, J. and R. Lataret. DEMONSTIRATON, BY DIRECT POTENTIOMETRIC MEASUR MEMT, OF THY 


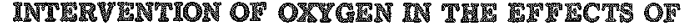
1-RAss. Compt. rend. soc. blol. 135, 1534-7(1041).

In saturated solutions at pIi 6 in water freed of air or 0 , hydroquinone is not oxidized by irradiation with $\mathrm{I}_{\alpha} \mathrm{X}-\mathrm{rays}$ from 2 Mo target. The Pt-electrode potential of the solution remains unchanged or decreases slightly. In water saturated wtth al $x$, oxidation occurs and the Pt-electrode potential gradually rises as more and more quinone is sormed. (CA)

\section{4}

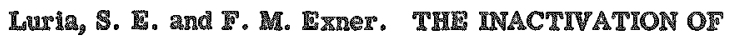
BACTEROPRAGES BW X-RAYS-INELUENCE OF THE MEDUUM. Rroc. Nat. Acad. Scl. U. \$. 27, 370-5(19A1).

X-rays have an "Indirect" effect in water or saline suspensions of bacteriophages. Probably some agent is produced in the medium by the irradiation which orddizes or reduces the phrges. In the presence of forelgn proteins bacteriophages are also inactivated by 8 -rays, but with reduced sensitivity. This seems to be a "direct" effect on phage itself. (BA)

1665

Mathis, Alice L., Robert Tr. Brooks and Rerman Schneiderman. STUDIRS IN TEY HYALURONDDASE-RTALURONC

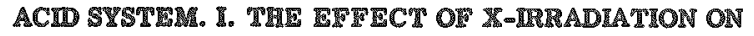
HII ALURONIC ACD. University of Callomia, Los

Angeles. Feb. 11, 1049. 10p. (ARCU-170; UCLA-11)

Hyaluronic acid is a mucopolysaccarde which is believed to be composed of equimolecular amounis of acetyl glucosamine and glucuronic acid. X-irradiation was lound to cause a change in potassium hyaluronate solution, which manifeste itgelf by a reduction in viscosiiy. Aiter cessathon of the irradiation, the viscosity decreased to some es 1666 tent for period of about five hours. (NSA)

Minder, W. THI PROBLIS OF TRANSTERENCE OF ENERG IN BIOLOGICAL MRAADITION. Radiologia Clin. Suppl, 15, 81-5(1946).

The effects of irradiation on substances of known chemical composition in aqueous aolutions are exerted in a sound about way through the agency of the solvent, water. A trangference of energy mugt take place from the $\mathrm{H}_{2} \mathrm{O}$ molecules activated by the radiation to the dissolved substance. As a result of radiation below $25^{\circ}, 35^{\circ}$, and $50^{\circ}$ respectively, solutions of bromoform, ortho-dichlorobenzene and ferrous sulphate in water show no temperature coefficient of reaction. Irradiation with $\mathrm{X}$ rays on radium does not afiect frozen (solid) solutions. This shows that the energy-transfer and therefore the radiation effect is ony possible where there is Ireedon of molecular movement (diffuston). (NSA)

\section{7}

Minder, W. RAOIO-CHIMISTRY OF ORCANC MALOCEN COMPOUNDS, p. 44 Of INTIRNATIONAL CONGRESS OF RADIOLOCY, Gth Congress, London. 1950.

The formation of halogen hydrides by irradiation of aqueous organic halogen compounds is certainly an indirect effect via the solvent nolecules. The yield is independent of the concentration of the irradiated solution, and the action is completely suppressed in the irozen state. The yleld is, whithout exception, amaller with aromatic than with aliphatic substances, and in the experiments described is of the order of 0.16 and 2.6 molecules per lon. The rem action 15 altered quantitatively and qualitatively by adding other substances (protection effect). From the lindting values of the independence of concentration on the one side, and the absolute values of protection on the other, the maximal distance of the diffusion of energy, free from loss, can be determined. The results give values which are in good agreement and, according to the substance concerned, vary between 20 and 100 times the diameter of the molecule of water. Halogen hydride is also formed when halogens containing hydrocarbons are irradiated in organic solutions (alcohol, acetone). The yield is proportional to the concentration in small concentrations, and reaches a limiling value in greater concentrations. The action of this Irradiation also muat be considered as an indirect effect although in the higher concentratlons a drect effect is superimposed. Addution of water up to a molecular matio $1: 1$ does not change the yield. The "effective volume" (ratio between lonic yield and concentration) is approximately 100 to 200 times greater than the dimension of the reacting molecules.

1668

Mosher, Wm. A. IONS AND RADICALS IN BIOCHEMICAL PROCESSES. Conf. on Biol. Antiowidants, Trans. Ath Conf. 13-44(1949); J. Fr ranklin Inst. 251, 665-74(1951).

A discussion of free radical formation, ionic oxidation of organic compounds, and effects of $\mathrm{x}$-irradiation and free radicals on mucleic acids. The gray is a producer of free radicals rather than a producer of ionized particles. $X$ rays, neutrons, ultraviolet light, and sonic vibrations have proved effective in the depolymerization of desoxyribonucleic acid in vitro. If radical chain-stopping agents, such as thiourea, are added to nucleic acid there is marked protecton from the destructive effect of $x-$ rays, chemically produced radicals, and other physical agents. This protection can be looked upon as a type of antionidation. (CA) 1669

Mullis, A., W. Minder, A. Liechli, and F. Wegmuller. FNPERIMENTS WTTH A MODEL ON THE BIOLOGICAL PRIMARI EFIECT OI IRRADITION. Radiologia Clin. 15, $295-312(1946)$.

The living cell is too complex a system for the study of the analytic effects of radiation, and therefore atiempis were made to examine the "physical primary effect", of rays on artificial models of simple construction. IEarlier experience has ghown that halogen compounds of benzene are hydrolyzed by $x-r a y s$, with production of an acid consisting of the halogen and hydrogen. Hydrolysis was observed to be proportional to the radiation applied, while it had no relation to the concentration of the compound. The rate of hydrolysis was conditioned on the dose of radiation and on the number of chlorine atoms in the molecule of the compound. In the work presented the radiation hydrolysis of $\mathrm{g}$-dichlorobenzene and of hexachlorobenzene was studied. When p-dichlorobenzene was dissolved in ether and the latter dissolved in water no hydrolysis occurred by radiation, but when a saturated aqueous solution of the compound was used, hydrolysis, production of hydrochloric acid, and a corresponding increase in the conductivity of the solution were observed. The ionic yield of the reaction was rather small (0.33 5\%), indicating that ony a minimal part of the substance had been converted. It is important. to note that in the case of mono-chlorobenzene approzimately half the amount of hydrochloric acd was produced in comparison with $\mathrm{g}-$ dichlorobenzene. In the fully substituted compound $\mathrm{C}_{5} \mathrm{Cl}_{6}$ no hydrolysis on irradiation was observed. Therefore it seems probable that production of hydrolysis should not be regarded as an isolated function of the chlorine atom, but that co-operation of hydrogen atome present in the compound may have some additional and important function. The fact that hydrolysis of $\mathrm{p}-$ dichorobenzene was hampered by the presence of ether is worth noting. (AWM)

1870

Pickels, 1. G. and R. \$. Anderson. MOLECULAR ASSOCIATION OF MEMOCYANDN PRODUCED BY IX-RATS AS 
OBSERVED IN THE ULTRACENTRIYUCE. J. GEn.

Physiol. 30, 83-99(1946).

When normal, monodisperse hemocyanin $(60.5$ \$) from Limulus polyphemus was irradiated in neutral buffer with T-rays, several new, more rapidly sedimenting ultracentrifugal components (86 S, $107 \mathrm{~S}, 122 \mathrm{~S}$ ) were produced, with a corresponding loss in the amount of the unaffected protein. The amount of the effect was roughly proportional to the amount of irradiation. The new resolvable components apparently represented an association of the primary particles into aggregates of 2,3 , and 4 primary particles, respectively. The proportional amount of hemocyanin affected decreased almost to the vanishing point as the conc. of the protein was raised to high levels. The absolute effect, i.e., the total number of particles a fected in a given wol., increased with the conen. of hemocyanin, at least for concris. below 15\%. The presence of 33\% horse serun during irradiation inhibited the effect on the hemocyanin almost completely, with hemocyamin concns. of both 0.8 and $14 \%$. The presence of $2.8 \% \mathrm{egg}$ alloumin during irradiation lowered the effect by about $70 \%$ in the cage of dilute preparations $(0.8 \%$ hemocyanin) but by only about $25 \%$ in the case of $14 \%$ golutions. A lowering of the solution's oxygen tengion during irradiation enhanced the effect, almost doubling it in come cases. (BA)

1671

Scholes, George and Joseph Weiss. FORMATION OF FYDROXYLAMINE FROM AMINO COMPOUNDS IN AQUEOUS SOLUTION BY IRPADIATION WITH X-RAYS.

Nature 167, 693(1851).

$\mathrm{NH}_{2}$ OH is produced upon irradiation of adenine, granine, cytosine, alanine, lysine, ethylamine, and histamine with $\mathrm{x}-\mathrm{ray}$. No $\mathrm{NH}_{2} \mathrm{OH}$ is obtained from hyoosanthine, uracil, uridine, and $\mathrm{NH}_{3}$, and only mall amounts are obtained from nucleic acids. Yields of $\mathrm{NH}_{2} \mathrm{OH}$ depend on the pH. Ir radiation of solutions of $\mathrm{NH}_{4}$ aalts by $\mathrm{x}$-rays results in oxidation to nitrate. (CA)

1672

Stein, G. and 3. Weiss. CHEMCAL FWECTS OT IONIZING RADIA TIONS. Nature 161, 650(1948).

Simple org. compounds, e.g., benzene and benzolc acid, in dilute aqueous systems have been exposed to penetrating radiations and the reaction products studied. When benzene was irradiated by $X-r_{a y s}$, about 0.1 millimol. of phenol was formed, also, a smaller quantity of biphenyl among other substances. In similar experiments with a neutron$\gamma$-ray source (corresponding to $1 \mathrm{~g}$ Ra mised with Be powder) benzene gave, in addition to the products mentioned above, catechol and products originating from the opening of the benzene ring. In all cases the total yield of the hydroxylated reaction products depends on the $\mathrm{BH}$ of the solutions. (CA)

1673

Stein, G. and J. WeIss. CHEMCAL ACTIONS OF IONIZING RADIATIONS ON AQUEOUS SOLUTIONS. IV. THE ACTION OF X-RAYS ON SOME AMTNO ACIDS. J. Chem. Soc. $3256-63(1249)$.

Glycine $(0.4 \mathrm{~g})$ in $\mathrm{H}_{2} \mathrm{O}(100 \mathrm{cc}$.) Irradated in vacuo with $\mathrm{x}$-rays $\left(10^{5}-10^{6}\right.$ ) gave $\mathrm{H}, \mathrm{NI}_{3}$ (0.1 millimole appros.) and HCHO (traces; recognized by isolation of the asdinitrophenylhydrazone). In comparable experiments alanine gave $\mathrm{H}_{,} \mathrm{NH}_{3}$ and $\mathrm{MeCHO}$; serine gave $\mathrm{H}_{3} \mathrm{NH}_{3}$, glyoral and glycolaldehyde. The amounts of $\mathrm{H}_{3} \mathrm{NH}_{3}$, and aldehydes were noninear function of the irradiation dose and both oxidative and reductive mechanisms contribute to the deamination. (CA)
1074

Svedberg, T. and \$. Brohult. SPLTTWING OF PROTEN MOLECULES BY ULTRA-VIOLTI IICHT AND ALPHA

RAIS. Nature 143, 038-011939).

Irradiation of hemocyanin, hemoglobin, and serum albumin with uv light and a-rays, both at room temp. and at the temp. of liqud alr slsows that in the case of hemo. cyanin the splitting into half mols. is a primary reaction. In the case of hemoglobin and gerum albumin the irregular breakdown is caused by secondary reactions. The amount of hemocyanin díssociated by a given no. of a-particles can be accounted for upon the assumption that each mol. struck by an a-ray is splist into halves. (CA)

1675

Tchaper of ; 1. C. C. DETERMNATION OF THE ERTET OF TRRAOLATON BY X-RAY ON P-AMMOBENZOIC ACID, USING CLOSTRIDIUM ACFTOBUTYLICUM POR ABSAT. Can. J. Research 24:

A study has been made on the effect of $\mathrm{z}-\mathrm{z}$ ay irgadiation by produeing inactivation of p-aminobenzole acid as determined by assay with $C$. acetobutylicum. it has been shown that p-aminobenzolc acid in concentration of $1 \times 10^{-6}$ Mgm per ce is inactivated by an X-ray dose as small as $50 \mathrm{~F}$ at $190 \mathrm{kw}$. The signinicance of the destruction of Paba in connection with the ireatment of Iniective lestons is discussed. (auth) 1676

Welss, Joseph. RECENT WORE ON THE $\triangle$ CTION OF IONISING RADLATONS ON AQUEOUS SYSTERS, p.165-6 OF INTERNATIONAL CONGRESS OF RADIOLOGY, Oth Congress, London. 1050 .

Chemical compounds of biological importance digeused are: (a) Amino-acids. The action of loniging radiations on amino-acids leads to deamination, and the process is in certain respects similar to the biological process of deamination. Ammonia, which is formed in this process, is capable of producing mutation effeets. A number of different amino-acids have been atudied in detall. The simplest of these (glyeine) is known to be a precurgor of the blood pigment in the human body. (b) Nucletc Acids (Nucleotides and Nucleosides). The action of J-Irays on different types of nucleic aclds in aqueous systems has been investigated. On irradiation of these solutions ammonia is formed and from the solution a number of low molecular products including nucleogides and Iree purine bases have been isolated and identified. The constituent bases are themselves also attacked as evidenced by the production of ammonia and by increase in the number of free anino-grouplings. The action of $\mathrm{X}$-rays on nucleotides and on some nucleosídes has recently been investigated. (c) Sterols. The irradiation of cholesterol by X-rays in aqueous systems leads principally to the formation of two substances, one of which seems to be of particular interest as it has been isolated from the arterlogelerotic humnan aorta, from pig's spleen and testis and from on liver, and it may be of some importance in sterold metabolism. The action of X-rays on pregnenolone and on 2 number of steroid hormones in agueous systems has also bees investigated.

\section{VI}

\section{GROWTH AND DEVELOPMENT}

1677

U. S. Department of Agriculture. 1948 IFIED RESULTS WITH CERTAIN RADIOACTVIE MATERELIS AS PLANT 
STIMULANTS; PROGRESS REPORT. May 1949. 43p. (M-4376)

Field experiments with radioactive materials as plant stimulants were conducted during the 1948 erop season by the Bureau of Plant Industry, Soils, and Agricultural Engineering, in cooperation with the State agricultural experiment stations of Arkansas, Ilinois, Kentucky, Michigan, Missigsippi, Montana, North Carolina, Ohio, New York, Washington, West Virginia, and the College of Agriculture, Univergity of Georgia. Three radioactive materials were used in the field experiments: Alphatron (commercial material) at the rate of 5,10 , and 20 pounds per acre; radium (as used, contained $30 \mu g$ of Ra per pound); and uranyl nitrate. The data from these field experiments is presented in this report in tabulated form. The general conclusion drawn from the data is that no effect of the radioactive materials was found, either beneficial or harmful. There were a few cases of differences that reached the $5 \%$ level of gignificance, but they were no more than would be expected from normal distributions. No comment is made 1678 with regard to the individual experiments. $(\mathrm{S} . \mathrm{V} . \mathrm{S}$. $)$

Alexander, Lyle T. RADIOACTIV MATERIALS AS PLANT STIMULANTS. FIELD RESULTS. Agron. J. 42, 252-5(1950).

Applications of alphatron, radium, and uranyl mitrate in fleld experiments with a number of crops showed that these materials had no effect, either beneficial or harmful. (CA) 1679

Amoroso, E. C. and A. S. Parkes. EFFECTS ON EMBRYONIC DEVELOPMENT OF I-IRRADIATION OF RABRIT SPIRMATOZOA IN VITRO. Proc. Roy. Soc. London, B $134,57-79(1347)$.

Rabbits in which ovulation was induced artificially by intravenous injection of $50 \mathrm{I}$. U. chorionic gonadotrophin were inseminated with spermatozoa exposed in vitro to doses of $x$-rays varying from $50-100,000 \mathrm{r}$, the ova being examined grossly, under visual microscopy with hematoxylin eosin and under ultraviolet microphotography. At $250 \mathrm{r}-1,000 \mathrm{r}$, the normal divisions dropped progressively below the normal of $80-90 \%$ down to $2 \%$ independently of intensity or type of irradiation and time of irradiation before ingemination (5-17 hours). Entry of spermatozoa into ova was effected at all dose levels. Cytological damage to the spermatozon nucleus appeared at $10,000 \mathrm{r}$, polyspermy appeared at 1,000 r. Activation occurred at all levels, but syngamy became irregular at $10,000 \mathrm{x}$ with suppression of spindle fiber formation at higher dosages. Occasional haploid and triploid ova were observed at the one-cell stage, but not in divided ova, and there was mo continued gynogenetic development. From 50-250 r, normal fertile young were born. At $500 x$, incidence of pregnancy and litter size were reduced, young were less viable, but. aurvivors were normally fertile. No implanted embryos, feluses or living young were obtained from spermatozoa exposed to $1,000 \mathrm{I}$ or over. (BA) 1680

Barr, J. S., J. R. Lingley, and E. A. Gall. THE EFTECT OF ROENTGEN IRRADIATION ON FPIPIYSEAL GROWTH. I. EXPLRIMENTAL STUDIES ON THE WHITE RAT. An. J. Roenthenol. Radium Therapy $49,104-15$ (1043).

Groups of 30,90 , and 180-day old albino rats were subjected to roentgen irradiation of one knee-joint, and the resulting changes atudied histologically and roentgenographically. Doses of $665-1,165 x$ produced moderately severe changes; doses of $1,335-1800 \mathrm{r}$ caused very severe damage, indicated by disruption of cartilage cell columns and degtruction of chondracytes. There was no evidence indicating stimulation and practically none of regeneration of epiphyseal cartilage. Other tissues showed only transitory changes. No degenerative or traumatic changes were found in the irradiated joints even after 9 months' observation. The doses used all produced retardation of growth, which varied roughly in proportion to the dose. Doses in excess of $1300 \mathrm{r}$ produced essentially complete arrest of growth of the treated epiphysis. (BA)

1681

Berk, Sigmund. BIOLOGCAL EFFECTS OF IONIZING RADIATIONS FROM RADIUM AND POLONIUM ON FUNGI.

PART III. THE PREVENTION OF MOLD GROWTH IN OPTICAL INSTRUMENTS SERIES. Pitman-Dunn Labs., Frankford Arsenal. July 1951. 89p. (NP-3798; IA Report $\mathbb{R - 1 0 1 6 )}$

Constant irradiation with various $7.5-\mu g$ sources of Ra inhibited the sporulation of Chaetomium globosum, Lenzites trabea, Aspergillus niger, A. flavus, and a species of Penicillium growing on potato-dextrose agar. There was an appreciable difference in radiosensitivity of the species. It was concluded that $a$ particles were primarily responsible for the effects observed. The biological effects of the irradiation of dry A. niger spores with $\mathrm{PO} \alpha$ particles was dependent on the dose: (a) the lowest dose showing any visible ffect (114 $\alpha$ or 26,900 rep) interfered with the sporulation of the fungus; (b) higher doses interfered with the extent of vegetative proliferation of the fungus; (c) larger doses (1000 or or 236,000 rep) inhibited the production of a germ tube; (d) still larger doses caused abnormally swollen spores which failed to produce germ tubes, and (e) doses in excess of $25,000 \alpha$ particles or $5,900,000$ rep were lethal. Isolates made from A. niger cultures that had been bombarded with Ra o particles yielded a number of mutant strains. Some of the variants were characterized by dwarf and giant conidial heads, others by a short conidiophore and a powdery type of sporulation. (NSA)

1682

Blanc, R. and C. Villee. DIF $\mathrm{CRENTIAL} \mathrm{REACTIONS} \mathrm{OF}$ VARIOUS SCUTE STOCKS OF DROSOPHILA MELANOGASTER TO $\mathrm{W}$-RADIATION. Genetics 26, 140(1941).

In a preliminary investigation of the phenotypic effects of X-rays on the bristles of different stocks of Drosophila melanogaster, different members of the scute series, sc, $\mathrm{sc}^{5}, \mathrm{sc}^{6}$, and $\mathrm{sc}^{10}$, a wild type, Canton $\mathrm{s}$, and various compounds of these were subjected, while prepupae, to 2115 r units of 5 -radiation. Fxperimental and control flies were left to hateh in petri dishes on moist filter paper at $25^{\circ} \mathrm{C}$. The imagines were recorded for bristle effects. A total of 1659 experimental and 1363 control flies were examined. The bristle effects produced were chiefly complete absence or great reduction in size, although a lew bent, curved, or doubled bristles occurred. The percentages of the different bristles affected in the various stocks were compared using the effect in Canton $\mathbf{S}$ as a "norm." It was found that the effects of X-rays on the bristles differed in the various stocls used, so that in each case a pattern of X-ray effects specific for the stock was super imposed on the bristle pattern characterigtic for that stock under control conditions. It was found that those bristles affected by the scute genotype under control conditions are more likely to be affected by $\mathrm{X}$-radiation than those not. In other words, the combined effect of genotype and X-radiation is greater than would be expected on the basis of simple summation. This suggests that $\mathrm{X}$-radiation and the scute genotype affect the same or closely integrated processes. (G)

1683

Bliss, C. I. and C. Packard. STABILITY OF TKE STAND- 
ARD DOSAGE-EFFET CURVE FOR RADIATION. Am. J. Roentgenol. Radium Therapy $46,400-4(1941)$.

The standard dosage-effect curve for Drosophila eggs and $\mathrm{X}$-irradiation was tested for statistical accuracy and possessed greater stability in both slope and position than had been generally reported in investigations of toric effects. (BA)

1684

Blum, Harold F., J. Courtiand Robinson, and Gordon M. LOOS. THE LOCI OF ACTION OF ULTRA-VIOLET AND X-RADIATION, AND OF PLOTORECOVERY, IN THE $\mathrm{EGG}$ ANO SPERM OF THE SEA URCHIN. Proc. Natl. Acad. Sci. U. S. 36, 623-7(1950!

Given in moderate doses, both ultraviolet radiation and x-rays delay cell division (cleavage) of the eggs of the gea urchin Arbacia punctulata. Experiments are summarized which indicate that the locus of this action is, in both cases, associated with cell nuclei, as contrasted to cell cytoplasm. There is recovery from the effect of the radiation, as indicated by return toward the mormal rate of cleavage. Following treatment with ultraviolet radiation, recovery is accelerated by illumination with "visible" radiation. This photorecovery has its locus in the cytoplasm of the egg. In the case of $x$-irradiation, visible radiation appears to be without effect on the recovery of cleavage rate. (NSA)

1685

Boell, E. J., O. M. Ray, and J.H. Bodine. EFTECT OF X-RAXS ON THE OXYGEN CONSUMPTION OF EMBRY ONIC CELLS. Radiology 29, 533-40(1937).

iffects of $x-$ rays on the growth, respiration and oxidation mechanism of the eggs and embryos of the grass hopper (Melanoplus differentialis) have been presented. X-rays in the dosage supplied (2040, $5000 \mathrm{r}$ units) do not affect the respiration $\left(\mathrm{O}_{2}\right.$ intake $)$ of diapause or blocked embryos. The stimulating action of 3,5-dinitro-0-cresol on 0 intake of the diapause embryos is not interfered with by $x$-irradiation. Actively developing eggs and embryos are affected by $x$-rays as indicated by changes in growth and respiration. I - -rays applied to actively developing egges and embryos in which growth has been stopged by exposure to $10^{\circ}$ produce no measurable effect on respiration, or stimulation of respiration by the addition of $2,4-$ dinitrophenol. Hatching of actively developing $\mathrm{z}-\mathrm{r}$ ayed eggs is greatly decreased and embryos hatching do not primarily affect the respiration mechanism of the embryonic cell but rather bring about fundamental changes in protoplasmic organization. (CA)

\section{6}

Bonham, Kelshaw. EFTECT OF X-RAYS UPON THE FRESH-WATER SNAIL, RADIX JAPONICA. University of Washington Applied Fisheries Lab. May 16, 1949. 33p. (AECU-309; UWFL-21)

Radix japonica (Jay) from a creek in Richland, Washington, were $x-r a y e d$ with a Coolidge tube at the approximate

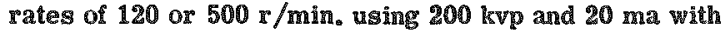
filters of $0.5 \mathrm{~mm}$ of copper and $1 \mathrm{~mm}$ of Al. In three experiments involving 162 adult snails and graduated series of doses up to $80,000 \mathrm{r}$, mortalities significantly higher than in the controls were observed in groups receiving 10,000 $\mathrm{r}$ or more. Egg masses similarly $x$-rayed with doses of 180 $r, 1,000 \mathrm{r}$, or $10,000 \mathrm{x}$ were retarded in their development as compared to the controls in the groups recelving 1,000 $r$ and $10,000 r$. Crawling activity of the newly hatched young was inhibited only in the $10,000 \mathrm{r}$ group. Ratio of hatched to unhatched was inversely related to exposure. (NSA)
1687

Bonham, Kelshaw, Lauren $\mathbb{R}$. Donaldson, Richard 1 . THE EFIECT OF X-RAY ON MORTALITY, WEIGHT, LENGTH, AND COUNTS OF ERYTHROCYTPS AND HEMATOPOIRTC CELLS IN FINGERLING CHNOOR SALMON, ONCORKY NCHUS TSCHAWTSCRA WALBAUM. Growth 12, 107-21(1948).

2ight groups of fingerling chinook salmon, Oncorhynchus tschawytscha Walbaum, were subjected to different $x$-ray doses graduated from 100 to $5,000 \mathrm{x}$, and one group was used as a control. The lowest exposure levels at which ir radiated groups were gigniffeantly adver zely affected 12 weeks after exposure were, in mortality $250 \mathrm{r}$, in weight $500 \mathrm{r}$, and in length 1,000 $\mathrm{F}$. Group means when tested against the mean of all nine groups combined showed gignificant decline in number of cells in the circulating blood at exposures of $750 \mathrm{r}$ and $1,250 \mathrm{r}$ two and three weeks after irradiation. Concentration of hematopoietic cells in the kidney showed rimilar decline in the $750 \mathrm{r}$ group one and two weeks after irradiation. (NSA)

1688

Brace, Rirkland C. WFFECTS OF X-RAYS ON SIZE OF

YEAST CELLS. Proc. Soe. Exptl. Biol. Med. 74, 751-5 (1950).

When yeast cells were exposed to $20,000 \mathrm{r}$ of $\mathrm{x}$-rays and cultured in liquid medium, they and their descendants attained a mean cell volume more than twice that of cells derived from nonirsadiated controls. On the other hand, there were no corresponding dufferences in specific gravity and nitrogen content per unit of cell wolume. It is accordingly concluded that enlargement of yeast cells after irradiation is largely a monselective increase in cell constituents rather than a swelling due mostly to uptake of water. (NSA)

1689

Brumfield, R. T. CELL-LINEAGE STUDIES IN ROOT MERISTEMS BY MEANS OF X-RAY TNDUCED CHROMOSOME RTARRANGEMENTS. Genetics 27, 135(1942).

Certain types of chromosome rearrangements induced by 18 -rays are pasaed on through subsequent cell division to all the descendants of the affected cell. Thus chromosome changes induced by X-rays offer a means of "tagging" a single cell and determining the kind and extent of tissues

derived from it. This paper reports results obtained by the use of this method in studying cell lineage in root meristems. - Germinating seed of Vicia laba and Crepis capillaris were X-rayed, allowed to grow for three weelrs after radiation, then primary root tips were fixed and sectioned transversely. Cytological observations revealed that many of the root meristems thus treated were chromosomal chimeras, both normal and rearranged complements (usually unequal translocations) being found in the same root. All chimeras so far observed have been sectorial ones. In most of the chimeras of both Vicia and Crepis the gector having the rearranged complement represents about one-thir d of the root and includes root cap, epidermis, cortex, endodermis, and ceniral cylinder. This indicates that in these roots a single cell $\mathrm{mm}$ give rise to all the above tissues and that the whole root develops from but few cells at the extreme tip. Sectorial chimeras would not be expected if the meristem consisted of "histogens" as described by Hanstein. (G) 1690

Bruns, K. Ho, W. Dittrich, W. Paul, and G. Schubert. CHANGES IN THE ROOT GROWTY OF RARLEY SEEDLINGS PRODUCED BY IAST IELECTRONS OF A G-MEV BETATRON. Strahlentherapie 81, 173-6(1250). 
The action of fast electrons (from a 6 -Mev betatron) on the growth of roots of barley seedlings was compared with that of $\mathrm{x}$-rays, the dose range being 1,000 to $6,000 \mathrm{r}$. The radiobiological action was measured by the percentage decreaze of the rate of growth of irradiated roots, as renlected in the root lengths $80 \mathrm{hr}$ atter the irxadiation. A statsitical treatment of the resulti revealed a weaker action of fast electrons as compared with that of $\mathrm{x}-\mathrm{r} 2 \mathrm{y}$. (NSA)

1691

Brunst, $V . V$. THE FFIECT OF X-RAY IRRADIATION UPON T"Y⿸厂二 (SRREDON MEXCANUM). Anat. Record 103, $430-1$ (1949).

The following is an abstract and is reproduced here in its entirely.

The lumbo-sacral regions of 235 axolotis (24 and 30 days after hatching) were locally treated with doses of $100 \mathrm{r}$. to 8000 1. Forty animals served as untreated controls. In the firgt series of experiments, the invegtigation of limed animals was made three months after treatment. The guppression of growth resulted in a more or loss prom nowneed waist-lille nar rowing of the treated part of the body. In much animals, the hindlimb skeleton, pelvic girdle, and some elements of the vertebral column were completely absent. In the cases where the animal was incorrectly oriented during irradiation, a partial development of the extremities was observed. These mmall limbs with arxested growth showed a relatively complete differentiation of skeletal and other tissues. In the second gerieg of experiments, the irradiated animals were fixed a variable period of time (from 4 days to 76 days after treatment, and in some cases, 6 months after treatment $\%$ The following results were obtained: A temporrary inhibition of development was observed $4-5$ after treatment. A primary acute reaction was observed $20-35$ dayg after treatment. The end of the acute reaction and late gecondary stimulation were observed 40-50 days after treatment. A secondary stable state was observed 60 days after irradiation. This state persisted for an unlimited per lod of time.

1692

Brunst, V. V. INILUENCE OF LOCAL X-RAY TREATMENT ON TEL DEVELOPMENT OF ZXTREMITES OF THE FOUNG AXOLOTL (SLPDDON METICANUM). J. REv1. Zoo̊l. 114, 1-35(1950).

In this papers a detalled description of the effects of $\mathrm{x}$ zadiation upon the develogment of the extremities of the axolotl is given. This paper consists of two parts. The first part describes the effects of irradiation upon the lumbosacral region in the aroloti. The gecond part deacribes the effects of irradiation th the lumbo-sacral region upon the gradual development of the extremities of arololls. The purpose of the experiments was to investigate the guccession of changes in the irradiated parts of the axrolot body during the process of development and to determine whether it is possible to stimulate development by means of weak irradiation. The effects of the irmadisfion un to 63 days following irradiation are discussed. 27 referencs: (NSA)

1603

Brunst, V.V. THE EFFECTS OF LOCAL X-RAF IRRADIATION ON THE TAIL DEVEIOPMENT ON YOUNG AXOLOTLS. 3. Morphol. 86, 115-41(1950).

Young arolotis were ir radiated while under anesthegia with $1,000,2,000,3,000$, or 6,000 r delivered by a $43-k v$ machine with no riltration at a target digtance of $17 \mathrm{~cm}$ to localized arean in the lail region, the regt of the body being carefully shielded; of the 120 animals tested, all recovered in water immediately after irradiation. The treated animals were photographed, described, fixed in a solution of bichloride of mercury, imbedded in paraffin, and sectioned 35 to 40 days and 85 to 100 days following irradiation. Two types of effects were noted: the primary type was characterized by inflammation, degeneration of the epithelium, notochord, and the neural tube, and inhibition of growth, in evidence 35 days after irradiation; the secondary type which followed the primary and was characterized by inhibition of growth of the irradiated portions (the tissue appearing normal but lacking the ability to divide) and the subsequent growth in many cases of a second tail (which never attained normal size and had varying anatomical characteristice). (NSA)

1604

Brunst, V. V. INFLUENCE OF X-RAYS ON LIMB REGENERATION IN URODELE AMPIIBIANS. Quart. Rev. Biol. 25, 1-29(1950).

A review is presented on the mode of action of $\mathrm{x}-\mathrm{r}$ ryg in inhibiting limb regeneration in urodele amphibians, on the source of cells for the regenerating blastema, and on the influence of $x-$ rays on growth and diferentiation in the regenerative process; the author discusses in detail the results of a number of important works on the subject, including some seldom-referred-to Russian papers. The primary reaction of the organism to the irradiation may consist in profound alterations in the cells, often seen as pathological degeneration and death; inflammation may also be the primary geaction. The surviving tissues recover their normal appearance, but not their potentialities; regeneration is lost. Postamputation ir radiation causes not only an arrest of regeneration, but also a rapid decrease in the size of the treated regenerate, due to an inflammatory process; irradiation prior to amputation causes no size reduction. Suppression of cell division does not affect the differentiation process; tissue and organ differentiation in irradiated animals may be completed despite a cessation of growth. Other results are discussed. 220 references. (NSA) 1695

Brunst, $V$. V. and Frank Higge. THE DEVELOPMENT OF SECONDARY TAILS IN FOUNG AXOLOTLS ATTER LOCAL IE-RAY IRRADLATION. J. Morphol. 89, 111-33 (1051).

High doses of $x$ radiation $(600$ to $1200 \mathrm{x}$ ) applied to localized axeas of tails of Axolotls, 10 days after their hatching, ${ }^{~ " S t i m u l a t e d " ~ t h e ~ f o r m a t i o n ~ o f ~ s e c o n d a r y ~ t a i l s . ~ T h e ~ i n d u c e d ~}$ cartlaginous skeletons were at first totally independent of the normal cartilaginous elements. Growth of the normal tails may be inhibited by $\mathrm{z}-\mathrm{r}$ adiation. (NSA) 1696

Chase, Herman B. GREYING INDUCED BY X-RAYS IN THE MOUS

Irregularitis in degree and pattern described by previous workers have been here controlled by obtaining groups of hairs at known stages of development and by using hard $\mathrm{X}$-rays in a phantom to give homogeneous dosage to the whole animal. The inbred $\mathrm{C}_{57}$ Black strain has been used. The threshold dosage is $250 \mathrm{~F}$ and about 50 percent loss of pigment occurs with $500 x$. A higher doge of 1000 r using soft rays on a prepared area gives, 100 per cent 10 ss of color. Plucking of hair on areas of 1.5 square centimeter 3 is essentially the method of obtaining new hair generations. Time of $X$-raying relative to times of plucking makes it posable to have on same animal several areas differing as to hair age. Maximum effect on the hair itself is when hair is growing rapidly; maximum effect on loss of pigment is ingtead at time pre- 
ceding hair growth. Fffect is on the reservoir of melanophores. This standardized method on $\mathrm{C}_{68}$ Black gives permanent readings within three weeks and has permitted biological assays of padiations from different sources (radium, betatron) and has been used in comparative studies on different genotypes and phenotypes. (G) 1687

\section{Chase, Herman B. GREYTMG OF IRAIR I. ETETCTS}

PRODUCED BY SINGLE DOSES OF X-RAYS ON MICE.

J. Morphol. 84, 57-76(1949).

Local areas on C57 Black gtrain mice were exposed to comparatively low dosages, $31 \mathrm{r}$ to $1000 \mathrm{r}$, but mostly between $200 \mathrm{r}$ and $500 \mathrm{r}$ at delinite times before hair was plucked, at plucking, and at definite times following plucking. Plucking hair by means of the fingerg from several separate areas of the coat is the method for obtaining areas in known stages of hair growth. Plucking of clubhair from an area stimulates the follicles of that area alone to produce the next hair generation. Inactive (Telogen) Iollicles when $\mathrm{x}-\mathrm{r}$ ayed given in the succeding hair generations the maximum greying response for the particular dose. Active follicles (Anagen) when $x-5$ ayed loge hair already pregent and the succeeding generations of hair are less grey for the particular doge than those from treated inactive follicles. Cpilation and growth retardation and delay effects are opposite in severity to the greying effects, with respect to active or inactive follicles at the time of treatment. The threshold response for follicles treated in the inactive phase occur $w$ with $200 \mathrm{~g}$ for $100 \mathrm{kv}$, $550 \mathrm{r} / \mathrm{min}$. irradiation and with $250 \mathrm{r}$ for $200 \mathrm{kv}, 102$ $\mathrm{r} / \mathrm{min}$. dradiation. There is a graded response. The greying effect is permanent; that is, persigts for succeding hair generations. A discusston of the literature is presented mainly in terms of the degree of sensitivity to greying of different animals and in terms of the irregular ity of the response. Considerable regularity of response, in the mouse at least, is obtainable by controlling the stage of hair growth at the time of treatment. A tentative hypothesis is presented involving inactivation or killing of mela noblasts of the dermal reservoir in relation to a melanoblast population cycle associated with epider mal follicle development. Haix development and pigmentation are shown to be separate processes. 26 references. (NSA) 1698

Chase, Herman B. EFIECTS OF X-RAY DOSES ON TIE CONTROLLED GREYING RESPONSE M MCE. ARta Unio Intern. contra Cancrum 6, 768-70(1949).

The normal pigmentation process of the hair follicles in mice and the gene-controlled variations of this procesm were investigated through a study of the responses to $\mathrm{x}-$ radiations. Controlled graying response in the highly inbred C-57 Black mouse strain could be obtained by plucking hair \$; radiation doses were delivered by 100 and 200 $\mathrm{kv} \mathrm{X}$-ray machines, by the 20 million volt betatron and by radium applications. It was found that age, sex, weight, and specific halg location do not influence a hair response to ir radiation; the stage of development of the hair does, however, affect the nature and degree of the regponse, maximum graying occurring when inactive or resting follicles are irradiated. The threshold for the graying effect was found to be $250 \mathrm{r}$; at $500 \mathrm{r}$, there was a $50 \%$ response, while at $1,000 \mathrm{r}$, a $95-100 \%$ response was elicited. The graying effect was found to be permanent for succeeding hair generations. It is concluded that this type of study is especially useful for comparative studies invol:ing different species and phenotypes, different irradiat.un sources, natural versus induced graying, and single vergus cumulative doses. (NSA)
1699

Clark Fancis E. and C. A. 1. Goring. GROWTH OF BACTERIA IN THE PRESENCE OF RADIOACTIV PHOSPHORUS. J. Bacterlol. 62, $352-4$ (1251).

A basal soll extract broth initially containing $0.2 \mathrm{ppm}$ inorganic $P$ and 0.6 ppm organic $P$ was enr iched with $0.5 \%$ glucose, $0.2 \% \mathrm{KNO}_{\mathrm{g}} 0.1 \% \mathrm{MgSO}_{\mathrm{g}}$ and $4 \mathrm{ppm} \mathrm{P}^{31}$ as $\mathrm{IKH}_{\mathrm{g}} \mathrm{PO}_{\mathrm{f}}$ The added phosphor us contained either $0,0.01,0.1,1.0,0$ or $5.0 \mathrm{mc}$ of $\mathbb{P}^{32}$ per $\mathrm{mg} \mathrm{P}^{31}$. Sterilized aliquots at each level of activity were given gtandard inocula of known microorganisms. The results suggest that although $\mathrm{P}^{32}$ ererts lethal effects on many individual cells, the murviving cells continue to multiply until their growth is limited by other factors, such as accumulation of staling products or substrate exhaustion. Consequently, turbidimetric or gravimetric differences, or interference with substrate transformation as indicated by measurementw of $\mathrm{pH}$ or of $C, \mathbb{N}$, or $\mathbb{P}$ mineralization may not be readily apparent. Nevertheles, in view of higher viable counts at 7 days for the 0.1-me level of activity than for the 0 level, it is believed that low levels of $\mathrm{P}^{32}$ muat to some extent delay normal aging or growth cycle of cultures. Low viable counts at $5.0 \mathrm{mc}$ and the cytological observations at $0.1 \mathrm{mc}$ or more of activity likewise indicated a deleter loug effect of such levels of $\mathrm{P}^{22}$ radioactivity. (NSA) 1700

Cottet, P. and W. Minder. TEE INELUENCE OF THE COMBNEDD ACTION OF LICHT AND TE-RAYS ON THE ROOT GROWTE OF VICLA FABA VULGARIS. Sehwelz. med. Wochschr。 77, 195-7(1847).

A study was made of the infiuence of 3 factors on the growth of Vicia faba vulgaris: benzopyrene (I), light, and X-rays. I was stabilized in glycerol with serum according to Graffi. The seeds were soaked in this solution for 24 hours. During this period they absorbed twice their welght of 1. This could be demonstrated by spectroscopic examimation of the acetone extract of the seedlings. I accelerated root growth by $40 \%$ during the 5 days after treatment of the geeds. This effect was partially depressed in the light as the result of a photosensisizing action. The inhibiting of fect of 8 -rays (200 roentgens) was more pronounced in those plants which had previously been treated with I. This was probably because I activated the life processes so that the tissue was rendered more sensitive to the radiation. (CA)

1701

Dent, J. N. and Robert L. Amy. DEVELOPMENTAL WFFECTS OASTRVED IN HABROBRACON AFTER SWPOSURE TO BETA BADLTION. GrOWh 14, 113-21 (1950).

Developing individuals of $\mathrm{H}$. juglandis were exposed to various levels of beta radiation for $48 \mathrm{ahr}$ periods just before hatching or during the early larval period. At the highest levels of radiation used, the wasps died before hatching or in the larval stage. At intermediate levels death usually occurred during pupation with considerable degree of inverge correlation between the extent of imaginal differentiation and the amount of radiation given off. At the lowest levels it was apparent that most of the animals attained adult form. The rate of development was somewhat reduced at the highest and intermediate levels but not at the lowest levels. Pupation without cocoon formation was a frequent occurrence at the intermediate levels. IDidence which indicates that ir radiated animals reaching adulthood were sterile is presented. (NSA) 1702

Dittrich, W., W. Paul, M. Riedel, and G. Schubert. BlOLOGICAL RADIATION EFFETS OF FAST ELECTRONS 
ON GERMINATING BARLEY, Strahlentherapie 80, 17-34 (1948).

After 24 hours of gwelling in wates, barley lsernels were aubjected to irradiation by last electrons of $4 \mathrm{Mev}$ energy. The results were conpared with nonirradiated and with s-ray-treated kernels. Measurements were made of the time it took the coleoptile to break through the soil, and the time that passed until the firgt leaf broke through the coleoptile-sheath, as well as the length of growth of the leaf. With increased dosages the inhibition of the germinal growth became greater and the percentage of coleoptiles breaking through the moil was smaller: the length of growth of the leaf under the same conditions became more and more retarded. Flectrons with difierent energies (2 to 5 Mev) did not produce any different biological radiation effect if equal dosages were used. Fast electrons were less retarding and less damaging than $x$-ray unit doses. Theoretical implications of this fact are discussed. Sman dosages $(1,600$ r and less) of fast electrons had a growthstimulating effect. (NSA)

1703

Drobkov, A. A. BIOLOGICAL FEFCTS OF NATURALLY RADIOACTIV TLEMENTS. Uspelkhi Sovremennoi Biol. 31, 82-100(1951).

A revlew of efiects of $\mathrm{Ra}, \mathrm{U}, \mathrm{Th}$ and other elements on plants. Radiophotographs are shown of sugar beets, virusinfested tobacco, tomato, and pea plants. 84 references. (CA)

1704

Fmmet, John. FIFECT OF X-RADIATON ON TRYPANOSOMA CEU21. J. Parasitol. 36, 45-7(1950).

Virulent cuitures of 'Trypanosoma cruzi in a medium were irradiated by $x$-ray doses of $10, \overline{000-100,000 ~} \mathrm{r}$ from a 180-kv machine using two wufiltered tubes and at a target distance of $14.8 \mathrm{~cm}$; with this apparatus 10,000 could be delivered in about 5 min. After varying exposures, $0.5 \mathrm{cc}$ amounts of the cultures were immediately injected intraperitoneally into white mice weighing about $10 \mathrm{~g}$; nonirradiated cultures were injected into control animals. It was found that with dosages up to 100,000 a the trypanosomes remained viable and morphologically unchanged; however, from 10,000 x upwards, a definite decrease in infective power of the organimms was induced. The earliest infection in an experimental animal was on the 5th day, but the majority of the animals did not become positive until several days later, as compared with positive control animals on the sth to the 7th day. Subcultures from the 100,000 r cullures were allowed to grow a week, checked for motility and numbers, and injected into mice; the approximate number of organ!mas was somewhat decreased from controls. It is concluded that the radiosensitivity of Trypanosoma crugl parallels that of $T$. gambiense and $T$. brucei, but, If is noted that the $T$. gambiense organisms became noninfectious after lower irradiation (12,000 g). (NSA)

1705

Fuler, R. von, L. Ahlstrom, and B. Flogherg. CHANGLS PRODUCED IN ITAST CFLLS BY ROENTGEN RAYS AND CHIMACAL SUESTANCES. $z$. physiol. Chem. 277, 1-17; 18-25(1942).

The damage suffered by the yeast cells (brewer's yeat) exposed to the rays at a distance of $20 \mathrm{~cm}$ from the Roentgen tubes was estimated from the number of dead celli which ranged after 5 hours from $5 \%$ to $5000 \mathrm{r}$ to $76 \%$ at 50,000 s. The surviving cells ghowed a reduced rate of maltiplication afterward as compared with non-irradiated colls. Up to $40 \%$ of the surviving cells whowed an increase In alwe. The content in nucleic acid of the yeat was re- duced by approx. 20\%. Large quantities of colchicine $(0.5$ to $1 \mathrm{~g}$ per $100 \mathrm{cc}$ ) inhibit the multiplication of yeast. The influence of 300 to $1000 \gamma$ is insignificant. Under the influence of colchicine the cells enlarge by 20 to $50 \%$. The treated yeast is more sensitive to Roentgen rays. Doses of 15,000 I have no influence on the fermentating power of the yeast. This is not altered by pretreatment with colchicme. An extrart from rat spleens added to yeast not only causes a decrease of growth but also cell degenerations. However, the fermenting effect of the yeast is increased by the spleen extract. (CA) 1706

Evans, T. C., J. C. Slaughter, F. P. Little, and G. Failla. INFLUENCE OF TUE MEDIUM ON RADLATION INJURY OF SPERM. Radiology $39,663-80(1242)$.

The same dose of $\mathrm{x}-\mathrm{y}$ ays administered to sperm of $\mathbb{A}$. punctulata in concentrated form and in sea water suspensions of increasing dilution, inactivated an increasingly larger of the sperm the more dilute the sumpension. Addition of seminal fluid, egg water, egg albumin, gelatin, germ arabic, glycylglycine (pH 8.2) and glycine (pH 8.2) produced decrease in the of of sperm inactivated by a given dose of $x$-rays. (BA) 1707

Forssberg, Arne. THE REACTIONS OF PHYCOMYCES BLARESLERANUS AFTER X- AND GAMMA-IRRADIATION. Acta Radiol. 22, 252-9(1941).

P. biakesleeanus was irradiated with $\mathrm{x}$-rays and gammarays over a wide range of doses and intensitles. Data with regard to the methods of irradiation, and of control of extraneous factors, are promised for a later paper. The author states that a reduction in growth rate can be observed after doses as small as $0.001 \mathrm{r}$. (BA) 1708

Foster, R. F., L. R. Donaldson, A. D. Welander, K. Bonham and A. H. Seymour. SOMT EFFECTS ON FMBRTO AND YOUNC RAINBOW TROUT (SALMO GALRDERII RICHARDSON) FROM ERPOSING THE PARENT FISH TO XRAYS. Growth 13, 110 A2: (1949).

One hundred and forty-eight rainbow trout approximately 20 months old and approaching sexual maturity were divided into a control group and seven irradiated groups. Total body irradiation doses of $50 \mathrm{r}, 100 \mathrm{r}, 500 \mathrm{r}, 750 \mathrm{r}$, $1,000 \mathrm{r}, 1,500 \mathrm{r}$, and 2,500 were administered at a distance of $48 \mathrm{in}$. from the target of the $\mathrm{x}$-ray tube, using 200 $\mathrm{kV}$ and $20 \mathrm{ma}$ and filters of $0.5 \mathrm{~mm}$ of $\mathrm{Cu}$ and $1.0 \mathrm{~mm}$ of $\mathrm{Al}$. The average radiation intensity received by the fish was $8.25 \mathrm{I} /$ rinin. When the fish became mature about three month after irradiation, they were apawned and a total of 115 , 454 egg were obtained from 67 females. The gametogenesis, egg development, abnormalities, fry mortality, early fingerling mortality, and growth of the fish are discussed at length and the results are tabulated. Photographs of apparatus used and development stages are furnished. Included are a series of photographs showing: the stages of development of the rambow trout egga; the abnormal trout egge from parents exposed to various doses of $x$-radiation; abnormal rainbow trout embryos reaching various stage: of development; and common types of abnormal fry found among rainbow trout fry. (NSA)

1708

Frtedewald, W. F. and R. S. Anderson. THR ERTCTS OR ROENTGEN RAYS ON CELL-VIRUS ASSOCLATONS. FINDINCS WITH VRTUS-INDUCED RABBIT PAPILLOMAS AND ITRROMAS. J. ISxphl. Med. 78, 285-304(1043).

The Hirus-induced papillomas of cottontail as well as. domestic rabbits regress completely within a few weeks when exposed to 5,000 I of X-ray irradiation. The X-rays 
do not immediately kill the papilloma cells, but lead to death by inhibiting cellular division and producing pathological changes in the cells which then continue to differentiate. The virus associated with the growths, however, not only persists in undiminished amount during regression, but often an increased yield of it can be obtained on extraction. The fibroma virus in crude extracts or in vivo is inactivated by far less irradiation than the papilloma virus. 10,000 re destroys $90 \%$ or more of the infectivity of the fibroma virus, whereas at least $100,000 \mathrm{I}$ is required to inactivate $50 \%$ of the papilloma virus in extracts containing about the same amount of protein. No variant of the papilloma virus or fibroma virus has been encountered 1710 as a result of the irradiation. (BA)

Goldfeder, Anna. FURTHER STUDIES ON THE RADIOSENSITIVITY OF THE ANALOGOUS MOUSE MAMMARY TUMORS dbrB AND C3L. Radiology 47, 845-63(19E1).

The growth rates of mammary adenocarcinomas of mice were studied before and after irradiation. Chalkley's method (J. Nat. Cancer Inst. $4,47-53(1943-44)$ ) was applied for quantitative evaluation of growth activity of the tumors, laking the mitotic index as the criterion. A depression in mitotic activity in the irradiated tumors was noted, the extent of which depended upon the dose of radiation applied and the lapse of time between exposure and removal of the tumor: for analysis. An earlier and more pronounced return of mitotic activity was noted in the fagter-growing dbrib type tumors following sublethal doses of radiation, as compared with the slower - growing C3H type. The levels of the phosphorylated intermediates in the glycolytic cycle for the two mouse mammaxy adenocaxcinomas were determined. The more rapidly growing mammary tumor (dbr B) shows significantly higher levels of glucose-1 1 phosphate, adenosine triphosphate, and possibly glycogen, and a lower level of inorganic phosphorus than the slower-growing $\mathrm{C} 3 \mathrm{H}$ tumor. These findings strongly suggest that a higher level of energy is available for vital function in the $d b r B$ tumor. Some of the compounds encountered in the phosphorylated glycolytic cyele, particularly adenosine triphosphate, may be more readily affected by irradiation in the C3H tumor than in the $\mathrm{dbr} B$ tumor. The metabolic processes were operative even after such large doser as 30,000 r for the dbrB tumor and 12,000 I for the $C 3 H$, although these doses proved to be lethal for their reapective growth activities. Successful application ts radiotherapy and advantages of applying large doses of $\mathrm{x}$ rays through amall portals exposing only the tumor is discussed. (NSA)

\section{1}

Gordon, Solon A. PHYTORADIOLOGY, p.124-5 of BIOLOGICAL AND MEDICAL DIVISONS; QUARTERLT REPORT FEBRARY, MARCH, APRL 1949. Austin M. Brues and Herman Lisco, eds. Argonne National Lab. [nd] 2p. (ANL-4281(p.124-5))

Inactivation of auxin by $x$ radiation is a first order rem action until most of the aurin if degtroyed, the lonic yield is 1 in contrast to a value of 25 reported by $\$$ lkoog. The source of the discrepancy is being invegtigated. Initial gesults indicate that radiation interferes with the production of indole acetic acid and its precursor indoleacetaldehyde. (R.D.B.)

\section{2}

Gordon, S. A., R. Stut, and R. Weber. ACTION OF X RADIATION ON GROWTH BY CELLULAR ELONGATION, p.144-51 of BIOLOGICAL ANO MIPDICAL DIVISIONS; QUARTERHY REPORT, NOVEMBER, DECEMBER, JANUARY, 1949-50. Austin M. Brues, ed. Argonne National Lab. [nd] 8p. (ANL-4401(p.144-51))
The first shoot of the oat seedling provides a thssue whereby one phase of the growth process, cellular expansion, can be separated from cellular division and differentiation. This phase of growth is apparently resistant to ionizing radiation, inhibition being manifest at rather high dose levels of $\mathrm{z}$ radiation. Whin relatively nargow limits of dose, growth inhibition can be reversed following irram diation by addition of growth hormone (auxin) and partly reversed by sulfhydryl activants. Such reversals could be interpreted as indicating an inactivation of the foregoing essential components of the growth process by ionizing radiation. A small quantity of $\mathrm{C}^{14}$-labeled growth hormone, indoleacetic acid, has been øynthesized. Synthetic procedures employed are detailed, particularly in view of some erroneous aspects of the published method for the synthesis of this radioauxin. Initial biological experiments using radioindoleacetate ohow the compound to be physiologically active and to have no deleterious effect on growth due to its emission. (Authos:s)

1713

Gray, L. T. and M. E. Scholes. THE RFECT OF IONIZWNG RADLATIONS ON THE BROAD BEAN ROOT. PART VIII. Brit. J. Radiol. 24, 82-92; 176-80; 228-36(1951).

A detailed study of the effect of $x$ and $a$ radiation on normal root growth of the broad bean, Vicia faba, has beem made. The morphological and histological aspects of root growth are discussed and equations are derived correlating root growth with mitotic activity and cell elongation. Mor phological observations on root growth as a function of time after irradiation are presented. Data obtained Irom histological studies of changes in roots exposed to 143 r of $\mathrm{x}$ rays are reported. The quantitative aspects of the observed cytological abmormalitics are considered with particular regard to cell elongation and the correlation of root growth with mitotic activity. Abstract of succeeding sections of this paper (Brit. J. Radiology 24, 285-91(1951) May and 1bid., 348-52(1951) June) appeared in Nucleas Sclence Abstracts as NSA 5-4317 a.d 5-4318, respectively. (NSA)

\section{4}

Gray, L. H. and M. R. Scholes. THE WISCT OF IONIZING RADIATIONS ON THE BROAD BRAN ROOT. PART VII. Brit. J. Radiol. 24, 285-91(1251).

After exposures lasting a few min to doses of $\gamma$ gadiation up to $250 \mathrm{r}, \mathrm{x}$ radiation up to $140 \mathrm{~s}$, and of a radiation up to 36 rep, the rate of growth of roots of Vicla faba falls to a minimum and then returns to normal. The $\gamma-$ and $x-r a y$ growth inhibition is dependent on the duration of exposure. The a-ray inhibition is independent of exposure times between $10 \mathrm{~min}$ and $24 \mathrm{hr}$. The minimum value of the growth rate relative to control roots, which occurs at about the 6th day after irradiation, is exponentially related to dose for a radiation. It is also exponential for $\gamma$ radiation delivered in 12 or $24 \mathrm{hr}$ and tends to exponential for long duration exposures to 8 radiation. The relation is sigmold for both $\gamma$ and $x$ radiation at higher dose rates. The relative biological eficiency of a radiation compared with $x$ radiation varies from about 4.5 to 12.5 , according to the dore level and duration of expowure at which the radiations are compared. Histological changer seen in roots from o to 10 days after exposure to $140 \mathrm{x}$ of $\mathrm{x}$ rays have been examined in detail. It is concluded that chronosome gtructural damage constitutes a major factor leading to growth inhibition by all three radiations. (NSA)

1715

Gray, L. I. and M. E. Scholes. TRE ING RADIATIONS ON THE BROAD BEAN ROOT. VUI.

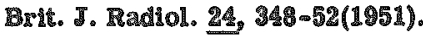


Two appendices to the previous articles appear. Appendix I gives a detailed deseription of the germination and culture of the Vicia laba used in the expeximenti, the method uged to measure roots with minimum handling, and the procedure for irradiation by a particles. Appendix II includes an explanation of how the number of cells per unit volume of tissue of a particular reglon can be estimated, the method of estimating $\tau$ (duration of mitosis) and $t$ (means intermitotic time at any point in the root), and a definition of resting nucleus, prophase, metaphase, and telophase. 20 references. (NSA)

1716

Handford, S. W. STUDIES ON THE EFTECT OF X-RADIATION ON MORPHOGENESIS OF AMBLYSTOMA PUNCTATUM. Anat. Record 103, 540(12)

The rollowing is an abstract and is presented here in its entirety.

Embryos at stages 11-12, 15-16, 20-21 and $38-37 \mathrm{re-}$ ceived an air dose of 245 . from a $200 \mathrm{kv}$ therapy machine. The 300 animals treated survived from 15-30 days and all showed pronounced developmental defects. It seems cleas that the earlier the stage the greater and more general are the effects. This leads to a shorter time of survival for animals at earlier developmental stages relative to more advanced animals upon which the effects are more apecific and slower to become manifeat. Outstanding In the individuals treated during early stages are abnormalities of the tail-bud, a general oedema which probably derives from damage of the pronephros; and the eye and gill-mound on one side only. Animala that received treatment at more advanced stages show reduction in the numbes of digits of the forelimb, failure of the hindimb to devolop normally, and a striking defect of jaws which disal low feeding at stage 46 . No complete morphologic study of the preserved material has yet been made.

$171 \%$

Henshaw, Raul \$. THE ACTION OF X-RAYS ON NUCLEATED AND NON-NUCLATTE RGG IFRAGMENTS. Am.

I. Cancer 3ิ3 258-64(1938).

Experiments have been carried out to determine whether a certain radiobiological effect results from changes produced in the nucleus or some other part of the cells irradiated. Iggs of Arbacia punctulata were used and the of sect observed was delay in cell division caused by $\mathrm{x}$-rays. Nucleated and non-nucleated egg fragments were obtained by the centrifuge method, both of which will undergo fertilization and development. These were irradiated and ingeminated, and the moment when $50 \%$ had divided was determined. It was found that for a given dose of radiation an appreciable delay of about the wame extent was produced in whole eggs and mucleated fragments, whereas no delay was produced in the non-nucleated iragments. Thus. since there is a direct correlation between the presenee of a nucleus and the manifestation of an irradiation effect and gince lrgadiation changes occurring in parts exclusive of the nucleus produce no observable effect, it appears that the glowing in cell division brought about in Arbacia eggs by X-rays is due to changes produced directly in the nucleus by the radiation. (Author's Summary)

1718

Menshaw, Paul S. RESPONSES OF DROSOPHILA PUPAE TO X-RAYS. Radiology 34, 721-30(1940).

The uge of Drosophila prpae as test object for quantitative radiobsological work has been considered and invegtigated. The relation between radiogensitivity, developmental activity, and respiratory rate at various stages during the pupal period has been detd. Using as a criterion of effect, development to the atage of pigmented bristles legs, and wings, and fallure of the imago to emerge, from the pupariun, it was found that duxing the first $18 \mathrm{hrs}$, the organisms maintain a strikingly uniform radiosensitivity, but that beyond this point their resistance increases at a rapid rate. Considering normal development alone, 3 fairly distinct phases were recognized: the first from 0 to 24 hrs, characterized mainly by destruction and removal of larval organs; the 2 nd from 24 to $48 \mathrm{hr}$, by relative quiescence, and $3 \mathrm{rd}, 48.96 \mathrm{hrs}$, by active organogenesis. The rate of $\mathrm{O}_{2}$ uptake in these form decreases during the $15 t$ phase, reaches a minimum duxing the and and increases during the $3 \mathrm{rd}$, reaching a level at the time of emergence about equal to that at the begimning of the pupal stage. There was therefore, a positive correlation between the rate of $\mathrm{O}_{2}$ uptake and developmental activity, but none between either of these and radiosensitivity. The reproducibility of the quantitative response of pupae 7 to 18 hro old to radiation was found to be within narrow limits. The general shape of the curve showing rate of $\mathrm{O}_{2}$ uptake during the pupal period was not changed appreciably when lethal doses of radiation were administered early in the period. Only a slight delay was manifested which probably has little significance. (BA)

1719

LLenshaw, Paul \$. PECULIAR GROWTH LESIONS $\mathbb{N}$ FROGS INDUCED BV IRRADLATION OF SPERM CELLS WITH X-RAYS. J. Natl. Cancer Inst. 3, 409-17(1043).

The changes responsible for growth lesions occur in the mucleus of the irradiated cells, the sperm nucleus after heavy irradiation will entex the egg (as part of the sperm cell) but will not fuse with the egg nucleus, growth abnormalities are not obtained in those cases in which the sperm nucleus is not functional. The ract that the sperm cell was free-living at the time of treatment, that it has a haploid number of chromosomes and that a normal egg fertilized with a treated sperm will give rise to growth abo normalities in later development, makes it clear that growth modifications may result from injury to only half of the chromosome complement of the cell. These findings nay be regarded as evidence that a radically changed pattern of multicellular growth may be set in motion by the sudden modiflcation of nuclear substances in a single cell. (Authors conclusion) (BA)

\section{0}

Henshaw, Paul S. and T. Turkowitz. SOME EFIFCTS OF ROENTGEN RAYS ON SACCHAROMYCES CEREVISIAT.

Am. J. Roentgenol. Radium Therapy 43, 93-106(1940).

Cultures of saccharomyces cerevisiae were exposed to intense beams of $200 \mathrm{kv}$ x-rays for varying times, and the subseguent rate of growth measured yhotometrically. A family of growth curves was obtained which indicated that the growth processes were delayed in some way by the irradiation, but not impaired. This delay appears to be due to organisms killed by the radiation rather than injured; the survivors appear to be as vigorous as the controls. The survival curve of the organisms tends toward the exponential form, with no sigmoid characteristic, indicating that this organism is in the "one-hit-to-kill" category. Microscopical examinations showed that cells affected lethally may undergo several divisions following exposura. but that they rarely, if ever, go beyond the 40 -cell stare. (BA)

1721

PZichs, Samuel P. ACUTE NECROSIS AND MALFORMATION OF DEVIZLOPING MAMMALIAN BRAIN CAUSED BY X-RAY. Proc. Soc. Exptl. Biol. Med. 75, 485-9(1950). (ACU-9A9) 
In the course of an investigation of the pathologic effects of ionizing radiation on the development of the mammalian nervous system it was found that growing central nervous tissues in fetal rats and mice could be selectively and extensively destroyed by $\mathrm{z}$ radiation with virtually no destructive effect on other organs. Irradiation of pregnant rati and mice with 200 and 600 r to the whole hody resulted in acute necrosis of the rapidly growing parts of the brain, apinal cord, and retinas of their setuses. This developed within a few hours, was highly selective for the central nervous system, and was often severe and extensive at 200 to $600 \mathrm{r}$. At $150 \mathrm{r}$ damage was less, and was followed in two days by malformations in the neuroblastic zones. Fetuses allowed to go to term and sacrificed after days or weeks showed severe maldevelopments of corpus callosum, hippocampus, striatum, and cerebral cortex. Extraneural lesions were absent below $200 \mathrm{r}$ and rare at $400 \mathrm{r}$. Below $100 \mathrm{r}$ no destructive lesions occurred. (NSA) 1722

KInkel, C. L. THE ERECT OF ROENTGEN RAYS UPON THE GROWING LONG BONES OF ALBHO RATS. 1. QUANTITATIVE STUDIES OF THE GROWTH LIMITATION FOLLOWING IRRADIATION. Am. J. Roentgenol. Radium Therapy 47 , 439-57(1942).

The growing epiphyseal regions of the xight hind leg of albino rats of various ages were irradiated with riltered $200 \mathrm{kv} x$-rays in various doses. Growth and development of the irradiated and control (opposite) femur were followed radiographically for 1 year. The irradiation produced quantitatively predictable clegrees of stunting, ranging from temporary setardation to permanent cessafion of growth, depending on the dose administered. The amount of radiation required to produce minimal stuntings bears a linear relationship to the age of the animal treated; it cannot be correlated with rapidity of growth, or with skin effect. The period of temporary retardation is followed by return to the normal growth rate. Young animals recover more quickly than old, and can tolerate quantities of radiation far in excegs of the minimal stunting doge. Months after Irradiation with large doses there is no evidence of bony decalcification. Microscopic studies show a return to normal cartilage and bone architecture and absence of permanent marrow or blood vessel injurles. (BA)

\section{3}

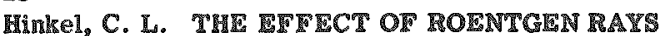
UPON THE GROWING LONG BONES OF ALBMNO RATS. II. HISTOPATHOLOGICAL CHANGES INVOLVING ENDOCHONDRAL GROWTH CENTERS. Am. J. RoentgenOl. Radiun Therapy 49 , 321-48(1943).

Female Sherman strain albino rats of various ager were anesthesized, immobolized, and irradiated with 600-2500 of filtered $200 \mathrm{kv} \mathrm{K}$ - rays over a field of $5 \mathrm{~mm}$ in diameter including the right distal femoral growth center. The left femur of each animal served as the unirradiated control. At various times after irradiation, the animals were sacrificed, the bones of the hind legs fixed, decalcified and studed histologically. Dodds classification of five zones occurring in actively growing cartilage (Am. J. Anat., 55: 139,1938 ) is used as the basis for the descriptions of observed changes. Microscopic abnormalities become evident before there is appreciable interference with growth. Stunting when it oceurs, is apparently the result of tamporary or permanent cessation of cartilage growth in a distal drrection. More lasting secondary effects are explained on the basis of injury to vessels, osteoblasts, and marrow cells. Histopathological changes, are related to dose, age, and interval after irradiation. The amount of osteoclasis and chondroclasis found proximal to the line of advaneing cartilage columns, and the degree of normal anatomical restitution are proportional to the completenegs of marrow and vascular regeneration. Osteoid deposition and true osteogenesis do not talse place in the absence of osteoblasts; in stead a brittle "pseudo bone" is formed. There is always an increase in mineral deposition following izradiation. The metaphyseal region of bones growing after irradiation contains more minesal and more bone than normal. The power of cartilage to regenerate is retained even atter large doses of $\mathrm{X}$-rays (2000 $\mathrm{g}$ ). Blood vessels are maxkedly reduced in number and rendered irregular in caliber and marrow cells are geriously affected by large doses. Possible mechanisms for the direct and indirect changes are discussed. -(BA)

1724

Irvine, Virgina C. J-RADIATION AND GEOWTER SUBSTANCES AS AFITCTHG PLANT PRIMORDLA TISSUIS. Proc. Soc. Expt1. Biol Med, 43, 453 5(1840).

In sunilower, zinma, and tomato seedlings after moderate $\mathrm{m}$ ray doses the meriatematic region becomes divided into 2 or 3 parts with separate groups of leaves, leading to distortion or fasciation of leaven, distur?ed phyllotoxy, and stunted growth of whole plant. B-naphthoxyacetic acid applied in lanolin paste gives stmilar, but not identical resulls. (BA)

1725

Jacobson, L. and R. Overstreet. THE UPTARE RI PLANT OIF PLUTONIUM AND $50 M$ PRO PRODCTS OF NUCLEAR FISIION ADSORBED ON SOIL COLLODS. Soil వెcL. 65, 129-34 (1948) (MDDC-1008)

The uptake of plutonim and the fission products $\mathrm{I}_{2} \mathrm{Ce}$, $\mathrm{Sr}, \mathrm{Te}$, and $\mathrm{Zx}+\mathrm{Cb}$ by two species of plants, barley and dwarf pea, was examined. Studies were made using bentonite clay suspensions (barley) and a montmorilinite goll (dwari pea). In all cases, the roots possess a marked ability to fix large proportions of the above elements even when trace amounts are intially adsorbed on clay part cles. With the exception of $\mathrm{Sr}$, the bulk of the activity remains in the roots. Sr is readily translocated to the tops. On the basis of greenhouse experiments, it is concluded that soll activities of the order of 0.1 microvurie per gram in the region of contact with roots will reaul in obvious injury to the plant. It is estimated that noticeable ingury to the plant will occur at much lower levels of goll activity than 0.1 microcurie per gram. (ADD) 1726

Jormlin, Francis M. THF ATOMC BOME'S EFTECTS ON

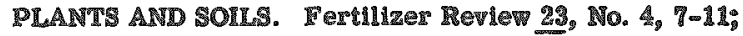
14, No. $5,7-9,11-13(1948)$.

The posalble effect of radioactivity on plants and colls are discussed, together with an investigation of the clams of Mr. Takeo Furuno in Nagasakl that the crop yjelds after the atomic bombing were accelerated. A description is given of the heat, radiations, and blasts produced by the bombs at Hiroshima and Nagasaki, with respect to theis immediate and residual influence on plants, seeds, and soli at various distances from the centers of the blasts. seeds and other organisms below the son surface received little irradiation; many plant abnormalities were noted in new sproutings soon after the radiations. The woll at 3,236 Ieet from the center of the blast ghowed little radioactivity two months after the bombing, and no activity one year after the bombing. The increased yields in Furuno's fields are belleved to be due to large amounts of fertilizer, in the form of potash and $\left(\mathrm{NH}_{4}\right)_{2} \mathrm{SO}_{4}$, that he added to the soll. Carefully controlled research is now in progress to es. Aablish more derinite facts concerming the blological effects of the bombing. (NSA) 
$172 \%$

Karnofsky, David A., Priscilla A. Patterson, and Lois P. Ridgway. THE TOXICITY OY ROENTGIEN RAYS TO THE CECK EMBRYO. Am. J. Roontgenol. Radium Therapy 64, 280-8(1950).

Roentgen rays have been found to exert both an acute and a delayed tozsc oflect on the chick embryo. The acute effect results in death of the embryo within 20 hours after irradiation. In embryos, eight days and older, the acute towic effect is dependent on al dose rate above 5 to 10 $\mathrm{s} / \mathrm{min}$, and is relatively independent of a total dose above $800 \mathrm{r}$. The embryo shows an imereasing gensitivity to this acute effect during the $3 \mathrm{rd}^{\mathrm{d}}$ to 7 th day of incubation, it is most sensitive at the oth to 10th day, and its response is stabinized at a slightly more resigtant level on the 12 th day and beyond. The acute death may be avoided, and delayed deaths produced, by lowering the dose rate appropriately for the age of the embryo at the time of irradiation. The delayed effects of roentgen rays are cumulative, and the LD-50 for chick enbryos of 2 to 12 days of age is in the range of 1,000 to $1,500 \mathrm{r}$, at dose rates varying from 1 to 43.45 . The effects of roentgen rays are gtill cumulative to produce delayed deaths at a dose rate of $0.55 \mathrm{~s} / \mathrm{min}$. The embryos succumbing to the delayed lethal effect of roentgen rays may show vascular injury and hemorrhage, generalized edena, consisting of either a watery or gelatinous fluid, necrosis of the liver and stunting of growth. Developmental abnormalities are common, and are chiefly evident in the beak, limbs, head, and eyes. (NSA)

1728

Kersten, R. Jog K. L. Miller, and C. F. Smith. STHMULA-

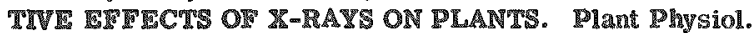
19-18(1843).

Investigations were made of stimulation of primary root growh in seedings from seeds given a small dose of solt X-rays. An apparatus was constructed which permitted 2,000 geeds to be germinated in water culture under controlled conditions. Observations were limited to the first five days of germination. Data on root length and wet and dry weights of roots of seedlings from 1,000 corri seeds

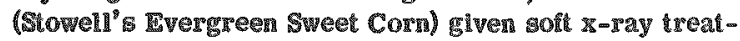
ments at voltages ranging from 15 to $25 \mathrm{kv}$ peals were compared to corresponding data obtained from seedings from 1,000 non-irmadiated corn seeds germinated at the same time ander the aame controlled conditions. Statistical analysis of the data indicates beyond a reasonable doubt that stimulation of primary root growh was obtained by irradiating dry aeeds of corn at vollages in the neighborhood of 17.5 to 20 peal $\mathrm{kv}$ but not at lower or higher voltages. (BA)

1729

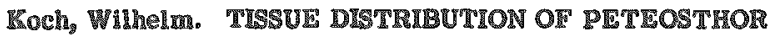
AND ITS COMPONHNYS THORIUA 4 AND PLATINUM IN TEIT GROWING ORGANISR AND THE INILUENCE OF

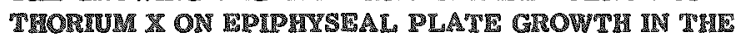

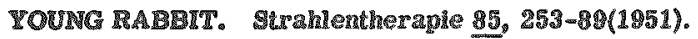

In the organism, ThX $\left(\mathrm{Ra}^{22}\right)$ and $\mathrm{Pt}$ deposits each, independently, favor particular organs. Due to the dysfunction of the enchondral osalication, a several weeks' ThX treatment in rabbits results in a retardation of the growth in length; an impovesishment of the bones in $C$ a malts; the occurrence of spontaneous iractures, a lesser weight increase and premature birth in the pregnant animal. The Belective Thr deposit in the newly calcifying and ozslfylmg tismes permits a Thx theragy in all cases where calcifications and ossiflcations are primarily involved. During pregnaney and in children, this treatment Is contra-indicated. (NSA)
1730

Koester, M. L. THE EFFECT OF CONTINUOUS GAMMA RADITION UPON STARCH FORMATION IN Z珄 MAYS

POLLEN. Genetics 36, 559(1951). (AECU-1463)

The report is reproduced here in its entirety.

Mature pollen from corn plants was tested with 0.2 per cent solution of iodine potassium iodide to determme those grains which were not completely filled with starch and for this study classed as abnormal. Plants used in this experiment received from 0.09 to 222.2 a per day throughout the growing season. A relationship was found between dosage received and percentage of abnormal pollen produced. Pollen from plants which received dosages up to $1.3 \mathrm{x}$ per day showed no increase over the control in the percentage abnormal, but for dosages above $2.6 \mathrm{r}$ per day there was observed a gradual increase in the percentage of abnormal pollen grains. The highest dosage studied gave the greatest percentage of abnomal pollen, 57.0 per cent. When the log of the dose was plotted against the percentage of abnormal pollen, the data were shown to fit a portion of a sigmoid curve. (NSA)

1781

Latarjet, Faymond. INTRACWLLULAR GROWTH OF BACTERIOPHAGE STUDIED BY ROENTGEN IRRADIATION. J. Gen. Plysiol. 31, 529-44(1948).

Growing Eschericha coli infected with bacteriophage T2 was $X$-rayed during the 21 minutes latent period which elapses between infection and lysis of the cells. Survival curves of the infected bacteria were determined almost from minute to minute; they disclosed the following facts which are related to the process of phage growth: During the first ininutes, the infective virus particle remains in the cell unique and genetically intact. The host cell synthesizes some ultraviolet-absorbing material probably devoted to building future particles. From the 7 th to 9 th minute the $X$-ray resigtance of the virus particle increases, probably because of some internal change. Then, multiplication starts and is completed at about the 13 th minute, when an average of 130 virulent units is present per cell, displaying an X-ray resistance twice as high as that of the extracellular virus particle. From 13 minutes to the end, the new units progressively recover the $\mathbf{X}$-ray sensitivity of the extracellular virus. Nothing can be said about either the rate of multiplication bet ween 9 and 13 minutes, or the nature of the multiplying units, except that they are more radiation-resistant (probably smaller) than the extracellular virus. The first steps of the growth process are favored by an unknown component of the lysate, different from the active particles. Several particles can grow in the same host cell. (NSA)

1732

Luee, W. M. REDUCTION IN FACET NUMBER IN FULLEYED (REVERTED BAR) DROSOPHILA BY K-RAYS. Genetics 36, 563(1951).

Larvae of an inbred full-eyed (reverted to full from Bar) strain of Drosophila melanogaster, kept at 28 degrees $C_{\text {. }}$ were treated with $\mathrm{x}$ - ray dosages ranging from 1000 to 3000 applied at the rate of 500 roez min. The conneas of the eyes of the imagoes which developed irom the treated larvae were dissected off, mounted an slides, projected, and the facets counted. The $\mathrm{X}$-rays produced a reduction in face number. The rate of reduction was approximately 0.075 facets per $x$. The $\mathrm{K}$-rays when applied before the larvae were 54 hours old (reckoned from the time when the larvae were laid as eggs) had no effect. Larvae treated at anytime when they were from 59 to 80 hour of age re gronded with essentially similar rates of reduction if facet number per $s$ agplied, with ame evidence that the rate was less for the lowest dosage used (1000 r). - The X-rays 
prolonged larval development. The rate of prolongation, approximately 0.011 hrs. per $\mathbb{x}$, appeased nearly constant for all $\mathrm{X}$-ray applications within the age limits (38-80 hr..) used in this experiment with the gualification that the 3000 r Ireatments had a slightly diminished effect on the rate of prolongation. (G)

\section{3}

Modigliani, Fanco. STATISTICAL ANALISIS OF THE FWPIRIMENTS ON THE INELUINCE OY RADIOACTIVE MATERIAL ON THE GROWTR OF CROPS CONDUCTED IN THEE U.S.A. IN TTHE YEARS 1044 TO 1946. Canadan Radium and Uranium Corp. \nd] 36p. (NP-332)

A statistical analysis has been made of 30 field and greenhouse experiments conducted over the year 1944 to 1946. Pesults of the calculations indicate that with radioactive material added to commercial fertilizer the odds are 10 to 1 that beneficial effect was derived by the following vegetables: pea, cucumber, tomato, lima bean, letuce, carrot, and sweet potato. A few small-scale experiments on the effect of radioactive material on vegetable crops grown on soil treated with manure failed to reveal any gifgnificant beneficial effect rasulting from treatment. A few scattered experiments, insurficient for raliable conclugions, on the af fect of radioactive material added to commercial fertilizer on crope other than vegetables, namely cereals, cotton, and tobacco, Fenerally failed to reveal any significant beneficial effect of radioactive material on the weight of crops. One experiment with tolsacco indicated an increase of $50 \%$ in nicotine content. (ACD)

O'Brien, JOhn P. STUDUES ON THE CELLULAR BASIS OF REGENPRATION IN NAIS PARAGUAYIRNSIS AND TWE EFIECTS OF X-RAYS THEREON. Growth 10, 25-44 (1946).

The cellular bases of anterior and posterior regeneration in Nais paraguayengis has been studied. Neoblasts, a apecial type of regenerative cell, constitute a persistent cell stock in the adult naid; they undergo proliferation and migralion, and together with the ectoderrs enter actively into the regenerative process. X-radiation inhibits regener ation, anteriorly and posteriorly, by way of a selective inhibitive effect upon those cellular lements through the activity of which regeneration is normally accomplished. The neoblasts are more sugceptible to irradiation than ectodermal epithelium; sufficient expogure causes their eventual disintegration. Small knobs, formed anteriorly and posteriorly in irradiated specimens, though often bearing stomodeal and proctodeal invaginations, do mot represent true regenerative activity. (BA) 1785

OUIS, EILeen M. INTRA-UTERINE DEATH TMME IN SEMISTERILE MICE. Anat. Record 105, 53S(1049).

Semi-sterile mouse pedigrees descended from males exposed to chronic x-radiation have been studied to determine intra-uterine death ilme of inviable zygotes. Inviability is presumably due to unbalanced chromosomes. Six pedigrees show developmental fallure before the 12 th day of pregnancy. Comparison of mouse and human developmental stages has been made using the literature on human development and tmed. A curve has been construeted by plotting the time of appearance and differentiation of individual organs or tissues in mouse embryos against the time of correspondings structures in human embryos. From this curve, it is judged that the time span of Antram uterine failure (7-12 days of mouse pregnancy) in the present sample of gemi-sterile pedigrees is probably comparable to the second to Bth weel of human pregnancy.
1736

Perxi, Teodoro, FPRE BIOLOGIC ACIION OF IIATIS ON PMBRYOS OF AMPYIBIANS. FXPERMMENTS OF

TRANSELANTATON. Riv. biol. $42,110-54$ (1D50).

In order to analyze the behavior of the different rudimentary organe of irradiated embryos, the author has transplanted such organs on normal embryos, in order to follow their destiny even after the death of the irradiated donors. The following conclusions are listed: (1) The optie wesicle of an enbryo, irradiated with certahn lethal doses, if it is transplarted on a normal embryo, outives by a long the irradiated embryo; in fact, it is gomethes in presty good condition at the death of the receiver. (2) The fore part of the encephalon is very ausceptible, much more than the hind parts. The rudimentary olfactory organ is more resistant than the fore encephalon, but generally leas than the optic vestcles. Very resigtant are the dorgal cord, the myotomes and the rudimentary fail. (3) In the transplamtation of the fore part of the head from irradiated to the normal embryos the disappearance of the tore parts of the encephalon causes the rudimentary olfactive organs to cone in mutual contact; the same thing happeng with the optic rudimentary organs. (A) The general conclusion is that the embryos irradiated with certan doses of $\mathrm{x}-\mathrm{ray}$ die, evidently on account of the death of organs indispensa ble to life, and because those conditions fall that render posalble the life of that unity of a superion order which is represented by the organim in its entrety. Some rudimentary organs, however, preserve the capacity to live and to develop themselves at least for a long time, which occurg if they are transplanted on normal enbryos. (5) Fsperiments of transplantation from normal to irradiated embryos and from irxadiated to normal embryos, have not. brought into evidence that the irradiated medium 18 in itself hurthul to a normal rudimentary organ, or that the normal medium is itself hur tul to an irradiated rudimentary organ. 35 references. (NSA)

$173 \%$

Quastler, Menry and Marianne Baer. INEITMTMON OF

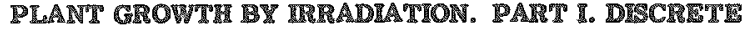

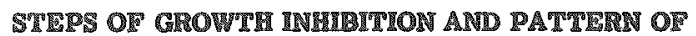
DOSE-RESPONS燚-RELATION. J. Cellular Comp. Physiol. 31, $213-34(1948)$.

Seed of Mung beang were treated whin varloug doses of roentgen rays and subsequently allowed to grow in lest tubes. The growth of primary root, hypocotyl, and epicotyl were observed and recorded. Fach of theae three parts grows ondy for a limited period of time reaching a limited length, even in normal plant:. The final lengh rneasure of growh. Ireadiation huhible growth. The relation between final length of primary root and dowge administered shows a patern characterized by geveral levals, where the root length remains stable in apite of congideram ble changes of dowage, and intermediate mones where the root length is labile and varies with dosage. The latter corregpond to relatively martow dorage zones. The doseresponse relations for hypocotyl and epicotyl are similar to but simpler than those for the root. It is assumed that the complex pattern of relations between dosage adminigtered and growth inhibition observed corresponds to an equally or more complex hierarchy of growth mechanismas. (NSA)

1738

Quastler, Henry and Mariana Baer. THLIBITYON OF PLANT GROWTII BY IRRADIATION. T. SENSITIVITY AND DEV V LOPMENT. J. Cellular Comp. Physiol. 33, 349-03(1940). 
A report is presented on the responses to $x$-irradiations administered to young growth bean seedlings. The concept of radiosensitivity is delined and methods for its calculation are presented in this report. The most sensitive type of response was elicited when the radiation doses were 1,500-3,500 r for the seedlings and were equivalent in their effect to $29,000-66,000 \mathrm{r}$ delivered to dry seeds: the response was characterized by fully developed side roots and atunted primary root. A series of experiments eliciting the severe reaction type, characterized by delayed primary growth arrest of the root and inhibition of the development of the side roots and the epicotyl, consisted of radations of $2,500-5,000 \mathrm{~m}$. It was concluded that the growing seedlings are more gensitive to roentgen rays than dry seeds. It was seen that with mild growth irhibition in the roots, the radiosensitivity rises rapidly, before any srowth occurs, and reaches a high value; in severe reactions to the roots, the sensitivity rises to a lesser degree. The developmental rise of senaltivity for the hypocotyl and epicotyl is similar to that for the roots. (NSA) 1730

Quastler, Henry and Marianne Baer. INHIBITION OF PLANT GROWTIY BY IRRADIATON. MI. SUCCESSTV RADITION EIT ECTE; HOMOLOCOUS RESPONSES.

J. Cellular Comp. Physiol. 35, 75-94(1950).

Growing scedlings of Mung beans (Phaseolus aureus) were mubjected to roentgen irradiation. The resuiting regponses showed great diversity but they can be analyzed into a small number of distinct components, most of which are related in nature, or homologous, to gome reaction found in dry aeeds. Different radiation effects characterized by their thming, and this serves as an additional means of distinguighing distinct growh mechanimms by their differential response to irradiation. (BA)

1740

Quaster, Heny and Mariane Baer. TNMBITON OF PLANT GROWTIY BV IRRADLATION. V. RADIATION

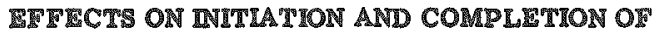
GROWTH. Cancer Research 10, 604-12(1050).

The observations reported concern linear growth in Mung beang (Phaseolug aureug), including the growth of individual segments and of the entire hypocotyl and the first Befraent of the epicotyl in normal plants, after resection of various parts, and after total or partial irradiation with various doses of $x$ radiation. Irradiation with $x$ rays causes three digtinct effects: temporary inhibition of growth completion and permanent reduction of the capacities both for initiating and completing growth. Permanent stunting is an effect of local irradiation. An analysis of dose-response relations Bhows that the apparatus for growth initiation is more vulnerable than the apparatus concerned with actual growth. (NSA)

\section{1}

Rollason, G. S. X-RADATION OF EGGS OF RANA PIPIENS AT VARIOUS RATURATION STAGES. BIOL. Bull. 97, 169-86(1049).

Eggs of Rana pipiens in the germinal vesicle stage (ovarian egger), metaphase of the second maturation division (uterine eggs) and during completion of the second maturation divirion (egge twenty minutes after fertilization) were irradiated with doses of roentgen units ranging from 100-41,600 x. The irradiation was carried out by means of two Coolidge-type water-cooled tubes at $180 \mathrm{kv}$, through filters of $2 \mathrm{~mm}$ Cu and $1 \mathrm{~mm} \mathrm{Al}$; no futration was used with the highest dosage given, $41,600 \mathrm{~F}$. Counts were made of cleaved eggs, gatrulae, neuralae, hatched embryos, and normal tadpoles. The jelly ourrounding the eggs was apparently unaffected except on the ovarian eggs at the highest irradiation doses $(25,600 \times$ and $41,600 \mathrm{x})$; damage at these doses may have been an indirect effect of the xrays on the oviduct. The cleavage rate and pattern were not affected; the percentage of cleavage was very much lowered by the irradiation. The lower doses of $x-r a y s$ had relatively liftle effect on the ovarian eggs; the uterine eggs were more sensitive to $\mathrm{x}$-radiation than were the ovarian eggs, while the fertilized eggs were by far the most sensilive of the three groups tested; when a dose of $1000 \mathrm{r}$ was administered to the fertilized eggs, only $9.6 \%$ of the ani mals gastrulated, 1.49 of these showed evidence of neurulation and non hatched; a dose of 41,600 $\mathrm{r}$ inactivated the uterine eggs, while at this latter dosage, although only a small percentage of the ovarian eggs cleaved, of those that cleaved, $87.5 \%$ gastrulated, $62.5 \%$ neurulated, and $10.71 \%$ hatched. The types of abnormalities produced by irradialion of the egge are similar to those resulting from irradiation of the sperm of the same species. A delay of 24 hours between irradiation and fertilization of the uterine eggs, to simulate the required delay for the ovarian eggs, appeared to have no effect; there seemed to be no recovery from the effects of the $\mathrm{x}$-radiation. There was no difference in the effectiveness of the different $x$-ray intensities used in this work as long as the total roentgen exposure remained the same. 43 references. (NSA)

\section{2}

Rugh, Roberts. HISTOLOGICAL EFIRCTS ON THE EMBRYO FOLLOWING X-RADIATION. J. Morphol. 85 , 683-95(1949). 27p. (AECD-2596)

Larvae of Amblygtoma punctatum (a salamander) measuring $2.2 \mathrm{~cm}$ in total length were given a single dose of $15,000 \mathrm{x}$-rays for the purpose of studying the resultant histo-and cytological changes after a period of one week, when they were killed. Mitosis was abruptly inhibited in all cells which had not started the process. Those cells which were in mitosis at the time of irradiation were either able to complete the process or the nuclei and chromatin were so severely damaged that the typical intermitotic "resting cell" stage could not be achieved. The tissues most resistant to $x-$ ray damage in the larva were cartilage and muscle, probably because they were the farther developed. There was no evidence of damage to the intercellular matrix of the cartilage or to the myoplasm or myofibrillae of the muscle. The tissue most sensitive to $x$-ray damage in the larva was that of the central nervous system and the related sense organs. Neuroblasts were sloughed of into the brain cavity and neurocoel, cells and nuclel were in all stages of degeneration, and there was no evidence of growth. This tissue is the most actively differentiating tissue at the time of irradiation. The larval or embryonic tissues are vareiously sensitive to $x$-ray damage in a manner quite different from the adult tissues. Discussion is included which points to the possibility that severe damage is impinged upon the so-called resting cell, as compared with the more obvious mitotic phases, since there was complete inhibition of mitoses in all non-mitotic cellis. (NSA)

\section{3}

Rugh, Roberts. SOME PRE-LETRAL EFTECTS OF AMBLYSTOMA OPACUM LARVAE EXPOSED TO 25,000 X-RADIATION. Anat. Record 103, 500-1(1949).

The following is an abstract and is presented here in its entirely.

Larvae of 'A. opacum measuring from 1.7 to $2.6 \mathrm{~cm}$. in total body length were irradiated at $25,000 \mathrm{r}$, single dose, and atudied for morphological and physiological corollaries of irradiation-induced death during the ensuing 9 days. Neuromuscular and cardiac responses showed alight im- 
mediate increase, then slow decline with partial recovery at approximately 3 -day intervals. The prior ingestion of food increased slightly the peaks of initial activity. Irradi ated larvae never ingested food. Chromatophore, gill, and hindlimb development were abruptly stopped as of the time of irradiation. Hiatological analysis indicated the total absence of mitotic figures after 1 week, with many pycnotic and framental nuclei. This implieg radiation damage to the nuclei of so-called resting cells. Growth (cell proliferation) was stopped in all tissues. The central nervous system and related aense organs showed the greatest radiation damage. The cells of the brain and cord were disar ranged, sloughed off into the neurocoel, or the nuclei extremely abnormal. The cartilage and muscle showed the least radiation danage. It is evident that the most actively proliferating tis1744 sue was the most sensitive.

Rugh, Roberts. INMIBITION OF GROWTH AND THE PRODUCTION OF ORDEMA BY $\triangle$ IRRADLATON. J. Expil. Zö̈l. 114, 197-57(1950).

Incidental to studies on the histopathological effects of x-rays on the early frog (Rana pipiens) embryo, it was discovered that at certain stages of development and certain $x$-ray doses, edema would develop within 24 hours in the majority of cases, and this edena would persist until the death of the embryo. This finding ind icated the necessity for trying to determine the exact stage and dose neces. sary to reproduce the edematous conditions to the maximum extent and the possible origin of the edemas. The rem sults of single exposures to $\mathrm{x}$-1rradiation doses of 10,000 to 50,000 r of tadpoles at various stages of development are discussed with respect to the inhibition or stoppage of growth and the production of lordosis (curved back), edema, 1745 and other morphological changes. (INSA)

Russell, Liane Brauch. X-RAY INDUCED DEVELOPMENT ABNORMALITIES IN THE MOUSE AND THELR USE IN TTIE ANALYSIS OF TMBRYOLOGICAL PATTERNS. I. EXTERNAL AND GROSS VISCEEAL CHANGES. J. Exptl. Zoöl. 114, 545-601(1950) (ORNL-595)

Four-hundred and twenty newborn nice, irradiated with 250 kvp x-rays during intrauterine life, were studied externally and by gross dissection. Treated stages differed by 24 hour intervals and ranged from day $1 / 2$ to day $13 \frac{1}{2}$ after fertilization. Dosages employed were $200 \mathrm{x}$ (100 $\mathrm{x}$ in a few cases) for a survey of all stages; 300 and $400 \mathrm{r}$ (or dosage comparisons at the last five stages. The irradiated embryos and controls were hybrids between two pure strains (C57 black female $\mathrm{x}$ Nb male) and came excluaively from second litters. Irradiation with $200 \mathrm{r}$ before implantation $(1 / 2-4 / 2$ days), and with $300 \mathrm{~m}$ at $9 / 2$ days increases prenatal mortality in early and late stages, respectively. The peak incidence of natal death follows irradiation on days $91 / 2$ and $10^{1} / 2$. Considerable depression of birth weight occurs as a consequence of irradiation between days $8 / 2$ and $10 \%$. Considerable depression of birth weights occurs as a consequence of irradiation between days $8 / 2$ and $13 \frac{1}{2}$. Birth weight curves for different doses are unimodal and roughly parallel. They indicate that maximum depression would follow irradiation at a stage intermediate between days $10^{1 / 2}$ and $11 \frac{1}{2}$. Considering two main types of effect following irradiation - namely, prenatal mortality and abnormality at birth - the intrauterine development of the mouse seems to be divisible into three broad phases: (1) days $1 / 2$ to $4 \frac{1}{2}$ (possibly $5 \frac{1}{2}$ ) - considerable incidence of probably early death but $28 \%$ ( $100 \%$ in external characters) normality among survivors to ferm; (2) days $6 \frac{1}{2}$ to $13^{3} / 2-$ little or no prenatal death but high incidence of abnormali ties at birth; (3) days $14 \%$ to birth (data from preliminary experiment) - neither prenatal death nor visible abnormalities at term with doses of $300 \mathrm{x}$ and below; abnormalities (cataract, hydrocephalus, slin defects) do, however, develop later in life. Irradiation during the $6^{1 / 2}$ to $13^{1 / 2}$ day phase reveals a definite patern of critical periods in the formation of a variety of characters of the newborn. Externally apparent and visceral abnomalities produced with considerable incidence in consequence of treatment on a definite day (or days) and abnormalities, each of which occurred in only one animal are listed. Using the evidence of critical periods, some of the induced abnormalities have been linked with certain phases, or physical events, in the chain of processes known to lead to the formation of affected characters. Sensitivity in some cases occurs at the the of visibly katest degree of change in a primordium le.g., the critical period for digital reductions corresponds to the time of growth of limb buds; the aecond phase of ausceptbility to tall reduction is coineident with early tail growth). In other cases, sensitivity is not predictable from inown facts of descrigtive embryology (e.g., critical period for overgrowth in feet, firgt phase of susceptibility to tall reduction - both occurring before appearance of visible primordia). Increase in incidence of abnormality with increase in dose indicates that, even where there is an allor-none effect (actually rarer than graded segponse), animals are continuously distributed with regard to sengitivity. The Bensitivities of certain processes are low and show liftle scatter. There are both close gimilarities and atriking differences between radiation induced abnormalities and the effects of known genes. (NSA)

\section{6}

PRussell, Llane Brauch and W. L. Russell. CHANCES IN THE RELATVE PROPORTIONS OF DIFTERENT AXMAL SRELETAL TYPES WITHIN INBRED STRAINS OF MCE BROUGHO ABOUT BY X-IRRADIATION AT CRITCAL STAGES IN EMBRYNIC DEVELOPMENT. Cenetics $35_{3}$ 280(1950).

This paper is reproduced here in its entirety.

An extensive study on the effects of single doses of $x$ rays on (C57 $\times$ NB) ${ }_{1}$ hybrid mouse embryos, at atages ranghtng from days $\% / 2$ to $13 \frac{1}{2}$ after ferturation, revealed that the eritical period lor a posterlor shift in the thoracolumbar border was from days $7^{1 / 2}$ to $9^{1} / 2$, with day $8 / 2$ exhibiting peak sensitivity. Two gtraing, one (129) showing considerable natural variability in number of presacral vertebrae and the other (B alb G) showing matural variability in this and in the thoracolumbar border, were chosen, as being probably more gensitive than the specified uniform hybrids, for an extersive dosage-comparian gtudy. Almost 600 newborn mice irradiated during the critical period with doses of $25,50,100$, or $200 \mathrm{r}$ and 947 controls have been obtained. In the $\mathrm{B}$ alb $\mathrm{C}$, an increase in courteenth-rib development occurs with 25 on day $8 \%, 2$, is greater with $50 \mathrm{~g}$, and complete with 100 or $200 \mathrm{x}$. (Number of sternebrae and number of ribs articulating with the sternum are also increased, but correlstion with the thoracolumbar shift incomplete.) Both $\mathbb{B}$ alb $C^{\prime} \$$ and 1293 , whose normal presacral complement is 26 - or-27 and 25-or -26 , respectively, are shifted toward the higher number by irradiation on day $8 / 2$ (50 and, posably, even $25 \mathrm{r}$ ) and, less markedly, on days $7 / 2$ and $9 \% 3$. As had been anticipated, in strains where there is no natural variability, characters are considerably more resistant to radiation-induced shift. This is true of the position of the thoracolumbar border in strain 128 and of the presacral number in the $(\mathrm{C} 57 \times \mathrm{NB}) \mathrm{I}_{\mathrm{i}}$ hybrid.

\section{7}

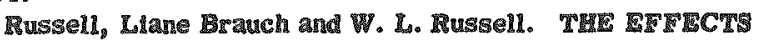


OF RADLTION ON THE PREMMLANTATION STAGES OF TIETE MOUSE EMBRYO. Anat. Record 108, 521(1950).

This paper is reprodiced here in its entirety. Ir radiation during preimplaniation (eleavage) stages causes high prenatal morality but only a negligible incidence of abnormalities in animals surviving to term, the relative magnitude of these two effects boing the reverse of that found following irradiation of postimplantation stages. To determine time of death inbred females which had received $200 \mathrm{r}$ on day $1 / 3,1 / 2,2 / 2,3 / 2$, or $4 / 2$ after mating were dissected on day $10^{1} / 2$ or $13^{1} / 2$ of gestat ion. A control was handled simultaneonsly with each irradiated female. Altogether 831 embryos and resorbing bodies were observed. In over-all effect, the earliest stages are more sensilive: the average number of living embryos per treated female is approximately $20 \%$ of the controls in groups irradiated on days $1 / 2,1 / 2$, or $2 / 2$, but 31 and $57 \%$ following treatment on days $3^{1 / 2} / 2$ and $4 / 2$, respectively. In groups irradiated on days $2^{1 / 2}$ and $3 / 2$, the major part $(91$ and $53 \%$, respectively) of the reduction in live embryo count is accounted for by death after implantation; wher as in grougs irradiated on day $1 / 2,1 / 2$, and $1 / 2$, the majority of the deaths $(71,71$, and $75 \%$, respectively) occurs before implantation. Postimplantation death probably occurs considerably before day $10 \%$. The main or sole expresaion of prewaplantation dealh is on entire litters in groups other than those irradiated on day $1 / 2$ or $1 \frac{1}{2}$ where 39 and $22 \%$, respectively, of the preimplantation death is due to selective mortalily within litters. Total-litter death may be due to radiation effect on the mother rather than direct lethal action on embryos.

\section{4}

Russell, Liane Brauch, W. L. Russell, and Mary M. Major. THE EFECT OF HYPOXIA ON THE RADIATON INDUCTION OF DEVELOPMISNTAL ABNORMALITIES IN THE MOUSE. Anat. Record 11, 455(1951).

This report is an abstract and is reproduced here in its entirely.

Several investigations have shown that hypoxia protects against various types of radiation damage (chromosome breakgge, sex-linked lethal mutations, immediate lethality). To determine whether protection can also be achieved against radiation induetion of developmental abnormalities (previously studied by us in a survey of the entire gestation period), mouse exubryos at equal developmental stages were erposed to various doses of $\mathrm{x}$-rays either in $5 \% \mathrm{O}+$ $95 \%$ He or $21 \% \mathrm{O}_{2}+79 \%$ He (i.e., oxygen per cent equivinlent to air), having also been preflushed with the respective gas mixture 10 min. prior to raying. Doses used were 0 , $100,200,300$, or 400 g. Irradiation was administered by whole-body exposure to the pregnant mothers: C5\%B females fertilized $11^{1 / 2}$ days before by $N$ B males. Our earlier work on critical periods had shown this stage 10 be convenient for quick asaays of differences. The 460 newborns ontained were eramined externally, then processed for delalled skeletal study. Hyporia in ilseli has no effect on the embryo under the conditions administered. Combined with irradiation, however, it exerts marked protection against damage in all the character radiosensitive on day $111 / 2$ that have so far been studied. These include birth weight, viability, tail length, tail shape, forefoot and hind soot giructure. Detailed gleletal findings will be described in future. The magnitude of protection is similar for all the characters so far tabulated, the median abnormality doses in the $5 \% O_{2}$ wixture ranging from roughly 100 to $160 \%$ above those in the $21 \% \mathrm{O}_{2}$ mixture.
1749

Sadun, Elvio R., Joseph N. Ane, Frances Willard Fuller, and Ruth Lewh. THE EFFECT OF X-RAX UPON THE GROWTI AND INFECITITY OF ENDAMOEBA HISTOLYTICA. Am. J. Trop. Med. 30, 635-40(1250).

$A$ brief study of the effect of $x$-ray treat ment in vitro upon the culturability, reproduction, and infectivity of Endarnoeba histolytica was carried out. It was observed that single irradiations up to $120,000 \mathrm{r}$ failed to kill or mactivate the organisms in Shaffer-Hrye and Lalamuth media. Fixposure of the "streptobacillus" up to $120,000 \mathrm{r}$ failed to produce any change in its ability to permut growth of amebate in $\mathbf{S}-\mathrm{F}$ medium. Exposure of amebat in Balamuth culture up to 30,000 ro did not seem to affect visubly the normal cycle of population growth. On the other hand, escposures 1060,000 and $120,000 \mathrm{r}$ seemed to inhibit, somewhat, arnebic nopulation yruwth. Viable amebae ware whserved for 16 transfers after irradiation with $120,000 \mathrm{~s}$. Injection of the progeny of the $x$-rayed amehas $(120,000 \mathrm{p})$ mit normal guinea pigg produced severe intestinal leswons just as did the unexposed controls. (NSA)

1750

Salzeber, Berthe. STHPILIRATION ANI INTLRSEXUALITY OBTAINED IN CHICREN EMERYOS BY X-IRIADIATION. PRELIMINAFY REPORT. Bull. biol. France of Belg. 84, 225-33(1050).

Lovealized arradiation of the gonads of chick embryos with 900 to $1200 \mathrm{r}$ in the 24 to 37 somite stage, followed 1 to 2 days later by total inradiation with $300 \mathrm{r}$, catused some sterilizations in male and female chicks, and 13 of 21 malss became interseses. Irradiathon-produced disturbances in hormone production probably caused these abnormalities. (NSA)

17551

Singleton, W. Ralph. NO STPMULATION FROM X-RAYS OR GAMMA IFAYS. Brookhaven National Lab. [nd] 2 p. (ABCU-804)

The document is in abstrat form; it is reproduced beslow in its entirely.

Various workers have reported at different times preliminary data showing stimulation of slight amounts of radi ation. Most, if not all, of these reports are based on limited data and, so far as we are aware, no extensive experiments have corroboraled these preliminary indicat 10 nis of st mulation.

Last cummer an experiment was planned to determme any atimulating effecls of x-rays and gannma xays. The $x$ ray used was set at $160 \mathrm{kv}$ and $10 \mathrm{ma}$. Doses given were $250,500,1,000$, and 2,000 r. Comparable doses of gamma rays were given by placing the seeds (in Petri dishes) at a distance of approximatel $1 / 2$ meter from a 16 of $\mathrm{Co}^{68}$. Four different 4 by 4 latin squares were planted with seed given the four different dosages of $x$-rays and gamma rays. In two latin squares standard early forld corn was grown. In the other two, short hybrids with the reduced grane (rd) were grown. These were used buseanse it wa thought that small differences in height might be more readily detected. In all the latn squares, 4 -row plots were planted, using a split-plot lechnique whereby two rows were checks with no treatment and for two rows the seed was radiated.

Height measurements were made al weekly mtervals on one of the four replications of each lat in square, and tolal lneight was obtained on the matures plants on all plots. There was no significant dufference in height of any of the 4 x-ray treatments or the 4 gamma-ray treatnents in 
comparison with the cont rols. lloikewnese the yrolds showed no significant dıference for any treatment. If xperiments will be run an 1950, using dosess of $2,000,4,000,8,000$, and $16,000 \mathrm{r}$ of both $\mathrm{x}$ - and ganma rays. On the basis of present information it seems unlikely that at stimulatmig effect will be found. Certamly none was found, using dosts of $250,500,1,000$, and $2,000 \mathrm{r}$ of elther $x$-rays or gamma rays. Neither was a depressing affect formed for any of the dosages used.

\section{2}

Shoog, Folke. THE EFIECT OF X-IRPADLATION ON AUXIN AND PLANT GROWTH. J. Cellular Comp. Physiol. 7. $227-70(1935)$.

By the application of hard $x$-rays to the plant growth hormone, ausm, in solution and to the plant the hormone is inactivated. The mechanssm is through the oxudation by strong oxiduding agents formed by irradiation. Thus in a

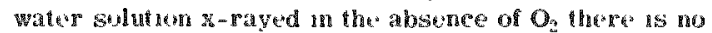
hor mone destroyed. A compapable unact a ation can be carreved ant also un white light by the use of small concentrathons of ensun as a catalyst. Extracted and synthetic heteroumun, beta-indulyl aeftic al'ad, as well as ausn $\mathbb{A}$ are sumlarly mactivated in aqueous solutum. In groen plants such as Pisum and Viro ia the subsequent formation of

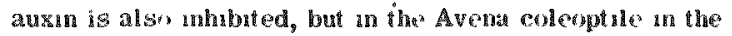
darts there us nu last ing effect on the formation of ausun. The mechansm of transport of auxin in the plant 15 not affected by rradiatum. The applicentom of ausin to treat fed plants shows that the caparily for growth 193 as great in irradiated as in cont rol plants. The development of lateral buds in irradated plants as correlated directly with the removal of auxin and 18 not due to any stumulation of growth by the $x$-rays. It is centelucted that the effect of urradiatnon on ausun and on the processs of its formation is the major

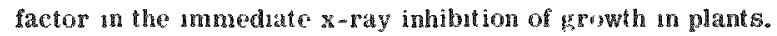
(BA)

1753

Smith, G. F. and H. Kersten. ROOT MODHICATIONS INDUCED IN ZEA MAYS SE EDLINGS BY IRRADIATING DRY SELDS WTT SOFT X-RA IS. Plant Phys301. 1\% $455-64$ (1242).

Unsoaked seeds were treated whth radratuen from a fastype Cu larget $\mathrm{x}$-ray tube operated at 30 peal $\mathrm{Al}$., $10 \mathrm{ma}$. for 100 muntes. Fiternal modifications nelude: decreased root-length; fanlure of lateral root formation; growth cessaton after is short pernod of gepmination. Histological modificatrons anclude: distortien of the primary root tup meristem; distortion of the pericycle in lateral root umt ia tron and of cells of adventitious root-primorida in the first int exode; early and rapld formation of lysigenous cavitres In the pith region of modised roots as compared with their formation in normal roots; formalion of schizugrenus cavitus due to cell wall gpliting in various portions of the pith. (BA)

\section{4}

Sparrow, A. R. and H. Christensen. IFFECTS OF X-RAY, NEUTHON, AND CHRONIC GAMMA IRRADIATON ON GROWTY AND YIELD OK POTATORS. Am. J. Botany 37, $667(1950)$.

Ths document is reproduced beluw in its entirety.

Lal ni-square plots of Katahdin potatoes were planted follownger cuposure of the tubers $1018.75,75,300,1200$, and $4600 \mathrm{r}$ of $200 \mathrm{kvp}$ x-rays. Control pieces were laken from gach luber before ir radiation and included in oach loatin square. Irradiation was given at a constan rate of about 4600 ger hour. Statistrcal analysis showed no signufficant effect on yeld following $18.75,75$, and $300 \mathrm{~g}$, but did show a signifunt adverse effert following 1200 and $4800 \mathrm{t}$.
"These yelded, respertuvely, $75 \%$ and $4 \%$ of the controls. The atter dosegge is shown to be near the lethal limit for Ratahdin tubers. True sted was collected from the 18.75 , 75 , and $300 \mathrm{rr}$ groups. Statistical tests of germination showed a symfirant trend toward better germination in the seed from the 300 rgroup over the 18.75 and $75 \mathrm{r}$ groups. I'urther lests are under way. Latin-square plots of the same variety were planted in a field where a centrally lofated gramma source of approsimately $16 \mathrm{c}$ of $\mathrm{Co}^{60}$ provided centruuous ar radation of $0.26,1.15,4.8,19.5$, and $79.7 \mathrm{r}$ per day to give full-season lotals of approximately 28,123 , 516,2046 , and $8529 \mathrm{r}$, respectively. Chronic gamma irradiation over this range of dosages produced no significant redation betwen dosage and yield. There was no adverse effects on urowih or yield even at the highest dosage. The efferts of fast-neutron acute irradiation are also under in1755 vesthgation. Siwndicant data will be reported. (NSA)

Tahmisian, Theodor N. THE FFFECT OF X-RADLATION ON TUE METABOLIC PROCESSES OF THE RESTING CELL. J. IExpL. Z001. 112, 449-63(1949).

Studies on the effect of high dosages of $x$-rays on respiratuon and the relationship of nuclear degeneration to the anabolic processes of the cell were conducted utilizing grasshopper eggs (Melanoplus differentalis) of known chronologgal, morphological, and physiological ages. After reachng the age of 40 days, and when in diaphase, the eggs were divided into sis groups, five of which were igradiated at $200 \mathrm{kv}$ with no filliration except the pet $\mathrm{r}$ dish, at a target dustance of $2 \mathrm{~cm}$ and at the dosage rate of 1,563 to 1,588 $\mathrm{r} / \mathrm{mm}$; doses were $25,000 \mathrm{r}, 50,000 \mathrm{r}, 100,000 \mathrm{r}, 150,000 \mathrm{r}$, and 200,000 ror the different groups. Immediately follow Ing the irradiation each group was equally divided and unehalf was maintained at $0^{\circ} \mathrm{C}$ and the other half at $25^{\circ} \mathrm{C}$ while respiration was measured; physiological, and morphological cxaminations were carried for a period of 40 days aster the ir radiat ion. It was found that dosages of 25,000 to $200,000 \mathrm{r}$ did not completely inhibit respiration. Anabolic encymes were found to be associated with the nuclear material and were more susceptible to the radiation damage than the catabolic enzymes; in a resting cell a nucleus was found to be necessary for the maintenance of the status quo. Pyknosis of the resting nueleus was found lo depend upon the s-ray dosage, the state of metabolism and the time. Repression of irradiation damage to the cell could be obtained by subjecting the cells to submetabolic temperatures. (NSA)

1756

Tahmisian, Theodore N. and Dorothy M. Adamson. THE EFFECT OF ANOXIA ON X-RAY-INDUCED INJURY IN

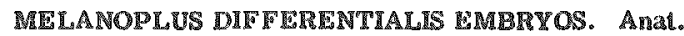
Record 108, 516(1950).

This paper is reproduced here in its entirety.

Melanoplus differentialis grasshopper embryos placed in an atmosphere of $\mathrm{N}_{2} / \mathrm{CO}_{2}(95 \% / 5 \%)$ for $24 \mathrm{hr}$ developed normally. If these anoxic embryos are irradiated at 25,000 I after 24 hr of anoxia and placed at $25^{\circ} \mathrm{C}$ in $\mathrm{ar}$, negative growth does not aceur as with eggs irradiated in arr. Upon interrupting diapause by fold ireatmont for 3 months, when returned to $25^{\circ} \mathrm{C}$ theses cmbryos irradiated under $\mathrm{N}_{2} / \mathrm{CO}_{2}$ unlike those irradiated in air show development. Namely, they undereg blastolsinesis, increase in size, resting nuclei appear normal, respiration is nornal, mitosis resumes, the embryos grow, and the oxidative enzyme due to $\mathrm{x}$ radiation dues not appear. These do not hat foh, however. Sinee any oxygen wheh is dissolved an the egg th theoretically used up in $1 / 2$ hr the irradiation effect on these embryos under $\mathrm{N}_{2} / \mathrm{CO}_{2}$ is in corrplete metabolir standstill. Evi- 
dently, in orthoptera no anaerobic glycolysis takes place, so that no form of energy release known to us is possible. Since the oxygen substrate moiety is interrupted, we may regard metabolism stopped, and under such conditions irradiation damage is at a miximum.

1757

TOOlan, H. W. PROLIFERATION AND VASCULARIRATION OF ADULT HUMAN EPITHELIUM IN SUBCUTANEOUS TISSUES OF X-IRRADIATED HETEROLOGOUS MOSTS.

Proc. Soc. Exptl. Biol. Med. 78, 540-3(1951).

In 6 experiments, minced fragment s of normal adult human epithelium implanted subcutaneousiy in previously $\mathrm{x}-$ irradiated heterologous hosts (rats and hamsters) becane vascularized, proliferated and increased for approximately 15 days. After this period they died suddenly. In unirradiated control animals the epithelial material survived in poor condition for $3-7$ days but did not become vascularized. When transferred every 10 days to new groups of $8-$ irradiated animals, however, epithelium remained viable and in active mitosis for the duration of the experiment (3 generations or 31 days). (Author's Summary) 1758

Toole, E. Kilmer, and I. T. Alexander. TFFECTS OF" ALPRATRON" AND OTHER RADIOACTIVE MATRIRALS ON GERMINATION AND EARLY GROWTI OF VARIOUS CROPS. U. 3. Department of Agriculture. Apr. 24, 1950. 12p. (AECU-852)

The effect of alphatron ( $\alpha-$ emilting material) ongermination and early growth of seedlings of wheat, alta fescue, soybean, cucumber, cabbage, radish, tomato, pepper, and com was studied. The results indicated no consistent trend of any effect sither on amount of germination or on carly growth of the young seedlings. (NSA)

\section{9}

Umanskŭi, E. E., B. M. Varshavskĭi, and V. P. Kudokotsev. SENSITIZATION OF TISSUES TO X-RAYS. Doklady Akad. Nauk S.S.S.R. 65, 581-3(1949).

Injection of fluorescein, Neutral red, or Congo red into specimens of Triturus cristatus with amputated legs, followed by $x$-ray ir radiation of various levels, showed that fluorescein is the most active sensilizer, so that even a $1000 \mathrm{x}$ dosage of $\mathrm{x}$-rays almost completely represses regeneration of the limb. The other 2 dyes showed very little effect and only at higher $\mathrm{x}$-ray dosages. (CA)

\section{0}

Villé, Claude A. SOME EFIECTS OF X-RAYS ON DEVELOPMENT IN DROSOPHLA. J. Expt1. ZOö1. 101, 261-80(1946).

Drosophila larvae, prepupae, and pupae of 11 genotypes and a variety of ages were irradiated with dosages ranging from 1,096 to $7,000 \mathrm{I}$ at several different intensities. The phenocopies produced varied with the age of the irradiated fly, the stock used, and the intensity and total dosage of the irradiation. Pupation was retarded by all but the smallest doses used. An irradiation given at high intensity $(5,540$ $r /$ min.) caused a much higher lethality, both before and after pupation, than an irradiation of comparable lotal dosage given at low intensity $(78 \mathrm{r} / \mathrm{min}$. $)$. Flies irradiated 28 hours after puparium formation showed only the suppression of some microchaetes. Flies irradiated as late larvae or early prepupae showed the suppression of macrochaetes, small, rough, or Lobe eyes, crippled legs, and abnormalities in the formation of mesonotum, scutellum and abdomen. The main effects of irradiating larvae 70-100 hours old were the overgrowth or duplication of parts and examples of abnormal histogenesis: leg-like antenne, formation of palps in the eye, prothoracic palps or wings, and others. No evidence was found of an interaction between irradiation and the homoetic genes in causing changes in development. Waddington's hypothesis of the indreet artion of z-rays in producing their pervasive effects is disfussed. (BA)

1761

Warren, Shelds and Frank J. Dixon. EFPECTS OF CONTINUOUS RADIATION ON CHICR IMBRYYOS AND DEVLLOPING CHICKS. 1. GROWTH RATE, GONADS, AND BONE. II. BONE MAREOW, LYMPHOD TISSUE, AND PERIPLERAL BLOOD. Radiology 52, 714-29(1949).

Three sets of experiments were performed on chck embryos and young chicks utilizing $\mathbf{P}^{32}$ combined as $\mathrm{KH}_{2} \mathrm{PO}_{4}$ in order to study the effects of radiation on these organisms. In the first group, white Leghorn embryos in the fourth to fourteenth day of incubation were given single injections, varying in amounts of $\mathbb{P}^{32}$ from 47.5 to $300 \mu \mathrm{c}$. In the second group, chicks 1 to 37 days old were given single subcutaneous injections with isotonic solutions of $\mathrm{p}^{22}$, in concentrations of 115-1,050 $\mu \mathrm{c}$. The third group consisted of chicks receiving muluiple $\mathbb{P}^{\text {Sa }}$ injections, the total dosage being 180-3,696 $\mu \mathrm{c}$. Part one deals with the effect of radi ation on the bones, growth and sex organs of the chicks and embryos. The results show that the $\mathrm{P}^{32}$ was mainly concentrated in the long bones of both the embryos and the chicks, and caused considerable over-all growth retardation, resulting in small but well proportioned birds. Both the testes and the ovaries were found to be among the most radiosensitive of the organs and were the least able to recover from the injury caused by continuous radiations. The primitive sex cells in either gonad were the most sensutuve element. The spermatogenic cells of the testes remainud extremely radiosensitive throughout their development, while the ova became more radioresistant as they matured. The somatic cells of the gonads were much more radioresistant than the sex cells, but their rate of growth was inhibited following injury to the sex cells. In bone growth retardation, the cartilage cells of the epiphysis were much more radiosengilive than the osteoblasts and osteoclast: After radiation censed, the bones regained a normal histologic structure but remained dwarfed. Part two deals with the effects of radiation on the bone marrow, lymphoid tissue and the peripheral blood of the Leghorn chicks and embryos. The radiations had erucial effeets on the bloodforming tissues, since the $\mathbb{P}$ was concentrated most heavily in the bone marrow; maturation of the hematopoietic cells was inhibited and mitotic activity was reduced. These changes were revergible and after the radiation had diminished, the bone marrow returned to normal. The larger doses of radiation, which eventually caused a fatal anemia, produced an inmediate and virtually complete cessation of mitotic activity and maturation. In this group, after the radiation had decreased significantly, the few resmaining hematopoietic and reticulo-endothelial cells began to show mitotic activity and maturation, but in an amount insufficient to prevent the development of an anemia which proved fatal several days later. The lymphoid tissue of the thymus was also very gensitive to radiation and showed an almost immediate marked reduction of mitotic activity, which lasted until most of the retained $\mathrm{P}^{32}$ had been concentrated in the bones. Recovery was rapid, and all but the most severe of these injuries to the lymphoid tissue were reversible. Peripheral blood counis followed a definite pattern during and following irradiation: the lymphocyte count fell first and recovered before the other cell iypes; the granulocyle count fell nest most rapidly and recovered most slowly; the red cell count dropped most slowly and returned to normal midway botween lymphocyte and granulucyte counts. (NSA) 
1762

Weber, R. P. and S. A. Gurdon. THE RADIOSENSITIVITY OF AUXIN BIOSYNTHESIS, p.79-80 of DIVISION OF BIOLOGICAL AND MEDICAL RESEARCH; QUARTERLY REPORT, MAX, JUNE, JULY, 1951, Austin M. Brues, ed. [nd] ?p. (ANL-4676(p.79-80))

The capacity of the plant tissues to produce the growth hormone auxin is rapidly decreased by low doses of ionzing radiation (25 to $100 \mathrm{r}$ ). Our experiments also indreate that the radiosensituvity lies in the lability of the entynes in volved in ausin production rather than a sensitivity oither of the intermediates of auxin biogenesis, or of auxin itself. (Authors)

1763

Weber, R. P. and S. A. Cordon. THE RADIOSENSITIVITY OF AUXIN BIOSYNTHESTS, P.91-3 of DIVISION OF BIOLOGICAL AND MEDICAL RESEAICH; QUARTERLY REPORT, AUGUST, SEPTEMBRR, OCTOBER, 1951, Austin M. Brues, ed. [nd] 3p. (ANL-4713(p.91-3))

Groups of seven-day mung bean seedlings were s-irradiated with dosages ranging from 50 to $1000 \mathrm{r}$. DetermuaHons were made of the capacity of these plants for converting infiltrated tryptophane to auxin immediately after and for several days followng arradiation. Evidence of enzyme damage is apparent immediately after irradiation with low doses; the extent of mhibition of the tryptophan convertng system becomng greater as the $\mathrm{X}$ ray dose inereases. With dosages of $500 \mathrm{r}$ or less, recovery from the inhbitory effect occurs within two weoks; even with doses of $1000 \mathrm{x}$, recovery is virtually complete at the end of the same period. These fundings strikingly correlate an enzymatic radiosensitivity with the effect of low radiation dosages on native auxin levels in kidney bean and cocklebur piants. (Authors)

1764

Welander, A. D., el al. THE EFECTS OF ROENTGEN RAYS ON THE EMBRYOS AND LARVAE OF THE CHINOOK SALMON. Growth 12, 203-42 (1948).

An evaluation was made of the effects of $x$-rays of 250 , $500,1,000,2,500,5,000$, and 10,000 r on the eyed embryos of chinook salmon, Oncorhyuchus tsehawytseha, using criteria such as death rate; change in growh rate, of both body weight and length; development of the fins, eyes, coloration and other structures; edematous or other degenerative effects; and histopathological effects on the hernopoietic tissue of the kidney, spleen, gonads, and interenal bodies. Cell counts and of her quantitalive measures revealed that the gonads were the most sensitive to x-rays with the hemopoietic tissue second. Most tissues and organs rew acted to $\mathrm{x}$-rays of $1,000 \mathrm{r}$ or more, indicating temporary retardation of developnent for the most part. Mortalities of $52.5 \%$ occurred in the $1,000 \mathrm{r}$ group over the period of 125 days of investigation. Severe damage to the organs and tissues and $100 \%$ mortalities resulted from doses of $2,500 \mathrm{r}$ or more. (ADD)

1765

Welander, Arthur D., Lauren R. Donaldson, Richard Foster, Kelshaw Bonham, Allyn $\mathrm{Il}$. Seymour, and Fank G. Lowman. THE EFFECTS OF ROENTGEN RAYS ON ADULT RAINBOW TROUT. University of Washington Applied Fisheries Lab. [nd] 20p. (AECU-188; UWFL-17)

Nearly mature adult rainbow trout were subjected to $25 \mathrm{ma}_{2} 200 \mathrm{kv}$, copper and aluminum filtered $\mathrm{x}$-rays of 50 , $100,500,750,1,000,1,500$, and $2,500 \mathrm{r}$ at $8.25 \mathrm{r} / \mathrm{min}$. A total of 148 fish was used in the experiment which lasted 64 weeks. When compared with an unirradiated control group mortalities were significantly higher in fish exposed to $1,000 \mathrm{r}$ or more. Duses of $2,500 \mathrm{r}$ killed all fish in a perind of 13 weeks with the majority dying during the 8th week. Exposures to $1,500 \mathrm{r}$ killed $56 \%$ in 13 weeks and $87 \%$ in 64 weeks. Mortality in the contruls was $15 \%$. Injuries symutomatic of radiatisn were seen in autopsied fish fredted with $500 \mathrm{r}$ or more. The injuries, such as hemorrhage, necrosis, growth of fungus and intestinal damage were generally proportional to the amount of x-ray given. Growth in length and weight was retarded in all irradiated groups as compared with controls. The growth increment during the fastest growing period of the experiment was significantly less in a fish irradiated with 750 r or more and proved to be a sensitive measurement of radiation damage and directly proportional to the amount of radratrus given. (NSA)

1766

Whiting, Anna R. FAILURE OF PUPATHON OF ERHESTIA LARVAE FOLLOWHAG EXPOSURE TO X-IRAYS. Anat.

Record 108, $009(1250)$

This paper is reproduced here in its entirety.

The Mediterranean flour moth $\mathrm{g}_{\text {. }}$ kuhniella, belongs to the group of insects (homodynamic) in which a continuous succession of generations occurs so long as conditions are favorable. In the course of geveral experiments full-grown larvae were irradiated with doses ranging from 40,000 to $160,000 \mathrm{r}$. Following a short period of inactivity they moved about normally and were readily stung and fed upon by the parasitic wasp Ihabrobracon. Those not exposed to the parasite continued to crawl, and none pupated. All Inved for at least a few days and some $(41 / 177$ or $23.16 \%)$ were still alive 30 to 40 days after exposure. Control larvae pupated 3 days after time of exposure of ireated larvae. 'The behavior of the irradiated larvae resembles that of duapause larvae in forms (heterodynamic) which show a prolonged arrest of growth in this stage. Whether the $\mathrm{x}-\mathrm{ray}-1 \mathrm{nduced}$ inhibition is due to an interference will secretory processes controlling pupation or to injury to cells of the larval imaginal disks vital to pupal formation is not known. Two irradiated in prepupal stage pupated but have failed to close.

1767

Wi1SOn, C. W. SOME EFTECTS OF THE GAMMA RAYS OF RADIUM ON THE DEVELOPING CHICK EMBRYO. THE DEPLNDENCE OF THE DELAYED LETHAL DOSE ON THE AGE OF THE EMBRYO. II. THE RECOVERY AND OEVELOPMENT OF CHICK EMBRYOS AFTER IRRADIATION AT VARIOUS AGES. Acta Radiol. 16, 719-34(1935).

Chuck embryos of $1-6$ days' incubation were uniformly irradiated with $\gamma$-rays at an intensity $5.3 \mathrm{x} / \mathrm{min}$. The dose given by 3 hrs. exposure (appros, $1000 \mathrm{r}$ ) is near the borderline which permits the complete recovery of an irradiated embryo, with subsequent hatching and this dose is the same for all ages between 1 and 6 days. The radiosensitivity of the chick decreases regulaty for the first 3 days of incubation, decreases more rapidly from 3 to 5 days and then remains fairly stationary. There is a possible differential action of roentgen rays and ymays on the developing chick embryo. (BA)

1768

Wilson, James C. EFICCTS OF X-IRRADIATON ON

EMBRYONIC DEVELOPMENT IN THE RAT. Anat.

Record 103, 520-1(1949).

The following is an abstract and is presented here in its chtirety.

On the 10 th day of gestation rate were opened surgically and shielded with lead plates in such a manner as to permit irradiation of 2 to 5 implantation sites, without exposure of the mother or remaining implantation sites. After post-irradiation intervals of $24,48,72,96$, or 120 hours 
both irradiated and nonirradiated embryos were removed, weighed and prepared for histologic study. Exposure to 50 $r$ had no effect on embryonic development. Dosage with $100 \mathrm{r}$. increased slightly the incidence of intrauterine death, reduced temporarily the rate of growth, and caused retarded or anomalous development of eyes in $60 \%$ of embryos. These effects were apparent within 24 hours but did not become appreciably more pronounced after longer intervals. Treatment with $200 \mathrm{r}$. caused similar but more intense effects; higher death rate, slower growth, more severe developmental defects; after progressively longer intervals, death rate and developmental abnormalities increased. Exposure to 400 r. always caused embryonic death within 24 hours after exposure. The eye was the organ most sensitive to the deleterious effects of $x-$ ray, and was the only structure frequently malformed or completely suppressed by irradiation. The brain was second in order of sensitivity to irradiation but it was more often retarded than aberrant in development. Parts of the urinary system, i.c., mesonephros and urogenital sunus, were affected al1769 most as often but rarely as severely as the brain.

Wilson, James G. and John W. Karr. DIFIERENCE IN THE EFFECTS OF X-IRRADIATION IN RAT EMBRYOS OF DIFTERENT AGES. Anat. Record 106, 259-60(1950).

Pregnant rats were opened surgically on the 9th and 10th days postovulation and shielded with lead plates so as to permit direct irradiation of selected implantation sites without exposure of remaining implantation sites or the mother. Irradiated and nonirradiated embryos were removed 3 or 4 days later and prepared for histologic study.

Subsequent development was altered much more severely by $x$-irradiation on the 9th than on the 10th day of gestation. Exposure to $200 \mathrm{r}$ on the 9th day usually resulted in the death of all embryos, whereas on the 10th day a dosage of $400 \mathrm{r}$ was required to obtain a similar lethal effect. Sublethal dosages at either age caused specific malformations in the survivors, but to produce defects comparable to those produced by $50 \mathrm{x}$ on the 9th day, it was necessary to use $200 \mathrm{r}$ on the 10 th day. In both instances the eye was the most common site of maldevelopment (anophthalmia and microphthalmia), the brain was often hypoplastic, and the heart, urinary tract, and brain were occasionally malformed. Situs inversus and malformation of the spinal cord, however, were noted only after irradiation on the 9th day.

Also more prevalent after the earlier exposure were abnormal growths of compact masses of nervous-like cells in the mesenchyme surrounding the brain. Although apparently of neural origin, these islands of aberrant tissue were growing independently of the neural tube. (NSA)

\section{0}

Wilson, James G. and John W. Karr. EFFECTS OF

IRRADIATION ON EMBRYONIC DEVELOPMENT. I. X-RAYS ON THE 1OTH DAY OF GESTATION IN THE RAT, Am. J. Anat. 88, 1-33(1951).

Doses of $50,100,200$, or 400 r were given at a single exposure to intrauterine rat embryos the 10th day of gestation, with shielding of the mother animal as well as of the remaining portions of the uterus. Four types of biological ef fects resulted: general retardation of growth, localized retardation of growth, aber ant growth or malformation, and embryonic death. Exposure to $400 \mathrm{r}$ always caused embryonie death within $24 \mathrm{hr}$. Malformations of brain, urinary organs, liver, limbs, and inversion of aortic arches occurred after exposure to 100 or $200 \mathrm{r}$. Improvement or recovery by later accelerated mitotic rate can occur. Malformation of eyes was prevalent and complete regression may occur. 29 figures. (NSA)
1771

Wort, D. J. X.RAY EFEECTS ON THE GROWTH AND REPRODUCTION OF WHEAT. Plant Physiol. 16, 373-83 (1941).

Twenty-four hour-old seedlings of Marquiss spring wheat grown from seeds 57 months old and 9 months old were ir radiated by $x$-rays of 19 to $228 \mathrm{r}$-units, using a $1 \mathrm{~mm} \mathrm{Al}$ screen. Controls were plants receiving no treatment. Irrespective of the age of the seed greatest growth rate and height were produced by $114 \mathrm{r}$-unit treatments. Greatest fresh and dry weights of plants grown from the older seeds resulted from the usc of $76 \mathrm{r}$-units; of fresh seeds, from $57 \mathrm{r}$-units. $\mathrm{X}$-radiation of from 76 to $114 \mathrm{r}$-units accelerated heading and flowerung of plants grown from old seed by as much as three days, but retardation of heading and flowering occurred when fresh seed was irradiated. Height and weight of Fulhio winter wheat seedlings were increased considerably by all doses of $x$-rays used, the maxima occurring when the dosage was $114 \mathrm{r}$-units. (BA)

\section{GENETICS}

1772

Anderson, E. G. ON THE FREQUENCY AND TRANSMITTED CHROMOSOME ALTERATIONS AND GENE MUTATIONS INDUCED BY ATOMIC BOMB RADIATIONS IN MAIZE. Proc. Natl. Acad. Sci. U. \$. 34, 387-90(1948).

Samples of maize seed of the single cross $\mathrm{L} 289 \times 1205$ were exposed to the atom bomb radiations at Bikini. Other samples were exposed to $15,000 \mathrm{r}$ units $\mathrm{x}$-ray. They were then tested for hereditary chromosomal alterations and gene nutations. The seeds exposed at Bikini were found to have received nearly $15,000 \mathrm{r}$ units $\mathrm{x}$-ray. In maize, chromosomal alterations can be readily detected by partial pollen sterility. In this study the frequency of alteration was found to be $4.7-6.4 \%$ in progenies not entirely randon. Random frequency was estimated to be between 4.5 and $5.0 \%$. Gene ruutation frequency was very high with probably more than $50 \%$ of the gametes of the exposed generation carrying one or more gene mutations. The frequencies of chromosomal alterations and of gene mutations in the Bikini group were roughly equal to those in the $\mathrm{x}$-rayed group. (NSA)

1773

Anderson, E. G., A. E. Longley, C. H. Li, and K. L. Retherford. HEREDTARY WITCTS PRODUCED IN MAIZE BI RADIATIONS FROM BIKINI ATOMIC BOMB. I. STUDIES ON SEEDLINGS AND POLLEN OF THE EXPOSED GENERATION. Genetics 34, 639-46(1949).

This report deals with the immediate generation of plants grown from five lots of maize seed exposed to atomic radiation, and includes observations on the effects shown in the seedling stage and observations and analyses of the effects shown in the pollen stage; the lots were compared with lots exposed to $5,000 \mathrm{r}, 10,000 \mathrm{r}, 15,000 \mathrm{r}$, and $20,000 \mathrm{x}$ of $\mathrm{x}$-radiations. Bikini Lot $\mathrm{A}$ showed effects slightly less than those observed in the $15,000 x$ lot; the effects on the Bikini Lot were, however, more uniform. The percentages of central spikes of the tassels of the plants showing one or more sectors of partially sterile pollen were $4.0-63.1 \%$ for the Bikini series, and 29.5$70.4 \%$ for the $x$-ray series. It is estinated that there were between two and three partially sterile sectors per plant of average size of about two branches in the Bikini Lot $A$. It is concluded that the data indicate that cell competition in the growth of the apical meristem eliminates the nore affected cells, thus modifying the dosage frequency relations in the plants. (NSA) 
1774

Anderson, Edward H. THE EFIPCT OF OXYGIS ON MUTATION INDUCTION BY K-RAYS. Proc. Natl. Acad. Sei. U. S. $37,340-9(1951)$.

The effect of a high oxygen tension on the induction of mutations by $\mathrm{x}$-rays has been examined for a streptomycin dependent strain and for a purineless strain of E.coli. Back mutation rates to streptomycin nondependence were studied under comparative exposures to $x$-rays in the presence of oxygen and nitrogen. The sensitivity of both strains to the lethal effects of $x$-rays was similarly increased by the presence of oxygen during irradiation. Mutation rates of both strains were increased with length of exposure to $x$-rays in the presence of either oxygen or nitrogen. Oxygen was found to have but slight additional effect over nitrogen on $x-r a y$ induction of back mutations in the streptomyein-dependent strain. On the other hand the back mutation rate of the purine-dependent strain was found to be profoundly increased by the presence of oxygen during irradiation. The dose-reduction value of oxygen for the genetic effect for this strain is the same as that for the lethal effect. This is interpreted as an indication that the same mechanismus are involved in the lethal and this specific genetic effect of $\mathrm{x}$-rays in the purineless strain. Different mechanisms appear to be involved in these two effects of $x$-rays on the streptomycin-dependent strain. (NSA)

1775

Atwood, K. C. RADIATION INDUCED MUTATIONS IN MICROORGANHSMS, p.17-20 of CONEERENCE ON BIOLOGLAL APPLICATION OF NUCLEAR PHYSICS, JULX 12-27, 1948. Brookhaven National Lab. July 1948. 154p. (BNL-C-4(p. 17-20))

Data suggessting factors, other than lethal mutations, that contribute to the lethal effects of radiation. It remains to be seen whether many of the findings in microorganisms 1776

can be brought into line with the target theory. (S.V.S)

Auerbach, Charlotte. INDUCTION OF GENETICAL CHANGE BY CHEMICAL AGENTS, P.44-5 Of INTERNATIONAL CONGRESS OF RADIOLOGY, 6h Congress, London. 1950.

At the beginning of the war, research on the pharmocological properties of mustard gas and related poisons showed that these substances, like X-rays, have a prefexential effect on dividing cells. This is being used in cancer therapy. Genelical tests with Drosophila, and subsequently with other organismus, showed that the mustards, like $\mathrm{X}$-rays, produce their effects in part through an action on chromosomes and genes. The similarity between the genetical effects of these "radiomimetic" poisons and ionising radiations is indeed striking; but closer malysis has revealed certain differences, study of which may lead to a better understanding of the mutation process. Search is being made in several laboratories for other substances which aifect chromosomes, in order (a) to test the validily of certain theories about the production of mutations and chronocome breaks (attack on -\$H groups; production of OH radicals; depolymerization of aucleic acid; crosslinkage of protein chains), and (b) to find a chemical property shared by all these substances which might be presumed the essential factor for producing nuclear effects of this type. In recent years, several effective substances have been discovered, but their chemical diversity is so great that their action cannot be covered by any existing theory. A further dirfieulty in this work on the genetical elfects of chemtcal substances is the selective response of certain organisms, cell types, and developmental stages. Thus, formalin which produces muations in Drosophila, acts only on male germ cells of a definite developmental stage. Diazomethane is highly effective in producing mutations in Neurospora, but not in Drosophila. With ethyleneimine, the situation is reversed.

1777

Baker, Willan K. TRYE PRODUCTION OF CRROMOSOME INTERCHANGES IN DROSOPMLA VIRUIS. Genetics 33, $603-4$ (1948).

Males of $\mathrm{D}$. virilis were treated with $\mathrm{X}-\mathrm{ray}$ dosages of $1000,2000,3000$, and $4000 \mathrm{x}$ units. Any translocations inm duced in the sperm of these males between chromosomes $2,3,4,5$, and $Y$ were detected by a conventional genetic analysis. In strictly comparable experiments, the temperature at the time of irradiation was maintained at either $3^{\circ}$ or $28^{\circ} \mathrm{C}$. A significantly greater number of translocations are produced at the lower temperature. The results of the cold series, in regard to both the percent of translocations induced and the minimun number of indueed breals, agree very closely at all dosage levels with the mathematical expectations based on the following main assumptions: (1) the number of breaks per sperm follows the Poisson distribution, (2) each of the major autosomes is equally likely to be broken, (3) the mean number of breaks increases linearly with dosage, and (4) the broken chromosome ends unite independently and at randonn. The decrease in the number of interchanges produced at the warm temperature cannof be attributed to an alteration in the process of joining of the broken ends since the relative numbers of tranglocat lons involving two, three, or four chromosones in both the warm and the cold temperature experiments agree with the expectation. If appears, therefore, that this dif ferential effect is caused by a direct or indirect action of heat on the breakage of the chromosone thread. (G) 1778

Baker, Willam $\mathbb{K}$. and Elizabeth Sgourakis. ALTREATION OF TME X X-RAY SENSITIVITY OF DROSOPRILLA BY MEANS OF RESPIRATORY INMUBHTORS. Genetics 35, 26(1950).

Males of the Oregon-R strain of $D$. melanogaster were subjected to X radiation (1000, 3000, or $5000 \mathrm{r}$ untts, 250 lswp, $15 \mathrm{ma}$ ) while in an almosphere of either $\mathrm{O}_{2}$ or $\mathrm{N}_{2}$ at an approximate temperature of either $27^{\circ}$ or $2^{\circ} \mathrm{C}$. The number of X-chromosomes which bear recessive lethal mutations was determined by mating the treated males to the females of Muller-5 stock using the techmique cutlimed by Spencer and Stern (Geneties 33: $43-74,1948)$. Denending upon the dosage, a 40-70 per cent reduction in the mumber of serlinked lethal mutations is observed in the sperm from the flies irradiated in $\mathrm{N}_{2}$ as compared to those treated in $\mathrm{O}_{2}$. Indirect evidence also indicates that the induced frequeney of dominant lethals is greatly reduced when the flies are X-rayed in $\mathbb{N}_{2}$. A significantly greater number of mutations is induced in the flles treated in $\mathrm{O}_{2}$ at the cold temperature than in those maintained in $\mathrm{O}_{2}$ at the warm temperature. "This "temperature effect" is not evident when the Ales are irradiated in $\mathrm{N}_{2}$ thus indicating that this effect is related to the $\mathrm{O}_{2}$ concentration in the sperm. The data are compatible with the anferences in solubility of $\mathrm{O}_{2}$ at the temperatures used. Control experiments were performed in which series of flies were subjected to each gas at the two temperatures without irradiation. These experiments show no alteration of the spontaneous mutation rate. (G) 1779

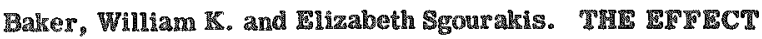

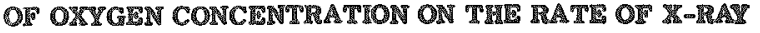
INDUCED MUTATIONS IN DROSOPUILA. PrOe. NaE.ACad. Sei. U. S. 36, 176-84(1950).

A striking reduction has been found in the number of recessive sex-linked lethal mutations induced in Drosopho 
ila melanogaster males when they are exposed to $\mathrm{x}-\mathrm{r}$ ray while in an atmosphere of low oxygen concentration. Although an increased number of mutations were induced in flies irradiated in oxygen at $2^{\circ} \mathrm{C}$ over those treated in oxygen at $27^{\circ} \mathrm{C}$, this increase was not due to the temperatures per se but rather it was apparently caused by a higher oxygen tension within the ixradiated sperm at the lower $1 \mathrm{en}$ perature. Additional evidence also indicates that fewer doninant lethal mutations and chromosone aberrations are induced in flies maintained in a near oxygen-free atros phere during irradiation. (NSA) 1780

Bateman, A. J. and W.K. Sinclair. MUTATIONS INDUCED IN DROSOPHILA BY INGESTED PHOSPHORUS-32.

Nature 165, 117-18(1950).

Radioactive phosphorus, $\mathrm{P}^{32}$, was administered to the larvae of Drosophila melanogaster in order to determine the number of mutations which could be induced by the radioisotope. The highest specisic amount introduced into the larvae bottles was 1 me/bottle; this resulted in the death of all the insects before pupation. A 0.1 me amount resulted in the death of an appresiable number of the specimens, while the introduction of $0.01 \mathrm{me}$ of $\mathrm{P}^{22} \mathrm{xe}-$ sulted in no appreciable number of deaths. Flies emerging from the $0.1 \mathrm{me}$ botlles were further studied; their activity was measured and, though it was variable, the mean figure was $0.0122 \mu \mathrm{c} / \mathrm{fly}$ two to three days after emergence. The over-all lethal mutation rate observed $(7.3 \%)$ is the rate which would be expected for an $\mathrm{x}$-ray dose of $2,500 \mathrm{r}$. The lethal mutation-rate/g-r was derived for the $\mathrm{P}^{32}$ and for a dose of 2,500 $x$ of x-rays and was found to be similar, but the author cautions that much significance should not be attached to this similarity. The high mutation-rate of visibles produced by the $\mathbb{P}^{32}$ corresponds to the expectation from $12,000 \mathrm{r}$ of $\mathrm{x}$-rays. It is concluded that additional work is necessary to confirm whether $\mathrm{P}^{32}$ is really four times as efficient as $\mathrm{X}-\mathrm{r}$ ays in the production of visible mutations. Further work on the general effects of the radioisotopes on mutations is in progress. (NSA)

\section{1}

Beadle, G. W. and E. L. Tatum. GENETC CONTROL OF BIOCHEMICAL REACTIONS IN NEUROSPORA. Proc. Natl. Acad. Sci. U. S. 27, 499-506(1941).

By means of $x$-rays, nutant strains were induced in Neurospor which are charactericed by their inability to carry out specific biochemical processes. In one the ability to synthesize vitamin $B_{6}$ is largely or wholly lost; in another strain the ability to synthesize the thiazole half of the vitamin $\mathbb{B}_{1}$ molecule is absent; in the third, paraaminobenzoic acid is not synthesized. Inability to synthesize vitamin $B_{6}$ is apparently differentiated by a single gene from the ability of the ability of the organism to elaborate this essential growth substance. It was later established that inability to synthesize both thiazole and p-aminobenzoic acid is also inherited as though differentiated from normal by single genes. (BA) 1782

Beal. J. M. NEGATIVE RESULTS FOLLOWING EXPOSURE OF SEVERAL IINDS OF SEEDS TO COSMIC RAYS AND OTHER RADIATIONS A'T HIGH ALTITUDES. Botan. Gaz. 112, 533-4(1951).

Seed from several species of angiosperms were exposed to cosmic radiations at high altitudes. No mutagenie effects were observed in seedlings grown from these seeds. 1783

Blano, Richard and Werner Braun. PHENOCOPLES AND X-RADIATION IN DROSOPHLA MELANOGASTER. Univ. Calif. (Berkeley) Pubs. Zoöl. 49, 31-50(1942).
Pupae of inbred Oregon-R stock were treated when $n-12$ hours of age, from $1 / 4$ to $4 \frac{1}{2}$ minutes at about $\$ 40 \mathrm{r}$-units per minute. percentage of flies showing phenocopies increased with increasing exposures to $100 \%$ at $3,290 \mathrm{r}-$ units, with the S-curve showing a linear relation from 235 to $2,115 \mathrm{x}$-units. The increase in phenocoples per fly is in linear proportion to radiation increase from 1,410 to 3,290 $x$-units. Frequencies of individual phenocopies were witudied, especially reduction of macrochaetes. Antertor and post dorsocentral bristles showed different rates of increase in numbers affected, but anterior and posterior scutcllars were similarly affected as dosage increased. Specific $x$-ray sensitive periods were suggested, but were different from the temperature-effective periods for the same phenocopies. The action of $\mathrm{x}$-rays in producing phenoconies is discussed. A statistical appendix is given. (BA)

\section{4}

Blano, Richard and Claude A. Villee, $J_{\mathrm{r}}$. THE EFFECT OF X-RADIATION UPON BRISTLE PATTERN IN OROSOPHILA MELANOGASTER. Univ. Calif. (Berkeley) Pubs. Zoö1. 49, 51-60(1942).

The effects of $\mathrm{x}$-radiation on bristle reduction are compared for wild Canton-S controls, and several scute and heterozygous scute stocks and compounds with genetic suppression of various bristles. The number and percentages of bristle suppression effects and the differences between treated and control for males and females are shown in tables. Most cases of extreme increase following treatment occur for suppression of these bristles which are genetically affected in a proportion of the controls. The influence of factors other than the scute alleles is suggested in different lines of males with the same seute x-chromosome. (BA)

1785

Blumel, Johanna. THE ACTION OF RADIOACTIVE PHOSPRORUS IN DROSOPHLA. Science 111, 205 6(1950).

In the present investigation, radioactive $\mathrm{P}^{32}$ was used to study the action of $\beta$ rays on Drosophila melanoraster and Drosophila virilis. Pairs of mature flies were placed in shell vials contaning a culture medium, consisting of a standard Drosophila culture medium and added radioactive $\mathrm{H}_{3} \mathrm{PO}_{3}$, the original volume (about $300 \mathrm{ml}$ ) of medium, with a count of $265,000 \mathrm{cpm}$ ml, was distributed among 50 vals in $6 \mathrm{ml}$ amounts. Twelve days after exposure, the distribution of radioactivity in the various tissues of the insects was determined; few of the D. virilis generation hatched in the radioactive solution survived, and none of the adults were tested, but in the case of $D$. melanogaster, the hatch was normal and all present, adults and larvac, were examined. All hatched individuals of $D$. virilis strain were morphologically abnormal, the abnormalitties pertaining mostly to the eyes, legs, abdomen, wings, and genitalia. Other data indicate that radioactive $\mathbb{P}^{32}$ not only produces mutations in $\mathbf{D}$. virilis but also chromosomal rearrange ments. The tolerance to such irradiation is considered to be high. (NSA)

1786

Bonner, David. RADIATION, GENES, AND ENZYMES, p.51-4 of BIOLOGICAL APPLICATIONS OF NUCLEAR PHYSICS, JULY 12-27, 1948. Brookhaven National Lab. July 1948. 3p. (BNL-C-4.2(p.51-4))

Since the famous experiments of $H$. J. Muller, it has been known that organisms subjected to irradiation with $\mathrm{x}$ rays or ultraviolet light frequently show heritable morphological changes. In the course of the past decade a great deal of work has been carried out in an effort to obtain a clearer understanding of this phenomenon, since the prob- 
lem of the effects of irradiation are intimately associated with the general problem of the nature of gene action. While we are, as yet far indeed from a complete under standing of the mechanism of gene action, the progress of the past decade is most encouraging. This paper is a brief review of the work carried out on this general problem with the fungus Neurospora crassa, and includes a discussion of the question of how irradiation with $x$-rays and ultraviolet light affects this action. (Author's Summary) 1787

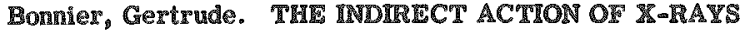
ON CHROMOSOMES, p.45 Of INTERNATIONAL CONGRESS OF RADIOLOGY, th Congress, London. 1950.

In accord with the theory of direct mutagenic action of X-rays (target theory), Bonnier and Linning found that when males of 'Drosophila melanogaster were irradiated, the rate of mutations, in specified loci, was linearly dependent upon dose. However, when extrapolating to the dose $r=0$ the "expected" rate thus calculated was distinetly higher than the rate actually found in controls. This indicates that besides the direct action there is some action of an indi reet kind. Patterson (1931) showed that after the irradiation of Drosophila females gynandromorphs occur due to elimination of the untreated paternal as well as of the treated maternal X-chromosome. The elimination of the untreated chromosome must thus be due to indirect action of X-rays. He found about equal numbers of maternal and paternal eliminations. We have mated irradiated females to untreated males using two different sets of marker genes. One of these gave a great excess of eliminations of the untreated chromosome, the other about equal numbers of treated and untreated. The indirect action in the elimination process may be due to disturbances in the spindle forming substances and/or breaks in the untreated chromosome. After irradiating white-eyed females and mating to non-white males we found 5 mutations from red to white in a progeny of 267,115 females as compared with 1 mutation in 123,263 controls. This finding taken with the other results indicates an indirect mutagenic effect of $\mathrm{X}$-rays. Tentatively we suggest that the ionising action of $X$-rays produces chemlcal mutagens which are capable of indueing gene mutations and chromosome breals.

1788

Bonnier, Gertrude and K. G. Lining. STUDIES ON XRAY MUTATIONS IN THE WHTTE AND FORYXD LOCI OF DROSOPHILA MELANOGASTER, I. A STATISTRCAL ANALYSIS OF MUTATION FREQUENCIFS. Hereditas 35, 163-89(1949).

x-ray experiments on a wild vagiety of Drosophla melanogaster, involving reasonably low doses and observa tions on a few of the most easily discernible loct, are described. The method used permitted estimates of variations in group doses and statistical analysis of the experimental results. Doses of $727 \mathrm{r}, 1,454 \mathrm{r}, 2,181 \mathrm{r}$, and $2,908 \mathrm{r}$ were administered to different groups by means of Sievert's ionization chamber. The $1,216,628$ female progeny of the irradiated males formed the basis of the statistical investigation. Results of control experiments indicated a nonlinear relationship between mutations and dose. No final conclusions are drawn in this paper. (NSA) 1789

Bonnier, Gertrude, K. G. Lüning, and Ann Margaret Perje. STUDIES ON X-RAY MUTATIONS IN THE WHTTE AND FOREED LOCI OF DROSOPHILA MELANOGASTER. II. A STUDY OF THE FORMATION OF GYNANDROMORPHS AND OTHER KINDS OF MOSAICS. Hereditas 35, 301-35 (1949).
This paper discusses certain aspects of the formation of mosaics of different kinds after I-1rradiation of wild males of Drosophila melanogaster. A study was made of the 140 gyandromorphs oeeurzing after erossing yellow white singed females to wild males irradiated with $2,008 \mathrm{r} ;$ cases with small female (wild type) areas isolated within male areas were especialy investigated. Cases of gmandromorphs with sexcombs on wild-type forelegs are described: other results are summarized, and the results of a previous investigation are reviewed. (NSA) 1790

Born, H. J. and K. G. ZImmer. INCREAST OF IR $-\mathrm{RAK}$ INDUCED MUTATION RATE, AS AFFECTED BZI IIEAVY ATOM SALTS INTRODUCED INTO TRE DODY. Z. Indult. Abstamm. Vererbungsl. 78, 246-50(1940).

Chemical analysis for Fe (colorimetric method with "Rhodankalium") and for U (through reaction with $\mathrm{K}$ fer" rocyanide) shows that flies fed with salts of these metals show a slight increase in their content over controls. Analysis for Fe of ash from gonads alone show an instgnficant increase of fed over control flies. Similar desults were obtained from analysis for $\mathrm{Pb}$. The inerease in $\mathrm{x}$-ray absorbing abilty caused by the increased metal absorption is about $1 \%$. Since mutation rates in fed llies were increased by $30-50 \%$, this mutational increase cannot be explained by the greater ablity of the treated flies to absorb x-rays. (BA) 1791

Brown, Meta S. ABERRATIONS FOUND IN THE PROGENHES OT PLANTS GROWN IROM IRRADLATED COTTON SERD. Naval Medical Research Section, Joint Task Force One. [nd] 10p. (NP-1003)

A report has been presented previously (NSA A-5568) giving a cytological description of 14 cotton, Gossypium hirsutum, plants grown from seeds exposed to $\gamma$ madiation at Bikini. Plants grown from seeds harvested from these 14 cotton plants have now been studied for possible mutations and other transmitted changes. It is concluded that, although chromosome breaks are readily induced, gene 1792 mutations are infrequent. (N\$A)

Buchmann, W. and G. Sydow. THE WFFCT OF HEAVY METAL SALTS ON X-PAY INDUCED MUTATIONS IN OROSOPHILA MELANOGASTER. EXPERIMERTS USING URANII ACETATE. Biol. Zentr.60, 137-42(1940).

About $40 \%-50 \%$ of 1 - to 2-day-old wild-type of $\sigma^{\circ}$ died after $3-4$ days on food containing $1 \%$ uranylacetate. For the survivor's the ses-linised mutation rate (CIB method) increased significantly from $8.6 \%$ to $14-9 \%$, after an $\mathrm{X}-\mathrm{ray}$ treatment of $3000 \mathrm{x}$. The few visible mutations affected mostly the wings. (BA)

1793

Buchman, Walter and Karl G. Zimmer. TNCREASE OF X-RAY INDUCED MUTATION RATE AS AFTECTED BY HIEAVY ATON SALTS INTRODUCED INTO THE ORGANISM. Z. indukt. Abstamm. Vererbungs1. 79, 192-8(1941).

Feeding of $\mathrm{Pb}$ acetate to Drosophila in larval and imaginal stages increases the rate of lethal mutations induced by subsequent $X$-ray treatment, the increase varying with wave length in accordance with the mass absorption coeff. of $\mathrm{Pb}$. This is in contrast with the absence of wave length dependence of increased mutational susceptibility due to iron-feeding and hence supports the contention that the results with Fe cannot be explained by a mere increase in physical absorption. (BA) 


\section{4}

Burkholder, P. R. and Norman H. Glles, Jr. INDUCED BIOCHEMICAL MUTATIONS IN BACLLUS SUBTILIS.

An. J. Botany 34, 345-8(1947).

Irradiation of spores or vegetative cells of $B$. Subtilis with either ultraviolet or $x-r a y$ has resulted in the production of numerous biochemical mutan strins differing from the parent strain in their inabilily to synthesize specific growth factors. From 4,260 isolates, 143 singly deficient mutants have been identified. The number of nutants occurring in each of the three general classification categories is as follows: 33 strains requiring specific $B$ witamins; four requiring nucleic acid components; and 106 requiring amino acids. The growth of the singly deficient mutants which have been tested is proportional to the amount of the growth substance supplied. A few isolates having multiple requirements have been obtained. Nine independent mutant strains requiring riboflavin at $30^{\circ} \mathrm{C}$ were found to be lemperature insensitive lypes, since these strains also require the vitamin at lower temperatures. The relatively high yield of mutants which was obtained will irradiation and with immediate plating of spores supports the view that the spores of $\mathbb{B}$. subtilis are unimucleate 1795 and haploid. (Author's Sammaxy)

Caldecott, Richard S. and Luher Smith. THE INILUENCE OF BEAT TREATMENTS ON THE INJURY AND CYTOGRNETIC RFFECTS OF X-RAYS ON BARLEY. Genetics 36, 546(1951).

Dormant seeds of Himalaya barley were subjected to the following treatments: 1) Control; 2) X-Fadiation only $(15,000 \mathrm{r})$; 3) Heal only (ranging from $75^{\circ} \mathrm{C}$ to $85^{\circ} \mathrm{C}$ for 30 minutes in different tests); 4) X-radiation followed immediately by heat; 5 ) Heat lollowed immediately by X-radiation. - The effects of these treatments were measured by: 1) Injury (height of seedlings at 14 days); 2) Surviwal to malurity; 3) Frequencies of chromosomal aberrations in root-tip cells and in pollen mother cells of $\mathrm{x}_{\mathrm{I}}$ plants (those grown from the (reated seeds); 4) Frequencies of seedling mutation in $\mathrm{X}_{2}$ head progenies. - The heat treatments alone had no perceptible influence on the injury, chromosomal aberrations, or mutations. However, such heal treatments applied before the $\mathrm{X}$-radiation significantly reduced the injury resulting from the irradiation. The effect on injury was more variable if the treatments were applied in the reverse order, that is, $\mathrm{X}$-radiation followed by heat. In some of these comparisons the injury was more and in others less than that resulting from $\mathrm{X}$-radiations alone. Chromosomal aberrations (as measured by chromatinic bridges at anaphase in root-tip cells and by interchanges at the first meiolic division in pollen mother cells were reduced by $30-50$ per cent, regardless of whether the heat treatment was applied before or atter the irradiation. On the other hand, the mutation frequency was increased from $30-50$ per cent in the seeds given these combination heat and $\mathrm{X}$-ray treatments. Thus, the same combination ircatments resulted in: 1) A decrease in chromosomal aber nations, and 2) an inerease in mutations - a clear lack of parallelism between these two effects of ionizing radiations. (G)

1796

Carter, I. C. RADIATON-INDUCED MUTATIONS IN MAMMATION TTSSUE: THE IFIRCT OF CHRONIC LOW DOSAGE-RATE GAMMA-IRRADITION ON THE MUTATPON RATE OF VHSHBLE MUTANTS IN MALE MCE, p.46 OF INTPREATIONAL CONGLESS OF RADHOLOGY, 6h Congress, London. 1050.
A pilot investigation into the effect of chronic lowdosage-rate whole-body gamma-irradiation (radium) on the mutation rate of visible mutants in male CDA-strain mice is being made. Dosage rates of $6 \mathrm{r}, 1.2 \mathrm{r}, 0.24 \mathrm{r}^{\circ}$. and $0.0 \mathrm{r}$. per 16-hour day are used and irradiation is continued for 25 five-day weeks. Spermatozoa from irradiated males are analysed for recessive visible mutants by one of two methods, nanely (i) treated males are mated to females homozygous for several recessive visibles and the $\mathbb{F}_{1}$ mice are examined for recessive phenotypes, (ii) treated males are mated to CDA females and the $F_{1}$ mice (each repre senting one irradiated spermatozoon) are analysed for recessive visibles by the backcross technique used by Paula Hertwig. Four visible mutants have been found, including two recurrences of known mutants and at least one new recessive visible ("ataxia"); but in each case it has been possible to shew that the mutation was spontaneous and not radiation-induced. Much valuable information has been gained about the problems of planning and executing work of this typo, but the conclusions to be drawn about induced mutation rates are purely negative. The results suggest an unexpectedly high spontaneous mutation rate.

1797

Caspari, Ernst and Curt Stern. THE INFLUENCE OF CMRONIC IRRADIATON WITH GAMMA RAYS AT LOW DOSAGES ON THE MUTATION RATE IN DROSOPHLA MELANOGASTER. CeneLICS 33, T5-94(1948).

The rate of Iethal sex-linked nutations in Drosophila exposed to gamma-rays of $2.5 \mathrm{r}$ units per day through 21 days (total $52.5 \mathrm{r}$ ) was determined. In a total of 108,215 chromosomes tested, no significant difference between experimental and controls was found. The difference between experimental and controls found in the present in vestigation is significantly diferent $(P=0.012)$ from that found by Spencer and Stern with the same amount of $x$ radiation given as one dose. By comparing the present results with those obtained by Spencer and Stern and by Raychoudhure, it is concluded that it is unlikely that the relalive inactivity of the irradiation is due to wave length and temperature of glass containers. As possible causes for the inactivity of irradiation, different sensitivity of sperm at different stages of aging, and dependence of induced mutation rate of low dosages on a time factor are considered. (NSA)

\section{8}

Caspari, Irnst. PRODUCTION OF MUTATIONS BY IONIZWNG RADLATIONS, p.21-6 Of CONFLRENCE ON BIOLOCICAL APPLICATIONS OF NUCL PAR PHYSICS, JULY 12-2\%, 1948. Brookhaven National Lab. July 1948 . 154p. (BNL-C -4 )

Author concludes: main reason for assuming the theory of direct action of radiation has been the linear propor tionality lsetween dosage and mutation rate. But this result orlly indicates a characteristic statistical connection between the primary effect which must follow the hit theory and the observable biological end effect. It would, therefore, only be necessary to assume that any intermediate effect would not be sensitive to changes in environmental and intracelluhar factors. It must be concluded that, as far as gene mutations go, the difference between direct and indirect action is not as large as would appear at first glance. (S.V.S.)

\section{9}

Catcheside, D. G. GENRTIC EFPECTS OF RADHATON. Brit. Med. Bull. 4, 18-23(1946). 
The following topies are discussed: 1) Stability in chromosomes and genes; 2) radiation-induced mutation in Drosophila melanogaster; 3) induced chromosome aberpalions. A diagram shows the mode of production of some chromosome structural changes, namely chromosome break, symmetrical interchange and asymmetrical inter change. For each of these three types the chromosomes are shown as unbroken, broken, in reunion, metaphase con1800

figuration and anaphase configuration. (BA)

Catcheside, D. G. GENE'TC EFTCTS OF RADIATIONS, p.271-358 of ADVANCES IN GENETICS, Volume $\mathrm{I}_{2}$ M. Demerec, ed. Academic Press, Inc., New York. 1948.

In this review the author is concerned only with the genetic effects of radiation in so far as these throw light on the mechanisms involved or upon the nature of the genetic changes and of the gene itself. No detailed consideration is afforded genetic effects of radiation such as the induction of non-disjunction, of attached-X detachment, of oogonial and spermatogonial crossing -over in Drosophila, of phenocopies, or of position effects. Author discusses general cytological effects of radiations; types of structural change; sequence of induced structural change in the mitotic eycle; quantitative relations: relative frequency of inter - and intrachanges, distribution amongst nuclei, location of breaks in chromosomes, restitution, modifying factors, dependence of yield on radiation intensity, dependence of yield on dose, relative efficiencies of different wavelengths and types of lonizing radiations, structural changes induced by ultraviolet light; time of split of chromosomes; mechanism of induction of chromosome structural changes: direct action breakage, initial separation of exchange breaks, proportion of breaks that restitute; relative efficiencies of different radiations; structural change in Drosophila; production of unutations by radiations; recessive lethal mutations in Drosophila; other mutagenic agents; cosmic rays and mutations; radiations and gene size; the nature of sex-linked recessive lethals in Drosophila; the nature of visible mutations; and genetical effects of ultraviolet light. (S.V.S.)

\section{1}

Catcheside, D. G. and D. E. Lea. THE RATE OF INDUCTION OF DOMINANT LIETHALS IN DROSOPHILA MELANOGASTER SPERM BY X-RAYS. J. Genet. 47, 1-9(1945).

Dominant lethals were induced by $5 \mathrm{~g}-\mathrm{ray}$ doses over the range from 1,124-11,420 r units in Oregon-R wild type and in w mutant llies. The results checked well with those obtained by other workers, and amount, at low doses, to $12 \%$ per 1,000 $\mathrm{x}$ for death in the embryo stage and $20 \% \mathrm{ger}$ $1,000 \mathrm{r}$ for death at any stage between zygote and adult. At higher doses of $x-$ rays both rates increase, so that over the whole dose range the dose-action curve does not fit a single-hit type of action, olthough this is approximated at low doses. Ratio of females to males declines about $2.5 \%$ per $1,000 x$. (BA)

1802

Catsch, A. A. Kanellis, G. H. Radu, and P. Welt. INDUCTION OF CHROMOSOME MUTATIONS OF D. MELANOGASTER BY ROENTGEN RAYS OF VARIOUS WAVE LENGTUS. Naturwissenschaften 32, 228(1944).

The literature to 1942 contains no definite proof that chromosome mutation induced by $x$-rays is a function of wavelength. The author proposes to study the problem more statistically. He chose the rate of the $\mathbb{I} / \mathbb{M I}$-translocation as a test, with developed sperm of $D$. melanogaster. Doses of $1,000 \mathrm{r}, 2,000 \mathrm{r}$, and $4,000 \mathrm{r}$ were given at each of the effective wavelengths $0.5 \mathrm{~A}, 0.4 \mathrm{~A}$, and $0.15 \mathrm{~A} . \mathrm{EX}$ citation potential of $\mathrm{x}$-ray tube $35 \mathrm{KV}(0.5 \mathrm{~mm}$, Al filter, H.V.L. $=0.5 \mathrm{~mm} \mathrm{Al)} 70 \mathrm{KV}$ (ditto, H.V.L. $=0.9 \mathrm{~mm} \mathrm{Al}$ ) and $180 \mathrm{KV}(0.5 \mathrm{~mm} \mathrm{Cu}=0.5 \mathrm{~mm}$ Al filter, H.V.L. $=1.1 \mathrm{~mm}$ Cu). An analysis of the results from the 213 to $2,342 \mathrm{sam}-$ ples at a given wavelength showed that the effectiveness of soft and hard $\mathrm{x}$-rays was not statistically differentiable. Neither the mutation constant nor the form $\mathrm{T}=\mathrm{CD} \mathrm{D}^{1,5}$ of his lethal-dosage-curve (Catsch, Radu, and Kanellis, Naturwiss. 31/32, 1943) was changed. In the above reference T is the translocation rate, $D$ the dose in $r$ unts, and $C$ a proportionality constant. (BA) 1803

Catsch, A., O. Petex, and P. Welt. A COMPARISON OF THE RFICTS OF ROENTGEN RAYS AND HLH SPEED NEUTRONS ON THE CHROMOSOME MUTATON RATE OF D. MELANOCASTER. Naturwissenschaften $32,230-1$ (1944).

Do x-rays and neutrons differ in their ability to change the rate of chromosome nutations? The II/III translocation, expressed in per cent, was established as a function of the dose in anits to which the chromosomes of nature sperm of $\mathrm{D}$. melanogaster were exposed. The radiationdose of high speed $\mathrm{Li}+\mathrm{D}$-neutrons was evaluated in $\mathrm{r}$ equivalent units after the method of $\mathrm{K}$. G. Zimmer (Strahlenther., 63, 1938); (Physik. Zeltschr., d2, 1941). Neutron doses ( $r$-units) were $1,050,2,160,4,480$. Numbers examined at each dose were $3,287,3,159,2.665$. Percentages of observed II/III-translocations were $1.07 \pm 0.18,1.67 \pm$ $0.24,4.02 \pm 0.38$. X $x$-ray doses $(r$-units $)$ were $1,006,2,000$, 4,000 . Numbers examined were $6,016,5,775$, and 10,223 . Per cent observed II-II-Lranslocations, $1.33 \pm 0.15,3.73$ $0.25,11.09$ t 0.30 . Conclusions: The efflclency of neutrons is less than the $\mathrm{x}-\mathrm{r}$ ay radiations. The dosage proportionality curve (the lethal effectiveness) for $\mathrm{z}-\mathrm{may}$ is best represented by $\mathrm{T}=\mathrm{CD}^{1,5}$ where $\mathrm{T}=$ translocation rate, $\mathrm{D}=$ dose and $\mathrm{e}$ an experimental constant. The shape of the curve allows the author to deduce that a muitiple hit (target theory) process is involved. The data from neutrons (where $\mathrm{T}=\mathrm{cD}$ ) imply a stigle hit process involved. (BA)

1804

Cavalli, L. I. and N. Visconti dI Modrone. BACTERIAL MUTATIONS FOR RESISTANCE TO RADIATIONS AND TO NITROGEN MUSTARD. Proc. Intern. Congr. Genetics 8th Congress, Stockholm 1948 (Suppl. to Hereditas) $550-$ 1 (1999).

E. coli $B$, producing mutants resistant to radiation $(B / \mathrm{r})$ were investigated. Regarding reastance to radiations, the resistant cells appearing in cultures of the $B$ gtraln are very similar to $\mathrm{B} / \mathrm{s}$ cells. They are not mutants but phenocopies. When single resistant $B$ cells are subcultured, they show a gradual loss of resistance, even before division, and when lrept in saline after irradiation. Thus resiatance occurring in the largest proportion of cells is not inherited, but is simply of a physiological nature; only a very small Iraction of these resistant cells seem to be the true, stable, resistant mutant $\mathrm{B} / \mathrm{r}$, which is spontaneously produced at a rate between $10^{-8}$ and $10^{-7}$ per cell per generation. Tests with $B$ and $B / a^{*}$ strains for resistance to nitrogen mustard (Ny) showed the latter stralns to be considerably more resistant than the original $B$ strain. Simllarly. the B strain cutrured an the presence of Ny showed an increase of resistance due to the selection of resistant mutants $(\mathbb{B} / \mathrm{N} y)$ which proved to be resigtant to radiation as well. No further increase in resistance was obtained with $B / x$. If appear 5 that $B / x$ and $B / N y$ are the same 
mutation, isolated through the different selective agents. (CA)

1805

Charles, Donald R. SUMMARX OF MOUSE GENETICS

WORK. Univ. of Rochester. [nd] 2p. (ARCU-378)

The objectives of the work of this laboratory have been:

(1) to determine how oflen dominant mutations occur in the sperm of mice exposed daily to $\mathrm{x}-\mathrm{radiation}$ as a function of the daily dosage and number of exposures; (2) to find a way of judging whether certain of these mutations (semisterlities) would, if they occurred in humans, have serious consequences for individuals inheriting them; (3) to investigate the extent and duration of chromosome damage, produced by single $x$-ray doses ranging from $58-229 \mathrm{r}$, in certain "poietic" tissues whose main activity is constant and rapid production of new cells, as a possible cause of the reduction, after $x$-ray exposure, in the cell populations normally replenished by the poietic lissues. Resulls have been obtained as follows: (1) At least twenty mutations are found to have occurred in the sperm of exposed and control males. Of the twenty mutations, nine are semi-sterilities, three are sex-linked lethals, and eight are dominant gene mutations. (2) Uterine deaths of offspring from semi w sterile humans should occur before the end of the seventh week and thus should not be a serious medical problem. (3) Broken chromosones are increasingly frequent in cells dividing within the first hour after exposure. Two days after exposure few or no broken chromosones are to be found. (NSA)

1806

Charles, Donald R. RADIATON-INDUCED MUTATIONS

IN MAMMALS. Radiology 55, 579-81(1950).

The seriousness of the effects of radiation-induced muta tions is considered. Data on the mutation rates and types of genetic changes induced in mice by repeated small exposures of $x-p$ pays and neutrons is presented. New mutathons oceur in about $1 \%$ of mouse sperm for each $100 \mathrm{r}$ of exposure at low-dosage rate. Of these mutations, about $40 \%$ would perhaps not be a real health hazard to individuals inheriting them. Another $40 \%$, the recessives, would not be apparent for probably many generations. Only $20 \%$, the dominants, would have immediate effects on of spzing receiving them. The death rate caused by semi-sterility mutations in humans has been estimated by studying the time equivalence between mouse and human development. The tentative conclusion is reached that women with inherited semi-sterility would lose half their offspring in the embryonic period, before the rth week, and so presumably without really gerious effects. (NSA)

\section{7}

Chatters, R. M. INDUCTION BY TAST NEUTRONS OF' MUTATIONS IN ANTIRRHINUM. 1]. GENETICS. Am. Naturalist $74,283-5(1940)$.

Mutations were produced (or hastened) by irradiating dry seeds with fast neutrons. The mutants, aiter self pollination, produced like progeny in a significantly large number of cases. (BA)

1808

Cole, Leon J. and Harold E. Finley. THE PRODUCTION OF SOMATIC MUTATONS IN TEE PIGEON WITI X-RAYS. Genetics 27, 138(1942).

The exhibit shows flight feathers from ash-red pigeons of genotype $B^{A} B$. Feather's on the right panel are from the right wing; on the left are the corresponding feather from the lef wing. In the top row are feathers plucked before any treatment; they show gelatively little lecking. Immediately after plueking the emply follicles in the right wing were exposed to 750 $\mathrm{x}$ and $1000 \mathrm{r}$ of $\mathrm{X}$-rays; those in the left wing received no treatment. In the second row are the feathers regenerated from these follicles. It is evident that the anount of flecking has been greatly increased in the treated feathers. That a permanent change has been produced is indicated by the tendeney to reproduce the flecking pattern in three subsequent feather-generations from these same follicles (rows 3,4 and 5 ) without further treatment. This increase in flecking may be attributed to mutations produced in the feather germ by the $X-1$ ays, elther by change of the $\mathrm{B}^{\mathrm{A}}$ gene to $\mathrm{B}$ or $\mathrm{b}$, or by the production of a deficiency in that region; the present experi1809

Corberi, E. PRODUCTION OF NEW FORMS OF EUTORULOPSIS DUBIA BY ACTION OF X-RAYS. Boll. Soc. ital. biol. sper. $25,526-8(1949)$.

A lype of Torula rosea, renamed Eutorulopsis by Ciferri and Redaelli, was submitted to $8-x a y s$, and certain new forms were produced, with somewhat different pignentation. These proved to have the same properties as far as gelatin-hydrolytic and sugar-fermentative powers were concerned, but differed in the production of smaller 1810 amounts of catalase and in 0 metabolism. (CA)

Crouse, Helen V. THE RESISTANCE OF SCIARA (DIPTERA) TO THE MUTAGENIC REFECTS OF IRLADIATION. Biol. Bull. 97, 311-14(1949).

A review is presented on data from previous workers in which it was noted that gross and minute chromosome rearrangements could be induced by irgadiation of the germ cells of the Sciara (Diptera) but visible mutations appeared at a negligible frequency. The experiments reported in this paper were originally performed in connection with cytogenctic studies on the unusual behavior of the ser chronosome of the Sciara; in one experiment, nine females of the Sciara coprophila strain were bred singly to adult males which had been ir radiated with 4,000 x of $x$ rays, and it was found that the dominant lethal value for the number of of spring conpared almost identically with that found in Drosophila irradiated at the same intensity. Several factors are offered as possible reasons for the great difference between Sciara and Drosophila in the visible mutation rates: the external appearance of this fly (bristle pattern, pigmentation, ete.) is such that only the most conspicuous visible changes are likely to be noticed; and, there is a distinct difference between the autosonal and sex-linked mutations, suggesting that the induced mutation rate in this genus is considerably greater than the detected mutation rate. (NSA)

\section{1}

Davis, B. D. PHENOMC DELAY IN RADIATION-INDUCED REYERSTON OF E. COLI AUXOTHEOPHS TO NUTRTION AL INDEPENDENCE. Genetics 35, 661-2(1950).

We have reported, in connection with the penicillin method, that phenotypic expression of UV-induced auxotrophic mutations requires growth. UV-induced reversions (without reference to genetic location) have given similar results. Tryp- strain 19-2, irradiated in washed suspenslon (survival $50 \%$ to $1 \%$ ) and plated on minimal medium, shows little induced reversion. A trace of tryptophan $(0.002-0.1 \mathrm{\gamma} / \mathrm{ml}$.$) , however, produces up to 300-$ fold increases in reversion rate with irradiated inocula, but none with unirradiated controls. Similar effects of trace enrich $=$ ment have been obtained with other amino acid, purine, and vitamin ausotrophs. The phenomenon could be due to requirement of growth for the action of a mutagen produced by UV irradiation. Similar delay, however, is encountered with X-irradiation, which, in contrast to UV, does not pro- 
duce a photochemically reversible mutagen. True phenomic delay therefore appears more likely, according to which the gene has mutated but cannot start to form the previously deficient enzyme until the cell is supplied with the product of that enzyme. This "pump-priming" effect implies that the metabolic turnover of the non-growing cell fails to provide a substrate, or exhaust an inhibitor, of some reaction essential for the synthesis of the previously defieient enzyme. With most strains reversions are heterogenous with respect to growth rate, as are prototrophs obtained by genetic recombination. Reversions were induced by irradiation in all auxotrophs studied, but not in a lac " strain (Lederberg's $Y-87$ ) with a sponlaneous reversion frequency of $2 \times 10^{-6}$, despite the opportunity for phenomic development. No explanation is available. (RG) 1812

Dean, A. C. R. and Cyril Hinshelwood. INDUCED AND OTHER VARIATIONS IN BACTERLAL CULTURES. PART II. GENERAL SURVEX OF CHANGES PRODUCED IN BACT. LACTIS AEROGENES (AEROBACTER AEROGENES) BY ULTRA-VIOLET LIGHT AND SOME OTHER MUTAGENIC AGENTS. J. Chem. Soe. 1159-68(1951)

A general survey is made of the changes induced in Bact. lactis aerogenes by exposure to ultraviolet light, to $\beta$ and $\gamma$ radiation from $S^{35}$, and to certain chemical agents (proflavine and Fenton's reagent). A significant proportion of mutants appears only when the fraction of survivors is very small. The chief effects are reduction in growth rate in a minimal medium, and modification of sugar-fermentation test.s. An almost continuous spectrum of behavior is manifested. Specific amino acid requirements seldom appeared and were never absolute with this organism (which is normally very nonselective as regards nitrogen source). (NSA)

1813

Demerec, M. INDUCED MUTATIONS AND POSSIBLE MECPANISMS OF THE TRANSMISSION OF HEREDITY IN ESCRERICHIA COLI. Proc. Nat1. Acad. Sci. U. \$. 32, $36-46(1946)$.

The rate of mutation from sensitivity to resistance to bacteriophage $T_{1}$ in the $B$ strain (resistant to radiations) of $\mathrm{E}$. coli was measured after treatment with ultraviolet radiation (wave length $2537 \mathrm{~A}, 4.2$ ergs per sec. per sq. mm. for 1-4 min.) and with $x$-rays $(10,000-50,000$ ro $)$. Both radiations produced a substantial increase in the mutation rate, especially with the higher doses of radiation. The induced mutations were indistinguishable from spontaneous $B / 1$ mutations. Sone of the induced mutants showed up immediately after treatment, while the appearance of a large proportion of then was delayed until after several bacterial generations. After approximately 13 generations the mutation rate had decreased to normal. Several possible interpretations are discussed. Difficulties are found with the simple hypothesis that these bacteria were diploid and with the hypothesis that there is a certain proportion of haploid, diploid, and polyploid individuals. Alternatively, it is suggested that there may be delay in the utilization of some substrate, or there may be two efiects of irradiation, one producing gene changes and the other increasing mutability. (BA)

1814

Demerec, M. and U. Fano. FREQUENCW OF DOMINANT LETHALS INDUCED BY RADIATION IN SPERMS OF DROSOPHILA MELANOGASTER. Ceneties 29, $348-60$ (1944).

The dependence of the frequency of dominant lethals on the radiation dosage aggees with Pontecorvo's interpretation of the origin of dominant lethals. Dependence of the sex ratio on the dosage, however, requires some modifica- tion of this interpretation. The proportion of organisms that die during the larval stage is approximately the same for different radiation treatments of the parent males. Equal treatments induce approximately the same irequency of dominant lethals in different stocks of flies. The results obtained with the same inbred stock at different times are qualitatively reproducible, but quantitative differences are noticeable. The uncertainty involved in the determimations of the frequeney of dominant lethals may be considerably greater than expected on the basis of the sampling error alone. (BA)

\section{5}

Demerec, M., B. P. Isaufmann, and E. Sutton, GENRTC EFFECTS PRODUCED BY NEUTRONS IN DROSOPHILA MELAROGASTER. Genetics 27, 140(1942).

Oregon-R, wild-type males were treated with neutrons using the Institution's electrostatic generator, with a lithium target. During the treatment, which lasted several hours, flies were kept at constant temperature and humidity, and shielded from beta rays by one-fourth inch of lead. Dosage measurements were made with a 25 r Victoreen chamber and are expressed in arbitrary $\mathbf{n}$-units. - Two groups of males were treated with about $1500 \mathrm{n}$-units, and two with about 2000 n-units. Part of the males from each group were mated with CBB females in order to determine the frequency of induced X-chromosome lethals. The remaining males were mated with wild-type females, and the salivary gland chromosomes of the $F_{1}$ female larvae were analyzed for breaks. - The lower dosage produced $23 / 1221=1.88$ percent lethals, and the higher dosage gave $30 / 1068=2.81$ percent. From $X$-ray studies of the Ore $-\mathbb{R}$ line by Demerec, and from Timofeef -Ressovsky's and Zimmer" $\&$ gtudies on the production of lethals by neutrons it is inferred that in this experimen gi-units can be transferred into $\mathrm{r}$-unit equivalents by multiplying values by a factor of about two. Cytological analysis showed for the lower dosage $18.43 \pm 2.43$ percent altered sperms and 46.66 percent breaks per total sperms, and for the higher dose $20.2+2.33$ percent altered aperms, and 53.5 percent breaks. Comparable values for equivalent treatment with $X$-rays are: $18.82 \pm 1.60,49.23 ; 29.8 \pm 3.12$, and 85.58 percent respectively. These results indicate that the frequency of breaks induced by neutrons does not differ appreciably from the frequency induced by an equivalent dose of $\mathrm{X}$-rays. Breaks are also distributed at random throughout the chromosomes. (G)

1816

Demerec, M. and R. Latarjet. MUTATIONS IN BACTERIA INDUCED BY RADIATION. Cold Spring Harbor Symposia Quant. Biol. 11, 39(1946).

Using bacterial mutation resulting in resistance to a bacteriophage as a criterion, the authors tested the genetic effect of radiations immediately following treatment and also after the treated cells had been allowed to divide. Two lines of the $\mathrm{B}$ strain of $\mathrm{E}$. coll were used in these experi ments. Bacteriophage TI, known also as "alpha" and "p28", was used to isolate mutants resistant to this phage. Whth this technique, two types of effect were observed: one immediate, producing phenotypic changes in the treated cells, and the other delayed, responsible for phenotypic changes appearing in the of spring of these cells. All detectable induced mutations were expressed after about 13 bacterial generations (end-polnt mutations). The results shown indicate a complez situation for ultraviolet radiation and a simpler one for $\mathrm{x}-\mathrm{p}$ adiation. Similar results were obtained with resting and with growing bacteria, and with the two different strains, which differ in their resistance to radiations. The results show no evidence of any relation between the ability of a radiation to produce sterilization 
and its ability to produce mutations. The highest rate of mutation, 2.8 per 100 survivors, was obtained after ultraviolet ireatment with 6,500 ergs $\mathrm{x} \mathrm{mm}^{-2}$. When simmilar amounis of energy are absorbed by the cell, either from ultraviolet or from $\mathbf{x}$-gadiation, similar rates of mutation are observed; while the sterilization rate is considerably $181 \%$ higher with $\mathrm{X}$-rays than with ultraviolet. (S.V.S.)

Dempster, Everett R. DOMINANT VS. RECESSIVE: MUTATION. Proc. Nat1. Acad. Sci. U. \$. 27, 249-50(1941).

After $X-r a y$, the Oregon-R strain of $D$. melanogaster exhibits both a lower recessive lethal mutation rate and a higher dominant lethal mutation rate than the Swedish-b strain. Sensitivity of the sperm chronosomes to induction of dominant lethal mutations increased with the age of the males, being about $1 \%$ when males were one day old, and $60 \%$ when 16 days old. Neutron produced ions used in the induction of recessive sex-linked mutations were only 0.75 as efficient as $x$-ray produced ions. (BA)

1818

Dempster, Fverett $R$. ABSENCE OF A TMM FACTOR IN THE PRODUCTION OF TRANSLOCATIONS IN DROSOPHLLA SPERM BY X-IRRADLATION. AM. Naturalist 75, 184-7(1941).

Genetical tests of translocations produced in Drosophila sperm by 5,000 to units of $x-$ rays at 220 kvp filtered will $1 / 2 \mathrm{~mm}$ Cu and $1 \mathrm{~mm} A \mathbb{A}$ showed 117 translocations 17.2 : $1.46 \%$ ) when the irradiation was applied at $167 \mathrm{r} / \mathrm{min}$ and 136 translocations $(17.0+1.33 \%)$ when applied at 12.5 $8 / \min .(B A)$

1819

Deringer, M. K. W. W. Heston, and L. Lorent. BLOLOGICAL EFFECTS OF LONG CONTINUED WHOLE BODY IRRADIATION WITH GAMMA RAYS ON MCE, GUINEA PIGS, RABBIS. IV. BIOLOGIC ACTION OF GAMMA RADIATION ON THE BREEDING BERAVIOR OF MICE. Oct. 25, 1946. Decl. Aug. 4, 1947. 30p. (MDDC-1247; CH-3729)

Female mice exposed to total doses of $770 \mathrm{r}$ and $880 \mathrm{r}$ on the 8.8 Ind on the 4.4 ger 24 hour's per day, and on the 8.8 a per hous per day levels showed sterility affects, some producing litters of reduced size and others being sterile. Females expossed to a total dose of $550 \mathrm{r}$ on the 4.4 $\mathrm{x}$ per 8 hours per day level bred but with reduced litter size. Male mice receiving a total dose of $1.760 \mathrm{r}$ at the rate of 8.8 ger 8 hours per day were sterile on removal from the ineld but regained fertility in two months. Males receiving total dose of $880 \mathrm{r}$ and $1,1000 \mathrm{~g}$ on the $8.8 \mathrm{~s}$ per 8 hours per day level bred normal síze. Males receiving total doses up to $1,100 \mathrm{r}$ on the $4.4 \mathrm{x}$ per 8 hours per day and on the $4.4 \mathrm{x}$ per 24 hours per day levels bred comparably to the controls. Breeding results obtained for fenale mice on $1 \mathrm{hr} ., 8 \mathrm{hr}$., and $24 \mathrm{hr}$. exposures do not differ significantly and failed to demonstrate any effect on the rate of administration of the dose. Female mice exposed to a single dose of $800 \mathrm{r}$ whole body $\mathrm{x}$-radiation at the rate of 41 r per minute produced one litter of reduced sice and were sterile therafter. The sterilized females did not recover their fertility but the males did. Breeding results for female mice exposed to a total dose of $440 \mathrm{r}$ at the rate of 4.4 tr per 24 hours per day entering the field at 3,4 , and 5 months of age falled to show any age effects. No evidence for the production of genetic chmeges was obtained in mice receiving chronic exposures, or for the production of translocations in the of spring of male mice receiving a total dose of $1,100 \mathrm{~s}$ at the rate of $8.8 \mathrm{x}$ per 8 hours per day or of female mice receiving a lotal dose of $770 \mathrm{r}$ at the rate of $8.8 \mathrm{x}$ per 24 hrs. per day. (ADD)

\section{0}

Devi, P., G. Pontecorvo, and C. Hilgginbottam. X-RAY INDUCED MUTATIONS IN DRED BACTERIA. Nature $160,503-4(1947)$.

X-ray irradiation of a strain of Bacterium aerogenes produced mutants at ates of up to $\overline{1^{6 / 2} \text {. Irradiation of bac- }}$ teria preserved in the dry state was effective, showing that it is not necessary for organisnus to be in artive division for mutants to be induced. Mutants differed in having some additional nutritional requirement, presumably owing to the mutant having lost the ability lo perform one step in the synthesis of an essential netabolite. A high proportion of the mutants were of the "adaptable" type. Whereas a non-adaptable nutant would never grow in a medium of glucose and inorganic salts witheut the addition of a particular nutrient, an "adaptable" mutant would grow after a lag of some 24 hours. In the mutant an enzyme system may be changed or suppressed, allowing an alternative enzyme system to function; alternatively, a gene may be inactivated which normally controlled the rate of growth of at plasmogene identical with, or a precursor of, the enzyme. It may be possible to discover whether adaptability is a property of the enzyme or of the eell producing the enzyme. (AWM) 1821

EHis, Frank. THE CENETIC EFEECTS OF NON-STERILIRING DOSES OF PRNETRATING RADIATION. Brit. J. Radiol. 21, 1-4(1948).

Non-sterilizing doses of radiation which cause no denonstrable deleterions effect on the health of the individual may cause serious changes in the chromosones of the spermatozoa or ova, resulfing in manifest disability of various types in subsequent generations. Irradiation changes the hereditary matexials, no matter how small the dose. The effects produced are all cumulative with increasing dose, being simply additive for some type of change, but increasing geometrically for others. These changes are almost entirely harmful and therefore undesirable. This information has been gained from the study of organisms other than man. A discussion is given of its application to man and permissible dosage. (NSA) 1822

Evans, Robley D. QUANTITATIVE INTERENCES CONCERNING THE CENETIC EFFECTS OF IADIATION ON IUMAN BEINGS. Brit. J. Radiol. 23, 175-83(1950).

By extrapolation from known facts of human geneties the author finds it unlikely that any increase in hereditary anomalies will occur, even after many generations, from daily doses of radiation up to $0.1 \mathrm{r}$ given to a small fraction of the population. (R)

1823

Evans, Robley D. QUANTITATIV INIPRENCES CONCERNING THE GENETIC EFFECTS OF RADIATHON ON HUMAN BEINCS. Science 109, 299-304(1949).

Genetic concepts and experiments pertinent to the questhon of mutalions induced by irradiation are reviewed and calculations are made. If is noted that the induced nutaltions, which are similar in type to those appearing spontaneously in nature, have an average probability of about $3 \times 10^{-3} \mathrm{per}$ gene per roentgen for an irradiation of 0.6 roentgens per hour, higher. Fixperimental results sugrgest that the effective average radiation sensitivity of immature sperm and exgrs may be less than the sensiltivity of mature sperm and eggs. Dominant mutations may become visible in the first generation of spring. Recessive mutations may appear only in homozygous individuals of a later generation, and therefore recessive mutations accumulate in the population as a result of both spontaneous and induced mutations. From the appropriate mathematical theory, and 
the experimental data now available, it seems safe cnough to conclude that no detectable increase in hereditary abnormalities is likely to result, even after many generations, if a small fraction of the population receives daily radiation doses up to 0.1 roentgen per day. It is also noted that it is only rarely that any individual receives the maximum permitted dose of $0.1 \mathrm{r}$ per day and that the average daily dose at installations like the Ianord Engineer Works and the Oak Ridge National Laboratory is about $0.005 \mathrm{r}$ per day. 29 references. (NSA) 1824

FANO, U. ON THE INTERPRETATION OF RADIATION EXPERIMENTS IN GENETTCS. Quart. Rev. Biol. 17, $244-52(1942)$.

Lack of influence of the wave-length of $x$-rays on their genetic effects can be understood independently of the hit theory. A generalized treatment of the dependence of the effectiveness of radiation on the ion-density enables us to consider the influence of several hitherto neglected factors. A modified pattern of biophysical analysis of the $\mathrm{x}-\mathrm{ray}$ action is suggested. (BA) 1825

Fan, U. NEUTRON-INDUCED LETHALS IN DROSOPH11. Genetics $28,74-5(1943)$

Wild type males of Drosophila melanogaster were treated with approximately 600 and 1200 neut ron units from the COIUMBIA UNIVERSITY cyclotron, and their of spring were tested for dominant and recessive lethals. One neutron mit is energetically equivalent to about $2.5 \vec{Z}$-rays. The frequency of dominant lethals was found to be approximately 73 percent and 96 percent for the two treatments the same effect is produced by about 3000 and $6000 \mathrm{r} X$ rays, respectively. Thus the ratio between the energies delivered to tissue by $X-r$ ay and neutron doses having equal dominant lethal refect approaches 2 ; the value 1.5 was horled previously for this ratio by DEMPSTER. Among the of fspring of 170 mallos treated with 1200 units, $998 \mathrm{CLn}$ tests were performed, yielding 60 recessive lethals: no "grouping" of lethals was observed in the of chpring of indi vidual males. All but one of the lethal-bearing chromosomes were further tested genefically and cylalogically. Only two chromosomes were clearly shown to carry two separate lethals, a coincidence that may be attributed to chance. Genetic analysis indicates that more than 30 per cent of the recessive lethals are associated with gross chromosomal reargangements. According to partially completed cytological analysis, this estimate may be slightly increased; no association of lethals with minute deficiencies has thus far been revealed. (G)

\section{6}

Fano, U. EXPRRIMENTS ON MUTATIONS INDUCED BV NEUTRONS IN DROSOPMILA MELANOGASTER SPERMS. Genetics $29,361-9(1944)$

New experiments on the phenomena of "grouging" of sexlnked lethals reported by Nagai and Locher (1938) and Nishina and Moriwaki (1941) seem to disprove their exist ence. The theoretical difficulties raised by the interpretafion of those phenonena are thus removed. A large per centage of neutron-induced lethals is associated with chromosomal rearrangements; cartain difficulties that had previously been met in diseussing this assoeiation are thus considerably increased. A new mensurement of the frequency of neutron-induced dominant lethals is reported, but the comparative efficiency of neutrons and $\mathrm{x}-\mathrm{m}$ ays in prohucing various types of chromusomal change in Drosophila sperms is not satisfactorily elucidated therehy. (Author's Summary)
1827

Tano, U. NOTE ON THE THEORY OF RADLATON INDUCED LETEALS IN DROSOPRILA. Science 106, $87-8(1947)$

A rather complete theory of induced lethals in Drosophila which was proposed by Lea and Catcheside (1945) has met wilh objections. In radiation-induced lethals there is dif ficuly in the interpretation of the dependence of frequency on dose. There is a nonlinear effect, leading to a very significant discrepancy between this theory and the experimental data. An estinate of the great importance of this discrepancy is discussed. (BA) 1828

Nano, U. and M. Demerec. MEASUREMENTS OF TPE FREQUENCY OF DOMINANT LETHALS INDUCED IN SPERM OF DROSOPHLA MELANOGASTER BY X-RAIS. Genetics 26,151 (1941)

A large amount of data on the frequency of production of dominant lethals has been collected. The fraction of eggs which after fertilization by X-zayed sperms fail to give rise to adul flies has been taken as an indicator of dominant lethals. The results of 50 independent measurements will be demonstrated, corresponding to 13 different $X$-ray doses varying between 0 and 11,000 roentgens and includàng counts of about 60,000 eggs. A large amount of work has been devoted to experiments at very low dosage. The production of dominant lethals is apparently proportional to the $\mathrm{X}$-ray dose for small dose and the survival ratio decreases exponentially for high dose. On the whole, however, the "dose-action" curve does not seem to fit the "Singyle hit" lype very well. This would indicate that the phenomenon is not simple, which is very plausible. About 1 percent of the eggs still gave rise to adult flies in the case of 11,000 roentgens. The percentage of dominant lethals produced by 1000 roentgens (about 15 percent) is so larrge that it cannot be accounted for by the production of inviable chromosone aberrations, which should appers at a rate corresponding to that of vinble inversions or translocations. (G) 1829

Fries, N. T-RAY INDUCED MUTATHONS IN THP PIYST OLOGY OF OPHIOSTOMA. NaLTRe 155, 757-811945).

Twelve physiological mutations (strains) were induced in the fungus $\mathrm{Q}$. Mulliannulatum, by $\mathrm{x}$-rays. The manner in which they differed is shown in a table. The physiological properties were heritable. Some of the properties of the different strains are discussed. (BA) 1830

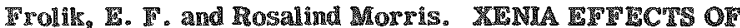
IRRADIATING CORN POLISN IN A NUCLEAR REACTOR. Agronomy 3. 42, $293-7(1050)$.

Xenia effects in corn crosses involving tassels irradiated in a muclear reactor were determined in comparison with crosses involving $\mathrm{x}-\mathrm{k}$ ayed and nonirradiated tassels. Irradiations were made in the thermal neutron column of the heavy water pile at the Argonne National Laboratory for periods of $0.5,1,2,4,8$, and 16 min, respectively. The sray dosage consisted of $1200 \mathrm{x}$ units. In general, the percentage of entire mutations increased with increase in length of time of syposure in the nuclear reactor. The ef fectiveness of pile radiation varied matkediy with date of treat ments. There was considerable wniformity in the response of individual plants to comparable segadiation treatments. The effectiveness of $x-$ ray treatment varted somewhere between $0.5 \mathrm{~min}$ and 4 min of pile irradiation, depending on date of treatment and on the character inwolved. The gerceatage of mutations for both sources of radiation 
varied for the characters under study in the following decreasing order of frequency: (a) defective kernels, (b) colorless aleurone, (c) sugary endosperm, and (d) red aleurone. The frequency of chimeras was affected far less than that of entire mutations by the pile radiations. No association was apparent between frequency of recoveries and either pile radiation or $\mathrm{x}-\mathrm{radiation}$. No qualitative differences between the two types of radiation were noted in these tests. 13 refarences. (NSA)

1831

Frolik, E. Ind Rosalind Morris. EFIFCTS OF IRYADIATING MAIZE POLLEN IN A NUCLEAR REACTOR ON THE P PLANTS. Sclence 111, 153-4(1950).

This paper constitutes a preliminary report on the inherited changes induced by izradiating maize pollen in a nuclear reactor. Mature maize tassels were placed in the thermal neutron column of the Argonne pile, which was operated at a power in the neighborhood of 300 kev. The average flux of neutrons at the position of the tassels was $7 \times 10^{10}$ neutrons $/ \mathrm{cm}^{2} / \mathrm{sec}$, and the exposure times varied from 0-16 minutes. Crosses were made by collecting pollen from the tassels and placing it on the untreated female plants. The seeds were then planted and the percentage stands of the different seed groups is tabulated. Other ef fects of the irradiation, such as the types of abnormalities produced, are described in the article. (NSA)

\section{2}

Fryer, P. C. and John W. Gowen. AN ANALYSTS OF DATA

ON X-RAIIINDUCED VISIBLE GENE MUTATIONS IN DROSOPHILA MELANOGASTRR. Genetics 27, 212 27 (1042).

This paper presents some new data on $x-r a y$ induced, visible gene mutations and shows how a satisfactory anal$y$ sis of such data can be made by means of the $\mathrm{x}^{2}$ distribu1833 tion. (S.V.S.)

Genter, Clarence $F$. and Hubert M. Brown. X-RAY STUDIES ON THE FIELD BEAN. J. Reredity $32,39-44$ (1941).

Lots of dormant and germinated (18 and 36 hours) seeds of Michelite white pea beans, Phaseolus vulgaris were $\mathrm{x}$ rayed with four dosages, $2,160,6,500,13,000$, and 26,000 r-units. Of the 1,434 and generation progenies grown from ireated seed, 150 showed mutations which were classified in 32 types. Ninety per cent of the mutants were distinguished within ive weels after emergence and of these $67 \%$ were chlorophyll abnormalities. Other characters affected were plant size; branching; leaf size, shape and texture; fertility; and earliness of maturity. The rate of rutation varied roughly with the dosage and length of germination period prior to treatment and tended to increase with the later harvest dates and with sige of progeny. The great variability of the mutant ratios indicated that the s-rays affected only limited sectors of the treated embryos rather than the entire embryos. (BA)

1834

Giles, Norman H., Jr., Anne F. F'lym, and Dorohy L. Giles. INDUCED REVIRSIONS OF INOSITOL-REQUIRING MUTANTS IN NEUROSPORA CRASSA. Records Genet. Soc. Am. 17, 38-9(1948).

Treatments of microconidial suspensions from inositolless mutants of Neurospora crassa with ultra-violet (2537 A) or X-5ys produce a greatly increased frequency of adaptation (inositol-independent cultures) in certain strains. Genetic studies of several independently produced adaptations indicate that these are the result of reverse mutation at the inositolless locus to the original wild-type allele controlling inositol synthesis. There is no evidence that gross chromosomal rearrangements are present in the induced inositol-independent stocks. The relation be tween frequency of reversions snd radiation dosage is being investigated. Present results indicate that the percentage of reverse mutations with ultra-violet continues to increase, even at dosages well beyond the point of one percent microconidial survival. Previous studies of the frequencies of induced reversions in several independently produced, apparently allelic, inositolless mutants, have indicated that some of these strains differ markedly in their mutability. An extensive comparison has been made of two strains: S-37401, which regularly reverts with radiation treatment, and $\mathrm{S}-46802$, which has failed to revert with any treatment so far attempted. Crosses of these two mutants, followed by serial ascospore isolations, yield cultures which are phenotypically indistinguishable. Such progeny can however be separated by reversion studies since, of the 8 cultures from a single ascus, four regularly adapt when conidial suspensions are treated with ultraviolet or $\mathrm{X}$-rays while the remaining four do not. (R.G.) 1835

Glass, H. Bentley. DIPFERETTAL SUSCEPTIBILITY OF THE SIXXS OF DROSOPHLA TO THE ETIECT OF XRATS IN PRODUCING CHROMOSOME ABERRATIONS. Genetics 25, 117(1940).

Treatment of mated fernales of $\mathbb{D}$. melanogaster by $\mathrm{X}$ rays provides simultaneous exposure of sperms and premeiotic eggs to identical doses. Offspring from eggs laid in the first five days following treatment yield approximately equal frequencies of lethal mutations in of and $\$$ germ-cells. 55 translocations were all produced in $\sigma^{\circ}$ germ-cells. This disproportion is significantly greater than would be expected from the elimination of translocations in eggs undergolng meiosis. Inversions have been obtained from both of and $\&$ germ-cells. They are more frequent in the former, but the significance of the diference is not yet astablished. (G)

\section{6}

Glass, Bentley. THE EFECT OF X-RAYS UPON THE ACTION OF A SPECIFIC GENE IN DROSOPRTLA MELANOGASTER. Genetics 29, $436-46(1944)$.

Embryos, 10 hours old at $26 \mathrm{C}$, of a brown searlet (bw; st) stock were given $1,000 \mathrm{r}$ units. Of $221 \mathrm{flies}$ emerging as adults, 94.1\% had a growth from the center of one or both eyes, varying from a mere protrusion of the eye in the central area to an eruption of a palp-like structure bearing bristles and sometimes segmented. The effect of the $x$-rays on this stock was confirmed in a second experiment. No such effect was found when other stocks were given similar treatments. This effect has been shown, by means of appropriate crosses, to be due to a semi-dominant gene erupt (er) in chromosome 3 of the bw; st stock, near $\mathrm{e}^{3}$; and to a semi-dominant gene, Suppressor-erupt (Su-er), in chromosome 2 of the same stock, the effect of which upon $e r$ is inactivated by the $x$-ray treatment. Erupt is not an allele of the nearby, similar homoeotic mutant, kidney. The significance of the specific inactivation of a gene affecting the eye by a treatment given before even the cephalic rudiment is invaginated is diseussed. Various stocks tested, including those lacking the mutant er, carry the same or a similar suppressor of erupt. Such inhibitory modifier systems are to be regarded as widespread and of considerable evolutionary significance. (BA)

\section{7}

Glass, H. Bentley. THE FFECTS OF SUPRLEMENTALY TREATMENT WITH INERARED RADIATION ON X-RAY INDUCED LFTHALS AND CMROMOSOME ABERRATIONS IN FEMALES OF DROSOPHILA MELANOGASTER.

Genetics $35,109-\overline{10(1950)}$ 
Infra-red pretreatments and posttreatments of 4 hours have been applied to of and 99 of $\mathrm{D}$. melanogaster given a dose of $X$-rays amounting to ca. $2000 \mathrm{r}$ units, the dose varying slightly for different series, but being always identical for $c f^{\prime}$ and $q 9$ of the same series. Translocations induced in $2 \%$ were 6 in 85 tested gametes (7.1\%) when pretreated with infra-red, and 7 in $113(6.2 \%)$ when posttreated. The diferences are not significant, a fact at variance with the results of Kaufmann, who found no effect of postreatment with infra-red. The rate in controls is about two-thirds as great. In 5 , 0 transhorations were found in 218 gametes tested when pretreated with infra-red, 1 in $169(0.6 \%)$ when posttreated. The translocation is the first one ever found by the author in treated $D$. melanogaster Yq, in series amounting to a total of $183 \overline{2}$ tested gametes. The sallvary gland chromosomes show that this translocation involved a high number of breaks. - Sex-linked lethal mutations have been scored in both $0^{\circ} \circ$ and $\$ \%$, with and without infram red posttreatment: $15 / 562(2.64 \%)$ in ofo with $X$-rays enly; $10569(1.76$ ) in fo with X-rays only; $14 / 281$ $\left(5.0^{\circ}\right)$ in $0^{\circ} \mathrm{O}^{\circ}$ with $\mathrm{X}$-rays plus infra-red; $2.222\left(0.9^{\prime}\right)$ in if with $\mathrm{X}$-rays plus inira-red. The difference between the two groups of $0^{\circ} \circ$ and the similar difference between $q 9$ are not significant; but the difference between of (combined) and $\$$ (combined) is significant ( $2 / 2$ level). The frequency 1838

of induced lethals in of is about half that in $\%$. (G)

Gowen, John W. MUTATION IN DROSOPHLLA, BACTERIA, AND VRUSES. Cold Spring Harbor Symposia Quant. Biol. $9,187-93(1941)$.

This paper presents evidence on the spontaneous frequency of variants in viruses, bacteria, and Drosophila. The rate of spontaneous vaxiation is shown to be small. The frequency of variants in the three forms is increased when they are exposed to comparable $\mathrm{x}$-ray dosages. The rates at which variants appear are of the same order of magnitude. This evidence is interpreted as indicating a common basle structure for inheritance in each group, the changes of which are in the nature of mutation. (S.V.S.) 1898

Gowen, John W. and Marie S. Gowen. CONSTITUTION OF THE SECOND CHROMOSOME OF DROSOPHILA MELANOGASTER AS INDICATED BY X-RAY ANALYSTS. Genetics $32,89(1947)$.

Newly produced lethal mutations in the second chromosome were examined for possible allelism, position within the chromosome, and association with translocations or inversions. The data obtained were analyzed in terms of the chronosomal size of $X-r a y$ effects and the relation these effects have to gene number in the second chromosome. Males were subjected to 2000 and $6000 \mathrm{I}$. Of 153 lethal bearing chromosomes obtained, 8 had incompleted tests, 16 carried two lethals. The 161 lethals treated had 128 occur once, 10 twice, 3 trice and 1 four thmes. The lethals were distributed in all chromosome reglons. Close agreement between loci occupied by visible genes and the lethals was found for dumpy black, curved plexus, and plexus speck regions. wethals were in excess in aristaless dumpy, and black purple regions. About one-third the expected lethals were found in the gurple curved region. Estimates of total number of regions capable of showimg lethal effects may be had from the relation

$\left(\frac{\mathrm{n}}{142}\right) \frac{161 !}{(4 !)(3 !)^{3}(2 !)^{10}} \frac{1}{\mathrm{n}^{16 !}}$ representing the probability of

our sample. The number of these regions is 623 . The variance of this estimate is 131 . The fiducial limits are 426 as the lower limit and 970 as the upper limit. These results are not to be considered as estimates of gene loct bat rather as average reglonal effects of the $x$-mays. The bearing of these results on gene loci in the second chromosome whl be discussed. (G)

\section{0}

Gowen, John W. and M. R. Zelle. IRRADIATON EFIECTS ON GENETIC RESISTANCE OF MCE TO MOUSE TYPMOD. J. Infect. Diseases 77, 85-91(1045).

This paper reports data on the effects of Irradiating mice with $\mathrm{x}$-rays and then determining the survival value of these mice to inoculations of mouse typhoid, Salmonella typhimurium, dose 200,000 organisms. The mice belong to six strains, each characterized by a spectic genetic resistance to mouse typhoid. The results of these observations may be explained by the hypothesis that the resistance of these mice decreases exponentially with the increased dosage of $x-82 y s$ to which they are exposed. The rate of this decrease per roentgen of $x-r$ ays is small and is statistically similar for the six different strains of mice although these strains are genetically very different in theis natural resistance to mouse typhoid. These facts may be rationalized if the leucocytes in the mouse blood also decrease exponentially with increased dosages of $\mathrm{g}-\mathrm{mays}$. The fact that even strains of mice which we now consider very susceptible to mouse typhoid may be greatly increased in susceptibility though 8 -rays shows that mice, in general, are probably much more resistant to this disease than when the disease first contacted the species. (BA)

1841

Granhall, I., A. Gustassson, F. NIIsson, and E. J. Oldên. X-RAY FTECTS IN FRUTT TREES. Hereditas' 35, 269$79(1949)$.

Radiation dosages of 500-25,000 r were administered to the scions of several varieties of apples, pears, and sweet cherries in order to study the lethality of $x$ rays and to determine the gossibility of inducing favorable mutations by means of the zadiations. The sclons were grafted on parent plants and observed for growth and other character istics. At 10,000 I none of the pears and agples surwived; the cher ries suffered a simllar fate at $5,000 \mathrm{r}$. The ideal dose for the peas and apples appeared to be $5,000 \mathrm{x}$ at which abnotrallities appeared without excessive mortality; $2,500 \mathrm{r}$ was the optimum dosage for the cherries. The most typical primary effects were bifurcations of shoots and leaf midribs; other effects, such as change in color and shape of the fruit also occurred. It is concluded that since the apparent effect of the $x-$ radiations is to increase the probabilty of oceurence of abnormalities ordinarly seen in the fruit (chmges in size, shape, and color), that this is a method ior the possible production of better fruit of increased size, flavor, resistance to disease and other desidrable qualities. (NSA)

1842

Gray, C. H. and S. L. Taturn. X-RAY INDUCED GROWTH FACTOR REQUIREMENTS IN BACTERIA. PEOE. NaUl. Acad. Sei. U. $2.30,404-10(1044)$.

Escherichia coll and Acetobacter melanogenum wer treated with $\mathrm{s}$-ray unti $99.99^{\circ}$ of the cells were killed. From 800 isolations of survivors of $\mathrm{E}$. colli, two strains differ from the parent, one by requiring the addition of biotin to the basic medium, the other by requiring theonine. srom 57g isolations, four strains of A. melanogenum have bert oblained which fail to grow in the bastc medum. The first requires a supplement of serine or glycine, the second requires adenine or adenosine, the third glycine and the fourth shows some response to aldition of leucine. As contrasted to the excellent growth of the parent strain in the appropriate minimal medium, the $\mathrm{x}$-ray treatment 
modified the capacty of bacteria for carrying out specific biochemical reactions, the growth of each mutant strain being a function of the concentration of the required supplement. Ultraviolet light produced no similar effects. The production by $x$-ray of new strains of 2 spp. of bacteria offers indirect support for the existence of specific genes controlling biosynthesis in bacteria. (BA)

1843

GRHATE, A. B. THE BS TRANSLOCATION IN D. MELANOGASTYR AND MODIFICATIONS OF THT BAR FPFET THROUGM IRRADLATION. Genetics 26, 154-5(1941).

$T(1 ; A) A-B^{S}$ is a translocation involving the $X$ and IV chromosomes with breaks at $102 F_{2} / 102 F_{3}$ and between the repeat sections of the $B$ duplication which was present in the original stock. The $\mathbb{B}^{\text {effect }}$ is confined to the $\mathrm{XP}$ fragment, while the $\mathrm{XL}$ has no $\mathrm{B}$ effeet although it bears one of the original members of the duplication. Irsadiation of $\underline{B}^{s}$ males has produced further facet reduction and complete returns to normal eye shape; some of these changes are associated with visible changes in the IV-XR fragnent and some appear to be simple mutations without visible rearrangement. In one case a $\mathrm{B}$ effect has been induced in the XL framment through deletion of the facet-iorked inter val, thes causing an association of white $\left({ }_{3} \mathrm{C}_{2}\right)$ and the first band $\left(16 \mathbb{A}_{1}\right)$ of the leit $\mathrm{B}$ sectlon; this new XL effect is independent from that of XPR. The mutant cases are instances of duplication of chromosome material with loss of visible effectw and are cxamples of one method for increasing the $18 \mathrm{~s} 4$ amount of genic material in the species. (G)

Grîfen, A. B., Mary Warters, and James D. Mohler.

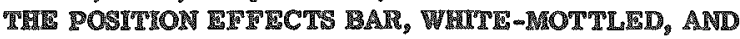
CUBITUS INTPEREUPTUS IN DROSOPHICA MELANOGASTER. Genetics 35, 112(1950).

Comparisons of $X-1$ ay induced reversals of position effects at the Bar, while, and ci loci indicate that each locus may revert to wild type action either by mutation or by virtue of new chromomere associations resulting from new chromosome rean'mugements. In the case of $\mathrm{B}^{5}$, mutation accounts for approximately one/third of the phemotyptc changes from $\mathrm{B}^{\mathrm{S}}$ to wild type. (G)

1845

Gustaisson, ARE. MUTATION FXPERIMENTS IN BARLEY. Preditas $207,225-42(1841)$.

Seed amples of the pure Ine "Golden" barley were $x-$ rayed with 5,000 and 10,000 r. Three degrees of seed hydration were used: 10 mnd $15 \%$ moisture and seed soaked for 24 hours in water or in auxin solution. The author believes that the direct proporthonality in barley between dosage and mutation rate is only apparent. Those associated with profound chromosomal disturbances (highy sterile) and those arising in entirely fertile plant progenies do mot show direct proportionality when considered alone. Mutations associated with small but evident changes do show a direct proportionality. The intentional direction of the process of mutation may take place to a certain extent in the following ways: (1) Abima mutations arise more frequently than other chlorophyll types at low dosages and to a greater extent in fertile progenies. They occur about three times as often in water-soaked seed as in those of lower moisture. (2) Kantha mutations are produced most often at the higher dosage in slightly sterile $\mathrm{x}_{\mathrm{g}}$ plants. (3) Alboviridis mutations occur as frequently in dry as in soaled series, but chiefly in progeny of sterile planis. (4) Albowantha mutations occur oniy in soaked seed. (5) VIridis mutations oceur in highny sterile plants which resulf from high dosage, and soaked seed. (6) Irinally, Ti grina arose exclusively from dry seed. In addition to the chlorophyll mutations a number of morphological forms have arisen which fall within the range of known variations in cultivated barley. Some of these mutations, such as straw-stifiness, size of frernel, and earliness, are thought to constitute practical improvements as compared with the original variations, although their comparative yielding capacity has not bean determined. (BA) 1846

Gustafsson, AlRe. PRELIMINARY TIELD RIXPRIMENTS WTTH TEN INDUCED MUTATIONS IN BARLET. Hereditas 27, $337-59(1941)$

Ten fertile mutations obtained from $x$-raying Golden barley were compared with the Golden and Maja variations for yield and other characteristics. Seven mutant forms yielded less, but three equalled or surpassed the mother strain. Other characteristics which were charged in both diyections from Golden were grain size and weight, number of ripe gremels, germination ripeness and protein and stareh content. A general discussion concerning the signif icance of mutations to evolution as well as induced nutations to plant breeding, followed. (BA)

1847

Gustatsson, Ake and Ewert Aberg. TWO ERTREME XRAY MUTATIONS OF MORPHOLOGICAL INTEREST. Hereditas 26, 257-61(1940).

In irradiated barley of the Golden vartation (Mordeum distichum var. mutang) the authors found (1) a 2flowered mutant (i.e., 2 llowers were formed within each lemma) ylelding a segregation of 3 normal to 1 mutant, the latter being sterile; and (2) a mutant in which the glumes were lemma-like with a long awn, whereas the normal glume in Golden barley is linear with short awns. (BA)

\section{8}

Gustaisson, Ake and Nils Nybon. COLCHCINE, X-RAYS AND THE MUTATION PROCESS. REReditas 35, 280-4 (1909).

A special strain of Ymer barley seed was tested for the effects of irradiation and colchicine. Twenty-four hours before irradiation, seeds were set to germinate in five dîfferent colchicine solutions of $0.1,0.05,0.005,0.01$, and $0.001 \%$ strength; the $x$-ray dosages were 5,000, 10,000 and $15,000 \mathrm{x}$. It. was found that the four highest concentrations of colchicine caused a complete - mitosis, while the lowest concentration was ineffective. From the results it is concluded that: the course of the mutation process is considerabiy altered by the application of colchicine previous to the irradiation; the santha and alboviridis types, as well as very rare mutants, inerease in number as a result of such a pretreatment; the viridis mutants become rarer; and, the different groups of mutation, at least with regard to albina, santha, and vizidis types, show a conspicuous connection with the sterility conditions of the ancestor plants. (NSA)

1849

Haldane, J. B. S. TPRP FORMAL GENETICS OF MAN. Proc. Roy. Soc. (London) D, 135, 18\%-70(1948).

With sublethal genes such as hemophilia, achondioplastic dwarfism, epiloia, retinoblastoma, Pelger anomaly and aniridia, mutation rate lie between $8 \times 10^{-5}$ and $4 \times 10^{-6}$ per loeus per generation. Lass common anomalies have mutation rates much lower: lobster claw $10^{-7}$, and porcupine men, $10^{-10}$. These rates suggest that much human mutation may be due to natural radiations. (BA partial summary)

1850

Hanson, H. J. W. C. Myers, G. L. Stahly, and J.M. Birkeland. VARIATIONS IN PENCCILIIUM NOTATATUM 
INDUCWD BY BOMBARDMFNT OF SPORES WITH

NEUTRONS. J. Bacteriol. 51, 9-18(1946).

Marked variations appeared in cultures from spore suspensions of $\underline{P}$. notatum which were exposed to a bombardment with slow neutrons for periods ranging from one hour to 134 days. The number of variants increased as time of exposure lengthened. Actively growing cultures which were treated with slow neutrons for three hours showed no definite evidence of induced variation. Variations similar to those uduced by slow neutrons occurred when moist or dry spores were bombarded with fast neutrons for 15 and 30 minutes. However, an exposure of 180 minutes was lethal to all spores. Isolation and study was made of $\mathbf{1 5 0}$ varieties which were characterized by differences in color, texture, rate and quantity of vegetative growth and sporulation, color and colonies. The variant strains differ greatly in their ability to produce penicillin. Some lost this property and some had it markedly increassed over the parent sirain. None of the variants showed great differences when their effect was tested against 10 different bacteria. (CA)

1851

Hayden, Barbara and Luther Smith. THE RELATION OF ATMOSPHERE TO RIOLOGICAL FFFECTS OF X-RAXS. Grneties 34, 26-43(1949).

Barley seeds $\mathrm{x}-\mathrm{r}$ ayed while in a vacuum consistently showed beter germination and growth and a lower frequency of chromosomal aberrations than seeds $x$-rayed in air. In one test the seedling mutation rate from test of plants grown from germinating seeds $x$-rayed in a vacum was $2.1+0.32$ as compared with $3.4+0.35 \%$ for seeds $x$-rayed in anx. This difference was slightly more than three tumes the standard error. Root-tip cells from seeds irradiated in air averaged 4.7 times as many chromatinic bridges as root-tip cells from seeds irradiated in a vacuum. (NSA)

1852

Heidenthal, Gertrude. THE OCCURRENCE OF X-RAY INDUCED DOMINANT LETHAL MUTATIONS IN MABROBIACON. Genetics 30, 197-205(1945).

Haploid males of the parasilic wasp Habrobracon were $x$-rayed with dosages ranging from 500 a to 10,000 re unis. Dominant lethals induced in the sperm were detected by counting the $F_{1}$ female and male progeny and calculating the ser ratios. In the 3 -allele cross involved, all $p_{1}$ male progeny were haploid and all female progeny diploid. Under the conditions of the experiment the number of $F_{1}$ males remained constant, as expected, but the number of $F_{1}$ females decreased with increasing $\mathrm{x}$-ray dosage. The curve of mortalily due to dominant lethals was found to be similar to conparable curves for Drosophila. Tests at very high dosages (approx, 152,000 and $168,000 r$ units) indicated not only that every sperm had at least one dominant lethal (no female progeny occurred), but that fairly high per cent (roughly 40 w) were not able to take part in the fertilization process. (BA) 1853

Herskowitz, Irwin H. THE RELATIONSMIP OF X-RAY INDUCED RECESSIV LETHALS TO CHROMOSOME BREAKAGE. Am. Naturalist 80, 588-92(1946).

Employing the CIB technique, recessive lothal changes were obtained in the Canton $\$$ stock of Drosuphila melanogaster after $\mathrm{x}-\mathrm{ray}$ dosage at $1000 \mathrm{r}$ and $5000 \mathrm{r}$. Lethal cultures were then tested in a crossover generation for their association with chromosomal pearrangements. These were detailed by a reduction in $\mathrm{crossing}^{\circ}$ over of 50 " or more in either or buth regions of the $X$ chromosome tested. Of the $1-2$ cultures $0.3+0.21 \%$ at $1000 \mathrm{r}$ and
$3.57+1.3 \%$ at $5000 \mathrm{r}$ showed lethals associated with the regions of chromosomal rearangement. Of 43 recessive lethals tested, none showed chromosomal rearrangements unassociated with a lethal. Analysis of the available data suggests $x-$ ray induced lethals are produced primarily at breakage points and relegate to position effect and nonbreakage mutation a relatively small part as sources of recessive lethals. (BA)

1854

Herskowitz, Irwin H. THE GENETIC BASIS OR X-RAT INDUCED RECESSTE LETHAI MUTATIONS. Genetics 36, $357-63(1351)$.

Several hypotheses coneerning the origin of $\mathrm{x}-\mathrm{ray}$ induced recessive lethals are discussed. It is found that those postulating a single, os even a double, origin for recessive lethals are not consistent with the avalable data and theory. An hypothesis is suggested that recognimes lethals arising from three origins: point mutation, independent of breakage; breakage alone; and position effect following rearrangement of breaks. When the number of X chromosome break-free point mutation lethals in 100 viable sperm per $1000 \mathrm{I}$ is assumed to be 0.6 , the chance for a break not involved in gross rearrangement to bear a lethal 23 per cent, and the chance for lethallity for a break included in a viable gross rearrangement 31.6 per cent. the number of breaks involved corresponds to the number postulated by rialdane and Lea (J. Genetics $48,1-10(1947)$ ) for different doses, and a linear increase in recessive lethals with dose is obtained for viable sperm. (NSA) 1855

Heriwig, Paula. HERIDITARY SEMISTERILTTI IN MCE CAUSED BY RECIBROCAL CIROMOSOME TRANSLOCATIONS APTER I IRRADIATON. Z. indukt. Abstamm. Vererbungsl. 70, 1-27(1940).

Studies of progeny from male mice of which the sperm were irradiated showed reduction of fertilty due to lechal ity both of gametes and zygotes. Litter size was reduced to one half or more in all of the $F_{1}$, and in one half of the of fspring of the survivors. Transmission ratios of the semisterility indicate the presence of a translocation, and that some individuals homozygous for the translocation survive. There is no evidence for the survival of hyperand hypoploid zygotes. No cytological studies were made. (BA)

1856

Mollaender, Alexander, W. $\mathrm{F}$. Baker, and F. Anderson. EFPECT OF OXYGEN TENSION AND CERTAIN CPRMICALS ON THE X-RAY SENSITIVIT OF MUTATION PRODUCTION AND SURVIVAL. Cold Spring Harbor Symposia Quant. Blol. 16, 315-26(1951).

This paper deals with some of the work which has been published during the last two or three years together with some work which has not been published. The major part of the discussion involves the effect of oxygen tension on x-ray sensitivity as measured by the lethal effects, chromosome changes, and bacterial mutations. The better part of the paper is concerned with the effects of chemicals on x-ray sensitivity. (S.V.S.) 1857

Hollaender, Alexander and C. W. Emmons. INDUCED MUTA TIONS AND SPECIATION IN FUNGE. Cold Spring Harbor Symposia Quant. Biol. 11, 78-84(1946).

Studies on the ffects of ultraviolet radiation and $x-r a y s$ on fungi revealed lethal effects, temporary physiological changes, and up to $40-80 \%$ mutants in the surviving spores. Representative mutants, selected from several hundred mutants of Trichophyton mentagrophytes induced by ultraviolet radiation and carried in culture for six years, still 
exhiblt the characteristic features that led to their selection. When the conidia of the fungus (strain of the variable species Trichophyton mentagraphytes) were exposed to sufficient ultraviolet radiation (2537 or $2650 \mathrm{~A}$ ) so that 90 $99 \%$ of them were killed, a high percentage (up to $40 \%$ ) of the surviving conidia gave rise to colonies strikingly dipferent from the parent. These mutations were permanent. Some of the mutants produced by radiation resemble closely cerhain naturally occurring fungi which have been generally accepted as sep. "species". Other mutants age of a type that could be classified as new "species" if one did not know that they had originated from certain fungus cultures. Since it has been possible to produce mutants in certain fungi by irradiation with wave lengths in the ullraviolet spectrum that are present in sufficient intensity in sunlight coming to the earth's surface, the question is raised whether they may be a factor in the production of mutations in nature. Besides Trichophyton, a number of other fungi have been studied, particularly Aspergllus terreus and Penicillum notatum, and the same results were observed. A detailed investigation of the effects of monochromatic radiation of 2967 and $3130 \mathrm{~A}$ was made. These wave lengths were especially interesting because they are present in the biologically effective part of sunlight reaching the surface of the earth, that is 2900 to $3150 \mathrm{~A}$. The two wave lengths that were tested were found to be effective in the production of mutations in A. terreus. The biologically effective radiation on an average bright and sunny day in June amounts to about 2,000 milliw. sec./sq. cm. It requires approximately 230 milliw. sec./ sq. cr. to produce a $6 \%$ mutation rate in the $10 \%$ of spores of A. terreus surviving 2967 A radiation. It should thus be possible in about one hour's exposure to bright sunight around noon in June to produce in this fungus a significant increase in mutation rate. (CA)

\section{8}

Elollaender, Alexander and C. P. Sพranson. MODIFICATION OF TRE X-RAY INDUCED MUTATION RATE TN FUNGI BY PRETREATMENT WITP NISAR INFRARED. CenetLCs 32, $90(1047)$.

In general the mutation rate in fungi increases linearly with increased exposure to $\mathrm{X}$-Tays. A significant increase of the X-ray produced mutations is found if the spores are irradiated with near infrared radiation before $X$-ray exposure. The infrared used covered the region from 7000 to $18000 \AA$ with a maximum at $10000 \AA$. The experimental arrangement for infrared irradiation is such that the temperature rise in the culture is not more than 2 to $5^{\circ}$ above room temperature. Infrared alone does not induce mutation. This increase of mutation rate has been observed with Aspergillus terreus and Trichophyton mentagrophytes. The significance of these findings will be discussed in relation to the increased percentage of deletions found in Tradescantia if infraged is given before or aiter $\mathrm{K}$-ray treatment (SWANSON and HOLLAENDER, in press) and the increased percentage of chromosome rearrange-in Drosophla if infrared is given before $\mathrm{x}$-ray exposure. (KAUFMANN, HOLLAENDER and GAY; Genetics 31: 1946). (G)

\section{9}

Tollaender, Alexander and F. M. Zimmer. THE RFECT OF ULTRAVIOLET RADIATION AND X-RAXS ON MUTATION PRODUCTION IN PENICILLIUM NOTATUM. Cenetics 30, 8(1245).

Spore suspensions of Penicllimm notatum were irradiated with monochromatic ultraviolet radiation between 2280 and 2967 and medium hard $\mathrm{x}$-rays. The mutation zate Increased with increasing ultraviolet energy up to a maximun, and dropped with further irradiation. The most efficient wave length in the production of stable alterations was $2650 \mathrm{~A}$. Such results have been previously observed in Trichophyton mentageophytes, Aspergillus toneus and Neurospora crassa. In the x-ray series the pattern of mutation production followed a linear relation with in creasing energy. The highest (morphological) mutation rate obtained was about $40 \%$ of the surviving spores. More often a sate of $20 \%$ occurred at the peak of the curve in the ultraviolet. Different strains showed a varied response to ultraviolet. The morphological types of mutants will be demonstrated.

1860

Hungate, . $\mathbb{P}$. and T. Mannell. SULTUR-35 AS A

MUTAGENC AGENT. Genetics 36, 555-6(1951).

Radioactive sulfur was chosen as a desirable isotope to test for mutagenic properties. Sulfur is a part of the sulfhydryl grotp which is known to be essential to some enzymes. The possibility that genes and enzymes are similar suggests that sulfur may be necessary in the functioning of zome genes. Sulfur-35 is easy to use in the laboratory due to its weal beta particle and its convenient half-life of 87 days. - Neurospopa crassa was grown on Fries Medium in which sulfur -35 as sulfate had been substituted for the normal sulfur. After growing the mold for varying periods of time, the conidiospores were suspended and plated according to the method of Tatum, Barratt, and Cutter (Se ience 1949). - When the radioactive sulfur was undiluted with nonradioactive sulfur, a recovery of more than $10 \%$ biochemical mutants was obtained even when the radiation absorbed by the mold was well below 10,000 reps. When non-radioactive sources of sulfur were also present, this rate was reduced. The fact that the presence of carrier sulfur reduces the mutation rate suggests that the mutations may be induced not only by the lonizing beta particle but also by the transmutation itself - The mutants identified have in most part shown an inability to synthesize some amino acid. Those whose requirements have been identified include argmineless, methionineless, and leucineless, as well as some 1861 having more complex requirements. (G)

Hunter, Matthew C., Grant L. Stahy, and Wm. G. Myer's. VARIATIONS OF LISTERIA MONOCYTOGENES PRODUCED BY BETA PARTICLES FROM RADIOPHOSPHORUS. Ohio J. Sีe1. 50, 253-9(1950).

Cells of Listeria monocytogenes bacteria ware suspended in a medium containing radioactive phosphorus at a total radiation level of approximately 45,000 equiv. $r$ over an 11-day period. At 24-hr intervals samples were removed and streaked on suitable media. Nineteen strains were isolated which showed variations from the parent in their colonial characteristics. Some of these also showed variations in physiological characteristics, in their antigenicity, and in their capacity to produce disease. (NSA) 1862

Kerschner, Jean. THE INTLUENCE OF THE $\mathbb{X}$-CHROMOSOME ON X-RAY INDUCED MUTATION RATE IN DROSOPHLLA MELANOGASTER. Proe. Nat1. Acad. Sci. U. \$. $35,847-51(1949)$

The experiments described were performed in order to determine the effect of an extra $Y$-chromosome.on $x$-ray induced visible and ser-linked recessive lethal mutation frequency in Drosophila melanogaster. A medium-voltage $(135 \mathrm{kv})$ therapy machine was used with no fllter at a target distance of $17 \mathrm{~cm}$ and at an average output of $450 \mathrm{r} / \mathrm{min}$; the total dosage was 2,000-4,000 r. The extra $Y$-chromosome, when present was shown to decrease $x$-ray induced visible and sex-linked lethal mutation frequency in the male fly; in the female, it appeared to be without effect. The possible: mode of action of the $Y$-chromosome was discussed and future lines of investigation were proposed. (NSA) 


\section{3}

Kimball, R. I. INHERTTANCE OF MUTATIONAL CHANGES INDUCED BY RADIATION IN PARAMECIUM AURELIA. Oct. 1, 1948. 27p. (ORNL-175).

A study has been carried out in Paramecium aurelia of the inheritance of effects of beta and $\mathrm{x}$-radiation which first expressed themselves as reductions in vigor of some of the exautogamous progeny of ir radiated animals. The technique used in investigating the inheritance was to cross the irradiated animals to unirradiated ones by conjugation. In some cases, the products of the cross were crossed to unirradiated animals. The genotypes of the products of the crosses were tested by sending their descendants through autogamy to bring about complete homozygosis. The clones from both members of the conjugant pairs were in the majority of the cases of normal vigor. The exautogamous progeny from both members of the pairs often included a high proportion of clones of reduced vigor indicating that the effect was transmitted to both members as would be expected if nuclear mutations were involved. There was clear evidence that the two members of a pair produced exautogamous generations which were more alike, on the average, than were those from different pairs from the same irradiated animal. This finding is in agreement with the fact that the two members of a conjugant pair should be of identical genotype as a result of conjugation.

It was possible to calculate the average number of mutations per micronucleus in the irradiated animals and in the animals resulting from the cross of irradiated to unir radiated. The average number in the former was approximately twice that in the latter as would be expected if gene mutations were involved. In one experiment an anomalous type of inheritance was found for which no adequate expla nation can be offered. Viable homozygous clones of lowered fission rate have been obtained by autogamy in the descendant $\mathrm{S}$ of irradiated animals. The lowered fission rate can be shown to be transmitted through both members of conjugrant pairs between the low fission rate clones and normal animals. A noticeable feature of such viable but low fission rate clones is the great intraclonal variability in respect to fission rate and even viability itself. The conclusion was drawn that non-genic changes were not a major contributing factor to be redueed vigor resulting from radiation. It was also concluded that the results of the crosses have confirmed the assumption that reduced vigor in the exautogamous progeny of irradiated animals is an expression of micronuclear mutations induced by the radiation. (NSA)

1864

Kimball, R. F. THE INDUCTION AND INHILRTANCE OF MUTATIONS IN PARAMECIUM AURELIA. Cenetics $33_{3}$ 616(1948).

Beta radiation up to 13,000 r.e.p. has no immediate effects on stock 90 of variety 1 of Paramecium aurelia. When the descendants of rayed animals are sent through autogamy to make them homozygous, many are of reduced vigor. Evidence from inheritance studies indicates that the reduced vigor results from segregation into homozygous form of mutational changes induced in the micronucle. Doses given in small daily fractions were just as effective as those given as a single exposure. Thus twohit chromosomal aberrations could not have made a major contribution to the effect. However, the relation of the average fraction of exautogamous clones of reduced vigor to dose showed that a multiple hit phenomenon was involved. This is interpreted to mean that the majority of the effect is due to the combined action of mutant genes which are individually undetectable. A formula was developed relating the average fraction of reduced vigor to the mean number of mutations per micronucleus. The formula gives results consistent with the expectation that animals resulting from a cross of rayed to non-sayed should have on the average half as many mutants per micronucleus as the original rayed animals. From the formula, it was calculated that approximately two mutations per micronucleus were induced per 1,000 r.e.p. This rate is quite high and suggests that the method can detect mutations in the great 1865 majority of all loci in paramecium. (G)

Kimball, $\mathbb{R}$. F. TRE INDUCTION OF MUTATIONS IN PARAMECIUM AURELIA BY BETA RADIATION. Genetics 34, 210-22(1949).

Beta radiation from an external $\mathrm{P}-32$ source in doses up to 13,000 rep has no immediate marked effect upon vegetatively multiplying Paramecium aurelia. When the progeny of rayed animals are sent through autogamy, many of the exautogamous clones are of reduced vigor as judged by total growth in 4 days. This reduced vigor is interpreted as the expression of mutational changes in the micronuclel partly because it first appears when the animals are made homozygous by autogamy, partly because data on inher itance to be published in a separate paper support this view. IRadation given in a period of about an hour before eytoplasmic division is less effective than radiation given at other stages of the fission cycle. No adequate explanation is available for this phenomenon. The relation of the percentage of exautogamous clones of reduced vigor to dose shows that most of the mutational changes cannot be gene mutations with individually detectable effects. His belief that most of the detectable reduction in vigor is due to the combined effect of mutant genes with individual effects 100 small to be detected. The mathematical relation between the average fraction of exautogamous clones of reduced vigor and the mean number of mutations per micronucleus has been worked out for this hypothesis. By approximation calculations, a value of about two mutations per micromucleus per 1000 rep has been obtained. (Authors Summary)

1866

REmball, R. F. THE EFFECT OF RADIATIONS ON GENETIC MECHANISMS OF PARAMECIUM AURELIA. J. Cellular Comp. Physiol. Suppl. 1, 35, 157-69(1950).

Paramecium presents several advantages for analyzing the effect of radiation on genetic mechanisms. One of these advantages is the possibility of studying the effects of pradiation on both the nuclear and the cytoplasmic mechanisms of inheritance. Cytoplasmio inherilance has been studied fairly extensively in this organism previousiy. The purpose of this paper is to present some results of studies on the effects of radiation on both types of inheritance, and in so doing, give some idea of the possibulities of the material. Inactivation studies have been carried out, in a preliminary way, using ultraviolet from a germicidal lamp having its major output around 2537 . Inactivalion oceurs but the data are still not sufficient to define with any exactmess the form of the curve. There is a possibility that it will turn out to be of the exponential type expected for single event occurrence. The rest of the work is concerned with mutations induced in the micronucleus by $\beta$ radiation. An external source of $\beta$ radiation was used by means of the phosphorus plaque technique developed in this laboratory. (NSA)

1867

Kimbal, R. T. and Nenita T. Gaither. PROTORECOVRTY OF THE EFFECTS OF ULTRAVIOLET RADIATION ON PARAMECIUM AURELIA. Genetics 35, 118(1950).

Four different effects of ultrawiolet radiation and one of X-rays upon Paramecium aurelia have been examined for 
the action upon them of intense illumination with a lamp (GE H-5) having its major oulput in the long ultraviolet and short visible. The animals were exposed to ultraviolet of wave length $2650 \AA$ or $2804 \AA$ or to $\mathrm{X}$-rays and immediately afterwards exposed to the H-5 lamp. Except duxing this expowure, dark conditions were maintained as far as possible. The effects studied were as follows: (1) mutation as measured by death and reduced division rate after autogamy; (2) temporary reduction in division rate in the first few days after irradiation and in the absence of autogamy: (3) death, presumably nongenic, in the first few days after irradiation and in the absence of autogamy; (4) a miteroseopically visible change in the structure of the macronucleus requiring a few hours to reach complete expression. In all four cases, light from the 5 -5 lamp greatly reduced the effects produced by the shorter ultraviolet. The induction of mutations by $\mathrm{X}$-rays was not affected. The lact that light from the H-3 lamp afiects several kinds of changes produced by short ultraviolet suggests that it may act upon mome process basic to all the changes. The failure to detect an effect upon $X-r a y$ induced mutations suggests that they may be different in some way 1868 rom those indueed by ultraviolet. (G)

KImball, R. F. and Nenta Gaither. MODIFICATION OF THE ACTION OF X-RAIS UPON PARAMECIUM AURELIA Cenetics 36, 558(1951).

A number of agents which previously have been shown to modíy one or more effects of X irradiation have been tested on Paramecium aurelia. The mutagenic action of $\mathrm{X}$ rays in doses of one to síx lijoroentgens was found lo be reduced when the irradiation was gerformed in an atmosphere of nitrogen or in the presence of $0.7 \mathrm{M}$ ethyl alcohol. On the other hand, $0.001 \mathrm{M}$ sodium hydrosulfite, a reducing agent, had no influence on mutagenesis. No effect of the metabolic state was found since irradiation of either fed or starved parameeis, with of without incubation with $0.01 \mathrm{M}$ sodum formate, produced identical amounts of mutation. Likewise the rapidty of multiplication of the cells after irradiation, as influenced by temperature, was without inIluence. Two nongenetic actions, division retardation and death within twenty-four hours, were studied using doses of 100 to 300 kilorentgens. Both the amount of division retardation and of death were decreased by 0.7 m ethyl alcohol present during irradiation. Sodium hydrosulfite $(0.001 \mathrm{M})$ clearly protected against the lethal action but had no influence upon division delay or, perhaps, enhanced it. Nelther starvation nor incubation with formate was found to have any effect. Thus there is at least one agent which affects a variety of different kinds of radiation damage in Paramecium. If may be suggested that some step which is common to the various effects is involved. However, it is possible that some agents influence the steps leadimg to certain luins of damage only or else act more effectively uvon one rand than the other. (G) 1860

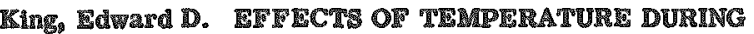
X-IRADLATION ON DEOSOPHITAA. CenetICS 32, 93-4(1947).

Males of $\mathrm{D}$. melanogaster were $\mathrm{X}$-mayed at dosages mul-

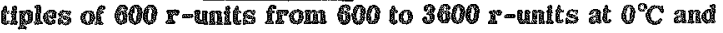
room tenperature respectively. At low temperatures for all dosages the frequencies of induced mutations were approximately twice as great as those for the same dosages at room temperature. The wablity li the cold series dropecd sharply at increashng dosages. (G) 1870

IRIng Ed

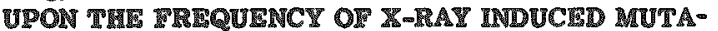
TIONS. Genetics 32, 161-4(1047).
Special stocks of Drosophila were irradiated at various dosages from 600 to $3,600 \times$ at room temperature and at $0.5^{\circ} \mathrm{C}$. For all dosages the frequency of induced lethal mutations was greater at the low temperature, with an average increment of $x 2.3$. The relation between dosage and frequency of lethal mutations was linear at both temperatures. (BA)

1871

King, R. C. GENETIC CHANGES ACCOMPANYING THE UPTARE OF PI2 $^{31}$ DI DROSOPRLLA MELANOGASTER. Genetles 35, 118-19(1950).

Adults or larvae of Drosophila melanogaster are found to incorporate large amounts of $\mathbf{P}^{32}$ into their tissue after feeding on yeasted cormmeal-molasses-agar media to which various anounts of $\mathrm{H}_{3} \mathrm{p}^{32} \mathrm{O}_{4}$ had been added previously. $\mathrm{P}^{32}$ is distributed among the head, thoras, gut, gonad and abdominal shell in the ratio of 20:50:10:10:10 for males fed as adults on the $\mathrm{P}^{32}$-containing medium regardless of the total activity of the fly. Radioautographs made of the spermathecae and assoeiated organs of untreated females fertilized by treated males gave direct evidence that the sperm were radioactive. Some treatments have produced sex-linked lethal mutation rates as high as $26 \%$ as measured in adult males of an inbred Canton-\$ stock by the M5 technique. Among a combined total of some 1,951 treated $X$ 's so far tested from all experiments 00 sexlinked recessive lethals and 11 viable mutants have been recovered. (G) 1872

Knudson, LEWIS. PERMANENT CRANGES OF CRLOROPLASTS INDUCED BY X-RAYS IN THE GAMETOPHYTE OF" POLYPODHUM AURIUM. Botan. Gaz. 101, 721-58 (1940).

The spores of $\mathrm{P}$. aureum may be treated with Ca hypochlorite up to 50 minutes at least, with no deleterious effect on germination and growth. Ir radiation of spores with dosages as high as $30,000 \mathrm{r}$ units is without ffect on the per cent of germination. With higher dosages the per cent of spores germinating decreases. The growth rate is decreased with dosages as low as $10,000 \mathrm{r}$-units, and progressively decreases with increased dosage. From 72 cultures in which were sown mearly 300,000 spores, 51 prothallia having abnormal hloroplasts were isolated.

Twenty-seven grothallia survived. These have been classified according to the characteristics of the chloroplasts. Including the normal, 11 types were noted. These prothallia have been propagated vegetatively during a period of seven years. The plastid types persist. Certain types are transmitted to the sporophyte as a result of sexual reproduction and retransmitted to the new generation of the gametophyte. The grouping of the plastids (type B) disappears in the adult leaves of the sporophyte and does not reappear in the new gametophyte generation. On theoretical grounds it is suggested that the plastid changes may be independent of any genic changes, and that certain plant mutations may be due solely to plastid changes. (BA) 1873

INoller, P. C. SEGMENTAL INTERCINANGE IN MCE. Genetics 29, 247-63(1944).

By exposing male mice to $750-900 \mathrm{r}$, three different 'semi-sterile' lines with varying sterility were obtained. The chromosome mechanism responsible for semi-sterility is analysed and described in detail. The degree of semi-sterility depend on the size and position of the translocated chromosome segments. (BA) 1874

Lea, D. W. WTECTS OF RADIATON ON GERM CELLS, DOMLNANT LFTHALS AND HERISDITARY PARTIAL STERTLITY. Brit. J. Radiol. Sumpi. 1, 120-41(1947). 
Chromosome structural changes induced by the irradiation of sperm or eggs may be viable or non-viable. Nonviable structural changes account for dominant lethals, e.l., for fallures of zygotes to develop to maturity when the sperm or the egg has been irradiated before fertllization. Viable structural changes give rise (in the mouse) to hereditary partial sterility. Exrperimental data on the induction of dominant lethals by $x$-rays and neutrons in Drosophila, Habrobracon, the mouse, rat, guinea pig, rabbit, and frog, and on the induction of viable chromosome structural changes in Drosophila and the mouse, are reviewed. The relation to be expected between the dose of radiation and the yield of viable and non-viable types of chromosome structural change is discussed on the assumptions that: the mean number of chromosome breaks primarily produced in a germ cell is proportional to the dose; a proportion, $p$, of the breaks primarily produced neither restitute nor exchange with other breaks, but behave as dominant lethals, probably as a gesult of sisterunion of chromatids occurring; and union is randon among the remaining breakage ends. (NSA) 1875

Lefevre, George, Jr. TRE RELATWE IPFTECTIVENESS OF FAST NEUTRONS AND GAMMA RAXS IN PRODUCING SOMATIC MUTATION IN DROSOPMILA. Genetics $33,112(1948)$.

Following exposure to gamma rays and fast neutrons 26 hours after egg laying, the ineidence of white and light eye color mosalcs was four times as high in eosin males as in wild-type males, and twice as high as in cosin with mutations at various other loci, rather than from a high mutability of the eosin gene. Using the data from wild-tyoe males, a mutation constant for somatic mutation at the white locus was calculated, showing the same order of . magnitude as that for germinal mutation. Mosaic ineidence following neutron exposures was higher than expected compared to gamma ray exposures, indicating that meutrons are not less effective per ionization, in producing mosaics. (NSA)

\section{6}

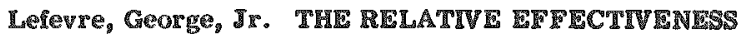
OF FAST NEUTRONS AND GAMMA RAYS IN PRODUCING SOMATIC CROSSING OVER IN DROSOPMLA. Genetics 33, 113(1948).

Heterozygous white and eosin-white female larvae were expozed 24 to 30 hours after egg laying to gamma rays and fast neutrons. The incidence of resulting eye color mosaics was much higher than in males, and was dependent upon the rate at which the total dose was administered. Heterozygous fenales with a double inversion in one x-chromosome ahowed no increase in mosaic incidence. Neutrons were relatively much more effective, per ionization, than gamma rays. Bvidence was found that mosaic size and age at exposure are not simply related. Twin mosaics in eosinwhite females shoved great inequality in size. Possible mechanisms for radiation induced somatic crossing over that require two hits within a limited length of time are considered. (NSA)

\section{9}

Lafevre, George, JI. W-RAT-INDUCED GRNIRTC ERTECTS IN CERMENAL AND SOMATIC TISSUE OR DROSORHILA MELANOGASTER Am. Naturalist 84, 341-65(1950).

Attempts were made to induce reverge mutations in both germinal and somatic tissue of Drogophila melanogastar. No evidence of reverse germinal mutation was found folm lowing irradiation with 5,000 $\mathrm{r}$ of some 166,000 recessive X-chromosome loci. In the somatic studies no reverge mutations of white were found in tests equivalent to the exposure of 600,000 white loci to $5,000 \mathrm{r}$. White alleles of three diverse origing were used: (a) spontaneous, (b) z-ray induced, and (c) mustard-gas-induced. Serious question is thus thrown on the reliability of the early reports of $\bar{x}$-rayinduced reverge mutation in Drosophila. The conclusion was also reached that $\mathrm{x}$-ray-induced rutability of the wt locus is not significantly affected by the kind of cell in which it is located. A comparison of the influence of various intrinsic and extrinsic Pactors on $x-5 a y-i n d u c e d$ and gpontaneous mutation indicates that the two mutation processes are qualitatively different. Fin all likelihood mum tations induced by lonizing radiation in Drosophila, as in maize, are losses or destructions of genetic material; and unlike spontaneous mutations, induced mutations are incapable of further change. (NSA)

\section{8}

Lefevre, George, Jx. X-RAY INDUCED SOMATC CROSSING OVER IN DROSOPHILA MELANOGASTER. Anat. Fecord 108, 623-4(1950).

This paper is reproduced here in its enthety.

Eye color mosaics were produced by irradatimg larval Btages of Drosophila with $1300 \mathrm{x}$ at the rate of $59 \mathrm{u} /$ min $48 \mathrm{hr}$ after egg Inying. The mosaic incidence in heterozy gous white females wa 0.65 per eye. In similar 1emales, but also bearing a double inversion in one X-chromosome, the mosaic incidence was 0.06 per eye. In males an imcidence of less than 0.02 white mosaics per eye was found. In males, gnosaics result mainly from somatic mutation; in females, delefion, chromosome loss, translocat ion, abnormal disjunction, and aomatic crossing over also may produce mosaics. The presence of inversions should not alter the effectivenss of any of the mosaic-producing mechanisms, except the last. Thus, in normal heterozygons females the great majority of mosaics resut from somatic crossing over, or possibly from nonhomologous segmental interchange. The distribution of induced somatic crossoverg was studied by comparing the mosaic imcidence in heterozygous white (locus 1.5), heterozygous garnet (44), and heterozygous carnation (62.5) females. The remutts indicate that an appreciable amount of somatic crossing over must take place in the heterochromatic portion of the X chromosome; but it is not equally distributed throughout the $\mathbb{X}$ chromosome, being greater in the right than in the left end. A similar distortion of the mormal pattern of crosing over is found when various agents are used in order to induce excessive germinal crossimg over. (AR) 1879

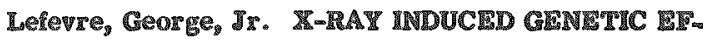
FECTS IN GERMINAL AND SOMATIC TRSUIP OF DROSOPMILA MIE.ANOGASTER. CEnetics 35, 120(1950).

The rate of X-ray induced direet mutation was compared following irrediation of germinal and amatic tigsue. Varlous data regarding the trequency of mutation to white after exposure of adult $w^{*}$ males indicate that the average germinal mutation constant for the mutation of $w^{t}$ to $w$ about $12.5 \times 10^{-8}$. Possible errors inherent in the estimathon of a somatic mutation constant can be evaluated, and by employing proper techniques reliable data on the somatic mutation rate of $w^{*}$ to w wan be oltained. Irrom the incidence and average aide of white eye-color mosalcs produced by irradiation of + male larvae, a somatic mutation constant of $14.2 \times 10^{-8}$ was calculated. Thus, $\mathrm{X}-\mathrm{rag}$ lnduced mutability of the w locus is not significantly affected by the kind of cell in which is is located. Altempts were made to induce reverge mutations in both germinal and somatic tisgue. No evidence of reverge germinal mutation wag found following irradiation with 5000 10 to 166,000 recessive

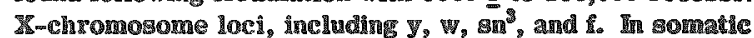
studies no reverse mutations of white were found in terts 
equivalent to the exposure to $5000 \mathrm{r}$ of $600,000 \mathrm{w}$ alleles of three diverse origins: (a) spontaneous, (b) X-ray induced, and (c) mustard gas induced. Serious question is thrown on the reliability of early reports of X-ray induced reverse mutation in Drosophila. A cormparison of the published data regarding the influence of various intrinsic and extrinsic factors on $\mathrm{X}-\mathrm{ray}$ induced and spontaneous mutation suggests that the two mutation processes are qualitatively 1880 different. (G)

Lefevre, George, Jr. and F. I. Ratty, Jr. X-RAY INDUCED EFITCTS IN THE WHTE-NOTCH REGION OF WILD-TYPE AND WUTE-MOTTLED-A CHROMOSOMESS OF DROSOPHILA MELANOGASTER. Genetics 36 , 560-1(1951).

Canton- + and $w^{m 4}$ males were exposed to similax $\mathrm{X}_{\mathrm{s}}$. ray doses of approximately $5000 x$. The relative frequen cies of viable white mutations, inviable white changes, notches, and simultaneous losses of both the white and Notch loci were determined. Examination of nearly 35,000 ofispring disclosed that the incidence of white lethals was gensibly the same in the two series, likewise the Notches. lowever, viable whites were twice as numerous in $w^{\text {Mnd }}$ as in Canton-\$ + , and white-Notch deletions about $16 \mathrm{x}$ more frequent in $w$ m. In each geries the number of Notches was almost exactly equal to the sum of the number of viable white plus the white lethals. - The failure to find more white lethals in $\mathrm{w}^{\mathrm{m}}$ than in Canton-S $t$, even though the W-N deficiencies were enormously more frequent, implies that many of the viable whites induced in wm" were actually deficiencies that would have been inviable if a loss of - imilar extent had been produced in a normal chromosome where the $w^{t}$ locus is surrounded by euchromatin. A consistent interprefation of the results can be made on the assumption that all $\mathrm{X}$-ray induced effects, viable and inviable, actually are the result of destruction of genetic material. When viable, the loss may consist of only one gene or less, and be cytologically undetectable. Longer euchromatic deficiencies will be almost invariably lethal. The Notch locus may well be more "important" than the $\mathrm{w}^{+}$ locus. Any loss in the former, however small, is homozygous inviable. (G)

1881

Lincoln, Ralph E. PRODUCTION AND RATE OT MUTATION IN BHYTOMONAS ST WWAPTT BV X-RADIATION. Genetics 25, 125-6(1940).

Broth cultures of Phytomonas stewartii were treated with $\mathrm{X}$-radiation of $0.7 \mathrm{~A}$ and $1.5 \mathrm{~A}$ at intensities which reduced a culture of $100,000,000$ viable cells to less than 1,000 viable cells within a period of 25 minutes. Inactivation curves for this organism are of the single absorption type - that is semilogarithmically linear. Variation was observed in colony type, colony color, virulence and mucoid characters. Mutation increases in proportion to exgosure time. Under conditions where only one out of every 100,000 cells treated remains viable, the mutation rate is about 200 times higher than the natural mutation rate after growth in broth, or about 10,000 mutalions per million treated cells as compared to 55 per willion cells after growth in broth. In general the same pattern and type of variation was found after - radiation as after growth in broth. Most mutations observed are stable, hereditable and transmissible from parent to daughter cell, although certain stocks have been isolated in which it is impossible to stabilize the variant type. (G)

1882

Lincoln, Ralph E. and John W. Gowen. MUTATION OF PHXTOMONAS STEWARTI BY X-RAY IRRADIATION. Genetics $27,441-62(1942)$.
This paper presents a comparative study of mutations in Phytomonas stewartii under natural conditions and under the gtimulus of $X$-irradiation of low quantum energy. Irradiation was at an intensity such that $100,000,000$ viable cells suspended in broth were reduced to about 1,000 in 25 min. of treatment. Survival during irradiation follows the simple exponential function-survival ratio $=\mathrm{e}^{\text {-ar }}$. Mutations were observed in colony color, surface appearance and size. The rate of mutation following $x$-irradiation is greater than the observed spontaneous rate. X-ray-induced mutation differs from spontaneous mutation only in frequency of occurrence, no differences in the kind of mutation produced being observed. The pattern of mutation observed in two widely different stocks of Ph. stewartil was similar. Mutations of colony characters may be accompanied by either increased or decreased virulence for maize. Except for mutations to an unstable form, mutants appear to be as stable as the parent strain from which they were derived. From evidence secured in this study it would appear that the terms "mutant", "variant," "Saltant," and "dissociant," as applied to bacteria are synonymous and are applied to phenomena resulting from gene mutation. From this point of view the physical basis of inheritance of Ph. stewartii is 1883 similar to that of higher organisms. (Authors' Summary)

Lindegren, Carl C. and Gertrude Lindegren. X-RAY

AND ULTRA-VIOLET INDUCED MUTATIONS IN

NEUROSPORA. J. Heredity 32, 404-12(1941).

$X-$ ray treatment of the uninucleate spermatia of $\mathbb{N}$. crassa induced both gene mutations and chromosomal aberrations, one of which was specifically identified as an inversion; ultra-violet treatment induced gene mutations and many of the ultra-violet treated variants were degenerate phenotypes which did not yield to genetical analysis. No chromosomal aberrations were found among the ultraviolet treated variants. These experiments followed by breeding tests are the first which specifically test induced fungal variants as to their genic and chromosomal nature. In most of the previous abundant work on the induction of mutation in fungi, the source material has not been properly purified, and it has not been possible to distinguish between induced mutations and mutations al ready present in the stock, or selected by the treatment, as compared to mutations actually induced by the reatment. (BA) 1884

Lindegren, Carl $\mathrm{C}$. and Certrude Lindegren. X-RAY AND ULTRA-VIOLET INDUCED MUTATIONS IN NEUROSPORA. II. ULTRA-VIOLET MUTATIONS. J. Heredity 32. $435-40(1941)$.

Ultra-violet treatment of the uninucleate spermatia of $\mathbb{N}$. crassa induced many stable simple gene mutations. None of the ultra-violet mutants showed the diminished fertility characteristic of chromosone inversion. Nearly half of the mutants were degenerate phenotypes which did not yield to genetical analysis. (BA) 1805

Lindegren, Carl C. and Gertrude Lindegren. $X-R A Y$ AND ULTRAVIOLET INDUCED MUTATIONS IN NEUROSPORA. Genetics $2 \%, 154(1942)$.

The uninucleate thin-walled spores (spermatia) of Neurospora crassa were treated by ultraviolet and $X$-rays cultured individually in separate test tubes and compared with an adequate number of controls. The variants induced were true gene mutations, for when mated with mycelia of opposite sex, factors differentiating the variations segregated at meiosis. A considerable number of the mutations, from both sources, reverted. Reverse mutations are much more easily brought into expression and have an opportunity to obtain supremacy in the fungi because the nuclei 
are haploid and the thallus is structureless and coencytial. A reverse mutation in any one of the nuclei in the thallus may change the phenotype. It is possible that the so-called "temporary changes" found by Emmons and Hollaender are likewise mutations which reverted. Fifty percent of the spermatia treated with $\mathrm{X}-\mathrm{rays}$ were killed and over 99 percent of those treated with ultraviolet were killed at the dosages used. A considerable number of the $\mathrm{X}$-ray variants gave evidence of the presence of chromosomal aberrations, while this was not true of any of the ultraviolet variants. (G)

1886

Luce, Wilbur M., H. B. Chase, and Henry Quastler. FFECTS OF GRADED DOSAGE OF X-RAYS ON THE PRODUCTION OF EYE FACETS IN THE BAR-EYED RACE OF DROSOPHILA MELANOGASTER. Genetics $33,115(1948)$.

The eggs and larvae of an inbred strain of the Bar-eyed mutant of Drosophila were treated with $\mathrm{x}$-rays of dosages of $115,460,920,1380 \mathrm{r}$ delivered from $5,20,40,60 \mathrm{sec}-$ onds respectively. The flies were kept at $28^{\circ} \mathrm{C}$. The eggs and larvae were in radiated at different ages ranging from 5 to 86 hours after deposition. The irradiation caused a reduction in facet number provided the time of treatment occurred during an effective period with limits between 48 and 72 hours of egg-larval life. The extent of the reduction increases with the $\mathrm{x}$-ray dosage ranging from 6.75 per cent with 115 to $41.20 \%$ with $1,380 \mathrm{r}$ in males, and from $2.83 \%$ with $115 \mathrm{r}$ to $34.76 \%$ with $138 \mathrm{r}$ in females. Irradiation outside the effective period had no significant effect on facet number except with the lowest dosage, $155 \mathrm{r}$. In this case treatment before the beginning of the effective period increased the facet number $7.30 \%$ in males and $5.92 \%$ in females. A conparison of the location of the effective period for $x$-rays with that for temperature indicates that the former begins earlier. Near the time of the beginning of the $x-r a y$ effective period the frontal sacs of the larvae are separating into the optic and antennal lobes. This suggesti that the $\mathrm{x}$-rays can act only after these areas are determined. After their separation the optic and antennal lobes begin to grow rapidly. The $\mathrm{x}$-rays produce their effects during this period. (NSA)

1887

Luce, Wilbur M., Henry Quastler, and Herman B. Chase. REDUCTION IN FACEI NUMBER IN BAR-EYED DROSOPIILA BY X-RAYS. Genetics 36, 488-98(1951).

Larvae of Bar-eyed Drosophila, kept at a temperature of $28^{\circ} \mathrm{C}$., which were irradiated with $\mathrm{X}$-rays of dosages varying from 115 to $1840 \mathrm{r}$, developed into adult flies whose compound eyes had a smaller number of facets than untreated controls. With a dosage of $920 \mathrm{r}$ the effect of $\mathrm{x}-$ rays was largely confined to a radiosensitive period exterding from about 55 to 70 hours of egg-larvae life with a maximum reduction occurring between 57 and 67 hours. The radiosensitive period closely corresponds to similar sensitive periods for other environmental agents affecting facet number in Bar-eyed Drosophila. The reduction in face number following application of $\mathrm{x}-\mathrm{rays}$ during the sensitive stage was nearly proportional to the doaage applied. In males the reduction in facets per unit dose was always greater than in females. The biological effects of $\mathrm{x}$-rays and the nature of the mode of action of the Bar mutants are discussed. (Authors' Summary)

\section{8}

Luce, Wilbur M., Henry Quastler, and Lester S. Skaggs. BIOLOGICAL RVALUTION OF 20 MLLION VOLT ROENTGEN RATS. III. RECESSIVE SEX-LINIED

LETRALS IN DROSOPHILA MELANOGASTER. Am. J. Roentgenol. Radium Theregy 62, 555-8(1949).
The mutagenic effect of roentgen rays has been investigated by means of the C1B test in Drosophila melanogaster. Three types of apparatus were used: the betatron, and conventional machines for deep and superficial therapy. The results obtained are consistent with an effectiveness of $70 \%$ of the high energy rays, as compared to low energy rays, both referged to the $25 \mathrm{r}$ condenser thimble as the common standard. (Authors' Summary)

1889

Mackey, James. NEUTRON AND X-RAY IXXPRIMENTS IN BARLEX. Hereditas 37, 421-64(1951).

Dry and presoaked seed of barley were treated with $\mathrm{x}$ rays and fast neutrons, and the biological effect of the in radiation was followed through the first and second generation. For dry, dormant seed the germinability was normal even after the heaviest dose applied. The retardation of the initial seedling growth was, however, pronounced, giving a sigmoid relation to $\mathrm{x}$-ray dose and an exponential one to meutron dose. The rrequeney of mature plants, i.e., the survival fraction, seems to be nearly equal in $\mathbb{X}_{1}$, and $\mathbb{N}_{1}$ at comparable tissue doses. The cytological response in the first mitotic cycle to irradiation showed an $\mathrm{N} / \mathrm{X}$ efficiency quotient of 10 , if the frequency of iragments and bridges were registered, whereas the fraction of affected seeds, the plant fertility, and the rate of induced chlorophyl mutations gave N/X ratios of $9 / \%$ g. For the two last-mentioned biological eventa a pronounced saturation effect was, however, intervening in $\mathrm{X}_{1}$, indicating that a contra-selection had set in. Presoaking of the seed did not change this difference in the biological action of $x$ rays and neutrons. In $\mathrm{X}_{1}$ the increased sensibility of the chromosomes was paralleled by an increased physiological killing. A more pronounced energy absorption due to the high moderating effect of water in the case of neutrons explains why the lethality after presoaking in $\mathbb{N}_{1}$ falls considerably below that of $\mathrm{x}_{\mathrm{i}}$. The types of induced chlorophyll mutations and their relative proportions were the same in $X_{8}$ and $\mathbb{N}_{2}$. At the high doses used in the present study no correlation between degrees of sterility in the first generation and different categories of chlorophyll mutation in the second has been found. After both types of radiation the mutations were to a great extent associated with chromosome disturbances. 108 references. (NSA)

\section{0}

\section{Markert, C. I. COMPARATIVE GENIS STABILIIY TN GLOMERELLA AND NEUROSPORA. Genetics 36, $564(1051)$}

More than 100 auxotrophic mutanto of Glomerella have been induced by the mutagenic radiations - ultraviolet light, X-rays, and fast neutrons. The gpectific nutrtional requirements of 74 of these mutanis have been determined by methods comparable to those commonly used with Neurospora. These data make possible (1) a comparison of the qualitative mutagenic effects of the three types of radiation, and (2) a comparison of the relative frequencles of the var ious tsinds of radiation-induced auxotrophic mutants in Neurospora and Glomerella. - The data do not reveal any qualitative differences in the types of mutants induced by the three radiations used. However, ultraviolet irradiation appears to induce the most easily repaired type of genetic damage, since ultraviolet-induced mutants revert to wild type much more frequently than $\mathrm{X}$-ray or neutron-induced mutants.- Since Glomerella and Neurospora are related fungi, it is not surprising that the commonly occurring mutants of Neurospora are also found in Glomerella and in similar relative proportions. Thus the genes which control homologous reactions in these two organisms appear to 1possess, in general, comparable stabilities. In contrast to Neurospora all auxotrophic mutants of Glomerella are 
visibly different from wild type on a complete medium. Two unusual types of Glomerella mulants are those requiring biotin and one which requires glutathione. $(G)$ 1891

Martin, Albert, $\mathbb{J}$. THE SIGNIFICANCE OF THE ACTION OF RADIOACTIVE PLOSPHORUS ON HABROBRACON JUGLANDIS ASHMEAD. Proc. Penm. Acad. Sei. 24, $60-4(1950)$.

A summary of the results of the effects of radioactive phosphorus on Habrobracon juglandis wasps is presented. Wild-type stock 33 were uged throughout the experiment and were subjected to $\beta$ rays of radioactive $\mathrm{Na}_{2} \mathrm{IP}^{32} \mathrm{O}_{4}$. Females were placed in exposure vials in contact with nonradioactive $\mathrm{p}$ salts while depositing eggs which became the control. They were then placed in exposure vials with the $\mathbb{P}^{32}$ salt on the basis that the $\beta$ radiation penet rates up to $1 \mathrm{~cm}$ of matter and would completely penetrate the body of the wasp. Among the progeny of each exposed female, virgin or nonvirgin, there were on the average $28 \% \mathrm{ab}$ normal individuals as against 1 or $2 \%$ in control cultures. Abnormalities such as misshanen wings, diferent numbers of antennae, short legs, and small over-all body size are 1822 described and discussed. (NSA)

Martin, Albert, Jx., J. N. Dent, and L. Joseph. EFF ECTS OF BETA-RAYS ON MARROBRACON JUGLANDIS.

Ceneties $33,612-20(1248)$.

A technique for exposing Habrobracon juglandis females to $\beta$-rays has been developed and utilized. Wild-type females exposed to dried radioactive phosphorous produce abnormal progeny with a frequency of approximately 25 percent, while untreated females produce abnormalities at the rate of about 5 percent. Some of the abnormalities in the test material have proved to be mutations, while none of the abnormalities in the controls have been transmitted to their of fspring. Frequency of abnormalities does not vary appreciably between progeny of treated virgins and non-virgins. Abnormalities occurring most frequently are those of the wings, antennae, and feet in that order. Mutations of wings and antennae have been checked through three generations. Reduplication of the right primary wing breeds true but with varying degrees of penetrance, even to the point of everlapping with wild-type. Wrinkled wings (primary and secondary) and drooping antennae appear in combination, indicating either a one-gene hit or a two-gene hit with genes so closely linked that they have so far shown no erossing segments of the left mesothoracic leg has not bred true, but the abnormality indieates the severity of possible effects of $\beta$-radiation. Current investigation indicates the need for extreme caution in the utilization of radioactive 1893 isotopes, particularly when used internally. (G)

McElroy, W. D. and C. P. Swanson. TPE THEORY OF RATE PROCESSSLS AND GENE MUTATION. Johns

Hopkins Univ. [nd] $42 \mathrm{p}$. (AECU-1713)

Genetic changes are pictured as being initiated by a chemical mutagen, which may be naturally occurring, radiation-induced, or artificially applied, and which in combination with a particular group in the genic structure initiates a series of reactions which give rise to an intermediate semi-activated state. This state may lead to a permanent change in the genic structure or it may, after a period of time, revert to a normal state. Environmental circumstances affecting the probability of the change's going one way or the other are discussed. The theory of absolute reaction rates as applied to gene mutation is geviewed and reevaluated. Finergy levels necessary for a molecule to reach the activated state are considered. Re- action rate and factors affect ing frequency of mutations are illustrated with reports of experimental work by the authors using plants. The potentiating effect of infrared radiation on changes induced by $\mathrm{x}$ rays, and changes produced by pressure and temperature on the mutagenic effects of nitrogen mustard are reviewed. (NSA)

\section{4}

Mitwoch, Urgula. STUDIES IN THE GENETICS OF SOME X-RAY-INDUCED MORPHOLOGICAL MUTANTS IN COPRINUS LAGOPUS. J. Genetics 50, 202-5(1951).

The method used for the culture of the hymenomycetes fungus Coprinus lagopus and for the production of $x-$ ray mutants is described. The mating type exhibited is outlined, and the independent inheritance of the mating-type genes is shown. Wour morphological mutants were found, one of which is linked with one of the mating-type genes. The action of aatural selection with regard to the binucleate 1895 condition of the secondary mycelium is discussed. (NSA)

Moh, C. C. and Euther Smith. AN ANALYSIS OF SEEDLING MUTANTS ISPONTANEOUS, ATOMIC BOMBRADLATION-, AND X RAY-INDUCED) IN BARLEY AND DURUM WHEAT. Genetics 36, 629-40(1951).

The study involved atomic bomb-and $x$ ray-induced, and spontaneous seedling mutants in barley and durum wheat. Progeny tests were made of $650 \mathrm{x}_{2}$ plants, representing 397 independent mutants; and of $5,653 \mathrm{X}_{3}$ plants, representing 581 mutants. It was concluded that chromosomal aberrations (possibly deficiencies) associated with a considerable portion of the mutations, or pleiotropic efiects of the mutan genes, best accounted for the fact that over 20 percent of the mutants tested segregated in ratios that deviated significantly from the theoretical $3: 1$. The results conld readily be accounted for if it were assumed that a considerable portion of the mutations were in fact deficiencies rather than gene or "point" mutations - an hypothesis which is being checked by further tests. (NSA) 1896

Mullex, H. J. AN ANALISIS OF THE PROCESS OF STRUCTURAL CHANGE IN CHROMOSOMES OF DROSOpHILA. J. Geneties 40, 1-66(1940).

The frequency of gross struetural changes induced by the irradiation of chromosomes in spermatozoa is proportional to the ionization produced, regardless of $\lambda$ of the radiation, from $50 \mathrm{kv} x$-rays to $\gamma$-rays; concentration of the dose in time, whether continuous treatment or fractionation over three weeks; fertilization immediately or after one month's delay; radiation intensity from $0.05-250 \mathrm{r} / \mathrm{min}$.; temperature from $5^{\circ}$ to $37^{\circ} \mathrm{C}$. These data constitute evidence that the "primary effects" of individual ionizations accumulate independently antil fertilization, the number of struchural changes produced depending on the final total. Since the frequency of rearrangements varies as the $3 / 2$ power of the total dosage from $1,000-4,000 \mathrm{r}$, the primary effects are breakages, followed secondarily by rearrangements only some of which survive. At lower doses, the exponent appears to approach 2 , as expected if all rearrangements zepresent combinations of independent primary changes. Evidence that the latter are actually breathages comes from data showing that whole chromosome losses of the $x_{1}$ presumably resulting from simple breakage followed by attachment of sister fragments, follow a linear proporlionality. It is suggested that gynandromorphs and mosaics arise not from treatment of occasional spernatozoa in which chromosomes are already split, but from a delay in the union of broken ends until after chromosome division, followed by differential behavior of the broken sister chromatids. Multiple exchange translocations (invol ving a ring chromosome) increase in frequency with dosage more 
rapidly than ordinary translocations (not involving a ring chromosome), and are more irequent al all dosages than expected on a basis of random attachment of broken ends. These data support the hypothesiss of initial breakages followed by unions favored by the original proximity of broken ends. Minute rearrangements have a linear dosage-frequency relation. Their breaks are interdependent, demonstrating the spread of the effect of a single ionization to nearby points on the chromonema spiral. This renders insecure the assumption that the genetic effects of radiation result solely from the ionization of atoms in the genetic material itself. The "sensitive volume" is not to be identified with the gene. The mortality of cells from treated sperm can be accounted for as due to the sum of induced gross and minute structural changes plus dominant lethal gene mutations. Part of the necrosis in irradiated prolifcrating cells must arise from these genetic causes, but not all, for the relative mortalities of irradiated triploid equal those of diploid D. embryos. The demonstration of the "breakage first" character of induced structural rearrangements is considered as throwing light, by analogy, on the mechanism of crossing over. The interpretation of all gene mutations as minute or ultraminute structural rearrangements of the chromosomes is also considered. It is shown that ulira-violet produces gene mutations without first brealing the chromosomes, since ultra-violet produces mutations but not translocations. (BA) 1897

Muller, H. J. INDUCED MUTATIONS IN DROSOPHILA. Cold Spring Harbor Symposia Quant. Biol. 9, 151-65(1941).

There is pvidence that gene mutalions start as changes in individual atoms, in consequence of which by transfer of energy or chain of reactions gene mutation results. Hadiation acts through single atom changes and a resulting chain of reactions to break the chromosema. If fusion of broken chromosome ends has not occurred before chromatid formation, two sister fragments can unite with subsequent loss of dicentric and acentric chromosomes. This is usually lethal. There is a tendency to restitution as opposed to rearrangement and chromosome loss. IVidence shows that broken Drosophila chromonemata seldom sheal" except by the union of broken ends with one another. It is shown that in minute rearrangements one individual atom-change must initiate a chain of reactions in two or more directions so that two or more different but nearby breaks of the chromonema are induced with subsequent fusion of ends from different breaks. The atom whose change initiates a muta tion dose not necessarily lie within the gene. Some evidence appears to favor the idea of a certan similarity between minute rear rangements and gene mutations, other evidence gives reasons for inferring that breaks occurring in both gross and minute rearrangements do not separate individual amino acid units but take place between much larger structures. (Mr)

1898

Muller, H. J. THE ROLE PLAYED BY RADIATION MUTATIONS IN MANISIND. Science 93, 438(1941).

This paper is an abstract and is reproduced here in its entirety.

Calculations are presented of the average number of generations to be expected between the production of a recessive gene mutation in man and its manifestation in a homozygous individual. The chance of the mutated gene meeting another like it that originated by an independent mutation depends on the frequency of such mutations, on the survival value of the abnormal type and on the randomness of breeding. 'Taking the maximal values for these factors (the values favoring the highest frequencies of recessive abnormalities) it turns out that at least 30 or, more likely, over 100 generations would on the average oecur -i.e., 750 to 3000 years or more - before a seriously harmful recessive abnormality (one having less than $90 \%$ of normal survival) would manifest itself by this process. Another mechanism of manifestation involves the meeting of two genes, each descended from the same original mutated gene. The frequency of this depends upon the total degree of inbreeding. A new method for gauging this is presented, involving the principle that the frequency of homozygosis from this cause is one fourth of the frequency of marriages between two related individuals having the same name. From statistics on this point and on cousin marriages it is found that, even in the most closely inbred groups in modern civilized society, the chance of a mutated gene manifesting itself by this process is not over $0.5 \%$. This represents a "latent period" of some 5000 years. Both processes together would give over 600 and probably thousands of years of latency. The large number of mu tated genes accidentally dying out while thus "under cover" are compensated for by those aceidentally multiplying. Thus the inherited damage from irradiation, though so long postponed, is in no wise prevented. Judging by results in mice (P. Hertwig) we may provisionally take the order of frequency of seriously harmiul recessive mutations induced by 300 is as one to several per cent (perhaps doubling the natural (requency). This chance of damage would seldom overweigh the direet benefits of diagnostic or therapeutic irxadiations, excepting those designed to stim ulate or temporarily inhibit reproduction. However, in irradiation intended for other parts, the gonads should be shielded. Watural radioactivity, while of no consequence in flies, may appreciably influence human mutation frequency. For the long duration of the human generation sometimes allows the reception of ten or more $r$. Thus under special conditions, the amount might conceivably be enough to be significant in evolution.

1890

Muller, H. J. THE PRODUCTION OF MUTATIONS.

3. Heredity $38,259-70(1947)$.

This is the text of the Nobel prize lecture delivered by the author before the Caroline Inst., Stockholm, Sweden, December 12, 1946. He reviews modern techniques for the marking, 1dentification, and manipulation of mutations in breeding experiments. Comparing mutations to chemical reactions, he points out that single mutations correspond with individual molecula changes. An extended series of mutations in a great number of identical genes in a population, spread out over thousands of years corresponds with the course of an ordinary chemical reaction that takes place in a fraction of a second or a few seconds. Chromosome analysis, radiation effects, somatic radiation effects, and frequency of mutations are other lopies discussed. Induced and natural mutations are compared, and the importance of selection is emplasized, since we are unable to control and direct mutations at will, even though the frequency of thein occurrence can be stimulated. The address is concluded with a warning to radiologists of their obligation to protect human germplasm against the danger of injury during medical treatment, industrial use of radiation and peace-time uses of atomic energy. (BA)

1900

Muller, H. J. THE PRODUCTION, ACCUMULATION, AND AVOIDANCE OF MUTATION. Argonne National Laboratory. Aug. 14, 1947. 2\%p. (ANL-4023)

A comprehensive discussion of mutations is presented. Though the mutant may be called "abnormal" in the given respect, we carefully define our term abnormal, nevertheless the phenomenon of mutation itself, i.e., the occurrence of changes which become reproduced in subsequent 
generations, is in a sense the most normal thing about all living matter, being the property that most basically distinguishes living matter from non-living and that has allowed living matter to develop, in its evolution, all the further peculiarities of its marvelous organization. For, on the modern genetic view of evolution, our entire bodily structure, with all its complicated physiology and biochemistry, has been built up through hundreds of millions of years by means of a long succession of mutations, probably millions of them in all. The thousands of different kinds of genes which we now contain, and which we call normal genes, cooperate in most complicated ways in the formation of every bodily organ and characteristic. Our whole complicated bodily organization is thus built up of a fabric of genes all of which were mutant in their day, but which happened to succeed and so are called "normal" by this time. Techniques using the Drosophila fruit fly are used as examples together with warnings against incidental esposure to $\mathrm{x}-\mathrm{r}$ ays handled by uniformed individuals and possible effects of atomic bomb warfare. (TIS)

1901

Muller, H. J. RADIATION DAMAGE TO THE GENETIC

MATERLAL. Am. Seientist 28, 33-59(1950).

Germinal genetic changes rather than the somatic effects of radiation are described and suggested in this paper. The origin of mutations is reviewed along with their general effect on the population. Approx. $92 \%$ of the mutations are harmul in some way, and as to be expected, eventually die out because of the lower viability of the individuals containing these mutated genes. Radiation is the only type of agent yet known to which human beings are likely to be exposed in quantity sufficient to cause any considerable production of mutations in them. that mutations resulting from radiation are similar to those arising naturally but the speed of their production is greatly increased, being as much as 100 times as many per generation in fruit flies. Danger to future human generations is implied when the amount of radiation is considered that received by women for such things as inducing ovulation; and the use of fluoroscopy and X-ray for diagnosis of disease in both men and women, even when the sex organs are not located immediately in front of the plate. The accumulated effects of these radiations may be observed in later generations by mutated genes. Few physicians or technicians keep a record of the total amount of radiation received by a patient and when the patient moves about the country and receives similar treatments in a number of places the danger is enhanced. We are much more concerned with the daily and present-generation somatic conditions than with the future development of detrimental genes. In this method of treatment the type of selection is lacking which would be needed to counteract the harmful eifects of an increase of the mutation frequency, and that increase, if it occurred, would spell retrogression rather than progreas. (BA)

\section{2}

Muller, H. J. RADIATION DAMAGE TO TME GENETIC MATEREL. I. IEITETS MANIFETED MANLT IN THE REPOSED INDIVIDUALS. Am. Scientist 38, 399$425(1950)$.

This article reviews some of the more recent work in the field. Some of the topics discussed are the induction of gene mutations in somatic cells, chromosome breakage by radiation, and its effects, the validity of the distinction between gene and chromosome changes, factors predisposing to the production of chromosone changes, the accumulation of radiation damage to the individual emphasizing the risk of repeated, low dosage exposures, and the danger of using what are normally considered reliable criteria, such as change in blood count, loss of sperm mobility, reddening of the skin, and loss of hair in estimating radiation damage to an individual, and other biologieal effects of radiation, such as the inhibition and regression of mitosis caused by low doses and the influence of certain chemicals on the damage of biological material by radiation. (NSA) 1903

Muller, H. J. SOME PRESENT PROBLEMS IN THE GENETIC EFFETS OF RADIATION. J. Cellular Comp. Physiol. Suppl. 1, 35, 9-70(1950).

The frequency of the primary genetic effects of ionizing radiation, gene mutations and chromosome breaks, is proportional to that of the ionizations produced, and, within wide limits, it is independent of wavelength, and of the time-intensity distribution of treatment. It is not, however, indegendent of the genetic and envixonmental conditions accompanying treat ment. Since gross arrangements of chromosome parts require two or more chromosome breaks, which in the case of $x$ and $\gamma$ rays are usually independent, their frequency follows a higher power of the dose than one. In Drosophila, but probably relatively little in most organisms other than Diptera, these rearrangements are accompanied by changes in the functioning of genes, often including lethal effects, near the sites of breakage. The question is considered why the frequency of lethals appears to remain proportional to dose even at high doses, despite the existence at such doses of a considerable class of lethals caused by the position effect of rearrangements whose increase in frequency follows a higher power of the dose than one. It is pointed out that this is probably because the rise in lethal frequency which would be caused in this way is at least partly compensated for by the presence of another kind of lethals, due to small rearrangements, especially deficiencies, which as the dose rose would increasingly come into combination with gross rearrangements and so become in part irrecoverable and in part lost to view as separate lethals. Not enough is yet known about the preferences of broken chromosome ends for one rather than another type of union to permit raliable calculations to be made of the expectations for different types of lethals at high doses, where there is much competition for union. Examples of some of the as yet unsolved problems concerning the manner of origination of chromovome changes are given together with some new evidence concerning them. It is shown that a high proportion of induced small deficiencies must result from two breaks in the same chromosome or chromatid, followed by deletion of the interstitial piece rather than by exchange of unequal segments between sister chromatids. Fvidence of the high frequency of cases of restitution is presented, based on data from ring chromosomes, but the total frequency of restitutions is still unknown. Grounds are given for concluding that the distinction between chromosome structural changes, even sinall ones and gene mutations, is valid, despite the fact that these processes are in some ways related. Some problems are considered concerning the amount and the mode of manifestation of radiation damage to the genetic material of the somatic cells of an exposed individual. (NSA)

\section{4}

Muller, H. J. PARTIAL DOMINANCE IN RELATION TO THE NIEED FOR STUDYING INDUCED MUTATIONS INDIVIDUALLI. J. Cellular Comp. Physiol. Suppl. 1, $35,205-10(1950)$.

There is evidence for the existence of a significant amount of dominance on the part of apparently recessive mutant genes. At first sight this dominance seems to make it possible to estimate the lotal genetic effects by studying the firgt few generations following exposure to radiation 
but the more analytical method of detecting individual mutant genes and comparng their effects in homozygous and heterozygous condition is indispensable. The most direct evidence for the existence of a small but significant degree of dominance of most "recessive" mutant genes is derived from cases like that of miniature wing in which measurement of a group of heterozygotes has demonstrated a significant statistical difference from normal in the direction of the homozygous mutants. Further evidence has been derived from consideration of the limitation in size of deficiencies that are recoverable heterozygously. Fividence for the ancomplete dominance of normal genes ("haploinsufficiency") lies also in the phenomenon of dosage compensation. There nust be an important difference between one dose and two doses of the normal gene in the same sex or there would be no selective pressure to readjust the effects in the two sexes in the manner observed. "Recessives" that are not fully lethal but only detrimental are harder to detect than lethals. But unless a sample of the "recessives" can be detected as individual mutants no idea of their numbers, amount of dominanee or consequent rate of extinction in the population can be obtained. The only fair criterion of the amount of genetic damage done to a population by a given treatment is the sum total of that damage throughout all succeeding generations. (MF) 1905

Muller, H. J. and G. Pontecorvo. THE SUPPISINGLY HIGH IREQUENCY OF SPONTANEOUS AND INDUCED CHROMOSOME BREARAGE IN DROSOPMILA, AND ITS EXPRESSION THROUGH DOMINANT LETRALS. Genetics $27,157-8(1942)$.

The unusually low ratio of daughters to sons of irradated males having ring $X$-chromosomes is shown to be mainly due to death of zygotes with affected X's. For, at. $4000 \mathrm{x}$, only about $3 \%$ of females are found to be converted into males by induced loss of an $\mathrm{X}$, whereas the relative number of females is reduced by about $35 \%$. Allowing for deaths from $\mathrm{X}$-translocations (some $10 \%$ ) most of this dominant lethal effeet on the females must be due to "simple breakage," which in rings must often be followed, in cases which would otherwise be restitutional, by formation of dicentrics, occasioned by twisting of the chromonema or chromonema-pair before re-union. The results show over $75 \%$ of these sister-dicentrics to be lethal (translocational dicentrics being still oftener lethal). Breaks in non-rings are presumably induced by irradiation as often in rings, but here the great majority are followed by perfect restitution. Thus at $4000 \mathrm{r}$ nearly all of fspring contain one or more restituted chromosomes nevertheless the lethal losses of non-ring $X$ 's are frequent enough (ca. $5 \%$ at $4000 \mathrm{r}$ ) to show that a considerable part of the dominant lethal effect of sperm irradiation results from "simple breakage" with sister-dicentric formation. The frequency of spontaneous lethal and viable losses of rings (ca. 13\% and $2.5 \%$ is likewise surprisingly high, and may be due to spontaneous breakage. This probably occurs similarly in non-rings but is followed by perfect restitution. Since so few spontaneous structural changes arise, this breakage would usually occur in pre-spermatozoon stages, allowing prompt restitution. (G)

\section{6}

Mullex, H. I. and J. I. Valencia. ThI LOCALIZATION OF THE MUTAGENIC ACTION OF NEUTRON-INDUCED IONIZA TIONS IN DROSOPHILA. Genetics 36, 567-8 (1951).

The frequency of translocations induced by fast neutron irradiation of Drosophila spermatozoa was found to vary linearly with dose even at doses sufficient to produce multiple proton tracks per sperm. This shows that broken chromosome ends derived from different breaks caused by the same track undergo recombination with one another much oftener than with those of different tracks. Our interpretation is that breaks caused by the same track tend to occur near together, this proximity favoring union between the broken ends. Thus the pieces would usually unite before greatly changing their relative positions. It must further be inferred that in this material a break usually occurs close to the point of origin of the ionization that induces it, i.e., that remote breakrage efiects, resulting from migration of ionization-induced, relatively stable mutagens over mîcroscopically appreciable distanees, are unconmon. - Further evidence for this conclusion is provided by the finding that lori, like that for white eyes, which with $\mathrm{X}$ rays give "visible mutations" seldom accompanied by a lethal effect unless there is a microscopically visible deficieney or other rearrangement, give with neutrons "visible mutations" that are usually accompanied by a lethal effect, even when there is no cytologically demonstrable alteration. This concatention of two mutagenic effecta in close proximity (whether in these cases usually breaks or gene mutations or both is not yet decided) would result from the crowding of ionizations in proton tracks, provided the mutagenic action of the ionizations remained narrowly localized. - Work supported by grant from Amertcan 1807 Cancer Society. (G)

Muller, H. J., R. M. Valencia, and J. 1. Valencia. THE PRODUCTION OF MUTATIONS AT INDTVDUAL LOCI IN DROSOPPILA BY IRRADIATION OF OOCYTISS AND OOGONLA. Genetics $35,126(1950)$.

To study mutagenesis in interphase nuclei females undergoing semi-gtarvation were $x$-rayed with 4600 Ind then passed through a series of cultures, usually at $1-d a y$ intervals. Offspring from firgt cultures represented irradiated later oocytes, with chromosomes relatively condensed, and those from later cultures "resting" oogonia or, more rarely, early oocytes, with chromosomes attenuated. The genetic technique deseribed in our parallel paper on spontaneous mutation was used, mutations at the 11 gpectfied loci being looked for in non-disjunctionally produced daughters. 8,865 such daughters from the first cultures were observed, 94,380 from later cultures. Over 200 mutants were obtained, and about 100 bred and analyzed cytogenetically. In the attenuated chromosomes, gene-muta tions were induced with a frequency apparently somewhat below but at least half as high as that previously obtained from spermatozoon irradiation. Presumptive deficiencies were however far less abundant than from treated mpermatozoa. Their frequency, in relation to gene-mutations, seemed like that in the untreated material. Only of of presumptive deficiencies examined proved cytologically visible. None of the mutations analyzed involved gross rearrangements. The more condensed chromosomes llater oocytes) gave frequencies of both gene-mutations and presumptive deficiencies sensibly similar to results from treated spermatozoa. For deficiencies, the difference from oogona was highy aignificant. But again, gross rearrangements were absent. Chromosome brealage evidently occurs as frequently here as in spermatozoa, but union of fragments must occur fairly promptly, 1.e., prior to the movements of the meiotic diviolons. Mosaid mutants appeared with only the spontaneous frequency, there being no evidence of delayed mutation. (Work aided by grant from American Cancer society, recommended by Committee on Growh of National Research Counell). (G) 1908 MUIYZ, M.J. AN ATTEMPT TO OBTAIN SEX-LINKED MUTATIONS IN A DIOECIOUS PLANT. GenetiCs 25 128(1940). 
A normal female plant of Acnida tamariscina (Nutt.) Wood was pollinated with $\mathrm{x}$-rayed pollen. Seed from 474 different $\mathbb{F}_{1}$ open-pollinated females were grown. No visible sex-linked mutations were found in over $250,000 \mathrm{~F}_{2}$ seedlings and approximately 10,000 mature $F_{2}$ plants. This suggests that the $Y$ chromosome has a full complement of allelomorphs. However, ten chimeras were obtained. These were chlorophyll changes associated with fifty percent bad pollen in the affected areas. (G) 1909

Myers, W. G. and Hazel Jean hanson. NEW STRAINS OF PENICILLIUM NOTATUM INDUCED BY BOMBARDMENT WITH NEUTRONS. Science 101, 357-8(1945).

Spore suspensions of a stable variant of $P$. notatum in normal saline in test -tubes were bombarded with neut rons from a cyclotron. About 100 new strains or mutations were produced. The antibiotic activity of many of the new strains were qualitatively similar to the parent strain. The number of new strains was fairly proportional to the 1910 amount of bombardment. (BA)

\section{Newcombe, H. B. SECTORING IN COLONIES FROM} IRRADIATED CELLS OF ESCMERICHIA COLI, Genetics 36, $570(1951)$.

Colonies from ultraviolet- or gamma-irradiated cells of I. coli, grown on mannitol-tetrazolium agar, are frequently sectored for colour response. With ultraviolet doses of 500 to 1000 ergs per sq. mm. 15 to 20 per cent of the colonies were sectored, and with gamma doses of 40,000 to $80,000 \times 6$ to 7 per cent. This was at first thought to be due to the presence of more than one nucleus in the treated cells. However, if this were the correct interpretation one would expect to eliminate the sectoring by selecting the descendents from a single nucleus. To do this, selection for induced nutations to phage resistance was carried out on the mannitol-tetrazolium agar, the treated cells being allowed a short period of growth and then sprayed with mixture of phages $\mathrm{T} 1$ and $\mathrm{T} 5$. Contrary to expectation, a proportion of the colonies arising after further incubation were still sectored for colour. This proportion, however, was roughly half that observed in the non-selection experiments. Essentially similar, although less extensive, results were obtained using streptomycin as the selective agent. - The sectoring which survives selection could be due to: (1) delayed mutation, (2) genetic interchange between two nuclei in the treated cell, (3) mutation in incompletely split chromosomes, or (4) mutation in hereditary particles other than the chromosomal genes. (G)

\section{1}

Novitski, E. and C. Stern. THE VIABILITY OF INDTVIDUALS HETEROZYGOUS FOR RECESSIVE LETHALS. Science 108, $538-9(1948)$.

Tests of daughters of females known to be heterozygous for a sex-linked lethal show that the viability of individuals heterozygous for a lethal is not equivalent to that of individuals free from a lethal. The percent of lethal-bearing daughters should, within statistical limits, fall equally about 50 percent. Thirty three lethals showed a marked inequality of distribution with 5 lethals above and 23 below the expected midclass. The range of values is also unequal, the lowest being 42.1 percent of lethal-bearing daughters and the highest 53.7 percent. It is pointed out that de creased viability of lethal heterozygotes in populations where the frequency of the lethal gene is very low, might. be more important than the loss of rarely occurring homozygotes. (NSA)
1912

OKami, Yoshiro and Hamao Umezawa. STUDIES ON THE MUTATION OF STREPTOMYCES GRISEUS NO. 200 by X-RAX IRRADIATION. J. Antibiotics (Japan) Suppl. B, 3. $7-9(1950)$.

S. griseus No. 200 which produced streptomycin abundantly in Waksman's medium was $\mathrm{x}$ ir xadiated at the rate of $300,000 \mathrm{r} / \mathrm{hr}$ (length of time not given). Five blackpigment-producing mutants and one albino-type mutant were obtained. These morphological mutants did not produce streptomycin. After $x$-ray treatment strains which produced more than $1000 \mu \mathrm{g} / \mathrm{ml}$ of streptomycun were found, but they were not physiological mutants because they were not stable in their streptomycin-producing ability. Both English and Japanese versions of this article are presented. (NSA)

\section{3}

Opatowski, I. ON THE MUTA TYON OF THE TOBACCO MOSAIC VIRUS. J. Gen. Physiol. 33, 171-6(1949).

Experiments on $x$-ray-induced mutations of tobaceo mosaic virus with radiation duses of 0 to $49,000 \mathrm{r}$ and mutation rates of 0.079 to $5.275 \%$ are analyzed on the bass of ionization theory. Three alte rnate assumptions are used in calculating the size of the volume in which the primary mutation process develops: this volume is a protein, from which it is found that the volume is identical with the hexagonal cell unit described by x-ray diffraction studies on virus protein; this volume is nucleic acid, from which it is found that one-fourth of the total nucleic acid content is involved in the mutation process; this volume is phosphorus, from which it is found that half of the total $P$ content is involved in the mutation process. The relation between induced and spontaneous mutation is examined, and the author concludes that natural ionizing radiations are insignificant for the spontaneous mutation of the virus. (NSA) 1914

Opatowski, I. ON THE INTERPRETATION OF THE DOSEFREQUENCY CURVE IN RADIOGENETICS. Genetics, 35 , 56-9(1950).

If is shown that assumptions opposite to those which are made in the single event theory of induction of mutations by radiation, lead to a relation between radiation dosage and frequency of induced mutation which is practically linear within its experimentally pertinent range. It is concluded from this that the apparent linearity of the experimental data on the dependence of the mutation frequency upon the administered dose cannot be considered as a proof that the mutation is caused by a single primary event. (NSA)

\section{5}

Opatowski, 1. and Alice M. Christiansen. ON THE SINGLE EVENT HY POTHESIS IN RADIOGENETICS. Bull. Math. Biophys. 12, 19(1950).

It is shown that the assumption of a cumulative action of many independent randon events as the primary cause of induction of a mutation fits the experimental data at least as well as the single event hypothesis. The theory is illustrated on the existing data of $x-r a y$ induced lethals in the X-chromosone of Drosophila. Possible shortcomings of the single event theory in connection with these experimental data and alternative ways of its modification are indicated. (Author)

\section{6}

Otani, Shohei and Hyuga Okada. ON THE VARLATION OF PENICILLIUM CHRYSOGENUM Q 176 INDUCED BY RADIUM IRRADIATION. J. Antibiotics (Japan) 3, 819-32 (1950). 
To induce saltation or mutation of microbes by radioactivity, the spores of $P$. chrysogenum $Q 176$ were irradiated with radium. Germination was prevented to the extent of about 2 , by 5-day irradiation. Various variants appeared by the end of this irradiation. The variation rate was $15 \%$. From the variants the authors isolated one st rain without specific yellow pigment. This strain was called "RaO." No particular variants which would yield higher potency could be obtained. The isolated variants were investigated by monospore culfure and other methods as to whether they are induced by saltation or by fluctuation and also were examined for penicillin yield. It was found that variations of the color of spores and of the form of colonies were induced by saltation; variation of spore numbers was induced by fluctuation; the strains which have few spores produce little penicillin, and there were intermediate forms between saltation and fluctuation. By shake culture and tank fermentation the pigmentless stratin "RaO" was found to be a saltant. It produced $470 \mu \mathrm{cc}$, the same potency as the parent strain, by shake culture. The potency in the test tank was $267 \mu$ ce. This strain may be a promising one. Filtered broth was always pigmentless, and the

1917 resultung a morphous penicillin was white. (NSA)

Plough, Harold K. PENICLLLIN ISOLATED BIOCHEMICAL MUTATIONS IN SALMONELLA TYPHMURIUM: THEIR PROPORTIONALITY TO X-RAY DOSAGE, AND THEIR FALURE TO SHOW RECOMBINATION. Gentics $35,130(1950)$.

Blochemically deficignt gtrains of Salmonella typhimurnum werf isolated from suspensions subjeeted to X-radiation and then screened with penicullin according to the method of Davis. The original strain was used by Gowen in his studics of the grenetic basis of virulenee, and was derived from a single cell isolation by zelle. Successive suspensions are $\mathrm{X}$-rayed at dosages from $11,000 \mathrm{R}$ to $57,000 \mathrm{r}$ units, incubated 24 hours in complete medum, centrifuged, washed, resuspended in minimal medum (salls plus glucose) containing 200 unils of penieillin per ml. and again incubated for 48 hours. This ireatment kills most of the parent type organisms and from dilution plates made on complete medium 100 colonies at random are isolated. Preliminary platings showed that the X-radiation killed from $60^{\circ}$ at 11,000 R to more than $99^{\circ} 0^{\circ}$ at 57,000 R. Tests by the paper dise method of assay of the clones 1solated as noted gave $9 \%$ biochenical mutants from the unradiated controls increasing up to $80 \%$ of mutations from the suspensions receiving the maximum $\mathrm{X}$-radiation dosage. Over 250 mutant strains resulted each of wheh requires one or more specific nutrilites for growth which the parent strain does not need. Specific amino acid requirements are most numerous with purine requirements second. The most irequent amino acid requirements are cysteine, histidine, methionine, proline, tyrosine, levsine, threonine, and valine. Several tests for possible sexual fusion and recombination by the method of Tederberg have proved entirely negative perhaps indicating that Salmonellat is a haploid uninucleate cell. Many interesting biochemical interactions are being further investigated. (Author)

\section{8}

Plough, Harold H. RADIATION INDUCED MUTATIONS IN CHEMICAL REQUIREMENTS IN SALMONELLA TYPHMURIUM. J. Cellular Comp. Physiol. Suppl. 1 , $35,141-55(1950)$.

Attention is directed to the desirability of pathogenic bacteria of the genus Salmonella as objects of genetic study using radiation as a mutagenic agent. Isolation methods are described and four different classes of mutations, iso- lated and investigated in Salmonella, are depicted. At least one other class, i.e., antigenic variants, is also indicated. Differences in the spontaneous frequency of these classes of mutations are suggested by the relative numbers isolated from ir radiated lines. (NSA)

1919

Plough, pharold Ho, Helen N. Young, and Madelon R. Grimm. PENICILLIN-SCREENED AUXOTROPHIC MUTATIONS IN SALMONELLA TYPHIMURLM AND THEIR RETATION TO X-RAY DOSAGE. J. Bacteriol. 60, 145-57(1950).

Suspensions of 5 . typhimurium have been exposed to $\mathrm{K}$ radiation up to 57,000 r unis and then sereened with penicillin on minimal medium containing essential salts and glucose. Of the 457 strains isolated and tested, 234 proved to be auxotrophic mutant strains having a constant requirement for one or more food substances lihat the parent strain can synthesize for itself. The frequencies of these mutations are directly related to the x-may dosage, suggesting that thoy are gene mutations similar to those found ir higher organisms. Most of the mutant strains required one of the amino acids or one of the purines, but altarnative and multiple requirements were found. The data indicate that at ratiation dosages up to 35,000 a a cysten - requin ing nutant is the most numerous, wheresas above that level the mutan most frequently isolated requires histidine. A test of the efficacy of the penicillin sereening of mixtures of will-type and s-1ay-induced nutations is giver. The value of auxotrophic mutations for gtudies of the steps in bacterial synthetic processes is suggested. (NSA)

1920

Pomper, Seymour. RECENT DEVELOPMENTS IN YEAST GENETICS. Wallerstein Labs, Commun. 14, (44) 31-41

(1951) (ORNL-696)

This review paper is limited, in the main, to work that has been performed in the past decade. Among the topics discussed are the early genetic studies with Saccharomyceg; mating types, crossing procedures, and sporulation; genetic markers (unique characteristics which can be demonstrated to be heritable); and biochemical mutants involving such agents as $\mathrm{z}$-radiation and compounds such as potassium cyanide, lithium chloride, and camphor. (NSSA)

\section{1}

Pontecorvo, G. THE INDUCTION OF CHROMOSOME LOSSES IN DROSOPHLA SPERM AND TRRIR LINEAR DEPENDENCE ON DOSAGES OF IRRADITION. \$. Genet. 41, 195-215(1941).

Non-lethal losses of the $\mathrm{X}$ and $\mathrm{X}$ chromosomes, produced by $x$-ray treatment of mature sperms, were detected by testing exceptional y $\mathrm{y}$ f females obtained from crosses of

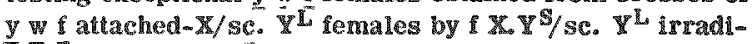
ated males. The tests indicated that $64-71 \%+7-9 \%$ of the exceptional yellow ( $w$ f) females had arisen from a complete loss of either the X.YS or the so. Yl chromosome. These losses are not lethal to the rygote, but a comparimon with data showing the amount of change in the sex ratio caused by equivalent dosages indicates that lethal losses are probably three times as numerous as non-lethal. At dosages of $0,1,000 x$, and 4,000 $\mathrm{r}$ the frequenciss of the non-lethal losses vary approximately as a linear function of the dosage and certainly less than the 1.5 power of the latter, like gross rearragements. The mechanism whereby these losses originate is therefore held to be through single breakage, followed by a lateral fusion of sister chromatids at the breakage point. This results in the formation of dicentric chromosomes that immediately or subsequently become lost. (BA) 
1922

Pontecorvo, G. and H. J. Muller. THE LETHALTTE OF DICENTRIC CHROMOSOMES IN DROSOPHLA. GeneticS 26. 165(1941).

The fact that viable translocations formed after 8 -raying apermatozon are proportional in frequency only to the $3 / 2$ power of the dose has been shown to signify that union of broken ends of chromosomes occurs without reference to presence or absence of centromere on ragment, the zygote with aneucentric rearrangements, i.e., with chromosomes other than monocentric, dying. If death is due solely to the genic unbalance resulting directly from the loss of the non-monocentric chromosomes, then, if extra chromosomes homologous to those lost were introduced, in compensation for the losses, the resulting zygote would survive. This can be tested, in Drosophila melanogaster, by crossing irradiated males to triploid females; homozygous recessive markers in the triploids allow recognition of the exceptional offapring. Thus, losses of a single paternal autosome, as by simple breakage, will allow one autosomal recessive to show in the while losses of two autosomes, as by aneucentric translocation, will "uncover" two recessives sinnultaneously. Among $8,880 \mathrm{~F}$, from crosses of brown ebony triploids by non-brown non-bony males given 4,000 I, 133 showed just one autosomal recessive, and only 3 (one being the approximate expectation for the coincldence of two independent losses) showed both recessives. But the frequency of aneucentric translocations produced must have been such as to have resulted in over 800 zygotes showing bol h recessives, had these zygotes lived. Hence over 92 percent of these aneucentric rearrangements are lethal per se even though many aneucentrics formed from union of sister chromatid fragments after simple chromosome breakage are not lethal. Investigation of the mechanism involved is proceeding. (G)

1023

Powers, E. L. Ir. DFATH ATTER AUTOGAMX IN PARAMECIUM AURELIA FOLLOWING DXPOSURE IN SOLUTION TO THE RADIOACTIVE ISOTOPES $\mathrm{P}^{92}$ AND

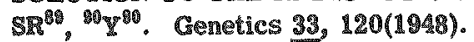

Sister animals derived from a single exautogamous organism were grown in graded specific activities of $\mathbb{P}^{32}$ and of $\$ r^{39}, 8 y^{90}$, all beta-emitters. After 48 hours' exposure, 7 to 8 cell divisions had occurred, sufficient to incorporate the gralioactive substances into the protoplasm to the extent of more than $99 \%$ of the theoretical maximum. At this time a series of isolations fron each of the solutions was made, and the lines so obtained were allowed to go into autogamy. Survival after autogamy was measured by isolating 108 autogamous animals and noting the proportion that survived aix divisions after isolation. The physical parameter was determined by measuring the current output from a vibrating reed-air ionization chamber system for equal volumes of each of the and of the Sr solutions. Each activity is expressed in terms of a conm gtant uranium source measured by the same system. In the low dose range, the effect of radioactive $\mathbf{P}$ on post-autogany death is much more marked than that of equivalent

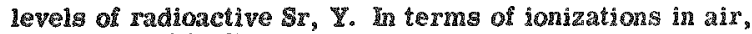
levels of $\mathrm{Sr}^{80,80} \mathrm{Y}^{80}$ necessary to produce a degree of death after autogamy exceed by a factor of 4 to 6 the level of $\mathrm{p}^{32}$ necessary to produce the same amount of death after antogany. (NSA) 1924.

Powers, E. L. Jr. and Deborah Shefner. LETHAL CHANGES INDUCED BY X-RAYS IN PARAMECIUM AURELIA. Genetics 33, 624-5(1948).
Cultures of $\mathrm{P}$. aurelia, stock 51, "killer" animals of varioty 4 , mating type VIII were irradiated with $250 \mathrm{KV} \mathrm{X}-$ rays at about $450 \mathrm{r} / \mathrm{minute}$ at 17 dose levels from 500 to $25,000 \mathrm{r}$. Lines derived from isolates from each of the irradiated cultures were allowed to go into autogamy and the fractions of the surviving exautogamous organism were noted. Death following autogamy inereases with increasing dose. Above 12,000 $\mathrm{x}$ the curve approaches 100 percent death very slowly, total death not being achieved in these experiments. Organisms which survived autogamy showed only control death following the next autogamy regardless of the degree of effect. Conjugation of irradiated with nonirradiated organisms resulted generally in increased survival following the next autogamy. These two results are expected if the effect of $\mathrm{X}$-rays at these dose levels are genetic ones in the micronucleus. No significant difference can be seen between the genetic damage induced by X-rays as demonstrated here and that caused by 3 particles of radioactive Strontium and Yttrium in solution as reported previously. (G) 1225

Randolph, L. T. CETOLOGICAL AND PHENOTYPICAL EFTECTS INDUCED IN MAIZE BY X-RAYS AND THE BHINI TEST ABLE ATOMC BOMB. J. Cellular Comp. Physiol. Suppl. 1, 35, 103-17(1950).

The cytological and phenotypical effects of the Bikini atomic bomb were qualitatively similar to those induced by x-rays. With respect lo retardat ion of plant growth, seedling mottling, partial sterility, and the production of leaf sectors involving morphological abnormalities and chlorophyll deficiencies, most heavily irradiated Bikini sample was comparable to an $\mathrm{x}-\mathrm{rayed}$ sample that had received 15,000 x units, except that a significantly higher frequency of chlorophyll sectors and dead tissue occurred in the bombed sample. Chromosomal mutations including reciprocal itranslocations, deletions, and inversions were obgerved with approximately the same frequency in the plants grown from the bombed and $x-r a y e d$ seed, and in both lot there were many more translocations than there were other types of chromosomal alterations. (NSA)

1926

Randolph, L. F., A. Longley, and C. Li. CYTOGENETIC EFIECTS IN CORN EXPOSED TO ATOMIC BOMB IONIZING RADIATION AT BIKINI. Science 108, 13-15 (1948).

The experiments included a comparison of the affects of the radiation of the bomb with measured doses of $x$-ray. seed germinability was not affected by the radiations from the bomb or by the s-ray treatments. A comparison of other effects of radiation from the bomb and $\mathrm{x}$-ray treatment is given. (N)

\section{7}

Ray-Chaudhuri, S. P. THE VALIDITX OF THE BUNSENROSCOE LAW IN THE PRODUCTION OF MUTATIONS BY RADIATION OF EXTREMELY LOW INTENSTTY. Proc. Roy. Soc. Edinburgh B 62, 66-72(1944).

Sperm of Drosophila were irradiated in the body of impregnated females with $70 \mathrm{mg}$ of radium for 720 hours at two dosages of $2,000 \mathrm{r}$ and $400 \mathrm{r}$ with an intensity of $0.556 \mathrm{r}$ and $2.78 \mathrm{x}$ per hour respectively. The frequency of sexlinked lethals produced was found to be directly proportional to the dosage independent of the intensity of irradia.m tion. The mutation rate was independent also of the duration of the treatment when flies were radiated with an intensity of $29 \mathrm{r}$ per hour, giving a total dose of $1,300 \mathrm{r}$. (BA) 
1928

Regehr, Hulda, T. J. Arnason, and H. E. Johns. INDUCTION OF MUTATION BY HIGH-ENERGY X-PADIATION PRODUCED BY A 23-MEV BETATRON. Nature 166, $228-9(1950)$.

Male Drosophila melanogaster received a single dose of 2000,5000 , or $7000 \mathrm{r}$ of $\mathrm{x}-\mathrm{radiation}$ at $100 \mathrm{r} / \mathrm{min}$. All the irradiated X-chromosomes which were tested for mutations were obtained from sperms wheh functioned in fertilization within 20 days of the treatment. The average percentage mutation per $1000 \mathrm{r}$ high-energy $\mathrm{x}-\mathrm{rays}$ is $1.70 \%$. The corresponding figure for ordinary $x$-rays as given in the literature is $2.89 \%$. This would indicate that the highenergy radiations are only 0.59 as efficient as ordinary $\mathrm{x}$-rays for the production of lethal mutations. The reason for the difference in effectiveness is discussed. (NSA) 1929

Rick, Charles M. THE GENETIC NATURE OF X-RAY INDUCED CHANGES IN POLLEN. Proc. Nat. Acad. Sei. U. S. $28,518-25(1942)$.

X-radiation of microspores of diploid spp. of Tradescantia shortly alter meiosis increases variability in pollen grain length and per cent of aborted grains, indicating that size and viability are due in part to the genotype of the pollen itsclf. The effect is greater after treatment at $3^{\circ}$ than at $33^{\circ}$. Similar radiation of autotetrapolid spp. confirms this conclusion by falling to give similar results, since unaffected dominant alleles mask the recessive mutations. Statistical analysis in the pea, Pisum sativum, where four kinds of hybrid pollen are intermediate in size and more variable than either parent further confirms the theory of self determination of pollen size by a large number of genes. (BA)

1930

Rick, Charles M. THE X-RAY INDUCED MUTATION RATE IN POLLEN IN RELATION TO DOSAGE AND THE NUCLEAR CYCLE. Genetics 28, 237-52(1943).

Mean length and variation of length of pollen grains and per cent of aborted grains were studied for a period of two months following application of $62.5,125,250$, and $500 \mathrm{r}$ to inflorescences of diploid Tradescantia. The inter- and intra-treatment variation was studied statistically. The mutational response of pollen abortion after treatment during the post-meiotic period varied approximately linearly with dose; the response of variance of length was intermediate between a linear and squared relationship. Length changes were all or nearly all toward smaller grains. Number of changes involved greatly exceeded total number of gross chromosomal aberrations induced by the same doses. Resemblance between these changes and mutational effects in other organisms is considered further evidence that the changes in pollen are the phenolypic expression of mutation induced by the $x-$ ray treatment. High pollen sterility was induced by treatment during meiosis and the first gametophytic mitosis. According to evidence presented these stexility responses correspond more closely to chromosomal aber ration than to intragenic change. Pollen changes of smaller degree result from treatment before meiosis, although measurements are complicated by a very irregular retum to normal sterility. (BA)

1931

Rowell, J. B., E. C. Stakman, and E. E. Butler. THE MUTAGENIC ACTION OF DILUTE COLLOIDAL POLONIUM ON FUNGI. Minnesota Univ. [nd] 6p.

(AECU-1823)

Extremely dilute quantities of polonium $210(0.5$ to 1.0 $\mu \mathrm{c} / \mathrm{ml}$ ) readily induced mutation in vegetative cells or spores of various fung in aqueous suspensions held at $8^{\circ} \mathrm{C}$ for appropriate periods of time. Spores germinated direct$1 \mathrm{y}$ in water containing $1 \mu \mathrm{c}$ of polonium per $\mathrm{ml}$ will grow and produce normal colonies. Lethal and mutagenic effects do not appear until the cells are held in this mixture at low temperatures over a long period of time. The effective length of time of exposure varied with the species of organism tested. In simullaneous trials with Ustilago zeae, Saccharomyces cereviseae, Chaetomium globosum, and Penicillium roquefortii the respective exposures in days for LD99 were $21,4,9$, and 2 ; and the percentages of visible mutation were $1.0,6.1,10.1$ and 3.5 . The most numerous mutants obtained have been those having reduced growth or reproductive rates. The amount of radiation associated with the cells in this method approximates dosages of $\mathrm{x}$ rays similarly affecting fungi as one $\mu \mathrm{c}$ of polonium produces 326 rep per day. (NSA)

1932

Rubin, B. A. THE MECHANISM OF GENETIC EFICT OF $\mathrm{P}^{32}$ IN BACTERLA. Brookhaven National Lab. [nd] 13p. (BNL-1069)

The biological effect of absorbed unstable isotopes is related, in some cases, to the chemical changes resulting from radioactive decay rather than to the accompanying radiations. The special geometric and metabolic characteristics of bacterial cultures are employed to show that genetic effects of $\mathrm{p}^{32}$ may be attributable to the rupture of the phosphate bond in desoxyribose nucleic acid. Other cxamples illustrate the use of the nuclear disintegration technique as an indicator of the specific chemical changes which can produce biological and genetic efiects. Possible evolutionary significances are discusged. (NSA)

\section{3}

Rubin, B. A. THE RELATION OF NUCLEIC ACID METABOLISM TO THE GENETIC EFIECT OF RADIATION IN BACTERLA. Genetics 36, 573-4/(1951).

Levels of continuous radiation (x - rays or $\beta-\mathrm{rays}$ ) which rapidly kill resting bacteria (15. coli $B / r)$ germit the growth of metabolizing organisms. When resting cells are inoculated into a fresh medium the inception of growth (lag) is delayed as a direct function of the radiation dose rate; but when multiplication beging, the rate of increase of cell mass during continuous radiation is equal to that in unirradiated controls and the same final culture densities are reached. Average cell sime increaseg with the number of generations that have occurred during irradiation, but this accounts for only a fraction of the cell mass, the logarithmic increase in viable count makes up the rest. - An examination of the mutant population (resistant to streptomyein) in cultures which have grown from 0 (resting) to 14 generations during irradiation indicate that the mutation rate is affected by the radiation dose, but not by the number of genetic reduplications during irradiation. Under the same conditions, changes in nuclei acid metabolism appear which are related to the extent of synthesis that has occurred during irradiation. (G)

1934

Rubin, B. A. and P. Stelnglass. THE GENETICS OF STREPTOMY CIN RESISTANCE INDUCED BY ABSORBED RADIOACTVE PHOSPHORUS (P $\left.{ }^{32}\right)$. [Brookhaven National Lab.] [nd] 2p. (AECU-765)

The document is in abstract form; it is reproduced below in its entirety.

In the study of the mechanism of genetic effect induced by $\mathrm{F}^{2 \mathrm{2}}$ in $\mathrm{E}$. coll $\mathrm{B} / \mathrm{r}$, the use of the phenomenon of streptomycin resistance has proved to be both a good quantitative tool and an interesting subject in itself. 
As a genetic tool, it has been possible to develop techniques similar to those used in the phage studies. Rapid and accurale estimates of the background mulation rates are now possible, which also provide simple methods for the isolation of the various types of resistant mutants. In this maner it has been possible to readily demonstrate the additional biochemical dependence of the mutants, their streptomycin requirements, and relative growth rates. Many of the objections to similar phage studies are eliminated by the stability of the drug and its lack of response to mutagenic agents - in this case, specifically $\beta$ radiation and the products thereof.

In the radiation experiments the variations in delay of phenotypic expression have necessitated kinetic studies which could interrelate the pattern of genetic events. The kinetics were userul in their ability to demonstrate qualita tive as well as quantitative genetic changes, produced by variations in the proportion of rintant cypes.

In these experiments the mutants varied with regard to their acquired need for the drug, but all organisms selected in 200 units/ml were resistant to 100,000 units/ml. Such characteristics are of significane in understanding the problems of chemotherapy. The responses of bacterial populations to atreptomycin have helped elucidate quantitative aspects of drug resistance. Good predictions can now be made of the likelihood of producing resistant organisms and of methods for preventing this difficulty under conditions of practical therapy. (INSA)

1935

Russell, W. L. THE INCLDENCE OF STERILITY AND PAPTLAL STERILITY IN THE DESCENDANTS OF XIRRADIATED MICE. Genetics 35, 689-90(1950).

This paper is reproduced here in its entirety.

The data reported here were obtained incidentally in the courge of another investigation. Adult male mice were given a single, whole-body, $80 \mathrm{r} / \mathrm{min}$ dose of 500,750 , or $1000 \mathrm{r}$, and then were mated. 22 male and 15 femalle of spring, all concelved within 2 to 30 days after irradiation of the sire, were tested for fertility. Averaging the results over wide dose ranges is not justifiable, because of nonlinearity of effect with dose, but is perhaps permissible, over the range used here, for a rough comparison with the regults of other workers. Of the 37 animals tested, and whose simes received a mean dose of $669 \mathrm{r}, 8.1 \%$ were found to be completely aterile and $27.0 \%$ partially gterile. These percentages are in remarkably close agreement with the results of Snell and Hertwig for similar dose ranges. All of the 10 partially sterile animals obtained in this experiment, and one other from another test, were found to transmit partial sterility to some of their descendants. Two of the 11 lines, however, do not int the simple interpretation of reciprocal translocation which Snell and others have established as the cause of the partial sterility in at least some cases. Both of these lines are producing some completely sterile descendanis, one also yielding some almost sterile descendants. The data obtained so far are not adequate to determine whether a different type of change is involved or whether an additional translocation or other chromosomal or gene change has occurred. 1936

Russell. W. I. X-RAY-INDUCED MUTATIONS IN MCE. Cold Spring Harbor Symposia Quant. Biol. 16, 327-6 (1951).

In an attempt to obtain information that would be useful in the estimation of the genetic hazards of ionizing radiahion in man, wild-type male mice were exposed to a single dose of $x$ rays and mated to a atock homozygous for seven autosomal recessive visible genes. Control, unexposed animals were also mated to the test stock. The offspring were observed carefully for mutations at the seven specific loci and cursorily for dominant visible mutations at any locus. Dominant mutations with obvious visible effects proved to be rare. Six have so far been found in the sradiated group and rone in the controls. However, a dominant fifect on size was found in about one hall of the mutations at one of the specific loci. Approximately 61 mutations at the specific loci have been observed in the irradiated group, while only two were found in the control group. The frequencies of mutation at the seven loci in the irradiated group ranged from none at the A locus to 28 or more at the S10eug. Fach of nine mutations in the irradiated group has now been lested for its phenotypic effect when homozygons. Two were found to be lethal, two sublethal and five viable. Three mutations to intermediate alleles were found in the irradiated group. Out of a tolal of eight mutations at either the $D$ locus or the closely linked Se locus in the irradiated group, none showed a change at the associated locus. The mean observed mutation rate, for irradiated spermatogonia, at the severn loci is $25.0 \times 3.7 \times 10^{-8}$ per roentgen。 per locus. The rate is not appreciably dependent on the length of the interval between irradiation and fertilization. Comparison with similar data from Drosophila shows a higher induced mutation rate in the mouse. This indicates that estimates of human hazards based on Drosophila mutation rates may be too low. 1037

Russell, W. L., J. C. Kile, Jr. and Liane B. Russell. TAILURE OF HYPOXIA TO PROTECT AGAINST THE RADIATION INDUCTION OF DOMINANT LETHALS IN MCE. Genetics 36, 574(1951).

To test whether hypoxia would protect against radiation induction of dominant lethals in mice, groups of malles were: (a) X-irradiated with $800 \mathrm{r}$ in air, (b) non-ixradiated in air, (c) $\mathrm{x}$-irriadiated with $800 \mathrm{r}$ in a $5 \%$ oxygen, $95 \%$ helium mixture, or (d) nonirradiated in the same mixture. The gas was passed through the exposure chamber for five minutes prior to ir radiation and during irradiation in exposed groups and for the equivalent time in non-irradiated groups. Females fertilized by these males shortly after treatment were opened just before term and their uterine contents examined. Classifying exbryos as alive or dead (including resorption sites) gave the following mean nuxnbers of the two respective lypes per litter, with from 47 to 50 litters in each group: (a) 3.9 and 3.6, (b) 8.2 and 0.98 , (c) 3.6 and 3.5, (d) 8.3 and 0.96 . Repetitions of the esperiment with 30 minutes pretreatment in the same gas misture and irradiation in this or in $2 \%$ oxygen $98 \%$ helium gave similar resulls. These data give no evidence for protection by hypoxia against the induction of dominant lethals to which the excess death of embryos from ir radiated sires is attributable. This is in contrast to the protection against genetic damage found by others in various organisms. It is also in contrast to protection by hypoxia, in this experiment, against direct radiation effects on the exposed males: acute radiation death reduced from $92 \%$ to $0 \%$, length of sterile period and graying of hair decreased. (G) 1938

Sansome, Eva R., M. Demerec, and Alexander Hollaender. QUANTITATIVE IRRADIATION EXPERIMENTS WITH NEUROSPORA CRASSA. I. EXPRERMETS WTTH X-RAYS. Am. J. Botany. 32, 218-26(1945).

Microconidia were irradiated with $\mathrm{x}-\mathrm{says}$. The increase is mulation rate with increase in energy followed a straight-line relationship at low dosages and reached $78.5 \%$ at 126,000 roentgens. Increase in intensity led to an increase in the mutation rate. Phenotypic normal and num 
tant cultures obtained from irradiated spores often showed partial sterility when crossed with a standard wild type, but this was more frequent in phenotypic mutants than in phenotypic normals. Partial sterility is believed to result from chromosomal aberration. An analys is of the sterility and mutation rates at different dosages and intensities indicates that there are two types of mutants, one caused by chromosomal aberration and the other not. However, the latter class may consist party or entirely of changes that are potentially capable of being associated with aberrations. (BA)

1939

Sansome, Eva R., Alexander Hollaender, E. Zimmer, and M. Demerec. QUANTTATIVE RFECTS OF RADIATION ON MUTATION PHODUCTION IN NEUROSPORA CRASSA. Genetics 30, 19-20(1945).

(This report is an abstract and is reproduced here in its entirety).

The heritable nature of alterations produced in fungi by monochromatic ultraviolet irradiation was verified by genetic analysis of a fungus having a sexual stage. Microspores of the "fluffy" type of Neurospora crassa were used in this study. The effects of wave length and dosage on the mutation rate resembled those reported for some Fungi Imperfecti. To eliminate contaminants, the mutants were routinely crossed with the conidial "wild" type. Certain of the ascospore progeny were germinated and studied in more detail. Of the 40 crosses, where the ascospores were sown at random, more than one type of "fluffy-mutant" was recovered from about hall. This suggests a high incidence of mutations. Unstable mutants were subjected to microspore analysis and classified. - Experiments with X-rays, indicate that visible mutants were produced with a 18 quency which increased linearly with dosage. For dosages given at an intensity of $270 \mathrm{r} / \mathrm{min}$, the frequency of mutants increased from $2.8 \%$ at $2,250 \mathrm{r}$ to $24.6 \%$ at $\$ 1,500 \mathrm{r}$. About half the spores survived the treatment of $31,500 \mathrm{r}$. When dosages up to $126,000 \mathrm{r}$ were given at $5,400 \mathrm{r} / \mathrm{min}$, the mutation rate increased to $78.5 \%$ with a survival of $0.01 \%$ The observation of the Lindegrens that $X-r a y$ induced mutants, when crossed with the wild type, often gave more aborted ascospores than the control crosses was confirmed. It was found that treated culures which were phenotypically normal were also sometimes sterile, but that the proportion of steriles among the mutants was higher than among the normals.

Drew Schwartz. 1. GAMETE FACTOR ON CHROMOSOME 9. 2. THE EFTECT OF OXXGEN TENSION ON THE RADIOSENSTTIVTY OF CMROMOSOMES. Oak Ridge National Lab. [nd] Ip. (AECU-1876)

Gamete factor on chromosome 9. A gamete factor was found which shows close linkage with the waxy gene. In back crosses of heterozygous Ga wr/ga WX as the male to homozygous waxy plants, only $10 \%$ starchy kernols are obtained. Further testing of these $B_{1}$ starchy kernels revealed that $30 \%$ of them are due to functioning of $\mathrm{ga} \mathrm{Wx}$ pollen and 70\% are crossovers. These data indicate that there is $3 \%$ functioning of ga pollen in competition with Ga and $7 \%$ crossing over between the Ga and $\mathrm{Wx}$ loci. The effect of oxygen tension on the radiosensitivity of chromosomes. Experiments conducted on the irradiations of pollen in different atmospheres revealed that there is a $3.2-f o l d$ decrease in chromosome aberrations when the exposures are made in $100 \% \mathrm{~N}$ as compared with exposure in air. The $\mathbb{A}_{1}-\mathbb{S h}_{2}$ region was used in this study. $\mathbb{A}_{1} \mathbb{S h}_{2}$ pollen was irradiated and used to pollinate $a_{1} a_{1} s \bar{h}_{2} s \overline{h_{2}}$ tester plants. Aberrations were detected in the endosperm as entire losses of the dominant characters and as mosaics. Irradiation in nitrogen decreased the frequency of entire losses by a lactor of 2.7 while the mosalcs were decreased by a factor of 9.9 . This difference is statistically significant and strongly suggest that the effect of oxygen tension during irradiation is on the recovery procegs of broken ends rather than on the initial breakage mechanism ag had previously been postulated. (Intire report.)

1941

singleton, W.R. EFFECT OF CONTINUOUS GAMMA RADIATION ON MUTATION RATE IN MAIZE. Genetics 36, 575-6(1951) (AECU-1461)

The report is reproduced here in its entirety.

Continuous gamma radiation from $\mathrm{CO}^{2}$ was used in studying the mutations induced in maize pollen. The intensity varied from 125 to $0.09 \mathrm{x} /$ day. Four endosperm characters were studied: $\mathbb{R}, \mathbb{P r}$, Su, and Sh. Pollen from the dominant stock $\mathrm{A}, \mathrm{B}$ Pl C R P Su Sh prowing in the radiation field was placed on silks of a multiple recessive A b pl C r pr su sh growing in a field with only background radiation. The following results were obtained: (1) There was no increase in mutation rate over the control when the radiation was less than $5 \mathrm{r} /$ day. (2) Above $5 \mathrm{r} /$ day there was a gradual increase up to $31 \mathrm{r} /$ day with a more marked increase from 31 to $55 \mathrm{r} / \mathrm{day}$. From 55 to $125 \mathrm{r} / \mathrm{day}$ the increase in mutations was even more marked. The increase in mutation rate at the higher intensities of radiation was greater than expected if the increase is linearly proportional to dose. (3) The logarithm of the mutation rate seemed to show a linear relationship with dose received. (4) The gene with the greatest spontaneous nutation frequency $(R)$ showed a smaller increase in induced changes than the other three genes $\mathrm{Su}, \mathrm{Sh}$, and $\mathrm{Pr}_{\mathrm{r}}$. (5) In control plots the mutations detected in the microspore were appreciably higher than those found in the megaspore. This is expected since only those mutations that occur at or before the first mitotic divigion of the megaspore mother cell will be detected because of the triple fusion forming the endosperm. (G)

1942

Smith, Luther. A COMPARISON OF THE EFFETS OF TEST ABLE ATOMC BOMB IONIZING RADLTION AND X-RAYS ON SEEDS OF BARLEY, WHEAT, AND OATS; APPENDW NO. 25 TO TIE FINAL REPORT. NAVAL Medical Research Station, Joint Task orce One. [nd] 17p. (NP-1900)

Comparisons were made of the biological effects on dor mant seeds of barley, rye, and durum and common wheats of radiations from an atomic bomb and from known dosages of $\mathrm{x}$ rays. Effects studied included germination, flecking or mottling on the first leaves, chimeras, interchanges in $X_{1}$ plants grown from the irradiated seeds, mutation frequencies in $\mathrm{X}_{2}$ seedlings, and types of mutants obtained in $\mathrm{X}_{\mathrm{g}}$. seeds most heavily irradiated from the bomb burst were estimated to have recelved irradiation equivalent to 16,000 $r$ of $x$ ray. Plants from seeds which were subjected to $16,000 \times$ irradiation were used for comparison. There were no clear-cut differences in the types of seedling mulants obtained from the 2 sources of radiation nor in the relative proportions of these types. (NSA)

\section{3}

Smith, Luther. FEREDTARY SUSCEPTIBILITY TO XRAY INJURY IN TRITICUM MONOCOCCUM. Am. J. Botany 29, 189-91(1942).

A mutant in einkorn wheat was found which is more severely injured by $\mathrm{X}$-ray treatment of the dormant seeds $(4,000-20,000 \mathrm{r}$ units $)$ than other mulants or normal vars. 
tested. In one trial the germination of a normal line was reduced $12 \%$ by $20,000 \mathrm{r}$ while the susceptible was reduced $84 \%$. The injury exhibits itself in greater flecking on the leaves, slower growth, and death from smaller doses than are required to produce similar effects in non-susceptible geeds. Preliminary tests indicate that the susceptibility is inherited as a simple recessive character, the factor has no effect on mutation rate and does not reduce the ability of seeds to withstand heat injury, and the moisture content of the seeds and the size of the chromosomes in the susceptible line are normal. (BA)

\section{4}

Smith, Luther. EFFECTS OF ATOMC BOMB RADIATIONS AND X-RAYS ON SEEDS OF CEREALS. J. Heredity $41,125-30(1950)$.

The biological effects of radiations from an atomic bomb and from known dosages of $\mathrm{x}$-rays on dormant seeds of barley, durum, and common wheats have been determined. The relation of age of barley seeds to susceptibility to radiation effects also has been studied and limited macroscopic observations have been made on plants grown from seeds of oats that had been exposed to atomic-bomb ionizing radiation. (NSA)

\section{5}

Smith, Luther and R. S. Caldecott. MODFICATION OF X-RAY EFFECTS ON BARLEY BY PRE- AND POSTTREATMENT WTH HEAT. J. Heredity $39,173-6(1948)$.

By applying a heat treatment immediately before or after x-radiation, it was possible to modify the biological effects of a given dose of irradiation on dormant seeds on barley. A heat treatment that was just short of killing the seeds, reduced by hall the frequency of chromatic bridges in meristematic cells of roots grown from treated seeds. This was true whether the heat treatment was applied before or after the irradiation. Such a heat treatment after $x-r$ adiation resulted in an increase in the seedling mutation rate from $5.8 \pm 0.6$ to $9.1 \pm 0.6 \%$; whereas the equivalent heat treatment applied just before the irradiation resulted in a lesser (not statistically significant) increase to $6.2+$ $0.6 \%$. There was not a close correlation between injury symptorns and the frequencies of chromatimic bridges or mutation rates resulting from the various treatments. (BA) 1946

Snell, George D. INDUCTION BY ROENTGEN RAYS OF HEREDITARY CHANGES IN MICE. Radiology 36, 18996(1941).

A review of previousily reported experiments on the induction of hereditary changes (especially translocations) by irradiation of males with either $\mathrm{X}-\mathrm{rays}$ or neutrons and of females with $\mathrm{x}$-rays. (BA)

1847

Sommermeyer, $\mathbb{K}$. RADIATION BIOLOGICAL DETER MINATION OF GENE NUMERS IN THE X CHROMOSOME OF DROSOPHILA, Z Z indukt. Abstamm. Vererbungsl. $79,240-4(1941)$.

The "saturation effect" of Timofeef-Ressovsky and Zimmer's neutron-induced lethal mutations is probably related to a greater size of those radiation-sensitive volumes pertaining to chromosome injury than of those pertaining to recessive lethal mutation; $D$. E. Lea's calculations of gene number are based on the untenable assumption that an ionization within a gene's padiation-sensitive volume would practically always induce a recessive lethal mutation. (BA)

1948

Sparrow, Arnold H. TOLERANCE OF TRADESCANTIA TO CONTINUOUS EXPOSURES TO GAMMA RADIATION FROM COBALT ${ }^{50}$. Genetics 35, 135(1950).
Plants of a clonal line of Tradescantia paludosa were exposed under field conditions to continuous gamma radiation (1.1 and $1.3 \mathrm{Mev}$ ) of approximately 122, 30.4, 7.5, 1.8, 0.41 and 0.084 r per day, for $8,16,32$ and 64 days. Dosage rates of 122 and $30.4 \mathrm{r}$ per day permitted little or no growth, while fairly normal growth occurred at $7.5 \mathrm{r}$ per day or less. Complete pollen abortion was produced after 16 days at $122 x$ per day. Chromosome breaks as such were not scored, but micronuclei at the microspore resting stage were counted. These are assumed to represent acentric fragments which lagged and remained in the cytoplasm at second telophase. A significant increase in the frequency of micronuclei was obtained with as low as 84 mr per day after 32 days exposure (total dose $2.69 \mathrm{~F}$ ). Dosage curves for various exposure times will be presented. An attempt will be made to relate growth-inhibiting and lethal doses to the percentage of microspores containing wicro-nuclei. (G)

1949

Spencer, W, P. and Curt Stern. EXPERIMENTS TO TEST THE VALIDITY OF THE LINEAR $r$ DOSE. MUTATION FREQUENCY RELATION IN DROSOPHILA AT LOW DOSAGE. Cenetics 33, 43-74(1948).

$200,801 \mathrm{x}$-chromosomes of mature sperm of a Canton Special stock of $D$. melanogaster were tested for the origin of new lethal mutations. Of these chromosomes, 73,901 were controls, 51,907 were subjected to $\mathrm{x}$-rays at a dosage of $25 \mathrm{r}$ units, 31,560 at $50 \mathrm{r}, 23,195$ at $150 \mathrm{r}, 6,634$ at $500 \mathrm{r}$, 6977 at $1000 \mathrm{r}$, and smaller numbers at $2000 \mathrm{r}$, $3000 \mathrm{r}$, and $4000 \mathrm{r}$. There were 72 control lethals found, 88 at $25 \mathrm{r}, 77$ at $50 \mathrm{r}, 74$ at $159 \mathrm{r}, 87$ at $500 \mathrm{r}$, and 147 at $1000 \mathrm{r}$. It is concluded that even for $x$-ray dosages as low as $25 \mathrm{r}$ and $50 \mathrm{r}$, the linear $\mathrm{r}$-dose/mutation frequency relation holds. The data showed a doubling of the control mutation frequency at an exposure of about $50 \mathrm{x}$. Several cases of control "Iethal clusters" were found and interpreted as mutation in early stages in the germ tract. The possible implications of the findings in connection with the exposure of large numbers of the human race to low dosages of $x$-rays are considered briefly. A parallel, but less extensive, study of visible mutation rates gave an over-all lethalvisible mutation ratio of 5.2:1. Linkage studies on 229 lethals and visibles showed these to be distributed over the length of the genetic map of the $x$-chromosome, with high frequencies near the scute and forked loci, and a low frequency near region 10. (Authors' Summary)

1950

Stadler, L. J. THE EFFECT OF X-RAYS UPON DOM-

INANT MUTATION IN MAIZE. Proc. Nat. Acad. Sci. U. S. 30, $123-8(1944)$.

In order to estimate the frequency of a dominant mutation in maize, a gene (a, colorless aleurone) was studied which rarely if ever shows spontaneous mutation but which mutates frequently in the presence of another (Dt). The frequency of losses of color and the number of cells involved were counted on other seeds of the same ears and provide a basis for estimation of the number of opportunities for occurrence of detectable mutation. X-rays failed to induce mutation of a to $\mathrm{A}$ (or to any other aleurone allele) in populations large enough to yield approximately 400,000 mutations to A under the influence of homozygous Dt or 900,000 losses of $A$ by deficiency or by mutation to a colorless allele. $(B A)$

\section{1}

Stadler, L. J. and Herschel Roman. THE GENETIC NATURE OF X-RAY AND ULTRAVIOLET INDUCED MUTATIONS AFFETING THE GENE A IN MAIZ. Genetics 28, 92(1943). 
The genetic nature of $\mathrm{X}$ - ray and ultraviolet induced mutations affecting the gene $A$ in maize. - Among a large number of alterations affecting $A$ induced by $X-1$ ay treatment, and a smaller number induced by ultraviolet treatment, those most nearly approaching the typical genetic behavior of gene mutations were selected for detailed study and comparison. With $\mathrm{X}$-rays, only a very small proportion of A-alterations are haplo-viable, and only two cases were found with normally developed pollen. The $X-$ ray mutants studied included these two $\left(a-X_{1}\right.$ and $\left.a-X_{2}\right)$ and a defective pollen type, $a-x_{3}$, which is haplo-viable in the female grametophyte. The ultraviolet mutants studied, all of which produce normal pollen, include $\mathrm{a}-\mathrm{U}_{1}, \mathrm{a}-\mathrm{U}_{2}$ and $\mathrm{a}-\mathrm{U}_{3}$, with phenotypic effects similar to a, and Alt, with phenotypic effects intermediate between $A$ and $a_{2}$ - All three $X$ ray mutants show lowered haplo-viability, as indicated by transmission of the mutant a (general designation a*) through $o$ and $q$ germ cells of $\mathrm{A} \mathrm{a}^{*}$ individuals. Transmission of $a-X_{1}$ is lowered thru $o$, gametophytes and normal thru $\% ; a-x_{2}$ much lowered thru $\sigma$, somewhat lowered thru 9 ; and $\mathrm{a}-\mathrm{X}_{3}$ lackng thru $o$, much lowered thru $q$. Both $\mathrm{a}-\mathrm{X}_{1}$ and $\mathrm{a}-\mathrm{X}_{2}$ are lethal when homozygous, and compounds of the $X$-ray mutants in all three possible combinations are lethal. Crossing over in $\mathrm{a}^{*}$ plants is approximately nor mal with $a-x_{1}$, lowered with $a-x_{2}$, and much lowered with a- $\mathbb{X}_{3}$. In all four ultraviolet mutants, $\sigma$ and $q$ transmission is normal, homozygotes and compounds are viable and are normal in development, and crossing over is normal. - By the use of an unstable duplication covering the $\mathbb{A}$ region, homozygotes and compounds of the various mutants were produced and sectors resulting from loss and alteration of the duplication segment studied. These studies show that all three $X$-ray mutants involve loss of the effects not only of $A$ but of additional genes affecting chlorophyll development and cell viability. All four ultraviolet mutants are free from these associated effects. (G)

1952

Stadler, L. J. and Herschel Roman. THE EFFECT OF X-RAYS UPON MUTATION OF THE GENE A IN MAIZE. Genetics 33, 273-303(1948).

In order to study the nature of the mutations at the $A$ locus induced by $\mathrm{z}-\mathrm{rays}$, selected $\mathbb{F}_{1}$ progenies produced by an aa $x A A$ cross after irradiation of the male parent just before pollination with a $1,000 \mathrm{r}$ dose were extensively studied for mutations, gametophyte viability, homozygote and compound viability, crossing-over effects, pachytene cytological changes, and effects noted from unstable duplications. From 415 A losses, two normals which were free of segregating pollen defects and one which showed segre gation of subnormal, but not aborted pollen, were selected for thorough studies on lethality, transmission, and crossing-over. The results are extensively reported and discussed. 18 references. (NSA)

1953

Stroman, Govan N. and Thomas H. Lewis. A STUDY OF GENETIC EFFECTS OF COSMIC RADIATION ON COTTON SERD. J. Heredity $42,210-13(1951)$.

Samples of seed from individual coton plants known to be heterozygous for certain seedling defects were exposed to high-altitude radiation in an experinental type $V-2$ rocket. An altitude of 90.5 miles was reached during $595.5-\mathrm{sec}$ flight. The exposed seed were grown through the second generation against controls from the same parent plants. Changes noted in the radiated material remain to be finally evaluated, and cannot as yet be designated as cosmic radiation effects. (NSA)
1954

Strómaes, istein. THE PRODUCTION OR DOMINANT LETHALS WITH X-RAYS IN AGED DROSOPHILA MELANOGASTER SPERM. Genetics 34, 462-74(1949).

Experiments to determine the effect of the age of the gametes at the time of $\bar{x}$-ray treatment on the percentage of dominant lethals induced in Drosophila melanogaster sperm are presented. The dosages were delivered at $200 \mathrm{kv}$ through $1 \mathrm{~mm}$ Al and 0.25 Cu filters at a distance of $31-41 \mathrm{~cm}$ and consisted of doses of 2,219-2,308 $\mathrm{x}$; the sperm was aged 1 23 days. It was found that the sensitivity to $\mathrm{X}$-rays increases with an increase in age; the age effect was also found when eggs were aged. No age effect was noted when the males were stored with the females; the age effect, moreover, was noted only in the sperms from early developing males. 15 references. (NSA)

1955

Strómnaes, бistein. X-RAY INDUCED LETHAL MUTATIONS IN SEVERAL STRAINS OF DROSOPHILA MILANOGASTER. Hereditas 37, 532-59(1951).

Males from 31 strains of Drosophila melanogaster were tested in regard to their sensitivity to the induction of dominant lethal mutations by $2300-\mathrm{r} \times$ rays. Genetic differences in the sensitivity to induction of dominant lethal mutations by $x$ rays were found to exist between unrelated strains. (NSA)

1956

Swanson, C.P. THE DIFTERENTIAL EFPECTS OF COMBINED RADITIONS ON CHROMOSOME BREARAGE AND MUTATION RATE. Science 107, 458(1948).

One of the important problems of cytogenetic s is the re. lationship between chromosome change and gene mutations. The solution of the problem is difficuls, particularly bince the usual mutagenic agents, such as s-rays and short ultraviolet light, also alter chromosome structure. A possible avenue of approach is through the use of additional agents which, while inefiective of themselves, can, when combined with s-rays, or ultraviolet, increase or decrease the rate of chromosome change without effecting mutation production, or vice versa. Infrared, when combined with 28 -rays, increases the rate of chromosone change (in Tradescanta) and the rate of morphological mutations (in Aspergillus). The chromosome effect is probably due to the infrared ai fecting the rate of recombination of broken ends of chromosomes but not the initial rate of chromosome breakage. It is likely that the increased rate of mutations is the result of additional chromosome changes having phenotypic effects, since infrared, when combined with ultraviolet $(2,537$ A) is ineffective in altering the mutation rate. Infrared, therefore, does not affect the stability of the gene. Ultraviolet, when combined with $\mathrm{x}$-rays, does not alter the s-rayinduced chromosome changes. This selectivity of radiations permits the separation of gene mutation from chromosome changes. The former result from initial absorptions of energy which are not affected by combined treatments, while the chromosome changes which depend upon initial breakage of chromosomer followed by recombination of broken ends, are influenced by addition agents which effect the recombination rather than the initial breakage phase of chromosome alteration. (NSA) $195 \%$

Swanson, C. P., Ale ander Hollaender, and B. N. Kauimann. MODIYICATION OF TEL X-RAY AND ULTRAVIOLET. INDUCED MUTATION RATE IN ASPRECILLUS TERRETS BT PRTTREATMLNT WTY NEAR INTRARED RADATOFT. Genetics 33 , 422-37(1948). 
To determine the effect on mutation rates of near-infresed ixradiation from $250 \mathrm{w}, 115 \mathrm{y}$ sources, fillered by $1.5 \mathrm{~cm}$ of jodine saturated $\mathrm{CCl}_{\mathrm{A}}$ and heat resistant glass filters, spore suspensions of Aspergilus terreus were continuously irradiated for three days after which they were $\mathrm{x}$ irradiated by two Coolidge-type tubes operating at $170 \mathrm{kv}$, with 0.25 Cu and $1.06 \mathrm{~mm}$ Al flters delivering $8,500 \mathrm{r} / \mathrm{min}$ for 1 to 20 minutes, or were ir radiated by ultraviolet light delivered at $15 \mathrm{w}, 115 \mathrm{w}$ with $200 \%$ radiation below $3,000 \mathbb{A}$. The per cent mutation rates for ultraviolet or x-irradiation alone are compared with rates for these combined with in ixared pretreatment. The dats and results are given and extensively discussed. The authors conclude that with $x-$ ray induced mutations infrared pretreatment causes a significanty increased mutation rat throughout the range tested, but that this does not effect the mutation induced by low ultraviolet doses although it does increase the mutation rate of high doses; an unknown mechanism is postulated whereby the mutated spores can utilize the infrared energy to recover and grow. (NSA)

1058

Tatum, L. L. FISCTS OF RADATON ON FUNGI. J. Cellular Comp. Physiol. Suppl. $1,35,119-31(1950)$.

The evidence so far suggests that in Neurospora, sensitivity to mutation is dependent on the inherent properties of the gene rather than on the nature of the mutagen, although specific action of particular mulagens will remain as a possible cause of certain inf requent mutant types unil more adequate data are obtained. The results of mutational treat ment of microconidia indicate that in Neurospora, the extranuclear determination of biochemical characters is gare, if not absent. This supports the current views on the over-all rarity of cytoplasmically inherited characters. Al present hopes for specific mutagenic activity seem poor, and what evidence there is seems to point only to the possibihity of quantilative, rather thas qualitative specificity in mutagenic activity of different agents in different organIsms. Tt would seom theoretically possible that genes may similarly differ in susceptibility to particular agents, and the discovery of mutagens with a high degree of genic gpecificity seems hopeful. (NSA)

1059

Taum, E. L.g. R. W. Barrat. Nils Iries; and David Bonner. BIOCHFMICAL MUTANT SLMANS OF NEUROSPORA PRODUCED BY PHYSICAL AND CMEMICAL TEEATMENT. An. J. Botany 37, 38-46(1950).

Plating techniques and cultures have bern developed which permit the efficient isolation of homocarytic strains of Neurospora cassa from unimucieate microconidia. With these techniques, x-radiation, ultraviolet radiation, bisand tri - mitrogen mustards, and methylcholanthuene hve been examined for their effects in producing biochemical mutants in Neurospora. Trestment with $x$ - rays was carried out by placing a drop of a dense suspension of microconidia on the surface of solidified $4 \%$ agax in a petri plate and allowing the water to be imbibed by the medium. The layer of microconidis remaining was treated directly with $-\mathrm{rays}$ following an exposure to about $20,000 \mathrm{r}$, about $0.01 \%$ of the organisms survived. Treatment of either microconidia or macroconidia with nitrogen mustards induces mutations which apoear quantitatively and qualitatively similar to those induced by ir radiation; the types of biochemically deficient mutants obtained are described. No indication has yet bern found for extranuclear control of biochemical deficiencies in Neurospora. (NSA)

1960

Thomas, Lyel $\mathrm{J}$. and Henry Quastler. PRELIMHARY REPONT OF -RAY EFTECTS ON THE NEMATODE RHADDTIS STRONGYLOTES. Science 112,356-7(1950).
The nonlethal effects of x-irradiation on the charactexs of adult individuals of a bisexual nematude, Rhabditis strongyloides, have been investigated. This worm appears to be different, in respect to observable changes and genetic response, from animals, plants, and bacteria used in conventional genetic studies. Exposures of 100 to $10,000 \times$ produced no visible physical changes in the worms, $20,000 \mathrm{r}$ applied to wet cultures caused the appearance of larvae with marked changes in the refractive index of the rhabditin granules of the intestine, and $40,000 r$ produced vacuolate, probably sterile reproductive systems, looped or spirallike intestines, variations in egg size and shell structure, and the appearance of "dauer" like worms. The same ef lects were obtained with dry cultures exposed to $40,000 \mathrm{r}$ as were obtained with 20,000-roir radiated wet cultures. These changes are shown to be genetic and to be perpetu1961 ated for generations in crowded mass cultures. (NSA)

Thompson, IF. F., J. Mackey, A. Gustafsson, and L. Ehrenberg. THE MUTAGENIC EFIECT OF RADIOPHOSPLORUS IN BARLEY. Hereditas 36, 220-3(1950).

The mutagenic effect of $\mathbb{P}^{32}$ on barley was studied by soaking seeds in water containing various quantities of radioactive phosphate ions and by subsequent measuring of the chlorophyl-mutation rates per spike progeny in the second generation. Radiophosphorus was found to be highly mutagenic, its mode of action lying between those of $x$-rays and neutrons and its mutation range being wides than that of $\mathrm{x}$-rays. Since $\mathrm{P}^{32}$ is easy to handle, it is expected to become very useful in mutation work and plant breeding. (NSA) 1962

Timoféev-Ressovski, N. W. SPONTANEOUS AND RADIATION INDUCED MUTABILITY IN GEOGRAPHICALLY DIFTERENT RACES OF DROSOPHILA MELANOGASTER.

None of elght stocks from widely scattered geographic regions deviated significantly from the average of the spontaneous mutation rate $(0.143 \%)$ nor from the average of the x-ray $(3,000 \mathrm{r})$ induced rate $(8.35 \%)$ for sex-linked mutations, as determined by the CIB-method. No conrelation was found between the spontareous and the $x-x a y$ indured rates. The normal allele at the white locus for a Russian and an American stock showed a difference on the border of statistical significance. If the diference is real in lies in the gtructural difference at the white locus and not in an influence of the rest of the genone. The extensive data show that stoch conditioned differences in the total mutation rate can be neither large nor frequent. (BA)

\section{3}

Uphoff, Delta E. and Curt Stern. THE GENETC EFFETS OF LOW INTENSITY IRIADIATION. Science 109, 609-10 (1949).

In contrast to previous findings no significant difference in mutation rates in the sperm of Drosophila melanogaster was obtained between controls and experimentals which had been subjected to a dose of $52.5 \mathrm{r}$ in gamma rays administered continuously for 21 days at a rate of $2.5 x$ per day. This unexpected result required further tests. After consideration of various factors, the following were regarded as possible causes for the apparent inartivity of ir radiation in the experiment: low sensitivity to irradiation of aged sperm, dependence of induced mutation frequency at low dosages on a time factor, and errors of sampling which might have obscured a true difference between control and experimental rates. Three new tests which are described gave an increased frecuency of mutations in the treated sperm as opposed to the controls. Viewing all experiments together, it appeared that irradiation at low dosages, administered at low intensity induces mutations in Drosophilat sperm. (NSA) 
1964

Wagner, Carroll E. and G. W. Beadle. MUTATIONS PRODUCED BY ATOMC BOMB IRRADIATION OF NEUROSPORA CRASSA; APPENDIX NO. 16. Naval Medical Research Institute, Bethesda and California Institute of Technology. Max. 7, 1949. 8p. (NP-1904)

Cighty biochemical mutants observed in red bread mold, Neurospora crussa, exposed to Tests Able and Baker have been classified. Of these, 32 are classified as unknowns, meaning that these mutants require for growth, compound not yet ddentified. No attempt was made to classify the various morphological mutants found. The numbers of recovered mutants are so small that no attempt has been made to correlate them with dosages measured by physical means at the various stations. (NSA)

\section{5}

Wallace, Bruce. AUTOSOMAL LETHALS IN EXPERIMENTAL POPULATIONS OF DROSOPMILA MELANOGASTER. Evolution 4 172-4(1950) (AECU-1716)

Preliminary work indicating that a large proportion of induced lethals in the second chromosome of $D$. melanogaster are incompletely recessive is reported. Three experimental populations of $\mathrm{D}$. melanogaster were analyzed for lethal second chromosomes. The original flies of two of these populations were treated with $x$ rays. Frequencies of lethals in experimental populations is presented in tabular form. Data indicate that less than one-half of the original induced lethals persist through six generations. (NSA) 1966

Wallace, Bruce. MUTATIONS INDUCED BY CMRONIC RADLATON. Ceneties $35,398(1950)$.

Males and females of the ore- $R$ strain of $D$. melanogaster have been subjected to chronic irradiation in a number of experiments. (1) Chronic $x$-ray treatment of approximately 108 per day (7min per (reatment) for $5,10,15,20$, and 25 days after deposition of eggs gave, in males, $1.21+$ $0.22 \%, 3.99+0.59 \%, 4.60 \pm 0.42 \%, 6.14 \pm 0.66 \%$, and 5.20 .6 $1.23 \%$ sex-linked lethals. The control rate was $0.19 \pm 0.10 \%$. The proportion of recoverable lethals increased linearly with dose for 20 days; there was no inerease after that time. (2) Males and femaler carrying lethal-iree second chromosones were exposed contimouly to $\gamma$ rays lor 18 days $(\sim 2200 \mathrm{x})$ at $25^{\circ} \mathrm{C}$ while they developed from eggs to adults. In all, 3772 second chromosomes 1 rom these treated males were tested for lethals; $456(12.1 \pm 0.5 \%)$ were recovered. No attempt was made to test for clusters. Likewise, 1187 second chromosomes from treated females were also treated; $69(5.8 \pm 0.7 \%)$ lethals were oblaned. The difference between the frequencies of recoverable lethals in the two sexres is significant. Preliminary tegts for translocations coincident with these lethals indicate that they occur with a low irequency; no tranglocations were coincident with the 69 lethals derived from lemales, 3 were present among 99 independent, "randomy" chosen lethals from ireated males. A test for allelism made with 100 lethals of independent origim (obtained rom diferent males) gave 14 cases of allelism in 4950 tests. A minimum of 400 (23418) loci on the gecond chromosome are capable of mutating to lethality under these conditions. (G)

\section{7}

Wallace, Bruce. GENETIC CAANGES WTITH POPULATONS ATIER X IRRADIATON. Cenetics 3B, 612-28 (1951) (ALCU-1107)

Genetic analyses have been made of populations of Drosophila melanogaster exposed to single s-may treatments. The parental males of population 1 received $7125 \mathrm{~F}$ females of population 1 and males and females of population 2 recelved $1012.5 \mathbb{F}_{0}^{\circ}$ the llies of population 3 were untreated.
Before treatment, all flies carried second chromosomes that were free of lethals and drastic subvitals. The recessive lethals of population 1 decreased from an original 18.3 to $10.1 \%$ in approximately four generations and then increased at a rate comparable to that of the control. The early elimination of lethals from population 1 indicated selective disadvantage of heterozygotes of 0.5 , and was adequately explained by lethal-translocation association. Analyses of lethals, semilethals, average viabilitis of 1 lies homotygoug for " "normal" chromosomes, and variances of the array of "normal" viabilities indicate that changes occurred at a more rapid rate in population 1 than in the control. The discussion touches briefly on general problems of ir adiated populations. (NSA)

1968

Wallace, Bruce and J. C. Hing. GFNETC CHANGES IN POPULATION UNDER IRRADIATION. Am. Naturallst 85 , 209-22(1951) (ABCU-1442)

A general account is given of studies made on experimental populations of Drosophila melanogaster which are exposed to radiations. A consideration of the irequencles of lethals, semilethals, and aubvitals within the populations and of the estimates of the adaptive values of the populations suggests that heterotic gene comblmations have been developed within some of these populations. The bearing these results have on estimation of radiation damage regulting from gene mutation is brtefly mentioned. (NSA)

\section{9}

Whiting Anna R. IMMATERNATE MALES AND HTGM VISIBLE MUTATION RATE FROR ECGS IRRADITIDD IN PROPHASE 1. Genelles 31, 235-6(1046).

Unmated wild type females of Habrobracon'were heavily X-rayed and mated to recessive unireated males. Surviving progeny (about 10 percent for 28,000 r) were from egga 1 irradiated in prophase I. They consisted of the expected whid type, haploid impaternate males and diploid biparental heterozygous females and, in addition, recessive haploid immaternate (and rogenetic) males. The last resembled thel fathers in every respect, vere fully fertile and mosm mosaic. Percentages of androgenesim (immaternate do $x$ $100 /$ mmaternate $\left.0^{\circ}+98\right)$ were 421 (15/356) for 28,000 : $23.06(3 / 13)$ (corrected 13.30) for 29,300 I and 12.50 (1/3) for $42,000 \mathrm{n}$. Correction is applied to second group because crosses made were two-allele and therefore 50 percent of zygotes should be diploid males and inviable. Production of normal individuals irom untreated sperm in heavily Irradiated cytoplasm strengthens wiews that $\mathrm{X}-\mathrm{ray}$ injury is directly chromosomal. The heteroxygons females were bred unmated. 232 cested from 28,000 x treatment produced meven or more sons. Among these, heterozygosis for vislble mutations was 12.69 percent. Tests of 10ur from 29,300 r. yielded no mutations and four from $42,000 \mathrm{I}$ ylelded one. There results suggest possiblity of obtaining readiy doae action curves for visibles for comparison whth those already avallable for lethals in this materal. (G) 1870

Whimang AnR R. MOTHERE ATED EGGS. Science 103, 210-20(1048).

Unmated females of an inbred wilo type stod of rabrobracon were heavily x-rayed, then mated to untreated names with recegsive mutant traits. The progeny included among the expected wild type males and temales, a few males mormal in structure and behavior and with recesaive mutant traits. Breeding tests shower that thet gonads were almo paternal in origin. Their mormal fertility was proot of thelp haploldy. (BA)

1971

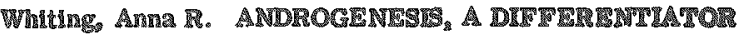


OF CYTOPLASMIC INJURY INDUCED BY X-RAYS IN HABROBRACON EGGS. Biol. Bull. 97, 210-20(1949).

Wild type parasitic wasp females, Habrobracon juglandis, were $x$-rayed and mated to untreated males with one or more types recessive to the wild type. Two kinds of haploid males were produced, the gynogenetic with $x$-rayed chromosomes in $x-$ rayed cytoplasm, and the androgenetic with untreated chromosomes in $x$-rayed cytoplasm. A simultaneous cross-firing technique was used to deliver the radiations at a $\mathrm{kv}$ of 182 with a filtering value of $0.2 \mathrm{Cu}$ at an intensity of $7,210 \mathrm{r} / \mathrm{min}$ and a target distance of $9.5 \mathrm{~cm}$. Doses were 50-144,200 $\mathrm{r}$ and were fractionated in some cases. It was found that at doses of 50-15,000 r death of the eggs was due to chromosomal injury, and was not reduced by fractionation or changes in intensity. Above $15,000 \mathrm{rg}$, s-rays induced chromosomal injury which may have exerted 2 lethal effect on developing embryos; the lethal effect was reduced by fractionation of the dose. Gynogenetic males developed from the unfertilized eggs, androgenetic males from the fertilized eggs; the lethal dose for the gynogenetic males was about $2,400 \mathrm{r}$ and about $54,000 \mathrm{r}$ for the androgenetic males. It was determined that injured cytoplasm acts in a direct manner in killing the embryo and not indirectly through injury to the untreated chromosomes. The author concludes that the evidence supports the target 1072 theory of chromosomal injury. (NSA)

Whiting, Anna R. TRE NON-INDUCTION OF MUTATIONS BY X-RAYED CYTOPLASM. Genetics 35, 139-40(1950).

Uniertilized Habrobracon eggs develop into haploid gynogenetic males. Of several thousand irradiated in first. meiotic metaphase, none have hatched after any dose higher than 2,500 $r$. Cytological observations demonstrate a consistent pattern of ehromosome injury. When severe, this injury retards movement of egg pronucleus after which the $\mathrm{egg}$, fertilized by an untreated sperm, may develop into a haploid androgenetic male. cggs are fertilized between one and seven hours alter exposure. From $6,714 \mathrm{x}-\mathrm{rayed}$ un mated wild type females crossed to untreated recessive males, 170 recessive androgenetic males have been obtained. These developed in egg cytoplasm exposed to doses ranging Irom 1,350 to $65,000 \mathrm{r}$, the latter dose about twentyfive times as great as that fatal to egg chromosomes in this stage. No mutations appeared in any of these males and of thirty-two tested, all were fully fertile and transmitted only paternal straits. Of 2,389 half-sisterg of androgenetic males developed from eggs $\mathrm{X}$-rayed in firgt meiotic prophase and fertilized by untreated sperm, 129 or $5.39 \%$ produced mutant sons. This differs significantly from the results obtained on androgenetic males. Evidence supports the theory that $x-$ rays induce visible mutations directly in the chromosomes. (G)

\section{9}

Whiting, Anna R. and Bernice Goldis. DOSE-ACTION CURVES FOR X-RAT-INDUCED DOMINANT LETHAL AND VISIBLE MUTATIONS IN HABROBRACON EGGS. Cenetics 32, 112(1947).

Data are being collected for visible mutation rate of eggs $X$-rayed in prophase 1 with doses ranging from $6,400 \times$ to 37,200 s. The dose-action curve suggests a direct relation to dose, thereby agreeing with work of previous investigators on this subject. Percentages of surviving eggs producing visibles range from 3.7 for $6,400 \mathrm{r}$ to 15.6 for 37,200 1. This curve is compared with that of dominant lethality previously published by senior author for prophase I. The latter is complex and tends to become exponential with iractionation of dose which increases resistance to treatment. (G)

\section{4}

Whittinghill, Maurice. SOME EFFECTS OR GAMMA RAYS UPON RECOMBINATION FREQUENCIES AND ON CROSSING OVER IN DROSOPHILA MELANOGASTER. Genetics 35, 140-1(1950).

In testcrosses of heterozyguus $\mathrm{eu} / \mathrm{ruh}$ th $\mathrm{st}+\mathrm{sr}^{\mathrm{s}} \mathrm{ca}$ females irradiation by $4,000 \mathrm{r}$ of gamma rays increased recombinations in the spindle attachment region to $2 \frac{1}{3}$ times the control value. Other increases of successively smaller magnitudes relative to control values were found in the flanking regions grading down to terminal decreases. The coincidence curve for all treated testcrosses showed a similar regular shape when the absulute changes were divided by the possible increases. Coincidences were higher in all regions in treated material. The se increases also graded up to a peak at the spindle region. Both these curves are qualitatively predictable from the hypothesis that irradiation induced a rather small amount of genetic crossing over in oogonial cells. There was a considerable variation between females in the amount of response and also in the place of response to irradiation, resembling previous results from treated males. 4,120-r gamma-ray treatment of S L/Cy Pid females produced "clusters" of crossovers, of appreciable sige in some families. A similar treatment of males produced crossovers also in a nonrandom manner appropriate to spermatogonial crussing over. The implications to the collection of data for standard maps are considered. (G)

1975

Whitinghill, Maurice. SOME EFECTS OF GAMMA RAYS ON RECOMBINATION AND ON CROSSING OVER IN DROSOPHILA MELANOGASTER. Genetics 36, 352-5(1951)。

$\gamma-r a y$ treatment of $4000 \mathrm{r}$ given to adult Drosophila melanogaster females altered crossover values in regular pattern in rucuca heterozygotes. Increases were greatest, relative to controls, in the spindle-attachment region in the middle of the third chromosome, progressively less in each successive region farther out, and negative in the two most distal regions. Coincidence of double crossover combinations was increased relative to control coincidence values in at similar curve but without distal decreases. Great individual variation in crossover production after $\gamma$-ray treatment was shown by regular females, by inversion females, and by regular males. The changes might have originated in meiotic or in gonial cells. The distribution of crossovers among families from -ray-treated Curly Lobe inversion heterozygotes had a probability of 0.001 of resulting from meiotic crossing over. It has been attributed to oögonial crossing over in these females. The $4000-r$ treatment induced crossovers in 14 of 59 treated males in an even greater nonrandom distribution which was due to spermatogonial crossing over. A new crossover-selector system of testing was employed here. Expression in ratio form of the regionally different cfects of starvation of larvae upon crossover production shows a maximum increase in the spindle region and other graded increases as found here using $\gamma$ rays. During three different 1 -hr periods of the day irradiation of adult rucuca heterozygotes seemed to be equally effective in producing changed recombination values. (NGS)

1976

Wijsman, R.A. ON THE INTERPRETATION OF THE DOSE FREQUENCY CURVE IN RADIOGENETICS, Geneties $38,478-87(1951)$.

The question whether a linear relationship between mutation frequency and radiation dose is a strong indication that a mutation is caused by a single event has been investigated in several cases, and answered in the affirmative. 
The results of the ealculations, supporting this conclusion, are shown in graphs, in which the curves for multiple-event models are conpared with the curve for a single mevent model. An exception has to be made for the possibility that some events may occur with much higher probability than any of the others in which case a multiple-event model could give rise to a singlemevent type curve. However, this case 1s physically unlikely. Tinally it is shown that Opatawski's curve, which is supposed to represent a many-event mecha nism, actually represents a lone-event mechanism, so that his conclusion that a many-event theory can give rise to a linear curve is unjustificed, (auth)

1977

Witkin, Evelyn M. A CASE OF INHERITED RESISTANCE TO RADIATION IN BACTERIA. Genetics 31, 236(1946).

When a sample of Strain $B$ of IEcherichia coli is exposed to ult re-violet radiation, the last survivors prove to be relatively resistant to both uitra-violet and $\mathrm{X}$-rays. The resistance is stable and heritable. - Survival curves for the radiation-resistant strain at various doses of ultra-violet differ markedly in shape from similar curves with $X$-rays. Survival curves for the normal strain with $\mathrm{X}$-rays and ultra-violet are identical in shape. - The lag phase of the resistant gtrain in broth is only one-hall the length of the normal lag phase. This apparent selective advantage disappears, however, when the two strains are mised and in cubated together for some time-the lag phase of the resistant strain becomes the same as that of the normal strain.-Macroscopic examination of ultra-violet irradiated cells of the normal and resistant strains reveals striking differences. At low doses, normal cells elongate for many hours without dividing while resistant cells begin to divide soon after treatment. This difference is the basis of a technique for the accurate determination of the number of resistant cells in a sample, without which genetic analysis would be very difficult. - Resistance to zadiation is in this case a mutation arising spontaneously in the bacterial culture, independently of treatment with radiation. "This was demongtrated by following directly the increase with time of the proportion of resistants in a nomal culture. (RG)

1978

Witkin, E.M. GENETICS OF RESISTANCE TO RADIATION IN ESCHEHICHIA COLI. Genetics 32, 221-47(1947).

Strain $B$ of 1 . coll gives rise to a variant which is characterized by relative resistance to the lethal effects of both ult raviolet radiation and 8 -rays. This character is stable and heritable. Some additional properties of radiation resistant, strain, $B / r$, derived from the parent strain by culturing a last survivor of an irradiation with ultraviolet, are desc ribed. This strain exhibits relative resistance to the division-inhibiting effect of radiation, as well as to it lethal effect, and is also relatively resistant to the lethal and division-inhibiting action of penicillin and sodium sulfathiazole. Strain $\mathrm{B} / \mathrm{r}$ exhibits a shorter lag phase in broth at $37^{\circ} \mathrm{C}$ than the parent strain, and a somewhat lower final tit re. Survival curves of strains $B$ and $B / Y$ with ultraviolet radiation and $\mathrm{x}$-rays are compared. A technique is described whereby radiation resistant mutants can be detected quantitatively in samples from the parent strain. This technique designated as the double-irradiation treatment, provides an absolute selective procedure for the detection of the variant. Registance to radiation is shown to be due to a spontaneous mutation, occurring in cultures of atrain $B$ at a rate of about $10^{-6}$ mutations per bacteri um per generation. The rate of occurrence of the mutation to radation-resist ance can be increased considerably by treatment of the parent strain with high doses of ultraviolet radiation. At least 4 independeni nutations are demonstrated, each of which results in resistance to radiation, but distinguishable by the presence or absence of associated resistance to penicillin, or sodium sulfathiazole or both. The mutation to radiation-resistance, when it is accompanied by reaistance to penicillin, act as a modifier of an independent mutation to penicillin-resistance, adding quantitats vely to its effect. Some preliminary experiments in population dynamics are deseribed. When atrains $B$ and $B / r$ are artifically mixed and carried by daily subculture neither strain is at a selective disadvantage. When the mixtures are aged without subculture, however, the mutant strain is at a distinct selective disadvantage approaching complete lethality. Interpretations are offered for certain features of the results. (Author'B Summary)

1979

Wright, SEwall. DISCUSSION ON POPULATION GENETICS AND RADLATION. J. Cellular Comp. Physiol. Suppl. 1, $35,187-205$ (1950).

A strong possibility exigts that cumulative doses of the order of 300 r may have important effects on the ofigpring and descendants of those affected and that doses as small as $30 \mathrm{r}$ may not be negligible. There is little or no threat to the persistance of a population as a whole from the cause. However, there are such enormous gaps in the knowledge of the subject that no judgements of the genetic consequences 1980 of radiation in man can be taken very seriously. (NSA)

Zimmer, I. G. and N. W. Timoféev-Ressovgkil. DOSIMETRIC AND RADIATION BIOLOGICAL FXPERIMISTS WITH $\mathrm{AST}$ NEUTRONS. Strahlentherapie 63, 528-36 (1038).

The authors studied the effect of fast neutrons on muthtions produced in the fruithy. There was a definite increase in the rate following exposure to fast neutrons which was directly proportional to the dose. The effect per dose unt or per pair of ions produced in tissues was less than that of roentgen or $\gamma$ rays. The experimental results were in good agreement with those calculated according to the tar get theory. (BA)

\section{CVTOLOGY}

1981

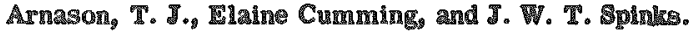
CHROMOSOME BREAKAGI INDUCED BI ABSORBED

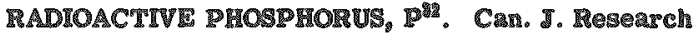
26, 109-1A(1848)

Radioactive phosphorus $\left(\mathbb{P}^{32}\right)$ was made avallable to individual germinating seeds and seeding of 'Triticum vulgare, T. durum, T. monococcum, and Hordeum digtichon. Cach treated plant was provided whe olther 0.18 or 0.018 of $\mathrm{g}^{92}$. Both concentrations were effective in causing chromosome breakage and rearrangements. Many aberrant chromosome configurations occurred in microsporocytes of treated tetraploid and hesaplold glants. Only one chromosome aberwation was found in T. monococcum and none in barley. Structural changes occurzed also in chronosomes of vulgas wheat rrown in soll to which fertilizer contalning $\mathrm{P}^{32}$ was added. It is possible that the effective ness of small amounts of $\mathrm{p}^{\text {s2 }}$ in induclng mutations is ingcreased by its inclusion in chromosome molecules. Emiteo ted $\beta$ particles would then be shot of very close to the senstive target. Further, the recollng atomic mucleus in almost ceriain to have enough energy to break any chemical bond. In any case since there is a change In atomic numbes (from 15 to 16) and in valence (P valence 5, 5 valence 2 ), molecular bonds must be released in the process. (NSA) 
1982

Beal, J. M. SOME HISTOLOGICAL EFIECTS OF CARBON 14 ON THE LISAVES OF CERTAIN MEDICINAL PLANTS. Botan. Gaz. 110, $800-3(1949)$.

A cytological description is given of the leaves of Digitalis purpurea, Digitalis lanata, Papaver somniferum, Nicotians rustica, and Atropa belladonna which had been grown in a $\mathrm{C}^{\mathrm{IA}} \mathrm{O}_{2}$ atmosphere for various periods of tirme. The atmospheres contained 400,200 , and 1,400 pe of the $\mathrm{C}^{16} \mathrm{O}_{3}$ in the various experiments; the author's conclude on the basis of these observations that the $\mathbb{C}^{14}$ does not effect the protoplasm with which it comes in contact, but that the differences noted are all alterations in the pattern of tissue differentiation. (NSA)

1283

Beal, J. M. and N. I. Scully. CHROMOSOMAL ABERRATIONS IN ONION ROOTS FROM PLANTS GROWN IN AN ATMOSPHERE CONTANING C ${ }^{14} \mathrm{O}_{2}$. Botan Gaz. 112. 232-5 (1950).

The roots showed both chromosome and chromatid breaks and fragments in dividing cells, bridging, and micronucleí. (CA)

1984

Bishop, Charles J. DIFPRENTIAL X-RAY SENSITIVITY OF TRADESCANTLA CMROMOSOMES DURING TRE MTOTIC CICLE. Genetics 35, 175-87(1950).

The results of an investigation to determine the stage and range of sensitivity in the mitotic divisions of pollen grains of Tradescantia are described. A drect analysis of chromosome alterations was performed. X-radiations were delivered at $160 \mathrm{kv}$ through inherent filtration at a Iarget distance of about $65 \mathrm{~cm}$, the dosage used was $250 \mathrm{z}$. Both betore and after $\mathrm{x}-\mathrm{ray}$ treatment the plants were grept in the greenhouse, remaining there until the buds to be analyzed were opened. The $\mathrm{x}$-ray effects were analyzed at the metaphase stage of the pollen division from slides prepared over a period extending from the second to the seventh day after treatment. The data obtained indicate there was a period of low sensidivity during the postmitotic resting stage, and two points of high sengitivity, one at the time of chromosome doubling during the prophase of the pollen tube division and the other at metaphase-anaphase of the polien grain division, the latter being the greater of the two. It is ruggested that the prophase peak of chromosome sensitivity to $\mathrm{x}$-ray brealage occur red about 3 days after actual chromosone doubling. It is also suggested that the metaphase-anaphase peak of sensitivity was due to the prevention of restitution by (a) factors of longitudinal tension induced with the chromonema by changes during the spiralization cycle and later by the spindle mechanism, and (b) the chromosome matrix which reduced iragment movernent after breakage and separation. X-ray breaks indiced during the stages before the prophase of the generative nucleus division were shown to remain open and to be capable of sister chromatid reunion when the chromosome became double, which was as long as 2 days after the x-ray treatment. 24 references. (NSA)

\section{5}

BIshop, Charles I. IHE INT LUENCL OF POLYPLOTY ON THE II-RAY SISMSITIVITY OF CELLS. Cenetics 35 ,

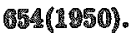

The report is in abstract form; it is reproduced here in its entirety.

A study of comparative sensitivity to $\mathrm{x}$ radiation has been made by treating developing microspores and pollen grains of diploid and tetraploid species of Tradescantia with equal doses of 8 rays. Treatments were given at a time just previous to the prophase of the pollen grain divi- sion and at a stage before chromosome doubling in the generative nucleus division. The aberrations produced were analyzed at the pollen grain division and the pollen tube division, respectively. Results indicate that the sensitivity of the two types of cells is the same if the comparison is based on the number of $x$-ray-induced breaks per chromosome. A comparison of the number of breaks per cell shows that the cells from the tetraplod plants have approximately double the number of breaks found in those from the diploid plants. (NSA)

1006

Bishop, Charles J., Victor D. McLaughlin, and Donald

F. Tapley. X-RAX TOLERANCE OF LIVING CELLS

AS MPASURED BY CYTOPLASMLC STREAMING. CAN.

J. Research 27, 262-8(1949).

An investigation was undertaken to determine the immediate effects of $x$-radiations on living, non-dividing cells, using the rate of cyloplasmic streaming as an indication of cell vitality. The observations were carried out on the stamen hairs and pollen tubes of Tradescantia paludosa, a diploid species, and on Tradescantia virginiana, a tetraploid species. In each experiment cultures were checked for rates of streaming and then were subjected to large, uninter rupted dosages of $250,000-450,000 x$ for the stamen halrs and 100,000-125,000 $x$ for the pollen tubes: subsequent dosages of 25,000 were given until cytoplasmic streaming ceased. The radiations were administered by a standard Coolidge $x-$ ray tube having a filtration of $1 \mathrm{~mm}$ Al and operated at $140 \mathrm{kv}$ with a beam intensity of 1200 r/min. In both digloid and tetraploid stamen hairs, 700,000 $r$ was recquired to stop all streaming, while in the pollen tubes of diploid and tetraplodd plants, streaming was stopped by $250,000 \mathrm{r}$ and $200,000 \mathrm{r}$, respectively; greater sensitivity was found with an increased initial dosage of continuous irradiation. 12 references. (NSA)

1987

Bishop, D. WV. CYTOLOGICAL DEMONSTRATIONS OF CMROMOSOME BREARDOWN SOON ATTER XIRAOIATION. Genetics 27, 132(1042).

"This paper is reproduced here in its entirety.

First spermatocyte prophase stages of Orthoptera are used to demonstrate $x-$ ray induced chromosome breaks. Three minutes after irradiation breaks in one of the four diakinetic chromatids can be demonstrated (Chortophaga viridifasciata treated with $540 \mathrm{r}$ ). In Melanoplus bivitalus, 17xed sis minutes after irradiation (270 r) many cases of breaks are found in one of the two visible threads of pachytene chromosomes and in one of the chromatids of diaknetic chromosomes. Within this time interval no cases of simultaneous breakage of two visible threads occurs. Howewer, within 30 minutes $(270 \mathrm{r})$ two-thread as well as one-thread breaks occur in diakinetic chronosomes. A few double breaks were found within 8 minutes at 540 g. These results are evidence for an almost immediate effect of the ixrradiation in contrast to a delayed effect. However, the breakage raction seems to be a gradual rather than an instantaneous event, judging from the longer time required for two-thread breaks at the same dosage. Chromosome fragments are found in spermatogonial and spermatocyte anaphases within 30 minutes after irradiation. The point of breakage in prophase chromosomes is at random. In normal and in treated cells neither the pachy tenc nor the diakinetic chronosomes are in contact with one another; if the breakage mechanism is the same as that leading to inversions and translocations, the evidence supports the breakage-first as opposed to the contact hypothesîs. 
1988

Bishop, D. W. SENSITIVITY OF SPERMATOCYTE CHROMOSOMES TO ABERRATIONS INDUCED IN THE EMBRYOS BY X-RADLATION. Genetics 27, 132-3(1942).

Gonads were removed from adult and late nymphal grasshoppers (Circotettix verruculatus) which had been irradiated ( 150 to $750 \mathrm{x}$ ) as embryos stull within the chorion. In the first meiotic anaphase there are chromosome alterations, not found in any other mitotic stage, and not included among the usual cytogenetic changes. These aberrations affect one or both of the diad components of the tetrad, and include constrictions, attenuations, and breaks. They are invariably located between the point of spindle attachment and the nearest region of association of the arms of the homologs ("chiasm"). The second spermatocytes thereby produced frequently contain large duplications and deficiencres and are inviable. All of the cells within an affected cyst have been derived from a single treated cell by at least seven divisions. Yet no abnormalities in the prevous spexmatogonial, diakinetic, or first spermatocyte metaphase chromosomes predict the aberrations that will occur at meiotic anaphase. Com parasons with chromosome rearrangements involving reciprocal translocations (rimltuples), inversion-crossovers (dicentrics and acentrics), and small deletions, demonstrate that some other mechanism of chromosome alteration pertains. The distribution of aberrations is nonrandom, concerning the regions affected within particular chromosomes as well as different chromosomes within a cell. A large proportion of aberrant chromosomes show homologous breaks, symmel rically placed, in the two members of a tetrad. The conclusion is drawn that the X-ray effect is general and the chromosomes become fragmented at points of natural weakness. (G) 1989

Bozemann, Martha L. and Charles W. Metz. FURTMER STUDIES ON SENSITTVITY OF CHROMOSOMES TO IRRADIATION AT DIFFERENT MEIOTIC STAGES IN OOCYTIS OF SCIAPA. Genetics 34, 285-314(1949).

$X$-ray doses of $1,100 \mathrm{r}$ were used to determine the chromosome sensitivity of maturing oocytes of Sciara ocellaris to radiations. The results indicate that the chromosome sensitivity is almost zero for a long period of time preceding the breakdown of the nuclear membrane at the beginning of the first melotic division. Then it rises rapidly to a peak, in anaphase, apparently dropping off somewhat in the late anaphase, at which time the mitotic activity is arrested, pending fertilization. Different types of chromosome rearrangements differ greatly in actual frequency. Only one translocation was secured. The author points out that the data suggest the intervention of a cytoplasmic agent which reaches the chromosomes after breakdown of the nuclear membrane and which either induces rearrangements direclly, by transmitting an influence already produced by the irradiation, or sensitizes the chromosomes to irradiation so that treatment is effective after, but not before, the entrance of the agent. A third possibility is also considered. (NSA)

\section{0}

Brown, Meta S. CHROMOSOME IRREGULARITIES PRODUCED BY ATOMC IRRADITION. Genetics 33, $98(1948)$.

Acetocamine smears were made of the P.M.C. of eleven cotton plants grown from seed exposed to atomic bomb radiation at Bikini during "Operation Crossroads". In four plants quadrivalents were found at metaphase, one plant having two such multivalent associations per nucleus. Three plants were mosaic for normal and altered chromo- some constitution. In two of these a chromosome had been lost, producing monosomic tissue, and in the other a quadrivalent was present. In one plant two chromosomes were frequently found as univalents, or paired with a chiasma only. No chromosome abnormalities were detected in four plants, which had 26 pairs of chromosomes, as was true for plants grown from unexposed seed of the same lot. The types of meiotic irregularities found indicate that gamma ray irradiation had produced translocations, loss of chromosomes, and possibly an inversion in the embryonic tissue of the cotton seed. Morphologically no plants showed any deviation from normal, except one plant which was slightly fasciated at an early seedling stage. (NSA)

1901

Brown, Meta 5. COTTON FOM BUINI. CMPOMOSOME IRREGULARITISS FOUND IN PLANTS GROWN FIROM SRDD EXPOSED TO GAMMA RADHATION. J. Heredity 41, $115-21(1950)$.

Cytological analyses of cotton plants grown from seed exposed to an atomic bomb explosion have been made. Irregularities in chromosome pairing have been found and attributed to changes in chromosome structure. (NSA) 1922

Brues, Austin M. and Leola Rietz. EFFCTs OF EXTERNAL AND INTERNAI RADIATION ON CELL OIVI\$ION. Am. N. Y. Acad. SeI. 51, 1497-1507(1951).

The effects of radiation upon cell division were studied, using regenerating rat liver. After partial hepatectomy, the liver was exposed to $x$ radiation given in a single dose (10 to $3000 \mathrm{r}$ ), injected $\mathrm{Pu}^{238}$ and $\mathrm{P}^{32}$, and continuous exposure to a point source of $\beta$ rays, using small glass beads containing caxrier-free $Y^{B 1}$. The $Y^{01}$ bead experi ment was of most quantitative value in deternining the degree of suppression of mitosis. Reffects of ionizing radiations on mitosis were shown to include reversible delay in cell division, followed by visible chromosome damage and changes in the mitotic figure. The amount of p radiation required to reduce liver mitosis by one-hal was shown to be of the order of $1 \mathrm{rep} / \mathrm{hr}$ over a $48 \mathrm{~m}$ hr period. (NSA)

1993

Brunfleld, Robert T. FFECT OF COLCHICINE PRETREATMENT ON THE FREQUENCY OF CEROMOSOMAL ABERRATIONS INDUCED BY X-RADIATION. Proc. Nat1. Acad. Scl. v. \$. 29, 190-3(1343).

Treatment of onion roots for 45 minutes with $0.05 \%$ colchicine solution prior to roentgen irradiation, resulted in less than $1 / 3$ as many chromotid aberrations as found in non-colchicine-treated controls. (CA) 1994

Buchsbaum, Ralph and Raymond E. Zirkle. SRRIMENG AND SWELLING AFTER ALPHA IRPADLTION OF VARIOUS PARTS OF LARGE IRYTMROCYTIS. PYOC. Soc. Expt1. Biol. Med. 72, 27-9(1949).

Erythrocytes from Amphiuma and from human blood were irradiated in vitro with Po a-particles from an extended source (4 mm diameter) and also with a small beam $20 \mu$ in diameter. The cells first shrans, then swelled and hemolyzed. Local Irradiation of part of a single cell resulted in shrinkage only in the irradated portion. This was followed by swelling of the whole cell and hemolysis. (CA) 1995

Carlson, J.G. IMMEDLATE ERECTS OF 250 R OF X-IRAYS ON THE DIFTERENT STAGES OF MITOSIS IN NEUROBLASTS OF THE GRASSHOPPER, CHORTOPHA- 
GA VIRUIFASCLATA. J. Morphol. 66, 11-2\$(1940).

Two hours after $\mathrm{x}-$ radiation of neuroblasts with $250 \mathrm{r}$ all middle prophase through early telophase stages have disappeared. Statistical and observational evidence indicates that middle prophase is the stage most susceptible to the 8 - ray effect that causes cessation of mitosis; for after treatment some of the cells in this stage, instead of entering late prophase, revert to earlier stages or at least undergo changes in their chromatin that stimulate reversion. Late prophases and metaphases deerease steadily in numbers by passing into successively later staqes unti1 they have disappeared at the end of 75 minutes. Both anaphases and early telophases decrease in numbers by passing into successively later stages during the first 30 minutes, increase at 45 minutes, and then fall steadily, tho former reaching zero at 90 minutes, the latter at 120 minutes. The simultaneous fall in the numbers of late prophase and metaphase stages and the increase simultaneously in the numbers of anaphases and early telophases at 45 minutes inciicate that $x$-rays alter the rate of change from stage to stage in cells undergoing mitosis at the time of treatment and that this change in rate differs with the stage of mitosis and with the time elapsing after irradiation. (EA)

1996

Carlson, J. G. AN ANALYSIS OF X-RAY INDUCED SINGLE BREARS IN NEUROBLAST CHROMOSOMES OF THE GRASSHOPPER, CHORTOPHAGA VIRIOIFASCIATA. Proc. Natl. Acad. Sci. U. \$. 27, 42-7(1941).

The single chromosonal break is considered the result of a single event - a single ionization or excitation - which occurs at random. This hypothesis is supported by (1) the linear proportionality existing between dosage measured in $r$-units and number of chromosomal breals, (2) the apparent absence of any threshold below which breaks do not occur, (3) the close correspondence between the observed and expected distribution of the breaks among different cells after each of the doses. The analysis of single breaks in terms of ion pairs per chromosome indicates that only a small proportion of the ionizations produced by X-rays is effective in the production of breaks. (BA) 1907

Carlson, J.G. IMMEDIATE EFECTS OF $31 \mathrm{R}$ OF XRAYS ON THE DIFERENT STAGES OF MTOSIS IN MCROBLASTS OF CHORTOPHAGA. J. Morphol. ${ }_{1}$, $449-62(1942)$.

Treatment of neuroblasts of the grasshopper, C. viridifasciata with $31 \mathrm{r}$ of $\mathrm{x}$-rays at the rate of $200 \mathrm{r} / \mathrm{min}$. leads to alterations in the average number of middle prophases, late prophases, metaphases, anaphases, and early telophases per embryo. After a brief latent period mitotic cessation of cells in advanced middle prophase and initial late prophase with less or no effect on those in early prophase, metaphase, anaphase, and early telophase leads to the accumulation of large numbers of middle prophases and the disappearance of advanced late prophases, meta-. phases, anaphases, and early telophases. The simultaneous recovery of a large proportion of the middle prophase cells and their mitotic progress into successively later stages result subsequently in abnormally large numbers of late prophases, metaphases, anaphases, and early telophases. Certain evidence suggests that the mitotic progress of cells phases. Certain evidence suggests that the mitotic progress of cells may be altered mainly through effects of 1958 the $x$-rays on the prophase chromosomes. (BA)

Carlson, J. Gordon. EFECTS OF RADIATION ON MTOSIS. J. Cellular Comp. Physiol. 35, Suppl. 1,89101(1950) (ORNL-570)
The effects of $\mathrm{x}$ - and ultravolet radiations upon the mitosis of grasshopper embryo neuroblasts is discussed. The approach is primarily cytological and is concerned with how the individual cell is affected by radiation. The inhibition of mitosis by $x-i$ rradiation is noted, and the effects of total dosage, dosage rates, and the mitotic stage at which the $\mathrm{x}$-radiation as administered is pointed out. A certain "critical period" for inhubiting mitosis-late prophase - has been noted. The effects of uit raviolet radim ation are compared with $\mathrm{x}$-radration. Instead of a clearcut mitotic stoppage at a "critical period", cells throughout most of interphase and prophase may be retarded mitotically by relatively low dosages of ultraviolet radiation. The author concludes that although there is much information avalable on the manner in which various radiations afrect mitusis, the mechansm by which excitation and ionization of atoms withn the cell are transformed into large scale mitotic effects is not understood. He states that the effectiveness of very low ionizing radiation dosages in interfering with enzyme systems is highly suggastive. (NSA)

1999

Carlson, J. Gordon, Mary Louise Snyder, and Alexander Hollaender. MELATION OF GAMMA-RAY DOSAGE RATT TO MTOTIC EFFECT IN THE GRASSHOPPER NEUROBLAST. J. Cellular Comp. Physiol. 33, 365-72 (1949). (AECU-243; ORNL-348)

Eggs of the grasshopper, Chortophaga viridifasciata (De Geer), containing 14 -day embryos (at $26^{\circ} \mathrm{C}$ ) were exposed to known doses of $\gamma$ rays given at certain selected dosage rates and then made into hanging-drop culture preparations. 'Treatment was at room temperature, (about $22^{\circ} \mathrm{C}$ ). Treated and control embryos were prepared from the same pod of eggs. They were studied at $38+0.3^{\circ} \mathrm{C}$ in an incubator enclosing the lower portion of a microscope. A gamma-ray dose of 8,32 , or $64 \mathrm{r}$ produced the same deggee of mitotic mhibition, whether administered at a rate of $2 \mathrm{r}$ per minute or $32 \mathrm{r}$ per minute. A $\gamma$-ray dose of 128 or $256 \mathrm{r}$ was more effective in depressing mitosis if given at $32 \mathrm{r}$ per minute than at $2 \mathrm{r}$ per minute. This document is a revision and amplification of MDDC -1576 . (NSA)

\section{0}

Carothers, E. E. A CXTOLOGICAL STUDY OF X-RAYIED GRASSHOPPER EMBRYOS. J. Morphol. 66, 529-59 (1940).

Melanoplus differentialis embryos after receiving 250 or $300 \mathrm{r}$ in a single dose in early predispause, developed. The immediate effect of radiation is direct injury to the chromatin of cells actually undergoing mitosis at the time of treatment. This injury is of such a nature that these cells die in amphase with pycnotic muclei. Twenty-four hours later these nuclei are found scattered throughout the germband. Later such cells are either engulfed by ofher embryonic cells, absorbed in sifu or extruded from the embryo. Within 48 hours certain cells show liquefaction of the cytoplasm and within five days lesions due to cytolosis appear. This cytolosis may be due to direct injury to the cytoplasm by the x-rays or to a toxic effect from the phagocytized necrotic cells. Mitotic activity is increased perhaps due to liberation of intercellular hormones. Yolk cells phagocytize injured cells and remove them from the vicinity of the embryo. They are also capable of assuming the function of serosa cells and secreting impervious yellow cuticle thus walling off neorotic areas. There is some evidence that the chromosomes respond differentially to general injury of the organism. About the onset of diapause the nuelei of groups of cells become pycnotic due probably to lack of necessary cellular associations. The terata 
Seem to be due to the isolation of groups of healthy cells from their normal associations in the embryo. Many of the chromosonal complexes in such misplaced parts are normal. Finally the embryos die due to lack of organization rather than to absence of differentiation since all types of tissue may be present. (Author's Summary)

\section{1}

Catcheside, D. G. EFFECTS OF IONIZING RADIATIONG ON CHROMOSOMES Biol. Revs. Cambridge Phil. Soc. 20, 14-28(1245).

The information about the process of chromosome breakage and structural change in somatic chromusomes, especially of Tradescantia, is reviewed. The number of primary breaks induced at resting stage of prophase greatly exceeds the total number of breaks observed at the subsequent metaphase. The majority of the breaks restitute after being open for a few minutes, during which time the formation of exchanges is possible. The yield of $x-$ ray induced exchanges is proportional to the square of the dose if the radiation is given at a high intensity. The yield is proportional to a lower power of the dose if the dose is given at a lower intensity. Only breaks with an initial separation of $1 \mu$ or less have an appreciable chance of taking part in an exchange. In neutron and $\alpha$-ray experiments, the yield of exchanges is linearly proportional to dose and independent of intensity; thus the majority of exchanges are between pairs of breaks produced simultaneously by the same ionizing partiele. An ionizing particle may break both chromatids of a split chromosome at the same locus and an isochromatid break usually results. A proportion of the primary breaks are unjoinable; hence a proportion of interchanges are incomplete, a proportion of isoehromatid breals show non-union, and a proportion of breaks not taking part in exchanges persist as visible chromatid breaks instead of restituting. The number of primary chromatid breaks differs for equal doses of different radiations. A proton of $\alpha-$ ray traversing a chromatid has almost unit probability of causing a break. An electron is likely to cause a break only if the last $0.3 \mu$ of its ionized track traverses the chromatid; hence a minimum of $15-20$ ionizations must be produced in a chromatid of diameter $0.1 \mu$ for the breakage probability to approach unity. The relative efficiencies of different wavelengths and types of radiation can be explained on this basis. (BA)

\section{2}

Catcheside, D. G. and D. E. Lea. THE EFTECT OF IONIZATION DISTRIBUTION ON CHROMOSOME BREAKAGE BY X-RAYS. Genetics $45,186-96(1943)$.

Chromatid breakage is not equal in frequency for equal doses of $x$-rays of different wave-lengths, dosage being calculated as ionization in the tissue. The effect is a maximum at $\lambda=4.1 \mathrm{~A}$, falls of slowly with decreasing wave-length $(\lambda=1.5 \mathrm{~A}-\lambda=0.15 \mathrm{~A})$ and rapidly with increasing wave-length $(\lambda=8.3 A)$. This is interpreted as meaning that only the densely ionizing tails of the electron tracks are effective in breaking chromosomes and only then if they completely traverse the chromosome, since the $\mathbb{A} I-K$ radiation $(\lambda=8.3 \mathrm{~A})$, for which the electron track is shorter than a chromatid diameter, is relatively ineffective. The probability of survival of chromatid breaks is equal for pollen grain and pollen tube nuclei. Since isochromatid breaks are far less frequent $(27 \%)$ in the latter, sister-chromatids must be in contact 24 hours before metaphase of the pollen tube division. The probability of interchange in the elongated cylindrical pollen tube nucleus is greatly reduced (ca. $75 \%$ ) by the different packing of the chromosomes. (BA)
2003

Catcheside, D. G. and D. E. Lea. DOMINANT LETHALS AND CHROMOSOME BREAKS IN IRNG X-CHROMOSOMES OF DROSORHILA MELANOGASTER. J. Genetics $47,25-40(1.945)$.

Ring x-chromosomes treated with $4,000 \mathrm{r}$ units of $\mathrm{x}-$ rays were examined cytologically. Among 749 chromosomes, 23 inversions, 9 intercalations, 4 complex interchanges, and 3 deficiencies were found, but no case of a simple rod-shaped chromosome resulting from a break. There is therefore no gositive evidence from this experiment that broken chromosome ends can heal. The signnificance of the absence of healed breaks was estimated by deriving the number of non-restituted breaks in the $\mathrm{x}-$ chromosome from the observed frequencies of induced structural changes in rod and ring $x$ 's and the different $x$ ray-induced depressions of the sex ratio in rod and ring 8 -chromosome stocks. It was first coneluded that the chance of restitution of a break in the $\mathrm{X}$ was 0.74 , the chance of remaining open 0.26 ; then, that there woud be 16 primary breaks per 100 rod $\mathrm{X}^{2} \mathrm{~s}$ per $1,000 \mathrm{x}$, or T per 100 sperms per 1,000 r. "This estimate agreed well with those based on other considerations. It was finally concluded that 3.8 non-restituted breaks were produced per 100 ring $\mathrm{X}$ 's per $1,000 \mathrm{x}$, and therefore 105 in the 749 analyzed chromosomes. Since recovery of rod chromsome from a broken ring chromosome would require simultaneous healm ing greater than 0.17 per chromosome end is excluded by these data. A terminal inversion in chromosome 2 was found, and also a complicated interchange between ring and chromosome 3 in which a new end was grovided by the ring $\mathrm{X}$ and the right and of 3 became intercalary. This is held to provide no support for Muller's conception of the special character of the chromosome end, or talomere. (BA)

2004

Catcheside, D. G., D. E. Lea, and J. M. Thoday. TYPES OF CHROMOSOME STRUCTURAL CHANGE INDUCED BII THE IRRADIATION OF TRADESCANTIA MCROSPORES. J. Genetics $47,113-36(1946)$.

Tradescantia microspores were inradiated with 78 and with gamma-rays, using different doses, exposure times and temperatures, and fimed after 18-23 hours. Chromatid breaks, isochromatid breaks, intrachanges and interchanges were observed. Symmetrical and asymmetrical types of exchange occur with about equal frequency, sug gesting that there is no sort of polarization favoring the union of proximal with distal fragments against the union of proximal with proximal or distal with distal. There is a correlation between the facts that a proportion of chromatid breaks remain visible and do not restitute, that a proportion of interchanges are incomplete, and that sisterunion dose not occur at some isochromatid breaks. The determination of the proportions of interchanges which are incomplete and of isochromatid breaks which show nonunion enables an estimate to be made of the proportion (I) of breaks which are unjoinable, and which therefore remain visible as chromatid breaks. As well as exchanges between chromatid breaks (c/c), exchanges occur between chromatid and isochromatid breaks (i/c), and the relative proportions of these two types of exchange must be related to the numbers of chromatid and isochromatid breaks primarily produced. From this observation a second estâmate is obtained of the proportion of chromatid breabs primarily produced which remain visible at the time of fissation. The two estimates of 1 agree in giving values about 0.09 for $x$-rays. The number of chromatid breaks primarily produced is calculated to be 0.09 per nucleus per roentgen of 8 -rays. (From Authors' Summary) 
2005

Catcheside, D. G., D. E. Lea, and J.M. Thoday. THE PRODUCTION OF CHROMOSOME STRUCTURAL CHANGLS IN TRADESCANTIA MICROSPORES IN RELATION TO DOSAGE, INTENSITY AND TEMPERATURE. J. Genetics 47, 137-49(1946).

The yield of chromatid breaks is proportional to dose, independent of exposure time at a given dose, and only slightly dependent (between 1 and $30^{\circ}$ ) upon the temperature at which the irradiation is made. The yield of isochromatid breaks increases slightly more rapidly than the first power of the dose, possibly decreases slightly with increase of exposure time at a given dose, and diminishes markedly with increase of irradiation temperature (especially between 20 and $\left.30^{\circ}\right)$. Most of the isochromatid breaks are 1-hit, e.e. produced by a single ionizing particle which traverses both sister chromatids, but a sman proportion are 2-hit, e.e. produced by separate ionizing particles. The yield of interchanges produced by 8 -rays increases slightly less rapidly than the square of the dose when the doses are given in a constant time. The yield produced by a given dose diminishes with increase of exposure time at 1 and $20^{\circ}$. The yield diminishes with rise of temperature as with isochromatid breaks. Interchanges produced by $x$-rays are mainly 2 -hit, but a small proportion are $1-$ hit, and this proportion is relatively more important when long exposure times are used. The separation at the moment of formation of breaks which exchange is of the order of $1 \mu$. Most of the exchanges occur within a few minutes of irradiation, but a few take much longer. For the same dose in roentgens, $\alpha$-rays produce slightly fewer interchanges than do s-rays. (Authors' Summary)

2000

Conger, A. D. X-RAY SEMSITIVITY OF THE STAGES OF MUTOSHS. Am. J. Botany 34, 582(1947).

The X-ray sensitivity of the stages of mitosis was studied in onion root tip cells. The production of polyploid cells by colchicine treatment was used to distinguish cells which were in meta-anaphase stage from the cells in the other stages when $\mathrm{x}$-rayed; cells in meta-anaphase when $\mathrm{x}$-rayed were the first to progress to the $4 \mathbb{N}$ Metaphase, when chromosomal aberxations were analyzed. The relative sensitivity was determined by comparing the chromosonal aberration frequencies resulting from \&-raying at the different stages of mitosis. Aber ration frequency of cells rayed in resting stage was assigned a sensitivity value of 1 . Other stages compared with resting stage showed relative sengitivities of approximately 1.7 to 3.4 from early to late prophase, 11.8 in anaphase, and 37 times as great in metaphase. The high sensitivity of metaphase chromosomes is believed to be due to an inerease in the factors which have been found to operate in the other stages to increase aberration frequency, as stresses of movement and colling and spatial relationships, and possibly the additional sensitivity of the process of duplication of the gene string which may be operative at this stage only. The occurrence of single chromatid deletions in $4 \mathrm{~N}$ cells which were $\mathrm{x}$-rayed at diploid metaphase is evidence that the chromosome can be effectively four strand to $x$-rays at metaphase, or two $200 \%$ strand at anaphase. (GPR)

\section{Conger, Alan D. THE CWTOGENETIC EFFET OF} SONIC ENERGY ADPLIED SIMULTANEOUSLY WITH X-RAYS. Ceneties 33, 607(1048) (ORNL-101)

An experiment has been performed to test the influence of sonic energy on the yield of $\mathrm{x}$-ray induced chromosomal aberrations in a clone of Tradescantia paludosa. Chromogoral aberration frequency was determined at the micro- spore mitosis from aceto-carmine amears made four days after treatment. Inflorescences were immersed in water contained in a steel cup used for the sonic tratment. The bottom of the cup was a vibrating daphragm which transmitted the sonic energy to the plant buds via the water. The inst rument used was Raytheon MIE. Co. magnetustiction oscillator, run at 9,100 cycles/sec., and at a power of 30 watts. Two treatments were given to inflorescences inside the sonic cup: (a) $250 \mathrm{r}$-rays $(78 \mathrm{r} / \mathrm{min}$. as measured in air outside the cup, and (b) $250 \mathrm{x}$-rays (as above) plus sonic treatment begun with and continued for five minutes after the $x$-radiation ceased. The frequency of all types of chromosome aber rations was higher when the sonic reatment was given in addition to the $x$ rays. The ratio of $\mathrm{x}$-ray plus sonic energy/ $\mathrm{x}-\mathrm{ray}$ abe $\mathrm{r}-$ rations was $1.3 / 1$ for both exchanges and simple deletions. The sonic treatment alone, as given here, did not cause aberrations. This increased yield of abexrations is probably due to an increased amount of chromosome movement caused by the luansmission of sonic snergy through the calls during and after the $\mathrm{x}$-radiation; movement would separate the broken ends from a single chromosome break (reducing restitution) and bring together broken ends from different breaks (increasing new reunions) - both would increase aberration frequency. (ACD)

\section{8}

Conger, Alan D. THE CYTOGENETC FFIECT OF SONIC ENERGV APPLIED SIMULTANEOUSLI WITM X-RAYS. Proc. Natl. Acad. Sei. 34, 470-4(1948).

The cytogenetic effects of simultaneous treatment of Tradescantia inflorescences with $x-$ rays (250 r) and sonce energy (9100 cycles/second) are described. Simultaneous treatment with sontc energy and $\mathrm{x}-\mathrm{rays}$ increases the yidd of x-ray induced chromosomal aberration about 1.3 times the yield obtained with the same amount of $\mathrm{x}-\mathrm{rays}$ alone. The sonic treatment alone did not cause aber $x^{2}$ tions. The increased yield is probably due to an increased movement of chromosomes and chromosome fragments caused by the sonic treatment resulting in a decrease in the amount of restitution and an increase in the amount of detectable new reunions between the broken ends of chromosomes. (MSA)

\section{9}

Conger, Alan D. THE E MENT ON SLOW NEUTHON IRTADIATED TISSUES. Genatics 35, 102(1950).

The affect of slow neutrons on tissue is an indirect one, and results from the jonizing radiations emitted by atoms which have captured a slow neutron. A previous investigation of the effect of slow neutrons on ${ }^{\text {I }}$ radescantia chromosomes had indicated that 99 per cent of the ionization dose absorbed in tissue was due to only three elements, hydrogen (emitting a $\gamma$ ray), nitrogen (proton) and boron (alpha particle). Boron alone was responsible for 32 per cent of the ionization dose absorbed. The considerable importance of boron in these slow neutron exposures was surprising in view of its minute mounts in t15sue, only $2.9 \mathrm{p} . \mathrm{p} . \mathrm{m}$. of the wet weight in this case, but was due to two factors: (1) the very high probability that a boron atom will capture a neutron, and (2) the fact that the a paricle emitted loses all its energy within the tissue. The reaction is: $B^{10}$ \& neut ton $\rightarrow\left[B^{11}\right] \rightarrow \mathbb{L i}^{7}+a$ particle. To test and confirm the importance of boron in tissues. inflorescences from Tradescantia plants grown in boric acid solution for about two months were exposed to slow neutrons, and the frequency of chromatid aberrations induced was compared with the frequency from normal nonenriched inflorescences. lt was found that, for the same 
dose of slow neutrons, the boron enriched plants showed from four to five times as many chromatid aberrations as the nonenriched plants, confirming the expectation that boron, in spite of its micro amounts, is one of the most damaging elements when small volumes of tissue are exposed to slow neutrons. (G) 2010

Conger, A. D. and L. M. Fairchild. THE INDUCTYON OF CHROMOSOMAL ABERRATIONS BY OXYGEN.

Genetics 36, 547-8(1951).

Treatment of dry pollen grains of the plant Tradescantia with oxygen (greater than $99.5 \%$ pure) produces chromosomal aber rations which can be observed at the pollen tube division on pollen culture slides. The aberrations produced are identical with those found after irradiation with $\mathrm{X}$ or $\gamma$ rays. Pollen treated with oxygen from two different cylinders (evaruated 5 times, gas Slushed 5 times) and pollen merely flushed with oxygen, all had aberration frequencies equivalent to that produced by about $600 \mathrm{r}$ of $X$ rays in air. Control pollen (not evacuated) and air pollen (evacuated 5 times, air flushed 5 times) had almost no aberrations. - Exposure of oxygen-lreated pollen to but $2 r$ of $\gamma$ rays caused so many chromosomal breaks that an analysis could be made of fragment irequency only. The fragment frequency was higher than oxygen alone, and roughly about what would be expected from $1000 \mathrm{r}$ of $\gamma$ rays in air. (G)

\section{1}

Conger, Alan D. and Nor man $\mathrm{H}$. Giles, Jr. THE CYTOGENIC EFTECT OF SLOW NEUTRONS. Genetics 35 , $397-419(1950)$. (ORNL-409)

The cylogenetic effect of thermal neutrons from a special facility in the thermal column of the Oak Ridge nuclear reactor has been investigated. The production of chromosonal aberrations, both chromatid and chromosome types, in Tradescantia microspores has been compared with the production of the same aberrations by $x$-rays. Calculations of the number of rep absorbed from thermal neut ron capture in tissue indicate that three elements, hydrogen, nitrogen, and boron are responsible for about $99 \%$ of the ionization dose produced by neutron canture, and that about $77 \%$ of this dose is due to the protons and $\alpha$ rays from nitrogen and boron capture reactions. These conclusions are substantiated biologically by the cobserved linear increase of exchange aberrations with dose, which is characteristic of particulate radiation (with electromagnetic radiation, exchange aberrations increase as a power of the dose). An equivalence of thermal neut rons/x-rays has been derived by comparing the production of chromosomal aberrations by the fwo radiations. The best value seems to be $\sim 5 \times 10^{8}$ thermal neutrons per $\mathrm{cm}^{2}=1 \mathrm{r}$ of $x$-rays in biological effect; this would make the 8-hour tolerance dose (the same amount of effect as $0.1 \mathrm{r}$ of $\mathrm{x}^{-}-$ rays) as based on this effect $\sim 5 \times 10^{2}$ thermal neutrons per $\mathrm{cm}^{2}$. A comparison of the $\mathrm{x}-\mathrm{ray}$ equivalent effect produced by unit dose of thermal neutrons with the calculated number of repgenerated by the same dose shows that thermal neutrons are alout 11 times as efficient as $x$-rays per unit dose in producing this biological effect. If only the nitrogen protons and boron $a$ rays from thermal neutron capture are considered, their efficiency compared with $\mathrm{x}$-rays seens to be about $15 / 1$. These biological efficiencies are about 3 to 4 times as great as have been observed when the same types of radiations were delivered from external sources. It is believed that this difference in the effectivem ness of protons and rays when they are administered to a tissue from an external source and these same radiations when they originate fron the atoms of the tissue itself is not due to any difference in the effect of the protons and a rays themselves, but is due to a difference in their place and manner of origin. (NSA) 2012

Costello, Donald $\mathrm{P}$. and Catherine Henley. EFIFCTS OF RADIO-PHOSPRORUS (P ${ }^{\$ 2}$ ) ON MTTOSIS IN LARVAL TAIL-TIPS OF AMELYSTOMA PUNCTATUM. AnaA. Record 108, 578-9(1950).

Young larvae (of appropriate age) of Amblystoma punctatum, from which tail-tips had been clipped, were permitted to degenerate in media containing various concentrations of radiophosphorus. The exact dosage of $\beta$ radiation within the epithelial cells of the regenerating tail-tip cannot be calculated, but a suficicient range of dosage was used to give variations in effects on mitosis from zero to complete inhibition. Submaximal dosages produced a wide variety of mitotic abnormalities, some types of which have never before been observed by the authors with other types of treatment. Many deletions (production of chromosome fragments) resuited similar to those induced in classical plant material, such as Tradescantia, by irradiation. Several extreme muitiple deletions were obtained, showing 30 or 40 or more chromosome fragments within a single cell. There was a paucity of multipolar mitoses among the abnormalities produced by the irradiation. Multipolar mitoses are among the most common of the mitotic anomalies produced by temperature effects. The demonstration that Amblystoma punctatum larval tail-fips are a favorable material (comparable, in some respects, to Tradescantial for the study of the effects of radioisotopes on cells and mitosis is of considerable importance. Investigations of the effects of radiations on animal cells have lagged considerably behind similar work on plants, due largely to a lack of suitable material. Tailtips of 580 treated larvae have been studied to date, with adequate numbers of controls. (NSA)

2013

Creighton, M. THE EFFECTS OF X-RAYS ON MUTOTRC AND MEIONIC CHROMOSOMES AND CELL DIVISION IN CHORTHIPPUS LONGICORNS (ORTYOPTERA). J. Exptl. Zool. 87. 347-69(1941).

Spermatogonia, embryo cells, and primary spermatocytes were treated with graded doses of $\mathrm{x}$-rays and the chromosomes were examined at varying intervals thereafter. The kinds of breaks were noted and the frequency of chromosome abnormalities in terms of per cent normal anaphases was recorded. It was not possible to determine the time of chronosome doubling by the order of appearance of chromatid and chromosome breaks after irradiation. The most sensitive stage for the mitotic chromosomes is three hours before anaphase which corresponds to a period just prior to prophase or in early prophase. The most sensitive stage for the meiotic chromosomes is 9 hours before anaphase. The slopes of the dose effect curves for the spermatogonia and embryo cells were identical and more than six times as great as the slope for the primary spermatocytes. The spermatogonia and enbryo cells are very sensitive to inhibition of mitosis by X-rays, the primary spermatocytes much less so. In the ambryo cells those cells which have the most sensitive chromosomes, i.e. those in stages just prior to prophase or in early prophase, are also the most sensitive to inhibition of mitosis. They are delayed longer than other cells and on reaching anaphase produce a characteristic dip in the curves for per cent normal anaphases at dif2014 ferent times after in radiation. (BA)

Creighton, M. and B. H. Evans. SOME RIPECTS OF 
X-RAYS ON THE GERM CELLS OF CHORTHIPPUS LONGICORNIS (ORTHOPTERA). J. MOSphol. 69, 187 $202(1841)$.

Male grasshoppers of the sp. C. longicornis were given 2 dose of 3,000 rof $x-$ rays and their testes examined at intervals thereafter. Spermatogonia and secondary spermatocytes show many abnormalities of the chromosomes during division within 90 minutes after irradiattion and by 12 hours they are no longer dividing. Dividing primary spermatocytes show many abnormalities soon after irxadation. X-rays inhibit the division of primary spermatocytes but in this dose do not cause a complete cessation of division. The number of dividing primary spermatocytes decreases rapidiy up to six hours after Irradiation and increases thereafter to normality at 48 hours. The inhibition is effective in preventing cells from entering prophase. As a result of the inhibition of division, primay and secondary spermatocytes begin to differentiate directly into spermatozoa, forming giant spermatids. The capacisy to difierentiate into spermatozoa is evidently independent of the maturation divisions. (BA)

\section{5}

Crouse, Helen $V$. THE DNFERENTIL RESPONST OF

MALE AND FIMALIE GERM CELLS OF SCLARA COPROPRILA (DIPTERA) TO IRRADIATION. Am. Naturalist st 84, 195-202(1950).

Comparative studies have been made on the response of male and female germ cells of $\mathbf{S}$. coprophila to irradiation. The following results were obtained: (1) When adult females are exposed to $\mathrm{x}-\mathrm{rays} 43$ to $45.5 \mathrm{hr}$ after eclosion, no gross rearrangements of any iype are recovered; but oocytes Irradiated between 46 and 72 hr of adult life yield inversions. (2) Approximately the same number of breaks leading to gross rearrangements are induced by $2,000 \mathrm{r}$ in oocytes and $4,000 \mathrm{r}$ in sperm. (3) Irradiation of sperm results in both reciprocal translocations and inversions. (4) Tests for reciprocal translocations involving the $x$ chromosome ylelded 9 such translocations among 108 sperm tested but none among the 42 oocytes examined. The failure to recover interchromosomal exchanges following irradiation of oocytes is discussed. (NSA)

2016

Da Câmara, A., M. Wagner, and A. Gardé. LOCATION OF BREAIS INDUCED BY X-RAYS IN CHFOMOSOM

OF TRITICUM. Proc. Intern. Conge. Geneties 8th

Congr. Stockholn 1948 (Suppl. to Hereditas) 555(1949).

investigations made on chromosomes of 3 groups of the genus Triticum indicate that ruptures induced by $x$ - rays are more frequent near the centromere or near the free ends. It is suggested that the points particularly vulnerable to 8 -radiation show this condition as a consequence of the chemical activities near the centromere and not because of a special susceptibility of the chromonemata in those regions. (CA)

2017

Daniels, E. W. STUDIS ON TIE PFCT OF X-IRRADIATION UPON PELOMYXA CAROLINENSIS WITH SPECIAL RETERENCE TO NUCLFAR DVISION AND PLASMOYOMY. J. Expt1. Zoö. 117, 189-209(1951).

The effect of $\mathrm{x}$ irradiation upon reproduction of Pelonywa carolinensis (an amoeboid Protozoa) was studied. The median lethal dose was determined to be $96,000 \mathrm{r}$, and the shape of the survival curve to be sigmoid. With dosages between 15,000 and $140,000 \mathrm{r}$, a pronounced delay was shown in the first plasmotomy (division) following $x$ irradiation during interphase. Greater delay was produced as the dosage was increased. $X$ irradiation did not change the rrequency of simple plasmotomies. Plas- mogamy (fusion) of $x$-irradiated halves to their nonirradiated sisters tended to prevent the delay ofherwise observed between exposure and the first mitosis. In cellular systems containing both $\mathrm{x}$-1 rradiated and nonirradiated nuclei (and cytoplasm), the nuclei still, as in a normal individual, were observed to undergo mitosis at precisely the same time. (NSA)

2018

Demerec, M. A COMPARISON BETWEEN TME X-RAY INDUCED AND THE SPONTANEOUS NOTCHES.

Genetics 25, 115-16(1940).

Salivary gland chromosome study of an unselected series of $27 \mathrm{X}$-ray induced Notches shows that 3 had the full complement of bands, in 7 cases one band was missing, in 4 cases 2 to 5 bands were missing and in 13 cases more thin 6 bands were missing. Similax study of a series of 10 spontaneous Notehes shows 1 case in the firgt group, and 3 cases in each of the other three groups. The spontaneous series consisted of Notches kept in stock at various laboratories and obtained from them for this study. Since long Notch deficiencies have poor viability it is probable that in this series natural selection may have eliminated some of the long deficiencies. Such a possibility finds support in the genetic analysis by $L i$ and Bridges of spontaneous Notches collected at randon: it was found that 4 out of 25 involved the white locus as well and were thus long deficiencies extending to the left of Notch. In my $\mathrm{X}$-ray series 7 out of 27 involve white, while in the spontaneous series only one out of ten is white, suggesting the working of natural selection in the case of the spontaneous series. When this is taken into consideration the two series show a striking similarity in the frequency distribution between the four groups differing in the length of the deficient segment. (G)

2019

Diderer, P. H. THE PIODUCTION OF GIANT SPERMATOCYTSS IN PHILOSAMIS CYNTIIA BY MEANS OF X-RAYs. J. Morphol. 67, 159-73(1040).

Irradiation of testes of Philosamis cynthia was undertaken to determine if there might be some visible effect of x-rays on the chromosomes of the spermatocytes, such as the clumping of two together, or an increase in their number. Pupae were irradiated under the following conditions: distance from target, $35 \mathrm{~cm}$, iliter of $2.5 \mathrm{cel}-$ luloid, $0.5 \mathrm{Al}$, and $0.16 \mathrm{Cu} ; 200 \mathrm{kv}, 32 \mathrm{ma}$, for periods of $11,27-1 / 2$, and 44 minutes at the rate of $180 \mathrm{r} / \mathrm{min}$, calculated as amounting to a total of $2,000,5,000$, and 8,000 $r$, respectively. This irradiation caused the formation of giant cells within the cysts of growing spermatocytes. The number of affected cysts increased with the dosage, being relatively few after a dosage of $2,000 \mathrm{r}$ units, and increasing considerably after a dosage of 5,000 and 8,000 $r$ units. Correlated with this, the number of empty cysts and the amount of cellular debris also increased. Some giant cells were monocucleate; others, binucleate or multinucleate, were probably produced by the fusion of cells. The spermatogonia remained apparently unaffected by low and medium doses. After high dosage usually only a very few cells remained, but among them some divisions occurred. No distinctive giant spermatogonia were ob. served. There was no visible effect of irradiation upon the chromosomes of the spermatocytos. In all favorable metaphase plates, the chromosomes were normal in number and in appearance. Irradiation affected similar cells in a testis very unequally, allowing some to complete the formation of sperrnatozoa, causing others to degenerate as spermatids, while those which became giant cells did not survive beyond the growth period. (SVS) 
2020

Duryea, William R. THE NATURE OF RADIATION INJURY TO AMPHIBIAN CELL NUCLEL. J. Natl.

Cancer Inst. 10, 735-86(1949).

In order to deternine the physiological mechanisms by which cell nuclei are damaged by ionizing radiations, the ovarian eggs of salamanders and frogs and whole animals were irradiated with various doses of $\mathrm{x}$-rays. Two Coolidge tubos were mounted above each other and operated from the same transformer at 182 to $194 \mathrm{kva}_{\mathrm{a}}$ no filters were employed, and the target distance from the center of the irradiated area was $35 \mathrm{~cm}$ for whole animals and $9.5 \mathrm{~cm}$ and $12.7 \mathrm{~cm}$ in the case of eggs and nucler; the irradiation rate was 6,100 to $7,600 \mathrm{r} / \mathrm{min}$. for the isolated cells and 450 to $500 \mathrm{r} / \mathrm{min}$, and 2,800 to $6,100 \mathrm{r} / \mathrm{min}$ for the whole animals. The total doses were 300 to $100,000 \mathrm{~g}$ with the great majority of the experiments being performed at the 30,000 to $60,000 \times$ level for the eggs and at the $300 \mathrm{r}$ level for the whole animals. The details of the experimental procedure are presented. At 3,000 $\mathrm{r}, 98 \%$ of the small eggs showed int ranuclear damage within 10 days. Nuclear components ir radiated in situ showed dif ferential susceptibility; exposures to low temperature $\left(5^{\circ} \mathrm{C}\right)$ delayed the nuclear injury up to 18 days but were unable to prevent pyknosis unoin rewarning of the animals. Nuclear and chromosomal injuries in ovarian eggs were roughly paralleled by those in the theca cell nuclei: typical radiation damage in nonirradiated cells could be produced by micro-injections of cyloplasmic material withdrawn from irradiated eggs. The conclusion drawn from these and other results is that cellular radiation damage is a triple phenomenon, consisting of: primary physical or radiochemeal changes in the cytoplasm; chemical metabolic processes which allow protoplasmic nuclear toxins to form or accumulate in the cytoplasm; and, Iransmission of toxic substances into the nueleus in 10 to 30 minutes at $23^{\circ} \mathrm{C}$. (NSA)

2021

Ehrenberg, I., A. Gustafison, A. Levan, and U. von Wettstein. RADIOPHOSPHORUS, SEEDLING LETHALITY, AND CHROMOSOME DISTURBANCES. HeTeditas 35, 469-89(1949).

The cytogenetical effects of radiophosphorus, $\mathbb{P}^{32}$, on the cereal seed of two-rowed barley, hexaploid spring wheat (triticum vulgare), and tetraploid wheat (triticum dicoccum) were investigated. In the different experiments the concentrations of the isotopic were $0.02-25.0 \mu \mathrm{c} / \mathrm{seed}$, usually in $5 \mathrm{ml}$ of solution for 72 hours. It was found that doses of $5 \mu \mathrm{c} / \mathrm{seed}$ or more were lethal to the tetraploid wheat and nearly so to the heraploid wheat; the cytogenetical disturbances compared with those produced by $\mathrm{x}-$ irradiation with $25,000 \mathrm{r}$. Barley was found to be less susceptible but with a dose of $5 \mu \mathrm{c}$, more than half of the cell divisions analyzed contained chromosome disturbances. Even with $2.5 \mu \mathrm{e}$ in $100 \mathrm{ml}$ of solution it was found that a treatment lasting three days produced chromosome fragmentation, and higher dosages demonstrated an increasing amount of such disturbances as pseudochiasmata, fragments, bridges due to stickiness (in the first three hours) or reunions and finally, pronounced chromosome erosions leading to full lethality. The greater susceptibility of the wheat seeds compared to the barley seeds is explained and discussed. (NSA) 2022

Fabergé, A. C. AN EXPERMENT ON CHROMOSOME FRAGMENTATION IN TRADESCANTIA BY X-RAYS.

J. Genetics $39,229-48(1940)$.
Twelve treatments were used on T. bracteata buds: Three dosages $(1320,2640,3960 \mathrm{~F}$ units) $\mathrm{X}$ continuous (18 min.) vs. fractionated (3 hr) exposure $\mathrm{X} 15^{\circ} \mathrm{C}$ va. $30^{\circ} \mathrm{C}$. The number of chromosomal objects (whole chromosones and fragments) per cell was adapted as an objective basis for scoring. To get a valld estimate of error, replicatlon was used. This proves necessary in such exphs. since variance between slides made from different buds treated alike was 10-12 times greater than variance within slides. The analysis of variance within the data shows that the effect of increasing dosage is linear"; fractionating the dose dem creases the number of chr. objects, more so at higher dosage; higher temp. reduces the number of chr. objects, more so at higher dosage; the effect of fractionation is alike at boh temps.; and there is mo significant effect at higher order interactions. The simplest interpretation is that ir radiation causes breaks and rejoining takes place afterwards. Unlike Drosophila, the amount of rejoining is an inverse function of the initial number of breaks, and also depends on the total dose. Higher temps. allow more breaks to rejoln, again unlike Drosophila. Many repairs probably restore the original sequence. (BA)

\section{3}

Faberge, A. C. ACTION OF TEMPERATURE DURING X-RAY TREATMENT OF TRADESCANTIA CRROMOSOME\$. Genetics 33, 104(1848).

There is agreement among a number of workers that in Tradescantia, the number of ehromosome aberrations is smaller if is radiation is conducted at higher temperature. It is plausible to think that it is an effect on rejoining, but attempts to demonstrate this directly by changing the temperature after treat ment have not been successful. An experiment is reported in which the time of treatment and the time of temperature change were reduced to a minimum (500 $\mathrm{x}$ units in 80 seconds; half period of temperature change 15 seconds), and were short relative to the time that a breal remains free, about four minutes. The results show that within these time limits, temperature is effective during treatment only, and not if applied before or after treatment. It was also found that change of temperature in either direction immediately preceding treatment increases considerably the number of aberrations. A temperature gradient may subsist in the material as a result of such a temperature change, and this gradient can cause thermal diffusion leading to movement of the chromosome threads. The effect may thus be parallel to that observed when cells are centrifuged during irradiation. (NSA)

Fabergé, A.C. CHROMOSOME ABRRRATIONS IN TRADESCANIIA PRODUCED BY X-RAY TREATMENY AT LIQUID AIR TEMPERATURE. Genetics 33,609 (1948).

A technique has been deviged for freering ripe Tradescantia pollen down to liquid air temperature, about $-192^{\circ} \mathrm{C}$. This pollen can be subsequently germinated on standard medium, and metaphase chromosomes observed at the division of the generative nucleus in the tube. Liquid air treatment by itself has no observable effect on subsequent division, which appears guite normal in every way. If X-ray treatment is applied to pollen while it is in liquid air, chromosome breaks and aberrations are produced which, qualitatively, do not seem to differ from those obtained by $\mathrm{x}$-raying at room temperature. The number of breaks and aberrations is, however, several times less than would be the case at ordinary temperature, and to get a comparable irequency, a very much heavier $\mathrm{x}$-ray dose 
has to be used. This decreased sensitivity is quite different from the effects within the physiological range of temperature observed in Tradescantia microspore divisions. In this range (about $+2^{\circ} \mathrm{C}$ to $+35^{\circ} \mathrm{C}$ ) nore aberrations are produced at lower temperatures, as has been shown by several workers. It is hoped that this method. by eliminating chromosome movement and presumably also restitution, may simplify some of the problems of radiation cytology. (G)

2025

Faberge, A. C. CHROMOSOME BREAKAGE BY X-RAYS AT LOW TEMPERATURE AND THE RADIODECOM-

POSITION OF WATER. Genetics 35, 104-5(1950).

A year ago data were presented at the meeting of this Society, showing that when Tradescantia pollen is X-rayed at liquid air temperature, the sensitivity of the chromosomes (as measured by the number of breaks observed in pollen tube divistons) is about one fifth of that at $+25^{\circ} \mathrm{C}$. Several intermediate temperatures have not been explored. The sensitivity curve has a dip in the region of $0^{\circ} \mathrm{C}$., and thereafter falls gradually. Its general character resembles the curve of $\mathrm{H}_{2} \mathrm{O}_{2}$ production plotted against temperature, when water containing oxygen is $\mathrm{X}$-rayed (Bonet-Maury and Le Fort, 1948, Nature 162: 381-382) but differs in that $\mathrm{H}_{2} \mathrm{O}_{2}$ production stops completely below $-116^{\circ} \mathrm{C}$., whereas chromosome breaks are still produced at $-192^{\circ} \mathrm{C}$. All previously reported effects of temperature on the $\mathrm{X}-\mathrm{xay}$ sensitivity of Tradescantia chromosome could be explained in terms of restitution and rejoining. The author believes that the effect now described is not of the kind, and occurs largely or entirely at the level of the chemical steps which intervene between ionization and chromosome breakage. I-ray action can gtill usefully be regarded as particulate, and a target theory is still possible, but a factor must be added to take into account the spread of effect 2026 around ionization columns. (G)

Pabergé, A. C. RELATION BETWTEN THE ACTION OF COLD AND OF NITROGRN IN DECREASING THE FREQUENCY OF CHROMOSOME ABERRATIONS. Genetics 35 , 663(1950).

Two methods drastically reduce chromosome breaks produced by $X$-rays in Tradescantia. One can irradiate at a very low temperature (Faberge, 1348 , Genetics 33:609) or in the absence of oxygen (Thoday and Read, 1947, $\overline{\text { Nature }}$ 160:608; Giles and Riley, 1949, P.N.A.S. 35:640-646). The order of magnitude is the same. There is a parallelism between the amount of $\mathrm{H}_{2} \mathrm{O}_{2}$ produced by $\mathrm{X}$-rays at various low temperatures and chromosome sensitivity (Faberge 1950, Genetics 35:104-105); $\mathrm{H}_{2} \mathrm{O}_{2}$ production also depends on oxygen. Experiments are now reported in which both agencies are applied, the material being Tradescantia pollen, later germinated to observe tube mitoses. Pollen was placed in a stream of either dry air or nitrogen, at room temperature, for 10 minutes before irradiation. During irradiation the gas streams continued. Nitrogen reduces gensitivity if irradiation is conducted at $+20^{\circ} \mathrm{C}$, but has no detectable action if irradiation is cone at $-191^{\circ} \mathrm{C}$. It is the writer's view that information accumulated from the irradiation of Tradescantia shows the traditional antithesis between direct and indirect action to lack realism. Experiments with oxygen and wilh extreme cold gtrongly suggest an indirect chemical step. This might represent spread of effect around an ionisation cluster. In terms of formal Target theory, a factor of $5 \times$ in sensitivsty represents a change of only $1.7 x$ in linear dimensions so that there is no occasion to abandon geometrical theories, at least in principle. (G)
2027

Tabergé, A. C. ULTRAVIOLET INDUCED CHROMOSOME ABERRATTONS IN MAIZR. Genetics $36,548-9$ (1951).

A comparison has been made between the effects of 2537A. Ultraviolet radiation and of medium hard X-rays on the chromosomes of Zea mays endosperm. Pollen carrying four linked dominant markers, $1,5 h, \mathrm{Bz}$, Wr, all affecting endosperm characters, was treated and used for pollinating plants recessive at these loci. By the detalled analysis of the mosaic patterns obtained, a method due to Meclintock, several kinds of chromosome aberrations ran be identified. Information obtained by this method is different from that given by standard cytological examination. All four markers are localed in the short arm of the 9 th chromosome, which represents only $2.7 \%$ of the pachytene length of the entire complement. Only aber rations in. volving this marked arm are identified. No aberrations are missed becausi of selection since even the complete absene of this entire arm is viable under these cromstances. Simple deficiencit's can be recognzed, and broakage-fusion-brodge cycles initiated at any pont distal to Wx. With certain limitations, rings, dicentric translocations and inversions if accompanied by a breakagefusion-bridge cycle can be identified. Ultraviolet radiation produces all of these aberrations in large numbers. One of the most common effects is the production of breakagefusion-bridge cycles. The same is true of $\mathrm{x}$-rays. At the time of writing this summary, no consistent differences between $X$-rays and ultraviolet have appeared, though the analysis of further data may reveal such differences. It is particularly desired to emphasize that these conclusions 2028 apply only to maize endosperm. (G)

Ford, C. E. CHARACTERISTIC DIFTERERCES IN THE PRODUCTION OF CHROMOSOME STRUCTURAL CHANGES BI NITROGRN MUSTARD TREATMENT AND BY IONISING RADIATIONS, p.46-7 Of INTERNATIONAL CONGRESS OF RADIOLOGY, th Congress, London. 1950.

A detailed comparison of the chromosome breaks and rearrangements (structural changes) produced in Vicia faba root tips by nitrogen mustard and by gamma rays has revealed a number of characteristic differences which are considered to be incompatible with a common mode of action of the two agents. These differences are as follows: Although the two agents cannot be distinguished by the types of structural changes produced, there are marked differences in the ratios of the frequences of the different typse. For instance, nit rogen mustard ireatment yields a much lower proportion of simple chromatid breaks and a much higher proportion of minute fragments as compared with gamma radiation. Structural changes occur as early as $1 \%$ hours after irradiation, whereas they are not found until 4 hours after nitrogen mustard treatment. At temperatures of $1^{\circ}-5^{\circ} \mathrm{C}$. the usual types of structural change are found after irradiation; after nit rogen mustard treatment none have been found so long as the low temperature is maintained. But if seedlings treated with nitrogen mustard at $1^{\circ}-5^{\circ} \mathrm{C}$. are subsequently warmed to $20^{\circ} \mathrm{C}$. struc tural changes soon appear in the metaphase chromosomes. These facts are accounted for if: (i) after the onset of prophase the nitrogen nustard (unlike $X$-rays) is unable to bring about structural changes identifiable at the immediately following metaphase; (ii) at low temperature only those nuclei which have already entered prophase complete mitosis; (iii) at low temperature milosis is greatly protracted. By far the great majority of the chromosome 
breaks induced by nitrogen mustard treatment occur in one or other of a number of short regions which appear to concide with the median heterochromat in of the short chromosomes. Gamma ray breaks, to a first approximation, are distributed umformly over the whole chromosome complement.

\section{9}

Garde, Alberto. SOME CYTOLOGICAL OBSERVATIONS OF X-RAY ACTION ON TRITICUM MONOCOCCUM.

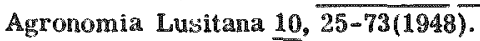

The author makes a survey of the literature dealing both with natural and artificial $x$ adiations and their genetic and cytological effects. The importance of the use of $\mathrm{x}$-rays together with other agencies is discussed, putting special emphasis on chenical substances. The writer in his experiments used lead acetate before $x$-irradiation. He concluded that: (1) Lead acetate per se, leads only to anglutination and total or partial dissolution of the matrix stbstance. (2) The supplementary treatment of Triticum monococcum root-tips with $1 \%$ aqueous solutions of lead acetate decreases the total percentage of chromosome aberrations due to $x$-rays. Fragment percentage df creases, whereas translocations percentage is not affected or very slighly uncreased. (3) The main effect of the secondary radiation of lead submitted to $x$-rays seems to be mutagenc. Dufferent cases of chromosome aberrations are descrubed (delay of division after treatment, cells with an abnormal number of chromosomes, tri-and tetrapolar spindles, fragments, dicentric chromosomes, anaphase bridges, etc.). From these observations the writer concludes that: (1) The type of dicentrics with the two centrometes very near one another seem to be more persistent than any other throughout cell division. The writer thmiss that perhaps the closeness of the two centromeres may produce an effect leading to their behavior as a single element. (2) Resistance of the chromosomes of Triticum monococcum $L$. to $x$-rays seems to be different for different pars. SAT chromosomes are the most resistant. (3) The centromere plays a very important part in chromosome reaction to $\mathrm{x}$-rays. 113 references. (NSA) 2030

Gaulden, Mary Esther and Marjorie Nix. EFECTS OF OXYGEN TENSION ON X-RAY INDUCED MITOTIC INMIBITION. Genetics 35, 665(1950).

This paper is reproduced here in its entirety.

The large neuroblast cells of the embryo of the grasshopper Chortophagra vi ridudase iata were given $64 \mathrm{r}$ of $\mathrm{x}$ rays while exposed to different tensions of oxygen, namely $100 \%, 21 \%$ (air), 2\% and $0 \%$ (nit rogen, carbon dioxide, or vacuum. The e mbryos were made into culture preparations. The number of cells in midmitosis (prometaphase, metaphase, and anaphase) was recorded every 22 minutes for $6 \mathrm{hr}$ after treatment. Thus the number of cells completing mitosis was determined, smee 22 min as the average duration of mid-mitosis (at $38^{\circ} \mathrm{C}$ ) and since the treatments used do not affect this duration. The results indirate that the sensitivity of mitosis to radiation is reduced when cells are sradiated at the lower oxygen tensions. In other words, the duration of the period of complete mitotic inhibition (period after irradiation during which there are no cells in the mid milotic stages) is shorter when cells are irradiated in 0 or $2 \%$ oxysen than when they are irradiated 121 or $100 \%$ oxygen. Experiments are now in progress to determine the sensitivity of cells to radia2031

tion in 5 and $10 \%$ oxygen. (NSA)

Gelin, O. E. V. THE' CYTOLOGICAL EFFECT OF DIF FERENT SEED TREATMENTS IN X-RAYED BARLEY.
The author uses exclusively Golden Barley series 1939: I: $21-24$, irradiated with $10,000 \mathrm{r}$ (voltage $160 \mathrm{kv}, 7.5 \mathrm{ma}$, $4 \mathrm{Al}, 72 \mathrm{r} / \mathrm{min}$ ). The pretreatments were as follows: I:21, the seeds contained $10 \%$ water; $1: 24,15 \%$ water; 1:22, the seeds were treated with distilled water for 23 hour $\$$ previous to the \&-raying in order to induce germination; 1:23 the seeds were treated with $0.01 \%$ of a heteroauxin solution for 23 hours for the purpose of still further enhancing gemination. The reaction of the chromosomes is directly influenced by the physiological condition of the seeds. Seeds containing $10 \%$ water show $12.66 \%$ disturbed divisions, seeds with $15 \%$ water $27.99 \%$ and seeds soaked 23 hours in water or in $0.01 \%$ hetero-auxin solution 53.80 and $50.99 \%$ disturbances, respectively. The disturbed cells show probably a random dist ribution in different parts of the roots. The cells that first enter into division show the highest irequency of disturbances. If the percentage of rells with fragments and the percentage of cells with bridges are computed in proportion to the total number of analyzed cases, two numerical series are obtained showing good proportionality, approximately the ratio is 1: 2: 4: 4. The frequency of bridges seems to be $1 / 3$ of the disturbed cells. The proportion of bridges and fragments is, generally speaking, the same in all division classes. There is a marked parallelism between the percentages of disturbed cells, sternlity and mutation rate. The most evident feature is that the same proportionality existing between the percentages of disturbed cells in the different series is also found in the percentage of mutations, viz. approximately 1: 2: 4: 4. (S.V.S.) 2032

Giese, A. C. RADIATIONS AND CELL DIVISION.

Quart. Rev, Biol. 32, 253-82(1947).

A revew with about 300 references, including chernical mechanism of cell division. (NSA) 2033

Giles, Norman $\mathrm{H}, \mathrm{J}_{\mathrm{r}}$. THE EFPCTS OF FAST NEUTRONS ON THE CMROMOSOMES OF TRADESCANT IA. Proc. Natl. Acad. Sci. U. S. 26, 567-75(1940).

Qualitatively there is no difference in neutron and $x-$ ray treatment. Quantitatively, for equal total doses in terms of ionization as measured with a bakelite Victoreen " $r$ " meter, neutrons are from 16-17 times as effective as $\mathrm{x}$-rays in producing chronatid dicentric aberrations. Also, exchange break aberrations, producing chromatid and chromosome rings and dicentrics, show an approx. linear relationship to dosage instead of the exponential relation found with $\mathrm{x}$-rays. This difference may result from the great difference in the types of ionization paths produced by neutrons and $\mathrm{x}-\mathrm{rays}$. (BA)

2034

Giles, Norman H., Ir. COMPARATIV STUDIES OF THE CYTOCENETCAL WFECTE OF NIUTRONS AND X-RAYS. Genetics 28, 398-418(1943).

Comparative effects of neutrons and 8 -rays were investigated in Tradescantia paludosa by a cytological analysis of chromosomal rearrangements induced in the developing microspores. Neutrons and $x$-rays produced the same qualitative types of rear rangements, but differed in their quantitative effects. For equal total tissue doses of ionization, neutrons were more efficient than $x$-rays in breaking chromosomes. Furthermore, under the conditions of dosimetry used, neutrons of lower average energy (7.5 Mev) were more efficient than those of higher average energy (15 Mev). Apparently, several ionizations are necessary to break a Tradescantia chromosome. For all types of grearrangements induced by neutrons, the relation beween dosage and effect was approximately linear, and 
there was no time-intensity factor. These results differ from those obtained with $\mathrm{x}$-rays and indicate that most neutron-induced exchanges are produced by single proton iondzation paths. Comparative ratios of certain aberation Aypes induced during resting stage and grophase provided evidence that space factors limit the reunion of both neuron and $\mathrm{x}-\mathrm{s}$ ay induced brolsen chromosome ends. In Drosophila melanogaster, neutrons were less efficient than $\mathrm{x}-\mathrm{ray}$ in inducing sex-linked lethal mutations. This effect is discussed and intergreted in comparison with the reauts in Tradescanta and whth those obtained by previous 9025

Giles, Norman 5. CHROMOSOME STRUCTVRAL CFANGES IN TIADRSCANTLA MCROSPORES PRODUCED DY AISORBED IRADIOPHOSPHORUS. DOC. Nath. Acad. ScL. U. 5. 33, 283-7(104\%).

Cut I. paludosa inflorescences were inserted in various concentrations of radiophosphorus ( $150 m$ loo $10 \mathrm{c} / \mathrm{ml}$ to $0.1 \mu c / \mathrm{ml}$ ) for mersods of 1 to 9 day. A count was made of cytological changes at the first post-meiotic (mic rospore) mitosis. The types of aber rations resulting from betasadiations inside the plant were similar to those carsed by neutrons and $\mathrm{x}-\mathrm{rays}$. Their frequency shows an increase with tine and correlates with he increase in radioactivity measured with an electroscope. Within 24 hours chromat îd aberrations were observed and remaned the principle 1ype. Chromatide exchanges occurred with a relatively high Irequency. It was not unil the fourth day that chromosome breaks were observed. At the highest concentraton (100 He ml) the frequancy of one hit chromatid aberration types aftes 24 hours was equivalent to that produced by about 40 of $x$-rays; the very high breakge rate prevented aberation frequency couts after about 48 hours. (N)

\section{6}

Giles, Norman H., Jr. RECENT EVIDENCE ON THE MECHANISM OF CHIOMOSOML ABERRATION PRO DUCTION BY IONIZWG RADIATIONS. OAK Ridge National Lab. Aug. 29, 1950, (ORNL-811)

Recent experiments have demonstrated that oxygen has a marled effect in increasing the sensitivity of chromosomes in Tradescantia and other plants to $x$-rays, as measured by the frequency of cytologically detected aberrations. It has been shown that this is not an effect of oxygen itself on the behavior of broken ends of chromosomes. The effect apparently arises from the production by $\mathrm{x}-\mathrm{rays}$, as a regult of the radiodecomposition of water in cells containing oxygen, of some substance which causes an increase in aberration srequency. Several independent lines of evidence indicate that this substance may be $\mathrm{H}_{2} \mathrm{O}_{2}$. It appears lincly that the increased frequency of aber rations arises from an increased production of chromozome breaks when oxygen is present during irradiation, zather than from a modification of the recovery process. Thus a major fraction of the radiation effect on Tradescantia chromoscomes is to be considered indirect rather than direct. (NSA) 2037

Giles, Norman H. and A. V. Beatty. THE EFECT OF X-IREADIATION IN OXIGEN UNDER PRESSURE ON CHROMOSOME ABERRATION FREQUENCY IN TRADESCANTIA MLCROSPORES. Cenetics 35, 666-7(1950).

Inflorescences of Tradescantia paludosa, clone 5, were exposed to $400 \mathrm{~g}$ of $x$-ray at $50 \mathrm{r}$ per minute in atmospheres of $5 \%$ and $10 \%$ at normal atrnospheric pressure $(740 \mathrm{~mm}$.) and at positive pressures of 1,2 , and 3 atmos pheres above normal. Similar exposures were made in He and in air $(21 \%$ o) at normal pressure and at 3 atmos pheres above normal. The frequency of both chromosone interchanges (diccntrics and centric rings) and interstitial deletions were determined from slides of the 1 st postmeiotic mitosis prepared on the 4th and 5 th days following irradiation. The exposures in Ne indicated that these pressures alone did not alter aberration frequencies. Exposures in $5 \%, 10 \%$, and $21 \%$ at the different pressures produced aberration frequencies similar to those obtained in comparable partial pressures of 0 . For example, the effect of $5 \%$ at 1 atmosphere above normal is comparable to that in $10 \% 0$ at normal pressure. From these and previous reaults it is evident that $O$ increases $\mathrm{s}$-ray induced chromosome aberrations and that the $\mathrm{x}$ amount of $O$ present at the lime of exposure determines the extent of the effect. Since no pressure effect was ontained in these experiments in the absence of 0 , the effect of pressure in the presence of $O$ apparently is, in reality, an $O$ effect. 2038

Giles, Norman H., Jr. and A. V. Beatty. EFECT OF X-IRRADIATION IN OXYGEN AND IN GYDROGEN AT NORMAL AND POSITIVE PRESSURES ON CRROMOSOME ADERRATION IREQUENCY IN TRADESCANTIA MCROSPORES. Science 112, 643-5(1950).

Aberrations are produced by irradiation in a gystem containing microspores of Tradescantia paludosa in the complete absence of $\mathrm{O}_{2}$. Alnear increase in the frequency of aberration occurrence results with $\mathrm{O}_{2}$ between 0 " ond $10 \%$, leveling of at $20 \%$ concentration. At pressures of 1 , 2 , and 3 at mospheres no further change in aberration frequency occurred following irradiation at all $\mathrm{O}_{2}$ concentrations employed. Results of $\mathrm{H}_{2}$ gas in place of the He under the same conditions were similar The failure to find a significant decrease in aberration frequency when s-irradiation is performed in $\mathrm{H}_{2}$ may mean that all the residual aberrations induced by 8 -rays in the absence of $O_{2}$ arise as a result of a direct effect of the radiations on the chromosomes. (CA) 2030

Giles, Norman R., J1., Alvin V. Beatty, and Herbert Parkes Riley. TRE RELATION BETWIEN THE EF FECTS OF TEMPERATURE AND OF OXYGEN ON THE FREQUENCY OF X-RAY-INDUCED CIROMOSOME ABFRRATIONS IN TRADESCANTIA MCROSPORES. Genetics 36, 552-3(1951).

Inflorescences were irradiated in experiments in which the amount of dissolved oxygen was varied over a similar range in terms of volumes per cent in two different ways: (1) by using a constant percentage of $O_{2}\left(5 \% O_{2}\right.$ plus $95 \%$ helium) and making a series of exposures at several different lemperatures, between $0.6^{\circ}$ and $40^{\circ} \mathrm{C}$., and (2) by using a constant temperature $\left(27^{\circ} \mathrm{C}\right.$.) and varying the percentage of $\mathrm{O}_{2}$ in the exposure chamber. Cytological analysetwere made of chromosomal aberration types at the fous - to five-day interval following irradiation. Rexposures were also performed in the absence of oxygen (in pure nitrogen) at various temperatures. The experiments in $O_{2}$ demonstrate that the increased yield of aberxations at low temparatures cannot be attributed entirely to increased oxygen solubility. There is a considerable additional effect of temperature itself. In the absence of oxygen, the unerpected result was obtained that the effect of temperature is exactly opposite to that noted in the presence of oxygen the yield of aberations is considerably higher than at low temperatures. This situation is true not only for chromocome aberrations but also for all types of chromatid effects. It seems probable that temperature exerts its offect, at least in the presence of oxygen, on either the formation or affectiveness of some substance (or substances) produced in the cell by the radiation and responsible for chromosome breakage. (G) 
2040

Giles, Norman H., Jr. and Rene A. Bolomey. CXTOGENETICAL EF FECTS OF INTERNAL RADIATIONS FROM RADTOISOTOPES. Cold Spring Harbor Sumposia Quant. Biol. 13, 104-12(1948).

The results of a cytological analysis to determine the amount of change caused by radations from isotopes in Tradescantia buds and mirrospore nuclei grown on radiophosphorus, $1^{32}$, media and in some cases $C^{14}$ media are presented. The rates of uptaks of $\mathrm{P}^{32}$ and $\mathbb{C}^{14}$, the chromosomal rear rangements produced by the 3 radiation from these isotopes, and relability of the avallable techniques are discussed. (NSA)

2041

Giles, Norman H., $J r$. and Alan D. Conger. CHROMOSOMAL INTERCHANGES INDUCED IN TR ADESCANTIA MCROSPORES BY FAST NEUTRONS FROM URANIUM FISSION. J. Cellular Comp. Physiol. 35, (Suppl. 1) 83(1950).

Inflorescences of Tradescantia paludosa (Sax, clone No. 5) were exposed to fast neutrons from uranium fission, utilizing a special suposure device in the Oak Ridge pile, and the yicld of chromosomal interchanges (dicentries and centrac rings) was determined in microspore nuclei on the 4 th and 5 th day following in radiation. A linear relationship was found between neutron dose and interchange frequency. confirming previous evidence that fast neutron induced interchanges are one-hil phenomena. Further evidence has been obtained that the interchange yield for a guen ionization dose is inversely related to the energy of the fast neutrons utulized. This relationship can be interpreted in terms of differences in ionization distribution within nuclei. The greater efficiency of less energete fast neutrons is in accord wh the vew that several ionizations are required to produce a chromosome break in Tradescantia. (NSA) 2042

Giles, Norman H. and Nebel, B. R. AN ANALISIS OF THE INT SOMAL ABERPATIONS IN TRADESCANTIA. Genetics 27, $142-3(1942)$.

In a series of experiments developing microspores of Tradescantia were given equal total doses (308 roentgens) of X-rays at widely different intensities, from 1.3 to $1200 \mathrm{r} / \mathrm{min}$. At the $4-5$ day period following irradiation smears were made for an analysis of the percentages of translocations (dicentric and ring types) and of interstitial delections - small acentric rings according to Rick and Sax (isodiametric fragments in the terminology of Nebel, Wilson and Marinelli) induced during the resting stage at the various intensities. At $50 \mathrm{r} / \mathrm{min}$. and above $(150,600$, and $1200 \mathrm{r} / \mathrm{min}$.) the percentage of aberrations in both classes remains approximately constant - there is little or no intensity effect. At lower intensities (20, 10.3, 5.2, 2.5, and $1.3 \mathrm{r} / \mathrm{min}$.) the percentage of aber rations in both classes decreases progressively, but not linearly with decreasing intensity, until at $1.3 \mathrm{r}$ 'min. the number of aberrations per cell is somewhat less than half that found at intensities of $50 x$, min. and higher. These results are in accord with the hypothesis that the chromosomal aberrations types studied result from two independent potential breaks, and that the reunion of broken ends, occurs during the resting stage. Within a certain time following the initial breakage. When this time is exceeded restitution occurs. (G)

2043

Giles, Norman H., Ir. and Herbert Parkes Riley. THE EFECT OF ONYGEN ON THE TREQUENCV ON X-RAY
INDUCED CRROMOSOMAL REARRANGEMENTS IN TRADESCANTIA MIROSPORRS. Proc. Natl. Acad. Sci. U. S. $35,640-6(1949)$.

Comparative exposures of Tradescantia paludosa to xh-rays were made in air, holium, argon, nitrogen and oxygen, and observations were made on chromosomal changes (interchanges and interstitial deletions) at the firgt postmeiotio mitosis in the microspores by means of acetocarmine smear preparations at the 4 th or 5 th day after irradiation. The $x$-ray source was a Coolidge self-rectifying tube with a tungsten target and operated at $250 \mathrm{kv}$; the filtration was $3 \mathrm{~mm}$ Al plus added filtration through a $0.25 \mathrm{in}$. lucate exposure chamber, and the dosage rate was usually $45 \mathrm{r} / \mathrm{min}$. Total doses of $90,180,270$, or $360 \mathrm{r}$ were given. It was found that the frequency of aberrations was gtrikingly reduced when nitrogen replaced air and increased when oxygen replaced air as the medium; the number of aber rat 1ons in the oxygen medium was five times that in the nitrogen medium; aber ration frequeneies were also reduced in additional exposures in other gases auch as helium and argon. The availability of oxygen is considered an important factor in the production of chromosomal changes by irradiation. (NSA)

\section{4}

Giles, Norman H., Jx. and Hexbert Parkes Riley. STUDIES ON THE MECHANISM OF THE OXYGEN LTFECT ON THE RADIOSENSITIVITY OF TRADESCANTIA CRROMOSOMES. Proc. Nat1. Acad. Sci. U. \$. 36, 337-44 (1950) (ORNL-688)

Previous experiment by the authors have demonstrated that the radiosensitivity of Tradescantia chromosomes, as measured by the occurrence of $x$-ray induced aber rations in microspores, is markedly influenced by the anount of oxygen present. Further experiments are reported here. Exposures of inflorescences to a single constant $x$-ray dose, but in atmospheres containing seven different percentages of oxygen, indicate that there is a rapid rise in aberration frequency between 2 and $21 \%$ oxygen, with a gradual increase thereafter. Further studies are being made to clarify the effect of oxygen at levels between 0 and $2 \%$. Other experiments have been performed to determine whether the oxygen effect is exerted by way of the initial brealigge mechanism or on the reunion process. These consisted of comparative exposures to a single dose of $300 \mathrm{r}$ in one minute of inflorescences in a vacum or in oxygen with the addition or removal of oxygen either immediately after or duxing part of the irradiation period. The experiments show that the presence or absence of oxygen during the actual exposure to $x$-rays rather than during the post-irradiation period is the important factor, thus demonstrating that the effect of oxy gen in increasing the radiosensitivity of Tradescantia chromosomes results from an increased production of initial breaks rather than from an influence on the seunion of broken ends. (NSA)

\section{5}

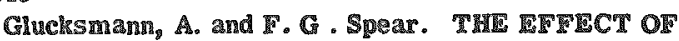
GAMMA RADIATION ON CELIS IN VIVO. II. (1) SINGLE EXPOSURES OF THE FASTING TADPOL: AT ROOM TEMPERATURE. (2) SINGLE EXPOSURES OF TYIF NORMAL ANMAL AT LOW TEMPERATURE. Brit. J. Radiol. 12, 486-98(1939).

In the tadpole, miotic activity can be varied by fasting and by low temps. Tadpoles were used 3 to 6 weeks after hatching, when they were 1.5 to $2.0 \mathrm{~cm}$ long, and all at about the same stage of development. They were irradiated with 268 x of $\gamma$ rays throughou their vol, and then complete serial sections were made of the fixed specimen. Mitotic counts 
were made and also counts of the number of degenerate cells (assumed to be he resul of adiation). After 1 week' fasting, when these is gtill some mitosis present, ir radiaton is followed by the appearance of degeneration in the germinative zone. Afte 2 weers fasting, when mitosis is Gurther reduced, sadiation is followed by very much less degeneration. Feeding is followed by the appearance of degereration with the first $15 \mathrm{hrg}$, of giving food and by the appearance of mitosis after an interval of 5 days. In the absence of radiation, mitosis reaches a higher level on this 5th day. In the chinings expts. alteration in the milotic count is proportional to the reduction in temp. With a dose of radiation sufificient to produce a marked degeneration in animals at soom temp., no degenerate cells are seen as long as the animal is kepechiled. If the animal is brought to room temperature, degenerate cells appear after about 6 hours. Desults are similar whether animals are irradiated 2046 at low or at room temp. (BA)

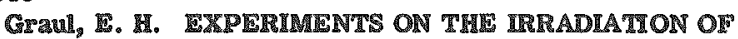
LIVMG PROTOPLASM OF THE CRLL: X-RAY IFIPCTS ON FWTRANUCLEAR CELL CONSTITUENTS, 1.172 of INTIRNATONAL CONGRESS OF RADIOLOCY, Congress, London. 1950.

The primary biological processes caused by lipadiation (uliraviolet and X-nays) are discussed in terms of nuclear and extranclear processes. A series of axperimental regults shows that with UV radiation extranuclear effects predominate. The cell memonane, the miciosomes, mitochondria and chondrosomes are the structures chiefly involved and our results are discussed in relation to the target theory of Timoferf-Ressovsky. Changes in the -lectric properties of the cell membrane are also described. The significance of the amount of current lowing a audiofrequencies in relation to the primary biological response is considered. By UV indadiation of the sharply defined germ layers of Triton alpestris (using our modifica thon of the fine beam method) (um ther evidence is given of the importance of the reaction of extranuclear cell structures to radiation. This evidence is supported by a discus3ilon of results of experiments on hemolysis. 2047

Gray, L. H. and John Read. THE ETEYCT OF IONHZING RADLATINS ON THE BROAD BEAN ROOT. VI. THE INHIBITION OF MTOSIS BV ALPHA RADIATION. Brit. J. Radiol. 23, 300-3(1950).

Broad bean roots were irradiated with $\alpha$ particles by immersion in radon colutions: the dose was 12 to 237 energy units $(1$ energy unit $=1.1$ roentgen equivalent physical $=$ $93 \mathrm{ergs} / \mathrm{g}$ tissue) delivered over a period of $201090 \mathrm{~min}-$ ates. Tips were fixed $3 \mathrm{hr}$ afterward and the number of cells ceen in mitosis compared with control roots. When the logarithm of the percentage residual mitosis was plotted against the dose, the points defined a straight line. These results are compared with similar results obtained previously with neutron and $\gamma$ rays. The ratio of the sloges of the neutron and $\alpha$ ray lines is the inverse of the ratio of the linear ion dengities, suggestive of an effect brought about by a aingle recoll proton or an a particle. The relative efficiencies of $\gamma$ rays, neutrons, and $\alpha$ rays in reducing the mumber of cells geen in mitosis in the bean root $3 \mathrm{hr}$ after irmadiation are $1: 2.1: 0.6$, whereas the corresponding figures for the lethal effect are $1: 0: 9$. (NSA)

\section{8}

Reinsich, H.L., W. Paul, and G. Schubert. THE E PECT OF X RADATION AND RAPID ELETRONS (ASAMVI) ON MUTOSIS IN ROOT ENDS OF VICLA FABA. Waturwissenschatten $3 \%, 544-5(1950)$.
Roots of Vicia faba were X-radiated with $150,300,450$, and $600 \mathrm{r}, 250 \mathrm{r} / \mathrm{min}$ or exposed to rapid electrons of Göttingen's atom reactor, and investigated after $72 \mathrm{hr}$. Chromosomes suffered increasing shortening, mitoses decreased in number, and number of damages of cells in telo- and anaphases increased with increasingly stronger doses. Rapid electrons were less effective than $x$ rays. (NSA)

2049

Helfer, R. G. A COMPARISON OF X-RAY INDUCED AND NATURALLY OCCURING CHROMOSOMAL VARIATIONS IN DROSOPHILA PSEUDOOBSCURA. GENETics 26, 1-22(1941).

The distribution of 347 breaks induced by $x-$ ray treatment in the chromosomes of Drosophila pseudoobseura was studied. The frequencies of the breaks in the different chromosomes are in proportion to their relative lengths. The induced breaks in the third chromosome are not distributed entirely at randon. The frequency of the breaks in the heterochromatin as compared with those in the euchromatin is much greater than would be expected on the basis of the lengths of the heterochromatic portions in the salivary gland cells but probably smaller than would be expected on the basis of its length in the mitotic chromosomes. Within the enchromatic protions the frequency of breaks increases slightly from the proximal to the distal end. Aside from the regularity mentioned above, the breaks in the third chromosome ghow no tendency to be concentrated around and "Weak points." In any case, a comparison of the induced breaks with those observed in the naturally occurring chromosomal aberrations shows very few coincidences. None of the inversions induced by $\mathrm{x}$-ray treatment proved gimilar to any of the naturally occurring inversions. The reunion of the chromosome fragments produced by $\mathrm{x}-\mathrm{ray}$ treatment is not at random, inversions being more and translocations less frequent than expected. Several mosaic chromosomal aberrations are described. An analysis of these aberrations seems to argue in favor of the breakage first, thather than the "contact" hypothesis of the origin of 2050

Denshaw, Paul S. TYYE ACTION OF X-RAYS ON NUCLEATED AND NONNUCLEATED EGG FRAGMENTS. Am. J. Cancer 33, 258-64(1938).

Igg of Arbacia punclulata were irradiated with the following conditions: $195 \mathrm{kv}, 150 \mathrm{pv}, 30 \mathrm{ma}, 4 \mathrm{~mm}$ Bakelite filter filter, $9.5 \mathrm{~cm}$ from target to matertal, making the untensilv of irradiation at the point of exposure approximately $7,600 \mathrm{r} / \mathrm{min}$. Usually 45 sec or a dose of $5,700 \mathrm{r}$ was selec ted as the duration of exposure. Three tyres of material were used: normal whole eggs, mucleated light parts, and non-nueleated heavy parts. These were irradiated and inseminated and the moment when $50 \%$ had divided was determined. It was found that for a given doge of radiation an apprecuable delay in cell division of about the same extent was produced in whole eggs and rucleated fragments, whereas no delay was produced in the non-nucleated fragments. It appears that he slowing in cell division brought about in Arbacia eggs by $x$-rays is due to changes produced directly in the nucleus by radiation. (S.V.S.)

\section{1}

Henshaw, Paul S. FURTHER STUDIES ON THE ACTION OF ROENTGEN RAYS ON GAMETES OF AREACLA PUNCTULATA. D. DELY IN CRLL DVISION CAUSED BY EXPOSURE OF SEIRM TO ROENTGEN RAYS. I. MODITCATION OT THE MTOTIC TME SCHEDULE IN THE EGGS BY EXPOSURE OF THE GAMETLS TO 
ROENTGEN RAYS. II IIIATION OF IRRADIATION EFFECT BY FERTILIZATION IN THE EGGS. IV. CHANGRS IN RADIOSENSETIVITY DURING TUE IIRST CLTAVAGE CYCLE (with cooperation of T. Cohen). V. THE INPLUENCE OF LOW TEMPERATURE ON RECOVERY FROM ROENTGEN RAY EFFCTS IN THE EGGS. VI. PRODUCTION OF MULTIPOLAR CLEAVAGR IN THE EGGS BY EXPOSURE OF THE GAMETES TO ROENTGEN RAYS. Am. J. Roentgenol. Radium Therapy 43 , 899-933(1940).

Normal eggs of Arbacia punctulata were iertilized with sperm exposed to doses of hard $\mathrm{x}$-rays up to $500,000 \mathrm{r}$, and the time between insemination and first cleavage noted. The interval was increased by urradiation, the amount of the delay varying exponentially with the exposure. Fertilization was prevented only by doses in excess of $300,000 \mathrm{r}$. The exposure of either the sperm or the eggs of Arbacia punctulata to $62.400 \mathrm{r}$ of hard $\mathrm{x}$-rays did not retard the entrance of the sperm into the egg or its movement through the egg cytoplasm and fusion with the egg nucleus. All phases of the milotic part of the 1st cleavage cycle were delayed, the prophase to the greatest extent. The eggs were irradiated with 28,800 or $14,400 \mathrm{r}$ of soft $\mathrm{x}$-rays, and fertilized at varying intervals up to 3 hours after irradiation; the delay was somewhat less when the fertilization did not take place until some time after the irradiation, indicating a recovery from the radiation effect. All possible combinations of normal and $x$-1rraduated eggs and spern of Arbacta punctulata, and the zygote irradiated at various minutes up to 45 min. after insemination, were sfudied for delay in cleavage time. The eggs were more susceptule than the sperm, for doses of 7800-31,200 r. For equal doses of radiation the effect on the zygote was greater than on either gamete and about equal to that on both of them. The zygote increased in radiosensitivity until the pronucle: fused, then decreased during the prophase stage. There was no apparent correlation between change of radiosensitivity and rate of $\mathrm{O}_{2}$ uptake. Irradiated eggs kept at $\mathrm{O}^{\circ} \mathrm{C}$ showed longer delay in cleavage time after insemination than those kept at $24^{\circ} \mathrm{C}$. In addition to the delay in cleavage time, described in preceding studies, abnormal cleavage was often produced by 8 -irradiation; multipolar cleavage being produced in a high $\%$ of individuals if the dose was large enough. Since chromatin deficiency usually results in cell death, it is possible that one of the mechanisms by which radiation causes cell death is the production of accessory asters, resulting in abnormal chromatin distribution at mitosis. Chromatin modification may be a plausible explanation of cancer; this may be an indication of the mechanism by which radiation may produce cell death on the one hand, and cancer on the other. (BA) 2052

Hofiman, R. S. and S H. Wollman. EFFECT OF X-RAYS ON THE MIGRATION OF CELLS FROM ADULT TISSUE EXPLANTS. Proc. Soc. Expth. Biol. Med. 70, 38-4011949).

To lest the effect of radiation dosages on the latent period before the appearance of cell migration, tissue preparations from adult chicken cardiac musele were ixradiated at a rate of $25 \mathrm{r} / \mathrm{min}$. by a $180 \mathrm{kw}$ machine, filtration of $0.5 \mathrm{~mm}$ Cu and $1.65 \mathrm{~mm} \mathrm{Al}$, at a distance of $50 \mathrm{~cm}$ giving a total dose of $010500 \mathrm{r}$. Results are presented graphically showing the per cent of explants showing a single migratory cell between 15 and 45 hours after in radiation and the variation of time before aypearance of a migratory cell with the dosage. The authors conclude that irradiation with less than $30 \mathrm{r}$ stimulates migration of cells from fresh explants and, irradiation of more than 30 ir shows a gradually more extended latent period: this suggests a superimposed inhibition, which does not exceed the excitation effect until dosages go over a $1,000 \mathrm{r}$ level. (NSA)
2053

Hohl, 1. COMPARATIE INVESTIGATIONS OF THE EFIECTS OF X RAYS AND CHEMICAL SUBSTANCES ON MTTOSIS. Radiol. C1n. 17, 302(1948).

Experiment established the possibility of disturbing the process of mitosis without damaging resting cells. The ef fects of radiation and several antimitotic chemicals are described in detail. The antimitotic ehemicals attack either specific chemical reactions in the mitotic sequence (colchicine) or the albumin metabolism in an unknown way. The target effect is common to both $\mathrm{x}$-rays and chemical substances (nitrogen mustard). (M. F.) 2054

Kaufnam, Berwind P. INDUCED CMROMOSOMAL BREARS IN DROSORNLLA. Cold Spring Harbor Symyocisis Quant. Biol. 19, 82-92(1941).

Salvary gland chromosomes of the Diptera provide unique and unparalleled material for quantitative studies of the effects of irradiation. In Drosophila such analyses have shown that breaks are distributed at random along the chromosomes except for the proximal heterochromatic region and for similar intercalary regions. The data are in harmony with the theory that brealss in a given region occur in proportion to the length represented by that region during late prophase or metaphase of mitosis. Changes induced in the chromosomes of the sperm are not affective at once in producing rearrangements and probaloly not until the male pronucleus is formed within the egg. Several lines of evidence indicate that these changes represent potential breaks. The suggestion is offered that they may only be effective if combination with othex similar regional alterations is realized. Recombination is not entirely at random but is apparently favored by propinquity of the potenilal breaks so that when only a few breaks are involved it appears that a restricted recombination zone exists. Evidence from distribution of chromosome and chromatid breals indicates that the chromosomes of the mature sperm consist of two separate chromatids, both of which can be severed at the same level by the elfects of a single "ionization". The number of altered sperms does not increase linearly with the dosage. The freguency of breaks and the frequency of independent rearrangements produced at different dosage levels indicate that the increase follows more nearly the square of the dosage in the range between 1,000 and 5,000 roentgens. All of these findings support the concept that breakage procedes recombination rather than the concept that brealrage and recombination are part of a single process and occur simultaneously. Neutrons give a similar or somewhat lower, but not a higher break frequency than $x-$ rays of comparable dosage. These results may be due to a saturation effect being reached al lower doses with neutrons than with $\mathrm{x}$-rays. (SVS)

\section{5}

Kaufmann, Berwind P. EVIDENCE OF DRLAY IN RE ATTACHMHNT OF CMROMOSOME IRAGMENTS FOLLOWING X-RADIATION OF SPERM OF DROSOPRILA MLANOGASTIR. Genetics 26, 157(1911).

In order to determine thd duration of any interval ocenxring between the time when chromosome breaks are induced and the time of secombination of the fragments, comparison has been made between the effects of a given dosage when administered continuously and in a series of fruction. Salivay chromosome analysis was used to measure frequency of breakage and types of rearsangements groduced. The number of breals detectable in the fraction-treated material should be lower than in contro material if in the interval between successive doses some of the breaks "heal" to restore the orighal sequences and are therefore unable to participate with subsequently pro- 
duced broken ends in the formation of chromosone rearrangements. Moreover, if recombination should occur between succesive treatments, the fraction-treated mateIral should show relatively fewer of the complex rearrangements involving a large number of broken ends than the controls. Results of the experiments (in which effects of a series of 1000 roentgen fractions at 24 hour intervals, and 2000 roentgen fractions at 16 day intervals were compared with the controls) indicate that such differences do not exist. The close correspondence between fraction and continuous treatment with respect to numbers of breaks guggests that no appreciable "healing" occurs even when the interval between successive treatments is 16 days. The presence of multiple-break, complex rear rangements following the fractionated dosage strengthens the evidnece for the theory that breakage precedes recombination. (G) 2056

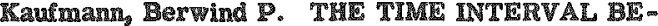
TWEEN X-RADIATIONS OF SPERM OF DROSOPHILA AND CLITOMOSOME RECOMEINATION. Proc. Natl. Acad. Scl. U. \$. 27. 18-24(1241).

Sperm of $D$. melanogaster were s-radiated with two equivalent doses, one applied continuously, the other fractonaly. Comparable irequencies obtained with both types of treatment indicate that no appreciable "healing" occurs, even when the interval between successive fractions is 16 days. The presence of multiple-break, complex rearzangements following the fractionated treatment strengthens the avidence for the theory that breakage precedes reunion. (BA)

$205 \%$

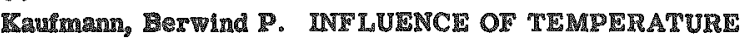
ON CPROMOSOMI RECOMBNATION FOLLOWNG X-RAIIMDUCRD RREARAGIR. Records Genetics Soc. Arm. 12, 50(1043).

Previous studies have indicated that the potenial brealss Induced in the chronowomes of the sperm of Drosophila by y-ray treatment are not utiliged in producing new rearrangements unttl after the sperm has entered the egg. This weparation in time between the periods of irradiation and recombination affords an opportunity to test experimentally the effects of varlous types of aupplementary treatment on the chromosomal changes induced by the $X$-rays. In this ahor note are presented gome of the results obtained by oxposing females, after inseminalion by sperm from males troated with 4000 roentgens of X-radiation, to controlled emperatures of either $18^{\circ} \mathrm{C}$ or $28^{\circ} \mathrm{C}$, or to the heating ef lects of near infrared irradiation. (The latter type of treatment was derigned by Dr. A. Hollaender, wnd will be deacribed in detall in a longer paper.) The effect produced by the combination treatment was measured by analysis of the galivary-gland chromosomes of the $F_{1}$ larval progeny of the treated parents. - The percentages of altered sperms and of chromomome brealss were found to be lowest $(29.5$ and 72.4 per cent respectively) following the $18^{\circ}$ treatment, womewhat higher (31 and 80.8 per cent) after exposure to $28^{\circ}$, and highest (3\%.5 and 100.8 per cent) following the near infrared treatment. These results suggest, therefore, that increased tomperature, within the range tegted, is effective during the thme that chromosome recombination is occurring in facilitating those movements that lead to the reunion of bromen ends, or to the utilization of potential brears induced by the X-ray treatment. (RG)

Kantmann, Berwind P. MODLICATION OI THE FREQUENCY OF CMROMOSOMAL REARRANGEMENTS INDUCED BY X-RAYS IN DROSOPHILA. TI. IFFECT
OF SUPPLEMENTARY TREATMENT AT THE TIME OF CHROMOSOME RECOMBINATION. Genetics 31 , $449-53(1946)$

Fggs deposited by females of D. mclanogaster that has been inseminated by males previously treated with $4,000 \mathrm{r}$ of $\mathrm{x}$-rays were exposed to near infrared radiation ( $A$ ca. $10,000 \mathrm{~A}$ ) during the period of syngany and early cleavage. A statistically significant increase, as compared with the effect of temperatures in the range from 18 to $28^{\circ} \mathrm{C}$, was obtained in the frequency of chromosomal rearrangements detected by analysis of salivary-gland nuclei. The action of the near infrared in facilitating recombination among the breaks induced by the $x$-rays is attributed to selective action on certain cellular components rather than to an extension of the range of temperature within which fertility and viability may be maintained. (BA)

\section{9}

Iaufmann, Berwind P. RADIATION INDUCED CHROMOSOME ABERRATIONS p.27-36 of CONFRERCE ON BIOLOGICAL APPLICATIONS OF NUCLEAR PHYSICS, JULY 12-27, 1948. Brookhaven National Lab. 10p. (BNL-C-4. (p.27-36))

In this report an effort has been made to outline some aspects of the problem of induced structural rearrangement, especially as revealed by cytological studies of salivary gland chromosomes of Drosophila. The various types of induced breaks have been described, together with the patterns of recombination effected by the breakage ends. Analysia of break distribution has provided information concerning the organization of the chromosome, particularly with respect to its subdivision into euchromatic and heterochromatic regions. By using such agents as u-v and near-infrared rays in combination with $x$-rays, progress has been made in evaluating the breakage and recombination aspects of the process of structural alteration. These studies indicate that breaks fall into qualitatively different groups, and that the differences are reflected in the subsequent behavior of the breakage ends. Of the various types of supplementary treatment utilized, near-infrared radiation has proved most serviceable in analysis of the cycle of breakage and recombination. It has been found that this agent modifies the processes that lead to st ructural rearrangement of chromosomes, but not those responsible for gene mutation. Accordingly, near-infrared radiation may serve as a useful diagnostic tool, if applied to organisms in which cytological analysis is not feasible in distinguishing between genetic changes that are attributable to chromosomal alterations, and those that are not. The potentialities of this portion of the spectrum for further cytogenetic studies lie in the prospect of securing intense monochromatic radiation of known physical properties. (auth)

\section{0}

TKaufjann, Berwind P. and Alexander Mollaender. ALTERATION OF THE FREQUENCY OF X-RAY INDUCED CMROMOSOMAL BREATS BY USE OF ULTRAVIOLET AND NEAR INIRARED RADIATION. Genetics 30, 1112(1945).

(The following $\mathrm{i}$ an abstract and is reproduced here in its entirety.)

It appears possible, on theoretical grounds, to modify the capacity for reattachment of endis of chromosomes disrupted by lonizing radiation, if other types of supplementary treatment are given prior to the time that new combinations are established. Drosophila is well suited for experiments of this type, since the interval between irradiation of the spermatozoa and chromosome recom - 
bination may be prolonged for many days by witholding the males from copulation. An extended set of experiments designed to measure such alterations in the frequency of detectable chromosomal breaks has now been largely completed. Ultraviolet and near infrared radiations were used following administ ration of the tolal $x$-ray dosage or in the interval between two iractional treatments. Determination of the frequency of breaks was made by analysis of salivary gland chromosomes. Ultraviolet radiation of wave length $2537 \AA$ following $x-$ ray treatment induces a reduction in the percentage of altered sperms and in the number of breaks as compared with the controls, which received only an equivalent dose of -rays. Similar reductions in the percentages of altered sperms and of chromosome breaks were obtained in some experiments in which the $x$-ray treatment was supplemented by near infrared radiation. The complete data cannot be presented here, but will form the basis of the demonstration, together with the designs of the special apparatus constructed for administering the ultraviolet and near infrared radiation.

2061

Kaumann, Berwind P. and Alexander Prollaender. MODIFICATION OF THE FREQUENCY OF CRROMOSOMAL REARRANGEMENTS INDUCEO BY X-RAYS IN DROSOPHLLA. II. USE OF ULTRAVIOLET RADIATION. Genetics 31, 368-76(1946).

Uitraviolet radiation $(\lambda 2537 \mathrm{~A})$ used subsequent to $\mathrm{x}$-rays in the treatment of the spermatozoa of 1 . melanogaster effected a decrease, as compared with the controls receiving an equivalent dose of $x$-rays, in the Irequency of chromosomal rear rangements. About $19 \%$ of the eggs failed to hatch that were deposited by females that had been inseminated by males treated with $A 2537 \mathrm{~A}$, as compared with $7 \%$ in untreated controls. The difference between the two groups is attributable to induction by the ultraviolet of the single-break type of dominant lethal. Other studies of hatchability of eggs deposiled by females that had been inseminated by males treated with either 2,000 I of $x$-rays, or 2,000 I +2537 A suggest that the effect of post-treatment in reducing the frequency of chromosomal reaxrangements is attributable to increased restitution rather than to an increase in the frequency of 2062

the single-break type of chromosomal derangenent. (BA)

Kaufmann, Berwind P., Alexander Hollaender, and $\mathrm{H}$. Gay. MODIFICATION OF THE FRYQUENCY OF CHROMOSOMAL REARRANGEMENTS INDUCED BY X-RAYS IN DROSOPHILA. I. USE OF NEAR INFRARED RADIATION. Genetics 31, 349-67(1946).

Modification of the frequency of $x$-ray-induced chromosomal rearrangements detected by analysis of the salivary gland chromosomes of D-melanogaster was effected by the use of near infra-red ( ca $10,000 \AA)$. Post-treatment of males with near infra-red accelerated the mitotic processes that make cells that were not mature sperm at the time of $x$-ray treatment avallable for transfer in copulation. Pretreatment with near infra-red increased significantly the frequency of detectable sa ray induced rearrangements as compared with the $x$-rayed controls. The higher frequency is not attributable to selective sensitization of any particular chromosome or portion thereof. Determination of hatchability of eggs deposited by females that had been inseminated by males exposed successively to near infrared and $x-r a y$ radiation suggests that pretreatment does not modify greatly the frequency of dominant lethals. Various possible modes of action of the near infrared radiation were considered. (BA)
2063

Traumann, Berwind P. and Katherine Wulson. MODIFICATION OF THIS FREQUENCY ON CHROMOSOMAL REARRANGEMENTS INDUCED BI I SOPHILA.IV. POST-TREATMENT WITH NEAR INTRARED RAOLATON. Genetics 34, 425-36(1949).

Drosophila melanogaster males of the Swedish-b stock were selected, three to five days betore their emergence, and exposed to about $5,000 x$ of $\mathbb{x}-12 a y s$, using a Coolidge tube at $85 \mathrm{kv}$. They were then mated with virgin females and discarded; hal of the females were subjected to ultraviolet radiations and the frequency of chromosomal rearrangements was determined. There was no increase in the frequency of the reas rangements due to the application of the ultraviolet pays after $\mathrm{x}$-irradiation. A comparison between these results and those obtained in similar experiments is made and a theory is postulated. 28 references. (NSA)

\section{4}

Kirby -\$S mith, J. S. EFTECTS OF INFRARED IRRADIATION ON THE IREQUENCY OF X- AND GAMMA-RAXINDUCED CHROMOSOMAL ABERRATIONS IN TRADESCANTIA POLLEN TURES. Genetics 36, 558-O(1951).

Tradescantia pollen has been treated with $250 \mathrm{kv} X$ rays and 1.3 mev gamma rays in combination with near infrared radiation in the $9500-30,000 \AA$ region. The temperature of the material during infrared radiation has been closely controlled to eliminate the possibility of thermal effects. Measurements of the infrared absorption spectra of Tradescantia pollen show that, in the preaent erperiments, an appreciable amount of infrared energy is reaching the nuclei. Typical near-infrared absorption curves will be preacnted. The chromosomal aberration irequency in the pollen tube division following irradiation has been studied. No significant difference in the aberration frequency, including interchanges, has been observed between material treated with $\mathrm{X}$ or gamma rays alone and material given near infrared radiation immediately before or after exposure to $\mathrm{X}$ rays or gamma rays.

\section{5}

Inowlton, Norman $\mathrm{P} ., J_{r}$. and Louis H. Hempelman. THE EFFECT OF X-RAYS ON THE MITOTIC ACTIVITY OT TIE ADRENAL GLAND, JEJUNUM, LYMPII NODE, AND EPIDERMIS OF THE MOUSE. J. Cellular Comp. Physiol. 33, 73-91(1849).

The effects of $250 \mathrm{KV}$ X-rays on mitotic activity of the adrenal gland, lymph node, jejunum, and epidermis of the mouse have been investigated. The data obtained are summarized in seven curves and four tables. In general a quantitive response to irradiation was observed although there is a lack of correlation between the sensitivity of the mitotic activity to ionizing radiation and the radio-sensitivity of different tissues. The mitotic activity of most radio-sensitive tissues apparently responds less to irradiation than that of tissues showing a lower degree of histologic response to the radiations. In several tissues "overcompensation" was observed in the response of the mitotic indes to irradiation; the possible mechanism for this is discussed. An attempt has been made to explain the abortive rise in the mitotic indices of the adronal gland and the jejunum by the use of the direct and indirect concept of radiation injury previously reported by llance (J. Morphology, 66, 409-422, 1940). The possibility of using the depression in mitotic activity subsequent to irradiation as a measure of the biological effects of radiations of different types is suggested. (NSA) 
2066

Kollex, P. C. and I. A. R. S. Anmed. X-RAY INDUCED STRUCTURAL CHANGES IN TME CHROMOSOMES OT DROSORHILA PSEUDO-OBSCURA. J. Genet. 44,537a(1942).

Males were treated with $4,500 \mathrm{r}$ units. Salivary grands of 425 larval of spring were then examined for induced chromosomal breaks. Changes were found in $40 \%$ of the treated sperms. The frequency of breaks per sperm is not signilicantly different from that found following 5 milar treatment of D. melanogaster. No 1-break abertations were found. Sperms with an odd number of detected breaks were less frequent than expected in comparison to those with an even number of breaks, probably because of the effects of prowimity and competition on reunion. The ratio of inversions within a single arm to interehanges between arms is higher than expected on the assumptions of equal arm lengths, equal chromosome breakability, and random distribution of breals. This is probably due to the greater inviability of interarm rearrangements. Multiple breaks within the stame sperm tend to produce several independent rear rangements rather than a single complex exchange involving all the altered chromosomes. The relative scarcity of duplications and deficiencies is attributed to their lower survival value. The XR chromosome has a lower and chromosome 4 a higher freguency of breaks than would be expected were breaks proportional to unit chromosome length. There was no significant difference in the breaks in successive broods from treated males over the age period of 10 to 25 days. (BA)

2067

IOSIn, 1. L. SOME ASPECTS OF THL BIOLOCICAL ACTION OF X-RAYS ON COCK SPRRMATOZOA.

Physiol. Z0öl. 17, 289-319(1944).

The fertilizing capacity of cock ænermatozon, as indicated by the available methods of detection, is adversely affected by the soft type of $\mathrm{x}-\mathrm{ray}$ irradiation. It is com pletely lost between 5,544 and $6,488 \mathrm{r}$. The inverse type of relationship between the fertilizing capacity and the $x$-ray dose can be expressed by a straight line of regression. In the present case the equation for the line was calculated to be $25-0.3268$ y. The coeficient of correlation between the two variables is -0.9631 . The type of fertilization is not responsible for the reduced fertilizing power of treated spermatozoa. The injurious effect of $x-r a y$ radiation is meted out directly to the spermatozoa and is not mediated via the fluid portion of the semen. The pll of the irradiated gemen is unaffected by the treatment. During the first 10 days following irradiation, longevity of the treated spermatozoa compares favorably with that of controls. The total functional life-span, however, is shortened. Motility and morphology of the treated spermatozon are not altered within the biotic range of radiation. They are modified, however, by more intensive treatment. The s-ray treatment of male gametes results in a preponderance of male chicks at hatching time irom zygotes fathered by such gametes. Zygotic mortality is closely related i, the intensity of radiation applied to the paternal germ cells. The bulk of deaths occurs during the early part of the incubation period. Lethality of the embryos fathered by the Irradiated spermatozoa is associated with increased frequency of abnormal development. Irradiation of spermatozoa with $10,000 \mathrm{r}$ has no detectable effect on their $\mathrm{QO}_{2}, \mathrm{QCO}_{2}$, and succinic dehydrogenase system. It is concluded that the ir radiation of cock spermatozoa with doses as high as $152,000 \mathrm{r}$ does not produce Hortwig phenomenon in the chicken ova exposed to such spermatozoa. The reduced fertilizing capacity is due to the increasing proportion of injured germ cells with the rising intensity of irradiation. This "dilution" of still functional gametes with functionally inert, though motile, spermatozoa eventually leads to the complete loss of fertilizing capacity by the specimen. (Author)

2068

Kostoff, Dontcho. HOMOZYGOUS TIRANSLOCATION OBTANIED IN THE SECOND GENERATION FROM MATERIAL TREATED WITH NEUTRONS. Proc. Indian Acad. Sci. 30D, 259-63(1949).

Dry seed from Crepis capillaris were irradiated with neutrons $(20,000 \mathrm{r})$. The effect on the chromosomes are reported, and compared with chromosomal damage due to x-rays. Abnormalities can be classified as follows: 1. Chromosomal dislocations. 2. Chromosomal heteroploidy. 3. Polyploidy. (NSA) 2069

Rotval, J. P. STRUCTURAL CHANGES PRODUCRD IN MICROSPORES OF TRADESCANTIA BY ALPHA-RADIATION. J. Genet. A3, 135-544(1947).

This work represents an extention of previous $x-r a y$ and $\gamma$-ray studies reported by Calcheside, Lea, Thoday, and Tolval, using o rays as the agent for production of strurtural changes. Anthers of Tradescantia were immersed in a radon soln. of known conc. for measured periods of time. Material was prepd. for microscopic study $24 \mathrm{hr}^{\mathrm{v}}$. after ipradiation using Benda's fixative and crystal-violet iodine stain. Structural changes noted resemble those observed following $x$-radiation and $\gamma$-radiation. In number they increase linearly with the dose. The number of changes in which there is an incomplete fusion of broken ends, how ever, is far greater than observed following other types of radiation. Since the total number of aberrations primarily produced is greater following a given dose of alpha-radialion than with other types, it is suggested that alpha particles passing close to the chromatid thread may be effective in producing breaks, as well as those which pass directly through it. 2070

Kotval, J. P. and L. M. Gray. STRUCTURAL CHANGES PRODUCED IN MCROSPORES OF TRADESCANTEA BI ALPHA-RADIATION. J. Genet. 48, 135-54(1947).

The structural changes seen at metaphase 24 hour after the Irradiation of microspores of Tradescantia bracteata by known doses of $a$-radiation have been studied. The types of structural change produced resemble those seen at the same interval after $\gamma$-ray, $x$-ray and neutron treatment, and have been classified as chromatid and isochromatid breaks, or chromatid exchanges, following Catcheside, Lea, and Thoday. The number of structural changes of all three types increases linearly with the dose. The proportion of structural changes in which fusion of broken chromatid ends is incomplete is considerably greater than observed previously with any other types of radiation. Even when allowance has been made for this fact the number of structural changes primarily produced by a given dose of $a$-radiation is estimated to be much larger than would have been anticipated from earlier studies with $x-1$ ays and neutrons, and suggests that those $a$-particles which pass close to the chromatid thread as well as those which pass through it have a significant probability of beeaking the thread. The new experimental data direct attention to $\alpha$-rays arising from the radioactivity of soil and living tissue as the possible, origin of certain types of "spontaneous" chromosome aber rations. (Authors" Summary)

2071

Lasnitzki, I. THE EFECT OF $\$$-RAYS ON CELLS 
CULTIVATED IN VITRO. II. RECOVERY FACTOR.

Brit. J. Radiol. 16, 61-7(1943).

Material from the choroid and sclerotic of $9-11$ day chick cmbryo was grown in a medium of fowl plasma and chick embryo extract. Cultures for test were paired on a basiss of mitotic activity; one of each pair was irradiated, the other used as control. Exposures of $100 \mathrm{r}$ of $160 \mathrm{kv} x-$ rays were made singly, twice separated by $48 \mathrm{hr}$. and twice separated by 96 hrs.; exposures of $1000 \mathrm{r}$ were made singly or twice separated by 10 days. Changes were observed for 10 days -5 sub-rultures. Irradiation was followed by a narhed fall in normal cell division with a temporary small wave of abnormal mitosis. Subscquently there was stme rewecurrence of fell division, the appearance of degenerate cells, and then a 2nd wave of abnormal mitosis. Recovery occurred in 48 hrs. after a dose of 100 $r$, and in 10 days after $1000 \mathrm{r}$. If previously irradiated cultures were stbjected to a 2nd dose equal to the first as soon ais the culture showed apparent recovery, the effect of the 2 nd doss was greater than that of the first. If the intreval was prolonged, the effects of the 1 st and 2 nd doses were apparently equal. (BA)

2072

LAsnitzki, I. THE RESPONSE OF CELLS IN VITRO TO VARIATONS IN X-RAY DOSAGE. Brit. J. Radiol. 16, $137-41(1943)$.

Cells from the choroid and selorotic of $9-11$ day old chek embryos, grown in equal parts of fowl plasma and chick embryo extract, were 1 rradiated with $160 \mathrm{kv}$ lightly filtered $x$-rays, and subsequently fixed. The culls in several fields just mbide the periphery we pes counted and classifind as resting, degenerative, and mitotie. For doses from $100 \mathrm{~m} 1000 \mathrm{~g}$, temporary inhibition of matosis was observed; for 2500,5000 , and $10,000 \mathrm{r}$ there was no mitotic recovery, but large numbers of degenerated cells. The time dt which these latter appear indicates that the degeneration is due th the breakdown of cells in the resting condition. (BA)

2073

LasnitzRi, I. RECOVERY OF CELLS FROM RADIATION INJURY, P.163 Of INTERNATIONAL CONGPESS OF RADIOLOGY, 6th Congress, London. 1950.

The restor ation of normal cell counts does not necessarily mean full recovery has becurred. A second dose, identical with the first, given as soon as mitotic activity reaches normil, sometimes produces a greater effect than the first, suggesting that the cells are still damaged at the time the second dose is given. Thus, recovery of chick fibroblasts exposed in vitro to a dose of $100 \mathrm{r} \gamma$ rays is apparently complete 48 hours after radiation. A second dose of $100 \mathrm{r}$ units given at this point when the cell counts have returned to normal produces a greater effect than the first one. Complete recovery, as indicated by a response to the second dose similar in all resperts to that obtained after the tirst, is observed only if the interval between the exposures is extended to 96 hours. The rate of recovery depends not only on the dose given but also on the doserate used and whether cells are irradiated in vivo or in vitro. Doses of 30 to $60 \mathrm{x}$ units given at the rate of $1 \mathrm{x}, 4 \mathrm{r}$ and $15 \mathrm{r}$ per day failed to produce any effect on chick fibroblasts exposed in vitro, suggesting that at these low dose rates of recovery keeps pace with the rate at which the radiation changes are produced within the cells. Cells exposed to duses of $100 \mathrm{r}$ and $2,500 \mathrm{r}$ given at $9 \mathrm{r}, 30 \mathrm{r}$ and $100 \mathrm{r}$ /minute show a rate of recovery which varies directly with the dose-rate after the smaller dose and inversely with the dose-rate after the hyph dose. Tumour cells exposed in vivo recover more quickly after a small dose which does not involve the vascular system than similar cells exposed in vitro. If the dose is sufficient to damage the blood circulation, recovery is delayed in vivo as compared with the same type of tumour cells irradiated in vitro.

2074

Lea, D. E. and D. G. Catcheside. THE MECHANISM OF THE INDUCTION BY RADIATION OF CHROMOSOME ABERRATIONS IN TRADESCANTUA. J. Genet. 44, 216$45(1942)$.

Experimental data from $\mathrm{x}$-ray and neutron experiments are used to develop a quantitative theory of the production of chromosome aberrations in Tradescantia by irradiation during mitotic prophase or resting stage. The data avallable show that neutrons are more efficient than $\mathrm{x}-\mathrm{rrays}$ for equal numbers of ionizations in the tissue. From the data it is calculated that approsimately 17 ionizations must occur within the chromatid to produce a break, 8 rays become important if hard radiation is used. Isochromatid bre ; are produced, according to this theory, by simultar.. as breakage of sister chromatids traversed by the same ionizing particle. Hence, as the data show, they are produced with far less frequency by $x$-rays than by an equivalent dose of neutrons, it being relatively unlikely that a given elec. ron tail will traverse both chromatids plus the space, if any, between them. It is calculated that probably $45 \%$ of the isochromatid breaks are detectable, but only $10-15 \%$ of the primary chromatid breaks and only about $5 \%$ of the primary chromosome breaks. The remainder rejoin or form interchanges not detectable cytologically. The period of rejoining is variable but averages about $3-\frac{1}{2} \mathrm{~min}-$ utes. The distance between breaks that take part in the same interchange may be as great as 1 or $2 \mu$, the probability of interchange varying inversely with the initial dis tance between the breaks. With $x$-rays the two breaks are usually caused by separate ionizing particles; with neutrons by the same one, although at high dosages the proportion of 2-hit interchanges becomes of importance. On the basis of the theory, $x$-rays of $4.4 \mathrm{~A}$ should be a maximum efficiency in producung chromatid breaks, with a decrease of relative $20 \% 5$ efficiency to one half at approximately $5.2 \mathrm{~A}$ and $2.5 \mathrm{~A}$. (BA)

Lindemann, B. INVESTICATION OF X-RAX HAEMOLY SIS BY THE ELECTRON MCROSCOPF, p.166 of INTERNATIONAL CONGRESS OF RADIOLOGY, Oth COngress, London. 1950.

Two interpretations of X-ray haemolysis are possible; either the erythrocyte membrane is denatured by the rays, or the membrane is sensilised against active ions. This problem has been investigated by electron-microseopic observations of irradiated erythrocytes. It has been found that radiation produces changes in the membrane similar to those produced when proteins are denatured. X-ray haemolysis is an osmotic, i.e., a diffusion phenomenon. Investigations with the electron microscope show that this haemolysis can be measured not only by the number of cells affected but also by the amount of haemoglobin liberated from each individual red cell. The "all or nothing" law, therefore, does rot apply to an osmotic haemolysis. The threshold dose for X-ray haemolysis is of the order of $6,000 \mathrm{~g}$. Calculating from cell volume on the basis of the target theory, the amoun of absorbed irradiation necessary to cause haemolysis leads to the conclusion that hapmoglobin loss in an individual red blood cell is not due to a "direct hit" but is an expression of the biological variation among the cells of the population exposed. 2070

Longley, A. C. CXTOLOGICAL ANALYSIS OF TRANS- 
LOCATIONS IN CORN CHROMOSOMES RESULTING FROM IONIZING RADIATION OF" THE TEST ABLE ATOMC BOMB AND X-RAXS AND OF TRANSLOCATIONS FROM OTHER SOURCES. Naval Medical Research Section, Joint Task Force One. June 10, 1950. 60p. (NP-3072)

Of the 588 chromosome translocations in corn described, 220 came from various sources, 179 from seeds treated with measured dosages of $\mathrm{x}$ rays, and 172 from seeds exposed to ionizing radiations of the Test Able bomb. The nonrandom distribution of the Bikini-induced translocations was different from that of the $\mathrm{x}$-ray-treated controls. Chromosome arms staining heavily adjacent to the fiber attachment had more breals than expected when treated with $x$-rays and fewer breaks than expected when exposed to the Bikini radiation. Chromosome arms staining lightly throughout their length had fewer breaks than expected when treated with $x$ rays and more than expected when exposed to Bikini radiations. A comparison of the position of breaks resulting from low, medium, and high dosages of $x$ rays indicates that the proportion of breaks in heavily staining areas is greatest when the dosage is highest, and in lightly staining areas is greatest after low dosages. The position of the breaks in Bikini-treated material indicates that the dosage intensity was intermediate between the low and medium $\mathrm{x}$-ray dosages studied. Among the whole population of translocations there was a tendency for breaks the same distance from the fiber attachment to be associ2077 ated more frequentiy than expected on a random basis.

Marinelli, L. D., B. R. Neble, N. Giles, and D. R. Charles. CHROMOSOMAL EFFECTS OF LOW X-RAY DOSES ON IPIVE DAI TRADESCANTIA MICROSPORES. Am. $J$.

\section{Botany 29, 866-74(1242).}

Quantitative studies have been made on the chromosome aberations, i.e. dicentrics (D), rings $(R)$, and isodiametric fragments (I. F.) produced by $x-$ radiation on the $m i-$ crospores of $T$. canaliculata. The material was rayed at interphase previous to the onset of nuclear enlargement and observations were made at metaphase and anaphase. The time factor was investigated by delivering different doses of radiations at constant intensity or in constant time and by delivering a given dose at different intensities. The results obtained as well as some of the data of other authors, are consistent with assumption that each D. R. or I. F. is the result of 2 chromosome breals and that each break has a definite probability of healing. This probability seems to be independent of the radiation dose. In the course of the mathematical analysis it is shown that 2 irradiations at high intensity separated by a variabie rest period will yield the most direct information of the rate of recovery. (Authors) 2078

Marquardt, Hans. THE DISTRIBUTION OF X-RAY INDUCED CHANGES IN THE CHROMOSOMES OF BELLIEVALIA ROMANA. Ber. deut. botan. Ges. 60, 98 124(1942).

Roman hyacinth was $x$-rayed several days before nuclear division, and 2,700 of the subsequent mitoses studied. The chromosomes are large and easily studied. All chromosomes are equally liable to alteration by $\mathrm{x}$-rays, except that the frequency of breaks and translocations is proportional to the length of the chromosomes. Breaks tend to occur more often in the commissure-to-subterminal end than in the subterminal-to-median region. Similarly, translocations increase in frequency from the subterminal end to the commissure, in all four chromosomes. Fxchange of similar regions between homologous chromosomes is more likely than are translocations within a chromosome. (BA)
2079

Marshak, A. ALTERATION OF CHROMOSOME SENSI-

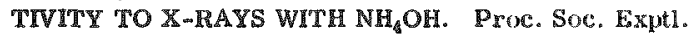
Biol. Med. 38, 705-13(1938).

Seedlings of Vicia faba and Allium copa wh treated with different concen. of $\mathrm{NH}_{4} \mathrm{OH}$ before and duming irradiation with $\mathrm{x}$-rays. The \% chromosome abnormalities in anaphase at 3 hrs, after irradiation was greatly reduced. Curves for $\%$ abnormalities as a function of ammonia concentration had a plateau in the middle range although the curves for abnormalities produced by ammonia alone did not show any. The maximum o abnormalities after treatment with ammonia alone was observed in $1 / 2 \mathrm{hr}$., while with $\mathrm{x}$-rays alone the maximum was observed in $2^{1 / 2}-3 \mathrm{hrs}$. after irradiation. Generalized swelling of chromosomes and "unraveling" of chromonemata were not found. $\mathrm{CO}_{2}$ in concns. up to 80 vols. \% produced no alteration in the \% abnormalities induced by $\mathrm{x}-\mathrm{rays}$. Abnormalities were apparently produced by $x$-rays throuph the action of electrons on positively changed groups of the separating surfaces of the dividing histories. From the ammonia curves it might be possible to determine the amino groups involved in the $x$-ray response. (BA) 2080

Marshak, Alfed. RELATIVR EFIECTS OF X-RAYS AND NEUTRONS ON CHROMOSOMES IN DIFFERENT PARTS OF THE "RESTING STAGE". Proc. Natl. Acad. \$ci. U. S. 28, 29-35(1942).

The per cent normal anaphase chromosomes is a negative exponential function of the dose of neutrons or $\mathrm{X}$-rays in different parts of the nuclear cycle. Chromosome abnormalities are, if allowance is made for multiple hits, therefore directly proportional to the dose at all stages studied. 2. The ratio of neutron to $x$-ray efficiency $(n / x)$ is constant for different species at the onset of the prophase, but increases by actor of at least two during the nuclear resting stage of single species. 3 . Increase in $n / x$ during the resting stage indicates: Conditions for chromosomes response are not constant during resting stage. The intrachromosomal structure responding to ionization may be different for the different parts of the nuclear cycle and resting stage; a theory invoking a single mechanism for the production of chromosome abnormalities is not adequate. The relative effect of neutrons on chromosomes in the resting nucleus is greater than with $x$-rays. Neutron therapy may produce regression in tumors which do not respond to $\mathrm{x}-\mathrm{z}$ ays. The daily "safe dose" for exposure to neutrons may be considerably less than $x$-rays. 4. The use of neutrons and $\mathrm{x}$-rays as described furnishes a method for identifying the functional stages which cannot otherwise be identified. (BA)

2081

Marshak, A. and M. Bradley. X-RAY INHIBITIONS OF MTTOSIS IN RELATION TO CHROMOSOME NUMBER.

Proc. Natl. Acad. Sci. U. S. 30, 231-37(1944).

The authors report evidence that inhibition of mitosis is dependent upon the number of chromosomes in the cell. Root tips of three species of Triticum (T. monococcur, T. dicoccum, and $T$. vulgare) and two species of Bromus (B. carinatus and $\bar{B}$. arizonicus) were used. Source of $\mathrm{x}-\mathrm{rays}$ was an oil-cooled tube with a tungsten target in G. E. Company Maximar apparatus, operated at $220 \mathrm{kvp}$ and $15 \mathrm{ma}$. The beam was filtered through $1 \mathrm{~mm} \mathrm{Al}$, and a lead diaphragm $1 \mathrm{~cm}$ thick and with a $10 \times 10 \mathrm{~cm}$ aperture was used at the base of the cone which was $17.5 \mathrm{~cm}$ in length. The target distance was $85 \mathrm{~cm}$. Irradiation was given at the rate of $43 \mathrm{r} / \mathrm{min}$. Results show that inhibition of milosig by $\mathrm{x}-\mathrm{r}$ ays is inversely proportional to the number of chromosomes in the species studied; inhibition of 
mitosis is neither directly nor inversely proportional to the chromosome length. The evidence indicates that in hibition of mitosis by $x$-rays oceurs through action upon the centromeres. The significance of these findings in connection with sensitive volume calculations is discussed. (S.V.S.)

2082

Maxshak, A. and W. S. Malloch. THE IFIFCT OF FAST NEUTRONS ON CHROMOSOMES IN MEIOSIS AND ITS BDARING ON PACHYTENE PAIRING. Genetics 27, 57683(1942).

Microsporocytes of Vioia faba were treated in pachytene with various doses of neutrons and the freguency of chromosome abnormalities in anaphase I determined. A method of estimating the duration of the different neiotic stages is deseribed. The log per cent cells, with no chromosome abnormalities, is a negative exponential function of the dose. The slope of this "survival curve" is the same within the statistical limits of error as for mitotic chromosomes at the onset of prophase. Comparison with previous results shows that the ratio of neutron to x-ray sensitivity is approximately for somatic chromosomes of several spp. but varies by more than a factor of 2 in the same sp. depending on the stage of mitosis treated. Since the ratio is approximately 6 for both meiotic and mitotic chromosomes of $\mathrm{V}$. faba, it is concluded that a similar functional condition with respect to lonizing paricles exists in the chromosomes at pachytene and at the onset of the mitotic prophase. This condition may be the presence of closely approsimated chromonematic surfaces at both these stages. In seeking explanations of chromosome pairing and crossing-over, consideration should be given to forces approm priate for structures of these dimensions. (BA) 2083

Mikaelsen, Knut. THE PROTRCTVE ERECT OF GLUTATHIONE AGAINST RADIATION INDUCED CHROMOSOME ABERRATIONS. Brookhaven National Lab. [nd] 8p. (BNL-1111)

Cuttings of Tradescantia paludosa were grown in solutions of different concentrations of reduced glutathione during a 48-hour exposure to continuous $\gamma$-radiation from a 3-curie $\mathrm{CO}^{60}$ source al a dose of 25 r per day. The results illustrated clearly that there was a positive correlation between increased glutathione concentrations and decreased fragment frequency between $1 \times 10^{-5}$ and $3 \times 10^{-4}$ M. The latter concentrations reduced the number of frogments produced to about 50 per cent of that in the irradiated controls. (NSA)

Mottram, J. C. VARTATIONS IN THE SENSITVVITI OF THE CELL TO RADIATON IN RELATION TO MTTOSIS. Brit. J. Radiol. 8, 643-51(1935).

Tumor cells vary greatly in sensitivity to radium irradiation when submitted to conditions which affect cellular respiration, exposure to cold or to $\mathrm{HCN}$ increasing the radiosensilivity, and $\mathbb{N}$ or $C O$ anaerobiosis decreasing The mitotie activity of the cells has been observed under these controlled conditions to determine whether there is any correlation between sensitivity and state of mitosis at the time of irradiation. None was found. HCN and anaerobiosis both cause great decrease in the number of dividing cells; cold has no effect on this number. (BA) 2085

Nabour's, Phobert $\mathrm{K}$. and Iorence M. Stebbins. CRROMO SOME ABERRATIONS AND VIABILITY WN APOTETTIX EURYCRPHALUS HANCOCK. Genetics 25, 129-30(1940).

The X-ray induced translocations of chromatin have been reported, one with the exchange between first and fourth autosumes, with depletion in amount of chromatin for the former and augmentation for the latter (1933); the other between a first autosome and a sex-chromosome, with increase in chromatin and probably an extra traction fiber attachment for the latter. The autosome of this translocation was lost (1935 and unpublished). The three suxviving aberrator's are designated tentatively as I. donor(a), 2. receptor(a4), and 3. receptor(X). The genetic and cytological data correlate (Robertson, 1935, and unpublished). Zygotes carrying donor(al), and otherwise normal, hatch but rarely survive to $3-4$ instar when they are recorded (14 donor(aI): 2443 normals). Receptor(ady) are 13 percent, and receptor(X) 14 percent, less viable than controls. Donor(aI) +receptor(X) are 38.5 percent below normal. Donor(aI) + receptor(aA) + receptor(x) fall 17 percent below controls. The data comprise approximately 50 other items of comparison of 15,088 aberrators with 49,000 normal, and with each other. In practically all cases augmentation of chromatin is accompanied by less mortality among males than females, and vice versa when there is a depletion of chromatin. There is indication, but not yet definite proof, that the XO(absence of $\mathrm{Y}$ ) chromosome condition in grouse locusts is responsible for the significantly lower viability, and perhaps for the much smaller size, of the males among the controls in grouse locust breeding. (G) 2086

Nebel, B. R., G. B. Wilson, and L. Marinelli. X-RAY DOSAGE CURVES IN TRADESCANTIA. Genetics 27, 158 (1942).

Three independent dosage curves have been plotted for the action of $\mathrm{X}$-rays on Tradescantia microsporocytes observed $4-5$ days after raying. The first set of exposures was made by G. B. Wilson in Geneva, N. Y. early in 1940 and scored in Montreal in 1941. The second set of data was collected by $B$. R. Nebel also in Ceneva from plants grown in constant environment. The third set of data was collected from radiation applied to cut heads of Tradescantia at the Menorial Hospital by L. Marinelli. Within the limits of the three experiments environmental differences were lound to be unimportant. The method of radiation is held responsible for the results obtained. At Geneva the radiation was intermittent the of period being from $30^{\prime \prime}$ to $90^{\circ}$ with the on period of equal or shorter duration. The intensity of the on period varled between 50 and $200 \mathrm{r} / \mathrm{m}, 100$ $\mathrm{r} / \mathrm{m}$ being the most common intensity used. - The WilsonGeneva and the Nebel-Geneva data are identical. They were scored independently. For rings and dikinetic translocations above 100 io the Geneva data show a straight line ( $\left.\mathrm{K}^{-1}\right)$ for the relationship between dose and effect. For isodiametric fragments the independent data of Wilson and Nebel show a more than linear rise for dose and effect $-K^{2}$ aps. $3^{-}$ A more than linear rise $\left(\mathrm{K}^{\mathrm{l}}{ }^{\circ}\right)$ for rings and dikinetic translocations plotted against dosage was obtained from the Nebel-Memorial Hospital experiment. The same experiment showed a value of $\mathrm{K}^{2}$ for isodiametric fragments. These results were obtained by apolying $110,200,300$ and 400 r with varying intensity and continuous exgosure over a total time of two minutes for every application. (G) 2087

Newcombe, H. B. THE ACTION ON X-RATS ON THE CELI. TI THE EXTERNAL VARIABLE. T. Genetics 43 , $237=48(1942)$.

A study of $x$-ray induced chromosomal changes in the pollen-grain mitosis of Tradescantia shows that simple chromosome breaks (fractures) vary as the square of the dose or above; while 2-break changes (new reunions) vary only as the 1.5 power, approximately. Minute fragments, which from the evidence include a large proportion of small zings behave in an intermediate fashion. All the ob- 
served types vary at a higher power of the dose when the duration of the dose is kept constan than when the intensity is constant. The intensity effect, since it applies to both 1- and 2-breals changes, cannot be due simply to healing of broten ends or to competition between restitution and reunion. If is attributed to an inimbicion of both the restitution and the new reunions of broken ends by the irradiation, the inhibitory sfect increasing with dosage more rapidly when 2089

the dose is administered more rapidly. (BA)

Nichols, Charles, Jr. THE EFPTCTS OF AGE AND IRRADIATION ON CHROMOSOMAI ABERRATIONS IN ALLIUN SEIDD. Am. J. Lotany 29, F55-2(1942).

Chromosomal aberrations appearing in the primary divisions of root tip cells of five variations of A. cepa were analyzed. Aging of the seed was found to result in an increase in the number of aberrant cells. Ir tadiation of dry seed also caused an increase in the number of aberrations. No correlation was found between the age of the seed and its radio-sensitivity. Delayed germination of seeds following irradiation caused a significant increase in the number of aberrations except in cases where iresh seed were used. The number of aberrations was found to be greater when the moisture content of the seed was increased during the period of delayed germination. In addition to $\mathrm{x}$-rays, physiological factors such as age of seed and moisture content are of importance in bringing about structural changes în the chromosomes. (BA) 2089

Parker, D. R. OBSRRVATIONS ON CROSSTNG OVER INDUCED BY X-RAYS IN MALES OF DROSOPHILA. Cenetics 33, 304-10(1948).

Relationship between dosage and frequeney of induced crossing over was shielded. Inducing crossing over in the male was found quite restricted in that only a short region of the chromosome can be involved at any time. This is indicated by the preponderance of doubles involving adjacent regions of crossing over, as well as by the occurrence of clusters of crossovers involving adjacent regions. The irequency of double crossing over failed to increase greally with increased dose. This is to be expected if crossing over is restricted to a region shorter than one marlsed region. The occurence of several crossovers in the progeny of others is correlated with the presence of growth stages in some testes and absence from the testes of other males of similar age. The cluster effect may be correlated with the similarity of stage of maturation of all cells in a cyst. All cells in one cyst might be similar in their crossing over restrictions at any time. Induced crossing over may be interpreted as a result of chromosome breakage by $\mathrm{X}$-rays, with the frequency/dose reIationshp indicating that both breaks involved in one exchange are produced by a single particle. The absence of lethal position effects does not rule out the explanation of direct breakage by X-rays if both chromatids are broken at the same locus. (NSA)

\section{0}

Rasch, Filen Myrberg. NUCLIPAR AND CELL DIVSION IN ALLIUM CEPA AS INITUENCED BY SLOW NEUTRONS AND X-RAYS. Botan. Gaz. 112, 331-48(1951).

Bulbs of Allium cepa, variety chbenezer, were exposed to x-ray dosages of $100 \mathrm{r}, 1000 \mathrm{r}$, or $10,000 \mathrm{r}$ or to slow neutrons produced in the Argonne heavy-water pile. The neutron-treated material was exposed to an average neutron flux of $5.8 \times 10^{10}$ slow neutrons/sq $\mathrm{cm} / \mathrm{sec}$ for periods of $2,4,8$, or 16 minutes. Plants from treated bulbs in both series were markedly retarded in growth with respect to conirol plants. All plants from bulbs treated with slow neutrons died as did also those treated with the highest dosage of $\mathrm{x}$-rays. Bulbs from control and treated serieg were sprouted in soil. Cytological analysis of root tips collected at 48-, 52-, and 96-hour intervals showed that the effects of both types of treatment followed a pattern that was chronologically determined: a general, but temporary, inhibition of mitosis; abnormal nuelear division necessitated by structural chromosome aberrations; abnormal cell division directly following aberrant nuclear division; and, lastly, degeneration of nuclei and cells which had been severely damaged either by the original treatment or by disorientation in normal cell processes during the preceding stages. Chromosomal aberrations of all types - dicentries, centric and acentric rings, acentric iragments, and dof deletions - were observed in root-tip cells from both treatments. Dosage had no agparent effect on type of aberrancy induced. Pycnosis of many nuclei at the higher dosages was of common occurrence. Aberration frequency induced by 2-, 4-, or 8-min. exposures to slow neutrons was higher in all cases than that produced by either $100 \mathrm{r}$ or $1000 \mathrm{x}$. Frequencies in the 10,000-r series approximated those found for the 16-minute dosages with slow neutrons. (Author's Summary)

2091

Rîck, Charles, M. X-RAY INDUCED CHROMOSOME DELETIONS IN RELATION TO MUTATION FATE IN TRADISCANTHA. Cenetics $25,132(1940)$.

Small spherical fragments, independent of other chromosome aberrations, result from $\mathrm{X}$-radiation of Tradescantia microspores. Evidence from alze and shape indicates most of these to be interstitial deletions of ring structure. Their frequency within the dosage range of $100 \mathrm{r}$ to $600 \mathrm{x}$ indicates that they are conditioned mostly by 2 hits and that they are produced at randon. $\mathrm{X}$-ray treatment at $3^{\circ} \mathrm{C}$ induces significantly more deletions than the same dose at $30^{\circ} \mathrm{C}$. The effect of $\mathrm{X}$-radiation during the interkinesis preceding and the one following meiosis on the size of subsequently developed pollen grains was studied. Variability following both treatments is significantly greater than in controls. Variability at low temperature exceeds that at high temperature. Pollen size is interpreted as, at least in part, genetically self-determined. Since minute deficiencies are inseparable in genet ic behavior from point mutations, the close parallel between fragment frequency and variability in pollen size suggests that the latter is a measure of mutation rate. (G)

2092

Rick, C. M. ON THE NATURE OF X-RAY INDUCED DELETIONS IN TRADESCANTIA CHROMOSOMES.

Genetics 25, $466-81(180)$.

Small spherical tragments, independent of other chromosome aberrations, result from $x$-radiation of Tradescantia microspores. Evidence from size and shape indicates most of these to be interstitial deletions of ring structure. Then frequency within the cosage range of $100 \mathrm{r}$ to $600 x$ indicates that they are conditioned mostly by 2 hits and that they are produced at random. X-ray trealment at $3^{\circ} \mathrm{C}$. Induces significantly more deletions than the same dose at. $30^{\circ} \mathrm{C}$. The effect of $\mathrm{z}$-radiation during the interhinesis preceding and the one following meiosis on the gize of subsequently developed pollen grains was studied. Variability following both treatments is significantly greater than in controls. Variability at low temperature exceeds that at high temperature. Pollen size is interpreted as, at least in part, genetically seli-determined. Since minute deficiencies are inseparable in genetic behavior from point mutations, the close parallel between fragment frequency and variability in pollen size suggests that the latter is a measure of mutation rate. (C) 
2093

Rick, Charles. CYTO-GENETIC CONSEQUENCES OF X-RAI TREATMENT OF POLLEN IN PETUNIA.

Botan. Car. 104, 528-40(1943).

Pollent of petunia $(2 \mathrm{n}-14)$ treated with 10,000 10 100,000 ir $x$-rays was applied to stigmas of untreated plants. Pollen exposed to $50,000 \mathrm{I}$ functioned to produce viable seed. The half-kill dose for the number of plants produced per flowers pollinated was ca. 9,000 r. Tests applied to $\mathrm{X}_{1}$ and $\mathrm{X}_{2}$ showed no evidence of pseudogamous development of the egg or of diploid indwiduals from eggs in which a doubling of the chromosome number had occur sed. Thirty-three $\mathrm{X}_{1}$ plants exhibited gross chromosomal aberrations; $1 / 3$ of these were root-shoot chmeras, the difference usually involving the presence or absence of a fragment chromosome. Of three monosomics obtained the odd chromosome bohaved as a univalent in one and was usually associated in a trivalent in the other. "The trivalent pairing is attributed to duplication in the normal comple2094 ment of Petunia. (BA)

Riley, Herbert Parkes and Norman H. Giles, Jr. THE EFFECT OF OXYGEN ON THE IREQUENCY OF X-RAY INDUCED CHPOMOSOMAL ABERRATIONS IN TRADESCANTIA MICROSPORES. Genetics 35, 131-2(1950).

A study has been made of the effect of oxygen on the frequeney of $\mathrm{X}$-ray induced chromosomal aberrations (interchanges and interstitial deletions) observed at the four-tofive-day interval following irradiation of microspores. Initial comparative exposures made in a $\mathrm{r}^{\circ}$, in nitrogen, and in oxygen demonstrated that aberration frequencies were matkedly reduced in nitrogen and increased in oxygen as compared with air. Furher test exposures in a vacuum and in other gases such as helium and argon also resulted in greatly reduced aberration frequencies, indicating that the availability of oxygen has a marked effect in increasing the radiosensitivity of Tradescantia chromosomes. Additional experiments have been performed comparing the aberration frequencies in two sets of inflorescences one kept in a vacuum both during and following irradiation and the other irradiated in a vacuum with pure oxygen being introduced under pressure immediately following the radiation exposure. The fallure to find any inereased aberration frequency in the second set, to which oxygen was added after ir radiation, suggests that the effect of oxygen in increasing aberration frequency is exerted on the initial breaksge mechanism rather than on the recovery proeess. (G)

\section{5}

Riley, Herbert Parkes, Norman H. Giles, Jr. and Alvin

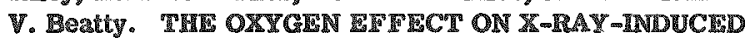
CHROMATD ABIRRATIONS IN TRADESCANTIA MICROSPORES. Genetics 36, 572-3(1951).

Comparative $\mathrm{X}-\mathrm{ray}$ dosage curves for the various types of ehromatid aberratins (mierospores exanimed at 24 hours following ir radiation) have been obtained following exposures of influorescences in atmospheres of pure oxygen and of pure helium. A constant $X-$ ray intensity of 300 3. per minule was used, and four dosages were administered - between 25 and $150 \mathrm{r}$ in oxygen, and between 50 and $450 \mathrm{r}$ in helium. A linear relationship between aberration frequency and dose was obtained for both isochromatid and chromatid aberrations in both the presence and the absence of oxygen. Abertation frequencies were higher in oxygen in both instances, but the magnitude of the difference was much greater for isochromatid types $(2.6 \mathrm{k})$ than for chromatid types (1.4 K). Noninear relationships were obtained for chromatid interchanges in both the presence and the absence of oxygen, the exponents of the dosage curves being approximately the same (1.7) in both cases. For equivalent dosages, chromatid interchanges were almost four times as frequent in oxygen as in helium. Exxperiments have aiso been performed on the effect of intensity on aberration yield in the presence and in the absence of oxygen. In oxygen, a constant dose of $150 \mathrm{~s}$ was administered in $1 / 2$ minute, 3 minutes, 15 minutes, and 30 minutes. In the absence of oxygen (in mitrogen), the same times of exposure were used, but a constant dose of $400 \mathrm{~g}$ was given in order to obtain conparable levels of biolog:cal response. There was no effect on intensity on the frequencies of chromatid and isochromatid aberrations. Chromatid exchanges decreased in frequency at lower intensities, generally similar types of curves being obtained for the two exposures. The results to date of the intensity experiments suggest that the average restitution time for broken ends is the same in the presence and in the absence of oxygen, and thus support the view that oxygen exerts its effect by increasing the initial frequency of breaks rather than by modifying the behavior of broken ends during the 2096 recovery process.

Rothfels, K. $_{\text {. }}$. SPONTANEOUS AND INDUCED CHROMOSOME ABERRATIONS IN THE GRASSEOPRER CHLOEALTIS CONSPERSA. Genetics 35, 687(1950).

An abstract of this paper appeared in Genetics, 35: 687 $687(1950)$ Nov. and is reproduced here in its entirety.

The incidence of spontaneous chromosome aberrations in first meiotie metaphases of Chloealtis males is estimated to be 0.1 incomplete breaks, 0.01 complete breaks, and 0.01 interchanges per cell. After an 8 -ray dose of 100 $r$ the following classes of induced chromosome aberrations come successively into maximum: (1) incomplete breaks (24 hr), (2) "Glickiness" (5 days), (3) complete breals (10 days), (4) interchanges (15 days), (5) incomplete nuclei (17 days). Over the range 2 to $300 \mathrm{r}, 0.05$ incomplete breaks are obtained per nucleus per $r$. If the dose-effect curve remains linear at even lower doses, natural ionizing radiations cannot account for more than $1 \%$ of the spontaneous abnormalities of this type. Similar conelusions were obtained with regard to the complete breaks and interchanges. These findings were tested directly by exposing grasshoppers at various distances from a $\mathrm{Co}^{60}$ source for 20 days. A dose rate of $500 \times$ background was required to produce a clearly significant increase in chromosome aberrations over control animals. (NSA) 2097

Rugh, Roberts. THE IMMRDEATE AND DRLAYTD MORP MEIOTC CHROMOSOMES. J. Cellular Comp. Physiol. 36, 185-203(1950).

The mitotic and meiotic stages in spermatogenesis of the Japanese fire salamander, Triturus pyrthogaster, were sludied Immediately and after an interval of 48 hours following $x$-radiation at 5,000 and 50,000 r. The chromosomes of this salamander are among the largest knowr, and, since spermatogenesis is a rather continuous cyelic process, it is possible to observe the effect of -irreadiation on chromosomes at various stages of their synthesis. X-ixradiation inmediately affects the formed chromosomes in that it makes them less viscous, more thid, and sticky. Their fluid nature is seen in the lact that they tend to flow out along the attached spindle fibers, losing their identily in shape. Their sticky nature is seen in the raet that the chromosomes beeome clumped logether. The spindle tibers are normally very difficull to locate and identify but following $\mathrm{x}$-inpradiation and the Peulgen lest 
for thymonucleic acid, they are heavily stainable due to the fact that they are coated by the very fluid chromosomal material to which they are attached. The chromosomes, once formed, seem to show movement, even following $x-$ irradiation, because the end point of the post-inradiation damage in most cells is the pycrotic nueleus. This is most frequently seen in the secondary apermatocytes. There is no evidence that chromosomes, $x$-irradiated to 5,000 r or more, even seturn to the premelotic on non-discrete phase. Stages in spermatogenesis which show no formed chromosomes at the time of irradiation show some fragmentation of the linin network, but this is difficult to quantitate or evaluate. It is the contention of this author that since the intermitotic stages never enter mitosis, following $\mathrm{x}-\mathbf{i r r a}$ diation to 5,000 r or more, that they must also be radiogensitive, as are the mitotic (e.g., metaphase) stages, and the criterion of visible chromosome damage is not exclusive evidence that the non-discrete phases in chromosome synthesis are not also radio-sensitive. (NSA)

2098

Sax, ILAR. AN ANALYSIS OF X-RAT INDUCED CHROMOSOMAL ABERRATIONS IN MRADESCANTIA.

Cenetics 25, 41-68(1940).

Irradiation of Tradescantia microspores produces 1-hit and $2-$ hit aberrations in the chromatids and chromosomes. The chromosomes in the resting stage react as single threads; those in prophase react as double threads. The frequency of 1-hit aberrations shows a linear relation to dosage; 2-hit aberdations increase exponentially with dosage. When losage is increased by lengthening the time of exposure the equation for frequency of 2 -hit aberrations is $(\mathrm{D} / \mathrm{K})^{\mathrm{H} .5}$, but when dosage is increased with time of exposure constant the equation is $(D / \mathrm{T})^{2}$. The time-intensily factor is important because the 2-hit aberrations are dependent upon $2-$ independent breals limited in both time and space. The frequeney of initial breaks induced by $x$-rays is relatively independent of temperature but the frequency of aberrations is greater in cells subjected to low temperatures during or following irradiation due to greater re union of broken ends in the original position at high temperatures. (BA)

2099

Sax, Kar1. X-RAY INDUCED CMROMOSOME ABERRATIONS AND THLIR SUBSEQUENT BEHAVIOR. Genetics 25, 134 (1940).

Both chromosome and chromatid aberrations are produced by $\mathrm{x}$-rays. The frequency of aberrations produced by angle breaks shows a linear relation to dosage, while those dependent upon two breaks increase as the square of the dosage. Many of these aberrations produce single or double chromatid bridges at anaphase. When these bridges break new fusions of broken ends occur, although some of 2100 the broken ends appear to behave as nor mal ends. (G)

Sax, I. TYPES AND FREQUENCIES OF CHROMOSOMAL ABERRATIONS INDUCED BY X-RAYS. Cold Spring Harbor Symposia Quant. Biol. 9, 93-103(1941).

Irradiation of Tradescantia microspore chromosomes with $x$-rays produces only chromatid aberrations during the first 25 hours after raying, when the period of nuclear cycle is at a minimum. Between 27 and 31 hours both chromatid and chromosome breaks are found in considerable numbers, and from 34 hours to 5 days after raying only chromosome aberrations appear in the division figures. Single chromatid deletions are most frequent shortly after raying when the sister chromatids are further apart at late prophase, while double deletions are most frequent following irradiation of early prophase when the sister chromatids are closely associated. However, a single hit can break both sister chromatids throughout prophase, even when the chromatids are easily differentiated in microscopic preparations. The relation between x-ray dosage and aberration frequency depends on the nature of the aberration and the intensity of the radiation. The relation between dosage and the frequency of one-hit aberrations is linear regardless of the time-intensity factor. With short exposure times, the two-hit aberrations increase in frequency as the square of the dosage, but as radiation intensity is decreased the aber ration frequency declines and the dosage curve approaches linearity at $2.7 \mathrm{r} / \mathrm{min}$. The effect of the time-intensity factor is due to regtitution of broken ends so that fewer adjacent breaks occur within the critical limits of time and space. The evidence from varying intensity and from fractional dosage indicates that breaks can remain open for only 26-60 minutes. Due to limitations in time and space oniy a small proportion of the original breaks must be involved in the production of aberrations. Broken ends usually fuse and if illegitimate fusion does not take place, restitution occurs so that there is no evidence of previous breakage. (Author's Summary)

\section{1}

Sax, Karl. THE BERAVIOR OF X-RAY INDUCED CHROMOSOMAL ABERRATIONS IN ALLIUM ROOT TIP CELLS. Genetics 26, 418-25(1941).

Irradiation of allium root tip cells with $x$-rays produces a "primary" effect which results in fusion bridges. The "gecondary" effect produces breaks usually followed by the union of broken ends to produce rings, dicentrics, invergions, translocations, and interstitial deletions. Terminal deletions are produced when a break is not followed by union of broken ends of sister chromatids. The ring and dicentric chromosomes tend to persist for several cell generations but are soon eliminated, and only cells with normal or balanced genoms survive. The elimination of ring and dicentric chromosomes appears to be due to the deleterious effect of the deletions associated with these aberrations so that they are unable to compete with normal or balanced cells. Delayed germination of irradiated onion seed increases the frequency of chromosomal aberrations due to a physiological effect similar to the effects of age or heat, but delayed germination of irradiated onion bulbs has no effect on aberration irequency. (Author's Summary)

\section{2}

Sax, LEAI. THE MECHANISMS OF X-RAY EFFECTS ON CELLS. I. Gen. Physiol. 25, 533-7(1942).

Irradiatlon of resting nuclei of Tradescantia microspores did not increase the sensitivity of the same nuclei when they reached the prophase stage. The frequency of $x$-ray induced chromosomal aberrations at prophase was slightly reduced by previous irradiation at the resting stage, due to the production of acentric chromosome fragments which were less sensitive to subsequent irradiation. So far as the individual cell was concerned the deleterious effect of $\mathrm{x}$-rays was effected by a "direct hit" mechanism, but the reaction of tissues might be more dependent upon 2103 more indirect physiological factors. (BA)

Sax, IRarl. THE DISTRIBUTION OF X-RAY INDUCED CHROMOSOMAL ABERRATIONS. Proc. Nat. Acad. Sci. U. 5. $28,229-33(1942)$.

The relative frequencies of various lypes of chromosomal aberrations indicate that secondary factors play an important part in determining the frequency of effective breaks induced by x-rays. These secondary factors include (1) the spatial relations of the chromosomes, (2) the 
stresses imposed by the centromere in maintaining polarity and (3) the stage of chromosome development in the nucleas cycle. (BA)

2104

Sax, Karl. TEMPERATURE EFIFCTS ON X-RAY INDUCED CLROMOSOME ABERRATIONS. Genetics 32, (1) 75-8(1947).

The frequency of ring and dicentric chromosomes was more than doubled when Tradescantia microspores were irradiated at $3^{\circ} \mathrm{C}$. compared with the same $\mathrm{s}-\mathrm{ray}$ dosage at $36^{\circ} \mathrm{C}$. Temperature differences had little or no effect on the frequency of rod deletions. The subjection of microspores to high and low temperatures before and after irradiation produced no consistent differences in $\mathrm{x}-\mathrm{ray}$ 2105 induced aberrations. (BA)

Sax, Karl. THE EFTECT OF X-RAXS ON CHROMOSOME STRUCTURE. J. Cellular Comp. Physiol., Suppl. 1, 35, T1-81(1950).

Chromosomal breaks in the Tradescantia caused by zradiation and the union of broken ends are described. Breaks induced during the resting stage produce chromosome aberrations, while those induced during prophase produce chromatid aberrations. The relation between $x-r a y$ dosage and aberration frequency depends upon the stage of ruclear development and the type of aberration involved. The intensity of the dosage is also considered. The effect of temperature and infrared and ultra-violet xadiation during $x$-ray exposure is discussed. Comparison is made with the effects of neutron radiation. (NSA) 2106

Sax, Karl. THE CYTOLOGICAL IFIECTS OF LOWINTENSTTY RADIATION. Science 112, 332-3 (1950).

The effects of long-continued exposure to low-intensity radiation have been studied by exposing potted plants of Tradescantia paludosa to low intensities of $\gamma$ radiation for several months. Exposure to $1.7 \mathrm{r} /$ day increased the aber ration frequency up to the end of three weeks expowure, after which time there was no continued increase. At the end of 22 weeks the plants had received 262 i of $\gamma$ radiation, but showed only $0.5 \%$ breals in the microspore chromosomes. This dose of $\mathrm{x}-\mathrm{rays}$, given in a few minutes at the prophase stage, would have produced more than $30 \%$ breaks. The fact that both chromosome aberrations and pollen sterility level of after a few weeks of exposure indicates that the plants can survive and reproduce after months, or perhaps even years, of exposure. Unfortunately, these results cannot be expected to apply to the higher animals, lneluding man. The factors of determinate growth, high sensitivity of critical tissues, the absence of haploid mitosis in gametophylic development, and the lack of rapid somatic divisions preceding egg formation, should render animals much more vulnerable to low intensities of ionizing radiation. (NSA)

2107

Sax, Rarl and Robert T. Brumfield. THE RELATION BETWEEN X-RAY DOSAGE AND THE FREQUENCY OF CHROMOSOMAL ABERRATIONS. Am. J. Botany 30, $564-70(1943)$.

The frequency of simple chromosomal deletions induced in Tradescantia microspores by $x$-rays tends to increase in direct proportion to dosage while aberrations involving two chromosomes or two loci of the same chromosome tend to increase as the square of the dosage if the time of exposure is constant, and the $\mathrm{x}-\mathrm{ray}$ intensity is high. The relation between aber ration frequency and $x$-ray dosage for meiotic division in Tradescantia is approximately linear, apparently due to the prevalence of 1 -hit aberrations. The frequency of 2-hit aberrations in root-tip cells in Vicia tends to increase in direct proportion to dogage. The difference in the dosage curve relations in Tradescantia microspores and Vicia root-tipg is attributed to difierences in the physiological response to $x$-rays in the two types of cells. (BA)

2108

Sax, Karl and E. V. Enz Mann. THE EFTECT OF TEMPERATURE ON X-RAY INDUCED CHROMOSOME

ABERRATIONS. Proc. Natl. Acad. Sci. U. S. 25, 397-405 (1939).

Tradescantia microspores $\mathrm{x}$-rayed at low temperatures show more chromosome aberrations than those irradiated at high temperatures. At high temperatures fusion of broken ends is accelerated and many of the breaks are reunited in the original position, while at low temperatures fusion is delayed and broken ends may fuse in Illegitimate unions to produce many chromosome aberrations. This interpretation applies to nuclei irradiated at the resting stage and at mid-prophase, but the reversed temperature effect on early prophase chromosomes is difficult to explain. The temperature effect is maximal at a critical temperature which appears to vary with the geasonal effect on the nuclear cycle. The varied response of different types of cells to temperature effects during irradiation can be attributed to the relative effects of physiological and genetic factorg. (Author's Summary)

2109

Sax, Rarl and C. P. Swanson. DIFTERENTIAL SENSITIV ITY OF CELLS TO X-RAYS. Am. J. Botany 28, 52-9(1941).

Cell sensitivity to $\mathrm{x}$-rayg was measured by the frequency of induced chromosomal aberrations. In Tradescantia microspores the sengitivity is greatest shortly before midprophase. Of the various types of cells in Tradescantia the microsporocytes are most sensitive and the generative cell is most resistant to $\mathrm{x}$-rays. Haploid microspores are more sensitive than diploid microspores. Differential sensitivity is attributed to differences in chromosome structure and freedom of chromosome movement. (BA)

2110

Schjeide, Ole Arne and Bennet M. Allen. THE RILATION OF MITOSIS TO THE MANITESTATION OF X-RAY DAMAGE IN ILMATOPOIETIC CELLS OF TADPOLES.

J. Celluar Comp. Physiol. 38, 51-68(1951).

This work is a quantitative test of the concept that hematopoietic cells of the tadpole of Rana catesbiana show visible damage from previously administered $500 \mathrm{r}$ hard x rays only upon entering into mitosis. The mitotic inhibitor colchicine is utilized as an index of the rate of division in tadpole tissue. Comparison is made between total cells entering mitosis during a given period and the number of cells developing $\mathrm{x}-\mathrm{ray}$ damage in the vame length of time following exposure. Tadpoles are subjected to several accurately controlled body temperatures for these determinations. Hematopoietic cell destruction following $500 \mathrm{r}$ x rays is found to be directly correlated with rate of cell division during this same time. Rate of mitosis, as well as rate of cell destruction by 500 r $x$ rays is proportional to temperature. However, at 10,000 and 20,000 ithe rate of hematopoietic cell destruction exceeds the amount of cell division. Hematopoletic cells appear to sustain intial invisible damage by 500 I $\times$ rays during the resting state and perhaps all cellular phases. The visible cell destruction is, however, apparently expressed as a secondary characteristic of the initial damage and seems to appear only at the time of cell division. The mitotic phase at which the first visible indications of damage occur is probably the prophase. Hematopoletic cells held in the late prophase by colchicine are destroyed by $500 \mathrm{x} \times \mathrm{xays}$. (NSA) 
2111

Schmermund, H. J., H. Bleck, and H. L. Heinrich. CHROMOSOME MODIFICATIONS PRODUCED MN BARLEY SEEDLINGS BY FAST ELECTRONS OF A 6-MEV BTTATRON. Strahlentheraple 81, 177-86(1950).

A comparative study was made of fast electrons and $x-$ rays as radiobiological agents. Various doses of 4-Mev electrons obtained in $6-\mathrm{Mev}$ betatron, and of $\mathrm{x}$-rays whose half-value layer was $0.25 \mathrm{~mm}$ Cu, produced changes in the mitosis pictures of the end cella in the roots of harley seedlings. These changes are fragmentations of the chromosomes plus a sticking together of the latter to form a bridge between the two chromatin masses of the dividing nucleus in its ana- and telo-phases. The frequeney of such changes among the dividing cells was taken as a measure of the extent of the lesion produced by the irradiation. It was found that with both kinds of rays the structural changes grow with the dose, the fast electrons being somewhat less active, per dose unit, than $x$-rays. This difference is explained by the inequalities in the differential ionization and in the size of the ion groupings produced by the two kinds of rays. If the ion groupings are considered the "hists" or "events" of the target theory of ray action, the lesion curves for both kinds of rays show that the number of "hits" involved in the reaction studied here is 3 to 5. (MSA)

2112

Sieburth, Louise R. THE INTERACTION OF NUCLEIC ACID AND X-RADIATIONS ON THE CHROMATTM OF RYE AND PEA. Northwest Seì. 25, 132-6(1051).

Rye and pea seeds in the energetic stage were treated with $\mathrm{x}$ radiation and/or sodium ribose nucleate. Cells treated with 5000 showed pycnosis and multinucleoli. The nucleolus had such dense clumping of the chromatin as to be solid in outline. The nucleolus of the rye cells was much larger than in the pea cells, but the nature of the pycnosis of the rye and pea cells was observed to be the same. The material treated with $200 \mathrm{r}$ was similarly affected except that the nucleolus in rye cells showed the chromatin in distince "bands," whereas pea cells were normal. Nornal prophase of mitosis was observed in rye and pea cells treated wath nucleate and followed by $200 \mathrm{r}$. The cells of rye and pea treated with $200 \mathrm{r}$ and followed by nucleate showed different characteristics. In the rye cells, the chromatin was evenly dispersed but was somewhat pycnotic in nature. No nucleolus differentiation was observed. The pea cells on the other hand showed normal granular chromatin, except that the nucleolus showed distinct even clumping of the chromatin, (NSA)

2113

Simon-Reäss, T. and F. G. Spear. EFPTET OF GAMMA RADIATION ON MITOSIS IN VITRO. I. Brit. J. Radiol. 20, 63-70(1947).

Cells from the choroid and aclerotic of chick embryos in culiure were irradiated with doses from 55 to $1,320 \mathrm{r}$ at the rate of $22 \mathrm{~m} / \mathrm{min}$, and the subsequent effect on mitosis was observed by photographic methods. At $55 \mathrm{x}$ there was lithe alteration in cell behavior. At 88 I about half the observed dividing cell were affected. After 1,320 mitosid was very seanty, no cells completing division normally. There was little recovery after one hour. The chief difficulty in studies of this type is lo determine the beginning of the prophase. In the present study timing was begun at 2114 the beginnimg of metaphase to avoid this difficulty. (R)

5imgleton Ralph w. GENETHC HFPCTS OF RADIATHON, D.40-50 OF CONF BRENCE ON BIOLOGICAL APPLICATIONS OF NUCLEAR PHYSICS. Brookhaven National Laboratory. July 1948. 10p. (BNito-C-4(p.40m50))
Studies have been made (with Lea collaborating) on the effects on the chromosomes of Tradescantia at the first and second microspore division of a) different doses and intensities of $X$-rays and $\gamma$ rays at various temperatures, b) different wave lengths of $x-r a y s$, and c) neutrons and $\alpha$-rays. The main results are as follows: Neutrons are more efficient than $x-$ rays in producing breaks. Twobreak aberrations, e.g., interchanges, are proportional to the first power of the dose in the case of neutrons, but to the square of the dose in the case of $x$-rays and $\gamma$-rays. Interchanges produced by 8 -rays are therefore due mainly to two separate hits by ionizing particles, while those produeed by neutrons are mainly one hit. The neutron work enables one to show that a fairly considerable number of ionizations, spread across the chromosome, are necessary to breal It; and that s-ray brealage is due mainly to the densely ionized tail of the electron track. This agrees with the finding that soft $\mathrm{x}$-rays are more efficient than medium and hard ones, there being an optimum of about 4 A. About $90 \%$ of chromatid breaks primarily produced by $x$-rays, undergo restitution, their lives as free breaks averaging about 4 minutes at $20^{\circ} \mathrm{C}$. The separation at the time production of breaks which take part in the interchanges is in the order of one micron or less. This pretty well sums up the work on exchanges produced by lonizing radiations. (Author's Summary)

2115

\$lizynskin, B. M. PARTIAL BREAIKAGE OF SALIVARY GLAND CRIROMOSOMES. Genetics 35, 279-87(1950).

Low dosage $x-$ ray treatment ( 400 to $600 \mathrm{x}$ units) of Drosophila melanogaster embryos when applied in early development stages produces in the salivary gland chromosomes partial structural changes affecting only a few of the chromonemata of which the chromosome is composed. Partial structure changes include deficiencies, inversions, translocations and ring chromosones; partial structural changes may also appear in mutual combination. Structural changes in which a few fibrils are thus separated in vivo from the remaining mass of the chromonemata form confirmatory experimental evidence of the polytene nature of salivary gland chromosomes of Diptera. 13 references. (IVSA)

2116

Smith, Luther. A COMPARISON OF THE EFP ETS OF HEAT AND XI-RAY ON DORMANT SEEDS OF CEREALS, WYTH SPECIAL REFERENCE TO POLYPLOTYY.J. Agr. Research $73_{3}$ 137-58(1946).

Diploids were as tolerant of high temperatures as polyploids, whether the duration of treatment was a few hour or several days. Polyploids showed greater tolerance to T- -5yys. Seeds of diploid wheat given heat treatments after irradiation were injured slightly more than those given irradiation only. Comparable seeds given heat treatments before irradiation were injured less than those given irradiation only. Data from such combination treatments on diploid barley were not conclusive, but indicated silmilar results. Heat had little if any effect on the irequency of chromatinic bridges in root-tip cells of diploid, tetraploid, and hexaploid wheat, and diploid and autotetraploid barley. X-ray treatment of the same stocks resulted in a marked increase in the irequency of bridges in root-tip cells. Heat treatment of seeds of diploid and tetraploid wheat had little if any fifect on the frequency of translocations. X-12ay lreatments, on the other hand, produced a marked increase 到 the frequency of transiocations. Heat treatments of seeds of diploid wheal did not agpreciably affect the mutation rate, whereas $x$-rays distinctly increased 1 . (CA) 2117

SMith, W.W. BFEECT OF TIYROID HORMONE AND 
RADIATION ON TER MITOTIC INDEX OF MOUSE

EPIDELMIS. J. Cellular Comp. Physiol. 38, 4l-9(1951).

Thyroid administration caused an increase in the mitotic index of mouse ear epidermiss sampled in midmorning, which reached full development at the same time as other manifestations of thyroid action. Thyroid arministration did not affect the response of mitotic activity, as measured by the mitntic indes of the mouse ear epidermis, to sublethal whole -body irradiation. Conversely, radiation did not affect the response of mitotic activity to thyroid 2118

administration. (Author's Summary)

Sparrow, A. H. RADIATION SENSITIVITY OF CELLS DURNG MTOTC AND MEIOTIC CXCLES WITH EMPHASIS ON POSSIBLE CYTO-CHEMCAL CHANGES. Ann. N. Y. Acad. Sci. 51, 1508-40(1951) (AECU-160)

Original data on sensitivity changes to $\mathrm{X}$-rays during the nuelear cycle in Trillium erectum are presented. "They show late prophase and motaphase to be highly sensitive to $x$-ray breakage and early interphase to be of low sensitivity. The tundency of broken ends to rejoin seems to be inversely related to the amount of fragmentation per unit dosage. The general significance of these conelusions is discused in the light of other work on sensilivity changes during nuclear division. The distribution of chromosome breaks within chromosomes and the relationship of breakage to mutation are also discussed. The factors affecting nuclear radiosnsitivity are considrored under the following topies: morphotogical and structural differentiation within the nueleus; sub-microserpic, cytochemical and cytophysiological fartors; effects of mitotic inhibitors, mitotic poicons and other miscellaneous substances; and efiects of physical factors. It appears that the condensed stafe of the chromosome (late prophase, metaphase, anaphase) is one of hich sensituvity to breakrage and that the diffuse stages such as telophast, interphase, and carly prophase are relatively much Iess sensitive. Possible reasons for these differences are considered and possible mechanisms whereby the processes initiated by ionizations produce effective chromosome breals are presented and discussed. (NSA)

\section{9}

Sparrow, Arnold H. A CYTOLOGCAL AND CYTOCHEMICAL APPROACH TO AN UNDERSTANDING OF RADIATION DAMAGE IN DTVIDING CELLS. Brookhaven

National Laboratory. [nd] 2p. (AECU-821)

The document is in abstract form; it is reproduced below in its entirety.

Analysis of induced chromosome fragmentation following irradiation at different stages in the nuclear cycle (miosis) of Trullium erectum indicates that major changes in the radiation sensitivity of the chromosomes occur. Two lypes of Iragrments have been observed and srored: (a) those which become visible at the metaphase or anaphase immediatcly following prophase ir radiation, and (b) those which are detectable only after the chromosomes have passed through an interphase and entered the succeeding methphase. When both types are considered, it is found that urradiation at late prophase (diplotene-diakinesis) and metaphase stages yields a very large number of fragments, and 1 radiation at late telophase and carly interphase produces relatively few ragments. Since loss of nuclear material is proportional to the induced fragmentation, it may be assumed that the amount of chromosome fragmentation is a fair index of eventual cell lethality (and probably also of mutation). It therefore becomes of considerable significance to try to determine the factors controllings the degree and manner in which the chromosomes react to ioni ing zadiation. Morphological changes and physical factors (stress, repulsion, efc.) are known to be important Submisroscopic, cythehemical, and cytophysiological changes are also very important but, unfortunately, less well known.

Measurements of desoxypentose nucleic acid (DNA) at stapes of high and low sensitivity indicate that total DNA is not related to radiation sensitivity; however, its rate of synthesis or ils distribution within the nucleus may be. other factors known to influence radiation affect such as oxygen tension and sulfydryl oxidation also will be briefly discussed, as well as evidence of disturbances in nuclear metabolism following irradiation.

Results of continuous exposure of baeteria to beta and of various species of higher plants to $\gamma$ radiation will be reported briefly. (NSA)

2120

Sparrow, Armold H. CONSIDERATION OF TTHE RELATIONSHIP BETEYEEN CHPOMOSOME BREAKAGE AND MITOTIC INHIBITION INDUCPD BI IONIZING RADLATION. Brookhaven National Lab. [nd] 1p. (AECU-822)

The document is in abstract form; it is reproduced below in its entirety.

Cells irradiated in mid to late prophase are known to show a high degree of mitotic inhibition. This is also a stage of very high sensitivity to radiation-induced chromosome breakage. Subsequent loss of chromatin resulting from fragmentation leads to the production of a wecondary mitotic inhibition. Two distinct mechanisms of frabibition seem to be involved. The question then arises as to whethe the two stem from a common initiatrug phenomenon. If they do, it should be possible to relate the mechanisms of primary inhibition and chromosome fragmentation. The following working hypothesis is proposed.

Ionizing radiations are lnown lo depolymerize DNA and denature proteins. Either of these changes in the nucleoprotein complex comprising chromosomes (or genes) can be visualized as sufficient to inhibit self-reproduction of the complex. Further, such changes seem sufficient to presume increased susceptibility of the complex to destruction by enzymatic processes. Denatured proteins are known to be more susceptible to proteolysis, and alkaline phosphatase is known to attack only the partly depolymerized form of nucleic acids. The preventing of selforeproduction is synonymous with mitotic inhibition and breakdown of changed chromatin, through attack by physiological processes, with resultant chromosome breakage. Thus it appears that both inhbition and breakage may be related to the same primary changes produced in the nucleoprotein by jonizing radiation. (NSA)

2121

Spartow, Arnold Pt. SOME CYTOLOGICAL AND MORPHOLOGICAL CEANGES INDUCED IN PLANTS BY IONIZING RADIATION. Brookhaven National Lab. [nd] 1p. (AICU. 16A5)

The report is reproduced here in its entirety. Several species of plants have been exposed to chronte $\gamma$ irradiation from $\mathrm{Co}^{6}$ for periods up to four months. There was considerable variation in the radiosensitivity of different species, but in one instance no significant ef fect was detectable after 2000 yer day for six weeks. Generally, however, much lower dosages were suficient to produce one or more characteristic morphological responses. These included, in addition to growth inhibition, some lasciation and various unusual shapes and sizes of leaves of reproductive sf certain structures was frequently produced in some species. In others tumor-like growths as well as somatic mutations were observed. In Tradescantia paludesa a dramatic increase in the number of both vegetative and 
flower buds was noted after chronic irradiation of about $30 \mathrm{r}$ per day.

Cytological observations were made on both meiotic and aomatic cells of irradiated T. paludesa. The expected types of chromosomal aber rations were found and their frequency in both tissues determined. Meiotic chromosomes were showa to be much more radiosensitive than chromosomes of somatic cells. This agrees with the obgervation that pollen gterility and morphological abnormalities in the anthers occur at dosages which have relatively little gross effect on somatic tissue.

Dosages which caused marked morphological changes also produced a considerable anount of chromosomal aberratlon. However, since recovery to a normal growth pattern occurred in many plants which had previously shown severe radiation damage, it would seem that most of the morphological aberrations were not of a genetic nature. It is considered that they were probably the result of radiation induced physiological changes, although the possibility that they are somehow related to chromosomal aberrations cannot yet be excluded. (NSA)

Sparrow, Arnold H. X-RAY SENSITIVITY OF MEIOTIC AND MCROSPORE CHROMOSOMES OF TRILLIUM. Records Genet. Soc. Am. 12, 54-5(1943).

Trillium erectum is favorable material for studying chromosome sensitivity to X-rays at various stages, because at low temperatures melosis proceeds very slowly. It was thus possible to irradiate at known stages and subsequently to determine the number of aberrations. The number of fragments induced by a given dosage was used as an index of the $\mathrm{x}$-ray sensitivity of the stage rayed. Farly results indicated that plants rayed at first metaphase and first anaphase were highly resistant, as they showed very few chromosome breaks in meiotic stages. However, a large number of tetraploid and diploid pollen grains appeared following ir radiations of first metaphase and fir st anaphase respectively. Examinations of these revealed a very large number of fragments, indicating that the great majority of X-ray "hits" on meiolic metaphase and anaphase chromosomes do not induce visible breaks until the following microspore division. Their high sensitivity is then apparent. The least sensitive stages were early meiotic prophase (leptotene-zygotene) and pollen grain resting stage. These were of about equal sensitivity. There was an increase at pachytene to a little less than twice the number of fragments. First metaphase was still more sensitive, with about 6 times as many fragments; and anaphase was even higher, giving 9 times the number found after leptotene-zygotene raying. This large change in sensitivity cannot be correlated with known length changes of the chromonemata, but it is suggested that it may be related to the nucleic-acid and spiralization cycles. The above results indicate that colchicine treatment preceding irradiation conld be used to increase tissue sensitivity. (RG)

2123

Sparrow, Arnold H. X-RAY SENSIVITY CMANGES IN MEIOTIC CHROMOSOMES AND TYR NUCLEIC ACID CYCLE. Proc. Natl. Acad. Sci. U. S. 30, 147-55(1944).

The relative effects of $\mathrm{x}$-rays on different stages of meiosis and on microspore resting nuclei have been investigated in Trillium erectum. The ratio of chromosome aberrations was roughly $1: 2: 6: 9: 1$ for early meiotic prophase, pachytene, metaphase, anaphase, and postmeiotic resting nuclei respectively. It is argued that since stages of high sensitivity to $\mathrm{x}-\mathrm{rays}$ also have a high content of desoxyribose nucleic acid and $v . v$, sensitivity to radiation may be correlated with nucleic acid metabolism. The relationship of the findings to tumor sensitivity is discussed.

\section{4}

Sparrow, Arnold H. REDUCED CHROMONEMA ELONGATION AND ABNORMAL SPIRALIZATION FOLLOWING X-RAY TREATMENT OF MEIOTIC CHROMOSOMES. Genetics 30, 23(1045).

In Trilium, the formation of the major coll between early diakinesis and first anaphase is normally accompanied by an elongation of the chromonema estimated to be not less than threefold. However, in pollen mother cells $\mathrm{x}$-rayed at meiotic prophase the major spiral in many cells is very poorly developed even as late as second anaphase. Measurements of chromonema length at first and second anaphase in such cases indicate that elongation has been reduced to considerably less than half that found in control material. Reduced spiralization in other cases is known to be associated with low nucleic acid concentrations, e.g., in negatively heterochromatic chromosomes or regions and in cold-induced "differential reactivity". Ph is allo shown that 8 -rays inhibit the formation of desoxyribose nucleic acid. It is therefore Buggested (1) that the abnormal spiralization and redeced chromonema elongation following $\mathrm{x}$-ray treatment may be aswociated with an x-ray induced deficiency of desoxyribose nucleic acid, and (2) that the normal prophase increase in desoryrdbose nucleic acid may play an important role in the mechanic of spiralization.

2125

Sparrow, Arnold 1 . TOLERANCE OF TRADESCANTLA TO CONTINUOUS IXXOSURES TO GAMMA RADLATION FROM CORALTEO. Genetics 35, 135(1950). (AECU-681)

Plants of a clonal line of Tradescantia paludosa were exposed under field conditions to continuous $\gamma$ radiation (1.1 and 1.3 Mev) of approximately $122,30.4,7.5,1.8,0.41$, and $0.084 \mathrm{r}$ per day, for $8,16,32$, and 64 days. Dosage rates of 122 and $30.4 x$ per day permitted little or no growth, while fairly normal growth occurred at $7.5 \mathrm{x}$ per day or less. Complete pollen abortion was produced after 16 days at 122 r per day. Chromosome breaks as such were not scored, but micro-nucled at the microspore resting stage were counted. These are assumed to represent acentric fragments which lagged and remained in the cytoplasm at second telophase. A significant increase in the frequency of micronuclei was obtained with as low as $84 \mathrm{mr}$ per day after 32 days of exposure (total dose 2.69 r). Dosage curves for various exposure times will be presented. An attempt will be made to relate growth-inhibiting and lethal doses to the percentage of microspores containing micro-nuclei. (NSA)

2126

Sparrow, Arnold H. and E. Christensen. NON-RANDOM DISTRIBUTION OF CHROMOSOME IPRAMENTATION IN UNIRRADIATED TRILLIUM. Geneties 35, 135(1950)

An unusual type of spontaneous fragmentation has been found in a plant of Trillium erectum. There is considerable variation from another to another though some appear normal. In the most extreme case about $4 \%$ of the microsporocytes show aberrations. However, within this small group of cells chromosome fragmentation reached an extreme of about 30 breaks per cell. This is equivalent to that produced by a dose of at least $100 \mathrm{r}$ at a highly sensitive stage or of not less than 5,000 $\mathrm{r}$ at a stage of low sensitivity. Statistical analysis shows the distribution of break frequency in the dbnormal cells to differ significantly from that cxpected in irradiated material. The reason for this difference is not understood. Other $\mathrm{ab}$ - 
normalities such as "fused" microsporocytes indicate that a physiological disturbance may be responsible. The seriousness of this type of spontaneous fragmentation in radiation experiments involving the use of chromosome breaks as a measure of radiation effect is obvious. (G) 2127

Sparrow, Arnold H. and Marc Maldawer. DIF RRENTAL REJONNING AS A FACTOR IN APPARENT SENSITIVITY OF CHROMOSOMES TO X-RAY BREAKAGE. Proc. Natl. Acad. Sci. U. S. 36, 636-43(1950) (AECU-989)

Differential rejoining has been studied in microspores of Trillium erectum following $x$ irradiation at stages of high and low apparent sensitivity to breakage. The stages irradiated were meiotic first metaphase and early postmeiotic interphase. Fragments, dicentrics, and rings were scored at microspore anaphase. The numbers of dicentrics and rings observed were used as a measure of rejoining. The data indicate a significantly higher amount of rejoining in cells 1 radiated at early interphase than in those irradiated at meiotic metaphase. However, the increase in rejoining is not sufficient to account for the observed decrease in fragmentation. It is, therefore, concluded that the observed difference in sensitivity of the two stages studied is due in part to a difference in number of primary breaks (or potential breaks) and in part to a difference in the amount of rejoining which subsequently occurs. In the material studied a hiph sensitivity to primary brealiage is associated with a low frequency of rejoining and vice versa. Certain implications of these conclusions on calculations of mutation rate and gene size are discussed. (NSA)

2128

Sparrow, Arnold H., M.J. Moses, and R. Steele. A CYTOLOGICAL AND CYTOCHEMCAL APPROACH TO AN UNDERSTANDING OF RADIATION DAMAGE IN DIVIDING CELLS. Brit. J. Radiol. 25, 182-9(1952) (AECU-1438)

The amount of chromosome fragmentation induced by a dosage of $50 \mathrm{r}$ of $\mathrm{x}$ radiation has been determined at various stages in the meiotic nuclear cycle of Trillium erectum. Diplotene seems to be the most sensitive, and early interphase least sensitive. (Diakinesis and first metaphase are also highly sensitive.) The sensitivity change is at least 60 -fold. Higher rejoining is apparently a significant factor in reducing the amount of definitive fragmentation at the least sensitive stage, but does not account for the whole difference in sensitivity. Desoxypentose (DNA) and pentose (PNA) nucleic acid determinations show that the DNA per pollen-mother-cell increases up to diplotene and remains constant thereafter until early post-meiotic interphase. Synthesis starts again during later interphase. PNA remains constant from pachytene through second division to early interphase. The sharp rise in the sensitivity curve during the periods when DNA content increases indicates that increasing sensitivity may be associated with synthesis of DNA. However, the fall in breakage sensitivity between first metaphase and early post-meiotic interphase when DNA content does not change indicates that DNA content does not determine cellular radiosensitivity. (NSA)

\section{9}

Sparrow, Arnold H. and L. F. Nims. A CYTOLOGICAL AND CYTOCUEMICAL APPROACH TO AN UNDERSTANDING OF RADIATION DAMAGE IN DIVIDING CELLS, p.47 Of INTERNATIONAL CONGRESS OF RADIOLOGY, 6th Congress, London. 1950.

Since loss of nuclear material is proportional to the induced fragmentation, it may be assumed that the amount of chromosome rragmentation is a fair index of eventual cell lethality (and probably also of mutation). It therefore becomes of considerable significance to try to determine the factors controlling the degree and manner in which the chromogomes react to ionising radiation. Morphological changes and physical factors (stress, repulsion, etc.) are known to be important. Submicroscopic, cytochemical and cytophysiological changes are also very important but unfortunately, less well known. Measurement of desoxypentose nucleic acid (DNA) at stages of high and low sensitivity indicate that total DNA is not related to radiation sensitivity. Other factors known to influence radiation ef fect such as oxygen tenslon and sulphydryl oxidation are also discussed, as well as evidence for disturbances in nuclear metabolism following Irradiation. Results of continuous exposure of bacteria to beta and of various spectsas of higher plants to $\gamma$ radiation are briefly reported. 2130

\section{Spear, F. G. and A. GLucksmann. THE ERTECT OF} GAMMA RADIATION ON CELLS IN VTVO. PART III. SPACED RADIATION. Brit. J. Radiol. 14, 65-76(1941).

The experiments described here were conducted to dem termine whether the amount of degeneration seen a a ter exposure could be increased by delivering the second of the two exposures at the moment when mitosis was least active ag a result of the first exposure. In addition, "gpread ovar" irradiations were delivered at such lowered intensity as would give the same total dose. Tadpoles were used three to sis weels after hatching. Two sources of radiation were used: (1) radium needles containing a total of $175.5 \mathrm{mg}$, and (2) 90 thin-walled, $2 \mathrm{mg}$ Ra needles (total Ra content, $360 \mathrm{mg}$ ). The methods of irradiation were: (a) dose of 268 $\mathrm{r}$ delivered over 70.5 minutes at an Intensity of $3.8 \mathrm{r} / \mathrm{mm}$. (b) spread-over dose of $536 \mathrm{r}$ delivered in 1,381 minutes at an intensity of $0.34 \mathrm{r} / \mathrm{min}$; (c) spread wover dose of $536 \mathrm{r}$ delivered in 261 minutes at an intensity of $2.05 \mathrm{x} / \mathrm{min}$ (d) two exposures of 70.5 minutes at $3.8 \mathrm{r} / \mathrm{min}^{\mathrm{n}}$, separated by an interval of 24 hours; (e) two exposures of 70.5 minutes at $3.8 \mathrm{r} / \mathrm{min}$, separated by an interval of 20 minutes; (f) single irradiation of $536 x$ delivered over 141 minutes at $3.8 \mathrm{r} / \mathrm{min}$; and $(\mathrm{g})$ two exposures of 70.5 minutes at 3.8 $\mathrm{r} / \mathrm{min}$, separated by an interval of 10 days. In every experiment the effect of radiation is to reduce mitotic activity. The following sequence of biological response is seen: Very small doses: no recognizable change in behavior of irradiated cell. Threshold dose: sorae cells prevented from dividing, but normal mitosis occur later on. No degenerate cells produced. Larger doses: some cells reach prophase and then break down. ? some slowing-up of prophase. Maximum dose $(536 \mathrm{r}$ ) delivered as one exposure: temporary arrest in prophase, subsequent breakdown of cells. Increase in number of differentiating cells. Maximum dose delivered in two exposures at optimum spacing: mitosis reduced almost to zero. Large amounts of degeneration. (S. V. S.)

\section{1}

Speidel, Carl Caskey. AN EZPRRIMIRTAL STUDY WTTH THE AID OF CINE-PHOTOMICROGRAPHY OF NUCLEAR CRANGES IN IRRADIATED AND INJURED CELLS IN LIVING FROG TADPOLES. Anat. Records 103, 560(1949).

(The following is an abstract and is reproduced here in its entirety.)

Histological observations have been made on the nuclel of cells in irog tadpoles, as the animals are subjected to various kinds of injury. The changes have been watched in moribund cells, also in cells that recover. A few gimilar observations have been made on dividing cells. Illustrative motion pictures of both the normal and fast-motion type have been obtained. Among the injurious agents used are treatments with alcohol, metrazol, colchicine, podophyllum, 
mitrogen mustard, electric shocks, potassium citrate. various other chemicals, and mechanical wound inflictions. Changes in the nucleus include marked swelling in some cases, marked contraction in others, coarse ring formations, and disintegrations into coarse or fine granules. Some of the kinds of naturally occurring degeneration that accompany tail reduction during ladpole metamorphosis have also been photographed. Long drawn trophic injuries of cells have been induced by destroying the relationship between two structures that normally function as a unit, such as the lateral-line sense organs and their specific nerves. The sensory cells of nerveless organs undergo atrophy, dedifferentiation, and degeneration. Organless nerves also degenerate. Sheath cells that remain permanently stranded on such nerves likewise atrophy and degenerate.

2132

Swanson, C.P. THE EFIECTS OF ULTRAVIOLET AND X-RAY TREATMENT ON THE POLLEN TUBE CHROMOSOMES OF TRADESCANTIA. Genetics 27, 491-503(1942).

In addition to extending information on the capacity of ultraviolet to produce simple breaks in pollen tube chromosomes, the present study nas brought out the following new evidence: The frequency of chromatid breaks induced by ultraviolet at $2537 \mathrm{~A}$ is directly proportional to dosage. A similar situation appears to hold for the longer wave lengths (2967 and 3022), but the scantiness of the data permit of no definite conclusion. A non-random distribution of chromatid breaks was found when nuclei in the pollen tubes were treated with ultraviolet and $\mathrm{x}$-rays. Breaks were more frequent in the medial and distal regions contrary to that previously reported in Tradescantia and Gasteria. A delayed series of uv treatments show that the prophase chromosomes became progressively more resistant as judged from breakage data. In the 2- to 4-hour period after germination, the chromosome appears to become more susceptible to $\mathrm{x}-\mathrm{ray}$ breakage, but with uv, the reverse was true. Following heavy doses of short wave length uv treatment at prophase, the metaphase chromosomes showed a marked shortening accompanied by the appearance of a hyaline sheath surrounding them. Longer wave lengths produced no such change. (Author's Summary) 2133

Swanson, C. P. DIPTERENTIAL SENSITIVITY OF PROPIASE POLLEN TUBE CHROMOSOMES TO X-RATS AND ULTRAVIOLET RADIATION. J. Gen. Physiol. 26, 485-94 (1943).

This study indicates that the pollen tube chromosomes of Treadescantia show a varying sensitivity to both $\mathrm{z}$-rays and ultraviolet as they pass through prophase stages, using the rate of induced breaks as a criterion of sensitivity. Following ultraviolet (nearly monochromatic $2537 \mathrm{~A}$ ), a rise in sensitivity is indicated up to two hours after the pollen grains are sown on an artificial medium, after which a steady decline follows. However, since the generative nucleus at the 0 and 1 -hour stages were irradiated through the pollen wall which absorbs strongly, it appears litrely that the resting stage is more sensitive than are the succeeding prophase stages. Following $x$ radiation, a rise in total chromosome breaks continues to the 4-hour period after which a decline sets in. Each type of break varies in sensitivit at different periods. At late prophase stages, neither $z-$ rays nor ultraviolet leads to an increased production of breaks over the spontaneous rate. The uptake of water and the movement of the chromosomes (due to spiralization and readjustments within the nucleus) readily account for the observed differential sensitivity to $x-r$ ays, the most active stages being the most sensitive. Differ- ential breakage to ultraviolet is probably related to one or more of three factors: 1) nucleic acid accumulation on the chromonema, 2) development of the matrix, and 3) subdivisions of the chromonema. Their relative importance to this problem is conjectural. (BA)

2134

Swanson, C. P. X-RAY AND ULTRA-VIOLET STUDIES ON POLLEN TUBE CHROMOSOMES. I. THE EFTCT OF ULTRAVIOLET ON X-RAY INDUCED CHROMOSOMAL ABERRATIONS. Genetics 29, 61-8(1944).

A study of the effect of short wave length uv (2537A) on the production of $x$-ray breaks in the pollen tube chromosomes of Tradescantia test to the following conclusions. Pre-treatment one hour before $x$-raying, leads to an inhibition of all types of visible $x$-ray breaks. Single deletions and translocations are more affected than are double deletions. The degree of inhibition depends upon the dosage of ultraviolet used, although the exact proportionality is not known. Post-treatment has no inhibitor effect on double deletions regardless of time of application. Translocations show considerable inhibition if the post-treatment is given immediately after $\mathrm{X}$-radiation, slight inhibition if given one-half hour later, no inhibition if given one hour later. Single deletions are inhibited even after the one hour post-treatment suggesting that the ultraviolet facilitates restitution. It is suggested that the action of ultraviolet is not immediate, but that a certain time interval is required before it becomes effective. A further suggestion is made that the inhibition caused by the ultraviolet results from an effect produced on the chromosome matrix which leads to its greater resistance to $X$-ray breakage. (Author's Summary)

\section{5}

Swanson, C. P. and A. Follaender. THE FREQUENCY OF X-RAY-INDUCED CHROMATID BREAKS IN TRADESCANTIA AS MODIFIED BY NEAR-INFRARID RADIATIONS. Proc. Natl. Acad. Sci. U. S. 32, 295-302(1946).

The frequency of $x$-ray-induced chromatid breaks in Tradescantia can be significantly increased by treatment of the inflorescences with near infrared radiation. Pretreatment with near infrared radiation for seven hours prior to $\mathrm{x}$-radiation increased the frequency of single deletions, double (isochromatid) deletions, and translocations between and within chromosomes; post-treatment increased only single deletions and translocations. A delay of 21 hours between treatment with infrared and $x$-rays did not appreciably decrease the effectiveness of the infrared, suggesting that the changes within the cell induced by the infrared were of a relatively permanent nature. At the present time, the nature of the effect of infrared is not clearly understood.

2136

Swanson, C. P. and Henry T. Yost, Jr. THE ACTION OF INERARED RADIATION ON THE CHROMOSOMES OF TRADESCANTLA. Genetics 36, 579(1951).

By analogy with certain physical phenomena such as phosphorescence it seems possible that infrared might act by raising the molecular structures of the chromosome to a metastable condition, in which state these structures, as yet unidentified, react with increased frequency to subsequent doses of $\mathrm{K}$-rays. This was tested by subjecting in frared-treated buds to heat shock $\left(48^{\circ} \mathrm{C}\right.$. for 30 sec.), a treatment designed to return a metastable state back to an original ground state. We have shown that such heat treatments, interposed between infrared and $x$-rays, remove the inf rared effect, the chromosomes behaving to $x$-rays as though no infrared had been given. A similar effect was obtained when the order of treatment was $x$-rays - heat - 
infrared. From these iwo types of experiments, plus additional studies which revealed that the effectiveness of infrared was a function of the temperature of the buds at the time of infrared treatment, we have concluded that the primary action of infrared is to induce metastable states in the chromosomes. Such chromosomes react more readily with the free radicals released in the surrounding area by the ionizing radiation to yield a higher frequency of primary breaks per given dose of $\mathrm{x}$-rays. (G)

2137

Tahmisian, Theodore N. THE EFFECT OF X-RADIATION ON THE METABOLIC PROCESSES OF THE RESTING

CELL. J. Exptl. Biol. 112, 449-64(1949) (AECU-539; J-539) UAC-128)

It is reported that $\mathrm{x}$-radiation dosages ranging from $10,000 \mathrm{r}$ to $200,000 \mathrm{r}$ do not completely inhibit respiration in the diapause grasshopper embryo (Melanoplus differentialis). Respiratory, morphological, and cytological studies revealed that anabolic enzymes are associated with the nuclear material and are nore susceptible to $x$-irradiation damage than the catabolic enzymes. In a resting cell a nucleus was necessary for maintenance of the status quo of the cell. Following $x$-irradiation a resting nucleus was found not to have to undergo mitosis to become pycnotic. Pycnosis of the resting nucleus depended upon the $\mathrm{x}$-ray dosage, upon the state of metabolism, and upon time. Repression of irradiation damage to the cell was obtained by subjecting the cells to submetabolic temperatures. The integrity of the nuclear chromatin mate138 rial was dependent upon its funetional potency. (NSA)

Tahmisian, Theodore N. and Dorthy M.Adamson. A PHASE CONTRAST MCROSCOPY STUDY OF X-RAY INDUCED PYCNOSIS IN THE LIVING CELL. AnaL. Record 108, 572-3(1950). (ANL-4.488(p.54)

This section of the report is in abstract form, and is reproduced here in its entirety.

The effect of $x$ radiation ranging from 25,000 to 200,000 $x$ can be kept latent in Melanoplus differentialis embryos for 6 months by maintaining them at submetabolic temperatures. If diapause eggs are irradiated and left at $25^{\circ} \mathrm{C}$, pycnosis of the nuclei takes place by 8 days. When the eggs are irradiated and placed immediately at $0^{\circ} \mathrm{C}$ and kept at this lemperature for 6 months, no morphological or physiological changes are observed upon returning them to $25^{\circ} \mathrm{C}$. However, pycnosis occurs by 8 days at $25^{\circ} \mathrm{C}$ depending not only on the radiation dosage but also on the metabolic activity of the cell. Pyenosis in the living cell, as observed by phase-contrast microseopy, resembles fixed material morphologically. Physiologically there is a difference in the physical state of the nuclei when observed in the living condition. Most nuclei show a drop in viscosity at the time of pyenosis, as judged by the increase in Brownian movement. Vital staining with Janus green indicates that the mitochondria usually clump as a separate body, whereas the chromatin material becomes pyenotic, containing lipoid-impregnated bodies. (NSA) 2139

Tahmisian, Theodore $\mathbb{N}$. and Jean Gasvoda. MORPHOLOCICAL AND RESPIRATORY CRANGES INDUCHD IN MPLANOPLUS DIFTERENTTALIS BY BPTA IRRADITION. Argone National Laboratory. Feb. 1951. $1 \mathrm{p}$.

(AECU-1258; UAC-350)

The report is reproduced here in its entirety.

Prediapause eggs of the grasshopper, Melanoplus differentialis, were exposed to $\beta$ radiation emanating from $\mathrm{p}^{32}$ of $\mathrm{Sr}^{89}$ 'solutions to determine the morphological and respiratory changes that occur during and following such irradiation. Total dosages delivered to the embryos ranged from 3.07 to $1215 \mathrm{r}$. Eixtensive damage was seen in the cells at all exposure levels. Respiration was increased during irradiation but was inhibited immediately thereafter. By use of Flemming's triple stain it was shown that $f$ irradiation caused pylnotic chromatin to appear as though it were in the metaphase condition. An osmophilic material, associated with the chromosomes, was observed following exposure. This material is a lipoid that undoubtedly is a degradation product of lipoproteins known to be present in chromosomes. 2140

Thoday, J. M. THE EFTECT OF IONIZING RADIATIONS ON THE BROAD BEAN ROOT. PART IX. CONCLUDED. Brit. J. Radiol. 24, 622-8(1951).

A technique is shown by which estimations can be made of the percentage of expected dificient daughter cells of a certain number of cells in mitosis. Rstimates of the number of singly and doubly deficient cells in Vicia faba root meristems per 100 cells after various dosages of $x$ radiation and of $\alpha$ particles and percentages of fragmented nuclei are tabulated. Various mitotic stages are considered. The ratio of relative efficiencies of highest doses of a particles and of $x$ rays in producing chromosome deficiencies is shown to be about homogeneous and both are also roughly equivalent in their inhibiting effects on tissue growth of root tips. This may support the hypothesis that the structural changes produced by ionizing radiations in Vicia root-tip cells contribute largely to inhibition of root growth caused by these radiations, for these doses are not equivalent in their effects on mitotic rate and probably not in their effect on gene mutation. (NSA)

2141

Thoday, J. M. and D. E. Lea. THE EFFECTS OF TONIZING RADIATINS ON TUE CHROMOSOMES OF TRADESCANTTA BRACTEATA. A COMPARISON BETWE EN

NEUTRONS AND X-RAYS. J. Genetics 43, 189-210(1942).

Neutrons produce the same types of chromosome aber. zations as $\mathbb{X}$-rays, but are more effective per ionization produced. The ratio of $x$-ray dose to neutron dose (in roentgens), required to produce an equivalent effect, is not the same for the several types of aberrations: 2.4 for chromatid breaks, 3.6 for isochromatic rejoins, 4.5 for chromosome breaks, and between 5 and 10 for interm changes. Many of the latter, when produced by neutrons, are caused by singe "hits", i.e., single ionization paths. Neutrons and $x$-rays differ in effect insofar as the former, with a denser ionization path and with fewer paths per unit volume than equivalent doses of $x$-rays, produce a greater number of primary breals having a different distribution. Sinee neutrons produce more primary breaks per ionization than $\mathrm{X}-\mathrm{rays}>1$ conization is needed to break a Tradescantia chromosome thread. (BA)

\section{2}

Thoday, J. M. and John Read. EFIECT OF OXXGEN ON TUE TREQUFNCY OF CHROMOSOME ABERRATIONS PRODUCED BY X-RAYS. Nature 160, 608(1947).

Groups of beans, will their roots in a "perspex" cell of water through which nitrogen was bubbled, were irradiated with $\mathrm{X}$-rays. Other groups were treated in the same manner except that air replaced nitrogen. Growth curves demonstrated that there was a definite decrease in radiosensitivity when nitrogen was employed. This decreased radiosensitivily was accompanied by a parallel decrease in the proportion of cells exhibiting mitotic aber rations. A similar reduchion in radiosensitivity as measured by growth after irradiation occurred when nitrous oxide, car bon dioxide, and hydrogen were substituted for nitrogen. 
Since anaerobiosis reduces the radiosensitivity of tumors, it is suggested that the increased radiosensitivity in the presence of oxygen is the result of the larger number of 2149 mitotic aberrations which are induced. (AWM)

Thoday, J. M. and John Read. EFFCTS OF OXIGEN ON THRE FREQUENCY OF CHROMOSOME ABERRATION PRODUCED BY ALPHA RAYS. Nature 163, 133-4(1949).

Four graded doses of $\alpha$ radiation were given to groups of 8 beans (Vicia faba) in oxygen-free water, and four in water containing $\overline{12 \mathrm{cc}}$. O per liter, and the mean growth of the roots compared. O has a much greater effect on the effect of $x$-rays than on the effect of $\alpha$-rays, the dose ratios for equal growth "without/with oxygen" being $\sim 2.5$ and $\sim 1$, 3 respectively.

Cytological studies of root-tips treated in a similar manner show that while there is a somewhat larger yield of aberrations in the presence of oxygen than in its absence, the increase is far less than when $\mathrm{x}$-rays are used. These effects of $O$ parallel its effect in promoting the decomposition of water, and in view of the ability of $\alpha$-rays to produce $\mathrm{H}_{2} \mathrm{O}_{2}$ whether oxygen is present or not, suggest that $\mathrm{H}_{2} \mathrm{O}_{2}$ has some influence on the processes involved in chromosome structural change. (VE)

Whiting, Anna R. X-RAY SENSITIVITY OF FIRST MEIOTIC PROPHASE AND METAPHASE IN HABROBRACON EGGS. Geneties 27, 174-5(1942).

Hatchability percentages of unfertilized eggs $X-r a y e d$ in late prophase I with dosages up to $400 \mathrm{r}$ are significantly higher than of controls. Above $400 \mathrm{r}$ dosage-hatchability curve indicates that death depends upon the concurrence of two or more events the chance of which increases disproportionately with increased radiation. The lethal dose is about $35,000 \mathrm{r}$. In contrast, dosage-hatchability curve of eggs X-rayed in late metaphase I indicates that death results from one event. It is unchanged by timeintensity differences or by fractionated dosages. These eggs are sensitive to $50 \mathrm{r}$ and the lethal dosage is about $1250 \mathrm{r}$. After $1864 \mathrm{r}$ all suitable stages show fragments (terminal deletions ?) within the telophase I spindle or between equatorial plates at metaphase il. With $500 \mathrm{r}$, $750 \mathrm{r}$ and $1000 \mathrm{r}$, percentages without visible fragments lorm a curve parallel to and somewhat higher than dosagehatchability curve. Mean fragment number per egg increases with dosage. No eggs treated in prophase I with $1864 \mathrm{r}$ show fragments. Hggs treated in metaphase I with $2500 \mathrm{x}$ pass at once into telophase I when laid (like controls). $97.5 \pm 1.35$ percent complete meiosis and begin cleavage at approximately normal rates. When egg-laying is temporarily restrained to keep chromosomes relatively inactive after treatment hatchability is not significantly changed. If stresses peculiar to metaphase I chromosomes sensitize this stage to irradiation, treated prophase I chromosomes, which later undergo these stresses, must receive but slight injury at dosages lethal to former or recover almost completely before entering metaphase $I_{\text {. }}$ Data indicate that metaphase injury, like prophase, is due to chromosome breaks correlated with ionizations and not to "physiological" causes, stickiness and clumping of chromosomes. (G)

2145

Whiting, Anna R. HATCHABLLTY AND CHROMOSOME CHANGIS OF HABROBRACON EGGS X-RAYED IN PROPHASE I AND METAPHASE I. Genetics 28, 94-5(1943).

Dosage-hatchability curve of unfertilized eggs $\mathrm{X}$-rayed in metaphase I indicates that death results from one event. (Lethal dosage $1250 \mathrm{r}$.) There is no evidence for recovery.
Absence of a low dosage threshold for injury and of a high one for chromosome clumping and retardation (up to 15,000 r) eliminates chromosome stickiness as a factor. After treatment, fragments (double, terminal deletions?) are found in telophase I spindle or between metaphase II plates; occasionally smaller ones (single?) are found in one or both telophase II spindles. No bridges have been observed before cleavage. Percentages of eggs without fragments are higher than corresponding hatchability percentages. The two curves are roughly parallel. Fragments per treated nucleus vary as the 1.59 power of dosage for $250 \mathrm{r}$ and $500 \mathrm{r}$. This decreases with each increase in dosage, but data are not statistically significant. These phenomena suggest that broken metaphase chromosomes, like those in spermatozoa, undergo neither restitution nor recombination while condensed, - Eggs X-rayed between synapsis and diakinesis (prophase 1 ) have lethal dosage of about $40,000 \mathrm{r}$. Chromosomes in these stages do not react with Feulgen. Dosage-hatchability curve, almost identical in three independent experiments, depends upon unknown combinations of I-hit and multiple-hit events, is complex and has not been analyzed. No cytological effects appear at low dosages. This removes possibility that high resistance depends upon extensive elimination of dicentrics in division I. At high dosages bridges have been seen in divisions I and II. - Cleavage of eggs treated in either stage may show bridges or may show fragments produced by two breaks in a bridge. (G)

2146

Whiting, Anna R. EFTECTS OF X-RAYS ON HATCHABILITY AND ON CHROMOSOMES OF HABROBRACON EGGS THREATED IN FIRST MEITOTIC PROPHASE AND METAPHASE. Am. Naturalist 79, 193-227(1945).

Because of their definite order in the ovariole, Kabrobracon eggs may be treated at specific meiotic stages and then allowed to develop parthenogenetically. Those treated at metaphase I give hatchability percentages which slow a linear decline with dosage from 50 to $1,400 \mathrm{r}$, but with no changes following aging after treatment, time-intensity differences, or fractionation of dose. The eggs treated in prophase I give hatchability percentages equal to or higher than controls for doses from 50 to $750 \mathrm{r}$. At medium doses, a linear relationship is apparent between dosage and hatchability but this relationship becomes obscured at the higher doses. The lethal dose for prophase I is about $44,800 \mathrm{r}$. For prophase I, hatchability increases with fractionation of dose but does not change following time-intensity changes. Eggs treated at either stage with lethal doses will continue meiosis and early cleavage at normal rates. Those treated in metaphase I lack bridges in Division $\mathbb{1}$, but otherwise eggs treated at both stages show fragments and bridges at both division I and II and also in cleavage. Fertilization with untreated spermatozoa does not change the hatchability percentages of eggs treated at either stage. The correlation of cytological aberrations with hatchability percentages seems to prove that death is caused by single hit chromosome breaks. The high sensitivity of the metaphase I eggs indicates that ionization effects may depend upon chromosome tension. Other explanations for differential sensitivity at various stages are discussed and found not to apply. (BA)

2147

Whiting, Anna R. DOMINANT LETHALITY AND CORRELATED CHROMOSOME EFTECTS IN HABROBRACON EGGS X-RAYED IN DIPLOTENE AND IN LATE METAPHASE. Biol. Bull. 89, 61-71(1945). 
Unlaid Habrobracon eggs $x$-rayed in diplotene (lethal dose about $45,000 \mathrm{r}$ ) and allowed to develop parthenogenetically, show fragments, bridges or both in division I; either or both in division 1 . Bridges in division I may be permanent. Unlaid eggs $x$-rayed in late metaphase $I$ (lethal dose about $1,400 \mathrm{r}$ ) show fragments but no bridges in division 1; bridges, fragments or both in division 11 . An explanation of difference in cytological effects of $x$-rays on these stages and of differences between them in sensitivity and in nature of survival curves is attempted through comparison with studies on forms with larger chromosomes. (BA)

2148

Wilbur, Karl M. THE RADIOSENSITIVITY OF EGGS OF ARBACIA PUNCTULATA IN VARIOUS SALT SOLUTIONS. Biol. Bull. 85, 193-200(1943).

Treatment of Arbacia eggs with 0.35 M K citrate inhibited the retarding action of $x$-radiation on cell division. However, the inhibition by citrate was slight, and appeared mainly with high $x$-ray doses $(30,400$ and $53,200 \mathrm{r})$. The radiosensitivily was unaffected by increasing the Ca or $\mathrm{Mg}$ content of the sea-water medium. The $\mathrm{K}$ citrate treatment increased the viscosity of the unfertilized $\mathrm{egg}$. The viscosity was decreased slightly in the sea-water solutions of increased $\mathrm{Ca}$ and Mg content.' The data presented indicate that changes in the ionic composition and viscosity of the protoplasm may occur without altering the sensitivity of the egg to $\mathrm{x}$-radiation. Doses of $\mathrm{x}$-radiation which markedly altered the rate and normal course of cell division produced no detectable change in the viscosity of the unfertilized egg. (BA)

2149

Wilson, G. B. and B. R. Nebel. CHANGES IN CHROMOSOME SENSITIVITY TO X-RAYS. Genetics 25, 140(1940).

Microspores of Tradescantia reflexa Raf. were rayed at various stages preceding first pollen-grain metaphase. Sensitivity was measured by the degree of chromosome alteration at first pollen-grain metaphase. Results indicate a positive correlation between the speed of mitotic development and the degree of response called "sensitivity." Several agents which either stop or refard division are being tested; first, for their own effect on chromosome and cell morphology and second, for their effects on sensitivity to $X$-rays as judged by comparisons with normal sensitivity curves. (G) 2150

Yamashita, H., K. Mori, and M. Miwa. THE ACTION OF IONIZING RAYS ON SEA-URCHINS. V. THE MTTOTIC OBSERVATIONS ON THE EFFECTS OF ROENTGEN RAYS UPON THE UNFERTLIZED EGGS AND SPERM. Gann 34, $239-45(1940)$.

The difference in the mitotic events between normal and irradiated eggs in the developing cycle from fertilization to first cleavage has been investigated numerically in histological studies. Irradiations were made with roentgen rays of moderate intensity. It was found that delay in time of the first cleavage of irradiated eggs occurred essentially in the nuclear streak phase and prophase. Considering these facts in connection with previous studies (Yamashita, H., Mori, K., and Miwa, M., Gann, 33, 117-121, 1939) that the radiological susceptibility of the fertilized eggs is highest in the phase of nuclear streak, we suggest that this particular mitiotic phase is importantly involved in the damaging of living cells by irradiation. (Author's Summary) 2151

Yost, H. T., Jr. AN ANALYSIS OF COMBINED INFRARED
AND X-RAY EFIECTS ON TRADESCANTIA CHROMO-

SOMES. Geneties 35, 700(1950).

$\mathrm{X}$-rayed Tradescantia microspores have been given preand post-treatments with inf ra-red radiations. In all cases, percentage increases of $50-120 \%$ in chromosomal aber rations were observed in the infra-red-treated cells. Post-1reatments have been given as late as 96 hours after $\mathrm{X}$-radiation with no apparent decrease in the effectiveness of the infra-red radiations. From these data the conclusion may be drawn that the determination of the type of aberration recovered (chromosome or chromatid) is independent of the time of recombination, and dependent solely upon the singleness or doubleness of the chromosomes at the time of X-radiation. Studies of Tradescantia chromatid aberrations indicate that the infra-redinduced increases show a non-linear relationship to the dosage of infra-red given. These studies and other concerned chiefly with $X$-ray dosage relationships and spectral dependency permit some conclusions concerning the mechanism by which infra-red radiations produce their effect. (G)

2152

Yost, Henry T., Jr. THE FREQUENCY OF X-RAY INDUCED CHROMOSOME ABERRATIONS IN TRADRSCANTIA AS MODIFIED BY NEAR INFRARED RADIATION.

Genetics 36, 176-84(1951).

From the data presented on the effects of infrared radiations when used as supplementary treatments with $x$ radiation, the following conclusions have been made: (1) pretreatment and posttreatment with infrared radiations increase the frequency of chromosome aberrations induced by $x$ radiation; (2) the posttreatment may be delayed 93 to $96 \mathrm{hr}$ after $\mathrm{x}$ radiation with no decrease in the effectiveness of the infrared radiations; (3) the type of aberration induced is dependent upon the time at which the $x$ radiation is administered, and is independent of the time of infrared radiation and the time of recombination. The various hypotheses which have been advanced to explain the effect of infrared treatment are discussed. The potential break hypothesis is supported by this data. (NSA)

\section{3}

Zirkle, R. E. SOME WFECTS OF ALPHA RADIATION UPON PLANT CELLS. J. Cellular Comp. Physiol. 2, 251-74(1932).

c rays from polonium retard and inhibit, but never accelerate, 3 distinct processes in the germination of the spores of Pteris longifolia: cracking of the spore wall, development of chlorophyll, and cell division. The doses producing $50 \%$ inhibition of these processes are, respectively, 33,000; 13,000; and 4000 a particles per protoplast, which correspond to somewhat smaller amounts of energy per unit volume than those used by others to produce hemolysis and $50 \%$ inhibition of cell division in yeast. When $4000 a$-particles per protoplast are used, about $9 \%$ of the protoplast is liable to direct alteration by ionization. All 3 processes are more easily inhibited in germinating than in air-dry spores, but increase in inhibition of cracking appears earlier in germination than that of greening or of division. Irradiation of definite fractions of the protoplast indicates the following conclusions: cracking, greening, and division may each be inhibited by extranuclear injury, but all are much more easily inhibited if the nucleus is irradiated; the extranuclear injury which inhibits greening is not direct injury to plastids or proplastids; imbibition is inadequate for production of normal cracking; and extranuclear irradiation produces a high frequency of a type of induced twinning. (BA) 


\section{AUTHOR INDEX}

Abderhalden, R., 303, 1556

Abele, Adolph, 70

Abels, J. C., 869

Abelson, Philip H., 186, 187

Aberg, Ewert, 1847

Abersold, P. C., 188, 189, 211, 382, 403,405

Abrams, Herbert L., 244, 304

Abrams, Richard, 748, 809, 916, 918, 1515

Ackerman, H., 997

Adams, S. N., 905

Adams, Williams, 644, 707, 1293

Adamson, Dorthy M., 1756, 2138

Aggeler, Paul, M., 626

Ahlstrom, L. H., 1516, 1517, 1705

Ahmed, I. A. R. S., 2066

Albaum, tharry G., 1514

Alexander, Lyle T., 1678,1758

Allen, A. C., 252

Allen, Alexander J., 1637

Allen, A. O., 1354, 1638

Allen, Bennet M., 645, 1619, 1620 , 2110

Allen, Carlene M., 124

Allen, J. Garrott, 535, 708, 709

$710,711,712,713,714,720,721$, $725,726,727,728,734,1140$

Allen, R. P., 997

Allen, Roberta, 787

Allsopp, C. B., 1355

Alper, Tikvah, 1607

Alland, Paul D., 96

Altman, Kurot $\mathbb{I}_{0}, 1411,1484,1485$, 1490

Amaniera, G., 700

Ambrosi, $F ., 715$

Anes, Stanley $\mathbb{R}_{\text {. }}, 1294$

Amoroso, E. C., 1679

Amory, Harold I., 556

Amy, Robert L., 1701

Andem, Margaret R., 1113

Andersen, Else Krag, 525

Anderson, C. T., 1412

Anderson, $\mathbb{E}_{0}, 360,646$

Anderøon, $\mathrm{E}$. A., 325

Ander won, Ernest C., 1011

Anderson, E. G., 1772, 1773

Ander gon, E. H., 1774,1856

Anderson, Rubert $S_{\circ}, 305,313,314$, $1639,1670,1709$

Andrews, G. A., 665

Ane, Joseph $\mathbb{N}_{\text {, }} 1798$

Anger, $\mathrm{Hal}, 133$

Anger, $\mathrm{H} .0 ., 398$

Anslow, $G_{0}$ A., 188

Anthony, D. S., 785, 807, 870, 975, 276,1030

Ariel, Irving M., 245
Arkin, Alvin, 246, 965

Armstrong, $W$. D. $792,795,796$

Arnason, T. J., 1928, 1981

Amold, Anna C., 1330

Arnold, James S., 774, 917, 1016

Arons, Iridore, 1152, 1193

Artorn, Camillo, 874, 1297

Arvy, Lucre, 647, 1557

Ashler, F. M., 1599

Asling, C. W., 830, 977

Atwood, $\mathbb{K}_{0}$ C., 1356,1775

Axelrod, Dorothy J., 757, 037, 934

Axelrod-Heller, D., 830

Auerbach, Charlotte, 1776

Austing, Mary E., 1095

Azevedo, M. Deodata, 1576

Bachofer, C. S., 1621

Back, A., 117, 306, 316, 1443, 1651

Baclesse, $F_{0}, 1320$

Bacq, Z. M., 247, 280, 1183, 1184, $1185,1190,1191,1192,1321$, $1334,1335,1357$

Badell, M., 1181, 1182

Baer, Marianne, 1737, 1738, 1739, 1740

Baidins, A., 248

Bakex, William Ko, 325, 1777, 1778 , 1779,1856

Baldini, Mario, 1558

Ballantyne, R. M., 1306

Bancroft, Geo. H., 1413

Barnard, R. D., 1559

Barnes, S. W., 620,621

Barnes, W. A., 648, 716

Barnet, Wenry L., 1079, 1080, 1081. 1083

Barnett, James C., 105

Barnett, T. B., 448

Barr, J. S., 1680

Barrate, R. W., 1959

Barron, $\mathbb{2}$. S. Guzman, 918, 978, $1414,1415,1416,1417,1418$

$1419,1420,1432,1475,1560$,

$1561,1562,1563,1564,1565$,

$1608,1609,1610,1611,1640$

Barrow, Jack, 410, 1421

Bassett, R. C., 557

Bateman, A. \$., 1780

Bauer, Frederick C., Jr., 1017

Baur, H., 1422

Baxter, J. G., 129

Bazin, Suzanne, 600

Beadle, G. W., 1781, 1964

Beal, J. M. 1782, 1982, 1983

Bean, William B., 1042

Beard, C., aro

Beatty, A. V., 203\%, 2038, 2039, 2095
Beatty, Clarissa, 1480

Beck, J. S. P., 1061

Becker, Robert M., 514

Beeler, John W., 1167

Behrens, C. F., 1, 724

Bellamy, Albert W., 320

Beller, F. K., Jr., 701

Bender, A. E., 249

Benedek, Agnes L., 730

Benkwith, K. B., 1062

Bennett, Leslie R., 515, 1094, 1145, $1146,1147,1158,1423,1424$, 1425,1473

Bennett, Vera C., 1423, 1424

Benmison, B. E., 307

Berg, Marvin, 777

Berls, Sigmund, 1681

Berner, F., 1426

Bernier, J. L., 1063

Bertiglia, B., 700

Bethard, W. F., 1279, 1281, 1284

Betz, H., 97, 649, 1186, 1187, 1188, $1189,1191,1122,1206,1207$, 1322

Beyl, Gertrude E., 602

Bier, M., 1566

Bigelow, R. R., 411

Biles, C. R., 106

Billen, Daniel, 351, 1427, 1567

Billings, Martha S., 250

Bingham, Hal, 955

Biram, M. B., 190

Birkeland, J. M., 1850

Bischoff, F., 251

Bisgard, J. D., 516

Bishop, Charles J., 1984, 1985, 1986

Bishop, D. W., 1987, 1988

Bishop, F. W., \$8, 228, 361

Blackford, M. E., 1264, 1265, 1268, 1274

Blagg, Friedrich J., 1221

Blair, Henry A., 735, 1323

Blane, Richard, 1783, 1784

Blanc, $R_{\text {o, }}$ 1682

Blanda, R. $J_{.,}^{4} 48$

Blaney, Loren $F \cdot 178$

Blaschlo, I., 1412

Bleck, H., 2111

Bless, A. A., 70a, 736, 744

Bliss, C. $\mathbb{H}_{0,} 1683$

Bliss, R. W. 2

Block, Matthew H., 650, 672, 725, 986, 1304

Blokhin, N. N., 1428

Bloom, Margaret A., 308, 651, 910, 341

Bloom, William, 3, 651, 652, 797, $798,919,941$

Bloomfield, đ. R., 462 
Blount, Henry C., Jr., 1208, 1633

Blum, Harold $\mathrm{F} ., 1684$

Blume, James M., 871

Blumel, Johanna, 1785

Blumethal, Gertrude, 1597, 1598

Boche, R. D., 98, 226, 228

Bodine, J. H., 1685

Boell, E. I., 1685

Boffil, J., 653

Bohlander, Adam, 1459, 1460

Boiffard, Jacques-Andre, 647, 1557

Bojanowsky, Rudolph, 1358

Boloney, Rene A., 2042

Bond, V. P., 252, 517, 550, 551, 594, 1328

Bonet-Maury, $\mathbb{P}_{\circ}, 99,362,543,877$, $1611,1612,1641$

Bonham, Kelshew, 100, 1686, 1687, 1708,1765

Bonner, David, 1786,1958

Bonner, G., 997

Bonnier, Gertrude, 1787, 1788, 1789

Bonte, Frederick J., $49 \%$

Bonzell, Veronica, 1414

Booth, J., 481

Borak, J., 12

Born, H. J., 1790

Borowskaja, D., 518

Botkin, A. L., 1095

Bouchard, Jean, 1401

Bould, C., 872

Bourret, J., 610

Bowers, John Z., 1058, 1064, 1096, 1429

Bowman, M. C., 1430

Boyland, E., 468, 1431

Boys, F., 1324

Bozemann, Martha L., 1989

Braasch, Nancy, 253

Brace, Kirkland C., 1688

Bradley, M., 2081

Braestrup, Carl B., 158

Brandt, C. L., 1568

Brasch, Arno, 70b

Braude, R., 810

Braun, Werner, 1783

Brawner, H. P., 655

Brean, Henry, 549

Brecher, George, 10, 654, 655, $1108,1252,1253,1255$

Brennam, James T., 1100

Brent, Robert L., 467, 512

Bretscher, E., 1373

Brick, Irving, 556

Brinkhaus, K. M., 729

Brohult, S., 1674

Brooks, Robert E., 1496, 1569, 1665

Brooks, W., 1221

Brown, Barrett James, 1276, 1277

Brown, Crawford S., 591

Brown, Hubert M., 1833

Brown, Mary B., 491, 492, 493, 494

Brown, Meta S., 1791, 1990, 1991

Brown, W. H., 1339

Brown, W. M. Court, 1043, 1168

Browning, Ithel, 611, 942

Bruce, Karl W., 558

Brucer, Marshall, 838
Brues, Austin M., 5, 130227,468 , $469,470,656,738,739,740,741$, $742,750,751,752,753,781,799$, $920,1010,1013,1031,1032,1044$, $1052,1097,1098,1099,1141$, $1325,1504,1613,1992$

Brumfield, Robert T., 1689, 1983, 2107

Bruner, H. D., 844

Brung, I. H., 1690

Brunst, v. V., 70c, 1691,1622 , $1693,1694,1695$

Brusa, Adolfo, 1018

Bryan, Carl E., 805, 806

Bryan, Fred A., 1045

Buchanan, Donald L., 754, 800, 801, 978,1432

Buchmann, Walter, 1792, 1793

Buchsbaum, Ralph, 1994

Buckaloo, G. Warren, 1343

Buesseler, John A., 584

Bujard, E., 440

Bukantz, S. C., $519,530,531$

Bunker, J. W. M., 944

Burkell, C. C., 180

Burkholder, R. R., 1794

Burlingame, Louise, 250

Burn, J. H., 1412

Burnett, W. T., Jr., 302, 326, 855

Burnham, E. M., 728

Burrows, H., 471

Burrows, William, 520, 521, 522, 523

Burstone, M. S., 254, 845, 873

Burton, Milton, 1642

Busch, Elizabeth, 1296

Bush, F., 755

Butler, Carrie L., 1441, 1580

Butler, E. E., 1931

Butler, G. C., 1518

Butler, J. A. V., 1519, 1520, 1521

Butler, Nell, 1046

Butterworth, J. S., 472

Buzell, W. C., 1434

Cafiaratti, Enrico, 1326

Caldecott, Richard \$., 1795, 1945

Calkins, Larry L., 584

Cameron, Gordon H., 158

Camillo, Artom, 1295

Campbell, Berry, 168413

Campbell, Ira L., 1327

Cantril, S. T., 612

Carbonaro, Lynn, 1017

Card, L. E, 685

Carlson, J. Gordon, 1995, 1996, $1997,1298,1999$

Carothers, E. E., 2000

Carpender, James W. J., 892

Carralho, S. de, 169

Carter, C. $\mathrm{E} ., 1570$

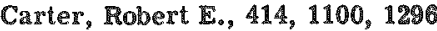

Carter, T. C., 1796

Casarett, George W., 71, 1490

Casati, Annibale, 1643

Case, A. C., 924

Caspari, Enrst, 1797, 1798

Cassel, Chester, 998
Catcheside, D. G., 1799, 1800, 1801, 2001, 2002, 2003, 2004, 2005, 2074

Cathie, 1. A. B., 559

Catsch, A., 191, 1802, 1803

Cavalli, L. L., 1804

Cayer, David, 874, 1297, 1457

Chace, M. J., 923

Chailkoff, I., 995

Chaikofi, I. L.., 853, 857, 858, 859, 979,980

Chambers, Francis W., 167, 1195, 1196

Chambers, Francis W., J1., 102, $106,134,1251,1421$

Chanutin, Alfred, 592, 593, 1470, 1593,1504

Chapman, E. M., 852

Chapman, William $\mathrm{R}_{\text {. }}, 11,102$, $1195,1196,1210,1250,1251$, $1252,1253,1254,1255$

Charipper, H. A., 386

Charles, Donald R., 1805, 1806, 2077

Chase, Herman B., 136, 1602, 1603, $1697,1698,1696,1886,1887$

Chastian, Sarah Mo, 1145, 1147, 1425

Chatters, R. M., 180 \%

Chriatensen, E。, 1754, 2126

Chrigtensen, William R., 999

Christian, Emily J. B., 92, 93, 1504

Christiansen, Alice M., 1915

Christie, James H., 777, 881, 891, 1162

Chrom, Sv. A., 1101

Ciaranfi, E., 1360

Cipollaro, A. C., 80, 147

Claesson, L., 248

Clark, A. M., 310,311

Clark, Francis E., 1689

Clark, George $\mathrm{L}_{a}, 6,685$

Clark, J. B., 1618

Clark, Janet Howell, of

Clark, Robert K., 153

Clark, W. G., 101, 1194

Clarke, Grace, 1111

Clemmesen, Johannes, 524, $52 \mathrm{a}$

Closon, J., 846

Cloudman, Arthur S., 473

Coatney, G. Robert, 307

Cochran, Kenneth W., 18,1438

Cogan, David G., 192, 255, 560 , 1065

Cohen, Lionel, 1299

Cohen, Sheldon G., 526, 529

Cohn, Stanton H., 717, 952

Cole, Jack W., 723

Cole, Leon s., 1328, 1808

Collier, Vera, 515

Collinson, $2 ., 1571$

Comar, C. L., 811, 812, 813, 814

Comer, Ralph, 955

Comstock, Carl R, 269

Conger, Alan D., 2006, 2007, 2008, $2009,2010,2011,2041$

Conrad, Robert A., 1102

Conway, B. $\mathbb{E}_{\circ}, 1519,1521$

Cook, Evelyn V., 1622

Cook, S. F., 907 
Coon, Julius M., 1352

Cooper, F. S., 222, 223

Copeland, M. M., 138

Copp, D. Harold, $8,757,788,831$, $837,923,925,977,987,988$

Corberi, $\mathrm{E}_{\text {. }}, 1809$

Cornatzer, W. E., 874, 1295, 1297

Cornisield, Jerome, 966

Corrigan, K. E., 771

Coss, J. A, Jro, 1483

Costello, Donald P., 2012

Cottet, 1700

Coulter, Molly P., 73, 1103 , $1154,1155,1156,1159,1160$, 1161, 1348

Cowan, Frederick P., 881

Cowing, Russell $F ., 426,427,911$, 312

Crabtree, Herbert $G_{\circ}, 137,1433$

Craddock, C. G., 696

Craddock, Charles G., Jr., 527, 528

Cramer, C., 788

Craver, B. N., 758, 1209

Craver, L., 868

Creighton, M., 2013, 2014

Crenshaw, James $F_{.,} 1046$

Criep, Leo H., 526, 529

Cronkite, Eugene $\mathrm{B} ., 9,10,11,102$, $134,613,654,657,708,718,724$, $729,1047,1104,1105,1106,1107$, $1108,1119,1195,1196,1210$, $1250,1251,1252,1253,1254$, 1255,1298

Crouse, Helen V., 1810, 2015

Crovisier, C., 1662

Crowley, Josephine $\mathbb{F}^{\circ}, 786,934$

Crowther, J. A., 12

Crubin, A. F., 1483

Culbertson, W. F. J., 815

Cullen, George, 792

Cumming, 黑Iaine, 1981

Cupp, M. N., 170, 1200

Curl, $\mathrm{H}$. 292

Curtis, Howard Jo, 13, 14, 193, 217, 360, 798,982

Cuthbertson, E. M., 923

Caech, H., 363

Czech, $\mathbf{R}_{\text {. }}, 363$

Da Câmara, A., 2016

Dainton, F. S., 1571, 1644

Dale, Walter M., 1572, 1573, 1574, $1575,1645,1646,1647,1648$, 1649

Dammin, G. J., 519, 530

Daniels, E. W., 2017

Darby, R. K., 160

da Silveira, Marieta, 1576, 1587, $1588,1589,1590$

Daudel, Pascaline, 945

Davidoff, L. M., 256

Davies, J. V., 1574, 1646, 1647, 1648

Davies, W. D., 163

Davis, B. D., 1811

Davis, George $\mathrm{ZK}_{\mathrm{og}}$ 811, 812, 813, 814

Davis, $\mathbb{R}$. Wendell, 658

Davison, Bo, 194
Davison, R. A., 1434

Day, Paul L., 1577

Dean, A. C. R., 1812

Dean, A. Ls, 581

DeBruyn, Peter P. H., 262, 259, 660

de Carlos, R., 1329

de Carvajal-Forero, J., 272

Decker, Arthur Bo, 1425, 1435, 1473

DeCoursey, Elbert, 1066, 1076

DeFeo, Edward, 1169

Deho, G. G., 1436

de Landt sheer, L., 1256

del Buono, Giuseppe, 640

Delong, C. W., 924

Demerec, M.s 1813, 1814, 1815, $1816,1828,1938,1939,2018$

Dempster, Everett R., 405, 1817 , 1818

de Nicola, Marina, 1522, 1523

Dennis, Richard H., 584

Denniston, Rollin Henry, 415

Denstad, T., 614

Dent. J. N., 876, 1701, 1892

Deringer, Margaret $\mathbb{K}_{\text {, }}$ 79, 499, 1819

Desaive, $\mathbb{P} ., 846$

Deupree, Norma G., 521, 522, 523

Devi, P., 1820

Devik, Finn., 364, 416, 939

Deysine, A., 877

Dickie, A., 615

Dickinson, $\mathbb{R}^{\mathrm{H}}$ H., 516

Dickman, Sherman, 1562, 1563, 1564

Diderer, P. H., 2019

Dieckmann, C., 365

Diethelm, Lothar, 616

Dietz, A. A., 1437

Dillard, G. H. L., 1108

Dinning, James S., 1577

Dittrich, W., 365, 1690, 1702

Dixon, Frank J., 519, 530, 531, 661, $719,914,915,1761$

Doan, C. A., 878, 958

Dobbs, W. H. H., 257, 258

Doluson, E. L., 1032

Dobson, R. Lowry, 15, 133, 366

Dobyns, B. M., 789

Doherty, David G., 1578

Dole, N., 658

Donaldson, David D., 255

Donaldson, Lauren R., 100,1687 , 1708,1765

Donlan, C. P., 970, 1483

Donnelly, A. J., 297

Dooley, Robert, 1151

Dorrance, G. M., 297

Dougherty, T. F., 662

Douglas, D. M., 171

Doull, John, 818, 1438

Dowdy, Andrew H., 16, 17, 228, 250, $678,679,1146,1147,1227,1228$, 1229

Downes, H. R., 138

Downey, Hal, 663

Downs, W. L., 997, 1020

Doyle, Wiliam L., 1579
Draeger, R. H., 18, 1093, 1109

Dreblow, W., 46

Drescher, H., 562

Drobliov, A. A., 1703

DuBois, Kenneth $\mathrm{P}_{*}, 818,1438$

DuBow, $\mathbb{R} .1543$

Ducoff, Howard, 780,781

Ducuing, J., 664

Dudley, H. C., 839, 841, 842, 843

Dudley, R. A., 789

Duffy, B. J., Jr., 856

Duffy, J. J., 138

Dufresene, $0 ., 1361$

Duggar, Benjamin M., 19

Dunlap., C. E., 1650

Dunn, Cecil G., 108

Dunn, Thelma B., 446

Dunning, 刃. R., 223

Duryee, William R., 2020

Dustin, Pierre, Jr., 1362, 1614

Dutcher, Raymond, 1193, 1204, 1205

Dwyer, Cecelia Marie, 1466

Dyke, C. G., 256

Easton, Thomas W., 1000

Eck-Vermande, G. J. Van, 1248

Eddy, Walter H., 1203

Edelmann, Abraham, 20, 21, 386, $1110,1211,1212,1213,1214$, $1215,1232,1439$

Efrati, E., 1651

Ehlers, O. C., 677

Ehrenberg, I., 2021

Ehrenberg, L., 1961

Ehret, Charles F., 124, 1621

Eldredge, J. H., 1282, 1283

Elghammer, Richard M., 708

Ellinger, Friedrich, 22, 103, 104, $105,106,159,417,1216,1217$, $1218,1219,1220,1221,1222,1363$, 1440

Ellis, Frank, 1461, 1821

Ellis, Hobart R., 776

Ellis, William W., 597

Elsberg, C. A., 256

Elson, L. A., 474

Eltzholtz, D. C., 657, 1196, 1251

Eltzholtz, Dean, 102

Ely, J. O., 1488, 1489, 1524, 1525, 1600

Ely, Ray E., 814

Emerson, G. A., 1346

Emily, J. B., 352

Emmett, John, 1704

E'mmons, C. W., 1857

Endicott, K. M., 655

Enerson, D. M., 711, 714, 720, 721, 1140

Engelhard, Hermann, 107

Engels, Alfred, 1001, 1002, 1005

Engelstad, R. B., 260, 418, 563, 564

Engle, Eat $\mathbb{T}_{0}, 209$

Englert, Mary Elizabeth, 1466

English, James A., 419, 843

Enloe, C. F., 1067

Entenman, Cecil, 601, 605, 979, 980, 1494,1495

Enudson, Lewis, 1872 
Enzmann, E. V., 1512, 2108

Ephrat1, Erela, 337, 532

Ephrussi, B., 338

Ezf, L. A., 879

Ermala, P., 1501

Errera, M., 1526

Eschenbrenner, Allen B., 79,195 , $229,234,420,421,422,423,424$, 499

Escher, G. C., 431

Essenberg, J. M., 261, 425

Euler, Hans von, 1516, 1553, 1554, 1555,1705

Evans, B. Ho, 2014

Evans, H. B., 953

Evans, Robley D., 23, 367, 752, 943, $944,968,1822,1823$

Evans, $\mathbb{R}$. Winston, 533

Evans, Titus C., 196, 197, 312, 368, $970,1111,1615,1623,1624,1625$, 1706

Eversole, W. J., 1110

Ewing, Paul 1., 1346

Exner, F. M., 1664

Faberge, A. C., 2022, 2023, 2024, $2025,2026,2027$

Failla, G., 24, 138, 1364, 1706

Fairchild, L. M., 2010

Falkenheim, Marlene, 790

Fano, U., 1814, 1824, 1825, 1826, 1827,1828

Furabee, Lawrence B., 981

Faraghan, W. G., 1022

Farmer, F. T., 1652

Farr, Richard S., 262

Farris, Edmond J., 430

Feinstein, Robert No, 1441, 1527, 1580

Feldman-Muhsam, B., 743

Feller, D. D., 853, 859

Fels, S. S., 74

Felsenfield, Osear, 1153

Ferkel, R. L., 1599

Ferri, L., 1310

Fershing, J. L., 1444

Fetzer, H., 1170

Field, John B., 1171, 1197, 1198, 1202

Figge, Frank H. J., 475, 476, 1695

Findlay, D., 854

Fink, Ko, 1442

Fink, Robert M., 25

Finkel, Mriam $\mathbf{P}_{\text {. }} 469,470,880$, $893,921,931,936,937,983,984$, 1032

Finkle, R. D. 783, 785, 918, 922, 971,975

Finley, Harold E., 1808

Fischel, Edward E., 534

Fischer, $P_{0}, 545,1185,1256$

Fisher, H., 808, 833, 868

Fishler, Maurice C., 252, 335, 517, $594,979,1328,1493$

Fishman, Harold C., 946

Fitzgerald, Patrick J., 856

Flint. Jerry S., 1145
Flood, Veronica, 1415, 416, 1418, $1565,1608,1640$

Florence, Stebbins M., 2085

Flyn, Anne E., 1834

Fogg, Lloyd C., 426, 427

Folsom, Fleming, B., 1259, 1266

Ford, C. E., 2028

Foreman, Harry, 1033

Forkner, C. E., 958

Forro, F., Jr, 1386

Forse, R. Armour, 172

Forssberg, Arne, 26, 72, 90, 1257 , $1365,1581,1582,1707$

Foster, Richard F., 1687, 1708, 1785

Fram, Harvey, 108

France, H. O., 227

France, Owen, 1112

Francis, D. S., 138, 321

Frankenthal, L., 1443

Franklin, Rachel G., 1637

Franks, W. R., 1430

Fredell, Herbert, 535

Frederic, J., 428, 429, 1583

Free, Audrey A., 815

Freed, John H., 430

Freeman, J. W., 1152

Freeman, P. J., 1568

Freud, J., 259, 1248

Fricke, H., 1653

Friedell, Hymer L., 497, 777, 847, 881, 1343

Friedewald, W. F., 313, 314, 1709

Friedman, Milton, 1345,1366

Friedman, N. B., 263

Friedman, W. B., 264

Friedmann, A. B., 565

Friedrich-F relss, H., 1395

Fries, Nils, 1829, 1959

Frilley, Marcel, 456, 457, 1641, 1654

Frolik, E. F., 393, 1830, 1831

Fruhling, L., 1187, 1188

Fryer, H. C., 1832

Fryer, Minot P., 1276, 1277

Fuerst, R., 1616

Fuller, Frances Willard, 1749

Fuilon, John D., 1330

Furth, Frank Wo, 73, 1103, 1154, $1155,1156,1159,1160,1161$, 1353

Furth, Jacob, 230, 477, 478, 479, $604,665,855$

Furth, O. B., 479, 648

Gabe, Manfred, 647, 1557

Gaines, J. A., 431

Gaither, Nenita $T_{0}, 334,1867,1868$

Gall, E. A。, 139, 140, 145, 1680

Galluzzi, N. J., 721, 728

Garcia-Calderon, J., 583

Garcia, J. F., 1279

Garde, Alberto, 2029

Gardiner, Donald, 937

Gardner, W. V., 480, 1247

Garrison, W. M., 830

Garrott, J., 34
Gastaldi, Gildo, 265

Gaston, Evelyn 0., 650, 666, 672, $1280,1283,1284$

Gaston, J. G., 725

Gaswoda, B., 978, 1414, 1417, 1418, 1608,1609

Gasvoda, Jeanne, 182, 2139

Gates, Allan A., 948

Gaudin, P., 440

Gaulden, Mary Ester, 2030

Gauwerky, Friedrich, 566

Gay, Douglas M., 203

Gay, H., 2062

Geffen, Abraham, 882

Geger, J. H. 878

Geist, S. H., 431

Gelber, Louis J., 1172

Gelin, O. E. V., 2031

Genter, Clarence $\mathbb{R}, 1833$

George, E. P., 481

George, M., 481

Gersch, Manfred, 432

Gershon-Cohen, J., 74, 266

Gerstner, Herbert B., 1367

Gest, H., 885

Getzendanex, M. E., 924

Ghent, $W_{\text {W. R. }} 171$

Gibson, John G., JI., 789

Gierlach, I. L., 443

Gierlach, Z. S., 1584

Giese, A. C., 2032

Gilbert, C., 1647,1648

Gilbert, Is. Aa, 1520, 1521

Giles, Dorothy L., 1834

Giles, H. H., Jr., 2033

Glles, N., 2077

Giles, Nornan H., Jr., 1794, 1834, 2001, 2034, 2035, 2036, 2037 , 2038,2039 , 2040, 2041, 2042, 2043, 2044, 2094, 2095

Gill, Dan C., 178

Gilmore, R. F., 727

Gjessing, Erland C c $_{\circ} 598$

Glass, H. Bentley, 1835, 1836, 1837

Glasser, $0 ., 27$

Glasser, S. M., 1222

Glasstone, Samuel, 28

Glenn, John C., Jr., 536, 1173

Glotzer, D. J., 462, 721

Glover, John KL, 1260

Gluelssmann, A., $75,1368,2045$, 2130

Godwin, 1. D., 729

Godwin, John T., 856

Goff, John L., 192

Gofman, John W., 1453

Goldberg, R. C., 857, 858, 859

Goldfeder, Anna, $76,1299,1444$, 1710

Goldhaber, G., 743

Goldie, Horace, 848,1258

Goldis, Bernice, 1973

Goldman, Douglas, 1445

Goldman, M. 0., 537

Goldscheider, $\mathrm{G}_{\text {. }}, 1446$

Goldschmidt, Leontine, 594,680 , 1270

Goldsmith, H. H, 29 
Gompel, C., 1614

Goodell, J. P. G., 849

Goodrich, J. P., 1623, 1624, 1625

Gorbman, Aubrey, 860, 861, 862

Gordon, A. S., 386

Gordon, E. Leiter, 462

Gordon, Solon Ao, 1711, 1712, 1762, 1763

Goring, C. A. I., 1699

Gounaris, I., 1447

Govaerts, J., 802

Gowen, John W., 538, 539, 1832, $1838,1839,1840,1882$

Gowen, Marie S., 1839

Grachus, Theresa, 1424

$\mathrm{Grad}_{2} \mathrm{~B}_{\text {. }}, 883$

Graevskaya, B. M., 1428

Graff, W. S., 884

Graffeo, A. J., 1224

Graham, J. B., 1223, 1224

Graham, R. M., 1223, 1224

Granhall, 1., 1841

Gratzek, F。R. 567

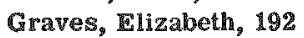

Grawl, E. II., 2046

Gray, C. H., 1842

Gray, Joe, 844

Gray, John L., 1225

Gray, L. H., 30, $\mathbf{3 1}, 109, \mathbf{1 1 0}, 111$, $112,137,149,198,215,369,370$, $371,387,464,1369,1575,1655$, $1713,1714,1715,2047,2070$

Greeley, Paul W., 1278

Greenberg, D. M., 819, 923, 985

Greenfield, Maurice M., 568, 682

Greening, J. R., 963

Greenstein, Jesse P., 1551, 1552

Grenan, Marie M., 348

Griffen, A. B., 1843, 1844

Griffith, J. Q., Jr., 266, 290, 1199 , 1294

Grimm, Madelon R., 1919

Grimmett, L。. G., 1656

Gros, Charles, 1448, 1628

Gross, $R_{\text {., }} 159$

Grossman, Burton J., 708, 720, 728

Grotts, John H., 1275

Growley, J., 761

Grundhauser, W., 773, 1032

Gump, H., 655

Gunz, F. W., 617, 681

Gurian, Joan, 1166

Gustafison, Gordon 1157

Gustafsson, Ake, 315, 1841, 1845, $1846,1847,1848,1961,2021$

Haas, F., 1618

Haddox, C. $1 ., 1616$

Hagen, C. E. 871

Ifagen, C. W., Jr., 113, 114, 115, $116,372,675,773$

Hahn, L. 1553

Hahn, Paul F., 848, 849, 850, 1258

Haines, R. B., 1373

Halberstaedter, L., 117, 306, 316 , $433,595,1226,1651$

Haldane, J. B. S., 1849

Haley, J. J., 317
Haley, Thomas J., 1113, 1174, 1227, 1228,1229

Hall, B. Vincent, $318,319,1331$

Hall, John J., 1496

Hall, R. H., 1003

Hall, Thomas, 540

Halpern, B., 718

Halsey, J. Bagg., 138

Halter, C. Robert, 138

Hamilton, Joseph G., 38, 757, 761, $782,786,788,820,821,822,823$, $824,825,826,827,828,829,830$, $831,837,923,934,977,1033$

Hamilton, Katherine, 473

Liammer, J. M., 771

Hammond, Carolyn W., 1158, 1164 , 1165,1166

Hammond, E. Cuyler, 1079, 1080, 1083

Hanford, S. W., 1718

Hans, Marguardt, 2078

Hansen, Robert A., 1145, 1146

Hanson, Hazel Jean, 1850, 1909

Harber, Alvin, 1345

Hardenbergh, Esther, 591

Harrell, George 'T,, Jr., 874, 1295 , 1297

Harrington, Helen, 1464,1528

Harrington, Nyra J., 1529

Harris, D. Harriette, 317, 1174

Harris, 1. D., 1324

Harris, Payne S., 1100

Harris, R. S., 944

Harrison, B., 1639

Harvey, R. A., 268, 269

Haskins, C. P., 1512

Hatcher, C. H., 569

Hauschildt, James D., 605, 1449

Hawmond, Carolyn W., 1121, 1122

Hayden, Baxbara, 1851

Hayden, H. S., 771

Hayes, Thomas L., 1453

Heald, A. H., 270

Hedin, Raymond $F^{\circ}, 173$

Heeren, I. G., 77

Heidenthal, Gert rude, 373,1852

Heinrirh, H. L., 2048, 2111

Heisler, Charles, 1343

Held, A., 722

Held, Edward E., 320

Helde, Matts, 618

Helfer, R. G., 2049

Heller, D. A., 977

Helmke, Roderich, 570

Hempelmann, Louis H., 178, 442, $615,1048,1626,2065$

Hendley, Daniel D., 1580

Henley, Catherine, 2012

Hennessy, Thomas G., 596, 1259, 1260

Henri, Fajerman, 945

Henry, J. A., 1332

Henry, Katherine $\mathbb{E}$. 842

Henshaw, C. T., 138

Henshaw, Paul S., 32, 122, 138, 174, $199,224,321,374,375,434,435$, $436,437,438,439,482,483,484$, $485,667,1049,171 \%, 1718,1719$, $1720,2050,2051$
Herde, K. E., 760

Hermel, M. B., 266

Hershey, A. D., 885

Herskowitz, Irwin H., 1853, 1854

Hertwig, P., 1855

Herve, Andre, 247, 280, 1183, 1184, $1185,1189,1190,1191,1192$,

$1300,1301,1321,1333,1334$, $1335,1336,1476$

Heston, W.B., 1819

Heston, Walter E., 79, 234, 235, 499

Heusghem, C., 1476

Hevesy, Georg V. 1450, 1451, 1452, $1516,1517,1530,1531,1554$, 1555

Hewitt, H. B., 322

Hewitt, John E., 1453

Hicks, Samuel, 1721

Hiemsth, Willi, 1175

Higginbottam, C., 1820

Highman, Benjamin J., 1633

Higinbotham, Noe, 1454

Hilcken, J. A., 139, 140, 145

Hills, T. H., 571

Hilscher, Wolfgang, 616

Hine, G. H., 765

Hinkel, C. L.o, 271, 1722, 1723

Hinshelwood, Cyril, 1812

Hirsch, Edwin F。, 1017

Hirsch, Gertrude M., 893, 931, 936, 937

Hobbs, Arthur A., J., 572

Hoch-Ligeti, Cornelia, 486

Hochwald, Lynette B., 1619, 1620

Hodes, P. J., 290

Hodge, Harold C., 790, 997, 1019 , 1020,1029

Hodges, Fred Jenner, 381

Hodges, G. R. V., 1263

Hoecker, Hrank E., 947, 956,957

Hoff man, Joseph $\mathbb{G}_{0}, 200,442$

Hoffman, R. S., 2052

Hogberg, B., 1705

Hogness, John R., 178

Hohl, K., 1337, 2053

Holden, William D., 723

Hollaender, Alexander, 309, 323 , $324,325,326,327,351,1427$, $1551,1552,1838,1839,1856$, $1857,1858,1859,1957,1999$, $2060,2061,2062,2135$

Hollc roft, Joanne Weikel, 828,886 , 967,1114

Holle, Fritz, 1585

Hollungsworth, đ. W. 540

Holmes, Barbara $\mathbb{E}_{\text {. }}, 1338,1374$, $1455,1532,1533,1571,1586$

Holmes, S. G. 887

Holmstrom, $\mathbb{E}$. G., 567

Holstrom, Vera, 964

Holl, Margaret W., 913

Horn, Edward C., 376

Horning, $\mathbb{E}$. S., 481

Hornyliewytsch, Th., 1456

Houtermans, Thea, 107

Howard, A., 1370

Howland, Joe W., 73, 1068, 1094, $1103,1155,1156,1159,1160$, 1161,1423 
Hsu, C. L., 282, 668

Hu, F.N., 887

Tuant, $\mathrm{E}, 1302$

Huber, Wolfgang, 70B

Hubert, Walther, 573

Hudson, J. C., 141

Hueper, W. C., 33, 272

Hufi, Rex L., 596, 699, 1138, 1279

Huffman, C. $F_{0}, 814$

Hughes, $W . F_{a g} J \mathrm{r} ., 175,176$

Hulback, H., 722

Humphreys, Eleanor M., 582

Hungate, F. P., 1860

Hunt, H. B., 516

Hunter, Mathew C., 1861

Hunter, R. B., 1168

Hurgh, John B., 81, 273, 938, 948

Hurwitz, Leon, 1021

Hyman, Arthur, B., 964

Ickowicz, M., 433, 1226, 1230

IIiff, C. EE., 175,176

Imirie, G. W., Jr., 839

Imlex, A. E'., 1303

Inda, Frank A., 239

Ingraham, $\mathbb{L}$. P., 251

Ingram, $M_{*}, 619,620,621,669,670$

Irvine, Virginia C., 1724

Ishibashi, $\mathbb{K}_{\circ}, 639$

Ishihara, Sachiro Janet, 1153

Istock, J. T., 106, 839

IEgO, M. U., 658

\section{Jackson, D.P., 718, 724}

Jacleson, Eugenia M., 1266, 1502

Jackson, Marvin A., 848,850

Jacobs, G. J., 724

Jacobsoln, Kurt, 1587, 1588, 1589, 1590

Jacobson, Leon 0., 34, 113, 236, $237,274,329,377,450,537,541$, $542,612,622,628,650,652,666$, $671,672,673,674,675,691,692$, $693,708,709,712,713,725,781$, 808, 833, 834, 868, 949, 972, 986, $992,995,1034,1279,1280,1281$, $1282,1283,1284,1304,1316$, 1351,1725

Jacox, Harold W., 973,

Jacquez, John A, 118

Janeway, Charles A., 549

Janzen, A. M., z75

Jarris, J. L., 1457

Jelatis, Demetrius G., 173

Jellinke, N., 454

Jenkinson, E. L., 1339

Jennings, $\mathrm{F}$. Lamont, 330

Jensen, Hans $F_{0}, 597,1225$

Jensen, $7 ., 1085$

Joel, C. A, 1231

John, E. S., 1240

Johns, H. I. 160,1928

Johnson, C. Ga, 1115

Johnson, Grant C., 1017

Jolles, Benjamin, 276, 277, 278, 441

Jones, Arthur, 574
Jones, D. C., 335, 788, 987, 988, 1116, 1117

Jones, Hardin $\mathbb{B}_{0}, 853,888,1453$, 1536,1537

Jones, M. W., 744

Jordan, M. L., 1194

Jordon, H. Charles, 467, 512

Jornlin, Francis M., 1726

Joseph, I., 1892

Jowsey, J. R., 899

Joyet-Lavergne, P., 331

Judassohn, $W ., 440$

Jude, $\mathbb{A}_{4}, 5 \mathbb{4} 3$

Jungner, Ingmar, 1534

Ifabakjian, D. $\mathbb{H}_{\text {. }, 230}$

Kabat, Elvin A., 534

Rahlau, G., 46

Kahn, J. B., Jr., 1340

Kamen, M. D., 35, 885, 1535

IRanee, B., 636

Kanellis, A., 1802

Kaplan, Henry So, 244, 332, 487, $488,489,490,491,492,493,494$, 495, 1237

Kaplan, N., 985

Tarnofsky, David A., 118

Karr, John W., 1769, 1770

Iar rasch, R. J., 425

Iraser, $0 ., 1472$

Kashehenko, L. A., $165 \%$

Katsh, Seymour, 1215, 1232

Katz, J., 924

Ifauf mann, B. N., 1957

Kaumann, Berwind P., 1815, 2054, $2055,2056,2057,2058,2059$, $2060,2061,2062,2063$

Rauf mann, J., 702

Kawin, Bergene, 831,925

政eating, Fichard P., 1135

Keilina, R. Ya, 1428

Keith, Cecilia $\mathbb{K}_{0}, 1577$

Keller, Mildred E., 1130

Teller, Paul D., 1069

Kelly, E. Mo, 310

Kelly, Lola Sa, 1536

Rempton, J. H., 1627, 1630

Kennedy, J. W. 885

Renney, J. M., 889,1471

Kepp, Richard Kurt, 378, 379, 380

Kerr, H. D., 312

Kerschner, Jean, 1862

Inerst, D. W., 162

TSersten, H. 刃., 1728, 1753

Tidman, Barbara, 1035

Kikuchi, T. ${ }^{\circ} 623,1070,1071$

Kille, J. C., Jr., 1937

Kilmer, Victor" d., 1758

Kimball, R. F., 333, 334, 1863, $1864,1865,1866,1867,1868$

Kineldorf, D. J., 335, 1116, 1117

Kimura, \$。 J。, 1065

KKing, Edward D., 1869, 1870

政ing, E. R., 844

King, 刃. C., 1968

King, R. Co, 1871

Kinsey, Victor $\mathbb{I E}_{0}, 1413$

Kirby-Smith, J. S., 2064
Kirk, Majorie, 540

KKirschbaum, Arthur, 496, 498

Rirschner, Leonard B., 1456

Kirschon, Alice, 535, 713

J3irslis, Marian, 1234

Kirsner, Joseph B., 582

IKisieleski, Walter K., $^{769,} 783$, $785,794,889,926,974,990$, 1022,1036

Kivel, B., 744

IKigerman, Morton M., 890

Kimecky, U1, 608

Tlumpar, $\mathbb{\pi} ., 161$

Knowhon, Norman P., Jr。, 178,442 , 624, 625, 1626, 2065

Koch, H. W., 162

Koch, $\mathbb{R} ., 1459$

Roch, Wilhelm, 1729

Kocholaty, Walter, 597

Koenig, Virgil L。, 1658

Koester, M. I., 1730

Koets, $P_{\circ}, 1434$

Kohn, Henry Io, 170, 544, 598, 607, $1118,1200,1233,1234,1341$

Koletsky, Simon, 497, 891, 1157,1162

Foller, P. C., 1873, 2066

Rordecki, Roman, 733

Kormblum, $\mathbb{K}, 1124,1125$

Kosin, 1. L. $208 \%$

Rostoff, Dontcho, 2068

Kotval, J. P., 2069, 2070

Koza, Robert W., 1529

Kraemer, E. O., 1491

Trebs, A. T. 46, 443, 1004, 1584

Trrejci, Io. E., 1481

Krenz, ${ }^{2}$. H., 1652

Kresage, Marion, 515

Kretzschmar, Carol H., 1461

Kreyberg, Leiv, 939

Krisek, H., 1462

Kroier, $\mathbb{X}_{\text {., }} 315$

rruger, $\mathbb{P}_{\text {a. }}$. 187

Ruck, Katherine D., 407

Kudolotsev, V. P. 1759

Kullander, S., 1176

Lacassagne, A., 142, 1142, 1148

LaFrance, Leo J., 1023

Lomarque, Paul, 78, 1828

Lambert, $\mathcal{G} ., 119,1163,1235,1342$

Lamberton, L. F, 164, 474

Lampe, Imadore, 381,406

Lamport, Juanita, 250

Lamaon, Baldwin G., 44

Landau, $\mathrm{H}_{\circ} \mathrm{G}_{\circ}, 1390,1660$

Lange, 鿊。, 968

Langendorf

Langham, W., 927

Lanz, 猡。 C. 761, 832

Ioanz, Henry, Jr

Lanz, R。, 1472

Langl, $\mathbb{E}, \mathbb{F}_{0}, 146,154,1130$

Larkin, John C., 201, 745, 1072, $117 \%$

Lartigue, Odette, 1120, 146\%, 1468, 1469

Lasnitcki, I1פe, 143, 179, 445, 2071, 2072,2073 
Latarjet, Raymond, $336,337,338$, $1371,1463,1629,1663,1731$, 1861

Lathrop, Katherine, $710,726,975$, 976

Lathrop, K. A., 711,727, 728, 807

Latta, J. S., 677

Laughlin, J. S., 163

Lavedan, Jacques, 36

Lavik, Paul S., 1343, 1464, 1528

Lawrason, F. Douglas, 1119

Lawrence, Enest 0., 202, 382

Lawrence, Geo. Ho, 1465

Lawrence, John H., 15, 37, 38, 189, $202,382,383,398,884,892,908$, $1247,1279,1537$

Lawrence, John $\mathrm{S}_{.}, 527,644,678$, $679,696,698,1293$

Lawrence, William $N_{.}, 528$

Layne, D. A., 766, 1372

Lea, D. E. 39, 143, 144, 339, 384, $1373,1374,1801,1874,2002$, $2003,2004,2005,2074,2141$

Lexch, J. E., 279, 575

Lear, P., 113

Leblond, C. P., 854

Lecomte, J., 247, 280, 545, 649, $1185,1256,1301,1336$

Lee, C. C., 899

Lefevre, George, \#r., 1875, 1876 , $1877,1878,1879,1880$

Le rort, M., 1612

Leibowitz, J., 595

Leifer, $\mathrm{g}_{\mathrm{g}} \mathrm{dgat}, 178$

Leitch, James L., 203

LeMay, Marjorie, 534, 1591, 1592

EeMay, M. I. 970

Lenz, M, 970

Leonard, Clark, 373

Leonards, Jack R., 1343

LeRoy, Geogge $V ., 718,1073,1074$, $1079,1080,1081,1082,1083$

Less2, Z, T., 1305

Leucutia, T., 1075

Levan, A., 2021

Lewis, Alvin E, 1145

Lewis, Ruth, 1748

Levis, Thomas H., 1953

L'Heureux, Maurice V., 791

$\mathrm{Li}, \mathrm{C}$. H., 1773,1925

Lichstein, Herman C., 1567

Lick, Louis, 498

Liebow, Averill A., 1076, 1079, $1080,1081,1083$

Liechti, A., 1669

Lienke, R. I., 792

Lih, B. 1308

Limperos, George I., 1149, 1261 , $1538,1539,1540$

Lincoln, Raloh E, 1802

Lindegren, Carl C., 1883, 1884 , 1885

Lindegren, Gertrude, 1883, 1884 , 1885

Lindemann, Bruno, 599, 2075

Lindenbaum, B., 281

Lindsay, Stuart, 859

Lingley, J. R, 139, 140, 145, 1680

Lisco, Jerman, 469, 762, 1037
Little, E. P., 1706

Livingood, C. S., 887

Loevinger, Robert, 882

Loewenthal, Lois A., 461

Lofstrom, J. E., 1178

Loiscleur, Jean, 1236, 1249, 1262, $1320,1463,1661,1662,1663$

Longley, A. E., 1773, 1926, 2076

Loofbourow, Dorothea G., 1541

Loofbourow, John R., 1466

Looney, William B., 950

Loos, Gordon M., 1684

Lorenz, Egon, 79, 120, 121, 231, 232, 233 , 234, 235, 236, 237, 238, $328,424,446,499,673,680,886$, $962,987,1114,1237,1285,1819$

Lorenz, K. P., 122

Lourau, Marguerite, 1120, 1467, 1468,1469

Love, Robert A., 981

Low-Beer, Bertram V.A., 626, $892,824,895,896$

Lowenberg-Scharenberg, $\mathbb{K} ., 557$

Lowman, Frank G., 1765

Luce, Wilbur M., 146, 1732, 1886, 1887,1888

Ludewig, Stephan, 582, 1470, 1593, 1594

Ludin, M., 1422

Lukens, R. M., 576

Luning, K. G., 1788, 1789

Luria, S. E., 1664

Lutwak-Mann, C., 681

Lyons, W. R., 270, 888

McClellan, Walter \$., 969

McClement, Patricia, 158

MeConmell, ㅍ. P. 887,940

McDonald, IE., 204

McDonnel, G. M., 41, 1159

McDowell, Frank, 1276, 1277

McElroy, W. D., 1893

McHenry, 区. W., 1306

Mellwain, A. J., 1307

Mckeen, Charles L., 708

McKendry, J. B. R., 172, 180

McLaughlin, Victor D., 1986

McLean, F. C., 798

MeNattin, R. F。, 138

McNunis, Ruth, 937

Ma, W. C., 282, 668

Maas, Jerome M., 577

MacComb, W. S., 283, 580

MacDuffee, R. C. 687

Mac Farland, M. L., 1306

Macht, D. I., 704

Mackee, G., 80, 147

Mackey, James, 1889, 1961

Macirie, Ruth W., 871

MacMillan, Jane C., 914, 915

MacNider, W. deB., 1024

Maddox, G. E., 841

Madison, Martha, 955

Magill, Mary A., 1637

Maisin, J., 119, 1163, 1179, 1235 , 1342

Major, Mary H., 1748

Maldawer, Marc, 2127
Malloch, W. S., 2082

Mandart, M., 119, 1163, 1235, 1342

Mandel, Paul, 1448

Mann, Samuel, 1227, 1228, 1229

Mannell, T., 1860

Manollov, S. E. 1595

March, H. C., 500, 501

Marchbanks, Dorothy F., 407

Marder, Sumner N., 494, 1237

Marinelli, L. D., 138, 763, 764, 765, $869,1471,2077,2086$

Markees, S., 1472

Markert, C. L., 1890

Markham, R., 1374

Marks, ㄹdna $\mathbb{K}_{.}, 274,377,541,542$, $622,627,628,650,668,672,673$, $674,675,725,1280,1281,1282$, 1283,1284

Marques, $\mathbb{P} ., 664$

Marshak, Alfred, 385, 2079, 2080, 2081, 2082

Marti, Norman F., 684, 1350

Martin, A., 897

Martin, Albert, Jr., 1891, 1892

Martin, F. L., 327

Martin, J.R., 40, 766

Martin, R. P., 905

Martin, S. F., 1065

Martland, Harrison S., 502, 951

Martland, Harrison S., Jr., 951

Masini, Vincenzo, 703

Mason, Karl E., 447, 460

Mason, Michael I。, 586, 1286

Mason, W. B., 669, 670

Masturzo, Aldo, 1025, 1026

Mateyko, G. M., 386

Matheson, Don R., 866

Mathis, Alice I., 1665

Mathews, Joyce J., 1347

Matthews, Marion, 328

Mattis, Paul A., 1008

Maurer, Werner, 1001, 1002, 1005

Maxileld, J. R., Jr., 1307

Maxwell, Louis R., 1627, 1630

Maxwell, Roy D., 1135

Mayer, Lloyd D., 526, 529

Mayer, S., 1621

Mayer, Sylvia D., 124

Maynard, E. A., 997, 1020

Maynard, Russell M., 1139

Mayneord, W. V., 164, 471, 503, 766

Mazia, Daniel, 1596, 1597, 1598

Mazur, Marcella, 818

Mead, James F., 1425, 1435, 1473

Meersseman, $F_{\text {. }}, 119,1235$

Meersseman, M., 1342

Mefferd, Roy B., Jr., 353

Meissner, W. A., 1061

Mendeleefi, P., 504

Meredith, W. J., 1574, 1575, 1649

Meschan, 1., 1577

Metcalf, Roger G., 239, 448

Metz, Charles W., 1989

Meyer, H. L., 667

Meynard, J. M., 345

Meymel, Gaston, 600

Mika, Edward S., 1454

Mikaelsen, $K$ nut, 2083

Miki, K., 1071 
Miletzky, $0 ., 653,644$

Milham, Mary, 535, 713

Miller, Charlotte, 1299

Miller, C. Phillip, 1121, 1122, 1158, $1164,1165,1166$

Miller, Earl R., 863

Miller, Eliza, 195, 421, 422, 423, 424

Miller, Esther S., 924

Miller, H. L., 1728

Miller, J., 684

Miller, Joseph H., 1350

Miller, Winston R., 173

Milne, John, 1474

Milton, Burton, 1359

Minder, W., 1375, 1376, 1686, 166\%, 1669,1700

Mitchell, C. J., 311

Mitchell, Jack H., Jr., 1548

Mitchell, J. R., 1633

Mitchell, J. S., 42, 148, 205, 206, 207,1542

Mitchell, Roland B., 1330

Mitchell, R. G. 278

Mitra, B. M., 900

Mittwoch, Ursula, 1894

Miwa, N., 2150

Miser, Harry, 498

Mixer, W. H., 496

Mobius, Wulfied, 432

Modigliani, Franco, 1733

Moh, C. C., 1895

Moheny, John B., 273

Mohler, James D., 1844

Moir, W. W., 455

Mole, R. H., 1263, 1344, 1412

Moll, Frederick C., 549

Molomut, Norman, 1345

Montag, C., 449

Montgomery, Philip O'B., 1123

Moon, V. H., 1124, 1125

Moore, Dorothy E., 521, 523

Moore, Jeffries P., 511

Moore, M. C., 241, 1127, 1128

Moos, Walter $S_{.}, 165$

Morgan, D. R, 1124, 1125

Morgan, Jasper E., 106, 167

Morgan, K. Z., 43, 767, 768

Mori, Akiko, 1088

Mori, IF., 2150

Morowitz, Harold J., 1377

Morris, Rosalind, 1830, 1831

Morrison, P., 162

Morse, M. L., 309

Moses, Campbell, 629

Moses, Montrose J., 1543, 1549, 2128

Mosher, W. A., 1261, 1539, 1540, 1668

Moshman, Jack, 505, 630

Mosley, Vernon M., 1630

Mossberg, H., 631, 632, 633

Motacek, D., 608

Mottram, J. C., 149, 198, 340, 387, $506,1051,2084$

Moulden, Elizabeth J., 1225

Moulder, Peter P., 714

Moulder, Peter V., 708, 711, 721, 728,1140
Muir, R. M., 898

Muller, H. J., 1896, 1897, 1898 , $1899,1900,1901,1902,1903$, $1904,1905,1906,1907,1922$

Müller, Kurt, 380

Mullis, A., 1669

Mulryan, B. \#., 1027

Munn, J.L., 842

Muntz, John A., 1420, 1475, 1563, 1564

Murphy, Douglas P., 430

Murphy, Edwin D., 495

Murray, M. J., 1908

Murray, Raymond, 113, 450

Mutscheller, A。, 80, 147

Muxart, Roland, 945

Myers, J.W., 878

Myers, W., 878

Myers, W. C., 1850, 1861, 1900

Myers, Wm. G., 1861, 1909

Nabours, Robert K., 2085

Nadal, R., 578

Nagelsen, Margaret G., 47

Nalaidzum, Masanori, 1077

Nathan, M. Herbert, 1169

Natsumoto, Shigeko, 1088

Neal, William B., 781

Nebel, B. R., 2042, 2077, 2086, 2149

Neel, James V., 630

Nelson, Eugene J., 989

Nettleship, $\mathbb{A}_{\circ}, 451$

Neu, Ladislaus, 1378, 1379

Neu, V., 1379

Neuman, M. W., 1027

Neuman, W. F., 787, 987, 1027

Neve, R. A., 601

Neville, George A., 203

Newcombe, H. B., 1910, 2087

Nicholas, D. J. D., 872

Nichols, Charles, Jr., 2088

Nichols, $0 ., 402$

Nichson, J. J., 612, 634

Nichson, M., 286, 278, 288

Nickson, Margaret J., 253

Niklas, Amnemarie, 1001, 1002, 1005

Nims, Leslie, $F_{0}, 45,731,732,1477$, 2129

Nishina, Yoshio, 208, 210

Nisson, F., 1841

Nix, Marjorie, 2030

Nizet, R. $\mathbb{E} ., 1476$

Nolan, Constance, 804

Noonan, T., 81

Noonan, T. $\mathbb{R}, 1490$

Norby, Barbara, 349

Nord, $F_{0}$. F., 1566

Nordenson, Nils G., 635

Nordenstrom-Blomqvist, Bjorn, E. W., 1008

Norman, A., 1356

Norris, William P., 769, 793, 794, $889,952,953,990,1022$

Norsen, Jeannette, 1153

North, No, 1477

Note, S., 1071

Novick, Rosalind, 168, 289, 413

Novitski, E., 191
Novy, Frederick Ceorge, $\mathfrak{I r}, 1078$ Nurnberger, C. E, 1178

Nuttal, John R., 1030, 1051

Nybom, Nis, 1848

Oakes, WHItam R., 178

O'Brien, John $\mathrm{P}_{\circ, 1734}$

Oddie, T. H., 77o

Okada, IIyuga, 1816

Okami, Yoshiro, 1912

Olden, E. J., 1841

O'Neil, J. B., 899

Opatowski, 1., 1380, 1381, 1382,

$1913,1914,1915$

Oppenheim, A., 1308

Oppenheim-Errera, A., 1541

Orlov, S. M., 1478

Osborne, Janea W., 1130

Osgood, Edwin E., 888,1383

Oshry, Eleanor, 964

Ostro, M., 704

Otani, Shohel, 1916

Otis, Eileen M., 1735

Oughterson, Ashley W., 1070, 1020, $1081,1082,1083$

Overstreet, Jacobson $\mathbb{R}_{\text {. }}, 895$

Overstreet, R., 808, 833, 834, 868, 1040,1725

Packard, C., 123, 150, 1683

Paillard, R., 40

Painter, Elizabeth E., 1052, 1126, 1127

$1127,1128,1129,1470,1508$

Pal, R. $\mathbb{R}, 900$

Palmer, Walter Lincoln, 582

Panzironi, P. E., 1180

Proletti, Mario, 1544

Papageorge';, N., Jr., 84

$\mathbb{P}_{\text {ape, }} \mathbb{R}$, 452, 453, 454

Parcher, $\mathrm{F}_{\text {. }} 636$

Paris, R., 1201

Park, Harold, $F_{0}, 50 \%$

Parker, D. R., 2089

Parker, H. M., 181

Parker, James Preston, 1346

Parkes, A. S., 1679

Parrish, Mary Lllen, 408

Parsons, Dorothy Lo, 503

Paterson, Edith, 82, 83, 84, 1347

Patt, Harvey M., 341, 1134, 1130, $1238,1239,1240,1241,1244$, $1245,1264,1285,1268,1267$, $1268,1269,1271,1272,1273$, 1274, 1502, 1621, 1632

Patterson, Priscilla A., 1727

Patti, $\mathbb{F}, 99,362$

Paul, W., 1690, 1702, 2045

Payne, Thomas J., 1084

Paynter, M。, 110

Pearce, Morton Lee, 682, 697, 698

Pearlman, N., 321, 1351

Pearse, Herman E., 1084

Pearson, I. A., 771

Pendergrass, Eugene P., 290, 430, 1199

Penick, G. D., 729

Pennybacker, Joe, 579 
Perje, Ann Margaret, 1789

Perkuson, Jesse D., Jr., 844

Pexrî, Teodoro, 1736

Perrings, J. D., 1658

Perryman, $\mathbb{R}_{\circ} 1199$

Pervinsek, William, 774

Peter, 0., 191, 1803

Peters, M. V., 1306

Petersen, B. W., 1653

Peterson, Ruth D., 1480

Peterson, Stanley C., 413

Petrakis, N. L., 1599

Petrova, J., 342

Philipp, Kuxt, 1007

Phillips, Michael D., 47

Philpot, J. St. L., 1263

Philpott, Osgoode \$., 590

Biccirillo, Ronald, 645

Pickels, G. G., 1670

Pickering, Bryant lo, 686, 1270

Pieguet, J., 578

Pierce, Frank T., 1453

Plerce, Milla, 450

Pierce, Milda, 708

Pietra, R., 1481

Pinney, Edmund, 1384

Pino, J. A., 297

Pinson, Rrmest A., 1011, 1292

Plasencia, H. T., 1482

Platt, Madeline, 629

Plat, W. R., 901

Plough, Harold B., 1917, 1918

Plough, H. H., 1919

Pluygers, 1., 119, 1163, 1235, 1342

Podio, G., 119, 1163, 1235, 1342

Pohle, 沓. A, 455

Pollard, E., 1385

Pollard, E. C., 1386

Pomerat, C. M., 887

Pomper, Seymoux, 1920

Pontecorvo, C., 1820, 1905, 1921, 1922

Popp, Walter C., 1167

Poppe, H., 961

Porter, Lille Mae, 1498

Portmann, A. F., 723

Posin, Daniel Q., 72\%

Potter, J. Craig, 151

Poulges, B. Ran $_{\text {, }} 85$

Power, J. M., 1758

Rowers, E. L., 124, 125, 1012

Powers, L. L., J7., 902, 1923, 1924

Praytor, 12, 1095

Price, C. H. G., 62\%, 683

Price, T. L., 1085

Proctor, Bermard, 108

Prosser, C. Ladd., 241, 390, 773, 774, 994, 1128, 1129, 1351, 1458, 1475

Bullman, 監. W., 1479

Quasller, Henry, 126, 136, 146, 152, $153,154,343,1458,1737,1738$, $1739,1740,1886,1887,1828$, 1860

Quimby, I. H., 2\%, 138, 283, 368, $580,581,765$
Raab, W., 291

Radu, G. H., 1802

Ragan, C., 1483

Rajewslky, B., 46, 508, 1395

Raleigh, Gordon W., 1275

Ralston, H. 『. 127

Randolph, L. F., 1925, 1926

Raper, J. R., 185, 409

Rasch, Ellen Myrberg, 2090

Ratty, F. J., Jr., 1880

Rawson, Rulon W., 850

Tay, O. M., 1685

Ray-Chavelhuri, S. P., 1927

Raynaud, Albert, 456, 457

Read, John, 109, 110, 111, 112,198 , $215,322,344,371,954,1387$, $1388,1389,2047,2142,2143$

Reboul, J., 345

Redd, James B., 1203, 1204, 1205

Redileld, Betty, 1610

Reeves, Robert J., 998, 1173, 1309

Regehr, Hulda, 1228

Reich, Herbert, 380

Reid, A. T., 1390

Reid, T. $\mathbb{R}_{0}, 1285$

Reitman, Paul I., 1169

Reit 2 , Leola, 656

Reker'\$, 10,684

Rekers, Paul E., 128, 515, 1094, $1131,1171,1197,1198,1202$, $1287,1348,1350$

Retherford, K. L., 1773

Reviei, Emanuel, 1349

Rhoades, R. Po, 918

Rhoads, C. P., 869

Richard, M. J., 902

Richards, $0 . W_{0}, 546$

Richards, Paul D., 775

Richmond, J., 1411

Richmond, Jonas E., 1484, 1485

Richter, Ester, 1486

Rirk, Charles M., 1929, 1930, 2091 , 2092, 2093

Ricketts, William 토., 582

Ridgway, Lois Po, 172\%

Riedel, M, 1702

Rietz, Lsola, 1010, 1031,1992

Rigdon, R. R., 292

Rifyler, L。 Gog 567

Riley, IE. Fo, 174, 360,409, 484

Riley, Herberl Parkes, 2039, 2043, 2044, 2094, 2095

Riley, Richard F., 1113, 1486

Ripple, Richard C., 584

Ritchie, G., 455

Ritter, Oskar Ls., 1367

Robbie, W. A., 1625

Robbins, C., 1650

Roberts, B. M., 1279

Roberts, E., 685

Robertson, James IE., 1307, 1407

Robertson, R. C., 660

Robertson, W. J., 1234

Robinell, R. W., 1200

Robinson, Courtland Jo, 1684

Robinson, David W., 1288

Robinson, J. M., 156

Robninson, John N., 209
Robinson, Radeliffe F., 47

Robson, M., 1280, 1283, 1284

Robson, Melba J., 274, 537, 541, 542,1281

Rollason, G. S., 1741

Roman, Herschel, 1951, 1952

Roofe, Paul G., 947, 955, 956, 957

Rose, J. E., 138

Rosenbaum, Jack D., 1079, 1080, 1081,1083

Rosenberg, R., 600

Rosenfield, Florence M., 1550

Rosenthal, Robert L., 584, 686, 730, 1132,1270

Ross, Joan, M., 509

Ross, Mary H., 1327, 1488, 1489, 1524, 1525, 1600

Ross, Milton S., 984

Ross, W. C. J., 1391

Rossi, Harald Ho, 48, 776, 1392

Roswit, B., 1222

Rothfels, I. H., 2096

Rovello, F., 1310

Rowell, J. B., 1931

Rowlands, S., 171

Rubin, Benjamin A., 50, 775, 903 , $1545,1932,1933,1934$

Rudolph, Pape, 240

Ruf, Fritz, $100 \%$

Ruffin, Julian M., 998

Rugh, Roberts, 864, 365, 1742, 1743, 1744,2097

Ruprecht, Koch, 1460

Russ, Clem, 004

Russ, s., 86, 1051

Russell, Dorothy \$., 293, 579

Russell, Liane Branch, 1745, 1746 , $1747,1748,1837$

Russell, R. S., 905

Russell, W. L., 1746, 1747, 1748 $1935,1936,1937$

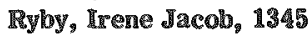

Sabin, 正. R., 958

Sacher, George A., 87, 88, 114, 115, $129,130,131,227,242,391,470$, $628,991,1166,1393$

Sacher, R. H., T84

Sackis, John, 130, 473

Sadun, EIvio H, 1749

Salerno, Paul R, 77\%, 1008

Sallisbury, $\mathbb{P} ., 684$

Salisbury, Peter T., 1350

Sallman, Ludwig von, 294, 346

Balomon, Eurt, 141, 1484,1485, 1490

Salva, J. A., 1181, 1182

Salzgeber, Berther, 1750

Sanden, IJurt von, 1324

Sanderson, M. K., 711, 712, 713, 728

Sanderson, Margaret M., 535, 710

Sanigax, E. B., 1491

Sansome, Eva R., 1938, 1939

Şarasin, Re, 583

Sargent, S., 1431

Sarian, Ji raix, No, 547 , 548 
Sasse, Kanshi, 1086

Sato, Keiji, 210

Sato, O., 208

Sato, Richard, 124, 902

Savage, L. J., 714

Sax, Karl, 2098, 2099, 2100, 2101, $2102,2103,2104,2105,2106$, 2107, 2108, 2109

Saxun, K. P., 296

Scartozz1, Cesare, 1492

Schack, J. A., $68 \%$

Srehaefer, $\mathrm{H}_{\text {. }}, 46$

Schaefer, Hermann J., 392

Schechmelster, I. L., 517, 550,551

Scheie, Harola $\mathrm{G} ., 584$

Scheragra, II. A., 731, 732

Schevessthal, R., 120

Schjeide, Ole Arne, 645, 1619, 1620, 2110

Schlaegel, T. G., JP., 1087

Schmermund, H. J., 363,2111

Schmid, Clarence $F_{0,}, 906$

Schumat, John W., 393

Schneider, B. Aubrey, 1079, 1080 , 1081, 1083

Schnelder, Lor rane, 1498

Schnerder, W., 585

Schnenderman, Herman, 1496, 1569 , 1665

Sohoenberg, Melvin D., 1496

Scholes, George, 1546, 1547, 1671

Scholes, M. E., 1713, 1714, 1715

Schon, M., 1395

Sehork, P. R., 657

Schottelndreyer, H., 89

Schraub A., 46

Schrek, Robert, $347,458,459$

Suhubert, Gerhard, 388, 389, 394, $1396,1690,1702,2048$

Schubert, Jack, 778, 929, 930, 931, 932, 1009

Schwab, Louis, 549

Schwartz, Drew, 1940

Schwartz, S., 638, 1462, 1497, $1498,1499,1510$

Schwe1sthal, R., 237, 238

Scott, C. M., 1397

Scott, G. M., 86, 1311

Scott, J. K., 787, 997

Scott, K.. 808, 833, 834, 868, 933, 1038

Scott, Kannel G., 395, 761, 786, $830,835,836,837,884,207,908$, $934,1096,1429$

Scott, O. K., 183

Scully, N. Jo, 1983

Segre, Mario, 705

Seibert, H. C., 809

Selhi, Louise, 1414, 1419

Selle, W. A., 1144

Selling, Lawrence, 688

Semenov, L. F., 1595

Servant, P., 543

Setala, Kai, 1500, 1501

Setlow, R. B., 1601

Seymour, Allyn H., 100, 1687, 1708, 1765

Sgourakis, Elizabeth, 1778, 1779

Shacter, Bernard, 1133, 1193, 1494, 1495
Shafer, Charles Lo, 178

Shapi ro, J. R., 496

Shaver, Alpha, 1424

Shaver, S. L., 447

Shaver, Samuell, 460

Shay, Harry, 74

Shêner, Deborah, 124, 902, 1012 , 1224

Sheline, Glenn E., 863

Shelton, Emma, 1285

Sheppard, C. W., 302, 602, 603, 849

Sheremetiev-Brunst, E. A., 70c

Sherman, Frederirk G., 1602, 1603

Shorter, Gertrude, 1166

Shorvon, L.. M., 1313, 1314

Sieburth, Louise R., 349, 2112

Sievert, R. M., 90

Siffert, Robert, 246

Silberstein, Hannah E., 959

Silverbach, I., 1462

Silvestri, Giovanni, 1492

Sima, $\mathbb{1}, 689$

Simen-Reiss, 1., 2113

Sîmmons, Lric Lo., 116, 554, 672, $674,675,691,692,693,949,972$ $986,992,1034,1281,1282,1283$, 1284, 1289, 1304, 1351

Simon, Norman, $246,909,965$

Simpson, Linda, 804

Sinclär, W. KR., 1780

Singer, Leon, 795

Singer, Margaret, 1299

Singer, Thomas $\mathbb{P} ., 1563,1564$

Singletor, Ralph W., 2114

Singleton, W. Ralph, 1751, 1941

Sinto, Y., 208

Sipe, Clyde $\mathbb{R}_{.}, 102,657,1196,1251$

Skaggs, Lester S. 136, 166, 1888

Slinpper, Howard E., 803, 804, 805, 806,1548

\$lkoog, T. O O, 1752

Slaughter, D. P., 581

Slaughter, J. C., 1623, 1624, 1625, 1706

Sl1zynsk1, B. M., 2115

Smith, Christianna, 461

Smith, Douglas F, $_{0}, 1134,1150,1238$, $1239,1244,1264,1265,1266,1267$ $1268,1269,1271,1272,1273,1502$

Smith, Falconer, 348, 1242, 1243, 1503

Smith, G. F, 1728, 1753

Smith, K. A., 1520

Smith, K. M., 1374

Smith, Luther, 349, 1795, 1851, 1895, 1242, 1943, 1944, 1945, 2216

Smith, Taylor R., 708

Smith, Willie W., 1151, 1208, 1242, $1243,1503,1633,2117$

Smyth, Henry De Wolf, 48,49

Snell, Fred M., 639

Sincll, George D., 211, 1946

Snider, R. S., 174, 185, 212, 213, $438,439,485$

Snowman, R. T., 867

Snyder, Mary Louise, 1999

Snyder, R., 976

Snyder, R. $\mathrm{H}_{0}, 783,784,785,870,974$
Sobel, Estelle, 111

Soberman, Robert J., 1135

Sokolofi, Boris, 1193, 1203, 1204, 1205

Sommermeyer, K., 1395, 1398, 1399 , 1400,1247

Sommers, Sheldon C., 913,999

Somenblick, B. P., 1604

Soule, A. B., 291

Soyka, 0., 706

Soykova-Pachnerova, E., 708

Sparago, B., 462

Sparks, A., 997

Sparrow, Arnold H., 50, 350, 1543, 1549, 1550, 1754, 1948, 2118 , $2119,2120,2121,2122,2123$ $2124,2125,2126,2127,2128$, 2129

Spear, F. G., 51, 52, 198, 214, 215, 216, 464, 2045, 2113, 2130

Speidel, Carl Caskey, 2131

Spencer, W. P., 1949

Spiers, $F$. W., 155

Spies, Tom D., 1042, 1115

Spiess, Peinz, 960, 961

Spinks, J. W. T., 898, 1981

Stadler, Is. J., 1950, 1951, 1952

Staine, Edward C., 558

Stahly, Grant Lo, 1850, 1861

Stakman, E. C., 1931

Stanford, R. W., 571

Stanski, Feliks, 733

Stapleton, G. A., 225

Stapleton, George E., 170, 217, 224, $309,326,327,351,396,409,484$, 1427

Stark, Frederick M., 568

Steadman, L. T., 1505

Stearner, S. Phyllis, 91, 92, 93, 132, $352,473,690,691,692,693,1304$, $1315,1316,1504$

Steele, Robert, 1549, 2128

Steggerda, . R. 1511

Steiger, Robert E., 1637

Stein, Gabriel, 1546, 1652, 1672, 1673

Steinberg, B., 1437

Steinglass, $\mathrm{p}_{\text {., }} 1934$

Sterlin, G. S. 463

Stern, C., 1797, 1911, 1949, 1963

Stevans, C. E., 910

Stevens, C. E., 883

Stevenson, Thomas W., 1290

Stewart, L. N., 1401

Stewart, M., 603

Stewart, W. B., 867

Stokinger, H. E. $99 \%$

Stone, Robert A., 156

Stone, Robert S., 34, 53, 54, 397

Stone, W. S., 1616, 1618

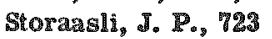

Storer, John B., 1352

Storey, R. H. 411, 604, 365

Strang, Verda, 1286

Straube, Robert L。, 781, 1238, 1239, $1240,1244,1264,1265,126 \%$

$1268,1269,127 \%, 1273,1274$

Stroman, Govan N., 1953

Stromnaes, distein, 1054, 1055 
Stroud, Agnes, 799, 1010, 1013

Sturkie, P. D., 297

Stutz, R., 1712

Suguira, K., 157, 279, 1634

Supplee, Melen, 605, 1449, 1494, 1495

Suskind, S. R., 1635

Suter, G. M., 694

Sutherland, J. M., 734

Sutton, ⿷., 1815

Sutton, Harriet, 120, 121, 230, 237, 238

Svedberg, T., 1674

Svihla, George, 774, 1134, 1274

Swanson, C. P., 1851, 1893, 1956 , $1957,2109,2132,2133,2134$, 2135,2136

Swenson, P. A., 1568

Swift, Marguerite N., 252, 341, 390, $517,551,993,994,1129,1238$ $1239,1240,1241,1245,1631$, 1132

Swingley, Nancy, 1234

Swisher, . N., 1353

Sydow, G., 1792

Tadahiro, Miyalkawa, 284

Tahmisian, Theodore $N_{0}, 182,1605$, $1755,1756,2137,2138,2139$

Tait, J. H., 218, 219, 220

Tatrahara, T., 1088

Talbot, John M., 1292

Taliaferro, Lucy Graves, 552, 553, 554

Taliaferro, Willam H, 552, 553, 554

Talmage, D. W., 519,531

T'Ang, W. H., 695

Tangherlini, $\mathrm{F}, \mathrm{R}, 1382$

Tamnebaum, A, 1028

Tansley, Katharine, 75, 216, 293, 464

Tapley, Donald F., 1986

Tarleton, G. J., 1312

Tarleton, Gadson $J_{\circ,} \mathrm{Ix}_{\circ}, 1258$

Tgarlov, I. M., 258

Tatum, E. L., 1701, 1842, 1958, 1959

Taนrog, Alvin, 853

Taylor, Babette, 1551, 1552

Taylor, L. S., 27

T2ylor, Ruth E., 13, 814

Tchaperofs, T.C.C., 1675

Teloh, H. A., 586

Tennant, Robert, 333

Teresi, J. D., 199, 832

Tessmer, Carl F, 134,1298

Tew, John T., 1225

Thoday, J. M, 2004, 2005, 2140, 2141, 2142, 2143

Thomas, Lyell, 1960

Thomas, W. D. E., 872

Thompeon, Edwin C., 1151, 124

Thompson, H. E., 1505

Thompaon, J. W., 667

Thompson, T. T, 1961

Thompson, M. V., 83

Thompson, R. C., 1018, 1015
Thompson, T. I., 353

Thomson, D. M., 788

Thornton, Doreen M., 815

Thyresson, Nils, 996

Tillsch, Jan H., 1167

Timmes, Joseph J., 1089

Timofeev-Ressovski, N. W., 1402, 1962,1980

Ting, T. P., 606

Tinsley, Mary, 1498

Tobias, Cornelias A, 133, 225, 398, 1403

Tomplins, Marianne, 1121, 1122, 1164, 1165,1166

Tompling, P. C., 778

Toolan, H. W., 1757

Toole, E. H., 1758

Toole, Vivian $K_{\circ}, 1758$

Toosy, M. H., 298

Torda, Clara, 1246

Torese, J. D., 784

Torgersen, $0 ., 418$

Treadwell, A., 1247

Tripodo, Cesare, 640

Txudova, R. G., 1506, 1636

Trujillo, Teodore T., 1626

Trunnell, ฮ. . 856

Tullis, John L. 134, 167, 35็, 410, $419,444,465,591,1136,1137$ 1298,1421

Turkowitz, H., 305, 1720

Tutt, Margaret, 1035

Tweedie, M. C. K., 1649

Tweedy, Wilbur R., 791

Tyler, Sylvanus A. 131, 135, 1166

Tyree, Ella B., 341, 1150,1238 , $1239,1240,1241,1244,1245$, $1267,1269,1271,1272,1273$ 1502,1632

Uber, F. M., 55

Uchimura, T., $150 \%$

Ullmann, H. J., 251

Ullrich, $F$. W., 657, 1298

Umanskii, E. E., 1759

Umezawa, Hamao, 1912

Uncapher, R. P. 101, 1194

Underwood, Elizabeth E., 790

Uphoff, Delta E., 120, 121, 234, 238, 1285,1963

U. S. Army Medical Depi., 1053, $1054,1055,1056$

Vairel, Edmond, 1201

Valencia, J. I., 1906, 1907

Valencia, R. M., $190 \%$

Valentine, William No, 528, 678, $679,682,696,697,698$

Vallee, Bert L., 989

van Cauwenberge, H., 1322

Van Cleave, C. D., 816

Van der Werff, J. T., 68, 1404

Van Dilla, Marvin A., 989

Van Dylke, D. C., 699, 1138

van Eck-Vermande, G.J., 258

Van Haltern, H. L., 1317

Van Middlesworth, I., 923,935
Van Roojen, J., 94

Van Slyke, F。, 81

Van Valkenburg, Priscilla A., 273

Van Zandt Hawn, Clinton, 549

Varshavskii, B. M., 1759

Varteresz, V., 555

Vaughan, J. M., 1051

Vaughan, Janet, 1035

Vavasseur, J., 1201

Velley, Georges, 1236, 1249, 1262

Venters, K. D., 1508

Vesin, M. S., 510

Vieten, H., 299

Villee, $C_{\text {, }}, 1682$

Villee, Claude A., 1760

Villee, Claude A., Jr., 1784

Viller, C. F., 1115

Vilter, R.W., 1042

Vining, Dorothy, 473

Visconti di Modrone, N., 1804

Voegtlin, Carl, 1029

Vogel, Howard H., Jr., 243, 300

Vogt, Alfred, 587

Volkin, Ellot, 607

Voluter, C., 583

Wagner, Carroll, 1964

Wagner, M., 2016

Wagner, R. P., 1616

Wahlberg, 'T., 779

Wakisalia, G., 1071

Waid, B., 555

Waldo, Cohn 2,756

Wallace, Bruce, 1265, 1966, 1967, 1968

Wallace, Jeon, 1498

Wallace, Herschel, Jx., 1009

Wallace, Patricia C., 782

Wallace, W. S., 1318

Walker, Leonard A., 1039

Walter, J., 588

Wammock, H., 1303

Wang, T., 195

Warner, L., 288

Warren, Shields, 18, 62, 63, 64, 65, $86,183,263,275,641,911,912$, $913,914,915,1052,1053,1076$, $1090,1091,1092,1093,1109,1123$, $1137,1405,1761$

Warren, Stafford L., 67, 95, 1068, 1348

Warters, Mary, 1844

Wasserman, L. R., 225

Waters, Thor, 1153

Watson, Cecil James, 1498

Wation, James Dewey, 1406, 1407

Watson, T. A., 160

Wattenberg, L., 1509, 1510

Wattenwyl, H. von, 301

Watts, N. P., 940

Watts, William E., 866

Weatherwas, J. I. 27, 297

Weber, R., 1712

Weber, R. P. 1511, 1762, 1763

Webster, D. R., 172

Wegmuller, $18 ., 166$

Weikel, J., $\$ 62$

Weintraub, S., 1193 
Weiss, đ., 1408, 1546, 1547, 1652, $1671,1672,1673,1676$

Weiss, Joseph, J., 1617

Welander, A. D., 1708

Welander, Arthur D., 100, $168 \%$, 1764,1765

Welt, P., 1802, 1803

Wenger, $P .440$

Werle, E., 1170

Werner, Sidney C., 581

West, Edward S., 1480

Westman, A., 248

Wettstein, U. von, 2021

Weymouth, P. P., 133, 225

Wheeler, Brownell, 850

Wheelock, M. C., 586

Whipple, G. H., 95, 867

Whitby, Lionel, 642

White, Abraham, 662, 1474

White, Locke, Jr., 805, 806

White, Marcia, 931 , 932

Whiting, Anna R., 1766, 1969, 1970 ,

$1971,1972,1973,2144,2145$, 2146,2147

Whitmore, W. H., 1319

Whittinghill, Maurice, 1974, 1975

Wijsman, R. A., 1409, 1976

Wilbur, Karl M., 2148

Wilde, W. S., 302

Wilder, Helenor Campbell, 1139

Williams, Idamae, 1113
Williams, George Z., 511513

Wilson, C. W., 75, 293, 963, 1767

Wilson, G. B., 2086, 2149

Wilson, James G., 512, 1768, 1769, 1770

Wilson, 甚. A., 221

Wilson, Katherine, 2063

Windholz, F., 355, 356, 589

Wiseman, B. K., 878

Wish, Leon, 604, 665

Withrew, Robert B., 1454

Witkin, Evelyn M., 1977, 1978

Witten, Victor H., 964

Wolf, Bernard S., 882, 1059

Wolff, Harold G., 1246

Wolfgang, Paul, 388, 389

Wolkowitz, W., 1420

Wollman, S. H., 2052

Wood, Betty, 96

Woadard, Helen Quincey, 138, 1606

Woodburne, Arthur Re, 590

Woodruff, L., 926

Woods, M. C., 411

Woodward, G. E., 1512

Works, Lee, 1275

Wort, D. J., 1771

Wright, C. S., 878

Wright, I., 1193

Wright, Sewall, 1979

Wrobel, C. J., 888

Wunsche, $0 ., 961$
Wyss, Orville, 353,1618

Yamada, H., 1071

Yamashita, $\mathrm{H}_{0}, 2150$

Yeats, Constance A., 1541

Yoda, T., 1071

Yost, Henry T., Jr., 2136, 2151, 2152

roung, Helen N., 1918

Young, L. E., 658

Young, Nelson F., 511, 513

Yuile, C. L., 867

Zacek, Jan, 608, 609

Zagaria, Ralph, 1499

Zahl, Paul A., 222, 223, 1514

Zbarsky, S. H., 790

Zelle, M.R., 1840

Zeller, John H., 167

Zerahn, K., 1452, 1517

Zilli, E., 1180

Zimmer, E., 1939

Zimmer, E. A., 643

Zimmer, E. M., 1859

Zimmer, Karl G., 191, 1402, 1780 , 1793,1980

Zirkle, Raymond E., 69, 184, 274 , $357,358,359,360,372,396,400$, $401,402,403,405,406,407,408$, $409,606,675,1280,1410,1994,2153$ Zorn, Elinor M., 791 


\section{NUMERICAL INDEX}

This section lists the code and number and the abstract number of reports issued by the atomic energy research organizations and other research organizations of the United States and foreign countries. The identification of the various codes is given in the Glossary at the end of this section.

The avallability of the reports referred to in this compilation varies according to type or origin of the reports.

A detailed discussion of $\mathrm{AEC}$ report availability is given in TID-4550(Revision No. 1) AVAILABILITY OF USAEC RESEARCH AND DEVELOPMENT REPORTS, which is free upon request from

Office of Technical Services

Department of Commerce

Washington $25, \mathrm{D} . \mathrm{C}$.

Price lists of $A E C$ reports on sale by the Office of Technical Services are also free upon request. All AEC sale reports and many AEC reports not sold by the Office of Technical Services may be examined at or borrowed from the AEC depository libraries, and microfilm or photostat copies may be purchased from them. Copies of reports not contained in their files will be secured through the proper channels. A list of these libraries is contained in the afore-mentioned TID-4550.

The non-Project reports referred to herein are generally available from their issuing sources.

\section{GLOSSARY}

MDDC- A code assigned to declassified reports released by the Manhattan Engineer District and USAEC before March 1, 1948.

ALCD- A code assigned to declassilied reports released by the USAEC after February 29, 1948.

AECU- A cone assigned to AEC unclassified reports. AD- Naval Radiological Defense Laboratory.

AERE- Atomic Energy Research Establishment, Harwell, Berks (England).

MMRL- Army Medical Research Laboratory, Fort Knox.

ANL- Argonne National Laboratory.

BNL- Brookhaven National Laboratory.

CH- Metallurgical Laboratory, University of Chicago.

HW - Hanford Engineer Works.

LA- Los Alamos Selentific Laboratory.

M- TIS USAEC nLe number.

NIEA- Nuclear Energy for Propulsion of Aircrait (Fairchild Engine and Arplane Corp.)
NM- Naval Medical Research Institute, Bethesda, Maryland.

NP. A file designation assigned by the Technical Information Service, USAEC, to nonproject reports whose codes, if present, are not practical for TIS use.

NYO- USAEC, New York Operations Office

ORNL- Oak Ridge National Laboratory.

ORO- USAEC, Oak Ridge Operations Office

RAD- Rand Corporation

TID- Technical Information Service

UCLA - University of California, Los Angeles

UCRL- Radiation Laboratory, University of California, Berkeley

UR- Atomic Inergy Project, University of Rochester

USAF - United States Air Force School of Aviation Medicine.

UWFL- University of Washington Applled Fisheries Laboratory
Report No.

AD-126 (B)

AD-131 (B)

AD-199 (B)

AD-214 (B)

$\mathrm{AD}-215$ (B)

AD-233 (B)

AD-241 (B)

AD-266 (B)

AD-267 (B)

AD-273-B

AD-279 (B)

AD-298 (B)

AD-307 (B)
Reference No.

1599
1449
594
1493
1270
550
1116
1259
1260
517
551
717
601

1599

1449

1493

1270

550

1259

1260

517

551

601
Report No.

AD-318 (B)

AD-321 (B)

AD-331 (B)

AD-333 (B)

AD-334 (B)

AECD-1962

AECD-1965

AECD-1966

AECD-2006

AECD-2009-A

AECD-2009-E

AECD-2009-F

AECD-2011
Reference No.

605
1117
1328
1494
1495
987
953
1287
644
990
893
926
971

Report No.

AECD-2013

$A E C D-2014$

AECD-2020

AECD-2022

AECD-2023

AECD-2024

AECD-2024-D

AECD-2024 -

AECD-2024-H

$A E C D-2024$-I

AECD-2024-P

AECD-2036

AECD-2037
Reference No. 
Report No.

AECD-2053

AECD-2055

AECD-2085

AECD-2099-A

AECD-2108

AECD-2114

AECD-2122

AECD-2132

AECD-2133

$A \mathrm{CD}-2176$

AECD-2219

AECD-2254

AECD-2283

AFCD-2303

AECD-2316

AECD-2326

AECD-2328

AECD-2358

$A E C D-2372$

AECD - 2432

AECD -2483

AECD-2596

AECD-2621

AECD-2814

AFCD -2828

AECD-2832

AECD-2852

AECD-2857

AECD - 2859

AECD-2872

AECD - 2901

AECD-3009

AECD-3057

AECD -3176

AECD-3191

AECD-3247

AECU-51

AECU-95

AECU-108

AECU-131

AECU -160

AECU-170

AECU -188

AECU-229

AECU-231

AECVI-23:

$A E C U-243$

AECU-309

AECU-325

AECU-353

AECU-357

AECU-378

AECU-379

AECU-397

AECU-416

AECU-425

AECU -487

AECU -488

AECU-496

AECU-524

AECU-529

AECU-531

AECU-535

$A E C U-539$

AECU-599

AECU-610

AECU-681

AECU-738
Referance No.

941

1032

673

133

991

200

959

666

628

1200

170

786

997

450

1560

213

409

929

949

634

923

1742

1009

925

390

61

938

1021

181

193

928

439

1022

1016

917

1033

851

790

994

1112

2118

1665

1765

650

1570

1626

1999

1686

1211

17

317

1805

1048

624

1296

1569

1239

130

710

1238

1244

308

274

2137

1267

1341

2125

1165
Report No.

Reference No.

AECU-739

AECU-747

AECU 748

AECU-765

AECU-790

AECU-804

AECU-821

AECU-822

AECU-852

AECU-873

AECU-889

AECU-937

AECU-949

AECU-968

AECU-989

AECU-1021

AECU-1081

AECU-1091

AECU-1107

AECU-1134

AECU-1137

AECU-1162

AECU-1169

AECU-1183

AECU-120?

AECU-1239

AECU-1241

AECU-1253

AECU-1258

AECU-1262

AECU-1368

AECU-1436

AECU - 1438

AECU-1442

AECU-1461

AECU-1463

AECU-1470

AECU-1485

AECU-1488

AECU-1508

AECU-1513

AECU-1532

AECU-1535

AECU-1543

AECU-1551

AECU-1599

AECU-1628

$A E C U-1645$

AECU-1689

$A E C U-1690$

AECU-1699

AECU -1703

AECU-1705

AECU-1713

A ECU -1716

AECU-1725

AECU-1730

$\triangle A E C U-1743$

AECU -1769

AECU -1794

AECU-1801

AECU-1811

AECU-1816

$A E C U-1819$

AECU-1820

AECU-1821

ARCU-1866

AECU-1876

1295

1164

328

262

1419

318

1940

$\begin{aligned} 1121 & \text { AERE-T } / M-13 \\ 460 & \text { AERE }-T / R-273 \\ 1160 & \text { AERE-T } / R-416 \\ 1934 & \text { AERE-T } / R-443 \\ 1549 & \text { AM-2837 }\end{aligned}$

AMRL-64

AMRL-66

AMRL-71

ANL-4001

ANL-4017

ANL-4023

ANL-4078

ANL-4100

ANL-4125

ANL-4163

ANL-4205

ANL-4206

ANL-4227

ANL-4253

ANL-4291

ANL-4333

ANL-4360

ANL-4401

ANL-4451

ANL-4474

AN1,-4488

ANL-453

ANL-4601

ANL-4625

ANL-4666

ANL-4676

ANL- 1713

ANL-4799

ANL-HDY-A66

ANL-HDY-496

ANL-HDY-592

BNL,-132

BNL-1069

BNL-1111

BNL-C-A

Reference No.

$$
\begin{gathered}
194 \\
218 \\
219 \\
190 \\
1079,1080, \\
1081,1082, \\
1083 \\
1225 \\
1095 \\
597 \\
691 \\
773 \\
1900 \\
902 \\
692 \\
1304 \\
87,1454,1640 \\
1417 \\
930 \\
182,420,656, \\
726,884, \\
1237 \\
135,475,780, \\
794,1415 \\
124,1036, \\
1711 \\
121,936 \\
1520
\end{gathered}
$$
520

$92,236,237$, 919,937 , 1265,1271 . 1279,1565 , 1605,1609 , 1621,1712

$120,129,195$, 


\begin{tabular}{|c|c|c|c|c|c|}
\hline Report No. & Reference No. & Report No. & Reference No. & Report No. & Reference No \\
\hline CH-775 & 825 & $\mathrm{CH}-3903$ & 372 & MDDC-1143-D & 1040 \\
\hline CH -821 & 221 & CH -3904 & 408 & MDDC-1143-E & 819 \\
\hline $\mathrm{CH}-848$ & 820 & CH-3907 & 115 & MDDC-1158 & 100 \\
\hline $\mathrm{CH}-946$ & 401 & HW-20092 & 1014 & $\mathrm{MDDC}-1174$ & 675 \\
\hline $\mathrm{CH}-1049$ & 833 & HW-20102 & 760 & MDDC-1197 & 227 \\
\hline $\mathrm{CH}-1572$ & 821 & HW-20941 & 924 & MDDC-1207 & 783 \\
\hline CH-1659 & 822 & HW-21494 & 1015 & MDDC- 1210 & 116 \\
\hline $\mathrm{CH}-2258$ & 995 & LA-1075 & 1100 & MDDC-1217 & 288 \\
\hline $\mathrm{CH}-2571$ & 409 & LAMS-816 (Rev.) & 625 & MDDC-1221 & 1499 \\
\hline $\mathrm{CH}-2576$ & 79 & M-2009 & 1171 & MDDC-1240 & 1030 \\
\hline $\mathrm{CH}-2673$ & 823 & MDDC-203 & 361 & MDDC-1241 & 1420 \\
\hline CKI-2844 & 832 & MDDC-204 & 226 & MDDC-1247 & 1819 \\
\hline CH-3329 & 1351 & MDDC-211 & 1442 & MDDC-1252 & 114 \\
\hline CH-3368 & 286 & MDDC -215 & 268 & MDDC-1253 & 374 \\
\hline CH-3485 & 817 & $M D D C-216$ & 269 & MDDC-1254 & 375 \\
\hline CH-3539 & 809 & MDDC -248 & 817 & MDDC-1269 & 287 \\
\hline CH -3541 & 1510 & MDDC-250 & 98 & MDDC-1270 & 286 \\
\hline CH -3590 & 836 & $\mathrm{MDDC}-254$ & 228 & MDDC-1271 & 241 \\
\hline CII-3591 & 923 & $\mathrm{MDDC}-329$ & 809 & MDDC-1272 & 1129 \\
\hline $\mathrm{CH}-3629$ & 748 & MDDC -418 & 360 & MDDC-1275 & 835 \\
\hline CH-3653 & 231 & MDDC -419 & 982 & MDDC -1276 & 836 \\
\hline $\mathrm{CH}-3654$ & 1560 & MDDC -444 & 401 & MDDC-1277 & 1351 \\
\hline CH-3655 & 916 & MDDC -490 & 824 & MDDC-1278 & 212 \\
\hline CH-3658 & 241 & $M D D C-496$ & 774 & MDDC-1279 & 785 \\
\hline $\mathrm{CH}-3661$ & 1509 & MDDC-516 & 798 & MDDC-1301 & 1068 \\
\hline $\mathrm{CH}-3698$ & 232 & MDDC-534 & 646 & MDDC-1319 & 691 \\
\hline CH-3719 & 1499 & MDDC-569 & 438 & MDDC-1326 & 807 \\
\hline CH-3725 & 1420 & MDDC-584 & 396 & MDDC-1337 & 391 \\
\hline $\mathrm{CH}-3727$ & 1128 & MDDC-642 & 229 & MDDC-1342 & 638 \\
\hline CH-3729 & 1819 & MDDC -648 & 761 & MDDC-1343 & 784 \\
\hline $\mathrm{CH}-3730$ & 212 & MDDC-653 & 235 & MDDC-1363 & 975 \\
\hline CH-3738 & 1129 & MDDC-654 & 231 & MDDC-1364 & 976 \\
\hline CH -3753 & 288 & MDDC -655 & 232 & MDDC-1372 & 377 \\
\hline CH-3754 & 114 & MDDC-677 & 916 & MDDC-1387 & 992 \\
\hline CH-3766 & 213 & MDDC-696 & 217 & MDDC-1388 & 993 \\
\hline CH-3783 & 922 & MDDC-700 & 1510 & $M D D C-1418-B$ & 1316 \\
\hline CH-3797 & 992 & MDDC-701 & 1508 & $M D D C-1418-C$ & 1037 \\
\hline $\mathrm{CH}-3798$ & 675 & MDDC-719 & 927 & $M D D C-1418-D$ & 1432 \\
\hline CH-3806 & 1564 & MDDC-754 & 199 & MDDC-1418-F & 978 \\
\hline CH -3815 & 116 & MDDC-761 & 1128 & $M D D C-1480$ & 773 \\
\hline CH-3824 & 807 & MDDC-795 & 748 & MDDC-1647 & 1462 \\
\hline CH-3825 & 783 & MDDC-824 & 694 & MDDC-1653 & 918 \\
\hline $\mathrm{CH}-3826$ & 785 & MDDC -836 & 712 & MDDC -1658 & 1171 \\
\hline CH-3832 & 784 & MDDC-881 & 870 & $M-1695$ & 959 \\
\hline CH-3833 & 287 & MDDC-907 & 79 & NEPA-1127 & 1144 \\
\hline CH -3835 & 227 & MDDC-912 & 627 & NM-006-012.04.34 & 419 \\
\hline CH-3838 & 1030 & MDDC-954 & 832 & $N M-006-012.04 .36$ & 724 \\
\hline CH-3839 & 377 & MDDC-991 & 613 & NM-006-012.04.37 & 444 \\
\hline CH-3843 & 993 & MDDC-999 & 113 & NM-006-012.04.38 & 465 \\
\hline CH-3845 & 976 & MDDC- 1000 & 820 & NM-006-012.04.43 & 106 \\
\hline CHI-3846 & 975 & MDDC-1001 & 822 & NM-006-012.08.26 & 657 \\
\hline CH-3849 & 918 & MDDC-1002 & 825 & NM-007-039 & $9,657,410$ \\
\hline CH-3851 & 1462 & MDDC-1005 & 995 & NM-008-009 & 843 \\
\hline CH-3852 & 953 & MDDC-1006 & 1725 & (Report 1) & \\
\hline CH-3855 & 1498 & MDDC-1011 & 833 & $\mathrm{NP}-302$ & 1077 \\
\hline $\mathrm{CH}-3856$ & 971 & MDDC-1018 & 933 & NP-303 & 1086 \\
\hline CH-3857 & 972 & MDDC-1022 & 935 & $N P-305$ & 623 \\
\hline CH-3859 & 1034 & MDDC-1025 & 221 & $(\mathrm{NP}-305(J-6))$ & 1070 \\
\hline CH-3864 & 673 & MDDC-1061 & 821 & NP-306 (J-7) & 1071 \\
\hline CKI-3866 & 991 & MDDC-1062 & 823 & NP-313 & 1088 \\
\hline CH-3868 & 634 & MDDC-1131 & 1564 & NP-332 & 1733 \\
\hline $\mathrm{CH}-3870$ & 666 & MDDC- 1140 & 922 & $\operatorname{NP}=516$ & 1537 \\
\hline $\mathrm{CH}-3873$ & 450 & MDDC-1142-A & 868 & NP-573 & 9 \\
\hline CH-3884 & 949 & MDDC-1142-B & 808 & NP-574 & 1104 \\
\hline $\mathrm{CH}-3888$ & 994 & MDDC-1142-D & 979 & NP-589 & 102 \\
\hline CH -3889 & 1112 & MDDC-1142-E & 985 & NP-1156 & 4 \\
\hline CH -3890 & 650 & $M D D C-1143-B$ & 980 & NP-1202 & 1060 \\
\hline CH -3900 & 80 & $M D D C-1143-C$ & 834 & $\mathrm{NP}-1411$ & 410,657 \\
\hline
\end{tabular}




\begin{tabular}{|c|c|}
\hline Report No. & Reference No. \\
\hline NP- 1419 & 1584 \\
\hline$N P^{-1762}$ & 591 \\
\hline NP-1826 & 186 \\
\hline$N P-1833$ & 1250 \\
\hline$N P-1876$ & 1252 \\
\hline$N P-1887$ & 167 \\
\hline$N P-1900$ & 1942 \\
\hline NP- 1902 & 1107 \\
\hline NP-1903 & 1791 \\
\hline NP-1904 & 1964 \\
\hline NP-3036 & 1079 \\
\hline NP-3037 & 1080 \\
\hline NP-3038 & 1081 \\
\hline NP-3039 & 1082 \\
\hline$N P-3040$ & 1083 \\
\hline NP-3072 & 2076 \\
\hline NP-3162 & 419 \\
\hline NP-3564 & 392 \\
\hline$N P-3600$ & 465 \\
\hline$N P-3610$ & 47 \\
\hline NP-3642 & 1441 \\
\hline NP-3667 & 444 \\
\hline NP-3796 & 10 \\
\hline NP-3798 & 1681 \\
\hline NYO-1609 & 881 \\
\hline NYO-1614 & 1162 \\
\hline NYO-1624 & 777 \\
\hline NYO-1626 & 1464 \\
\hline NYO-1627 & 1528 \\
\hline NYO-1637 & 1343 \\
\hline NYO-1639 & 847 \\
\hline ORNL-101 & 2007 \\
\hline ORNL-175 & 1863 \\
\hline ORNL-348 & 1999 \\
\hline \multicolumn{2}{|l|}{ ORNL -386} \\
\hline ORNL-391 & 544 \\
\hline ORNL-409 & 2011 \\
\hline ORNL -464 & 1118 \\
\hline ORNL -570 & 1998 \\
\hline
\end{tabular}

Report No.

ORNL-595

ORNL-656

ORNL-687

ORNL-688

ORNL-696

ORNL-734

ORNL-811

ORNL-832

ORNL-878

ORNL -884

ORNL-1193

ORO-13

ORO-54

RAD-202

Report No. 1

TID-77

TID-364

UCLA-11

UCLA-22

UCLA-36

UCLA-52

UCLA-61

UCLA-69

UCLA-81

UCLA-90

UCLA-93

UCLA-112

UCLA-123

UCLA-124

UCLA-125

UCLA-137

UCLA-149

UCLA-152

UCLA-154

UCLA-156

UCLA-181

UCLA-171

UCRL-60
Reference No.

1745

1234

604

2044

1920

607

2036

327

665

323

1327

630

747

16

1367

505

743

1665

17

1569

1435

1227

320

1228

1473

1619

1229

697

698

682

1425

645

250

1145

1146

1113

1486

987
Report No.

UCRL-92

UCRL -148

UCRL-332

UCRL-812

UCRL-882

UCRL-1101

UCRL-1140

UCRL-1464

UCRL-1549

UR-11

UR-13

UR -46

UR-53

UR-83

UR -88

UR-94

UR-113

UR -117

UR-121

UR-122

UR-123

UR-137

UR-140

UR-142

UR-146

UR-150

UR-152

UR-159

UR-161

UR -166

UR-169

UR-183

UR-186

UR-189

UWFL-17

UWFL-21
Reference No.

1032

786

828

831

1096

366

788

1453

1287

997

515

790

938

239

1159

71

1021

669

870

448

621

1094

447,1003

1154

467

619,1103 , 1155,1156 , 1505

684

81

1023

1411

512

1353

735,1323

1765

1686 


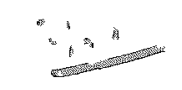

\section{SOURCE OF ABSTRACTS INDEX}

E.G. Edith Goldarb

M. F. Margaret Fieldhouse

R.D.B. Robert D. Boche

S.V.S. Sophie V. Stephens

V.E. Valesta Evertsbusch

ACD Abstracts of Classified Documents (Technical Information Service, USAEC)

ADD Abstracts of Declassified Documents (Technical Information Service, USAEC)

AJR American Journal of Roentgenology and Radium Therapy

AP Archives of Pathology

AR Anatomical Record

AWM Abstracts of World Medic me

BA Biological Abstracts

BCPA British Chemical and Physiological Abstracts

BEMP The British Encyclopedia of Medical Practice, Surveys and Abstracts

BJR British Journal of Radiology

CA Chemical Abstracts

CR Cancer Research

LiM Lexcerpta Medica

G Genetics

GPR Guide to Published Research in Atomic Energy (Technical Information Service, USAEC)

N Nucleonics

NSA Nuclear Science Abstracts

PA Physics Abstracts

R Radiology

RG Records of the Genetic Societv of America

TIS Technical Information Service, USAEC, Oaf Ridge Extension

YBR Year Book of Radiology 